
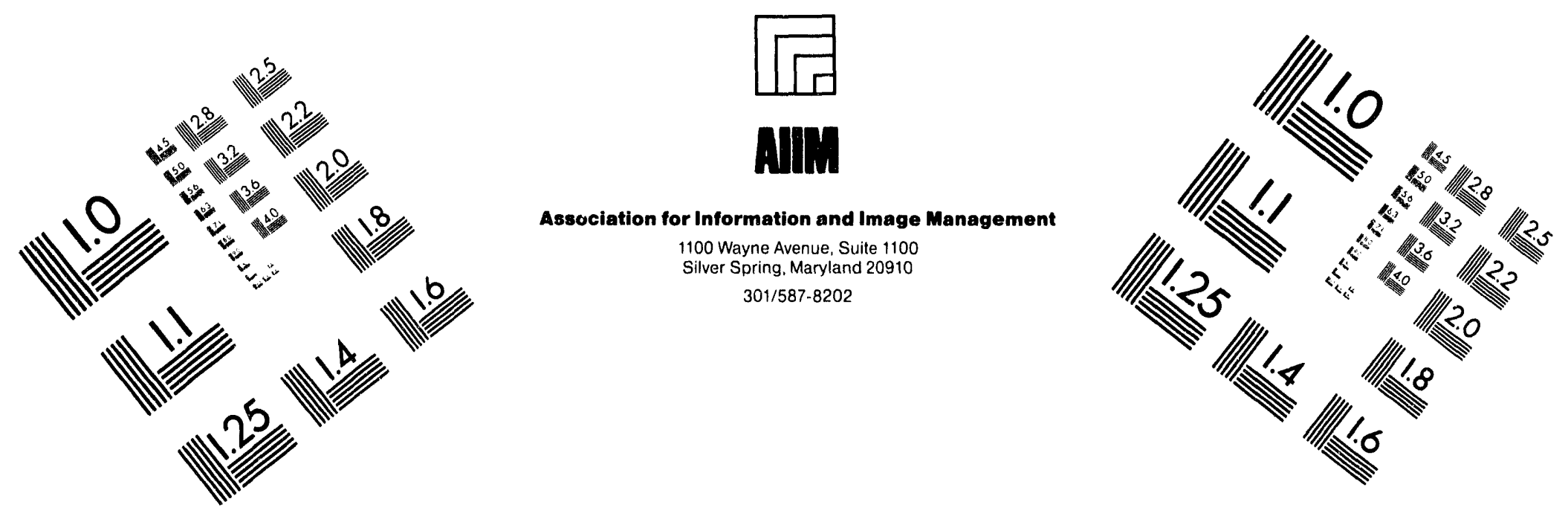

\title{
Centimeter
}

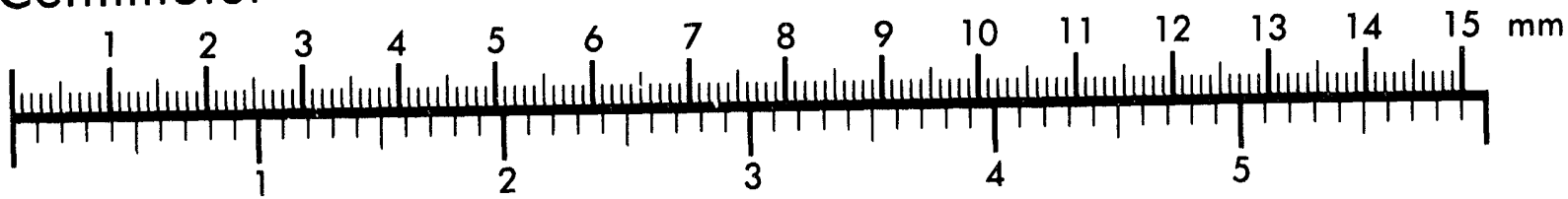
Inches
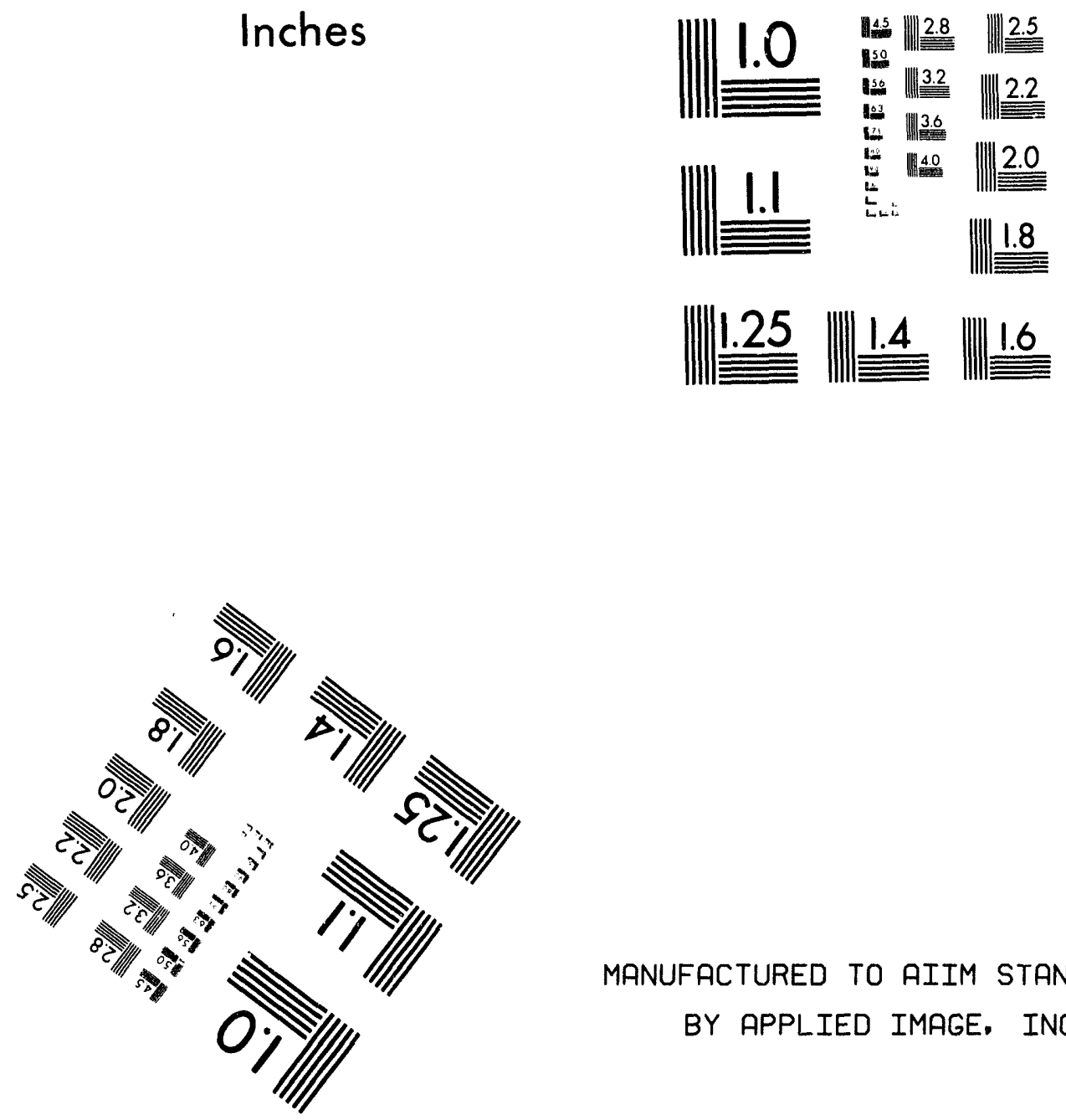

MANUFACTURED TO AIIM STANDARDS

BY APPLIED IMAGE. INC.

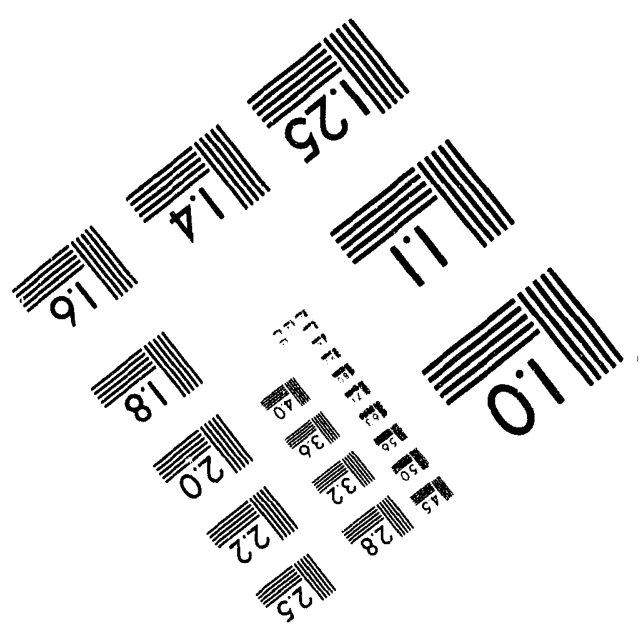



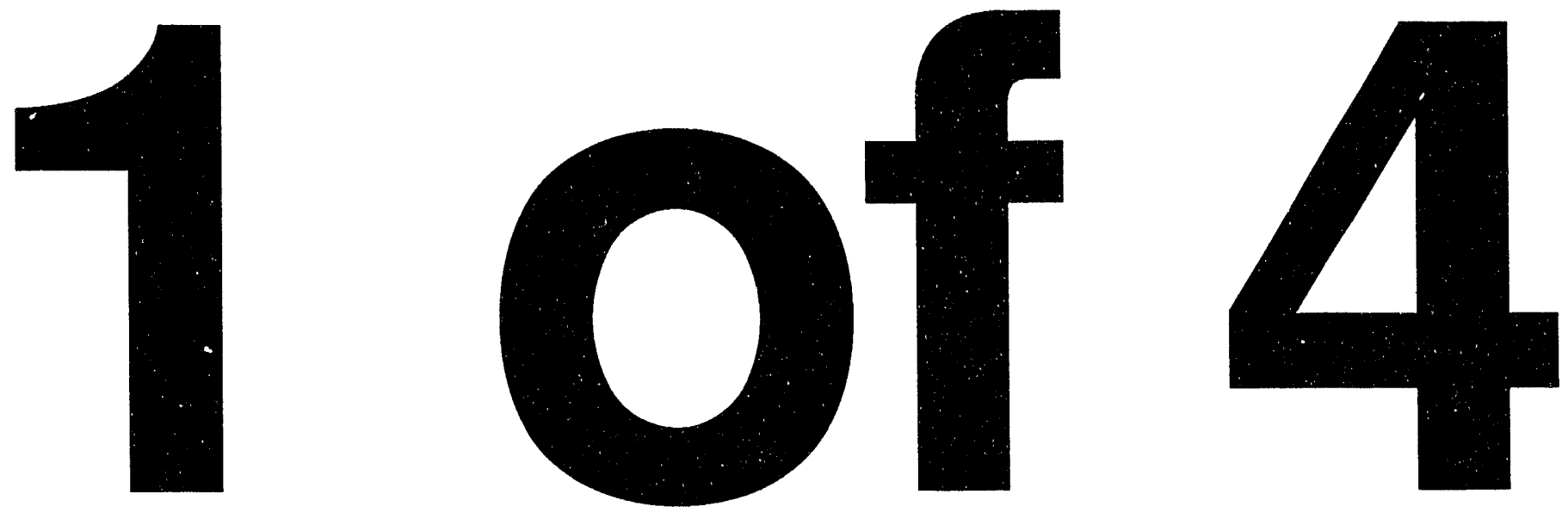


\section{SAMPLING AND ANALYSIS OF WATER FROM UPPER THREE RUNS AND ITS WETLANDS NEAR MIXED WASTE MANAGEMENT FACILITY}

by

Dixon

Westinghouse Savannah River Company

Savannah River Site

Aiken, South Carolina 29808

Cummins

This paper was prepared in connection with work done under the above contract number with the U.S.

Department of Energy. By acceptance of this paper, the publisher and/or recipient acknowledges the U.S. Government's right to retain a nonexclusive, royalty-free license in and to any copyright covering this paper, along with the right to reproduce and to authorize others to reproduce all or part of the copyrighted paper. 


\section{DISCLAIMER}

This report was prepared as an account of work sponsored by an agency of the United States Government. Neither the United States Government nor any agency thereof, nor any of their employees, makes any warranty, express or implied, or assumes any legal liability or responsibility for the accuracy, completeness, or usefulness of any information, apparatus, product, or process disclosed, or represents that its use would not infringe privately owned rights. Reference herein to any specific commercial product, process, or service by trade name, trademark, manufacturer, or otherwise does not necessarily constitute or imply its endorsement, recommendation, or favoring by the United States Government or any agency thereof. The views and opinions of authors expressed herein do not necessarily state or reflect those of the United States Government or any agency thereof.

This report has been reproduced directly from the best available copy.

Available to DOE and DOE contractors from the Office of Scientific and Technical Information, P.O. Box 62, Oak Ridge, TN 37831; prices available from (615) 576-8401.

Available to the public from the National Technical Information Service, U.S. Department of Commerce, 5285 Port Royal Road, Springfield, VA 22161. 


\section{CONTENTS}

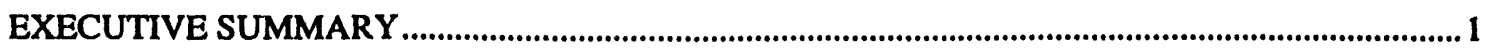

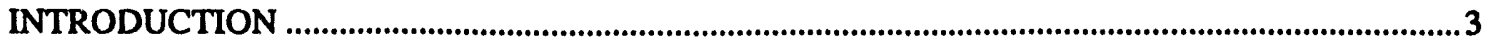

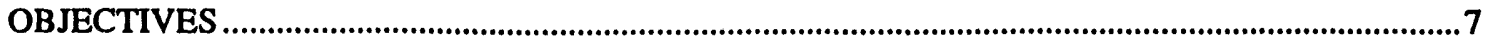

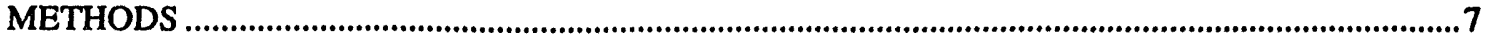

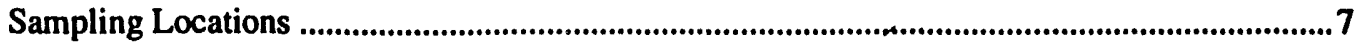

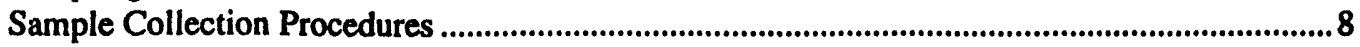

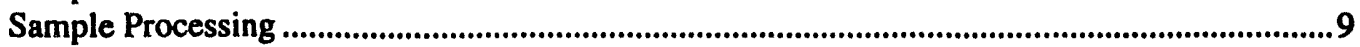

Quality Assurance/Quality Control (QA/QC) .......................................................................9

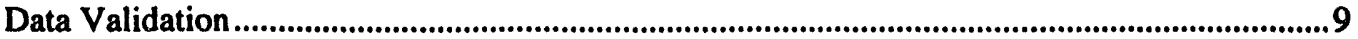

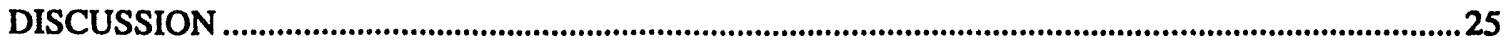

Field Measurements ..........................................................................................................225

Comparison of Wetland and Stream Sampling Locations to Established Standards.....................25

Tank 16 Impacts on UTR Wetland Water Quality...................................................................227

SUMMARY.

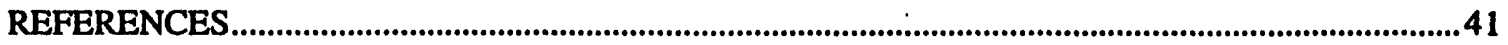

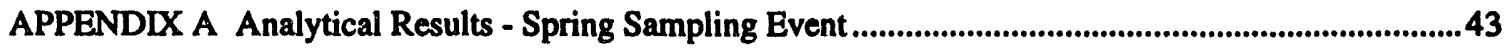

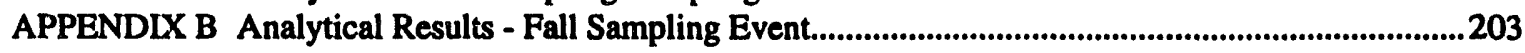

APPENDIX C Shipping Trip Blanks ................................................................................................3 363

APPENDIX D Information Provided to ESS on Electronic Data Diskettes ..........................................367 


\section{LIST OF FIGURES}

Figure 1. General Location Map for the Upper Three Runs Sampling Project . .5

Figure 2. Upper Three Runs Wetland Sampling Stations Map Pocket

\section{LIST OF TABLES}

Table 1. PDWS, MCL, and SDWS Standards Used in Data Comparisuns .11

Table 2. Methods and Method Detection Limits for Weston Analytics (WA) and TMA/Eberline (TM). . .14

Table 3. Methods and Method Detection Limits for General Engineering Laboratories (GE) and Environmental Physics (EP) .......................................................................................... 17

Table 4. Wetland and Stream Sampling Locations for the Spring Sampling Event..............................20

Table 5. Wetland and Stream Sampling Locations for the Fall Sampling Event ....................................21

Table 6. Dry Sampling Locations for the Fall Sampling Event ............................................................222

Table 7. QA/QC Blanks, Trip Blanks, Blind Replicates, and Split Replicates for the Spring

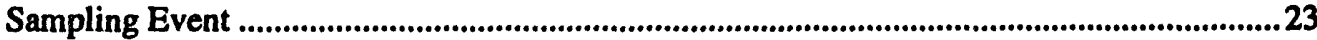

Table 8. QAVC Blanks, Trip Blanks, Blind Replicates, and Split Replicates for the Fall Sampling Event................................................................................................................................ 24

Table 9. Minimum, maximum, and average values for selected field measurements made during the spring sampling event ..........................................................................................................29

Table 10. Minimum, maximum, and average values for selected field measurements made during the fall sampling event..............................................................................................................30

Table 11. Comparison of Analytes Detected at Wetland and Background Locations During Spring Sampling Event

Table 12. Comparison of Analytes Detected at Stream and Background Locations During Spring

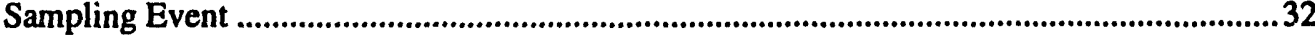

Table 13. Analytes Detected above the SDWS during the Spring Sampling Event...............................33

Table 14. Analytes Detected above the PDWS or MCL during the Spring Sampling Event................... 34

Table 15. Comparison of Analytes Detected at Wetland and Background Locations During Fall Sampling Event

Table 16. Comparison of analytes detected at Stream and Background Locations During Fall Sampling Event 36

Table 17. Analytes Detected above the SDWS during the Fall Sampling Event ...................................3

Table 18. Analytes Detected above the PDWS or MCL during the Fall Sampling Event ........................38 


\title{
Sampling And Analysis Of Water From Upper Three Runs And Its Wetlands Near Tank 16 and the Mixed Waste Management Facility(U)
}

\author{
K. L. Dixon and C. L. Cummins \\ Savannah River Technology Center \\ Westinghouse Savannah River Company \\ Aiken, SC 29802

\section{EXECUTIVE SUMMARY}

In April and September 1993, sampling was conducted to characterize the Upper Three Runs (UTR) wetland waters near the Mixed Waste Management Facility to determine if contaminants migrating from MWMF are outcropping into the floodplain wetlands. This study was developed to supplement an initial characterization by Haselow et al. (1992).

For the spring sampling evert, 37 wetland and five stream water samples were collected. Thirty-six wetland and six stream water samples were collected for the fall sampling event. Background seepline and stream water samples were also collected for both sampling events. All samples were analyzed for RCRA Appendix IX volatiles, inorganics appearing on the Target Analyte List, tritium, gamma-emitting radionuclides, and gross radiological activity.

Most of the analytical data for both the spring and fall sampling events were reported as below method derection limits. The primary exceptions were the routine water quality indicators (e.g., turbidity, alkalinity, total suspended solids, etc.), iron, manganese, and tritium. Iron and manganese were detected above the secondary drinking water standards (SWDS) at several wetland and stream locations (including background locations) during both the spring and fall sampling events. However, the levels reported are typical of wetland and stream water in this area (Dixon and Rogers, 1993; Haselow et al., 1990; Haselow et al., 1992). Tritium was detected during the spring and fall above the maximum contaminant level (MCL) at 13 ( 9 wetland and 4 stream) and 18 (13 wetland and 5 stream) locations, respectively.

During the spring, cadmium, gross alpha, nonvolatile beta, potassium-40, ruthenium-106, and trichloroethylene were also detected above the MCLs from at least one location. Cadmium was detected above the MCL at four wetland locations. Gross alpha was detected above the MCL at two wetland locations, one of which was a background location. Nonvolatile beta was detected above the MCL at three wetland locations, two of which were background locations. Trichloroethylene was detected at one wetland location at $5.09 \mu \mathrm{g} / \mathrm{L}$, which is slightly above the method detection limit and MCL of $5 \mu \mathrm{g} / \mathrm{L}$. None of these contaminants were detected above the MCLs during the fall.

The results of this study are comparable to the data reported by Haselow, et al. (1992) and suggest that tritium is outcropping in the Upper Three Runs wetlands.

A secondary objective of this project was to identify any UTR wetland water quality impacts resulting from leaks from Tank 16 located at the H-Area Tank Farm. In September 1960, a maximum of 16 gallons of waste (86\% Cs$134,137)$ leaked from Tank 16 into the soil. Using cesium-137 results from groundwater samples collected in the vicinity of the tank, Poe (1974) estimated in 1974 that the vaste had moved only a few feet since the leak. Due to low groundwater flow in the area $(0.01-0.02 \mathrm{ft} /$ day $)$ and the sorptive characteristics of the soil and waste, the waste would not be expected to move very far from the tank (Poe, 1974). Results of the UTR wetland sampling project did not suggest that the wetlands were being impacted by leaks from Tank 16.

The information in this report was developed during the course of work under Contract No. DE-AC09-89SR18035 with the U. S. Department of Energy. 
WSRC-TR-94-0277 
Sampling And Analysts Of Water From Upper Three Runs And its Wetlands Near Tank 16 and the Mixed Waste Management Facility

\section{INTRUDUCTION}

The radioactive waste burial grounds are central waste storage sites used for disposing of onsite and offsite radioactive and nonradiocative wastes (Jaegge et al., 1985). The burial grounds are divided into three areas: Building 643-E (Old burial ground); which received wastes from 1952 through 1972; Building 643-7E, which has been receiving wastes since 1969; and Building 643-28E which is a closure area within 643-7E that is termed the Mixed Waste Management Facility (MWMF) (Jaegge et al., 1985). Waste disposal into the MWMF portion of 6437E was discontinued in March, 1986. MWMF received mixed wastes including scintillation fluids, waste lubricating oils held in absorbent material contained in drums, cadmium, lead, and silver (Jaegge, et al., 1985). The area has been capped and closed with closure being completed in December, 1990 and accepted by the South Carolina Department of Health and Environmental Control (SCDHEC) in April, 1991 (SAIC, 1992).

In the fall of 1991 and the spring of 1992, the Environmental Restoration Department (ERD) and the Savannah River Technology Center (SRTC) conducted a study to characterize the groundwater outcropping into UTR and its wetland system (Haselow et al., 1992). As part of this project, soil and water samples were collected from numerous locations in the UTR wetlands bordering MWMF and other facilities in the area. These samples were analyzed for selected radionuclides, metals, inorganic compounds, and volatile compounds. The results from this study indicated that the primary constituents in the shallow groundwater and soils were tritium, gross alpha, radium-226, total radium, and strontium-90. This project was developed to supplement the previous study by further characterizing the UTR wetland waters near MWMF to determine if contaminants migrating from MWMF are outcropping into the floodplain wetlands.

The sampling zone for this project lies within the wetlands of Upper Three Runs (UTR) which lies on the northern side of MWMF (Figure 1). MWMF is located on a plateau in an interstream area bounded by UTR to the north and Fourmile Branch (FMB) to the south. The hydrogeologic setting of the General Separations Area (GSA; which includes both MWMF and Tank 16) has been described in detail in several regulatory documents (WSRC, 1990; WSRC, 1991a; WSRC, 1991b; WSRC, 1991c). A brief description of the groundwater system relative to MWMF and Tank 16 is presented here; however, for specific details, the aforementioned references should be consulted.

In the vicinity of MWMF and Tank 16, a groundwater divide occurs in the water table aquifer (Aquifer IIB 2 ) and trends in an east-west direction passing beneath MWMF. Lateral groundwater flow to the south of this divide is towards Fourmile Branch (FMB) and to the north flow is towards UTR. The Tan Clay confining unit separates the water table aquifer from the Barnwell/McBean aquifer (Aquifer IIB 1 ). A groundwater divide also occurs in the Barnwell/McBtan aquifer and trends in an east-west direction passing slightly to the south of MWMF. Lateral groundwater flows are similar to those in the water table aquifer. Head differences across the Tan Clay confining unit indicate that there exists a potential for downward migration from the water table aquifer to the Barnwell/McBean aquifer (WSRC, 1990).

The Green Clay confining unit separates the Barnwell/McBean aquifer from the Congaree aquifer (Aquifer IIA). Due to discontinuities in the Green Clay confining unit, the Congaree aquifer has been described as "semi-confined and hydraulically connected to the overlying Barnwell/McBean aquifer" (WSRC, 1990). Therefore, potential exists for downward migration from the Barnwell/McBean aquifer to the Congaree aquifer. Lateral groundwater flow in the Congaree aguifer is in a northwesterly direction and towards UTR.

The Congaree aquifer is separated from the Ellenton aquifer (Aquifer IB) by the Ellenton Clay confining unit. The potential for downward migration across the Ellenton Clay confining unit has been shown to be small (WSRC, 1990) in the GSA. Thus, the Ellenton Clay confining unit is considered to be an effective hydraulic barrier preventing contaminant migration from the Congaree aquifer to the Ellenton aquifer. Any contaminants that have entered the water table aquifer and Barnwell/McBean aquifer from MWMF or Tank 16 would travel laterally towards either FMB or UTR depending on which side of the groundwater divide the contaminants entered the water table.

Contaminants migrating across the Green Clay confining unit into the Congaree aquifer would be transported towards UTR. Model simulations have shown that the most likely contaminant transport path from Tank 16 is vertically through the water table aquifer and the Barnwell/McBean aquifer and then laterally through the Congaree aquifer towards UTR (WSRC, 1990). Flow times to UTR from Tank 16 have been estimated to range in the hundreds of years (WSRC, 1990; Poe, 1974). 
A secondary objective of this project was to identify any UTR wetland water quality impacts resulting from leaks from Tank 16 (241-916H) located at the H-Area Tank Farm (Figure 1). The H-Area Tank Farm is located eastsoutheast of MWMF on the same plateau between UTR and FMB. This facility consists of several steel tanks designed to store liquid radioactive wastes. Tank 16, which has a storage capacity of 1,030,000 gallons of liquid waste, is constructed of steel and is situated in a secondary cuntainment pan also constructed of steel. The tank and containment pan are housed in a concrete encasement to further reduce the potential for release of radioactive wastes to the environment (Poe, 1974). In September 1960, a maximum of 700 gallons of high level waste whiçh had leaked from Tank 16 (via small cracks in the steel tank) rose above the top of the steel secondary containment pan and spilled into the concrete encasement. Before the waste could be transferred to another tank, a small volume leaked through the concrete encasement and into the water table zone (Poe, 1974). The precise amount of waste that leaked into the soil was not measured directly, but Poe (1974) estimated the amount to be a maximum of about 16 gallons containing approximately $7.4 \mathrm{Ci}$ of radionuclides $(86 \% \mathrm{Cs}-134,137)$ per gallon.

For this project, wetland and stream water samples were collected and analyzed for RCRA Appendix IX volatiles, inorganics appearing on the Target Analyte List, tritium, gamma-emitting radionuclides, and gross radiological activity. Analytical data from this project should allow for an assessment of UTR wetland water quality impacts resulting from MWMF operations and Tank 16 leaks. 


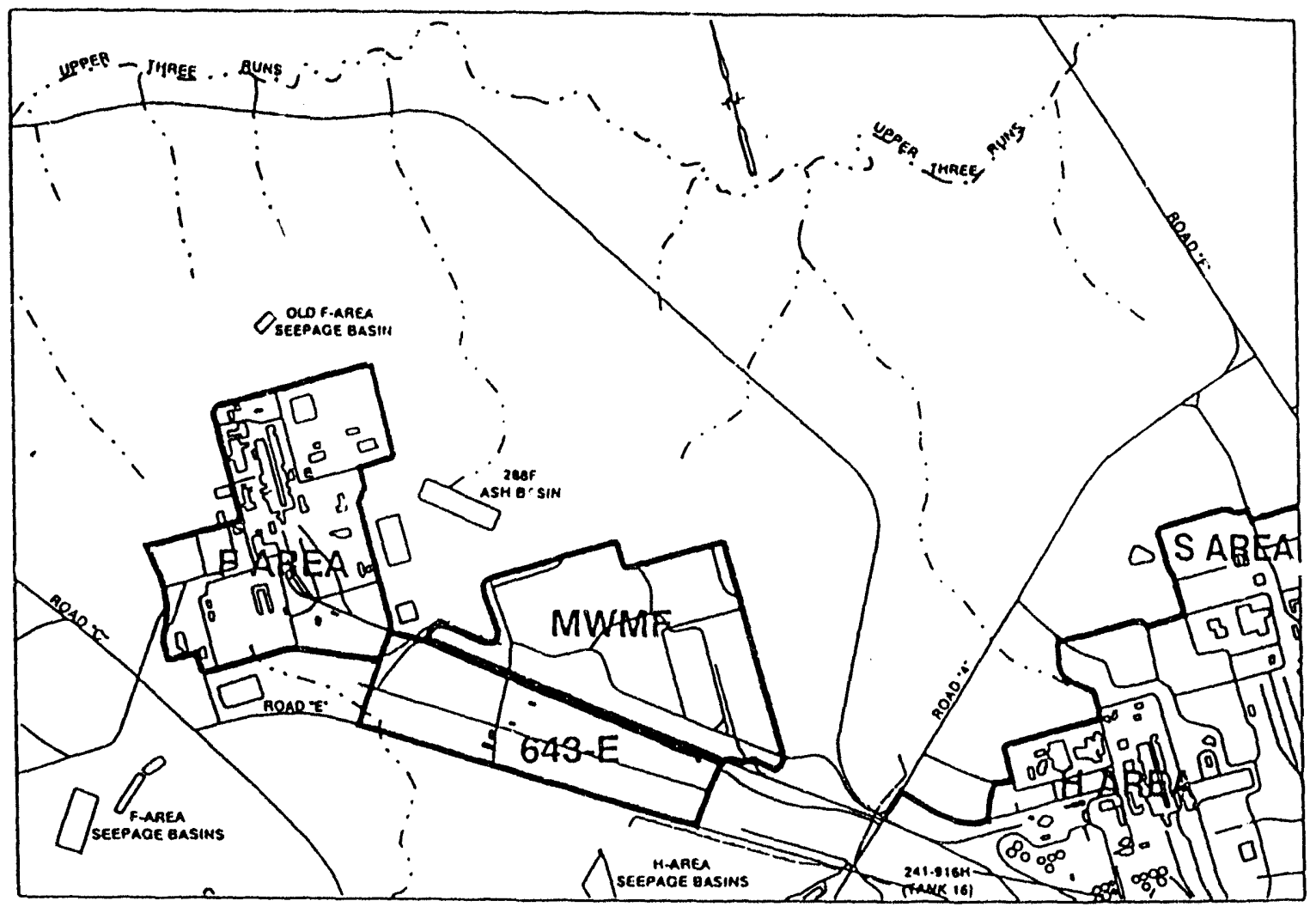

Figure 1. General Location Map for the Upper Three Runs Wetlands Sampling Project. 


\section{OBJECTIVES}

The objectives of this report are to:

1) summarize the results from the spring and fall sampling event of UTR and its wetlands

2) compare, where possible, these results to related standards (Table 1)

3) distinguish, if possible, UTR wetland impacts due to Tank 16 contaminant releases from any impacts resulting from MWMF operations.

\section{METHODS}

Metcalf \& Eddy, Inc. (M\&E) provided wetland sampling services for WSRC via task order under contract AA46327P (Task 39). M\&E subcontracted the field sampling to Normandeau Associates, Inc. (NAI). Samples were collected during the spring and fall to coincide with seasonal precipitation extremes. The spring and fall sampling events occurred April 13 - April 27 and August 23- September 02, 1993. These sampling activities are described in detail in Metcalf and Eddy (1993a and 1993b) and are reproduced, in pa:t, here for this discussion.

Analytical services fer the reference samples were obtained via task order under contract AA01244N with Weston Analytics (WA). Weston Analytics subcontracted radiological analyses to TMA/Eberline, Inc. Samples analyzed by Weston Analytics and/or their subcontractors were considered to be the reference samples for this project. WSRC Environmental Monitoring Section (EMS) arranged for General Engineering Laboratories, Inc. (GE; nonradiological) and Environmental Physics (EP; radiological) to serve as QA laboratories for split sample analyses. Tables 2 and 3 list the constituents analyzed, the laboratory performing the analyses, the methods used, and method detection limits for each analyte. Results from the spring and fall sampling events are presented in Appendices A and $B$, respectively.

The laboratories provided all sample containers with preservatives, blue ice, and coolers necessary to collect and ship the samples. Chain-of-Custody forms and packing materials were provided by SRTC Environmental Sciences Section.

\section{Sampling Locations}

Seven stream and 41 wetland locations were identified for the spring sampling event and eight stream and 49 wetland locations were identified for the fall sampling event. Tables 4 and 5 identify the spring and fall sampling locations and the corresponding SRS coordinates. Figure 2 shows the wetland and stream sampling locations. Each stream and wetland sampling location was marked with a PVC stake. Identification numbers were written on each stake to serve as the sample location $\mathrm{ID}$.

Both sampling events were scheduled during periods of hydrologic stability in order to relate analytical results and water quality measurements with stable conditions. No samples were collected within three days of 0.5 inches of rainfail.

A two foot deep hole was excavated within a three foot radius of the PVC stake using a decontaminated stainless steel shovel. A five-gallon plastic bucket screened on the sides and bottom was placed in the hole and covered with a lid. At locations where replicates and splits were to be collected, additional buckets were installed to provide additional sample volume.

For the spring sampling event, the buckets were installed 24 hours prior to the sample collection in order to allow suspended matter resulting from site preparation to settle. The time between installing the buckets and sample collection was recorded in the field notebook. The $\mathrm{pH}$, conductivity, reduction/oxidation potential, and dissolved oxygen concentration of the water in the bucket were measured and recorded at the time the bucket was set.

For the fall sampling event, the buckets (from the spring sampling) were bailed one day before sample collection using a one gallon plastic bucket. New sampling locations were prepared in the same manner as during the spring 
event. The $\mathrm{pH}$, conductivity, reduction/oxidation potential, and dissolved oxygen concentration of the water in the bucket were measured and recorded immediately after the bucket was bailed.

If by the next day water had not seeped into the bucket, the sampling location was identified as a "dry hole" and no sample was collected at that location. There were no dry holes during the spring sampling event. The fall sampling event was conducted during a period of seasonally low rainfall. As a result, groundwater levels were depressed and several dry holes were identified. In several cases, alternative sampling locations were identified and sampled. Table 6 lists the dry holes encountered during the fall sampling event.

\section{Sample Collection Procedures}

Wetland and stream water samples (bucket samples) were collected according to procedure ESSOP 2-104 (WSRC-L-14.1, 1993a). A brief description of the sampling procedure is given here; however, for a detailed discussion of the sampling procedure, ESSOP 2-104 should be reviewed.

\section{Wetland Water}

Vials for volatiles organic analyses (VOA) were filled first by dipping them into the bucket, taking care not to disturb the sediment in the bottom of the bucket. The VOA vial was filled completely such that no headspace remained. The other sample aliquots for volatile constituents (i.e. total organic carbon, sulfide, etc.) were pumped from the bucket using a peristaltic pump equipped with Tygon tubing. Before collecting samples, the pump was allowed to discharge for 20 seconds in order to flush any residue in the pump tubing. The remaining samples were also collected by pumping the water from the bucket using the peristaltic pump; however, a $10 \mu \mathrm{m}$ capsule filter was installed at the end of the tubing to remove particulates. New tubing and a new filter were used at each sampling location.

The temperature, $\mathrm{pH}$, conductivity, reduction/oxidation potential, and dissolved oxygen concentration of the water was measured in situ at the time of sampling and the resulting data were recorded on the field data sheets and in the field logbook. The probes were rinsed with deionized water before and after taking the measurements at each sampling location.

\section{Stream Water}

Each stream location was approached and sampled from the downstream direction to minimize turbidity from the field crew's activities. To minimize further cross-contamination from resuspension of contaminated sediments into the water column and downstream transport of such sediments, downstream sampling locations were sampled first.

Collection of stream water samples was performed by dipping the sample containers directly into the stream. These grab samples were collected from the middle of the stream at the top of the water column. At each location, the containers were placed into the water with the mouth facing upstream so that water flowed directly into the container. Care was taken when opening and filling the containers to prevent the loss of preservatives.

With the exception of the metals samples, stream samples were not filtered. For the metals samples, stream water was collected in an unpreserved sample container and filtered using the peristaltic pump and a $10 \mu \mathrm{m}$ filter capsule.

The temperature, $\mathrm{pH}$, conductivity, reduction/oxidation potential, and dissolved oxygen concentration of the water was measured in situ at the time of sampling and the resulting data were recorded on the field data sheets and in the field logbook. The probes were rinsed with deionized water before and after taking the measurements at each sampling location.

Stream velocity measurements were collected at each stream location by immersing a Marsh-McBirney current meter into the stream. The measurements were taken at approximate 0.1 to 0.2 meter intervals across the channel, or by dividing the channel into a minimum of 4 sections. When the water depth was greater than 0.5 meters, the velocity measurements were taken at $20 \%$ and $80 \%$ of the depth, as measured from the water surface. When the water depth was less than 0.5 meters, the velocity measurements were taken at $60 \%$ of the water depth, as measured from the 
Sampling And Analysis Of Water From Upper Three Runs And lis Wetlands Near Tank 16 and the Mixed Waste Management Facility

water surface. Stream discharge at each location was calculated by summing across the channel the products of the section areas and their respective velocities (WSRC, 1993b).

\section{Sample Processing}

Once all sample containers had been filled for a sampling location, they were stored in a cooler containing blue ice for transport to a staging area. At the staging area, the samples were placed in a refrigerator and stored at $4^{\circ} \mathrm{C}$ until they were packaged for shipping. All samples were packaged and shipped according to the procedures "Packaging Non-Radioactive Water Samples for Offsite Shipment" (WSRC 3Q5 Chapter 12). Chain of cistody procedures were followed at all times for all samples. Copies of the completed chain of custody forms for each sampling location have been presented by Metcalf \& Eddy (1993a and 1993b).

\section{Quality Assurance/Quality Control (QA/QC)}

Blind replicates, split replicates, equipment blanks (rinsates), trip blanks, and shipping trip blanks were collected and sent to the laboratories as QA/QC checks for both the spring and fall sampling events. Tables 7 and 8 provide a summary of the QAVC samples collected. Field blind replicate samples were collected using the same sampling methods at the same locations as the reference samples and were submitted to Weston Analytics for the same analytical parameters as the associated reference sample. The split replicates were collected at the designated locations and sent to General Engineering and Environmental Physics. The equipment blanks were collected by rinsing decontaminated equipment with deionized water and bottling the rinsate for analysis. A trip blank sample was also submitted to the laboratories. The trip blank bottles for volatiles analysis were prepared by the subcontracted laboratories, and the trip blank bottles for the other aliquots were prepared by M\&E. The trip blank sample was composed of one of each type of sample bottle filled with deionized water. The trip blank sample bottles were filled before any samples were collected, transported to the sampling locations, handled like a sample, and returned to the laboratory for analysis. Shipping trip blanks (for volatile organic analyses only) were placed in each cooler prior to shipment to the laboratories during both sampling events. For the spring event, shipping trip blanks were not uniquely numbered and records were not kept by $M \& E$ as to which samples corresponded to each shipping trip blank. The only means of identifying which sample locations correspond to a particular shipping trip blank would be to match the sample ship date (M\&E field logs) with the sample date in the analysis records for the shipping trip blanks. Unique identification numbers were used for the shipping trip blanks during the fall event and records were kept by $M \& E$ to allow for correlation of shipping trip blanks with sample locations. This information is presented in Appendix $\mathbf{C}$ for the fall event.

\section{Data Validation}

Weston Analytics, General Engineering, and Environmental Physics submitted analytical data to ESS in hard copy and on electronic data diskettes (EDD). The hard copies were considered the laboratory-validated data packages and consisted of a case narrative for each batch, the result of the analyses, and QAVQC information (i.e. spike recoveries). The EDDs consisted of one flat ASCII computer file for each laboratory batch. The Weston Analytics data were formatted on the EDDs into the AN92 data format and the General Engineering and Environmental Physics data were formatted on the EDDs into the AN91 data format to provide the information given in Appendix D. The AN91 formatted files were converted to AN92 format to maintain database consistency.

Each EDD file was uploaded individually onto an IBM mainframe computer at SRS. The files were then merged to create one file to accommodate data review and validation activities.

The names of the blind replicate samples were changed to the correct location number and were marked as a replicate sample. Statistical Application Software (SAS) computer programs were run on each file to verify the data. The following data verification checks were made using the SAS programs:

- verification of location names and sample dates

- identification of data entry errors (incorrect methods or units)

- verification that samples were analyzed within hold times

- verification that all result and analysis qualifiers were valid 
Any suspected data entry errors were documented and submitted to Weston Analytics for verification or correction. Changes to the database were recorded in a logbook.

After all data entry errors had been resolved, the data were downloaded into a spreadsheet, where the data were screened to identify missing analyses and duplicate records. The laboratory was notified of any data that were missing and responded by submitting an EDD for the particular analysis. Any duplicate records were deleted from the database after the laboratory determined they had, in error, submitted the data twice.

The results from samples that had been used as laboratory replicates were averaged when both analyses were above the detection limit. Data below the detection limit and data presented with result or analysis qualifiers were not averaged and are noted in the comprehensive data tables (Appendices A and B) by "NA". Data that were averaged are noted with an " $A$ " in the averaged column.

Other data validation checks includej:

- verification that all surrogates and spikes were within acceptable recovery ranges

- determination of lab blanks which had results above detection limits and verifying that these results were qualified accordingly. 
Table 1. PDWS, MCL, and SDWS Standards Used in Data Comparisons

\begin{tabular}{|c|c|c|c|}
\hline Analyte & Standard & Units & Source \\
\hline 1,1,1,2-Tetrachloroethane & NS & & \\
\hline 1,1,2,2-Tetrachlorcethane & NS & & \\
\hline 1,1,1-Trichloroethane & 200 & $\mu g / L$ & EPA, 1993 \\
\hline 1,1,2-Trichloroethane & 5 & $\mu g / L$ & EPA, 1993 \\
\hline 1,1-Dichloroethane & NS & & \\
\hline 1,1-Dichloroethylene & 7 & $\mu \mathrm{g} / \mathrm{L}$ & EPA, 1993 \\
\hline 1,2,3-Trichloropropane & NS & & \\
\hline 1,2-Dibromo-3-chloropropane & 0.2 & $\mu g / L$ & EPA, 1993 \\
\hline 1,2-Dibromoethane & NS & & \\
\hline 1,2-Dichloroethane & 5 & $\mu g / L$ & EPA, 1993 \\
\hline 1,2-Dichloroethylene (total) & $70^{2}$ & $\mu g / L$ & EPA, 1993 \\
\hline 1,2-Dichloropropane & 5 & $\mu g / L$ & EPA, 1993 \\
\hline 2-Hexanone & NS & & \\
\hline 4-Methyl-2-pentanone & NS & & . \\
\hline Acetone & NS & & \\
\hline Acetonitrile & NS & & \\
\hline Acidity & NS & & \\
\hline Acrolein & NS & & \\
\hline Acrylonitrile & NS & & \\
\hline Alkalinity & NS & & \\
\hline Allyl chloride & NS & & \\
\hline Aluminum & NS & & \\
\hline Americium-241 & 6.34 & $\mathrm{pCi} / \mathrm{L}$ & EPA, 1991 \\
\hline Antimony & 6 & $\mu g / L$ & EPA, 1993 \\
\hline Arsenic & 50 & $\mu \mathrm{g} / \mathrm{L}$ & EPA, 1993 \\
\hline Barium & 2000 & ugL & EPA, 1993 \\
\hline Benzene & 5 & $\mu \mathrm{g} / \mathrm{L}$ & EPA, 1993 \\
\hline Beryllium & 4 & $\mu \mathrm{g} / \mathrm{L}$ & EPA, 1993 \\
\hline Bromodichloromethane & NS & & \\
\hline Bromoform & NS & & \\
\hline Cadmium & 5 & $\mu g / L$ & EPA, 1993 \\
\hline Calcium & NS & & \\
\hline Carbon disulfide & NS & & \\
\hline Carbon tetrachloride & 5 & $\mu \mathrm{g} / \mathrm{L}$ & EPA, 1993 \\
\hline Cerium-139 & NS & & \\
\hline Cesium-137 & 200 & $\mathrm{pCi} / \mathrm{L}$ & EPA, 1991 \\
\hline Chloride & NS & & \\
\hline Chlorobenzene & 100 & $\mu g / L$ & EPA, 1993 \\
\hline Chloroethane & NS & & \\
\hline Chloroform & 100 & $\mu \mathrm{g} / \mathrm{L}$ & EPA, 1991 \\
\hline Chloroprene & NS & & \\
\hline Chromium & 100 & $\mu g / L$ & EPA, 1993 \\
\hline cis-1,3-Dichloropropene & NS & & \\
\hline Cobalt & NS & & \\
\hline Cobalt -60 & 100 & $\mathrm{pCi} / \mathrm{L}$ & EPA, 197' \\
\hline Copper & 1000 & $\mu \mathrm{g} / \mathrm{L}$ & \\
\hline Cyanide & 200 & $\mu \mathrm{g} / \mathrm{L}$ & EPA, 1993 \\
\hline Dibromochloromethane & NS & & \\
\hline Dichlorodifluoromethane & NS & & \\
\hline
\end{tabular}


Table 1. Continued

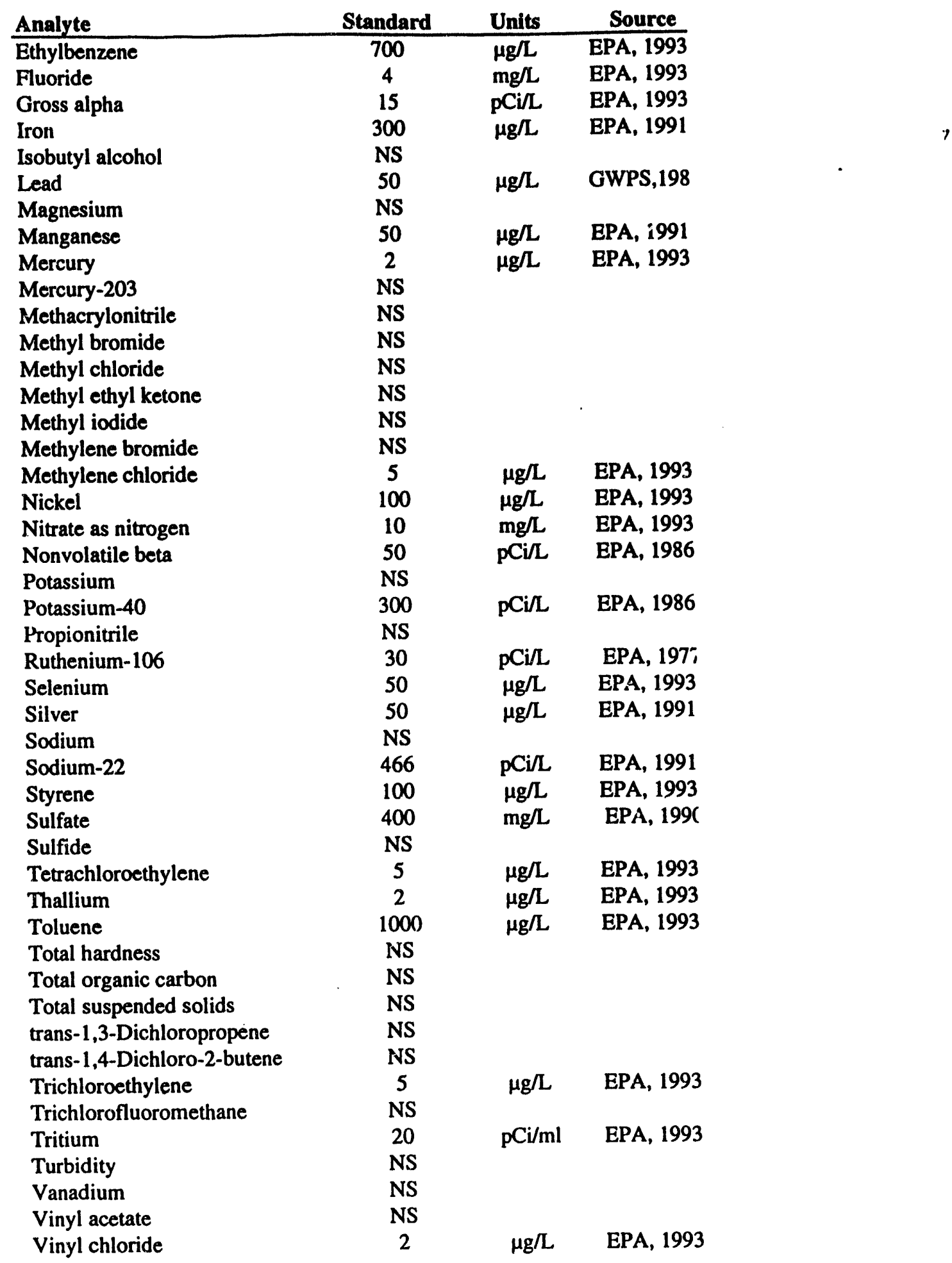


Table 1. Continued

\begin{tabular}{lccc} 
Analyte & Standard & Units & Source \\
\hline Xylenes (Total) & 10000 & $\mu \mathrm{g} / \mathrm{L}$ & $\mathrm{EPA}, 1993$ \\
Zinc & 5000 & $\mu \mathrm{g} / \mathrm{L}$ & $\mathrm{EPA}, 1991$ \\
Zinc-65 & 300 & $\mathrm{pCi} / \mathrm{L}$ & $\mathrm{EPA}, 197 \%$ \\
\hline
\end{tabular}

TNo Standard.

${ }^{2}$ MCL listed is for cis-1,2-dichloroethylene. 
Table 2. Methods and Method Detection Limits for Weston Analytics (WA) and TMA/Eberline (TM)

\begin{tabular}{|c|c|c|c|c|}
\hline Analyte & Lab & Method & MDL 1 & Units \\
\hline 1,1,1-Trichloroethane & WA & EPA8240 & 5 & $\mu g / L$ \\
\hline 1,1,2-Trichloroethane & WA & EPA8240 & 5 & $\mu g / L$ \\
\hline 1,1-Dichloroethylene & WA & EPA8240 & 5 & $\mu g / L$ \\
\hline 1,1-Dichloroethane & WA & EPA8240 & 5 & $\mu g / L$ \\
\hline 1,2,3-Trichloropropane & WA & EPA8240 & 10 & $\mu g / L$ \\
\hline 1,2 Dihromo-3-chloropropane & WA & EPA8240 & 20 & $\mu g / L$ \\
\hline 1,2 Dibromoethane & WA & EPA8240 & 20 & $\mu g / L$ \\
\hline 1,2-Dichloroethane & WA & EPA8240 & 5 & $\mu g / L$ \\
\hline 1,2-Dichloroethylene (total) & WA & EPA8240 & 5 & $\mu g / L$ \\
\hline 1,2-Dichloropropane & WA & EPA8240 & 5 & $\mu g / L$ \\
\hline cis-1,3-Dichloropropene & WA & EPA8240 & 5 & $\mu g / L$ \\
\hline trans-1,3-Dichloropropene & WA & EPA8240 & 5 & $\mu \mathrm{g} / \mathrm{L}$ \\
\hline trans-1,4-Dichloro-2-butene & WA & EPA8240 & 100 & $\mu g / L$ \\
\hline 1,1,2,2-Tetrachloroethane & WA & EPA8240 & 5 & $\mu g / L$ \\
\hline 1,1,1,2-Tetrachloroethane & WA & EPA8240 & 10 & $\mu g / L$ \\
\hline 2-Hexanone & WA & EPA8240 & 10 & $\mu g / L$ \\
\hline 4-Methyl-2-pentanone & WA & EPA8240 & 10 & $\mu g / L$ \\
\hline Acetone & WA & EPA8240 & 10 & $\mu g / L$ \\
\hline Acetonitrile & WA & EPA8240 & 20 & $\mu g / L$ \\
\hline Acidity & WA & SM402 & 0.5 & $\mathbf{m g} /$ \\
\hline Acrolein & WA & EPA8240 & 10 & $\mu g /$ \\
\hline Acrylonitrile & WA & EPA8240 & 10 & $\mu g / \Omega$ \\
\hline Alkalinity & WA & SM403 & 0.5 & mg/ \\
\hline Allyl chloride & WA & EPA8240 & 100 & $\mu g / L$ \\
\hline Aluminum & WA & EPA200.7 & 200 & $\mu g / L$ \\
\hline Americium-241 & TM & EMLC-02 & 10 & $\mathrm{pCi}$ \\
\hline Antimony & WA & EPA200.7 & 60 & $\mu g /$ \\
\hline Arsenic & WA & EPA206.2 & 10 & $\mu \mathrm{g} /$ \\
\hline Barium & WA & EPA200.7 & 200 & $\mu g /$ \\
\hline Benzene & WA & EPA8240 & 5 & \\
\hline Beryllium & WA & EPA200.7 & 5 & $\mu g /$ \\
\hline Bromodichloromethane & WA & EPA8240 & 5 & $\mu g /$ \\
\hline Bromoform & WA & EPA8240 & 5 & $\mu g /$ \\
\hline Cadmium & WA & EPA200.7 & 5 & $\mu \mathrm{g} /$ \\
\hline Calcium & WA & EPA200.7 & 5000 & $\mu g /$ \\
\hline Carbon disulfide & WA & EPA8240 & 5 & $\mu \mathrm{g} /$ \\
\hline Carbon tetrachloride & WA & EPA8240 & 5 & $\mu \mathrm{g}$ \\
\hline Cerium-139 & TM & EMLC-02 & * & $\mathrm{pCi}$ \\
\hline Cesium-137 & $\mathrm{TM}$ & EMLC-02 & 10 & $\mathrm{pCi}$ \\
\hline Chloride & WA & EPA325.2 & 0.25 & mg \\
\hline Chlorobenzene & WA & EPA8240 & 10 & \\
\hline Chloroethane & WA & EPA8240 & 10 & $\mu \mathrm{g} /$ \\
\hline Chloroform & WA & EPA8240 & 5 & $\mu g$ \\
\hline Chloroprene & WA & EPA8240 & 20 & $\mu \mathrm{g} /$ \\
\hline Chromium & WA & EPA200.7 & 10 & $\mu \mathrm{g} /$ \\
\hline Cobalt & WA & EPA200.7 & 50 & $\mu \mathrm{g}$ \\
\hline Cobalt -60 & TM & EMLC-02 & 10 & \\
\hline
\end{tabular}


Table 2. Continued

\begin{tabular}{|c|c|c|c|c|}
\hline Analyte & Lab & Method & MDL 1 & Units \\
\hline Copper & WA & EPA200.7 & 25 & $\mu \mathrm{g} / \mathrm{L}$ \\
\hline Cyanide & WA & EPA335.3 & 10 & $\mu g / L$ \\
\hline Dibromochloromethane & WA & EPA8240 & 5 & $\mu g / L$ \\
\hline Dithlorodifluoromethane & WA & EPA8240 & 10 & $\mu g / L$ \\
\hline Ethy lhenzene & WA & EPA8240 & 5 & $\mu g / L$ \\
\hline Fluoride & WA & EPA340.2 & 0.1 & $\mu g / L$ \\
\hline Gross alpha & TM & EPA900.0 & 1.5 & $\mathrm{pCi} / \mathrm{L}$ \\
\hline Iron & WA & EPA200.7 & 100 & $\mu \mathrm{g} / \mathrm{L}$ \\
\hline Isobutyl alcohol & WA & EPA8240 & 20 & $\mu \mathrm{g} / \mathrm{L}$ \\
\hline Lead & WA & EPA23?.2 & 3 & $\mu g / L$ \\
\hline Magnesium & WA & EPA200.7 & 5000 & $\mu g / L$ \\
\hline Manganese & WA & EPA200.7 & 15 & $\mu g / L$ \\
\hline Mercury & WA & EPA245.1 & 0.2 & $\mu g / L$ \\
\hline Methacrylonitrile & WA & EPA8240 & 20 & $\mu g / L$ \\
\hline Methyl bromide & WA & EPA8240 & 10 & $\mu g / L$ \\
\hline Methyl chloride & WA & EPA8240 & 10 & $\mu g / L$ \\
\hline Methylene bromide & WA & EPA8240 & 10 & $\mu g / L$ \\
\hline Methylene chloride & WA & EPA8240 & 5 & $\mu g / L$ \\
\hline Methyl ethyl ketone & WA & EPA8240 & 10 & $\mu g / L$ \\
\hline Methyl iodide & WA & EPA8240 & 10 & $\mu g / L$ \\
\hline Nickel & WA & EPA200.7 & 40 & $\mu g / L$ \\
\hline Nitrate as nitrogen & WA & EPA353.1 & 0.02 & $\mathrm{mg} / \mathrm{L}$ \\
\hline Nonvolatile beta & TM & EPA900.0 & 2 & $\mathrm{pCi} / \mathrm{L}$ \\
\hline Potassium & WA & EPA200.7 & 5000 & $\mu g / L$ \\
\hline Potassium-40 & TM & EMLC-02 & 50 & $\mathrm{pCi} / \mathrm{L}$ \\
\hline Propionitrile & WA & EPA8240 & 50 & $\mu g / L$ \\
\hline Ruthenium-106 & TM & EMLC-02 & $*$ & $\mathrm{pCi} / \mathrm{L}$ \\
\hline Selenium & WA & EPA270.2 & 5 & $\mu g / L$ \\
\hline Silver & WA & EPA200.7 & 10 . & $\mu g / L$ \\
\hline Sodium & WA & EPA200.7 & 5000 & $\mu g / L$ \\
\hline Sodium-22 & TM & EMLC-02 & $*$ & $\mathrm{pCi} / \mathrm{L}$ \\
\hline Sulfate & WA & EPA375.4 & 2.5 & $\mathrm{mg} / \mathrm{L}$ \\
\hline Styrene & WA & EPA8240 & 5 & $\mu \mathrm{g} / \mathrm{L}$ \\
\hline Sulfide & WA & EPA376.2 & 0.1 & $\mathrm{mg} / \mathrm{L}$ \\
\hline Tetrachloroethylene & WA & EPA8240 & 5 & $\mu \mathrm{g} / \mathrm{L}$ \\
\hline Thallium & WA & EPA279.2 & 10 & $\mu g / L$ \\
\hline Toluene & WA & EPA8240 & 5 & $\mu g / L$ \\
\hline Total hardness & WA & EPA 130.2 & 0.1 & $\mathrm{mg} / \mathrm{L}$ \\
\hline Total organic carbon & WA & EPA415.1 & 0.5 & $\mathrm{mg} / \mathrm{L}$ \\
\hline Trichloroethylene & WA & EPA8240 & 5 & $\mu \mathrm{g} / \mathrm{L}$ \\
\hline Trichloflouromethane & WA & EPA8240 & 5 & $\mu \mathrm{g} / \mathrm{L}$ \\
\hline Tritium & TM & EPA906.0 & 0.7 & $\mathrm{pCi} / \mathrm{mL}$ \\
\hline Total suspended solids & WA & SM160 & 5 & $\mathrm{mg} / \mathrm{L}$ \\
\hline Turbidity & WA & EPA180.1 & 0.2 & NTU \\
\hline Vanadium & WA & EPA200.7 & 50 & $\mu g / L$ \\
\hline Vinyl acetate & WA & EPA8240 & 10 & $\mu g / L$ \\
\hline
\end{tabular}


Table 2 Continued

\begin{tabular}{lcccc} 
Analyte & Lab & Method & MDL & Units \\
\hline Vinyl chloride & WA & EPA8240 & 10 & $\mu g / L$ \\
Xylene (total) & WA & EPA8240 & 5 & $\mu g / L$ \\
Zinc & WA & EPA200.7 & 20 & $\mu g / L$ \\
Zinc-65 & TM & EMLC-02 & $*$ & pCi/L \\
\hline
\end{tabular}

Method Detection Limit

*Weston Analytics did not report a method detection limit for these radiological parameters. 
Table 3. Methods and Method Detection Limits for General Engineering Laboratories (GE) and Environmental Physics (EP)

\begin{tabular}{|c|c|c|c|c|}
\hline Analyte & Lab & Method & MDL 1 & Units \\
\hline 1,1,1-Trichloroethane & GE & EPA8240 & 1 & $\mu g / L$ \\
\hline 1,1,2-Trichloroethane & GE & EPA8240 & 1 & $\mu g / L$ \\
\hline 1,1-Dichloroethlyene & $\mathrm{GE}$ & EPA8240 & 1 & $\mu g / L$ \\
\hline 1,1-Dichloroethane & GE & EPA8240 & 1 & $\mu g / L$ \\
\hline 1,2,3-Trichloropropane & GE & EPA8240 & 1 & $\mu g / L$ \\
\hline 1,2 Dihromo-3-chloropropane & GE & EPA8240 & 1 & $\mu g / L$ \\
\hline 1,2 Dibromoethane & GE & EPA8240 & 20 & $\mu g / L$ \\
\hline 1,2-Dichloroethane & GE & EPA8240 & 1 & $\mu g / L$ \\
\hline 1,2-Dichloropropane & GE & EPA8240 & 1 & $\mu g / L$ \\
\hline 1,2-Dichloroethylene (total) & GE & EPA8240 & 1 & $\mu g / L$ \\
\hline 1,1,2,2-Tetrachloroethane & GE & EPA8240 & 1 & $\mu g / L$ \\
\hline 1,1,1,2-Tetrachloroethane & GE & EPA8240 & 1 & $\mu g / L$ \\
\hline Acetone & GE & EPA8240 & 100 & $\mu g / L$ \\
\hline Acetonitrile & GE & EPA8240 & 1 & $\mu g / L$ \\
\hline Acrolein & GE & EPA8240 & 20 & $\mu g / L$ \\
\hline Acrylonitrile & GE & EPA8240 & 20 & $\mu g / L$ \\
\hline Actinium-228 & EP & HASL300 & 30 & $\mathrm{pCi} / \mathrm{L}$ \\
\hline Alkalinity & GE & EPA310.1 & 1 & $\mathrm{mg} / \mathrm{L}$ \\
\hline Allyl chloride & GE & EPA8240 & 50 & $\mu g / L$ \\
\hline Aluminum & GE & EPA6010 & 20 & $\mu \mathrm{g} / \mathrm{L}$ \\
\hline Antimony & GE & EPA7041 & 2 & $\mu g / L$ \\
\hline Antimony-125 & EP & HASL300 & 20 & $\mathrm{pCi} / \mathrm{L}$ \\
\hline Arsenic & GE & EPA7060 & 2 & $\mu g / L$ \\
\hline Barium & GE & EPA6010 & 3 & $\mu g / L$ \\
\hline Benzene & GE & EPA8240 & 1 & $\mu g / L$ \\
\hline Beryllium & GE & EPA6010 & 3 & $\mu g / L$ \\
\hline Bis(2-chloro-1-methylethyl)ether & GE & EPA8240 & 10 & $\mu g / L$ \\
\hline Bromodichloromethane & GE & EPA8240 & 1 . & $\mu g / L$ \\
\hline Bromoform & GE & EPA8240 & 1 & $\mu g / L$ \\
\hline Cadmium & GE & EPA6010 & 2 & $\mu g / L$ \\
\hline Calcium & GE & EPA6010 & 10 & $\mu g / L$ \\
\hline Carbon disulfide & GE & EPA8240 & 1 & $\mu g / L$ \\
\hline Carbon tetrachloride & GE & EPA8240 & 1 & $\mu g / L$ \\
\hline Cerium-144 & EP & HASL300 & 60 & $\mathrm{pCi} / \mathrm{L}$ \\
\hline Cesium-134 & EP & HASL300 & 10 & $\mathrm{pCi} / \mathrm{L}$ \\
\hline Cesium-137 & EP & HASL300 & 10 & $\mathrm{pCi} / \mathrm{L}$ \\
\hline Chloride & GE & EPA300.0 & 0.25 & $\mathrm{mg} / \mathrm{L}$ \\
\hline Chlorobenzene & GE & EPA8240 & 1 & $\mu \mathrm{g} / \mathrm{L}$ \\
\hline Chloroethane & GE & EPA8240 & 1 & $\mu g / L$ \\
\hline Chloroform & GE & EPA8240 & 1 & $\mu g / L$ \\
\hline Chloroprene & GE & EPA8240 & 200 & $\mu g / L$ \\
\hline Chromium & GE & EPA6010 & 4 & $\mu \mathrm{g} / \mathrm{L}$ \\
\hline cis-1,3-Dichloropropene & GE & EPA8240 & 1 & $\mu \mathrm{g} / \mathrm{L}$ \\
\hline Cobalt & GE & EPA6010 & 4 & $\mu \mathrm{g} / \mathrm{L}$ \\
\hline Cobalt-57 & EP & HASL300 & 10 & $\mathrm{pCi} / \mathrm{L}$ \\
\hline Cobalt-60 & EP & HASL300 & 10 & $\mathrm{pCi} / \mathrm{L}$ \\
\hline Copper & GE & EPA6010 & 4 & $\mu g / L$ \\
\hline
\end{tabular}


Table 3. Continued

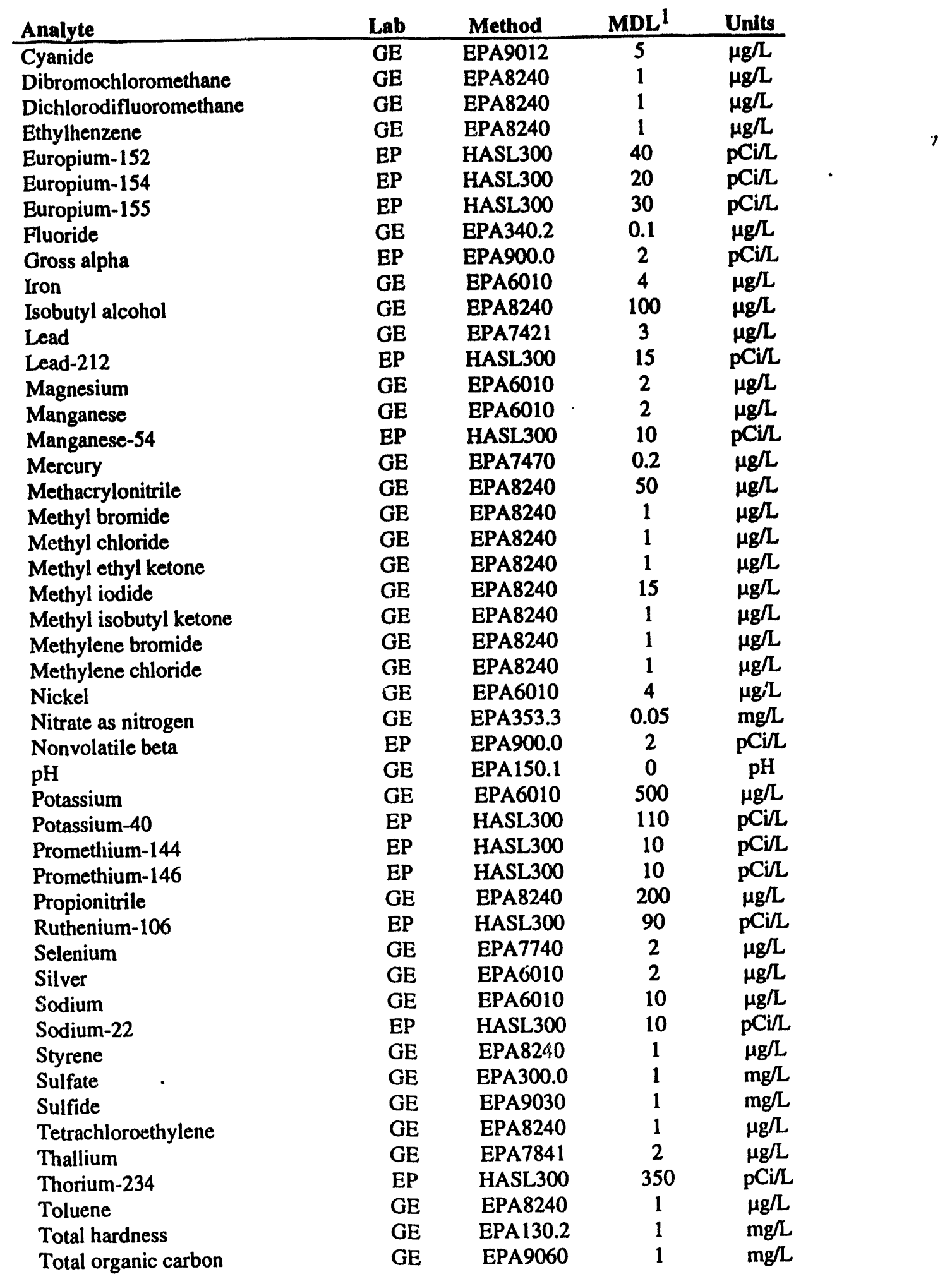


Table 3. Continued

\begin{tabular}{lcccc} 
Analyte & Lab & Method & MDL & Units \\
\hline Total suspended solids & GE & EPA160.2 & 1 & $\mathrm{mg} / \mathrm{L}$ \\
trans-1,3-Dichloropropene & GE & EPA8240 & 1 & $\mu \mathrm{g} / \mathrm{L}$ \\
trans-1,4-Dichloro-2-butene & GE & EPA8240 & 30 & $\mu \mathrm{g} / \mathrm{L}$ \\
Trichloflouromethane & GE & EPA8240 & 1 & $\mu \mathrm{g} / \mathrm{L}$ \\
Trichloroethylene & GE & EPA8240 & 1 & $\mu \mathrm{g} / \mathrm{L}$ \\
Tritium & EP & EPA906.0 & 0.7 & $\mathrm{pCi} / \mathrm{mL}$ \\
Turbidity & GE & EPA180.1 & 0.1 & $\mathrm{NTU}$ \\
Vanadium & GE & EPA6010 & 8 & $\mu \mathrm{g} / \mathrm{L}$ \\
Vinyl acetate & GE & EPA8240 & 1 & $\mu \mathrm{g} / \mathrm{L}$ \\
Vinyl chloride & GE & EPA8240 & 1 & $\mu \mathrm{g} / \mathrm{L}$ \\
Xylene (total) & GE & EPA8240 & 2 & $\mu \mathrm{g} / \mathrm{L}$ \\
Zinc & GE & EPA6010 & 2 & $\mu \mathrm{g} / \mathrm{L}$ \\
Zinc-65 & EP & HASL300 & 20 & $\mathrm{pCi} / \mathrm{L}$ \\
\hline
\end{tabular}

$1_{\text {Method Detection Limit }}$ 
Table 4. Wetland and Stream Sampling Locations for the Spring Sampling Event

\begin{tabular}{|c|c|c|c|c|c|c|c|c|}
\hline \multirow{2}{*}{$\begin{array}{l}\text { Sampling } \\
\text { Location }\end{array}$} & \multirow{2}{*}{$\begin{array}{c}\text { Sample } \\
\text { Type }\end{array}$} & \multicolumn{2}{|c|}{ SRS Coordinates } & \multirow{2}{*}{$\begin{array}{l}\text { Sampling } \\
\text { Location }\end{array}$} & \multirow{2}{*}{$\begin{array}{c}\text { Sample } \\
\text { Type }\end{array}$} & \multicolumn{3}{|c|}{ SRS Coordinates } \\
\hline & & North & East & & & North & & East \\
\hline UTR004 & Wetland & 76202 & 62158 & UTR109 & Wetland & 80036 & & 58791 \\
\hline UTR011 & Wetland & 76158 & 61630 & UTR 112 & Wetland & 79720 & & 58817 \\
\hline UTR024 & Wetland & 74694 & 60911 & UTR114 & Wetland & 79422 & , & 58975 \\
\hline UTR029 & Wetland & 74855 & 60792 & UTR116 & Wetland & .79665 & & 59050 \\
\hline UTR033 & Wetland & 75611 & 60946 & UTR120 & Wetland & 78967 & & 58753 \\
\hline UTR036 & Wetland & 75779 & 60664 & UTR122 & Wetland & 79278 & & 58742 \\
\hline UTR042 & Wetland & 76315 & 61161 & UTR123 & Wetland & 82105 & & 56338 \\
\hline UTR045 & Wetland & 77395 & 61382 & UTR 127 & Wetland & 81252 & & 56379 \\
\hline UTR051A & Wetland & 77864 & 60745 & UTR133 & Wetland & 79771 & & 56509 \\
\hline UTR053 & Wetland & 77998 & 60652 & UTR039 & Wetland & 78105 & & 56363 \\
\hline UTR056 & Wetland & 78197 & 60778 & UTR142 & Wetland & 79353 & & 56261 \\
\hline UTR059 & Wetland & 78499 & 60888 & UTR147 & Wetland & 80443 & & 56325 \\
\hline UTR060 & Wetland & 78588 & 60792 & UTR155 & Wetland & 81495 & & 55886 \\
\hline UTR062 & Wetland & 78742 & 60644 & BGSO01 & Wetland & 81517 & & 82198 \\
\hline UTR066 & Wetland & 78536 & 59972 & BGSOO2 & Wetland & 93847 & & 87239 \\
\hline UTR068 & Wetland & 78818 & 59861 & BGSO03 & Wetland & 94651 & & 82775 \\
\hline UTR071 & Wetland & 79142 & 60119 & BGS004 & Wetland & 18977 & & 55754 \\
\hline UTR075 & Wetland & 80070 & 59913 & UTD001 & Stream & 81340 & & 56280 \\
\hline UTR080 & Wetland & 80911 & 58678 & UTD002 & Stream & 80180 & & 59346 \\
\hline UT:.083 & Wetland & 81274 & 58131 & UTD003 & Stream & 78940 & & 60280 \\
\hline UTR091 & Wetland & 82528 & 56953 & UTD004 & Stream & 76400 & & 61270 \\
\hline UTR099 & Wetland & 81857 & 57447 & UTD005 & Stream & 75670 & & 62750 \\
\hline UTR100 & Wetland & 81351 & $5782 y$ & BGD001 & Stream & 91534 & & 86799 \\
\hline UTR102 & Wetland & 80744 & 58287 & BGD002 & Stream & 90875 & & 58168 \\
\hline
\end{tabular}


Table 5. Wetland and Stream Sampling Locations for the Fall Sampling Event

\begin{tabular}{|c|c|c|c|c|c|c|c|}
\hline \multirow{2}{*}{$\begin{array}{l}\text { Sample } \\
\text { Location }\end{array}$} & \multirow{2}{*}{$\begin{array}{c}\text { Sample } \\
\text { Type }\end{array}$} & \multicolumn{2}{|c|}{ SRS Coordinates } & \multirow{2}{*}{$\begin{array}{l}\text { Sample } \\
\text { Location }\end{array}$} & \multirow{2}{*}{$\begin{array}{c}\text { Sample } \\
\text { Type }\end{array}$} & \multicolumn{2}{|c|}{ SRS Coordinates } \\
\hline & & North & East & & & North & East \\
\hline UTR004 & Wetland & 76202 & 62158 & UTR100 & Wetland & 81351 & 57829 \\
\hline UTR011 & Wetland & 76158 & 61630 & UTR102 & Wetland & 80744 & 58287 \\
\hline UTR020 & Wetland & 74588 & 61552 & UTR108 & Wetland & 80127 & 758915 \\
\hline UTR022 & Wetland & 74990 & 61270 & UTR109 & Wetland & 80036 & 58791 \\
\hline UTR024 & Wetland & 74694 & 60911 & UTR112 & Wetland & 79720 & 58817 \\
\hline UTR029 & Wetland & 74855 & 60792 & UTR114 & Wetland & 79422 & 58975 \\
\hline UTR033 & Wetland & 75611 & 60946 & UTR116 & Wetland & 79665 & 59050 \\
\hline UTR036 & Wetland & 75779 & 60664 & UTR120 & Wetland & 78967 & 58753 \\
\hline UTR039 & Wetland & 75991 & 60890 & UTR122 & Wetland & 79278 & 58742 \\
\hline UTR042 & Wetland & 76315 & 61161 & UTR123 & Wetland & 82105 & 56338 \\
\hline UTR043 & Wetland & 77026 & 61307 & UTR127 & Wetland & 81252 & 56379 \\
\hline UTR045 & Wetland & 77395 & 61382 & UTR133 & Wetland & 79771 & 56509 \\
\hline UTR049 & Wetland & 77795 & 61142 & UTR039 & Wetland & 78105 & 56363 \\
\hline UTR051A & Wetland & 77864 & 60745 & UTR142 & Wetland & 79353 & 56261 \\
\hline UTR053 & Wetland & 77998 & 60652 & UTR145 & Wetland & 80068 & 56443 \\
\hline UTR056 & Wetland & 78197 & 60778 & UTR147 & Wetland & 80443 & 56325 \\
\hline UTR059 & Wetland & 78499 & 60888 & UTR155 & Wetland & 81495 & 55886 \\
\hline UTR060 & Wetland & 78588 & 60792 & BGSO01 & Wetland & 81517 & 82198 \\
\hline UTR062 & Wetland & 78742 & 60644 & BGSOO2 & Wetland & 93847 & 87239 \\
\hline UTR063 & Wetland & 78605 & 60453 & BGS003 & Wetland & 94651 & 82775 \\
\hline UTR066 & Wetland & 78536 & 59972 & BGS004 & Wetland & 18977 & 55754 \\
\hline UTR068 & Wetland & 78818 & 59861 & UTD001 & Stream & 81340 & 56280 \\
\hline UTR071 & Wetland & 79142 & 60119 & UTDO02 & Stream & 80180 & 59346 \\
\hline UTR072 & Wetland & 79232 & 60290 & UTD003 & Stream & 78940 & 60280 \\
\hline UTR075 & Wetland & 80070 & 59913 & UTD004 & Stream & 76400 & 61270 \\
\hline UTR080 & Wetland & 80911 & 58678 & UTD005 & Stream & 75670 & 62750 \\
\hline UTR083 & Wetland & 81274 & 58131 & UTD006 & Stream & 75540 & 63010 \\
\hline UTR091 & Wetland & 82528 & 56953 & BGD001 & Stream & 91534 & 86799 \\
\hline UTR099 & Wetland & 81857 & 57447 & BGD002 & Stream & 90875 & 58168 \\
\hline
\end{tabular}


Table 6. Dry Sampling Locations for the Fall Sampling Event ${ }^{1}$

\begin{tabular}{l|cc}
\multicolumn{1}{l}{$\begin{array}{l}\text { Sampling } \\
\text { Location }\end{array}$} & \multicolumn{2}{c}{ SRS Coordinates } \\
\hline UTR004 & 72602 & East \\
\hline UTR059 & 78499 & 62158 \\
UTR075 & 80070 & 60888 \\
UTR080 & 80911 & 59913 \\
UTR083 & 81274 & 58678 \\
UTR091 & 82528 & 58131 \\
UTR100 & 81351 & 56953 \\
UTR123 & 82105 & 57829 \\
UTR139 & 78105 & 56338 \\
UTR142 & 79353 & 56363 \\
BGS001 & 81517 & 82198 \\
BGS004 & 18977 & 55754 \\
\hline
\end{tabular}

There were no dry locations during the spring event. 
Table 7. QAVC Blanks, Trip Blanks, Blind Replicates, and Split Replicates for the Spring Sampling Event

\begin{tabular}{|c|c|c|c|}
\hline & $\begin{array}{l}\text { Associated } \\
\text { Sampling Locations }\end{array}$ & Blank ID & Source \\
\hline Equipment & NA & UQA006 & Filter \\
\hline \multirow[t]{4}{*}{ Blanks } & UTR102 & UQA007 & Shovel \\
\hline & UTR120 & UQA008 & Shovel \\
\hline & UTR133 & UQA009 & Shovel \\
\hline & BGSO03 & UQA010 & Shovel \\
\hline Trip Blank & All & UQA011 & \\
\hline \multirow[t]{5}{*}{ Blind Replicates } & UTR011 & & \\
\hline & UTR033 & & \\
\hline & UTR066 & & \\
\hline & UTR116 & & . \\
\hline & UTD004 & & ${ }^{\circ}$ \\
\hline \multirow[t]{10}{*}{ Split Replicates } & UTR011 & & \\
\hline & UTR033 & & \\
\hline & UTR045 & & \\
\hline & UTR066 & & \\
\hline & UTR068 & & \\
\hline & UTR114 & & \\
\hline & UTR116 & & \\
\hline & UTR 120 & & \\
\hline & UTR147 & & \\
\hline & UTD004 & & \\
\hline
\end{tabular}


Table 8. QAVQC Blanks, Trip Blanks, Blind Replicates, and Split Replicates for the

Fall Sampling Event

\begin{tabular}{|c|c|c|c|}
\hline & $\begin{array}{l}\text { Associated } \\
\text { Sampling Location }\end{array}$ & Blank ID & Source \\
\hline Equipment Blanks & $\begin{array}{l}\text { UTR022 } \\
\text { UTR108 } \\
\text { NA } \\
\text { NA } \\
\text { NA }\end{array}$ & $\begin{array}{l}\text { UQA006 } \\
\text { UQA007 } \\
\text { UQA008 } \\
\text { UQA009 } \\
\text { UQA010 }\end{array}$ & $\begin{array}{l}\text { Shovel } \\
\text { Shovel } \\
\text { Tubing/Filter } \\
\text { Tubing/Filter } \\
\text { Bucket }\end{array}$ \\
\hline Trip Blank & All & UQA011 & \\
\hline Blind Replicates & $\begin{array}{l}\text { UTR011 } \\
\text { UTR033 } \\
\text { UTR066 } \\
\text { UTR116 } \\
\text { UTD004 }\end{array}$ & & \\
\hline Split Replicates & $\begin{array}{l}\text { UTR045 } \\
\text { UTR068 } \\
\text { UTR114 } \\
\text { UTR147 } \\
\text { UTD003 }\end{array}$ & & \\
\hline
\end{tabular}




\section{DISCUSSION}

Analytical services were provided by several laboratories. Weston Analytics (and their subcontractor TMA/Eberline) was used as the reference laboratory for all analyses. Split samples were analyzed by General Engineering Laboratories, Inc. (GE; nonradiological) and Environmental Physics (EP; radiological). Samples analyzed by Weston Analytics were considered to be the reference samples on which all analyses and interpretations were based. Samples analyzed by General Engineering and Environmental Physics were considered to be quality assurance samples and were not used in the analyses and interpretations. This should prevent inter-lab variability of constituent concentrations from biasing the analyses and interpretation.

Analytical results for the spring and fall sampling events in the wetlands along UTR north of MWMF are presented in Appendices A and B. Location identification numbers ending with the suffix "D" (i.e. UTD or BGD) designate stream (or drainage way) water sampling locations. These drainage, or stream, samples were collected from perennial tributaries to UTR rather than the main channel of UTR. All other location IDs, excluding QA samples, designate wetland locations (i.e. UTR or BGS). Location identification numbers beginning with the prefix "BG" designate background locations. For each sampling location, the sampling date, field measurements including pH, conductivity, etc., and all laboratory analyses are presented (excluding lab blanks and standards). Also included are the analytical results, the labs that performed the analyses, the result and analytical qualifiers, the accuracy of the results (for radiological analyses only), any standards (i.e. PDWS, MCL, SDWS), and the units of measurement. A legend is given at the beginning of the appendices to provide explanations for the abbreviations and qualifiers used to present the data. For locations where split samples were collected, results from each lab are presented for each analyte. Laboratory duplicates were averaged with their corresponding reference samples except in cases where either the duplicate or reference analysis was reported at or below detection limit or in cases where results were qualified with a result or analysis qualifier.

\section{Field Measurements}

Spring and Fall Sampling Events

Conductivity, pH, reduction/oxidation potential, and dissolved oxygen content were measured at each stream and wetland location during both the spring and fall sampling events. Results are presented in Appendix A for the spring sampling event and in Appendix B for the fall sampling event. Field measurements presented in Appendices A and $B$ for both stream and wetland locations were made in the field at the time the samples were collected. Tables 9 and 10 provide minimum, maximum, and average values for selected field measurements made during the spring and fall sampling events.

\section{Comparison of Wetland and Stream Sampling Locations to Established Standards}

Since most of the analytical data, excluding routine water quality indicators (c.g., turbidity, alkalinity, total suspended solids, etc.), from the wetland and stream sampling locations were reported as below the method detection limit (MDL), and virtually all of the background data was reported as below the MDL, interpretation and analysis of the data is based completely upon observed trends rather than statistical analysis. The numerous data reported below the MDL make statistical analysis of the data impractical.

Spring Sampling Event

At the 37 UTR wetland locations that were sampled, 25 analytes occurred at concentrations greater than the MDL at least once. These analytes were reported without any type of result or analysis qualifiers and represented 35 percent of the total analyte list. A list of those analytes and the number of detected analyses are listed in Table 11. A total of 19 analytes were detected at the four background locations representing 19 percent of the total analyte list.

A total of 17 analytes (17 percent of the total analyte list) were detected at concentrations greater than the MDL from the seven UTR stream locations (including background locations). At the two background locations, twelve analytes were detected at the stream locations at concentrations greater than the MDL representing 12 percent of the total analyte list. A list of those analytes and the number of detected analyses are presented in Table 12. Most of the detected analytes were routine water quality indicators, with the exception of gross alpha, nonvolatile beta, 
americium-241, cerium-139, cesium-137, cobalt-60, potassium-40, ruthenium-106, sodium-22, tritium, and zinc-65. Potassium- 40 is a naturally occurring radionuclide commonly measured in wetland water samples in this area (Dixon and Rogers, 1993). Generally, for these samples, gross radiological indicators did not support the presence of these radionuclides.

Acetone was detected at several wetland and stream locations, including background locations. Acetone is a common laboratory contaminant which is routinely detected in both samples and method blanks. Even though the acetone results in Table 12 were not flagged by the lab to indicate method blank contamination (as several were), they are believed to be attributable to laboratory contamination.

Analytical results from the spring sampling event were compared to appropriate drinking water standands (PDWS, MCL, and SDWS) where possible. The results of these comparisons are shown in Tables 13 (SDWS) and 14 (PDWS and MCL).

Of the nonradiological inorganic parameters, only cadmium was elevated above the MCL or PDWS. Iron and manganese were elevated above the SDWS at several locations including some background locations. These results are typical of wetland and stream water in this area (Dixon and Rogers, 1993; Haselow et al., 1990; Haselow et al., 1992) and are not cause for concern; particularly since the SDWS for iron and manganese was established as a guideline to minimize staining from rust spots. Trichloroethylene was the only organic parameter reported above the MCL for the spring sampling event. It was detected at UTR155 slightly above the MCL ( $5.09 \mu \mathrm{g} / \mathrm{L})$, which for trichloroethylene, is equivalent to the $\operatorname{MDL}(5 \mu \mathrm{g} / \mathrm{L})$.

As shown in Table 14, most of the analytes reported above the MCL or PDWS are radiological parameters including, gross alpha, nonvolatile beta, potassium-40, ruthenium-106, and tritium. As mentioned earlier, gross radiological indicators did not support the presence of ruthenium-106. Gross alpha was reported above the MCL, at two locations, one of which was a background location. Gross alpha activities for the UTR and UTD locations ranged from 0.4 to $49.1 \mathrm{pCi} / \mathrm{L}$ and 0.4 to $1.5 \mathrm{pCi} / \mathrm{L}$, respectively with average activities of 2.5 and $0.8 \mathrm{pCi} / \mathrm{L}$, respectively. Nonvolatile beta was reported above the $\mathrm{MCL}$ at three locations, two of which were background locations. Nonvolatile beta activities for the UTR and UTD locations ranged from 0.7 to $79.0 \mathrm{pCi} / /$ and 1.5 to $6.1 \mathrm{pCi} / \mathrm{L}$ with average activities of 4.8 and $2.7 \mathrm{pCi} / \mathrm{L}$, respectively. Gross alpha and nonvolatile beta activity was detected in several equipment blanks including UQA009 (34 \pm 4 and $841 \pm 17 \mathrm{pCi} / \mathrm{L}$, respectively). The batch that included this sample also included the wetland location with the maximum gross alpha and nonvolatile beta activity (UTR142; $49.1 \pm 10.4$ and $79.0 \pm 12 \mathrm{pCi} / \mathrm{L}$, respectively). Excluding location UTR142, gross alpha and nonvolatile beta results were comparable to those measured at the background locations and were generally lower than those reported by Haselow et al. (1992) for the area. Tritium activities for the UTR and UTD locations ranged from 4.9 to 143.5 $\mathrm{pCi} / \mathrm{ml}$ and 9 to $68.2 \mathrm{pCi} / \mathrm{ml}$ with average activities of 20.5 and $36.0 \mathrm{pCi} / \mathrm{ml}$, respectively. Although tritium results were elevated above background, they were comparable to those measured by Haselow et al. (1992).

Americium-241 was detected (at concentrations greater than the PDWS) using gamma PHA analysis in three samples, UTD003, UTR053, and UTR062 during the spring sampling event. Americium is primarily an alpha emitter (5.49 Mev), but it is also a low energy gamma emitter $(0.06 \mathrm{Mev})$. The americium-241 peak on a gamma PHA spectrum is in an area commonly influenced by compton rays and background interference and for most analyses the instrument is not calibrated for energies below $0.088 \mathrm{Mev}$. Due to these potential interferences, Weston Analytics was requested to review the gamma PHA spectra associated with these three samples. Their review of the spectra showed no particular interferences that might have produced false positive americium-241 results; however, Weston confirmed that their instruments were not calibrated below $0.088 \mathrm{Mev}$. Gross alpha activities for these locations did not suggest the presence of americium-241 at the levels indicated by the gamma PHA scans.

Furthermore, although americium-241 was detected at one stream location during the fall event, it was not detected at the three spring locations. Sample aliquots from these three locations were provided to EMS for confirmatory gamma PHA. The results of these analyses showed no detectable americium-241. Therefore, americium-241 results are not presented in Table 14. 


\section{Fall Sampling Event}

At the 36 UTR wetland locations that were sampled, 22 analytes occurred at concentrations greater than the MDL at least once. These analytes were reported without any type of result or analysis qualifiers and represented 22 percent of the total analyte list. A list of those analytes and the number of detected analyses are listed in Table 15. A total of 14 analytes were detected at the two background locations representing 14 percent of the total analyte list, Most of the detected analytes were routine water quality indicators, with the exception of gross alpha, nonvolatile beta, americium-241, cesium-137, mercury-203, and tritium. Generally, for these samples, gross radiological indicators did not support the presence of these radionuclides.

A total of 17 analytes (17 percent of the total analyte list) were detected at concentrations greater than the MDL from the seven UTR stream locations. At the two background locations, 15 analytes were detected at concentrations greater than the MDL representing 15 percent of the total analyte list. A list of those analytes and the number of detected analyses are presented in Table 16.

Analytical results from the fall sampling event were compared to appropriate drinking water standards (PDWS, MCL, and SDWS) where possible. The results of these comparisons are shown in Tables 17 (SDWS) and 18 (PDWS and MCL). None of the nonradiological inorganic parameters or volatile organic parameters were elevated above the MCL or PDWS. Iron was elevated above the SDWS at the background locations and manganese was elevated above the SDWS at several locations including some background locations. These results are similar to those from the spring sampling event and are typical of wetland and stream water in this area (Dixon and Rogers, 1993; Haselow et al., 1990; Haselow et al., 1992). The iron and manganese results are not cause for concern, particularly since the SDWS for these two metals was established as a guideline to minimize staining from rust spots.

The only analytes that exceeded the MCL or PDWS for the fall sampling event were americium-241 and tritium (Table 18). Gross alpha and nonvolatile beta activities measured in the fall sampling event were considerably less than those for the spring sampling event. Gross alpha activities for the UTR and UTD locations ranged from 0.5 to $4.4 \mathrm{pCi} / \mathrm{L}$ and 0.4 to $1.4 \mathrm{pCi} / \mathrm{L}$ with average activities of 1.2 and $0.9 \mathrm{pCi} / \mathrm{L}$, respectively. Nonvolatile beta activities for the UTR and UTD locations ranged from 0.7 to $11 \mathrm{pCi} / \mathrm{L}$ and 0.6 to $4.6 \mathrm{pCi} / \mathrm{L}$ with average activities of 2.5 and $1.7 \mathrm{pCi} / \mathrm{L}$, respectively. Tritium activities for the UTR and UTD locations ranged from 4.6 to $272 \mathrm{pCi} / \mathrm{ml}$ and 7.0 to $103.5 \mathrm{pCi} / \mathrm{ml}$ with average activities of 31.5 and $45.7 \mathrm{pCi} / \mathrm{ml}$, respectively. Tritium was also detected in one equipment blank above the MCL (UQA009; $38.76 \pm 1.31$ ). This equipment blank was collected from deionized water pumped through new tubing and filter. As with the spring tritium results, fall tritium results were slightly elevated above background but comparable to those measured by Haselow et al. (1992).

Americium-241 was detected at three locations during the spring sampling event (two wetland and one stream location). For the fall sampling event, it was only detected at one stream location (UTD005), which was different than the location of the spring detect. The reported concentration was greater than the PDWS, although gross radiological indicators did not support the presence of americiun-241 in the sample. For the same reasons discussed in the section detailing results from the spring event, it is believed that this is a false positive result. Therefore, americium-241 results are not presented in Table 18. Mercury-203 was detected at one wetland location (UTR043) during the fall event. This result is considered a false positive since the half life of mercury-203 is only 46.5 days.

\section{Tank 16 Impacts on UTR Wetland Water Quality}

In September 1960, approximately 16 gallons of high level waste leaked from Tank 16 into the soil (Poe, 1974; Carlton et al., 1992). The waste contained approximately $7.4 \mathrm{Ci}$ of radionuclides per gallon (118 Ci total). Approximately $86 \%$ of the activity was cesium-134,137. Other radioactive constituents of the waste included strontium-89,90 (1.7\%), zirconium-95 and niobium-95 (7.3\%), ruthenium-103,106 (4.3\%), and plutonium, americium-243, and neptunium-237 (<1\%; Poe, 1974).

Immediately following the leak, the ratio of cesium-137 to cesium-134 was used to positively identify Tank 16 as the source of the radioactivity in the groundwater. The ratio of cesium-137 to cesium-134 in the groundwater was 8 to 1 , which corresponded to a cesium-137 to cesium-134 ratio of 9 to 1 in Tank 16 waste. The ratios of cesium-137 to 
cesium-134 in Tanks 13 and 14 (tanks near Tank 16) were more than 20 to 1 , thus, eliminating Tanks 13 and 14 as the source of the radioactivity (Poe, 1974).

Current potential UTR wetland impacts from Tank 16 leaks cannot be identified using the cesium-137 - cesium-134 ratio initially used to characterize the Tank 16 wastes. Cesium-134 has a half-life of 2.1 years. In the approximately 30 years following the leak, most of the cesium-134 has likely decayed making using of the ratio impractical. Furthermore, waste migration away from the tank has been estimated to be only a few feet (Poe, 1974). The slow movement results from the placement of the Tank Farm in an area of very low lateral groundwater flow $(0.01-0.02$ ft per day) and from the ion exchange capacity of the soil. Transport of radioactive constituonts would be extremely slow since they would be adsorbed to the exchange complex of the soil. Travel times for groundwater flow from the tank to the nearest creek was estimated to range from 70 - 350 years while the travel time for the leading edge of the radionuclides to reach the nearest creek was estimated to range from 200,000 to 1,200,000 years (Poe, 1974). During this time period, any radioactivity would have decayed to insignificant levels.

Examination of the analytical data from both the spring and fall sampling events did not reveal any evidence that UTR wetlands are being impacted by waste migrating from Tank 16. Gamma PHA scans of each sample did not show any detectable cesium-134. Cesium-137 was detected at low levels (3.5 \pm 3.6 to $13.8 \pm 10.9 \mathrm{pCi} / \mathrm{L}$ spring and $0.39 \pm 7.8$ to $9.8 \pm 7.6 \mathrm{pCi} / \mathrm{L}$ fall) in a few wetland samples during both the spring and fall events. Generally, nonvolatile beta results did not support the presence of cesium-137 in the samples at the levels reported. This, coupled with the high analytical uncertainty associated with the results from both the spring and fall sampling events, makes it unlikely that cesium- 137 in significant quantities was present in any of the samples. 
Table 9. Minimum, maximum, and average values for selected field measurements made during the spring sampling event

\begin{tabular}{|c|c|c|c|c|}
\hline & Parameter & Minimum & Maximum & Average \\
\hline Wetland $(\mathrm{N}=37)$ & $\begin{array}{l}\text { pH } \\
\text { Conductivity ( } \mu \mathrm{S} / \mathrm{cm}) \\
\text { Reduction/oxidation }(\mathrm{mV}) \\
\text { Dissolved oxygen (mg/L) }\end{array}$ & $\begin{array}{l}4.5 \\
17 \\
-204 \\
0.8\end{array}$ & $\begin{array}{l}8.7 \\
133 \\
212 \\
5.7\end{array}$ & $\begin{array}{l}5.9 \\
41 \\
105 \\
2.5\end{array}$ \\
\hline Background wetland $(N=4)$ & $\begin{array}{l}\mathrm{pH} \\
\text { Conductivity }(\mu \mathrm{S} / \mathrm{cm}) \\
\text { Reduction/oxidation }(\mathrm{mV}) \\
\text { Dissolved oxygen }(\mathrm{mg} / \mathrm{L})\end{array}$ & $\begin{array}{l}5.4 \\
20 \\
106 \\
0.9\end{array}$ & $\begin{array}{l}6.2 \\
60 \\
165 \\
8.3\end{array}$ & $\begin{array}{l}5.7 \\
37 \\
147 \\
3.2\end{array}$ \\
\hline Stream $(N=5)$ & $\begin{array}{l}\text { pH } \\
\text { Conductivity }(\mu \mathrm{S} / \mathrm{cm}) \\
\text { Reduction/oxidation }(\mathrm{mV}) \\
\text { Dissolved oxygen }(\mathrm{mg} / \mathrm{L})\end{array}$ & $\begin{array}{l}6.5 \\
25 \\
79 \\
.5 .4\end{array}$ & $\begin{array}{l}7.4 \\
49 \\
196 \\
10.0\end{array}$ & $\begin{array}{l}6.8 \\
36 \\
142 \\
7.7\end{array}$ \\
\hline Background stream $(\mathrm{N}=2)$ & $\begin{array}{l}\text { pH } \\
\text { Conductivity }(\mu S / \mathrm{cm}) \\
\text { Reduction/oxidation }(\mathrm{mV}) \\
\text { Dissolved oxygen }(\mathrm{mg} / \mathrm{L})\end{array}$ & $\begin{array}{l}6.3 \\
13 \\
111 \\
5.4\end{array}$ & $\begin{array}{l}7.1 \\
30 \\
196 \\
8.6\end{array}$ & $\begin{array}{l}* \\
* \\
* \\
*\end{array}$ \\
\hline
\end{tabular}

*Because there were only two data points, averages were not computed. 
Table 10. Minimum, maximum, and average values for selected field measurements made during the fall sampling event

\begin{tabular}{|c|c|c|c|c|}
\hline cremi & Parameter & Minimum & Maximum & Average \\
\hline Wetland $(\mathrm{N}=36)$ & $\begin{array}{l}\text { pH } \\
\text { Conductivity ( } \mu \mathrm{S} / \mathrm{cm}) \\
\text { Reduction/oxidation }(\mathrm{mV}) \\
\text { Dissolved oxygen }(\mathrm{mg} / \mathrm{L})\end{array}$ & $\begin{array}{l}4.0 \\
15 \\
-180 \\
0.6\end{array}$ & $\begin{array}{l}7.7 \\
115 \\
224 \\
5.5\end{array}$ & $\begin{array}{l}6.2 \\
37 \\
131 \\
2.2\end{array}$ \\
\hline Background wetland $(\mathrm{N}=2)$ & $\begin{array}{l}\text { pH } \\
\text { Conductivity ( } \mu S / \mathrm{cm}) \\
\text { Reduction/oxidation }(\mathrm{mV}) \\
\text { Dissolved oxygen }(\mathrm{mg} / \mathrm{L})\end{array}$ & $\begin{array}{l}5.5 \\
41 \\
170 \\
1.0\end{array}$ & $\begin{array}{l}7.5 \\
51 \\
281 \\
2.1\end{array}$ & $\begin{array}{l}* \\
* \\
*\end{array}$ \\
\hline Stream $(N=6)$ & $\begin{array}{l}\mathrm{pH} \\
\text { Conductivity }(\mu \mathrm{S} / \mathrm{cm}) \\
\text { Reduction/oxidation }(\mathrm{mV}) \\
\text { Dissolved oxygen }(\mathrm{mg} / \mathrm{L})\end{array}$ & $\begin{array}{l}5.7 \\
38 \\
25 \\
3.2\end{array}$ & $\begin{array}{l}7.8 \\
79 \\
359 \\
8.0\end{array}$ & $\begin{array}{l}6.4 \\
55 \\
161 \\
6.6\end{array}$ \\
\hline Background stream $(\mathrm{N}=2)$ & $\begin{array}{l}\mathrm{pH} \\
\text { Conductivity }(\mu \mathrm{S} / \mathrm{cm}) \\
\text { Reduction/oxidation }(\mathrm{mV}) \\
\text { Dissolved oxygen }(\mathrm{mg} / \mathrm{L})\end{array}$ & $\begin{array}{l}5.3 \\
31 \\
161 \\
3.7\end{array}$ & $\begin{array}{l}5.4 \\
79 \\
197 \\
3.9\end{array}$ & $\begin{array}{l}* \\
* \\
*\end{array}$ \\
\hline
\end{tabular}

* Because there were only two data points, averages were not computed. 
Table 11. Comparison of Analytes Detected at Wetland and Background Locations During Spring Sampling Event

\begin{tabular}{|c|c|c|c|c|c|c|}
\hline Analyte & $\begin{array}{l}\text { Number of } \\
\text { Wetland } \\
\text { Locations }\end{array}$ & $\begin{array}{c}\text { Number of } \\
\text { Detects }\end{array}$ & Percen & $\begin{array}{c}\text { Number of } \\
\text { Backgrounc } \\
\text { Locations }\end{array}$ & $\begin{array}{c}\text { Number of } \\
\text { Detects }\end{array}$ & Percen \\
\hline Acetone & 37 & 10 & 27 & 4 & 2 & 50 \\
\hline Acidity & 37 & 37 & 100 & 4 & 4 &, 100 \\
\hline Alkalinity & 37 & 29 & 78 & 4 & 0 & 0 \\
\hline Aluminum & 37 & 10 & 27 & 4 & 4 & 100 \\
\hline Americium-241 1 & 37 & 2 & 5 & 4 & 0 & $\mathbf{0}$ \\
\hline Barium & 37 & 1 & 3 & 4 & 0 & 0 \\
\hline Cadmium & 37 & 4 & 11 & 4 & 0 & 0 \\
\hline Calcium & 37 & 4 & 11 & 4 & $\mathbf{0}$ & 0 \\
\hline Cerium-1391 & 37 & 1 & 3 & 4 & $\mathbf{0}$ & 0 \\
\hline Cesium-137 & 37 & 9 & 24 & 4 & 1 & 25 \\
\hline Chloride & 37 & 37 & 100 & 4 & 4 & 100 \\
\hline Chromium & 37 & 1 & 3 & 4 & 0 & 0 \\
\hline Cobalt-60 1 & 37 & 1 & 3 & 4 & 0 & $\mathbf{0}$ \\
\hline Copper & 37 & 0 & 0 & 4 & 2 & 50 \\
\hline Gross alpha & 37 & 20 & 54 & 4 & 4 & 100 \\
\hline Iron & 37 & 32 & 87 & 4 & 4 & 100 \\
\hline Lead & 37 & 5 & 14 & 4 & 1 & 25 \\
\hline Manganese & 37 & 29 & 78 & 4 & 4 & 100 \\
\hline Mercury 2 & 37 & 1 & 3 & 4 & 0 & 0 \\
\hline Nickel & 37 & 3 & 8 & 4 & 0 & $\mathbf{0}$ \\
\hline Nitrate as nitrogen & 37 & 27 & 73 & 4 & 2 & 50 \\
\hline Nonvolatile beta & 37 & 26 & 70 & 4 & 4 & 100 \\
\hline Potassium-40 & 37 & 6 & 17 & 4 & 0 & 0 \\
\hline Sodium & 37 & 2 & 5 & 4 & 0 & 0 \\
\hline Sodium-22 1 & 37 & 1 & 3 & 4 & 0 & 0 \\
\hline Sulfate & 37 & 17 & 46 & 4 & 4 & 100 \\
\hline Total hardness & 37 & 30 & 81 & 4 & 4 & 100 \\
\hline Total organic carbon & 37 & 35 & 95 & 4 & 4 & 100 \\
\hline Total suspended solids & 37 & 23 & 62 & 4 & 4 & 100 \\
\hline Trichloroethylene & 37 & 1 & 3 & 4 & 0 & 0 \\
\hline Tritium & 37 & 37 & 100 & 4 & 4 & 100 \\
\hline Turbidity & 37 & 35 & 95 & 4 & 4 & 100 \\
\hline Vanadium & 37 & 1 & 3 & 4 & 0 & 0 \\
\hline Zinc & 37 & 20 & 54 & 4 & 4 & 100 \\
\hline Zinc-65 1 & 37 & 1 & 3 & 4 & 0 & 0 \\
\hline
\end{tabular}

TGross radiological indicators did not support the presence of any of these radionuclides in the samples.

2 The MDL for mercury reported by Weston Analytics was $0.2 \mu \mathrm{g} / \mathrm{L}$. The reported mercury concentration for UTR 142 was $0.203 \mu \mathrm{g} / \mathrm{L}$. 
Table 12. Comparison of Analytes Detected at Stream and Background Locations During Spring Sampling Event

\begin{tabular}{|c|c|c|c|c|c|c|}
\hline Analyte & $\begin{array}{l}\text { Number of } \\
\text { Stream } \\
\text { Locations }\end{array}$ & $\begin{array}{c}\text { Number of } \\
\text { Detects }\end{array}$ & Percen & $\begin{array}{c}\text { Number of } \\
\text { Backgrounc } \\
\text { Locations }\end{array}$ & $\begin{array}{l}\text { Number of } \\
\text { Detects }\end{array}$ & Percen \\
\hline Acetone & 5 & 0 & 0 & 2 & 1 & 50 \\
\hline Acidity & 5 & 5 & 100 & 2 & 2 & 100 \\
\hline Alkalinity & 5 & 3 & 60 & 2 & 1 & 750 \\
\hline Americium-241 ${ }^{1}$ & 5 & 1 & 20 & 2 & 0 & 0 \\
\hline Chloride & 5 & 5 & 100 & 2 & 2 & 100 \\
\hline Gross alpha & 5 & 4 & 80 & 2 & 0 & 0 \\
\hline Iron & 5 & 5 & 100 & 2 & 2 & 100 \\
\hline Manganese & 5 & 5 & 100 & 2 & 2 & 100 \\
\hline Nonvolatile beta & 5 & 5 & 100 & 2 & 1 & 50 \\
\hline Nitrate & 5 & 3 & 60 & 2 & $\mathbf{0}$ & $\mathbf{0}$ \\
\hline Sodium & 5 & 2 & 40 & 2 & 0 & 0 \\
\hline Sulfate & 5 & 2 & 40 & 2 & 1 & 50 \\
\hline Total hardness & 5 & 5 & 100 & 2 & 1 & 50 \\
\hline Total organic carbon & 5 & 5 & 100 & 2 & 2 & 100 \\
\hline Total suspended solids & 5 & 4 & 80 & 2 & 2 & 100 \\
\hline Tritium & 5 & 5 & 100 & 2 & 0 & 0 \\
\hline Turbidity & 5 & 5 & 100 & 2 & 1 & 50 \\
\hline Zinc & 5 & 2 & 40 & 2 & 0 & $\mathbf{0}$ \\
\hline
\end{tabular}

$T_{G}$ ross radiological indicators did not support the presence of any of these radionuclides in the samples. 
Sampling And Analysis Of Water From Upper Three Runs And Its Wetlands Near Tank 16 and the Mixed Wasse Mamagement Facilioy

Table 13. Analytes Detected above the SDWS during the Spring Sampling Event

\begin{tabular}{|c|c|c|c|c|c|}
\hline Analyte & Lab & Result & Standard & Units & Location \\
\hline Iron & WA & 1340 & 300 & $\mu g / L$ & BGD002 \\
\hline Iron & WA & 478 & 300 & $\mu g / L$ & BGS001 \\
\hline Iron & WA & 12400 & 300 & $\mu g / L$ & BGSOO2 \\
\hline Iron & WA & 1300 & 300 & $\mu \mathrm{g} / \mathrm{L}$ & BGSO03 \\
\hline Iron & WA & 1460 & 300 & $\mu g / L$ & BGSO04 \\
\hline Iron & WA & 348 & 300 & $\mu g / L$ & UTD002 \\
\hline Iron & WA & 345 & 300 & $\mu g / L$ & UTD003 \\
\hline Iron & WA & 386 & 300 & $\mu g / L$ & UTD004 \\
\hline Iron & WA & 1080 & 300 & $\mu \mathrm{g} / \mathrm{L}$ & UTDOOS \\
\hline Iron & WA & 4320 & 300 & $\mu \mathrm{g} / \mathrm{L}$ & UTR004 \\
\hline Iron & WA & 17700 & 300 & $\mu g / L$ & UTR029 \\
\hline Iron & WA & 2360 & 300 & $\mu g / L$ & UTR036 \\
\hline Iron & WA & 507 & 300 & $\mu \mathrm{g} / \mathrm{L}$ & UTR045 \\
\hline Iron & WA & 969 & 300 & $\mu g / L$ & UTR056 \\
\hline Iron & WA & 451 & 300 & $\mu \mathrm{g} / \mathrm{L}$ & UTR071 \\
\hline Iron & WA & 597 & 300 & $\mu \mathrm{g} / \mathrm{L}$ & UTR075 \\
\hline Iron & WA & 905 & 300 & $\mu g / L$ & UTR080 \\
\hline Iron & WA & 34500 & 300 & $\mu \mathrm{g} / \mathrm{L}$ & UTR083 \\
\hline Iron & WA & 1560 & 300 & $\mu g / L$ & UTR091 \\
\hline Iron & WA & 16400 & 300 & $\mu \mathrm{g} / \mathrm{L}$ & UTR099 \\
\hline Iron & WA & 427 & 300 & $\mu g / L$ & UTR102 \\
\hline Iron & WA & 3100 & 300 & $\mu g / L$ & UTR109 \\
\hline Iron & WA & 26900 & 300 & $\mu g / L$ & UTR112 \\
\hline Iron & WA & 2760 & 300 & $\mu g / L$ & UTR114 \\
\hline Iron & WA & 349 & 300 & $\mu \mathrm{g} / \mathrm{L}$ & UTR116 \\
\hline Iron & WA & 1160 & 300 & $\mu \mathrm{g} / \mathrm{L}$ & UTR122 \\
\hline Iron & WA & 631 & 300 & $\mu \mathrm{g} / \mathrm{L}$ & UTR123 \\
\hline Iron & WA & 1310 & 300 & $\mu \mathrm{g} / \mathrm{L}$ & UTR133 \\
\hline Iron & WA & 53100 & 300 & $\mu \mathrm{g} / \mathrm{L}$ & UTR142 \\
\hline Iron & WA & 415 & 300 & $\mu \mathrm{g} / \mathrm{L}$ & UTR155 \\
\hline Manganese & WA & 233 & 50 & $\mu g / L$ & BGD002 \\
\hline Manganese & WA & 120 & 50 & $\mu g / L$ & UTD003 \\
\hline Manganese & WA & 125 & 50 & $\mu \mathrm{g} / \mathrm{L}$ & UTD005 \\
\hline Manganese & WA & 272 & 50 & $\mu \mathrm{g} / \mathrm{L}$ & UTR004 \\
\hline Manganese & WA & 1900 & 50 & $\mu \mathrm{g} / \mathrm{L}$ & UTR029 \\
\hline Manganese & WA & 52 & 50 & $\mu \mathrm{g} / \mathrm{L}$ & UTR056 \\
\hline Manganese & WA & 79 & 50 & $\mu \mathrm{g} / \mathrm{L}$ & UTR071 \\
\hline Manganese & WA & 52.1 & 50 & $\mu \mathrm{g} / \mathrm{L}$ & UTR075 \\
\hline Manganese & WA & 1810 & 50 & $\mu \mathrm{g} / \mathrm{L}$ & UTR083 \\
\hline Manganese & WA & 568 & 50 & $\mu \mathrm{g} / \mathrm{L}$ & UTR091 \\
\hline Manganese & WA & 402 & 50 & $\mu \mathrm{g} / \mathrm{L}$ & UTR099 \\
\hline Manganese & WA & 295 & 50 & $\mu \mathrm{g} / \mathrm{L}$ & UTR100 \\
\hline Manganese & WA & 91.2 & 50 & $\mu \mathrm{g} / \mathrm{L}$ & UTR109 \\
\hline Manganese & WA & 136 & 50 & $\mu \mathrm{g} / \mathrm{L}$ & UTR 112 \\
\hline Manganese & WA & 58.8 & 50 & $\mu \mathrm{g} / \mathrm{L}$ & UTR114 \\
\hline Manganese & WA & 138 & 50 & $\mu \mathrm{g} / \mathrm{L}$ & UTR122 \\
\hline Manganese & WA & 247 & 50 & $\mu \mathrm{g} / \mathrm{L}$ & UTR 123 \\
\hline Manganese & WA & 84.8 & 50 & $\mu \mathrm{g} / \mathrm{L}$ & UTR127 \\
\hline Manganese & WA & 1550 & 50 & $\mu \mathrm{g} / \mathrm{L}$ & UTR 142 \\
\hline Manganese & WA & 133 & 50 & $\mu \mathrm{g} / \mathrm{L}$ & UTR155 \\
\hline
\end{tabular}


Table 14. Analytes Detected above the PDWS or MCI. during the Spring Sampling Event

\begin{tabular}{|c|c|c|c|c|c|}
\hline Analyte & Lab & Result & Standard & Units & Location \\
\hline Cadmium & WA & 6.5 & 5 & $\mu \mathrm{g} / \mathrm{L}$ & UTR004 \\
\hline Cadmium & WA & 9.1 & 5 & $\mu \mathrm{g} / \mathrm{L}$ & UTR036 \\
\hline Cadmium & WA & 10.7 & 5 & $\mu \mathrm{g} / \mathrm{L}$ & UTR066 \\
\hline Cadmium & WA & 7.5 & 5 & $\mu \mathrm{g} / \mathrm{L}$ & UTR!16 \\
\hline Gross alpha & TM & 27.8 & 15 & $\mathrm{pCi} / \mathrm{L}$ & BGSOO2 \\
\hline Gross alpha & TM & 49.1 & 15 & $\mathrm{pCi} / \mathrm{L}$ & UTR142 \\
\hline Nonvolatile beta & TM & 67.3 & 50 & $\mathrm{pCi} / \mathrm{L}$ & BGS002 \\
\hline Nonvolatile beta & TM & 61.2 & 50 & $\mathrm{pCi} / \mathrm{L}$ & BGS004 \\
\hline Nonvolatile beta & TM & 79 & 50 & $\mathrm{pCi} / \mathrm{L}$ & UTR142 \\
\hline Potassium-40 & TM & 2023 & 300 & $\mathrm{pCi} / \mathrm{L}$ & UTR100 \\
\hline Potassium-40 & TM & 797.3 & 300 & $\mathrm{pCi} / \mathrm{L}$ & UTR102 \\
\hline Potassium-40 & TM & 766 & 300 & $\mathrm{pCi} / \mathrm{L}$ & UTR109 \\
\hline Potassium-40 & $\mathbf{T M}$ & 2023 & 300 & $\mathrm{pCi} / \mathrm{L}$ & UTR112 \\
\hline Potassium-40 & TM & 2084 & 300 & $\mathrm{pCi} / \mathrm{L}$ & UTR114 \\
\hline Potassium-40 & TM & 851.7 & 300 & $\mathrm{pCi} / \mathrm{L}$ & UTR116 \\
\hline Ruthenium-106 & TM & 52.4 & 30 & $\mathrm{pCi} / \mathrm{L}$ & UTR075 \\
\hline Trichloroethylene & WA & 5.09 & 5 & $\mu \mathrm{g} / \mathrm{L}$ & UTR155 \\
\hline Tritium & TM & 41.3 & 20 & $\mathrm{pCi} / \mathrm{ml}$ & UTD002 \\
\hline Tritium & TM & 68.2 & 20 & $\mathrm{pCi} / \mathrm{ml}$ & UTD003 \\
\hline Tritium & TM & 39.35 & 20 & $\mathrm{pCi} / \mathrm{ml}$ & UTD004 \\
\hline Tritium & TM & 22.3 & 20 & $\mathrm{pCi} / \mathrm{ml}$ & UTD005 \\
\hline Tritium & TM & 22.3 & 20 & $\mathrm{pCi} / \mathrm{ml}$ & UTR004 \\
\hline Tritium & TM & 33.1 & 20 & $\mathrm{pCi} / \mathrm{ml}$ & UTR024 \\
\hline Tritium & TM & 21.6 & 20 & $\mathrm{pCi} / \mathrm{ml}$ & UTR029 \\
\hline Tritium & TM & 20.6 & 20 & $\mathrm{pCi} / \mathrm{ml}$ & UTR033 \\
\hline Tritium & TM & 32.6 & 20 & $\mathrm{pCi} / \mathrm{ml}$ & UTR068 \\
\hline Tritium & TM & 134.2 & 20 & $\mathrm{pCi} / \mathrm{ml}$ & UTR114 \\
\hline Tritium & TM & 143.5 & 20 & $\mathrm{pCi} / \mathrm{ml}$ & UTR116 \\
\hline Tritium & TM & 24.85 & 20 & $\mathrm{pCi} / \mathrm{ml}$ & UTR133 \\
\hline Tritium & TM & 30.6 & 20 & $\mathrm{pCi} / \mathrm{ml}$ & UTR155 \\
\hline
\end{tabular}


Table 15. Comparison of Analytes Detected at Wetland and Background Locations During Fall Sampling Event

\begin{tabular}{|c|c|c|c|c|c|c|c|}
\hline Analyte & $\begin{array}{l}\text { Number of } \\
\text { Wetland } \\
\text { Locations }\end{array}$ & $\begin{array}{c}\text { Number of } \\
\text { Detects }\end{array}$ & Percen & $\begin{array}{c}\text { Number of } \\
\text { Backgrounc } \\
\text { Locations }\end{array}$ & $\begin{array}{c}\text { Number } 0 \\
\text { Detects }\end{array}$ & & Percen \\
\hline Acidity & 36 & 36 & 100 & 2 & 2 & & 100 \\
\hline Alkalinity & 36 & 25 & 69 & 2 & 0 & 7 & 0 \\
\hline Aluminum & 36 & 12 & 33 & 2 & 2 & & 100 \\
\hline Calcium & 36 & 3 & 8 & 2 & 0 & & 0 \\
\hline Cesium-137 & 36 & 3 & 8 & 2 & 0 & & $\mathbf{0}$ \\
\hline Chloride & 36 & 35 & 97 & 2 & 2 & & 100 \\
\hline Chromium & 36 & 3 & 8 & 2 & 0 & & $\mathbf{0}$ \\
\hline Gross alpha & 36 & 23 & 64 & 2 & 2 & & 100 \\
\hline Iron & 36 & 32 & 89 & 2 & 2 & & 100 \\
\hline Lead & 36 & 2 & 6 & 2 & 1 & & 50 \\
\hline Manganese & 36 & 22 & 61 & 2 & 2 & & 100 \\
\hline Mercury-2031 & 36 & 1 & 3 & 2 & 0 & & 0 \\
\hline Nonvolatile beta & 36 & 26 & 72 & 2 & 1 & & 50 \\
\hline Sodium & 36 & 4 & 11 & 2 & $\mathbf{0}$ & & $\mathbf{0}$ \\
\hline Sulfate & 36 & 10 & 28 & 2 & 1 & & 50 \\
\hline Total hardness & 36 & 27 & 75 & 2 & 2 & & 100 \\
\hline Total organic carbon & 36 & 36 & 100 & 2 & 2 & & 100 \\
\hline Total suspended solids & 36 & 30 & 83 & 2 & 2 & & 100 \\
\hline 1,2-Dichloroethylene (total) & 36 & 1 & 3 & 2 & 0 & & 0 \\
\hline Tritium & 36 & 36 & 100 & 2 & 2 & & 100 \\
\hline Turbidity & 36 & 20 & 56 & 2 & 0 & & 0 \\
\hline & 36 & 20 & 56 & 2 & 2 & & 100 \\
\hline
\end{tabular}

1The half-life of mercury-203 is $\mathbf{4 6 . 5}$ days. Therefore, this result is believed to be a false positive. 
Table 16. Comparison of analytes detected at Stream and Background Locations During Fall Sampling Event

\begin{tabular}{|c|c|c|c|c|c|c|}
\hline Analyte & $\begin{array}{c}\text { Number of } \\
\text { Stream } \\
\text { Locations } \\
\end{array}$ & $\begin{array}{c}\text { Number of } \\
\text { Detects }\end{array}$ & Percen & $\begin{array}{c}\text { Number of } \\
\text { Backgrounc } \\
\text { Locations }\end{array}$ & $\begin{array}{c}\text { Number of } \\
\text { Detects } \\
\end{array}$ & Percen \\
\hline Acidity & 6 & 6 & 100 & 2 & 2 & 100 \\
\hline Alkalinity & 6 & 6 & 100 & 2 & 2 & 100 \\
\hline Aluminum & 6 & 1 & 17 & 2 & 2 & 100 \\
\hline Americium-241 1 & 6 & 1 & 17 & 2 & $\cdot 0$ & 0 \\
\hline Arsenic & 6 & 0 & 0 & 2 & 1 & 50 \\
\hline Chloride & 6 & 6 & 100 & 2 & 2 & 100 \\
\hline Gross alpha & 6 & 5 & 83 & 2 & 2 & 100 \\
\hline Iron & 6 & 6 & 100 & 2 & 2 & 100 \\
\hline Lead & 6 & 1 & 17 & 2 & 1 & 50 \\
\hline Manganese & 6 & 4 & 67 & 2 & 2 & 100 \\
\hline Nonvolatile beta & 6 & 3 & 50 & 2 & 2 & 100 \\
\hline Sodium & 6 & 4 & 67 & 2 & 0 & 0 \\
\hline Sulfate & 6 & 3 & 50 & 2 & 0 & 0 \\
\hline Total hardness & 6 & 5 & 83 & 2 & 2 & 100 \\
\hline Total organic carbon & 6 & 6 & 100 & 2 & 2 & 100 \\
\hline Total suspended solids & 6 & 6 & 100 & 2 & 2 & 100 \\
\hline Tritium & 6 & 6 & 100 & 2 & 2 & 100 \\
\hline Zinc & 6 & 2 & 33 & 2 & 1 & 50 \\
\hline
\end{tabular}

Tross radiological indicators did not support the presence of any of these radionuclides in the samples. 
Table 17. Analytes Detected above the SDWS during the Fall Sampling Event

\begin{tabular}{lccccc} 
Analyte & Lab & Result & Standard & Units & Location \\
\hline Iron & WA & 1745 & 300 & $\mu \mathrm{g} / \mathrm{L}$ & BGD001 \\
Iron & WA & 146000 & 300 & $\mu \mathrm{g} / \mathrm{L}$ & BGD002 \\
Iron & WA & 7690 & 300 & $\mu \mathrm{g} / \mathrm{L}$ & BGS002 \\
Iron & WA & 580 & 300 & $\mu \mathrm{g} / \mathrm{L}$ & BGS003 \\
Manganese & WA & 90.6 & 50 & $\mu \mathrm{g} / \mathrm{L}$ & BGD001 \\
Manganese & WA & 278 & 50 & $\mu \mathrm{g} / \mathrm{L}$ & BGD002 \\
Manganese & WA & 96.1 & 50 & $\mu \mathrm{g} / \mathrm{L}$ & $\mathrm{BGS003}$ \\
Manganese & WA & 67.9 & 50 & $\mu \mathrm{g} / \mathrm{L}$ & UTD003 \\
Manganese & WA & 79.1 & 50 & $\mu \mathrm{g} / \mathrm{L}$ & UTD005 \\
Manganese & WA & 103 & 50 & $\mu \mathrm{g} / \mathrm{L}$ & UTD006 \\
Manganese & WA & 308 & 50 & $\mu \mathrm{g} / \mathrm{L}$ & UTR022 \\
Manganese & WA & 795 & 50 & $\mu \mathrm{g} / \mathrm{L}$ & UTR029 \\
Manganese & WA & 52.9 & 50 & $\mu \mathrm{L} / \mathrm{L}$ & UTR051A \\
Manganese & WA & 53.1 & 50 & $\mu \mathrm{g} / \mathrm{L}$ & UTR071 \\
Manganese & WA & 215 & 50 & $\mu \mathrm{g} / \mathrm{L}$ & UTR072 \\
Manganese & WA & 188 & 50 & $\mu \mathrm{g} / \mathrm{L}$ & UTR099 \\
Manganese & WA & 57.8 & 50 & $\mu \mathrm{g} / \mathrm{L}$ & UTR108 \\
Manganese & WA & 73.9 & 50 & $\mu \mathrm{g} / \mathrm{L}$ & UTR112 \\
Manganese & WA & 143 & 50 & $\mu \mathrm{g} / \mathrm{L}$ & UTR122 \\
Manganese & WA & 60.7 & 50 & $\mu \mathrm{g} / \mathrm{L}$ & UTR127 \\
\hline
\end{tabular}


Table 18. Analytes Detected above the PDWS or MCL during the Fall Sampling Event

\begin{tabular}{lccccc} 
Analyte & Lab & Result & Standard & Units & Location \\
\hline Tritium & TM & 55.2 & 20 & $\mathrm{pCi} / \mathrm{ml}$ & UTD002 \\
Tritium & TM & 103.5 & 20 & $\mathrm{pCi} / \mathrm{ml}$ & UTD003 \\
Tritium & TM & 53.45 & 20 & $\mathrm{pCi} / \mathrm{ml}$ & UTD004 \\
Tritium & TM & 28.22 & 20 & $\mathrm{pCi} / \mathrm{ml}$ & UTD005 \\
Tritium & TM & 26.76 & 20 & $\mathrm{pCi} / \mathrm{ml}$ & UTD006 \\
Tritium & TM & 32.24 & 20 & $\mathrm{pCi} / \mathrm{ml}$ & UTR020 \\
Tritium & TM & 132.05 & 20 & $\mathrm{pCi} / \mathrm{ml}$ & UTR022 \\
Tritium & TM & 28.4 & 20 & $\mathrm{pCi} / \mathrm{ml}$ & UTR024 \\
Tritium & TM & 23.1 & 20 & $\mathrm{pCi} / \mathrm{ml}$ & UTR029 \\
Tritium & TM & 20.2 & 20 & $\mathrm{pCi} / \mathrm{ml}$ & UTR033 \\
Tritium & TM & 27.3 & 20 & $\mathrm{pCi} / \mathrm{ml}$ & UTR066 \\
Tritium & TM & 73.84 & 20 & $\mathrm{pCi} / \mathrm{ml}$ & UTR068 \\
Tritium & TM & 44.4 & 20 & $\mathrm{pCi} / \mathrm{ml}$ & UTR071 \\
Tritium & TM & 34.79 & 20 & $\mathrm{pCi} / \mathrm{ml}$ & UTR072 \\
Tritium & TM & 272 & 20 & $\mathrm{pCi} / \mathrm{ml}$ & UTR114 \\
Tritium & TM & 111.9 & 20 & $\mathrm{pCi} / \mathrm{ml}$ & UTR116 \\
Tritium & TM & 53.48 & 20 & $\mathrm{pCi} / \mathrm{ml}$ & UTR120 \\
Tritium & TM & 21.18 & 20 & $\mathrm{pCi} / \mathrm{ml}$ & UTR147 \\
\hline
\end{tabular}




\section{SUMMARY}

The results of this study are comparable to the data reported by Haselow, et al. (1992). Results of the spring and fall sampling of the Upper Three Runs wetlands suggest that tritium is outcropping in the wetlands and surrounding drainages. Samples collected from 13 locations ( 9 wetland and 4 stream) in the spring and 18 locations (13 wetland and 5 stream) in the fall contained tritium above the MCL. However, evaluation of the analytical results for both the spring and fall sampling events did not reveal any evidence that the Upper Three Runs wetlands are being impacted by waste (Cs-134,137) migrating from Tank 16.

Most of the remaining analytical data for both the spring and fall sampling events were reported as below method detection limits. The primary exceptions were the routine water quality indicators ( e.g., turbidity, alkalinity, total suspended solids, etc.), iron and manganese. Iron and manganese were detected above the secondary drinking water standards at several wetland and stream locations (including background locations) during both the spring and fall sampling events. The levels reported are typical of wetland and stream water in this area (Dixon and Rogers, 1993; Haselow, 1990; Haselow et al., 1992).

During the spring, cadmium, gross alpha, nonvolatile beta, potassium-40, ruthenium-106, and trichloroethylene were also detected above SWDS or MCL from at least one location. Cadmium was detected above the MCL at four wetland locations. Gross alpha was detected above the MCL at two wetland locations, one of which was a background location. Nonvolatile beta was detected above the MCL at three wetland locations, two of which were background locations. Trichloroethylene was detected at one wetland location at $5.09 \mu \mathrm{g} / \mathrm{L}$, which is slightly above the detection limit and MCL of $5 \mu \mathrm{g} / \mathrm{L}$. None of these contaminants were detected above SWDS or MCL during the fall. 


\section{REFERENCES}

Carlton, W.H, L.R. Bauer, A.G. Evans, L.A. Geary, C.E. Murphy, J.E. Pinder, and R.N. Strom. 1992. Cesium in the Savannah River Site Environment. WSRC-RP-92-250. Westinghouse Savannah River Company, Savannah River Technology Center, Aiken SC.

Dixon, K.L. and V.A. Rogers. 1993. Semi-Annual Sampling of Fourmile Branch and its Seeplines in the F- and HAreas of SRS: July 1992. WSRC-TR-93-289, Westinghouse Savannah River Company, Savannah River Technology Center, Aiken SC.

Groundwater Protection Standard. 1982. Resource Conservation and Recovery Act. 40 CFR 264.94(a2), Table 1. Washington, D.C.

Haselow, J.S., M. Harris, B.B. Looney, N.V. Halverson, and J.B. Gladden 1990. Analysis of Soil and Water from the Four Mile Creek Seepline Near the F- and H-Areas of SRS. WSRC-RP-90-0591, Westinghouse Savannah River Company, Savannah River Technology Center, Aiken SC.

Haselow, L.A., V.A. Rogers, C.J. Riordan, G.W. Eidson, M.K. Herring. 1992. Analysis of Water and Soil from the Wetlands of Upper Three Runs Creek (U). WSRC-TR-92-0415, Westinghouse Savannah River Company, Savannah River Technology Center, Aiken SC.

Jaegge, W.J., N.L. Knolb, B.B. Looney, I.W. Marine, O.A. Towler, and J.R. Cook. 1985. Radioactive Waste Burial Grounds. DPST-85-694, Savannah River Laboratory, Aiken, SC.

Metcalf and Eddy. 1993a. Wetland and Stream Sampling of UTR: Spring Sampling - Final Report. Submitted to Westinghouse Savannah River Company, Aiken, SC.

Metcalf and Eddy, 1993b. Wetland and Stream Sampling of UTR: Fall Sampling - Final Report. Submitted to Westinghouse Savannah River Company, Aiken, SC.

Poe, W.L., J.W. Fenimore, J.H. Horton, I.W. Marine, W.E. Prout. 1974. Leakage from Waste Tank 16, Amount, Fate, and Impact. DP-1358, Savannah River Laboratory, E.I. duPont de. Nemours \& Co., Aiken, SC.

Science Applications International Corporation (SAIC). 1992. Hydrogeological Characterization of the Mixed Waste Management Facility. WSRC-RP-92-837, Rev 2.0, Westinghouse Savannah River Company, Savannah River Technology Center, Aiken SC.

U.S. Department of Health, Education, and Welfare. 1970. Radiological Health Handbook, Rockville, MD.

U.S. Environmental Protection Agency (EPA). 1977. National Interim Primary Drinking Water Regulations. EPA570/9-76-003, Washington, D.C.

U.S. Environmęntal Protection Agency (EPA). 1980. Prescribed Procedures for Measurement of Radioactivity in Drinking Water. EPA-600/4-80-032, Cincinati, $\mathrm{OH}$.

U.S. Environmental Protection Agency (EPA). 1983. Methods for Chemical Analysis of Water and Wastes. PB84128677, Cincinati, OH.

U.S. Environmental Protection Agency (EPA). 1986a. Water Pollution Control; National Primary Drinking Water Regulations; Radionuclides; Advance Notice of Proposed Rulemaking, Federal Register, September 30, 1986, pp. 34835-34862. Washington, D.C.

U.S. Environmental Protection Agency (EPA). 1986b. Test Methods for Evaluating Solid Waste, Volumes 1A, 1B, and $1 C$, third edition, SW-846 Washington, D.C.

U.S. Environmental Protection Agency (EPA). 1991a. National Primary Drinking Water Regulations, Code of Federal Regulations, Title 40, Part 141, pp. 578-715, Washington, D.C. 
U.S. Environmental Protection Agency (BPA). 1991b. National Secondary Drinking Water Regulations, Code of Federal Regulations, Title 40, Part 143, pp. 758-762, Washington, D.C.

U.S. Environmental Protection Agency (EPA). 1991c. National Primary Drinking Water Regulations; Radionuclides; Proposed, Federal Register, July 18, 1991, pp. 33052-33127, Washington, D.C.

U.S. Environmental Protection Agency (EPA). 1993a. National Primary Drinking Water Regulations -'Safe Drinking Water Act Maximum Contaminant Levels (MCL), Code of Federal Regulations, Title 40, Part 141, Washington, D.C.

U.S. Environmental Protection Agency (EPA). 1993b. "National Primary Drinking Water Regulations - Safe Drinking Water Act Maximum Contaminant Levels (MCL)", Federal Register, 57FR31776, Effective 1/17/1994, Washington, D.C.

WSRC-RP-90-497. 1990. RCRA Facility Investigation/Remedial Investigation Work Plan for Tank 16. Rev 2. Westinghouse Savannah River Company, Aiken SC.

WSRC-IM-91-53. 1991a. Mixed Waste Management Facility Corrective Action Plan - Final Draft. Westinghouse Savannah River Company, Aiken SC.

WSRC-IM-91-53. 1991b. F-Area Hazardous Waste Management Facility Postclosure, 1992 RCRA Part B Permit Renewal Application. Westinghouse Savannah River Company, Aiken SC.

WSRC-IM-91-53. 1991c. H-Area Hazardous Waste Management Facility Postclosure, 1992 RCRA Part B Permit Renewal Application. Westinghouse Savannah River Company, Aiken SC.

WSRC-3Q5 (Rev.1). 1992. Hydrogeologic Data Collection. Westinghouse Savannah River Company, Aiken SC.

WSRC-L14.1. 1993a. Procedure for Seepline and Wetland Surface Water Sampling. Procedure 2-104, Rev. 0. Westinghouse Savannah River Company, Aiken SC.

WSRC-L14.1. 1993b. Procedure for Stream Velocity and Discharge Measurement. Procedure 2-102, Rev. 0. Westinghouse Savannah River Company, Aiken SC. 
Appendix A

Analytical Results

Spring Sampling Event 


\section{APPENDIX A}

\section{Analytical Results - Spring Sampling Event}

$\gamma$

Analytical results from the spring sampling event of the UTR wetlands sampling project are presented in this section. The analytical data for each location (wetland and stream) are presented in tabular format with the background samples presented first followed by the stream samples, wetland samples, and QAVC samples. Locations ending with the suffix " $D$ " designate drainage, or stream sampling locations. These drainage, or stream, samples were collected from perennial tributaries to UTR rather than the main channel of UTR. All other location IDs, excluding QA samples, designate wetland locations (i.e. UTR or BGS). Location identification numbers beginning with the prefix "BG" designate background locations.

For each sampling location, the sampling date, field measurements including $\mathrm{pH}_{\text {, conductivity, etc., and all }}$ laboratory analyses are presented (excluding lab blanks and standards). Included in the laboratory analyses tables for each location and analyte is the lab that performed the analyses, any result or analytical qualifiers, the analytical results, the accuracy of the result (for radiological analyses only), any standard (i.e. PDWS, MCL, SDWS), and the units of measurement.

The following is a list of abbreviations used in the tables:

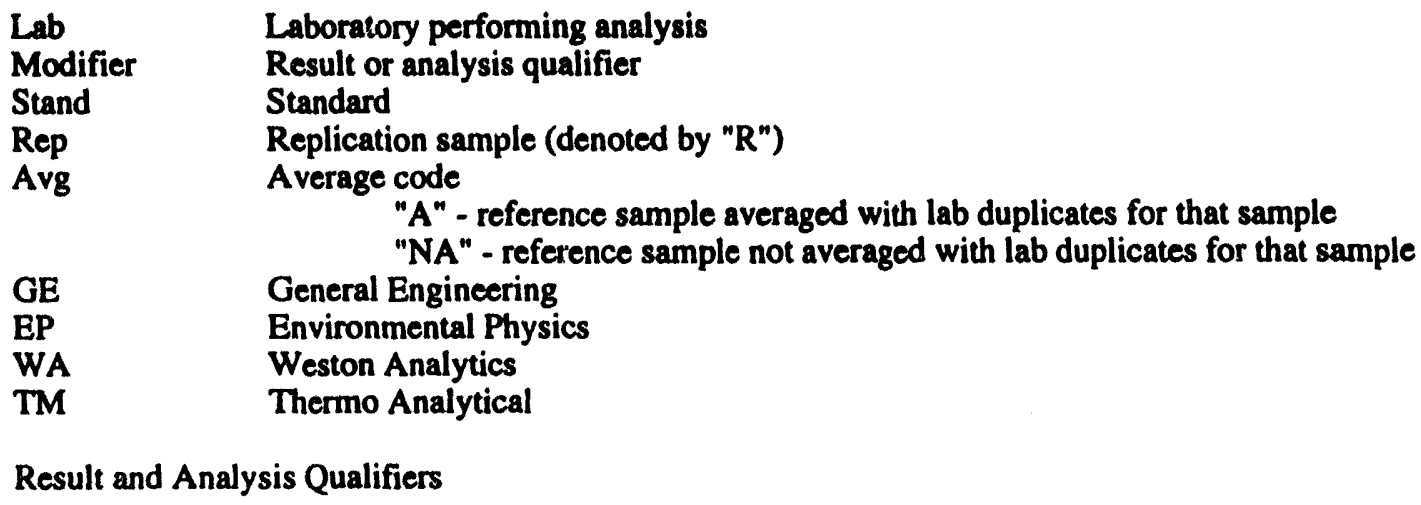

Result and Analysis Qualifiers

\begin{tabular}{|l|l|}
\hline Result Qualifiers \\
\hline CODE & DESCRIPTION \\
\hline (BLANK) & Data not remarked. Number should be interpreted exactly as reported. \\
\hline $\mathrm{J}$ & The analytical result is an estimated quantity. \\
\hline $\mathrm{R}$ & $\begin{array}{l}\text { Rejected because of performance requirements in the sample or associated quality contro } \\
\text { analyses were not met. The analyte may or may not be present. }\end{array}$ \\
\hline $\mathrm{U}$ & Material analyzed for but not detected. Value reported is the sample quantification limit. \\
\hline
\end{tabular}

\begin{tabular}{|l|l|}
\hline \multicolumn{2}{|l|}{ Analysis Qualifiers } \\
\hline CODE & DESCRIPTION \\
\hline $\mathrm{Q}$ & $\begin{array}{l}\text { Sample held beyond normal holding time. If holding time is exceeded by less than } 30 \text { das } \\
\text { used with "J". If holding time is exceeded by more than } 30 \text { days, used with "R". }\end{array}$ \\
\hline $\mathrm{L}$ & Analytical factor causing bias. The associated result may under estimate the true value. \\
\hline $\mathrm{H}$ & Analytical factor causing bias. The associated result may over estimate the true value. \\
\hline $\mathrm{P}$ & Analytical factor causing bias. The associated result may be of high variability.. \\
\hline $\mathrm{V}$ & Indicates the analyte was detected in both the sample and associated method blank. \\
\hline
\end{tabular}




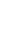




\section{BGD001}

Field Measurements:

Stream Discharge: $0.03 \mathrm{cfs}$

Sample Date: 4/28/93

pH: 7.12

Specific Conductance: $13 \mu \mathrm{S} / \mathrm{cm}$

Red/Oxid Potential: $196 \mathrm{mv}$

Dissolved Oxygen: $8.6 \mathrm{mg} / \mathrm{L}$

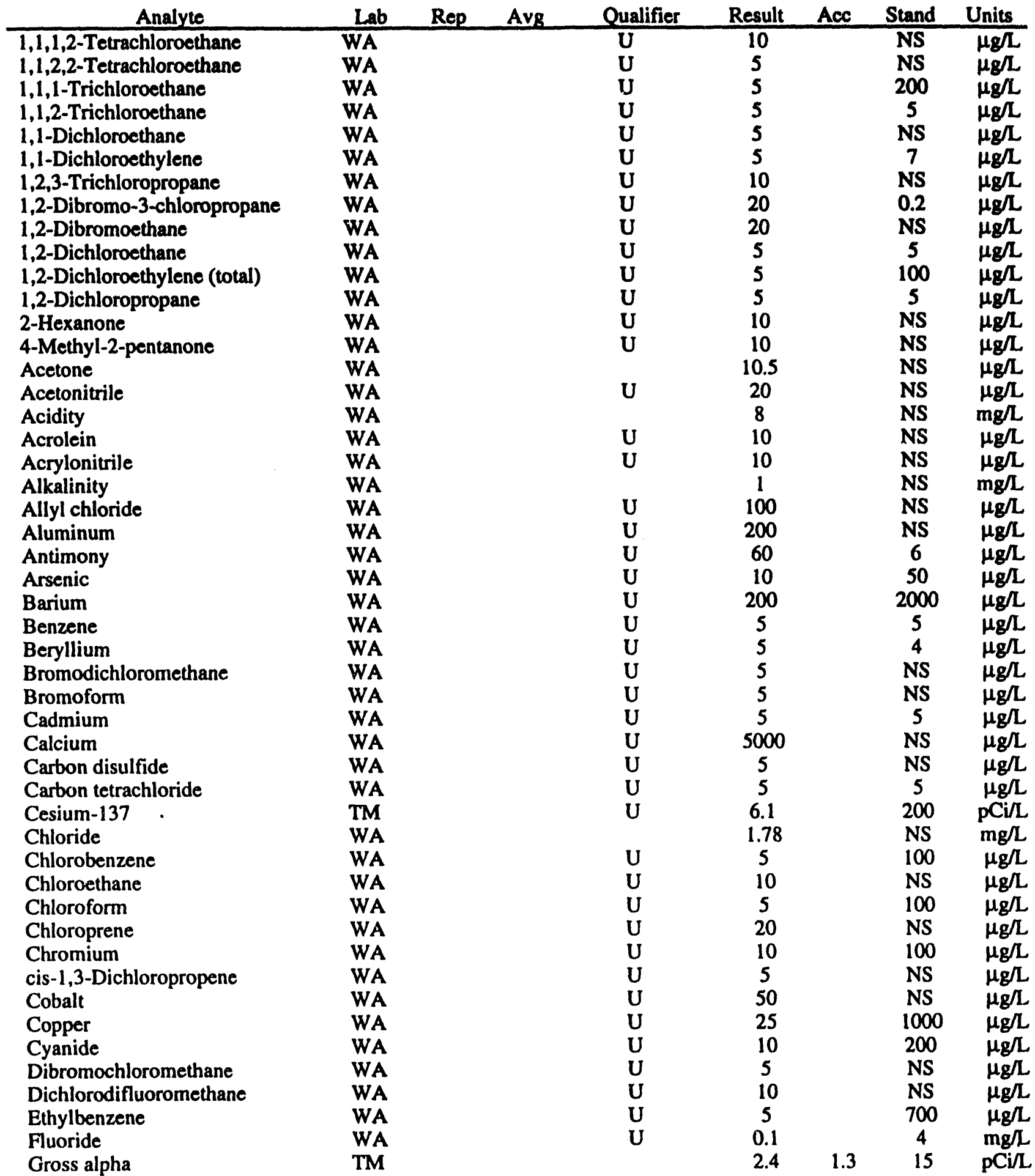


BGD001 Laboratory Analyses (cont'd)

\begin{tabular}{|c|c|c|c|c|c|c|c|c|}
\hline Analyte & Lab & Rep & Avg & Qualifier & Result & Acc & Stand & Units \\
\hline Iron & WA & & & & 114 & & 300 & $\mu g / L$ \\
\hline Isobutyl alcohol & WA & & & $\mathbf{U}$ & 20 & & NS & $\mu \mathrm{g} / \mathrm{L}$ \\
\hline Lead & WA & & & U & 3 & & 50 & $\mu \mathrm{g} / \mathrm{L}$ \\
\hline Magnesium & WA & & & $\mathbf{U}$ & 5000 & & NS & $\mu g / L$ \\
\hline Manganese & WA & & & & 27.9 & & 50 & $\mu g / L$ \\
\hline Mercury & WA & & & $\mathbf{U}$ & 0.2 & & 2 & $\mu g / L$ \\
\hline Methacrylonitrile & WA & & & $\mathbf{U}$ & 20 & & NS & $\mu g / L$ \\
\hline Methyl bromide & WA & & & $\mathbf{U}$ & 10 & & NS & $\mu \mathrm{g} / \mathrm{L}$ \\
\hline Methyl chloride & WA & & & $\mathbf{U}$ & 10 & & NS & $\mu g / L$ \\
\hline Methyl ethyl ketone & WA & & & $\mathbf{U}$ & 10 & & NS & $\mu \mathrm{g} / \mathrm{L}$ \\
\hline Methyl iodide & WA & & & $\mathbf{U}$ & 10 & & NS & $\mu \mathrm{g} / \mathrm{L}$ \\
\hline Methylene bromide & WA & & & $\mathbf{U}$ & 10 & & NS & $\mu g / L$ \\
\hline Methylene chloride & WA & & & $\mathbf{U}$ & 5 & & 5 & $\mu \mathrm{g} / \mathrm{L}$ \\
\hline Nickel & WA & & & $\mathbf{U}$ & 40 & & 100 & $\mu \mathrm{g} / \mathrm{L}$ \\
\hline Nitrate as nitrogen & WA & & & $\mathbf{U}$ & 0.02 & & 10 & $\mathrm{mg} / \mathrm{L}$ \\
\hline Nonvolatile beta & TM & & & & 13 & 2.7 & 50 & $\mathrm{pCi} / \mathrm{L}$ \\
\hline Potassium & WA & & & $\mathbf{U}$ & 5000 & & NS & $\mu g / L$ \\
\hline Propionitrile & WA & & & $\mathbf{U}$ & 50 & & NS & $\mu g / L$ \\
\hline Selenium & WA & & & $\mathbf{U}$ & 5 & & 50 & $\mu g / L$ \\
\hline Silver & WA & & & $\mathbf{U}$ & 10 & & 50 & $\mu g / L$ \\
\hline Sodium & WA & & & $\mathbf{U}$ & 5000 & & NS & $\mu \mathrm{g} / \mathrm{L}$ \\
\hline Styrene & WA & & & $\mathbf{U}$ & 5 & & 100 & $\mu \mathrm{g} / \mathrm{L}$ \\
\hline Sulfate & WA & & & $\mathbf{U}$ & 2.5 & & 400 & $\mathrm{mg} / \mathrm{L}$ \\
\hline Sulfide & WA & & & $\mathbf{U}$ & 0.1 & & NS & $\mathrm{mg} / \mathrm{L}$ \\
\hline Tetrachloroethylene & WA & & & $\mathbf{U}$ & 5 & & 5 & $\mu g / L$ \\
\hline Thallium & WA & & & $\mathbf{U}$ & 10 & & 2 & $\mu \mathrm{g} / \mathrm{L}$ \\
\hline Toluene & WA & & & $\mathbf{U}$ & 5 & & 1000 & $\mu g / L$ \\
\hline Total hardness & WA & & & $\mathbf{U}$ & 0.1 & & NS & $\mathrm{mg} / \mathrm{L}$ \\
\hline Total organic carbon & WA & & & & 1.42 & & NS & $\mathrm{mg} / \mathrm{L}$ \\
\hline Total suspended solids & WA & & & & 12 & & NS & $\mathrm{mg} / \mathrm{L}$ \\
\hline trans-1,3-Dichloropropene & WA & & & $\mathbf{U}$ & 5 & & NS & $\mu \mathrm{g} / \mathrm{L}$ \\
\hline trans-1,4-Dichloro-2-butene & WA & & & $\mathbf{U}$ & 100 & & NS & $\mu \mathrm{g} / \mathrm{L}$ \\
\hline Trichloroethylene & WA & & & $\mathbf{U}$ & 5 & & 5 & $\mu \mathrm{g} / \mathrm{L}$ \\
\hline Trichlorofluoromethane & WA & & & $\mathbf{U}$ & 5 & & NS & $\mu \mathrm{g} / \mathrm{L}$ \\
\hline Tritium & TM & & & & 1.9 & 0.89 & 20 & $\mathrm{pCi} / \mathrm{ml}$ \\
\hline Turbidity & WA & & & & 19.2 & & NS & NTU \\
\hline Vanadium & WA & & & $\mathbf{U}$ & 50 & & NS & $\mu \mathrm{g} / \mathrm{L}$ \\
\hline Vinyl acetate & WA & & & $\mathbf{U}$ & 10 & & NS & $\mu \mathrm{g} / \mathrm{L}$ \\
\hline Vinyl chloride & WA & & & $\mathbf{U}$ & 10 & & 2 & $\mu \mathrm{g} / \mathrm{L}$ \\
\hline Xylenes (total) & WA & & & $\mathbf{U}$ & 5 & & 10000 & $\mu \mathrm{g} / \mathrm{L}$ \\
\hline Zinc & WA & & & $\mathbf{U}$ & 20 & & 5000 & $\mu g / L$ \\
\hline
\end{tabular}




\section{BGD002}

Field Measurements:

Stream Discharge: $0.2 \mathrm{cfs}$

Sample Date: 4/27/93

pH: 6.25

Specific Conductance: $30 \mu \mathrm{S} / \mathrm{cm}$

Red/Oxid Potential: $111 \mathrm{mv}$

Dissolved Oxygen: $5.4 \mathrm{mg} / \mathrm{L}$

\begin{tabular}{|c|c|c|c|c|c|c|c|}
\hline Analyte & Lab & Avg & Oualifier & Result & Acc & Stand & Units \\
\hline 1,1,1,2-Tetrachloroethane & WA & & $\bar{U}$ & 10 & & NS & $\mu g / L$ \\
\hline 1,1,1-Trichloroethane & WA & & $\mathbf{U}$ & 5 & & 200 & $\mu g / L$ \\
\hline 1,1,2,2-Tetrachloroethane & WA & & $\mathbf{U}$ & 5 & & NS & $\mu g / L$ \\
\hline 1,1,2-Trichloroethane & WA & & $\mathbf{U}$ & 5 & & 5 & $\mu g / L$ \\
\hline 1,1-Dichloroethane & WA & & $\mathbf{U}$ & 5 & & NS & $\mu g / L$ \\
\hline 1,1-Dichloroethylene & WA & & $\mathbf{U}$ & 5 & & 7 & $\mu g / L$ \\
\hline 1,2,3-Trichloropropane & WA & & $\mathbf{U}$ & 10 & & NS & $\mu g / L$ \\
\hline 1,2-Dibromo-3-chloropropane & WA & & $\mathbf{U}$ & 20 & & 0.2 & $\mu g / L$ \\
\hline 1,2-Dibromoethane & WA & & $\mathbf{U}$ & 20 & & NS & $\mu g / L$ \\
\hline 1,2-Dichloroethane & WA & & $\mathbf{U}$ & 5 & & 5 & $\mu g / L$. \\
\hline 1,2-Dichloroethylene (total) & WA & & $\mathbf{U}$ & 5 & & 100 & $\mu g / L$ \\
\hline 1,2-Dichloropropane & WA & & $\mathbf{U}$ & 5 & & 5 & $\mu g / L$ \\
\hline 2-Hexanone & WA & & $\mathbf{U}$ & 10 & & NS & $\mu g / L$ \\
\hline 4-Methyl-2-pentanone & WA & & $\mathbf{U}$ & 10 & & NS & $\mu g / L$ \\
\hline Acetone & WA & & $\mathbf{U}$ & 10 & & NS & $\mu \mathrm{g} / \mathrm{L}$ \\
\hline Acetonitrile & WA & & $\mathbf{U}$ & 20 & & NS & $\mu \mathrm{g} / \mathrm{L}$ \\
\hline Acidity & WA & & & 22 & & NS & $\mathrm{mg} / \mathrm{L}$ \\
\hline Acrolein & WA & & $\mathbf{U}$ & 10 & & NS & $\mu g / L$ \\
\hline Acrylonitrile & WA & & $\mathbf{U}$ & 10 & & NS & $\mu g / L$ \\
\hline Alkalinity & WA & & $\mathbf{U}$ & 0.5 & & NS & $\mathrm{mg} / \mathrm{L}$ \\
\hline Allyl chloride & WA & & $\mathbf{U}$ & 100 & & NS & $\mu g / L$ \\
\hline Aluminum & WA & & $\mathbf{U}$ & 200 & & NS & $\mu g / L$ \\
\hline Antimony & WA & & $\mathbf{U}$ & 60 & & 6 & $\mu g / L$ \\
\hline Arsenic & WA & & $\mathbf{U}$ & 10 & & 50 & $\mu g / L$ \\
\hline Barium & WA & & $\mathbf{U}$ & 200 & & 2000 & $\mu g / L$ \\
\hline Benzene & WA & & $\mathbf{U}$ & 5 & & 5 & $\mu g / L$ \\
\hline Beryllium & WA & & $\mathbf{U}$ & 5 & & 4 & $\mu g / L$ \\
\hline Bromodichloromethane & WA & & $\mathbf{U}$ & 5 & & NS & $\mu g / L$ \\
\hline Bromoform & WA & & $\mathbf{U}$ & 5 & & NS & $\mu \mathrm{g} / \mathrm{L}$ \\
\hline Cadmium & WA & & $\mathbf{U}$ & 5 & & 5 & $\mu g / L$ \\
\hline Calcium & WA & & $\mathbf{U}$ & 5000 & & NS & $\mu \mathrm{g} / \mathrm{L}$ \\
\hline Carbon disulfide & WA & & $\mathbf{U}$ & 5 & & NS & $\mu g / L$ \\
\hline Carbon tetrachloride & WA & & $\mathbf{U}$ & 5 & & 5 & $\mu \mathrm{g} / \mathrm{L}$ \\
\hline Cesium-137 . & $\mathbf{T M}$ & & $\mathbf{U}$ & 5.8 & & 200 & $\mathrm{pCi} / \mathrm{L}$ \\
\hline Chloride & WA & & & 2.53 & & NS & $\mathrm{mg} / \mathrm{L}$ \\
\hline Chlorobenzene & WA & & $\mathbf{U}$ & 5 & & 100 & $\mu \mathrm{g} / \mathrm{L}$ \\
\hline Chloroethane & WA & & $\mathbf{U}$ & 10 & & NS & $\mu \mathrm{g} / \mathrm{L}$ \\
\hline Chloroform & WA & & $\mathbf{U}$ & 5 & & 100 & $\mu \mathrm{g} / \mathrm{L}$ \\
\hline Chloroprene & WA & & $\mathbf{U}$ & 20 & & NS & $\mu \mathrm{g} / \mathrm{L}$ \\
\hline Chromium & WA & & $\mathbf{U}$ & 10 & & 100 & $\mu \mathrm{g} / \mathrm{L}$ \\
\hline cis-1,3-Dichloropropene & WA & & $\mathbf{U}$ & 5 & & NS & $\mu \mathrm{g} / \mathrm{L}$ \\
\hline Cobalt & WA & & $\mathbf{U}$ & 50 & & NS & $\mu \mathrm{g} / \mathrm{L}$ \\
\hline Copper & WA & & $\mathbf{U}$ & 25 & & 1000 & $\mu \mathrm{g} / \mathrm{L}$ \\
\hline Cyanide & WA & & $\mathbf{U}$ & 10 & & 200 & $\mu \mathrm{g} / \mathrm{L}$ \\
\hline Dibromochloromethane & WA & & $\mathbf{U}$ & 5 & & NS & $\mu \mathrm{g} / \mathrm{L}$ \\
\hline Dichlorodifluoromethane & WA & & $\mathbf{U}$ & 10 & & NS & $\mu g / L$ \\
\hline Ethylbenzene & WA & & $\mathbf{U}$ & 5 & & 700 & $\mu g / L$ \\
\hline Fluoride & WA & & $\mathbf{U}$ & 0.1 & & 4 & $\mathrm{mg} / \mathrm{L}$ \\
\hline
\end{tabular}


BGD002 Laboratory Analyses (cont'd)

\begin{tabular}{|c|c|c|c|c|c|c|c|}
\hline Analyte & Lab & Rep & Qualifier & Result & Acc & Stand & Units \\
\hline Gross alpha & TM & & $\bar{U}$ & 0.4 & & 15 & $\mathrm{pCi} / \mathrm{L}$ \\
\hline Iron & WA & & & 1340 & & 300 & $\mu g / L$ \\
\hline Isobutyl alcohol & WA & & U & 20 & & NS & $\mu g / L$ \\
\hline Lead & WA & & U & 3 & & 50 & $\mu \mathrm{g} / \mathrm{L}$ \\
\hline Magnesium & WA & & $\mathbf{U}$ & 5000 & & NS & $\mu \mathrm{g} / \mathrm{L}$ \\
\hline Manganese & WA & & & 233 & & 50 & $\mu g / L$ \\
\hline Mercury & WA & & $\mathbf{U}$ & 0.2 & & 2 & $\mu g / L$ \\
\hline Methacrylonitrile & WA & & $\mathbf{U}$ & 20 & & NS & $\mu g / L$ \\
\hline Methyl bromide & WA & & $\mathbf{U}$ & 10 & & NS & $\mu g / L$ \\
\hline Methyl chloride & WA & & $\mathbf{U}$ & 10 & & NS & $\mu \mathrm{g} / \mathrm{L}$ \\
\hline Methyl ethyl ketone & WA & & $\mathbf{U}$ & 10 & & NS & $\mu g / L$ \\
\hline Methyl iodide & WA & & $\mathbf{U}$ & 10 & & NS & $\mu \mathrm{g} / \mathrm{L}$ \\
\hline Methylene bromide & WA & & $\mathbf{U}$ & 10 & & NS & $\mu g / L$ \\
\hline Methylene chloride & WA & & $\mathbf{U}$ & 5 & & 5 & $\mu g / L$ \\
\hline Nickel & WA & & $\mathbf{U}$ & 40 & & 100 & $\mu g / L$ \\
\hline Nitrate as nitrogen & WA & & $\mathbf{U}$ & 0.02 & & 10 & $\mathrm{mg} / \mathrm{L}$ \\
\hline Nonvolatile beta & TM & & $\mathbf{U}$ & 0.6 & & 50 & $\mathrm{pCi} / \mathrm{L}$ \\
\hline Potassium & WA & & $\mathbf{U}$ & 5000 & & NS & $\mu g / L$ \\
\hline Propionitrile & WA & & $\mathbf{U}$ & 50 & & NS & $\mu g / L$ \\
\hline Selenium & WA & & $\mathbf{U}$ & 5 & & 50 & $\mu g / L$ \\
\hline Silver & WA & & $\mathbf{U}$ & 10 & & 50 & $\mu g / L$ \\
\hline Sodium & WA & & $\mathbf{U}$ & 5000 & & NS & $\mu \mathrm{g} / \mathrm{L}$ \\
\hline Styrene & WA & & $\mathbf{U}$ & 5 & & 100 & $\mu g / L$ \\
\hline Sulfate & WA & & & 2.72 & & 400 & $\mathrm{mg} / \mathrm{L}$ \\
\hline Sulfide & WA & & $\mathbf{U}$ & 0.1 & & NS & $\mathrm{mg} / \mathrm{L}$ \\
\hline Tetrachloroethylene & WA & & $\mathbf{U}$ & 5 & & 5 & $\mu g / L$ \\
\hline Thallium & WA & & $\mathbf{U}$ & 10 & & 2 & $\mu \mathrm{g} / \mathrm{L}$ \\
\hline Toluene & WA & & $\mathbf{U}$ & 5 & & 1000 & $\mu g / L$ \\
\hline Total hardness & WA & & & 10 & & NS & $\mathrm{mg} / \mathrm{L}$ \\
\hline Total organic carbon & WA & & & 1.81 & & NS & $\mathrm{mg} / \mathrm{L}$ \\
\hline Total suspended solids & WA & & & 8 & & NS & $\mathrm{mg} / \mathrm{L}$ \\
\hline trans-1,3-Dichloropropene & WA & & $\mathbf{U}$ & 5 & & NS & $\mu g / L$ \\
\hline trans-1,4-Dichloro-2-butene & WA & & $\mathbf{U}$ & 100 & & NS & $\mu \mathrm{g} / \mathrm{L}$ \\
\hline Trichloroethylene & WA & & $\mathbf{U}$ & 5 & & 5 & $\mu g / L$ \\
\hline Trichlorofluoromethane & WA & & $\mathbf{U}$ & 5 & & NS & $\mu g / L$ \\
\hline Tritium & $\mathrm{TM}$ & & & 1.9 & 0.5 & 20 & $\mathrm{pCi} / \mathrm{ml}$ \\
\hline Turbidity & WA & & & Q $\quad 1.37$ & & NS & NTU \\
\hline Vanadium & WA & & $\mathrm{U}$ & 50 & & NS & $\mu \mathrm{g} / \mathrm{L}$ \\
\hline Vinyl acetate & WA & & U & 10 & & NS & $\mu g / L$ \\
\hline Vinyl chloride & WA & & $\mathbf{U}$ & 10 & & 2 & $\mu g / L$ \\
\hline Xylenes (total) & WA & & U & 5 & & 10000 & $\mu g / L$ \\
\hline Zinc & WA & & $\mathbf{U}$ & 20 & & 5000 & $\mu g / L$ \\
\hline
\end{tabular}




\section{BGS001}

Field Measurements:

Sample Date: 4/22/93

$\mathrm{pH}: .5 .52$

Specific Conductance: $28 \mu \mathrm{S} / \mathrm{cm}$

Red/Oxid Potential: $106 \mathrm{mv}$

Dissolved Oxygen: $1.1 \mathrm{mg} / \mathrm{L}$

\begin{tabular}{|c|c|c|c|c|c|c|c|c|}
\hline Analyte & Lab & Rep & Avg & Qualifier & Result & Acc & Stand & Units \\
\hline 1,1,1,2-Tetrachloroethane & WA & & & $\mathbf{U}$ & 10 & & NS & $\mu g / L$ \\
\hline 1,1,1-Trichloroethane & WA & & & $\mathbf{U}$ & 5 & & 200 & $\mu g / L$ \\
\hline 1,1,2,2-Tetrachloroethane & WA & & & $\mathbf{U}$ & 5 & & NS & $\mu g / L$ \\
\hline 1,1,2-Trichloroethane & WA & & & $\mathbf{U}$ & 5 & & 5 & $\mu g / L$ \\
\hline 1,1-Dichloroethane & WA & & & $\mathbf{U}$ & 5 & & NS & $\mu g / L$ \\
\hline 1,1-Dichloroethylene & WA & & & $\mathbf{U}$ & 5 & & 7 & $\mu g / L$ \\
\hline 1,2,3-Trichloropropane & WA & & . & $\mathbf{U}$ & 10 & & NS & $\mu g / L$ \\
\hline 1,2-Dibromo-3-chloropropane & WA & & & $\mathbf{U}$ & 20 & & 0.2 & $\mu g / L$ \\
\hline 1,2-Dibromoethane & WA & & & $\mathbf{U}$ & 20 & & NS & $\mu g / L$ \\
\hline 1,2-Dichloroethane & WA & & & $\mathbf{U}$ & 5 & & 5 & $\mu g / L$ \\
\hline 1,2-Dichloroethylene (total) & WA & & & $\mathbf{U}$ & 5 & & 100 & $\mu g / L$ \\
\hline 1,2-Dichloropropane & WA & & & $\mathbf{U}$ & 5 & & 5 & $\mu g / L$ \\
\hline 2-Hexanone & WA & & & $\mathbf{U}$ & 10 & & NS & $\mu g / L$ \\
\hline 4-Methyl-2-pentasione & WA & & & $\mathbf{U}$ & 10 & & NS & $\mu g / L$ \\
\hline Acetone & WA & & & & 10.3 & & NS & $\mu g / L$ \\
\hline Acetonitrile & WA & & & $\mathbf{U}$ & 20 & & NS & $\mu g / L$ \\
\hline Acidity & WA & & & & 42 & & NS & $\mathrm{mg} / \mathrm{L}$ \\
\hline Acrolein & WA & & & $\mathbf{U}$ & 10 & & NS & $\mu \mathrm{g} / \mathrm{L}$ \\
\hline Acrylonitrile & WA & . & & $\mathbf{U}$ & 10 & & NS & $\mu g / L$ \\
\hline Alkalinity & WA & & & $\mathbf{U}$ & 0.5 & & NS & $\mathrm{mg} / \mathrm{L}$ \\
\hline Allyl chloride & WA & & & $\mathbf{U}$ & 100 & & NS & $\mu g / L$ \\
\hline Aluminum & WA & & & & 2830 & & NS & $\mu g / L$ \\
\hline Antimony & WA & & & $\mathbf{U}$ & 60 & & 6 & $\mu g / L$ \\
\hline Arsenic & WA & & & $\mathbf{U}$ & 10 & & 50 & $\mu g / L$ \\
\hline Barium & WA & & & $\mathbf{U}$ & 200 & & 2000 & $\mu g / L$ \\
\hline Benzene & WA & & & $\mathbf{U}$ & 5 & & 5 & $\mu g / L$ \\
\hline Beryllium & WA & & & $\mathbf{U}$ & 5 & & 4 & $\mu g / L$ \\
\hline Bromodichloromethane & WA & & & $\mathbf{U}$ & 5 & & NS & $\mu g / L$ \\
\hline Bromoform & WA & & & $\mathbf{U}$ & 5 & & NS & $\mu g / L$ \\
\hline Cadmium & WA & & & $\mathbf{U}$ & 5 & & 5 & $\mu g / L$ \\
\hline Calcium & WA & & & $\mathbf{U}$ & 5000 & & NS & $\mu g / L$ \\
\hline Carbon disulfide & WA & & & $\mathbf{U}$ & 5 & & NS & $\mu g / L$ \\
\hline Carbon tetrachloride & WA & & & $\mathbf{U}$ & 5 & & 5 & $\mu g / L$ \\
\hline Cesium-137 & TM & & & $\mathbf{U}$ & 9.4 & & 200 & $\mathrm{pCi} / \mathrm{L}$ \\
\hline Chloride & WA & & $\mathbf{A}$ & & 5.265 & & NS & $\mathrm{mg} / \mathrm{L}$ \\
\hline Chlorobenzene & WA & & & $\mathbf{U}$ & 5 & & 100 & $\mu \mathbf{g} / \mathrm{L}$ \\
\hline Chloroethane & WA & & & $\mathbf{U}$ & 10 & & NS & $\mu g / L$ \\
\hline Chloroform & WA & & & $\mathbf{U}$ & 5 & & 100 & $\mu \mathrm{g} / \mathrm{L}$ \\
\hline Chloroprene & WA & & & $\mathbf{U}$ & 20 & & NS & $\mu g / L$ \\
\hline Chromium & WA & & & $\mathbf{U}$ & 10 & & 100 & $\mu g / L$ \\
\hline cis-1,3-Dichloropropene & WA & & & $\mathbf{U}$ & 5 & & NS & $\mu g / L$ \\
\hline Cobalt & WA & & & $\mathbf{U}$ & 50 & & NS & $\mu g / L$ \\
\hline Copper & WA & & & $\mathbf{U}$ & 25 & & 1000 & $\mu g / L$ \\
\hline Cyanide & WA & & & $\mathbf{U}$ & 10 & & 200 & $\mu g / L$ \\
\hline Dibromochloromethane & WA & & & $\mathbf{U}$ & 5 & & NS & $\mu g / L$ \\
\hline Dichlorodifluoromethane & WA & & & $\mathbf{U}$ & 10 & & NS & $\mu \mathrm{g} / \mathrm{L}$ \\
\hline Ethylbenzene & WA & & & $\mathbf{U}$ & 5 & & 700 & $\mu g / L$ \\
\hline Fluoride & WA & & & $\mathbf{U}$ & 0.1 & & 4 & $\mathrm{mg} / \mathrm{L}$ \\
\hline Gross alpha & TM & & & & 4.4 & 2.4 & 15 & $\mathrm{pCi} / \mathrm{L}$ \\
\hline
\end{tabular}


BGS001 Laboratory Analyses (cont'd)

\begin{tabular}{|c|c|c|c|c|c|c|c|c|}
\hline Analyte & Lab & $\operatorname{Rep}$ & Avg & Qualifier & Result & Acc & Stand & Units \\
\hline Iron & WA & & & & 478 & & 300 & $\mu g / L$ \\
\hline Isobutyl alcohol & WA & & & $\mathbf{U}$ & 20 & & NS & $\mu \mathrm{g} / \mathrm{L}$ \\
\hline Lead & WA & & & $\mathbf{U}$ & 3 & & 50 & $\mu \mathrm{g} / \mathrm{L}$ \\
\hline Magnesium & WA & & & $\mathbf{U}$ & 5000 & & NS & $\mu g / L$ \\
\hline Manganese & WA & & & & 26.4 & & 50 & $\mu g / L$ \\
\hline Mercury & WA & & & $\mathbf{U}$ & 0.2 & & 2 & $\mu g / L$ \\
\hline Methacrylonitrile & WA & & & $\mathbf{U}$ & 20 & & NS & $\mu g / L$ \\
\hline Methyl bromide & WA & & & $\mathbf{U}$ & 10 & & NS & $\mu g / L$ \\
\hline Methyl chloride & WA & & & $\mathbf{U}$ & 10 & & NS & $\mu g / L$ \\
\hline Methyl ethyl ketone & WA & & & $\mathbf{U}$ & 10 & & NS & $\mu g / L$ \\
\hline Methyl iodide & WA & & & $\mathbf{U}$ & 10 & & NS & $\mu g / L$ \\
\hline Methylene bromide & WA & & & $\mathbf{U}$ & 10 & & NS & $\mu g / L$ \\
\hline Methylene chloride & WA & & & $\mathbf{U}$ & 5 & & 5 & $\mu g / L$ \\
\hline Nickel & WA & & & $\mathbf{U}$ & 40 & & 100 & $\mu g / L$ \\
\hline Nitrate as nitrogen & WA & & & $\mathbf{U}$ & 0.1 & & 10 & $\mathrm{mg} / \mathrm{L}$ \\
\hline Nitrate as nitrogen & WA & & NA & $\mathbf{U}$ & 0.1 & & 10 & mg/L \\
\hline Nonvolatile beta & TM & & & & 4.3 & 2.6 & 50 & $\mathrm{pCi} / \mathrm{L}$ \\
\hline Potassium & WA & & & $\mathbf{U}$ & 5000 & & NS & $\mu g / L$ \\
\hline Propionitrile & WA & & & $\mathbf{U}$ & 50 & & NS & $\mu g / L$ \\
\hline Selenium & WA & & & $\mathbf{U}$ & 5 & & 50 & $\mu g / L$ \\
\hline Silver & WA & & & $\mathbf{U}$ & 10 & & 50 & $\mu g / L$ \\
\hline Sodium & WA & & & $\mathbf{U}$ & 5000 & & NS & $\mu g / L$ \\
\hline Styrene & WA & & & $\mathbf{U}$ & 5 & & 100 & $\mu g / L$ \\
\hline Sulfate & WA & & & & 0.137 & & 400 & $\mathrm{mg} / \mathrm{L}$ \\
\hline Sulfide & WA & & & UJ & 0.1 & & NS & $\mathrm{mg} / \mathrm{L}$ \\
\hline Sulfide & WA & & $\mathbf{N A}$ & UJ & 0.1 & & NS & $\mathrm{mg} / \mathrm{L}$ \\
\hline Tetrachloroethylene & WA & & & $\mathbf{U}$ & 5 & & 5 & $\mu g / L$ \\
\hline Thallium & WA & & & $\mathbf{U}$ & 10 & & 2 & $\mu g / L$ \\
\hline Toluene & WA & & & $\mathbf{J}$ & 1.32 & & 1000 & $\mu g / L$ \\
\hline Total hardness & WA & & $\mathbf{A}$ & & 3.5 & & NS & $\mathrm{mg} / \mathrm{L}$ \\
\hline Total organic carbon & WA & & & & 42.6 & & NS & $\mathrm{mg} / \mathrm{L}$ \\
\hline Total suspended solids & WA & & $\mathbf{A}$ & . & 69 & & NS & mg/L \\
\hline trans-1,3-Dichloropropene & WA & & & $\mathbf{U}$ & 5 & & NS & $\mu g / L$ \\
\hline trans-1,4-Dichloro-2-butene & WA & & & $\mathbf{U}$ & 100 & & NS & $\mu g / L$ \\
\hline Trichloroethylene & WA & & & $\mathbf{U}$ & 5 & & 5 & $\mu \mathrm{g} / \mathrm{L}$ \\
\hline Trichlorofluoromethane & WA & & & $\mathbf{U}$ & 5 & & NS & $\mu g / L$ \\
\hline Tritium & TM & & & & 1.7 & 0.8 & 20 & $\mathrm{pCi} / \mathrm{ml}$ \\
\hline Turbidity & WA & & & & 314 & & NS & NTU \\
\hline Vanadium & WA & & & $\mathbf{U}$ & 50 & & NS & $\mu g / L$ \\
\hline Vinyl acetate & WA & & & $\mathbf{U}$ & 10 & & NS & $\mu \mathrm{g} / \mathrm{L}$ \\
\hline Vinyl chloride & WA & & & $\mathbf{U}$ & 10 & & 2 & $\mu \mathrm{g} / \mathrm{L}$ \\
\hline Xylenes (total) & WA & & & $\mathbf{U}$ & 5 & & 10000 & $\mu g / L$ \\
\hline Zinc & WA & & & & 43.1 & & 5000 & $\mu \mathrm{g} / \mathrm{L}$ \\
\hline
\end{tabular}




\section{BGS002}

Field Measurements:

Sample Date: $4 / 22 / 93$

pH: 6.19

Specific Conductance: $60 \mu \mathrm{S} / \mathrm{cm}$

Red/Oxid Potential: $158 \mathrm{mv}$

Dissolved Oxygen: $2.6 \mathrm{mg} / \mathrm{L}$

$$
\text { Analyte }
$$

Lab Rep Av

1,1,1,2-Tetrachloroethane

1,1,1-Trichloroethane

1,1,2,2-Tetrachloroethane

1,1,2-Trichloroethane

1,1-Dichloroethane

1,1-Dichloroethylene

1,2,3-Trichloropropane

1,2-Dibromo-3-chloropropane WA

1,2-Dibromoethane

1,2-Dichloroethane

1,2-Dichloroethylene (total)

1,2-Dichloropropane

2-Hexanone

4-Methyl-2-pentanone

WA

WA

WA

WA

Acetone

WA

WA

WA

WA

WA

WA

WA

WA

Acetonitrile

Acidity

Acrolein

Acrylonitrile

Alkalinity

Allyl chloride

WA

Aluminum

WA

WA

WA

WA

WA

WA

Antimony

WA

Arsenic

Barium

Benzene

Beryllium

Bromodichloromethane

WA

WA

WA

WA

WA

WA

Bromoform

WA

WA

Cadmium

WA

Calcium

Carbon disulfide

WA

WA

Carbon tetrachloride WA

Cesium-137

TM

Chloride . WA

Chlorobenzene WA

Chloroethane WA

Chloroform WA

Chloroprene WA

Chromium

cis-1,3-Dichloropropene

WA

Cobalt

Copper

Cyanide

WA

WA

WA

Dibromochloromethane

WA

WA

Dichlorodifluoromethane

WA

Ethylbenzene

WA

Fluoride

WA

Gross alpha

TM

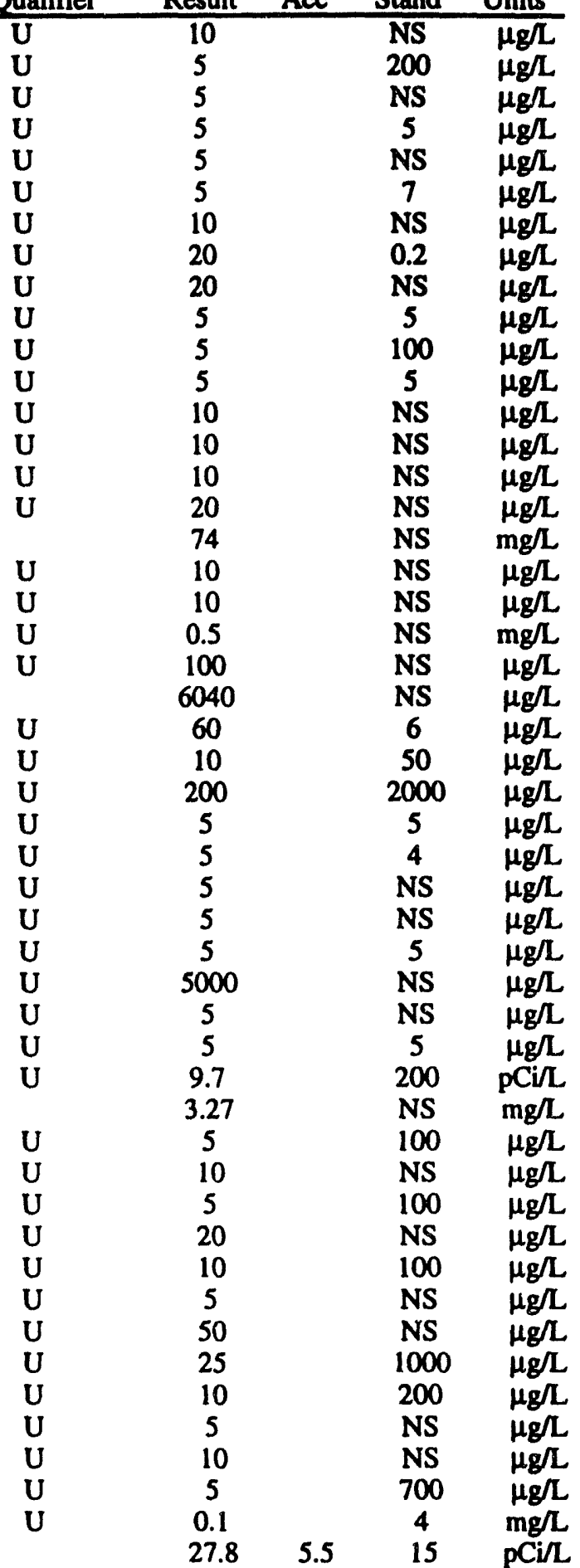


BGS002 Laboratory Analyses (cont'd)

\begin{tabular}{|c|c|c|c|c|c|c|c|c|c|}
\hline Analyte & Lab & Rep & Avg & Qualifier & & Result & Acc & Stand & Units \\
\hline Iron & $\overline{\text { WA }}$ & & & & & 12400 & & 300 & $\mu \mathrm{g} / \mathrm{L}$ \\
\hline Isobutyl alcohol & WA & & & $\mathbf{U}$ & & 20 & & NS & $\mu \mathrm{g} / \mathrm{L}$ \\
\hline Lead & WA & & & & & 4.3 & & 50 & $\mu g / L$ \\
\hline Magnesium & WA & & & $\mathbf{U}$ & & 5000 & & NS & $\mu \mathrm{g} / \mathrm{L}$ \\
\hline Manganese & WA & & & & & 36.7 & & 50 & $\mu \mathrm{g} / \mathrm{L}$ \\
\hline Mercury & WA & & & $\mathbf{U}$ & & 0.2 & & 2 & $\mu \mathrm{g} / \mathrm{L}$ \\
\hline Methacrylonitrile & WA & & & $\mathbf{U}$ & & 20 & & NS & $\mu \mathrm{g} / \mathrm{L}$ \\
\hline Methyl bromide & WA & & & $\mathbf{U}$ & & 10 & & NS & $\mu g / L$ \\
\hline Methyl chloride & WA & & & $\mathbf{U}$ & & 10 & & NS & $\mu g / L$ \\
\hline Methyl ethyl ketone & WA & & & $\mathbf{U}$ & & 10 & & NS & $\mu g / 2$ \\
\hline Methyl iodide & WA & & & $\mathbf{U}$ & & 10 & & NS & $\mu g / L$ \\
\hline Methylene bromide & WA & & & $\mathbf{U}$ & & 10 & & NS & $\mu g / L$ \\
\hline Methylene chloride & WA & & & $\mathbf{U}$ & & 5 & & 5 & $\mu g / L$ \\
\hline Nickel & WA & & & $\mathbf{U}$ & & 40 & & 100 & $\mu g / L$ \\
\hline Nitrate as nitrogen & WA & & & & & 0.116 & & 10 & $\mathrm{mg} / \mathrm{L}$ \\
\hline Nonvolatile beta & TM & & & & & 67.3 & 6.5 & 50 & pCi/L \\
\hline Potassium & WA & & & $\mathbf{U}$ & & 5000 & & NS & $\mu g / L$ \\
\hline Propionitrile & WA & & & $\mathbf{U}$ & & 50 & & NS & $\mu g / L$ \\
\hline Selenium & WA & & & $\mathbf{U}$ & & 5 & & 50 & $\mu g / L$ \\
\hline Silver & WA & & & $\mathbf{U}$ & & 10 & & 50 & $\mu g / L$ \\
\hline Sodium & WA & & & $\mathbf{U}$ & & 5000 & & NS & $\mu g / L$ \\
\hline Styrene & WA & & & $\mathbf{U}$ & & 5 & & 100 & $\mu g / L$ \\
\hline Sulfate & WA & & & & & 0.165 & & 400 & $\mathrm{mg} / \mathrm{L}$ \\
\hline Sulfide & WA & & & UJ & Q & 0.1 & & NS & $\mathrm{mg} / \mathrm{L}$ \\
\hline Tetrachloroethylene & WA & & & $\mathbf{U}$ & & 5 & & 5 & $\mu \mathrm{g} / \mathrm{L}$ \\
\hline Thallium & WA & & & U & & 10 & & 2 & $\mu g /$ \\
\hline Toluene & WA & & & $\mathbf{U}$ & & 5 & & 1000 & $\mu \mathrm{g} / \mathrm{L}$ \\
\hline Total hardness & WA & & & & & 4 & & NS & $\mathrm{mg} / \mathrm{L}$ \\
\hline Total organic carbon & WA & & & & & 35.5 & & NS & $\mathrm{mg} / \mathrm{L}$ \\
\hline Total suspended solids & WA & & & & & 120 & & NS & $\mathrm{mg} / \mathrm{L}$ \\
\hline trans-1,3-Dichloropropene & WA & & & $\mathbf{U}$ & & 5 & & NS & $\mu g / L$ \\
\hline trans-1,4-Dichloro-2-butene & WA & & & U & & 100 & & NS & $\mu g / L$ \\
\hline Trichloroethylene & WA & & & $\mathbf{U}$ & & 5 & & 5 & $\mu g / L$ \\
\hline Trichlorofluoromethane & WA & & & $\mathrm{U}$ & & 5 & & NS & $\mu g / L$ \\
\hline Tritium & TM & & & & & 1.3 & 0.63 & 20 & $\mathrm{pCi} / \mathrm{ml}$ \\
\hline Turbidity & WA & & & & & 97 & & NS & NTU \\
\hline Vanadium & WA & & & $\mathbf{U}$ & & 50 & & NS & $\mu g / L$ \\
\hline Vinyl acetate & WA & & & $\mathbf{U}$ & & 10 & & NS & $\mu g / L$ \\
\hline Vinyl chloride & WA & & & U & & 10 & & 2 & $\mu \mathrm{g} / \mathrm{L}$ \\
\hline Xylenes (total) & WA & & & $\mathbf{U}$ & & 5 & & 10000 & $\mu \mathrm{g} / \mathrm{L}$ \\
\hline Zinc & WA & & & & & 147 & & 5000 & $\mu g / L$ \\
\hline
\end{tabular}




\section{BGS003}

Field Measurements:

Sample Date: 4/22/93

pH: 5.38

Specific Conductance: $38 \mu \mathrm{S} / \mathrm{cm}$

Red/Oxid Potential: $159 \mathrm{mv}$

Dissolved Oxygen: $8.31 \mathrm{mg} / \mathrm{L}$

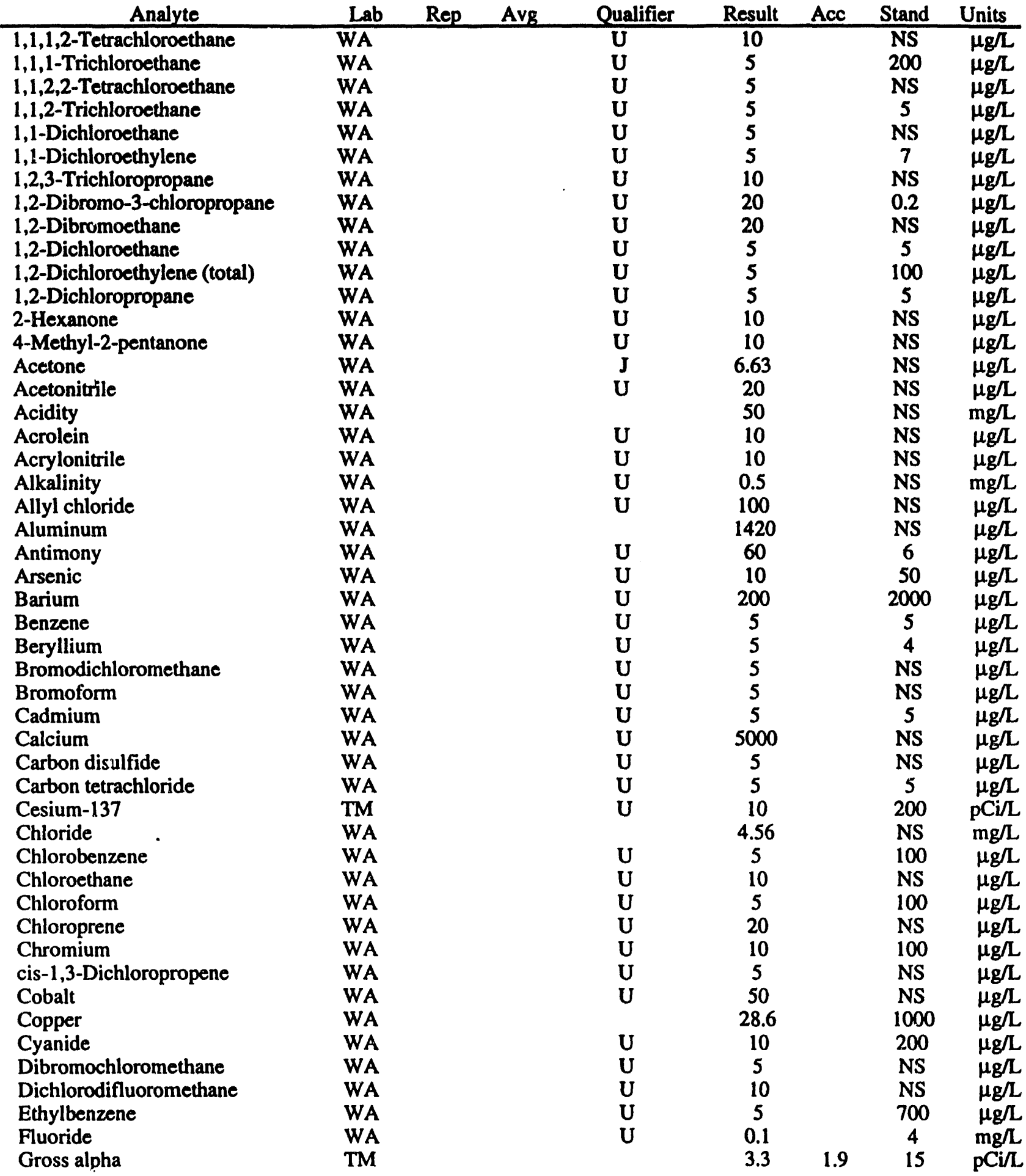


BGS003 Laboratory Analyses (cont'd)

\begin{tabular}{|c|c|c|c|c|c|c|c|c|}
\hline Analyte & Lab & Rep & Avg & Qualifier & Result & Acc & Stand & Units \\
\hline Iron & WA & & & & 1300 & & 300 & $\mu g / L$ \\
\hline Isobutyl alcohol & WA & & & $\mathbf{U}$ & 20 & & NS & $\mu g / L$ \\
\hline Lead & WA & & & $\mathbf{U}$ & 3 & & 50 & $\mu g / L$ \\
\hline Magnesium & WA & & & $\mathbf{U}$ & 5000 & & NS & $\mu g / L$ \\
\hline Manganese & WA & & & & 30.3 & & 50 & $\mu g / L$ \\
\hline Mercury & WA & & & $\mathbf{U}$ & 0.2 & . & 2 & $\mu_{g} / L$ \\
\hline Methacrylonitrile & WA & & & $\mathbf{U}$ & 20 & $\cdot$ & NS & $\mu g / L$ \\
\hline Methyl bromide & WA & & & $\mathbf{U}$ & 10 & & NS & $\mu g / L$ \\
\hline Methyl chloride & WA & & & $\mathbf{U}$ & 10 & & NS & $\mu g / L$ \\
\hline Methyl ethyl ketone & WA & & & $\mathbf{U}$ & 10 & & NS & $\mu g / L$ \\
\hline Methyl iodide & WA & & & $\mathbf{U}$ & 10 & & NS & $\mu g / L$ \\
\hline Methylene bromide & WA & & & $\mathbf{U}$ & 10 & & NS & $\mu g / L$ \\
\hline Methylene chloride & WA & & & $\mathbf{U}$ & 5 & & 5 & $\mu g / L$ \\
\hline Nickel & WA & & & $\mathbf{U}$ & 40 & & 100 & $\mu g / L$ \\
\hline Nitrate as nitrogen & WA & & & $\mathbf{U}$ & 0.1 & & 10 & $\mathrm{mg} / \mathrm{L}$ \\
\hline Nonvolatile beta & TM & & . & & 26.5 & 4.1 & 50 & pCi/L \\
\hline Potassium & WA & & & $\mathbf{U}$ & 5000 & & NS & $\mu g / L$ \\
\hline Propionitrile & WA & & & $\mathbf{U}$ & 50 & & NS & $\mu g / L$ \\
\hline Selenium & WA & & & $\mathbf{U}$ & 5 & & 50 & $\mu g / L$ \\
\hline Silver & WA & & & $\mathbf{U}$ & 10 & & 50 & $\mu g / L$ \\
\hline Sodium & WA & & & $\mathbf{U}$ & 5000 & & NS & $\mu g / L$ \\
\hline Styrene & WA & & & $\mathbf{U}$ & 5 & & 100 & $\mu g / L$ \\
\hline Sulfate & WA & & & & 5.1 & & 400 & $\mathrm{mg} / \mathrm{L}$ \\
\hline Sulfide & WA & & & $\mathbf{U}$ & 0.1 & & NS & $\mathrm{mg} / \mathrm{L}$ \\
\hline Tetrachloroethylene & WA & & & $\mathbf{U}$ & 5 & & 5 & $\mu g / L$ \\
\hline Thallium & WA & & & $\mathbf{U}$ & 10 & & 2 & $\mu g / L$ \\
\hline Toluene & WA & & & $\mathbf{J}$ & 1.29 & & 1000 & $\mu g / L$ \\
\hline Total hardness & WA & & & & 6 & & NS & $\mathrm{mg} / \mathrm{L}$ \\
\hline Total organic carbon & WA & & & & 15.2 & & NS & $\mathrm{mg} / \mathrm{L}$ \\
\hline Total suspended solids & WA & & & & 40 & & NS & $\mathrm{mg} / \mathrm{L}$ \\
\hline trans-1,3-Dichloropropene & WA & & & $\mathbf{U}$ & 5 & & NS & $\mu g / L$ \\
\hline trans-1,4-Dichloro-2-butene & WA & & & $\mathbf{U}$ & 100 & & NS & $\mu g / L$ \\
\hline Trichloroethylene & WA & & & $\mathbf{U}$ & 5 & & 5 & $\mu \mathrm{g} / \mathrm{L}$ \\
\hline Trichlorofluoromethane & WA & & & $\mathbf{U}$ & 5 & & NS & $\mu g / L$ \\
\hline Tritium & TM & & & & 1.2 & 0.58 & 20 & $\mathrm{pCi} / \mathrm{ml}$ \\
\hline Turbidity & WA & & & & 91.8 & & NS & NTU \\
\hline Vanadium & WA & & & $\mathbf{U}$ & 50 & & NS & $\mu g / L$ \\
\hline Vinyl acetate & WA & & & $\mathbf{U}$ & 10 & & NS & $\mu \mathrm{g} / \mathrm{L}$ \\
\hline Vinyl chloride & WA & & & $\mathbf{U}$ & 10 & & 2 & $\mu g / L$ \\
\hline Xylenes (total) & WA & & & $\mathbf{U}$ & 5 & & 10000 & $\mu g / L$ \\
\hline Zinc & WA & & & & 76.4 & & 5000 & $\mu \mathrm{g} / \mathrm{L}$ \\
\hline
\end{tabular}




\section{BGSO04}

Field Measurements:

Sample Date: 4/22/93

pH: 5.72

Specific Conductance: $20 \mu \mathrm{S} / \mathrm{cm}$

Red/Oxid Potential: $165 \mathrm{mv}$

Dissolved Oxygen: $0.9 \mathrm{mg} / \mathrm{L}$

\begin{tabular}{|c|c|c|c|c|c|c|c|}
\hline Analyte & Lab & Avg & Qualifier & Result & Acc & Stand & Units \\
\hline 1,1,1,2-Tetrachloroethane & WA & & $\mathbf{U}$ & 10 & & NS & $\mu g / L$ \\
\hline 1,1,1-Trichloroethane & WA & & $\mathbf{U}$ & 5 & & 200 & $\mu g / L$ \\
\hline 1,1,2,2-Tetrachloroethane & WA & & $\mathbf{U}$ & 5 & & NS & $\mu \varepsilon / 2$ \\
\hline 1,1,2-Trichloroethane & WA & & $\mathbf{U}$ & 5 & & 5 & $\mu g / L$ \\
\hline 1,1-Dichloroethane & WA & & $\mathbf{U}$ & 5 & & NS & $\mu g / L$ \\
\hline 1,1-Dichloroethylene & WA & & $\mathbf{U}$ & 5 & & 7 & $\mu g / L$ \\
\hline 1,2,3-Trichloropropane & WA & . & $\mathbf{U}$ & 10 & & NS & $\mu g / L$ \\
\hline 1,2-Dibromo-3-chloropropane & WA & & $\mathbf{U}$ & 20 & & 0.2 & $\mu g / L$ \\
\hline 1,2-Dibromoethane & WA & & $\mathbf{U}$ & 20 & & NS & $\mu g / L$ \\
\hline 1,2-Dichloroethane & WA & & $\mathbf{U}$ & 5 & & 5 & $\mu g / L$ \\
\hline 1,2-Dichloroethylene (total) & WA & & $\mathbf{U}$ & 5 & & 100 & $\mu g / L$ \\
\hline 1,2-Dichloropropane & WA & & $\mathbf{U}$ & 5 & & 5 & $\mu g / L$ \\
\hline 2-Hexanone & WA & & $\mathbf{U}$ & 10 & & NS & $\mu g / L$ \\
\hline 4-Methyl-2-pentanone & WA & & $\mathbf{U}$ & 10 & & NS & $\mu g / L$ \\
\hline Acetone & WA & & & 10.2 & & NS & $\mu g / L$ \\
\hline Acetonitrile & WA & & $\mathbf{U}$ & 20 & & NS & $\mu g / L$ \\
\hline Acidity & WA & & & 26 & & NS & $\mathrm{mg} / \mathrm{L}$ \\
\hline Acrolein & WA & & $\mathbf{U}$ & 10 & & NS & $\mu g / L$ \\
\hline Acrylonitrile & WA & & $\mathbf{U}$ & 10 & & NS & $\mu g / L$ \\
\hline Alkalinity & WA & & $\mathbf{U}$ & 0.5 & & NS & $\mathrm{mg} / \mathrm{L}$ \\
\hline Allyl chloride & WA & & $\mathbf{U}$ & 100 & & NS & $\mu g / L$ \\
\hline Aluminum & WA & & & 255 & & NS & $\mu g / L$ \\
\hline Antimony & WA & & $\mathbf{U}$ & 60 & & 6 & $\mu g / L$ \\
\hline Arsenic & WA & & $\mathbf{U}$ & 10 & & 50 & $\mu g / L$ \\
\hline Barium & WA & & $\mathbf{U}$ & 200 & & 2000 & $\mu g / L$ \\
\hline Benzene & WA & & $\mathbf{U}$ & 5 & & 5 & $\mu \mathrm{g} / \mathrm{L}$ \\
\hline Beryllium & WA & & $\mathbf{U}$ & 5 & & 4 & $\mu g / L$ \\
\hline Bromodichloromethane & WA & & $\mathbf{U}$ & 5 & & NS & $\mu g / L$ \\
\hline Bromoform & WA & & $\mathbf{U}$ & 5 & & NS & $\mu g / L$ \\
\hline Cadmium & WA & & $\mathbf{U}$ & 5 & & 5 & $\mu g / L$ \\
\hline Calcium & WA & & $\mathbf{U}$ & 5000 & & NS & $\mu g / L$ \\
\hline Carbon disulfide & WA & & $\mathbf{U}$ & 5 & & NS & $\mu g / L$ \\
\hline Carbon tetrachloride & WA & & $\mathbf{U}$ & 5 & & 5 & $\mu g / L$ \\
\hline Cesium-137 & TM & & & 6.4 & 5.3 & 200 & $\mathrm{pCi} / \mathrm{L}$ \\
\hline Chloride & WA & & & 3.87 & & NS & $\mathrm{mg} / \mathrm{L}$ \\
\hline Chlorobenzene & WA & & $\mathbf{U}$ & 5 & & 100 & $\mu g / L$ \\
\hline Chloroethane & WA & & $\mathbf{U}$ & 10 & & NS & $\mu g / L$ \\
\hline Chloroform & WA & & $\mathbf{U}$ & 5 & & 100 & $\mu \mathrm{g} / \mathrm{L}$ \\
\hline Chloroprene & WA & & $\mathbf{U}$ & 20 & & NS & $\mu \mathrm{g} / \mathrm{L}$ \\
\hline Chromium & WA & & $\mathbf{U}$ & 10 & & 100 & $\mu g / L$ \\
\hline cis-1,3-Dichloropropene & WA & & $\mathbf{U}$ & 5 & & NS & $\mu g / L$ \\
\hline Cobalt & WA & & $\mathbf{U}$ & 50 & & NS & $\mu g / L$ \\
\hline Copper & WA & & & 28.3 & & 1000 & $\mu g / L$ \\
\hline Cyanide & WA & & $\mathbf{U}$ & 10 & & 200 & $\mu g / L$ \\
\hline Dibromochloromethane & WA & & $\mathbf{U}$ & 5 & & NS & $\mu \mathrm{g} / \mathrm{L}$ \\
\hline Dichlorodifluoromethane & WA & & $\mathbf{U}$ & 10 & & NS & $\mu g / L$ \\
\hline Ethylbenzene & WA & & $\mathbf{U}$ & 5 & & 700 & $\mu g \Omega$ \\
\hline Fluoride & WA & & $\mathbf{U}$ & 0.1 & & 4 & $\mathrm{mg} / \mathrm{L}$ \\
\hline Fluoride & WA & NA & $\mathbf{U}$ & 0.1 & & 4 & $g / L$ \\
\hline
\end{tabular}


BGS004 Laboratory Analyses (cont'd)

\begin{tabular}{|c|c|c|c|c|c|c|c|c|}
\hline Analyte & Lab & Rep & Avg & Qualifier & Result & Acc & Stand & Units \\
\hline Gross alpha & TM & & & & 6.3 & 2.9 & 15 & $\mathrm{pCi} / \mathrm{L}$ \\
\hline Iron & WA & & & & 1460 & & 300 & $\mu g / L$ \\
\hline Isobutyl alcohol & WA & & & $\mathbf{U}$ & 20 & & NS & $\mu g / L$ \\
\hline Lead & WA & & & $\mathbf{U}$ & 3 & & 50 & $\mu g / L$ \\
\hline Magnesium & WA & & & $\mathbf{U}$ & 5000 & & NS & $\mu g /$ \\
\hline Manganese & WA & & & & 22.4 & . & 50 & $\mu g / L$ \\
\hline Mercury & WA & & & $\mathbf{U}$ & 0.2 & & 2 & $\mu g / L$ \\
\hline Methacrylonitrile & WA & & & $\mathbf{U}$ & 20 & & NS & $\mu g / L$ \\
\hline Methyl bromide & WA & & & $\mathbf{U}$ & 10 & & NS & $\mu g / L$ \\
\hline Methyl chloride & WA & & & $\mathbf{U}$ & 10 & & NS & $\mu g / L$ \\
\hline Methyl ethyl ketone & WA & & & $\mathbf{U}$ & 10 & & NS & $\mu g / L$ \\
\hline Methyl iodide & WA & & & $\mathbf{U}$ & 10 & & NS & $\mu g /$ \\
\hline Methylene bromide & WA & & & $\mathbf{U}$ & 10 & & NS & $\mu g /$ \\
\hline Methylene chloride & WA & & & $\mathbf{U}$ & 5 & & 5 & $\mu g / L$ \\
\hline Nickel & WA & & & $\mathbf{U}$ & 40 & & 100 & $\mu g / L$ \\
\hline Nitrate as nitrogen & WA & & & & 0.139 & & 10 & mg/L \\
\hline Nonvolatile beta & $\mathbf{T M}$ & & & & 61.2 & 6.4 & 50 & $\mathrm{pCi} / \mathrm{L}$ \\
\hline Potassium & WA & & & $\mathbf{U}$ & 5000 & & NS & $\mu g / L$ \\
\hline Propionitrile & WA & & & $\mathbf{U}$ & 50 & & NS & $\mu g / L$ \\
\hline Selenium & WA & & & $\mathbf{U}$ & 5 & & 50 & $\mu g / L$ \\
\hline Silver & WA & & & $\mathbf{U}$ & 10 & & 50 & $\mu g / L$ \\
\hline Sodium & WA & & & $\mathbf{U}$ & 5000 & & NS & $\mu g / L$ \\
\hline Styrene & WA & & & $\mathbf{U}$ & 5 & & 100 & $\mu g / L$ \\
\hline Sulfate & WA & & & & 0.127 & & 400 & $\mathrm{mg} / \mathrm{L}$ \\
\hline Sulfide & WA & & & UJ & 0.1 & & NS & mg/L \\
\hline Tetrachloroethylene & WA & & & $\mathbf{U}$ & 5 & & 5 & $\mu g / L$ \\
\hline Thallium & WA & & & $\mathbf{U}$ & 10 & & 2 & $\mu g / L$ \\
\hline Toluene & WA & & & J & 1.3 & & 1000 & $\mu g / L$ \\
\hline Total hardness & WA & & & & 12 & & NS & $\mathbf{m g} / \mathbf{L}$ \\
\hline Total organic carbon & WA & & & & 18.2 & & NS & $\mathbf{m g} / \mathbf{L}$ \\
\hline Total suspended solids & WA & & & & 94 & & NS & $\mathbf{m g} / \mathbf{L}$ \\
\hline trans-1,3-Dichloropropene & WA & & & $\mathbf{U}$ & 5 & & NS & $\mu g / L$ \\
\hline trans-1,4-Dichloro-2-butene & WA & & & $\mathbf{U}$ & 100 & & NS & $\mu g / L$ \\
\hline Trichloroethylene & WA & & & $\mathbf{U}$ & 5 & & 5 & $\mu g / L$ \\
\hline Trichlorofluoromethane & WA & & & U & 5 & & NS & $\mu g / L$ \\
\hline Tritium & $\mathbf{T M}$ & & & & 1.9 & 0.98 & 20 & $\mathrm{pCi} / \mathrm{ml}$ \\
\hline Turbidity & WA & & $\mathbf{A}$ & & 49.8 & & NS & NTU \\
\hline Vanadium & WA & & & $\mathrm{U}$ & 50 & & NS & $\mu g / L$ \\
\hline Vinyl acetate & WA & & & $\mathrm{U}$ & 10 & & NS & $\mu g / L$ \\
\hline Vinyl chloride & WA & & & $\mathbf{U}$ & 10 & & 2 & $\mu g / L$ \\
\hline Xylenes (total) & WA & & & U & 5 & & 10000 & $\mu g / L$ \\
\hline Zinc & WA & & & & 51.7 & & 5000 & $\mu g / L$ \\
\hline
\end{tabular}




\section{UTD001}

Field Measurements:

Stream Discharge: $0.4 \mathrm{cfs}$

Sample Date: 4/26/93

pH: 7.02

Specific Conductance: $49 \mu \mathrm{S} / \mathrm{cm}$

Red/Oxid Potential: $79 \mathrm{mv}$

Dissolved Oxygen: $7.9 \mathrm{mg} /$.

\begin{tabular}{|c|c|c|c|c|c|c|c|c|}
\hline Analyte & Lab & Rep & Avg & Qualifier & Result & Acc & Stand & Units \\
\hline 1,1,1,2-Tetrachloroethane & WA & & & $\bar{U}$ & 10 & & NS & $\mu g / L$ \\
\hline 1,1,1-Trichloroethane & WA & & & $\mathbf{U}$ & 5 & & 200 & $\mu g / L$ \\
\hline 1,1,2,2-Tetrachloroethane & WA & & & $\mathbf{U}$ & 5 & & NS & $\mu g / L$ \\
\hline 1,1,2-Trichloroethane & WA & & & $\mathbf{U}$ & 5 & & 5 & $\mu g / L$ \\
\hline 1,1-Dichloroethane & WA & & & $\mathbf{U}$ & 5 & & NS & $\mu \mathrm{g} / \mathrm{L}$ \\
\hline 1,1-Dichloroethylene & WA & & & $\mathbf{U}$ & 5 & & 7 & $\mu g / L$ \\
\hline 1,2,3-Trichloropropane & WA & & & $\mathbf{U}$ & 10 & & NS & $\mu g /$ \\
\hline 1,2-Dibromo-3-chloropropane & WA & & & $\mathbf{U}$ & 20 & & 0.2 & $\mu g / L$ \\
\hline 1,2-Dibromoethane & WA & & & $\mathbf{U}$ & 20 & & NS & $\mu g / L$ \\
\hline 1,2-Dichloroethane & WA & & & $\mathbf{U}$ & 5 & & 5 & $\mu \mathrm{g} / \mathrm{L}$ \\
\hline 1,2-Dichloroethylene (total) & WA & & & $\mathbf{U}$ & 5 & & 100 & $\mu g / L$ \\
\hline 1,2-Dichloropropane & WA & & & $\mathbf{U}$ & 5 & & 5 & $\mu g / \Omega$ \\
\hline 2-Hexanone & WA & & & $\mathbf{U}$ & 10 & & NS & $\mu g / L$ \\
\hline 4-Methyl-2-pentanone & WA & & & $\mathbf{U}$ & 10 & & NS & $\mu g / L$ \\
\hline Acetone & WA & & & $\mathbf{U}$ & 10 & & NS & $\mu g / L$ \\
\hline Acetonitrile & WA & & & $\mathbf{U}$ & 20 & & NS & $\mu g /$ \\
\hline Acidity & WA & & & & 8 & & NS & $\mathrm{mg} / \mathrm{L}$ \\
\hline Acrolein & WA & & & $\mathbf{U}$ & 10 & & NS & $\mu g / L$ \\
\hline Acrylonitrile & WA & & & $\mathbf{U}$ & 10 & & NS & $\mu \mathrm{g} / \mathrm{L}$ \\
\hline Alkalinity & WA & & & & 5 & & NS & $\mathrm{mg} / \mathrm{L}$ \\
\hline Allyl chloride & WA & & & $\mathbf{U}$ & 100 & & NS & $\mu g / L$ \\
\hline Aluminum & WA & & & $\mathbf{U}$ & 200 & & NS & $\mu g / L$ \\
\hline Antimony & WA & & & $\mathbf{U}$ & 60 & & 6 & $\mu g / L$ \\
\hline Arsenic & WA & & & $\mathbf{U}$ & 10 & & 50 & $\mu g / L$ \\
\hline Barium & WA & & & $\mathbf{U}$ & 200 & & 2000 & $\mu g / L$ \\
\hline Benzene & WA & & & $\mathbf{U}$ & 5 & & 5 & $\mu \mathrm{g} / \mathrm{L}$ \\
\hline Beryllium & WA & & & $\mathbf{U}$ & 5 & & 4 & $\mu \mathrm{g} / \mathrm{L}$ \\
\hline Bromodichloromethane & WA & & & $\mathbf{U}$ & 5 & & NS & $\mu \mathrm{g} / \mathrm{L}$ \\
\hline Bromoform & WA & & & U & 5 & & NS & $\mu g / L$ \\
\hline Cadmium & WA & & & $\mathbf{U}$ & 5 & & 5 & $\mu g / L$ \\
\hline Calcium & WA & & & $\mathbf{U}$ & 5000 & & NS & $\mu g / L$ \\
\hline Carbon disulfide & WA & & & $\mathbf{U}$ & 5 & & NS & $\mu \mathrm{g} / \mathrm{L}$ \\
\hline Carbon tetrachloride & WA & & & $\mathbf{U}$ & 5 & & 5 & $\mu \mathrm{g} / \mathrm{L}$ \\
\hline Cesium-137 & TM & & & $\mathrm{U}$ & 10 & & 200 & $\mathrm{pCi} / \mathrm{L}$ \\
\hline Chloride & WA & & & & 2.88 & & NS & $\mathrm{mg} / \mathrm{L}$ \\
\hline Chlorobenzene & WA & & & $\mathbf{U}$ & 5 & & 100 & $\mu g / L$ \\
\hline Chloroethane & WA & & & $\mathbf{U}$ & 10 & & NS & $\mu g / L$ \\
\hline Chloroform & WA & & & $\mathbf{U}$ & 5 & & 100 & $\mu g / L$ \\
\hline Chloroprene & WA & & & $\mathbf{U}$ & 20 & & NS & $\mu g / L$ \\
\hline Chromium & WA & & & $\mathbf{U}$ & 10 & & 100 & $\mu \mathrm{g} / \mathrm{L}$ \\
\hline cis-1,3-Dichloropropene & WA & & & $\mathbf{U}$ & 5 & & NS & $\mu g / L$ \\
\hline Cobalt & WA & & & $\mathbf{U}$ & 50 & & NS & $\mu \mathrm{g} / \mathrm{L}$ \\
\hline Copper & WA & & & $\mathbf{U}$ & 25 & & 1000 & $\mu g / L$ \\
\hline Cyanide & WA & & & $\mathbf{U}$ & 10 & & 200 & $\mu g / L$ \\
\hline Dibromochloromethane & WA & & & $\mathbf{U}$ & 5 & & NS & $\mu g / L$ \\
\hline Dichlorodifluoromethane & WA & & & $\mathbf{U}$ & 10 & & NS & $\mu g / L$ \\
\hline Ethylbenzene & WA & & & $\mathbf{U}$ & 5 & & 700 & $\mu \mathrm{g} / \mathrm{L}$ \\
\hline Fluoride & WA & & & $\mathrm{U}$ & 0.1 & & 4 & $\mathrm{mg} / \mathrm{L}$ \\
\hline Gross alpha & TM & & & U & 0.4 & & 15 & $\mathrm{pCi} / \mathrm{L}$ \\
\hline
\end{tabular}


UTD001 Laboratory Analyses (cont'd)

\begin{tabular}{|c|c|c|c|c|c|c|c|c|}
\hline Analyte & Lab & Rep & Avg & Qualifier & Result & Acc & Stand & Units \\
\hline Iron & WA & & & & 238 & & 300 & $\mu g / 2$ \\
\hline Isobutyl alcohol & WA & & & $\mathbf{U}$ & 20 & & NS & $\mu g / L$ \\
\hline Lead & WA & & & U & 3 & & 50 & $\mu_{g} / L$ \\
\hline Magnesium & WA & & & $\mathbf{U}$ & 5000 & & NS & $\mu g / L$ \\
\hline Manganese & WA & & & & 15.9 & & 50 & $\mu g / L$ \\
\hline Mercury & WA & & & $\mathbf{U}$ & 0.2 & . & 2 & $\mu_{g} / L$ \\
\hline Methacrylonitrile & WA & & & U & 20 & & NS & $\mu g / L$ \\
\hline Methyl bromide & WA & & & U & 10 & & NS & $\mu g / L$ \\
\hline Methyl chloride & WA & & & $\mathbf{U}$ & 10 & & NS & $\mu g / L$ \\
\hline Methyl ethyl ketone & WA & & & $\mathbf{U}$ & 10 & & NS & $\mu g / L$ \\
\hline Methyl iodide & WA & & & $\mathbf{U}$ & 10 & & NS & $\mu g / L$ \\
\hline Methylene bromide & WA & & & U & 10 & & NS & $\mu g / L$ \\
\hline Methylene chloride & WA & & & J & 1.37 & & 5 & $\mu g / L$ \\
\hline Nickel & WA & & & $\mathbf{U}$ & 40 & & 100 & $\mu g \alpha$ \\
\hline Nitrate as nitrogen & WA & & & & 0.111 & & 10 & $\mathrm{mg} / \mathrm{L}$ \\
\hline Nonvolatile beta & $\mathbf{T M}$ & & & & 1.5 & 1.5 & 50 & pCir/ \\
\hline Potassium & WA & & & $\mathbf{U}$ & 5000 & & NS & $\mu g / L$ \\
\hline Propionitrile & WA & & & $\mathbf{U}$ & 50 & & NS & $\mu g / L$ \\
\hline Selenium & WA & & & $\mathbf{U}$ & 5 & & 50 & $\mu g / L$ \\
\hline Silver & WA & & & $\mathbf{U}$ & 10 & & 50 & $\mu g / L$ \\
\hline Sodium & WA & & & & 6040 & & NS & $\mu g / L$ \\
\hline Styrene & WA & & & $\mathbf{U}$ & 5 & & 100 & $\mu_{g} / L$ \\
\hline Sulfate & WA & & & & 2.12 & & 400 & $\mathrm{mg} / \mathrm{L}$ \\
\hline Sulfide & WA & & & U & 0.1 & & NS & $\mathrm{mg} / \mathrm{l}$ \\
\hline Tetrachloroethylene & WA & & & U & 5 & & 5 & $\mu g /$ \\
\hline Thallium & WA & & & $\mathbf{U}$ & 10 & & 2 & $\mu g / L$ \\
\hline Toluene & WA & & & U & 5 & & 1000 & $\mu g / L$ \\
\hline Total hardness & WA & & & & 8 & & NS & $\mathrm{mg} / \mathrm{L}$ \\
\hline Total organic carbon & WA & & & & 1.44 & & NS & $\mathrm{mg} / \mathrm{L}$ \\
\hline Total suspended solids & WA & & & & 7 & & NS & $\mathrm{mg} / \mathrm{h}$ \\
\hline trans-1,3-Dichloropropene & WA & & & $\mathbf{U}$ & 5 & & NS & $\mu g / 2$ \\
\hline trans-1,4-Dichloro-2-butene & WA & & & $\mathrm{U}$ & 100 & & NS & $\mu g /$ \\
\hline Trichloroethylene & WA & & & U & 5 & & 5 & $\mu g /$ \\
\hline Trichlorofluoromethane & WA & & & $\mathbf{U}$ & 5 & & NS & $\mu_{8}$ \\
\hline Tritium & TM & & & & 9 & 1 & 20 & $\mathrm{pCi} / \mathrm{m}$ \\
\hline Turbidity & WA & & & & 6.89 & & NS & NTU \\
\hline Vanadium & WA & & & $\mathbf{U}$ & 50 & & NS & $\mu g /$ \\
\hline Vinyl acetate & WA & & & U & 10 & & NS & $\mu g /$ \\
\hline Vinyl chloride & WA & & & U & 10 & & 2 & $\mu \mathrm{g} /$ \\
\hline Xylenes (total) & WA & & & $\mathbf{U}$ & 5 & & 10000 & 1 \\
\hline Zinc & WA & & & U & 20 & & 5000 & $\mu \mathrm{g} /$ \\
\hline
\end{tabular}




\section{UTD002}

Field Measurements:

Stream Discharge: $0.74 \mathrm{cfs}$

Sample Date: 4/26/93

pH: 6.45

Specific Conductance: $25 \mu \mathrm{S} / \mathrm{cm}$

Red/Oxid Potential: $143 \mathrm{mv}$

Dissolved Oxygen: $8 \mathrm{mg} / \mathrm{L}$

\begin{tabular}{|c|c|c|c|c|c|c|c|}
\hline Analyte & Lab & Avg & Qualifier & Result & Acc & Stand & Units \\
\hline 1,1,1,2-Tetrachloroethane & WA & & $\bar{U}$ & 10 & & NS & $\mu g / L$ \\
\hline 1,1,1-Trichloroethane & WA & & U & 5 & & 200 & $\mu \mathrm{g} / \mathrm{L}$ \\
\hline 1,1,2,2-Tetrachloroethane & WA & & $\mathbf{U}$ & 5 & & NS & $\mu \mathrm{g} / \mathrm{L}$ \\
\hline 1,1,2-Trichloroethane & WA & & $\mathbf{U}$ & 5 & & 5 & $\mu \mathrm{g} / \mathrm{L}$ \\
\hline 1,1-Dichloroethane & WA & & $\mathbf{U}$ & 5 & & NS & $\mu g / L$ \\
\hline 1,1-Dichloroethylene & WA & & $\mathbf{U}$ & 5 & & 7 & $\mu g / L$ \\
\hline 1,2,3-Trichloropropane & WA & & $\mathbf{U}$ & 10 & & NS & $\mu g / L$ \\
\hline 1,2-Dibromo-3-chloropropane & WA & & $\mathbf{U}$ & 20 & & 0.2 & $\mu g / L$ \\
\hline 1,2-Dibromoethane & WA & & $\mathbf{U}$ & 20 & & NS & $\mu g / L$ \\
\hline 1,2-Dichloroethane & WA & & $\mathbf{U}$ & 5 & & 5 & $\mu g / L$ \\
\hline 1,2-Dichloroethylene (total) & WA & & $\mathbf{U}$ & 5 & & 100 & $\mu g / L$ \\
\hline 1,2-Dichloropropane & WA & & $\mathbf{U}$ & 5 & & 5 & $\mu \mathrm{g} / \mathrm{L}$ \\
\hline 2-Hexanone & WA & & $\mathbf{U}$ & 10 & & NS & $\mu g / L$ \\
\hline 4-Methyl-2-pentanone & WA & & $\mathbf{U}$ & 10 & & NS & $\mu g / L$ \\
\hline Acetone & WA & & $\mathbf{U}$ & 10 & & NS & $\mu g / L$ \\
\hline Acetonitrile & WA & & $\mathbf{U}$ & 20 & & NS & $\mu g / L$ \\
\hline Acidity & WA & & & 9 & & NS & $\mathrm{mg} / \mathrm{L}$ \\
\hline Acrolein & WA & & U & 10 & & NS & $\mu \mathrm{g} / \mathrm{L}$ \\
\hline Acrylonitrile & WA & & U & 10 & & NS & $\mu \mathrm{g} / \mathrm{L}$ \\
\hline Alkalinity & WA & & $\mathbf{U}$ & 0.5 & & NS & $\mathrm{mg} / \mathrm{L}$ \\
\hline Allyl chloride & WA & & $\mathrm{U}$ & 100 & & NS & $\mu \mathrm{g} / \mathrm{L}$ \\
\hline Aluminum & WA & & $\mathrm{U}$ & 200 & & NS & $\mu g / L$ \\
\hline Antimony & WA & & U & 60 & & 6 & $\mu \mathrm{g} / \mathrm{L}$ \\
\hline Arsenic & WA & & $\mathbf{U}$ & 10 & & 50 & $\mu g / L$ \\
\hline Barium & WA & & $\mathrm{U}$ & 200 & & 2000 & $\mu g / L$ \\
\hline Benzene & WA & & $\mathrm{U}$ & 5 & & 5 & $\mu g / L$ \\
\hline Beryllium & WA & & $\mathrm{U}$ & 5 & & 4 & $\mu g / L$ \\
\hline Bromodichloromethane & WA & & $\mathrm{U}$ & 5 & & NS & $\mu \mathrm{g} / \mathrm{L}$ \\
\hline Bromoform & WA & & $\mathrm{U}$ & 5 & & NS & $\mu g / L$ \\
\hline Cadmium & WA & & U & 5 & & 5 & $\mu \mathrm{g} / \mathrm{L}$ \\
\hline Calcium & WA & & U & 5000 & & NS & $\mu \mathrm{g} / \mathrm{L}$ \\
\hline Carbon disulfide & WA & & $\mathrm{U}$ & 5 & & NS & $\mu g / L$ \\
\hline Carbon tetrachloride & WA & & $\mathbf{U}$ & 5 & & 5 & $\mu g / L$ \\
\hline Cesium-137 & TM & & $\mathbf{U}$ & 9.8 & & 200 & $\mathrm{pCi} / \mathrm{L}$ \\
\hline Chloride & WA & & & 1.77 & & NS & $\mathrm{mg} / \mathrm{L}$ \\
\hline Chlorobenzene & WA & & U & 5 & & 100 & $\mu \mathrm{g} / \mathrm{L}$ \\
\hline Chloroethane & WA & & $\mathrm{U}$ & 10 & & NS & $\mu g / L$ \\
\hline Chloroform & WA & & U & 5 & & 100 & $\mu \mathrm{g} / \mathrm{L}$ \\
\hline Chloroprene & WA & & U & 20 & & NS & $\mu g / L$ \\
\hline Chromium & WA & & $\mathrm{U}$ & 10 & & 100 & $\mu g / L$ \\
\hline cis-1,3-Dichloropropene & WA & & U & 5 & & NS & $\mu g / L$ \\
\hline Cobalt & WA & & U & 50 & & NS & $\mu \mathrm{g} / \mathrm{L}$ \\
\hline Copper & WA & & U & 25 & & 1000 & $\mu g / L$ \\
\hline Cyanide & WA & & U & 10 & & 200 & $\mu g / L$ \\
\hline Dibromochloromethane & WA & & $\mathrm{U}$ & 5 & & NS & $\mu \mathrm{g} / \mathrm{L}$ \\
\hline Dichlorodifluoromethane & WA & & $\mathbf{U}$ & 10 & & NS & $\mu g / L$ \\
\hline Ethylbenzene & WA & & $\mathbf{U}$ & 5 & & 700 & $\mu \mathrm{g} / \mathrm{L}$ \\
\hline Fluoride & WA & & $\mathrm{U}$ & 0.1 & & 4 & $\mathrm{mg} / \mathrm{L}$ \\
\hline
\end{tabular}


UTD002 Laboratory Analyses (cont'd)

\begin{tabular}{|c|c|c|c|c|c|c|c|}
\hline Analyte & Lab & Avg & Qualifier & Result & Acc & Stand & Units \\
\hline Gross alpha & TM & & & 0.9 & 0.9 & 15 & $\mathrm{pCi} / \mathrm{L}$ \\
\hline Iron & WA & & & 348 & & 300 & $\mu g / L$ \\
\hline Isobutyl alcohol & WA & & $\mathbf{U}$ & 20 & & NS & $\mu \mathrm{g} \Omega$ \\
\hline Lead & WA & & U & 3 & & 50 & $\mu \mathrm{g} / \mathrm{L}$ \\
\hline Magnesium & WA & & $\mathbf{U}$ & 5000 & & NS & $\mu g / L$ \\
\hline Manganese & WA & & & 30.1 & . & 50 & $\mu g / L$ \\
\hline Mercury & WA & & $\mathrm{U}$ & 0.2 & & 2 & $\mu g / L$ \\
\hline Methacrylonitrile & WA & & $\mathbf{U}$ & 20 & & NS & $\mu g / L$ \\
\hline Methyl bromide & WA & & $\mathbf{U}$ & 10 & & NS & $\mu g / L$ \\
\hline Methyl chloride & WA & & $\mathbf{U}$ & 10 & & NS & $\mu g / L$ \\
\hline Methyl ethyl ketone & WA & & $\mathbf{U}$ & 10 & & NS & $\mu \mathrm{g} / \mathrm{L}$ \\
\hline Methyl iodide & WA & & $\mathbf{U}$ & 10 & & NS & $\mu g / L$ \\
\hline Methylene bromide & WA & & $\mathbf{U}$ & 10 & & NS & $\mu g / \mathrm{L}$ \\
\hline Methylene chloride & WA & & $\mathbf{J}$ & 1.01 & & 5 & $\mu g / L$ \\
\hline Nickel & WA & & $\mathbf{U}$ & 40 & & 100 & $\mu g / L$ \\
\hline Nitrate as nitrogen & WA & & $\mathbf{U}$ & 0.02 & & 10 & $\mathbf{m g} / \mathbf{L}$ \\
\hline Nonvolatile beta & TM & & & 1.5 & 1.5 & 50 & $\mathrm{pCi} / \mathrm{L}$ \\
\hline Potassium & WA & & $\mathbf{U}$ & 5000 & & NS & $\mu g / L$ \\
\hline Propionitrile & WA & & $\mathbf{U}$ & 50 & & NS & $\mu g / L$ \\
\hline Selenium & WA & & $\mathbf{U}$ & 5 & & 50 & $\mu g / L$ \\
\hline Silver & WA & & $\mathbf{U}$ & 10 & & 50 & $\mu g / L$ \\
\hline Sodium & WA & & $\mathrm{U}$ & 5000 & & NS & $\mu g / L$ \\
\hline Styrene & WA & & $\mathbf{U}$ & 5 & & 100 & $\mu g / L$ \\
\hline Sulfate & WA & & $\mathrm{U}$ & 0.25 & & 400 & $\mathrm{mg} / \mathrm{L}$ \\
\hline Sulfide & WA & & $\mathbf{U}$ & 0.1 & & NS & $\mathrm{mg} / \mathrm{L}$ \\
\hline Tetrachloroethylene & WA & & $\mathbf{U}$ & 5 & & 5 & $\mu \mathrm{g} / \mathrm{L}$ \\
\hline Thallium & WA & & $\mathbf{U}$ & 10 & & 2 & $\mu g / L$ \\
\hline Toluene & WA & & $\mathrm{U}$ & 5 & & 1000 & $\mu g / L$ \\
\hline Total hardness & WA & & & 21 & & NS & $\mathrm{mg} / \mathrm{L}$ \\
\hline Total or zanic carbon & WA & & & 3.49 & & NS & $\mathrm{mg} / \mathrm{L}$ \\
\hline Total suspended solids & WA & & & 14 & & NS & $\mathrm{mg} / \mathrm{L}$ \\
\hline trans-1,3-Dichloropropene & WA & & $\mathbf{U}$ & 5 & & NS & $\mu \mathrm{g} / \mathrm{L}$ \\
\hline trans-1,4-Dichloro-2-butene & WA & & $\mathbf{U}$ & 100 & & NS & $\mu \mathrm{g} / \mathrm{L}$ \\
\hline Trichloroethylene & WA & & $\mathbf{U}$ & 5 & & 5 & $\mu g / L$ \\
\hline Trichlorofluoromethane & WA & & $\mathrm{U}$ & 5 & & NS & $\mu \mathrm{g} / \mathrm{L}$ \\
\hline Tritium & TM & & & 41.3 & 3.9 & 20 & $\mathrm{pCi} / \mathrm{ml}$ \\
\hline Turbidity & WA & & & 15.6 & & NS & NTU \\
\hline Vanadium & WA & & $\mathbf{U}$ & 50 & & NS & $\mu \mathrm{g} / \mathrm{L}$ \\
\hline Vinyl acetate & WA & & $\mathbf{U}$ & 10 & & NS & $\mu g / L$ \\
\hline Vinyl chloride & WA & & $\mathbf{U}$ & 10 & & 2 & $\mu g / L$ \\
\hline Xylenes (total) & WA & & $U$ & 5 & & 10000 & $\mu \mathrm{g} / \mathrm{L}$ \\
\hline Zinc & WA & & $\mathbf{U}$ & 20 & & 5000 & $\mu \mathrm{g} / \mathrm{L}$ \\
\hline
\end{tabular}




\section{UTD003}

Field Measurements:

Stream Discharge: 0.14 cfs

Sample Date: 4/26/93

pH: 7.37

Specific Conductance: $33 \mu \mathrm{S} / \mathrm{cm}$

Red/Oxid Potential: $196 \mathrm{mv}$

Dissolved Oxygen: $7.2 \mathrm{mg} / \mathrm{L}$

\begin{tabular}{|c|c|c|c|c|c|c|c|c|}
\hline Analyte & Lab & Rep & Avg & Qualifier & Result & Acc & Stand & Units \\
\hline 1,1,1,2-Tetrachloroethane & WA & & & $\mathbf{U}$ & 10 & & NS & $\mu g / L$ \\
\hline $1,1,1,2$-Tetrachloroethane & WA & & NA & $\mathbf{U}$ & 10 & & NS & $\mu g / L$ \\
\hline $1,1,1,2$-Tetrachloroethane & WA & & $\mathbf{N A}$ & $\mathbf{U}$ & 10 & & NS & $\mu g / L$ \\
\hline 1,1,1-Trichloroethane & WA & & & $\mathbf{U}$ & 5 & & 200 & $\mu g / L$ \\
\hline 1,1,1-Trichloroethane & WA & & NA & $\mathbf{U}$ & 5 & & 200 & $\mu g / L$ \\
\hline 1,1,1-Trichloroethane & WA & & NA & $\mathbf{U}$ & 5 & & 200 & $\mu g / L$ \\
\hline 1,1,2,2-Tetrachloroethane & WA & & & $\mathbf{U}$ & 5 & & NS & $\mu \mathrm{g} / \mathrm{L}$ \\
\hline $1,1,2,2$-Tetrachloroethane & WA & & NA & $\mathbf{U}$ & 5 & & NS & $\mu g / L$ \\
\hline 1,1,2,2-Tetrachloroethane & WA & & NA & $\mathbf{U}$ & 5 & & NS & $\mu g / L$ \\
\hline 1,1,2-Trichloroethane & WA & & & $\mathbf{U}$ & 5 & & 5 & $\mu g / L$ \\
\hline 1,1,2-Trichloroethane & WA & & NA & $\mathbf{U}$ & 5 & & 5 & $\mu g / L$ \\
\hline 1,1,2-Trichloroethane & WA & & NA & $\mathbf{U}$ & 5 & & 5 & $\mu g / L$ \\
\hline 1,1-Dichloroethane & WA & & & $\mathbf{U}$ & 5 & & NS & $\mu \mathrm{g} / \mathrm{L}$ \\
\hline 1,1-Dichloroethane & WA & & NA & $\mathbf{U}$ & 5 & & NS & $\mu \mathrm{g} / \mathrm{L}$ \\
\hline 1,1-Dichloroethane & WA & & NA & $\mathbf{U}$ & 5 & & NS & $\mu \mathrm{g} / \mathrm{L}$ \\
\hline 1,1-Dichloroethylene & WA & & & $\mathbf{U}$ & 5 & & 7 & $\mu \mathrm{g} / \mathrm{L}$ \\
\hline 1,2,3-Trichloropropane & WA & & & $\mathbf{U}$ & 10 & & NS & $\mu g / L$ \\
\hline 1,2,3-Trichloropropane & WA & & NA & $\mathbf{U}$ & 10 & & NS & $\mu g / L$ \\
\hline 1,2,3-Trichloropropane & WA & & NA & $\mathbf{U}$ & 10 & & NS & $\mu g / L$ \\
\hline 1,2-Dibromo-3-chloropropane & WA & & & $\mathbf{U}$ & 20 & & 0.2 & $\mu \mathrm{g} / \mathrm{L}$ \\
\hline 1,2-Dibromo-3-chloropropane & WA & & NA & $\mathbf{U}$ & 20 & & 0.2 & $\mu g / L$ \\
\hline 1,2-Dibromo-3-chloropropane & WA & & NA & $\mathbf{U}$ & 20 & & 0.2 & $\mu \mathrm{g} / \mathrm{L}$ \\
\hline 1,2-Dibromoethane & WA & & & $\mathbf{U}$ & 20 & & NS & $\mu g / L$ \\
\hline 1,2-Dibromoethane & WA & & NA & $\mathrm{U}$ & 20 & & NS & $\mu g / L$ \\
\hline 1,2-Dibromoethane & WA & & NA & $\mathbf{U}$ & 20 & & NS & $\mu \mathrm{g} / \mathrm{L}$ \\
\hline 1,2-Dichloroethane & WA & & & $\mathbf{U}$ & 5 & & 5 & $\mu \mathrm{g} / \mathrm{L}$ \\
\hline 1,2-Dichloroethane & WA & & NA & $\mathbf{U}$ & 5 & & 5 & $\mu g / L$ \\
\hline 1,2-Dichloroethane & WA & & NA & $\mathbf{U}$ & 5 & & 5 & $\mu \mathrm{g} / \mathrm{L}$ \\
\hline 1,2-Dichloroethylene (total) & WA & & & $\mathbf{U}$ & 5 & & 100 & $\mu \mathrm{g} / \mathrm{L}$ \\
\hline 1,2-Dichloroethylene (total) & WA & & NA & $\mathbf{U}$ & 5 & & 100 & $\mu g / L$ \\
\hline 1,2-Dichloroethylene (total) & WA & & NA & $\mathbf{U}$ & 5 & & 100 & $\mu g / L$ \\
\hline 1,2-Dichloropropane & WA & & & $\mathbf{U}$ & 5 & & 5 & $\mu g / L$ \\
\hline 1,2-Dichloropropane & WA & & NA & U & 5 & & 5 & $\mu \mathrm{g} / \mathrm{L}$ \\
\hline 1,2-Dichloropropane & WA & & NA & $\mathrm{U}$ & 5 & & 5 & $\mu g / L$ \\
\hline 2-Hexanone & WA & & & $\mathbf{U}$ & 10 & & NS & $\mu g / L$ \\
\hline 2-Hexanone & WA & & NA & $\mathbf{U}$ & 10 & & NS & $\mu \mathrm{g} / \mathrm{L}$ \\
\hline 2-Hexanone & WA & & NA & $\mathbf{U}$ & 10 & & NS & $\mu \mathrm{g} / \mathrm{L}$ \\
\hline 4-Methyl-2-pentanone & WA & & & U & 10 & & NS & $\mu g / L$ \\
\hline 4-Methyl-2-pentanone & WA & & NA & $\mathbf{U}$ & 10 & & NS & $\mu g / L$ \\
\hline 4-Methyl-2-pentanone & WA & & NA & $\mathrm{U}$ & 10 & & NS & $\mu \mathrm{g} / \mathrm{L}$ \\
\hline Acetone & WA & & & $\mathbf{U}$ & 10 & & NS & $\mu g / L$ \\
\hline Acetone & WA & & NA & $\mathrm{U}$ & 10 & & NS & $\mu g / L$ \\
\hline Acetone & WA & & NA & $\mathbf{U}$ & 10 & & NS & $\mu \mathrm{g} / \mathrm{L}$ \\
\hline Acetonitrile & WA & & & $\mathbf{U}$ & 20 & & NS & $\mu g / L$ \\
\hline Acetonitrile & WA & & NA & $\mathbf{U}$ & 20 & & NS & $\mu \mathrm{g} / \mathrm{L}$ \\
\hline Acetonitrile & WA & & NA & $\mathbf{U}$ & 20 & & NS & $\mu g / L$ \\
\hline Acidity & WA & & & & 8 & & NS & $\mathrm{mg} / \mathrm{L}$ \\
\hline
\end{tabular}


UTD003 Laboratory Analyses (cont'd)

\begin{tabular}{|c|c|c|c|c|c|c|c|c|}
\hline Analyte & Lab & Rep & Avg & Qualifier & Result & Acc & Stand & Units \\
\hline Acrolein & WA & & & $\mathbf{U}$ & 10 & & NS & $\mu g / L$ \\
\hline Acrolein & WA & & NA & $\mathbf{U}$ & 10 & & NS & $\mu g / L$ \\
\hline Acrolein & WA & & NA & $\mathbf{U}$ & 10 & & NS & $\mu \mathrm{g} / \mathrm{L}$ \\
\hline Acrylonitrile & WA & & & $\mathbf{U}$ & 10 & & NS & $\mu g / L$ \\
\hline Acrylonitrile & WA & & NA & $\mathbf{U}$ & 10 & & NS & $\mu g / L$ \\
\hline Acrylonitrile & WA & & NA & $\mathbf{U}$ & 10 & & NS & $\mu g / L$ \\
\hline Alkalinity & WA & & & & 2 & & NS & $\mathrm{mg} / \mathrm{L}$ \\
\hline Allyl chloride & WA & & & $\mathbf{U}$ & 100 & & NS & $\mu g / L$ \\
\hline Allyl chloride & WA & & NA & $\mathbf{U}$ & 100 & & NS & $\mu g / L$ \\
\hline Allyl chloride & WA & & NA & $\mathbf{U}$ & 100 & & NS & $\mu \mathrm{g} / \mathrm{L}$ \\
\hline Aluminum & WA & & & $\mathbf{U}$ & 200 & & NS & $\mu g / L$ \\
\hline Americium-241 & $T M$ & & & & 40.6 & 8.1 & 6.34 & $\mathrm{pCi} / \mathrm{L}$ \\
\hline Antimony & WA & & & $\mathbf{U}$ & 60 & & 6 & $\mu \mathrm{g} / \mathrm{L}$ \\
\hline Arsenic & WA & & & $\mathbf{U}$ & 10 & & 50 & $\mu g / L$ \\
\hline Barium & WA & & & $\mathbf{U}$ & 200 & & 2000 & $\mu g / L$ \\
\hline Benzene & WA & & & U & 5 & & 5 & $\mu g / L$ \\
\hline Beryllium & WA & & & $\mathbf{U}$ & 5 & & 4 & $\mu g / L$ \\
\hline Bromodichloromethane & WA & & & $\mathbf{U}$ & 5 & & NS & $\mu g / L$ \\
\hline Bromodichloromethane & WA & & NA & $\mathbf{U}$ & 5 & & NS & $\mu g / L$ \\
\hline Bromodichloromethane & WA & & NA & $\mathbf{U}$ & 5 & & NS & $\mu \mathrm{g} / \mathrm{L}$ \\
\hline Bromoform & WA & & & $\mathbf{U}$ & 5 & & NS & $\mu g / L$ \\
\hline Bromoform & WA & & NA & $\mathbf{U}$ & 5 & & NS & $\mu g / L$ \\
\hline Bromoform & WA & & NA & $\mathbf{U}$ & 5 & & NS & $\mu g / L$ \\
\hline Cadmium & WA & & & $\mathbf{U}$ & 5 & & 5 & $\mu g / L$ \\
\hline Calcium & WA & & & $\mathbf{U}$ & 5000 & & NS & $\mu \mathrm{g} / \mathrm{L}$ \\
\hline Carbon disulfide & WA & & & $\mathbf{U}$ & 5 & & NS & $\mu g / L$ \\
\hline Carbon disulfide & WA & & NA & $\mathbf{U}$ & 5 & & NS & $\mu \mathrm{g} / \mathrm{L}$ \\
\hline Carbon disulfide & WA & & NA & $\mathbf{U}$ & 5 & & NS & $\mu \mathrm{g} / \mathrm{L}$ \\
\hline Carbon tetrachloride & WA & & & $\mathbf{U}$ & 5 & & 5 & $\mu g / L$ \\
\hline Carbon tetrachloride & WA & & NA & $\mathbf{U}$ & 5 & & 5 & $\mu \mathrm{g} / \mathrm{L}$ \\
\hline Carbon tetrachloride & WA & & NA & $\mathbf{U}$ & 5 & & 5 & $\mu \mathrm{g} / \mathrm{L}$ \\
\hline Cesium-137 & TM & & & $\mathbf{U}$ & 9.6 & & 200 & $\mathrm{pCi} / \mathrm{L}$ \\
\hline Chloride & WA & & & & 1.78 & & NS & $\mathrm{mg} / \mathrm{L}$ \\
\hline Chlorobenzene & WA & & & $\mathbf{U}$ & 5 & & 100 & $\mu g / L$ \\
\hline Chloroethane & WA & & & $\mathbf{U}$ & 10 & & NS & $\mu g / L$ \\
\hline Chloroethane & WA & & NA & $\mathbf{U}$ & 10 & & NS & $\mu \mathrm{g} / \mathrm{L}$ \\
\hline Chloroethane & WA & & NA & $\mathbf{U}$ & 10 & & NS & $\mu \mathrm{g} / \mathrm{L}$ \\
\hline Chloroform & WA & & & $\mathbf{U}$ & 5 & & 100 & $\mu \mathrm{g} / \mathrm{L}$ \\
\hline Chloroform & WA & & NA & $\mathbf{U}$ & 5 & & 100 & $\mu g / L$ \\
\hline Chloroform & WA & & NA & $\mathbf{U}$ & 5 & & 100 & $\mu \mathrm{g} / \mathrm{L}$ \\
\hline Chloroprene & WA & & & U & 20 & & NS & $\mu g / L$ \\
\hline Chloroprene & WA & & NA & $\mathrm{U}$ & 20 & & NS & $\mu \mathrm{g} / \mathrm{L}$ \\
\hline Chloroprene . & WA & & NA & $\mathbf{U}$ & 20 & & NS & $\mu \mathrm{g} / \mathrm{L}$ \\
\hline Chromium & WA & & & $\mathbf{U}$ & 10 & & 100 & $\mu g / L$ \\
\hline cis-1,3-Dichloropropene & WA & & & U & 5 & & NS & $\mu \mathrm{g} / \mathrm{L}$ \\
\hline cis-1,3-Dichloropropene & WA & & NA & $\mathbf{U}$ & 5 & & NS & $\mu g / L$ \\
\hline cis-1,3-Dichloropropene & WA & & NA & $\mathbf{U}$ & 5 & & NS & $\mu \mathrm{g} / \mathrm{L}$ \\
\hline Cobalt & WA & & & $\mathbf{U}$ & 50 & & NS & $\mu \mathrm{g} / \mathrm{L}$ \\
\hline Copper & WA & & & $\mathbf{U}$ & 25 & & 1000 & $\mu \mathrm{g} / \mathrm{L}$ \\
\hline Cyanide & WA & & & $\mathbf{U}$ & 10 & & 200 & $\mu \mathrm{g} / \mathrm{L}$ \\
\hline Dibromochloromethane & WA & & & $\mathbf{U}$ & 5 & & NS & $\mu g / L$ \\
\hline Dibromochloromethane & WA & & NA & $\mathbf{U}$ & 5 & & NS & $\mu \mathrm{g} / \mathrm{L}$ \\
\hline Dibromochloromethane & WA & & NA & $\mathbf{U}$ & 5 & & NS & $\mu g / L$ \\
\hline
\end{tabular}


UTD003 Laboratory Analyses (cont'd)

\begin{tabular}{|c|c|c|c|c|c|c|c|c|}
\hline Analyte & Lab & Rep & Avg & Qualifier & Result & Acc & Stand & Units \\
\hline Dichlorodifluoromethane & WA & & & $\mathbf{U}$ & 10 & & NS & $\mu g / L$ \\
\hline Dichlorodifluoromethane & WA & & NA & $\mathbf{U}$ & 10 & & NS & $\mu \mathrm{g} / \mathrm{L}$ \\
\hline Dichlorodifluoromethane & WA & & NA & $\mathbf{U}$ & 10 & & NS & $\mu g / L$ \\
\hline Ethylbenzene & WA & & & U & 5 & & 700 & $\mu g / L$ \\
\hline Ethylbenzene & WA & & NA & $\mathbf{U}$ & 5 & & 700 & $\mu g / L$ \\
\hline Ethylbenzene & WA & & NA & $\mathbf{U}$ & 5 & & 700 & $\mu g / L$ \\
\hline Fluoride & WA & & & $\mathbf{U}$ & 0.1 & & 4 & $\mathrm{mg} / \mathrm{L}$ \\
\hline Gross alpha & TM & & & & 0.6 & 0.8 & 15 & $\mathrm{pCi} / \mathrm{L}$ \\
\hline Iron & WA & & & & 345 & & 300 & $\mu \mathrm{g} / \mathrm{L}$ \\
\hline Isobutyl alcohol & WA & & & $\mathbf{U}$ & 20 & & NS & $\mu \mathrm{g} / \mathrm{L}$ \\
\hline Isobutyl alcohol & WA & & NA & $\mathbf{U}$ & 20 & & NS & $\mu g / L$ \\
\hline Isobutyl alcohol & WA & & NA & $\mathbf{U}$ & 20 & & NS & $\mu g / L$ \\
\hline Lead & WA & & & $\mathbf{U}$ & 3 & & 50 & $\mu \mathrm{g} / \mathrm{L}$ \\
\hline Magnesium & WA & & & $\mathbf{U}$ & 5000 & & NS & $\mu g / L$ \\
\hline Manganese & WA & & & & 120 & & 50 & $\mu \mathrm{g} / \mathrm{L}$ \\
\hline Mercury & WA & & . & $\mathbf{U}$ & 0.2 & & 2 & $\mu g / L$ \\
\hline Methacrylonitrile & WA & & & $\mathbf{U}$ & 20 & & NS & $\mu g / L$ \\
\hline Methacrylonitrile & WA & & NA & $\mathbf{U}$ & 20 & & NS & $\mu g / L$ \\
\hline Methacrylonitrile & WA & & NA & $\mathbf{U}$ & 20 & & NS & $\mu g / L$ \\
\hline Methyl bromide & WA & & & $\mathbf{U}$ & 10 & & NS & $\mu g / L$ \\
\hline Methyl bromide & WA & & NA & $\mathbf{U}$ & 10 & & NS & $\mu g / L$ \\
\hline Methyl bromide & WA & & NA & $\mathbf{U}$ & 10 & & NS & $\mu g / L$ \\
\hline Methyl chloride & WA & & & $\mathbf{U}$ & 10 & & NS & $\mu \mathrm{g} / \mathrm{L}$ \\
\hline Methyl chloride & WA & & NA & $\mathbf{U}$ & 10 & & NS & $\mu g / L$ \\
\hline Methyl chloride & WA & & NA & $\mathbf{U}$ & 10 & & NS & $\mu \mathrm{g} / \mathrm{L}$ \\
\hline Methyl ethyl ketone & WA & & & $\mathbf{U}$ & 10 & & NS & $\mu g / L$ \\
\hline Methyl ethyl ketone & WA & & NA & $\mathbf{U}$ & 10 & & NS & $\mu g / L$ \\
\hline Methyl ethyl ketone & WA & & NA & $\mathbf{U}$ & 10 & & NS & $\mu g / L$ \\
\hline Methyl iodide & WA & & & U & 10 & & NS & $\mu \mathrm{g} / \mathrm{L}$ \\
\hline Methyl iodide & WA & & NA & U & 10 & & NS & $\mu g / L$ \\
\hline Methyl iodide & WA & & NA & $\mathrm{U}$ & 10 & & NS & $\mu g / L$ \\
\hline Methylene bromide & WA & & & $\mathbf{U}$ & 10 & & NS & $\mu \mathrm{g} / \mathrm{L}$ \\
\hline Methylene bromide & WA & & NA & $\mathbf{U}$ & 10 & & NS & $\mu g / L$ \\
\hline Methylene bromide & WA & & NA & $\mathbf{U}$ & 10 & & NS & $\mu g / L$ \\
\hline Methylene chloride & WA & & & $\mathbf{U}$ & 5 & & 5 & $\mu \mathrm{g} / \mathrm{L}$ \\
\hline Methylene chloride & WA & & NA & $\mathbf{U}$ & 5 & & 5 & $\mu g / L$ \\
\hline Methylene chloride & WA & & NA & $\mathbf{U}$ & 5 & & 5 & $\mu g / L$ \\
\hline Nickel & WA & & & $\mathbf{U}$ & 40 & & 100 & $\mu \mathrm{g} / \mathrm{L}$ \\
\hline Nitrate as nitrogen & WA & & & $\mathbf{U}$ & 0.02 & & 10 & $\mathrm{mg} / \mathrm{L}$ \\
\hline Nonvolatile beta & TM & & & & 2.3 & 1.6 & 50 & $\mathrm{pCi} / \mathrm{L}$ \\
\hline Potassium & WA & & & $\mathbf{U}$ & 5000 & & NS & $\mu g / L$ \\
\hline Propionitrile & WA & & & $\mathbf{U}$ & 50 & & NS & $\mu g / L$ \\
\hline Propionitrile & WA & & NA & $\mathbf{U}$ & 50 & & NS & $\mu \mathrm{g} / \mathrm{L}$ \\
\hline Propionitrile & WA & & NA & $\mathbf{U}$ & 50 & & NS & $\mu \mathrm{g} / \mathrm{L}$ \\
\hline Selenium & WA & & & $\mathbf{U}$ & 5 & & 50 & $\mu \mathrm{g} / \mathrm{L}$ \\
\hline Silver & WA & & & $\mathbf{U}$ & 10 & & 50 & $\mu \mathrm{g} / \mathrm{L}$ \\
\hline Sodium & WA & & & $\mathbf{U}$ & 5000 & & NS & $\mu \mathrm{g} / \mathrm{L}$ \\
\hline Styrene & WA & & & $\mathbf{U}$ & 5 & & 100 & $\mu \mathrm{g} / \mathrm{L}$ \\
\hline Styrene & WA & & NA & $\mathbf{U}$ & 5 & & 100 & $\mu \mathrm{g} / \mathrm{L}$ \\
\hline Styrene & WA & & NA & $\mathbf{U}$ & 5 & & 100 & $\mu \mathrm{g} / \mathrm{L}$ \\
\hline Sulfate & WA & & & $\mathbf{U}$ & 0.25 & & 400 & $\mathrm{mg} / \mathrm{L}$ \\
\hline Sulfide & WA & & & $\mathbf{U}$ & 0.1 & & NS & $\mathrm{mg} / \mathrm{L}$ \\
\hline Tetrachloroethylene & WA & & & $\mathbf{U}$ & 5 & & 5 & $\mu g / L$ \\
\hline Tetrachloroethylene & WA & & NA & U & 5 & & 5 & $\mu g / L$ \\
\hline Tetrachloroethylene & WA & & NA & $\mathrm{U}$ & 5 & & 5 & $\mu \mathrm{g} / \mathrm{L}$ \\
\hline
\end{tabular}


UTD003 Laboratory Analyses (cont'd)

\begin{tabular}{|c|c|c|c|c|c|c|c|c|}
\hline Analyte & Lab & Rep & Avg & Qualifier & Result & Acc & Stand & Units \\
\hline Thallium & WA & & & $\bar{U}$ & 10 & & 2 & $\mu g / L$ \\
\hline Toluene & WA & & & $\mathbf{U}$ & 5 & & 1000 & $\mu \mathrm{g} / \mathrm{L}$ \\
\hline Total hardness & WA & & & & 7 & & NS & $\mathrm{mg} / \mathrm{L}$ \\
\hline Total organic carbon & WA & & & & 2.4 & & NS & $\mathrm{mg} / \mathrm{L}$ \\
\hline Total suspended solids & WA & & $\mathbf{A}$ & & 15.5 & & NS & $\mathrm{mg} / \mathrm{L}$ \\
\hline trans-1,3-Dichloropropene & WA & & & $\mathbf{U}$ & 5 & . & NS & $\mu g / L$ \\
\hline trans-1,3-Dichloropropene & WA & & NA & $\mathbf{U}$ & 5 & & NS & $\mu g / L$ \\
\hline trans-1,3-Dichloropropene & WA & & NA & $\mathbf{U}$ & 5 & & NS & $\mu \mathrm{g} / \mathrm{L}$ \\
\hline trans-1,4-Dichloro-2-butene & WA & & & $\mathbf{U}$ & 100 & & NS & $\mu \mathrm{g} / \mathrm{L}$ \\
\hline trans-1,4-Dichloro-2-butene & WA & & NA & $\mathbf{U}$ & 100 & & NS & $\mu g \Omega$ \\
\hline trans-1,4-Dichloro-2-butene & WA & & NA & $\mathbf{U}$ & 100 & & NS & $\mu g / L$ \\
\hline Trichloroethylene & WA & & & $\mathbf{U}$ & 5 & & 5 & $\mu g / L$ \\
\hline Trichlorofluoromethane & WA & & & $\mathbf{U}$ & 5 & & NS & $\mu g / 2$ \\
\hline Trichlorofluoromethane & WA & & NA & $\mathbf{U}$ & 5 & & NS & $\mu \mathrm{g} / \mathrm{L}$ \\
\hline Trichlorofluoromethane & WA & & NA & $\mathbf{U}$ & 5 & & NS & $\mu g / L$ \\
\hline Tritium & $\mathbf{T M}$ & & & & 68.2 & 6.4 & 20 & $\mathrm{pCi} / \mathrm{ml}$ \\
\hline Turbidity & WA & & & & 6.66 & & NS & NTU \\
\hline Vanadium & WA & & & $\mathbf{U}$ & 50 & & NS & $\mu g / L$ \\
\hline Vinyl acetate & WA & & & $\mathbf{U}$ & 10 & & NS & $\mu \mathrm{g} / \mathrm{L}$ \\
\hline Vinyl acetate & WA & & NA & $\mathbf{U}$ & 10 & & NS & $\mu g / L$ \\
\hline Vinyl acetate & WA & & NA & $\mathbf{U}$ & 10 & & NS & $\mu \mathrm{g} / \mathrm{L}$ \\
\hline Vinyl chloride & WA & & & $\mathbf{U}$ & 10 & & 2 & $\mu \mathrm{g} / \mathrm{L}$ \\
\hline Vinyl chloride & WA & & NA & $\mathbf{U}$ & 10 & & 2 & $\mu \mathrm{g} / \mathrm{L}$ \\
\hline Vinyl chloride & WA & & NA & $\mathbf{U}$ & 10 & & 2 & $\mu \mathrm{g} / \mathrm{L}$ \\
\hline Xylenes (total) & WA & & & $\mathbf{U}$ & 5 & & 10000 & $\mu \mathrm{g} / \mathrm{L}$ \\
\hline Xylenes (total) & WA & & NA & $\mathbf{U}$ & 5 & & 10000 & $\mu \mathrm{g} / \mathrm{L}$ \\
\hline Xylenes (total) & WA & & NA & $\mathbf{U}$ & 5 & & 10000 & $\mu g / L$ \\
\hline Zinc & WA & & & $\mathrm{U}$ & 20 & & 5000 & $\mu \mathrm{g} / \mathrm{L}$ \\
\hline
\end{tabular}




\section{UTD004}

Field Measurements:

Stream Discharge: $0.77 \mathrm{cfs}$

Sample Date: 4/28/93

pH: 6.71

Specific Conductance: $28 \mu \mathrm{S} / \mathrm{cm}$

Red/Oxid Potential: $134 \mathrm{mv}$

Dissolved Oxygen: $10 \mathrm{mg} / \mathrm{L}$

\begin{tabular}{|c|c|c|c|c|c|c|c|c|c|}
\hline Analyte & Lab & Rep & Avg & Qualifier & & Result & Acc & Stand & Units \\
\hline 1,1,1,2-Tetrachloroethane & WA & & & $\overline{\mathrm{U}}$ & & 10 & & NS & $\mu g / L$ \\
\hline 1,1,1,2-Tetrachloroethane & GE & & & $\mathbf{U}$ & & 1 & & NS & $\mu g / L$ \\
\hline 1,1,1,2-Tetrachloroethane & WA & $\mathbf{R}$ & & $\mathbf{U}$ & & 10 & & NS & $\mu g / L$ \\
\hline 1,1,1-Trichloroethane & WA & & & U & & 5 & & 200 & $\mu g / L$ \\
\hline 1,1,1-Trichloroethane & GE & & & U & & 1 & & 200 & $\mu g / L$ \\
\hline 1,1,1-Trichloroethane & WA & $\mathbf{R}$ & & $\mathbf{U}$ & & 5 & & 200 & $\mu \mathrm{g} / \mathrm{L}$ \\
\hline 1,1,2,2-Tetrachloroethane & WA & & & $\mathbf{U}$ & & 5 & & NS & $\mu g / L$ \\
\hline 1,1,2,2-Tetrachloroethane & GE & & & $\mathbf{U}$ & & 1 & & NS & $\mu g / L$ \\
\hline 1,1,2,2-Tetrachloroethane & WA & $\mathbf{R}$ & & $\mathbf{U}$ & & 5 & & NS & $\mu g / L$ \\
\hline 1,1,2-Trichloroethane & WA & & & U & & 5 & & 5 & $\mu g / L$ \\
\hline 1,1,2-Trichloroethane & GE & & & $\mathbf{U}$ & & 1 & & 5 & $\mu g / L$ \\
\hline 1,1,2-Trichloroethane & WA & $\mathbf{R}$ & & $\mathbf{U}$ & & 5 & & 5 & $\mu g / L$ \\
\hline 1,1-Dichloroethane & WA & & & $\mathrm{U}$ & & 5 & & NS & $\mu g / L$ \\
\hline 1,1-Dichloroethane & GE & & & $\mathbf{U}$ & & 1 & & NS & $\mu g / L$ \\
\hline 1,1-Dichloroethane & WA & $\mathbf{R}$ & & $\mathbf{U}$ & & 5 & & NS & $\mu g / L$ \\
\hline 1,1-Dichloroethylene & WA & & & $\mathbf{U}$ & & 5 & & 7 & $\mu g / L$ \\
\hline 1,1-Dichloroethylene & GE & & & $\mathrm{U}$ & & 1 & & 7 & $\mu g / L$ \\
\hline 1,1-Dichloroethylene & WA & $\mathbf{R}$ & & $\mathbf{U}$ & & 5 & & 7 & $\mu g / L$ \\
\hline 1,2,3-Trichloropropane & WA & & & U & & 10 & & NS & $\mu g / L$ \\
\hline 1,2,3-Trichloropropane & GE & & & U & & 1 & & NS & $\mu g / L$ \\
\hline 1,2,3-Trichloropropane & WA & $\mathbf{R}$ & & $\mathbf{U}$ & & 10 & & NS & $\mu g / L$ \\
\hline 1,2-Dibromo-3-chloropropane & WA & & & U & & 20 & & 0.2 & $\mu \mathrm{g} / \mathrm{L}$ \\
\hline 1,2-Dibromo-3-chloropropane & GE & & & $\mathbf{U}$ & & 1 & & 0.2 & $\mu g / L$ \\
\hline 1,2-Dibromo-3-chloropropane & WA & $\mathbf{R}$ & & $\mathbf{U}$ & & 20 & & 0.2 & $\mu \mathrm{g} / \mathrm{L}$ \\
\hline 1,2-Dibromoethane & WA & & & $\mathrm{U}$ & & 20 & & NS & $\mu \mathrm{g} / \mathrm{L}$ \\
\hline 1,2-Dibromoethane & GE & & & $\mathbf{U}$ & & 20 & & NS & $\mu \mathrm{g} / \mathrm{L}$ \\
\hline 1,2-Dibromoethane & WA & $\mathbf{R}$ & & $\mathbf{U}$ & & 20 & & NS & $\mu \mathrm{g} / \mathrm{L}$ \\
\hline 1,2-Dichloroethane & WA & & & U & & 5 & & 5 & $\mu \mathrm{g} / \mathrm{L}$ \\
\hline 1,2-Dichloroethane & GE & & & U & & 1 & & 5 & $\mu \mathrm{g} / \mathrm{L}$ \\
\hline 1,2-Dichloroethane & WA & $\mathbf{R}$ & & U & & 5 & & 5 & $\mu \mathrm{g} / \mathrm{L}$ \\
\hline 1,2-Dichloroethylene (total) & WA & & & $\mathbf{U}$ & & 5 & & 100 & $\mu \mathrm{g} / \mathrm{L}$ \\
\hline 1,2-Dichloroethylene (total) & GE & & & $\mathbf{U}$ & & 1 & & 100 & $\mu \mathrm{g} / \mathrm{L}$ \\
\hline 1,2-Dichloroethylene (total) & WA & $\mathbf{R}$ & & $\mathbf{U}$ & & 5 & & 100 & $\mu g / L$ \\
\hline 1,2-Dichloropropane & WA & & & U & & 5 & & 5 & $\mu \mathrm{g} / \mathrm{L}$ \\
\hline 1,2-Dichloropropane & GE & & & U & & 1 & & 5 & $\mu \mathrm{g} / \mathrm{L}$ \\
\hline 1,2-Dichloropropane & WA & $\mathbf{R}$ & & $\mathrm{U}$ & & 5 & & 5 & $\mu \mathrm{g} / \mathrm{L}$ \\
\hline 2-Hexanone & WA & & & $\mathbf{U}$ & & 10 & & NS & $\mu \mathrm{g} / \mathrm{L}$ \\
\hline 2-Hexanone & GE & & & U & & 1 & & NS & $\mu \mathrm{g} / \mathrm{L}$ \\
\hline 2-Hexanone & WA & $\mathbf{R}$ & & $\mathbf{U}$ & & 10 & & NS & $\mu \mathrm{g} / \mathrm{L}$ \\
\hline 4-Methyl-2-pentanone & WA & & & $\mathbf{U}$ & & 10 & & NS & $\mu g / L$ \\
\hline 4-Methyl-2-pentanone & WA & $\mathbf{R}$ & & $\mathbf{U}$ & & 10 & & NS & $\mu \mathrm{g} / \mathrm{L}$ \\
\hline Acetone & WA & & & $J$ & $\mathbf{V}$ & 1.42 & & NS & $\mu \mathrm{g} / \mathrm{L}$ \\
\hline Acetone & GE & & & $\mathbf{U}$ & & 100 & & NS & $\mu g / L$ \\
\hline Acetone & WA & $\mathbf{R}$ & & $\mathbf{U}$ & & 10 & & NS & $\mu \mathrm{g} / \mathrm{L}$ \\
\hline Acetonitrile & WA & & & $\mathbf{U}$ & & 20 & & NS & $\mu \mathrm{g} / \mathrm{L}$ \\
\hline Acetonitrile & GE & & & U & & 1 & & NS & $\mu g / L$ \\
\hline Acetonitrile & WA & $\mathbf{R}$ & & $\mathrm{U}$ & & 20 & & NS & $\mu g / L$ \\
\hline Acidity & WA & & & & & 8 & & NS & $\mathrm{mg} / \mathrm{L}$ \\
\hline Acidity & WA & $\mathbf{R}$ & & & & 2.5 & & NS & $\mathrm{mg} / \mathrm{L}$ \\
\hline
\end{tabular}


UTD004 Laboratory Analyses (cont'd)

\begin{tabular}{|c|c|c|c|c|c|c|c|c|}
\hline Analyte & Lab & Rep & Avg & Qualifier & Result & Acc & Stand & Units \\
\hline Acrolein & WA & & & $\mathbf{U}$ & 10 & & NS & $\mu g / L$ \\
\hline Acrolein & GE & & & $\mathbf{U}$ & 20 & & NS & $\mu \mathrm{g} / \mathrm{L}$ \\
\hline Acrolein & WA & $\mathbf{R}$ & & $\mathbf{U}$ & 10 & & NS & $\mu g / L$ \\
\hline Acrylonitrile & WA & & & $\mathbf{U}$ & 10 & & NS & $\mu g / L$ \\
\hline Acrylonitrile & GE & & & $\mathbf{U}$ & 20 & & NS & $\mu g / L$ \\
\hline Acrylonitrile & WA & $\mathbf{R}$ & & $\mathbf{U}$ & 10 & . & NS & $\mu \mathrm{g} / \mathrm{L}$ \\
\hline Actinium-228 & EP & & & $\mathbf{U}$ & 30 & & NS & $\mathrm{pCi} / L$ \\
\hline Alkalinity & WA & & & $\mathbf{U}$ & 0.5 & & NS & $\mathrm{mg} / \mathrm{L}$ \\
\hline Alkalinity & GE & & & & 7.2 & & NS & $\mathrm{mg} / \mathrm{L}$ \\
\hline Alkalinity & WA & $\mathbf{R}$ & & $\mathbf{U}$ & 0.5 & & NS & $\mathrm{mg} / \mathrm{L}$ \\
\hline Allyl chloride & WA & & & $\mathbf{U}$ & 100 & & NS & $\mu g / L$ \\
\hline Allyl chloride & GE & & & $\mathbf{U}$ & 50 & & NS & $\mu \mathrm{g} / \mathrm{L}$ \\
\hline Allyl chloride & WA & $\mathbf{R}$ & & $\mathbf{U}$ & 100 & & NS & $\mu g / L$ \\
\hline Aluminum & WA & & & $\mathbf{U}$ & 200 & & NS & $\mu g / L$ \\
\hline Aluminum & GE & & & & 102 & & NS & $\mu g / L$ \\
\hline Aluminum & WA & $\mathbf{R}$ & & $\mathbf{U}$ & 200 & & NS & $\mu g / L$ \\
\hline Antimony & WA & & & $\mathbf{U}$ & 60 & & 6 & $\mu g / L$ \\
\hline Antimony & $\mathbf{G E}$ & & & $\mathbf{U}$ & 2 & & 6 & $\mu g / L$ \\
\hline Antimony & WA & $\mathbf{R}$ & & $\mathbf{U}$ & 60 & & 6 & $\mu g / L$ \\
\hline Antimony-125 & EP & & & $\mathbf{U}$ & 20 & & 300 & $\mathrm{pCi} / L$ \\
\hline Arsenic & WA & & & $\mathbf{U}$ & 10 & & 50 & $\mu g / L$ \\
\hline Arsenic & GE & & & $\mathbf{U}$ & 2 & & 50 & $\mu g / L$ \\
\hline Arsenic & WA & $\mathbf{R}$ & & $\mathbf{U}$ & 10 & & 50 & $\mu g / L$ \\
\hline Barium & WA & & & $\mathbf{U}$ & 200 & & 2000 & $\mu \mathrm{g} / \mathrm{L}$ \\
\hline Barium & GE & & & & 10.4 & & 2000 & $\mu g / L$ \\
\hline Barium & WA & $\mathbf{R}$ & & $\mathbf{U}$ & 200 & & 2000 & $\mu g / L$ \\
\hline Benzene & WA & & & $\mathbf{U}$ & 5 & & 5 & $\mu g / L$ \\
\hline Benzene & GE & & & $\mathbf{U}$ & 1 & & 5 & $\mu g / L$ \\
\hline Benzene & WA & $\mathbf{R}$ & & $\mathbf{U}$ & 5 & & 5 & $\mu g / L$ \\
\hline Beryllium & WA & & & $\mathbf{U}$ & 5 & & 4 & $\mu g / L$ \\
\hline Beryllium & GE & & & $\mathbf{U}$ & 3 & & 4 & $\mu g / L$ \\
\hline Beryllium & WA & $\mathbf{R}$ & & $\mathbf{U}$ & 5 & & 4 & $\mu \mathrm{g} / \mathrm{L}$ \\
\hline Bis(2-chloro-1-methylethyl)ether & GE & & & $\mathbf{U}$ & 10 & & NS & $\mu \mathrm{g} / \mathrm{L}$ \\
\hline Bromodichloromethane & WA & & & U & 5 & & NS & $\mu \mathrm{g} / \mathrm{L}$ \\
\hline Bromodichloromethane & GE & & & $\mathbf{U}$ & 1 & & NS & $\mu g / L$ \\
\hline Bromodichloromethane & WA & $\mathbf{R}$ & & $\mathbf{U}$ & 5 & & NS & $\mu g / L$ \\
\hline Bromoform & WA & & & $\mathbf{U}$ & 5 & & NS & $\mu \mathrm{g} / \mathrm{L}$ \\
\hline Bromoform & GE & & & $\mathbf{U}$ & 1 & & NS & $\mu g / L$ \\
\hline Bromoform & WA & $\mathbf{R}$ & & $\mathbf{U}$ & 5 & & NS & $\mu \mathrm{g} / \mathrm{L}$ \\
\hline Cadmium & WA & & & U & 5 & & 5 & $\mu g / L$ \\
\hline Cadmium & GE & & & $\mathbf{U}$ & 2 & & 5 & $\mu g / L$ \\
\hline Cadmium & WA & $\mathbf{R}$ & & $\mathbf{U}$ & 5 & & 5 & $\mu g / L$ \\
\hline Calcium & WA & & & $\mathbf{U}$ & 5000 & & NS & $\mu g / L$ \\
\hline Calcium & GE & & & $\mathbf{J}$ & 1920 & & NS & $\mu g / L$ \\
\hline Calcium & WA & $\mathbf{R}$ & & $\mathbf{U}$ & 5000 & & NS & $\mu \mathrm{g} / \mathrm{L}$ \\
\hline Carbon disulfide & WA & & & $\mathbf{U}$ & 5 & & NS & $\mu g / L$ \\
\hline Carbon disulfide & GE & & & $\mathbf{U}$ & 1 & & NS & $\mu g / L$ \\
\hline Carbon disulfide & WA & $\mathbf{R}$ & & U & 5 & & NS & $\mu g / L$ \\
\hline Carbon tetrachloride & WA & & & $\mathbf{U}$ & 5 & & 5 & $\mu \mathrm{g} / \mathrm{L}$ \\
\hline Carbon tetrachloride & GE & & & $\mathbf{U}$ & 1 & & 5 & $\mu g / L$ \\
\hline Carbon tetrachloride & WA & $\mathbf{R}$ & & $\mathbf{U}$ & 5 & & 5 & $\mu g / L$ \\
\hline Cerium-144 & EP & & & $\mathbf{U}$ & 60 & & 261 & $\mathrm{pCi} / \mathrm{L}$ \\
\hline Cesium-134 & EP & & & $\mathbf{U}$ & 10 & & 81.3 & $\mathrm{pCi} / \mathrm{L}$ \\
\hline
\end{tabular}


UTD004 Laboratory Analyses (cont'd)

\begin{tabular}{|c|c|c|c|c|c|c|c|c|}
\hline Analyte & Lab & Rep & Avg & Qualifier & Result & Acc & Stand & Units \\
\hline Cesium-137 & TM & & NA & $\mathbf{U}$ & 5.7 & & 200 & $\mathrm{pCi} / \mathrm{L}$ \\
\hline Cesium-137 & TM & & & $\mathbf{U}$ & 6.1 & & 200 & $\mathrm{pCi} / \mathrm{L}$ \\
\hline Cesium-137 & EP & & & $\mathbf{U}$ & 10 & & 200 & $\mathrm{pCi} / \mathrm{L}$ \\
\hline Cesium-137 & TM & $\mathbf{R}$ & & $\mathrm{U}$ & 9.7 & & 200 & $\mathrm{pCi} / \mathrm{L}$ \\
\hline Chloride & WA & & & & 3.19 & & NS & $\mathrm{mg} / \mathrm{L}$ \\
\hline Chloride & $\mathrm{GE}$ & & & & 3.19 & & NS & $\mathrm{mg} / \mathrm{L}$ \\
\hline Chloride & WA & $\mathbf{R}$ & & & 2.98 & & NS & $\mathrm{mg} / \mathrm{L}$ \\
\hline Chlorobenzene & WA & & & $\mathbf{U}$ & 5 & & 100 & $\mu g / L$ \\
\hline Chlorobenzene & GE & & & $\mathbf{U}$ & 1 & & 100 & $\mu g / L$ \\
\hline Chlorobenzene & WA & $\mathbf{R}$ & & $\mathbf{U}$ & 5 & & 100 & $\mu g / L$ \\
\hline Chloroethane & WA & & & $\mathbf{U}$ & 10 & & NS & $\mu g / L$ \\
\hline Chloroethane & GE & & & $\mathbf{U}$ & 1 & & NS & $\mu g / L$ \\
\hline Chloroethane & WA & $\mathbf{R}$ & & $\mathbf{U}$ & 10 & & NS & $\mu g / L$ \\
\hline Chloroform & WA & & & $\mathbf{U}$ & 5 & & 100 & $\mu g / L$ \\
\hline Chloroform & GE & & & U & 1 & & 100 & $\mu \mathrm{g} / \mathrm{L}$ \\
\hline Chloroform & WA & $\mathbf{R}$ & & $\mathbf{U}$ & 5 & & 100 & $\mu \mathrm{g} / \mathrm{L}$ \\
\hline Chloroprene & WA & & & U & 20 & & NS & $\mu \mathrm{g} / \mathrm{L}$ \\
\hline Chloroprene & GE & & & $\mathrm{U}$ & 200 & & NS & $\mu g / L$ \\
\hline Chloroprene & WA & $\mathbf{R}$ & & $\mathbf{U}$ & 20 & & NS & $\mu g / L$ \\
\hline Chromium & WA & & & U & 10 & & 100 & $\mu g / L$ \\
\hline Chromium & GE & & & $\mathrm{U}$ & 4 & & 100 & $\mu \mathrm{g} / \mathrm{L}$ \\
\hline Chromium & WA & $\mathbf{R}$ & & U & 10 & & 100 & $\mu g / L$ \\
\hline cis-1,3-Dichloropropene & WA & & & U & 5 & & NS & $\mu g / L$ \\
\hline cis-1,3-Dichloropropene & GE & & & $\mathrm{U}$ & 1 & & NS & $\mu \mathrm{g} / \mathrm{L}$ \\
\hline cis-1,3-Dichloropropene & WA & $\mathbf{R}$ & & $\mathrm{U}$ & 5 & & NS & $\mu g / L$ \\
\hline Cobalt & WA & & & U & 50 & & NS & $\mu \mathrm{g} / \mathrm{L}$ \\
\hline Cobalt & GE & & & $\mathrm{U}$ & 4 & & NS & $\mu g / L$ \\
\hline Cobalt & WA & $\mathbf{R}$ & & $\mathrm{U}$ & 50 & & NS & $\mu g / L$ \\
\hline Cobalt-57 & EP & & & $\mathrm{U}$ & 10 & & 1000 & $\mathrm{pCi} / \mathrm{L}$ \\
\hline Cobalt -60 & EP & & & $\mathbf{U}$ & 10 & & 100 & $\mathrm{pCi} / \mathrm{L}$ \\
\hline Copper & WA & & & U & 25 & & 1000 & $\mu g / L$ \\
\hline Copper & GE & & & $\mathbf{U}$ & 4 & & 1000 & $\mu \mathrm{g} / \mathrm{L}$ \\
\hline Copper & WA & $\mathbf{R}$ & & $\mathrm{U}$ & 25 & & 1000 & $\mu \mathrm{g} / \mathrm{L}$ \\
\hline Cyanide & WA & & & $\mathrm{U}$ & 10 & & 200 & $\mu \mathrm{g} / \mathrm{L}$ \\
\hline Cyanide & GE & & & UJ & 2.5 & & 200 & $\mu g / L$ \\
\hline Cyanide & WA & $\mathbf{R}$ & & $\mathrm{U}$ & 10 & & 200 & $\mu g / L$ \\
\hline Dibromochloromethane & WA & & & $\mathbf{U}$ & 5 & & NS & $\mu \mathrm{g} / \mathrm{L}$ \\
\hline Dibromochloromethane & GE & & & $\mathbf{U}$ & 1 & & NS & $\mu \mathrm{g} / \mathrm{L}$ \\
\hline Dibromochloromethane & WA & $\mathbf{R}$ & & U & 5 & & NS & $\mu g / L$ \\
\hline Dichlorodifluoromethane & WA & & & U & 10 & & NS & $\mu g / L$ \\
\hline Dichlorodifluoromethane & GE & & & $\mathrm{U}$ & 1 & & NS & $\mu g / L$ \\
\hline Dichlorodifluoromethane & WA & $\mathbf{R}$ & & $\mathrm{U}$ & 10 & & NS & $\mu \mathrm{g} / \mathrm{L}$ \\
\hline Ethylbenzene & WA & & & $\mathrm{U}$ & 5 & & 700 & $\mu \mathrm{g} / \mathrm{L}$ \\
\hline Ethylbenzene. & GE & & & $\mathrm{U}$ & 1 & & 700 & $\mu \mathrm{g} / \mathrm{L}$ \\
\hline Ethylbenzene & WA & $\mathbf{R}$ & & $\mathrm{U}$ & 5 & & 700 & $\mu \mathrm{g} / \mathrm{L}$ \\
\hline Europium-152 & EP & & & U & 40 & & NS & $\mathrm{pCi} / \mathrm{L}$ \\
\hline Europium-154 & EP & & & $\mathrm{U}$ & 20 & & 200 & $\mathrm{pCi} / \mathrm{L}$ \\
\hline Europium-155 & EP & & & $\mathrm{U}$ & 30 & & 600 & $\mathrm{pCi} / 2$ \\
\hline Fluoride & WA & & & $U$ & 0.1 & & 4 & $\mathrm{mg} / \mathrm{L}$ \\
\hline Fluoride & WA & & NA & $\mathrm{U}$ & 0.1 & & 4 & $\mathrm{mg} / \mathrm{L}$ \\
\hline Fluoride & GE & & & $\mathrm{U}$ & 0.1 & & 4 & $\mathrm{mg} / \mathrm{L}$ \\
\hline Fluoride & WA & $\mathbf{R}$ & & $\mathbf{U}$ & 0.1 & & 4 & $\mathrm{mg} / \mathrm{L}$ \\
\hline Gross alpha & TM & & A & & 1.5 & 1.05 & 15 & $\mathrm{pCi} / \mathrm{L}$ \\
\hline Gross alpha & GE & & & U & 2 & & 15 & $\mathrm{pCi} / \mathrm{L}$ \\
\hline Gross alpha & TM & $\mathbf{R}$ & & & 1.2 & 0.9 & 15 & $\mathrm{pCi} / \mathrm{L}$ \\
\hline
\end{tabular}


UTD004 Laboratory Analyses (cont'd)

\begin{tabular}{|c|c|c|c|c|c|c|c|c|c|}
\hline Analyte & Lab & Rep & Avg & Oualifier & & Result & Acc & Stand & Units \\
\hline Iron & $\overline{W A}$ & & & & & 386 & & 300 & $\mu g / L$ \\
\hline Iron & GE & & & & & 323 & & 300 & $\mu g / L$ \\
\hline Iron & WA & $\mathbf{R}$ & & & & 380 & & 300 & $\mu_{g / h}$ \\
\hline Isobutyl alcohol & WA & & & $\mathbf{U}$ & & 20 & & NS & $\mu \mathrm{g} / \mathrm{L}$ \\
\hline Isobutyl alcohol & GE & & & $\mathbf{U}$ & & 100 & & NS & $\mu g / L$ \\
\hline Isobutyl alcohol & WA & $\mathbf{R}$ & & $\mathbf{U}$ & & 20 & & NS & $\mu g / 2$ \\
\hline Lead & WA & & & $\mathbf{U}$ & & 3 & & 50 & $\mu g /$ \\
\hline Lead & GE & & & $\mathbf{U}$ & & 3 & & 50 & $\mu g /$ \\
\hline Lead & WA & $\mathbf{R}$ & & $\mathbf{U}$ & & 3 & & 50 & $\mu g / L$ \\
\hline Lead-212 & EP & & & $\mathbf{U}$ & & 15 & & NS & pCi $/ L$ \\
\hline Magnesium & WA & & & $\mathbf{U}$ & & 5000 & & NS & $\mu_{g} / L$ \\
\hline Magnesium & GE & & & & & 461 & & NS & $\mu g / L$ \\
\hline Magnesium & WA & $\mathbf{R}$ & • & $\mathbf{U}$ & & 5000 & & NS & $\mu g / L$ \\
\hline Manganese & WA & & & & & 30 & & 50 & $\mu g \Omega$ \\
\hline Manganese & GE & & & & & 31.7 & & 50 & $\mu g / L$ \\
\hline Manganese & WA & $\mathbf{R}$ & & & & 28.8 & & 50 & $\mu g / L$ \\
\hline Manganese-54 & EP & & & $\mathbf{U}$ & & 10 & & 300 & $\mathrm{pCi} / \mathrm{L}$ \\
\hline Mercury & WA & & & $\mathbf{U}$ & & 0.2 & & 2 & $\mu g / L$ \\
\hline Mercury & GE & & & $\mathbf{U}$ & & 0.2 & & 2 & $\mu g / L$ \\
\hline Mercury & WA & $\mathbf{R}$ & & $\mathbf{U}$ & & 0.2 & & 2 & $\mu g / L$ \\
\hline Methacrylonitrile & WA & & & $\mathbf{U}$ & & 20 & & NS & $\mu g / 2$ \\
\hline Methacrylonitrile & GE & & & $\mathbf{U}$ & & 50 & & NS & $\mu g / L$ \\
\hline Methacrylonitrile & WA & $\mathbf{R}$ & & $\mathbf{U}$ & & 20 & & NS & $\mu g / L$ \\
\hline Methyl bromide & WA & & & $\mathbf{U}$ & & 10 & & NS & $\mu g / L$ \\
\hline Methyl bromide & GE & & & $\mathbf{U}$ & & 1 & & NS & $\mu g / L$ \\
\hline Methyl bromide & WA & $\mathbf{R}$ & & $\mathbf{U}$ & & 10 & & NS & $\mu g / L$ \\
\hline Methyl chloride & WA & & & $\mathbf{U}$ & & 10 & & NS & $\mu g / L$ \\
\hline Methyl chloride & GE & & & $\mathbf{U}$ & & 1 & & NS & $\mu g / L$ \\
\hline Methyl chloride & WA & $\mathbf{R}$ & & $\mathbf{U}$ & & 10 & & NS & $\mu g / L$ \\
\hline Methyl ethyl ketone & WA & & & $\mathbf{U}$ & & 10 & & NS & $\mu g / L$ \\
\hline Methyl ethyl ketone & GE & & & $\mathbf{U}$ & & 1 & & NS & $\mu g / L$ \\
\hline Methyl ethyl ketone & WA & $\mathbf{R}$ & & $\mathbf{U}$ & & 10 & & NS & $\mu g / L$ \\
\hline Methyl iodide & WA & & & $\mathbf{U}$ & & 10 & & NS & $\mu g / L$ \\
\hline Methyl iodide & GE & & & $\mathbf{U}$ & & 15 & & NS & $\mu g / L$ \\
\hline Methyl iodide & WA & $\mathbf{R}$ & & $\mathbf{U}$ & & 10 & & NS & $\mu g / L$ \\
\hline Methyl isobutyl ketone & GE & & & $\mathbf{U}$ & & 1 & & NS & $\mu g / L$ \\
\hline Methylene bromide & WA & & & $\mathbf{U}$ & & 10 & & NS & $\mu g / L$ \\
\hline Methylene bromide & GE & & & $\mathbf{U}$ & & 1 & & NS & $\mu \mathrm{g} / \mathrm{L}$ \\
\hline Methylene bromide & WA & $\mathbf{R}$ & & $\mathbf{U}$ & & 10 & & NS & $\mu g / L$ \\
\hline Methylene chloride & WA & & & $\mathbf{U}$ & & 5 & & 5 & $\mu g / \mathrm{L}$ \\
\hline Methylene chloride & GE & & & $\mathrm{U}$ & & 1 & & 5 & $\mu g / L$ \\
\hline Methylene chloride & WA & $\mathbf{R}$ & & $\mathbf{U}$ & & 5 & & 5 & $\mu g / L$ \\
\hline Nickel & WA & & & $\mathbf{U}$ & & 40 & & 100 & $\mu g / \mathrm{L}$ \\
\hline Nickel & GE & & & $\mathbf{U}$ & & 4 & & 100 & $\mu g / L$ \\
\hline Nickel & WA & $\mathbf{R}$ & & $\mathbf{U}$ & & 40 & & 100 & $\mu g / L$ \\
\hline Nitrate as nitrogen & WA & & & & & 0.158 & & 10 & $\mathrm{mg} / \mathrm{L}$ \\
\hline Nitrate as nitrogen & GE & & & & & 0.56 & & 10 & $\mathrm{mg} / \mathrm{L}$ \\
\hline Nitrate as nitrogen & WA & $\mathbf{R}$ & & & & 0.173 & & 10 & $\mathrm{mg} / \mathrm{L}$ \\
\hline Nonvolatile beta & TM & & $\mathbf{A}$ & & & 6.05 & 2.8 & 50 & $\mathrm{pCi} / \mathrm{L}$ \\
\hline Nonvolatile beta & GE & & & $\mathbf{U}$ & & 2 & & 50 & $\mathrm{pCi} / \mathrm{L}$ \\
\hline Nonvolatile beta & TM & $\mathbf{R}$ & & & & 2.3 & 1.8 & 50 & $\mathrm{pCi} / \mathrm{L}$ \\
\hline pH & GE & & & $\mathbf{J}$ & Q & 6.83 & & NS & PH \\
\hline Potassium & WA & & & $\mathbf{U}$ & & 5000 & & NS & $\mu g / L$ \\
\hline Potassium & GE & & & & & 806 & & NS & $\mu \mathrm{g} / \mathrm{L}$ \\
\hline Potassium & WA & $\mathbf{R}$ & & U & & 5000 & & NS & $\mu g / L$ \\
\hline
\end{tabular}


UTD004 Laboratory Analyses (cont'd)

\begin{tabular}{|c|c|c|c|c|c|c|c|c|}
\hline Analyte & Lab & Rep & Avg & Qualifier & Result & Acc & Stand & Units \\
\hline Potassium-40 & $\mathrm{EP}$ & & & $\mathbf{U}$ & 110 & & 300 & $\mathrm{pCi} / \mathrm{L}$ \\
\hline Promethium-144 & EP & & & $\mathbf{U}$ & 10 & & NS & $\mathrm{pCi} / \mathrm{L}$ \\
\hline Promethium-146 & EP & & & $\mathbf{U}$ & 10 & & NS & $\mathrm{pCi} / \mathrm{L}$ \\
\hline Propionitrile & WA & & & $\mathbf{U}$ & 50 & & NS & $\mu g / L$ \\
\hline Propionitrile & GE & & & $\mathbf{U}$ & 200 & & NS & $\mu g / L$ \\
\hline Propionitrile & WA & $\mathbf{R}$ & & U & 50 & . & NS & $\mu g / L$ \\
\hline $\begin{array}{l}\text { Ruthenium-106 } \\
\text { Selenium }\end{array}$ & EP & & & $\mathbf{U}$ & & & $\begin{array}{l}30 \\
50\end{array}$ & $\mathrm{pCi} / \mathrm{L}$ \\
\hline $\begin{array}{l}\text { Selenium } \\
\text { Selenium }\end{array}$ & WA & & & $\underset{\mathbf{U J}}{\mathbf{U}}$ & $\begin{array}{l}5 \\
2\end{array}$ & & $\begin{array}{l}50 \\
50\end{array}$ & $\begin{array}{l}\mu g / L \\
\mu \rho / L\end{array}$ \\
\hline $\begin{array}{l}\text { Selenium } \\
\text { Selenium }\end{array}$ & $\begin{array}{l}\text { GE } \\
\text { WA }\end{array}$ & & & $\underset{U}{U}$ & $\begin{array}{l}2 \\
5\end{array}$ & & $\begin{array}{l}50 \\
50\end{array}$ & $\begin{array}{l}\mu g / L \\
\mu g / \Omega\end{array}$ \\
\hline $\begin{array}{l}\text { Selenium } \\
\text { Silver }\end{array}$ & $\begin{array}{l}\text { WA } \\
\text { WA }\end{array}$ & $\mathbf{R}$ & & $\mathbf{U}$ & 10 & & 50 & $\begin{array}{l}\mu g / L \\
\mu g / L\end{array}$ \\
\hline Silver & GE & & & U & 2 & & 50 & $\begin{array}{l}\mu g / L \\
\mu g / L\end{array}$ \\
\hline Silver & WA & $\mathbf{R}$ & & $\mathbf{U}$ & 10 & & 50 & $\mu \mathrm{g} / \mathrm{L}$ \\
\hline Sodium & WA & & & $\mathbf{U}$ & 5000 & & NS & $\mu g / L$ \\
\hline Sodium & GE & & & & 4160 & & NS & $\mu g / L$ \\
\hline Sodium & WA & $\mathbf{R}$ & . & $\mathbf{U}$ & 5000 & & NS & $\mu g / L$ \\
\hline Sodium-22 & EP & & & $\mathbf{U}$ & 10 & & 466 & $\mathrm{pCi} / \mathrm{L}$ \\
\hline Styrene & WA & & & $\mathbf{U}$ & 5 & & 100 & $\mu g / L$ \\
\hline Styrene & GE & & & $\mathbf{U}$ & 1 & & 100 & $\mu g / L$ \\
\hline Styrene & WA & $\mathbf{R}$ & & $\mathbf{U}$ & 5 & & 100 & $\mu g / L$ \\
\hline Sulfate & WA & & & & 0.454 & & 400 & $\mathrm{mg} / \mathrm{L}$ \\
\hline Sulfate & GE & & & & 3.32 & & 400 & $\mathrm{mg} / \mathrm{L}$ \\
\hline Sulfate & WA & $\mathbf{R}$ & & $\mathbf{U}$ & 0.25 & & 400 & $\mathrm{mg} / \mathrm{L}$ \\
\hline Sulfide & WA & & & $\mathbf{U}$ & 0.1 & & NS & $\mathrm{mg} / \mathrm{L}$ \\
\hline Sulfide & GE & & & $\mathbf{U}$ & 1 & & NS & $\mathrm{mg} / \mathrm{L}$ \\
\hline Sulfide & GE & & NA & U & 1 & & NS & $\mathrm{mg} / \mathrm{L}$ \\
\hline Sulfide & WA & $\mathbf{R}$ & & U & 0.1 & & NS & $\mathrm{mg} / \mathrm{L}$ \\
\hline Tetrachloroethylene & WA & & & U & 5 & & 5 & $\mu g / L$ \\
\hline Tetrachloroethylene & GE & & & U & 1 & & 5 & $\mu g / L$ \\
\hline Tetrachloroethylene & WA & $\mathbf{R}$ & & $\mathbf{U}$ & 5 & & 5 & $\mu \mathrm{g} / \mathrm{L}$ \\
\hline Thallium & WA & & & $\mathbf{U}$ & 10 & & 2 & $\mu g / L$ \\
\hline Thallium & GE & & & $\mathbf{U}$ & 2 & & 2 & $\mu g / L$ \\
\hline Thallium & WA & $\mathbf{R}$ & & $\mathbf{U}$ & 10 & & 2 & $\mu g / L$ \\
\hline Thorium-234 & EP & & & $\mathbf{U}$ & 350 & & 401 & pCi/L \\
\hline Toluene & WA & & & $\mathrm{U}$ & 5 & & 1000 & $\mu g / L$ \\
\hline Toluene & GE & & & $\mathbf{U}$ & 1 & & 1000 & $\mu g / L$ \\
\hline Toluene & WA & $\mathbf{R}$ & & $\mathbf{U}$ & 5 & & 1000 & $\mu g / L$ \\
\hline Total hardness & WA & & & & 5 & & NS & $\mathrm{mg} / \mathrm{L}$ \\
\hline Total hardness & GE & & $\mathbf{A}$ & & 7.75 & & NS & $\mathrm{mg} / \mathrm{L}$ \\
\hline Total hardness & WA & $\mathbf{R}$ & & & 24 & & NS & $\mathrm{mg} / \mathrm{L}$ \\
\hline Total organic carbon & WA & & & & 1.26 & & NS & $\mathrm{mg} / \mathrm{L}$ \\
\hline Total organic carbon & GE & & & & 1.97 & & NS & $\mathrm{mg} / \mathrm{L}$ \\
\hline Total organic carbon & WA & $\mathbf{R}$ & & & 1.34 & & NS & $\mathrm{mg} / \mathrm{L}$ \\
\hline Total suspended solids & WA & & & U & 5 & & NS & $\mathrm{mg} / \mathrm{L}$ \\
\hline Total suspended solids & GE & & A & & 6 & & NS & $\mathrm{mg} / \mathrm{L}$ \\
\hline Total suspended solids & WA & $\mathbf{R}$ & A & & 10 & & NS & $\mathrm{mg} / \mathrm{L}$ \\
\hline trans-1,3-Dichloropropene & WA & & & $\mathbf{U}$ & 5 & & NS & \\
\hline trans-1,3-Dichloropropene & GE & & & $\mathrm{U}$ & 1 & & NS & $\mu g / L$ \\
\hline trans-1,3-Dichloropropene & WA & $\mathbf{R}$ & & $\mathbf{U}$ & 5 & & NS & $\mu g / L$ \\
\hline trans-1,4-Dichloro-2-butene & WA & & & $\mathbf{U}$ & 100 & & NS & $\mu g$ \\
\hline trans-1,4-Dichloro-2-butene & GE & & & $\mathbf{U}$ & 30 & & NS & $\mu g / L$ \\
\hline trans-1,4-Dichloro-2-butene & WA & $\mathbf{R}$ & & $\mathbf{U}$ & 100 & & NS & $\mu g$ \\
\hline Trichloroethylene & WA & & & $\mathbf{J}$ & 2.09 & & 5 & $\mu g / 2$ \\
\hline Trichloroethylene & GE & & & & 2.5 & & 5 & \\
\hline Trichloroethylene & WA & $\mathbf{R}$ & & $\mathbf{J}$ & 2.12 & & 5 & \\
\hline
\end{tabular}


UTD004 Laboratory Analyses (cont'd)

\begin{tabular}{|c|c|c|c|c|c|c|c|c|}
\hline Analyte & Lab & Rep & Avg & Qualifier & Result & Acc & Stand & Units \\
\hline Trichlorofluoromethane & WA & & & $\bar{U}$ & 5 & & NS & $\mu g /$ \\
\hline Trichlorofluoromethane & $\mathrm{GE}$ & & & $\mathbf{U}$ & 1 & & NS & $\mu g / L$ \\
\hline Trichlorofluoromethane & WA & $\mathbf{R}$ & & $\mathbf{U}$ & 5 & & NS & $\mu g \Omega$ \\
\hline Tritium & TM & & A & & 39.35 & 16.4 & 20 & $\mathrm{pCi} / \mathrm{ml}$ \\
\hline Tritium & $\mathbf{G E}$ & & & & 35.3 & & 20 & $\mathrm{pCi} / \mathrm{ml}$ \\
\hline Tritium & $\mathbf{T M}$ & $\mathbf{R}$ & & & 39 & .16 .2 & 20 & $\mathrm{pCi} / \mathrm{ml}$ \\
\hline Turbidity & WA & & $\mathbf{A}$ & & 7.24 & & NS & NTU \\
\hline Turbidity & GE & & & & 8.08 & & NS & NTU \\
\hline Turbidity & WA & $\mathbf{R}$ & & & 7.32 & & NS & NTU \\
\hline Vanadium & WA & & & $\mathbf{U}$ & 50 & & NS & $\mu g / L$ \\
\hline Vanadium & $\mathbf{G E}$ & & & $\mathbf{U}$ & 8 & & NS & $\mu g / L$ \\
\hline Vanadium & WA & $\mathbf{R}$ & & $\mathbf{U}$ & 50 & & NS & $\mu g / L$ \\
\hline Vinyl acetate & WA & & & $\mathbf{U}$ & 10 & & NS & $\mu g / L$ \\
\hline Vinyl acetate & $\mathrm{GE}$ & & & $\mathbf{U}$ & 1 & & NS & $\mu g / L$ \\
\hline Vinyl acetate & WA & $\mathbf{R}$ & & $\mathbf{U}$ & 10 & & NS & $\mu_{8}$ \\
\hline Vinyl chloride & WA & & & $\mathbf{U}$ & 10 & & 2 & $\mu$ \\
\hline Vinyl chloride & GE & & & $\mathbf{U}$ & 1 & & 2 & \\
\hline Vinyl chloride & WA & $\mathbf{R}$ & - & $\mathbf{U}$ & 10 & & 2 & \\
\hline Xylenes (total) & WA & & & $\mathbf{U}$ & 5 & & 10000 & \\
\hline Xylenes (total) & GE & & & $\mathbf{U}$ & 2 & & 10000 & \\
\hline Xylenes (total) & WA & $\mathbf{R}$ & & $\mathbf{U}$ & 5 & & 10000 & \\
\hline Yttrium-88 & EP & & & $\mathbf{U}$ & 60 & & NS & PC \\
\hline Zinc & WA & & & & 23.3 & & 5000 & $\mu g$ \\
\hline Zinc & GE & & & & 7.12 & & 5000 & \\
\hline Zinc & WA & $\mathbf{R}$ & & & 26.8 & & 5000 & \\
\hline Zinc-65 & EP & & & $\mathbf{U}$ & 20 & & 300 & \\
\hline
\end{tabular}




\section{UTD005}

Field Measurements:

Stream Discharge: 0.22 cfs

Sample Date: 4/27/93

pH: 6.47

Specific Conductance: $43 \mu \mathrm{S} / \mathrm{cm}$

Red/Oxid Potential: $156 \mathrm{mv}$

Dissolved Oxygen: $5.4 \mathrm{mg} / \mathrm{L}$

\begin{tabular}{|c|c|c|c|c|c|c|c|c|}
\hline Analyte & Lab & Rep & Avg & Qualifier & Result & Acc & Stand & Units \\
\hline 1,1,1,2-Tetrachloroethane & WA & & & $\mathrm{U}$ & 10 & & NS & $\overline{\mu g / L}$ \\
\hline 1,1,1-Trichloroethane & WA & & & $\mathbf{U}$ & 5 & & 200 & $\mu g / L$ \\
\hline $1,1,2,2$-Tetrachloroethane & WA & & & $\mathbf{U}$ & 5 & & NS & $\mu g / L$ \\
\hline 1,1,2-Trichloroethane & WA & & & $\mathbf{U}$ & 5 & & 5 & $\mu g / L$ \\
\hline 1,1-Dichloroethane & WA & & & $\mathbf{U}$ & 5 & & NS & $\mu g / L$ \\
\hline 1,1-Dichloroethylene & WA & & & $\mathbf{U}$ & 5 & & 7 & $\mu \mathrm{g} / \mathrm{L}$ \\
\hline 1,2,3-Trichloropropane & WA & & & $\mathbf{U}$ & 10 & & NS & $\mu g / L$ \\
\hline 1,2-Dibromo-3-chloropropane & WA & & & $\mathbf{U}$ & 20 & & 0.2 & $\mu g / L$ \\
\hline 1,2-Dibromoethane & WA & & & $\mathbf{U}$ & 20 & & NS & $\mu g / L$ \\
\hline 1,2-Dichloroethane & WA & & & $\mathbf{U}$ & 5 & & 5 & $\mu g / L$ \\
\hline 1,2-Dichloroethylene (total) & WA & & & $\mathbf{U}$ & 5 & & 100 & $\mu g / L$ \\
\hline 1,2-Dichloropropane & WA & & & $\mathbf{U}$ & 5 & & 5 & $\mu g / L$ \\
\hline 2-Hexanone & WA & & & $\mathbf{U}$ & 10 & & NS & $\mu g / L$ \\
\hline 4-Methyl-2-pentanone & WA & & & $\mathbf{U}$ & 10 & & NS & $\mu g / L$ \\
\hline Acetone & WA & & & $\mathbf{U}$ & 10 & & NS & $\mu g / L$ \\
\hline Acetonitrile & WA & & & $\mathbf{U}$ & 20 & & NS & $\mu g / L$ \\
\hline Acidity & WA & & & & 22 & & NS & $\mathrm{mg} / \mathrm{L}$ \\
\hline Acrolein & WA & & & $\mathbf{U}$ & 10 & & NS & $\mu g / L$ \\
\hline Acrylonitrile & WA & & & $\mathbf{U}$ & 10 & & NS & $\mu g / L$ \\
\hline Alkalinity & WA & & & & 8 & & NS & $\mathrm{mg} / \mathrm{L}$ \\
\hline Allyl chloride & WA & & & $\mathbf{U}$ & 100 & & NS & $\mu g / L$ \\
\hline Aluminum & WA & & & $\mathbf{U}$ & 200 & & NS & $\mu g / L$ \\
\hline Antimony & WA & & & $\mathbf{U}$ & 60 & & 6 & $\mu g / L$ \\
\hline Arsenic & WA & & & $\mathbf{U}$ & 10 & & 50 & $\mu g / L$ \\
\hline Barium & WA & & & $\mathbf{U}$ & 200 & & 2000 & $\mu g / L$ \\
\hline Benzene & WA & & & $\mathrm{U}$ & 5 & & 5 & $\mu g / L$ \\
\hline Beryllium & WA & & & $\mathbf{U}$ & 5 & & 4 & $\mu g / L$ \\
\hline Bromodichloromethane & WA & & & $\mathbf{U}$ & 5 & & NS & $\mu g / L$ \\
\hline Bromoform & WA & & & $\mathbf{U}$ & 5 & & NS & $\mu g / L$ \\
\hline Cadmium & WA & & & $\mathbf{U}$ & 5 & & 5 & $\mu g / L$ \\
\hline Calcium & WA & & & $\mathbf{U}$ & 5000 & & NS & $\mu g / L$ \\
\hline Carbon disulfide & WA & & & $\mathbf{U}$ & 5 & & NS & $\mu g / L$ \\
\hline Carbon tetrachloride & WA & & & $\mathbf{U}$ & 5 & & 5 & $\mu \mathrm{g} / \mathrm{L}$ \\
\hline Cesium-137 & TM & & & $\mathbf{U}$ & 9.9 & & 200 & $\mathrm{pCi} / \mathrm{L}$ \\
\hline Cesium-137 & TM & & & U & 9.9 & & 200 & $\mathrm{pCi} / \mathrm{L}$ \\
\hline Chloride & WA & & & & 3.01 & & NS & $\mathrm{mg} / \mathrm{L}$ \\
\hline Chlorobenzene & WA & & & $\mathbf{U}$ & 5 & & 100 & $\mu g / L$ \\
\hline Chloroethane & WA & & & $\mathbf{U}$ & 10 & & NS & $\mu g / L$ \\
\hline Chloroform & WA & & & $\mathbf{U}$ & 5 & & 100 & $\mu g / L$ \\
\hline Chloroprene & WA & & & $\mathbf{U}$ & 20 & & NS & $\mu g / L$ \\
\hline Chromium & WA & & & $U$ & 10 & & 100 & $\mu g / L$ \\
\hline cis-1,3-Dichloropropene & WA & & & $\mathbf{U}$ & 5 & & NS & $\mu \mathrm{g} / \mathrm{L}$ \\
\hline Cobalt & WA & & & $\mathbf{U}$ & 50 & & NS & $\mu g / L$ \\
\hline Copper & WA & & & $\mathbf{U}$ & 25 & & 1000 & $\mu \mathrm{g} / \mathrm{L}$ \\
\hline Cyanide & WA & & & $\mathbf{U}$ & 10 & & 200 & $\mu \mathrm{g} / \mathrm{L}$ \\
\hline Dibromochloromethane & WA & & & $\mathbf{U}$ & 5 & & NS & $\mu g / L$ \\
\hline Dichlorodifluoromethane & WA & & & $\mathbf{U}$ & 10 & & NS & $\mu \mathrm{g} / \mathrm{L}$ \\
\hline Ethylbenzene & WA & & & $\mathbf{U}$ & 5 & & 700 & $\mu g / L$ \\
\hline Fluoride & WA & & & $\mathbf{U}$ & 0.1 & & 4 & $\mathrm{mg} / \mathrm{L}$ \\
\hline
\end{tabular}


UTD005 Laburatory Analyses (cont'd)

\begin{tabular}{|c|c|c|c|c|c|c|c|c|}
\hline Analyte & Lab & Rep & Avg & Qualifier & Resule & Acc & Stand & Units \\
\hline $\begin{array}{l}\text { Gross alpha } \\
\text { Iron }\end{array}$ & TM & & & & 0.6 & 0.8 & 15 & $\begin{array}{l}\mathrm{pCi} / \mathrm{L} \\
\mu \rho / \mathrm{L}\end{array}$ \\
\hline $\begin{array}{l}\text { Iron } \\
\text { Isobutyl alcohol }\end{array}$ & $\begin{array}{l}\text { WA } \\
\text { WA }\end{array}$ & & & $\mathbf{U}$ & $\begin{array}{c}1080 \\
20\end{array}$ & & NS & $\begin{array}{l}\mu g / L \\
\mu g / L\end{array}$ \\
\hline Lead & WA & & & $\mathrm{U}$ & 3 & & 50 & $\mu g /$ \\
\hline Magnesium & WA & & & $\mathbf{U}$ & 5000 & & NS & $\mu_{g} / L$ \\
\hline Manganese & WA & & & & 125 & & 50 & $\mu g / \Omega$ \\
\hline Mercury & WA & & & $\mathbf{U}$ & 0.2 & & 2 & $\mu g$ \\
\hline Methacrylonitrile & WA & & & $\mathbf{U}$ & 20 & & NS & $\mu \mathrm{g}$ \\
\hline Methyl bromide & WA & & & $\mathbf{U}$ & 10 & & NS & $\mu g$ \\
\hline Methyl chloride & WA & & & $\mathbf{U}$ & 10 & & NS & $\mu \mathrm{g}$ \\
\hline Methyl ethyl ketone & WA & & & U & 10 & & NS & $\mu g /$ \\
\hline Methyl iodide & WA & & & $\mathbf{U}$ & 10 & & NS & $\mu g$ \\
\hline Methylene bromide & WA & & & $\mathbf{U}$ & 10 & & NS & $\mu \mathrm{g}$ \\
\hline Methylene chloride & WA & & & J & 1.05 & & 5 & $\mu g$ \\
\hline Nickel & WA & & & $\mathbf{U}$ & 40 & & 100 & $\mathrm{Hg}$ \\
\hline Nitrate as nitrogen & WA & & & & 0.027 & & 10 & $\mathrm{mg}$ \\
\hline Nonvolatile beta & TM & & & & 2.2 & 1.6 & 50 & $\mathrm{pCi}$ \\
\hline Potassium & WA & & & $\mathbf{U}$ & 5000 & & NS & 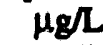 \\
\hline Propionitrile & WA & & & $\mathbf{U}$ & 50 & & NS & $\boldsymbol{\mu}$ \\
\hline Selenium & WA & & & $\mathbf{U}$ & 5 & & 50 & $\mu$ \\
\hline Silver & WA & & & $\mathbf{U}$ & 10 & & 50 & \\
\hline Sodium & WA & & & & 5300 & & NS & $\mu$ \\
\hline Styrene & WA & & & $\mathbf{U}$ & 5 & & 100 & \\
\hline Sulfate & WA & & & $\mathbf{U}$ & 0.25 & & 400 & In \\
\hline Sulfide & WA & & & $\mathbf{U}$ & 0.1 & & NS & $\mathrm{mg}$ \\
\hline Tetrachloroethylene & WA & & & $\mathbf{U}$ & 5 & & 5 & \\
\hline Thallium & WA & & & U & 10 & & 2 & $\mu$ \\
\hline Toluene & WA & & & $\mathbf{U}$ & 5 & & 1000 & \\
\hline Total hardness & WA & & & & 10 & & NS & $\mathbf{m g}$ \\
\hline Total organic carbon & WA & & $\mathbf{A}$ & & 1.17 & & NS & ms \\
\hline Total suspended solids & WA & & & & 43 & & NS & mg \\
\hline trans-1,3-Dichloropropene & WA & & & $\mathbf{U}$ & 5 & & NS & \\
\hline trans-1,4-Dichloro-2-butene & WA & & & U & 100 & & NS & \\
\hline Trichloroethylene & WA & & & U & 5 & & 5 & \\
\hline Trichlorofluoromethane & WA & & & U & 5 & & NS & \\
\hline Tritium & TM & & & & 22.3 & 2.3 & 20 & $p C i$ \\
\hline Turbidity & WA & & $\mathbf{A}$ & & 7.485 & & NS & \\
\hline Vanadium & WA & & & $\mathrm{U}$ & 50 & & NS & \\
\hline Vinyl acetate & WA & & & $\mathbf{U}$ & 10 & & NS & \\
\hline Vinyl chloride & WA & & & $\mathbf{U}$ & 10 & & 2 & $r$ \\
\hline Xylenes (total) & WA & & & U & 5 & & 10000 & \\
\hline Zinc & WA & & & & 22.1 & & 5000 & \\
\hline
\end{tabular}




\section{UTR004}

Field Measurements:

Sample Date: 4/14/93

pH: 4.54

Specific Conductance: $34 \mu \mathrm{S} / \mathrm{cm}$

Red/Oxid Potential: $89.7 \mathrm{mv}$

Dissolved Oxygen: $2.8 \mathrm{mg} / \mathrm{L}$

\begin{tabular}{|c|c|c|c|c|c|c|c|c|}
\hline Analyte & Lab & Rep & Avg & Qualifier & Result & Acc & Stand & Units \\
\hline 1,1,1,2-Tetrachloroethane & WA & & & $\mathbf{U}$ & 10 & & NS & $\mu g / L$ \\
\hline 1,1,1-Trichloroethane & WA & & & $\mathbf{U}$ & 5 & & 200 & $\mu g / L$ \\
\hline 1,1,2,2-Tetrachloroethane & WA & & & $\mathbf{U}$ & 5 & & NS & $\mu \mathrm{g} / \mathrm{L}$ \\
\hline 1,1,2-Trichloroethane & WA & & & $\mathbf{U}$ & 5 & & 5 & $\mu g / L$ \\
\hline 1,1-Dichloroethane & WA & & & $\mathbf{U}$ & 5 & & NS & $\mu g / L$ \\
\hline 1,1-Dichloroethylene & WA & & & $\mathbf{U}$ & 5 & & 7 & $\mu \mathrm{g} / \mathrm{L}$ \\
\hline 1,2,3-Trichloropropane & WA & & & $\mathbf{U}$ & 10 & & NS & $\mu g / L$ \\
\hline 1,2-Dibromo-3-chloropropane & WA & & . & $\mathbf{U}$ & 20 & & 0.2 & $\mu g / L$ \\
\hline 1,2-Dibromoethane & WA & & & $\mathbf{U}$ & 20 & & NS & $\mu g / L$ \\
\hline 1,2-Dichloroethane & WA & & & $\mathbf{U}$ & 5 & & 5 & $\mu \mathrm{g} / \mathrm{L}$ \\
\hline 1,2-Dichloroethylene (total) & WA & & & $\mathbf{U}$ & 5 & & 100 & $\mu \mathrm{g} / \mathrm{L}$ \\
\hline 1,2-Dichloropropane & WA & & & $\mathbf{U}$ & 5 & & 5 & $\mu g / L$ \\
\hline 2-Hexanone & WA & & & $\mathbf{U}$ & 10 & & NS & $\mu g / L$ \\
\hline 4-Methyl-2-pentanone & WA & & & $\mathbf{U}$ & 10 & & NS & $\mu g / L$ \\
\hline Acetone & WA & & & $\mathbf{U}$ & 10 & & NS & $\mu g / L$ \\
\hline Acetonitrile & WA & & & $\mathbf{U}$ & 20 & & NS & $\mu \mathrm{g} / \mathrm{L}$ \\
\hline Acidity & WA & & A & & 26 & & NS & $\mathrm{mg} / \mathrm{L}$ \\
\hline Acrolein & WA & & & $\mathbf{U}$ & 10 & & NS & $\mu \mathrm{g} / \mathrm{L}$ \\
\hline Acrylonitrile & WA & & & $\mathbf{U}$ & 10 & & NS & $\mu \mathrm{g} / \mathrm{L}$ \\
\hline Alkalinity & WA & & & & 11 & & NS & $\mathrm{mg} / \mathrm{L}$ \\
\hline Allyl chloride & WA & & & $\mathbf{U}$ & 100 & & NS & $\mu g / L$ \\
\hline Aluminum & WA & & & & 628 & & NS & $\mu g / L$ \\
\hline Antimony & WA & & & $\mathbf{U}$ & 60 & & 6 & $\mu \mathrm{g} / \mathrm{L}$ \\
\hline Arsenic & WA & & & $\mathbf{U}$ & 10 & & 50 & $\mu g / L$ \\
\hline Barium & WA & & & $\mathbf{U}$ & 200 & & 2000 & $\mu g / L$ \\
\hline Benzene & WA & & & $\mathbf{U}$ & 5 & & 5 & $\mu \mathrm{g} / \mathrm{L}$ \\
\hline Beryllium & WA & & & $\mathbf{U}$ & 5 & & 4 & $\mu \mathrm{g} / \mathrm{L}$ \\
\hline Bromodichloromethane & WA & & & $\mathbf{U}$ & 5 & & NS & $\mu \mathrm{g} / \mathrm{L}$ \\
\hline Bromoform & WA & & & $\mathbf{U}$ & 5 & & NS & $\mu g / L$ \\
\hline Cadmium & WA & & & & 6.5 & & 5 & $\mu g / L$ \\
\hline Calcium & WA & & & $\mathbf{U}$ & 5000 & & NS & $\mu \mathrm{g} / \mathrm{L}$ \\
\hline Carbon disulfide & WA & & & $\mathbf{U}$ & 5 & & NS & $\mu g / L$ \\
\hline Carbon tetrachloride & WA & & & $\mathbf{U}$ & 5 & & 5 & $\mu g / L$ \\
\hline Cesium-137 & TM & & NA & $\mathbf{U}$ & 10 & & 200 & $\mathrm{pCi} / \mathrm{L}$ \\
\hline Cesium-137 & TM & & & $\mathbf{U}$ & 10 & & 200 & $\mathrm{pCi} / \mathrm{L}$ \\
\hline Chloride . & WA & & & & 3.26 & & NS & $\mathrm{mg} / \mathrm{L}$ \\
\hline Chlorobenzene & WA & & & $\mathbf{U}$ & 5 & & 100 & $\mu \mathrm{g} / \mathrm{L}$ \\
\hline Chloroethane & WA & & & $\mathbf{U}$ & 10 & & NS & $\mu g / L$ \\
\hline Chloroform & WA & & & $\mathbf{U}$ & 5 & & 100 & $\mu \mathrm{g} / \mathrm{L}$ \\
\hline Chloroprene & WA & & & $\mathbf{U}$ & 20 & & NS & $\mu g / L$ \\
\hline Chromium & WA & & & $\mathbf{U}$ & 10 & & 100 & $\mu g / L$ \\
\hline cis-1,3-Dichloropropene & WA & & & $\mathbf{U}$ & 5 & & NS & $\mu g / L$ \\
\hline Cobalt & WA & & & $\mathbf{U}$ & 50 & & NS & $\mu g / L$ \\
\hline Copper & WA & & & $\mathbf{U}$ & 25 & & 1000 & $\mu g / L$ \\
\hline Cyanide & WA & & & $\mathbf{U}$ & 10 & & 200 & $\mu g / L$ \\
\hline Dibromochloromethane & WA & & & $\mathbf{U}$ & 5 & & NS & $\mu \mathrm{g} / \mathrm{L}$ \\
\hline Dichlorodifluoromethane & WA & & & $\mathbf{U}$ & 10 & & NS & $\mu g / L$ \\
\hline Ethylbenzene & WA & & & $\mathbf{U}$ & 5 & & 700 & $\mu g / L$ \\
\hline Fluoride & WA & & & $\mathbf{U}$ & 0.1 & & 4 & $\mathrm{mg} / \mathrm{L}$ \\
\hline
\end{tabular}


UTR004 Laboratory Analyses (cont'd)

\begin{tabular}{|c|c|c|c|c|c|c|c|}
\hline Analyte & Lab & Rep & Qualifier & Result & Acc & Stand & Units \\
\hline Gross alpha & TM & $\mathbf{A}$ & & 1.3 & 0.9 & 15 & $\mathrm{pCi} / \mathrm{L}$ \\
\hline Iron & WA & & & 4320 & & 300 & $\mu \mathrm{g} / \mathrm{L}$ \\
\hline Isobutyl alcohol & WA & & $\mathbf{U}$ & 20 & & NS & $\mu \mathrm{g} / \mathrm{L}$ \\
\hline Lead & WA & & $\mathbf{U}$ & 3 & & 50 & $\mu g / L$ \\
\hline Magnesium & WA & & $\mathbf{U}$ & 5000 & & NS & $\mu g / L$ \\
\hline Manganese & WA & & & 272 & . & 50 & $\mu g / L$ \\
\hline Mercury & WA & & $\mathbf{U}$ & 0.2 & & 2 & $\mu \mathrm{g} / \mathrm{L}$ \\
\hline Methacrylonitrile & WA & & $\mathbf{U}$ & 20 & & NS & $\mu g / L$ \\
\hline Methyl bromide & WA & & $\mathbf{U}$ & 10 & & NS & $\mu \mathrm{g} / \mathrm{L}$ \\
\hline Methyl chloride & WA & & $\mathbf{U}$ & 10 & & NS & $\mu g / L$ \\
\hline Methyl ethyl ketone & WA & & $\mathbf{U}$ & 10 & & NS & $\mu g / L$ \\
\hline Methyl iodide & WA & & $\mathbf{U}$ & 10 & & NS & $\mu g / L$ \\
\hline Methylene bromide & WA & & $\mathbf{U}$ & 10 & & NS & $\mu g / L$ \\
\hline Methylene chloride & WA & & $\mathbf{U}$ & 5 & & 5 & $\mu g / L$ \\
\hline Nickel & WA & & $\mathbf{U}$ & 40 & & 100 & $\mu g / L$ \\
\hline Nitrate as nitrogen & WA & & $\mathbf{U}$ & 0.02 & & 10 & mg/L \\
\hline Nitrate as nitrogen & WA & NA & $\mathbf{U}$ & 0.02 & & 10 & $\mathrm{mg} / \mathrm{L}$ \\
\hline Nonvolatile beta & $\mathbf{T M}$ & $\mathbf{A}$ & & 3.2 & 1.9 & 50 & $\mathrm{pCi} / \mathrm{L}$ \\
\hline Potassium & WA & & $\mathbf{U}$ & 5000 & & NS & $\mu g / L$ \\
\hline Propionitrile & WA & & $\mathbf{U}$ & 50 & & NS & $\mu g / L$ \\
\hline Selenium & WA & & $\mathbf{U}$ & 5 & & 50 & $\mu g / L$ \\
\hline Silver & WA & & $\mathbf{U}$ & 10 & & 50 & $\mu g / L$ \\
\hline Sodium & WA & & $\mathbf{U}$ & 5000 & & NS & $\mu g / L$ \\
\hline Styrene & WA & & $\mathbf{U}$ & 5 & & 100 & $\mu g / L$ \\
\hline Sulfate & WA & & $\mathbf{U}$ & 1 & & 400 & $\mathrm{mg} / \mathrm{L}$ \\
\hline Sulfide & WA & & $\mathbf{U}$ & 0.1 & & NS & $\mathrm{mg} / \mathrm{L}$ \\
\hline Tetrachloroethylene & WA & & $\mathbf{U}$ & 5 & & 5 & $\mu \mathrm{g} / \mathrm{L}$ \\
\hline Thallium & WA & & $\mathbf{U}$ & 10 & & 2 & $\mu g / L$ \\
\hline Toluene & WA & & $\mathbf{U}$ & 5 & & 1000 & $\mu g / L$ \\
\hline Total hardness & WA & & & 9 & & NS & $\mathrm{mg} / \mathrm{L}$ \\
\hline Total organic carbon & WA & & & 10.5 & & NS & $\mathrm{mg} / \mathrm{L}$ \\
\hline Total suspended solids & WA & & $\mathbf{U}$ & 5 & & NS & $\mathrm{mg} / \mathrm{L}$ \\
\hline trans-1,3-Dichloropropene & WA & & $\mathbf{U}$ & 5 & & NS & $\mu \mathrm{g} / \mathrm{L}$ \\
\hline trans-1,4-Dichloro-2-butene & WA & & $\mathbf{U}$ & 100 & & NS & $\mu g / L$ \\
\hline Trichloroethylene & WA & & $\mathbf{U}$ & 5 & & 5 & $\mu g / L$ \\
\hline Trichlorofluoromethane & WA & & $\mathbf{U}$ & 5 & & NS & $\mu g / L$ \\
\hline Tritium & TM & A & & 22.3 & 10.3 & 20 & $\mathrm{pCi} / \mathrm{ml}$ \\
\hline Turbidity & WA & $\mathbf{A}$ & & 1.095 & & NS & NTU \\
\hline Vanadium & WA & & $\mathbf{U}$ & 50 & & NS & $\mu g / L$ \\
\hline Vinyl acetate & WA & & $\mathbf{U}$ & 10 & & NS & $\mu \mathrm{g} / \mathrm{L}$ \\
\hline Vinyl chloride & WA & & $\mathbf{U}$ & 10 & & 2 & $\mu \mathrm{g} / \mathrm{L}$ \\
\hline Xylenes (total) & WA & & $\mathbf{U}$ & 5 & & 10000 & $\mu g / L$ \\
\hline Zinc & WA & & & 68.4 & & 5000 & $\mu \mathrm{g} / \mathrm{L}$ \\
\hline
\end{tabular}




\section{UTR011}

Field Measurements:

Sample Date: 4/14/93

pH: 5.83

Specific Conductance: $17 \mu \mathrm{S} / \mathrm{cm}$

Red/Oxid Potential: $206 \mathrm{mv}$

Dissolved Oxygen: $4.6 \mathrm{mg} / \mathrm{L}$

\begin{tabular}{|c|c|c|c|c|c|c|c|c|}
\hline Analyte & $\mathrm{Lab}$ & Rep & Avg & Qualifier & Result & Acc & Stand & Units \\
\hline 1,1,1,2-Tetrachloroethane & WA & & & $\mathrm{U}$ & 10 & & NS & $\mu g / L$ \\
\hline $1,1,1,2$-Tetrachloroethane & WA & & NA & $\mathbf{U}$ & 10 & & NS & $\mu g / L$ \\
\hline 1,1,1,2-Tetrachloroethane & WA & & NA & $\mathbf{U}$ & 10 & & NS & $\mu g / L$ \\
\hline 1,1,1,2-Tetrachloroethane & GE & & & $\mathbf{U}$ & 1 & & NS & $\mu g / L$ \\
\hline $1,1,1,2$-Tetrachloroethane & WA & $\mathbf{R}$ & & $\mathbf{U}$ & 10 & & NS & $\mu \mathrm{g} / \mathrm{L}$ \\
\hline $1,1,1$-Trichloroethane & WA & & & $\mathbf{U}$ & 5 & & 200 & $\mu g / L$ \\
\hline 1,1,1-Trichloroethane & WA & & NA & $\mathbf{U}$ & 5 & & 200 & $\mu g / L$ \\
\hline 1,1,1-Trichloroethane & WA & & NA & $\mathbf{U}$ & 5 & & 200 & $\mu g / L$ \\
\hline 1,1,1-Trichloroethane & GE & & & $\mathbf{U}$ & 1 & & 200 & $\mu g / L$ \\
\hline $1,1,1$-Trichloroethane & W.A & $\mathbf{R}$ & & $\mathbf{U}$ & 5 & & 200 & $\mu g / L$ \\
\hline $1,1,2,2$-Tetrachloroethane & WA & & & $\mathbf{U}$ & 5 & & NS & $\mu g / L$ \\
\hline $1,1,2,2$-Tetrachloroethane & WA & & NA & $\mathbf{U}$ & 5 & & NS & $\mu g / L$ \\
\hline 1,1,2,2-Tetrachloroethane & WA & & NA & $\mathbf{U}$ & 5 & & NS & $\mu g / L$ \\
\hline $1,1,2,2$-Tetrachloroethane & GE & & & $\mathbf{U}$ & 1 & & NS & $\mu g / L$ \\
\hline $1,1,2,2$-Tetrachloroethane & WA & $\mathbf{R}$ & & $\mathbf{U}$ & 5 & & NS & $\mu \mathrm{g} / \mathrm{L}$ \\
\hline 1,1,2-Trichloroethane & WA & & & $\mathbf{U}$ & 5 & & 5 & $\mu \mathrm{g} / \mathrm{L}$ \\
\hline 1,1,2-Trichloroethane & WA & & NA & $\mathrm{U}$ & 5 & & 5 & $\mu \mathrm{g} / \mathrm{L}$ \\
\hline 1,1,2-Trichloroethane & WA & & NA & $\mathbf{U}$ & 5 & & 5 & $\mu g / L$ \\
\hline 1,1,2-Trichloroethane & GE & & & $\mathbf{U}$ & 1 & & 5 & $\mu \mathrm{g} / \mathrm{L}$ \\
\hline 1,1,2-Trichloroethane & WA & $\mathbf{R}$ & & $\mathbf{U}$ & 5 & & 5 & $\mu \mathrm{g} / \mathrm{L}$ \\
\hline 1,1-Dichloroethane & WA & & & $\mathbf{U}$ & 5 & & NS & $\mu g / L$ \\
\hline 1,1-Dichloroethane & WA & & NA & $\mathbf{U}$ & 5 & & NS & $\mu g / L$ \\
\hline 1,1-Dichloroethane & WA & & NA & $\mathrm{U}$ & 5 & & NS & $\mu g / L$ \\
\hline 1,1-Dichloroethane & GE & & & $\mathbf{U}$ & 1 & & NS & $\mu g / L$ \\
\hline 1,1-Dichloroethane & WA & $\mathbf{R}$ & & U & 5 & & NS & $\mu g / L$ \\
\hline 1,1-Dichloroethylene & WA & & & $\mathbf{U}$ & 5 & & 7 & $\mu g / L$ \\
\hline 1,1-Dichloroethylene & $\mathbf{G E}$ & & & $\mathbf{U}$ & 1 & & 7 & $\mu \mathrm{g} / \mathrm{L}$ \\
\hline 1,1-Dichloroethylene & WA & $\mathbf{R}$ & & $\mathbf{U}$ & 5 & & 7 & $\mu \mathrm{g} / \mathrm{L}$ \\
\hline 1,2,3-Trichloropropane & WA & & & $\mathbf{U}$ & 10 & & NS & $\mu \mathrm{g} / \mathrm{L}$ \\
\hline 1,2,3-Trichloropropane & WA & & NA & $\mathbf{U}$ & 10 & & NS & $\mu \mathrm{g} / \mathrm{L}$ \\
\hline 1,2,3-Trichloropropane & WA & & NA & $\mathbf{U}$ & 10 & & NS & $\mu \mathrm{g} / \mathrm{L}$ \\
\hline 1,2,3-Trichloropropane & GE & & & U & 1 & & NS & $\mu g / L$ \\
\hline 1,2,3-Trichloropropane & WA & $\mathbf{R}$ & & $\mathbf{U}$ & 10 & & NS & $\mu g / L$ \\
\hline 1,2-Dibromo-3-chloropropane & WA & & & U & 20 & & 0.2 & $\mu g / L$ \\
\hline 1,2-Dibromo-3-chloropropane & WA & & NA & $\mathbf{U}$ & 20 & & 0.2 & $\mu g / L$ \\
\hline 1,2-Dibromo-3-chloropropane & WA & & NA & $\mathbf{U}$ & 20 & & 0.2 & $\mu g / L$ \\
\hline 1,2-Dibromo-3-chloropropane & GE & & & $\mathbf{U}$ & 1 & & 0.2 & $\mu g / L$ \\
\hline 1,2-Dibromo-3-chloropropane & WA & $\mathbf{R}$ & & $\mathbf{U}$ & 20 & & 0.2 & $\mu g / L$ \\
\hline 1,2-Dibromoethane & WA & & & $\mathbf{U}$ & 20 & & NS & $\mu \mathrm{g} / \mathrm{L}$ \\
\hline 1,2-Dibromoethane & WA & & NA & $\mathbf{U}$ & 20 & & NS & $\mu \mathrm{g} / \mathrm{L}$ \\
\hline 1,2-Dibromoethane & WA & & NA & $\mathbf{U}$ & 20 & & NS & $\mu \mathrm{g} / \mathrm{L}$ \\
\hline 1,2-Dibromoethane & GE & & & $\mathbf{U}$ & 20 & & NS & $\mu \mathrm{g} / \mathrm{L}$ \\
\hline 1,2-Dibromoethane & WA & $\mathbf{R}$ & & $\mathbf{U}$ & 20 & & NS & $\mu \mathrm{g} / \mathrm{L}$ \\
\hline 1,2-Dichloroethane & WA & & & $\mathbf{U}$ & 5 & & 5 & $\mu \mathrm{g} / \mathrm{L}$ \\
\hline 1,2-Dichloroethane & WA & & NA & $\mathbf{U}$ & 5 & & 5 & $\mu g / L$ \\
\hline 1,2-Dichloroethane & WA & & NA & $\mathbf{U}$ & 5 & & 5 & $\mu g / L$ \\
\hline 1,2-Dichloroethane & $\mathrm{GE}$ & & & $\mathbf{U}$ & 1 & & 5 & $\mu g / L$ \\
\hline 1,2-Dichloroethane & WA & & & $\mathbf{U}$ & 5 & & 5 & $\mu g / L$ \\
\hline
\end{tabular}


UTR011 Laboratory Analyses (cont'd)

\begin{tabular}{|c|c|c|c|c|c|c|c|c|}
\hline Analyte & Lab & Rep & Avg & Qualifier & Result & Acc & Stand & Units \\
\hline 1,2-Dichloroethylene (total) & WA & $\mathbf{R}$ & & $\mathbf{U}$ & 5 & & 100 & $\mu \mathrm{g} / \mathrm{L}$ \\
\hline 1,2-Dichloroethylene (total) & WA & & NA & $\mathbf{U}$ & 5 & & 100 & $\mu g / L$ \\
\hline 1,2-Dichloroethylene (total) & WA & & NA & $\mathbf{U}$ & 5 & & 100 & $\mu \mathrm{g} / \mathrm{L}$ \\
\hline 1,2-Dichloroethylene (total) & GE & & & $\mathbf{U}$ & 1 & & 100 & $\mu g / L$ \\
\hline 1,2-Dichloroethylene (total) & WA & $\mathbf{R}$ & & $\mathbf{U}$ & 5 & & 100 & $\mu g / L$ \\
\hline 1,2-Dichloropropane & WA & & & $\mathbf{U}$ & 5 & - & 5 & $\mu \mathrm{g} / \mathrm{L}$ \\
\hline 1,2-Dichloropropane & WA & & NA & $\mathbf{U}$ & 5 & $\theta^{\circ}$ & 5 & $\mu g / L$ \\
\hline 1,2-Dichloropropane & WA & & NA & $\mathbf{U}$ & 5 & & 5 & $\mu g / L$ \\
\hline 1,2-Dichloropropane & GE & & & $\mathbf{U}$ & 1 & & 5 & $\mu g / L$ \\
\hline 1,2-Dichloropropane & WA & $\mathbf{R}$ & & $\mathbf{U}$ & 5 & & 5 & $\mu \mathrm{g} / \mathrm{L}$ \\
\hline 2-Hexanone & WA & & & $\mathbf{U}$ & 10 & & NS & $\mu g / L$ \\
\hline 2-Hexanone & WA & & NA & $\mathbf{U}$ & 10 & & NS & $\mu \mathrm{g} / \mathrm{L}$ \\
\hline 2-Hexanone & WA & & NA & $\mathbf{U}$ & 10 & & NS & $\mu g / L$ \\
\hline 2-Hexanone & GE & & & $\mathbf{U}$ & 1 & & NS & $\mu g / L$ \\
\hline 2-Hexanone & WA & $\mathbf{R}$ & & $\mathbf{U}$ & 10 & & NS & $\mu g / L$ \\
\hline 4-Methyl-2-pentanone & WA & & & $\mathbf{U}$ & 10 & & NS & $\mu g / L$ \\
\hline 4-Methyl-2-pentanone & WA & & NA & $\mathbf{U}$ & 10 & & NS & $\mu \mathrm{g} / \mathrm{L}$ \\
\hline 4-Methyl-2-pentanone & WA & & NA & $\mathbf{U}$ & 10 & & NS & $\mu g / L$ \\
\hline 4-Methyl-2-pentanone & WA & $\mathbf{R}$ & & $\mathbf{U}$ & 10 & & NS & $\mu g / L$ \\
\hline Acetone & WA & & & $\mathbf{U}$ & 10 & & NS & $\mu \mathrm{g} / \mathrm{L}$ \\
\hline Acetone & WA & & NA & U & 10 & & NS & $\mu g / L$ \\
\hline Acetone & WA & & NA & & 13.9 & & NS & $\mu \mathrm{g} / \mathrm{L}$ \\
\hline Acetone & GE & & & $\mathbf{U}$ & 100 & & NS & $\mu \mathrm{g} / \mathrm{L}$ \\
\hline Acetone & WA & $\mathbf{R}$ & & $\mathbf{J}$ & 4.05 & & NS & $\mu g / L$ \\
\hline Acetonitrile & WA & & & $\mathbf{U}$ & 20 & & NS & $\mu g / L$ \\
\hline Acetonitrile & WA & & NA & $\mathbf{U}$ & 20 & & NS & $\mu g / L$ \\
\hline Acetonitrile & WA & & NA & $\mathbf{U}$ & 20 & & NS & $\mu g / L$ \\
\hline Acetonitrile & GE & & & $\mathbf{U}$ & 1 & & NS & $\mu \mathrm{g} / \mathrm{L}$ \\
\hline Acetonitrile & WA & $\mathbf{R}$ & & $\mathbf{U}$ & 20 & & NS & $\mu \mathrm{g} / \mathrm{L}$ \\
\hline Acidity & WA & & & & 17.5 & & NS & $\mathrm{mg} / \mathrm{L}$ \\
\hline Acidity & WA & $\mathbf{R}$ & & & 24 & & NS & $\mathrm{mg} / \mathrm{L}$ \\
\hline Acrolein & WA & & & $\mathbf{U}$ & 10 & & NS & $\mu \mathrm{g} / \mathrm{L}$ \\
\hline Acrolein & WA & & NA & $\mathbf{U}$ & 10 & & NS & $\mu \mathrm{g} / \mathrm{L}$ \\
\hline Acrolein & WA & & NA & $\mathrm{U}$ & 10 & & NS & $\mu \mathrm{g} / \mathrm{L}$ \\
\hline Acrolein & GE & & & $\mathrm{U}$ & 20 & & NS & $\mu \mathrm{g} / \mathrm{L}$ \\
\hline Acrolein & WA & $\mathbf{R}$ & & U & 10 & & NS & $\mu \mathrm{g} / \mathrm{L}$ \\
\hline Acrylonitrile & WA & & & $\mathbf{U}$ & 10 & & NS & $\mu g / L$ \\
\hline Acrylonitrile & WA & & NA & $\mathbf{U}$ & 10 & & NS & $\mu g / L$ \\
\hline Acrylonitrile & WA & & NA & U & 10 & & NS & $\mu g / L$ \\
\hline Acrylonitrile & GE & & & $U$ & 20 & & NS & $\mu \mathrm{g} / \mathrm{L}$ \\
\hline Acrylonitrile & WA & $\mathbf{R}$ & & $\mathbf{U}$ & 10 & & NS & $\mu \mathrm{g} / \mathrm{L}$ \\
\hline Actinium-228 & EP & & & U & 30 & & NS & $\mathrm{pCi} / \mathrm{L}$ \\
\hline Alkalinity & WA & & A & & 0.5 & & NS & $\mathrm{mg} / \mathrm{L}$ \\
\hline Alkalinity & GE & & & $\mathbf{U}$ & 1 & & NS & $\mathrm{mg} / \mathrm{L}$ \\
\hline Alkalinity & WA & $\mathbf{R}$ & & & 0.5 & & NS & $\mathrm{mg} / \mathrm{L}$ \\
\hline Allyl chloride & WA & & & U & 100 & & NS & $\mu \mathrm{g} / \mathrm{L}$ \\
\hline Allyl chloride & WA & & NA & $\mathrm{U}$ & 100 & & NS & $\mu \mathrm{g} / \mathrm{L}$ \\
\hline Allyl chloride & WA & & NA & $\mathrm{U}$ & 100 & & NS & $\mu g / L$ \\
\hline Allyl chloride & GE & & & $\mathrm{U}$ & 50 & & NS & $\mu \mathrm{g} / \mathrm{L}$ \\
\hline Allyl chloride & WA & $\mathbf{R}$ & & $\mathbf{U}$ & 100 & & NS & $\mu g / L$ \\
\hline Aluminum & WA & & & $\mathbf{U}$ & 200 & & NS & $\mu \mathrm{g} / \mathrm{L}$ \\
\hline Aluminum & GE & & A & & 78.75 & & NS & $\mu g / L$ \\
\hline Aluminum & WA & $\mathbf{R}$ & & $\mathbf{U}$ & 200 & & NS & $\mu g / L$ \\
\hline
\end{tabular}


UTR01 1 Laboratory Analyses (cont'd)

\begin{tabular}{|c|c|c|c|c|c|c|c|c|}
\hline Analyte & $\mathrm{Lab}$ & Rep & Avg & Qualifier & Result & Acc & Stand & Units \\
\hline Antimony & WA & & & $\mathbf{U}$ & 60 & & 6 & $\mu g / L$ \\
\hline Antimony & GE & & NA & $\mathbf{U}$ & 2 & & 6 & $\mu g / L$ \\
\hline Antimony & GE & & & $\mathbf{U}$ & 2 & & 6 & $\mu \mathrm{g} / \mathrm{L}$ \\
\hline Antimony & WA & $\mathbf{R}$ & & $\mathbf{U}$ & 60 & & 6 & $\mu \mathrm{g} / \mathrm{L}$ \\
\hline Antimony-125 & EP & & & $\mathbf{U}$ & 20 & & 3.00 & $\mathrm{pCi} / \mathrm{L}$ \\
\hline Arsenic & WA & & & $\mathbf{U}$ & 10 & & 50 & $\mu \mathrm{g} / \mathrm{L}$ \\
\hline Arsenic & $G E$ & & NA & $\mathbf{U}$ & 2 & - & 50 & $\mu \mathrm{g} / \mathrm{L}$ \\
\hline Arsenic & GE & & & $\mathrm{U}$ & 2 & & 50 & $\mu g / L$ \\
\hline Arsenic & WA & $\mathbf{R}$ & & $\mathbf{U}$ & 10 & & 50 & $\mu g / L$ \\
\hline Barium & WA & & & $\mathbf{U}$ & 200 & & 2000 & $\mu \mathrm{g} / \mathrm{L}$ \\
\hline Barium & GE & & $\mathbf{A}$ & & 8.26 & & 2000 & $\mu g / L$ \\
\hline Barium & WA & $\mathbf{R}$ & & $\mathbf{U}$ & 200 & & 2000 & $\mu \mathrm{g} / \mathrm{L}$ \\
\hline Benzene & WA & & & $\mathbf{U}$ & 5 & & 5 & $\mu \mathrm{g} / \mathrm{L}$ \\
\hline Benzene & GE & & & $\mathbf{U}$ & 1 & & 5 & $\mu \mathrm{g} / \mathrm{L}$ \\
\hline Benzene & WA & $\mathbf{R}$ & & $\mathbf{U}$ & 5 & & 5 & $\mu g / L$ \\
\hline Beryllium & WA & & & $\mathbf{U}$ & 5 & & 4 & $\mu g / L$ \\
\hline Beryllium & GE & & NA & $\mathbf{U}$ & 3 & & 4 & $\mu g / L$ \\
\hline Beryllium & GE & & & $\mathbf{U}$ & 3 & & 4 & $\begin{array}{l}\mu g / L \\
\mu g / L\end{array}$ \\
\hline Beryllium & WA & $\mathbf{R}$ & & $\mathbf{U}$ & 5 & & 4 & $\mu g / L$ \\
\hline Bis(2-chloro-1-methylethyl)ether & GE & & & $\mathbf{U}$ & 10 & & NS & $\mu g / L$ \\
\hline Bromodichloromethane & WA & & & $\mathbf{U}$ & 5 & & NS & $\mu g / L$ \\
\hline Bromodichloromethane & WA & & NA & $\mathbf{U}$ & 5 & & NS & $\mu g / L$ \\
\hline Bromodichloromethane & WA & & NA & $\mathbf{U}$ & 5 & & NS & $\mu \mathrm{g} / \mathrm{L}$ \\
\hline Bromodichloromethane & GE & & & $\mathbf{U}$ & 1 & & NS & $\mu \mathrm{g} / \mathrm{L}$ \\
\hline Bromodichloromethane & WA & $\mathbf{R}$ & & $\mathbf{U}$ & 5 & & NS & $\mu \mathrm{g} / \mathrm{L}$ \\
\hline Bromoform & WA & & & $\mathbf{U}$ & 5 & & NS & $\begin{array}{l}\mu g / L \\
\mu g / L\end{array}$ \\
\hline Bromoform & WA & & NA & $\mathbf{U}$ & 5 & & NS & $\mu g / L$ \\
\hline Bromoform & WA & & NA & $\mathbf{U}$ & 5 & & NS & $\begin{array}{l}\mu \mathrm{g} / \mathrm{L} \\
\mu \mathrm{g} / \mathrm{L}\end{array}$ \\
\hline Bromoform & $\mathrm{GE}$ & & & $\mathbf{U}$ & 1 & & NS & $\begin{array}{l}\mu g / L \\
\mu g / L\end{array}$ \\
\hline Bromoform & WA & $\mathbf{R}$ & & $\mathbf{U}$ & 5 & & NS & \\
\hline Cadmium & WA & & & $\mathbf{U}$ & 5 & & 5 & $\begin{array}{l}\mu g / L \\
\mu g / L\end{array}$ \\
\hline Cadmium & GE & & NA & $\mathbf{U}$ & 2 & & 5 & \\
\hline Cadmium & GE & & & $\mathbf{U}$ & 2 & & 5 & $\begin{array}{l}\mu g / L \\
\mu g / L\end{array}$ \\
\hline Cadmium & WA & $\mathbf{R}$ & & $\mathbf{U}$ & 5 & & 5 & $\begin{array}{l}\mu g / L \\
\mu g / L\end{array}$ \\
\hline Calcium & WA & & & $\mathbf{U}$ & 5000 & & NS & $\begin{array}{l}\mu g / L \\
\mu g / L\end{array}$ \\
\hline Calcium & GE & & $\mathbf{A}$ & & 218 & & NS & $\begin{array}{l}\mu g / L \\
\mu g / L\end{array}$ \\
\hline Calcium & WA & $\mathbf{R}$ & & $\mathbf{U}$ & 5000 & & NS & $\begin{array}{l}\mu g / L \\
\mu g / L\end{array}$ \\
\hline Carbon disulfide & WA & & & $\mathbf{U}$ & 5 & & NS & $\mu g / L$ \\
\hline Carbon disulfide & WA & & NA & $\mathbf{U}$ & 5 & & NS & $\mu \mathrm{g} / \mathrm{L}$ \\
\hline Carbon disulfide & WA & & NA & $\mathbf{U}$ & 5 & & $\begin{array}{l}\text { NS } \\
\text { NS }\end{array}$ & $\mu \mathrm{g} / \mathrm{L}$ \\
\hline Carbon disulfide & GE & & & $\mathbf{U}$ & 1 & & $\begin{array}{l}\text { NS } \\
\text { NS }\end{array}$ & $\mu \mathrm{g} / \mathrm{L}$ \\
\hline Carbon disulfide & WA & $\mathbf{R}$ & & $\mathbf{U}$ & 5 & & NS & $\mu \mathrm{g} / \mathrm{L}$ \\
\hline Carbon tetrachloride & WA & & & $\mathbf{U}$ & 5 & & & $\mu \mathrm{g} / \mathrm{L}$ \\
\hline Carbon tetrachloride & WA & & NA & $\mathbf{U}$ & 5 & & $\begin{array}{l}5 \\
5\end{array}$ & $\mu \mathrm{g} / \mathrm{L}$ \\
\hline Carbon tetrachloride & WA & & NA & $\mathbf{U}$ & $\begin{array}{l}5 \\
5\end{array}$ & & & $\mu \mathrm{g} / \mathrm{L}$ \\
\hline Carbon tetrachloride & GE & & & $\mathbf{U}$ & 1 & & $\begin{array}{l}5 \\
5\end{array}$ & $\mu g / L$ \\
\hline Carbon tetrachloride & WA & $\mathbf{R}$ & & $\mathbf{U}$ & 5 & & & $\mu \mathrm{g} / \mathrm{L}$ \\
\hline Cerium-144 & EP & & & $\mathbf{U}$ & 60 & & $\begin{array}{c}5 \\
261\end{array}$ & $\mu \mathrm{g} / \mathrm{L}$ \\
\hline Cesium-134 & $\mathrm{EP}$ & & & $\mathbf{U}$ & 10 & & $\begin{array}{l}261 \\
81.3\end{array}$ & $\mathrm{pCi} / \mathrm{L}$ \\
\hline Cesium-137 & TM & & & $\mathbf{U}$ & 7.7 & & $\begin{array}{l}81.3 \\
200\end{array}$ & $\mathrm{pCi} / \mathrm{L}$ \\
\hline Cesium-137 & EP & & & $\mathbf{U}$ & 10 & & $\begin{array}{l}200 \\
200\end{array}$ & $\mathrm{pCi} / \mathrm{L}$ \\
\hline Cesium-137 & TM & $\mathbf{R}$ & & $\mathbf{U}$ & 9.6 & & $\begin{array}{l}200 \\
200\end{array}$ & $\mathrm{pCi} / \mathrm{L}$ \\
\hline Chloride & WA & & & & 3.38 & & 200 & $\mathrm{pCi} / \mathrm{L}$ \\
\hline Chloride & GE & & & & 1.77 & & NS & $\mathrm{mg} / \mathrm{L}$ \\
\hline Chloride & WA & $\mathbf{R}$ & & & 3.55 & & NS & $\mathrm{mg} / \mathrm{L}$ \\
\hline & & & & & & & NS & $\mathrm{mg} / \mathrm{L}$ \\
\hline
\end{tabular}


UTR011 Laboratory Analyses (cont'd)

\begin{tabular}{|c|c|c|c|c|c|c|c|c|}
\hline Analyte & Lab & Rep & Avg & Qualifier & Result & Acc & Stand & Units \\
\hline Chlorobenzene & $\overline{\text { WA }}$ & & & $\mathbf{U}$ & 5 & & 100 & $\mu \mathrm{g} / \mathrm{L}$ \\
\hline Chlorobenzene & GE & & & $\mathbf{U}$ & 1 & & 100 & $\mu g / L$ \\
\hline Chlorobenzene & WA & $\mathbf{R}$ & & $\mathbf{U}$ & 5 & & 100 & $\mu g / L$ \\
\hline Chloroethane & WA & & & $\mathbf{U}$ & 10 & & NS & $\mu g / L$ \\
\hline Chloroethane & WA & & NA & $\mathbf{U}$ & 10 & & NS & $\mu \mathrm{g} / \mathrm{L}$ \\
\hline Chloroethane & WA & & NA & $\mathbf{U}$ & 10 & . & NS & $\mu g / L$ \\
\hline Chloroethane & GE & & & $\mathbf{U}$ & 1 & & NS & $\mu \mathrm{g} / \mathrm{L}$ \\
\hline Chloroethane & WA & $\mathbf{R}$ & & $\mathbf{U}$ & 10 & & NS & $\mu g / L$ \\
\hline Chloroform & WA & & & $\mathbf{U}$ & 5 & & 100 & $\mu \mathrm{g} / \mathrm{L}$ \\
\hline Chloroform & WA & & NA & $\mathbf{U}$ & 5 & & 100 & $\mu g / L$ \\
\hline Chloroform & WA & & NA & $\mathbf{U}$ & 5 & & 100 & $\mu g / L$ \\
\hline Chloroform & GE & & & $\mathbf{U}$ & 1 & & 100 & $\mu g / L$ \\
\hline Chloroform & WA & $\mathbf{R}$ & & $\mathbf{U}$ & 5 & & 100 & $\mu g / L$ \\
\hline Chloroprene & WA & & & $\mathbf{U}$ & 20 & & NS & $\mu g / L$ \\
\hline Chloroprene & WA & & NA & $\mathbf{U}$ & 20 & & NS & $\mu \mathrm{g} / \mathrm{L}$ \\
\hline Chloroprene & WA & & NA & $\mathbf{U}$ & 20 & & NS & $\mu g / L$ \\
\hline Chloroprene & GE & & & $\mathbf{U}$ & 200 & & NS & $\mu g / L$ \\
\hline Chloroprene & WA & $\mathbf{R}$ & & $\mathbf{U}$ & 20 & & NS & $\mu g / L$ \\
\hline Chromium & WA & & & $\mathbf{U}$ & 10 & & 100 & $\mu g / L$ \\
\hline Chromium & GE & & NA & $\mathbf{U}$ & 4 & & 100 & $\mu g / L$ \\
\hline Chromium & GE & & & $\mathbf{U}$ & 4 & & 100 & $\mu g / L$ \\
\hline Chromium & WA & $\mathbf{R}$ & & $\mathbf{U}$ & 10 & & 100 & $\mu g / L$ \\
\hline cis-1,3-Dichloropropene & WA & & & $\mathbf{U}$ & 5 & & NS & $\mu g / L$ \\
\hline cis-1,3-Dichloropropene & WA & & NA & $\mathbf{U}$ & 5 & & NS & $\mu g / L$ \\
\hline cis-1,3-Dichloropropene & WA & & NA & $\mathbf{U}$ & 5 & & NS & $\mu g / L$ \\
\hline cis-1,3-Dichloropropene & GE & & & $\mathbf{U}$ & 1 & & NS & $\mu \mathrm{g} / \mathrm{L}$ \\
\hline cis-1,3-Dichloropropene & WA & $\mathbf{R}$ & & $\mathbf{U}$ & 5 & & NS & $\mu \mathrm{g} / \mathrm{L}$ \\
\hline Cobalt & WA & & & $\mathbf{U}$ & 50 & & NS & $\mu \mathrm{g} / \mathrm{L}$ \\
\hline Cobalt & GE & & NA & $\mathbf{U}$ & 4 & & NS & $\mu \mathrm{g} / \mathrm{L}$ \\
\hline Cobalt & GE & & & $\mathbf{U}$ & 4 & & NS & $\mu g / L$ \\
\hline Cobalt & WA & $\mathbf{R}$ & & $\mathbf{U}$ & 50 & & NS & $\mu g / L$ \\
\hline Cobalt-57 & EP & & & $\mathbf{U}$ & 10 & & 1000 & pCi/L \\
\hline Cobalt- 60 & EP & & & U & 10 & & 100 & $\mathrm{pCi} / \mathrm{L}$ \\
\hline Copper & WA & & & $\mathbf{U}$ & 25 & & 1000 & $\mu g / L$ \\
\hline Copper & GE & & NA & $\mathrm{U}$ & 4 & & 1000 & $\mu g / L$ \\
\hline Copper & GE & & & $\mathbf{U}$ & 4 & & 1000 & $\mu g / L$ \\
\hline Copper & WA & $\mathbf{R}$ & & $\mathbf{U}$ & 25 & & 1000 & $\mu g / L$ \\
\hline Cyanide & WA & & & $\mathrm{U}$ & 10 & & 200 & $\mu \mathrm{g} / \mathrm{L}$ \\
\hline Cyanide & GE & & & $\mathbf{U}$ & 5 & & 200 & $\mu \mathrm{g} / \mathrm{L}$ \\
\hline Cyanide & WA & $\mathbf{R}$ & & $\mathbf{U}$ & 10 & & 200 & $\mu \mathrm{g} / \mathrm{L}$ \\
\hline Dibromochloromethane & WA & & & $\mathbf{U}$ & 5 & & NS & $\mu g / L$ \\
\hline Dibromochloromethane & WA & & NA & $\mathbf{U}$ & 5 & & NS & $\mu \mathrm{g} / \mathrm{L}$ \\
\hline Dibromochloromethane & WA & & NA & $\mathbf{U}$ & 5 & & NS & $\mu \mathrm{g} / \mathrm{L}$ \\
\hline Dibromochloromethane & GE & & & $\mathbf{U}$ & 1 & & NS & $\mu \mathrm{g} / \mathrm{L}$ \\
\hline Dibromochloromethane & WA & $\mathbf{R}$ & & $\mathbf{U}$ & 5 & & NS & $\mu g / L$ \\
\hline Dichlorodifluoromethane & WA & & & $\mathbf{U}$ & 10 & & NS & $\mu g / L$ \\
\hline Dichlorodifluoromethane & WA & & NA & $\mathbf{U}$ & 10 & & NS & $\mu \mathrm{g} / \mathrm{L}$ \\
\hline Dichlorodifluoromethane & WA & & NA & $\mathbf{U}$ & 10 & & INS & $\mu \mathrm{g} / \mathrm{L}$ \\
\hline Dichlorodifluoromethane & GE & & & $\mathbf{U}$ & 1 & & NS & $\mu \mathrm{g} / \mathrm{L}$ \\
\hline Dichlorodifluoromethane & WA & $\mathbf{R}$ & & $\mathbf{U}$ & 10 & & NS & $\mu \mathrm{g} / \mathrm{L}$ \\
\hline Ethylbenzene & WA & & & $\mathbf{U}$ & 5 & & 700 & $\mu \mathrm{g} / \mathrm{L}$ \\
\hline Ethylbenzene & WA & & NA & $\mathbf{U}$ & 5 & & 700 & $\mu \mathrm{g} / \mathrm{L}$ \\
\hline Ethylbenzene & WA & & NA & $\mathbf{U}$ & 5 & & 700 & $\mu g / L$ \\
\hline Ethylbenzene & GE & & & U & 1 & & 700 & $\mu \mathrm{g} / \mathrm{L}$ \\
\hline Ethylbenzene & WA & $\mathbf{R}$ & & $\mathbf{U}$ & 5 & & 700 & $\mu \mathrm{g} / \mathrm{L}$ \\
\hline
\end{tabular}


UTR011 Laboratory Analyses (cont'd)

\begin{tabular}{|c|c|c|c|c|c|c|c|c|}
\hline Analyte & $\mathrm{Lab}$ & Rep & Avg & Qualifier & Result & Acc & Stand & Units \\
\hline Europium-152 & EP & & & $\mathbf{U}$ & 40 & & NS & $\mathrm{pCi} / \mathrm{L}$ \\
\hline Europium-154 & EP & & & $\mathbf{U}$ & 20 & & 200 & $\mathrm{pCi} / \mathrm{L}$ \\
\hline Europium-155 & EP & & & $\mathbf{U}$ & 30 & & 600 & $\mathrm{pCi} / \mathrm{L}$ \\
\hline Fluoride & WA & & & $\mathbf{U}$ & 0.1 & & 4 & $\mathrm{mg} / \mathrm{L}$ \\
\hline Fluoride & GE & & & $\mathbf{U}$ & 0.1 & & 4 & $\mathrm{mg} / \mathrm{L}$ \\
\hline Fluoride & WA & $\mathbf{R}$ & & $\mathbf{U}$ & 0.1 & & 4 & $\mathrm{mg} / \mathrm{L}$ \\
\hline Gross alpha & TM & & & & 0.5 & 0.6 & 15 & $\mathrm{pCi} / \mathrm{L}$ \\
\hline Gross alpha & GE & & & $\mathbf{U}$ & 2 & & 15 & $\mathrm{pCi} / \mathrm{L}$ \\
\hline Gross alpha & TM & $\mathbf{R}$ & & & 0.8 & 0.9 & 15 & $\mathrm{pCi} / \mathrm{L}$ \\
\hline Iron & WA & & & $\mathbf{U}$ & 100 & & 300 & $\mu g / L$ \\
\hline Iron & GE & & $\mathbf{A}$ & & 230 & & 300 & $\mu g / L$ \\
\hline Iron & WA & $\mathbf{R}$ & & & 102 & & 300 & $\mu g / L$ \\
\hline Isobutyl alcohol & WA & & & $\mathbf{U}$ & 20 & & NS & $\mu g / L$ \\
\hline Isobutyl alcohol & WA & & NA & $\mathbf{U}$ & 20 & & NS & $\mu g / L$ \\
\hline Isobutyl alcohol & WA & & NA & $\mathbf{U}$ & 20 & & NS & $\mu g / L$ \\
\hline Isobutyl alcohol & GE & & & $\mathbf{U}$ & 100 & & NS & $\mu g / L$ \\
\hline Isobutyl alcohol & WA & $\mathbf{R}$ & & $\mathbf{U}$ & 20 & & NS & $\mu g / L$ \\
\hline Lead & WA & & & $\mathbf{U}$ & 3 & & 50 & $\mu g / L$ \\
\hline Lead & GE & & NA & $\mathbf{U}$ & 3 & & 50 & $\mu g / L$ \\
\hline Lead & GE & & & $\mathbf{U}$ & 3 & & 50 & $\mu \mathrm{g} / \mathrm{L}$ \\
\hline Lead & WA & $\mathbf{R}$ & & $\mathbf{U}$ & 3 & & 50 & $\mu g / L$ \\
\hline Lead-212 & EP & & & $\mathbf{U}$ & 15 & & NS & $\mathrm{pCi} / \mathrm{L}$ \\
\hline Magnesium & WA & & & $\mathbf{U}$ & .5000 & & NS & $\mu \mathrm{g} / \mathrm{L}$ \\
\hline Magnesium & $\mathrm{GE}$ & & $\mathbf{A}$ & & 340 & & NS & $\mu g / L$ \\
\hline Magnesium & WA & $\mathbf{R}$ & & $\mathbf{U}$ & 5000 & & NS & $\mu \mathrm{g} / \mathrm{L}$ \\
\hline Manganese & WA & & & $\mathbf{U}$ & 15 & & 50 & $\mu \mathrm{g} / \mathrm{L}$ \\
\hline Manganese & GE & & $\mathbf{A}$ & & 8.41 & & 50 & $\mu g / L$ \\
\hline Manganese & WA & $\mathbf{R}$ & & $\mathbf{U}$ & 15 & & 50 & $\mu \mathrm{g} / \mathrm{L}$ \\
\hline Manganese-54 & EP & & & $\mathrm{U}$ & 10 & & 300 & $\mathrm{pCi} / \mathrm{L}$ \\
\hline Mercury & WA & & & $\mathbf{U}$ & 0.2 & & 2 & $\mu g / L$ \\
\hline Mercury & GE & & & $\mathbf{U}$ & 0.2 & & 2 & $\mu \mathrm{g} / \mathrm{L}$ \\
\hline Mercury & WA & $\mathbf{R}$ & & $\mathbf{U}$ & 0.2 & & 2 & $\mu \mathrm{g} / \mathrm{L}$ \\
\hline Methacrylonitrile & WA & & & $\mathbf{U}$ & 20 & & NS & $\mu \mathrm{g} / \mathrm{L}$ \\
\hline Methacrylonitrile & WA & & NA & $\mathbf{U}$ & 20 & & NS & $\mu g / L$ \\
\hline Methacrylonitrile & WA & & NA & $\mathbf{U}$ & 20 & & NS & $\mu g / L$ \\
\hline Methacrylonitrile & GE & & & $\mathbf{U}$ & 50 & & NS & $\mu \mathrm{g} / \mathrm{L}$ \\
\hline Methacrylonitrile & WA & $\mathbf{R}$ & & $\mathrm{U}$ & 20 & & NS & $\mu g / L$ \\
\hline Methyl bromide & WA & & & $\mathbf{U}$ & 10 & & NS & $\mu g / L$ \\
\hline Methyl bromide & WA & & NA & $\mathrm{U}$ & 10 & & NS & $\mu \mathrm{g} / \mathrm{L}$ \\
\hline Methyl bromide & WA & & NA & $\mathbf{U}$ & 10 & & NS & $\mu g / L$ \\
\hline Methyl bromide & $\mathrm{GE}$ & & & $\mathbf{U}$ & 1 & & NS & $\mu g / L$ \\
\hline Methyl bromide & WA & $\mathbf{R}$ & & $\mathbf{U}$ & 10 & & NS & $\mu \mathrm{g} / \mathrm{L}$ \\
\hline Methyl chloride & WA & & & $\mathbf{U}$ & 10 & & NS & $\mu \mathrm{g} / \mathrm{L}$ \\
\hline Methyl chloride & WA & & NA & $\mathrm{U}$ & 10 & & NS & $\mu \mathrm{g} / \mathrm{L}$ \\
\hline Methyl chloride & WA & & NA & $\mathrm{U}$ & 10 & & NS & $\mu \mathrm{g} / \mathrm{L}$ \\
\hline Methyl chloride & $\mathrm{GE}$ & & & $\mathrm{U}$ & 1 & & NS & $\mu \mathrm{g} / \mathrm{L}$ \\
\hline Methyl chloride & WA & $\mathbf{R}$ & & $\mathbf{U}$ & 10 & & NS & $\mu \mathrm{g} / \mathrm{L}$ \\
\hline Methyl ethyl ketone & WA & & & $\mathbf{U}$ & 10 & & NS & $\mu \mathrm{g} / \mathrm{L}$ \\
\hline Methyl ethyl ketone & WA & & NA & $\mathrm{U}$ & 10 & & NS & $\mu \mathrm{g} / \mathrm{L}$ \\
\hline Methyl ethyl ketone & WA & & NA & $\mathbf{U}$ & 10 & & NS & $\mu \mathrm{g} / \mathrm{L}$ \\
\hline Methyl ethyl ketone & GE & & & $\mathbf{U}$ & 1 & & NS & $\mu \mathrm{g} / \mathrm{L}$ \\
\hline Methyl ethyl ketone & WA & $\mathbf{R}$ & & $\mathbf{U}$ & 10 & & NS & $\mu \mathrm{g} / \mathrm{L}$ \\
\hline
\end{tabular}


UTR011 Laboratory Analyses (cont'd)

\begin{tabular}{|c|c|c|c|c|c|c|c|c|c|}
\hline Analyte & Lab & Rep & Avg & Qualifier & & Result & Acc & Stand & Units \\
\hline Methyl iodide & WA & & & $\mathrm{U}$ & & 10 & & NS & $\mu g / L$ \\
\hline Methyl iodide & WA & & NA & $\mathbf{U}$ & & 10 & & NS & $\mu g / L$ \\
\hline Methyl iodide & WA & & NA & $\mathbf{U}$ & & 10 & & NS & $\mu g / L$ \\
\hline Methyl iodide & GE & & & $\mathbf{U}$ & & 15 & & NS & $\mu g / L$ \\
\hline Methyl iodide & WA & $\mathbf{R}$ & & $\mathbf{U}$ & & 10 & & NS & $\mu g / L$ \\
\hline Methyl isobutyl ketone & GE & & & $\mathbf{U}$ & & 1 & - & NS & $\mu g / L$ \\
\hline Methylene bromide & WA & & & $\mathbf{U}$ & & 10 & $\cdot$ & NS & $\mu g / L$ \\
\hline Methylene bromide & WA & & $\mathbf{N A}$ & $\mathbf{U}$ & & 10 & & NS & $\mu g / L$ \\
\hline Methylene bromide & WA & & NA & $\mathbf{U}$ & & 10 & & NS & $\mu g / L$ \\
\hline Methylene bromide & $\mathbf{G E}$ & & & $\mathbf{U}$ & & 1 & & NS & $\mu g / L$ \\
\hline Methylene bromide & WA & $\mathbf{R}$ & & $\mathbf{U}$ & & 10 & & NS & $\mu g / L$ \\
\hline Methylene chloride & WA & & & $\mathbf{U}$ & & 5 & & 5 & $\mu g / L$ \\
\hline Methylene chloride & WA & & $\mathbf{N A}$ & $\mathbf{U}$ & & 5 & & 5 & $\mu g / L$ \\
\hline Methylene chloride & WA & & NA & $\mathbf{U}$ & & 5 & & 5 & $\mu g / L$ \\
\hline Methylene chloride & GE & & & $\mathbf{U}$ & & 1 & & 5 & $\mu g / L$ \\
\hline Methylene chloride & WA & $\mathbf{R}$ & & $\mathbf{U}$ & & 5 & & 5 & $\mu g / L$ \\
\hline Nickel & WA & & & $\mathbf{U}$ & & 40 & & 100 & $\mu g / L$ \\
\hline Nickel & $\mathbf{G E}$ & & NA & $\mathbf{U}$ & & 4 & & 100 & $\mu g / L$ \\
\hline Nickel & GE & & & $\mathbf{U}$ & & 4 & & 100 & $\mu g / L$ \\
\hline Nickel & WA & $\mathbf{R}$ & & $\mathbf{U}$ & & 40 & & 100 & $\mu g / L$ \\
\hline Nitrate as nitrogen & WA & & & & & 0.219 & & 10 & $\mathrm{mg} / \mathrm{L}$ \\
\hline Nitrate as nitrogen & GE & & & & & 0.76 & & 10 & $\mathrm{mg} / \mathrm{L}$ \\
\hline Nitrate as nitrogen & WA & $\mathbf{R}$ & & & & 1.15 & & 10 & $\mathrm{mg} / \mathrm{L}$ \\
\hline Nonvolatile beta & TM & & & & & 1 & 1.6 & 50 & $\mathrm{pCi} / \mathrm{L}$ \\
\hline Nonvolatile beta & GE & & & $\mathbf{U}$ & & 2 & & 50 & $\mathrm{pCi} / \mathrm{L}$ \\
\hline Nonvolatile beta & TM & $\mathbf{R}$ & & & & 2 & 1.6 & 50 & $\mathrm{pCi} / \mathrm{L}$ \\
\hline $\mathrm{pH}$ & $\mathrm{GE}$ & & & $\mathbf{J}$ & $\mathbf{Q}$ & 5.5 & & NS & PH \\
\hline Potassium & WA & & & $\mathbf{U}$ & & 5000 & & NS & $\mu g / L$ \\
\hline Potassium & GE & & NA & $\mathbf{U}$ & & 500 & & NS & $\mu g / L$ \\
\hline Potassium & GE & & & $\mathbf{U}$ & & 500 & & NS & $\mu g / L$ \\
\hline Potassium & WA & $\mathbf{R}$ & & $\mathbf{U}$ & & 5000 & & NS & $\mu g / L$ \\
\hline Potassium-40 & $\mathbf{E P}$ & & & $\mathbf{U}$ & & 110 & & 300 & $\mathrm{pCi} / \mathrm{L}$ \\
\hline Promethium-144 & $\mathbf{E P}$ & & & $\mathbf{U}$ & & 10 & & NS & $\mathrm{pCi} / \mathrm{L}$ \\
\hline Promethium-146 & $\overrightarrow{\mathbf{E P}}$ & & & $\mathbf{U}$ & & 10 & & NS & $\mathrm{pCi} / \mathrm{L}$ \\
\hline Propionitrile & WA & & & $\mathbf{U}$ & & 50 & & NS & $\mu g / L$ \\
\hline Propionitrile & WA & & $\mathbf{N A}$ & $\mathbf{U}$ & & 50 & & NS & $\mu g / L$ \\
\hline Propionitrile & WA & & NA & $\mathbf{U}$ & & 50 & & NS & $\mu g / L$ \\
\hline Propionitrile & GE & & & $\mathbf{U}$ & & 200 & & NS & $\mu g / L$ \\
\hline Propionitrile & WA & $\mathbf{R}$ & & $\mathbf{U}$ & & 50 & & NS & $\mu \mathrm{g} / \mathrm{L}$ \\
\hline Ruthenium-106 & $\mathrm{EP}$ & & & $\mathbf{U}$ & & 90 & & 30 & $\mathrm{pCi} / \mathrm{L}$ \\
\hline Selenium & WA & & & $\mathbf{U}$ & & 5 & & 50 & $\mu \mathrm{g} / \mathrm{L}$ \\
\hline Selenium & GE & & NA & UJ & $\mathbf{L}$ & 2 & & 50 & $\mu g / L$ \\
\hline Selenium & GE & & & UJ & $\mathbf{L}$ & 2 & & 50 & $\mu \mathrm{g} / \mathrm{L}$ \\
\hline Selenium & WA & $\mathbf{R}$ & & $\mathbf{U}$ & & 5 & & 50 & $\mu g / L$ \\
\hline Silver & WA & & & $\mathbf{U}$ & & 10 & & 50 & $\mu \mathrm{g} / \mathrm{L}$ \\
\hline Silver & $\mathrm{GE}$ & & NA & $\mathbf{U}$ & & 2 & & 50 & $\mu \mathrm{g} / \mathrm{L}$ \\
\hline Silver & GE & & & $\mathbf{U}$ & & 2 & & 50 & $\mu g / L$ \\
\hline Silver & WA & $\mathbf{R}$ & & $\mathbf{U}$ & & 10 & & 50 & $\mu \mathrm{g} / \mathrm{L}$ \\
\hline Sodium & WA & & & $\mathbf{U}$ & & 5000 & & NS & $\mu g / L$ \\
\hline Sodium &. $\mathrm{GE}$ & & $\mathbf{A}$ & & & 1130 & & NS & $\mu \mathrm{g} / \mathrm{L}$ \\
\hline Sodium & WA & $\mathbf{R}$ & & $\mathbf{U}$ & & 5000 & & NS & $\mu \mathrm{g} / \mathrm{L}$ \\
\hline Sodium-22 & EP & & & $\mathbf{U}$ & & 10 & & 466 & $\mathrm{pCi} / \mathrm{L}$ \\
\hline
\end{tabular}


UTR011 Laboratory Analyses (cont'd)

\begin{tabular}{|c|c|c|c|c|c|c|c|c|}
\hline Analyte & Lab & Rep & Avg & Qualifier & Result & Acc & Stand & Units \\
\hline Styrene & $\overline{\text { WA }}$ & & & $\overline{\mathbf{U}}$ & 5 & & 100 & $\mu g / L$ \\
\hline Styrene & WA & & NA & $\mathbf{U}$ & 5 & & 100 & $\mu g / L$ \\
\hline Styrene & WA & & NA & $\mathbf{U}$ & 5 & & 100 & $\mu \mathrm{g} / \mathrm{L}$ \\
\hline Styrene & GE & & & U & 1 & & 100 & $\mu \mathrm{g} / \mathrm{L}$ \\
\hline Styrene & WA & $\mathbf{R}$ & & $\mathbf{U}$ & 5 & & 190 & $\mu g / L$ \\
\hline Sulfate & WA & & & U & 2.5 & & 400 & $\mathrm{mg} / \mathrm{L}$ \\
\hline Sulfate & GE & & & & 1.05 & & 400 & $\mathrm{mg} / \mathrm{L}$ \\
\hline Sulfate & WA & $\mathbf{R}$ & & $\mathbf{U}$ & 2.5 & & 400 & $\mathrm{mg} / \mathrm{L}$ \\
\hline Sulfide & WA & & & $\mathbf{U}$ & 0.1 & & NS & $\mathrm{mg} / \mathrm{L}$ \\
\hline Sulfide & GE & & & $\mathbf{U}$ & 1 & & NS & $\mathrm{mg} / \mathrm{L}$ \\
\hline Sulfide & WA & $\mathbf{R}$ & & $\mathbf{U}$ & 0.1 & & NS & $\mathrm{mg} / \mathrm{L}$ \\
\hline Tetrachloroethylene & WA & & & $\mathbf{U}$ & 5 & & 5 & $\mu g / L$ \\
\hline Tetrachloroethylene & WA & & NA & $\mathbf{U}$ & 5 & & 5 & $\mu \mathrm{g} / \mathrm{L}$ \\
\hline Tetrachloroethylene & WA & & NA & $\mathbf{U}$ & 5 & & 5 & $\mu g / L$ \\
\hline Tetrachloroethylene & GE & & & $\mathbf{U}$ & 1 & & 5 & $\mu g / L$ \\
\hline Tetrachloroethylene & WA & $\mathbf{R}$ & & $\mathbf{U}$ & 5 & & 5 & $\mu g / L$ \\
\hline Thallium & WA & & & $\mathbf{U}$ & 10 & & 2 & $\mu g / L$ \\
\hline Thallium & GE & & NA & $\mathbf{U}$ & 2 & & 2 & $\mu g / L$ \\
\hline Thallium & GE & & & $\mathbf{U}$ & 2 & & 2 & $\mu \mathrm{g} / \mathrm{L}$ \\
\hline Thallium & WA & $\mathbf{R}$ & & $\mathbf{U}$ & 10 & & 2 & $\mu \mathrm{g} / \mathrm{L}$ \\
\hline Thorium-234 & EP & & & $\mathbf{U}$ & 350 & & 401 & $\mathrm{pCi} / \mathrm{L}$ \\
\hline Toluene & WA & & & $\mathbf{U}$ & 5 & & 1000 & $\mu \mathrm{g} / \mathrm{L}$ \\
\hline Toluene & GE & & & $\mathbf{U}$ & 1 & & 1000 & $\mu \mathrm{g} / \mathrm{L}$ \\
\hline Toluene & WA & $\mathbf{R}$ & & $\mathbf{U}$ & 5 & & 1000 & $\mu \mathrm{g} / \mathrm{L}$ \\
\hline Total hardness & WA & & & $\mathbf{U}$ & 1 & & NS & $\mathrm{mg} / \mathrm{L}$ \\
\hline Total hardness & GE & & & & 1.5 & & NS & $\mathrm{mg} / \mathrm{L}$ \\
\hline Total hardness & WA & $\mathbf{R}$ & & U & 1 & & NS & $\mathrm{mg} / \mathrm{L}$ \\
\hline Total organic carbon & WA & & & & 1.75 & & NS & $\mathrm{mg} / \mathrm{L}$ \\
\hline Total organic carbon & GE & & & & 2.11 & & NS & $\mathrm{mg} / \mathrm{L}$ \\
\hline Total organic carbon & WA & $\mathbf{R}$ & & & 1.65 & & NS & $\mathrm{mg} / \mathrm{L}$ \\
\hline Total suspended solids & WA & & & $\mathbf{U}$ & 5 & & NS & $\mathrm{mg} / \mathrm{L}$ \\
\hline Total suspended solids & $\mathrm{GE}$ & & & $\mathrm{U}$ & 1 & & NS & $\mathrm{mg} / \mathrm{L}$ \\
\hline Total suspended solids & WA & $\mathbf{R}$ & & $\mathbf{U}$ & 5 & & NS & $\mathrm{mg} / \mathrm{L}$ \\
\hline trans-1,3-Dichloropropene & WA & & & $\mathbf{U}$ & 5 & & NS & $\mu \mathrm{g} / \mathrm{L}$ \\
\hline trans-1,3-Dichloropropene & WA & & NA & $\mathrm{U}$ & 5 & & NS & $\mu \mathrm{g} / \mathrm{L}$ \\
\hline trans-1,3-Dichloropropene & WA & & NA & $\mathrm{U}$ & 5 & & NS & $\mu g / L$ \\
\hline trans-1,3-Dichloropropene & GE & & & $\mathbf{U}$ & 1 & & NS & $\mu \mathrm{g} / \mathrm{L}$ \\
\hline trans-1,3-Dichloropropene & WA & $\mathbf{R}$ & & $\mathrm{U}$ & 5 & & NS & $\mu g / L$ \\
\hline trans-1,4-Dichloro-2-butene & WA & & & $\mathbf{U}$ & 100 & & NS & $\mu \mathrm{g} / \mathrm{L}$ \\
\hline trans-1,4-Dichloro-2-butene & WA & & NA & $\mathbf{U}$ & 100 & & NS & $\mu \mathrm{g} / \mathrm{L}$ \\
\hline trans-1,4-Dichloro-2-butene & WA & & NA & $\mathbf{U}$ & 100 & & NS & $\mu \mathrm{g} / \mathrm{L}$ \\
\hline trans-1,4-Dichloro-2-butene & GE & & & $\mathbf{U}$ & 30 & & NS & $\mu \mathrm{g} / \mathrm{L}$ \\
\hline trans-1,4-Dichloro-2-butene & WA & $\mathbf{R}$ & & $\mathbf{U}$ & 100 & & NS & $\mu g / L$ \\
\hline Trichloroethylene & WA & & & $\mathbf{U}$ & 5 & & 5 & $\mu \mathrm{g} / \mathrm{L}$ \\
\hline Trichloroethylene & GE & & & $\mathbf{U}$ & 1 & & 5 & $\mu \mathrm{g} / \mathrm{L}$ \\
\hline Trichloroethylene & WA & $\mathbf{R}$ & & $\mathbf{U}$ & 5 & & 5 & $\mu \mathrm{g} / \mathrm{L}$ \\
\hline Trichlorofluoromethare & WA & & & $\mathbf{U}$ & 5 & & NS & $\mu \mathrm{g} / \mathrm{L}$ \\
\hline Trichlorofluoromethane: & WA & & NA & $\mathbf{U}$ & 5 & & NS & $\mu \mathrm{g} / \mathrm{L}$ \\
\hline Trichlorofluoromethane & WA & & NA & $\mathbf{U}$ & 5 & & NS & $\mu \mathrm{g} / \mathrm{L}$ \\
\hline Trichlorofluoromethane & GE & & & U & 1 & & NS & $\mu \mathrm{g} / \mathrm{L}$ \\
\hline Trichlorofluoromethane & WA & $\mathbf{R}$ & & $\mathbf{U}$ & 5 & & NS & $\mu \mathrm{g} / \mathrm{L}$ \\
\hline Tritium & TM & & & & 19.6 & 9 & 20 & $\mathrm{pCi} / \mathrm{ml}$ \\
\hline Tritium & GE & & & & 17.8 & & 20 & $\mathrm{pCi} / \mathrm{mi}$ \\
\hline Tritium & TM & $\mathbf{R}$ & & & 18.9 & 5.2 & 20 & $\mathrm{pCi} / \mathrm{ml}$ \\
\hline
\end{tabular}


Sampling and Analysis of Water From Upper Three Runs And lis Wetlands Near Tank 16 and the Mixed Wasce Management Facillity

UTR011 Laboratory Analyses (cont'd)

\begin{tabular}{|c|c|c|c|c|c|c|c|c|}
\hline Analyte & Lab & Rep & Avg & Qualifier & Result & Acc & Stand & Units \\
\hline Turbidity & WA & & & & 0.43 & & NS & NTU \\
\hline Turbidity & GE & & & $\mathbf{U}$ & 0.1 & & NS & NTU \\
\hline Turbidity & WA & $\mathbf{R}$ & & & 0.6 & & NS & NTU \\
\hline Vanadium & WA & & & $\mathbf{U}$ & 50 & & NS & $\mu g / L$ \\
\hline Vanadium & GE & & NA & $\mathbf{U}$ & 8 & & NS & $\mu g / L$ \\
\hline Vanadium & $\overline{G E}$ & & & $\mathbf{U}$ & 8 & . & NS & $\mu g / L$ \\
\hline Vanadium & WA & $\mathbf{R}$ & & $\mathbf{U}$ & 50 & & NS & $\mu g / L$ \\
\hline Vinyl acetate & WA & & & $\mathbf{U}$ & 10 & & NS & $\mu g / L$ \\
\hline Vinyl acetate & WA & & NA & $\mathbf{U}$ & 10 & & NS & $\mu g / L$ \\
\hline Vinyl acetate & WA & & NA & $\mathbf{U}$ & 10 & & NS & $\mu g / L$ \\
\hline Vinyl acetate & $\mathbf{G E}$ & & & $\mathbf{U}$ & 1 & & NS & $\mu g / L$ \\
\hline Vinyl acetate & WA & $\mathbf{R}$ & & $\mathbf{U}$ & 10 & & NS & $\mu g / L$ \\
\hline Vinyl chloride & WA & & & $\mathbf{U}$ & 10 & & 2 & $\mu g / L$ \\
\hline Vinyl chloride & WA & & NA & $\mathbf{U}$ & 10 & & 2 & $\mu g / L$ \\
\hline Vinyl chloride & WA & & NA & $\mathbf{U}$ & 10 & & 2 & $\mu g / L$ \\
\hline Vinyl chloride & GE & & & $\mathbf{U}$ & 1 & & 2 & $\mu g / L$ \\
\hline Vinyl chloride & WA & $\mathbf{R}$ & & $\mathbf{U}$ & 10 & & 2 & $\mu g / L$ \\
\hline Xylenes (total) & WA & & & $\mathbf{U}$ & 5 & & 10000 & $\mu g / L$ \\
\hline Xylenes (total) & WA & & NA & $\mathbf{U}$ & 5 & & 10000 & $\mu g / L$ \\
\hline Xylenes (total) & WA & & NA & $\mathbf{U}$ & 5 & & 10000 & $\mu g / L$ \\
\hline Xylenes (total) & GE & & & $\mathbf{U}$ & 2 & & 10000 & $\mu g / L$ \\
\hline Xylenes (total) & WA & $\mathbf{R}$ & & $\mathbf{U}$ & 5 & & 10000 & $\mu g / L$ \\
\hline Yttrium-88 & EP & & & $\mathbf{U}$ & 60 & & NS & $\mathrm{pCi} / \mathrm{L}$ \\
\hline Zinc & WA & & & $\mathbf{U}$ & 20 & & 5000 & $\mu g / L$ \\
\hline Zinc & GE & & $\mathbf{A}$ & & 16.95 & & 5000 & $\mu \mathrm{g} / \mathrm{L}$ \\
\hline Zinc & WA & $\mathbf{R}$ & & $\mathbf{U}$ & 20 & & 5000 & $\mu g / L$ \\
\hline Zinc-65 & EP & & & $\mathbf{U}$ & 20 & & 300 & $\mathrm{pCi} / \mathrm{L}$ \\
\hline
\end{tabular}




\section{UTR024}

Field Measurements:

Sample Date: 4/14/93

pH: 5.36

Specific Conductance: $29 \mu \mathrm{S} / \mathrm{cm}$

Red/Oxid Potential: $211.5 \mathrm{mv}$

Dissolved Oxygen: $3.2 \mathrm{mg} / \mathrm{L}$

\begin{tabular}{|c|c|c|c|c|c|c|c|c|}
\hline Analyte & Lab & Rep & Avg & Qualifier & Result & Acc & Stand & Units \\
\hline 1,1,1,2-Tetrachloroethane & WA & & & $\mathbf{U}$ & 10 & & NS & $\mu g / L$ \\
\hline 1,1,1-Trichloroethane & WA & & & $\mathbf{U}$ & 5 & & 200 & $\mu g / L$ \\
\hline $1,1,2,2$-Tetrachloroethane & WA & & & $\mathbf{U}$ & 5 & & NS & $\mu g / L$ \\
\hline 1,1,2-Trichloroethane & WA & & & $\mathrm{U}$ & 5 & & 5 & $\mu g / L$ \\
\hline 1,1-Dichloroethane & WA & & & $\mathbf{U}$ & 5 & & NS & $\mu g / L$ \\
\hline 1,1-Dichloroethylene & WA & & & $\mathbf{U}$ & 5 & & 7 & $\mu \mathrm{g} / \mathrm{L}$ \\
\hline 1,2,3-Trichloropropane & WA & & & $\mathbf{U}$ & 10 & & NS & $\mu g / L$ \\
\hline 1,2-Dibromo-3-chloropropane & WA & & & $\mathbf{U}$ & 20 & & 0.2 & $\mu g / L$ \\
\hline 1,2-Dibromoethane & WA & & & $\mathbf{U}$ & 20 & & NS & $\mu g / L$ \\
\hline 1,2-Dichloroethane & WA & & & $\mathbf{U}$ & 5 & & 5 & $\mu \mathrm{g} / \mathrm{L}$ \\
\hline 1,2-Dichloroethylene (total) & WA & & & $\mathbf{U}$ & 5 & & 100 & $\mu \mathrm{g} / \mathrm{L}$ \\
\hline 1,2-Dichloropropane & WA & & & $\mathbf{U}$ & 5 & & 5 & $\mu g / L$ \\
\hline 2-Hexanone & WA & & & $\mathbf{U}$ & 10 & & NS & $\mu \mathrm{g} / \mathrm{L}$ \\
\hline 4-Methyl-2-pentanone & WA & & & U & 10 & & NS & $\mu \mathrm{g} / \mathrm{L}$ \\
\hline Acetone & WA & & & & 15.7 & & NS & $\mu g / L$ \\
\hline Acetonitrile & WA & & & $\mathbf{U}$ & 20 & & NS & $\mu g / L$ \\
\hline Acidity & WA & & & & 28 & & NS & $\mathrm{mg} / \mathrm{L}$ \\
\hline Acrolein & WA & & & $\mathbf{U}$ & 10 & & NS & $\mu g / L$ \\
\hline Acrylonitrile & WA & & & U & 10 & & NS & $\mu g / L$ \\
\hline Alkalinity & WA & & & $\mathbf{U}$ & 0.5 & & NS & $\mathrm{mg} / \mathrm{L}$ \\
\hline Allyl chloride & WA & & & $\mathbf{U}$ & 100 & & NS & $\mu g / L$ \\
\hline Aluminum & WA & & & $\mathbf{U}$ & 200 & & NS & $\mu g / L$ \\
\hline Antimony & WA & & & $\mathbf{U}$ & 60 & & 6 & $\mu g / L$ \\
\hline Arsenic & WA & & & $\mathbf{U}$ & 10 & & 50 & $\mu \mathrm{g} / \mathrm{L}$ \\
\hline Barium & WA & & & U & 200 & & 2000 & $\mu g / L$ \\
\hline Benzene & WA & & & $\mathbf{U}$ & 5 & & 5 & $\mu g / L$ \\
\hline Beryllium & WA & & & $\mathbf{U}$ & 5 & & 4 & $\mu \mathrm{g} / \mathrm{L}$ \\
\hline Bromodichloromethane & WA & & & $\mathrm{U}$ & 5 & & NS & $\mu g / L$ \\
\hline Bromoform & WA & & & U & 5 & & NS & $\mu g / L$ \\
\hline Cadmium & WA & & & U & 5 & & 5 & $\mu g / L$ \\
\hline Calcium & WA & & & $\mathbf{U}$ & 5000 & & NS & $\mu \mathrm{g} / \mathrm{L}$ \\
\hline Carbon disulfide & WA & & & $\mathbf{U}$ & 5 & & NS & $\mu \mathrm{g} / \mathrm{L}$ \\
\hline Carbon tetrachloride & WA & & & $\mathbf{U}$ & 5 & & 5 & $\mu \mathrm{g} / \mathrm{L}$ \\
\hline Cesium-137 & TM & & & & 6.4 & 5.3 & 200 & $\mathrm{pCi} / \mathrm{L}$ \\
\hline Chloride & WA & & & & 2.86 & & NS & $\mathrm{mg} / \mathrm{L}$ \\
\hline Chlorobenzene & WA & & & $\mathbf{U}$ & 5 & & 100 & $\mu \mathrm{g} / \mathrm{L}$ \\
\hline Chloroethane & WA & & & $\mathbf{U}$ & 10 & & NS & $\mu \mathrm{g} / \mathrm{L}$ \\
\hline Chloroform & WA & & & $\mathrm{U}$ & 5 & & 100 & $\mu \mathrm{g} / \mathrm{L}$ \\
\hline Chloroprene & WA & & & $\mathbf{U}$ & 20 & & NS & $\mu \mathrm{g} / \mathrm{L}$ \\
\hline Chromium & WA & & & $\mathbf{U}$ & 10 & & 100 & $\mu \mathrm{g} / \mathrm{L}$ \\
\hline cis-1,3-Dichloropropene & WA & & & $\mathrm{U}$ & 5 & & NS & $\mu \mathrm{g} / \mathrm{L}$ \\
\hline Cobalt & WA & & & $\mathbf{U}$ & 50 & & NS & $\mu \mathrm{g} / \mathrm{L}$ \\
\hline Copper & WA & & & $\mathbf{U}$ & 25 & & 1000 & $\mu \mathrm{g} / \mathrm{L}$ \\
\hline Cyanide & WA & & & $\mathbf{U}$ & 10 & & 200 & $\mu \mathrm{g} / \mathrm{L}$ \\
\hline Dibromochloromethane & WA & & & $\mathbf{U}$ & 5 & & NS & $\mu g / L$ \\
\hline Dichlorodifluoromethane & WA & & & $\mathbf{U}$ & 10 & & NS & $\mu \mathrm{g} / \mathrm{L}$ \\
\hline Ethylbenzene & WA & & & $\mathbf{U}$ & 5 & & 700 & $\mu g / L$ \\
\hline Fluoride & WA & & & $\mathbf{U}$ & 0.1 & & 4 & $\mathrm{mg} / \mathrm{L}$ \\
\hline Gross alpha & TM & & & & 1 & 0.8 & 15 & $\mathrm{pCi} / \mathrm{L}$ \\
\hline
\end{tabular}


UTR025 Laboratory Analyses (cont'd)

\begin{tabular}{|c|c|c|c|c|c|c|c|c|}
\hline Analyte & Lab & Rep & Avg & Qualifier & Result & Acc & Stand & Units \\
\hline Iron & WA & & & $\bar{U}$ & 100 & & 300 & $\mu g / L$ \\
\hline Isobutyl alcohol & WA & & & $\mathbf{U}$ & 20 & & NS & $\mu \mathrm{g} / \mathrm{L}$ \\
\hline Lead & WA & & & U & 3 & & 50 & $\mu g / L$ \\
\hline Magnesium & WA & & & $\mathbf{U}$ & 5000 & & NS & $\mu \mathrm{g} / \mathrm{L}$ \\
\hline Manganese & WA & & & $\mathbf{U}$ & 15 & & 50 & $\mu \mathrm{g} / \mathrm{L}$ \\
\hline Mercury & WA & & & U & 0.2 & • & 2 & $\mu g / L$ \\
\hline Methacrylonitrile & WA & & & U & 20 & & NS & $\mu g / L$ \\
\hline Methyl bromide & WA & & & $\mathbf{U}$ & 10 & & NS & $\mu g / L$ \\
\hline Methyl chloride & WA & & & $\mathbf{U}$ & 10 & & NS & $\mu \mathrm{g} / \mathrm{L}$ \\
\hline Methyl ethyl ketone & WA & & & $\mathbf{U}$ & 10 & & NS & $\mu g / L$ \\
\hline Methyl iodide & WA & & & $\mathbf{U}$ & 10 & & NS & $\mu g / L$ \\
\hline Methylene bromide & WA & & & $\mathbf{U}$ & 10 & & NS & $\mu g \Omega$ \\
\hline Methylene chloride & WA & & & $\mathbf{J}$ & 1.42 & & 5 & $\mu \mathrm{g} / \mathrm{L}$ \\
\hline Nickel & WA & & & $\mathbf{U}$ & 40 & & 100 & $\mu g / L$ \\
\hline Nitrate as nitrogen & WA & & & $\mathbf{U}$ & 0.02 & & 10 & $\mathrm{mg} / \mathrm{h}$ \\
\hline Nonvolatile beta & TM & & & $\mathbf{U}$ & 0.7 & & 50 & $\mathrm{pCi} / \mathrm{L}$ \\
\hline Potassium & WA & & & $\mathbf{U}$ & 5000 & & NS & $\mu g / L$ \\
\hline Propionitrile & WA & & & $\mathbf{U}$ & 50 & & NS & $\mu \mathrm{g} / \mathrm{L}$ \\
\hline Selenium & WA & & & $\mathbf{U}$ & 5 & & 50 & $\mu g / L$ \\
\hline Silver & WA & & & $\mathbf{U}$ & 10 & & 50 & $\mu \mathrm{g} / \mathrm{L}$ \\
\hline Sodium & WA & & & $\mathbf{U}$ & 5000 & & NS & $\mu g / L$ \\
\hline Styrene & WA & & & $\mathbf{U}$ & 5 & & 100 & $\mu g / L$ \\
\hline Sulfate & WA & & & $\mathbf{U}$ & 1 & & 400 & $\mathrm{mg} / \mathrm{L}$ \\
\hline Sulfate & WA & & NA & $\mathbf{U}$ & 1 & & 400 & $\mathrm{mg} / \mathrm{L}$ \\
\hline Sulfide & WA & & & $\mathbf{U}$ & 0.1 & & NS & $\mathrm{mg} / \mathrm{L}$ \\
\hline Tetrachloroethylene & WA & & & $\mathbf{U}$ & 5 & & 5 & $\mu g / L$ \\
\hline Thallium & WA & & & $\mathbf{U}$ & 10 & & 2 & $\mu \mathrm{g} / \mathrm{L}$ \\
\hline Toluene & WA & & & $\mathbf{U}$ & 5 & & 1000 & $\mu g / L$ \\
\hline Total hardness & WA & & & & 14 & & NS & $\mathrm{mg} / \mathrm{L}$ \\
\hline Total organic carbon & WA & & & $\mathbf{U}$ & 0.5 & & NS & $\mathrm{mg} / \mathrm{L}$ \\
\hline Total organic carbon & WA & & NA & $\mathbf{U}$ & 0.5 & & NS & $\mathrm{mg} / \mathrm{L}$ \\
\hline Total suspended solids & WA & & & $\mathbf{U}$ & 5 & & NS & $\mathrm{mg} / \mathrm{L}$ \\
\hline trans-1,3-Dichloropropene & WA & & & $\mathbf{U}$ & 5 & & NS & $\mu \mathrm{g} / \mathrm{L}$ \\
\hline trans-1,4-Dichloro-2-butene & WA & & & $\mathbf{U}$ & 100 & & NS & $\mu \mathrm{g} / \mathrm{L}$ \\
\hline Trichloroethylene & WA & & & $\mathbf{U}$ & 5 & & 5 & $\mu g / L$ \\
\hline Trichlorofluoromethane & WA & & & $\mathbf{U}$ & 5 & & NS & $\mu g / L$ \\
\hline Tritium & TM & & & & 33.1 & 15.3 & 20 & $\mathrm{pCi} / \mathrm{ml}$ \\
\hline Turbidity & WA & & & & 0.51 & & NS & NTU \\
\hline Vanadium & WA & & & $\mathbf{U}$ & 50 & & NS & $\mu g / L$ \\
\hline Vinyl acetate & WA & & & $\mathbf{U}$ & 10 & & NS & $\mu \mathrm{g} / \mathrm{L}$ \\
\hline Vinyl chloride & WA & & & $\mathbf{U}$ & 10 & & 2 & $\mu \mathrm{g} / \mathrm{L}$ \\
\hline Xylenes (total) & WA & & & $\mathbf{U}$ & 5 & & 10000 & $\mu g / L$ \\
\hline Zinc & WA & & & U & 20 & & 5000 & $\mathrm{~L}$ \\
\hline
\end{tabular}




\section{UTR029}

Field Measurements:

Sample Date: $1 / 27 / 93$

pH: 6.15

Specific Conductance: $131 \mu \mathrm{S} / \mathrm{cm}$

Red/Oxid Potential: $-171 \mathrm{mv}$

Dissolved Oxygen: $3.6 \mathrm{mg} / \mathrm{L}$

\begin{tabular}{|c|c|c|c|c|c|c|c|c|}
\hline Analyte & Lab & Rep & Avg & Qualifier & Result & Acc & Stand & Units \\
\hline 1,1,1,2-Tetrachloroethane & WA & & & $\mathbf{U}$ & 10 & & NS & $\mu g / L$ \\
\hline 1,1,1-Trichloroethane & WA & & & $\mathbf{U}$ & 5 & & 200 & $\mu g / L$ \\
\hline 1,1,2,2-Tetrachloroethane & WA & & & $\mathbf{U}$ & 5 & & NS & $\mu g / L$ \\
\hline 1,1,2-Trichloroethane & WA & & & $\mathbf{U}$ & 5 & & 5 & $\mu g / L$ \\
\hline 1,1-Dichloroethane & WA & & & $\mathbf{U}$ & 5 & & NS & $\mu g / L$ \\
\hline 1,1-Dichloroethylene & WA & & & $\mathbf{U}$ & 5 & & 7 & $\mu g / L$ \\
\hline 1,2,3-Trichloropropane & WA & & & $\mathbf{U}$ & 10 & & NS & $\mu g / L$ \\
\hline 1,2-Dibromo-3-chloropropane & WA & & & $\mathbf{U}$ & 20 & & 0.2 & $\mu g / L$ \\
\hline 1,2-Dibromoethane & WA & & & $\mathbf{U}$ & 20 & & NS & $\mu g / L$ \\
\hline 1,2-Dichloroethane & WA & & & $\mathbf{U}$ & 5 & & 5 & $\mu g / L$ \\
\hline 1,2-Dichloroethylene (total) & WA & & & $\mathbf{U}$ & 5 & & 100 & $\mu g / L$ \\
\hline 1,2-Dichloropropane & WA & & & $\mathbf{U}$ & 5 & & 5 & $\mu g / L$ \\
\hline 2-Hexanone & WA & & & $\mathbf{U}$ & 10 & & NS & $\mu g / L$ \\
\hline 4-Methyl-2-pentanone & WA & & & $\mathbf{U}$ & 10 & & NS & $\mu g / L$ \\
\hline Acetone & WA & & & $\mathbf{U}$ & 10 & & NS & $\mu g / L$ \\
\hline Acetonitrile & WA & & & $\mathbf{U}$ & 20 & & NS & $\mu g / L$ \\
\hline Acidity & WA & & & & 100 & & NS & $\mathrm{mg} / \mathrm{L}$ \\
\hline Acrolein & WA & & & $\mathbf{U}$ & 10 & & NS & $\mu g / L$ \\
\hline Acrylonitrile & WA & & & $\mathbf{U}$ & 10 & & NS & $\mu g / L$ \\
\hline Alkalinity & WA & & & & 20 & & NS & $\mathrm{mg} / \mathrm{L}$ \\
\hline Allyl chloride & WA & & & $\mathbf{U}$ & 100 & & NS & $\mu g / L$ \\
\hline Aluminum & WA & & & & 5930 & & NS & $\mu g / L$ \\
\hline Antimony & WA & & & $\mathbf{U}$ & 60 & & 6 & $\mu g / L$ \\
\hline Arsenic & WA & & & $\mathbf{U}$ & 10 & & 50 & $\mu g / L$ \\
\hline Barium & WA & & & $\mathbf{U}$ & 200 & & 2000 & $\mu g / L$ \\
\hline Benzene & WA & & & $\mathbf{U}$ & 5 & & 5 & $\mu g / L$ \\
\hline Beryllium & WA & & & $\mathbf{U}$ & 5 & & 4 & $\mu g / L$ \\
\hline Bromodichloromethane & WA & & & $\mathbf{U}$ & 5 & & NS & $\mu g / L$ \\
\hline Bromoform & WA & & & $\mathbf{U}$ & 5 & & NS & $\mu g / L$ \\
\hline Cadmium & WA & & & $\mathbf{U}$ & 5 & & 5 & $\mu g / L$ \\
\hline Calcium & WA & & & $\mathbf{U}$ & 5000 & & NS & $\mu g / L$ \\
\hline Carbon disulfide & WA & & & $\mathbf{U}$ & 5 & & NS & $\mu \mathrm{g} / \mathrm{L}$ \\
\hline Carbon tetrachloride & WA & & & $\mathbf{U}$ & 5 & & 5 & $\mu g / L$ \\
\hline Cesium-137 & TM & & & $\mathbf{U}$ & 6.1 & & 200 & $\mathrm{pCi} / \mathrm{L}$ \\
\hline Cesium-137 & TM & & & $\mathbf{U}$ & 6.1 & & 200 & $\mathrm{pCi} / \mathrm{L}$ \\
\hline Chloride & WA & & & & 3.4 & & NS & $\mathrm{mg} / \mathrm{L}$ \\
\hline Chlorobenzene & WA & & & $\mathbf{U}$ & 5 & & 100 & $\mu g / L$ \\
\hline Chloroethane & WA & & & $\mathrm{U}$ & 10 & & NS & $\mu \mathrm{g} / \mathrm{L}$ \\
\hline Chloroform & WA & & & $\mathbf{U}$ & 5 & & 100 & $\mu g / L$ \\
\hline Chloroprene & WA & & & $\mathbf{U}$ & 20 & & NS & $\mu \mathrm{g} / \mathrm{L}$ \\
\hline Chromium & WA & & & $\mathbf{U}$ & 10 & & 100 & $\mu \mathrm{g} / \mathrm{L}$ \\
\hline cis-1,3-Dichloropropene & WA & & & $\mathbf{U}$ & 5 & & NS & $\mu \mathrm{g} / \mathrm{L}$ \\
\hline Cobalt & WA & & & $\mathbf{U}$ & 50 & & NS & $\mu g / L$ \\
\hline Copper & WA & & & $\mathrm{U}$ & 25 & & 1000 & $\mu g / L$ \\
\hline Cyanide & WA & & & $\mathbf{U}$ & 10 & & 200 & $\mu \mathrm{g} / \mathrm{L}$ \\
\hline Dibromochloromethane & WA & & & $\mathbf{U}$ & 5 & & NS & $\mu \mathrm{g} / \mathrm{L}$ \\
\hline Dichlorodifluoromethane & WA & & & $\mathbf{U}$ & 10 & & NS & $\mu \mathrm{g} / \mathrm{L}$ \\
\hline Ethylbenzene & WA & & & $\mathbf{U}$ & 5 & & 700 & $\mu g / L$ \\
\hline Fluoride & WA & & & $\mathbf{U}$ & 0.1 & & 4 & \\
\hline
\end{tabular}


UTR029 Laboratory Analyses (cont'd)

\begin{tabular}{|c|c|c|c|c|c|c|c|c|c|}
\hline Analyte & Lab & Rep & Avg & Qualifier & & Result & Acc & Stand & Units \\
\hline Gross alpha & TM & & & & & 4.4 & 2.5 & 15 & $\overline{\mathrm{pCi} / \mathrm{L}}$ \\
\hline Iron & WA & & & & & 17700 & & 300 & $\mu g / L$ \\
\hline Isobutyl alcohol & WA & & & U & & 20 & & NS & $\mu g / L$ \\
\hline Lead & WA & & & & & 6 & & 50 & $\mu_{g} / 2$ \\
\hline Magnesium & WA & & & $\mathbf{U}$ & & 5000 & & NS & $\mu g / L$ \\
\hline Manganese & WA & & & & & 1900 & - & 50 & $\mu g /$ \\
\hline Mercury & WA & & & $\mathbf{U}$ & & 0.2 & & 2 & $\mu g / L$ \\
\hline Methacrylonitrile & WA & & & $\mathbf{U}$ & & 20 & & NS & $\mu g / L$ \\
\hline Methyl bromide & WA & & & $\mathbf{U}$ & & 10 & & NS & $\mu g / L$ \\
\hline Methyl chloride & WA & & & $\mathbf{U}$ & & 10 & & NS & $\mu g / L$ \\
\hline Methyl ethyl ketone & WA & & & $\mathbf{U}$ & & 10 & & NS & $\mu g / L$ \\
\hline Methyl iodide & WA & & & $\mathbf{U}$ & & 10 & & NS & $\mu g / L$ \\
\hline Methylene bromide & WA & & & $\mathbf{U}$ & & 10 & & NS & $\mu g / 2$ \\
\hline Methylene chloride & WA & & & $\mathbf{U}$ & & 5 & & 5 & $\mu g / L$ \\
\hline Nickel & WA & & & $\mathbf{U}$ & & 40 & & 100 & $\mu g / \Omega$ \\
\hline Nitrate as nitrogen & WA & & & $\mathbf{U}$ & & 0.02 & & 10 & $\mathrm{mg} / \mathrm{h}$ \\
\hline Nonvolatile beta & TM & & & & & 7.2 & 3.2 & 50 & $\mathrm{pCi} / \mathrm{L}$ \\
\hline Potassium & WA & & & $\mathbf{U}$ & & 5000 & & NS & $\mu \mathrm{g} / \mathrm{L}$ \\
\hline Propionitrile & WA & & & U & & 50 & & NS & $\mu \mathrm{g} / \mathrm{L}$ \\
\hline Selenium & WA & & & $\mathbf{U}$ & & 5 & & 50 & $\mu g / L$ \\
\hline Silver & WA & & & $\mathbf{U}$ & & 10 & & 50 & $\mu \mathrm{g} / \mathrm{L}$ \\
\hline Sodium & WA & & & & & 5000 & & NS & $\mu \mathrm{g} / \mathrm{L}$ \\
\hline Styrene & WA & & & $\mathbf{U}$ & & 5 & & 100 & $\mu \mathrm{g} / \mathrm{L}$ \\
\hline Sulfate & WA & & & & & 12.9 & & 400 & $\mathrm{mg} / \mathrm{L}$ \\
\hline Sulfide & WA & & & $\mathbf{U}$ & & 0.1 & & NS & $\mathrm{mg} / \mathrm{h}$ \\
\hline Tetrachloroethylene & WA & & & $U$ & & 5 & & 5 & $\mu \mathrm{g} / \mathrm{L}$ \\
\hline Thallium & WA & & & $\mathbf{U}$ & & 10 & & 2 & $\mu g / \mathrm{L}$ \\
\hline Toluene & WA & & & $\mathbf{J}$ & & 1.54 & & 1000 & $\mu g /$ \\
\hline Total hardness & WA & & $\mathbf{A}$ & & & 59 & & NS & $\mathrm{mg} / \mathrm{L}$ \\
\hline Total organic carbon & WA & & & & & 8.57 & & NS & $\mathrm{mg} / \mathrm{L}$ \\
\hline Total suspended solids & WA & & & & & 308 & & NS & $\mathrm{mg} / \mathrm{L}$ \\
\hline trans-1,3-Dichloropropene & WA & & & $\mathbf{U}$ & & 5 & & NS & $\mu \mathrm{g} / \mathrm{L}$ \\
\hline trans-1,4-Dichloro-2-butene & WA & & & $\mathbf{U}$ & & 100 & & NS & $\mu \mathrm{g} \Omega \mathrm{L}$ \\
\hline Trichloroethylene & WA & & & $\mathbf{U}$ & & 5 & & 5 & $\mu \mathrm{g} / \mathrm{L}$ \\
\hline Trichlorofluoromethane & WA & & & $\mathbf{U}$ & & 5 & & NS & $\mu \mathrm{g} / \mathrm{L}$ \\
\hline Tritium & TM & & & & & 21.6 & 2.1 & 20 & $\mathrm{pCi} / \mathrm{ml}$ \\
\hline Turbidity & WA & & & $\mathbf{J}$ & $\mathbf{Q}$ & 270 & & NS & NTU \\
\hline Vanadium & WA & & & $\mathbf{U}$ & & 50 & & NS & $\mu \mathrm{g} / \mathrm{L}$ \\
\hline Vinyl acetate & WA & & & $\mathbf{U}$ & & 10 & & NS & $\mu \mathrm{g} / \mathrm{L}$ \\
\hline Vinyl chloride & WA & & & $\mathbf{U}$ & & 10 & & 2 & $\mu \mathrm{g} / \mathrm{L}$ \\
\hline Xylenes (total) & WA & & & $\mathbf{U}$ & & 5 & & 10000 & $\mu \mathrm{g} / \mathrm{L}$ \\
\hline Zinc & WA & & & & & 32.5 & & 5000 & $\lg / \mathrm{L}$ \\
\hline
\end{tabular}



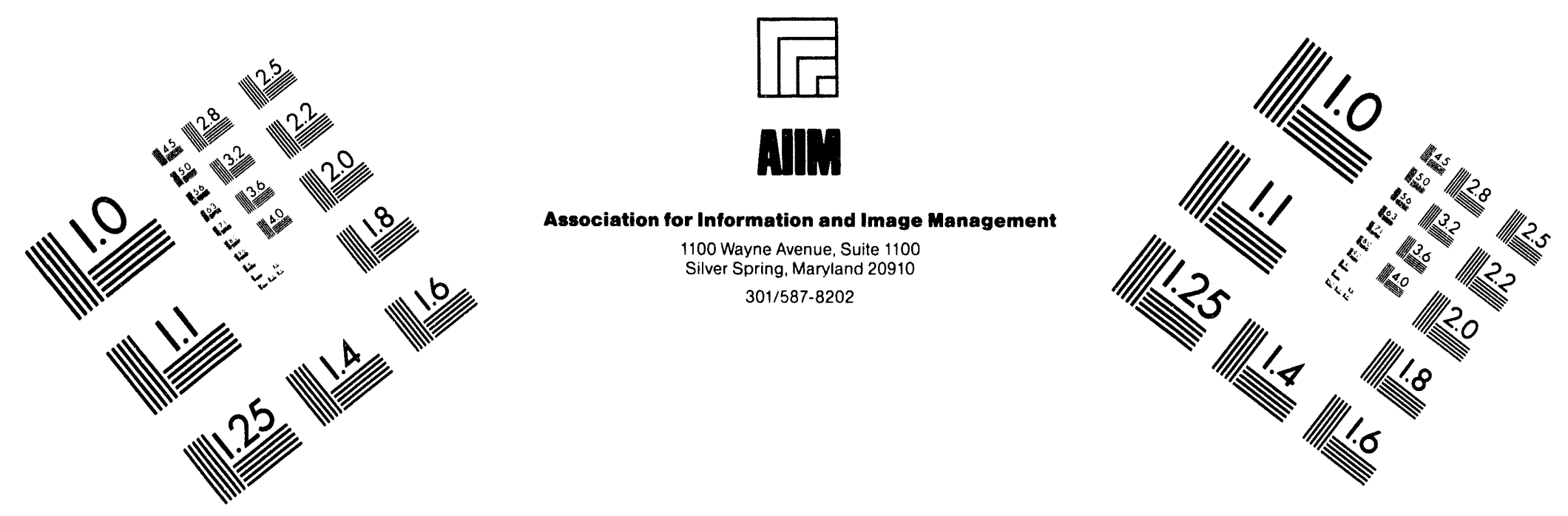

\section{Centimeter}

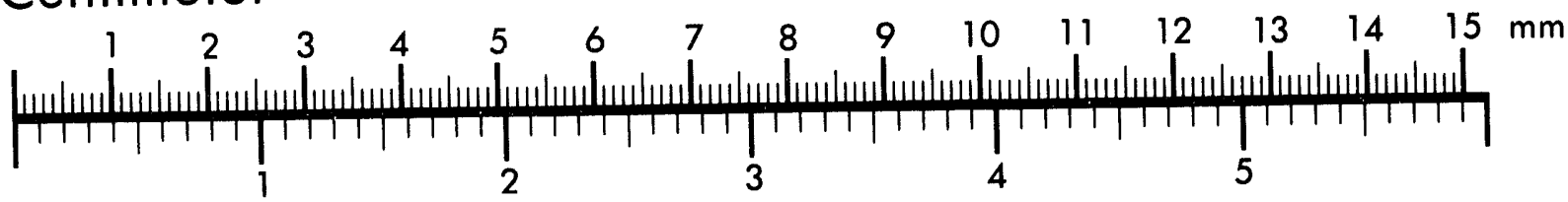
Inches
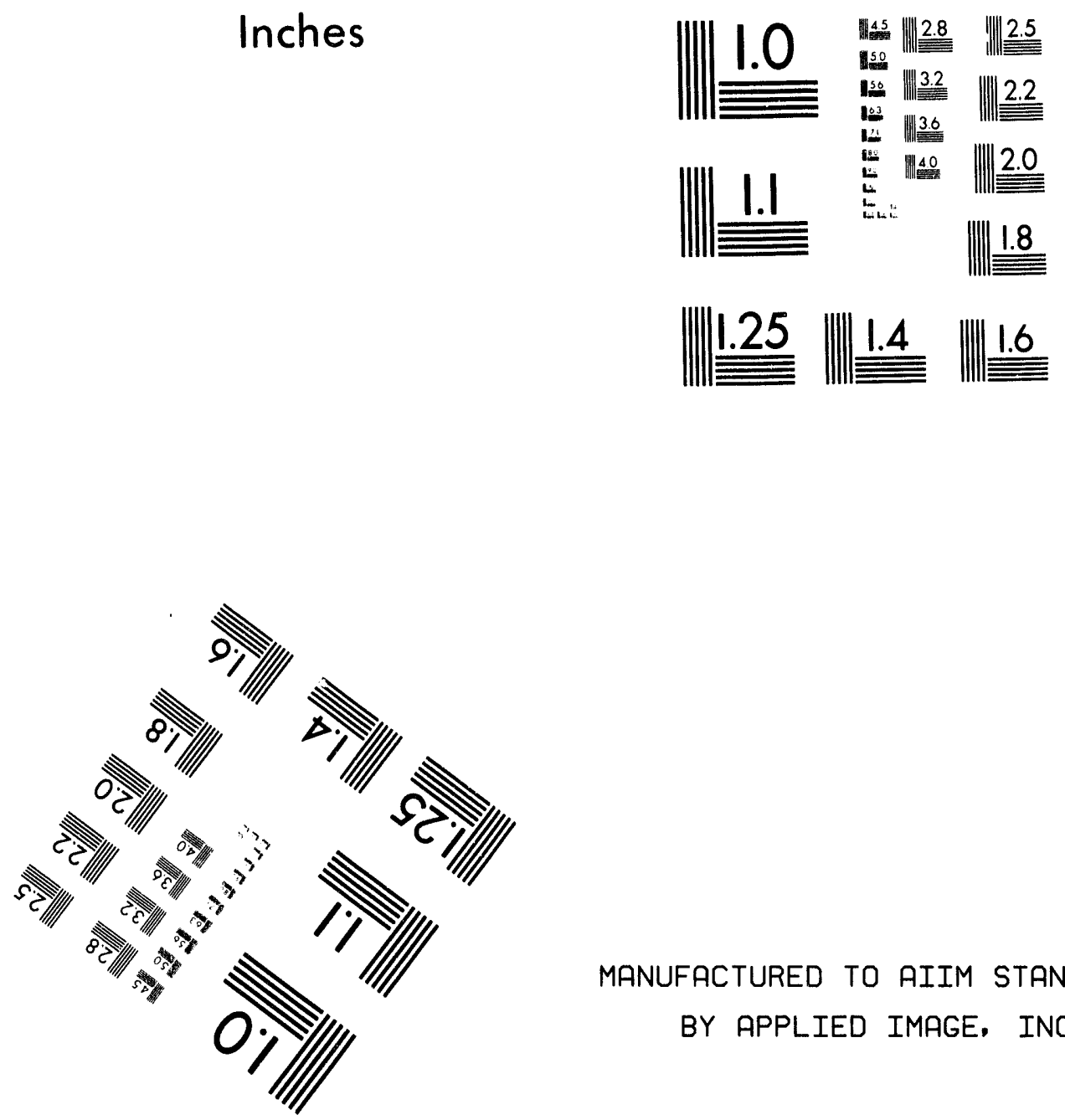

MANUFACTURED TO AIIM STANDARDS BY APPLIED IMAGE, INC.

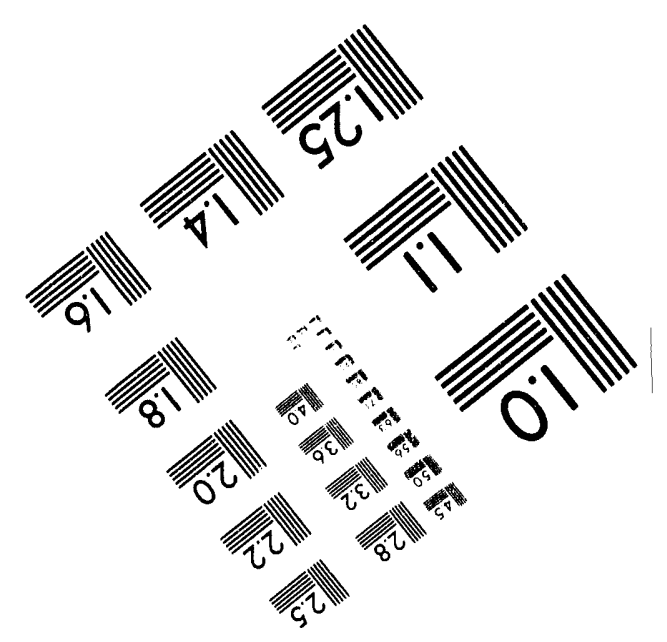



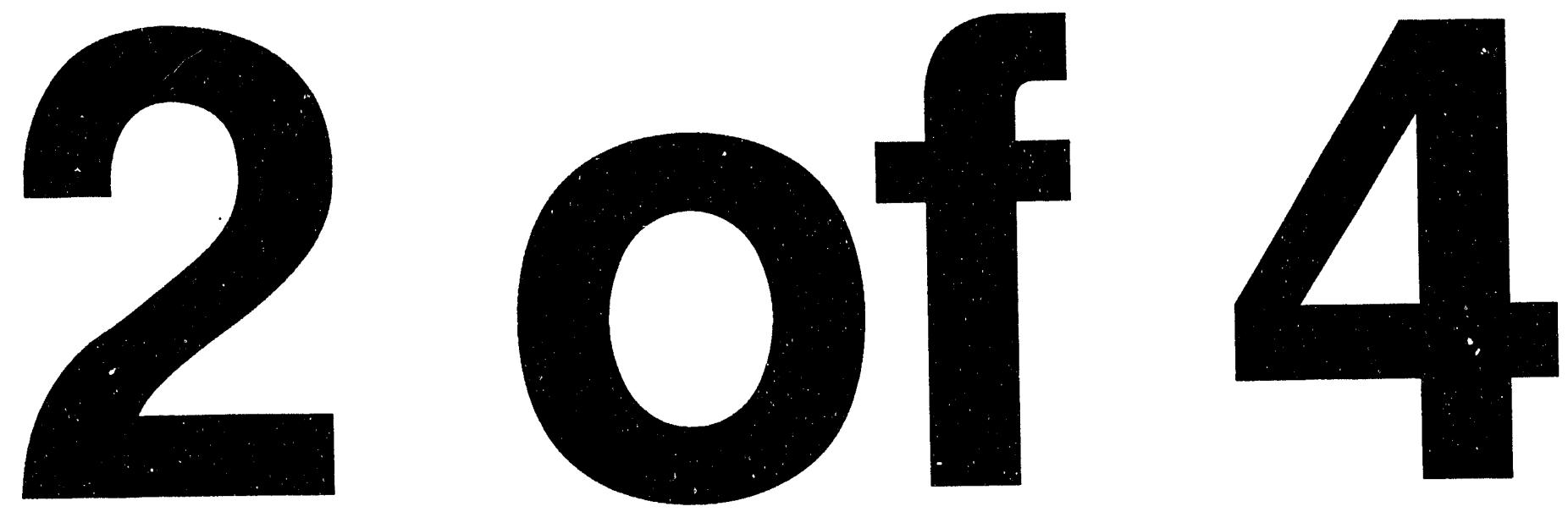


\section{UTR033}

Field Measurements:

Sample Date: 4/14/93

pH: 5.38

Specific Conductance: $22 \mu \mathrm{S} / \mathrm{cm}$

Red/Oxid Potential: $190 \mathrm{mv}$

Dissolved Oxygen: $3.6 \mathrm{mg} / \mathrm{L}$

\begin{tabular}{|c|c|c|c|c|c|c|c|c|}
\hline Analyte & Lab & Rep & Avg & Qualifier & Result & Acc & Stand & Units \\
\hline 1,1,1,2-Tetrachloroethane & WA & & & $\mathrm{U}$ & 10 & & NS & $\mu g / L$ \\
\hline 1,1,1,2-Tetrachloroethane & GE & & & $\mathbf{U}$ & 1 & & NS & $\mu g / L$ \\
\hline $1,1,1,2$-Tetrachloroethane & WA & $\mathbf{R}$ & & $\mathbf{U}$ & 10 & & NS & $\mu g / L$ \\
\hline 1,1,1-Trichloroethane & WA & & & $\mathbf{U}$ & 5 & & 200 & $\mu g / L$ \\
\hline 1,1,1-Trichloroethane & $\mathrm{GE}$ & & & $\mathrm{U}$ & 1 & & 200 & $\mu g / L$ \\
\hline 1,1,1-Trichloroethane & WA & $\mathbf{R}$ & & $\mathbf{U}$ & 5 & & 200 & $\mu \mathrm{g} / \mathrm{L}$ \\
\hline 1,1,2,2-Tetrachloroethane & WA & & & $\mathbf{U}$ & 5 & & NS & $\mu g / L$ \\
\hline 1,1,2,2-Tetrachloroethane & GE & & 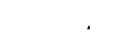 & $\mathbf{U}$ & 1 & & NS & $\mu g / L$ \\
\hline 1,1,2,2-Tetrachloroethane & WA & $\mathbf{R}$ & & $\mathbf{U}$ & 5 & & NS & $\mu g / L$ \\
\hline 1,1,2-Trichloroethane & WA & & & $\mathbf{U}$ & 5 & & 5 & $\mu g / L$ \\
\hline 1,1,2-Trichloroethane & GE & & & $\mathbf{U}$ & 1 & & 5 & $\mu g / L$ \\
\hline 1,1,2-Trichloroethane & WA & $\mathbf{R}$ & & $\mathbf{U}$ & 5 & & 5 & $\mu g / L$ \\
\hline 1,1-Dichloroethane & WA & & & $\mathrm{U}$ & 5 & & NS & $\mu \mathrm{g} / \mathrm{L}$ \\
\hline 1,1-Dichloroethane & $\mathrm{GE}$ & & & $\mathbf{U}$ & 1 & & NS & $\mu g / L$ \\
\hline 1,1-Dichloroethane & WA & $\mathbf{R}$ & & $\mathrm{U}$ & 5 & & NS & $\mu g / L$ \\
\hline 1,1-Dichloroethylene & WA & & & U & 5 & & 7 & $\mu \mathrm{g} / \mathrm{L}$ \\
\hline 1,1-Dichloroethylene & GE & & & $\mathbf{U}$ & 1 & & 7 & $\mu g / L$ \\
\hline 1,1-Dichloroethylene & WA & $\mathbf{R}$ & & $\mathbf{U}$ & 5 & & 7 & $\mu g / L$ \\
\hline 1,2,3-Trichloropropane & WA & & & $U$ & 10 & & NS & $\mu g / L$ \\
\hline 1,2,3-Trichloropropane & GE & & & $U$ & 1 & & NS & $\mu \mathrm{g} / \mathrm{L}$ \\
\hline 1,2,3-Trichloropropane & WA & $\mathbf{R}$ & & $\mathbf{U}$ & 10 & & NS & $\mu g / L$ \\
\hline 1,2-Dibromo-3-chloropropane & WA & & & U & 20 & & 0.2 & $\mu g / L$ \\
\hline 1,2-Dibromo-3-chloropropane & GE & & & $\mathbf{U}$ & 1 & & 0.2 & $\mu g / L$ \\
\hline 1,2-Dibromo-3-chloropropane & WA & $\mathbf{R}$ & & $\mathrm{U}$ & 20 & & 0.2 & $\mu \mathrm{g} / \mathrm{L}$ \\
\hline 1,2-Dibromoethane & WA & & & U & 20 & & NS & $\mu g / L$ \\
\hline 1,2-Dibromoethane & GE & & & $\mathbf{U}$ & 20 & & NS & $\mu g / L$ \\
\hline 1,2-Dibromoethane & WA & $\mathbf{R}$ & & $\mathbf{U}$ & 20 & & NS & $\mu g / L$ \\
\hline 1,2-Dichloroethane & WA & & & $\mathbf{U}$ & 5 & & 5 & $\mu \mathrm{g} / \mathrm{L}$ \\
\hline 1,2-Dichloroethane & GE & & & $\mathbf{U}$ & 1 & & 5 & $\mu \mathrm{g} / \mathrm{L}$ \\
\hline 1,2-Dichloroethane & WA & $\mathbf{R}$ & & $\mathrm{U}$ & 5 & & 5 & $\mu \mathrm{g} / \mathrm{L}$ \\
\hline 1,2-Dichloroethylene (total) & WA & & & $\mathbf{U}$ & 5 & & 100 & $\mu \mathrm{g} / \mathrm{L}$ \\
\hline 1,2-Dichloroethylene (total) & GE & & & $\mathbf{U}$ & 1 & & 100 & $\mu \& L$ \\
\hline 1,2-Dichloroethylene (total) & WA & $\mathbf{R}$ & & $\mathbf{U}$ & 5 & & 100 & $\mu \mathrm{g} / \mathrm{L}$ \\
\hline 1,2-Dichloropropane & WA & & & $\mathbf{U}$ & 5 & & 5 & $\mu g / L$ \\
\hline 1,2-Dichloropropane & GE & & & $\mathbf{U}$ & 1 & & 5 & $\mu \mathrm{g} / \mathrm{L}$ \\
\hline 1,2-Dichloropropane & WA & $\mathbf{R}$ & & $U$ & 5 & & 5 & $\mu \mathrm{g} / \mathrm{L}$ \\
\hline 2-Hexanone & WA & & & $\mathrm{U}$ & 10 & & NS & $\mu \mathrm{g} / \mathrm{L}$ \\
\hline 2-Hexanone & GE & & & $\mathbf{U}$ & 1 & & NS & $\mu g / L$ \\
\hline 2-Hexanone & WA & $\mathbf{R}$ & & $\mathbf{U}$ & 10 & & NS & $\mu \mathrm{g} / \mathrm{L}$ \\
\hline 4-Methyl-2-pentanone & WA & & & $\mathbf{U}$ & 10 & & NS & $\mu g / L$ \\
\hline 4-Methyl-2-pentanone & WA & $\mathbf{R}$ & & $\mathrm{U}$ & 10 & & NS & $\mu \mathrm{g} / \mathrm{L}$ \\
\hline Acetone & WA & & & $\mathbf{U}$ & 10 & & NS & $\mu \mathrm{g} / \mathrm{L}$ \\
\hline Acetone & GE & & & $\mathbf{U}$ & 100 & & NS & $\mu \mathrm{g} / \mathrm{L}$ \\
\hline Acetone & WA & $\mathbf{R}$ & & & 20.8 & & NS & $\mu g / L$ \\
\hline Acetonitrile & WA & & & $\mathbf{U}$ & 20 & & NS & $\mu \mathrm{g} / \mathrm{L}$ \\
\hline Acetonitrile & GE & & & $\mathbf{U}$ & 1 & & NS & $\mu \mathrm{g} / \mathrm{L}$ \\
\hline Acetonitrile & WA & $\mathbf{R}$ & & $\mathbf{U}$ & 20 & & NS & $\mu g / L$ \\
\hline Acidity & WA & & & & 22 & & NS & $\mathrm{mg} / \mathrm{L}$ \\
\hline Acidity & WA & $\mathbf{R}$ & A & & 43 & & NS & $\mathrm{mg} / \mathrm{L}$ \\
\hline
\end{tabular}


UTR033 Laboratory Analyses (cont'd)

\begin{tabular}{|c|c|c|c|c|c|c|c|c|}
\hline Analyte & Lab & Rep & Avg & Qualifier & Result & Acc & Stand & Units \\
\hline Acrolein & WA & & & $\overline{\mathbf{U}}$ & 10 & & NS & $\overline{\mu g / L}$ \\
\hline Acrolein & GE & & & $\mathbf{U}$ & 20 & & NS & $\mu \mathrm{g} / \mathrm{L}$ \\
\hline Acrolein & WA & $\mathbf{R}$ & & $\mathbf{U}$ & 10 & & NS & $\mu \mathrm{g} / \mathrm{L}$ \\
\hline Acrylonitrile & WA & & & $\mathbf{U}$ & 10 & & NS & $\mu \mathrm{g} / \mathrm{L}$ \\
\hline Acrylonitrile & GE & & & $\mathbf{U}$ & 20 & & NŚ & $\mu \mathrm{g} / \mathrm{L}$ \\
\hline Acrylonitrile & WA & $\mathbf{R}$ & & $\mathbf{U}$ & 10 & . & NS & $\mu g / L$ \\
\hline Actinium-228 & EP & & & $\mathbf{U}$ & 30 & & NS & $\mathrm{pCi} / \mathrm{L}$ \\
\hline Actinium-228 & EP & & NA & $\mathbf{U}$ & 30 & & NS & $\mathrm{pCi} / \mathrm{L}$ \\
\hline Alkalinity & WA & & & & 1.25 & & NS & $\mathrm{mg} / \mathrm{L}$ \\
\hline Alkalinity & GE & & & $\mathbf{U}$ & 1 & & NS & $\mathrm{mg} / \mathrm{L}$ \\
\hline Alkalinity & WA & $\mathbf{R}$ & & & 1 & & NS & $\mathrm{mg} / \mathrm{L}$ \\
\hline Allyl chloride & WA & & & $\mathbf{U}$ & 100 & & NS & $\mu \mathrm{g} / \mathrm{L}$ \\
\hline Allyl chloride & GE & & & $U$ & 50 & & NS & $\mu g / L$ \\
\hline Allyl chloride & WA & $\mathbf{R}$ & & $\mathbf{U}$ & 100 & & NS & $\mu \mathrm{g} / \mathrm{L}$ \\
\hline Aluminum & WA & & & U & 200 & & NS & $\mu g / L$ \\
\hline Aluminum & GE & & & & 75.8 & & NS & $\mu g / L$ \\
\hline Aluminum & WA & $\mathbf{R}$ & & $\mathbf{U}$ & 200 & & NS & $\mu g / L$ \\
\hline Antimony & WA & & & $\mathbf{U}$ & 60 & & 6 & $\mu \mathrm{g} / \mathrm{L}$ \\
\hline Antimony & GE & & & $\mathbf{U}$ & 2 & & 6 & $\mu g / L$ \\
\hline Antimony & WA & $\mathbf{R}$ & & $\mathbf{U}$ & 60 & & 6 & $\mu g / L$ \\
\hline Antimony-125 & EP & & & $\mathbf{U}$ & 20 & & 300 & $\mathrm{pCi} / \mathrm{L}$ \\
\hline Antimony-125 & EP & & NA & $\mathbf{U}$ & 20 & & 300 & $\mathrm{pCi} / \mathrm{L}$ \\
\hline Arsenic & WA & & & $\mathbf{U}$ & 10 & & 50 & $\mu \mathrm{g} / \mathrm{L}$ \\
\hline Arsenic & GE & & & $\mathbf{U}$ & 2 & & 50 & $\mu g / L$ \\
\hline Arsenic & WA & $\mathbf{R}$ & & U & 10 & & 50 & $\mu g / L$ \\
\hline Barium & WA & & & $\mathbf{U}$ & 200 & & 2000 & $\mu g / L$ \\
\hline Barium & GE & & & & 6.1 & & 2000 & $\mu g / L$ \\
\hline Barium & WA & $\mathbf{R}$ & & $\mathbf{U}$ & 200 & & 2000 & $\mu \mathrm{g} / \mathrm{L}$ \\
\hline Benzene & WA & & & $\mathbf{U}$ & 5 & & 5 & $\mu g / L$ \\
\hline Benzene & GE & & & $\mathbf{U}$ & 1 & & 5 & $\mu g / L$ \\
\hline Benzene & WA & $\mathbf{R}$ & & $\mathbf{U}$ & 5 & & 5 & $\mu \mathrm{g} / \mathrm{L}$ \\
\hline Beryllium & WA & & & $\mathbf{U}$ & 5 & & 4 & $\mu g / L$ \\
\hline Beryllium & GE & & & $\mathbf{U}$ & 3 & & 4 & $\mu g / L$ \\
\hline Beryllium & WA & $\mathbf{R}$ & & $\mathbf{U}$ & 5 & & 4 & $\mu g / L$ \\
\hline Bis(2-chloro-1-methylethyl)ether & GE & & & $\mathbf{U}$ & 10 & & NS & $\mu g / L$ \\
\hline Bromodichloromethane & WA & & & $\mathbf{U}$ & 5 & & NS & $\mu g / L$ \\
\hline Bromodichloromethane & GE & & & $\mathrm{U}$ & 1 & & NS & $\mu g / L$ \\
\hline Bromodichloromethane & WA & $\mathbf{R}$ & & $\mathbf{U}$ & 5 & & NS & $\mu g / L$ \\
\hline Bromoform & WA & & & $\mathbf{U}$ & 5 & & NS & $\mu g / L$ \\
\hline Bromoform & GE & & & $\mathbf{U}$ & 1 & & NS & $\mu g / L$ \\
\hline Bromoform & WA & $\mathbf{R}$ & & $\mathbf{U}$ & 5 & & NS & $\mu \mathrm{g} / \mathrm{L}$ \\
\hline Cadmium & WA & & & $\mathbf{U}$ & 5 & & 5 & $\mu g / L$ \\
\hline Cadmium & GE & & & $\mathbf{U}$ & 2 & & 5 & $\mu g / L$ \\
\hline Cadmium & WA & $\mathbf{R}$ & & $\mathrm{U}$ & 5 & & 5 & $\mu g / L$ \\
\hline Calcium & WA & & & U & 5000 & & NS & $\mu \mathrm{g} / \mathrm{L}$ \\
\hline Calcium & GE & & & & 284 & & NS & $\mu \mathrm{g} / \mathrm{L}$ \\
\hline Calcium & WA & $\mathbf{R}$ & & $\mathbf{U}$ & 5000 & & NS & $\mu \mathrm{g} / \mathrm{L}$ \\
\hline Carbon disulfide & WA & & & $\mathrm{U}$ & 5 & & NS & $\mu \mathrm{g} / \mathrm{L}$ \\
\hline Carbon disulfide & GE & & & $\mathbf{U}$ & 1 & & NS & $\mu g / L$ \\
\hline Carbon disulfide & WA & $\mathbf{R}$ & & $\mathbf{U}$ & 5 & & NS & $\mu g / L$ \\
\hline Carbon tetrachloride & WA & & & $\mathbf{U}$ & 5 & & 5 & $\mu g / L$ \\
\hline Carbon tetrachloride & GE & & & $\mathrm{U}$ & 1 & & 5 & $\mu \mathrm{g} / \mathrm{L}$ \\
\hline Carbon tetrachloride & WA & $\mathbf{R}$ & & $\mathbf{U}$ & 5 & & 5 & $\mu g / L$ \\
\hline Cerium-144 & EP & & & $\mathbf{U}$ & 60 & & 261 & $\mathrm{pCi} / \mathrm{L}$ \\
\hline Cerium-144 & EP & & NA & $\mathrm{U}$ & 60 & & 261 & $\mathrm{pCi} / \mathrm{L}$ \\
\hline
\end{tabular}


UTR033 Laboratory Analyses (cont'd)

\begin{tabular}{|c|c|c|c|c|c|c|c|c|}
\hline Analyte & Lab & Rep & Avg & Qualifier & Result & Acc & Stand & Units \\
\hline Cesium-134 & EP & & & $\mathbf{U}$ & 10 & & 81.3 & $\mathrm{pCi} / \mathrm{L}$ \\
\hline Cesium-134 & EP & & NA & & 10 & & 81.3 & $\mathrm{pCi} / \mathrm{L}$ \\
\hline Cesium-137 & TM & & & & 5.4 & 4.6 & 200 & $\mathrm{pCi} / \mathrm{L}$ \\
\hline Cesium-137 & EP & & & $\mathrm{U}$ & 10 & & 200 & $\mathrm{pCi} / \mathrm{L}$ \\
\hline Cesium-137 & EP & & NA & $\mathrm{U}$ & 10 & & 200 & $\mathrm{pCi} / \mathrm{L}$ \\
\hline Cesium-137 & TM & $\mathbf{R}$ & & $\mathrm{U}$ & 9.4 & & 200 & $\mathrm{pCi} / \mathrm{L}$ \\
\hline Chloride & WA & & & & 3.51 & & NS & $\mathrm{mg} / \mathrm{L}$ \\
\hline Chloride & GE & & $\mathbf{A}$ & & 1.91 & & NS & $\mathrm{mg} / \mathrm{L}$ \\
\hline Chloride & WA & $\mathbf{R}$ & & & 4.6 & & NS & $\mathrm{mg} / \mathrm{L}$ \\
\hline Chlorobenzene & WA & & & $\mathbf{U}$ & 5 & & 100 & $\mu \mathrm{g} / \mathrm{L}$ \\
\hline Chlorobenzene & GE & & & $\mathbf{U}$ & 1 & & 100 & $\mu g / L$ \\
\hline Chlorobenzene & WA & $\mathbf{R}$ & & U & 5 & & 100 & $\mu g / L$ \\
\hline Chloroethane & WA & & & $\mathbf{U}$ & 10 & & NS & $\mu \mathrm{g} / \mathrm{L}$ \\
\hline Chloroethane & GE & & & U & 1 & & NS & $\mu \mathrm{g} / \mathrm{L}$ \\
\hline Chloroethane & WA & $\mathbf{R}$ & & $\mathbf{U}$ & 10 & & NS & $\mu \mathrm{g} / \mathrm{L}$ \\
\hline Chloroform & WA & & & $\mathbf{U}$ & 5 & & 100 & $\mu g / L$ \\
\hline Chloroform & GE & & & $\mathrm{U}$ & 1 & & 100 & $\mu \mathrm{g} / \mathrm{L}$ \\
\hline Chloroform & WA & $\mathbf{R}$ & & U & 5 & & 100 & $\mu \mathrm{g} / \mathrm{L}$ \\
\hline Chloroprene & WA & & & $\mathbf{U}$ & 20 & & NS & $\mu g / L$ \\
\hline Chloroprene & GE & & & $\mathbf{U}$ & 200 & & NS & $\mu \mathrm{g} / \mathrm{L}$ \\
\hline Chloroprene & WA & $\mathbf{R}$ & & $\mathbf{U}$ & 20 & & NS & $\mu g / L$ \\
\hline Chromium & WA & & & $\mathbf{U}$ & 10 & & 100 & $\mu g / L$ \\
\hline Chromium & GE & & & $\mathbf{U}$ & 4 & & 100 & $\mu \mathrm{g} / \mathrm{L}$ \\
\hline Chromium & WA & $\mathbf{R}$ & & $\mathrm{U}$ & 10 & & 100 & $\mu \mathrm{g} / \mathrm{L}$ \\
\hline cis-1,3-Dichloropropene & WA & & & $\mathrm{U}$ & 5 & & NS & $\mu \mathrm{g} / \mathrm{L}$ \\
\hline cis-1,3-Dichloropropene & GE & & & $\mathrm{U}$ & 1 & & NS & $\mu g / L$ \\
\hline cis-1,3-Dichloropropene & WA & $\mathbf{R}$ & & $\mathrm{U}$ & 5 & & & $\mu \mathrm{g} / \mathrm{L}$ \\
\hline $\begin{array}{l}\text { Cobalt } \\
\text { Cobalt }\end{array}$ & WA & & & $\mathrm{U}$ & 50 & & $\begin{array}{l}\text { NS } \\
\text { NS }\end{array}$ & $\mu g / L$ \\
\hline & $\begin{array}{l}\text { GE } \\
\text { WA }\end{array}$ & & & $\begin{array}{l}U \\
U\end{array}$ & $\begin{array}{l}4 \\
50\end{array}$ & & $\begin{array}{l}\text { NS } \\
\text { NS }\end{array}$ & $\begin{array}{l}\mu g / L \\
\mu g / L\end{array}$ \\
\hline $\begin{array}{l}\text { Cobalt } \\
\text { Cobalt-57 }\end{array}$ & $\begin{array}{l}\text { WA } \\
\text { EP }\end{array}$ & $\mathbf{R}$ & & U & 10 & & 1000 & $\begin{array}{l}\mu \mathrm{g} / \mathrm{L} \\
\mathrm{pCi} / \mathrm{L}\end{array}$ \\
\hline Cobalt-57 & $\mathrm{EP}$ & & NA & $\mathbf{U}$ & 10 & & 1000 & $\mathrm{pCi} / \mathrm{L}$ \\
\hline Cobalt -60 & $\mathbf{E P}$ & & & $U$ & 10 & & 100 & $\mathrm{pCi} / \mathrm{L}$ \\
\hline Cobalt-60 & EP & & NA & $\mathrm{U}$ & 10 & & 100 & $\mathrm{pCi} / \mathrm{L}$ \\
\hline Copper & WA & & & $\mathrm{U}$ & 25 & & 1000 & $\mu g / L$ \\
\hline Copper & GE & & & $\mathbf{U}$ & 4 & & 1000 & $\mu \mathrm{g} / \mathrm{L}$ \\
\hline Copper & WA & $\mathbf{R}$ & & $\mathrm{U}$ & 25 & & 1000 & $\mu \mathrm{g} / \mathrm{L}$ \\
\hline Cyanide & WA & & & $\mathrm{U}$ & 10 & & 200 & $\mu \mathrm{g} / \mathrm{L}$ \\
\hline Cyanide & GE & & & $\mathrm{U}$ & 5 & & 200 & $\mu g / L$ \\
\hline Cyanide & WA & $\mathbf{R}$ & & U & 10 & & 200 & $\mu \mathrm{g} / \mathrm{L}$ \\
\hline Dibromochloromethane & WA & & & $\mathbf{U}$ & 5 & & NS & $\mu \mathrm{g} / \mathrm{L}$ \\
\hline Vibromochloromethane & $\mathrm{GE}$ & & & $\mathrm{U}$ & 1 & & NS & $\mu \mathrm{g} / \mathrm{L}$ \\
\hline Dibromochloromethane & WA & $\mathbf{R}$ & & $\mathbf{U}$ & 5 & & NS & $\mu \mathrm{g} / \mathrm{L}$ \\
\hline Dichlorodifluoromethane & WA & & & $\mathbf{U}$ & 10 & & NS & $\mu g / L$ \\
\hline Dichlorodifluoromethane & GE & & & U & 1 & & NS & $\mu g / L$ \\
\hline Dichlorodifluoromethane & WA & $\mathbf{R}$ & & $\mathrm{U}$ & 10 & & NS & $\mu g / L$ \\
\hline Ethylbenzene & WA & & & $\mathrm{U}$ & 5 & & 700 & $\mu g / L$ \\
\hline Ethylbenzene & GE & & & $\mathbf{U}$ & 1 & & 700 & $\mu \mathrm{g} / \mathrm{L}$ \\
\hline Ethylbenzene & WA & $\mathbf{R}$ & & $\mathrm{U}$ & 5 & & 700 & $\mu \mathrm{g} / \mathrm{L}$ \\
\hline Europium-152 & EP & & & $\mathrm{U}$ & 40 & & NS & $\mathrm{pCi} / \mathrm{L}$ \\
\hline Europium-152 & EP & & NA & $\mathbf{U}$ & 40 & & NS & $\mathrm{pCi} / \mathrm{L}$ \\
\hline Europium-154 & EP & & & $\mathbf{U}$ & 20 & & 200 & $\mathrm{pCi} / \mathrm{L}$ \\
\hline Europium-154 & EP & & NA & $\mathrm{U}$ & 20 & & 200 & $\mathrm{pCi} / \mathrm{L}$ \\
\hline Europium-155 & $\mathrm{EP}$ & & & U & 30 & & 600 & $\mathrm{pCi} / / \mathrm{L}$ \\
\hline Europium-155 & EP & & NA & $\mathbf{U}$ & 30 & & 600 & $\mathrm{pCi} / \mathrm{L}$ \\
\hline
\end{tabular}


UTR033 Laboratory Analyses (cont'd)

\begin{tabular}{|c|c|c|c|c|c|c|c|c|}
\hline Analyte & Lab & Rep & Avg & Qualifier & Result & Acc & Stand & Units \\
\hline Fluoride & $\overline{W A}$ & & & $\overline{\mathrm{U}}$ & 0.1 & & 4 & $\overline{m g / h}$ \\
\hline Fluoride & WA & & NA & $\mathbf{U}$ & 0.1 & & 4 & $\mathrm{mg} / \mathrm{L}$ \\
\hline Fluoride & GE & & & $\mathbf{U}$ & 0.1 & & 4 & $\mathrm{mg} / \mathrm{L}$ \\
\hline Fluoride & WA & $\mathbf{R}$ & & $\mathbf{U}$ & 0.1 & & 4 & $\mathrm{mg} / \mathrm{L}$ \\
\hline Gross alpha & TM & & & & 0.6 & 0.7 & $15^{2}$ & $\mathrm{pCi} / \mathrm{L}$ \\
\hline Gross alpha & GE & & & $\mathbf{U}$ & 2 & & 15 & $\mathrm{pCi} / \mathrm{L}$ \\
\hline Gross alpha & TM & $\mathbf{R}$ & & & 1 & 0.9 & 15 & $\mathrm{pCi} / \mathrm{L}$ \\
\hline Iron & WA & & & & 162 & & 300 & $\mu g / L$ \\
\hline Iron & GE & & & & 67.6 & & 300 & $\mu \mathrm{g} / \mathrm{L}$ \\
\hline Iron & WA & $\mathbf{R}$ & & U & 100 & & 300 & $\mu \mathrm{g} / \mathrm{L}$ \\
\hline Isobutyl alcohol & WA & & & $\mathbf{U}$ & 20 & & NS & $\mu \mathrm{g} / \mathrm{L}$ \\
\hline Isobutyl alcohol & GE & & & U & 100 & & NS & $\mu \mathrm{g} / \mathrm{L}$ \\
\hline Isobutyl alcohol & WA & $\mathbf{R}$ & & $\mathbf{U}$ & 20 & & NS & $\mu g / L$ \\
\hline Lead & WA & & & $\mathbf{U}$ & 3 & & 50 & $\mu g / L$ \\
\hline Lead & GE & & & $\mathbf{U}$ & 3 & & 50 & $\mu g / L$ \\
\hline Lead & WA & $\mathbf{R}$ & & $\mathbf{U}$ & 3 & & 50 & $\mu g / L$ \\
\hline Lead-212 & EP & & & $\mathbf{U}$ & 15 & & NS & pCi $/ L$ \\
\hline Lead-212 & EP & & NA & $\mathbf{U}$ & 15 & & NS & $\mathrm{pCi} / \mathrm{L}$ \\
\hline Magnesium & WA & & & $\mathbf{U}$ & 5000 & & NS & $\mu g / L$ \\
\hline Magnesium & GE & & & & 766 & & NS & $\mu \mathrm{g} / \mathrm{L}$ \\
\hline Magnesium & WA & $\mathbf{R}$ & & $\mathbf{U}$ & 5000 & & NS & $\mu \mathrm{g} / \mathrm{L}$ \\
\hline Manganese & WA & & & & 16 & & 50 & $\mu g / L$ \\
\hline Manganese & GE & & & & 9.97 & & 50 & $\mu g / L$ \\
\hline Manganese & WA & $\mathbf{R}$ & & $\mathbf{U}$ & 15 & & 50 & $\mu \mathrm{g} / \mathrm{L}$ \\
\hline Manganese-54 & $\mathrm{EP}$ & & & $\mathbf{U}$ & 10 & & 300 & $\mathrm{pCi} / \mathrm{L}$ \\
\hline Manganese-54 & EP & & NA & $\mathbf{U}$ & 10 & & 300 & $\mathrm{pCi} / \mathrm{L}$ \\
\hline Mercury & WA & & & U & 0.2 & & 2 & $\mu \mathrm{g} / \mathrm{L}$ \\
\hline Mercury & $\mathrm{GE}$ & & & $\mathbf{U}$ & 0.2 & & 2 & $\mu g / L$ \\
\hline Mercury & WA & $\mathbf{R}$ & & $\mathbf{U}$ & 0.2 & & 2 & $\mu g / L$ \\
\hline Methacrylonitrile & WA & & & $\mathbf{U}$ & 20 & & NS & $\mu g / L$ \\
\hline Methacrylonitrile & GE & & & U & 50 & & NS & $\mu \mathrm{g} / \mathrm{L}$ \\
\hline Methacrylonitrile & WA & $\mathbf{R}$ & & $\mathrm{U}$ & 20 & & NS & $\mu g / L$ \\
\hline Methyl bromide & WA & & & $\mathbf{U}$ & 10 & & NS & $\mu \mathrm{g} / \mathrm{L}$ \\
\hline Methyl bromide & GE & & & $\mathbf{U}$ & 1 & & NS & $\mu g / L$ \\
\hline Methyl bromide & WA & $\mathbf{R}$ & & $\mathbf{U}$ & 10 & & NS & $\mu g \Omega$ \\
\hline Methyl chloride & WA & & & U & 10 & & NS & $\mu \mathrm{g} / \mathrm{L}$ \\
\hline Methyl chloride & $\mathrm{GE}$ & & & $\mathbf{U}$ & 1 & & NS & $\mu g / L$ \\
\hline Methyl chloride & WA & $\mathbf{R}$ & & $\mathbf{U}$ & 10 & & NS & $\mu g / L$ \\
\hline Methyl ethyl ketone & WA & & & $U$ & 10 & & NS & $\mu g / L$ \\
\hline Methyl ethyl ketone & $\mathrm{GE}$ & & & U & 1 & & NS & $\mu g / L$ \\
\hline Methyl ethyl ketone & WA & $\mathbf{R}$ & & $\mathbf{U}$ & 10 & & NS & $\mu g / L$ \\
\hline Methyl iodide & WA & & & $\mathbf{U}$ & 10 & & NS & $\mu g / L$ \\
\hline Methyl iodide & GE & & & $\mathbf{U}$ & 15 & & NS & $\mu g / L$ \\
\hline Methyl iodide & WA & $\mathbf{R}$ & & $\mathbf{U}$ & 10 & & NS & $\mu g / L$ \\
\hline Methyl isobutyl ketone & GE & & & U & 1 & & NS & $\mu \mathrm{g} / \mathrm{L}$ \\
\hline Methylene bromide & WA & & & $\mathrm{U}$ & 10 & & NS & $\mu g / L$ \\
\hline Methylene bromide & $\mathrm{GE}$ & & & $\mathbf{U}$ & 1 & & NS & $\mu \mathrm{g} / \mathrm{L}$ \\
\hline Methylene bromide & WA & $\mathbf{R}$ & & $\mathbf{U}$ & 10 & & NS & $\mu g / L$ \\
\hline Methylene chloride & WA & & & $\mathrm{U}$ & 5 & & 5 & $\mu g / L$ \\
\hline Methylene chloride & GE & & & $\mathbf{U}$ & 1 & & 5 & $\mu g /$ \\
\hline Methylene chloride & WA & $\mathbf{R}$ & & $\mathbf{U}$ & 5 & & 5 & $\mu g / L$ \\
\hline Nickel & WA & & & $\mathbf{U}$ & 40 & & 100 & $\mu g / L$ \\
\hline Nickel & GE & & & $\mathbf{U}$ & 4 & & 100 & $\mu \mathrm{g} /$ \\
\hline Nickel & WA & $\mathbf{R}$ & & $\mathbf{U}$ & 40 & & 100 & 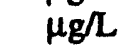 \\
\hline
\end{tabular}


UTR033 Laboratory Analyses (cont'd)

\begin{tabular}{|c|c|c|c|c|c|c|c|c|c|}
\hline Analyte & Lab & Rep & Avg & Qualifier & & Result & Acc & Stand & Units \\
\hline Nitrate as nitrogen & WA & & & & & 0.987 & & 10 & $\mathrm{mg} / \mathrm{L}$ \\
\hline Nitrate as nitrogen & GE & & & & & 2.58 & & 10 & $\mathrm{mg} / \mathrm{L}$ \\
\hline Nitrate as nitrogen & WA & $\mathbf{R}$ & A & & & 0.3215 & & 10 & $\mathrm{mg} / \mathrm{L}$ \\
\hline Nonvolatile beta & TM & & & U & & 0.7 & & 50 & $\mathrm{pCi} / \mathrm{L}$ \\
\hline Nonvolatile beta & $\mathrm{GE}$ & & & $U$ & & 2 & & so & $\mathrm{pCi} / \mathrm{L}$ \\
\hline Nonvolatile beta & TM & $\mathbf{R}$ & & & & 1 & 1.5 & 50 & $\mathrm{pCi} / \mathrm{L}$ \\
\hline pH & GE & & & $\mathbf{J}$ & $\mathbf{Q}$ & 4.82 & & NS & PH \\
\hline Potassium & WA & & & $\mathbf{U}$ & & 5000 & & NS & $\mu g / L$ \\
\hline Potassium & GE & & & $\mathrm{U}$ & & 500 & & NS & $\mu \mathrm{g} / \mathrm{L}$ \\
\hline Potassium & WA & $\mathbf{R}$ & & $\mathbf{U}$ & & 5000 & & NS & $\mu \mathrm{g} / \mathrm{h}$ \\
\hline Potassium-40 & EP & & & $\mathbf{U}$ & & 110 & & 300 & $\mathrm{pCi} / \mathrm{L}$ \\
\hline Potassium-40 & EP & & NA & $\mathrm{U}$ & & 110 & & 300 & $\mathrm{pCi} / \mathrm{L}$ \\
\hline Promethium-144 & EP & & & $\mathbf{U}$ & & 10 & & NS & $\mathrm{pCi} / \mathrm{L}$ \\
\hline Promethium-144 & EP & & NA & $\mathbf{U}$ & & 10 & & NS & $\mathrm{pCi} / \mathrm{L}$ \\
\hline Promethium-146 & EP & & & $\mathbf{U}$ & & 10 & & NS & $\mathrm{pCi} / \mathrm{L}$ \\
\hline Promethium-146 & EP & & NA & $\mathbf{U}$ & & 10 & & NS & $\mathrm{pCi} / \mathrm{L}$ \\
\hline Propionitrile & WA & & & U & & 50 & & NS & $\mu g / L$ \\
\hline Propionitrile & GE & & & $\mathbf{U}$ & & 200 & & NS & $\mu g / L$ \\
\hline Propionitrile & WA & $\mathbf{R}$ & & $\mathbf{U}$ & & 50 & & NS & $\mu g / L$ \\
\hline Ruthenium-106 & EP & & & $\mathbf{U}$ & & 90 & & 30 & $\mathrm{pCi} / \mathrm{L}$ \\
\hline Ruthenium-106 & EP & & NA & U & & 90 & & 30 & $\mathrm{pCi} / \mathrm{L}$ \\
\hline Selenium & WA & & & $\mathbf{U}$ & & 5 & & 50 & $\mu g / L$ \\
\hline Selenium & GE & & & UJ & $\mathrm{L}$ & 2 & & 50 & $\mu g / L$ \\
\hline Selenium & WA & $\mathbf{R}$ & & $\mathbf{U}$ & & 5 & & 50 & $\mu \mathrm{g} / \mathrm{L}$ \\
\hline Silver & WA & & & $\mathbf{U}$ & & 10 & & 50 & $\mu g / L$ \\
\hline Silver & GE & & & $\mathbf{U}$ & & 2 & & 50 & $\mu \mathrm{g} / \mathrm{L}$ \\
\hline Silver & WA & $\mathbf{R}$ & & $\mathbf{U}$ & & 10 & & 50 & $\mu g / L$ \\
\hline Sodium & WA & & & $\mathbf{U}$ & & 5000 & & NS & $\mu g / L$ \\
\hline Sodium & GE & & & & & 1560 & & NS & $\mu g / L$ \\
\hline Sodium & WA & $\mathbf{R}$ & & $\mathbf{U}$ & & 5000 & & NS & $\mu g / L$ \\
\hline Sodium-22 & EP & & & $\mathbf{U}$ & & 10 & & 466 & $\mathrm{pCi} / \mathrm{L}$ \\
\hline Sodium-22 & EP & & NA & $\mathbf{U}$ & & 10 & & 466 & $\mathrm{pCi} / \mathrm{L}$ \\
\hline Styrene & WA & & & $\mathbf{U}$ & & 5 & & 100 & $\mu g / L$ \\
\hline Styrene & GE & & & $\mathbf{U}$ & & 1 & & 100 & $\mu \mathrm{g} / \mathrm{L}$ \\
\hline Styrene & WA & $\mathbf{R}$ & & $\mathrm{U}$ & & 5 & & 100 & $\mu \mathrm{g} / \mathrm{L}$ \\
\hline Sulfate & WA & & & $\mathbf{U}$ & & 2.5 & & 400 & $\mathrm{mg} / \mathrm{L}$ \\
\hline Sulfate & GE & & NA & $\mathbf{U}$ & & 1 & & 400 & $\mathrm{mg} / \mathrm{L}$ \\
\hline Sulfate & GE & & & $\mathbf{U}$ & & 1 & & 400 & $\mathrm{mg} / \mathrm{L}$ \\
\hline Sulfate & WA & $\mathbf{R}$ & & $\mathbf{U}$ & & 2.5 & & 400 & $\mathrm{mg} / \mathrm{L}$ \\
\hline Sulfide & WA & & & $\mathbf{U}$ & & 0.1 & & NS & $\mathrm{mg} / \mathrm{L}$ \\
\hline Sulfide & GE & & & $\mathbf{U}$ & & 1 & & NS & $\mathrm{mg} / \mathrm{L}$ \\
\hline Sulfide & WA & $\mathbf{R}$ & & $\mathbf{U}$ & & 0.1 & & NS & $\mathrm{mg} / \mathrm{L}$ \\
\hline Tetrachloroethylene & WA & & & $\mathrm{U}$ & & 5 & & 5 & $\mu g / L$ \\
\hline Tetrachloroethylene & GE & & & U & & 1 & & 5 & $\mu \mathrm{g} / \mathrm{L}$ \\
\hline Tetrachloroethylene & WA & $\mathbf{R}$ & & $\mathbf{U}$ & & 5 & & 5 & $\mu \mathrm{g} / \mathrm{L}$ \\
\hline Thallium & WA & & & $\mathrm{U}$ & & 10 & & 2 & $\mu \mathrm{g} / \mathrm{L}$ \\
\hline Thallium & GE & & & U & & 2 & & 2 & $\mu \mathrm{g} / \mathrm{L}$ \\
\hline Thallium & WA & $\mathbf{R}$ & & U & & 10 & & 2 & $\mu g / L$ \\
\hline Thorium-234 & EP & & & $\mathrm{U}$ & & 350 & & 401 & $\mathrm{pCi} / \mathrm{L}$ \\
\hline Thorium-234 & EP & & NA & $\mathrm{U}$ & & 350 & & 401 & $\mathrm{pCi} / \mathrm{L}$ \\
\hline Toluene & WA & & & U & & 5 & & 1000 & $\mu g / L$ \\
\hline Toluene & GE & & & U & & 1 & & 1000 & $\mu g / L$ \\
\hline Toluene & WA & $\mathbf{R}$ & & $\mathrm{U}$ & & 5 & & 1000 & $\mu \mathrm{g} / \mathrm{L}$ \\
\hline
\end{tabular}


UTR033 Laboratory Analyses (cont'd)

\begin{tabular}{|c|c|c|c|c|c|c|c|c|}
\hline Analyte & Lab & Rep & Avg & Qualifier & Result & Acc & Stand & Units \\
\hline Total hardness & WA & & & $\mathrm{U}$ & 1 & & NS & $\mathrm{mg} / \mathrm{L}$ \\
\hline Total hardness & GE & & & & 2 & & NS & $\mathrm{mg} / \mathrm{L}$ \\
\hline Total hardness & WA & $\mathbf{R}$ & & & 2 & & NS & $\mathrm{mg} / \mathrm{L}$ \\
\hline Total organic carbon & WA & & $\mathbf{A}$ & & 1.17 & & NS & $\mathrm{mg} / \mathrm{L}$ \\
\hline Total organic carbon & $\mathrm{GE}$ & & & & 1.06 & & NS̆ & $\mathrm{mg} / \mathrm{L}$ \\
\hline Total organic carbon & WA & $\mathbf{R}$ & & & 0.837 & . & NS & $\mathrm{mol}$ \\
\hline Total suspended solids & WA & & & $U$ & 5 & & NS & $\mathrm{mg} / \mathrm{L}$ \\
\hline Total suspended solids & WA & & NA & $\mathbf{U}$ & 5 & & NS & $\mathrm{mg} / \mathrm{L}$ \\
\hline Total suspended solids & $\mathbf{G E}$ & & & $\mathbf{U}$ & 1 & & NS & $\mathrm{mg} / \mathrm{L}$ \\
\hline Total suspended solids & WA & $\mathbf{R}$ & & $\mathbf{U}$ & 5 & & NS & $\mathrm{mg} / \mathrm{L}$ \\
\hline trans-1,3-Dichloropropene & WA & & & $\mathbf{U}$ & 5 & & NS & $\mu g \Omega$ \\
\hline trans-1,3-Dichloropropene & $\mathbf{G E}$ & & & $\mathbf{U}$ & 1 & & NS & $\mu \mathrm{g} / \mathrm{L}$ \\
\hline trans-1,3-Dichloropropene & WA & $\mathbf{R}$ & & $\mathbf{U}$ & 5 & & NS & $\mu \mathrm{g} / \mathrm{L}$ \\
\hline trans-1,4-Dichloro-2-butene & WA & & & $\mathbf{U}$ & 100 & & NS & $\mu g / L$ \\
\hline trans-1,4-Dichloro-2-butene & GE & & & $\mathbf{U}$ & 30 & & NS & $\mu g / L$ \\
\hline trans-1,4-Dichloro-2-butene & WA & $\mathbf{R}$ & & $\mathbf{U}$ & 100 & & NS & $\mu \mathrm{g} / \mathrm{L}$ \\
\hline Trichloroethylene & WA & & & $\mathbf{U}$ & 5 & & 5 & $\mu \mathrm{g} / \mathrm{L}$ \\
\hline Trichloroethylene & GE & & & U & 1 & & 5 & $\mu \mathrm{g} / \mathrm{L}$ \\
\hline Trichloroethylene & WA & $\mathbf{R}$ & & $\mathbf{U}$ & 5 & & 5 & $\mu \mathrm{g} / \mathrm{L}$ \\
\hline Trichlorofluoromethane & WA & & & $\mathbf{U}$ & 5 & & NS & $\mu \mathrm{g} / \mathrm{L}$ \\
\hline Trichlorofluoromethane & GE & & & $\mathbf{U}$ & 1 & & NS & $\mu \mathrm{g} / \mathrm{L}$ \\
\hline Trichlorofluoromethane & WA & $\mathbf{R}$ & & $\mathbf{U}$ & 5 & & NS & $\mu g / L$ \\
\hline Tritium & TM & & & & 20.6 & 9.5 & 20 & $\mathrm{pCi} / \mathrm{ml}$ \\
\hline Tritium & $G E$ & & & & 24.3 & & 20 & $\mathrm{pCi} / \mathrm{ml}$ \\
\hline Tritium & TM & $\mathbf{R}$ & & & 19.3 & 5.3 & 20 & $\mathrm{pCi} / \mathrm{ml}$ \\
\hline Turbidity & WA & & & & 0.39 & & NS & NTU \\
\hline Turbidity & GE & & & $\mathbf{U}$ & 0.1 & & NS & NTU \\
\hline Turbidity & WA & $\mathbf{R}$ & & U & 0.2 & & NS & NTU \\
\hline Vanadium & WA & & & $\mathbf{U}$ & 50 & & NS & $\mu \mathrm{g} / \mathrm{L}$ \\
\hline Vanadium & GE & & & $\mathbf{U}$ & 8 & & NS & $\mu \mathrm{g} / \mathrm{L}$ \\
\hline Vanadium & WA & $\mathbf{R}$ & & $\mathrm{U}$ & 50 & & NS & $\mu g \alpha$ \\
\hline Vinyl acetate & WA & & & $\mathbf{U}$ & 10 & & NS & $\mu g / \mathrm{L}$ \\
\hline Vinyl acetate & GE & & & U & 1 & & NS & $\mu g / \mathrm{L}$ \\
\hline Vinyl acetate & WA & $\mathbf{R}$ & & $\mathbf{U}$ & 10 & & NS & $\mu \mathrm{g} / \mathrm{L}$ \\
\hline Vinyl chloride & WA & & & $\mathrm{U}$ & 10 & & 2 & $\mu g / L$ \\
\hline Vinyl chloride & $G E$ & & & U & 1 & & 2 & $\mu \mathrm{g} / \mathrm{L}$ \\
\hline Vinyl chloride & WA & $\mathbf{R}$ & & $\mathbf{U}$ & 10 & & 2 & $\mu \mathrm{g} / \mathrm{L}$ \\
\hline Xylenes (total) & WA & & & $\mathbf{U}$ & 5 & & 10000 & $\mu g / L$ \\
\hline Xylenes (total) & GE & & & $\mathbf{U}$ & 2 & & 10000 & $\mu \mathrm{g} / \mathrm{L}$ \\
\hline Xylenes (total) & WA & $\mathbf{R}$ & & $\mathbf{U}$ & 5 & & 10000 & $\mu g / L$ \\
\hline Yttrium-88 & EP & & & $\mathbf{U}$ & 60 & & NS & $\mathrm{pCi} / \mathrm{L}$ \\
\hline Yttrium-88 & EP & & NA & $\mathbf{U}$ & 60 & & NS & $\mathrm{pCi} / \mathrm{L}$ \\
\hline Zinc & WA & & & & 41 & & 5000 & $\mu g / L$ \\
\hline Zinc & GE & & & & 31.3 & & 5000 & $\mu \mathrm{g} / \mathrm{L}$ \\
\hline Zinc & WA & $\mathbf{R}$ & & & 41.5 & & 5000 & $\mu \mathrm{g} / \mathrm{L}$ \\
\hline Zinc-65 & EP & & & $\mathbf{U}$ & 20 & & 300 & $\mathrm{pCi} / \mathrm{L}$ \\
\hline Zinc-65 & $\mathrm{EP}$ & & NA & $\mathrm{U}$ & 20 & & 300 & $\mathrm{pCi} / \mathrm{L}$ \\
\hline
\end{tabular}




\section{UTR036}

Field Measurements:

Sample Date: 4/14/93

pH: 5.57

Specific Conductance: $32 \mu \mathrm{S} / \mathrm{cm}$

Red/Oxid Potential: $180.2 \mathrm{mv}$

Dissolved Oxygen: $1 \mathrm{mg} / \mathrm{L}$

\begin{tabular}{|c|c|c|c|c|c|c|c|c|}
\hline Analyte & Lab & Rep & Avg & Qualifier & Result & Acc & Stand & Units \\
\hline 1,1,1,2-Tetrachloroethane & $\overline{W A}$ & & & $\mathrm{U}$ & 10 & & NS & $\mu g / L$ \\
\hline 1,1,1-Trichloroethane & WA & & & $\mathbf{U}$ & 5 & & 200 & $\mu g / L$ \\
\hline 1,1,2,2-Tetrachloroethane & WA & & & $\mathbf{U}$ & 5 & & NS & $\mu g / L$ \\
\hline 1,1,2-Trichloroethane & WA & & & $\mathbf{U}$ & 5 & & 5 & $\mu g / L$ \\
\hline 1,1-Dichloroethane & WA & & & $\mathbf{U}$ & 5 & & NS & $\mu g / L$ \\
\hline 1,1-Dichloroethylene & WA & & & $\mathbf{U}$ & 5 & & 7 & $\mu \mathrm{g} / \mathrm{L}$ \\
\hline 1,2,3-Trichloropropane & WA & & & U & 10 & & NS & $\mu g / L$ \\
\hline 1,2-Dibromo-3-chloropropane & WA & & & $\mathbf{U}$ & 20 & & 0.2 & $\mu g / L$ \\
\hline 1,2-Dibromoethane & WA & & & $\mathbf{U}$ & 20 & & NS & $\mu g / L$ \\
\hline 1,2-Dichloroethane & WA & & & $\mathbf{U}$ & 5 & & 5 & $\mu g /$ \\
\hline 1,2-Dichloroethylene (total) & WA & & & $\mathbf{U}$ & 5 & & 100 & $\mu g / L$ \\
\hline 1,2-Dichloropropane & WA & & & $\mathbf{U}$ & 5 & & 5 & $\mu g / L$ \\
\hline 2-Hexanone & WA & & & $\mathrm{U}$ & 10 & & NS & $\mu g / L$ \\
\hline 4-Methyl-2-pentanone & WA & & & $\mathrm{U}$ & 10 & & NS & $\mu g / L$ \\
\hline Acetone & WA & & & U & 10 & & NS & $\mu g / L$ \\
\hline Acetonitrile & WA & & & $\mathbf{U}$ & 20 & & NS & $\mu g / L$ \\
\hline Acidity & WA & & & & 22 & & NS & $\mathrm{mg} / \mathrm{L}$ \\
\hline Acrolein & WA & & & $\mathbf{U}$ & 10 & & NS & $\mu g / L$ \\
\hline Acrylonitrile & WA & & & $\mathbf{U}$ & 10 & & NS & $\mu g / L$ \\
\hline Alkalinity & WA & & & & 1.25 & & NS & $\mathrm{mg} / \mathrm{L}$ \\
\hline Allyl chloride & WA & & & $\mathbf{U}$ & 100 & & NS & $\mu g / L$ \\
\hline Aluminum & WA & & & & 287 & & NS & $\mu g / L$ \\
\hline Antimony & WA & & & $\mathbf{U}$ & 60 & & 6 & $\mu g / L$ \\
\hline Arsenic & WA & & & $\mathbf{U}$ & 10 & & 50 & $\mu g / L$ \\
\hline Barium & WA & & & $\mathbf{U}$ & 200 & & 2000 & $\mu g / L$ \\
\hline Benzene & WA & & & $\mathbf{U}$ & 5 & & 5 & $\mu g / L$ \\
\hline Beryllium & WA & & & $\mathbf{U}$ & 5 & & 4 & $\mu g / L$ \\
\hline Bromodichloromethane & WA & & & $\mathbf{U}$ & 5 & & NS & $\mu \mathrm{g} / \mathrm{L}$ \\
\hline Bromoform & WA & & & $\tilde{U}$ & 5 & & NS & $\mu g / L$ \\
\hline Cadmium & WA & & & & 9.1 & & 5 & $\mu \mathrm{g} / \mathrm{L}$ \\
\hline Calcium & WA & & & $\mathbf{U}$ & 5000 & & NS & $\mu g / L$ \\
\hline Carbon disulfide & WA & & & $\mathbf{U}$ & 5 & & NS & $\mu g / L$ \\
\hline Carbon tetrachloride & WA & & & $\mathbf{U}$ & 5 & & 5 & $\mu \mathrm{g} / \mathrm{L}$ \\
\hline Cesium-137 & TM & & NA & $\mathbf{U}$ & 9.2 & & 200 & $\mathrm{pCi} / \mathrm{L}$ \\
\hline Cesium-137 & $\mathrm{TM}$ & & & $\mathbf{U}$ & 9.2 & & 200 & $\mathrm{pCi} / \mathrm{L}$ \\
\hline Chloride & WA & & & & 4.34 & & NS & $\mathrm{mg} / \mathrm{L}$ \\
\hline Chlorobenzene & WA & & & $\mathrm{U}$ & 5 & & 100 & $\mu \mathrm{g} / \mathrm{L}$ \\
\hline Chloroethane & WA & & & U & 10 & & NS & $\mu \mathrm{g} / \mathrm{L}$ \\
\hline Chloroform & WA & & & U & 5 & & 100 & $\mu g / L$ \\
\hline Chloroprene & WA & & & $\mathbf{U}$ & 20 & & NS & $\mu g / L$ \\
\hline Chromium & WA & & & $\mathrm{U}$ & 10 & & 100 & $\mu \mathrm{g} / \mathrm{L}$ \\
\hline cis-1,3-Dichloropropene & WA & & & $\mathrm{U}$ & 5 & & NS & $\mu \mathrm{g} / \mathrm{L}$ \\
\hline Cobalt & WA & & & $U$ & 50 & & NS & $\mu g / L$ \\
\hline Copper & WA & & & $\mathrm{U}$ & 25 & & 1000 & $\mu \mathrm{g} / \mathrm{L}$ \\
\hline Cyanide & WA & & & $\mathrm{U}$ & 10 & & 200 & $\mu g / L$ \\
\hline Dibromochloromethane & WA & & & $\mathrm{U}$ & 5 & & NS & $\mu g / \Lambda$ \\
\hline Dichlorodifluoromethane & WA & & & $\mathrm{U}$ & 10 & & NS & $\mu \mathrm{g} / \mathrm{L}$ \\
\hline Ethylbenzene & WA & & & $\mathrm{U}$ & 5 & & 700 & $\mu g / L$ \\
\hline Fluoride & WA & & & $\mathbf{U}$ & 0.1 & & 4 & $\mathrm{mg} / \mathrm{h}$ \\
\hline
\end{tabular}


UTR036 Laboratory Analyses (cont'd)

\begin{tabular}{|c|c|c|c|c|c|c|c|c|}
\hline Analyte & Lab & Rep & Avg & Qualifier & Result & Acc & Stand & Units \\
\hline Gross alpha & TM & & $\mathbf{A}$ & & 0.9 & 0.9 & 15 & $\mathrm{pCi} / / \mathrm{L}$ \\
\hline Iron & WA & & & & 2360 & & 300 & $\mu \mathrm{g} / \mathrm{L}$ \\
\hline Isobutyl alcohol & WA & & & $\mathbf{U}$ & 20 & & NS & $\mu \mathrm{g} / \mathrm{L}$ \\
\hline Lead & WA & & & $\mathbf{U}$ & 3 & & 50 & $\mu \mathrm{g} / \mathrm{L}$ \\
\hline Magnesium & WA & & & $\mathbf{U}$ & 5000 & & $\mathrm{NS}$ & $\mu \mathrm{g} / \mathrm{L}$ \\
\hline Manganese & WA & & & & 39.5 & . & 50 & $\mu g / L$ \\
\hline Mercury & WA & & & $\mathbf{U}$ & 0.2 & & 2 & $\mu \mathrm{g} / \mathrm{L}$ \\
\hline Methacrylonitrile & WA & & & $\mathbf{U}$ & 20 & & NS & $\mu g / L$ \\
\hline Methyl bromide & WA & & & $\mathbf{U}$ & 10 & & NS & $\mu \mathrm{g} / \mathrm{L}$ \\
\hline Methyl chloride & WA & & & $\mathbf{U}$ & 10 & & NS & $\mu g / L$ \\
\hline Methyl ethyl ketone & WA & & & U & 10 & & NS & $\mu \mathrm{g} / \mathrm{L}$ \\
\hline Methyl iodide & WA & & & $\mathbf{U}$ & 10 & & NS & $\mu g / L$ \\
\hline Methylene bromide & WA & & & $\mathbf{U}$ & 10 & & NS & $\mu g / L$ \\
\hline Methylene chloride & WA & & & $\mathbf{U}$ & 5 & & 5 & $\mu \mathrm{g} / \mathrm{L}$ \\
\hline Nickel & WA & & . & $\mathbf{U}$ & 40 & & 100 & $\mu \mathrm{g} / \mathrm{L}$ \\
\hline Nitrate as nitrogen & WA & & & & 0.126 & & 10 & $\mathrm{mg} / \mathrm{L}$ \\
\hline Nonvolatile beta & TM & & $\mathbf{A}$ & & 4.05 & 1.9 & 50 & $\mathrm{pCi} / \mathrm{L}$ \\
\hline Potassium & WA & & & $\mathbf{U}$ & 5000 & & NS & $\mu g / L$ \\
\hline Propionitrile & WA & & & $\mathbf{U}$ & 50 & & NS & $\mu \mathrm{g} / \mathrm{L}$ \\
\hline Selenium & WA & & & $\mathbf{U}$ & 5 & & 50 & $\mu \mathrm{g} / \mathrm{L}$ \\
\hline Silver & WA & & & $\mathbf{U}$ & 10 & & 50 & $\mu g / L$ \\
\hline Sodium & WA & & & $\mathbf{U}$ & 5000 & & NS & $\mu g / L$ \\
\hline Styrene & WA & & & $\mathbf{U}$ & 5 & & 100 & $\mu g / L$ \\
\hline Sulfate & WA & & & $\mathbf{U}$ & 2.5 & & 400 & $\mathrm{mg} / \mathrm{L}$ \\
\hline Sulfide & WA & & & $\mathrm{U}$ & 0.1 & & NS & $\mathrm{mg} / \mathrm{L}$ \\
\hline Tetrachloroethylene & WA & & & $\mathbf{U}$ & 5 & & 5 & $\mu g / L$ \\
\hline Thallium & WA & & & $\mathbf{U}$ & 10 & & 2 & $\mu g / L$ \\
\hline Toluene & WA & & & $\mathbf{U}$ & 5 & & 1000 & $\mu g / L$ \\
\hline Total hardness & WA & & & $\mathrm{U}$ & 1 & & NS & $\mathrm{mg} / \mathrm{L}$ \\
\hline Total organic carbon & WA & & & & 7.39 & & NS & $\mathrm{mg} / \mathrm{L}$ \\
\hline Total suspended solids & WA & & & & 6 & & NS & $\mathrm{mg} / \mathrm{L}$ \\
\hline trans-1,3-Dichloropropene & WA & & & U & 5 & & NS & $\mu \mathrm{g} / \mathrm{L}$ \\
\hline trans-1,4-Dichloro-2-butene & WA & & & U & 100 & & NS & $\mu \mathrm{g} / \mathrm{L}$ \\
\hline Trichloroethylene & WA & & & $\mathbf{U}$ & 5 & & 5 & $\mu g / L$ \\
\hline Trichlorofluoromethane & WA & & & $\mathbf{U}$ & 5 & & NS & $\mu \mathrm{g} / \mathrm{L}$ \\
\hline Tritium & $\mathbf{T M}$ & & $\mathbf{A}$ & & 15.75 & 4.45 & 20 & $\mathrm{pCi} / \mathrm{ml}$ \\
\hline Turbidity & WA & & $\mathbf{A}$ & & 1.31 & & NS & NTU \\
\hline Vanadium & WA & & & $\mathrm{U}$ & 50 & & NS & $\mu g / L$ \\
\hline Vinyl acetate & WA & & & $\mathrm{U}$ & 10 & & NS & $\mu \mathrm{g} / \mathrm{L}$ \\
\hline Vinyl chloride & WA & & & $\mathrm{U}$ & 10 & & 2 & $\mu \mathrm{g} / \mathrm{L}$ \\
\hline Xylenes (total) & WA & & & $\mathrm{U}$ & 5 & & 10000 & $\mu \mathrm{g} / \mathrm{L}$ \\
\hline Zinc & WA & & & & 48.6 & & 5000 & $\mu g / L$ \\
\hline
\end{tabular}




\section{UTR039}

Field Measurements:

Sample Date: 4/28/93

pH: 5.51

Specific Conductance: $20 \mu \mathrm{S} / \mathrm{cm}$

Red/Oxid Potential: -204 mv

Dissolved Oxygen: $2.4 \mathrm{mg} / \mathrm{L}$

\begin{tabular}{|c|c|c|c|c|c|c|c|c|}
\hline Analyte & Lab & Rep & Avg & Qualifier & Result & Acc & Stand & Units \\
\hline 1,1,1,2-Tetrachloroethane & WA & & & $\mathbf{U}$ & 10 & & NS & $\mu g / L$ \\
\hline 1,1,1-Trichloroethane & WA & & & $\mathbf{U}$ & 5 & & 200 & $\mu g / L$ \\
\hline $1,1,2,2$-Tetrachloroethane & WA & & & $\mathbf{U}$ & 5 & & NS & $\mu g / L$ \\
\hline 1,1,2-Trichloroethane & WA & & & $\mathbf{U}$ & 5 & & 5 & $\mu g / L$ \\
\hline 1,1-Dichloroethane & WA & & & $\mathbf{U}$ & 5 & & NS & $\mu \mathrm{g} / \mathrm{L}$ \\
\hline 1,1-Dichloroethylene & WA & & & $\mathbf{U}$ & 5 & & 7 & $\mu g / L$ \\
\hline 1,2,3-Trichloropropane & WA & & & $\mathbf{U}$ & 10 & & NS & $\mu \mathrm{g} / \mathrm{L}$ \\
\hline 1,2-Dibromo-3-chloropropane & WA & & . & $\mathbf{U}$ & 20 & & 0.2 & $\mu \mathrm{g} / \mathrm{L}$ \\
\hline 1,2-Dibromoethane & WA & & & $\mathbf{U}$ & 20 & & NS & $\mu g / L$ \\
\hline 1,2-Dichloroethane & WA & & & $\mathbf{U}$ & 5 & & 5 & $\mu g / L$ \\
\hline 1,2-Dichloroethylene (total) & WA & & & $\mathbf{U}$ & 5 & & 100 & $\mu g / L$ \\
\hline 1,2-Dichloropropane & WA & & & $\mathbf{U}$ & 5 & & 5 & $\mu g / L$ \\
\hline 2-Hexanone & WA & & & $\mathbf{U}$ & 10 & & NS & $\mu g / L$ \\
\hline 4-Methyl-2-pentanone & WA & & & $\mathbf{U}$ & 10 & & NS & $\mu g / L$ \\
\hline Acetone & WA & & & $\mathbf{J}$ & 7.87 & & NS & $\mu g / L$ \\
\hline Acetonitrile & WA & & & $\mathbf{U}$ & 20 & & NS & $\mu g / L$ \\
\hline Acidity & WA & & & & 22 & & NS & $\mathrm{mg} / \mathrm{L}$ \\
\hline Acrolein & WA & & & $\mathbf{U}$ & 10 & & NS & $\mu \mathrm{g} / \mathrm{L}$ \\
\hline Acrylonitrile & WA & & & $\mathbf{U}$ & 10 & & NS & $\mu g / L$ \\
\hline Alkalinity & WA & & & $\mathbf{U}$ & 0.5 & & NS & $\mathrm{mg} / \mathrm{L}$ \\
\hline Allyl chloride & WA & & & $\mathbf{U}$ & 100 & & NS & $\mu \mathrm{g} / \mathrm{L}$ \\
\hline Aluminum & WA & & & $\mathbf{U}$ & 200 & & NS & $\mu \mathrm{g} / \mathrm{L}$ \\
\hline Antimony & WA & & & $\mathbf{U}$ & 60 & & 6 & $\mu \mathrm{g} / \mathrm{L}$ \\
\hline Arsenic & WA & & & $\mathbf{U}$ & 10 & & 50 & $\mu g / L$ \\
\hline Barium & WA & & & $\mathbf{U}$ & 200 & & 2000 & $\mu g / L$ \\
\hline Benzene & WA & & & $\mathbf{U}$ & 5 & & 5 & $\mu \mathrm{g} / \mathrm{L}$ \\
\hline Beryllium & WA & & & $\mathbf{U}$ & 5 & & 4 & $\mu g / L$ \\
\hline Bromodichloromethane & WA & & & $\mathbf{U}$ & 5 & & NS & $\mu g / L$ \\
\hline Bromoform & WA & & & $\mathbf{U}$ & 5 & & NS & $\mu g / L$ \\
\hline Cadmium & WA & & & $\mathbf{U}$ & 5 & & 5 & $\mu g / L$ \\
\hline Calcium & WA & & & $\mathbf{U}$ & 5000 & & NS & $\mu g / L$ \\
\hline Carbon disulfide & WA & & & $\mathbf{U}$ & 5 & & NS & $\mu \mathrm{g} / \mathrm{L}$ \\
\hline Carbon tetrachloride & WA & & . & $\mathbf{U}$ & 5 & & 5 & $\mu \mathrm{g} / \mathrm{L}$ \\
\hline Cesium-137 & $\mathrm{TM}$ & & & $\mathbf{U}$ & 9.7 & & 200 & $\mathrm{pCi} / \mathrm{L}$ \\
\hline Chloride & WA & & & & 3.07 & & NS & $\mathrm{mg} / \mathrm{L}$ \\
\hline Chlorobenzene & WA & & & $\mathbf{U}$ & 5 & & 100 & $\mu g / L$ \\
\hline Chloroethane & WA & & & $\mathbf{U}$ & 10 & & NS & $\mu \mathrm{g} / \mathrm{L}$ \\
\hline Chloroform & WA & & & $\mathbf{U}$ & 5 & & 100 & $\mu \mathrm{g} / \mathrm{L}$ \\
\hline Chloroprene & WA & & & $\mathbf{U}$ & 20 & & NS & $\mu \mathrm{g} / \mathrm{L}$ \\
\hline Chromium & WA & & & $\mathbf{U}$ & 10 & & 100 & $\mu \mathrm{g} / \mathrm{L}$ \\
\hline cis-1,3-Dichloropropene & WA & & & $\mathbf{U}$ & 5 & & NS & $\mu \mathrm{g} / \mathrm{L}$ \\
\hline Cobalt & WA & & & $\mathbf{U}$ & 50 & & NS & $\mu \mathrm{g} / \mathrm{L}$ \\
\hline Copper & WA & & & $\mathrm{U}$ & 25 & & 1000 & $\mu \mathrm{g} / \mathrm{L}$ \\
\hline Cyanide & WA & & & $\mathbf{U}$ & 10 & & 200 & $\mu \mathrm{g} / \mathrm{L}$ \\
\hline Dibromochloromethane & WA & & & $\mathrm{U}$ & 5 & & NS & $\mu g / L$ \\
\hline Dichlorodifluoromethane & WA & & & $\mathbf{U}$ & 10 & & NS & $\mu \mathrm{g} / \mathrm{L}$ \\
\hline Ethylbenzene & WA & & & $\mathbf{U}$ & 5 & & 700 & $\mu g / L$ \\
\hline Fluoride & WA & & & $\mathbf{U}$ & 0.1 & & 4 & $\mathrm{mg} / \mathrm{L}$ \\
\hline
\end{tabular}


Sampling and Analysis of Water From Upper Three Runs And Its Wellands Near Tank I6 and the Mixed Waste Management Facility

UTR039 Laboratory Analyses (cont'd)

\begin{tabular}{|c|c|c|c|c|c|c|c|c|c|}
\hline Analyte & Lab & Rep & Avg & Qualifier & & Result & Acc & Stand & Units \\
\hline Gross alpha & $\overline{T M}$ & & & & & 1.8 & 1.3 & 15 & $\mathrm{pCi} / \mathrm{L}$ \\
\hline Iron & WA & & & & & 101 & & 300 & $\mu g / L$ \\
\hline Isobutyl alcohol & WA & & & $\mathbf{U}$ & & 20 & & NS & $\mu \mathrm{g} / \mathrm{L}$ \\
\hline Lead & WA & & & $\mathbf{U}$ & & 3 & & 50 & $\mu \mathrm{g} / \mathrm{L}$ \\
\hline Magnesium & WA & & & $\mathbf{U}$ & & 5000 & & NS & $\mu g / L$ \\
\hline Manganese & WA & & & & & 24.7 & . & 50 & $\mu g / L$ \\
\hline Mercury & WA & & & $\mathbf{U}$ & & 0.2 & & 2 & $\mu \mathrm{g} / \mathrm{L}$ \\
\hline Methacrylonitrile & WA & & & $\mathbf{U}$ & & 20 & & NS & $\mu \mathrm{g} / \mathrm{L}$ \\
\hline Methyl bromide & WA & & & $\mathbf{U}$ & & 10 & & NS & $\mu \mathrm{g} / \mathrm{L}$ \\
\hline Methyl chloride & WA & & & $\mathbf{U}$ & & 10 & & NS & $\mu \mathrm{g} / \mathrm{L}$ \\
\hline Methyl ethyl ketone & WA & & & $\mathbf{U}$ & & 10 & & NS & $\mu g / L$ \\
\hline Methyl iodide & WA & & & U & & 10 & & NS & $\mu \mathrm{g} / \mathrm{L}$ \\
\hline Methylene bromide & WA & & & $\mathbf{U}$ & & 10 & & NS & $\mu g / L$ \\
\hline Methylene chloride & WA & & & $\mathbf{J}$ & V & 3.49 & & 5 & $\mu g / L$ \\
\hline Nickel & WA & & & $\mathbf{U}$ & & 40 & & 100 & $\mu \mathrm{g} / \mathrm{L}$ \\
\hline Nitrate as nitrogen & WA & & $\cdot$ & & & 0.628 & & 10 & $\mathrm{mg} / \mathrm{L}$ \\
\hline Nonvolatile beta & $\mathrm{TM}$ & & & & & 1.8 & 1.7 & 50 & $\mathrm{pCi} / \mathrm{L}$ \\
\hline Potassium & WA & & & $\mathbf{U}$ & & 5000 & & NS & $\mu g / L$ \\
\hline Propionitrile & WA & & & $\mathbf{U}$ & & 50 & & NS & $\mu g / L$ \\
\hline Selenium & WA & & & $\mathbf{U}$ & & 5 & & 50 & $\mu g / L$ \\
\hline Silver & WA & & & $\mathbf{U}$ & & 10 & & 50 & $\mu \mathrm{g} / \mathrm{L}$ \\
\hline Sodium & WA & & & $\mathbf{U}$ & & 5000 & & NS & $\mu \mathrm{g} / \mathrm{L}$ \\
\hline Styrene & WA & & & $\mathbf{U}$ & & 5 & & 100 & $\mu g / L$ \\
\hline Sulfate & WA & & & $\mathbf{U}$ & & 2.5 & & 400 & $\mathrm{mg} / \mathrm{L}$ \\
\hline Sulfide & WA & & & $\mathbf{U}$ & & 0.1 & & NS & $\mathrm{mg} / \mathrm{L}$ \\
\hline Tetrachloroethylene & WA & & & $\mathbf{U}$ & & 5 & & 5 & $\mu \mathrm{g} / \mathrm{L}$ \\
\hline Thallium & WA & & & $\mathbf{U}$ & & 10 & & 2 & $\mu \mathrm{g} / \mathrm{L}$ \\
\hline Toluene & WA & & & $\mathbf{J}$ & & 1.45 & & 1000 & $\mu \mathrm{g} / \mathrm{L}$ \\
\hline Total hardness & WA & & $\mathbf{A}$ & & & 4 & & NS & $\mathrm{mg} / \mathrm{L}$ \\
\hline Total organic carbon & WA & & & & & 3.01 & & NS & $\mathrm{mg} / \mathrm{L}$ \\
\hline Total suspended solids & WA & & & $\mathbf{U}$ & & 5 & & NS & $\mathrm{mg} / \mathrm{L}$ \\
\hline trans-1,3-Dichloropropene & WA & & & $\mathbf{U}$ & & 5 & & NS & $\mu g / L$ \\
\hline trans-1,4-Dichloro-2-butene & WA & & & $\mathbf{U}$ & & 100 & & NS & $\mu \mathrm{g} / \mathrm{L}$ \\
\hline Trichloroethylene & WA & & & $\mathbf{U}$ & & 5 & & 5 & $\mu g / L$ \\
\hline Trichlorofluoromethane & WA & & & $\mathbf{U}$ & & 5 & & NS & $\mu \mathrm{g} / \mathrm{L}$ \\
\hline Tritium & TM & & & & & 15.6 & 1.6 & 20 & $\mathrm{pCi} / \mathrm{ml}$ \\
\hline Turbidity & WA & & & & & 0.559 & & NS & NTU \\
\hline Vanadium & WA & & & $\mathbf{U}$ & & 50 & & NS & $\mu g / L$ \\
\hline Vinyl acetate & WA & & & $\mathbf{U}$ & & 10 & & NS & $\mu g / L$ \\
\hline Vinyl chloride & WA & & & $\mathbf{U}$ & & 10 & & 2 & $\mu g / L$ \\
\hline Xylenes (total) & WA & & & $\mathbf{U}$ & & 5 & & 10000 & $\mu g / L$ \\
\hline Zinc & WA & & & & & 23.3 & & 5000 & $\mu g / L$ \\
\hline
\end{tabular}




\section{UTR042}

Field Measurements:

Sample Date: 4/14/93

pH: 5.94

Specific Conductance: $26 \mu \mathrm{S} / \mathrm{cm}$

Red/Oxid Potential: $208.6 \mathrm{mv}$

Dissolved Oxygen: $2.5 \mathrm{mg} / \mathrm{L}$

\begin{tabular}{|c|c|c|c|c|c|c|c|c|}
\hline Analyte & Lab & Rep & Avg & Qualifier & Result & Acc & Stand & Units \\
\hline 1,1,1,2-Tetrachloroethane & WA & & & $\mathbf{U}$ & 10 & & NS & $\mu g / L$ \\
\hline 1,1,1-Trichloroethane & WA & & & $\mathbf{U}$ & 5 & & 200 & $\mu g / L$ \\
\hline $1,1,2,2$-Tetrachloroethane & WA & & & $\mathbf{U}$ & 5 & & NS & $\mu \mathrm{g} / \mathrm{L}$ \\
\hline 1,1,2-Trichloroethane & WA & & & $\mathbf{U}$ & 5 & & 5 & $\mu \mathrm{g} / \mathrm{L}$ \\
\hline 1,1-Dichloroethane & WA & & & $\mathbf{U}$ & 5 & & NS & $\mu g / L$ \\
\hline 1,1-Dichloroethylene & WA & & & $\mathbf{U}$ & 5 & & 7 & $\mu g / L$ \\
\hline 1,2,3-Trichloropropane & WA & & & $\mathbf{U}$ & 10 & & NS & $\mu g / L$ \\
\hline 1,2-Dibromo-3-chloropropane & WA & & . & $\mathbf{U}$ & 20 & & 0.2 & $\mu g / L$ \\
\hline 1,2-Dibromoethane & WA & & & $\mathbf{U}$ & 20 & & NS & $\mu g / L$ \\
\hline 1,2-Dichloroethane & WA & & & $\mathbf{U}$ & 5 & & 5 & $\mu g / L$ \\
\hline 1,2-Dichloroethylene (total) & WA & & & $\mathbf{U}$ & 5 & & 100 & $\mu g / L$ \\
\hline 1,2-Dichloropropane & WA & & & $\mathbf{U}$ & 5 & & 5 & $\mu g / L$ \\
\hline 2-Hexanone & WA & & & $\mathbf{U}$ & 10 & & NS & $\mu \mathrm{g} / \mathrm{L}$ \\
\hline 4-Methyl-2-pentanone & WA & & & $\mathbf{U}$ & 10 & & NS & $\mu g / L$ \\
\hline Acetone & WA & & & & 12.6 & & NS & $\mu g / L$ \\
\hline Acetonitrile & WA & & & $\mathbf{U}$ & 20 & & NS & $\mu g / L$ \\
\hline Acidity & WA & & & & 7.5 & & NS & $\mathrm{mg} / \mathrm{L}$ \\
\hline Acrolein & WA & & & $\mathbf{U}$ & 10 & & NS & $\mu \mathrm{g} / \mathrm{L}$ \\
\hline Acrylonitrile & WA & & & $\mathbf{U}$ & 10 & & NS & $\mu \mathrm{g} / \mathrm{L}$ \\
\hline Alkalinity & WA & & & & 1 & & NS & $\mathrm{min} / \mathrm{L}$ \\
\hline Allyl chloride & WA & & & $\mathbf{U}$ & 100 & & NS & $\mu \mathrm{g} / \mathrm{L}$ \\
\hline Aluminum & WA & & & $\mathbf{U}$ & 200 & & NS & $\mu g / L$ \\
\hline Antimony & WA & & & $\mathbf{U}$ & 60 & & 6 & $\mu \mathrm{g} / \mathrm{L}$ \\
\hline Arsenic & WA & & & $\mathbf{U}$ & 10 & & 50 & $\mu g / L$ \\
\hline Barium & WA & & & $\mathbf{U}$ & 200 & & 2000 & $\mu g / L$ \\
\hline Benzene & WA & & & $\mathbf{U}$ & 5 & & 5 & $\mu \mathrm{g} / \mathrm{L}$ \\
\hline Beryllium & WA & & & $\mathbf{U}$ & 5 & & 4 & $\mu g / L$ \\
\hline Bromodichloromethane & WA & & & $\mathbf{U}$ & 5 & & NS & $\mu \mathrm{g} / \mathrm{L}$ \\
\hline Bromoform & WA & & & $\mathbf{U}$ & 5 & & NS & $\mu g / L$ \\
\hline Cadmium & WA & & & $\mathbf{U}$ & 5 & & 5 & $\mu \mathrm{g} / \mathrm{L}$ \\
\hline Calcium & WA & & & $\mathbf{U}$ & 5000 & & NS & $\mu g / L$ \\
\hline Carbon disulfide & WA & & & $\mathbf{U}$ & 5 & & NS & $\mu g / L$ \\
\hline Carbon tetrachloride & WA & & & $\mathbf{U}$ & 5 & & 5 & $\mu \mathrm{g} / \mathrm{L}$ \\
\hline Cesium-137 & TM & & & $\mathbf{U}$ & 6.3 & & 200 & $\mathrm{pCi} / \mathrm{L}$ \\
\hline Chloride & WA & & & & 3.78 & & NS & $\mathrm{mg} / \mathrm{L}$ \\
\hline Chlorobenzene & WA & & & $\mathbf{U}$ & 5 & & 100 & $\mu \mathrm{g} / \mathrm{L}$ \\
\hline Chloroethane & WA & & & $\mathbf{U}$ & 10 & & NS & $\mu \mathrm{g} / \mathrm{L}$ \\
\hline Chloroform & WA & & & $\mathbf{U}$ & 5 & & 100 & $\mu \mathrm{g} / \mathrm{L}$ \\
\hline Chloroprene & WA & & & $\mathbf{U}$ & 20 & & NS & $\mu \mathrm{g} / \mathrm{L}$ \\
\hline Chromium & WA & & & $\mathbf{U}$ & 10 & & 100 & $\mu \mathrm{g} / \mathrm{L}$ \\
\hline cis-1,3-Dichloropropene & WA & & & $\mathbf{U}$ & 5 & & NS & $\mu \mathrm{g} / \mathrm{L}$ \\
\hline Cobalt & WA & & & $\mathbf{U}$ & 50 & & NS & $\mu \mathrm{g} / \mathrm{L}$ \\
\hline Copper & WA & & & $\mathbf{U}$ & 25 & & 1000 & $\mu \mathrm{g} / \mathrm{L}$ \\
\hline Cyanide & WA & & & $\mathbf{U}$ & 10 & & 200 & $\mu \mathrm{g} / \mathrm{L}$ \\
\hline Dibromochloromethane & WA & & & $\mathbf{U}$ & 5 & & NS & $\mu \approx / L$ \\
\hline Dichlorodifluoromethane & WA & & & $\mathbf{U}$ & 10 & & NS & $\mu g / L$ \\
\hline Ethylbenzene & WA & & & $\mathbf{U}$ & 5 & & 700 & $\mu g / L$ \\
\hline Fluoride & WA & & & $\mathbf{U}$ & 0.1 & & 4 & $\mathrm{mg} / \mathrm{L}$ \\
\hline
\end{tabular}


UTR042 Laboratory Analyses (cont'd)

\begin{tabular}{|c|c|c|c|c|c|c|c|}
\hline Analyte & Lab & Avg & Qualifier & Result & Acc & Stand & Units \\
\hline Gross alpha & $\overline{T M}$ & & $\bar{U}$ & 0.6 & & 15 & $\overline{\mathrm{pCi} / \mathrm{L}}$ \\
\hline Iron & WA & & & 143 & & 300 & $\mu g / L$ \\
\hline Isobutyl alcohol & WA & & $\mathbf{U}$ & 20 & & NS & $\mu g / L$ \\
\hline Lead & WA & & $\mathbf{U}$ & 3 & & 50 & $\mu g / L$ \\
\hline Magnesium & WA & & $\mathbf{U}$ & 5000 & & NS & $\mu g / L$ \\
\hline Manganese & WA & & & 31.1 & . & 50 & $\mu \mathrm{g} / \mathrm{L}$ \\
\hline Mercury & WA & & $\mathbf{U}$ & 0.2 & & 2 & $\mu g / L$ \\
\hline Methacrylonitrile & WA & & $\mathbf{U}$ & 20 & & NS & $\mu g / L$ \\
\hline Methyl bromide & WA & & $\mathbf{U}$ & 10 & & NS & $\mu g / L$ \\
\hline Methyl chloride & WA & & $\mathbf{U}$ & 10 & & NS & $\mu g / L$ \\
\hline Methyl ethyl ketone & WA & & $\mathbf{U}$ & 10 & & NS & $\mu \mathrm{g} / \mathrm{L}$ \\
\hline Methyl iodide & WA & & $\mathbf{U}$ & 10 & & NS & $\mu g / L$ \\
\hline Methylene bromide & WA & & $\mathbf{U}$ & 10 & & NS & $\mu g / L$ \\
\hline Methylene chioride & WA & & $\mathbf{U}$ & 5 & & 5 & $\mu g / L$ \\
\hline Nickel & WA & & $\mathbf{U}$ & 40 & & 100 & $\mu g / L$ \\
\hline Nitrate as nitrogen & WA & & & 0.125 & & 10 & $\mathrm{mg} / \mathrm{L}$ \\
\hline Nonvolatile beta & TM & & $\mathbf{U}$ & 0.8 & & 50 & $\mathrm{pCi} / \mathrm{L}$ \\
\hline Potassium & WA & & $\mathbf{U}$ & 5000 & & NS & $\mu \mathrm{g} / \mathrm{L}$ \\
\hline Propionitrile & WA & & $\mathbf{U}$ & 50 & & NS & $\mu \mathrm{g} / \mathrm{L}$ \\
\hline Selenium & WA & & $\mathbf{U}$ & 5 & & 50 & $\mu g / L$ \\
\hline Silver & WA & & $\mathbf{U}$ & 10 & & 50 & $\mu g / L$ \\
\hline Sodium & WA & & $\mathbf{U}$ & 5000 & & PSS & $\mu g / L$ \\
\hline Styrene & WA & & $\mathbf{U}$ & 5 & & 100 & $\mu g / L$ \\
\hline Sulfate & WA & & $\mathbf{U}$ & 2.5 & & 400 & $\mathrm{mg} / \mathrm{L}$ \\
\hline Sulfide & WA & & $\mathbf{U}$ & 0.1 & & NS & $\mathrm{mg} / \mathrm{L}$ \\
\hline Tetrachloroethylene & WA & & $\mathbf{U}$ & 5 & & 5 & $\mu \mathrm{g} / \mathrm{L}$ \\
\hline Thallium & WA & & $\mathbf{U}$ & 10 & & 2 & $\mu g / L$ \\
\hline Toluene & WA & & $\mathbf{U}$ & 5 & & 1000 & $\mu g / L$ \\
\hline Total hardness & WA & & & 2 & & NS & $\mathrm{mg} / \mathrm{L}$ \\
\hline Total organic carbon & WA & & & 6.12 & & NS & $\mathrm{mg} / \mathrm{L}$ \\
\hline Total suspended solids & WA & & $\mathbf{U}$ & 5 & & NS & $\mathrm{mg} / \mathrm{L}$ \\
\hline trans-1,3-Dichloropropene & WA & & $\mathbf{U}$ & 5 & & NS & $\mu \mathrm{g} / \mathrm{L}$ \\
\hline trans-1,4-Dichloro-2-butene & WA & & $\mathbf{U}$ & 100 & & NS & $\mu \mathrm{g} / \mathrm{L}$ \\
\hline Trichloroethylene & WA & & $\mathbf{U}$ & 5 & & 5 & $\mu g / L$ \\
\hline Trichlorofluoromethane & WA & & $\mathbf{U}$ & 5 & & NS & $\mu g / L$ \\
\hline Tritium & TM & & & 14.6 & 4.5 & 20 & $\mathrm{pCi} / \mathrm{ml}$ \\
\hline Turbidity & WA & & & 1.29 & & NS & NTU \\
\hline Vanadium & WA & & $\mathbf{U}$ & 50 & & NS & $\mu \mathrm{g} / \mathrm{L}$ \\
\hline Vinyl acetate & WA & & $\mathbf{U}$ & 10 & & NS & $\mu \mathrm{g} / \mathrm{L}$ \\
\hline Vinyl chloride & WA & & $\mathbf{U}$ & 10 & & 2 & $\mu \mathrm{g} / \mathrm{L}$ \\
\hline Xylenes (total) & WA & & U & 5 & & 10000 & $\mu \mathrm{g} / \mathrm{L}$ \\
\hline Zinc & WA & & & 54.5 & & 5000 & $\mu \mathrm{g} / \mathrm{L}$ \\
\hline
\end{tabular}




\section{UTR045}

Field Measurements:

Sample Date: 4/14/93

pH: 6.03

Specific Conductance: $34 \mu \mathrm{s} / \mathrm{cm}$

Red/Oxid Potential: $165.2 \mathrm{mv}$

Dissolved Oxygen: $2.1 \mathrm{mg} / \mathrm{L}$

\begin{tabular}{|c|c|c|c|c|c|c|c|c|}
\hline Analyte & Lab & Rep & Avg & Qualifier & Result & Acc & Stand & Units \\
\hline 1,1,1,2-Tetrachloroethane & WA & & & $\mathbf{U}$ & 10 & & NS & $\mu g / 2$ \\
\hline 1,1,1,2-Tetrachloroethane & GE & & & $\mathbf{U}$ & 1 & & NS & $\mu g / L$ \\
\hline 1,1,1-Trichloroethane & WA & & & $\mathbf{U}$ & 5 & & 200 & $\mu g / L$ \\
\hline 1,1,1-Trichloroethane & GE & & & $\mathbf{U}$ & 1 & & 200 & $\mu g / L$ \\
\hline 1,1,2,2-Tetrachloroethane & WA & & & $\mathbf{U}$ & 5 & & NS & $\mu g / L$ \\
\hline 1,1,2,2-Tetrachloroethane & GE & & & $\mathbf{U}$ & 1 & & NS & $\mu g / L$ \\
\hline 1,1,2-Trichloroethane & WA & & & $\mathbf{U}$ & 5 & & 5 & $\mu g / L$ \\
\hline 1,1,2-Trichloroethane & GE & & & $\mathbf{U}$ & 1 & & 5 & $\mu g / L$ \\
\hline 1,1-Dichloroethane & WA & & & $\mathbf{U}$ & 5 & & NS & $\mu g / L$ \\
\hline 1,1-Dichloroethane & GE & & & $\mathbf{U}$ & 1 & & NS & $\mu g / L$ \\
\hline 1,1-Dichloroethylene & WA & & & $\mathbf{U}$ & 5 & & 7 & $\mu \mathrm{g} / \mathrm{L}$ \\
\hline 1,1-Dichloroethylene & GE & & & $\mathbf{U}$ & 1 & & 7 & $\mu g / L$ \\
\hline 1,2,3-Trichloropropane & WA & & & $\mathbf{U}$ & 10 & & NS & $\mu g / L$ \\
\hline 1,2,3-Trichloropropane & GE & & & $\mathbf{U}$ & 1 & & NS & $\mu g / L$ \\
\hline 1,2-Dibromo-3-chloropropane & WA & & & $\mathrm{U}$ & 20 & & 0.2 & $\mu \mathrm{g} / \mathrm{L}$ \\
\hline 1,2-Dibromo-3-chloropropane & GE & & & $\mathbf{U}$ & 1 & & 0.2 & $\mu g / L$ \\
\hline 1,2-Dibromoethane & WA & & & $\mathbf{U}$ & 20 & & NS & $\mu g / L$ \\
\hline 1,2-Dibromoethane & GE & & & $\mathbf{U}$ & 20 & & NS & $\mu g / L$ \\
\hline 1,2-Dichloroethane & WA & & & U & 5 & & 5 & $\mu g / L$ \\
\hline 1,2-Dichloroethane & GE & & & $\mathbf{U}$ & 1 & & 5 & $\mu \mathrm{g} / \mathrm{L}$ \\
\hline 1,2-Dichlornethylene (total) & WA & & & U & 5 & & 100 & $\mu \mathrm{g} / \mathrm{L}$ \\
\hline 1,2-Dichloroethylene (total) & GE & & & $\mathbf{U}$ & 1 & & 100 & $\mu g / L$ \\
\hline 1,2-Dichloropropane & WA & & & $\mathrm{U}$ & 5 & & 5 & $\mu g / L$ \\
\hline 1,2-Dichloropropane & GE & & & $\mathbf{U}$ & 1 & & 5 & $\mu g / L$ \\
\hline 2-Hexanone & WA & & & $\mathbf{U}$ & 10 & & NS & $\mu g / L$ \\
\hline 2-Hexanone & GE & & & $\mathbf{U}$ & 1 & & NS & $\mu g / L$ \\
\hline 4-Methyl-2-pentanone & WA & & & U & 10 & & NS & $\mu g / L$ \\
\hline Acetone & WA & & & $\mathbf{U}$ & 10 & & NS & $\mu g / L$ \\
\hline Acetone & GE & & & $\mathbf{U}$ & 100 & & NS & $\mu g / L$ \\
\hline Acetonitrile & WA & & & U & 20 & & NS & $\mu g / L$ \\
\hline Acetonitrile & GE & & & $\mathbf{U}$ & 1 & & NS & $\mu g / L$ \\
\hline Acidity & WA & & & & 22 & & NS & $\mathrm{mg} / \mathrm{L}$ \\
\hline Acrolein & WA & & & $\mathrm{U}$ & 10 & & NS & $\mu g / L$ \\
\hline Acrolein & GE & & & $\mathrm{U}$ & 20 & & NS & $\mu g / L$ \\
\hline Acrylonitrile & WA & & & $\mathbf{U}$ & 10 & & NS & $\mu g / L$ \\
\hline Acrylonitrile & GE & & & $\mathbf{U}$ & 20 & & NS & $\mu g / L$ \\
\hline Actinium-228 & EP & & & $U$ & 30 & & NS & $\mathrm{pCi} / \mathrm{L}$ \\
\hline Alkalinity & WA & & & U & 0.5 & & NS & $\mathrm{mg} / \mathrm{L}$ \\
\hline Alkalinity & $\mathrm{GE}$ & & & & 1.92 & & NS & $\mathrm{mg} / \mathrm{L}$ \\
\hline Allyl chloride & WA & & & U & 100 & & NS & $\mu \mathrm{g} / \mathrm{L}$ \\
\hline Allyl chloride & GE & & & $\mathrm{U}$ & 50 & & NS & $\mu g / L$ \\
\hline Aluminum & WA & & & U & 200 & & NS & $\mu g / L$ \\
\hline Aluminum & GE & & & & 163 & & NS & $\mu g / L$ \\
\hline Antimony & WA & & & $\mathbf{U}$ & 60 & & 6 & $\mu g / L$ \\
\hline Antimony & GE & & & $\mathbf{U}$ & 2 & & 6 & $\mu g / L$ \\
\hline Antimony-125 & EP & & & $\mathbf{U}$ & 20 & & 300 & $\mathrm{pCi} / \mathrm{L}$ \\
\hline Arsenic & WA & & & U & 10 & & 50 & $\mu g / L$ \\
\hline Arsenic & GE & & & U & 2 & & 50 & $\mu \mathrm{g} / \mathrm{L}$ \\
\hline
\end{tabular}


Sampling and Analysis of Water From Upper Three Runs And Its Wetlands Near Tank 16 and the Mixed Waste Management Facility

UTR045 Laboratory Analyses (cont'd)

\begin{tabular}{|c|c|c|c|c|c|c|c|}
\hline Analyte & Lab & Rep & Qualifier & Result & Acc & Stand & Units \\
\hline Barium & WA & & $\bar{U}$ & 200 & & 2000 & $\mu g / L$ \\
\hline Barium & $\mathrm{GE}$ & & & 10.6 & & 2000 & $\mu g / L$ \\
\hline Benzene & WA & & U & 5 & & 5 & $\mu g / L$ \\
\hline Benzene & GE & & U & 1 & & 5 & $\mu g / L$ \\
\hline Beryllium & WA & & $\mathbf{U}$ & 5 & & 4 & $\mu g / \mathrm{L}$ \\
\hline Beryllium & GE & & $\mathbf{U}$ & 3 & . & 4 & $\mu g / L$ \\
\hline Bis(2-chloro-1-methylethyl)ether & GE & & $\mathbf{U}$ & 10 & & NS & $\mu g / L$ \\
\hline Bromodichloromethane & WA & & $\mathbf{U}$ & 5 & & NS & $\mu \mathrm{g} / \mathrm{L}$ \\
\hline Bromodichloromethane & $\mathrm{GE}$ & & $\mathbf{U}$ & 1 & & NS & $\mu \mathrm{g} / \mathrm{L}$ \\
\hline Bromoform & WA & & $\mathbf{U}$ & 5 & & NS & $\mu \mathrm{g} / \mathrm{L}$ \\
\hline Bromoform & GE & & $\mathbf{U}$ & 1 & & NS & $\mu \mathrm{g} / \mathrm{L}$ \\
\hline Cadmium & WA & & $\mathbf{U}$ & 5 & & 5 & $\mu \mathrm{g} / \mathrm{L}$ \\
\hline Cadmium & GE & & $\mathbf{U}$ & 2 & & 5 & $\mu g / L$ \\
\hline Calcium & WA & & $\mathbf{U}$ & 5000 & & NS & $\mu g / L$ \\
\hline Calcium & GE & & & 1080 & & NS & $\mu \mathrm{g} / \mathrm{L}$ \\
\hline Carbon disulfide & WA & . & $\mathbf{U}$ & 5 & & NS & $\mu g / L$ \\
\hline Carbon disulfide & GE & & $\mathbf{U}$ & 1 & & NS & $\mu g / L$ \\
\hline Carbon tetrachloride & WA & & $\mathbf{U}$ & 5 & & 5 & $\mu g / L$ \\
\hline Carbon tetrachloride & GE & & $\mathbf{U}$ & 1 & & 5 & $\mu g / L$ \\
\hline Cerium-144 & EP & & $\mathbf{U}$ & 60 & & 261 & $\mathrm{pCi} / \mathrm{L}$ \\
\hline Cesium-134 & EP & & $\mathbf{U}$ & 10 & & 81.3 & $\mathrm{pCi} / \mathrm{L}$ \\
\hline Cesium-137 & TM & & $\mathbf{U}$ & 6.5 & & 200 & $\mathrm{pCi} / \mathrm{L}$ \\
\hline Cesium-137 & EP & & & 40.2 & & 200 & $\mathrm{pCi} / \mathrm{L}$ \\
\hline Chloride & WA & & & 3.26 & & NS & $\mathrm{mg} / \mathrm{L}$ \\
\hline Chloride & GE & & & 2.4 & & NS & $\mathrm{mg} / \mathrm{L}$ \\
\hline Chlorobenzene & WA & & $\mathbf{U}$ & 5 & & 100 & $\mu g / L$ \\
\hline Chlorobenzene & GE & & $\mathbf{U}$ & 1 & & 100 & $\mu \mathrm{g} / \mathrm{L}$ \\
\hline Chloroethane & WA & & $\mathbf{U}$ & 10 & & NS & $\mu \mathrm{g} / \mathrm{L}$ \\
\hline Chloroethane & GE & & $\mathbf{U}$ & 1 & & NS & $\mu g / L$ \\
\hline Chloroform & WA & & $\mathbf{U}$ & 5 & & 100 & $\mu g / L$ \\
\hline Chloroform & GE & & U & 1 & & 100 & $\mu g / \mathrm{L}$ \\
\hline Chloroprene & WA & & $\mathbf{U}$ & 20 & & NS & $\mu g / L$ \\
\hline Chloroprene & $\mathrm{GE}$ & & $\mathbf{U}$ & 200 & & NS & $\mu \mathrm{g} / \mathrm{L}$ \\
\hline Chromium & WA & & $\mathbf{U}$ & 10 & & 100 & $\mu g / L$ \\
\hline Chromium & GE & & $\mathbf{U}$ & 4 & & 100 & $\mu g / L$ \\
\hline cis-1,3-Dichloropropene & WA & & $\mathbf{U}$ & 5 & & NS & $\mu g / L$ \\
\hline cis-1,3-Dichloropropene & GE & & U & 1 & & NS & $\mu g / L$ \\
\hline Cobalt & WA & & $\mathbf{U}$ & 50 & & NS & $\mu g / L$ \\
\hline Cobalt & GE & & U & 4 & & NS & $\mu g / L$ \\
\hline Cobalt-57 & $\mathrm{EP}$ & & U & 10 & & 1000 & $\mathrm{pCi} / \mathrm{L}$ \\
\hline Cobalt -60 & EP & & $\mathbf{U}$ & 10 & & 100 & $\mathrm{pCi} / \mathrm{L}$ \\
\hline Copper & WA & & $\mathrm{U}$ & 25 & & 1000 & $\mu \mathrm{g} / \mathrm{L}$ \\
\hline Copper & GE & & $\mathbf{U}$ & 4 & & 1000 & $\mu \mathrm{g} / \mathrm{L}$ \\
\hline Cyanide & WA & & $\mathbf{U}$ & 10 & & 200 & $\mu \mathrm{g} / \mathrm{L}$ \\
\hline Cyanide & GE & & $\mathbf{U}$ & 5 & & 200 & $\mu \mathrm{g} / \mathrm{L}$ \\
\hline Dibromochloromethane & WA & & U & 5 & & NS & $\mu g / L$ \\
\hline Dibromochloromethane & GE & & $\mathrm{U}$ & 1 & & NS & $\mu g / L$ \\
\hline Dichlorodifluoromethane & WA & & $\mathbf{U}$ & 10 & & NS & $\mu \mathrm{g} / \mathrm{L}$ \\
\hline Dichlorodifluoromethane & GE & & $\mathbf{U}$ & 1 & & NS & $\mu \mathrm{g} / \mathrm{L}$ \\
\hline Ethylbenzene & WA & & $\mathbf{U}$ & 5 & & 700 & $\mu \mathrm{g} / \mathrm{L}$ \\
\hline Ethylbenzene & $\mathrm{GE}$ & & $\mathbf{U}$ & 1 & & 700 & $\mu \mathrm{g} / \mathrm{L}$ \\
\hline Europium-152 & EP & & $\mathbf{U}$ & 40 & & NS & $\mathrm{pCi} / \mathrm{L}$ \\
\hline Europium-154 & EP & & $\mathbf{U}$ & 20 & & 200 & $\mathrm{pCi} / \mathrm{L}$ \\
\hline Europium-155 & EP & & $\mathrm{U}$ & 30 & & 600 & $\mathrm{pCi} / \mathrm{L}$ \\
\hline Fluoride & WA & & $\mathbf{U}$ & 0.1 & & 4 & $\mathrm{mg} / \mathrm{L}$ \\
\hline Fluoride & GE & & $\mathrm{U}$ & 0.1 & & 4 & $\mathrm{mg} / \mathrm{L}$ \\
\hline
\end{tabular}


UTR045 Laboratory Analyses (cont'd)

\begin{tabular}{|c|c|c|c|c|c|c|c|c|}
\hline Analyte & $\underline{L a b}$ & Rep & Avg & Qualifier & Result & Acc & Stand & Units \\
\hline Gross alpha & $\mathrm{TM}$ & & & $\bar{U}$ & 0.6 & & 15 & $\mathrm{pCi} / \mathrm{L}$ \\
\hline Gross alpha & GE & & NA & U & 2 & & 15 & $\mathrm{pCi} / \mathrm{L}$ \\
\hline Gross alpha & GE & & & $\mathbf{U}$ & 2 & & 15 & $\mathrm{pCi} / \mathrm{L}$ \\
\hline Iron & WA & & & & 507 & & 300 & $\mu g / L$ \\
\hline Iron & GE & & & & 547 & & 300 & $\mu g / L$ \\
\hline Isobutyl alcohol & WA & & & $\mathbf{U}$ & 20 & - & NS & $\mu \mathrm{g} / \mathrm{L}$ \\
\hline Isobutyl alcohol & GE & & & $\mathbf{U}$ & 100 & & NS & $\mu \mathrm{g} / \mathrm{L}$ \\
\hline Lead & WA & & & $U$ & 3 & & 50 & $\mu \mathrm{g} / \mathrm{L}$ \\
\hline Lead & GE & & & U & 3 & & 50 & $\mu g / L$ \\
\hline Lead-212 & EP & & & $\mathbf{U}$ & 15 & & NS & $\mathrm{pCi} / \mathrm{L}$ \\
\hline Magnesium & WA & & & $\mathbf{U}$ & 5000 & & NS & $\mu g / L$ \\
\hline Magnesium & GE & & & & 611 & & NS & $\mu g / L$ \\
\hline Manganese & WA & & & & 18.5 & & 50 & $\mu g / L$ \\
\hline Manganese & GE & & & & 16.4 & & 50 & $\mu g / L$ \\
\hline Manganese-54 & EP & & & $\mathbf{U}$ & 10 & & 300 & $\mathrm{pCi} / \mathrm{L}$ \\
\hline Mercury & WA & & & $\mathbf{U}$ & 0.2 & & 2 & $\mu \mathrm{g} / \mathrm{L}$ \\
\hline Mercury & GE & & & U & 0.2 & & 2 & $\mu \mathrm{g} / \mathrm{L}$ \\
\hline Methacrylonitrile & WA & & & $\mathbf{U}$ & 20 & & NS & $\mu g / \Omega$ \\
\hline Methacrylonitrile & GE & & & $\mathbf{U}$ & 50 & & NS & $\mu g / L$ \\
\hline Methyl bromide & WA & & & U & 10 & & NS & $\mu \mathrm{g} / \mathrm{L}$ \\
\hline Methyl bromide & GE & & & $\mathbf{U}$ & 1 & & NS & $\mu \mathrm{g} / \mathrm{L}$ \\
\hline Methyl chloride & WA & & & $\mathbf{U}$ & 10 & & NS & $\mu g / L$ \\
\hline Methyl chloride & GE & & & U & 1 & & NS & $\mu g / L$ \\
\hline Methyl ethyl ketone & WA & & & U & 10 & & NS & $\mu g / L$ \\
\hline Methyl ethyl ketone & GE & & & U & 1 & & NS & $\mu g / L$ \\
\hline Methyl iodide & WA & & & $\mathbf{U}$ & 10 & & NS & $\mu g / L$ \\
\hline Methyl iodide & GE & & & $\mathbf{U}$ & 15 & & NS & $\mu g / L$ \\
\hline Methyl isobutyl ketone & GE & & & $\mathbf{U}$ & 1 & & NS & $\mu g / L$ \\
\hline Methylene bromide & WA & & & $\mathbf{U}$ & 10 & & NS & $\mu g / L$ \\
\hline Methylene bromide & GE & & & $\mathbf{U}$ & 1 & & NS & $\mu g / L$ \\
\hline Methylene chloride & WA & & & U & 5 & & 5 & $\mu g / L$ \\
\hline Methylene chloride & GE & & & $\mathbf{U}$ & 1 & & 5 & $\mu g / L$ \\
\hline Nickel & WA & & & U & 40 & & 100 & $\mu \mathrm{g} / \mathrm{L}$ \\
\hline Nickel & GE & & & $\mathbf{U}$ & 4 & & 100 & $\mu g / L$ \\
\hline Nitrate as nitrogen & WA & & & & 0.275 & & 10 & $\mathrm{mg} / \mathrm{L}$ \\
\hline Nitrate as nitrogen & GE & & & & 2.1 & & 10 & $\mathrm{mg} / \mathrm{L}$ \\
\hline Nonvolatile beta & TM & & & U & 0.8 & & 50 & pCi/L \\
\hline Nonvolatile beta & GE & & NA & $\mathbf{U}$ & 2 & & 50 & $\mathrm{pCi} / \mathrm{L}$ \\
\hline Nonvolatile beta & GE & & & U & 2 & & 50 & $\mathrm{pCi} / \mathrm{L}$ \\
\hline pH & GE & & & $\mathbf{J}$ & 5.2 & & NS & PH \\
\hline Potassium & WA & & & $\mathrm{U}$ & 5000 & & NS & $\mu \mathrm{g} / \mathrm{L}$ \\
\hline Potassium & GE & & & $\mathbf{U}$ & 500 & & NS & $\mu g / L$ \\
\hline Potassium-40 & EP & & & $\mathbf{U}$ & 110 & & 300 & $\mathrm{pCi} / \mathrm{L}$ \\
\hline Promethium-144 & EP & & & $\mathbf{U}$ & 10 & & NS & $\mathrm{pCi} / \mathrm{L}$ \\
\hline Promethium-146 & EP & & & U & 10 & & NS & $\mathrm{pCi} / \mathrm{L}$ \\
\hline Propionitrile & WA & & & $\mathrm{U}$ & 50 & & NS & $\mu \mathrm{g} / \mathrm{L}$ \\
\hline Propionitrile & GE & & & U & 200 & & NS & $\mu \mathrm{g} / \mathrm{L}$ \\
\hline Ruthenium-106 & EP & & & $\mathbf{U}$ & 90 & & 30 & $\mathrm{pCi} / \mathrm{L}$ \\
\hline Selenium & WA & & & $\mathrm{U}$ & 5 & & 50 & $\mu g / L$ \\
\hline Selenium & GE & & & UJ & L & & 50 & $\mu g / L$ \\
\hline Silver & WA & & & $\mathbf{U}$ & 10 & & 50 & $\mu g / L$ \\
\hline Silver & GE & & & $\mathbf{U}$ & 2 & & 50 & $\mu \mathrm{g} / \mathrm{L}$ \\
\hline Sodium & WA & & & U & 5000 & & NS & $\mu g / L$ \\
\hline Sodium & GE & & & & 1260 & & NS & $\mu g / L$ \\
\hline Sodium-22 & EP & & & U & 10 & & 466 & $\mathrm{pCi} / \mathrm{L}$ \\
\hline
\end{tabular}


Sampling and Analysis of Water From Upper Three Runs And lis Wellands Near Tank 16 and the Mixed Waste Management Facility

UTR045 Laboratory Analyses (cont'd)

\begin{tabular}{|c|c|c|c|c|c|c|c|c|}
\hline Analyte & Lab & Rep & Avg & Qualifier & Result & Acc & Stand & Units \\
\hline Styrene & $\overline{\text { WA }}$ & & & $\overline{\mathbf{U}}$ & 5 & & 100 & $\mu \mathrm{g} / \mathrm{L}$ \\
\hline Styrene & GE & & & $\mathbf{U}$ & 1 & & 100 & $\mu \mathrm{g} / \mathrm{L}$ \\
\hline Sulfate & WA & & & $\mathbf{U}$ & 2.5 & & 400 & $\mathrm{mg} / \mathrm{L}$ \\
\hline Sulfate & GE & & & & 2.1 & & 400 & $\mathrm{mg} / \mathrm{L}$ \\
\hline Sulfide & WA & & & $\mathbf{U}$ & 0.1 & & NS & $\mathrm{mg} / \mathrm{L}$ \\
\hline Sulfide & GE & & NA & $\mathbf{U}$ & 1 & . & NS & $\mathrm{mg} / \mathrm{L}$ \\
\hline Sulfide & GE & & & $\mathbf{U}$ & 1 & & NS & $\mathrm{mg} / \mathrm{L}$ \\
\hline Tetrachloroethylene & WA & & & $\mathbf{U}$ & 5 & & 5 & $\mu \mathrm{g} / \mathrm{L}$ \\
\hline Tetrachloroethylene & GE & & & $\mathbf{U}$ & 1 & & 5 & $\mu g / L$ \\
\hline Thallium & WA & & & $\mathbf{U}$ & 10 & & 2 & $\mu \mathrm{g} / \mathrm{L}$ \\
\hline Thallium & GE & & & $\mathbf{U}$ & 2 & & 2 & $\mu g / L$ \\
\hline Thorium-234 & EP & & & $\mathbf{U}$ & 350 & & 401 & $\mathrm{pCi} / \mathrm{L}$ \\
\hline Toluene & WA & & & $\mathbf{J}$ & 1.09 & & 1000 & $\mu g / L$ \\
\hline Toluene & GE & & & & 1 & & 1000 & $\mu \mathrm{g} / \mathrm{L}$ \\
\hline Total hardness & WA & & & $\mathbf{U}$ & 1 & & NS & $\mathrm{mg} / \mathrm{L}$ \\
\hline Total hardness & GE & & & & 11 & & NS & $\mathrm{mg} / \mathrm{L}$ \\
\hline Total organic carbon & WA & & & & 3.9 & & NS & $\mathrm{mg} / \mathrm{L}$ \\
\hline Total organic carbon & $\mathrm{GE}$ & & & & 4.59 & & NS & $\mathrm{mg} / \mathrm{L}$ \\
\hline Total suspended solids & WA & & & $\mathbf{U}$ & 5 & & NS & $\mathrm{mg} / \mathrm{L}$ \\
\hline Total suspended solids & GE & & A & & 2 & & NS & $\mathrm{mg} / \mathrm{L}$ \\
\hline trans-1,3-Dichloropropene & WA & & & $\mathbf{U}$ & 5 & & NS & $\mu \mathrm{g} / \mathrm{L}$ \\
\hline trans-1,3-Dichloropropene & GE & & & $\mathbf{U}$ & 1 & & NS & $\mu g / L$ \\
\hline trans-1,4-Dichloro-2-butene & WA & & & $\mathbf{U}$ & 100 & & NS & $\mu \mathrm{g} / \mathrm{L}$ \\
\hline trans-1,4-Dichloro-2-butene & GE & & & $\mathbf{U}$ & 30 & & NS & $\mu \mathrm{g} / \mathrm{L}$ \\
\hline Trichloroethylene & WA & & & $\mathbf{U}$ & 5 & & 5 & $\mu \mathrm{g} / \mathrm{L}$ \\
\hline Trichloroethylene & $\mathrm{GE}$ & & & $\mathbf{U}$ & 1 & & 5 & $\mu \mathrm{g} / \mathrm{L}$ \\
\hline Trichlorofluoromethane & WA & & & $\mathbf{U}$ & 5 & & NS & $\mu g / L$ \\
\hline Trichlorofluoromethane & GE & & & $\mathrm{U}$ & 1 & & NS & $\mu \mathrm{g} / \mathrm{L}$ \\
\hline Tritium & TM & & & & 17.1 & 4.7 & 20 & $\mathrm{pCi} / \mathrm{ml}$ \\
\hline Tritium & GE & & A & & 15.05 & & 20 & $\mathrm{pCi} / \mathrm{ml}$ \\
\hline Turbidity & WA & & & & 1.51 & & NS & NTU \\
\hline Turbidity & GE & & $\mathbf{A}$ & & 2.07 & & NS & NTU \\
\hline Vanadium & WA & & & $\mathbf{U}$ & 50 & & NS & $\mu \mathrm{g} / \mathrm{L}$ \\
\hline Vanadium & GE & & & $\mathbf{U}$ & 8 & & NS & $\mu \mathrm{g} / \mathrm{L}$ \\
\hline Vinyl acetate & WA & & & $\mathbf{U}$ & 10 & & NS & $\mu \mathrm{g} / \mathrm{L}$ \\
\hline Vinyl acetate & $G E$ & & & $\mathbf{U}$ & 1 & & NS & $\mu \mathrm{g} / \mathrm{L}$ \\
\hline Vinyl chloride & WA & & & $\mathrm{U}$ & 10 & & 2 & $\mu \mathrm{g} / \mathrm{L}$ \\
\hline Vinyl chloride & $\mathbf{G E}$ & & & $\mathbf{U}$ & 1 & & 2 & $\mu \mathrm{g} / \mathrm{L}$ \\
\hline Xylenes (total) & WA & & & U & 5 & & 10000 & $\mu \mathrm{g} / \mathrm{L}$ \\
\hline Xylenes (total) & $\mathrm{GE}$ & & & U & 2 & & 10000 & $\mu \mathrm{g} / \mathrm{L}$ \\
\hline Yttrium-88 & $\mathrm{EP}$ & & & $\mathrm{U}$ & 60 & & NS & $\mathrm{pCi} / \mathrm{L}$ \\
\hline Zinc & WA & & & & 32.6 & & 5000 & $\mu g / L$ \\
\hline Zinc & GE & & & & 38.8 & & 5000 & $\mu \mathrm{g} / \mathrm{L}$ \\
\hline Zinc-65 & EP & & & $U$ & 20 & & 300 & $\mathrm{pCi} / \mathrm{L}$ \\
\hline
\end{tabular}




\section{UTR051A}

Field Measurements:

Sample Date: 4/13/93

pH: 6.88

Specific Conductance: $23 \mu \mathrm{S} / \mathrm{cm}$

Red/Oxid Potential: $191.5 \mathrm{mv}$

Dissolved Oxygen: $5.7 \mathrm{mg} / \mathrm{L}$

\begin{tabular}{|c|c|c|c|c|c|c|c|c|}
\hline Analyte & Lab & Rep & Avg & Qualifier & Result & Acc & Stand & Units \\
\hline 1,1,1,2-Tetrachloroethane & WA & & & $\mathbf{U}$ & 10 & & NS & $\mu \mathrm{g} / \mathrm{L}$ \\
\hline $1,1,1-$ Trichloroethane & WA & & & $\mathbf{U}$ & 5 & & 200 & $\mu \mathrm{g} / \mathrm{L}$ \\
\hline 1,1,2,2-Tetrachloroethane & WA & & & $\mathbf{U}$ & 5 & & NS & $\mu g / L$ \\
\hline 1,1,2-Trichloroethane & WA & & & $\mathbf{U}$ & 5 & & 5 & $\mu g / L$ \\
\hline 1,1-Dichloroethane & WA & & & $\mathbf{U}$ & 5 & & NS & $\mu g / L$ \\
\hline 1,1-Dichloroethylene & WA & & & U & 5 & & 7 & $\mu g / L$ \\
\hline 1,2,3-Trichloropropane & WA & & & $\mathbf{U}$ & 10 & & NS & $\mu g / L$ \\
\hline 1,2-Dibromo-3-chloropropane & WA & & . & $\mathbf{U}$ & 20 & & 0.2 & $\mu g / L$ \\
\hline 1,2-Dibromoethane & WA & & & U & 20 & & NS & $\mu g / L$ \\
\hline 1,2-Dichloroethane & WA & & & U & 5 & & $\begin{array}{c}5 \\
100\end{array}$ & $\mu g / L$ \\
\hline $\begin{array}{l}\text { 1,2-Dichloroethylene (total) } \\
\text { 1.2-Dichloropropane }\end{array}$ & WA & & & $\begin{array}{l}\mathbf{U} \\
\mathbf{U}\end{array}$ & $\begin{array}{l}5 \\
5\end{array}$ & & $\begin{array}{c}100 \\
5\end{array}$ & $\mu g / L$ \\
\hline 1,2-Dichloropropane & WA & & & $\begin{array}{l}\mathbf{U} \\
\mathbf{U}\end{array}$ & & & $\stackrel{5}{\text { NS }}$ & $\mu g / L$ \\
\hline $\begin{array}{l}\text { 2-Hexanone } \\
\text { 4-Methyl-2-pentanone }\end{array}$ & $\begin{array}{l}\text { WA } \\
\text { WA }\end{array}$ & & & $\begin{array}{l}\mathbf{U} \\
\mathbf{U}\end{array}$ & $\begin{array}{l}10 \\
10\end{array}$ & & NS & $\begin{array}{l}\mu g / L \\
\mu g / L\end{array}$ \\
\hline $\begin{array}{l}\text { 4-Methyl-2-pentanone } \\
\text { Acetone }\end{array}$ & $\begin{array}{l}\text { WA } \\
\text { WA }\end{array}$ & & & $\mathbf{U}$ & 10 & & NS & $\begin{array}{l}\mu g / L \\
\mu g / L\end{array}$ \\
\hline $\begin{array}{l}\text { Acetone } \\
\text { Acetonitrile }\end{array}$ & $\begin{array}{l}\text { WA } \\
\text { WA }\end{array}$ & & & $\mathbf{U}$ & 20 & & NS & $\mu g / L$ \\
\hline $\begin{array}{l}\text { Acetonitrile } \\
\text { Acidity }\end{array}$ & WA & & & & 7.5 & & NS & $\mathrm{mg} / \mathrm{L}$ \\
\hline $\begin{array}{l}\text { Acidity } \\
\text { Acrolein }\end{array}$ & WA & & & $\mathbf{U}$ & 10 & & NS & $\mu g / h$ \\
\hline $\begin{array}{l}\text { Actolen } \\
\text { Acrylonitrile }\end{array}$ & WA & & & U & 10 & & NS & $\mu g / L$ \\
\hline Alkalinity & WA & & & & 1 & & NS & $\mathrm{mg} / \mathrm{L}$ \\
\hline Allyl chloride & WA & & & $\mathbf{U}$ & 100 & & NS & $\mu g / L$ \\
\hline Aluminum & WA & & & $\mathbf{U}$ & 200 & & NS & $\mu g / L$ \\
\hline Antimony & WA & & & $\mathbf{U}$ & 60 & & 6 & $\mu g / L$ \\
\hline Arsenic & WA & & & $\mathbf{U}$ & 10 & & 50 & $\mu \mathrm{g} / \mathrm{L}$ \\
\hline Barium & WA & & & $\mathbf{U}$ & 200 & & 2000 & $\mu \mathrm{g} / \mathrm{L}$ \\
\hline Benzene & WA & & & $\mathbf{U}$ & 5 & & 5 & $\mu g / L$ \\
\hline Beryllium & WA & & & $\mathbf{U}$ & 5 & & 4 & $\mu g / L$ \\
\hline Bromodichloromethane & WA & & & U & 5 & & NS & $\mu \mathrm{g} / \mathrm{L}$ \\
\hline Bromoform & WA & & & $\mathbf{U}$ & 5 & & NS & $\mu g / L$ \\
\hline Cadmium & WA & & & $\mathbf{U}$ & 5 & & 5 & $\mu \mathrm{g} / \mathrm{L}$ \\
\hline Calcium & WA & & & $\mathbf{U}$ & 5000 & & NS & $\mu g / \mathrm{L}$ \\
\hline Carbon disulfide & WA & & & $\mathbf{U}$ & 5 & & NS & $\mu g / L$ \\
\hline Carbon tetrachloride & WA & & & U & 5 & & 5 & $\mu \mathrm{g} / \mathrm{L}$ \\
\hline Cesium-137 & TM & & NA & $\mathbf{U}$ & 8.6 & & 200 & $\mathrm{pCi} / \mathrm{L}$ \\
\hline Cesium-137 & TM & & & U & 8.9 & & 200 & $\mathrm{pCi} / \mathrm{L}$ \\
\hline Cnloride & WA & & A & & 3.465 & & NS & $\mathrm{mg} / \mathrm{L}$ \\
\hline Chlorobenzene & WA & & & $\mathbf{U}$ & 5 & & 100 & $\mu g / L$ \\
\hline Chloroethane & WA & & & $\mathbf{U}$ & 10 & & NS & $\mu g / L$ \\
\hline Chloroform & WA & & & U & 5 & & 100 & $\mu \mathrm{g} / \mathrm{L}$ \\
\hline Chloroprene & WA & & & $\mathrm{U}$ & 20 & & NS & $\mu g / L$ \\
\hline Chromium & WA & & & $\mathbf{U}$ & 10 & & 100 & $\mu g / L$ \\
\hline cis-1,3-Dichloropropene & WA & & & U & 5 & & NS & $\mu g / L$ \\
\hline Cobalt & WA & & & $\mathbf{U}$ & 50 & & NS & $\mu g / L$ \\
\hline Copper & WA & & & $\mathbf{U}$ & 25 & & 1000 & $\mu g / L$ \\
\hline Cyanide & WA & & & $\mathbf{U}$ & 10 & & 200 & $\mu g / L$ \\
\hline Dibromochloromethane & WA & & & U & 5 & & NS & $\mu g / L$ \\
\hline Dichlorodifluoromethane & WA & & & $\mathbf{U}$ & 10 & & NS & $\mu g / L$ \\
\hline Ethylbenzene & WA & & & $\mathbf{U}$ & 5 & & 700 & $\mu g / L$ \\
\hline
\end{tabular}


UTR051A Laboratory Analyses (cont'd)

\begin{tabular}{|c|c|c|c|c|c|c|c|c|}
\hline Analyte & Lab & Rep & Avg & Qualifier & Result & Acc & Stand & Units \\
\hline Fluoride & WA & & & $\bar{U}$ & 0.1 & & 4 & $\mathrm{mg} / \mathrm{L}$ \\
\hline Gross alpha & TM & & & & 0.8 & 0.75 & 15 & $\mathrm{pCi} / \mathrm{L}$ \\
\hline Iron & WA & & & $\mathbf{U}$ & 100 & & 300 & $\mu g / L$ \\
\hline Isobutyl alcohol & WA & & & $\mathbf{U}$ & 20 & & NS & $\mu g / L$ \\
\hline Lead & WA & & & $\mathbf{U}$ & 3 & & 50 & $\mu \mathrm{g} / \mathrm{L}$ \\
\hline Magnesium & WA & & & $\mathbf{U}$ & 5000 & • & NS & $\mu \mathrm{g} / \mathrm{L}$ \\
\hline Manganese & WA & & & & 17.1 & & 50 & $\mu \mathrm{g} / \mathrm{L}$ \\
\hline Mercury & WA & & & $\mathbf{U}$ & 0.2 & & 2 & $\mu \mathrm{g} / \mathrm{L}$ \\
\hline Methacrylonitrile & WA & & & $\mathbf{U}$ & 20 & & NS & $\mu \mathrm{g} / \mathrm{L}$ \\
\hline Methyl bromide & WA & & & $\mathbf{U}$ & 10 & & NS & $\mu g / L$ \\
\hline Methyl chloride & WA & & & $\mathbf{U}$ & 10 & & NS & $\mu g / L$ \\
\hline Methyl ethyl ketone & WA & & & $\mathbf{U}$ & 10 & & NS & $\mu g / L$ \\
\hline Methyl iodide & WA & & & $\mathbf{U}$ & 10 & & NS & $\mu g / L$ \\
\hline Methylene bromide & WA & & & $\mathbf{U}$ & 10 & & NS & $\mu \mathrm{g} / \mathrm{L}$ \\
\hline Methylene chloride & WA & & & $\mathbf{U}$ & 5 & & 5 & $\mu \mathrm{g} / \mathrm{L}$ \\
\hline Nickel & WA & & & $\mathbf{U}$ & 40 & & 100 & $\mu g / L$ \\
\hline Nitrate as nitrogen & WA & & & & 0.259 & & 10 & $\mathrm{mg} / \mathrm{L}$ \\
\hline Nonvolatile beta & TM & & A & & 1.15 & 1.65 & 50 & $\mathrm{pCi} / \mathrm{L}$ \\
\hline Potassium & WA & & & $\mathbf{U}$ & 5000 & & NS & $\mu \mathrm{g} / \mathrm{L}$ \\
\hline Propionitrile & WA & & & $\mathbf{U}$ & 50 & & NS & $\mu g / L$ \\
\hline Selenium & WA & & & $\mathbf{U}$ & 5 & & 50 & $\mu \mathrm{g} / \mathrm{L}$ \\
\hline Silver & WA & & & $\mathbf{U}$ & 10 & & 50 & $\mu \mathrm{g} / \mathrm{L}$ \\
\hline Sodium & WA & & & $\mathbf{U}$ & 5000 & & NS & $\mu \mathrm{g} / \mathrm{L}$ \\
\hline Styrene & WA & & & $\mathbf{U}$ & 5 & & 100 & $\mu \mathrm{g} / \mathrm{L}$ \\
\hline Sulfate & WA & & & & 17.2 & & 400 & $\mathrm{mg} / \mathrm{L}$ \\
\hline Sulfide & WA & & & $\mathbf{U}$ & 0.1 & & NS & $\mathrm{mg} / \mathrm{L}$ \\
\hline Sulfide & WA & & NA & $\mathbf{U}$ & 0.1 & & NS & $\mathrm{mg} / \mathrm{L}$ \\
\hline Tetrachloroethylene & WA & & & U & 5 & & 5 & $\mu \mathrm{g} / \mathrm{L}$ \\
\hline Thallium & WA & & & U & 10 & & 2 & $\mu g / L$ \\
\hline Toluene & WA & & & $\mathbf{U}$ & 5 & & 1000 & $\mu g / L$ \\
\hline Total hardness & WA & & & & 5 & & NS & $\mathrm{mg} / \mathrm{L}$ \\
\hline Total organic carbon & WA & & & & 0.778 & & NS & $\mathrm{mg} / \mathrm{L}$ \\
\hline Total suspended solids & WA & & & & 7 & & NS & $\mathrm{mg} / \mathrm{L}$ \\
\hline trans-1,3-Dichloropropene & WA & & & $\mathbf{U}$ & 5 & & NS & $\mu g / L$ \\
\hline trans-1,4-Dichloro-2-butene & WA & & & $\mathbf{U}$ & 100 & & NS & $\mu g / \Lambda$ \\
\hline Trichloroethylene & WA & & & $\mathbf{U}$ & 5 & & 5 & $\mu \mathrm{g} / \mathrm{L}$ \\
\hline Trichlorofluoromethane & WA & & & $\mathbf{U}$ & 5 & & NS & $\mu g / L$ \\
\hline Tritium & TM & & A & & 12.35 & 1.9 & 20 & $\mathrm{pCi} / \mathrm{ml}$ \\
\hline Turbidity & WA & & A & & 0.775 & & NS & NTU \\
\hline Vanadium & WA & & & $\mathbf{U}$ & 50 & & NS & $\mu g / L$ \\
\hline Vinyl acetate & WA & & & U & 10 & & NS & $\mu \mathrm{g} / \mathrm{L}$ \\
\hline Vinyl chloride & WA & & & $\mathbf{U}$ & 10 & & 2 & $\mu g / L$ \\
\hline Xylenes (total) & WA & & & U & 5 & & 10000 & $\mu g / L$ \\
\hline Zinc & WA & & & U & 20 & & 5000 & $\mu \mathrm{g} / \mathrm{L}$ \\
\hline
\end{tabular}




\section{UTR053}

Field Measurements:

Sample Date: 4/13/93

pH: 6.48

Specific Conductance: $30 \mu \mathrm{S} / \mathrm{cm}$

Red/Oxid Potential: $164.6 \mathrm{mv}$

Dissolved Oxygen: $7.6 \mathrm{mg} / \mathrm{L}$

\begin{tabular}{|c|c|c|c|c|c|c|c|}
\hline Analyte & Lab & Avg & Qualifier & Result & Acc & Stand & Units \\
\hline 1,1,1,2-Tetrachloroethane & WA & & $\mathrm{U}$ & 10 & & NS & $\mu g / L$ \\
\hline 1,1,1-Trichlorcethane & WA & & $\mathbf{U}$ & 5 & & 200 & $\mu g / L$ \\
\hline 1,1,2,2-Tetrachloroethane & WA & & $\mathbf{U}$ & 5 & & NS & $\mu g / L$ \\
\hline 1,1,2-Trichloroethane & WA & & $\mathbf{U}$ & 5 & & 5 & $\mu g / L$ \\
\hline 1,1-Dichloroethane & WA & & $\mathbf{U}$ & 5 & & NS & $\mu g / L$ \\
\hline 1,1-Dichloroethylene & WA & & $\mathbf{U}$ & 5 & & 7 & $\mu g / L$ \\
\hline 1,2,3-Trichloropropane & WA & & $\mathrm{U}$ & 10 & & NS & $\mu g / L$ \\
\hline 1,2-Dibromo-3-chloropropane & WA & & $\mathbf{U}$ & 20 & & 0.2 & $\mu g / L$ \\
\hline 1,2-Dibromoethane & WA & & $\mathbf{U}$ & 20 & & NS & $\mu g / L$ \\
\hline 1,2-Dichloroethane & WA & & $\mathbf{U}$ & 5 & & 5 & $\mu g / L$ \\
\hline 1,2-Dichloroethylene (total) & WA & & U & 5 & & 100 & $\mu g / L$ \\
\hline 1,2-Dichloropropane & WA & & $\mathbf{U}$ & 5 & & 5 & $\mu g / L$ \\
\hline 2-Hexanone & WA & & U & 10 & & NS & $\mu g / L$ \\
\hline 4-Methyl-2-pentanone & WA & & $\mathbf{U}$ & 10 & & NS & $\mu g / L$ \\
\hline Acetone & WA & & $\mathbf{U}$ & 10 & & NS & $\mu g / L$ \\
\hline Acetonitrile & WA & & $\mathbf{U}$ & 20 & & NS & $\mu g / L$ \\
\hline Acidity & WA & & & 2 & & NS & $\mathrm{mg} / \mathrm{L}$ \\
\hline Acrolein & WA & & $\mathbf{U}$ & 10 & & NS & $\mu \mathrm{g} / \mathrm{L}$ \\
\hline Acrylonitrile & WA & & $\mathbf{U}$ & 10 & & NS & $\mu g / L$ \\
\hline Alkalinity & WA & & & 5 & & NS & $\mathrm{mg} / \mathrm{L}$ \\
\hline Allyl chloride & WA & & $\mathbf{U}$ & 100 & & NS & $\mu \mathrm{g} / \mathrm{L}$ \\
\hline Aluminum & WA & & $\mathbf{U}$ & 200 & & NS & $\mu \mathrm{g} / \mathrm{L}$ \\
\hline Americium-241 & TM & & & 35.6 & 7.6 & 6.34 & $\mathrm{pCi} / \mathrm{L}$ \\
\hline Antimony & WA & & $\mathbf{U}$ & 60 & & 6 & $\mu g / L$ \\
\hline Arsenic & WA & & $\mathbf{U}$ & 10 & & 50 & $\mu g / L$ \\
\hline Barium & WA & & $\mathbf{U}$ & 200 & & 2000 & $\mu_{g} / L$ \\
\hline Benzene & WA & & $\mathbf{U}$ & 5 & & 5 & $\mu g / L$ \\
\hline Beryllium & WA & & $\mathbf{U}$ & 5 & & 4 & $\mu \mathrm{g} / \mathrm{L}$ \\
\hline Bromodichloromethane & WA & & U & 5 & & NS & $\mu \mathrm{g} / \mathrm{L}$ \\
\hline Bromoform & WA & & $\mathbf{U}$ & 5 & & NS & $\mu g / L$ \\
\hline Cadmium & WA & & $\mathbf{U}$ & 5 & & 5 & $\mu \mathrm{g} / \mathrm{L}$ \\
\hline Calcium & WA & & & 5100 & & NS & $\mu g / L$ \\
\hline Carbon disulfide & WA & & $\mathbf{U}$ & 5 & & NS & $\mu g / L$ \\
\hline Carbon tetrachloride & WA & & $\mathbf{U}$ & 5 & & 5 & $\mu g / L$ \\
\hline Cesium-137 & $\mathrm{TM}$ & & $\mathbf{U}$ & 10 & & 200 & $\mathrm{pCi} / \mathrm{L}$ \\
\hline Chloride & WA & & & 2.86 & & NS & $\mathrm{mg} / \mathrm{L}$ \\
\hline Chlorobenzene & WA & & $\mathbf{U}$ & 5 & & 100 & $\mu g / L$ \\
\hline Chloroethane & WA & & $\mathrm{U}$ & 10 & & NS & $\mu \mathrm{g} / \mathrm{L}$ \\
\hline Chloroform & WA & & $\mathbf{U}$ & 5 & & 100 & $\mu \mathrm{g} / \mathrm{L}$ \\
\hline Chloroprene & WA & & $\mathbf{U}$ & 20 & & NS & $\mu \mathrm{g} / \mathrm{L}$ \\
\hline Chromium & WA & & $\mathbf{U}$ & 10 & & 100 & $\mu \mathrm{g} / \mathrm{L}$ \\
\hline cis-1,3-Dichloropropene & WA & & $\mathbf{U}$ & 5 & & NS & $\mu g / L$ \\
\hline Cobalt & WA & & $\mathbf{U}$ & 50 & & NS & $\mu \mathrm{g} / \mathrm{L}$ \\
\hline Copper & WA & & $\mathbf{U}$ & 25 & & 1000 & $\mu \mathrm{g} / \mathrm{L}$ \\
\hline Cyanide & WA & & $\mathbf{U}$ & 10 & & 200 & $\mu g / L$ \\
\hline Dibromochloromethane & WA & & $\mathbf{U}$ & 5 & & NS & $\mu g / L$ \\
\hline Dichlorodifluoromethane & WA & & $\mathbf{U}$ & 10 & & NS & $\mu g / L$ \\
\hline Ethylbenzene & WA & & $\mathbf{U}$ & 5 & & 700 & $\mu \mathrm{g} / \mathrm{L}$ \\
\hline Fluoride & WA & & $\mathbf{U}$ & 0.1 & & 4 & $\mathbf{m g} / \mathbf{L}$ \\
\hline
\end{tabular}


Sampling and Analysis of Water From Upper Three Runs And Its Wetlands Near Tank 16 and the Mixed Waste Management Facility

UTR053 Laboratory Analyses (cont'd)

\begin{tabular}{|c|c|c|c|c|c|c|c|c|}
\hline Analyte & Lab & Rep & Avg & Qualifier & Result & Acc & Stand & Units \\
\hline Gross alpha & $T M$ & & & $\mathrm{U}$ & 0.5 & & 15 & $\overline{\mathrm{pCi} / \mathrm{L}}$ \\
\hline Iron & WA & & & $\mathbf{U}$ & 100 & & 300 & $\mu g / L$ \\
\hline Isobutyl alcohol & WA & & & $\mathbf{U}$ & 20 & & NS & $\mu g / L$ \\
\hline Lead & WA & & & U & 3 & & 50 & $\mu g / L$ \\
\hline Magnesium & WA & & & $\bar{U}$ & 5000 & & NS & $\mu \mathrm{g} / \mathrm{L}$ \\
\hline Manganese & WA & & & $\mathbf{U}$ & 15 & . & 50 & $\mu g / L$ \\
\hline Mercury & WA & & & $\mathbf{U}$ & 0.2 & & 2 & $\mu g / L$ \\
\hline Methacrylonitrile & WA & & & $\mathbf{U}$ & 20 & & NS & $\mu g / L$ \\
\hline Methyl bromide & WA & & & $\mathbf{U}$ & 10 & & NS & $\mu g / L$ \\
\hline Methyl chloride & WA & & & $\mathbf{U}$ & 10 & & NS & $\mu g / L$ \\
\hline Methyl ethyl ketone & WA & & & $\mathbf{U}$ & 10 & & NS & $\mu g / L$ \\
\hline Methyl iodide & WA & & & $\mathbf{U}$ & 10 & & NS & $\mu g / L$ \\
\hline Methylene bromide & WA & & & $\mathbf{U}$ & 10 & & NS & $\mu g / L$ \\
\hline Methylene chloride & WA & & & $\mathbf{U}$ & 5 & & 5 & $\mu g / L$ \\
\hline Nickel & WA & & & U & 40 & & 100 & $\mu g / L$ \\
\hline Nitrate as nitrogen & WA & & & & 0.402 & & 10 & $\mathrm{mg} / \mathrm{L}$ \\
\hline Nonvolatile beta & $\mathbf{T M}$ & & & $\mathbf{U}$ & 0.7 & & 50 & $\mathrm{pCi} / \mathrm{L}$ \\
\hline Potassium & WA & & & $\mathbf{U}$ & 5000 & & NS & $\mu g / L$ \\
\hline Propionitrile & WA & & & $\mathbf{U}$ & 50 & & NS & $\mu g / L$ \\
\hline Selenium & WA & & & $\mathbf{U}$ & 5 & & 50 & $\mu g / L$ \\
\hline Silver & WA & & & $\mathbf{U}$ & 10 & & 50 & $\mu g / L$ \\
\hline Sodium & WA & & & $\mathbf{U}$ & 5000 & & NS & $\mu g / L$ \\
\hline Styrene & WA & & & $\mathbf{U}$ & 5 & & 100 & $\mu g / L$ \\
\hline Sulfate & WA & & & $\mathbf{U}$ & 2.5 & & 400 & $\mathrm{mg} / \mathrm{L}$ \\
\hline Sulfide & WA & & & $\mathbf{U}$ & 0.1 & & NS & $\mathrm{mg} / \mathrm{L}$ \\
\hline Tetrachloroethylene & WA & & & $\mathbf{U}$ & 5 & & 5 & $\mu g / L$ \\
\hline Thallium & WA & & & $\mathbf{U}$ & 10 & & 2 & $\mu g / L$ \\
\hline Toluene & WA & & & $\mathbf{U}$ & 5 & & 1000 & $\mu g / L$ \\
\hline Total hardness & WA & & & & 7 & & NS & $\mathrm{mg} / \mathrm{L}$ \\
\hline Total organic carbon & WA & & & $\mathbf{U}$ & 0.5 & & NS & $\mathrm{mg} / \mathrm{L}$ \\
\hline Total suspended solids & WA & & & $\mathbf{U}$ & 5 & & NS & $\mathrm{mg} / \mathrm{L}$ \\
\hline trans-1,3-Dichloropropene & WA & & & $\mathbf{U}$ & 5 & & NS & $\mu \mathrm{g} / \mathrm{L}$ \\
\hline trans-1,4-Dichloro-2-butene & WA & & & $\mathbf{U}$ & 100 & & NS & $\mu g / L$ \\
\hline Trichloroethylene & WA & & & $\mathbf{U}$ & 5 & & 5 & $\mu \mathrm{g} / \mathrm{L}$ \\
\hline Trichlorofluoromethane & WA & & & $\mathbf{U}$ & 5 & & NS & $\mu \mathrm{g} / \mathrm{L}$ \\
\hline Tritium & $\mathrm{TM}$ & & & & 11.1 & 1.8 & 20 & $\mathrm{pCi} / \mathrm{ml}$ \\
\hline Turbidity & WA & & & & 0.78 & & NS & NTU \\
\hline Vanadium & WA & & & $\mathbf{U}$ & 50 & & NS & $\mu \mathrm{g} / \mathrm{L}$ \\
\hline Vinyl acetate & WA & & & $\mathbf{U}$ & 10 & & NS & $\mu g / L$ \\
\hline Vinyl chloride & WA & & & $\mathbf{U}$ & 10 & & 2 & $\mu g / L$ \\
\hline Xylenes (total) & WA & & & $\mathbf{U}$ & 5 & & 10000 & $\mu \mathrm{g} / \mathrm{L}$ \\
\hline Zinc & WA & & & $\mathrm{U}$ & 20 & & 5000 & $\mu \mathrm{g} / \mathrm{L}$ \\
\hline
\end{tabular}




\section{UTR056}

Field Measurements:

Sample Date: 4/13/93

pH: 6.61

Specific Conductance: $43 \mu \mathrm{S} / \mathrm{cm}$

Red/Oxid Potential: $\mathbf{4 1 . 5} \mathrm{mv}$

Dissolved Oxygen: $3.1 \mathrm{mg} / \mathrm{L}$

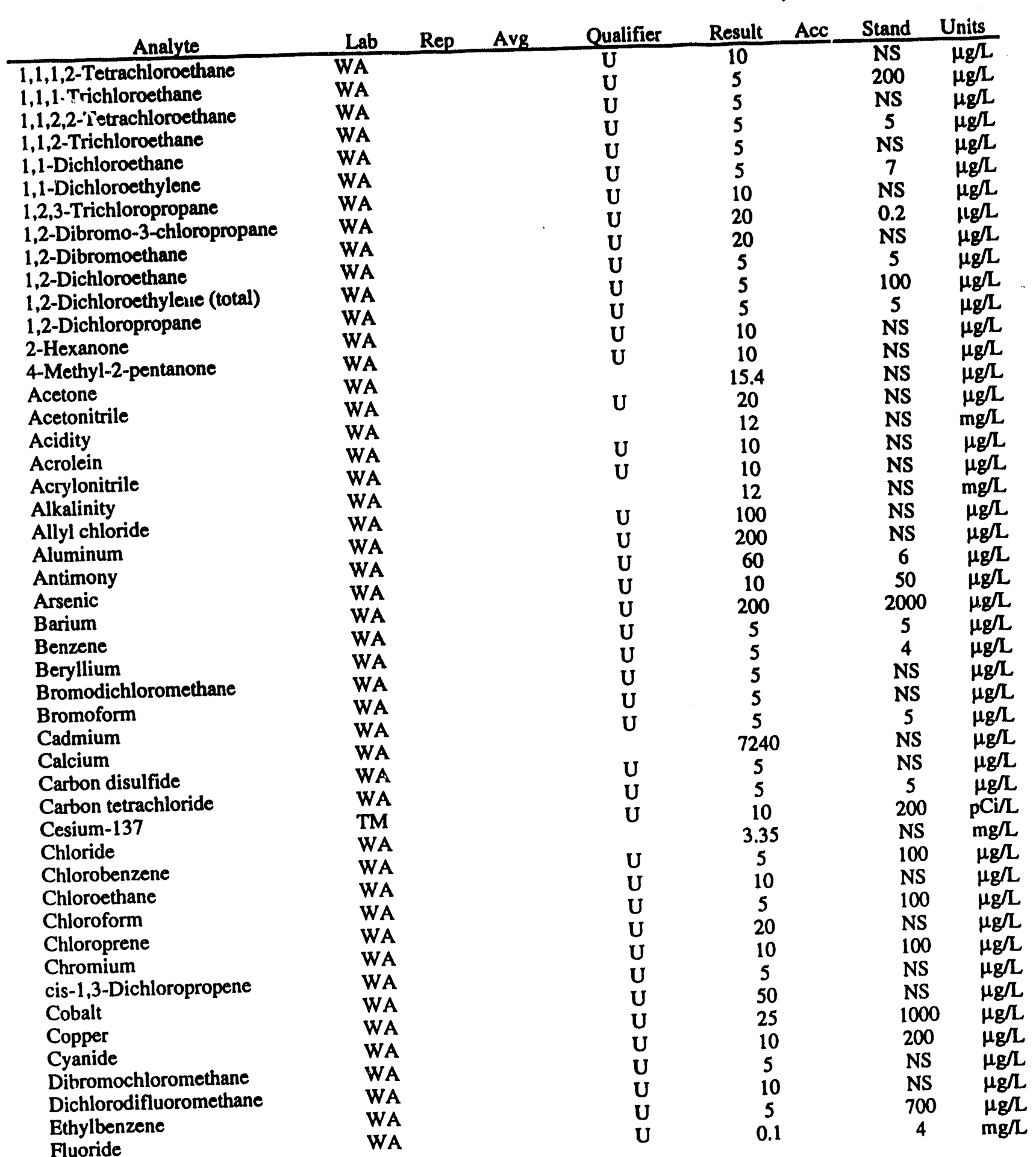


UTR056 Laboratory Analyses (cont'd)

\begin{tabular}{|c|c|c|c|c|c|c|c|}
\hline Analyte & Lab & Avg & Qualifier & Result & Acc & Stand & Units \\
\hline Gross alpha & $\mathrm{TM}$ & & $\mathbf{U}$ & 0.5 & & 15 & $\overline{\mathrm{pCi} / \mathrm{L}}$ \\
\hline Iron & WA & & & 969 & & 300 & $\mu g / L$ \\
\hline Isobutyl alcohol & WA & & $\mathbf{U}$ & 20 & & NS & $\mu g / L$ \\
\hline Lead & WA & & $\mathbf{U}$ & 3 & & 50 & $\mu g / L$ \\
\hline Magnesium & WA & & $\mathbf{U}$ & 5000 & & NS & $\mu g / L$ \\
\hline Manganese & WA & & & 52 & . & 50 & $\mu g / L$ \\
\hline Mercury & WA & & $\mathbf{U}$ & 0.2 & & 2 & $\mu g / L$ \\
\hline Methacrylonitrile & WA & & $\mathbf{U}$ & 20 & & NS & $\mu g / L$ \\
\hline Methyl bromide & WA & & $\mathbf{U}$ & 10 & & NS & $\mu g / L$ \\
\hline Methyl chloride & WA & & $\mathbf{U}$ & 10 & & NS & $\mu g / L$ \\
\hline Methyl ethyl ketone & WA & & $\mathbf{U}$ & 10 & & NS & $\mu g / L$ \\
\hline Methyl iodide & WA & & $\mathbf{U}$ & 10 & & NS & $\mu g / L$ \\
\hline Methylene bromide & WA & & $\mathbf{U}$ & 10 & & NS & $\mu g / L$ \\
\hline Methylene chloride & WA & & $\mathbf{U}$ & 5 & & 5 & $\mu g / L$ \\
\hline Nickel & WA & & $\mathbf{U}$ & 40 & & 100 & $\mu g / L$ \\
\hline Nitrate as nitrogen & WA & & & 0.11 & & 10 & $\mathrm{mg} / \mathrm{L}$ \\
\hline Nonvolatile beta & TM & & $\mathbf{U}$ & 0.7 & & 50 & $\mathrm{pCi} / \mathrm{L}$ \\
\hline Potassium & WA & & $\mathbf{U}$ & 5000 & & NS & $\mu g / L$ \\
\hline Propionitrile & WA & & $\mathbf{U}$ & 50 & & NS & $\mu g / L$ \\
\hline Selenium & WA & & $\mathbf{U}$ & 5 & & 50 & $\mu g / L$ \\
\hline Silver & WA & & $\mathbf{U}$ & 10 & & 50 & $\mu g / L$ \\
\hline Sodium & WA & & $\mathbf{U}$ & 5000 & & NS & $\mu g / L$ \\
\hline Styrene & WA & & $\mathbf{U}$ & 5 & & 100 & $\mu g / L$ \\
\hline Sulfate & WA & & $\mathbf{U}$ & 2.5 & & 400 & $\mathrm{mg} / \mathrm{L}$ \\
\hline Sulfide & WA & & $\mathbf{U}$ & 0.1 & & NS & $\mathrm{mg} / \mathrm{L}$ \\
\hline Tetrachloroethylene & WA & & $\mathbf{U}$ & 5 & & 5 & $\mu g / L$ \\
\hline Thallium & WA & & $\mathbf{U}$ & 10 & & 2 & $\mu g / L$ \\
\hline Toluene & WA & & $\mathbf{J}$ & 2.38 & & 1000 & $\mu g / L$ \\
\hline Total hardness & WA & & & 9 & & NS & $\mathrm{mg} / \mathrm{L}$ \\
\hline Total organic carbon & WA & & & 4.18 & & NS & $\mathbf{m g / L}$ \\
\hline Total suspended solids & WA & & & 6 & & NS & $m g / L$ \\
\hline trans-1,3-Dichloropropene & WA & & $\mathbf{U}$ & 5 & & NS & $\mu g / L$ \\
\hline trans-1,4-Dichloro-2-butene & WA & & $\mathbf{U}$ & 100 & & NS & $\mu g / L$ \\
\hline Trichloroethylene & WA & & $\mathbf{U}$ & 5 & & 5 & $\mu g / L$ \\
\hline Trichlorofluoromethane & WA & & $\mathbf{U}$ & 5 & & NS & $\mu g / L$ \\
\hline Tritium & $\mathbf{T M}$ & & & 10.5 & 1.6 & 20 & $\mathrm{pCi} / \mathrm{ml}$ \\
\hline Turbidity & WA & & & 1.68 & & NS & NTU \\
\hline Vanadium & WA & & $\mathbf{U}$ & 50 & & NS & $\mu g / L$ \\
\hline Vinyl acetate & WA & & $\mathbf{U}$ & 10 & & NS & $\mu g / L$ \\
\hline Vinyl chloride & WA & & $\mathbf{U}$ & 10 & & 2 & $\mu g / L$ \\
\hline Xylenes (total) & WA & & $\mathbf{U}$ & 5 & & 10000 & $\mu g / L$ \\
\hline Zinc & WA & & $\mathbf{U}$ & 20 & & 5000 & $\mu g / L$ \\
\hline
\end{tabular}




\section{UTR059}

Field Measurements:

Sample Date: 4/13/93

pH: 8.7

Specific Conductance: $27 \mu \mathrm{S} / \mathrm{cm}$

Red/Oxid Potential: $179 \mathrm{mv}$

Dissolved Oxygen: $3.3 \mathrm{mg} / \mathrm{L}$

\begin{tabular}{|c|c|c|c|c|c|c|c|}
\hline Analyte & Lab & Rep & Qualifier & Result & Acc & Stand & Units \\
\hline 1,1,1,2-Tetrachloroethane & WA & & $\mathbf{U}$ & 10 & & NS & $\mu g / L$ \\
\hline 1,1,1-Trichloroethane & WA & & $\mathbf{U}$ & 5 & & 200 & $\mu g / L$ \\
\hline $1,1,2,2$-Tetrachloroethane & WA & & $\mathbf{U}$ & 5 & & NS & $\mu g / L$ \\
\hline 1,1,2-Trichloroethane & WA & & $\mathbf{U}$ & 5 & & 5 & $\mu \mathrm{g} / \mathrm{L}$ \\
\hline 1,1-Dichloroethane & WA & & $\mathbf{U}$ & 5 & & NS & $\mu g / L$ \\
\hline 1,1-Dichloroethylene & WA & & $\begin{array}{l}\mathbf{U} \\
\mathbf{U}\end{array}$ & 5 & & 7 & $\mu g / L$ \\
\hline 1,2,3-Trichloropropane & WA & & $\underset{\mathbf{U}}{\mathbf{U}}$ & 10 & & NS & $\mu g / L$ \\
\hline 1,2-Dibromo-3-chloropropane & WA & & $\underset{\mathbf{U}}{\mathbf{U}}$ & $\begin{array}{l}20 \\
20\end{array}$ & & 0.2 & $\mu \mathrm{g} / \mathrm{L}$ \\
\hline & $\begin{array}{l}\text { WA } \\
\text { WA }\end{array}$ & & $\begin{array}{l}\mathbf{U} \\
\mathbf{U}\end{array}$ & $\begin{array}{c}20 \\
5\end{array}$ & & $\begin{array}{c}\text { NS } \\
5\end{array}$ & $\mu g / L$ \\
\hline $\begin{array}{l}\text { 1,2-Dichloroethane } \\
\text { 1,2-Dichloroethylene (total) }\end{array}$ & $\begin{array}{l}\text { WA } \\
\text { WA }\end{array}$ & & $\mathbf{U}$ & 5 & & $\begin{array}{c}5 \\
100\end{array}$ & $\begin{array}{l}\mu g / L \\
\mu g / L\end{array}$ \\
\hline & WA & & $\mathbf{U}$ & 5 & & 5 & $\begin{array}{l}\mu \mathrm{g} / \mathrm{L} \\
\mu \mathrm{g} / \mathrm{L}\end{array}$ \\
\hline 2-Hexanone & WA & & $\mathbf{U}$ & 10 & & NS & $\mu \mathrm{g} / \mathrm{L}$ \\
\hline 4-Methyl-2-pentanone & WA & & $\mathbf{U}$ & 10 & & NS & $\mu g / L$ \\
\hline Acetone & WA & & $\mathbf{U}$ & 10 & & NS & $\mu g / L$ \\
\hline Acetonitrile & WA & & $\mathbf{U}$ & 20 & & NS & $\begin{array}{l}\mu g / L \\
\mu g / L\end{array}$ \\
\hline Acidity & WA & & & 4 & & NS & $\begin{array}{l}\mu \mathrm{g} / \mathrm{L} \\
\mathrm{mg} / \mathrm{L}\end{array}$ \\
\hline Acrolein & WA & & $\mathbf{U}$ & 10 & & NS & $\begin{array}{l}\mathrm{mg} / \mathrm{L} \\
\mu \mathrm{g} / \mathrm{L}\end{array}$ \\
\hline $\begin{array}{l}\text { Acrolein } \\
\text { Acrylonitrile }\end{array}$ & WA & & $\mathbf{U}$ & 10 & & NS & \\
\hline $\begin{array}{l}\text { Acryloniture } \\
\text { Alkalinity }\end{array}$ & WA & & & 1 & & NS & $\begin{array}{l}\mu \mathrm{g} / \mathrm{L} \\
\mathrm{mg} / \mathrm{L}\end{array}$ \\
\hline $\begin{array}{l}\text { Alkalinity } \\
\text { Allyl chloride }\end{array}$ & WA & & $\mathbf{U}$ & 100 & & NS & $\begin{array}{l}\mathrm{mg} / \mathrm{L} \\
\mu \mathrm{g} / \mathrm{L}\end{array}$ \\
\hline $\begin{array}{l}\text { Allyi cnionae } \\
\text { Aluminum }\end{array}$ & WA & & $\mathbf{U}$ & 200 & & NS & $\mu g / L$ \\
\hline $\begin{array}{l}\text { Aluminum } \\
\text { Antimony }\end{array}$ & WA & & $\mathbf{U}$ & 60 & & 6 & $\mu g / L$ \\
\hline $\begin{array}{l}\text { Antimony } \\
\text { Arsenic }\end{array}$ & WA & & $\mathbf{U}$ & 10 & & $\begin{array}{l}0 \\
50\end{array}$ & $\mu g / L$ \\
\hline $\begin{array}{l}\text { Arsenic } \\
\text { Barium }\end{array}$ & WA & & $\mathbf{U}$ & 200 & & $\begin{array}{c}50 \\
2000\end{array}$ & $\mu g / L$ \\
\hline $\begin{array}{l}\text { Barium } \\
\text { Benzene }\end{array}$ & WA & & $\mathbf{U}$ & 5 & & $\begin{array}{c}2000 \\
5\end{array}$ & $\mu g / L$ \\
\hline $\begin{array}{l}\text { Benzene } \\
\text { Beryllium }\end{array}$ & WA & & $\mathbf{U}$ & $\begin{array}{l}5 \\
5\end{array}$ & & $\begin{array}{l}5 \\
4\end{array}$ & $\mu g / L$ \\
\hline & WA & & $\mathbf{U}$ & & & 4 & $\mu g / L$ \\
\hline $\begin{array}{l}\text { Bromodichloromethane } \\
\text { Bromoform }\end{array}$ & WA & & $\mathbf{U}$ & $\begin{array}{l}5 \\
5\end{array}$ & & NS & $\mu \mathrm{g} / \mathrm{L}$ \\
\hline $\begin{array}{l}\text { Bromoform } \\
\text { Cadmium }\end{array}$ & WA & & $\mathbf{U}$ & $\begin{array}{l}5 \\
5\end{array}$ & & NS & $\mu g / L$ \\
\hline $\begin{array}{l}\text { Cadmium } \\
\text { Calcium }\end{array}$ & WA & & $\mathbf{U}$ & $\begin{array}{c}5 \\
5000\end{array}$ & & $\begin{array}{l}5 \\
\text { NS }\end{array}$ & $\mu g / L$ \\
\hline & WA & & $\mathbf{U}$ & $\begin{array}{c}5000 \\
5\end{array}$ & & NS & $\mu g / L$ \\
\hline $\begin{array}{l}\text { Carbon disulfide } \\
\text { Carbon tetrachloride }\end{array}$ & WA & & $\mathbf{U}$ & $\begin{array}{l}5 \\
5\end{array}$ & & NS & $\mu g / L$ \\
\hline $\begin{array}{l}\text { Carbon tetrachloride } \\
\text { Cesium-137 }\end{array}$ & $\mathrm{TM}$ & & $\mathbf{U}$ & $\begin{array}{c}5 \\
9.6\end{array}$ & & 5 & $\mu g / L$ \\
\hline $\begin{array}{l}\text { Cesium-137 } \\
\text { Chloride }\end{array}$ & WA & & & $\begin{array}{l}9.6 \\
3.51\end{array}$ & & 200 & $\mathrm{pCi} / \mathrm{L}$ \\
\hline & WA & & $\mathbf{U}$ & & & NS & $\mathrm{mg} / \mathrm{L}$ \\
\hline $\begin{array}{l}\text { Chlorobenzene } \\
\text { Chloroethane }\end{array}$ & WA & & U & $\begin{array}{c}5 \\
10\end{array}$ & & 100 & $\mu g / L$ \\
\hline $\begin{array}{l}\text { Chloroethane } \\
\text { Chloroform }\end{array}$ & WA & & $\mathrm{U}$ & & & NS & $\mu \mathrm{g} / \mathrm{L}$ \\
\hline $\begin{array}{l}\text { Chloroform } \\
\text { Chloroprene }\end{array}$ & WA & & $\mathbf{U}$ & $\begin{array}{c}5 \\
20\end{array}$ & & 100 & $\mu g / L$ \\
\hline $\begin{array}{l}\text { Chloroprene } \\
\text { Chromium }\end{array}$ & WA & & $\mathrm{U}$ & 20 & & NS & $\mu g / L$ \\
\hline $\begin{array}{l}\text { Chromium } \\
\text { cis-1,3-Dichloropropene }\end{array}$ & WA & & $\mathrm{U}$ & 10 & & 100 & $\mu \mathrm{g} / \mathrm{L}$ \\
\hline $\begin{array}{l}\text { cis-1,3-Dichloropropene } \\
\text { Cobalt }\end{array}$ & WA & & U & 5 & & NS & $\mu \mathrm{g} / \mathrm{L}$ \\
\hline Cobalt & WA & & $\begin{array}{l}U \\
U\end{array}$ & 50 & & NS & $\mu g / L$ \\
\hline Copper & WA & & U & 25 & & 1000 & $\mu g / L$ \\
\hline Cyanide & WA & & U & 10 & & 200 & $\mu g$ \\
\hline Dibromochloromethane & $\begin{array}{l}\text { WA } \\
\text { WA }\end{array}$ & & U & 5 & & NS & $\mu \mathrm{g} / \mathrm{L}$ \\
\hline Dichlorodifluoromethane & WA & & U & 10 & & NS & $\mu g / L$ \\
\hline Ethylbenzene & WA & & U & 5 & & 700 & $\mu g$ \\
\hline Fluoride & WA & & $\mathbf{U}$ & 0.1 & & 4 & $\mathrm{mg} / \mathrm{L}$ \\
\hline
\end{tabular}


UTR056 Laboratory Analyses (cont'd)

\begin{tabular}{|c|c|c|c|c|c|c|c|c|}
\hline Analyte & Lab & Rep & Avg & Qualifier & Result & Acc & Stand & Units \\
\hline Gross alpha & TM & & & $\mathrm{U}$ & 0.5 & & 15 & $\overline{\mathrm{pCi} / \mathrm{I}}$ \\
\hline Iron & WA & & & $\mathbf{U}$ & 100 & & 300 & $\mu g \Omega$ \\
\hline Isobutyl alcohol & WA & & & $\mathbf{U}$ & 20 & & NS & $\mu g / L$ \\
\hline Lead & WA & & & $\mathbf{U}$ & 3 & & 50 & $\mu \mathrm{g} / \mathrm{L}$ \\
\hline Magnesium & WA & & & $\mathbf{U}$ & 5000 & & NS & $\mu g / L$ \\
\hline Manganese & WA & & & $\mathbf{U}$ & 15 & . & 50 & $\mu g / L$ \\
\hline Mercury & WA & & & $\mathbf{U}$ & 0.2 & & 2 & $\mu g / L$ \\
\hline Methacrylonitrile & WA & & & $\mathbf{U}$ & 20 & & NS & $\mu g / L$ \\
\hline Methyl bromide & WA & & & $\mathbf{U}$ & 10 & & NS & $\mu g / L$ \\
\hline Methyl chloride & WA & & & $\mathbf{U}$ & 10 & & NS & $\mu g / L$ \\
\hline Methyl ethyl ketone & WA & & & $\mathbf{U}$ & 10 & & NS & $\mu g / L$ \\
\hline Methyl iodide & WA & & & $\mathbf{U}$ & 10 & & NS & $\mu g / L$ \\
\hline Methylene bromide & WA & & & $\mathbf{U}$ & 10 & & NS & $\mu g / L$ \\
\hline Methylene chloride & WA & & & $\mathrm{U}$ & 5 & & 5 & $\mu g / L$ \\
\hline Nickel & WA & & & $\mathbf{U}$ & 40 & & 100 & $\mu g / L$ \\
\hline Nitrate as nitrogen & WA & & & & 0.134 & & 10 & $\mathrm{mg} / \mathrm{L}$ \\
\hline Nonvolatile beta & TM & & & $\mathbf{U}$ & 0.7 & & 50 & $\mathrm{pCi} / \mathrm{L}$ \\
\hline Potassium & WA & & & $\mathbf{U}$ & 5000 & & NS & $\mu g / L$ \\
\hline Propionitrile & WA & & & $\mathbf{U}$ & 50 & & NS & $\mu g / L$ \\
\hline Selenium & WA & & & U & 5 & & 50 & $\mu g / L$ \\
\hline Silver & WA & & & $\mathbf{U}$ & 10 & & 50 & $\mu g / L$ \\
\hline Sodium & WA & & & $\mathbf{U}$ & 5000 & & NS & $\mu g / L$ \\
\hline Styrene & WA & & & $\mathbf{U}$ & 5 & & 100 & $\mu g / L$ \\
\hline Sulfate & WA & & & & 3.93 & & 400 & $\mathrm{mg} / \mathrm{L}$ \\
\hline Sulfide & WA & & & $\mathbf{U}$ & 0.1 & & NS & $\mathrm{mg} / \mathrm{L}$ \\
\hline Tetrachloroethylene & WA & & & $\mathrm{U}$ & 5 & & 5 & $\mu \mathrm{g} / \mathrm{L}$ \\
\hline Thallium & WA & & & $\mathbf{U}$ & 10 & & 2 & $\mu \mathrm{g} / \mathrm{L}$ \\
\hline Toluene & WA & & & $\mathbf{U}$ & 5 & & 1000 & $\mu g / L$ \\
\hline Total hardness & WA & & & & 9 & & NS & $\mathrm{mg} / \mathrm{L}$ \\
\hline Total organic carbon & WA & & & & 1.85 & & NS & $\mathrm{mg} / \mathrm{L}$ \\
\hline Total suspended solids & WA & & & & 10 & & NS & $\mathrm{mg} / \mathrm{L}$ \\
\hline trans-1,3-Dichloropropene & WA & & & $\mathbf{U}$ & 5 & & NS & $\mu g / L$ \\
\hline trans-1,4-Dichloro-2-butene & WA & & & $\mathbf{U}$ & 100 & & NS & $\mu \mathrm{g} / \mathrm{L}$ \\
\hline Trichloroethy!ene & WA & & & $\mathbf{U}$ & 5 & & 5 & $\mu \mathrm{g} / \mathrm{L}$ \\
\hline Trichlorofluoromethane & WA & & & $\mathbf{U}$ & 5 & & NS & $\mu g / L$ \\
\hline Tritium & $\mathrm{TM}$ & & & & 12.3 & 1.9 & 20 & $\mathrm{pCi} / \mathrm{ml}$ \\
\hline Turbidity & WA & & & & 0.72 & & NS & NTU \\
\hline Vanadium & WA & & & $\mathbf{U}$ & 50 & & NS & $\mu \mathrm{g} / \mathrm{L}$ \\
\hline Vinyl acetate & WA & & & $\mathbf{U}$ & 10 & & NS & $\mu g / L$ \\
\hline Vinyl chloride & WA & & & U & 10 & & 2 & $\mu \mathrm{g} / \mathrm{L}$ \\
\hline Xylenes (total) & WA & & & U & 5 & & 10000 & $\mu \mathrm{g} / \mathrm{L}$ \\
\hline Zinc & WA & & & & 21 & & 5000 & $\mu g \Omega$ \\
\hline
\end{tabular}




\section{UTR060}

Field Measurements:

Sample Date: 4/13/93

pH: 6.56

Specific Conductance: $21 \mu \mathrm{S} / \mathrm{cm}$

Red/Oxid Potential: $155 \mathrm{mv}$

Dissolved Oxygen: $3.3 \mathrm{mg} / \mathrm{L}$

\begin{tabular}{|c|c|c|c|c|c|c|c|c|}
\hline Analyte & Lab & Rep & Avg & Qualifier & Result & Acc & Stand & Units \\
\hline 1,1,1,2-Tetrachloroethane & $\overline{\text { WA }}$ & & & $\mathrm{U}$ & 10 & & NS & $\mu \mathrm{g} / \mathrm{L}$ \\
\hline 1,1,1-Trichloroethane & WA & & & $\mathbf{U}$ & 5 & & 200 & $\mu g / L$ \\
\hline 1,1,2,2-Tetrachloroethane & WA & & & $\mathbf{U}$ & 5 & & NS & $\mu g / L$ \\
\hline 1,1,2-Trichloroethane & WA & & & $\mathbf{U}$ & 5 & & 5 & $\mu g / L$ \\
\hline 1,1-Dichloroethane & WA & & & $\mathbf{U}$ & 5 & & NS & $\mu \mathrm{g} / \mathrm{L}$ \\
\hline 1,1-Dichloroethylene & WA & & & $\mathbf{U}$ & 5 & & 7 & $\mu g / L$ \\
\hline 1,2,3-Trichloropropane & WA & & & $\mathbf{U}$ & 10 & & NS & $\mu g / L$ \\
\hline 1,2-Dibromo-3-chloropropane & WA & & . & $\mathbf{U}$ & 20 & & 0.2 & $\mu \mathrm{g} / \mathrm{L}$ \\
\hline 1,2-Dibromoethane & WA & & & $\mathbf{U}$ & 20 & & NS & $\mu \mathrm{g} / \mathrm{L}$ \\
\hline 1,2-Dichloroethane & WA & & & $\mathbf{U}$ & 5 & & 5 & $\mu g / L$ \\
\hline 1,2-Dichloroethylene (total) & WA & & & $\mathbf{U}$ & 5 & & 100 & $\mu \mathrm{g} / \mathrm{L}$ \\
\hline 1,2-Dichloropropane & WA & & & $\mathbf{U}$ & 5 & & 5 & $\mu \mathrm{g} / \mathrm{L}$ \\
\hline 2-Hexanone & WA & & & $\mathbf{U}$ & 10 & & NS & $\mu g / L$ \\
\hline 4-Methyl-2-pentanone & WA & & & $\mathbf{U}$ & 10 & & NS & $\mu g / L$ \\
\hline Acetone & WA & & & $\mathbf{J}$ & 4.8 & & NS & $\mu \mathrm{g} / \mathrm{L}$ \\
\hline Acetonitrile & WA & & & $\mathbf{U}$ & 20 & & NS & $\mu \mathrm{g} / \mathrm{L}$ \\
\hline Acidity & WA & & & & 4.5 & & NS & $\mathrm{mg} / \mathrm{L}$ \\
\hline Acrolein & WA & & & $\mathbf{U}$ & 10 & & NS & $\mu g / L$ \\
\hline Acrylonitrile & WA & & & $\mathbf{U}$ & 10 & & NS & $\mu \mathrm{g} / \mathrm{L}$ \\
\hline Alkalinity & WA & & & & 3.5 & & NS & $\mathrm{mg} / \mathrm{L}$ \\
\hline Allyl chloride & WA & & & $\mathbf{U}$ & 100 & & NS & $\mu g / L$ \\
\hline Aluminum & WA & & & $\mathbf{U}$ & 200 & & NS & $\mu g / L$ \\
\hline Antimony & WA & & & $\mathbf{U}$ & 60 & & 6 & $\mu g / L$ \\
\hline Arsenic & WA & & & $\mathbf{U}$ & 10 & & 50 & $\mu g / L$ \\
\hline Barium & WA & & & $\mathbf{U}$ & 200 & & 2000 & $\mu \mathrm{g} / \mathrm{L}$ \\
\hline Benzene & WA & & & $\mathbf{U}$ & 5 & & 5 & $\mu \mathrm{g} / \mathrm{L}$ \\
\hline Beryllium & WA & & & $\mathbf{U}$ & 5 & & 4 & $\mu g / L$ \\
\hline Bromodichloromethane & WA & & & $\mathbf{U}$ & 5 & & NS & $\mu \mathrm{g} / \mathrm{L}$ \\
\hline Bromoform & WA & & & $\mathbf{U}$ & 5 & & NS & $\mu g / L$ \\
\hline Cadmium & WA & & & $\mathbf{U}$ & 5 & & 5 & $\mu g / L$ \\
\hline Calcium & WA & & & $\mathbf{U}$ & 5000 & & NS & $\mu g / L$ \\
\hline Carbon disulfide & WA & & & $\mathbf{U}$ & 5 & & NS & $\mu \mathrm{g} / \mathrm{L}$ \\
\hline Carbon tetrachloride & WA & & & $\mathbf{U}$ & 5 & & 5 & $\mu g / L$ \\
\hline Cesium-137 & TM & & & $\mathbf{U}$ & 10 & & 200 & $\mathrm{pCi} / \mathrm{L}$ \\
\hline Chloride & WA & & & & 3.66 & & NS & $\mathrm{mg} / \mathrm{L}$ \\
\hline Chlorobenzene & WA & & & $\mathbf{U}$ & 5 & & 100 & $\mu g / L$ \\
\hline Chloroethane & WA & & & $\mathbf{U}$ & 10 & & NS & $\mu g / L$ \\
\hline Chloroform & WA & & & $U$ & 5 & & 100 & $\mu \mathrm{g} / \mathrm{L}$ \\
\hline Chloroprene & WA & & & $\mathbf{U}$ & 20 & & NS & $\mu \mathrm{g} / \mathrm{L}$ \\
\hline Chromium & WA & & & $\mathbf{U}$ & 10 & & 100 & $\mu \mathrm{g} / \mathrm{L}$ \\
\hline cis-1,3-Dichloropropene & WA & & & $\mathbf{U}$ & 5 & & NS & $\mu \mathrm{g} / \mathrm{L}$ \\
\hline Cobalt & WA & & & $\mathbf{U}$ & 50 & & NS & $\mu g / L$ \\
\hline Copper & WA & & & $\mathbf{U}$ & 25 & & 1000 & $\mu \mathrm{g} / \mathrm{L}$ \\
\hline Cyanide & WA & & & $\mathbf{U}$ & 10 & & 200 & $\mu \mathrm{g} / \mathrm{L}$ \\
\hline Dibromochloromethane & WA & & & $\mathbf{U}$ & 5 & & NS & $\mu g / L$ \\
\hline Dichlorodifluoromethane & WA & & & $\mathbf{U}$ & 10 & & NS & $\mu \mathrm{g} / \mathrm{L}$ \\
\hline Ethylbenzene & WA & & & $\mathbf{U}$ & 5 & & 700 & $\mu g / L$ \\
\hline Fluoride & WA & & & $\mathbf{U}$ & 0.1 & & 4 & $\mathrm{mg} / \mathrm{L}$ \\
\hline
\end{tabular}


UTR060 Laboratory Analyses (cont'd)

\begin{tabular}{|c|c|c|c|c|c|c|c|c|}
\hline Analyte & Lab & Rep & Avg & Qualifier & Result & Acc & Stand & Units \\
\hline Gross alpha & $\mathrm{TM}$ & & & & 0.9 & 0.8 & 15 & $\mathrm{pCi} / \mathrm{L}$ \\
\hline Iron & WA & & & & 263 & & 300 & $\mu g / L$ \\
\hline Isobutyl alcohol & WA & & & $\mathbf{U}$ & 20 & & NS & $\mu \mathrm{g} / \mathrm{L}$ \\
\hline Lead & WA & & & $\mathbf{U}$ & 3 & & 50 & $\mu g / L$ \\
\hline Magnesium & WA & & & $\mathbf{U}$ & 5000 & & NS & $\mu \mathrm{g} / \mathrm{L}$ \\
\hline Manganese & WA & & & $\mathbf{U}$ & 15 & . & 50 & $\mu \mathrm{g} / \mathrm{L}$ \\
\hline Mercury & WA & & & $\mathbf{U}$ & 0.2 & & 2 & $\mu \mathrm{g} / \mathrm{L}$ \\
\hline Methacrylonitrile & WA & & & $\mathbf{U}$ & 20 & & NS & $\mu g / L$ \\
\hline Methyl bromide & WA & & & $\mathbf{U}$ & 10 & & NS & $\mu \mathrm{g} / \mathrm{L}$ \\
\hline Methyl chloride & WA & & & $\mathbf{U}$ & 10 & & NS & $\mu \mathrm{g} / \mathrm{L}$ \\
\hline Methyl ethyl ketone & WA & & & $\mathbf{U}$ & 10 & & NS & $\mu \mathrm{g} / \mathrm{L}$ \\
\hline Methyl iodide & WA & & & $\mathbf{U}$ & 10 & & NS & $\mu \mathrm{g} / \mathrm{L}$ \\
\hline Methylene bromide & WA & & & $\mathbf{U}$ & 10 & & NS & $\mu \mathrm{g} / \mathrm{L}$ \\
\hline Methylene chloride & WA & & & $\mathbf{U}$ & 5 & & 5 & $\mu \mathrm{g} / \mathrm{L}$ \\
\hline Nickel & WA & & & $\mathbf{U}$ & 40 & & 100 & $\mu \mathrm{g} / \mathrm{L}$ \\
\hline Nitrate as nitrogen & WA & & & $\mathbf{U}$ & 0.1 & & 10 & $\mathrm{mg} / \mathrm{L}$ \\
\hline Nonvolatile beta & TM & & & & 1 & 1.7 & 50 & $\mathrm{pCi} / \mathrm{L}$ \\
\hline Potassium & WA & & & $\mathbf{U}$ & 5000 & & NS & $\mu g / L$ \\
\hline Propionitrile & WA & & & $\mathbf{U}$ & 50 & & NS & $\mu g / L$ \\
\hline Selenium & WA & & & $\mathbf{U}$ & 5 & & 50 & $\mu g / L$ \\
\hline Silver & WA & & & $\mathbf{U}$ & 10 & & 50 & $\mu g / L$ \\
\hline Sodium & WA & & & $\mathbf{U}$ & 5000 & & NS & $\mu g / L$ \\
\hline Styrene & WA & & & $\mathbf{U}$ & 5 & & 100 & $\mu \mathrm{g} / \mathrm{L}$ \\
\hline Sulfate & WA & & & $\mathbf{U}$ & 2.5 & & 400 & $\mathrm{mg} / \mathrm{L}$ \\
\hline Sulfide & WA & & & $\mathbf{U}$ & 0.1 & & NS & $\mathrm{mg} / \mathrm{L}$ \\
\hline Tetrachloroethylene & WA & & & $\mathbf{U}$ & 5 & & 5 & $\mu g / L$ \\
\hline Thallium & WA & & & $\mathbf{U}$ & 10 & & 2 & $\mu \mathrm{g} / \mathrm{L}$ \\
\hline Toluene & WA & & & U & 5 & & 1000 & $\mu \mathrm{g} / \mathrm{L}$ \\
\hline Total hardness & WA & & & & 10 & & NS & $\mathrm{mg} / \mathrm{L}$ \\
\hline Total organic carbon & WA & & & & 4.66 & & NS & $\mathrm{mg} / \mathrm{L}$ \\
\hline Total suspended solids & WA & & $\mathbf{A}$ & & 9 & & NS & $\mathrm{mg} / \mathrm{L}$ \\
\hline trans-1,3-Dichloropropene & WA & & & $\mathbf{U}$ & 5 & & NS & $\mu \mathrm{g} / \mathrm{L}$ \\
\hline trans-1,4-Dichloro-2-butene & WA & & & $\mathbf{U}$ & 100 & & NS & $\mu g / L$ \\
\hline Trichloroethylene & WA & & & $\mathbf{U}$ & 5 & & 5 & $\mu g / L$ \\
\hline Trichlorofluoromethane & WA & & & $\mathbf{U}$ & 5 & & NS & $\mu \mathrm{g} / \mathrm{L}$ \\
\hline Tritium & TM & & & & 13.7 & 2.3 & 20 & $\mathrm{pCi} / \mathrm{ml}$ \\
\hline Turbidity & WA & & & & 0.27 & & NS & NTU \\
\hline Vanadium & WA & & & $\mathbf{U}$ & 50 & & NS & $\mu g / L$ \\
\hline Vinyl acetate & WA & & & $\mathbf{U}$ & 10 & & NS & $\mu g / L$ \\
\hline Vinyl chloride & WA & & & $\mathbf{U}$ & 10 & & 2 & $\mu \mathrm{g} / \mathrm{L}$ \\
\hline Xylenes (total) & WA & & & $\mathrm{U}$ & 5 & & 10000 & $\mu g / L$ \\
\hline Zinc & WA & & & $\mathbf{U}$ & 20 & & 5000 & $\mu \mathrm{g} / \mathrm{L}$ \\
\hline
\end{tabular}




\section{UTR062}

Field Measurements:

Sample Date: 4/14/93

pH: 6.3

Specific Conductance: $44 \mu \mathrm{S} / \mathrm{cm}$

Red/Oxid Potential: $199.7 \mathrm{mv}$

Dissolved Oxygen: $1.3 \mathrm{mg} / \mathrm{L}$

\begin{tabular}{|c|c|c|c|c|c|c|c|c|}
\hline Analyte & $\underline{L a b}$ & Rep & Avg & Qualifier & Result & Acc & Stand & Units \\
\hline 1,1,1,2-Tetrachloroethane & WA & & & $\mathrm{U}$ & 10 & & NS & $\mu g / L$ \\
\hline 1,1,1-Trichloroethane & WA & & & $\mathbf{U}$ & 5 & & 200 & $\mu \mathrm{g} / \mathrm{L}$ \\
\hline 1,1,2,2-Tetrachloroethane & WA & & & $\mathbf{U}$ & 5 & & NS & $\mu g / L$ \\
\hline 1,1,2-Trichloroethane & WA & & & $\mathbf{U}$ & 5 & & 5 & $\mu g / L$ \\
\hline 1,1-Dichloroethane & WA & & & $\mathbf{U}$ & 5 & & NS & $\mu g / L$ \\
\hline 1,1-Dichloroethylene & WA & & & $\mathbf{U}$ & 5 & & 7 & $\mu g / L$ \\
\hline 1,2,3-Trichloropropane & WA & & & $\mathbf{U}$ & 10 & & NS & $\mu g / L$ \\
\hline 1,2-Dibromo-3-chloropropane & WA & & . & $\mathbf{U}$ & 20 & & 0.2 & $\mu g / L$ \\
\hline 1,2-Dibromoethane & WA & & & $\mathbf{U}$ & 20 & & NS & $\mu g / L$ \\
\hline 1,2-Dichloroethane & WA & & & $\mathbf{U}$ & 5 & & 5 & $\mu g / L$ \\
\hline 1,2-Dichloroethylene (total) & WA & & & $\mathbf{U}$ & 5 & & 100 & $\mu g / L$ \\
\hline 1,2-Dichloropropane & WA & & & $\mathbf{U}$ & 5 & & 5 & $\mu g / L$ \\
\hline 2-Hexanone & WA & & & $\mathbf{U}$ & 10 & & NS & $\mu g / L$ \\
\hline 4-Methyl-2-pentanone & WA & & & $\mathbf{U}$ & 10 & & NS & $\mu g / L$ \\
\hline Acetone & WA & & & $\mathbf{J}$ & 5.83 & & NS & $\mu g / L$ \\
\hline Acetonitrile & WA & & & $\mathbf{U}$ & 20 & & NS & $\mu g / L$ \\
\hline Acidity & WA & & & & 10 & & NS & $\mathrm{mg} / \mathrm{L}$ \\
\hline Acrolein & WA & & & $\mathbf{U}$ & 10 & & NS & $\mu \mathrm{g} / \mathrm{L}$ \\
\hline Acrylonitrile & WA & & & $\mathrm{U}$ & 10 & & NS & $\mu \mathrm{g} / \mathrm{L}$ \\
\hline Alkalinity & WA & & & & 1 & & NS & $\mathrm{mg} / \mathrm{L}$ \\
\hline Allyl chloride & WA & & & $\mathrm{U}$ & 100 & & NS & $\mu g / L$ \\
\hline Aluminum & WA & & & $\mathbf{U}$ & 200 & & NS & $\mu g / L$ \\
\hline Americium-241 & TM & & & & 31.9 & 6.9 & 6.34 & $\mathrm{pCi} / \mathrm{L}$ \\
\hline Antimony & WA & & & $\mathbf{U}$ & 60 & & 6 & $\mu g / L$ \\
\hline Arsenic & WA & & & $\mathbf{U}$ & 10 & & 50 & $\mu \mathrm{g} / \mathrm{L}$ \\
\hline Barium & WA & & & $\mathbf{U}$ & 200 & & 2000 & $\mu \mathrm{g} / \mathrm{L}$ \\
\hline Benzene & WA & & & $\mathbf{U}$ & 5 & & 5 & $\mu \mathrm{g} / \mathrm{L}$ \\
\hline Beryllium & WA & & & $\mathbf{U}$ & 5 & & 4 & $\mu \mathrm{g} / \mathrm{L}$ \\
\hline Bromodichloromethane & WA & & & $\mathbf{U}$ & 5 & & NS & $\mu \mathrm{g} / \mathrm{L}$ \\
\hline Bromoform & WA & & & $\mathbf{U}$ & 5 & & NS & $\mu \mathrm{g} / \mathrm{L}$ \\
\hline Cadmium & WA & & & $\mathrm{U}$ & 5 & & 5 & $\mu g / L$ \\
\hline Calcium & WA & & & $\mathrm{U}$ & 5000 & & NS & $\mu \mathrm{g} / \mathrm{L}$ \\
\hline Carbon disulfide & WA & & & $U$ & 5 & & NS & $\mu \mathrm{g} / \mathrm{L}$ \\
\hline Carbon tetrachloride & WA & & & $\mathrm{U}$ & 5 & & 5 & $\mu g / L$ \\
\hline Cesium-137 & TM & & & $\mathrm{U}$ & 9.5 & & 200 & $\mathrm{pCi} / \mathrm{L}$ \\
\hline Chloride & WA & & A & & 2.77 & & NS & $\mathrm{mg} / \mathrm{L}$ \\
\hline Chlorobenzene & WA & & & $\mathrm{U}$ & 5 & & 100 & $\mu g / L$ \\
\hline Chloroethane & WA & & & $\mathrm{U}$ & 10 & & NS & $\mu g / L$ \\
\hline Chloroform & WA & & & $\mathbf{U}$ & 5 & & 100 & $\mu g / L$ \\
\hline Chloroprene & WA & & & U & 20 & & NS & $\mu \mathrm{g} / \mathrm{L}$ \\
\hline Chromium & WA & & & U & 10 & & 100 & $\mu \mathrm{g} / \mathrm{L}$ \\
\hline cis-1,3-Dichloropropene & WA & & & $\mathbf{U}$ & 5 & & NS & $\mu \mathrm{g} / \mathrm{L}$ \\
\hline Cobalt & WA & & & $\mathbf{U}$ & 50 & & NS & $\mu \mathrm{g} / \mathrm{L}$ \\
\hline Copper & WA & & & $\mathbf{U}$ & 25 & & 1000 & $\mu \mathrm{g} / \mathrm{L}$ \\
\hline Cyanide & WA & & & $\mathbf{U}$ & 10 & & 200 & $\mu \mathrm{g} / \mathrm{L}$ \\
\hline Dibromochloromethane & WA & & & $\mathbf{U}$ & 5 & & NS & $\mu \mathrm{g} / \mathrm{L}$ \\
\hline Dichlorodifluoromethane & WA & & & $\mathbf{U}$ & 10 & & NS & $\mu g / L$ \\
\hline Ethylbenzene & WA & & & $\mathbf{U}$ & 5 & & 700 & $\mu \mathrm{g} / \mathrm{L}$ \\
\hline Fluoride & WA & & & $\mathbf{U}$ & 0.1 & & 4 & $\mathrm{mg} / \mathrm{L}$ \\
\hline
\end{tabular}


UTR062 Laboratory Analyses (cont'd)

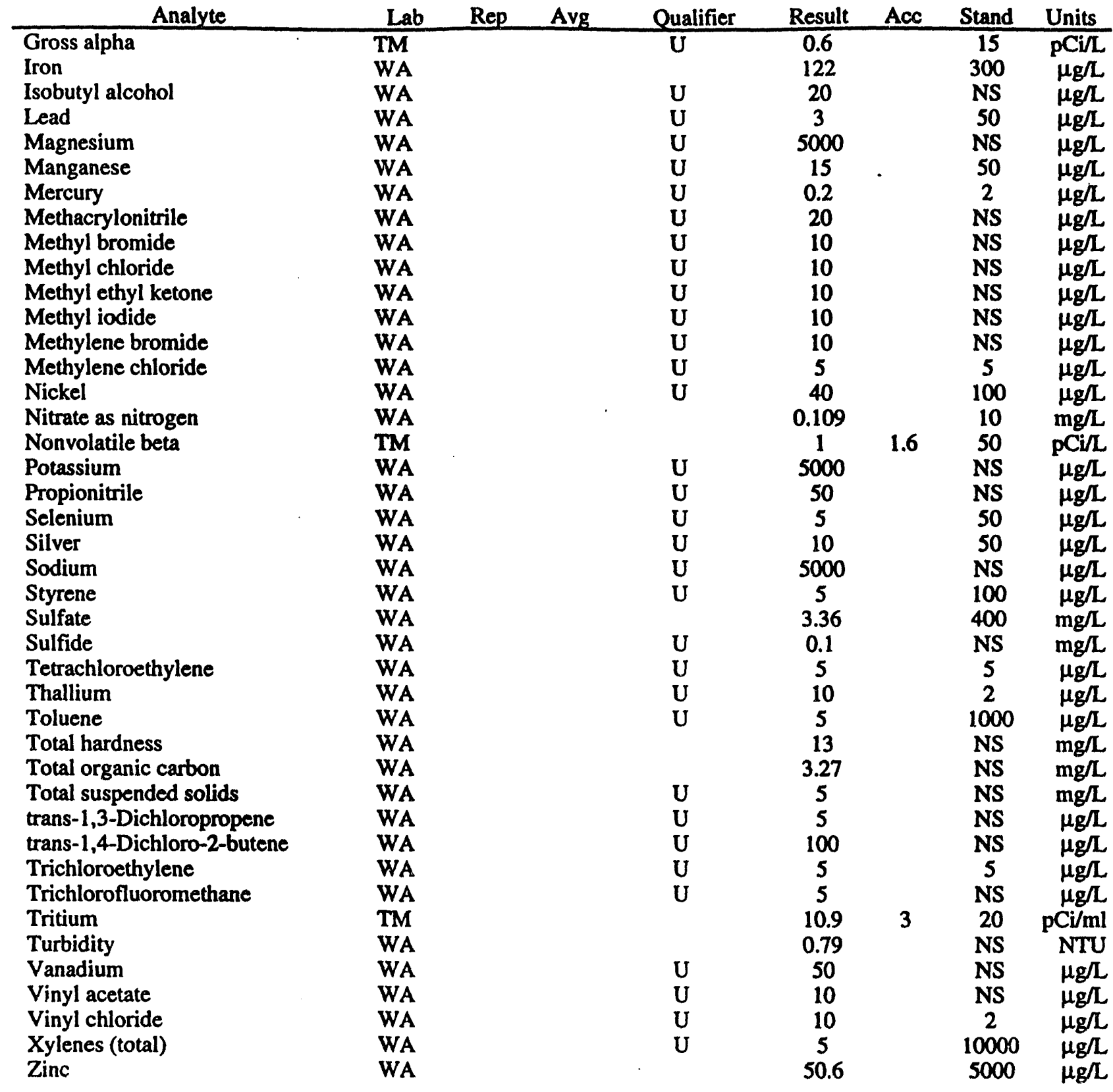




\section{UTR066}

Field Measurements:

Sample Date: 4/14/93

$\mathrm{pH}: 5.8$

Specific Conductance: $23 \mu \mathrm{S} / \mathrm{cm}$

Red/Oxid Potential: 204.7 mv

Dissolved Oxygen: $2.5 \mathrm{mg} / \mathrm{L}$

\begin{tabular}{|c|c|c|c|c|c|c|c|c|}
\hline Analyte & Lab & Rep & Avg & Qualifier & Result & Acc & Stand & Units \\
\hline 1,1,1,2-Tetrachloroethane & WA & & & $\mathbf{U}$ & 10 & & NS & $\mu g / L$ \\
\hline 1,1,1,2-Tetrachloroethane & GE & & & $\mathbf{U}$ & 1 & & NS & $\mu g / L$ \\
\hline 1,1,1,2-Tetrachloroethane & WA & $\mathbf{R}$ & & $\mathbf{U}$ & 10 & & NS & $\mu g / L$ \\
\hline 1,1,1-Trichloroethane & WA & & & $\mathbf{U}$ & 5 & & 200 & $\mu g / L$ \\
\hline 1,1,1-Trichloroethane & GE & & & $\mathbf{U}$ & 1 & & 200 & $\mu g / L$ \\
\hline $1,1,1$-Trichloroethane & WA & $\mathbf{R}$ & & $\mathbf{U}$ & 5 & & 200 & $\mu g / L$ \\
\hline $1,1,2,2$-Tetrachloroethane & WA & & & $\mathbf{U}$ & 5 & & NS & $\mu \mathrm{g} / \mathrm{L}$ \\
\hline 1,1,2,2-Tetrachloroethane & GE & & . & $\mathbf{U}$ & 1 & & NS & $\mu g / L$ \\
\hline 1,1,2,2-Tetrachloroethane & WA & $\mathbf{R}$ & & $\mathbf{U}$ & 5 & & NS & $\mu g / L$ \\
\hline 1,1,2-Trichloroethane & WA & & & $\mathbf{U}$ & 5 & & 5 & $\mu g / L$ \\
\hline 1,1,2-Trichloroethane & GE & & & $\mathbf{U}$ & 1 & & 5 & $\mu g / L$ \\
\hline 1,1,2-Trichloroethane & WA & $\mathbf{R}$ & & $\mathbf{U}$ & 5 & & 5 & $\mu g / L$ \\
\hline 1,1-Dichloroethane & WA & & & $\mathbf{U}$ & 5 & & NS & $\mu g / L$ \\
\hline 1,1-Dichloroethane & $\mathrm{GE}$ & & & $\mathbf{U}$ & 1 & & NS & $\mu g / L$ \\
\hline 1,1-Dichloroethane & WA & $\mathbf{R}$ & & $\mathbf{U}$ & 5 & & NS & $\mu g / L$ \\
\hline 1,1-Dichloroethylene & WA & & & $\mathbf{U}$ & 5 & & 7 & $\mu \mathrm{g} / \mathrm{L}$ \\
\hline 1,1-Dichloroethylene & GE & & & $\mathbf{U}$ & 1 & & 7 & $\mu \mathrm{g} / \mathrm{L}$ \\
\hline 1,1-Dichloroethylene & WA & $\mathbf{R}$ & & $\mathbf{U}$ & 5 & & 7 & $\mu \mathrm{g} / \mathrm{L}$ \\
\hline 1,2,3-Trichloropropane & WA & & & $\mathbf{U}$ & 10 & & NS & $\mu \mathrm{g} / \mathrm{L}$ \\
\hline 1,2,3-Trichloropropane & GE & & & $\mathbf{U}$ & 1 & & NS & $\mu \mathrm{g} / \mathrm{L}$ \\
\hline 1,2,3-Trichloropropane & WA & $\mathbf{R}$ & & $\mathbf{U}$ & 10 & & NS & $\mu g / L$ \\
\hline 1,2-Dibromo-3-chloropropane & WA & & & $\mathbf{U}$ & 20 & & 0.2 & $\mu g / L$ \\
\hline 1,2-Dibromo-3-chloropropane & GE & & & $\mathbf{U}$ & 1 & & 0.2 & $\mu g / L$ \\
\hline 1,2-Dibromo-3-chloropropane & WA & $\mathbf{R}$ & & $\mathbf{U}$ & 20 & & 0.2 & $\mu g / L$ \\
\hline 1,2-Dibromoethane & WA & & & $\mathbf{U}$ & 20 & & NS & $\mu \mathrm{g} / \mathrm{L}$ \\
\hline 1,2-Dibromoethane & GE & & & $\mathbf{U}$ & 20 & & NS & $\mu g / L$ \\
\hline 1,2-Dibromoethane & WA & $\mathbf{R}$ & & $\mathbf{U}$ & 20 & & NS & $\mu g / L$ \\
\hline 1,2-Dichloroethane & WA & & & $\mathbf{U}$ & 5 & & 5 & $\mu g / L$ \\
\hline 1,2-Dichloroethane & GE & & & $\mathbf{U}$ & 1 & & 5 & $\mu \mathrm{g} / \mathrm{L}$ \\
\hline 1,2-Dichloroethane & WA & $\mathbf{R}$ & & $\mathbf{U}$ & 5 & & 5 & $\mu \mathrm{g} / \mathrm{L}$ \\
\hline 1,2-Dichloroethylene (total) & WA & & & $\mathbf{U}$ & 5 & & 100 & $\mu g / L$ \\
\hline 1,2-Dichloroethylene (total) & GE & & & $\mathbf{U}$ & 1 & & 100 & $\mu \mathrm{g} / \mathrm{L}$ \\
\hline 1,2-Dichloroethylene (total) & WA & $\mathbf{R}$ & & U & 5 & & 100 & $\mu \mathrm{g} / \mathrm{L}$ \\
\hline 1,2-Dichloropropane & WA & & & $\mathrm{U}$ & 5 & & 5 & $\mu g / L$ \\
\hline 1,2-Dichloropropane & $\mathrm{GE}$ & & & $\mathbf{U}$ & 1 & & 5 & $\mu g / L$ \\
\hline 1,2-Dichloropropane & WA & $\mathbf{R}$ & & $\mathrm{U}$ & 5 & & 5 & $\mu g / L$ \\
\hline 2-Hexanone & WA & & & $\mathbf{U}$ & 10 & & NS & $\mu \mathrm{g} / \mathrm{L}$ \\
\hline 2-Hexanone & GE & & & $\mathbf{U}$ & 1 & & NS & $\mu g / L$ \\
\hline 2-Hexanone & WA & $\mathbf{R}$ & & $\mathbf{U}$ & 10 & & NS & $\mu \mathrm{g} / \mathrm{L}$ \\
\hline 4-Methyl-2-pentanone & WA & & & $\mathrm{U}$ & 10 & & NS & $\mu \mathrm{g} / \mathrm{L}$ \\
\hline 4-Methyl-2-pentanone & WA & $\mathbf{R}$ & & $\mathbf{U}$ & 10 & & NS & $\mu g / L$ \\
\hline Acetone & WA & & & $\mathbf{J}$ & 4.77 & & NS & $\mu g / L$ \\
\hline Acetone & $\mathrm{GE}$ & & & $\mathbf{U}$ & 100 & & NS & $\mu g / L$ \\
\hline Acetone & WA & $\mathbf{R}$ & & J & 4.16 & & NS & $\mu \mathrm{g} / \mathrm{L}$ \\
\hline Acetonitrile & WA & & & $\mathbf{U}$ & 20 & & NS & $\mu \mathrm{g} / \mathrm{L}$ \\
\hline Acetonitrile & GE & & & U & 1 & & NS & $\mu g / L$ \\
\hline Acetonitrile & WA & $\mathbf{R}$ & & $\mathbf{U}$ & 20 & & NS & $\mu g / L$ \\
\hline Acidity & WA & & & & 32 & & NS & $\mathrm{mg} / \mathrm{L}$ \\
\hline Acidity & WA & $\mathbf{R}$ & & & 13.5 & & NS & $\mathrm{mg} / \mathrm{L}$ \\
\hline
\end{tabular}


UTR066 Laboratory Analyses (cont'd)

\begin{tabular}{|c|c|c|c|c|c|c|c|}
\hline Analyte & Lab & Avg & Qualifier & Result & Acc & Stand & Units \\
\hline Acrolein & WA & & $\mathbf{U}$ & 10 & & NS & $\mu g / L$ \\
\hline $\begin{array}{l}\text { Acrolein } \\
\text { Acrolein }\end{array}$ & GE & & $\mathbf{U}$ & 20 & & NS & $\mu g / L$ \\
\hline Acrolein & WA & $\mathbf{R}$ & $\mathbf{U}$ & 10 & & NS & $\mu g / L$ \\
\hline $\begin{array}{l}\text { Acrylonitrile } \\
\text { Acrulonitrile }\end{array}$ & WA & & $\mathbf{U}$ & 10 & & NS & $\mu \mathrm{g} / \mathrm{L}$ \\
\hline $\begin{array}{l}\text { Acrylonitrile } \\
\text { Acrulonitrile }\end{array}$ & GE & & $\mathbf{U}$ & 20 & & NS & $\mu \mathrm{g} / \mathrm{L}$ \\
\hline Acrylonitrile & WA & $\mathbf{R}$ & $\mathbf{U}$ & 10 & & NS & $\mu g / L$ \\
\hline Actinium-228 & EP & & $\mathbf{U}$ & 30 & & NS & $\mathrm{pCi} / \mathrm{L}$ \\
\hline Alkalinity & WA & & & 0.5 & & NS & $\mathrm{mg} / \mathrm{L}$ \\
\hline Alkalinity & GE & & & 1.92 & & NS & $\mathrm{mg} / \mathrm{L}$ \\
\hline Alkalinity & WA & $\mathbf{R}$ & & 0.5 & & NS & $\mathrm{mg} / \mathrm{L}$ \\
\hline Allyl chloride & WA & & $\mathbf{U}$ & 100 & & NS & $\mu g / L$ \\
\hline Allyl chloride & GE & & $\mathbf{U}$ & 50 & & NS & $\mu g / L$ \\
\hline Allyl chloride & WA & $\mathbf{R}$ & U & 100 & & NS & $\mu g / L$ \\
\hline Aluminum & WA & & $\mathbf{U}$ & 200 & & NS & $\mu g / L$ \\
\hline Aluminum & GE & & & 112 & & NS & $\mu g / L$ \\
\hline Aluminum & WA & $\mathbf{R}$ & $\mathbf{U}$ & 200 & & NS & $\mu g / L$ \\
\hline Antimony & WA & & U & 60 & & 6 & $\mu g / L$ \\
\hline Antimony & GE & & U & 2 & & 6 & $\mu g / L$ \\
\hline Antimony & WA & $\mathbf{R}$ & $\mathrm{U}$ & 60 & & $\begin{array}{c}6 \\
300\end{array}$ & $\begin{array}{l}\mu \mathrm{g} / \mathrm{L} \\
\mathrm{pCi} / \mathrm{L}\end{array}$ \\
\hline $\begin{array}{l}\text { Antimony-125 } \\
\text { Arsenic }\end{array}$ & EP & & $U$ & $\begin{array}{l}20 \\
10\end{array}$ & & $\begin{array}{c}300 \\
50\end{array}$ & $\mathrm{pCi} / \mathrm{L}$ \\
\hline $\begin{array}{l}\text { Arsenic } \\
\text { Arsenic }\end{array}$ & WA & & $\mathbf{U}$ & $\begin{array}{c}10 \\
2\end{array}$ & & $\begin{array}{l}50 \\
50\end{array}$ & $\begin{array}{l}\mu g / L \\
\mu g / L\end{array}$ \\
\hline $\begin{array}{l}\text { Arsenic } \\
\text { Arsenic }\end{array}$ & GE & & $\begin{array}{l}\mathbf{U} \\
\mathbf{U}\end{array}$ & $\begin{array}{c}2 \\
10\end{array}$ & & 50 & $\begin{array}{l}\mu \mathrm{g} / \mathrm{L} \\
\mu \mathrm{g} / \mathrm{L}\end{array}$ \\
\hline $\begin{array}{l}\text { Arsenic } \\
\text { Barium }\end{array}$ & $\begin{array}{l}\text { WA } \\
\text { WA }\end{array}$ & $\mathbf{R}$ & $\mathbf{U}$ & 200 & & 2000 & $\begin{array}{l}\mu \mathrm{g} / \mathrm{L} \\
\mu \mathrm{g} / \mathrm{L}\end{array}$ \\
\hline & GE & & & 25.7 & & 2000 & $\begin{array}{l}\mu g / L \\
\mu g / L\end{array}$ \\
\hline $\begin{array}{l}\text { Barium } \\
\text { Barium }\end{array}$ & WA & $\mathbf{R}$ & $U$ & 200 & & 2000 & $\begin{array}{l}\mu g / L \\
\mu g / L\end{array}$ \\
\hline $\begin{array}{l}\text { Banum } \\
\text { Benzene }\end{array}$ & WA & & $\mathbf{U}$ & 5 & & 5 & $\mu g / L$ \\
\hline Benzene & GE & & $\mathbf{U}$ & 1 & & 5 & $\mu g / \mathrm{L}$ \\
\hline Benzene & WA & $\mathbf{R}$ & $\mathrm{U}$ & 5. & & 5 & $\mu g / L$ \\
\hline Beryllium & WA & & $\mathbf{U}$ & 5 & & 4 & $\mu g / L$ \\
\hline Beryllium & GE & & $\mathbf{U}$ & 3 & & 4 & $\mu g / L$ \\
\hline Beryllium & WA & $\mathbf{R}$ & u & 5 & & 4 & $\mu g / L$ \\
\hline Bis(2-chloro-1-methylethyl)ether & GE & & $\mathbf{U}$ & 10 & & NS & $\mu \mathrm{g} / \mathrm{L}$ \\
\hline Bromodichloromethane & WA & & $\mathbf{U}$ & 5 & & NS & $\mu g / L$ \\
\hline Bromodichloromethane & GE & & $\mathbf{U}$ & 1 & & NS & $\mu \mathrm{g} / \mathrm{L}$ \\
\hline Bromodichloromethane & WA & $\mathbf{R}$ & $\mathbf{U}$ & 5 & & NS & $\mu g / L$ \\
\hline Bromoform & WA & & $\mathbf{U}$ & 5 & & NS & $\mu g / L$ \\
\hline Bromoform & GE & & $\mathbf{U}$ & 1 & & NS & $\mu \mathrm{g} / \mathrm{L}$ \\
\hline Bromoform & WA & $\mathbf{R}$ & $\mathbf{U}$ & 5 & & NS & $\mu g / L$ \\
\hline Cadmium & WA & & & 10.7 & & 5 & $\mu g / L$ \\
\hline Cadmium & GE & & $\mathbf{U}$ & 2 & & 5 & $\mu g / \mathrm{L}$ \\
\hline Cadmium & WA & $\mathbf{R}$ & $\mathbf{U}$ & 5 & & 5 & $\mu \mathrm{g} / \mathrm{L}$ \\
\hline Calcium & WA & & $\mathbf{U}$ & 5000 & & NS & $\mu \mathrm{g} / \mathrm{L}$ \\
\hline Calcium & GE & & & 641 & & NS & $\mu \mathrm{g} / \mathrm{L}$ \\
\hline Calcium & WA & $\mathbf{R}$ & $\mathbf{U}$ & 5000 & & NS & $\mu g / L$ \\
\hline Carbon disulfide & WA & & $\mathrm{U}$ & 5 & & NS & $\mu g / L$ \\
\hline Carbon disulfide & $\mathrm{GE}$ & & U & 1 & & NS & $\mu g / L$ \\
\hline Carbon disulfide & WA & $\mathbf{R}$ & U & 5 & & NS & $\mu g / L$ \\
\hline Carbon tetrachloride & WA & & U & 5 & & 5 & $\mu g / L$ \\
\hline Carbon tetrachloride & GE & & U & 1 & & 5 & $\mu g / L$ \\
\hline Carbon tetrachloride & WA & $\mathbf{R}$ & $\mathbf{U}$ & 5 & & 5 & $\mu g / L$ \\
\hline Cerium-144 & EP & & $\mathbf{U}$ & 60 & & 261 & $\mathrm{pCi} / \mathrm{L}$ \\
\hline Cesium-134 & EP & & $\mathbf{U}$ & 10 & & 81.3 & $\mathrm{pCi} / \mathrm{L}$ \\
\hline Cesium-137 & TM & & & 4.4 & 4 & 200 & $\mathrm{pCi} / \mathrm{L}$ \\
\hline Cesium-137 & EP & & U & 10 & & $\begin{array}{l}200 \\
200\end{array}$ & $\begin{array}{l}\mathrm{pCi} / \mathrm{L} \\
\mathrm{pCi} / \mathrm{L}\end{array}$ \\
\hline Cesium-137 & TM & $\mathbf{R}$ & $\mathbf{U}$ & 6.3 & & & \\
\hline
\end{tabular}


UTR066 Laboratory Analyses (cont'd)

\begin{tabular}{|c|c|c|c|c|c|c|c|c|}
\hline Analyte & Lab & Rep & Avg & Qualifier & Result & Acc & Stand & Units \\
\hline Chloride & WA & & & & 3.28 & & NS & $\mathrm{mg} / \mathrm{l}$ \\
\hline Chloride & GE & & & & 1.7 & & NS & $\mathrm{mg} / \mathrm{L}$ \\
\hline Chloride & WA & $\mathbf{R}$ & & & 3.14 & & NS & $\mathrm{mg} / \mathrm{L}$ \\
\hline Chlorobenzene & WA & & & $\mathbf{U}$ & 5 & & 100 & $\mu \mathrm{g} / \mathrm{L}$ \\
\hline Chlorobenzene & GE & & & $U$ & 1 & & 100 & $\mu \mathrm{g} / \mathrm{L}$ \\
\hline Chlorobenzene & WA & $\mathbf{R}$ & & U & 5 & & 100 & $\mu \mathrm{g} / \mathrm{L}$ \\
\hline Chloroethane & WA & & & $\mathbf{U}$ & 10 & & NS & $\mu g / L$ \\
\hline Chloroethane & GE & & & $\mathbf{U}$ & 1 & & NS & $\mu g / L$ \\
\hline Chloroethane & WA & $\mathbf{R}$ & & $\mathbf{U}$ & 10 & & NS & $\mu g / L$ \\
\hline Chloroform & WA & & & $\mathbf{U}$ & 5 & & 100 & $\mu \mathrm{g} / \mathrm{L}$ \\
\hline Chloroform & GE & & & $\mathbf{U}$ & 1 & & 100 & $\mu g / L$ \\
\hline Chloroform & WA & $\mathbf{R}$ & & $\mathbf{U}$ & 5 & & 100 & $\mu g / L$ \\
\hline Chloroprene & WA & & & $\mathbf{U}$ & 20 & & NS & $\mu \mathrm{g} / \mathrm{L}$ \\
\hline Chloroprene & GE & & & $\mathbf{U}$ & 200 & & NS & $\mu g / L$ \\
\hline Chloroprene & WA & $\mathbf{R}$ & & $\mathbf{U}$ & 20 & & NS & $\mu g / L$ \\
\hline Chromium & WA & & . & $\mathbf{U}$ & 10 & & 100 & $\mu g / L$ \\
\hline Chromium & $\mathbf{G E}$ & & & $\mathbf{U}$ & 4 & & 100 & $\mu g / L$ \\
\hline Chromium & WA & $\mathbf{R}$ & & $\mathbf{U}$ & 10 & & 100 & $\mu g / L$ \\
\hline cis-1,3-Dichloropropene & WA & & & $\mathbf{U}$ & 5 & & NS & $\mu g / L$ \\
\hline cis-1,3-Dichloropropene & GE & & & $\mathbf{U}$ & 1 & & NS & $\mu g / L$ \\
\hline cis-1,3-Dichloropropene & WA & $\mathbf{R}$ & & $\mathbf{U}$ & 5 & & NS & $\mu \mathrm{g} / \mathrm{L}$ \\
\hline Cobalt & WA & & & $\mathbf{U}$ & 50 & & NS & $\mu \mathrm{g} / \mathrm{L}$ \\
\hline Cobalt & $\mathrm{GE}$ & & & $\mathbf{U}$ & 4 & & NS & $\mu \mathrm{g} / \mathrm{L}$ \\
\hline Cobalt & WA & $\mathbf{R}$ & & $\mathbf{U}$ & 50 & & NS & $\mu g / L$ \\
\hline Cobalt-57 & EP & & & $\mathbf{U}$ & 10 & & 1000 & $\mathrm{pCi} / \mathrm{L}$ \\
\hline Cobalt-60 & EP & & & $\mathbf{U}$ & 10 & & 100 & $\mathrm{pCi} / \mathrm{L}$ \\
\hline Copper & WA & & & $\mathbf{U}$ & 25 & & 1000 & $\mu \mathrm{g} / \mathrm{L}$ \\
\hline Copper & GE & & & $\mathbf{U}$ & 4 & & 1000 & $\mu \mathrm{g} / \mathrm{L}$ \\
\hline Copper & WA & $\mathbf{R}$ & & U & 25 & & 1000 & $\mu \mathrm{g} / \mathrm{L}$ \\
\hline Cyanide & WA & & & $\mathbf{U}$ & 10 & & 200 & $\mu g / L$ \\
\hline Cyanide & GE & & & $\mathbf{U}$ & 5 & & 200 & $\mu g / L$ \\
\hline Cyanide & WA & $\mathbf{R}$ & & $\mathbf{U}$ & 10 & & 200 & $\mu g / L$ \\
\hline Dibromochloromethane & WA & & & $\mathbf{U}$ & 5 & & NS & $\mu \mathrm{g} / \mathrm{L}$ \\
\hline Dibromochloromethane & GE & & & $\mathbf{U}$ & 1 & & NS & $\mu g / L$ \\
\hline Dibromochloromethane & WA & $\mathbf{R}$ & & $\mathrm{U}$ & 5 & & NS & $\mu \mathrm{g} / \mathrm{L}$ \\
\hline Dichlorodifluoromethane & WA & & & U & 10 & & NS & $\mu g / L$ \\
\hline Dichlorodifluoromethane & GE & & & $\mathbf{U}$ & 1 & & NS & $\mu g / L$ \\
\hline Dichlorodifluoromethane & WA & $\mathbf{R}$ & & U & 10 & & NS & $\mu \mathrm{g} / \mathrm{L}$ \\
\hline $\begin{array}{l}\text { Ethylbenzene } \\
\end{array}$ & WA & & & $\mathrm{U}$ & 5 & & 700 & $\mu \mathrm{g} / \mathrm{L}$ \\
\hline Ethylbenzene & GE & & & $\mathbf{U}$ & 1 & & 700 & $\mu \mathrm{g} / \mathrm{L}$ \\
\hline Ethylbenzene & WA & $\mathbf{R}$ & & $U$ & 5 & & 700 & $\mu \mathrm{g} / \mathrm{L}$ \\
\hline Europium-152 & EP & & & $\mathrm{U}$ & 40 & & NS & $\mathrm{pCi} / \mathrm{L}$ \\
\hline Europium-154 & EP & & & U & 20 & & 200 & $\mathrm{pCi} / \mathrm{L}$ \\
\hline Europium-155 & EP & & & $\mathrm{U}$ & 30 & & 600 & $\mathrm{pCi} / \mathrm{L}$ \\
\hline Fluoride & WA & & & $\mathrm{U}$ & 0.1 & & 4 & $\mathrm{mg} / \mathrm{L}$ \\
\hline Fluoride & $\mathrm{GE}$ & & & $U$ & 0.1 & & 4 & $\mathrm{mg} / \mathrm{L}$ \\
\hline Fluoride & WA & $\mathbf{R}$ & & $\mathrm{U}$ & 0.1 & & 4 & $\mathrm{mg} / \mathrm{L}$ \\
\hline Gross alpha & TM & & & $U$ & 0.6 & & 15 & $\mathrm{pCi} / \mathrm{L}$ \\
\hline Gross alpha & $\mathrm{GE}$ & & & $\mathrm{U}$ & 2 & & 15 & $\mathrm{pCi} / \mathrm{L}$ \\
\hline Gross alpha & TM & $\mathbf{R}$ & & $\mathbf{U}$ & 0.6 & & 15 & $\mathrm{pCi} / \mathrm{L}$ \\
\hline Iron & WA & & & & 181 & & 300 & $\mu \mathrm{g} / \mathrm{L}$ \\
\hline Iron & GE & & & & 317 & & 300 & $\mu g / L$ \\
\hline Iron & WA & $\mathbf{R}$ & & & 288 & & 300 & $\mu \mathrm{g} / \mathrm{L}$ \\
\hline Isobutyl alcohol & WA & & & $\mathbf{U}$ & 20 & & NS & $\mu \mathrm{g} / \mathrm{L}$ \\
\hline Isobutyl alcohol & GE & & & $\mathbf{U}$ & 100 & & NS & $\mu g / L$ \\
\hline Isobutyl alcohol & WA & $\mathbf{R}$ & & $\mathrm{U}$ & 20 & & NS & $\mu g / L$ \\
\hline
\end{tabular}


UTR066 Laboratory Analyses (cont'd)

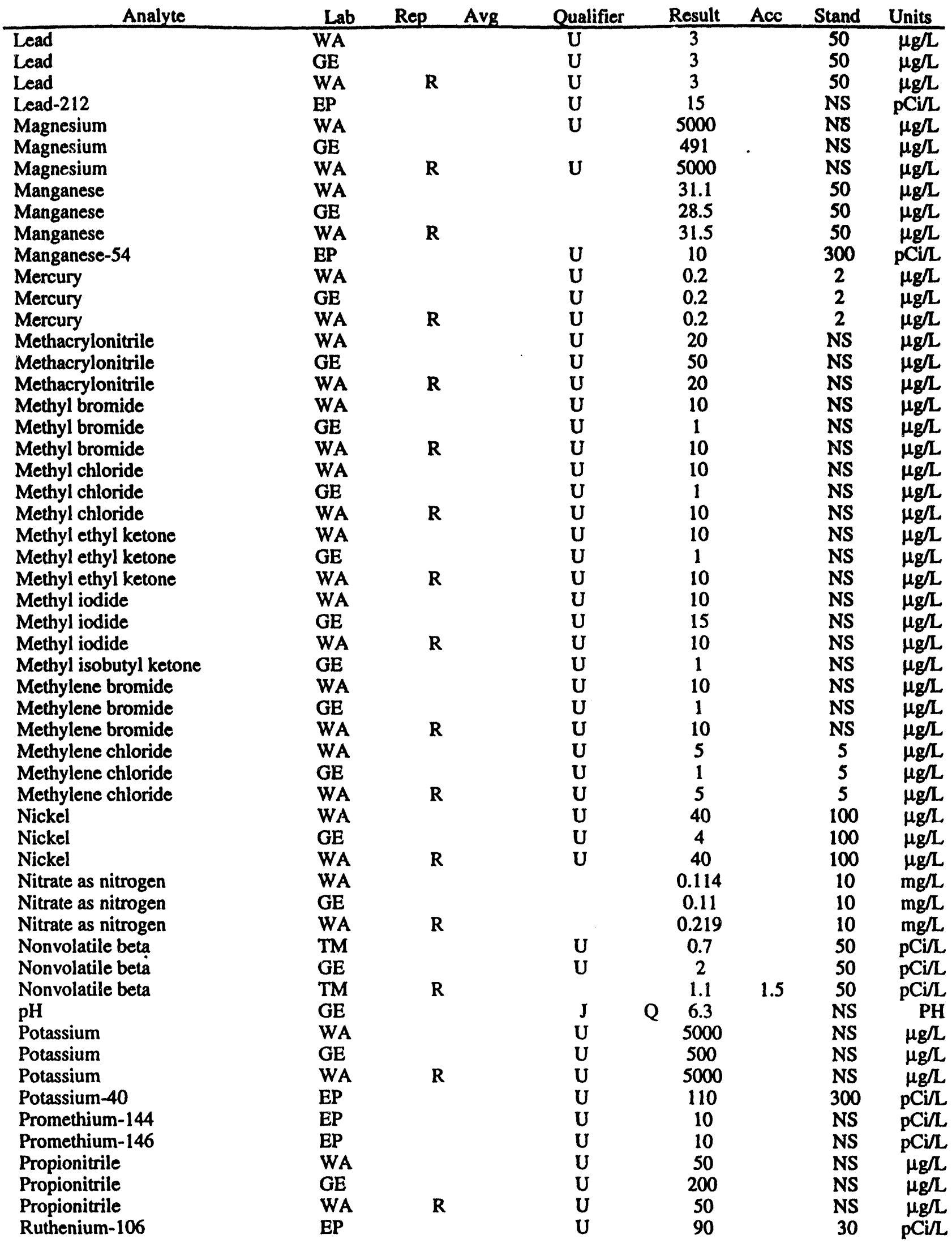


UTR066 Laboratory Analyses (cont'd)

\begin{tabular}{|c|c|c|c|c|c|c|c|c|}
\hline Analyte & Lab & Rep & Avg & Qualifier & Result & Acc & Stand & Units \\
\hline Selenium & WA & & & $U$ & 5 & & 50 & $\mu g / L$ \\
\hline Selenium & GE & & & UJ & L & & 50 & $\mu g /$ \\
\hline Selenium & WA & $\mathbf{R}$ & & U & 5 & & 50 & $\mu g / L$ \\
\hline Silver & WA & & & $\mathbf{U}$ & 10 & & 50 & $\mu g / L$ \\
\hline Silver & GE & & & $\mathbf{U}$ & 2 & & $\$ 0$ & $\mu \mathrm{g} / \mathrm{L}$ \\
\hline Silver & WA & $\mathbf{R}$ & & $\mathbf{U}$ & 10 & & 50 & $\mu g / L$ \\
\hline Sodium & WA & & & U & 5000 & $\cdot$ & NS & $\mu g / L$ \\
\hline Sodium & GE & & & & 1290 & & NS & $\mu g / L$ \\
\hline Sodium & WA & $\mathbf{R}$ & & $\begin{array}{l}\mathbf{U} \\
\mathbf{U}\end{array}$ & 5000 & & $\begin{array}{l}\text { NS } \\
466\end{array}$ & $\mu g / L$ \\
\hline $\begin{array}{l}\text { Sodium-22 } \\
\text { Styrene }\end{array}$ & EP & & & $\begin{array}{l}\mathbf{U} \\
\mathbf{U}\end{array}$ & $\begin{array}{c}10 \\
5\end{array}$ & & $\begin{array}{l}466 \\
100\end{array}$ & $\mathrm{pCi} / \mathrm{L}$ \\
\hline $\begin{array}{l}\text { Styrene } \\
\text { Styrene }\end{array}$ & $\begin{array}{l}\text { WA } \\
\text { GE }\end{array}$ & & & $\begin{array}{l}\mathbf{U} \\
\mathbf{U}\end{array}$ & $\begin{array}{l}0 \\
1\end{array}$ & & 100 & $\underset{\mu g / L}{\mu g / L}$ \\
\hline $\begin{array}{l}\text { Styrene } \\
\text { Styrene }\end{array}$ & $\begin{array}{l}\text { GE } \\
\text { WA }\end{array}$ & $\mathbf{R}$ & & $\mathbf{U}$ & 5 & & 100 & $\begin{array}{l}\mu g / L \\
\mu g / L\end{array}$ \\
\hline Sulfate & WA & $\mathrm{K}$ & & $\mathbf{U}$ & 2.5 & & 400 & mg $/ \mathrm{L}$ \\
\hline Sulfate & GE & & & & 2.35 & & 400 & $\mathrm{mg} / \mathrm{L}$ \\
\hline Sulfate & WA & $\mathbf{R}$ & & $\mathbf{U}$ & 2.5 & & 400 & $\mathrm{mg} / \mathrm{L}$ \\
\hline Sulfide & WA & & & $\mathbf{U}$ & 0.1 & & NS & $\mathrm{mg} / \mathrm{L}$ \\
\hline Sulfide & $\mathbf{G E}$ & & & $\mathbf{U}$ & 1 & & NS & $\mathrm{mg} / \mathrm{L}$ \\
\hline Sulfide & WA & $\mathbf{R}$ & & $\mathbf{U}$ & 0.1 & & NS & $\mathrm{mg} / \mathrm{h}$ \\
\hline Sulfide & WA & $\mathbf{R}$ & NA & $\mathbf{U}$ & 0.1 & & NS & $\mathrm{mg} / \mathrm{L}$ \\
\hline Tetrachloroethylene & WA & & & $\mathbf{U}$ & 5 & & 5 & $\mu g / \Omega$ \\
\hline Tetrachloroethylene & GE & & & $\mathbf{U}$ & 1 & & 5 & $\mu g / L$ \\
\hline Tetrachloroethylene & WA & $\mathbf{R}$ & & $\mathrm{U}$ & 5 & & 5 & $\mu g / L$ \\
\hline Thallium & WA & & & $\mathbf{U}$ & 10 & & 2 & $\mu \mathrm{g} / \mathrm{L}$ \\
\hline Thallium & GE & & & $\mathrm{U}$ & 2 & & 2 & $\mu \mathrm{g} / \mathrm{L}$ \\
\hline Thallium & WA & $\mathbf{R}$ & & U & 10 & & 2 & $\mu g / L$ \\
\hline Thorium-234 & EP & & & U & 350 & & 401 & $\mathrm{pCi} / \mathrm{L}$ \\
\hline Toluene & WA & & & $\mathbf{J}$ & 1.43 & & 1000 & $\mu g / L$ \\
\hline Toluene & GE & & & $\mathrm{U}$ & 1 & & 1000 & $\mu \mathrm{g} / \mathrm{L}$ \\
\hline Toluene & WA & $\mathbf{R}$ & & J & 1.06 & & 1000 & $\mu g / L$ \\
\hline Total hardness & WA & & & $\mathbf{U}$ & 1 & & NS & $\mathrm{mg} / \mathrm{L}$ \\
\hline Total hardness & GE & & & & 2 & & NS & $\mathrm{mg} / \mathrm{L}$ \\
\hline Total hardness & WA & $\mathbf{R}$ & & $\mathbf{U}$ & 1 & & NS & $\mathrm{mg} / \mathrm{L}$ \\
\hline Total organic carbon & WA & & & & 5.2 & & NS & $\mathrm{mg} / \mathrm{L}$ \\
\hline Total organic carbon & GE & & & & 4.27 & & NS & $\mathrm{mg} / \mathrm{L}$ \\
\hline Total organic carbon & WA & $\mathbf{R}$ & A & & 4.92 & & NS & $\mathrm{mg} / \mathrm{L}$ \\
\hline Total suspended solids & WA & & & $\mathbf{U}$ & 5 & & NS & $\mathrm{mg} / \mathrm{L}$ \\
\hline Total suspended solids & GE & & & & 2 & & NS & $\mathrm{mg} / \mathrm{L}$ \\
\hline Total suspended solids & WA & $\mathbf{R}$ & & $\mathbf{U}$ & 5 & & NS & $\mathrm{mg} / \mathrm{L}$ \\
\hline trans-1,3-Dichloropropene & WA & & & $\mathbf{U}$ & 5 & & NS & $\mu g / L$ \\
\hline trans-1,3-Dichloropropene & GE & & & $\mathrm{U}$ & 1 & & NS & $\mu g / L$ \\
\hline trans-1,3-Dichloropropene & WA & $\mathbf{R}$ & & $\mathrm{U}$ & 5 & & NS & $\mu \mathrm{g} / \mathrm{L}$ \\
\hline trans-1,4-Dichloro-2-butene & WA & & & U & 100 & & NS & $\mu \mathrm{g} / \mathrm{L}$ \\
\hline trans-1,4-Dichloro-2-butene & GE & & & $\mathrm{U}$ & 30 & & NS & $\mu g / L$ \\
\hline trans-1,4-Dichloro-2-butene & WA & $\mathbf{R}$ & & U & 100 & & NS & $\mu g / L$ \\
\hline Trichloroethylene & WA & & & $\mathrm{U}$ & 5 & & 5 & $\mu g / L$ \\
\hline Trichloroethylene & $\mathrm{GE}$ & & & $\mathrm{U}$ & 1 & & 5 & $\mu g / L$ \\
\hline Trichloroethylene & WA & $\mathbf{R}$ & & $\mathrm{U}$ & 5 & & 5 & $\mu g / L$ \\
\hline Trichlorofluoromethane & WA & & & $\mathbf{U}$ & 5 & & NS & $\mu g / L$ \\
\hline Trichlorofluoromethane & GE & & & $\mathrm{U}$ & 1 & & NS & $\mu \mathrm{g} / \mathrm{L}$ \\
\hline Trichlorofluoromethane & WA & $\mathbf{R}$ & & $\mathbf{U}$ & 5 & & NS & $\mu g / L$ \\
\hline Tritium & TM & & & & 15.6 & 4.3 & 20 & $\mathrm{pCi} / \mathrm{ml}$ \\
\hline Tritium & GE & & & & 18.2 & & 20 & $\mathrm{pCi} / \mathrm{ml}$ \\
\hline Tritium & TM & $\mathbf{R}$ & & & 15.6 & 4.3 & 20 & $\mathrm{pCi} / \mathrm{ml}$ \\
\hline
\end{tabular}


UTR066 Laboratory Analyses (cont'd)

\begin{tabular}{|c|c|c|c|c|c|c|c|c|}
\hline Analyte & $\mathrm{Lab}$ & Rep & Avg & Qualifier & Result & Acc & Stand & Units \\
\hline Turbidity & $\overline{\mathbf{W A}}$ & & & & 0.6 & & NS & NIU \\
\hline Turbidity & GE & & & & 0.31 & & NS & NTU \\
\hline Turbidity & WA & $\mathbf{R}$ & $\mathbf{A}$ & & 0.715 & & NS & NTU \\
\hline Vanadium & WA & & & $\mathbf{U}$ & 50 & & NS & $\mu g / L$ \\
\hline Vanadium & GE & & & $\mathbf{U}$ & 8 & & NS & $\mu g / L$ \\
\hline Vanadium & WA & $\mathbf{R}$ & & $\mathbf{U}$ & 50 & . & NS & $\mu g / \lambda$ \\
\hline Vinyl acetate & WA & & & $\mathbf{U}$ & 10 & & NS & $\mu g / L$ \\
\hline Vinyl acetate & GE & & & $\mathbf{U}$ & 1 & & NS & $\mu g / L$ \\
\hline Vinyl acetate & WA & $\mathbf{R}$ & & $\mathbf{U}$ & 10 & & NS & $\mu g / L$ \\
\hline Vinyl chloride & WA & & & $\mathbf{U}$ & 10 & & 2 & $\mu g / L$ \\
\hline Vinyl chloride & GE & & & $\mathbf{U}$ & 1 & & 2 & $\mu \mathrm{g} / \mathrm{L}$ \\
\hline Vinyl chloride & WA & $\mathbf{R}$ & & $\mathbf{U}$ & 10 & & 2 & $\mu g / L$ \\
\hline Xylenes (total) & WA & & & $\mathbf{U}$ & 5 & & 10000 & $\mu \mathrm{g} / \mathrm{L}$ \\
\hline Xylenes (total) & $\mathbf{G E}$ & & & $\mathbf{U}$ & 2 & & 10000 & $\mu g / L$ \\
\hline Xylenes (total) & WA & $\mathbf{R}$ & & $\mathbf{U}$ & 5 & & 10000 & $\mu g / L$ \\
\hline Yttrium-88 & EP & & & $\mathbf{U}$ & 60 & & NS & $\mathrm{pCi} / \mathrm{L}$ \\
\hline Zinc & WA & & & $\mathbf{U}$ & 20 & & 5000 & $\mu g / L$ \\
\hline Zinc & GE & & & & 2.82 & & 5000 & $\mu g / L$ \\
\hline Zinc & WA & $\mathbf{R}$ & & $\mathbf{U}$ & 20 & & 5000 & $\mu g / L$ \\
\hline Zinc-65 & EP & & & $\mathbf{U}$ & 20 & & 300 & $\mathrm{pCi} / \mathrm{L}$ \\
\hline
\end{tabular}




\section{UTR068}

Field Measurements:

Sample Date: 4/15/93

$\mathrm{pH}: \mathbf{4 . 5 5}$

Specific Conductance: $27 \mu \mathrm{S} / \mathrm{cm}$

Red/Oxid Potential: $174.2 \mathrm{mv}$

Dissolved Oxygen: $3.2 \mathrm{mg} / \mathrm{L}$

\begin{tabular}{|c|c|c|c|c|c|c|c|c|}
\hline Analyte & Lab & Rep & Avg & Qualifier & Result & Acc & Stand & Units \\
\hline 1,1,1,2-Tetrachloroethane & WA & & & $\mathbf{U}$ & 10 & & NS & $\mu g / L$ \\
\hline $\begin{array}{l}\text { 1,1,1,2-Tetrachloroethane } \\
\text { 1,1,1-Trichloroethane }\end{array}$ & GE & & & UJ & $\mathbf{Q}$ & & NS & $\mu g \Omega$ \\
\hline $\begin{array}{l}\text { 1,1,1-Trichloroethane } \\
\text { 1,1,1-Trichloroethane }\end{array}$ & WA & & & U & 5 & & 200 & $\mu g / L$ \\
\hline $\begin{array}{l}\text { 1,1,1-Trichloroethane } \\
\text { 1,1,2,2-Tetrachloroethane }\end{array}$ & GE & & & UJ & $\mathbf{Q}$ & & 200 & $\mu g / L$ \\
\hline $\begin{array}{l}\text { 1,1,2,2-Tetrachloroethane } \\
\text { 1,1,2,2-Tetrachloroethane }\end{array}$ & WA & & & $\underset{\mathbf{U}}{\mathbf{U}}$ & 5 & & NS & $\mu g / L$ \\
\hline $\begin{array}{l}\text { 1,1,2,2-Tetrachloroethane } \\
\text { 1,1,2-Trichloroethane }\end{array}$ & $\begin{array}{l}\text { GE } \\
\text { WA }\end{array}$ & & & $\begin{array}{r}\mathbf{U J} \\
\mathbf{U}\end{array}$ & $\mathbf{Q}$ & & $\begin{array}{c}\text { NS } \\
5\end{array}$ & $\mu g / L$ \\
\hline $\begin{array}{l}\text { 1,1,2-Trichloroethane } \\
\text { 1,1,2-Trichloroethane }\end{array}$ & GE & & . & UJ & $\begin{array}{l}3 \\
1\end{array}$ & & 5 & $\begin{array}{l}\mu g / L \\
\mu g / L\end{array}$ \\
\hline 1,1-Dichloroethane & WA & & & $\mathbf{U}$ & 5 & & NS & $\mu g / L$ \\
\hline $\begin{array}{l}\text { 1,1-Dichloroethane } \\
\text { 1,1-Dichloroethylene }\end{array}$ & GE & & & UJ & $\mathbf{Q}$ & & NS & $\mu g / L$ \\
\hline $\begin{array}{l}\text { 1,1-Dichloroethylene } \\
\text { 1,1-Dichloroethylene }\end{array}$ & WA & & & $\mathbf{U}$ & 5 & & 7 & $\mu g / L$ \\
\hline $\begin{array}{l}\text { 1,1-Dichloroethylene } \\
\text { 1,2,3-Trichloropropane }\end{array}$ & GE & & & UJ & $\mathbf{Q}$ & & $\begin{array}{l}7 \\
\text { NS }\end{array}$ & $\mu g / L$ \\
\hline & $\begin{array}{l}\text { WA } \\
\text { GE }\end{array}$ & & & $\underset{\mathbf{U J}}{\mathbf{U}}$ & $\begin{array}{lc} & 10 \\
0 & 1\end{array}$ & & $\begin{array}{l}\text { NS } \\
\text { NS }\end{array}$ & $\mu g / L$ \\
\hline $\begin{array}{l}\text { 1,2,3-Trichloropropane } \\
\text { 1,2-Dibromo-3-chloropropane }\end{array}$ & $\begin{array}{l}\text { GE } \\
\text { WA }\end{array}$ & & & U & $\begin{array}{c}1 \\
20\end{array}$ & & $\begin{array}{l}\text { NS } \\
0.2\end{array}$ & $\begin{array}{l}\mu g / L \\
\mu g / L\end{array}$ \\
\hline & $\mathrm{GE}$ & & & UJ & $\mathbf{Q}$ & & 0.2 & $\begin{array}{l}\mu g / L \\
\mu g / L\end{array}$ \\
\hline 1,2-Dibromoethane & WA & & & $\mathbf{U}$ & 20 & & NS & $\begin{array}{l}\mu g L \\
\mu g / L\end{array}$ \\
\hline 1,2-Dibromoethane & GE & & & UJ & 20 & & NS & $\mu g / L$ \\
\hline 1,2-Dichloroethane & WA & & & U & 5 & & 5 & $\mu g / L$ \\
\hline 1,2-Dichloroethane & GE & & & UJ & $\mathbf{Q}$ & & 5 & $\mu g / L$ \\
\hline 1,2-Dichloroethylene (total) & WA & & & $\mathbf{U}$ & 5 & & 100 & $\mu \mathrm{g} / \mathrm{L}$ \\
\hline 1,2-Dichloroethylene (total) & GE & & & UJ & $\mathbf{Q}$ & & 100 & $\mu g / L$ \\
\hline 1,2-Dichloropropane & WA & & & $\mathbf{U}$ & 5 & & 5 & $\mu g / L$ \\
\hline 1,2-Dichloropropane & GE & & & UJ & $\mathbf{Q}$ & & 5 & $\mu g / L$ \\
\hline 2-Hexanone & WA & & & $\mathbf{U}$ & 10 & & NS & $\mu g / L$ \\
\hline 2-Hexanone & GE & & & UJ & $\mathbf{Q}$ & & NS & $\mu \mathrm{g} / \mathrm{L}$ \\
\hline 4-Methyl-2-pentanone & WA & & & $\mathbf{U}$ & 10 & & NS & $\mu g / L$ \\
\hline Acetone & WA & & & $\mathbf{U}$ & 10 & & NS & $\mu g / L$ \\
\hline Acetone & GE & & & UJ & 100 & & NS & $\mu g / L$ \\
\hline Acetonitrile & WA & & & $\mathbf{U}$ & 20 & & NS & $\mu g / L$ \\
\hline Acetonitrile & GE & & & UJ & Q & & NS & $\mu \mathrm{g} / \mathrm{L}$ \\
\hline Acidity & WA & & & & 9 & & NS & $\mathrm{mg} / \mathrm{L}$ \\
\hline Acrolein & WA & & & $\mathrm{U}$ & 10 & & NS & $\mu \mathrm{g} / \mathrm{L}$ \\
\hline Acrolein & GE & & & UJ & 20 & & NS & $\mu \mathrm{g} / \mathrm{L}$ \\
\hline Acrylonitrile & WA & & & $\mathbf{U}$ & 10 & & NS & $\mu \mathrm{g} / \mathrm{L}$ \\
\hline Acrylonitrile. & GE & & & UJ & 20 & & NS & $\mu g / L$ \\
\hline Actinium-228 & EP & & & $\mathbf{U}$ & 30 & & NS & pCi/L \\
\hline Alkalinity & WA & & & & 1 & & NS & $\mathrm{mg} / \mathrm{L}$ \\
\hline Alkalinity & GE & & $\mathbf{A}$ & & 1.44 & & NS & $\mathrm{mg} / \mathrm{L}$ \\
\hline Allyl chloride & WA & & & $\mathbf{U}$ & 100 & & NS & $\mu g / L$ \\
\hline Allyl chloride & $\mathrm{GE}$ & & & UJ & 50 & & NS & $\mu \mathrm{g} / \mathrm{L}$ \\
\hline Aluminum & WA & & & $\mathbf{U}$ & 200 & & NS & $\mu g / L$ \\
\hline Aluminum & GE & & & & 85.6 & & NS & $\mu \mathrm{g} / \mathrm{L}$ \\
\hline Antimony & WA & & & $\mathbf{U}$ & 60 & & 6 & $\mu g / L$ \\
\hline Antimony & $\mathrm{GE}$ & & & $\mathbf{U}$ & 2 & & 6 & $\mu \mathrm{g} / \mathrm{L}$ \\
\hline Antimony-125 & EP & & & $\mathbf{U}$ & 20 & & 300 & $\mathrm{pCi} / \mathrm{L}$ \\
\hline Arsenic & WA & & & $\mathrm{U}$ & 10 & & 50 & $\mu g / L$ \\
\hline$\cdot$ & GE & & & $\mathbf{U}$ & 2 & & 50 & $\mu g / L$ \\
\hline
\end{tabular}


Sampling and Analysis of Water From Upper Three Runs And lis Wellands Near Tank 16 and the Mixed Waste Management Facilioy

UTR068 Laboratory Analyses (cont'd)

\begin{tabular}{|c|c|c|c|c|c|c|c|c|}
\hline Analyte & $L a b$ & Rep & Qualifier & & Result & Acc & Stand & Units \\
\hline Barium & WA & & $\mathbf{U}$ & & 200 & & 2000 & $\mu \mathrm{g} / \mathrm{L}$ \\
\hline Barium & $\mathbf{G E}$ & & & & 17.3 & & 2000 & $\mu g / L$ \\
\hline Benzene & WA & & $\mathbf{U}$ & & 5 & & 5 & $\mu \mathrm{g} / \mathrm{L}$ \\
\hline Benzene & GE & & UJ & $\mathbf{Q}$ & 1 & & 5 & $\mu g / L$ \\
\hline Beryllium & WA & & $\mathbf{U}$ & & 5 & & 4 & $\mu g / L$ \\
\hline Beryllium & GE & & $\mathbf{U}$ & & 3 & . & 4 & $\mu g / L$ \\
\hline Bis(2-chloro-1-methylethyl)ether & $\mathbf{G E}$ & & UJ & $\mathbf{Q}$ & 10 & & NS & $\mu g / L$ \\
\hline Bromodichloromethane & WA & & $\mathbf{U}$ & & 5 & & NS & $\mu g / L$ \\
\hline Bromodichloromethane & GE & & UJ & $\mathbf{Q}$ & 1 & & NS & $\mu g / L$ \\
\hline Bromoform & WA & & $\mathbf{U}$ & & 5 & & NS & $\mu g / L$ \\
\hline Bromoform & $\mathbf{G E}$ & & UJ & $\mathbf{Q}$ & 1 & & NS & $\mu g / L$ \\
\hline Cadmium & WA & & $\mathbf{U}$ & & 5 & & 5 & $\mu g / L$ \\
\hline Cadmium & $\mathrm{GE}$ & & $\mathbf{U}$ & & 2 & & 5 & $\mu g / L$ \\
\hline Calcium & WA & & $\mathbf{U}$ & & 5000 & & NS & $\mu g / L$ \\
\hline Calcium & GE & & & & 327 & & NS & $\mu g / L$ \\
\hline Carbon disulfide & WA & & $\mathbf{U}$ & & 5 & & NS & $\mu g / L$ \\
\hline Carbon disulfide & GE & & UJ & $\mathbf{Q}$ & 1 & & NS & $\mu g / L$ \\
\hline Carbon tetrachloride & WA & & $\mathbf{U}$ & & 5 & & 5 & $\mu g / L$ \\
\hline Carbon tetrachloride & GE & & UJ & $\mathbf{Q}$ & 1 & & 5 & $\mu g / 2$ \\
\hline Cerium-144 & $\overline{\mathbf{E P}}$ & & $\mathbf{U}$ & & 60 & & 261 & $\mathrm{pCi} / \mathrm{L}$ \\
\hline Cesium-134 & $\mathbf{E P}$ & & $\mathbf{U}$ & & 10 & & 81.3 & $\mathrm{pCi} / \mathrm{L}$ \\
\hline Cesium-137 & TM & & $\mathbf{U}$ & & 5.9 & & 200 & $\mathrm{pCi} / \mathrm{L}$ \\
\hline Cesium-137 & EP & & $\mathbf{U}$ & & 10 & & 200 & $\mathrm{pCi} / \mathrm{L}$ \\
\hline Chloride & WA & & & & 1.91 & & NS & $\mathrm{mg} / \mathrm{L}$ \\
\hline Chloride & GE & & & & 1.66 & & NS & $\mathrm{mg} / \mathrm{L}$ \\
\hline Chlorobenzene & WA & & $\mathbf{U}$ & & 5 & & 100 & $\mu g / L$ \\
\hline Chlorobenzene & GE & & UJ & $\mathbf{Q}$ & 1 & & 100 & $\mu \mathrm{g} / \mathrm{L}$ \\
\hline Chloroethane & WA & & $\mathrm{U}$ & & 10 & & NS & $\mu g / L$ \\
\hline Chloroethane & $\mathrm{GE}$ & & UJ & $\mathbf{Q}$ & 1 & & NS & $\mu \mathrm{g} / \mathrm{L}$ \\
\hline Chloroform & WA & & $\mathbf{U}$ & & 5 & & 100 & $\mu g / L$ \\
\hline Chloroform & GE & & UJ & $\mathbf{Q}$ & 1 & & 100 & $\mu \mathrm{g} / \mathrm{L}$ \\
\hline Chloroprene & WA & & $\mathbf{U}$ & & 20 & & NS & $\mu g / L$ \\
\hline Chloroprene & GE & & UJ & $\mathbf{Q}$ & 200 & & NS & $\mu \mathrm{g} / \mathrm{L}$ \\
\hline Chromium & WA & & $\mathbf{U}$ & & 10 & & 100 & $\mu g / L$ \\
\hline Chromium & GE & & $\mathbf{U}$ & & 4 & & 100 & $\mu \mathrm{g} / \mathrm{L}$ \\
\hline cis-1,3-Dichloropropene & WA & & $\mathbf{U}$ & & 5 & & NS & $\mu \mathrm{g} / \mathrm{L}$ \\
\hline cis-1,3-Dichloropropene & GE & & UJ & $\mathbf{Q}$ & 1 & & NS & $\mu \mathrm{g} / \mathrm{L}$ \\
\hline Cobalt & WA & & $\mathbf{U}$ & & 50 & & NS & $\mu g / L$ \\
\hline Cobalt & GE & & $\mathbf{U}$ & & 4 & & NS & $\mu g / L$ \\
\hline Cobalt-57 & $\mathbf{E P}$ & & $\mathbf{U}$ & & 10 & & 1000 & $\mathrm{pCi} / \mathrm{L}$ \\
\hline Cobalt-60 & EP & & $\mathrm{U}$ & & 10 & & 100 & $\mathrm{pCi} / \mathrm{L}$ \\
\hline Copper & $\overrightarrow{\text { WA }}$ & & $\mathbf{U}$ & & 25 & & 1000 & $\mu g / L$ \\
\hline Copper & GE & & $\mathbf{U}$ & & 4 & & 1000 & $\mu g / L$ \\
\hline Cyanide & WA & & $\mathrm{U}$ & & 10 & & 200 & $\mu g / L$ \\
\hline Cyanide & GE & & $\mathrm{U}$ & & 5 & & 200 & $\mu g / L$ \\
\hline Dibromochloromethane & WA & & $\mathbf{U}$ & & 5 & & NS & $\mu g / L$ \\
\hline Dibromochloromethane & GE & & UJ & $\mathbf{Q}$ & 1 & & NS & $\mu \mathrm{g} / \mathrm{L}$ \\
\hline Dichlorodifluoromethane & WA & & $\mathbf{U}$ & & 10 & & NS & $\mu \mathrm{g} / \mathrm{L}$ \\
\hline Dichlorodifluoromethane & GE & & UJ & $\mathbf{Q}$ & 1 & & NS & $\mu \mathrm{g} / \mathrm{L}$ \\
\hline Ethylbenzene & WA & & $\mathbf{U}$ & & 5 & & 700 & $\mu g / L$ \\
\hline Ethylbenzene & GE & & UJ & $\mathbf{Q}$ & 1 & & 700 & $\mu g / L$ \\
\hline Europium-152 & EP & & $\mathbf{U}$ & & 40 & & NS & $\mathrm{pCi} / \mathrm{L}$ \\
\hline Europium-154 & EP & & $\mathbf{U}$ & & 20 & & 200 & $\mathrm{pCi} / \mathrm{L}$ \\
\hline Europium-155 & EP & & $\mathbf{U}$ & & 30 & & 600 & $\mathrm{pCi} / \mathrm{L}$ \\
\hline Fluoride & WA & & $\mathbf{U}$ & & 0.1 & & 4 & $\mathrm{mg} / \mathrm{L}$ \\
\hline Fluoride & GE & & $\mathbf{U}$ & & 0.1 & & 4 & $\mathrm{mg} / \mathrm{L}$ \\
\hline
\end{tabular}


UTR068 Laboratory Analyses (cont'd)

\begin{tabular}{|c|c|c|c|c|c|c|c|c|c|}
\hline Analyte & Lab & Rep & Avg & Qualifier & & Result & Acc & Stand & Units \\
\hline Gross alpha & $T M$ & & & $\mathrm{U}$ & & 0.6 & & 15 & $\overline{\mathrm{pCi} / \mathrm{L}}$ \\
\hline Gross alpha & $\mathrm{GE}$ & & NA & $\mathbf{U}$ & & 2 & & 15 & $\mathrm{pCi} / \mathrm{L}$ \\
\hline Gross alpha & GE & & & $\mathbf{U}$ & & 2 & & 15 & $\mathrm{pCi} / \mathrm{L}$ \\
\hline Iron & WA & & & & & 186 & & 300 & $\mu g / L$ \\
\hline Iron & GE & & & & & 297 & & 300 & $\mu g / L$ \\
\hline Isobutyl alcohol & WA & & & $\mathbf{U}$ & & 20 & & NS & $\mu \mathrm{g} / \mathrm{L}$ \\
\hline Isobutyl alcohol & GE & & & UJ & $\mathbf{Q}$ & 100 & - & NS & $\mu g / L$ \\
\hline Lead & WA & & & $\mathbf{U}$ & & 3 & & 50 & $\mu \mathrm{g} \Omega$ \\
\hline Lead & $\mathrm{GE}$ & & & $\mathbf{U}$ & & 3 & & 50 & $\mu g / L$ \\
\hline Lead-212 & EP & & & $\mathbf{U}$ & & 15 & & NS & $\mathrm{pCi} / \mathrm{L}$ \\
\hline Magnesium & WA & & & $\mathbf{U}$ & & 5000 & & NS & $\mu g / L$ \\
\hline Magnesium & GE & & & & & 316 & & NS & $\mu g / L$ \\
\hline Manganese & WA & & & & & 41.6 & & 50 & $\mu g / L$ \\
\hline Manganese & GE & & & & & 44 & & 50 & $\mu g / L$ \\
\hline Manganese-54 & EP & & & $\mathbf{U}$ & & 10 & & 300 & $\mathrm{pCi} / \mathrm{L}$ \\
\hline Mercury & WA & & . & $\mathbf{U}$ & & 0.2 & & 2 & $\mu g / L$ \\
\hline Mercury & $\mathrm{GE}$ & & & U & & 0.2 & & 2 & $\mu g / \mathrm{L}$ \\
\hline Methacrylonitrile & WA & & & $\mathbf{U}$ & & 20 & & NS & $\mu \mathrm{g} / \mathrm{L}$ \\
\hline Methacrylonitrile & $\mathbf{G E}$ & & & UJ & $\mathbf{Q}$ & 50 & & NS & $\mu \mathrm{g} / \mathrm{L}$ \\
\hline Methyl bromide & WA & & & $\mathbf{U}$ & & 10 & & NS & $\mu g / L$ \\
\hline Methyl bromide & GE & & & UJ & $\mathbf{Q}$ & 1 & & NS & $\mu g / L$ \\
\hline Methyl chloride & WA & & & $\mathbf{U}$ & & 10 & & NS & $\mu \mathrm{g} / \mathrm{L}$ \\
\hline Methyl chloride & GE & & & UJ & $\mathbf{Q}$ & 1 & & NS & $\mu \mathrm{g} / \mathrm{L}$ \\
\hline Methyl ethyl ketone & WA & & & $U$ & & 10 & & NS & $\mu g / L$ \\
\hline Methyl ethyl ketone & GE & & & UJ & Q & 1 & & NS & $\mu \mathrm{g} / \mathrm{L}$ \\
\hline Methyl iodide & WA & & & $\mathbf{U}$ & & 10 & & NS & $\mu g / L$ \\
\hline Methyl iodide & GE & & & UJ & $\mathbf{Q}$ & 15 & & NS & $\mu g /$ \\
\hline Methyl isobutyl ketone & GE & & & UJ & $Q$ & 1 & & NS & $\mu \mathrm{g} / \mathrm{L}$ \\
\hline Methylene bromide & WA & & & $\mathbf{U}$ & & 10 & & NS & $\mu g / L$ \\
\hline Methylene bromide & GE & & & UJ & $\mathbf{Q}$ & 1 & & NS & $\mu \mathrm{g} / \mathrm{L}$ \\
\hline Methylene chloride & WA & & & J & & 1.39 & & 5 & $\mu g / \mathrm{L}$ \\
\hline Methylene chloride & GE & & & UJ & $Q$ & 1 & & 5 & $\mu \mathrm{g} / \mathrm{L}$ \\
\hline Nickel & WA & & & $\mathbf{U}$ & & 40 & & 100 & $\mu g / L$ \\
\hline Nickel & GE & & & $\mathbf{U}$ & & 4 & & 100 & $\mu g / L$ \\
\hline Nitrate as nitrogen & WA & & & & & 0.203 & & 10 & $\mathrm{mg} / \mathrm{L}$ \\
\hline Nitrate as nitrogen & GE & & NA & $\mathbf{U}$ & & 0.05 & & 10 & $\mathrm{mg} / \mathrm{L}$ \\
\hline Nitrate as nitrogen & GE & & & $\mathbf{U}$ & & 0.05 & & 10 & ing/L \\
\hline Nonvolatile beta & TM & & & $\mathbf{U}$ & & 0.7 & & 50 & $\mathrm{pCi} / \mathrm{L}$ \\
\hline Nonvolatile beta & $\mathbf{G E}$ & & NA & U & & 2 & & 50 & $\mathrm{pCi} / \mathrm{L}$ \\
\hline Nonvolatile beta & GE & & & U & & 2 & & 50 & $\mathrm{pCi} / \mathrm{L}$ \\
\hline $\mathrm{pH}$ & GE & & & $\mathrm{J}$ & $\mathbf{Q}$ & 5.2 & & NS & PH \\
\hline Potassium & WA & & & $\mathbf{U}$ & & 5000 & & NS & $\mu g / L$ \\
\hline Potassium & GE & & & U & & 500 & & NS & $\mu \mathrm{g} / \mathrm{L}$ \\
\hline Potassium-40. & EP & & & U & & 110 & & 300 & $\mathrm{pCi} / \mathrm{L}$ \\
\hline Promethium-144 & $\mathrm{EP}$ & & & U & & 10 & & NS & $\mathrm{pCi} / \mathrm{L}$ \\
\hline Promethium-146 & $\mathrm{EP}$ & & & $\mathbf{U}$ & & 10 & & NS & $\mathrm{pCi} / \mathrm{L}$ \\
\hline Propionitrile & WA & & & $\mathrm{U}$ & & 50 & & NS & $\mu \mathrm{g} / \mathrm{L}$ \\
\hline Propionitrile & GE & & & UJ & $\mathbf{Q}$ & 200 & & NS & $\mu \mathrm{g} / \mathrm{L}$ \\
\hline Ruthenium-106 & $\mathrm{EP}$ & & & U & & 90 & & 30 & $\mathrm{pCi} / \mathrm{L}$ \\
\hline Selenium & WA & & & $\mathrm{U}$ & & 5 & & 50 & $\mu g / L$ \\
\hline Selenium & GE & & & UJ & $\mathbf{L}$ & 2 & & 50 & $\mu \mathrm{g} / \mathrm{L}$ \\
\hline Silver & WA & & & $\mathbf{U}$ & & 10 & & 50 & $\mu \mathrm{g} / \mathrm{L}$ \\
\hline Silver & GE & & & U & & 2 & & 50 & $\mu \mathrm{g} / \mathrm{L}$ \\
\hline Sodium & WA & & & $\mathbf{U}$ & & 5000 & & NS & $\mu g / L$ \\
\hline Sodium & $\mathrm{GE}$ & & & & & 1060 & & NS & $\mu g / L$ \\
\hline Sodium-22 & EP & & & U & & 10 & & 466 & $\mathrm{pCi} / \mathrm{L}$ \\
\hline
\end{tabular}


UTR068 Laboratory Analyses (cont'd)

\begin{tabular}{|c|c|c|c|c|c|c|c|c|c|}
\hline Analyte & Lab & Rep & Avg & Qualifier & & Result & Acc & Stand & Units \\
\hline Styrene & WA & & & $\bar{U}$ & & 5 & & 100 & $\mu \mathrm{g} / \mathrm{h}$ \\
\hline Styrene & $\mathrm{GE}$ & & & UJ & Q & 1 & & 100 & $\mu g / L$ \\
\hline Sulfate & WA & & & & & 2.01 & & 400 & $\mathrm{mg} / \mathrm{L}$ \\
\hline Sulfate & $\mathrm{GE}$ & & & & & 2.78 & & 400 & $\mathrm{mg} / \mathrm{L}$ \\
\hline Sulfide & WA & & & $\mathbf{U}$ & & 0.1 & & NS & $\mathrm{mg} / \mathrm{L}$ \\
\hline Sulfide & GE & & NA & $\mathbf{U}$ & & 1 & . & NS & $\mathrm{mg} / \mathrm{L}$ \\
\hline Sulfide & GE & & & $\mathbf{U}$ & & 1 & & NS & $\mathrm{mg} / \mathrm{L}$ \\
\hline Tetrachloroethylene & WA & & & $\mathbf{U}$ & & 5 & & 5 & $\mu g / L$ \\
\hline Tetrachloroethylene & $\mathrm{GE}$ & & & UJ & $\mathbf{Q}$ & 1 & & 5 & $\mu g / L$ \\
\hline Thallium & WA & & & $\mathbf{U}$ & & 10 & & 2 & $\mu g / L$ \\
\hline Thallium & GE & & & $\mathbf{U}$ & & 2 & & 2 & $\mu g / L$ \\
\hline Thorium-234 & EP & & & $\mathbf{U}$ & & 350 & & 401 & pCi $/ 2$ \\
\hline Toluene & WA & & & $\mathbf{U}$ & & 5 & & 1000 & $\mu g / L$ \\
\hline Toluene & $\mathrm{GE}$ & & & UJ & $\mathbf{Q}$ & 1 & & 1000 & $\mu g / L$ \\
\hline Total hardness & WA & & & & & 14 & & NS & $\mathrm{mg} / \mathrm{h}$ \\
\hline Total hardness & GE & & A & & & 1.33 & & NS & $\mathrm{mg} / \mathrm{h}$ \\
\hline Total organic carbon & WA & & & & & 1.81 & & NS & $\mathrm{mg} / \mathrm{L}$ \\
\hline Total organic carton & GE & & & & & 2.04 & & NS & $\mathrm{mg} / \mathrm{L}$ \\
\hline Total suspended solids & WA & & & $\mathbf{U}$ & & 5 & & NS & $\mathrm{mg} / \mathrm{h}$ \\
\hline Total suspended solids & WA & & NA & $\mathbf{U}$ & & 5 & & NS & $\mathrm{mg} / \mathrm{h}$ \\
\hline Total suspended solids & $\mathbf{G E}$ & & $\mathbf{A}$ & & & 1 & & NS & $\mathrm{mg} / \mathrm{L}$ \\
\hline trans-1,3-Dichloropropene & WA & & & $\mathbf{U}$ & & 5 & & NS & $\mu g / L$ \\
\hline trans-1,3-Dichloropropene & GE & & & UJ & Q & 1 & & NS & $\mu g / L$ \\
\hline trans-1,4-Dichloro-2-butene & WA & & & $\mathbf{U}$ & & 100 & & NS & $\mu g / L$ \\
\hline trans-1,4-Dichloro-2-butene & GE & & & UJ & $\mathbf{Q}$ & 30 & & NS & $\mu g / L$ \\
\hline Trichloroethylene & WA & & & U & & 5 & & 5 & $\mu g / L$ \\
\hline Trichloroethylene & GE & & & UJ & $\mathbf{Q}$ & 1 & & 5 & $\mu g / L$ \\
\hline Trichlorofluoromethane & WA & & & $\mathbf{U}$ & & 5 & & NS & $\mu g / L$ \\
\hline Trichlorofluoromethane & GE & & & UJ & Q & 1 & & NS & $\mu g / L$ \\
\hline Tritium & $T M$ & & & & & 32.6 & 8.9 & 20 & $\mathrm{pCi} / \mathrm{ml}$ \\
\hline Tritium & GE & & & & & 32.4 & & 20 & $\mathrm{pCi} / \mathrm{ml}$ \\
\hline Turbidity & WA & & & $\mathbf{U}$ & & 0.2 & & NS & NTU \\
\hline Turbidity & $\mathbf{G E}$ & & NA & UJ & Q & 0.1 & & NS & NTU \\
\hline Turbidity & $\mathbf{G E}$ & & & UJ & Q & 0.1 & & NS & NTU \\
\hline Vanadium & WA & & & $\mathbf{U}$ & & 50 & & NS & $\mu g / L$ \\
\hline Vanadium & $\mathrm{GE}$ & & & U & & 8 & & NS & $\mu \mathrm{g} / \mathrm{L}$ \\
\hline Vinyl acetate & WA & & & $\mathbf{U}$ & & 10 & & NS & $\mu g / L$ \\
\hline Vinyl acetate & GE & & & UJ & $\mathbf{Q}$ & 1 & & NS & $\mu g / L$ \\
\hline Vinyl chloride & WA & & & U & & 10 & & 2 & $\mu g / L$ \\
\hline Vinyl chloride & $\mathrm{GE}$ & & & UJ & $Q$ & 1 & & 2 & $\mu \mathrm{g} / \mathrm{L}$ \\
\hline Xylenes (total) & WA & & & $\mathbf{U}$ & & 5 & & 10000 & $\mu \mathrm{g} / \mathrm{L}$ \\
\hline Xylenes (total) & GE & & & UJ & $Q$ & 2 & & 10000 & $\mu \mathrm{g} / \mathrm{L}$ \\
\hline Yttrium-88 & EP & & & $\mathbf{U}$ & & 60 & & NS & $\mathrm{pCi} / \mathrm{L}$ \\
\hline Zinc & WA & & & U & & 20 & & 5000 & $\mu \mathrm{g} / \mathrm{L}$ \\
\hline Zinc & GE & & & & & 22.7 & & 5000 & $\mu \mathrm{g} / \mathrm{L}$ \\
\hline Zinc-65 & EP & & & $U$ & & 20 & & 300 & $\mathrm{Ci} / \mathrm{L}$ \\
\hline
\end{tabular}




\section{UTR071}

Field Measurements:

Sample Date: $4 / 15 / 93$

pH: 5.38

Specific Conductance: $19 \mu \mathrm{S} / \mathrm{cm}$

Red/Oxid Potential: $171.5 \mathrm{mv}$

Dissolved Oxygen: $2.3 \mathrm{mg} / \mathrm{L}$

\begin{tabular}{|c|c|c|c|c|c|c|c|c|}
\hline Analyte & Lab & Rep & Avg & Qualifier & Result & Acc & Stand & Units \\
\hline 1,1,1,2-Tetrachloroethane & WA & & & $\mathbf{U}$ & 10 & & NS & $\mu g / L$ \\
\hline 1,1,1-Trichloroethane & WA & & & $\mathbf{U}$ & 5 & & 200 & $\mu g / L$ \\
\hline 1,1,2,2-Tetrachloroethane & WA & & & $\mathbf{U}$ & 5 & & NS & $\mu g / L$ \\
\hline 1,1,2-Trichloroethane & WA & & & $\mathbf{U}$ & 5 & & 5 & $\mu g / L$ \\
\hline 1,1-Dichloroethane & WA & & & $\mathbf{U}$ & 5 & & NS & $\mu g / L$ \\
\hline 1,1-Dichloroethylene & WA & & & $\mathbf{U}$ & 5 & & 7 & $\mu g / L$ \\
\hline 1,2,3-Trichloropropane & WA & & & $\mathbf{U}$ & 10 & & NS & $\mu g / L$ \\
\hline 1,2-Dibromo-3-chloropropane & WA & & & $\mathbf{U}$ & 20 & & 0.2 & $\mu g / L$ \\
\hline 1,2-Dibromoethane & WA & & & $\mathbf{U}$ & 20 & & NS & $\mu g / L$ \\
\hline 1,2-Dichloroethane & WA & & & $\mathbf{U}$ & 5 & & 5 & $\mu g / L$ \\
\hline 1,2-Dichloroethylene (total) & WA & & & $\mathbf{U}$ & 5 & & 100 & $\mu g / L$ \\
\hline 1,2-Dichloropropane & WA & & & $\mathbf{U}$ & 5 & & 5 & $\mu g / L$ \\
\hline 2-Hexanone & WA & & & $\mathbf{U}$ & 10 & & NS & $\mu g / L$ \\
\hline 4-Methyl-2-pentanone & WA & & & $\mathbf{U}$ & 10 & & NS & $\mu \mathrm{g} / \mathrm{L}$ \\
\hline Acetone & WA & & & $\mathbf{U}$ & 10 & & NS & $\mu g / L$ \\
\hline Acetonitrile & WA & & & $\mathbf{U}$ & 20 & & NS & $\mu g / L$ \\
\hline Acidity & WA & & & & 28 & & NS & $\mathrm{mg} / \mathrm{L}$ \\
\hline Acrolein & WA & & & $\mathbf{U}$ & 10 & & NS & $\mu g / L$ \\
\hline Acrylonitrile & WA & & & $\mathbf{U}$ & 10 & & NS & $\mu g / L$ \\
\hline Alkalinity & WA & & & & 0.5 & & NS & $\mathrm{mg} / \mathrm{L}$ \\
\hline Allyl chloride & WA & & & $\mathbf{U}$ & 100 & & NS & $\mu g / L$ \\
\hline Aluminum & WA & & & $\mathbf{U}$ & 200 & & NS & $\mu g / L$ \\
\hline Antimony & WA & & & $\mathbf{U}$ & 60 & & 6 & $\mu g / L$ \\
\hline Arsenic & WA & & & $\mathbf{U}$ & 10 & & 50 & $\mu g / L$ \\
\hline Barium & WA & & & $\mathbf{U}$ & 200 & & 2000 & $\mu g / L$ \\
\hline Benzene & WA & & & $\mathbf{U}$ & 5 & & 5 & $\mu g / L$ \\
\hline Beryllium & WA & & & $\mathbf{U}$ & 5 & & 4 & $\mu g / L$ \\
\hline Bromodichloromethane & WA & & & $\mathbf{U}$ & 5 & & NS & $\mu g / L$ \\
\hline Bromoform & WA & & & $\mathbf{U}$ & 5 & & NS & $\mu g / L$ \\
\hline Cadmium & WA & & & $\mathbf{U}$ & 5 & & 5 & $\mu g / L$ \\
\hline Calcium & WA & & & $\mathbf{U}$ & 5000 & & NS & $\mu g / L$ \\
\hline Carbon disulfide & WA & & & $\mathbf{U}$ & 5 & & NS & $\mu \mathrm{g} / \mathrm{L}$ \\
\hline Carbon tetrachloride & WA & & & $\mathbf{U}$ & 5 & & 5 & $\mu g / L$ \\
\hline Cesium-137 & TM & & & & 4.9 & 4.8 & 200 & $\mathrm{pCi} / \mathrm{L}$ \\
\hline Chloride & WA & & & & 4.97 & & NS & $\mathrm{mg} / \mathrm{L}$ \\
\hline Chlorobenzene & WA & & & $\mathbf{U}$ & 5 & & 100 & $\mu \mathrm{g} / \mathrm{L}$ \\
\hline Chloroethane & WA & & & $\mathbf{U}$ & 10 & & NS & $\mu \mathrm{g} / \mathrm{L}$ \\
\hline Chloroform & WA & & & $\mathbf{U}$ & 5 & & 100 & $\mu g / L$ \\
\hline Chloroprene & WA & & & $\mathbf{U}$ & 20 & & NS & $\mu \mathrm{g} / \mathrm{L}$ \\
\hline Chromium & WA & & & $\mathbf{U}$ & 10 & & 100 & $\mu \mathrm{g} / \mathrm{L}$ \\
\hline cis-1,3-Dichloropropene & WA & & & U & 5 & & NS & $\mu g / L$ \\
\hline Cobalt & WA & & & $\mathbf{U}$ & 50 & & NS & $\mu g / L$ \\
\hline Copper & WA & & & $\mathbf{U}$ & 25 & & 1000 & $\mu g / L$ \\
\hline Cyanide & WA & & & $\mathbf{U}$ & 10 & & 200 & $\mu g / L$ \\
\hline Dibromochloromethane & WA & & & $\mathbf{U}$ & 5 & & NS & $\mu g / L$ \\
\hline Dichlorodifluoromethane & WA & & & $\mathbf{U}$ & 10 & & NS & $\mu g / L$ \\
\hline Ethylbenzene & WA & & & $\mathbf{U}$ & 5 & & 700 & $\mu g / L$ \\
\hline Fluoride & WA & & & $\mathbf{U}$ & 0.1 & & 4 & $\mathrm{mg} / \mathrm{L}$ \\
\hline Gross alpha & TM & & & $\mathbf{U}$ & 0.6 & & 15 & $\mathrm{pCi} / \mathrm{L}$ \\
\hline
\end{tabular}


UTR071 Laboratory Analyses (cont'd)

\begin{tabular}{|c|c|c|c|c|c|c|c|}
\hline Analyte & Lab & Rep Avg & Qualifier & Result & Acc & Stand & Units \\
\hline Iron & WA & & & 451 & & 300 & $\mu g / L$ \\
\hline Isobutyl alcohol & WA & & $\mathbf{U}$ & 20 & & NS & $\mu \mathrm{g} / \mathrm{L}$ \\
\hline Lead & WA & & $\mathbf{U}$ & 3 & & 50 & $\mu g / L$ \\
\hline Magnesium & WA & & $\mathbf{U}$ & 5000 & & NS & $\mu g / L$ \\
\hline Manganese & WA & & & 79 & & 50 & $\mu g / L$ \\
\hline Mercury & WA & & $\mathbf{U}$ & 0.2 & . & 2 & $\mu g / L$ \\
\hline Methacrylonitrile & WA & & $\mathbf{U}$ & 20 & & NS & $\mu g / L$ \\
\hline Methyl bromide & WA & & $\mathbf{U}$ & 10 & & NS & $\mu g / L$ \\
\hline Methyl chloride & WA & & $\mathbf{U}$ & 10 & & NS & $\mu g / L$ \\
\hline Methyl ethyl ketone & WA & & $\mathbf{U}$ & 10 & & NS & $\mu g / L$ \\
\hline Methyl iodide & WA & & $\mathbf{U}$ & 10 & & NS & $\mu g / L$ \\
\hline Methylene bromide & WA & & $\mathbf{U}$ & 10 & & NS & $\mu g / L$ \\
\hline Methylene chloride & WA & & $\mathbf{U}$ & 5 & & 5 & $\mu g / L$ \\
\hline Nickel & WA & & $\mathbf{U}$ & 40 & & 100 & $\mu g / L$ \\
\hline Nitrate as nitrogen & WA & & & 0.237 & & 10 & $\mathrm{mg} / \mathrm{L}$ \\
\hline Nonvolatile beta & $\mathbf{T M}$ & · & $\mathbf{U}$ & 0.8 & & 50 & $\mathrm{pCi} / \mathrm{L}$ \\
\hline Potassium & WA & & $\mathbf{U}$ & 5000 & & NS & $\mu g / L$ \\
\hline Propionitrile & WA & & $\mathbf{U}$ & 50 & & NS & $\mu g / L$ \\
\hline Selenium & WA & & $\mathbf{U}$ & 5 & & 50 & $\mu g / L$ \\
\hline Silver & WA & & $\mathbf{U}$ & 10 & & 50 & $\mu g / L$ \\
\hline Sodium & WA & & $\mathbf{U}$ & 5000 & & NS & $\mu g / L$ \\
\hline Styrene & WA & & $\mathbf{U}$ & 5 & & 100 & $\mu g / L$ \\
\hline Sulfate & WA & & $\mathbf{U}$ & 2.5 & & 400 & $\mathrm{mg} / \mathrm{L}$ \\
\hline Sulfide & WA & & $\mathbf{U}$ & 0.1 & & NS & $\mathrm{mg} / \mathrm{L}$ \\
\hline Tetrachloroethylene & WA & & $\mathbf{U}$ & 5 & & 5 & $\mu g / L$ \\
\hline Thallium & WA & & $\mathbf{U}$ & 10 & & 2 & $\mu \mathrm{g} / \mathrm{L}$ \\
\hline Toluene & WA & & J & 1.77 & & 1000 & $\mu g / L$ \\
\hline Total hardness & WA & & $\mathbf{U}$ & 1 & & NS & $\mathrm{mg} / \mathrm{L}$ \\
\hline Total organic carbon & WA & & & 3.57 & & NS & $\mathrm{mg} / \mathrm{L}$ \\
\hline Total suspended solids & WA & & $\mathbf{U}$ & 5 & & NS & $\mathrm{mg} / \mathrm{L}$ \\
\hline trans-1,3-Dichloropropene & WA & & $\mathbf{U}$ & 5 & & NS & $\mu g / L$ \\
\hline trans-1,4-Dichloro-2-butene & WA & & $\mathbf{U}$ & 100 & & NS & $\mu \mathrm{g} / \mathrm{L}$ \\
\hline Trichloroethylene & WA & & $\mathbf{U}$ & 5 & & 5 & $\mu \mathrm{g} / \mathrm{L}$ \\
\hline Trichlorofluoromethane & WA & & $\mathbf{U}$ & 5 & & NS & $\mu g / L$ \\
\hline Tritium & TM & & & 11.1 & 3 & 20 & $\mathrm{pCi} / \mathrm{ml}$ \\
\hline Turbidity & WA & & & 0.6 & & NS & NTU \\
\hline Vanadium & WA & & U & 50 & & NS & $\mu g / L$ \\
\hline Vinyl acetate & WA & & $\mathbf{U}$ & 10 & & NS & $\mu \mathrm{g} / \mathrm{L}$ \\
\hline Vinyl chloride & WA & & $\mathbf{U}$ & 10 & & 2 & $\mu \mathrm{g} / \mathrm{L}$ \\
\hline Xylenes (total) & WA & & $\mathbf{U}$ & 5 & & 10000 & $\mu g / L$ \\
\hline Zinc & WA & & & 99 & & 5000 & $\mu g / L$ \\
\hline
\end{tabular}




\section{UTR075}

Field Measurements:

Sample Date: 4/14/93

pH: 6.12

Specific Conductance: $29 \mu \mathrm{S} / \mathrm{cm}$

Red/Oxid Potential: $141.7 \mathrm{mv}$

Dissolved Oxygen: $1 \mathrm{mg} / \mathrm{L}$

\begin{tabular}{|c|c|c|c|c|c|c|c|}
\hline Analyte & Lab & Avg & Qualifier & Result & Acc & Stand & Units \\
\hline 1,1,1,2-Tetrachloroethane & $\overline{\text { WA }}$ & & $\mathrm{U}$ & 10 & & NS & $\mu \mathrm{g} / \mathrm{L}$ \\
\hline 1,1,1-Trichloroethane & WA & & $\mathbf{U}$ & 5 & & 200 & $\mu g / L$ \\
\hline 1,1,2,2-Tetrachloroethane & WA & & $\mathbf{U}$ & 5 & & NS & $\mu g / L$ \\
\hline 1,1,2-Trichloroethane & WA & & U & 5 & & 5 & $\mu \mathrm{g} / \mathrm{L}$ \\
\hline 1,1-Dichloroethane & WA & & $\mathrm{U}$ & 5 & & NS & $\mu g / L$ \\
\hline 1,1-Dichloroethylene & WA & & U & 5 & & 7 & $\mu g / L$ \\
\hline 1,2,3-Trichloropropane & WA & & $\mathbf{U}$ & 10 & & NS & $\mu \mathrm{g} / \mathrm{L}$ \\
\hline 1,2-Dibromo-3-chloropropane & WA & & U & 20 & & 0.2 & $\mu \mathrm{g} / \mathrm{L}$ \\
\hline 1,2-Dibromoethane & WA & & $\mathbf{U}$ & 20 & & NS & $\mu g / L$ \\
\hline 1,2-Dichloroethane & WA & & U & 5 & & 5 & $\mu \mathrm{g} / \mathrm{L}$ \\
\hline 1,2-Dichloroethylene (total) & WA & & $\mathbf{U}$ & 5 & & 100 & $\mu \mathrm{g} / \mathrm{L}$ \\
\hline 1,2-Dichloropropane & WA & & $\mathbf{U}$ & 5 & & 5 & $\mu g / L$ \\
\hline 2-Hexanone & WA & & U & 10 & & NS & $\mu g / L$ \\
\hline 4-Methyl-2-pentanone & WA & & U & 10 & & NS & $\mu \mathrm{g} / \mathrm{L}$ \\
\hline Acetone & WA & & & 9.56 & & NS & $\mu \mathrm{g} / \mathrm{L}$ \\
\hline Acetonitrile & WA & & $\mathbf{U}$ & 20 & & NS & $\mu \mathrm{g} / \mathrm{L}$ \\
\hline Acidity & WA & & & 20 & & NS & $\mathrm{mg} / \mathrm{L}$ \\
\hline Acrolein & WA & & U & 10 & & NS & $\mu \mathrm{g} / \mathrm{L}$ \\
\hline Acrylonitrile & WA & & $\mathbf{U}$ & 10 & & NS & $\mu g / L$ \\
\hline Alkalinity & WA & & U & 0.5 & & NS & $\mathrm{mg} / \mathrm{L}$ \\
\hline Allyl chloride & WA & & $\mathbf{U}$ & 100 & & NS & $\mu g / L$ \\
\hline Aluminum & WA & & U & 200 & & NS & $\mu \mathrm{g} / \mathrm{L}$ \\
\hline Antimony & WA & & U & 60 & & 6 & $\mu g / L$ \\
\hline Arsenic & WA & & $\mathbf{U}$ & 10 & & 50 & $\mu g / L$ \\
\hline Barium & WA & & $U$ & 200 & & 2000 & $\mu g / L$ \\
\hline Benzene & WA & & $\mathbf{U}$ & 5 & & 5 & $\mu g / L$ \\
\hline Beryllium & WA & & $\mathbf{U}$ & 5 & & 4 & $\mu \mathrm{g} / \mathrm{L}$ \\
\hline Bromodichloromethane & WA & & $\mathbf{U}$ & 5 & & NS & $\mu g / L$ \\
\hline Bromoform & WA & & $\mathrm{U}$ & 5 & & NS & $\mu g / L$ \\
\hline Cadmium & WA & & $\mathbf{U}$ & 5 & & 5 & $\mu \mathrm{g} / \mathrm{L}$ \\
\hline Calcium & WA & & $\mathbf{U}$ & 5000 & & NS & $\mu g / L$ \\
\hline Carbon disulfide & WA & & $\mathbf{U}$ & 5 & & NS & $\mu \mathrm{g} / \mathrm{L}$ \\
\hline Carbon tetrachloride & WA & & $\mathbf{U}$ & 5 & & 5 & $\mu \mathrm{g} / \mathrm{L}$ \\
\hline Cerium-139139 & TM & & & 6.1 & 4.1 & & $\mathrm{pCi} / \mathrm{L}$ \\
\hline Cesium-137 & TM & & $\mathbf{U}$ & 6.1 & & 200 & $\mathrm{pCi} / \mathrm{L}$ \\
\hline Chloride & WA & & & 4.15 & & NS & $\mathrm{mg} / \mathrm{L}$ \\
\hline Chlorobenzene & WA & & $\mathrm{U}$ & 5 & & 100 & $\mu \mathrm{g} / \mathrm{L}$ \\
\hline Chloroethane & WA & & $\mathrm{U}$ & 10 & & NS & $\mu \mathrm{g} / \mathrm{L}$ \\
\hline Chloroform & WA & & $\mathbf{U}$ & 5 & & 100 & $\mu \mathrm{g} / \mathrm{L}$ \\
\hline Chloroprene & WA & & $\mathrm{U}$ & 20 & & NS & $\mu \mathrm{g} / \mathrm{L}$ \\
\hline Chromium & WA & & $\mathbf{U}$ & 10 & & 100 & $\mu g / L$ \\
\hline cis-1,3-Dichloropropene & WA & & $\mathbf{U}$ & 5 & & NS & $\mu g / L$ \\
\hline Cobalt & WA & & $\mathbf{U}$ & 50 & & NS & $\mu \mathrm{g} / \mathrm{L}$ \\
\hline Copper & WA & & $\mathbf{U}$ & 25 & & 1000 & $\mu g / L$ \\
\hline Cyanide & WA & & $\mathbf{U}$ & 10 & & 200 & $\mu \mathrm{g} / \mathrm{L}$ \\
\hline Dibromochloromethane & WA & & $\mathbf{U}$ & 5 & & NS & $\mu g / L$ \\
\hline Dichlorodifluoromethane & WA & & $\mathbf{U}$ & 10 & & NS & $\mu \mathrm{g} / \mathrm{L}$ \\
\hline Ethylbenzene & WA & & $\mathbf{U}$ & 5 & & 700 & $\mu \mathrm{g} / \mathrm{L}$ \\
\hline Fluoride & WA & & $\mathbf{U}$ & 0.1 & & 4 & $\mathrm{mg} / \mathrm{L}$ \\
\hline
\end{tabular}


UTR075 Laboratory Analyses (cont'd)

\begin{tabular}{|c|c|c|c|c|c|c|c|c|}
\hline Analyte & Lab & Rep & Avg & Qualifier & Result & Acc & Stand & Units \\
\hline Gross alpha & $\overline{T M}$ & & & $\mathbf{U}$ & 0.6 & & 15 & $\mathrm{pCi} / \mathrm{L}$ \\
\hline Iron & WA & & & & 597 & & 300 & $\mu g / L$ \\
\hline Isobutyl alcohol & WA & & & $\mathbf{U}$ & 20 & & NS & $\mu g / L$ \\
\hline Lead & WA & & & $\mathbf{U}$ & 3 & & 50 & $\mu \mathrm{g} / \mathrm{L}$ \\
\hline Magnesium & WA & & & $\bar{U}$ & 5000 & & NS & $\mu \mathrm{g} / \mathrm{L}$ \\
\hline Manganese & WA & & & & 52.1 & . & 50 & $\mu \mathrm{g} / \mathrm{L}$ \\
\hline Mercury & WA & & & $\mathbf{U}$ & 0.2 & & 2 & $\mu g / L$ \\
\hline Methacrylonitrile & WA & & & $\mathbf{U}$ & 20 & & NS & $\mu g / L$ \\
\hline Methyl bromide & WA & & & $\mathbf{U}$ & 10 & & NS & $\mu g / L$ \\
\hline Methyl chloride & WA & & & $\mathbf{U}$ & 10 & & NS & $\mu g / L$ \\
\hline Methyl ethyl ketone & WA & & & $\mathbf{U}$ & 10 & & NS & $\mu g / L$ \\
\hline Methyl iodide & WA & & & $\mathbf{U}$ & 10 & & NS & $\mu \mathrm{g} / \mathrm{L}$ \\
\hline Methylene bromide & WA & & & $\mathbf{U}$ & 10 & & NS & $\mu g / L$ \\
\hline Methylene chloride & WA & & & $\mathbf{U}$ & 5 & & 5 & $\mu \mathrm{g} / \mathrm{L}$ \\
\hline Nickel & WA & & & $\mathbf{U}$ & 40 & & 100 & $\mu g / L$ \\
\hline Nitrate as nitrogen & WA & & & $\mathbf{U}$ & 0.02 & & 10 & $\mathrm{mg} / \mathrm{L}$ \\
\hline Nonvolatile beta & TM & & & & 0.9 & 1.5 & 50 & $\mathrm{pCi} / \mathrm{L}$ \\
\hline Potassium & WA & & & $\mathbf{U}$ & 5000 & & NS & $\mu g / L$ \\
\hline Propionitrile & WA & & & $\mathbf{U}$ & 50 & & NS & $\mu g / L$ \\
\hline Ruthenium-106 & TM & & & & 52.4 & 45.2 & 30 & $\mathrm{pCi} / \mathrm{L}$ \\
\hline Selenium & WA & & & $\mathbf{U}$ & 5 & & 50 & $\mu g / L$ \\
\hline Silver & WA & & & $\mathbf{U}$ & 10 & & 50 & $\mu \mathrm{g} / \mathrm{L}$ \\
\hline Sodium & WA & & & $\mathbf{U}$ & 5000 & & NS & $\mu g / L$ \\
\hline Styrene & WA & & & $\mathbf{U}$ & 5 & & 100 & $\mu \mathrm{g} / \mathrm{L}$ \\
\hline Sulfate & WA & & & $\mathbf{U}$ & 2.5 & & 400 & $\mathrm{mg} / \mathrm{L}$ \\
\hline Sulfide & WA & & & $\mathbf{U}$ & 0.1 & & NS & $\mathrm{mg} / \mathrm{L}$ \\
\hline Tetrachloroethylene & WA & & & $\mathbf{U}$ & 5 & & 5 & $\mu g / L$ \\
\hline Thallium & WA & & & $\mathbf{U}$ & 10 & & 2 & $\mu \mathrm{g} / \mathrm{L}$ \\
\hline Toluene & WA & & & J & 1.77 & & 1000 & $\mu \mathrm{g} / \mathrm{L}$ \\
\hline Total hardness & WA & & & & 6 & & NS & $\mathrm{mg} / \mathrm{L}$ \\
\hline Total organic carbon & WA & & & & 6.71 & & NS & $\mathrm{mg} / \mathrm{L}$ \\
\hline Total suspended solids & WA & & & $\mathbf{U}$ & 5 & & NS & $\mathrm{mg} / \mathrm{L}$ \\
\hline trans-1,3-Dichloropropene & WA & & & $\mathrm{U}$ & 5 & & NS & $\mu g / L$ \\
\hline trans-1,4-Dichloro-2-butene & WA & & & $\mathbf{U}$ & 100 & & NS & $\mu g / L$ \\
\hline Trichloroethylene & WA & & & $\mathbf{U}$ & 5 & & 5 & $\mu \mathrm{g} / \mathrm{L}$ \\
\hline Trichlorofluoromethane & WA & & & $\mathrm{U}$ & 5 & & NS & $\mu g / L$ \\
\hline Tritium & $\mathrm{TM}$ & & & & 8.8 & 2.4 & 20 & $\mathrm{pCi} / \mathrm{ml}$ \\
\hline Turbidity & WA & & & & 0.49 & & NS & NTU \\
\hline Vanadium & WA & & & $U$ & 50 & & NS & $\mu g / L$ \\
\hline Vinyl acetate & WA & & & $\mathbf{U}$ & 10 & & NS & $\mu \mathrm{g} / \mathrm{L}$ \\
\hline Vinyl chloride & WA & & & $\mathrm{U}$ & 10 & & 2 & $\mu \mathrm{g} / \mathrm{L}$ \\
\hline Xylenes (total) & WA & & & $U$ & 5 & & 10000 & $\mu \mathrm{g} / \mathrm{L}$ \\
\hline Zinc & WA & & & & 65.6 & & 5000 & $\mu \mathrm{g} / \mathrm{L}$ \\
\hline
\end{tabular}




\section{UTR080}

Field Measurements:

Sample Date: 4/20/93

pH: 4.92

Specific Conductance: $42 \mu \mathrm{S} / \mathrm{cm}$

Red/Oxid Potential: $149 \mathrm{mv}$

Dissolved Oxygen: $2.4 \mathrm{mg} / \mathrm{h}$

\begin{tabular}{|c|c|c|c|c|c|c|c|c|}
\hline Analyte & Lab & Rep & Avg & Qualifier & Result & Acc & Stand & Units \\
\hline 1,1,1,2-Tetrachloroethane & WA & & & $\mathbf{U}$ & 10 & & NS & $\mu g / L$ \\
\hline 1,1,1-Trichloroethane & WA & & & $\mathbf{U}$ & 5 & & 200 & $\mu g / L$ \\
\hline 1,1,2,2-Tetrachloroethane & WA & & & $\mathbf{U}$ & 5 & & NS & $\mu g / L$ \\
\hline 1,1,2-Trichloroethane & WA & & & $\mathbf{U}$ & 5 & & 5 & $\mu g / L$ \\
\hline 1,1-Dichlorocthane & WA & & & $\mathbf{U}$ & 5 & & NS & $\mu g / L$ \\
\hline 1,1-Dichloroethylene & WA & & & $\mathbf{U}$ & 5 & & 7 & $\mu g / L$ \\
\hline 1,2,3-Trichloropropane & WA & & & $\mathbf{U}$ & 10 & & NS & $\mu g / L$ \\
\hline 1,2-Dibromo-3-chloropropane & WA & & . & $\mathbf{U}$ & 20 & & 0.2 & $\mu g / L$ \\
\hline 1,2-Dibromoethane & WA & & & $\mathbf{U}$ & 20 & & NS & $\mu g / L$ \\
\hline 1,2-Dichloroethane & WA & & & $\mathbf{U}$ & 5 & & 5 & $\mu g / L$ \\
\hline 1,2-Dichloroethylene (total) & WA & & & $\mathbf{U}$ & 5 & & 100 & $\mu g / L$ \\
\hline 1,2-Dichloropropane & WA & & & $\mathbf{U}$ & 5 & & 5 & $\mu g / L$ \\
\hline 2-Hexanone & WA & & & $\mathbf{U}$ & 10 & & NS & $\mu g / L$ \\
\hline 4-Methyl-2-pentanone & WA & & & $\mathbf{U}$ & 10 & & NS & $\mu \mathrm{g} / \mathrm{L}$ \\
\hline Acetone & WA & & & & 13.9 & & NS & $\mu g / L$ \\
\hline Acetonitrile & WA & & & $\mathbf{U}$ & 20 & & NS & $\mu g / L$ \\
\hline Acidity & WA & & $\mathbf{A}$ & & 82 & & NS & $\mathrm{mg} / \mathrm{L}$ \\
\hline Acrolein & WA & & & $\mathbf{U}$ & 10 & & NS & $\mu \mathrm{g} / \mathrm{L}$ \\
\hline Acrylonitrile & WA & & & $\mathbf{U}$ & 10 & & NS & $\mu g / L$ \\
\hline Alkalinity & WA & & & $\mathbf{U}$ & 0.5 & & NS & $\mathrm{mg} / \mathrm{L}$ \\
\hline Allyl chloride & WA & & & $\mathbf{U}$ & 100 & & NS & $\mu \mathrm{g} / \mathrm{L}$ \\
\hline Aluminum & WA & & & & 2000 & & NS & $\mu g / L$ \\
\hline Antimony & WA & & & $\mathbf{U}$ & 60 & & 6 & $\mu g / L$ \\
\hline Arsenic & WA & & & $\mathbf{U}$ & 10 & & 50 & $\mu g / L$ \\
\hline Barium & WA & & & $\mathbf{U}$ & 200 & & 2000 & $\mu \mathrm{g} / \mathrm{L}$ \\
\hline Benzene & WA & & & $\mathbf{U}$ & 5 & & 5 & $\mu \mathrm{g} / \mathrm{L}$ \\
\hline Beryllium & WA & & & $\mathbf{U}$ & 5 & & 4 & $\mu \mathrm{g} / \mathrm{L}$ \\
\hline Bromodichloromethane & WA & & & $\mathbf{U}$ & 5 & & NS & $\mu g / L$ \\
\hline Bromoform & WA & & & $\mathbf{U}$ & 5 & & NS & $\mu \mathrm{g} / \mathrm{L}$ \\
\hline Cadmium & WA & & & $\mathbf{U}$ & 5 & & 5 & $\mu \mathrm{g} / \mathrm{L}$ \\
\hline Calcium & WA & & & $\mathbf{U}$ & 5000 & & NS & $\mu \mathrm{g} / \mathrm{L}$ \\
\hline Carbon disulfide & WA & & & $\mathbf{U}$ & 5 & & NS & $\mu \mathrm{g} / \mathrm{L}$ \\
\hline Carbon tetrachloride & WA & & & $\mathbf{U}$ & 5 & & 5 & $\mu \mathrm{g} / \mathrm{L}$ \\
\hline Cesium-137 & TM & & NA & $\mathbf{U}$ & 10 & & 200 & $\mathrm{pCi} / \mathrm{L}$ \\
\hline Cesium-137 & TM & & & $\mathbf{U}$ & 9.7 & & 200 & $\mathrm{pCi} / \mathrm{L}$ \\
\hline Chloride . & WA & & & & 7.09 & & NS & $\mathrm{mg} / \mathrm{L}$ \\
\hline Chlorobenzene & WA & & & $\mathbf{U}$ & 5 & & 100 & $\mu g / L$ \\
\hline Chloroethane & WA & & & $\mathbf{U}$ & 10 & & NS & $\mu g / L$ \\
\hline Chloroform & WA & & & $\mathbf{U}$ & 5 & & 100 & $\mu \mathrm{g} / \mathrm{L}$ \\
\hline Chloroprene & WA & & & $\mathbf{U}$ & 20 & & NS & $\mu \mathrm{g} / \mathrm{L}$ \\
\hline Chromium & WA & & & $\mathbf{U}$ & 10 & & 100 & $\mu \mathrm{g} / \mathrm{L}$ \\
\hline cis-1,3-Dichloropropene & WA & & & $\mathbf{U}$ & 5 & & NS & $\mu \mathrm{g} / \mathrm{L}$ \\
\hline Cobalt & WA & & & $\mathbf{U}$ & 50 & & NS & $\mu \mathrm{g} / \mathrm{L}$ \\
\hline Copper & WA & & & $\mathrm{U}$ & 25 & & 1000 & $\mu g / L$ \\
\hline Cyanide & WA & & & $\mathbf{U}$ & 10 & & 200 & $\mu \mathrm{g} / \mathrm{L}$ \\
\hline Dibromochloromethane & WA & & & $\mathbf{U}$ & 5 & & NS & $\mu \mathrm{g} / \mathrm{L}$ \\
\hline Dichlorodifluoromethane & WA & & & $\mathbf{U}$ & 10 & & NS & $\mu \mathrm{g} / \mathrm{L}$ \\
\hline Ethylbenzene & WA & & & $\mathbf{U}$ & 5 & & 700 & $\mu \mathrm{g} / \mathrm{L}$ \\
\hline Fluoride & WA & & & $\mathbf{U}$ & 0.1 & & 4 & mg/L \\
\hline
\end{tabular}


UTR080 Laboratory Analyses (cont'd)

\begin{tabular}{|c|c|c|c|c|c|c|c|c|}
\hline Analyte & Lab & Rep & Avg & Qualifier & Result & Acc & Stand & Units \\
\hline Gross alpha & $\overline{\mathrm{TM}}$ & & & & 4.9 & 1.9 & 15 & $\overline{\mathrm{pCi} / L}$ \\
\hline Iron & WA & & & & 905 & & 300 & $\mu \mathrm{g} / \mathrm{L}$ \\
\hline Isobutyl alcohol & WA & & & $\mathbf{U}$ & 20 & & NS & $\mu g / L$ \\
\hline Lead & WA & & & & 4.2 & & 50 & $\mu g / L$ \\
\hline Magnesium & WA & & & $\mathbf{U}$ & 5000 & & NS & $\mu g / L$ \\
\hline Manganese & WA & & & U & 15 & . & 50 & $\mu \mathrm{g} / \mathrm{L}$ \\
\hline Mercury & WA & & & $\mathbf{U}$ & 0.2 & & 2 & $\mu \mathrm{g} / \mathrm{L}$ \\
\hline Methacrylonitrile & WA & & & U & 20 & & NS & $\mu \mathrm{g} / \mathrm{L}$ \\
\hline Methyl bromide & WA & & & $\mathbf{U}$ & 10 & & NS & $\mu \mathrm{g} / \mathrm{L}$ \\
\hline Methyl chloride & WA & & & $\mathbf{U}$ & 10 & & NS & $\mu g / L$ \\
\hline Methyl ethyl ketone & WA & & & $\mathbf{U}$ & 10 & & NS & $\mu g / L$ \\
\hline Methyl iodide & WA & & & $\mathbf{U}$ & 10 & & NS & $\mu \mathrm{g} / \mathrm{L}$ \\
\hline Methylene bromide & WA & & & $\mathbf{U}$ & 10 & & NS & $\mu g / L$ \\
\hline Methylene chloride & WA & & & $\mathbf{U}$ & 5 & & 5 & $\mu g / L$ \\
\hline Nickel & WA & & & & 48.2 & & 100 & $\mu \mathrm{g} / \mathrm{L}$ \\
\hline Nitrate as nitrogen & WA & & & $\mathbf{U}$ & 0.1 & & 10 & $\mathrm{mg} / \mathrm{L}$ \\
\hline Nonvolatile beta & $\mathbf{T M}$ & & & & 7.2 & 2.3 & 50 & $\mathrm{pCi} / \mathrm{L}$ \\
\hline Potassium & WA & & & $\mathbf{U}$ & 5000 & & NS & $\mu g / L$ \\
\hline Propionitrile & WA & & & $\mathbf{U}$ & 50 & & NS & $\mu \mathrm{g} / \mathrm{L}$ \\
\hline Selenium & WA & & & U & 5 & & 50 & $\mu g / L$ \\
\hline Silver & WA & & & $\mathbf{U}$ & 10 & & 50 & $\mu g / L$ \\
\hline Sodium & WA & & & U & 5000 & & NS & $\mu g / L$ \\
\hline Styrene & WA & & & $\mathrm{U}$ & 5 & & 100 & $\mu g / \mathrm{L}$ \\
\hline Sulfate & WA & & & & 8.71 & & 400 & $\mathrm{mg} / \mathrm{L}$ \\
\hline Sulfide & WA & & & $\mathbf{U}$ & 0.1 & & NS & $\mathrm{mg} / \mathrm{L}$ \\
\hline Sulfide & WA & & NA & $\mathbf{U}$ & 0.1 & & NS & $\mathrm{mg} / \mathrm{L}$ \\
\hline Tetrachloroethylene & WA & & & $\mathbf{U}$ & 5 & & 5 & $\mu \mathrm{g} / \mathrm{L}$ \\
\hline Thallium & WA & & & $\mathbf{U}$ & 10 & & 2 & $\mu g / L$ \\
\hline Toluene & WA & & & $\mathbf{U}$ & 5 & & 1000 & $\mu \mathrm{g} / \mathrm{L}$ \\
\hline Total hardness & WA & & & & 27 & & NS & $\mathrm{mg} / \mathrm{L}$ \\
\hline Total organic carbon & WA & & & & 37.4 & & NS & $\mathrm{mg} / \mathrm{L}$ \\
\hline Total suspended solids & WA & & & & 7 & & NS & $\mathrm{mg} / \mathrm{L}$ \\
\hline trans-1,3-Dichloropropene & WA & & & $\mathbf{U}$ & 5 & & NS & $\mu g / L$ \\
\hline trans-1,4-Dichloro-2-butene & WA & & & $\mathbf{U}$ & 100 & & NS & $\mu g / L$ \\
\hline Trichloroethylene & WA & & & $\mathbf{U}$ & 5 & & 5 & $\mu g / L$ \\
\hline Trichlorofluoromethane & WA & & & $\mathbf{U}$ & 5 & & NS & $\mu \mathrm{g} / \mathrm{L}$ \\
\hline Tritium & TM & & $\mathbf{A}$ & & 6.85 & 1.5 & 20 & $\mathrm{pCi} / \mathrm{ml}$ \\
\hline Turbidity & WA & & & & 144 & & NS & NTU \\
\hline Vanadium & WA & & & $\mathbf{U}$ & 50 & & NS & $\mu g / L$ \\
\hline Vinyl acetate & WA & & & $\mathbf{U}$ & 10 & & NS & $\mu g / L$ \\
\hline Vinyl chloride & WA & & & $\mathbf{U}$ & 10 & & 2 & $\mu \mathrm{g} / \mathrm{L}$ \\
\hline Xylenes (total) & WA & & & $\mathbf{U}$ & 5 & & 10000 & $\mu \mathrm{g} / \mathrm{L}$ \\
\hline Zinc & WA & & & & 36.9 & & 5000 & $\mu \mathrm{d}$ \\
\hline
\end{tabular}




\section{UTR083}

Field Measurements:

Sample Date: 4/20/93

pH: 6.4

Specific Conductance: $133 \mu \mathrm{S} / \mathrm{cm}$

Red/Oxid Potential: $-\mathbf{2 0} \mathrm{mv}$

Dissolved Oxygen: $1.4 \mathrm{mg} / \mathrm{L}$

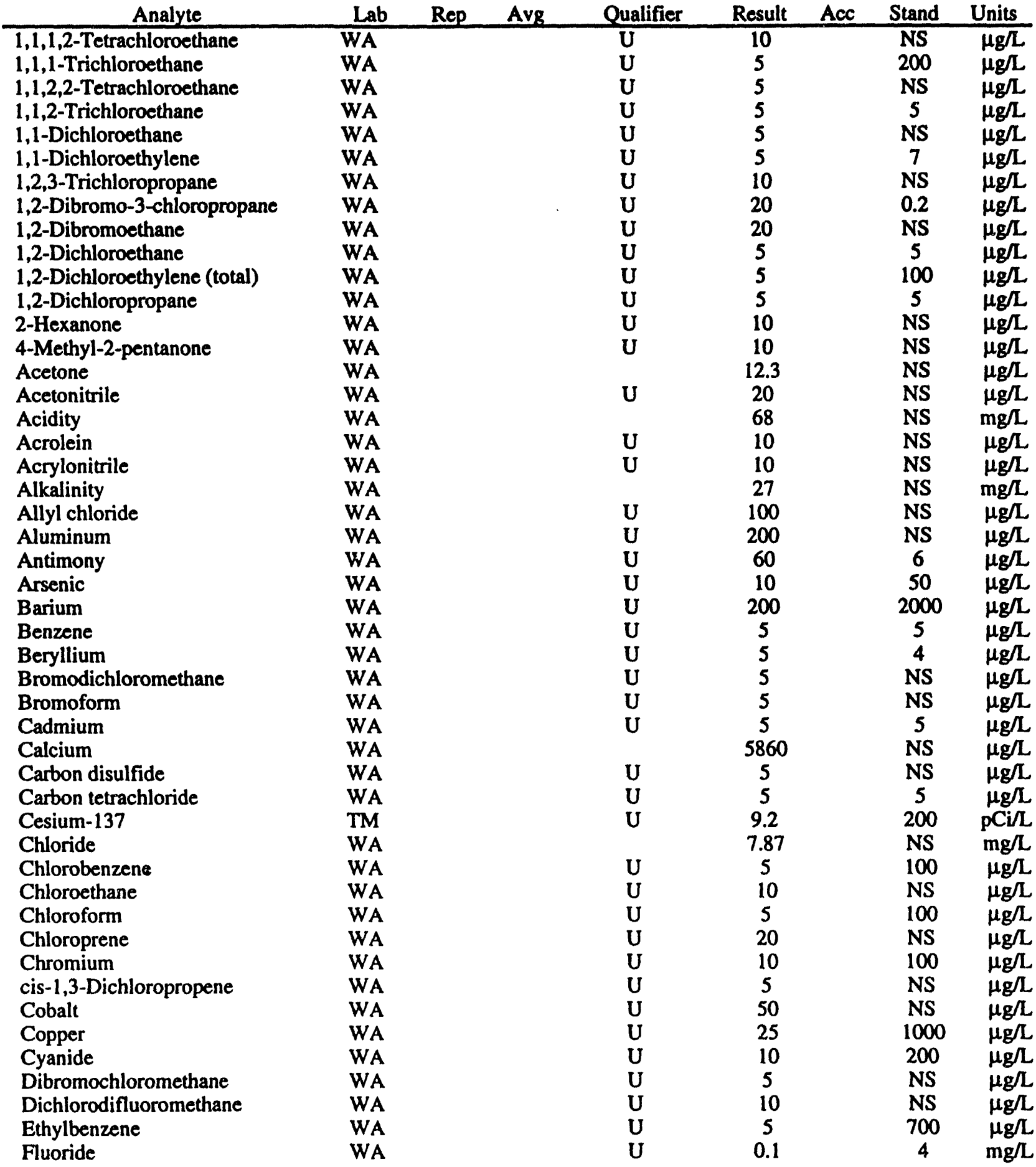


UTR083 Laboratory Analyses (cont'd)

\begin{tabular}{|c|c|c|c|c|c|c|c|}
\hline Analyte & Lab & Rep & Qualifier & Result & Acc & Stand & Units \\
\hline Gross alpha & TM & & & 0.9 & 1 & 15 & $\mathrm{pCi} / \mathrm{L}$ \\
\hline Iron & WA & & & 34500 & & & $\mu g / L$ \\
\hline Isobutyl alcohol & WA & & $\mathbf{U}$ & 20 & & NS & $\mu g / L$ \\
\hline Lead & WA & & $\mathbf{U}$ & 3 & & 50 & $\mu g / L$ \\
\hline Magnesium & WA & & $\mathbf{U}$ & 5000 & & NS & $\mu g / L$ \\
\hline Manganese & WA & & & 1810 & & 50 & $\mu g / L$ \\
\hline Mercury & WA & & $\mathbf{U}$ & 0.2 & & 2 & $\mu g / L$ \\
\hline Methacrylonitrile & WA & & $\mathbf{U}$ & 20 & & NS & $\mu g / L$ \\
\hline Methyl bromide & WA & & $\mathbf{U}$ & 10 & & NS & $\mu g / L$ \\
\hline Methyl chloride & WA & & $\mathbf{U}$ & 10 & & NS & $\mu g / L$ \\
\hline Methyl ethyl ketone & WA & & $\mathbf{U}$ & 10 & & NS & $\mu g / L$ \\
\hline Methyl iodide & WA & & $\mathbf{U}$ & 10 & & NS & $\mu g / L$ \\
\hline Methylene bromide & WA & & $\mathbf{U}$ & 10 & & NS & $\mu g / L$ \\
\hline Methylene chloride & WA & & $\mathbf{U}$ & 5 & & 5 & $\mu g / L$ \\
\hline Nickel & WA & & $\mathbf{U}$ & 40 & & 100 & $\mu g / L$ \\
\hline Nitrate as nitrogen & WA & & $\mathbf{U}$ & 0.1 & & 10 & $\mathrm{mg} / \mathrm{h}$ \\
\hline Nonvolatile beta & TM & & & 4.3 & 2 & 50 & $\mathrm{pCi} / \mathrm{L}$ \\
\hline Potassium & WA & & $\mathbf{U}$ & 5000 & & NS & $\mu g / L$ \\
\hline Propionitrile & WA & & $\mathbf{U}$ & 50 & & NS & $\mu g / L$ \\
\hline Selenium & WA & & $\mathbf{U}$ & 5 & & 50 & $\mu g / L$ \\
\hline Silver & WA & & $\mathbf{U}$ & 10 & & 50 & $\mu g / L$ \\
\hline Sodium & WA & & $\mathbf{U}$ & 5000 & & NS & $\mu g / L$ \\
\hline Styrene & WA & & $\mathbf{U}$ & 5 & & 100 & $\mu g / L$ \\
\hline Sulfate & WA & & & 4.97 & & 400 & $\mathrm{mg} / \mathrm{h}$ \\
\hline Sulfide & WA & & $\mathbf{U}$ & 0.1 & & NS & $\mathrm{mg} / \mathrm{L}$ \\
\hline Tetrachloroethylene & WA & & $\mathbf{U}$ & 5 & & 5 & $\mu g / L$ \\
\hline Thallium & WA & & $\mathbf{U}$ & 10 & & 2 & $\mu g / L$ \\
\hline Toluene & WA & & J & 1.84 & & 1000 & $\mu g / L$ \\
\hline Total hardness & WA & & & 26 & & NS & $\mathrm{mg} / \mathrm{L}$ \\
\hline Total organic carbon & WA & & & 16.3 & & NS & $\mathrm{mg} / \mathrm{h}$ \\
\hline Total suspended solids & WA & & & 39 & & NS & $\mathrm{mg} / \mathrm{L}$ \\
\hline trans-1,3-Dichloropropene & WA & & $\mathbf{U}$ & 5 & & NS & $\mu g / L$ \\
\hline trans-1,4-Dichloro-2-butene & WA & & $\mathbf{U}$ & 100 & & NS & $\mu g / L$ \\
\hline Trichloroethylene & WA & & $\mathbf{U}$ & 5 & & 5 & $\mu g / L$ \\
\hline Trichlorofluoromethane & WA & & $\mathbf{U}$ & 5 & & NS & $\mu \mathrm{g} / \mathrm{L}$ \\
\hline Tritium & TM & & & 5.2 & 1.2 & 20 & $\mathrm{pCi} / \mathrm{ml}$ \\
\hline Turbidity & WA & & & 64.2 & & NS & N1 \\
\hline Vanadium & WA & & $\mathbf{U}$ & 50 & & NS & \\
\hline Vinyl acetate & WA & & $\mathbf{U}$ & 10 & & NS & \\
\hline Vinyl chloride & WA & & $\mathrm{U}$ & 10 & & 2 & $\mu g^{\prime}$ \\
\hline Xylenes (total) & WA & & $\mathbf{U}$ & 5 & & 10000 & \\
\hline Zinc & WA & & $\mathbf{U}$ & 20 & & 5000 & \\
\hline
\end{tabular}




\section{UTR091}

Field Measurements:

Sample Date: 4/26/93

pH: 6.0

Specific Conductance: $42 \mu \mathrm{S} / \mathrm{cm}$

Red/Oxid Potential: $145 \mathrm{mv}$

Dissolved Oxygen: $1.3 \mathrm{mg} / \mathrm{L}$

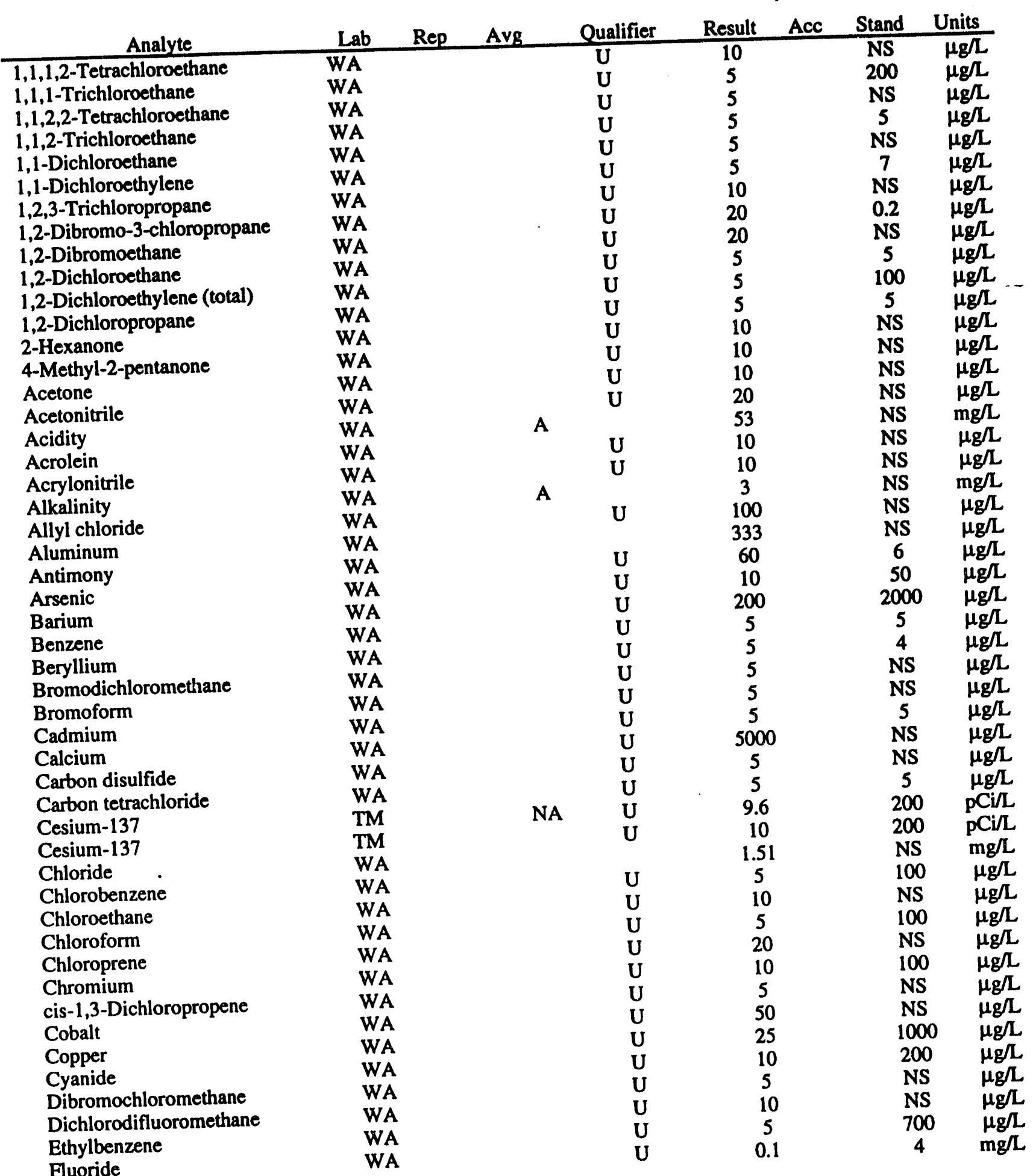


UTR091 Laboratory Analyses (cont'd)

\begin{tabular}{|c|c|c|c|c|c|c|c|}
\hline Analyte & Lab & Avg & Qualifier & Result & Acc & Stand & Units \\
\hline Gross alpha & TM & $\mathbf{N A}$ & & 0.7 & 0.8 & 15 & $\mathrm{pCi} / \mathrm{L}$ \\
\hline Gross alpha & TM & & $\mathbf{U}$ & 0.4 & & 15 & $\mathrm{pCi} / \mathrm{L}$ \\
\hline Iron & WA & & & 1560 & & 300 & $\mu g / L$ \\
\hline Isobutyl alcohol & WA & & $\mathbf{U}$ & 20 & & NS & $\mu g / L$ \\
\hline Lead & WA & & $\mathbf{U}$ & 3 & & 50 & $\mu g / L$ \\
\hline Magnesium & WA & & $\mathbf{U}$ & 5000 & . & NS & $\mu g / L$ \\
\hline Manganese & WA & & & 568 & & 50 & $\mu g / L$ \\
\hline Mercury & WA & & $\mathbf{U}$ & 0.2 & & 2 & $\mu g / L$ \\
\hline Methacrylonitrile & WA & & $\mathbf{U}$ & 20 & & NS & $\mu g / L$ \\
\hline Methyl bromide & WA & & $\mathbf{U}$ & 10 & & NS & $\mu g / L$ \\
\hline Methyl chloride & WA & & $\mathbf{U}$ & 10 & & NS & $\mu g / L$ \\
\hline Methyl ethyl ketone & WA & & $\mathbf{U}$ & 10 & & NS & $\mu g / L$ \\
\hline Methyl iodide & WA & & $\mathbf{U}$ & 10 & & NS & $\mu g / L$ \\
\hline Methylene bromide & WA & & $\mathbf{U}$ & 10 & & NS & $\mu g / L$ \\
\hline Methylene chloride & WA &. & $\mathbf{U}$ & 5 & & 5 & $\mu g / L$ \\
\hline Nickel & WA & . & $\mathbf{U}$ & 40 & & 100 & $\mu g / L$ \\
\hline Nitrate as nitrogen & WA & & $\mathbf{U}$ & 0.02 & & 10 & $\mathbf{m g} / \mathrm{L}$ \\
\hline Nonvolatile beta & TM & $\mathbf{A}$ & & 3.15 & 1.7 & 50 & $\mathrm{pCi} / \mathrm{L}$ \\
\hline Potassium & WA & & $\mathbf{U}$ & 5000 & & NS & $\mu g / L$ \\
\hline Propionitrile & WA & & $\mathbf{U}$ & 50 & & NS & $\mu g / L$ \\
\hline Selenium & WA & & $\mathbf{U}$ & 5 & & 50 & $\mu g / L$ \\
\hline Silver & WA & & $\mathbf{U}$ & 10 & & 50 & $\mu g / L$ \\
\hline Sodium & WA & & $\mathbf{U}$ & 5000 & & NS & $\mu \mathrm{g} / \mathrm{L}$ \\
\hline Styrene & WA & & $\mathbf{U}$ & 5 & & 100 & $\mu \mathrm{g} / \mathrm{L}$ \\
\hline Sulfate & WA & $\mathbf{A}$ & & 3.645 & & 400 & $\mathrm{mg} / \mathrm{L}$ \\
\hline Sulfide & WA & & $\mathbf{U}$ & 0.1 & & NS & $\mathrm{mg} / \mathrm{L}$ \\
\hline Tetrachloroethylene & WA & & $\mathbf{U}$ & 5 & & 5 & $\mu \mathrm{g} / \mathrm{L}$ \\
\hline Thallium & WA & & $\mathbf{U}$ & 10 & & 2 & $\mu \mathrm{g} / \mathrm{L}$ \\
\hline Toluene & WA & & $\mathbf{U}$ & 5 & & 1000 & $\mu \mathrm{g} / \mathrm{L}$ \\
\hline Total hardness & WA & & & 80 & & NS & $\mathrm{mg} / \mathrm{L}$ \\
\hline Total organic carbon & WA & A & & 5.51 & & NS & $\mathrm{mg} / \mathrm{L}$ \\
\hline Total suspended solids & WA & & & 5 & & NS & $\mathrm{mg} / \mathrm{L}$ \\
\hline trans-1,3-Dichloropropene & WA & & $\mathbf{U}$ & 5 & & NS & $\mu g / L$ \\
\hline trans-1,4-Dichloro-2-butene & WA & & $\mathbf{U}$ & 100 & & NS & $\mu \mathrm{g} / \mathrm{L}$ \\
\hline Trichloroethylene & WA & & $\mathbf{U}$ & 5 & & 5 & $\mu \mathrm{g} / \mathrm{L}$ \\
\hline Trichlorofluoromethane & WA & & $\mathbf{U}$ & 5 & & NS & $\mu \mathrm{g} / \mathrm{L}$ \\
\hline Tritium & TM & A & & 6.15 & 0.61 & 20 & $\mathrm{pCi} / \mathrm{ml}$ \\
\hline Turbidity & WA & A & & 17.4 & & NS & NTU \\
\hline Vanadium & WA & & $\mathbf{U}$ & 50 & & NS & $\mu \mathrm{g} / \mathrm{L}$ \\
\hline Vinyl acetate & WA & & $\mathbf{U}$ & 10 & & NS & $\mu g / L$ \\
\hline Vinyl chloride & WA & & $\mathbf{U}$ & 10 & & 2 & $\mu \mathrm{g} / \mathrm{L}$ \\
\hline Xylenes (total) & WA & & $\mathbf{U}$ & 5 & & 10000 & $\mu \mathrm{g} / \mathrm{L}$ \\
\hline Zinc & WA & & & 44.1 & & 5000 & $\mu g / L$ \\
\hline
\end{tabular}




\section{UTR099}

Field Measurements:

Sample Date: 4/20193

pH: 6.22

Specific Conductance: $60 \mu \mathrm{S} / \mathrm{cm}$

Red/Oxid Potential: - $199 \mathrm{mv}$

Dissolved Oxygen: $0.8 \mathrm{mg} / \mathrm{L}$

\begin{tabular}{|c|c|c|c|c|c|c|c|}
\hline Analyte & Lab & Avg & Qualifier & Result & Acc & Stand & Units \\
\hline 1,1,1,2-Tetrachloroethane & WA & & $\mathbf{U}$ & 10 & & NS & $\mu g / L$ \\
\hline 1,1,1-Trichloroethane & WA & & $\mathbf{U}$ & 5 & & 200 & $\mu g / L$ \\
\hline $1,1,2,2$-Tetrachloroethane & WA & & $\mathbf{U}$ & 5 & & NS & $\mu g / L$ \\
\hline 1,1,2-Trichloroethane & WA & & $\mathbf{U}$ & 5 & & 5 & $\mu g / L$ \\
\hline 1,1-Dichloroethane & WA & & $\mathbf{U}$ & 5 & & NS & $\mu g / L$ \\
\hline 1,1-Dichloroethylene & WA & & $\mathbf{U}$ & 5 & & 7 & $\mu g / L$ \\
\hline 1,2,3-Trichloropropane & WA & & $\mathbf{U}$ & 10 & & NS & $\mu \mathrm{g} / \mathrm{L}$ \\
\hline 1,2-Dibromo-3-chloropropane & WA & & $\mathbf{U}$ & 20 & & 0.2 & $\mu g / L$ \\
\hline 1,2-Dibromoethane & WA & & $\mathbf{U}$ & 20 & & NS & $\mu g / L$ \\
\hline 1,2-Dichloroethane & WA & & $\mathbf{U}$ & 5 & & 5 & $\mu g / L$ \\
\hline 1,2-Dichloroethylene (total) & WA & & $\mathbf{U}$ & 5 & & 100 & $\mu g / L$ \\
\hline 1,2-Dichloropropane & WA & & $\mathbf{U}$ & 5 & & 5 & $\mu \mathrm{g} / \mathrm{L}$ \\
\hline 2-Hexanone & WA & & $\mathbf{U}$ & 10 & & NS & $\mu g / L$ \\
\hline 4-Methyl-2-pentanone & WA & & $\mathbf{U}$ & 10 & & NS & $\mu g / L$ \\
\hline Acetone & WA & & & 11.3 & & NS & $\mu g / L$ \\
\hline Acetonitrile & WA & & $\mathbf{U}$ & 20 & & NS & $\mu g / L$ \\
\hline Acidity & WA & & & 48 & & NS & $\mathrm{mg} / \mathrm{L}$ \\
\hline Acrolein & WA & & $\mathbf{U}$ & 10 & & NS & $\mu g / L$ \\
\hline Acrylonitrile & WA & & $\mathbf{U}$ & 10 & & NS & $\mu g / L$ \\
\hline Alkalinity & WA & & & 14 & & NS & $\mathrm{mg} / \mathrm{L}$ \\
\hline Allyl chloride & WA & & $\mathbf{U}$ & 100 & & NS & $\mu g / L$ \\
\hline Aluminum & WA & & & 2530 & & NS & $\mu g / L$ \\
\hline Antimony & WA & & $\mathbf{U}$ & 60 & & 6 & $\mu \mathrm{g} / \mathrm{L}$ \\
\hline Arsenic & WA & & $\mathbf{U}$ & 10 & & 50 & $\mu \mathrm{g} / \mathrm{L}$ \\
\hline Barium & WA & & $\mathbf{U}$ & 200 & & 2000 & $\mu g / L$ \\
\hline Benzene & WA & & $\mathbf{U}$ & 5 & & 5 & $\mu g / L$ \\
\hline Beryllium & WA & & $\mathbf{U}$ & 5 & & 4 & $\mu \mathrm{g} / \mathrm{L}$ \\
\hline Bromodichloromethane & WA & & $\mathbf{U}$ & 5 & & NS & $\mu g / L$ \\
\hline Bromoform & WA & & $\mathbf{U}$ & 5 & & NS & $\mu \mathrm{g} / \mathrm{L}$ \\
\hline Cadmium & WA & & U & 5 & & 5 & $\mu \mathrm{g} / \mathrm{L}$ \\
\hline Calcium & WA & & $\mathbf{U}$ & 5000 & & NS & $\mu \mathrm{g} / \mathrm{L}$ \\
\hline Carbon disulfide & WA & & $\mathbf{U}$ & 5 & & NS & $\mu \mathrm{g} \Omega$ \\
\hline Carbon tetrachloride & WA & & $\mathbf{U}$ & 5 & & 5 & $\mu g / L$ \\
\hline Cesium-137 & $\mathrm{TM}$ & & $\mathbf{U}$ & 9.1 & & 200 & $\mathrm{pCi} / \mathrm{L}$ \\
\hline Chloride & WA & & & 3.66 & & NS & $\mathrm{mg} / \mathrm{L}$ \\
\hline Chlorobenzene & WA & & $\mathbf{U}$ & 5 & & 100 & $\mu \mathrm{g} / \mathrm{L}$ \\
\hline Chloroethane & WA & & $\mathbf{U}$ & 10 & & NS & $\mu g / L$ \\
\hline Chloroform & WA & & $U$ & 5 & & 100 & $\mu \mathrm{g} / \mathrm{L}$ \\
\hline Chloroprene & WA & & $\mathrm{U}$ & 20 & & NS & $\mu g / L$ \\
\hline Chromium & WA & & $\mathrm{U}$ & 10 & & 100 & $\mu g / L$ \\
\hline cis-1,3-Dichloropropene & WA & & $\mathbf{U}$ & 5 & & NS & $\mu g / L$ \\
\hline Cobalt & WA & & U & 50 & & NS & $\mu \mathrm{g} / \mathrm{L}$ \\
\hline Copper & WA & & $\mathbf{U}$ & 25 & & 1000 & $\mu \mathrm{g} / \mathrm{L}$ \\
\hline Cyanide & WA & & $\mathbf{U}$ & 10 & & 200 & $\mu g / L$ \\
\hline Dibromochloromethane & WA & & $\mathrm{U}$ & 5 & & NS & $\mu g / L$ \\
\hline Dichlorodifluoromethane & WA & & $\mathbf{U}$ & 10 & & NS & $\mu g / L$ \\
\hline Ethylbenzene & WA & & $\mathbf{U}$ & 5 & & 700 & $\mu g / L$ \\
\hline Fluoride & WA & & $\mathbf{U}$ & 0.1 & & 4 & $\mathrm{mg} / \mathrm{L}$ \\
\hline
\end{tabular}


UTR099 Laboratory Analyses (cont'd)

\begin{tabular}{|c|c|c|c|c|c|c|c|c|}
\hline Analyte & Lab & Rep & Avg & Qualifier & Result & Acc & Stand & Units \\
\hline Gross alpha & TM & & & & 9.4 & 2.8 & 15 & $\mathrm{pCi} / \mathrm{L}$ \\
\hline Iron & WA & & & & 16400 & & 300 & $\mu g / L$ \\
\hline Isobutyl alcohol & WA & & & $\mathbf{U}$ & 20 & & NS & $\mu g / L$ \\
\hline Lead & WA & & & & 3.4 & & 50 & $\mu \mathrm{g} / \mathrm{L}$ \\
\hline Magnesium & WA & & & $\mathbf{U}$ & 5000 & & NS & $\mu \mathrm{g} / \mathrm{L}$ \\
\hline Manganese & WA & & & & 402 & & 50 & $\mu g /$ \\
\hline Mercury & WA & & & $\mathbf{U}$ & 0.2 & & 2 & $\mu g / L$ \\
\hline Methacrylonitrile & WA & & & $\mathbf{U}$ & 20 & & NS & $\mu g / L$ \\
\hline Methyl bromide & WA & & & $\mathbf{U}$ & 10 & & NS & $\mu g / L$ \\
\hline Methyl chloride & WA & & & $\mathbf{U}$ & 10 & & NS & $\mu g / \alpha$ \\
\hline Methyl ethyl ketone & WA & & & $\mathbf{U}$ & 10 & & NS & $\mu \mathrm{g} / \mathrm{L}$ \\
\hline Methyl iodide & WA & & & $\mathbf{U}$ & 10 & & NS & $\mu g / L$ \\
\hline Methylene bromide & WA & & & $\mathbf{U}$ & 10 & & NS & $\mu \mathrm{g} / \mathrm{L}$ \\
\hline Methylene chloride & WA & & & $\mathbf{U}$ & 5 & & 5 & $\mu \mathrm{g} / \mathrm{L}$ \\
\hline Nickel & WA & & & $\mathbf{U}$ & 40 & & 100 & $\mu \mathrm{g} / \mathrm{L}$ \\
\hline Nitrate as nitrogen & WA & & & $\mathbf{U}$ & 0.1 & & 10 & $\mathrm{mg} / \mathrm{L}$ \\
\hline Nonvolatile beta & TM & & & & 10.5 & 2.7 & 50 & $p C i / L$ \\
\hline Potassium & WA & & & $\mathbf{U}$ & 5000 & & NS & $\mu g / L$ \\
\hline Propionitrile & WA & & &. $\mathrm{U}$ & 50 & & NS & $\mu \mathrm{g} / \mathrm{L}$ \\
\hline Selenium & WA & & & $\mathbf{U}$ & 5 & & 50 & $\mu g / L$ \\
\hline Silver & WA & & & $\mathbf{U}$ & 10 & & 50 & $\mu g / L$ \\
\hline Sodium & WA & & & $\mathbf{U}$ & 5000 & & NS & $\mu g / L$ \\
\hline Styrene & WA & & & $\mathbf{U}$ & 5 & & 100 & $\mu \mathrm{g} / \mathrm{L}$ \\
\hline Sulfate & WA & & & & 6.37 & & 400 & $\mathrm{mg} / \mathrm{L}$ \\
\hline Sulfide & WA & & & $\mathbf{U}$ & 0.1 & & NS & $\mathrm{mg} / \mathrm{L}$ \\
\hline Tetrachloroethylene & WA & & & $\mathbf{U}$ & 5 & & 5 & $\mu \mathrm{g} / \mathrm{L}$ \\
\hline Thallium & WA & & & $\mathbf{U}$ & 10 & & 2 & $\mu \mathrm{g} / \mathrm{L}$ \\
\hline Toluene & WA & & & $\mathbf{U}$ & 5 & & 1000 & $\mu \mathrm{g} / \mathrm{L}$ \\
\hline Total hardness & WA & & & & 28 & & NS & $\mathrm{mg} / \mathrm{L}$ \\
\hline Total organic carbon & WA & & $\mathbf{A}$ & & 6.71 & & NS & $\mathrm{mg} / \mathrm{L}$ \\
\hline Total suspended solids & WA & & & & 550 & & NS & $\mathrm{mg} / \mathrm{L}$ \\
\hline trans-1,3-Dichloropropene & WA & & & $\mathbf{U}$ & 5 & & NS & $\mu \mathrm{g} / \mathrm{L}$ \\
\hline trans-1,4-Dichloro-2-butene & WA & & & $\mathbf{U}$ & 100 & & NS & $\mu g / L$ \\
\hline Trichloroethylene & WA & & & $\mathbf{U}$ & 5 & & 5 & $\mu g / L$ \\
\hline Trichlorofluoromethane & WA & & & $\mathbf{U}$ & 5 & & NS & $\mu \mathrm{g} / \mathrm{L}$ \\
\hline Tritium & TM & & & & 5.2 & 0.92 & 20 & $\mathrm{pCi} / \mathrm{ml}$ \\
\hline Turbidity & WA & & & & 245 & & NS & NTU \\
\hline Vanadium & WA & & & $\mathbf{U}$ & 50 & & NS & $\mu g / L$ \\
\hline Vinyl acetate & WA & & & $\mathbf{U}$ & 10 & & NS & $\mu g / L$ \\
\hline Vinyl chloride & WA & & & $\mathbf{U}$ & 10 & & 2 & $\mu \mathrm{g} / \mathrm{L}$ \\
\hline Xylenes (total) & WA & & & $\mathbf{U}$ & 5 & & 10000 & $\mu g / L$ \\
\hline Zinc & WA & & & & 21 & & 5000 & $\mu g$ \\
\hline
\end{tabular}




\section{UTR100}

Ficld Measurements:

Sample Date: 4/20/93

pH: 6.53

Specific Conductance: $40 \mu \mathrm{S} / \mathrm{cm}$

Red/Oxid Potential: $139 \mathrm{mv}$

Dissolved Oxygen: $3.4 \mathrm{mg} / \mathrm{L}$

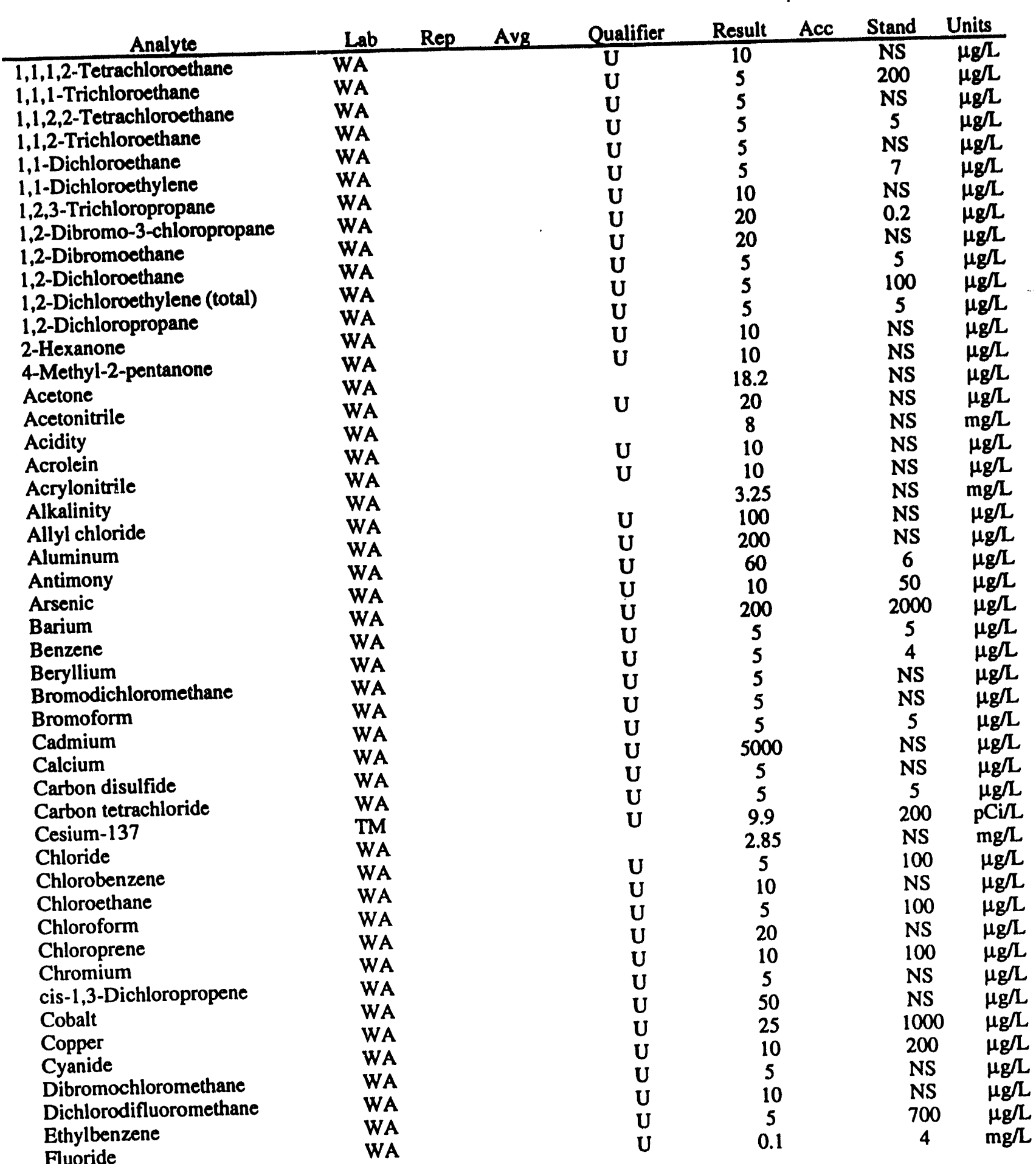


UTR100 Laboratory Analyses (cont'd)

\begin{tabular}{|c|c|c|c|c|c|c|c|c|}
\hline Analyte & Lab & Rep & Avg & Qualifier & Result & Acc & Stand & Units \\
\hline Gross alpha & $\mathrm{TM}$ & & A & & 1 & 1.15 & 15 & $\mathrm{pCi} / \mathrm{L}$ \\
\hline Iron & WA & & & & 290 & & 300 & $\mu g / L$ \\
\hline Isobutyl alcohol & WA & & & $\mathbf{U}$ & 20 & & NS & $\mu g / L$ \\
\hline Lead & WA & & & $\mathbf{U}$ & 3 & & 50 & $\mu g / L$ \\
\hline Magnesium & WA & & & $\mathbf{U}$ & 5000 & & NS & $\mu g / L$ \\
\hline Manganese & WA & & & & 295 & . & 50 & $\mu g / L$ \\
\hline Mercury & WA & & & $\mathbf{U}$ & 0.2 & & 2 & $\mu g / L$ \\
\hline Methacrylonitrile & WA & & & $\mathbf{U}$ & 20 & & NS & $\mu g / L$ \\
\hline Methyl bromide & WA & & & $\mathbf{U}$ & 10 & & NS & $\mu g / L$ \\
\hline Methyl chloride & WA & & & $\mathbf{U}$ & 10 & & NS & $\mu g / L$ \\
\hline Methyl ethyl ketone & WA & & & $\mathbf{U}$ & 10 & & NS & $\mu g / L$ \\
\hline Methyl iodide & WA & & & $\mathbf{U}$ & 10 & & NS & $\mu g / L$ \\
\hline Methylene bromide & WA & & & $\mathbf{U}$ & 10 & & NS & $\mu g / L$ \\
\hline Methylene chloride & WA & & & $\mathbf{U}$ & 5 & & 5 & $\mu g / L$ \\
\hline Nickel & WA & & & & 46.8 & & 100 & $\mu g / L$ \\
\hline Nitrate as nitrogen & WA & & & & 0.234 & & 10 & $\mathrm{mg} / \mathrm{L}$ \\
\hline Nonvolatile beta & TM & & $\mathbf{A}$ & & 2.3 & 2.2 & 50 & $\mathrm{pCi} / \mathrm{L}$ \\
\hline Potassium & WA & & & $\mathbf{U}$ & 5000 & & NS & $\mu g / L$ \\
\hline Potassium -40 & TM & & & & 2023 & 144 & 300 & $\mathrm{pCi} / \mathrm{L}$ \\
\hline Propionitrile & WA & & & $\mathbf{U}$ & 50 & & NS & $\mu g / L$ \\
\hline Selenium & WA & & & $\mathbf{U}$ & 5 & & 50 & $\mu g / L$ \\
\hline Silver & WA & & & $\mathbf{U}$ & 10 & & 50 & $\mu g / L$ \\
\hline Sodium & WA & & & $\mathbf{U}$ & 5000 & & NS & $\mu g / L$ \\
\hline Styrene & WA & & & $\mathbf{U}$ & 5 & & 100 & $\mu g / L$ \\
\hline Sulfate & WA & & $\mathbf{A}$ & & 10.4 & & 400 & $\mathrm{mg} / \mathrm{L}$ \\
\hline Sulfide & WA & & & $\mathbf{U}$ & 0.1 & & NS & $\mathrm{mg} / \mathrm{L}$ \\
\hline Tetrachloroethylene & WA & & & $\mathbf{U}$ & 5 & & 5 & $\mu g / L$ \\
\hline Thallium & WA & & & $\mathbf{U}$ & 10 & & 2 & $\mu g / L$ \\
\hline Toluene & WA & & & $\mathbf{U}$ & 5 & & 1000 & $\mu \mathrm{g} / \mathrm{L}$ \\
\hline Total hardness & WA & & & & 5 & & NS & $\mathrm{mg} / \mathrm{L}$ \\
\hline Total organic carbon & WA & & & & 1.39 & & NS & mg/L \\
\hline Total suspended solids & WA & & & & 14 & & NS & $\mathrm{mg} / \mathrm{L}$ \\
\hline trans-1,3-Dichloropropene & WA & & & $\mathbf{U}$ & 5 & & NS & $\mu g / L$ \\
\hline trans-1,4-Dichloro-2-butene & WA & & & $\mathbf{U}$ & 100 & & NS & $\mu \mathrm{g} / \mathrm{L}$ \\
\hline Trichloroethylene & WA & & & $\mathbf{U}$ & 5 & & 5 & $\mu \mathrm{g} / \mathrm{L}$ \\
\hline Trichlorofluoromethane & WA & & & $\mathbf{U}$ & 5 & & NS & $\mu g / L$ \\
\hline Tritium & $\mathbf{T M}$ & & & & 6 & 1.1 & 20 & $\mathrm{pCi} / \mathrm{ml}$ \\
\hline Turbidity & WA & & $\mathbf{A}$ & & 10.75 & & NS & NTU \\
\hline Vanadium & WA & & & $\mathbf{U}$ & 50 & & NS & $\mu g / L$ \\
\hline Vinyl acetate & WA & & & $\mathbf{U}$ & 10 & & NS & $\mu g / L$ \\
\hline Vinyl chloride & WA & & & $\mathbf{U}$ & 10 & & 2 & $\mu g / L$ \\
\hline Xylenes (total) & WA & & & $\mathbf{U}$ & 5 & & 10000 & $\mu \mathrm{g} / \mathrm{L}$ \\
\hline Zinc & WA & & & & 42.8 & & 5000 & $\mu \mathrm{g} / \mathrm{L}$ \\
\hline
\end{tabular}




\section{UTR102}

Field Measurements:

Sample Date: 4/20/93

pH: 6.08

Specific Conductance: $32 \mu \mathrm{S} / \mathrm{cm}$

Red/Oxid Potential: $143 \mathrm{mv}$

Dissolved Oxygen: $2.6 \mathrm{mg} / \mathrm{L}$

\begin{tabular}{|c|c|c|c|c|c|c|c|}
\hline Analyte & Lab & Rep & Qualifier & Result & Acc & Stand & Units \\
\hline 1,1,1,2-Tetrachloroethane & WA & & $\mathbf{U}$ & 10 & & NS & $\mu g / L$ \\
\hline 1,1,1-Trichloroethane & WA & & U & 5 & & 200 & $\mu g / L$ \\
\hline $1,1,2,2$-Tetrachloroethane & WA & & $\mathbf{U}$ & 5 & & NS & $\mu \mathrm{g} / \mathrm{L}$ \\
\hline 1,1,2-Trichloroethane & WA & & $\mathbf{U}$ & 5 & & 5 & $\mu g / L$ \\
\hline 1,1-Dichloroethane & WA & & $\mathbf{U}$ & 5 & & NS & $\mu g / L$ \\
\hline 1,1-Dichloroethylene & WA & & $\mathbf{U}$ & 5 & & 7 & $\mu g / L$ \\
\hline 1,2,3-Trichloropropane & WA & & $\mathbf{U}$ & 10 & & NS & $\mu g / L$ \\
\hline 1,2-Dibromo-3-chloropropane & WA & & $\mathbf{U}$ & 20 & & 0.2 & $\mu g / L$ \\
\hline 1,2-Dibromoethane & WA & & $\mathbf{U}$ & 20 & & NS & $\mu g / L$ \\
\hline 1,2-Dichloroethane & WA & & $\mathbf{U}$ & 5 & & 5 & $\mu g / L$ \\
\hline 1,2-Dichloroethylene (total) & WA & & $\mathbf{U}$ & 5 & & 100 & $\mu \mathrm{g} / \mathrm{L}$ \\
\hline 1,2-Dichloropropane & WA & & $\mathbf{U}$ & 5 & & 5 & $\mu g / L$ \\
\hline 2-Hexanone & WA & & $\mathbf{U}$ & 10 & & NS & $\mu g / L$ \\
\hline 4-Methyl-2-pentanone & WA & & $\mathbf{U}$ & 10 & & NS & $\mu \mathrm{g} / \mathrm{L}$ \\
\hline Acetone & WA & & $\mathbf{U}$ & 10 & & NS & $\mu \mathrm{g} / \mathrm{L}$ \\
\hline Acetonitrile & WA & & $\mathbf{U}$ & 20 & & NS & $\mu g / L$ \\
\hline Acidity & WA & & & 14 & & NS & $\mathrm{mg} / \mathrm{L}$ \\
\hline Acrolein & WA & & $\mathbf{U}$ & 10 & & NS & $\mu g / L$ \\
\hline $\begin{array}{l}\text { Acrolein } \\
\text { Acrylonitrile }\end{array}$ & WA & & U & 10 & & NS & $\begin{array}{l}\mu g L \\
\mu g / L\end{array}$ \\
\hline $\begin{array}{l}\text { Acrylonituile } \\
\text { Alkaliility }\end{array}$ & WA & & $\mathbf{U}$ & 0.5 & & NS & $\begin{array}{l}\mu g / L \\
m g / L\end{array}$ \\
\hline $\begin{array}{l}\text { Alkaliinity } \\
\text { Allyl chloride }\end{array}$ & WA & & $\mathbf{U}$ & 100 & & NS & $\begin{array}{l}\mathrm{mg} / \mathrm{L} \\
\mu \mathrm{g} / \mathrm{L}\end{array}$ \\
\hline $\begin{array}{l}\text { Allyl chloride } \\
\text { Aluminum }\end{array}$ & WA & & $\mathbf{U}$ & 200 & & NS & $\begin{array}{l}\mu g / L \\
\mu g / L\end{array}$ \\
\hline $\begin{array}{l}\text { Aluminum } \\
\text { Antimony }\end{array}$ & WA & & $\mathbf{U}$ & 60 & & 6 & $\begin{array}{l}\mu g / L \\
\mu g / L\end{array}$ \\
\hline $\begin{array}{l}\text { Antimony } \\
\text { Arsenic }\end{array}$ & WA & & $\mathbf{U}$ & 10 & & 50 & $\begin{array}{l}\mu g / L \\
\mu g / L\end{array}$ \\
\hline $\begin{array}{l}\text { Arsenic } \\
\text { Barium }\end{array}$ & WA & & $\mathbf{U}$ & 200 & & 2000 & $\begin{array}{l}\mu g / L \\
\mu g / L\end{array}$ \\
\hline $\begin{array}{l}\text { Barium } \\
\text { Benzene }\end{array}$ & WA & & $\mathbf{U}$ & 5 & & $\begin{array}{c}2000 \\
5\end{array}$ & $\mu g / L$ \\
\hline $\begin{array}{l}\text { Benzene } \\
\text { Beryllium }\end{array}$ & WA & & U & 5 & & 4 & $\mu g / L$ \\
\hline & WA & & U & 5 & & $\begin{array}{c}4 \\
\text { NS }\end{array}$ & $\mu g / L$ \\
\hline $\begin{array}{l}\text { Bromodichloromethane } \\
\text { Bromoform }\end{array}$ & WA & & $\mathbf{U}$ & 5 & & $\begin{array}{l}\text { NS } \\
\text { NS }\end{array}$ & $\mu \mathrm{g} / \mathrm{L}$ \\
\hline $\begin{array}{l}\text { Bromoform } \\
\text { Cadmium }\end{array}$ & WA & & $\mathbf{U}$ & 5 & & $\begin{array}{c}\text { NS } \\
5\end{array}$ & $\mu g / L$ \\
\hline $\begin{array}{l}\text { Cadmium } \\
\text { Calcium }\end{array}$ & WA & & $\mathbf{U}$ & 5000 & & $\begin{array}{l}5 \\
\text { NS }\end{array}$ & $\mu \mathrm{g} / \mathrm{L}$ \\
\hline & WA & & $\mathbf{U}$ & 5 & & $\begin{array}{l}\text { NS } \\
\text { NS }\end{array}$ & $\mu g / L$ \\
\hline $\begin{array}{l}\text { Carbon disulfide } \\
\text { Carbon tetrachloride }\end{array}$ & WA & & U & 5 & & $\begin{array}{c}\text { NS } \\
5\end{array}$ & $\mu g / L$ \\
\hline $\begin{array}{l}\text { Carbon tetrachloride } \\
\text { Cesium-137 }\end{array}$ & TM & & & 3.5 & 3.6 & $\begin{array}{c}5 \\
200\end{array}$ & $\mu g / L$ \\
\hline $\begin{array}{l}\text { Cesium-137 } \\
\text { Chloride }\end{array}$ & WA & & & 2.79 & & $\begin{array}{l}200 \\
\text { NS }\end{array}$ & $\mathrm{pCi} / \mathrm{L}$ \\
\hline & WA & & $\mathbf{U}$ & 5 & & $\begin{array}{l}\text { NS } \\
100\end{array}$ & $\mathrm{mg} / \mathrm{L}$ \\
\hline $\begin{array}{l}\text { Chlorobenzene } \\
\text { Chloroethane }\end{array}$ & WA & & $\mathbf{U}$ & 10 & & $\begin{array}{l}100 \\
\text { NS }\end{array}$ & $\mu \mathrm{g} / \mathrm{L}$ \\
\hline $\begin{array}{l}\text { Chloroethane } \\
\text { Chloroform }\end{array}$ & WA & & $\mathbf{U}$ & 5 & & $\begin{array}{l}\text { NS } \\
100\end{array}$ & $\mu g / L$ \\
\hline $\begin{array}{l}\text { Chloroform } \\
\text { Chloroprene }\end{array}$ & WA & & $\mathbf{U}$ & 20 & & $\begin{array}{l}100 \\
\text { NS }\end{array}$ & $\mu \mathrm{g} / \mathrm{L}$ \\
\hline $\begin{array}{l}\text { Chloroprene } \\
\text { Chromium }\end{array}$ & WA & & $\mathrm{U}$ & 10 & & $\begin{array}{l}\text { NS } \\
100\end{array}$ & $\mu \mathrm{g} / \mathrm{L}$ \\
\hline $\begin{array}{l}\text { Chromium } \\
\text { cis-1.3-Dichloropropene }\end{array}$ & WA & & $\mathbf{U}$ & $\begin{array}{c}10 \\
5\end{array}$ & & $\begin{array}{l}100 \\
N S\end{array}$ & $\mu \mathrm{g} / \mathrm{L}$ \\
\hline cis-1,3-Dichloropropene & WA & & $\mathbf{U}$ & 5 & & NS & $\mu \mathrm{g} / \mathrm{L}$ \\
\hline Cobalt & WA & & $\mathbf{U}$ & 50 & & NS & $\mu \mathrm{g} / \mathrm{L}$ \\
\hline Copper & WA & & & 25 & & 1000 & $\mu \mathrm{g} / \mathrm{L}$ \\
\hline Cyanide & & & U & 10 & & 200 & $\mu g / L$ \\
\hline Dibromochloromethane & $\begin{array}{l}\text { WA } \\
\text { WA }\end{array}$ & & U & 5 & & NS & $\mu g / L$ \\
\hline Dichlorodifluoromethane & WA & & U & 10 & & NS & $\mu \mathrm{g}$ \\
\hline Ethylbenzene & WA & & $\mathrm{U}$ & 5 & & 700 & $\mu \mathrm{g}$ \\
\hline Fluoride & WA & & $\mathbf{U}$ & 0.1 & & 4 & \\
\hline
\end{tabular}


UTR 102 Laboratory Analyses (cont'd)

\begin{tabular}{|c|c|c|c|c|c|c|c|c|}
\hline Analyte & Lab & Rep & Avg & Qualifier & Result & Acc & Stand & Units \\
\hline Gross alpha & TM & & & $\mathrm{U}$ & 0.6 & & 15 & $\overline{\mathrm{pCi} / \mathrm{L}}$ \\
\hline Iron & WA & & & & 427 & & 300 & $\mu \mathrm{g} / \mathrm{L}$ \\
\hline Isobutyl alcohol & WA & & & $\mathbf{U}$ & 20 & & NS & $\mu g / L$ \\
\hline Lead & WA & & & & 3.8 & & 50 & $\mu \mathrm{g} / \mathrm{L}$ \\
\hline Magnesium & WA & & & U & 5000 & & NS & $\mu \mathrm{g} / \mathrm{L}$ \\
\hline Manganese & WA & & & & 21.7 & & 50 & $\mu \mathrm{g} / \mathrm{L}$ \\
\hline Mercury & WA & & & $\mathbf{U}$ & 0.2 & & 2 & $\mu \mathrm{g} / \mathrm{L}$ \\
\hline Methacrylonitrile & WA & & & $\mathbf{U}$ & 20 & & NS & $\mu \mathrm{g} / \mathrm{L}$ \\
\hline Methyl bromide & WA & & & $\mathbf{U}$ & 10 & & NS & $\mu \mathrm{g} / \mathrm{L}$ \\
\hline Methyl chloride & WA & & & $\mathbf{U}$ & 10 & & NS & $\mu \mathrm{g} / \mathrm{L}$ \\
\hline Methyl ethyl ketone & WA & & & $\mathbf{U}$ & 10 & & NS & $\mu g / L$ \\
\hline Methyl iodide & WA & & & $\mathbf{U}$ & 10 & & NS & $\mu \mathrm{g} / \mathrm{L}$ \\
\hline Methylene bromide & WA & & & $\mathrm{U}$ & 10 & & NS & $\mu \mathrm{g} / \mathrm{L}$ \\
\hline Methylene chloride & WA & & & $\mathrm{U}$ & 5 & & 5 & $\mu \mathrm{g} / \mathrm{L}$ \\
\hline Nickel & WA & & & U & 40 & & 100 & $\mu \mathrm{g} / \mathrm{L}$ \\
\hline Nitrate as nitrogen & WA & & & & 0.364 & & 10 & $\mathrm{mg} / \mathrm{L}$ \\
\hline Nonvolatile beta & $\mathbf{T M}$ & & & & 2.4 & 1.7 & 50 & pCi/L \\
\hline Potassium & WA & & & U & 5000 & & NS & $\mu \mathrm{g} / \mathrm{L}$ \\
\hline Potassium- 40 & TM & & & & 797.3 & 81.1 & 300 & $\mathrm{pCi} / \mathrm{L}$ \\
\hline Propionitrile & WA & & & $\mathbf{U}$ & 50 & & NS & $\mu \mathrm{g} / \mathrm{L}$ \\
\hline Selenium & WA & & & $\mathbf{U}$ & 5 & & 50 & $\mu \mathrm{g} / \mathrm{L}$ \\
\hline Silver & WA & & & $\mathrm{U}$ & 10 & & 50 & $\mu g / L$ \\
\hline Sodium & WA & & & $\mathbf{U}$ & 5000 & & NS & $\mu \mathrm{g} / \mathrm{L}$ \\
\hline Styrene & WA & & & $\mathrm{U}$ & 5 & & 100 & $\mu \mathrm{g} / \mathrm{L}$ \\
\hline Sulfate & WA & & & & 2.73 & & 400 & $\mathrm{mg} / \mathrm{L}$ \\
\hline Sulfide & WA & & & $\mathrm{U}$ & 0.1 & & NS & $\mathrm{mg} / \mathrm{L}$ \\
\hline Tetrachloroethylene & WA & & & $\mathrm{U}$ & 5 & & 5 & $\mu \mathrm{g} / \mathrm{L}$ \\
\hline Thallium & WA & & & U & 10 & & 2 & $\mu \mathrm{g} / \mathrm{L}$ \\
\hline Toluene & WA & & & $\mathrm{U}$ & 5 & & 1000 & $\mu \mathrm{g} / \mathrm{L}$ \\
\hline Total hardness & WA & & A & & 4 & & NS & $\mathrm{mg} / \mathrm{L}$ \\
\hline Total organic carbon & WA & & & & 8.75 & & NS & $\mathrm{mg} / \mathrm{L}$ \\
\hline Total suspended solids & WA & & & & 16 & & NS & $\mathrm{mg} / \mathrm{L}$ \\
\hline trans-1,3-Dichloropropene & WA & & & $\mathrm{U}$ & 5 & & NS & $\mu \mathrm{g} / \mathrm{L}$ \\
\hline trans-1,4-Dichloro-2-butene & WA & & & U & 100 & & NS & $\mu \mathrm{g} / \mathrm{L}$ \\
\hline Trichloroethylene & WA & & & U & 5 & & 5 & $\mu \mathrm{g} / \mathrm{L}$ \\
\hline Trichlorofluoromethane & WA & & & $\mathrm{U}$ & 5 & & NS & $\mu \mathrm{g} / \mathrm{L}$ \\
\hline Tritium & TM & & & & 5.6 & 0.95 & 20 & $\mathrm{pCi} / \mathrm{ml}$ \\
\hline Turbidity & WA & & & & 5.59 & & NS & NTU \\
\hline Vanadium & WA & & & $\mathrm{U}$ & 50 & & NS & $\mu g / L$ \\
\hline Vinyl acetate & WA & & & $\mathrm{U}$ & 10 & & NS & $\mu g / L$ \\
\hline Vinyl chloride & WA & & & U & 10 & & 2 & $\mu \mathrm{g} / \mathrm{L}$ \\
\hline Xylenes (total) & WA & & & $\mathrm{U}$ & 5 & & 10000 & $\mu \mathrm{g} / \mathrm{L}$ \\
\hline Zinc & WA & & & $\mathrm{U}$ & 20 & & 5000 & $\mu \mathrm{g} / \mathrm{L}$ \\
\hline Zinc-65 & TM & & & & 12.8 & 9.4 & 300 & $p C i / L$ \\
\hline
\end{tabular}




\section{UTR109}

Field Measurements:

Sample Date: 4/20/93

pH: 5.69

Specific Conductance: $39 \mu \mathrm{S} / \mathrm{cm}$

Red/Oxid Potential: $-170 \mathrm{mv}$

Dissolved Oxygen: $1.2 \mathrm{mg} / \mathrm{L}$

\begin{tabular}{|c|c|c|c|c|c|c|c|c|}
\hline Analyte & Lab & Rep & Avg & Qualifier & Result & Acc & Stand & Units \\
\hline 1,1,1,2-Tetrachloroethane & $\overline{\text { WA }}$ & & & $\mathbf{U}$ & 10 & & NS & $\mu \mathrm{g} / \mathrm{L}$ \\
\hline $1,1,1$-Trichloroethane & WA & & & U & 5 & & 200 & $\mu g / L$ \\
\hline $1,1,2,2$-Tetrachloroethane & WA & & & $\mathbf{U}$ & 5 & & NS & $\mu g / L$ \\
\hline 1,1,2-Trichloroethane & WA & & & U & 5 & & 5 & $\mu g / L$ \\
\hline 1,1-Dichloroethane & WA & & & U & 5 & & NS & $\mu g / L$ \\
\hline 1,1-Dichloroethylene & WA & & & $\mathbf{U}$ & 5 & & 7 & $\mu \mathrm{g} / \mathrm{L}$ \\
\hline 1,2,3-Trichloropropane & WA & & & $\mathbf{U}$ & 10 & & NS & $\mu g / L$ \\
\hline 1,2-Dibromo-3-chloropropane & WA & & & $\mathbf{U}$ & 20 & & 0.2 & $\mu g / L$ \\
\hline 1,2-Dibromoethane & WA & & & $\mathbf{U}$ & 20 & & NS & $\mu g / L$ \\
\hline 1,2-Dichloroethane & WA & & & U & 5 & & 5 & $\mu g / L$ \\
\hline 1,2-Dichloroethylene (total) & WA & & & $\mathbf{U}$ & 5 & & 100 & $\mu \mathrm{g} / \mathrm{L}$ \\
\hline 1,2-Dichloropropane & WA & & & $\mathrm{U}$ & 5 & & 5 & $\mu g / L$ \\
\hline 2-Hexanone & WA & & & $\mathbf{U}$ & 10 & & NS & $\mu \mathrm{g} / \mathrm{L}$ \\
\hline 4-Methyl-2-pentanone & WA & & & $\mathbf{U}$ & 10 & & NS & $\mu g / L$ \\
\hline Acetone & WA & & & & V 14.4 & & NS & $\mu g / L$ \\
\hline Acetonitrile & WA & & & $\mathbf{U}$ & 20 & & NS & $\mu \mathrm{g} / \mathrm{L}$ \\
\hline Acidity & WA & & & & 17.5 & & NS & $\mathrm{mg} / \mathrm{L}$ \\
\hline Acrolein & WA & & & $\mathrm{U}$ & 10 & & NS & $\mu g / L$ \\
\hline Acrylonitrile & WA & & & $\mathrm{U}$ & 10 & & NS & $\mu \mathrm{g} / \mathrm{L}$ \\
\hline Alkalinity & WA & & A & & 1.75 & & NS & $\mathrm{mg} / \mathrm{L}$ \\
\hline Allyl chloride & WA & & & U & 100 & & NS & $\mu g / \mathrm{L}$ \\
\hline Aluminum & WA & & & U & 200 & & NS & $\mu \mathrm{g} / \mathrm{L}$ \\
\hline Antimony & WA & & & $\mathbf{U}$ & 60 & & 6 & $\mu \mathrm{g} / \mathrm{L}$ \\
\hline Arsenic & WA & & & $\mathbf{U}$ & 10 & & 50 & $\mu \mathrm{g} / \mathrm{L}$ \\
\hline Barium & WA & & & $\mathbf{U}$ & 200 & & 2000 & $\mu g / L$ \\
\hline Benzene & WA & & & $\mathbf{U}$ & 5 & & 5 & $\mu g / L$ \\
\hline Beryllium & WA & & & $\mathbf{U}$ & 5 & & 4 & $\mu g / L$ \\
\hline Bromodichloromethane & WA & & & $\mathrm{U}$ & 5 & & NS & $\mu g / L$ \\
\hline Bromoform & WA & & & $\mathrm{U}$ & 5 & & NS & $\mu \mathrm{g} / \mathrm{L}$ \\
\hline Cadmium & WA & & & $\mathrm{U}$ & 5 & & 5 & $\mu g / L$ \\
\hline Calcium & WA & & & $\mathbf{U}$ & 5000 & & NS & $\mu g / L$ \\
\hline Carbon disulfide & WA & & & $\mathrm{U}$ & 5 & & NS & $\mu \mathrm{g} / \mathrm{L}$ \\
\hline Carbon tetrachloride & WA & & & U & 5 & & 5 & $\mu \mathrm{g} / \mathrm{L}$ \\
\hline Cesium-137 & TM & & & U & 5.9 & & 200 & $\mathrm{pCi} / \mathrm{L}$ \\
\hline Chloride & WA & & A & & 3.035 & & NS & $\mathrm{mg} / \mathrm{L}$ \\
\hline Chlorobenzene & WA & & & $\mathbf{U}$ & 5 & & 100 & $\mu g / L$ \\
\hline Chloroethane & WA & & & $\mathrm{U}$ & 10 & & NS & $\mu \mathrm{g} / \mathrm{L}$ \\
\hline Chloroform & WA & & & $\mathrm{U}$ & 5 & & 100 & $\mu \mathrm{g} / \mathrm{L}$ \\
\hline Chloroprene & WA & & & $\mathbf{U}$ & 20 & & NS & $\mu g / L$ \\
\hline Chromium & WA & & & $\mathbf{U}$ & 10 & & 100 & $\mu g / L$ \\
\hline cis-1,3-Dichloropropene & WA & & & $\mathrm{U}$ & 5 & & NS & $\mu g / L$ \\
\hline Cobalt & WA & & & $\mathbf{U}$ & 50 & & NS & $\mu \mathrm{g} / \mathrm{L}$ \\
\hline Cobalt -60 & TM & & & & 5.1 & 3.5 & 100 & $\mathrm{pCi} / \mathrm{L}$ \\
\hline Copper & WA & & & $\mathrm{U}$ & 25 & & 1000 & $\mu \mathrm{g} / \mathrm{L}$ \\
\hline Cyanide & WA & & & $\mathbf{U}$ & 10 & & 200 & $\mu g / L$ \\
\hline Dibromochloromethane & WA & & & $U$ & 5 & & NS & $\mu g / L$ \\
\hline Dichlorodifluoromethane & WA & & & $\mathrm{U}$ & 10 & & NS & $\mu g / L$ \\
\hline Ethyibenzene & WA & & & $\mathbf{U}$ & 5 & & 700 & $\mu g / L$ \\
\hline & WA & & & U & 0.1 & & 4 & $\mathrm{mg} / \mathrm{L}$ \\
\hline
\end{tabular}


Sampling and Analysis of Water From Upper Three Runs And Iss Wetlands Near Tank 16 and the Mixed Waste Management Faciliny

UTR109 Laboratory Analyses (cont'd)

\begin{tabular}{|c|c|c|c|c|c|c|c|c|}
\hline Analyte & $L a b$ & Rep & Avg & Qualifier & Result & Acc & Stand & Units \\
\hline Gross alpha & $T M$ & & & $\mathrm{U}$ & 0.6 & & 15 & $\mathrm{pCi} / \mathrm{L}$ \\
\hline Iron & WA & & & & 3100 & & 300 & $\mu g / L$ \\
\hline Isobutyl alcohol & WA & & & $\mathbf{U}$ & 20 & & NS & $\mu g / L$ \\
\hline Lead & WA & & & $\mathbf{U}$ & 3 & & 50 & $\mu g / L$ \\
\hline Magnesium & WA & & & $\mathbf{U}$ & 5000 & & NS & $\mu g / L$ \\
\hline Manganese & WA & & & & 91.2 & & 50 & $\mu \mathrm{g} / \mathrm{L}$ \\
\hline Mercury & WA & & & $\mathbf{U}$ & 0.2 & & 2 & $\mu g / L$ \\
\hline Methacrylonitrile & WA & & & $\mathbf{U}$ & 20 & & NS & $\mu g / L$ \\
\hline Methyl bromide & WA & & & $\mathbf{U}$ & 10 & & NS & $\mu g / L$ \\
\hline Methyl chloride & WA & & & $\mathbf{U}$ & 10 & & NS & $\mu g / L$ \\
\hline Methyl ethyl ketone & WA & & & U & 10 & & NS & $\mu \mathrm{g} / \mathrm{L}$ \\
\hline Methyl iodide & WA & & & $\mathrm{U}$ & 10 & & NS & $\mu g / L$ \\
\hline Methylene bromide & WA & & & $\mathbf{U}$ & 10 & & NS & $\mu \mathrm{g} / \mathrm{L}$ \\
\hline Methylene chloride & WA & & & $\mathbf{U}$ & 5 & & 5 & $\mu g / L$ \\
\hline Nickel & WA & & & $\mathbf{U}$ & 40 & & 100 & $\mu g / L$ \\
\hline Nitrate as nitrogen & WA & & & & 0.293 & & 10 & $\mathrm{mg} / \mathrm{L}$ \\
\hline Nonvolatile beta & TM & & & & 1.1 & 1.6 & 50 & pCi/L \\
\hline Potassium & WA & & & $\mathbf{U}$ & 5000 & & NS & $\mu g / L$ \\
\hline Potassium -40 & $\mathrm{TM}$ & & & & 766 & 65.1 & 300 & $\mathrm{pCi} / \mathrm{L}$ \\
\hline Propionitrile & WA & & & $\mathbf{U}$ & 50 & & NS & $\mu g / L$ \\
\hline Selenium & WA & & & $\mathbf{U}$ & 5 & & 50 & $\mu g / L$ \\
\hline Silver & WA & & & $\mathbf{U}$ & 10 & & 50 & $\mu g / L$ \\
\hline Sodium & WA & & & $\mathbf{U}$ & 5000 & & NS & $\mu g / L$ \\
\hline Sodium-22 & $\mathbf{T M}$ & & & & 8.5 & 6.1 & 466 & $\mathrm{pCi} / \mathrm{L}$ \\
\hline Styrene & WA & & & $\mathbf{U}$ & 5 & & 100 & $\mu g / L$ \\
\hline Sulfate & WA & & & $\mathbf{U}$ & 2.5 & & 400 & $\mathrm{mg} / \mathrm{L}$ \\
\hline Sulfide & WA & & & $\mathbf{U}$ & 0.1 & & NS & $\mathrm{mg} / \mathrm{L}$ \\
\hline Tetrachloroethyliene & WA & & & $\mathbf{U}$ & 5 & & 5 & $\mu g / L$ \\
\hline Thallium & WA & & & $\mathbf{U}$ & 10 & & 2 & $\mu g / L$ \\
\hline Toluene & WA & & & I & 1.51 & & 1000 & $\mu g / L$ \\
\hline Total hardness & WA & & & & 15 & & NS & $\mathrm{mg} / \mathrm{L}$ \\
\hline Total organic carbon & WA & & & & 2.51 & & NS & $\mathrm{mg} / \mathrm{L}$ \\
\hline Total suspended solids & WA & & & & 14 & & NS & $\mathrm{mg} / \mathrm{L}$ \\
\hline trans-1,3-Dichloropropene & WA & & & $\mathbf{U}$ & 5 & & NS & $\mu g / L$ \\
\hline trans-1,4-Dichloro-2-butene & WA & & & $\mathbf{U}$ & 100 & & NS & $\mu \mathrm{g} / \mathrm{L}$ \\
\hline Trichloroethylene & WA & & & $\mathbf{U}$ & 5 & & 5 & $\mu \mathrm{g} / \mathrm{L}$ \\
\hline Trichlorofluoromethane & WA & & & $\mathbf{U}$ & 5 & & NS & $\mu g / L$ \\
\hline Tritium & $\mathrm{TM}$ & & & & 6.1 & 1.2 & 20 & $\mathrm{pCi} / \mathrm{ml}$ \\
\hline Turbidity & WA & & & & 2.7 & & NS & NTU \\
\hline Vanadium & WA & & & $\mathbf{U}$ & 50 & & NS & $\mu g / L$ \\
\hline Vinyl acetate & WA & & & $\mathbf{U}$ & 10 & & NS & $\mu \mathrm{g} / \mathrm{L}$ \\
\hline Vinyl chloride & WA & & & $\mathbf{U}$ & 10 & & 2 & $\mu g / L$ \\
\hline Xylenes (total) & WA & & & $\mathbf{U}$ & 5 & & 10000 & $\mu g / L$ \\
\hline Zinc & WA & & & $\mathbf{U}$ & 20 & & 5000 & $\mu g / L$ \\
\hline
\end{tabular}




\section{UTR112}

Field Measurements:

Sampls Date: 4/20/93

pH: 6.3

Specific Conductance: $84 \mu \mathrm{S} / \mathrm{cm}$

Red/Oxid Potential: $54 \mathrm{mv}$

Dissolved Oxygen: $1.3 \mathrm{mg} / \mathrm{L}$

\begin{tabular}{|c|c|c|c|c|c|c|}
\hline Analyte & Lab & Avg & Qualifier & Result Acc & Stand & Units \\
\hline 1,1,1,2-Tetrachloroethane & WA & & $\mathbf{U}$ & 10 & NS & $\overline{\mu g / L}$ \\
\hline 1,1,1-Trichloroethane & WA & & $\mathbf{U}$ & 5 & 200 & $\mu g / L$ \\
\hline $1,1,2,2$-Tetrachloroethane & WA & & $\mathbf{U}$ & 5 & NS & $\mu g / L$ \\
\hline 1,1,2-Trichloroethane & WA & & $\mathbf{U}$ & 5 & 5 & $\mu g / L$ \\
\hline 1,1-Dichloroethane & WA & & $\mathbf{U}$ & 5 & NS & $\mu g / L$ \\
\hline 1,1-Dichloroethylene & WA & & $\mathbf{U}$ & 5 & 7 & $\mu \mathrm{g} / \mathrm{L}$ \\
\hline 1,2,3-Trichloropropane & WA & & $\mathbf{U}$ & 10 & NS & $\mu g / L$ \\
\hline 1,2-Dibromo-3-chloropropane & WA & . & U & 20 & 0.2 & $\mu g / L$ \\
\hline 1,2-Dibromoethane & WA & & $\mathbf{U}$ & 20 & NS & $\mu g / L$ \\
\hline 1,2-Dichloroethane & WA & & $\mathbf{U}$ & 5 & 5 & $\mu g / L$ \\
\hline 1,2-Dichloroethylene (total) & WA & & $\mathbf{U}$ & 5 & 100 & $\mu \mathrm{g} / \mathrm{L}$. \\
\hline 1,2-Dichloropropane & WA & & $\mathbf{U}$ & 5 & 5 & $\mu g / L$ \\
\hline 2-Hexanone & WA & & $\mathbf{U}$ & 10 & NS & $\mu g / L$ \\
\hline 4-Methyl-2-pentanone & WA & & $\mathbf{U}$ & 10 & NS & $\mu g / L$ \\
\hline Acetone & WA & & J & 3.8 & NS & $\mu \mathrm{g} / \mathrm{L}$ \\
\hline Acetonitrile & WA & & $\mathbf{U}$ & 20 & NS & $\mu \mathrm{g} / \mathrm{L}$ \\
\hline Acidity & WA & & & 54 & NS & $\mathrm{mg} / \mathrm{L}$ \\
\hline Acrolein & WA & & $\mathbf{U}$ & 10 & NS & $\mu \mathrm{g} / \mathrm{L}$ \\
\hline Acrylonitrile & WA & & $\mathrm{U}$ & 10 & NS & $\mu \mathrm{g} / \mathrm{L}$ \\
\hline Alkalinity & WA & & & 13.2 & NS & $\mathrm{mg} / \mathrm{L}$ \\
\hline Allyl chloride & WA & & $\mathbf{U}$ & 100 & NS & $\mu g / L$ \\
\hline Aluminum & WA & & U & 200 & NS & $\mu \mathrm{g} / \mathrm{L}$ \\
\hline Antimony & WA & & $\mathbf{U}$ & 60 & 6 & $\mu \mathrm{g} / \mathrm{L}$ \\
\hline Arsenic & WA & & $\mathbf{U}$ & 10 & 50 & $\mu \mathrm{g} / \mathrm{L}$ \\
\hline Barium & WA & & $\tilde{U}$ & 200 & 2000 & $\mu \mathrm{g} / \mathrm{L}$ \\
\hline Benzene & WA & & $\mathbf{U}$ & 5 & 5 & $\mu \mathrm{g} / \mathrm{L}$ \\
\hline Beryllium & WA & & $\mathbf{U}$ & 5 & 4 & $\mu \mathrm{g} / \mathrm{L}$ \\
\hline Bromodichloromethane & WA & & $\mathbf{U}$ & 5 & NS & $\mu g / L$ \\
\hline Bromoform & WA & & $\mathbf{U}$ & 5 & NS & $\mu g / L$ \\
\hline Cadmium & WA & & $U$ & 5 & 5 & $\mu \mathrm{g} / \mathrm{L}$ \\
\hline Calcium & WA & & $\mathbf{U}$ & 5000 & NS & $\mu \mathrm{g} / \mathrm{L}$ \\
\hline Carbon disulfide & WA & & $\mathbf{U}$ & 5 & NS & $\mu g / L$ \\
\hline Carbon tetrachloride & WA & & $\mathbf{U}$ & 5 & 5 & $\mu g / L$ \\
\hline Cesium-137 & TM & & $U$ & 9.9 & 200 & $\mathrm{pCi} / \mathrm{L}$ \\
\hline Chloride & WA & & & 4.83 & NS & $\mathrm{mg} / \mathrm{L}$ \\
\hline Chlorobenzene & WA & & $\mathbf{U}$ & 5 & 100 & $\mu g / L$ \\
\hline Chloroethane & WA & & $\mathbf{U}$ & 10 & NS & $\mu g / L$ \\
\hline Chloroform & WA & & $\mathbf{U}$ & 5 & 100 & $\mu \mathrm{g} / \mathrm{L}$ \\
\hline Chloroprene & WA & & $\mathrm{U}$ & 20 & NS & $\mu \mathrm{g} / \mathrm{L}$ \\
\hline Chromium & WA & & $\mathrm{U}$ & 10 & 100 & $\mu \mathrm{g} / \mathrm{L}$ \\
\hline cis-1,3-Dichloropropene & WA & & $\mathbf{U}$ & 5 & NS & $\mu \mathrm{g} / \mathrm{L}$ \\
\hline Cobalt & WA & & $\mathbf{U}$ & 50 & NS & $\mu \mathrm{g} / \mathrm{L}$ \\
\hline Copper & WA & & $\mathbf{U}$ & 25 & 1000 & $\mu g / L$ \\
\hline Cyanide & WA & & $\mathbf{U}$ & 10 & 200 & $\mu \mathrm{g} / \mathrm{L}$ \\
\hline Dibromochloromethane & WA & & $\mathbf{U}$ & 5 & NS & $\mu \mathrm{g} / \mathrm{L}$ \\
\hline Dichlorodifluoromethane & WA & & $\mathbf{U}$ & 10 & NS & $\mu \mathrm{g} / \mathrm{L}$ \\
\hline Ethylbenzene & WA & & $\mathbf{U}$ & 5 & 700 & $\mu \mathrm{g} / \mathrm{L}$ \\
\hline Fluoride & WA & & $\mathbf{U}$ & 0.1 & 4 & $\mathrm{mg} / \mathrm{L}$ \\
\hline
\end{tabular}


UTR1 12 Laboratory Analyses (cont'd)

\begin{tabular}{|c|c|c|c|c|c|c|c|}
\hline Analyte & Lab & Rep & Qualifier & Result & Acc & Stand & Units \\
\hline $\begin{array}{l}\text { Iron } \\
\text { Isobutyl alcohol }\end{array}$ & WA & & $\mathbf{U}$ & 20 & & NS & $\mu g / L$ \\
\hline $\begin{array}{l}\text { Isobutyl alcohol } \\
\text { Lead }\end{array}$ & WA & & $\mathbf{U}$ & 3 & & 50 & $\begin{array}{l}\mu g / L \\
\mu g / L\end{array}$ \\
\hline $\begin{array}{l}\text { Lead } \\
\text { Magnesium }\end{array}$ & WA & & $\mathbf{U}$ & 5000 & & NS & $\mu g \mathrm{~g} / \mathrm{L}$ \\
\hline $\begin{array}{l}\text { Magnesium } \\
\text { Manganese }\end{array}$ & WA & & & 136 & . & 50 & $\mu g / L$ \\
\hline $\begin{array}{l}\text { Manganese } \\
\text { Mercury }\end{array}$ & WA & & $\mathbf{U}$ & 0.2 & & 2 & $\mu g / L$ \\
\hline $\begin{array}{l}\text { Mercury } \\
\text { Methacrylonitrile }\end{array}$ & $\begin{array}{l}\text { WA } \\
\text { WA }\end{array}$ & & $\mathbf{U}$ & 20 & & NS & $\mu g / L$ \\
\hline $\begin{array}{l}\text { Methacrylonitrile } \\
\text { Methyl bromide }\end{array}$ & $\begin{array}{l}\text { WA } \\
\text { WA }\end{array}$ & & $\mathbf{U}$ & 10 & & NS & $\mu g / \Lambda$ \\
\hline $\begin{array}{l}\text { Methyl bromide } \\
\text { Methyl chloride }\end{array}$ & $\begin{array}{l}\text { WA } \\
\text { WA }\end{array}$ & & $\mathbf{U}$ & 10 & & NS & $\mu g / L$ \\
\hline $\begin{array}{l}\text { Methyl chloride } \\
\text { Methyl ethyl ketone }\end{array}$ & WA & & U & 10 & & NS & $\mu g / L$ \\
\hline & WA & & U & 10 & & NS & $\mu g /$ \\
\hline $\begin{array}{l}\text { Methyl iodide } \\
\text { Methylene bromide }\end{array}$ & WA & & $\mathbf{U}$ & 10 & & NS & $\mu g / L$ \\
\hline $\begin{array}{l}\text { Methylene bromide } \\
\text { Methylene chloride }\end{array}$ & WA & & $\mathbf{U}$ & 5 & & 5 & $\mu g / L$ \\
\hline Nickel & WA & & $\mathbf{U}$ & 40 & & 100 & $\mu g / L$ \\
\hline Nitrate as nitrogen & WA & & & 0.21 & & $\begin{array}{l}10 \\
50\end{array}$ & $\begin{array}{l}\mathrm{mg} / \mathrm{L} \\
\mathrm{DC} / \mathrm{L}\end{array}$ \\
\hline Nonvolatile beta & TM & & & $\begin{array}{c}3.2 \\
5000\end{array}$ & 1.9 & NS & $\begin{array}{l}\mathrm{pCi} / \mathrm{L} \\
\mu \mathrm{g} / \mathrm{L}\end{array}$ \\
\hline Potassium & $\begin{array}{l}\text { WA } \\
\text { TM }\end{array}$ & & $\mathbf{U}$ & 2023 & 143 & 300 & $\mathrm{pCi} / \mathrm{L}$ \\
\hline Potassium-40 & $\begin{array}{l}\text { TM } \\
\text { WA }\end{array}$ & & $\mathbf{U}$ & 50 & & NS & $\mu g / L$ \\
\hline Propionitrile & $\begin{array}{l}\text { WA } \\
\text { WA }\end{array}$ & & $\mathbf{U}$ & 5 & & 50 & $\mu g / L$ \\
\hline $\begin{array}{l}\text { Selenium } \\
\text { Silver }\end{array}$ & $\begin{array}{l}\text { WA } \\
\text { WA }\end{array}$ & & $\mathbf{U}$ & 10 & & 50 & $\mu g / L$ \\
\hline Silver & WA & & $\mathbf{U}$ & 5000 & & NS & $\mu \mathrm{g} / \mathrm{L}$ \\
\hline $\begin{array}{l}\text { Sodium } \\
\text { Styrene }\end{array}$ & WA & & $\mathbf{U}$ & 5 & & 100 & $\mu g / L$ \\
\hline $\begin{array}{l}\text { Styrene } \\
\text { Sulfate }\end{array}$ & WA & & U & 2.5 & & 400 & $\mathrm{mg} / \mathrm{L}$ \\
\hline $\begin{array}{l}\text { Sulfate } \\
\text { Sulfide }\end{array}$ & WA & & $\mathbf{U}$ & 0.1 & & NS & $\mathrm{mg} / \mathrm{L}$ \\
\hline $\begin{array}{l}\text { Sulfide } \\
\text { Tetrachloroethylene }\end{array}$ & WA & & $\mathbf{U}$ & 5 & & 5 & $\mu \mathrm{g} / \mathrm{L}$ \\
\hline $\begin{array}{l}\text { Tetrachloroethylene } \\
\text { Thallium }\end{array}$ & WA & & $\mathbf{U}$ & 10 & & 2 & $\mu g / L$ \\
\hline Thallium & WA & & $\mathrm{U}$ & 5 & & 1000 & $\mu \mathrm{g} / \mathrm{L}$ \\
\hline $\begin{array}{l}\text { Toluene } \\
\text { Total hardness }\end{array}$ & WA & & & 20 & & NS & $\mathrm{mg} / \mathrm{L}$ \\
\hline $\begin{array}{l}\text { Total hardness } \\
\text { Total organic carbon }\end{array}$ & WA & & & 9.07 & & NS & $\mathrm{mg} / \mathrm{L}$ \\
\hline $\begin{array}{l}\text { Total organic carbon } \\
\text { Total suspended solids }\end{array}$ & WA & & & 52 & & NS & $\mathrm{mg} / \mathrm{L}$ \\
\hline $\begin{array}{l}\text { Total suspended solids } \\
\text { trans-13-Dichloropropene }\end{array}$ & WA & & $\mathbf{U}$ & 5 & & NS & $\mu g / L$ \\
\hline $\begin{array}{l}\text { trans-1,3-Dichloropropene } \\
\text { trans-1,4-Dichloro-2-butene }\end{array}$ & WA & & $\mathbf{U}$ & 100 & & NS & $\mu g / L$ \\
\hline $\begin{array}{l}\text { trans-1,4-Dichloro-2-butene } \\
\text { Trichloroethylene }\end{array}$ & WA & & $\mathbf{U}$ & 5 & & 5 & $\mu g / L$ \\
\hline $\begin{array}{l}\text { Trichloroethylene } \\
\text { Trichlorofluoromethane }\end{array}$ & WA & & $\mathbf{U}$ & 5 & & NS & $\mu g / L$ \\
\hline $\begin{array}{l}\text { Trichlorofluoromethane } \\
\text { Tritium }\end{array}$ & TM & & & 8.1 & 1.6 & 20 & $\mathrm{Ci} / \mathrm{ml}$ \\
\hline Tritium & WA & & & 7.84 & & NS & NTU \\
\hline Turbidity & WA & & $\mathbf{U}$ & 50 & & NS & $\mu g / L$ \\
\hline Vanadium & WA & & $\mathbf{U}$ & 10 & & NS & $\mu g / L$ \\
\hline Vinyl acetate & & & $\mathrm{U}$ & 10 & & 2 & $\mu g / L$ \\
\hline Vinyl chloride & WA & & $\mathrm{U}$ & 5 & & 10000 & $\mu \mathrm{g} / \mathrm{L}$ \\
\hline Xylenes (total) & WA & & & 56.7 & & 5000 & $\mu g / L$ \\
\hline Zinc & & & & & & & \\
\hline
\end{tabular}




\section{UTR114}

Field Measurements:

Sample Date: 4/20/93

pH: 5.5

Specific Conductance: $28 \mu \mathrm{S} / \mathrm{cm}$

Red/Oxid Potential: $59 \mathrm{mv}$

Dissolved Oxygen: $3.2 \mathrm{mg} / \mathrm{L}$

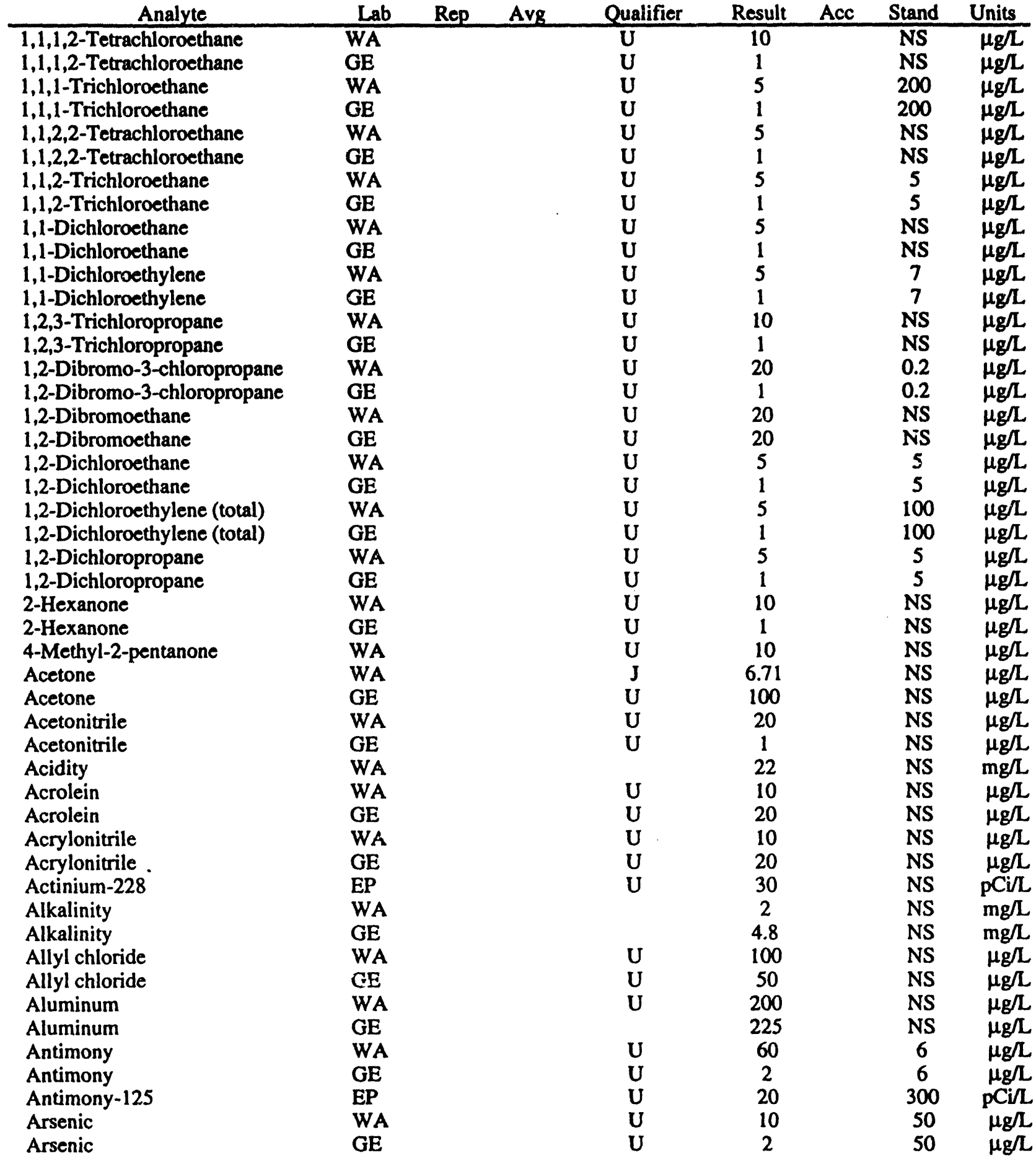


UTR 114 Laboratory Analyses (cont'd)

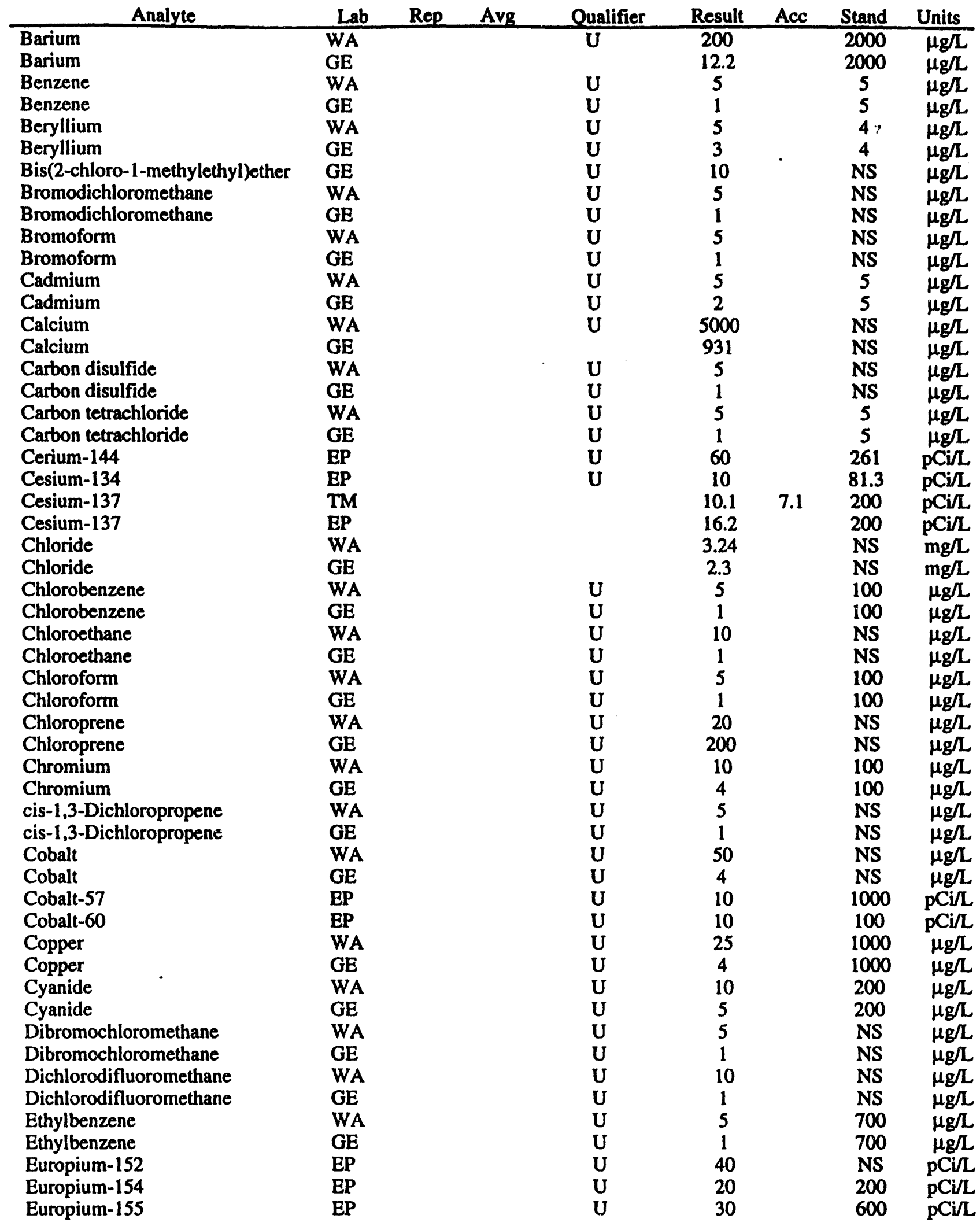


UTR1 14 Laboratory Analyses (cont'd)

\begin{tabular}{|c|c|c|c|c|c|c|c|c|}
\hline Analyte & Lab & Avg & Qualifier & & Result & Acc & Stand & Units \\
\hline Fluoride & WA & & $U$ & & 0.1 & & 4 & $\mathrm{mg} / \mathrm{L}$ \\
\hline Fluoride & WA & NA & $\mathrm{U}$ & & 0.1 & & 4 & $\mathrm{mg} / \mathrm{L}$ \\
\hline Fluoride & GE & & $\mathbf{U}$ & & 0.1 & & 4 & $\mathrm{mg} / \mathrm{L}$ \\
\hline Gross alpha & TM & & & & 1.1 & 1 & 15 & $\mathrm{pCi} / \mathrm{L}$ \\
\hline Gross alpha & GE & & $\mathbf{U}$ & & 2 & & 15 & $\mathrm{pCi} / \mathrm{L}$ \\
\hline Iron & WA & & & & 2760 & & 300 & $\mu g / L$ \\
\hline Iron & GE & & $\mathbf{J}$ & H & 3910 & - & 300 & $\mu \mathrm{g} / \mathrm{L}$ \\
\hline Isobutyl alcohol & WA & & $\mathbf{U}$ & & 20 & & NS & $\mu \mathrm{g} / \mathrm{L}$ \\
\hline Isobutyl alcohol & GE & & U & & 100 & & NS & $\mu \mathrm{g} / \mathrm{L}$ \\
\hline Lead & WA & & U & & 3 & & 50 & $\mu g / L$ \\
\hline Lead & GE & & U & & 3 & & 50 & $\mu g / L$ \\
\hline Lead-212 & $\overline{\mathbf{E P}}$ & & $\mathbf{U}$ & & 15 & & NS & $\mathrm{pCi} / \mathrm{L}$ \\
\hline Magnesium & WA & & $\mathbf{U}$ & & 5000 & & NS & $\mu g / L$ \\
\hline Magnesium & GE & & & & 455 & & NS & $\mu g / L$ \\
\hline Manganese & WA & & & & 58.8 & & $\begin{array}{l}50 \\
50\end{array}$ & $\mu g / L$ \\
\hline Manganese & $\mathbf{G E}$ & & & & 56.9 & & 50 & $\mu g / L$ \\
\hline Manganese-54 & EP & & $\mathbf{U}$ & & 10 & & 300 & $\mathrm{pCi} / \mathrm{L}$ \\
\hline Mercury & WA & & $\mathbf{U}$ & & 0.2 & & 2 & $\mu g / L$ \\
\hline Mercury & GE & & $\mathbf{U}$ & & 0.2 & & 2 & $\mu g / L$ \\
\hline Methacrylonitrile & WA & & $\mathbf{U}$ & & 20 & & NS & $\mu \mathrm{g} / \mathrm{L}$ \\
\hline Methacrylonitrile & GE & & $\mathbf{U}$ & & 50 & & NS & $\mu \mathrm{g} / \mathrm{L}$ \\
\hline Methyl bromide & WA & & U & & 10 & & NS & $\mu g / L$ \\
\hline Methyl bromide & GE & & U & & 1 & & NS & $\mu g / L$ \\
\hline Methyl chloride & WA & & $\mathbf{U}$ & & 10 & & NS & $\mu \mathrm{g} / \mathrm{L}$ \\
\hline Methyl chloride & GE & & $\mathbf{U}$ & & 1 & & NS & $\mu g / L$ \\
\hline Methyl ethyl ketone & WA & & $\mathbf{U}$ & & 10 & & NS & $\mu \mathrm{g} / \mathrm{L}$ \\
\hline Methyl ethyl ketone & GE & & $\mathbf{U}$ & & 1 & & NS & $\mu \mathrm{g} / \mathrm{L}$ \\
\hline Methyl iodide & WA & & $\mathbf{U}$ & & 10 & & NS & $\mu g / L$ \\
\hline Methyl iodide & GE & & $\mathbf{U}$ & & 15 & & NS & $\mu \mathrm{g} / \mathrm{L}$ \\
\hline Methyl isobutyl ketone & GE & & U & & 1 & & NS & $\mu g / L$ \\
\hline Methylene bromide & WA & & $\mathbf{U}$ & & 10 & & NS & $\mu g / L$ \\
\hline Methylene bromide & GE & & $\mathbf{U}$ & & 1 & & NS & $\mu \mathrm{g} / \mathrm{L}$ \\
\hline Methylene chloride & WA & & U & & 5 & & 5 & $\mu g / L$ \\
\hline Methylene chloride & GE & & $\tilde{U}$ & & 1 & & 5 & $\mu g / L$ \\
\hline Nickel & WA & & $\mathbf{U}$ & & 40 & & 100 & $\mu \mathrm{g} / \mathrm{L}$ \\
\hline Nickel & GE & & U & & 4 & & 100 & $\mu \mathrm{g} / \mathrm{L}$ \\
\hline Nitrate as nitrogen & WA & & & & 1.05 & & 10 & $\mathrm{mg} / \mathrm{L}$ \\
\hline Nitrate as nitrogen & GE & & & & 0.22 & & 10 & $\mathrm{mg} / \mathrm{L}$ \\
\hline Nonvolatile beta & TM & & & & 1.1 & 1.6 & 50 & $\mathrm{pCi} / \mathrm{L}$ \\
\hline Nonvolatile beta & $\mathrm{GE}$ & & $\mathbf{U}$ & & 2 & & 50 & $\mathrm{pCi} / \mathrm{L}$ \\
\hline pH & GE & & $\mathbf{J}$ & $\mathbf{Q}$ & 5.96 & & NS & PH \\
\hline Potassium & WA & & $\mathrm{U}$ & & 5000 & & NS & $\mu g / L$ \\
\hline Potassium & GE & & $\mathbf{U}$ & & 500 & & NS & $\mu g / L$ \\
\hline Potassium-40. & TM & & & & 2084 & 119 & 300 & $\mathrm{pCi} / \mathrm{L}$ \\
\hline Potassium-40 & EP & & $\mathbf{U}$ & & 110 & & 300 & $\mathrm{pCi} / \mathrm{L}$ \\
\hline Promethium-144 & EP & & $\mathbf{U}$ & & 10 & & NS & $\mathrm{pCi} / \mathrm{L}$ \\
\hline Promethium-146 & EP & & $\mathbf{U}$ & & 10 & & NS & $\mathrm{pCi} / \mathrm{L}$ \\
\hline Propionitrile & WA & & $\mathrm{U}$ & & 50 & & NS & $\mu \mathrm{g} / \mathrm{L}$ \\
\hline Propionitrile & GE & & $\mathrm{U}$ & & 200 & & NS & $\mu \mathrm{g} / \mathrm{L}$ \\
\hline Ruthenium-106 & EP & & $\mathbf{U}$ & & 90 & & 30 & $\mathrm{pCi} / \mathrm{L}$ \\
\hline Selenium & WA & & $\mathbf{U}$ & & 5 & & 50 & $\mu \mathrm{g} / \mathrm{L}$ \\
\hline Selenium & GE & & UJ & $\mathbf{L}$ & 2 & & 50 & $\mu \mathrm{g} / \mathrm{L}$ \\
\hline Silver & WA & & $\mathbf{U}$ & & 10 & & 50 & $\mu \mathrm{g} / \mathrm{L}$ \\
\hline Silver & GE & & $\mathbf{U}$ & & 2 & & 50 & $\mu g / L$ \\
\hline Sodium & WA & & U & & 5000 & & NS & $\mu g / L$ \\
\hline Sodium & GE & & & & 1300 & & NS & $\mu \mathrm{g} / \mathrm{L}$ \\
\hline
\end{tabular}


UTR 114 Laboratory Analyses (cont'd)

\begin{tabular}{|c|c|c|c|c|c|c|c|}
\hline Analyte & Lab & Avg & Qualifier & Result & Acc & Stand & Units \\
\hline Sodium-22 & $\mathrm{EP}$ & & $\mathrm{U}$ & 10 & & 466 & $\mathrm{pCi} / \mathrm{L}$ \\
\hline Styrene & WA & & $\mathbf{U}$ & 5 & & 100 & $\mu g / L$ \\
\hline Styrene & $\mathrm{GE}$ & & $\mathbf{U}$ & 1 & & 100 & $\mu g / L$ \\
\hline Sulfate & WA & & $\mathbf{U}$ & 2.5 & & 400 & $\mathrm{mg} / \mathrm{L}$ \\
\hline Sulfate & $\mathrm{GE}$ & & $\mathbf{U}$ & 1 & & 400 & $\mathrm{mg} / \mathrm{L}$ \\
\hline Sulfide & WA & & $\mathbf{U}$ & 0.1 & . & NS & $\mathrm{mg} / \mathrm{L}$ \\
\hline Sulfide & GE & & $\mathbf{U}$ & 1 & & NS & $\mathrm{mg} / \mathrm{L}$ \\
\hline Tetrachloroethylene & WA & & $\mathbf{U}$ & 5 & & 5 & $\mu g / L$ \\
\hline Tetrachloroethylene & GE & & $\mathbf{U}$ & 1 & & 5 & $\mu g / L$ \\
\hline Thallium & WA & & $\mathbf{U}$ & 10 & & 2 & $\mu g / L$ \\
\hline Thallium & GE & & $\mathbf{U}$ & 2 & & 2 & $\mu g / L$ \\
\hline Thorium-234 & EP & & $\mathbf{U}$ & 350 & & 401 & $\mathrm{pCi} / \mathrm{L}$ \\
\hline Toluene & WA & & $\mathbf{U}$ & 5 & & 1000 & $\mu g / L$ \\
\hline Toluene & GE & & $\mathbf{U}$ & 1 & & 1000 & $\mu g / L$ \\
\hline Total hardness & WA & & & 10 & & NS & $\mathrm{mg} / \mathrm{L}$ \\
\hline Total hardness & GE & & & 4.5 & & NS & $\mathrm{mg} / \mathrm{L}$ \\
\hline Total organic carbon & WA & & & 4.97 & & NS & $\mathrm{mg} / \mathrm{L}$ \\
\hline Total organic carbon & GE & & & 5.43 & & NS & $\mathrm{mg} / \mathrm{L}$ \\
\hline Total suspended solids & WA & & & 19 & & NS & $\mathrm{mg} / \mathrm{L}$ \\
\hline Total suspended solids & GE & & & 10 & & NS & $\mathbf{m g} / \mathbf{L}$ \\
\hline trans-1,3-Dichloropropene & WA & & $\mathbf{U}$ & 5 & & NS & $\mu g / L$ \\
\hline trans-1,3-Dichloropropene & $\mathbf{G E}$ & & $\mathbf{U}$ & 1 & & NS & $\mu g / L$ \\
\hline trans-1,4-Dichloro-2-butene & WA & & $\mathbf{U}$ & 100 & & NS & $\mu g / L$ \\
\hline trans-1,4-Dichloro-2-butene & GE & & $\mathbf{U}$ & 30 & & NS & $\mu g / L$ \\
\hline Trichloroethylene & WA & & $\mathbf{U}$ & 5 & & 5 & $\mu g / L$ \\
\hline Trichloroethylene & GE & & $\mathbf{U}$ & 1 & & 5 & $\mu g / L$ \\
\hline Trichlorofluoromethane & WA & & $\mathbf{U}$ & 5 & & NS & $\mu g / L$ \\
\hline Trichlorofluoromethane & GE & & $\mathbf{U}$ & 1 & & NS & $\mu g / L$ \\
\hline Tritium & TM & & & 134.2 & 22.5 & 20 & $\mathrm{pCi} / \mathrm{ml}$ \\
\hline Tritium & GE & & & 163 & & 20 & $\mathrm{pCi} / \mathrm{ml}$ \\
\hline Turbidity & WA & & & 7.49 & & NS & NTU \\
\hline Turbidity & GE & & & 17 & & NS & NTU \\
\hline Vanadium & WA & & $\mathbf{U}$ & 50 & & NS & $\mu g / L$ \\
\hline Vanadium & GE & & $\mathbf{U}$ & 8 & & NS & $\mu g / L$ \\
\hline Vinyl acetate & WA & & $\mathbf{U}$ & 10 & & NS & $\mu g / L$ \\
\hline Vinyl acetate & GE & & $\mathbf{U}$ & 1 & & NS & $\mu \mathrm{g} / \mathrm{L}$ \\
\hline Vinyl chloride & WA & & $\mathbf{U}$ & 10 & & 2 & $\mu g / L$ \\
\hline Vinyl chloride & GE & & $\mathbf{U}$ & 1 & & 2 & $\mu g / L$ \\
\hline Xylenes (total) & WA & & $\mathbf{U}$ & 5 & & 10000 & $\mu g / L$ \\
\hline Xylenes (total) & $\mathbf{G E}$ & & $\mathbf{U}$ & 2 & & 10000 & $\mu g / L$ \\
\hline Yttrium-88 & EP & & $\mathbf{U}$ & 60 & & NS & $\mathrm{pCi} / \mathrm{L}$ \\
\hline Zinc & WA & & & 20.9 & & 5000 & $\mu g / L$ \\
\hline Zinc & GE & & & 9.75 & & 5000 & $\mu g / L$ \\
\hline Zinc-65 & $\mathrm{EP}$ & & $\mathbf{U}$ & 20 & & 300 & $\mathrm{pCi} / \mathrm{L}$ \\
\hline
\end{tabular}




\section{UTR116}

Field Measurements:

Sample Date: 4/20/93

pH: 5.1

Specific Conductance: $38 \mu \mathrm{S} / \mathrm{cm}$

Red/Oxid Potential: $105 \mathrm{mv}$

Dissolved Oxygen: $1.9 \mathrm{mg} / \mathrm{L}$

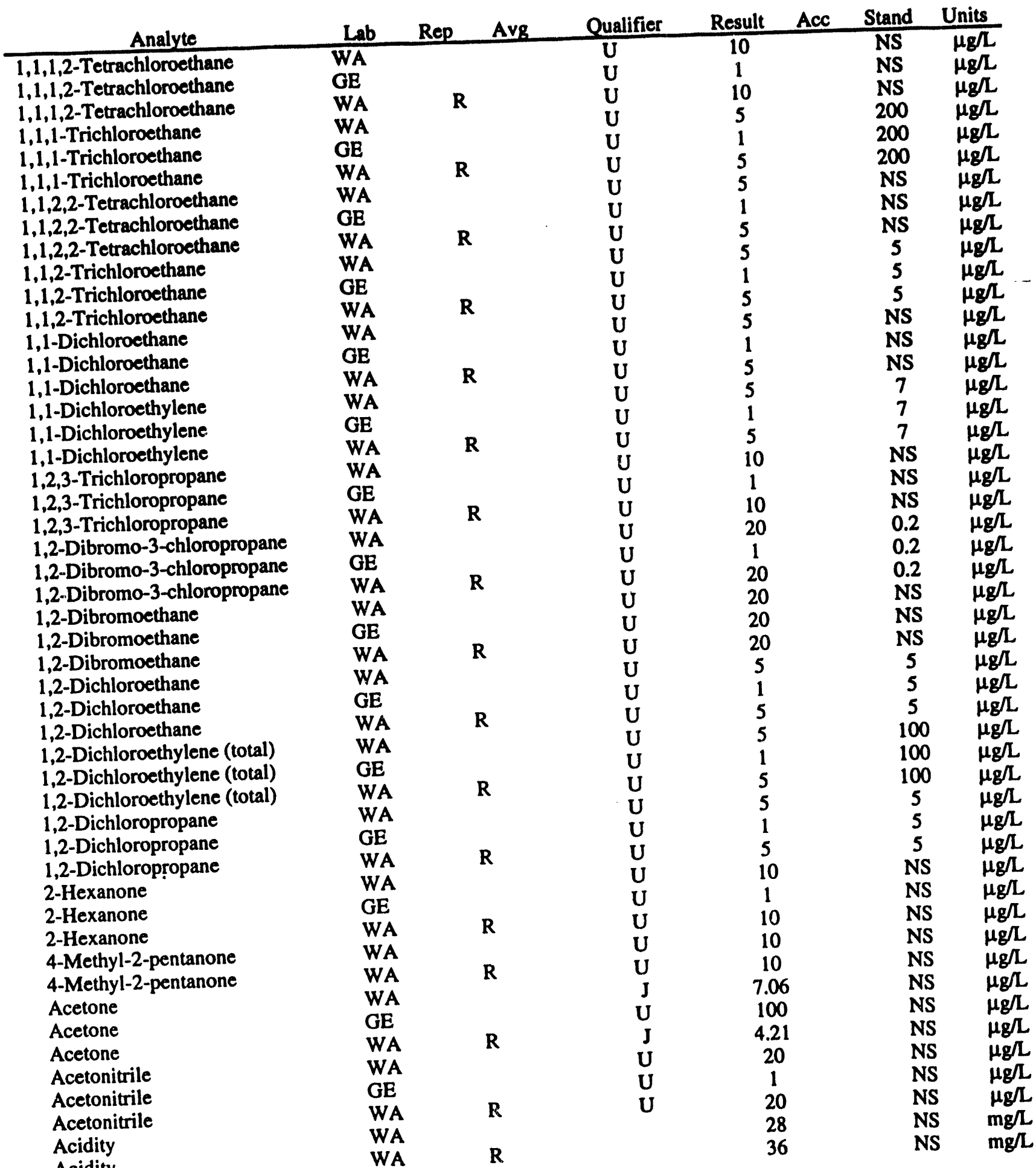


UTR 16 Laboratory Analyses (cont'd)

\begin{tabular}{|c|c|c|c|c|c|c|c|c|}
\hline Analyte & Lab & Rep & Avg & Qualifier & Result & Acc & Stand & Units \\
\hline Acrolein & WA & & & $\mathbf{U}$ & 10 & & NS & $\overline{\mu g / L}$ \\
\hline Acrolein & GE & & & $\mathbf{U}$ & 20 & & NS & $\mu g / L$ \\
\hline Acrolein & WA & $\mathbf{R}$ & & $\mathbf{U}$ & 10 & & NS & $\mu g / L$ \\
\hline Acrylonitrile & WA & & & $\mathbf{U}$ & 10 & & NS & $\mu g / L$ \\
\hline Acrylonitrile & GE & & & $\mathbf{U}$ & 20 & & NS & $\mu g / L$ \\
\hline Acrylonitrile & WA & $\mathbf{R}$ & & $\mathbf{U}$ & 10 & . & NS & $\mu g / L$ \\
\hline Actinium-228 & EP & & & $\mathbf{U}$ & 30 & & NS & $\mathrm{pCi} / \mathrm{L}$ \\
\hline Alkalinity & WA & & $\mathbf{A}$ & & 4 & & NS & $\mathrm{mg} / \mathrm{L}$ \\
\hline Alkalinity & GE & & & & 3.8 & & NS & $\mathrm{mg} / \mathrm{L}$ \\
\hline Alkalinity & WA & $\mathbf{R}$ & & & 0.5 & & NS & $m g / L$ \\
\hline Allyl chloride & WA & & & $\mathbf{U}$ & 100 & & NS & $\mu g / L$ \\
\hline Allyl chloride & GE & & & $\mathbf{U}$ & 50 & & NS & $\mu_{g} / L$ \\
\hline Allyl chloride & WA & $\mathbf{R}$ & & $\mathbf{U}$ & 100 & & NS & $\mu g / L$ \\
\hline Aluminum & WA & & & $\mathbf{U}$ & 200 & & NS & $\mu g / L$ \\
\hline Aluminum & $\mathbf{G E}$ & & & & 128 & & NS & $\mu g / L$ \\
\hline Aluminum & WA & $\mathbf{R}$ & . & $\mathbf{U}$ & 200 & & NS & $\mu g / L$ \\
\hline Antimony & WA & & & $\mathbf{U}$ & 60 & & 6 & $\mu g / L$ \\
\hline Antimony & GE & & & $\mathbf{U}$ & 2 & & 6 & $\mu g / L$ \\
\hline Antimony & WA & $\mathbf{R}$ & & $\mathbf{U}$ & 60 & & 6 & $\mu g / L$ \\
\hline Antimony-125 & EP & & & $\mathbf{U}$ & 20 & & 300 & $\mathrm{pCi} / \mathbf{L}$ \\
\hline Arsenic & WA & & & $\mathbf{U}$ & 10 & & 50 & $\mu g / L$ \\
\hline Arsenic & $\mathbf{G E}$ & & & $\mathbf{U}$ & 2 & & 50 & $\mu g / L$ \\
\hline Arsenic & WA & $\mathbf{R}$ & & $\mathbf{U}$ & 10 & & 50 & $\mu g / L$ \\
\hline Barium & WA & & & $\mathbf{U}$ & 200 & & 2000 & $\mu g / L$ \\
\hline Barium & GE & & & & 12 & & 2000 & $\mu g /$ \\
\hline Barium & WA & $\mathbf{R}$ & & $\mathbf{U}$ & 200 & & 2000 & $\mu g / L$ \\
\hline Benzene & WA & & & $\mathbf{U}$ & 5 & & 5 & $\mu g / L$ \\
\hline Benzene & $\mathrm{GE}$ & & & $\mathbf{U}$ & 1 & & 5 & $\mu g / L$ \\
\hline Benzene & WA & $\mathbf{R}$ & & $\mathbf{U}$ & 5 & & 5 & $\mu g / L$ \\
\hline Beryllium & WA & & & $\mathbf{U}$ & 5 & & 4 & $\mu g / L$ \\
\hline Beryllium & GE & & & $\mathbf{U}$ & 3 & & 4 & $\mu g / L$ \\
\hline Beryllium & WA & $\mathbf{R}$ & & $\mathbf{U}$ & 5 & & 4 & $\mu g / L$ \\
\hline Bis(2-chloro-1-methylethyl)ether & GE & & & $\mathbf{U}$ & 10 & & NS & $\mu g / L$ \\
\hline Bromodichloromethane & WA & & & $\mathbf{U}$ & 5 & & NS & $\mu g / L$ \\
\hline Bromodichloromethane & GE & & & $\mathbf{U}$ & 1 & & NS & $\mu g /$ \\
\hline Bromodichloromethane & WA & $\mathbf{R}$ & & $\mathbf{U}$ & 5 & & NS & $\mu g / L$ \\
\hline Bromoform & WA & & & $\mathbf{U}$ & 5 & & NS & $\mu g / L$ \\
\hline Bromoform & GE & & & $\mathbf{U}$ & 1 & & NS & $\mu g / L$ \\
\hline Bromoform & WA & $\mathbf{R}$ & & $\mathbf{U}$ & 5 & & NS & $\mu g / L$ \\
\hline Cadmium & WA & & & & 7.5 & & 5 & $\mu g / L$ \\
\hline Cadmium & GE & & & & 3.17 & & 5 & $\mu \mathrm{g} / \mathrm{L}$ \\
\hline Cadmium & WA & $\mathbf{R}$ & & $\mathrm{U}$ & 5 & & 5 & $\mu g / L$ \\
\hline Calcium & WA & & & $\mathbf{U}$ & 5000 & & NS & $\mu g / L$ \\
\hline Calcium & GE & & & & 893 & & NS & $\mu g / L$ \\
\hline Calcium & WA & $\mathbf{R}$ & & $\mathbf{U}$ & 5000 & & NS & $\mu g / L$ \\
\hline Carbon disulfide & WA & & & $\mathrm{U}$ & 5 & & NS & $\mu g / L$ \\
\hline Carbon disulfide & GE & & & $\mathbf{U}$ & 1 & & NS & $\mu g / L$ \\
\hline Carbon disulfide & WA & $\mathbf{R}$ & & $\mathbf{U}$ & 5 & & NS & $\mu g / L$ \\
\hline Carbon tetrachloride & WA & & & $\mathbf{U}$ & 5 & & 5 & $\mu g / L$ \\
\hline Carbon tetrachloride & GE & & & $\mathbf{U}$ & 1 & & 5 & $\mu g / L$ \\
\hline Carbon tetrachloride & WA & $\mathbf{R}$ & & $\mathbf{U}$ & 5 & & 5 & $\mu g / L$ \\
\hline Cerium-144 & EP & & & $\mathbf{U}$ & 60 & & 261 & $\mathrm{pCi} / \mathrm{L}$ \\
\hline Cesium-134 & EP & & & $\mathbf{U}$ & 10 & & 81.3 & $\mathrm{pCi} / \mathrm{L}$ \\
\hline Cesium-137 & TM & & & & 4.5 & 3.9 & 200 & $\mathrm{pCi} / \mathrm{L}$ \\
\hline Cesium-137 & EP & & & $\mathbf{U}$ & 10 & & 200 & $\mathrm{pCi} / \mathrm{L}$ \\
\hline Cesium-137 & $T \mathrm{TM}$ & $\mathbf{R}$ & & $\mathbf{U}$ & 9.7 & & 200 & $\mathrm{pCi} / \mathrm{L}$ \\
\hline
\end{tabular}


UTR116 Laboratory Analyses (cont'd)

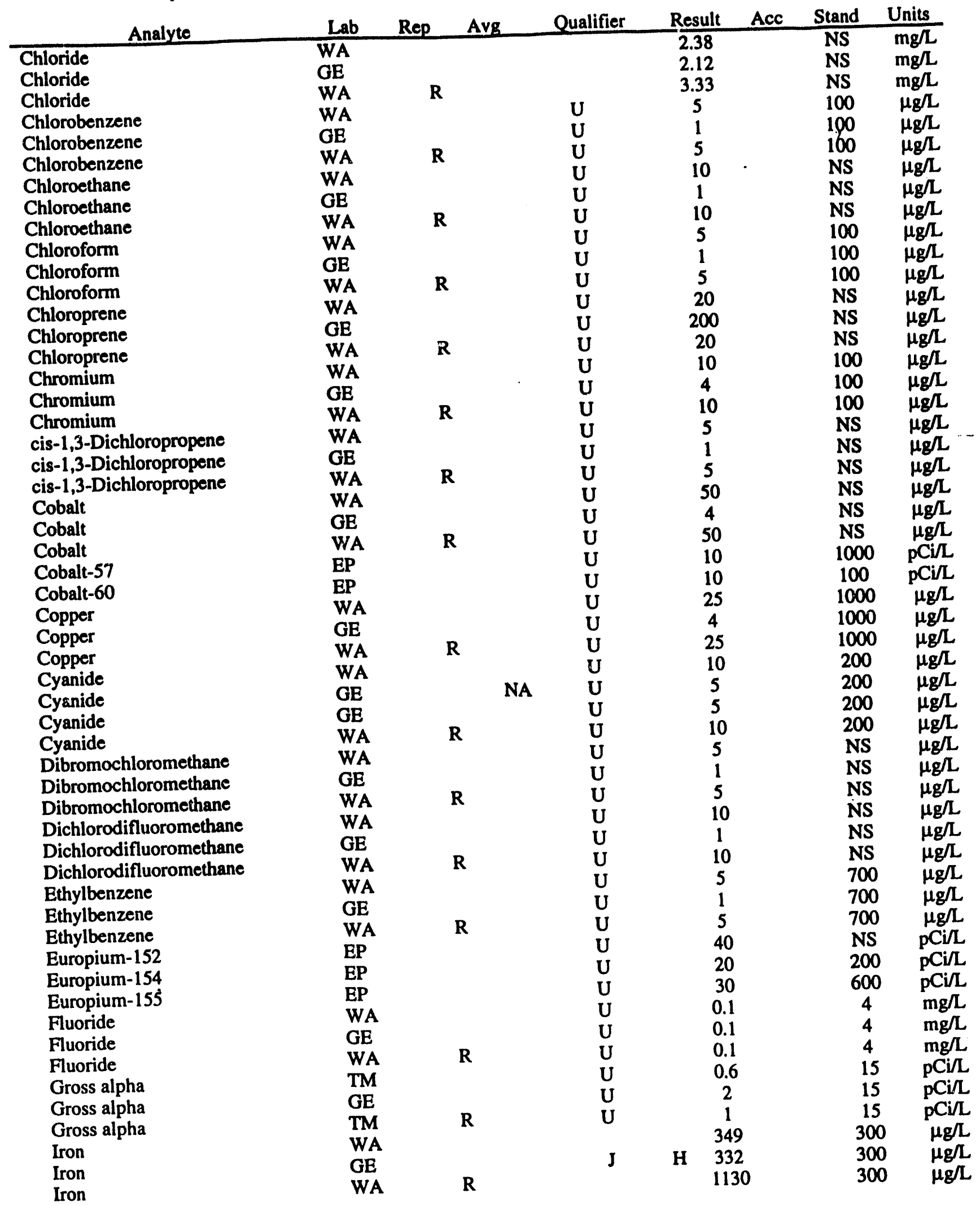


Sampling and Analysis of Water From Upper Three Runs And lis Wellands Near Tank 16 and the Mixed Waste Management Facillity

UTR 16 Laboratory Analyses (cont'd)

\begin{tabular}{|c|c|c|c|c|c|c|c|c|}
\hline Analyte & Lab & Rep & Avg & Qualifier & Result & Acc & Stand & Units \\
\hline Isobutyl alcohol & $\overline{\text { WA }}$ & & & $\mathbf{U}$ & 20 & & NS & $\mu g / L$ \\
\hline Isobutyl alcohol & GE & & & $\mathbf{U}$ & 100 & & NS & $\mu g / L$ \\
\hline Isobutyl alcohol & WA & $\mathbf{R}$ & & $\mathbf{U}$ & 20 & & NS & $\mu \mathrm{g} / \mathrm{L}$ \\
\hline Lead & WA & & & U & 3 & & 50 & $\mu g / L$ \\
\hline Lead & GE & & & U & 3 & & 50 & $\mu \mathrm{g} / \mathrm{L}$ \\
\hline Lead & WA & $\mathbf{R}$ & & U & 3 & & 50 & $\mu \mathrm{g} / \mathrm{L}$ \\
\hline Lead-212 & EP & & & $\mathbf{U}$ & 15 & & NS & $\mathrm{pCi} / \mathrm{L}$ \\
\hline Magnesium & WA & & & $\mathbf{U}$ & 5000 & & NS & $\mu g / L$ \\
\hline Magnesium & $\mathrm{GE}$ & & & & 418 & & NS & $\mu \mathrm{g} / \mathrm{L}$ \\
\hline Magnesium & WA & $\mathbf{R}$ & & $\mathbf{U}$ & 5000 & & NS & $\mu g / L$ \\
\hline Manganese & WA & & & & 28.8 & & 50 & $\mu g /$ \\
\hline Manganese & GE & & & & 25.7 & & 50 & $\mu g / L$ \\
\hline Manganese & WA & $\mathbf{R}$ & & & 56.4 & & 50 & $\mu g / L$ \\
\hline Manganese-54 & EP & & & $\mathbf{U}$ & 10 & & 300 & $\mathrm{pCi} / \mathrm{L}$ \\
\hline Mercury & WA & & & $\mathbf{U}$ & 0.2 & & 2 & $\mu \mathrm{g} / \mathrm{L}$ \\
\hline Mercury & GE & & & $\mathbf{U}$ & 0.2 & & 2 & $\mu g / L$ \\
\hline Mercury & WA & $\mathbf{R}$ & & $\mathbf{U}$ & 0.2 & & 2 & $\mu g / L$ \\
\hline Methacrylonitrile & WA & & & $\mathbf{U}$ & 20 & & NS & $\mu g /$ \\
\hline Methacrylonitrile & GE & & & $\mathbf{U}$ & 50 & & NS & $\mu g / L$ \\
\hline Methacrylonitrile & WA & $\mathbf{R}$ & & $\mathbf{U}$ & 20 & & NS & $\mu_{g} / L$ \\
\hline Methyl bromide & WA & & & $\mathbf{U}$ & 10 & & NS & $\mu g / L$ \\
\hline Methyl bromide & GE & & & $\mathbf{U}$ & 1 & & NS & $\mu \mathrm{g} / \mathrm{L}$ \\
\hline Methyl bromide & WA & $\mathbf{R}$ & & $\mathbf{U}$ & 10 & & NS & $\mu g / L$ \\
\hline Methyl chloride & WA & & & $\mathbf{U}$ & 10 & & NS & $\mu g / L$ \\
\hline Methyl chloride & GE & & & U & 1 & & NS & $\mu \mathrm{g} / \mathrm{L}$ \\
\hline Methyl chloride & WA & $\mathbf{R}$ & & $\mathbf{U}$ & 10 & & NS & $\mu g / L$ \\
\hline Methyl ethyl ketone & WA & & & $\mathbf{U}$ & 10 & & NS & $\mu g / L$ \\
\hline Methyl ethyl ketone & $\mathrm{GE}$ & & & U & 1 & & NS & $\mu \mathrm{g} / \mathrm{L}$ \\
\hline Methyl ethyl ketone & WA & $\mathbf{R}$ & & $\mathrm{U}$ & 10 & & NS & $\mu \mathrm{g} / \mathrm{L}$ \\
\hline Methyl iodide & WA & & & U & 10 & & NS & $\mu g / L$ \\
\hline Methyl iodide & GE & & & $\mathbf{U}$ & 15 & & NS & $\mu g / L$ \\
\hline Methyl iodide & WA & $\mathbf{R}$ & & $\mathbf{U}$ & 10 & & NS & $\mu g / L$ \\
\hline Methyl isobutyl ketone & GE & & & $U$ & 1 & & NS & $\mu g / L$ \\
\hline Methylene bromide & WA & & & U & 10 & & NS & $\mu g / L$ \\
\hline Methylene bromide & GE & & & U & 1 & & NS & $\mu g / L$ \\
\hline Methylene bromide & WA & $\mathbf{R}$ & & $\mathrm{U}$ & 10 & & NS & $\mu \mathrm{g} / \mathrm{L}$ \\
\hline Methylene chloride & WA & & & U & 5 & & 5 & $\mu \mathrm{g} / \mathrm{L}$ \\
\hline Methylene chloride & GE & & & $U$ & 1 & & 5 & $\mu g / L$ \\
\hline Methylene chloride & WA & $\mathbf{R}$ & & $\mathbf{U}$ & 5 & & 5 & $\mu g / L$ \\
\hline Nickel & WA & & & $\mathbf{U}$ & 40 & & 100 & $\mu g / L$ \\
\hline Nickel & GE & & & $\mathrm{U}$ & 4 & & 100 & $\mu g / L$ \\
\hline Nickel & WA & $\mathbf{R}$ & & & 77.4 & & 100 & $\mu \mathrm{g} / \mathrm{L}$ \\
\hline Nitrate as nitrogen & WA & & A & & 0.0365 & & 10 & $\mathrm{mg} / \mathrm{L}$ \\
\hline Nitrate as nitrogen & $\mathrm{GE}$ & & & & 1.05 & & 10 & $\mathrm{mg} / \mathrm{L}$ \\
\hline Nitrate as nitrogen & WA & $\mathbf{R}$ & & & 0.114 & & 10 & $\mathrm{mg} / \mathrm{L}$ \\
\hline Nonvolatile beta & TM & & & & 3.8 & 1.8 & 50 & $\mathrm{pCi} / \mathrm{L}$ \\
\hline Nonvolatile beta & GE & & & $\mathrm{U}$ & 2 & & 50 & $\mathrm{pCi} / \mathrm{L}$ \\
\hline Nonvolatile beta & TM & $\mathbf{R}$ & & & 1.1 & 1.6 & 50 & $\mathrm{pCi} / \mathrm{L}$ \\
\hline pH & GE & & & J & Q 5.57 & & NS & PH \\
\hline Potassium & WA & & & U & 5000 & & NS & $\mu g / L$ \\
\hline Potassium & GE & & & $U$ & 500 & & NS & $\mu g / L$ \\
\hline Potassium & WA & $\mathbf{R}$ & & $\mathbf{U}$ & 5000 & & NS & $\mu g / L$ \\
\hline Potassium-40 & TM & & & & 851.7 & 71.5 & 300 & $\mathrm{pCi} / \mathrm{L}$ \\
\hline Potassium -40 & EP & & & $\mathbf{U}$ & 110 & & 300 & $\mathrm{pCi} / \mathrm{L}$ \\
\hline Promethium-144 & EP & & & U & 10 & & NS & $\mathrm{pCi} / \mathrm{L}$ \\
\hline Promethium-146 & EP & & & $\mathbf{U}$ & 10 & & NS & $\mathrm{pCi} / \mathrm{L}$ \\
\hline
\end{tabular}


UTR116 Laboratory Analyses (cont'd)

\begin{tabular}{|c|c|c|c|c|c|c|c|}
\hline Analyte & Lab & Avg & Qualifier & Result & Acc & Stand & Units \\
\hline Propionitrile & WA & & $\mathbf{U}$ & 50 & & NS & $\mu g / L$ \\
\hline Propionitrile & GE & & $\mathbf{U}$ & 200 & & NS & $\mu g / L$ \\
\hline Propionitrile & WA & $\mathbf{R}$ & $\mathbf{U}$ & 50 & & NS & $\mu g / L$ \\
\hline Ruthenium-106 & $\mathbf{E P}$ & & $\mathbf{U}$ & 90 & & 30 & $\mathrm{pCi} / \mathrm{L}$ \\
\hline Selenium & WA & & $\mathbf{U}$ & 5 & & 50 & $\mu g / L$ \\
\hline Selenium & GE & & UJ & $\mathbf{L}$ & & 50 & $\mu g / L$ \\
\hline Selenium & WA & $\mathbf{R}$ & $\mathbf{U}$ & 5 & - & 50 & $\mu g / L$ \\
\hline Silver & WA & & $\mathbf{U}$ & 10 & & 50 & $\mu g / L$ \\
\hline Silver & GE & & $\mathbf{U}$ & 2 & & 50 & $\mu g / L$ \\
\hline Silver & WA & $\mathbf{R}$ & $\mathbf{U}$ & 10 & & 50 & $\mu g / L$ \\
\hline Sodium & WA & & $\mathbf{U}$ & 5000 & & NS & $\mu g / L$ \\
\hline Sodium & $\mathrm{GE}$ & & & 1230 & & NS & $\mu g / L$ \\
\hline Sodium & WA & $\mathbf{R}$ & $\mathbf{U}$ & 5000 & & NS & $\mu g / L$ \\
\hline Sodium-22 & $\mathrm{EP}$ & & $\mathbf{U}$ & 10 & & 466 & $\mathrm{pCi} / \mathrm{L}$ \\
\hline Styrene & WA & & $\mathbf{U}$ & 5 & & 100 & $\mu g / L$ \\
\hline Styrene & GE & & $\mathbf{U}$ & 1 & & 100 & $\mu g / L$ \\
\hline Styrene & WA & $\mathbf{R}$ & $\mathbf{U}$ & 5 & & 100 & $\mu g / L$ \\
\hline Sulfate & WA & & & 1.04 & & 400 & $\mathrm{mg} / \mathrm{L}$ \\
\hline Sulfate & GE & & $\mathbf{U}$ & 1 & & 400 & mg/L \\
\hline Sulfate & WA & $\mathbf{R}$ & & 1.14 & & 400 & $m g / L$ \\
\hline Sulfide & WA & & $\mathbf{U}$ & 0.1 & & NS & $m g / L$ \\
\hline Sulfide & WA & NA & $\mathbf{U}$ & 0.1 & & NS & $m g / L$ \\
\hline Sulfide & GE & & $\mathbf{U}$ & 1 & & NS & $\mathrm{mg} / \mathrm{L}$ \\
\hline Sulfide & WA & $\mathbf{R}$ & $\mathbf{U}$ & 0.1 & & NS & $\mathrm{mg} / \mathrm{L}$ \\
\hline Tetrachloroethylene & WA & & $\mathbf{U}$ & 5 & & 5 & $\mu g / L$ \\
\hline Tetrachloroethylene & GE & & $\mathrm{U}$ & 1 & & 5 & $\mu \mathrm{g} / \mathrm{L}$ \\
\hline Tetrachloroethylene & WA & $\mathbf{R}$ & $\mathbf{U}$ & 5 & & 5 & $\mu g / L$ \\
\hline Thallium & WA & & $\mathbf{U}$ & 10 & & 2 & $\mu g / L$ \\
\hline Thallium & GE & & $\mathbf{U}$ & 2 & & 2 & $\mu g / L$ \\
\hline Thallium & WA & $\mathbf{R}$ & $\mathbf{U}$ & 10 & & 2 & $\mu g / L$ \\
\hline Thorium-234 & EP & & $\mathbf{U}$ & 350 & & 401 & $\mathrm{pCi} / \mathrm{L}$ \\
\hline Toluene & WA & & $\mathbf{U}$ & 5 & & 1000 & $\mu g / L$ \\
\hline Toluene & $\mathrm{GE}$ & & $\mathbf{U}$ & 1 & & 1000 & $\mu g / L$ \\
\hline Toluene & WA & $\mathbf{R}$ & $\mathbf{J}$ & 1.01 & & 1000 & $\mu g / L$ \\
\hline Total hardness & WA & & & 6 & & NS & $\mathrm{mg} / \mathrm{L}$ \\
\hline Total hardness & GE & & & 5 & & NS & $\mathrm{mg} / \mathrm{L}$ \\
\hline Total hardness & WA & $\mathbf{R}$ & & 12 & & NS & $\mathrm{mg} / \mathrm{L}$ \\
\hline Total organic carbon & WA & & & 3.38 & & NS & $\mathrm{mg} / \mathrm{L}$ \\
\hline Total organic carbon & $\mathrm{GE}$ & & & 2.68 & & NS & $\mathrm{mg} / \mathrm{L}$ \\
\hline Total organic carbon & WA & $\mathbf{R}$ & & 2.66 & & NS & mg/L \\
\hline Total suspended solids & WA & & & 12 & & NS & $\mathrm{mg} / \mathrm{L}$ \\
\hline Total suspended solids & GE & & $\mathbf{U}$ & 1 & & NS & $\mathrm{mg} / \mathrm{L}$ \\
\hline Total suspended solids & WA & $\mathbf{R}$ & & 15 & & NS & $\mathrm{mg} / \mathrm{L}$ \\
\hline trans-1,3-Dichloropropene & WA & & $\mathbf{U}$ & 5 & & NS & $\mu g / L$ \\
\hline trans-1,3-Dichloropropene & GE & & $\mathbf{U}$ & 1 & & NS & $\mu g / L$ \\
\hline trans-1,3-Dichloropropene & WA & $\mathbf{R}$ & $\bar{U}$ & 5 & & NS & $\mu \mathrm{g} / \mathrm{L}$ \\
\hline trans-1,4-Dichloro-2-butene & WA & & $\mathbf{U}$ & 100 & & NS & $\mu \mathrm{g} / \mathrm{L}$ \\
\hline trans-1,4-Dichloro-2-butene & $\mathrm{GE}$ & & $\mathbf{U}$ & 30 & & NS & $\mu \mathrm{g} / \mathrm{L}$ \\
\hline trans-1,4-Dichloro-2-butene & WA & $\mathbf{R}$ & $\mathbf{U}$ & 100 & & NS & $\mu g / L$ \\
\hline Trichloroethylene & WA & & $\mathbf{U}$ & 5 & & 5 & $\mu g / L$ \\
\hline Trichloroethylene & GE & & $\mathbf{U}$ & 1 & & 5 & $\mu \mathrm{g} / \mathrm{L}$ \\
\hline Trichloroethylene & WA & $\mathbf{R}$ & $\mathbf{U}$ & 5 & & 5 & $\mu \mathrm{g} / \mathrm{L}$ \\
\hline Trichlorofluoromethane & WA & & $\mathbf{U}$ & 5 & & NS & $\mu g / L$ \\
\hline Trichlorofluoromethane & GE & & $\mathbf{U}$ & 1 & & NS & $\mu g / L$ \\
\hline Trichlorofluoromethane & WA & $\mathbf{R}$ & $\mathbf{U}$ & 5 & & NS & $\mu g / L$ \\
\hline
\end{tabular}


Sampling and Analysis of Water From Upper Three Runs And Its Wetlands Near Tank 16 and the Mixed Waste Management Facility

UTR 116 Laboratory Analyses (cont'd)

\begin{tabular}{|c|c|c|c|c|c|c|c|c|}
\hline Analyte & Lab & Rep & Avg & Qualifier & Result & Acc & Stand & Units \\
\hline Tritium & $\overline{T M}$ & & & & 143.5 & 24.2 & 20 & $\overline{\mathrm{pCi} / \mathrm{ml}}$ \\
\hline Tritium & GE & & & & 127 & & 20 & $\mathrm{pCi} / \mathrm{ml}$ \\
\hline Tritium & TM & $\mathbf{R}$ & & & 172.8 & 63.5 & 20 & $\mathrm{pCi} / \mathrm{ml}$ \\
\hline Turbidity & WA & & $\mathbf{A}$ & & 4.34 & & NS & NTU \\
\hline Turbidity & $\mathrm{GE}$ & & & & 5.09 & & NS & NTU \\
\hline Turbidity & WA & $\mathbf{R}$ & & & 10.1 & . & NS & NTU \\
\hline Vanadium & WA & & & $\mathbf{U}$ & 50 & & NS & $\mu g / L$ \\
\hline Vanadium & $\mathrm{GE}$ & & & $\mathbf{U}$ & 8 & & NS & $\mu g / L$ \\
\hline Vanadium & WA & $\mathbf{R}$ & & $\mathbf{U}$ & 50 & & NS & $\mu \mathrm{g} / \mathrm{L}$ \\
\hline Vinyl acetate & WA & & & $\mathbf{U}$ & 10 & & NS & $\mu \mathrm{g} / \mathrm{L}$ \\
\hline Vinyl acetate & GE & & & $\mathbf{U}$ & 1 & & NS & $\mu \mathrm{g} / \mathrm{L}$ \\
\hline Vinyl acetate & WA & $\mathbf{R}$ & & $\mathbf{U}$ & 10 & & NS & $\mu g / L$ \\
\hline Vinyl chloride & WA & & & $\mathbf{U}$ & 10 & & 2 & $\mu \mathrm{g} / \mathrm{L}$ \\
\hline Vinyl chloride & $\mathrm{GE}$ & & & $\mathbf{U}$ & 1 & & 2 & $\mu g / L$ \\
\hline Vinyl chloride & WA & $\mathbf{R}$ & & $\mathbf{U}$ & 10 & & 2 & $\mu \mathrm{g} / \mathrm{L}$ \\
\hline Xylenes (total) & WA & & & $\mathbf{U}$ & 5 & & 10000 & $\mu g / L$ \\
\hline Xylenes (total) & GE & & & $\mathbf{U}$ & 2 & & 10000 & $\mu g / L$ \\
\hline Xylenes (total) & WA & $\mathbf{R}$ & & $\mathbf{U}$ & 5 & & 10000 & $\mu \mathrm{g} / \mathrm{L}$ \\
\hline Yttrium-88 & EP & & & $\mathbf{U}$ & 60 & & NS & $\mathrm{pCi} / \mathrm{L}$ \\
\hline Zinc & WA & & & $\mathbf{U}$ & 20 & & 5000 & $\mu g / L$ \\
\hline Zinc & GE & & & & 8.24 & & 5000 & $\mu g / L$ \\
\hline Zinc & WA & $\mathbf{R}$ & & & 37.1 & & 5000 & $\mu \mathrm{g} / \mathrm{L}$ \\
\hline Zinc-65 & EP & & & $\mathbf{U}$ & 20 & & 300 & $\mathrm{pCi} / \mathrm{L}$ \\
\hline
\end{tabular}




\section{UTR120}

Field Measurements:

Sample Date: 4/20/93

$\mathrm{pH}: 7.06$

Specific Conductance: $25 \mu \mathrm{S} / \mathrm{cm}$

Red/Oxid Potential: $173 \mathrm{mv}$

Dissolved Oxygen: $1.6 \mathrm{mg} / \mathrm{L}$

\begin{tabular}{|c|c|c|c|c|c|c|c|c|}
\hline Analyte & Lab & Rep & Avg & Qualifier & Result & Acc & Stand & Units \\
\hline 1,1,1,2-Tetrachlore thane & WA & & & $\mathbf{U}$ & 10 & & NS & $\mu g / L$ \\
\hline 1,1,1,2-Tetrachloroethane & GE & & & $\mathbf{U}$ & 1 & & NS & $\mu g / L$ \\
\hline 1,1,1-Trichloroethane & WA & & & $\mathbf{U}$ & 5 & & 200 & $\mu g / L$ \\
\hline 1,1,1-Trichloroethane & GE & & & $\mathbf{U}$ & 1 & & 200 & $\mu g / L$ \\
\hline $1,1,2,2$-Tetrachloroethane & WA & & & $\mathbf{U}$ & 5 & & NS & $\mu g / L$ \\
\hline 1,1,2,2-Tetrachloroethane & GE & & & $\mathbf{U}$ & 1 & & NS & $\mu g / L$ \\
\hline 1,1,2-Trichloroethane & WA & & & $\mathbf{U}$ & 5 & & 5 & $\mu \mathrm{g} / \mathrm{L}$ \\
\hline 1,1,2-Trichloroethane & GE & & & $\mathbf{U}$ & 1 & & 5 & $\mu g / L$ \\
\hline 1,1-Dichloroethane & WA & & & $\mathbf{U}$ & 5 & & NS & $\mu g / L$ \\
\hline 1,1-Dichloroethane & GE & & & $\mathbf{U}$ & 1 & & NS & $\mu g / L$ \\
\hline 1,1-Dichloroethylene & WA & & & $\mathbf{U}$ & 5 & & 7 & $\mu g / L$ \\
\hline 1,1-Dichloroethylene & GE & & & $\mathbf{U}$ & 1 & & 7 & $\mu \mathrm{g} / \mathrm{L}$ \\
\hline 1,2,3-Trichloropropane & WA & & & $\mathbf{U}$ & 10 & & NS & $\mu g / L$ \\
\hline 1,2,3-Trichloropropane & GE & & & $\mathbf{U}$ & 1 & & NS & $\mu g / L$ \\
\hline 1,2-Dibromo-3-chloropropane & WA & & & $\mathbf{U}$ & 20 & & 0.2 & $\mu g / L$ \\
\hline 1,2-Dibromo-3-chloropropane & GE & & & $\mathbf{U}$ & 1 & & 0.2 & $\mu \mathrm{g} / \mathrm{L}$ \\
\hline 1,2-Dibromoethane & WA & & & $\mathbf{U}$ & 20 & & NS & $\mu g / L$ \\
\hline 1,2-Dibromoethane & GE & & & $\mathbf{U}$ & 20 & & NS & $\mu \mathrm{g} / \mathrm{L}$ \\
\hline 1,2-Dichloroethane & WA & & & $\mathbf{U}$ & 5 & & 5 & $\mu \mathrm{g} / \mathrm{L}$ \\
\hline 1,2-Dichloroethane & GE & & & $\mathbf{U}$ & 1 & & 5 & $\mu g / L$ \\
\hline 1,2-Dichloroethylene (total) & WA & & & $\mathbf{U}$ & 5 & & 100 & $\mu \mathrm{g} / \mathrm{L}$ \\
\hline 1,2-Dichloroethylene (total) & GE & & & $\mathbf{U}$ & 1 & & 100 & $\mu \mathrm{g} / \mathrm{L}$ \\
\hline 1,2-Dichloropropane & WA & & & $\mathbf{U}$ & 5 & & 5 & $\mu g / L$ \\
\hline 1,2-Dichloropropane & GE & & & $\mathbf{U}$ & 1 & & 5 & $\mu \mathrm{g} / \mathrm{L}$ \\
\hline 2-Hexanone & WA & & & U & 10 & & NS & $\mu \mathrm{g} / \mathrm{L}$ \\
\hline 2-Hexanone & GE & & & $\mathbf{U}$ & 1 & & NS & $\mu \mathrm{g} / \mathrm{L}$ \\
\hline 4-Methyl-2-pentanone & WA & & & $\mathbf{U}$ & 10 & & NS & $\mu \mathrm{g} / \mathrm{L}$ \\
\hline Acetone & WA & & & J & 7.69 & & NS & $\mu g / L$ \\
\hline Acetone & $\mathrm{GE}$ & & & $\mathbf{U}$ & 100 & & NS & $\mu \mathrm{g} / \mathrm{L}$ \\
\hline Acetonitrile & WA & & & U & 20 & & NS & $\mu \mathrm{g} / \mathrm{L}$ \\
\hline Acetonitrile & GE & & & $\mathbf{U}$ & 1 & & NS & $\mu g / L$ \\
\hline Acidity & WA & & $\mathbf{A}$ & & 22.5 & & NS & $\mathrm{mg} / \mathrm{L}$ \\
\hline Acrolein & WA & & & $\mathbf{U}$ & 10 & & NS & $\mu \mathrm{g} / \mathrm{L}$ \\
\hline Acrolein & GE & & & $\mathbf{U}$ & 20 & & NS & $\mu \mathrm{g} / \mathrm{L}$ \\
\hline Acrylonitrile & WA & & & $\mathbf{U}$ & 10 & & NS & $\mu g / L$ \\
\hline Acrylonitrile & GE & & & $\mathbf{U}$ & 20 & & NS & $\mu g / L$ \\
\hline Actinium-228 & $\mathrm{EP}$ & & & $\mathbf{U}$ & 30 & & NS & $\mathrm{pCi} / \mathrm{L}$ \\
\hline Alkalinity & WA & & & & 3.5 & & NS & $\mathrm{mg} / \mathrm{L}$ \\
\hline Alkalinity & GE & & $\mathbf{A}$ & & 4.8 & & NS & $\mathrm{mg} / \mathrm{L}$ \\
\hline Allyl chloride & WA & & & $\mathrm{U}$ & 100 & & NS & $\mu \mathrm{g} / \mathrm{L}$ \\
\hline Allyl chloride & GE & & & $\mathbf{U}$ & 50 & & NS & $\mu \mathrm{g} / \mathrm{L}$ \\
\hline Aluminum & WA & & & $\mathbf{U}$ & 200 & & NS & $\mu \mathrm{g} / \mathrm{L}$ \\
\hline Aluminum & GE & & $\mathbf{A}$ & & 66.85 & & NS & $\mu \mathrm{g} / \mathrm{L}$ \\
\hline Antimony & WA & & & $\mathbf{U}$ & 60 & & 6 & $\mu \mathrm{g} / \mathrm{L}$ \\
\hline Antimony & GE & & & $\mathbf{U}$ & 2 & & 6 & $\mu g / L$ \\
\hline Antimony-125 & EP & & & $\mathbf{U}$ & 20 & & 300 & $\mathrm{pCi} / \mathrm{L}$ \\
\hline Arsenic & WA & & & U & 10 & & 50 & $\mu \mathrm{g} / \mathrm{L}$ \\
\hline Arsenic & GE & & & $\mathbf{U}$ & 2 & & 50 & $\mu g / L$ \\
\hline
\end{tabular}


UTR 120 Laboratory Analyses (cont'd)

\begin{tabular}{|c|c|c|c|c|c|c|}
\hline Analyte & Lab & Rep & Qualifier & Result & Acc Stand & Units \\
\hline Barium & WA & & $\bar{U}$ & 200 & 2000 & $\mu \mathrm{g} / \mathrm{L}$ \\
\hline Barium & GE & $\mathbf{A}$ & & 12.3 & 2000 & $\mu \mathrm{g} / \mathrm{L}$ \\
\hline Benzene & WA & & U & 5 & 5 & $\mu g / L$ \\
\hline Benzene & GE & & $\mathrm{U}$ & 1 & 5 & $\mu g / L$ \\
\hline Beryllium & WA & & $\mathbf{U}$ & 5 & $4^{\prime}$ & $\mu \mathrm{g} / \mathrm{L}$ \\
\hline Beryllium & GE & & U & 3 & 4 & $\mu \mathrm{g} / \mathrm{L}$ \\
\hline Beryllium & GE & NA & $\mathbf{U}$ & 3 & 4 & $\mu \mathrm{g} / \mathrm{L}$ \\
\hline Bis(2-chloro-1-methylethyl)ether & GE & & U & 10 & NS & $\mu \mathrm{g} / \mathrm{L}$ \\
\hline Bromodichloromethane & WA & & $\mathrm{U}$ & 5 & NS & $\mu \mathrm{g} / \mathrm{L}$ \\
\hline Bromodichloromethane & GE & & $\mathbf{U}$ & 1 & NS & $\mu \mathrm{g} / \mathrm{L}$ \\
\hline Bromoform & WA & & $\mathbf{U}$ & 5 & NS & $\mu g / L$ \\
\hline Bromoform & GE & & U & 1 & NS & $\mu \mathrm{g} / \mathrm{L}$ \\
\hline Cadmium & WA & & U & 5 & 5 & $\mu \mathrm{g} / \mathrm{L}$ \\
\hline Cadmium & GE & & $\mathbf{U}$ & 2 & 5 & $\mu g / L$ \\
\hline Cadmium & GE & NA & $\mathbf{U}$ & 2 & 5 & $\mu g / L$ \\
\hline Calcium & WA & & $\mathbf{U}$ & 5000 & NS & $\mu \mathrm{g} / \mathrm{L}$ \\
\hline Calcium & GE & A & & 1620 & NS & $\mu g / L$ \\
\hline Carbon disulfide & WA & & $\mathbf{U}$ & 5 & NS & $\mu g / L$ \\
\hline Carbon disulfide & GE & & U & 1 & NS & $\mu g / L$ \\
\hline Carbon tetrachloride & WA & & $\mathbf{U}$ & 5 & 5 & $\mu g / L$ \\
\hline Carbon tetrachloride & GE & & $\mathbf{U}$ & 1 & 5 & $\mu g / L$ \\
\hline Cerium-144 & EP & & $\mathbf{U}$ & 60 & 261 & $\mathrm{pCi} / \mathrm{L}$ \\
\hline Cesium-134 & EP & & $\mathbf{U}$ & 10 & 81.3 & $\mathrm{pCi} / \mathrm{L}$ \\
\hline Cesium-137 & TM & NA & $\mathbf{U}$ & 9.9 & 200 & $\mathrm{pCi} / \mathrm{L}$ \\
\hline Cesium-137 & TM & & U & 9.6 & 200 & $\mathrm{pCi} / \mathrm{L}$ \\
\hline Cesium-137 & EP & & $\mathrm{U}$ & 10 & 200 & $\mathrm{pCi} / \mathrm{L}$ \\
\hline Chloride & WA & & & 3.28 & NS & $\mathrm{mg} / \mathrm{L}$ \\
\hline Chloride & GE & & & 2.08 & NS & $\mathrm{mg} / \mathrm{L}$ \\
\hline Chlorobenzene & WA & & $\mathbf{U}$ & 5 & 100 & $\mu \mathrm{g} / \mathrm{L}$ \\
\hline Chlorobenzene & GE & & $\mathbf{U}$ & 1 & 100 & $\mu \mathrm{g} / \mathrm{L}$ \\
\hline Chloroethane & WA & & U & 10 & NS & $\mu \mathrm{g} / \mathrm{L}$ \\
\hline Chloroethane & GE & & $\mathbf{U}$ & 1 & NS & $\mu g / L$ \\
\hline Chloroform & WA & & $\mathbf{U}$ & 5 & 100 & $\mu g / L$ \\
\hline Chloroform & GE & & $\mathbf{U}$ & 1 & 100 & $\mu \mathrm{g} / \mathrm{L}$ \\
\hline Chloroprene & WA & & $\mathbf{U}$ & 20 & NS & $\mu \mathrm{g} / \mathrm{L}$ \\
\hline Chloroprene & $\mathrm{GE}$ & & $\mathbf{U}$ & 200 & NS & $\mu g / L$ \\
\hline Chromium & WA & & $\mathrm{U}$ & 10 & 100 & $\mu \mathrm{g} / \mathrm{L}$ \\
\hline Chromium & GE & & $\mathrm{U}$ & 4 & 100 & $\mu g / L$ \\
\hline Chromium & GE & NA & $\mathbf{U}$ & 4 & 100 & $\mu g / L$ \\
\hline cis-1,3-Dichloropropene & WA & & $\mathrm{U}$ & 5 & NS & $\mu g / L$ \\
\hline cis-1,3-Dichloropropene & $\mathrm{GE}$ & & $\mathrm{U}$ & 1 & NS & $\mu g / L$ \\
\hline Cobalt & WA & & $\mathrm{U}$ & 50 & NS & $\mu \mathrm{g} / \mathrm{L}$ \\
\hline Cobalt & GE & & $U$ & 4 & NS & $\mu \mathrm{g} / \mathrm{L}$ \\
\hline Cobait & $\mathrm{GE}$ & NA & $\mathrm{U}$ & 4 & NS & $\mu \mathrm{g} / \mathrm{L}$ \\
\hline Cobalt-57 & EP & & U & 10 & 1000 & $\mathrm{pCi} / \mathrm{L}$ \\
\hline Cobalt-60 & EP & & U & 10 & 100 & $\mathrm{pCi} / \mathrm{L}$ \\
\hline Copper & WA & & $\mathbf{U}$ & 25 & 1000 & $\mu g / L$ \\
\hline Copper & $\mathrm{GE}$ & & $\mathrm{U}$ & 4 & 1000 & $\mu g / L$ \\
\hline Copper & $\mathrm{GE}$ & NA & $\mathbf{U}$ & 4 & 1000 & $\mu g / L$ \\
\hline Cyanide & WA & & $\mathbf{U}$ & 10 & 200 & $\mu g / L$ \\
\hline Cyanide & GE & & $\mathbf{U}$ & 5 & 200 & $\mu \mathrm{g} / \mathrm{L}$ \\
\hline Dibromochloromethane & WA & & $\mathbf{U}$ & 5 & NS & $\mu \mathrm{g} / \mathrm{L}$ \\
\hline Dibromochloromethane & GE & & U & 1 & NS & $\mu \mathrm{g} / \mathrm{L}$ \\
\hline Dichlorodifluoromethane & WA & & $\mathbf{U}$ & 10 & NS & $\mu g / L$ \\
\hline Dichlorodifluoromethane & GE & & $\mathbf{U}$ & 1 & NS & $\mu g / L$ \\
\hline
\end{tabular}


UTR120 Laboratory Analyses (cont'd)

\begin{tabular}{|c|c|c|c|c|c|c|c|c|c|}
\hline Analyte & Lab & Rep & Avg & Qualifier & & Result & Acc & Stand & Units \\
\hline Ethylbenzente & WA & & & $\mathbf{U}$ & & 5 & & 700 & $\mu g / L$ \\
\hline Ethylbenzene & GE & & & $\mathbf{U}$ & & 1 & & 700 & $\mu g / L$ \\
\hline Europium-152 & EP & & & $\mathbf{U}$ & & 40 & & NS & $\mathrm{pCi} / \mathrm{L}$ \\
\hline Europium-154 & EP & & & $\mathbf{U}$ & & 20 & & 200 & $\mathrm{pCi} / \mathrm{L}$ \\
\hline Europium-155 & EP & & & $\mathbf{U}$ & & 30 & & 600 & $\mathrm{pCi} / \mathrm{L}$ \\
\hline Fluoride & WA & & & $\mathbf{U}$ & & 0.1 & & 4 & $\mathrm{mg} / \mathrm{L}$ \\
\hline Fluoride & $\mathrm{GE}$ & & & $U$ & & 0.1 & $\cdot$ & 4 & $\mathrm{mg} / \mathrm{L}$ \\
\hline Gross alpha & TM & & NA & U & & 1 & & 15 & $\mathrm{pCi} / \mathrm{L}$ \\
\hline Gross alpha & TM & & & $\mathbf{U}$ & & 1 & & 15 & $\mathrm{pCi} / \mathrm{L}$ \\
\hline Gross alpha & GE & & & $\mathbf{U}$ & & 2 & & 15 & $\mathrm{pCi} / \mathrm{L}$ \\
\hline Iron & WA & & & & & 161 & & 300 & $\mu g / L$ \\
\hline Iron & GE & & & J & $\mathbf{H}$ & 243 & & 300 & $\mu \mathrm{g} / \mathrm{L}$ \\
\hline Iron & GE & & NA & J & $\mathbf{H}$ & 213 & & 300 & $\mu \mathrm{g} / \mathrm{L}$ \\
\hline Isobutyl alcohol & WA & & & $\mathrm{U}$ & & 20 & & NS & $\mu \mathrm{g} / \mathrm{L}$ \\
\hline Isobutyl alcohol & GE & & & $\mathbf{U}$ & & 100 & & NS & $\mu \mathrm{g} / \mathrm{L}$ \\
\hline Lead & WA & & & U & & 3 & & 50 & $\mu \mathrm{g} / \mathrm{L}$ \\
\hline Lead & $\mathrm{GE}$ & & . & $\mathrm{U}$ & & 3 & & 50 & $\mu g / L$ \\
\hline Lead-212 & EP & & & & & 23.5 & & NS & $\mathrm{pCi} / \mathrm{L}$ \\
\hline Magnesium & WA & & & $\mathbf{U}$ & & 5000 & & NS & $\mu g / L$ \\
\hline Magnesium & $\mathrm{GE}$ & & A & & & 741 & & NS & $\mu g / L$ \\
\hline Manganese & WA & & & $\mathbf{U}$ & & 15 & & 50 & $\mu g / L$ \\
\hline Manganese & GE & & $\mathbf{A}$ & & & 12.45 & & 50 & $\mu g / L$ \\
\hline Manganese-54 & EP & & & U & & 10 & & 300 & $\mathrm{pCi} / \mathrm{L}$ \\
\hline Mercury & WA & & & $\mathbf{U}$ & & 0.2 & & 2 & $\mu g / L$ \\
\hline Mercury & GE & & & $\mathrm{U}$ & & 0.2 & & 2 & $\mu g / L$ \\
\hline Methacrylonitrile & WA & & & $\mathbf{U}$ & & 20 & & NS & $\mu g / L$ \\
\hline Methacrylonitrile & GE & & & $\mathbf{U}$ & & 50 & & NS & $\mu \mathrm{g} / \mathrm{L}$ \\
\hline Methyl bromide & WA & & & $\mathbf{U}$ & & 10 & & NS & $\mu g / L$ \\
\hline Methyl bromide & GE & & & $\mathbf{U}$ & & 1 & & NS & $\mu g / L$ \\
\hline Methyl chloride & WA & & & $\mathbf{U}$ & & 10 & & NS & $\mu \mathrm{g} / \mathrm{L}$ \\
\hline Methyl chloride & $\mathrm{GE}$ & & & $\mathbf{U}$ & & 1 & & NS & $\mu \mathrm{g} / \mathrm{L}$ \\
\hline Methyl ethyl ketone & WA & & & $\mathbf{U}$ & & 10 & & NS & $\mu g / L$ \\
\hline Methyl ethyl ketone & $\mathrm{GE}$ & & & U & & 1 & & NS & $\mu \mathrm{g} / \mathrm{L}$ \\
\hline Methyl iodide & WA & & & $\mathbf{U}$ & & 10 & & NS & $\mu g / L$ \\
\hline Methyl iodide & GE & & & $\mathbf{U}$ & & 15 & & NS & $\mu g / L$ \\
\hline Methyl isobutyl ketone & GE & & & $\mathbf{U}$ & & 1 & & NS & $\mu g / L$ \\
\hline Methylene bromide & WA & & & $\mathrm{U}$ & & 10 & & NS & $\mu \mathrm{g} / \mathrm{L}$ \\
\hline Methylene bromide & GE & & & $\mathbf{U}$ & & 1 & & NS & $\mu g / L$ \\
\hline Methylene chloride & WA & & & $\mathrm{U}$ & & 5 & & 5 & $\mu g / L$ \\
\hline Methylene chloride & GE & & & $\mathbf{U}$ & & 1 & & 5 & $\mu g / L$ \\
\hline Nickel & WA & & & U & & 40 & & 100 & $\mu \mathrm{g} / \mathrm{L}$ \\
\hline Nickel & GE & & & $\mathbf{U}$ & & 4 & & 100 & $\mu \mathrm{g} / \mathrm{L}$ \\
\hline Nickel & GE & & NA & $\mathbf{U}$ & & 4 & & 100 & $\mu \mathrm{g} / \mathrm{L}$ \\
\hline Nitrate as nitrogen & WA & & & & & 1.07 & & 10 & $\mathrm{mg} / \mathrm{L}$ \\
\hline Nitrate as nitrogen & GE & & & & & 1 & & 10 & $\mathrm{mg} / \mathrm{L}$ \\
\hline Nonvolatile beta & TM & & A & & & 2 & 1.6 & 50 & $\mathrm{pCi} / \mathrm{L}$ \\
\hline Nonvolatile beta & GE & & & U & & 2 & & 50 & $\mathrm{pCi} / \mathrm{L}$ \\
\hline $\mathrm{pH}$ & $\mathrm{GE}$ & & & $\mathbf{J}$ & $\mathbf{Q}$ & 5.66 & & NS & PH \\
\hline Potassium & WA & & & $\mathrm{U}$ & & 5000 & & NS & $\mu \mathrm{g} / \mathrm{L}$ \\
\hline Potassium & GE & & A & & & 618 & & NS & $\mu \mathrm{g} / \mathrm{L}$ \\
\hline Potassium -40 & IP & & & $\mathbf{U}$ & & 110 & & 300 & $\mathrm{pCi} / \mathrm{L}$ \\
\hline Promethium-144 & EP & & & $\mathrm{U}$ & & 10 & & NS & $\mathrm{pCi} / \mathrm{L}$ \\
\hline Promethium-146 & EP & & & $\mathbf{U}$ & & 10 & & NS & $\mathrm{pCi} / \mathrm{L}$ \\
\hline Propionitrile & WA & & & $\mathbf{U}$ & & 50 & & NS & $\mu \mathrm{g} / \mathrm{L}$ \\
\hline Propionitrile & GE & & & $\mathbf{U}$ & & 200 & & NS & $\mu \mathrm{g} / \mathrm{L}$ \\
\hline Ruthenium-106 & $\mathbf{E P}$ & & & $\mathbf{U}$ & & 90 & & 30 & $\mathrm{pCi} / \mathrm{L}$ \\
\hline
\end{tabular}


UTR120 Laboratory Analyses (cont'd)

\begin{tabular}{|c|c|c|c|c|c|c|c|}
\hline Analyte & Lab & Avg & Qualifier & Result & Acc & Stand & Units \\
\hline Selenium & WA & & $\mathbf{U}$ & 5 & & 50 & $\mu g / L$ \\
\hline Selenium & GE & & UJ & $\mathbf{L}$ & & 50 & $\mu g / L$ \\
\hline Silver & WA & & $\mathbf{U}$ & 10 & & 50 & $\mu g / L$ \\
\hline Silver & $\mathrm{GE}$ & & $\mathbf{U}$ & 2 & & 50 & $\mu \mathrm{g} / \mathrm{L}$ \\
\hline Silver & $\mathbf{G E}$ & NA & $\mathbf{U}$ & 2 & & 50 & $\mu \mathrm{g} / \mathrm{L}$ \\
\hline Sodium & WA & & $\mathbf{U}$ & 5000 & & NS & $\mu \mathrm{g} / \mathrm{L}$ \\
\hline Sodium & GE & $\mathbf{A}$ & & 1540 & & NS & $\mu g / L$ \\
\hline Sodium-22 & EP & & $\mathbf{U}$ & 10 & & 466 & $\mathrm{pCi} / \mathrm{L}$ \\
\hline Styrene & WA & & $\mathbf{U}$ & 5 & & 100 & $\mu g / L$ \\
\hline Styrene & GE & & $\mathbf{U}$ & 1 & & 100 & $\mu g / L$ \\
\hline Sulfate & WA & & $\mathbf{U}$ & 2.5 & & 400 & $\mathrm{mg} / \mathrm{L}$ \\
\hline Sulfate & GE & & & 1.5 & & 400 & $\mathrm{mg} / \mathrm{L}$ \\
\hline Sulfide & WA & & $\mathbf{U}$ & 0.1 & & NS & $\mathrm{mg} / \mathrm{L}$ \\
\hline Sulfide & GE & $\mathbf{N A}$ & $\mathbf{U}$ & 1 & & NS & $\mathrm{mg} / \mathrm{L}$ \\
\hline Sulfide & $\mathbf{G E}$ & & $\mathbf{U}$ & 1 & & NS & $\mathrm{mg} / \mathrm{h}$ \\
\hline Tetrachloroethylene & WA & . & $\mathbf{U}$ & 5 & & 5 & $\mu g / L$ \\
\hline Tetrachloroethylene & GE & & $\mathbf{U}$ & 1 & & 5 & $\mu g / L$ \\
\hline Thallium & WA & & $\mathbf{U}$ & 10 & & 2 & $\mu g / L$ \\
\hline Thallium & GE & & $\mathbf{U}$ & 2 & & 2 & $\mu g / L$ \\
\hline Thorium-234 & EP & & $\mathbf{U}$ & 350 & & 401 & $\mathrm{pCi} / \mathrm{L}$ \\
\hline Toluene & WA & & $\mathbf{U}$ & 5 & & 1000 & $\mu g / L$ \\
\hline Toluene & $\mathrm{GE}$ & & $\mathbf{U}$ & 1 & & 1000 & $\mu g / L$ \\
\hline Total hardness & WA & & & 16 & & NS & $\mathrm{mg} / \mathrm{L}$ \\
\hline Total hardness & GE & $\mathbf{A}$ & & 10 & & NS & $\mathrm{mg} / \mathrm{L}$ \\
\hline Total organic carbon & WA & & & 3.84 & & NS & $\mathrm{mg} / \mathrm{L}$ \\
\hline Total organic carbon & GE & & & 4.1 & & NS & $\mathrm{mg} / \mathrm{L}$ \\
\hline Total suspended solids & WA & & & 12 & & NS & $\mathrm{mg} / \mathrm{L}$ \\
\hline Total suspended solids & GE & NA & & 2 & & NS & $\mathrm{mg} / \mathrm{L}$ \\
\hline Total suspended solids & GE & & $\mathbf{U}$ & 1 & & NS & $\mathrm{mg} / \mathrm{L}$ \\
\hline trans-1,3-Dichloropropene & WA & & $\mathbf{U}$ & 5 & & NS & $\mu \mathrm{g} / \mathrm{L}$ \\
\hline trans-1,3-Dichloropropene & GE & & $\mathbf{U}$ & 1 & & NS & $\mu g / L$ \\
\hline trans-1,4-Dichloro-2-butene & WA & & $\mathbf{U}$ & 100 & & NS & $\mu g / L$ \\
\hline trans-1,4-Dichloro-2-butene & GE & & $\mathbf{U}$ & 30 & & NS & $\mu g / L$ \\
\hline Trichloroethylene & WA & & $\mathbf{U}$ & 5 & & 5 & $\mu \mathrm{g} / \mathrm{L}$ \\
\hline Trichloroethylene & GE & & $\mathbf{U}$ & 1 & & 5 & $\mu \mathrm{g} / \mathrm{L}$ \\
\hline Trichlorofluoromethane & WA & & $\mathbf{U}$ & 5 & & NS & $\mu \mathrm{g} / \mathrm{L}$ \\
\hline Trichlorofluoromethane & GE & & $\mathbf{U}$ & 1 & & NS & $\mu g / L$ \\
\hline Tritium & TM & $\mathbf{A}$ & & 17.95 & 6.65 & 20 & $\mathrm{pCi} / \mathrm{ml}$ \\
\hline Tritium & $\mathrm{GE}$ & & & 16.3 & & 20 & $\mathrm{pCi} / \mathrm{ml}$ \\
\hline Turbidity & WA & & & 1.99 & & NS & NTU \\
\hline Turbidity & $\mathrm{GE}$ & $\mathbf{A}$ & & 1.58 & & NS & NTU \\
\hline Vanadium & WA & & $\mathbf{U}$ & 50 & & NS & $\mu g / L$ \\
\hline Vanadium & GE & & $\mathbf{U}$ & 8 & & NS & $\mu g / L$ \\
\hline Vanadium & GE & NA & $\mathbf{U}$ & 8 & & NS & $\mu g / L$ \\
\hline Vinyl acetate & WA & & $\mathbf{U}$ & 10 & & NS & $\mu \mathrm{g} / \mathrm{L}$ \\
\hline Vinyl acetate & GE & & $\mathrm{U}$ & 1 & & NS & $\mu \mathrm{g} / \mathrm{L}$ \\
\hline Vinyl chloride & WA & & $\mathbf{U}$ & 10 & & 2 & $\mu \mathrm{g} / \mathrm{L}$ \\
\hline Vinyl chloride & GE & & $\mathbf{U}$ & 1 & & 2 & $\mu g / L$ \\
\hline Xylenes (total) & WA & & $\mathbf{U}$ & 5 & & 10000 & $\mu \mathrm{g} / \mathrm{L}$ \\
\hline Xylenes (total) & $\mathrm{GE}$ & & $\mathbf{U}$ & 2 & & 10000 & $\mu \mathrm{g} / \mathrm{L}$ \\
\hline Yttrium-88 & EP & & $\mathbf{U}$ & 60 & & NS & $\mathrm{pCi} / \mathrm{L}$ \\
\hline Zinc & WA & & $\mathbf{U}$ & 20 & & 5000 & $\mu g / L$ \\
\hline Zinc & GE & A & & 8.95 & & 5000 & $\mu \mathrm{g} / \mathrm{L}$ \\
\hline Zinc-65 & EP & & $\mathbf{U}$ & 20 & & 300 & $\mathrm{pCi} / \mathrm{L}$ \\
\hline
\end{tabular}




\section{UTR122}

Field Measurements:

Sample Date: 4/20/93

pH: 5.7

Specific Conductance: $38 \mu \mathrm{S} / \mathrm{cm}$

Red/Oxid Potential: $161 \mathrm{mv}$

Dissolved Oxygen: $1 \mathrm{mg} / \mathrm{L}$

\begin{tabular}{|c|c|c|c|c|c|c|c|}
\hline Analyte & Lab & Rep & Qualifier & Result & Acc & Stand & Units \\
\hline 1,1,1,2-Tetrachloroethane & WA & & $\mathrm{U}$ & 10 & & NS & $\overline{\mu g / L}$ \\
\hline 1,1,1-Trichloroethane & WA & & U & 5 & & 200 & $\mu g / L$ \\
\hline 1,1,2,2-Tetrachloroethane & WA & & $\mathbf{U}$ & 5 & & NS & $\mu \mathrm{g} / \mathrm{L}$ \\
\hline 1,1,2-Trichloroethane & WA & & $\mathbf{U}$ & 5 & & 5 & $\mu g / \mathrm{L}$ \\
\hline 1,1-Dichloroethane & WA & & $\mathbf{U}$ & 5 & & NS & $\mu g / L$ \\
\hline 1,1-Dichloroethylene & WA & & $\mathbf{U}$ & 5 & & 7 & $\mu \mathrm{g} / \mathrm{L}$ \\
\hline 1,2,3-Trichloropropane & WA & & $\mathbf{U}$ & 10 & & NS & $\mu g / \hbar$ \\
\hline 1,2-Dibromo-3-chloropropane & WA & & $\mathbf{U}$ & 20 & & 0.2 & $\mu g / L$ \\
\hline 1,2-Dibromoethane & WA & & $\mathbf{U}$ & 20 & & NS & $\mu g / L$ \\
\hline 1,2-Dichloroethane & WA & & $\mathbf{U}$ & 5 & & 5 & $\mu g / L$ \\
\hline 1,2-Dichloroethylene (total) & WA & & $\mathbf{U}$ & 5 & & 100 & $\mu g / L$ \\
\hline 1,2-Dichloropropane & WA & & $\mathbf{U}$ & 5 & & 5 & $\mu g / L^{-}$ \\
\hline 2-Hexanone & WA & & $\mathbf{U}$ & 10 & & NS & $\mu g / L$ \\
\hline 4-Methyl-2-pentanone & WA & & $\mathbf{U}$ & 10 & & NS & $\mu g / L$ \\
\hline Acetone & WA & & $\mathbf{U}$ & 10 & & NS & $\mu g / \mathrm{L}$ \\
\hline Acetonitrile & WA & & $\mathbf{U}$ & 20 & & NS & $\mu \mathrm{g} / \mathrm{L}$ \\
\hline Acidity & WA & & & 34 & & NS & $\mathrm{mg} / \mathrm{L}$ \\
\hline Acrolein & WA & & $\mathbf{U}$ & 10 & & NS & $\mu \mathrm{g} / \mathrm{L}$ \\
\hline Acrylonitrile & WA & & U & 10 & & NS & $\mu \mathrm{g} / \mathrm{L}$ \\
\hline Alkalinity & WA & & & 0.5 & & NS & $\mathrm{mg} / \mathrm{L}$ \\
\hline Allyl chloride & WA & & $\mathbf{U}$ & 100 & & NS & $\mu g / \mathrm{L}$ \\
\hline Aluminum & WA & & & 269 & & NS & $\mu g / L$ \\
\hline Antimony & WA & & $\mathbf{U}$ & 60 & & 6 & $\mu g / L$ \\
\hline Arsenic & WA & & $\mathbf{U}$ & 10 & & 50 & $\mu g \Omega$ \\
\hline Barium & WA & & U & 200 & & 2000 & $\mu g / L$ \\
\hline Benzene & WA & & $\mathrm{U}$ & 5 & & 5 & $\mu g / \mathrm{L}$ \\
\hline Beryllium & WA & & U & 5 & & 4 & $\mu \mathrm{g} / \mathrm{L}$ \\
\hline Bromodichloromethane & WA & & U & 5 & & NS & $\mu g / L$ \\
\hline Bromoform & WA & & $\mathrm{U}$ & 5 & & NS & $\mu g / 2$ \\
\hline Cadmium & WA & & $\mathrm{U}$ & 5 & & 5 & $\mu \mathrm{g} / \mathrm{L}$ \\
\hline Calcium & WA & & $\mathbf{U}$ & 5000 & & NS & $\mu g / L$ \\
\hline Carbon disulfide & WA & & U & 5 & & NS & $\mu g / L$ \\
\hline Carbon tetrachloride & WA & & $\mathbf{U}$ & 5 & & 5 & $\mu \mathrm{g} / \mathrm{L}$ \\
\hline Cesium-137 & TM & & U & 5.7 & & 200 & $\mathrm{pCi} / \mathrm{L}$ \\
\hline Chloride & WA & & & 3.47 & & NS & $\mathrm{mg} / \mathrm{L}$ \\
\hline Chlorobenzene & WA & & $\mathrm{U}$ & 5 & & 100 & $\mu g / L$ \\
\hline Chloroethane & WA & & U & 10 & & NS & $\mu g / L$ \\
\hline Chloroform & WA & & $\mathrm{U}$ & 5 & & 100 & $\mu \mathrm{g} / \mathrm{L}$ \\
\hline Chloroprene & WA & & $\mathrm{U}$ & 20 & & NS & $\mu \mathrm{g} / \mathrm{L}$ \\
\hline Chromium & WA & & U & 10 & & 100 & $\mu \mathrm{g} / \mathrm{L}$ \\
\hline cis-1,3-Dichloropropene & WA & & $\mathrm{U}$ & 5 & & NS & $\mu \mathrm{g} / \mathrm{L}$ \\
\hline Cobalt & WA & & U & 50 & & NS & $\mu g / L$ \\
\hline Copper & WA & & U & 25 & & 1000 & $\mu g / L$ \\
\hline Cyanide & WA & & $\mathrm{U}$ & 10 & & 200 & $\mu g / L$ \\
\hline Dibromochloromethane & WA & & U & 5 & & NS & $\mu \mathrm{g} / \mathrm{L}$ \\
\hline Dichlorodifluoromethane & WA & & U & 10 & & NS & $\mu g / L$ \\
\hline Ethylbenzene & WA & & U & 5 & & 700 & $\mu g / L$ \\
\hline Fluoride & WA & & $\mathbf{U}$ & 0.1 & & 4 & mg \\
\hline
\end{tabular}


UTR122 Laboratory Analyses (cont'd)

\begin{tabular}{|c|c|c|c|c|c|c|c|c|}
\hline Analyte & $\mathrm{Lab}$ & Rep & Avg & Qualifier & Result & Acc & Stand & Units \\
\hline Gross alpha & TM & & & & 1.8 & 1.1 & 15 & $\mathrm{pCi} / \mathrm{L}$ \\
\hline Iron & WA & & & & 1160 & & 300 & $\mu g / L$ \\
\hline Isobutyl alcohol & WA & & & $\mathbf{U}$ & 20 & & NS & $\mu g / L$ \\
\hline Lead & WA & & & $\mathbf{U}$ & 3 & & 50 & $\mu g / L$ \\
\hline Magnesium & WA & & & $\mathbf{U}$ & 5000 & & NS & $\mu g / L$ \\
\hline Manganese & WA & & & & 138 & . & 50 & $\mu g / L$ \\
\hline Mercury & WA & & & $\mathbf{U}$ & 0.2 & & 2 & $\mu g / L$ \\
\hline Methacrylonitrile & WA & & & $\mathbf{U}$ & 20 & & NS & $\mu g / L$ \\
\hline Methyl bromide & WA & & & $\mathbf{U}$ & 10 & & NS & $\mu g / L$ \\
\hline Methyl chloride & WA & & & $\mathbf{U}$ & 10 & & NS & $\mu g / L$ \\
\hline Methyl ethyl ketone & WA & & & $\mathbf{U}$ & 10 & & NS & $\mu g / L$ \\
\hline Methyl iodide & WA & & & $\mathbf{U}$ & 10 & & NS & $\mu g / L$ \\
\hline Methylene bromide & WA & & & $\mathbf{U}$ & 10 & & NS & $\mu \mathrm{g} / \mathrm{L}$ \\
\hline Methylene chloride & WA & & & $\mathbf{U}$ & 5 & & 5 & $\mu g / L$ \\
\hline Nickel & WA & & & $\mathbf{U}$ & 40 & & 100 & $\mu g / L$ \\
\hline Nitrate as nitrogen & WA & & & & 0.325 & & 10 & $\mathrm{mg} / \mathrm{L}$ \\
\hline Nonvolatile beta & TM & & & & 9.1 & 2.3 & 50 & $\mathrm{pCi} / \mathrm{L}$ \\
\hline Potassium & WA & & & $\mathbf{U}$ & 5000 & & NS & $\mu g / L$ \\
\hline Propionitrile & WA & & & $\mathbf{U}$ & 50 & & NS & $\mu g / L$ \\
\hline Selenium & WA & & & $\mathbf{U}$ & 5 & & 50 & $\mu g / L$ \\
\hline Silver & WA & & & $\mathbf{U}$ & 10 & & 50 & $\mu g / L$ \\
\hline Sodium & WA & & & $\mathbf{U}$ & 5000 & & NS & $\mu g / L$ \\
\hline Styrene & WA & & & $\mathbf{U}$ & 5 & & 100 & $\mu g / L$ \\
\hline Sulfate & WA & & & & 4.37 & & 400 & $\mathrm{mg} / \mathrm{L}$ \\
\hline Sulfide & WA & & & $\mathbf{U}$ & 0.1 & & NS & $\mathrm{mg} / \mathrm{L}$ \\
\hline Sulfide & WA & & NA & $\mathbf{U}$ & 0.1 & & NS & $\mathrm{mg} / \mathrm{L}$ \\
\hline Tetrachloroethylene & WA & & & $\mathbf{U}$ & 5 & & 5 & $\mu g / L$ \\
\hline Thallium & WA & & & $\mathbf{U}$ & 10 & & 2 & $\mu \mathrm{g} / \mathrm{L}$ \\
\hline Toluene & WA & & & $\mathbf{U}$ & 5 & & 1000 & $\mu \mathrm{g} / \mathrm{L}$ \\
\hline Total hardness & WA & & & & 8 & & NS & $\mathrm{mg} / \mathrm{L}$ \\
\hline Total organic carbon & WA & & & & 4.46 & & NS & $\mathrm{mg} / \mathrm{L}$ \\
\hline Total suspended solids & WA & & & & 19 & & NS & $\mathrm{mg} / \mathrm{L}$ \\
\hline trans-1,3-Dichloropropene & WA & & & $\mathbf{U}$ & 5 & & NS & $\mu \mathrm{g} / \mathrm{L}$ \\
\hline trans-1,4-Dichloro-2-butene & WA & & & $\mathbf{U}$ & 100 & & NS & $\mu \mathrm{g} / \mathrm{L}$ \\
\hline Trichloroethylene & WA & & & $\mathbf{U}$ & 5 & & 5 & $\mu g / L$ \\
\hline Trichlorofluoromethane & WA & & & $\mathbf{U}$ & 5 & & NS & $\mu g / L$ \\
\hline Tritium & TM & & & & 12 & 4.4 & 20 & $\mathrm{pCi} / \mathrm{ml}$ \\
\hline Turbidity & WA & & $\mathbf{A}$ & & 14.7 & & NS & NTU \\
\hline Vanadium & WA & & & $\mathbf{U}$ & 50 & & NS & $\mu \mathrm{g} / \mathrm{L}$ \\
\hline Vinyl acetate & WA & & & $\mathbf{U}$ & 10 & & NS & $\mu \mathrm{g} / \mathrm{L}$ \\
\hline Vinyl chloride & WA & & & $\mathbf{U}$ & 10 & & 2 & $\mu g / L$ \\
\hline Xylenes (total) & WA & & & $\mathbf{U}$ & 5 & & 10000 & $\mu g / L$ \\
\hline Zinc & WA & & & $\mathbf{U}$ & 20 & & 5000 & $\mu \mathrm{g} / \mathrm{L}$ \\
\hline
\end{tabular}




\section{UTR123}

Field Measurements:

Sample Date: 4/20/93

pH: 5.76

Specific Conductance: $25 \mu \mathrm{S} / \mathrm{cm}$

Red/Oxid Potential: $121 \mathrm{mv}$

Dissolved Oxygen: $1.6 \mathrm{mg} / \mathrm{L}$

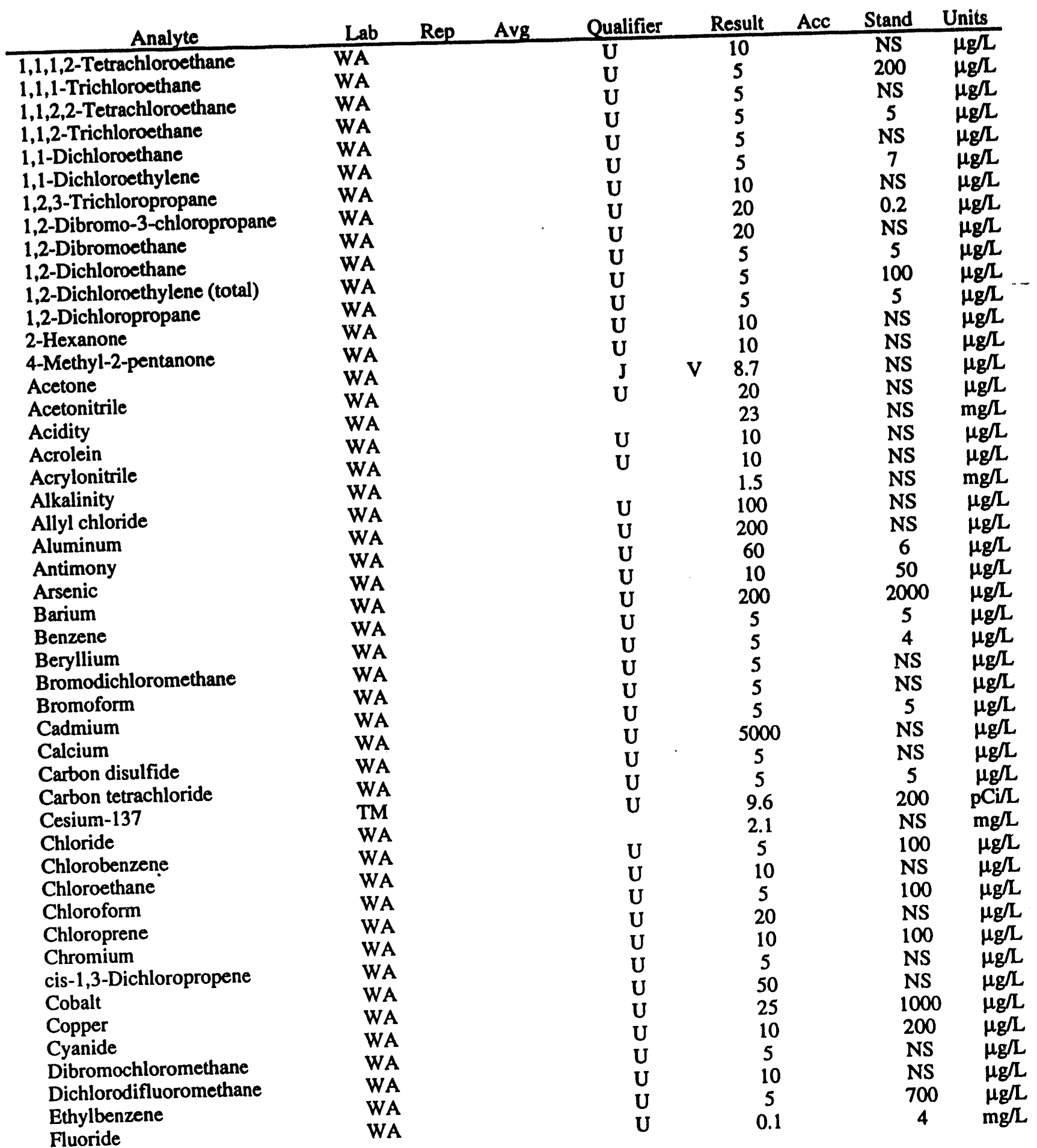


UTR123 Laboratory Analyses (cont'd)

\begin{tabular}{|c|c|c|c|c|c|c|c|}
\hline Analyte & Lab & Avg & Qualifier & Result & Acc & Stand & Units \\
\hline Gross alpha & TM & & $\mathrm{U}$ & 1 & & 15 & $\mathrm{pCi} / \mathrm{L}$ \\
\hline Iron & WA & & & 631 & & 300 & $\mu \mathrm{g} / \mathrm{L}$ \\
\hline Isobutyl alcohol & WA & & U & 20 & & NS & $\mu \mathrm{g} / \mathrm{L}$ \\
\hline Lead & WA & & $\mathbf{U}$ & 3 & & so. & $\mu g / L$ \\
\hline Magnesium & WA & & U & 5000 & & NS & $\mu g \Omega$ \\
\hline Manganese & WA & & & 247 & - & 50 & $\mu \mathrm{g} / \mathrm{L}$ \\
\hline Mercury & WA & & $\mathrm{U}$ & 0.2 & & 2 & $\mu \mathrm{g} / \mathrm{L}$ \\
\hline Methacrylonitrile & WA & & $\mathbf{U}$ & 20 & & NS & $\mu g / L$ \\
\hline Methyl bromide & WA & & $\mathbf{U}$ & 10 & & NS & $\mu g / L$ \\
\hline Methyl chloride & WA & & $\mathrm{U}$ & 10 & & NS & $\mu g / L$ \\
\hline Methyl ethyl ketone & WA & & $\mathbf{U}$ & 10 & & NS & $\mu \mathrm{g} / \mathrm{L}$ \\
\hline Methyl iodide & WA & & $\mathbf{U}$ & 10 & & NS & $\mu \mathrm{g} / \mathrm{L}$ \\
\hline Methylene bromide & WA & & U & 10 & & NS & $\mu g / L$ \\
\hline Methylene chloride & WA & & U & 5 & & 5 & $\mu g / L$ \\
\hline Nickel & WA & & $\mathrm{U}$ & 40 & & 100 & $\mu g / L$ \\
\hline Nitrate as nitrogen & WA & & & 0.212 & & 10 & $\mathrm{mg} / \mathrm{L}$ \\
\hline Nonvolatile beta & TM & & & 3.7 & 1.8 & 50 & $\mathrm{pCi} / \mathrm{L}$ \\
\hline Potassium & WA & & $\mathbf{U}$ & 5000 & & NS & $\mu g / L$ \\
\hline Propionitrile & WA & & $\bar{U}$ & 50 & & NS & $\mu \mathrm{g} / \mathrm{L}$ \\
\hline Selenium & WA & & $\mathbf{U}$ & 5 & & 50 & $\mu g / L$ \\
\hline Silver & WA & & $\mathbf{U}$ & 10 & & 50 & $\mu g / L$ \\
\hline Sodium & WA & & $\mathbf{U}$ & 5000 & & NS & $\mu g / L$ \\
\hline Styrene & WA & & $\mathrm{U}$ & 5 & & 100 & $\mu g /$ \\
\hline Sulfaie & WA & & & 2.9 & & 400 & $\mathrm{mg} / \mathrm{L}$ \\
\hline Sulfide & WA & & $\mathbf{U}$ & 0.1 & & NS & $\mathrm{mg} / \mathrm{L}$ \\
\hline Tetrachloroethylene & WA & & $\mathbf{U}$ & 5 & & 5 & $\mu \mathrm{g} / \mathrm{L}$ \\
\hline Thallium & WA & & U & 10 & & 2 & $\mu g / L$ \\
\hline Toluene & WA & & $\mathrm{U}$ & 5 & & 1000 & $\mu g / L$ \\
\hline Total hardness & WA & & & 6 & & NS & $\mathrm{mg} / \mathrm{L}$ \\
\hline Total organic carbon & WA & & & 4.15 & & NS & $\mathrm{mg} / \mathrm{h}$ \\
\hline Total suspended solids & WA & & & 13 & & NS & $\mathrm{mg} / \mathrm{h}$ \\
\hline trans-1,3-Dichloropropene & WA & & $\mathbf{U}$ & 5 & & NS & $\mu \mathrm{g} / \mathrm{h}$ \\
\hline trans-1,4-Dichloro-2-butene & WA & & $\mathbf{U}$ & 100 & & NS & $\mu \mathrm{g} / \mathrm{L}$ \\
\hline Trichloroethylene & WA & & $\mathbf{U}$ & 5 & & 5 & $\mu \mathrm{g} / \mathrm{L}$ \\
\hline Trichlorofluoromethane & WA & & $\mathbf{U}$ & 5 & & NS & $\mu \mathrm{g} / \mathrm{L}$ \\
\hline Tritium & TM & & & 5.5 & 2 & 20 & $\mathrm{pCi} / \mathrm{m}$ \\
\hline Turbidity & WA & & & 3.26 & & NS & NTU \\
\hline Vanadium & WA & & U & 50 & & NS & $\mu \mathrm{g} / \mathrm{L}$ \\
\hline Vinyl acetate & WA & & $\mathrm{U}$ & 10 & & NS & $\mu \mathrm{g} / \mathrm{L}$ \\
\hline Vinyl chloride & WA & & $\mathbf{U}$ & 10 & & 2 & $\mu \mathrm{g} / \mathrm{h}$ \\
\hline Xylenes (total) & WA & & $\mathrm{U}$ & 5 & & 10000 & $\mu g / L$ \\
\hline Zinc & WA & & U & 20 & & 5000 & $\mu g / \mathbf{L}$ \\
\hline
\end{tabular}




\section{UTR127}

Field Measurements:

Sample Date: 4/20/93

pH: 5.59

Specific Conductance: $41 \mu \mathrm{S} / \mathrm{cm}$

Red/Oxid Potential: $143 \mathrm{mv}$

Dissolved Oxygen: $3.1 \mathrm{mg} / \mathrm{L}$

\begin{tabular}{|c|c|c|c|c|c|c|c|c|}
\hline Analyte & Lab & Rep & Avg & Qualifier & Result & Acc & Stand & Units \\
\hline 1,1,1,2-Tetrachloroethane & WA & & & U & 10 & & NS & $\mu g / L$ \\
\hline 1,1,1-Trichloroethane & WA & & & $\mathbf{U}$ & 5 & & 200 & $\mu g / L$ \\
\hline $1,1,2,2$-Tetrachloroethane & WA & & & $\mathbf{U}$ & 5 & & NS & $\mu g / L$ \\
\hline 1,1,2-Trichloroethane & WA & & & $\mathbf{U}$ & 5 & & 5 & $\mu g / L$ \\
\hline 1,1-Dichloroethane & WA & & & $\mathbf{U}$ & 5 & & NS & $\mu g / L$ \\
\hline 1,1-Dichloroethylene & WA & & & $\mathbf{U}$ & 5 & & 7 & $\mu g / L$ \\
\hline 1,2,3-Trichloropropane & WA & & & $\mathbf{U}$ & 10 & & NS & $\mu g / L$ \\
\hline 1,2-Dibromo-3-chloropropane & WA & & & $\mathbf{U}$ & 20 & & 0.2 & $\mu g / L$ \\
\hline 1,2-Dibromoethane & WA & & & U & 20 & & NS & $\mu g / L$ \\
\hline 1,2-Dichloroethane & WA & & & $\mathbf{U}$ & 5 & & 5 & $\mu g / L$ \\
\hline 1,2-Dichloroethylene (total) & WA & & & $\mathbf{U}$ & 5 & & 100 & $\mu g / L$ \\
\hline 1,2-Dichloropropane & WA & & & $\mathbf{U}$ & 5 & & 5 & $\mu g / L$ \\
\hline 2-Hexanone & WA & & & $\mathbf{U}$ & 10 & & NS & $\mu g / L$ \\
\hline 4-Methyl-2-pentanone & WA & & & $\mathbf{U}$ & 10 & & NS & $\mu g / L$ \\
\hline Acetone & WA & & & $\mathbf{U}$ & 10 & & NS & $\mu g / L$ \\
\hline Acetonitrile & WA & & & $\mathbf{U}$ & 20 & & NS & $\mu g / L$ \\
\hline Acidity & WA & & & & 12 & & NS & mg/L \\
\hline Acrolein & WA & & & $\mathbf{U}$ & 10 & & NS & $\mu g /$ \\
\hline Acrylonitrile & WA & & & $\mathbf{U}$ & 10 & & NS & $\mu g / L$ \\
\hline Alkalinity & WA & & & $\mathbf{U}$ & 0.5 & & NS & $\mathrm{mg} / \mathrm{L}$ \\
\hline Allyl chloride & WA & & & $\mathbf{U}$ & 100 & & NS & $\mu g / L$ \\
\hline Aluminum & WA & & & $\mathbf{U}$ & 200 & & NS & \\
\hline $\begin{array}{l}\text { Alumimum } \\
\text { Antimony }\end{array}$ & WA & & & $\mathbf{U}$ & 60 & & 6 & $\begin{array}{l}\mu g / L \\
\mu g / L\end{array}$ \\
\hline $\begin{array}{l}\text { Anumony } \\
\text { Arsenic }\end{array}$ & WA & & & $\mathbf{U}$ & 10 & & 50 & \\
\hline $\begin{array}{l}\text { Arsenic } \\
\text { Barium }\end{array}$ & WA & & & $\mathbf{U}$ & 200 & & 2000 & $\begin{array}{l}\mu g / L \\
\mu g / L\end{array}$ \\
\hline $\begin{array}{l}\text { Barium } \\
\text { Benzene }\end{array}$ & WA & & & $\mathbf{U}$ & 5 & & 5 & $\begin{array}{l}\mu g / L \\
\mu g / L\end{array}$ \\
\hline $\begin{array}{l}\text { Benzene } \\
\text { Beryllium }\end{array}$ & WA & & & $\mathbf{U}$ & 5 & & 4 & $\begin{array}{l}\mu g / L \\
\mu g / L\end{array}$ \\
\hline $\begin{array}{l}\text { Beryllium } \\
\text { Bromodichloromethane }\end{array}$ & WA & & & $\mathbf{U}$ & $\begin{array}{l}3 \\
5\end{array}$ & & NS & $\begin{array}{l}\mu g / L \\
\mu g / L\end{array}$ \\
\hline $\begin{array}{l}\text { Bromodichloromethane } \\
\text { Bromoform }\end{array}$ & WA & & & $\mathbf{U}$ & 5 & & NS & $\mu g / L$ \\
\hline $\begin{array}{l}\text { Bromoform } \\
\text { Cadmium }\end{array}$ & WA & & & $\mathbf{U}$ & 5 & & 5 & $\mu g / L$ \\
\hline $\begin{array}{l}\text { Cadmium } \\
\text { Calcium }\end{array}$ & WA & & & $\mathbf{U}$ & 5000 & & $\begin{array}{l}5 \\
\text { NS }\end{array}$ & $\mu g / L$ \\
\hline $\begin{array}{l}\text { Calcium } \\
\text { Carbon disulfide }\end{array}$ & WA & & & $\mathbf{U}$ & 5 & & $\begin{array}{l}\text { NS } \\
\text { NS }\end{array}$ & $\mu g / L$ \\
\hline $\begin{array}{l}\text { Carbon disulfide } \\
\text { Carbon tetrachloride }\end{array}$ & WA & & & $\mathbf{U}$ & 5 & & & $\mu \mathrm{g} / \mathrm{L}$ \\
\hline $\begin{array}{l}\text { Carbon tetrachloride } \\
\text { Cesium-137 }\end{array}$ & TM & & & $\mathbf{U}$ & 6.4 & & $\begin{array}{c}5 \\
200\end{array}$ & $\mu g / L$ \\
\hline $\begin{array}{l}\text { Cesium-137 } \\
\text { Chloride }\end{array}$ & WA & & & & 3.34 & & $\begin{array}{l}200 \\
\text { NS }\end{array}$ & $\mathrm{pCi} / \mathrm{L}$ \\
\hline $\begin{array}{l}\text { Chloride } \\
\text { Chlorobenzene }\end{array}$ & WA & & & $\mathbf{U}$ & 5 & & $\begin{array}{l}\text { NS } \\
100\end{array}$ & $\mathrm{mg} / \mathrm{L}$ \\
\hline $\begin{array}{l}\text { Chlorobenzene } \\
\text { Chloroethane }\end{array}$ & WA & & & $\mathbf{U}$ & 10 & & & $\mu \mathrm{g} / \mathrm{L}$ \\
\hline & WA & & & $\mathbf{U}$ & 5 & & $\begin{array}{l}\text { NS } \\
100\end{array}$ & $\mu \mathrm{g} / \mathrm{I}$ \\
\hline $\begin{array}{l}\text { Chloroform } \\
\text { Chloroprene }\end{array}$ & WA & & & $\mathbf{U}$ & 20 & & $\begin{array}{l}100 \\
\text { NS }\end{array}$ & $\mu g / \Lambda$ \\
\hline & WA & & & $\mathbf{U}$ & & & $\begin{array}{l}\text { NS } \\
100\end{array}$ & $\mu g / L$ \\
\hline & WA & & & $\mathbf{U}$ & 5 & & 100 & $\mu g / L$ \\
\hline $\begin{array}{l}\text { cis-1,3-Dichloropropene } \\
\text { Cobalt }\end{array}$ & WA & & & $\mathbf{U}$ & 50 & & $\begin{array}{l}\text { NS } \\
\text { NS }\end{array}$ & $\mu \mathrm{g} / \mathrm{L}$ \\
\hline & WA & & & $\mathbf{U}$ & 25 & & NS & $\mu g / L$ \\
\hline $\begin{array}{l}\text { Copper } \\
\text { Cyanide }\end{array}$ & WA & & & $\mathbf{U}$ & $\begin{array}{l}25 \\
10\end{array}$ & & 1000 & $\mu g / L$ \\
\hline $\begin{array}{l}\text { Cyanide } \\
\text { Dibromochlorometh }\end{array}$ & WA & & & $\mathbf{U}$ & $\begin{array}{c}10 \\
5\end{array}$ & & 200 & $\mu g / L$ \\
\hline $\begin{array}{l}\text { Dibromochloromethane } \\
\text { Dichlorodifluoromethane }\end{array}$ & WA & & & $\mathbf{U}$ & $\begin{array}{c}5 \\
10\end{array}$ & & NS & $\mu g / L$ \\
\hline $\begin{array}{l}\text { Dichlorodifluoromethane } \\
\text { Ethylbenzene }\end{array}$ & WA & & & $\mathbf{U}$ & $\begin{array}{c}10 \\
5\end{array}$ & & NS & $\mu \mathrm{g} / \mathrm{L}$ \\
\hline Ethylbenzene & WA & & & $\mathrm{U}$ & $\begin{array}{c}5 \\
0.1\end{array}$ & & $\begin{array}{c}700 \\
4\end{array}$ & $\mu g / L$ \\
\hline & & & & & & & 4 & $\mathrm{mg} / \mathrm{L}$ \\
\hline
\end{tabular}


UTR127 Laboratory Analyses (cont'd)

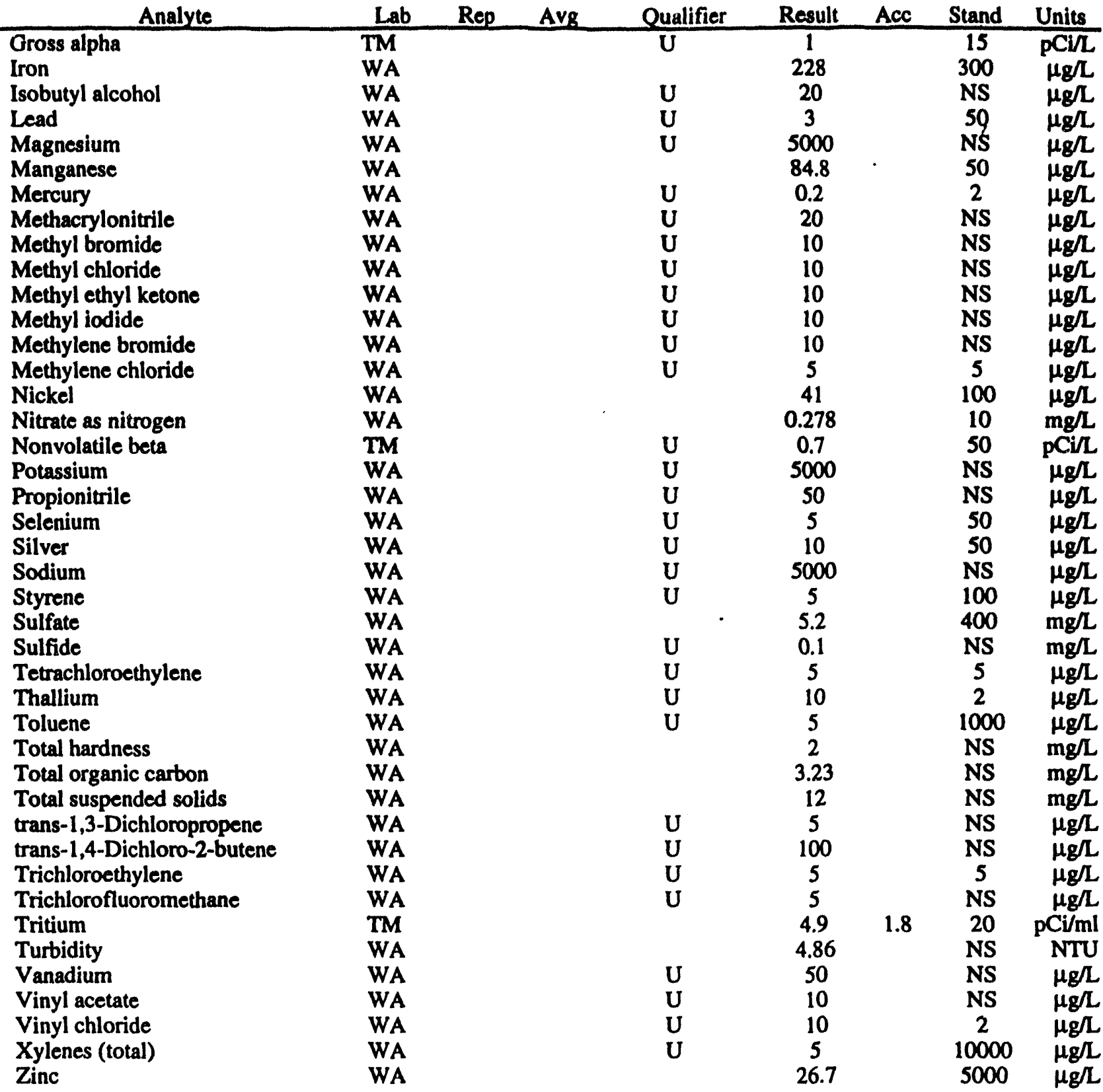




\section{UTR133}

Field Measurements:

Sample Date: 4/22/93

$\mathrm{pH}: 5.95$

Specific Conductance: $43 \mu \mathrm{S} / \mathrm{cm}$

Red/Oxid Potential: $63 \mathrm{mv}$

Dissolved Oxygen: $2.9 \mathrm{mg} / \mathrm{L}$

\begin{tabular}{|c|c|c|c|c|c|c|c|c|}
\hline Analyte & Lab & Rep & Avg & Qualifier & Result & Acc & Stand & Units \\
\hline 1,1,1,2-Tetrachloroethane & WA & & & $\mathbf{U}$ & 10 & & NS & $\mu g / L$ \\
\hline 1,1,1-Trichloroethane & WA & & & $\mathbf{U}$ & 5 & & 200 & $\mu \boldsymbol{g} /$ \\
\hline 1,1,2,2-Tetrachloroethane & WA & & & $\mathbf{U}$ & 5 & & NS & $\mu g / L$ \\
\hline 1,1,2-Trichloroethane & WA & & & $\mathbf{U}$ & 5 & & 5 & $\mu g /$ \\
\hline 1,1-Dichloroethane & WA & & & $\mathbf{U}$ & 5 & & NS & $\mu g / L$ \\
\hline 1,1-Dichloroethylene & WA & & & $\mathbf{U}$ & 5 & & 7 & $\mu g / L$ \\
\hline 1,2,3-Trichloropropane & WA & & & $\mathbf{U}$ & 10 & & NS & $\mu g / L$ \\
\hline 1,2-Dibromo-3-chloropropane & WA & & & $\mathbf{U}$ & 20 & & 0.2 & $\mu g /$ \\
\hline 1,2-Dibromoethane & WA & & & $\mathbf{U}$ & 20 & & NS & $\mu g / L$ \\
\hline 1,2-Dichloroethane & WA & & & $\mathbf{U}$ & 5 & & 5 & $\mu g / L$ \\
\hline 1,2-Dichloroethylene (total) & WA & & & $\mathbf{U}$ & 5 & & 100 & $\mu g / L$ \\
\hline 1,2-Dichloropropane & WA & & & $\mathbf{U}$ & 5 & & 5 & $\mu g / L$ \\
\hline 2-Hexanone & WA & & & $\mathbf{U}$ & 10 & & NS & $\mu g / L$ \\
\hline 4-Methyl-2-pentanone & WA & & & $\mathbf{U}$ & 10 & & NS & $\mu g / L$ \\
\hline Acetone & WA & & & & 12.1 & & NS & $\mu g / L$ \\
\hline Acetonitrile & WA & & & $\mathbf{U}$ & 20 & & NS & $\mu g / L$ \\
\hline Acidity & WA & & & & 30 & & NS & mg/L \\
\hline Acrolein & WA & & & $\mathbf{U}$ & 10 & & NS & $\mu g / L$ \\
\hline Acrylonitrile & WA & & & $\mathbf{U}$ & 10 & & NS & $\mu g / L$ \\
\hline Alkalinity & WA & & $\mathbf{A}$ & & 9 & & NS & mg/L \\
\hline Allyl chloride & WA & & & $\mathbf{U}$ & 100 & & NS & $\mu g / L$ \\
\hline Aluminum & WA & & & & 3900 & & NS & $\mu g / L$ \\
\hline Antimony & WA & & & $\mathbf{U}$ & 60 & & 6 & $\mu g / L$ \\
\hline Arsenic & WA & & & $\mathbf{U}$ & 10 & & 50 & $\mu g / L$ \\
\hline Barium & WA & & & $\mathbf{U}$ & 200 & & 2000 & $\mu g / L$ \\
\hline Benzene & WA & & & $\mathbf{U}$ & 5 & & 5 & $\mu g / L$ \\
\hline Beryllium & WA & & & $\mathbf{U}$ & 5 & & 4 & $\mu g / L$ \\
\hline Bromodichloromethane & WA & & & $\mathbf{U}$ & 5 & & NS & $\mu g / L$ \\
\hline Bromoform & WA & & & $\mathbf{U}$ & 5 & & NS & $\mu g / L$ \\
\hline Cadmium & WA & & & $\mathbf{U}$ & 5 & & 5 & $\mu g / L$ \\
\hline Calcium & WA & & & $\mathbf{U}$ & 5000 & & NS & $\mu g / L$ \\
\hline Carbon disulfide & WA & & & $\mathbf{U}$ & 5 & & NS & $\mu g / L$ \\
\hline Carbon tetrachloride & WA & & & $\mathbf{U}$ & 5 & & 5 & $\mu g / L$ \\
\hline Cesium-137 & TM & & NA & $\mathbf{U}$ & 6 & & 200 & pCi/L \\
\hline Cesium-137 & TM & & & $\mathbf{U}$ & 6 & & 200 & $\mathrm{pCi} / \mathrm{L}$ \\
\hline Chloride . & WA & & & & 7.16 & & NS & $\mathrm{mg} / \mathrm{L}$ \\
\hline Chlorobenzene & WA & & & $\mathbf{U}$ & 5 & & 100 & $\mu g / L$ \\
\hline Chloroethane & WA & & & $\mathbf{U}$ & 10 & & NS & $\mu g / L$ \\
\hline Chloroform & WA & & & $\mathbf{U}$ & 5 & & 100 & $\mu g / L$ \\
\hline Chloroprene & WA & & & $\mathbf{U}$ & 20 & & NS & $\mu g / L$ \\
\hline Chromium & WA & & & $\mathbf{U}$ & 10 & & 100 & $\mu g / L$ \\
\hline cis-1,3-Dichloropropene & WA & & & $\mathbf{U}$ & 5 & & NS & $\mu g /$ \\
\hline Cobalt & WA & & & $\mathbf{U}$ & 50 & & NS & $\mu g / L$ \\
\hline Copper & WA & & & $\mathbf{U}$ & 25 & & 1000 & $\mu \mathrm{g} / \mathrm{L}$ \\
\hline Cyanide & WA & & & $\mathbf{U}$ & 10 & & 200 & $\mu g / L$ \\
\hline Dibromochloromethane & WA & & & $\mathbf{U}$ & 5 & & NS & $\mu g / L$ \\
\hline Dichlorodifluoromethane & WA & & & $\mathbf{U}$ & 10 & & NS & $\mu g / L$ \\
\hline Ethylbenzene & WA & & & $\mathbf{U}$ & 5 & & 700 & $\mu g / L$ \\
\hline Fluoride & WA & & & $\mathbf{U}$ & 0.1 & & 4 & $\mathrm{mg} / \mathrm{L}$ \\
\hline
\end{tabular}


UTR133 Laboratory Analyses (cont'd)

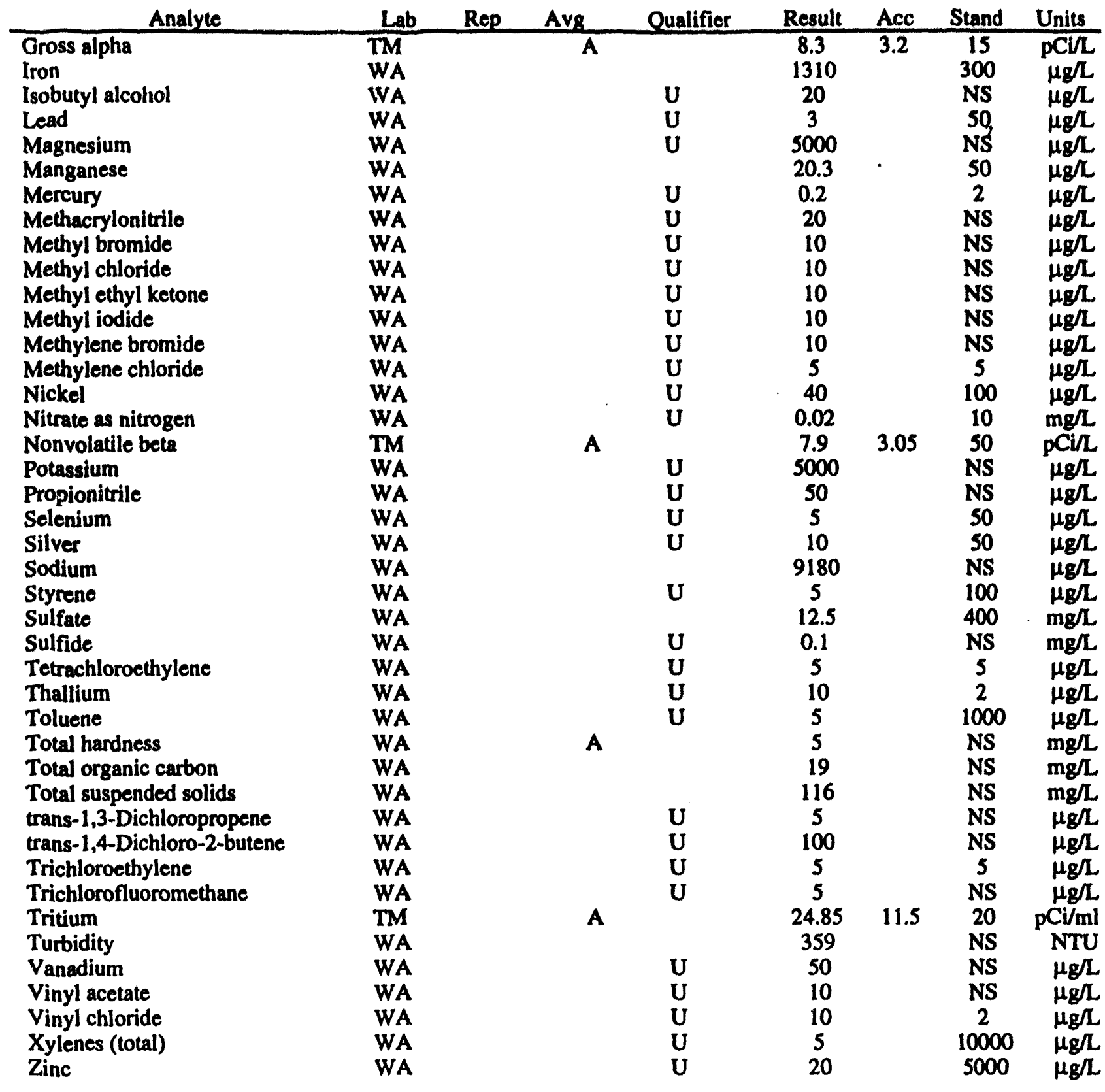




\section{UTR142}

Field Measurements:

Sample Date: 4/22/93

pH: 5.67

Specific Conductance: $86 \mu \mathrm{S} / \mathrm{cm}$

Red/Oxid Potential: $100 \mathrm{mv}$

Dissolved Oxygen: $1.2 \mathrm{mg} / \mathrm{L}$

\begin{tabular}{|c|c|c|c|c|c|c|c|c|}
\hline Analyte & Lab & Rep & Avg & Qualifier & Result & Acc & Stand & Units \\
\hline 1,1,1,2-Tetrachloroethane & WA & & & $\mathbf{U}$ & 10 & & NS & $\mu g / L$ \\
\hline 1,1,1-Trichloroethane & WA & & & $\mathbf{U}$ & 5 & & 200 & $\mu g / L$ \\
\hline $1,1,2,2$-Tetrachloroethane & WA & & & $\mathbf{U}$ & 5 & & NS & $\mu g / L$ \\
\hline 1,1,2-Trichloroethane & WA & & & $\mathbf{U}$ & 5 & & 5 & $\mu g / L$ \\
\hline 1,1-Dichloroethane & WA & & & $\mathbf{U}$ & 5 & & NS & $\mu g / L$ \\
\hline 1,1-Dichloroethylene & WA & & & $\mathbf{U}$ & 5 & & 7 & $\mu g / L$ \\
\hline 1,2,3-Trichloropropane & WA & & & $\mathbf{U}$ & 10 & & NS & $\mu g / L$ \\
\hline 1,2-Dibromo-3-chloropropane & WA & & & $\mathbf{U}$ & 20 & & 0.2 & $\mu g / L$ \\
\hline 1,2-Dibromoethane & WA & & & $\mathbf{U}$ & 20 & & NS & $\mu g / L$ \\
\hline 1,2-Dichloroethane & WA & & & $\mathbf{U}$ & 5 & & 5 & $\mu g / L$ \\
\hline 1,2-Dichloroethylene (total) & WA & & & $\mathbf{U}$ & 5 & & 100 & $\mu g / L$ \\
\hline 1,2-Dichloropropane & WA & & & $\mathbf{U}$ & 5 & & 5 & $\mu g / L$ \\
\hline 2-Hexanone & WA & & & $\mathbf{U}$ & 10 & & NS & $\mu g / L$ \\
\hline 4-Methyl-2-pentanone & WA & & & $\mathbf{U}$ & 10 & & NS & $\mu g / L$ \\
\hline Acetone & WA & & & $\mathbf{U}$ & 10 & & NS & $\mu g / L$ \\
\hline Acetonitrile & WA & & & $\mathbf{U}$ & 20 & & NS & $\mu g / L$ \\
\hline Acidity & WA & & & & 94 & & NS & $\mathrm{mg} / \mathrm{L}$ \\
\hline Acrolein & WA & & & $\mathbf{U}$ & 10 & & NS & $\mu g / L$ \\
\hline Acrylonitrile & WA & & & $\mathbf{U}$ & 10 & & NS & $\mu g / L$ \\
\hline Alkalinity & WA & & & & 11 & & NS & $\mathrm{mg} / \mathrm{L}$ \\
\hline Allyl chloride & WA & & & $\mathbf{U}$ & 100 & & NS & $\begin{array}{l}\mathrm{mg} / \mathrm{L} \\
\mu \mathrm{g} / \mathrm{L}\end{array}$ \\
\hline Aluminum & WA & & & & 34800 & & NS & $\mu g / L$ \\
\hline Antimony & WA & & & $\mathbf{U}$ & 60 & & 6 & $\mu g / L$ \\
\hline Arsenic & WA & & & $\mathbf{U}$ & 10 & & 50 & $\mu g / L$ \\
\hline Barium & WA & & & & 212 & & 2000 & $\mu g / L$ \\
\hline Benzene & WA & & & $\mathbf{U}$ & 5 & & 5 & $\mu g / L$ \\
\hline Beryllium & WA & & & $\mathbf{U}$ & 5 & & 4 & $\mu g / L$ \\
\hline Bromodichloromethane & WA & & & $\mathbf{U}$ & 5 & & NS & $\mu g / L$ \\
\hline Bromoform & WA & & & $\mathbf{U}$ & 5 & & NS & $\mu g / L$ \\
\hline Cadmium & WA & & & $\mathbf{U}$ & 5 & & 5 & $\mu g / L$ \\
\hline Calcium & WA & & & & 9940 & & NS & $\mu g / L$ \\
\hline Carbon disulfide & WA & & & $\mathbf{U}$ & 5 & & NS & $\mu g / L$ \\
\hline Carbon tetrachloride & WA & & & $\mathbf{U}$ & 5 & & 5 & $\mu g / L$ \\
\hline Cesium-137 & TM & & & & 13.8 & 10.9 & 200 & $\mathrm{pCi} / \mathrm{L}$ \\
\hline Chloride & WA & & $\mathbf{A}$ & & 5.195 & & NS & $\mathrm{mg} / \mathrm{L}$ \\
\hline Chlorobenzene & WA & & & $\mathbf{U}$ & 5 & & 100 & $\mu \mathrm{g} / \mathrm{L}$ \\
\hline Chloroethane & WA & & & $\mathbf{U}$ & 10 & & NS & $\mu g / L$ \\
\hline Chloroform & WA & & & $\mathbf{U}$ & 5 & & 100 & $\begin{array}{l}\mu \mathrm{g} / \mathrm{L} \\
\mu \mathrm{g} / \mathrm{L}\end{array}$ \\
\hline Chloroprene & WA & & & $\mathbf{U}$ & 20 & & NS & $\begin{array}{l}\mu g / L \\
\mu g / L\end{array}$ \\
\hline Chromium & WA & & & & 47.5 & & 100 & $\begin{array}{l}\mu g / L \\
\mu g / L\end{array}$ \\
\hline cis-1,3-Dichloropropene & WA & & & $\mathbf{U}$ & 5 & & NS & $\begin{array}{l}\mu \mathrm{g} / \mathrm{L} \\
\mu \mathrm{g} / \mathrm{L}\end{array}$ \\
\hline Cobalt & WA & & & $\mathbf{U}$ & 50 & & NS & $\begin{array}{l}\mu g / L \\
\mu g / L\end{array}$ \\
\hline Copper & WA & & & $\mathbf{U}$ & 25 & & 1000 & $\begin{array}{l}\mu g / L \\
\mu g / L\end{array}$ \\
\hline Cyanide & WA & & & U & 10 & & 200 & $\begin{array}{l}\mu g / L \\
\mu g / L\end{array}$ \\
\hline Dibromochloromethane & WA & & & $\mathbf{U}$ & 5 & & NS & $\begin{array}{l}\mu g / L \\
\mu g / L\end{array}$ \\
\hline Dichlorodifluoromethane & WA & & & $\mathbf{U}$ & 10 & & NS & $\mu g /$ \\
\hline Ethylbenzene & WA & & & $\mathbf{U}$ & 5 & & 700 & $\mu g / L$ \\
\hline Fluoride & WA & & & $\mathbf{U}$ & 0.1 & & 4 & $\begin{array}{l}\mu g / L \\
m g / L\end{array}$ \\
\hline Fluoride & WA & & NA & $\mathbf{U}$ & 0.1 & & 4 & $\mathrm{mg} / \mathrm{h}$ \\
\hline & & & & & & & & $\mathrm{mg}$ \\
\hline
\end{tabular}


UTR142 Laboratory Analyses (cont'd)

\begin{tabular}{|c|c|c|c|c|c|c|c|c|}
\hline Analyte & Lab & Rep & Avg & Qualifier & Result & Acc & Stand & Units \\
\hline Gross alpha & TM & & & & 49.1 & 10.4 & 15 & $\mathrm{pCi} / \mathrm{L}$ \\
\hline Iron & WA & & & & 53100 & & 300 & $\mu \mathrm{g} / \mathrm{L}$ \\
\hline Isobutyl alcohol & WA & & & $\mathbf{U}$ & 20 & & NS & $\mu \mathrm{g} / \mathrm{L}$ \\
\hline Lead & WA & & & & 27 & & 50. & $\mu g / L$ \\
\hline Magnesium & WA & & & $\mathbf{U}$ & 5000 & & NS $^{7}$ & $\mu g / L$ \\
\hline Manganese & WA & & & & 1550 & - & 50 & $\mu g / L$ \\
\hline Mercury & WA & & & & 0.203 & & 2 & $\mu g / L$ \\
\hline Methacrylonitrile & WA & & & $\mathbf{U}$ & 20 & & NS & $\mu g / L$ \\
\hline Methyl bromide & WA & & & U & 10 & & NS & $\mu g / L$ \\
\hline Methyl chloride & WA & & & $\mathbf{U}$ & 10 & & NS & $\mu \mathrm{g} / \mathrm{L}$ \\
\hline Methyl ethyl ketone & WA & & & $\mathbf{U}$ & 10 & & NS & $\mu g / L$ \\
\hline Methyl iodide & WA & & & $\mathbf{U}$ & 10 & & NS & $\mu g / L$ \\
\hline Methylene bromide & WA & & & $\mathbf{U}$ & 10 & & NS & $\mu \mathrm{g} / \mathrm{L}$ \\
\hline Methylene chloride & WA & & & $\mathbf{U}$ & 5 & & 5 & $\mu \mathrm{g} / \mathrm{L}$ \\
\hline Nickel & WA & & & U & 40 & & 100 & $\mu \mathrm{g} / \mathrm{L}$ \\
\hline Nitrate as nitrogen & WA & & & & 0.043 & & 10 & $\mathrm{mg} / \mathrm{L}$ \\
\hline Nonvolatile beta & $\mathrm{TM}$ & & & & 79 & 12.4 & 50 & $\mathrm{pCi} / \bar{L}$ \\
\hline Potassium & WA & & & $\mathbf{U}$ & 5000 & & NS & $\mu g / L$ \\
\hline Propionitrile & WA & & & $\mathbf{U}$ & 50 & & NS & $\mu \mathrm{g} / \mathrm{L}$ \\
\hline Selenium & WA & & & $\mathbf{U}$ & 5 & & 50 & $\mu g / L$ \\
\hline Silver & WA & & & $\mathbf{U}$ & 10 & & 50 & $\mu g / L$ \\
\hline Sodium & WA & & & $\mathbf{U}$ & 5000 & & NS & $\mu g / L$ \\
\hline Styrene & WA & & & $\mathbf{U}$ & 5 & & 100 & $\mu g / L$ \\
\hline Sulfate & WA & & $\mathbf{A}$ & & 22.4 & & 400 & $\mathrm{mg} / \mathrm{L}$ \\
\hline Sulfide & WA & & & $\mathbf{U}$ & 0.1 & & NS & $\mathrm{mg} / \mathrm{L}$ \\
\hline Tetrachloroethylene & WA & & & $\mathbf{U}$ & 5 & & 5 & $\mu g / L$ \\
\hline Thallium & WA & & & $\mathbf{U}$ & 10 & & 2 & $\mu \mathrm{g} / \mathrm{L}$ \\
\hline Toluene & WA & & & $\mathbf{U}$ & 5 & & 1000 & $\mu \mathrm{g} / \mathrm{L}$ \\
\hline Total hardness & WA & & & & 50 & & NS & $\mathrm{mg} / \mathrm{L}$ \\
\hline Total organic carbon & WA & & & & 18 & & NS & $\mathrm{mg} / \mathrm{L}$ \\
\hline Total suspended solids & WA & & & & 1700 & & NS & $\mathrm{mg} / \mathrm{L}$ \\
\hline trans-1,3-Dichloropropene & WA & & & $\mathbf{U}$ & 5 & & NS & $\mu g / L$ \\
\hline trans-1,4-Dichloro-2-butene & WA & & & $\mathbf{U}$ & 100 & & NS & $\mu g / L$ \\
\hline Trichloroethylene & WA & & & $\mathrm{U}$ & 5 & & 5 & $\mu \mathrm{g} / \mathrm{L}$ \\
\hline Trichlorofluoromethane & WA & & & $\mathbf{U}$ & 5 & & NS & $\mu g / L$ \\
\hline Tritium & $\mathrm{TM}$ & & & & 10.8 & 5 & 20 & $\mathrm{pCi} / \mathrm{ml}$ \\
\hline Turbidity & WA & & & & 2090 & & NS & NTU \\
\hline Vanadium & WA & & & & 123 & & NS & $\mu g / L$ \\
\hline Vinyl acetate & WA & & & $\mathbf{U}$ & 10 & & NS & $\mu \mathrm{g} / \mathrm{L}$ \\
\hline Vinyl chloride & WA & & & $\mathbf{U}$ & 10 & & 2 & $\mu \mathrm{g} / \mathrm{L}$ \\
\hline Xylenes (total) & WA & & & $\mathrm{U}$ & 5 & & 10000 & $\mu \mathrm{g} / \mathrm{L}$ \\
\hline Zinc & WA & & & & 165 & & 5000 & $\mu g / L$ \\
\hline
\end{tabular}




\section{UTR147}

Field Measurements:

Sample Date: 4/22/93

pH: 5.64

Specific Conductance: $23 \mu \mathrm{S} / \mathrm{cm}$

Red/Oxid Potential: $134 \mathrm{mv}$

Dissolved Oxygen: $4.6 \mathrm{mg} / \mathrm{L}$

\begin{tabular}{|c|c|c|c|c|c|c|c|}
\hline Analyte & Lab & Rep & Avg & Qualifier & Result & Stand & Units \\
\hline 1,1,1,2-Tetrachloroethane & WA & & & $\bar{U}$ & 10 & NS & $\mu \mathrm{g} / \mathrm{L}$ \\
\hline 1,1,1,2-Tetrachloroethane & GE & & & $\mathbf{U}$ & 1 & NS & $\mu g / L$ \\
\hline 1,1,1-Trichloroethane & WA & & & $\mathbf{U}$ & 5 & 200 & $\mu \mathrm{g} / \mathrm{L}$ \\
\hline 1,1,1-Trichloroethane & GE & & & $\mathbf{U}$ & 1 & 200 & $\mu g / L$ \\
\hline 1,1,2,2-Tetrachloroethane & WA & & & $\mathbf{U}$ & 5 & NS & $\mu \mathrm{g} / \mathrm{L}$ \\
\hline 1,1,2,2-Tetrachloroethane & GE & & & $\mathbf{U}$ & 1 & NS & $\mu g / L$ \\
\hline 1,1,2-Trichloroethane & WA & & & $\mathbf{U}$ & 5 & 5 & $\mu \mathrm{g} / \mathrm{L}$ \\
\hline 1,1,2-Trichloroethane & GE & & & $\mathbf{U}$ & 1 & 5 & $\mu \mathrm{g} / \mathrm{L}$ \\
\hline 1,1-Dichloroethane & WA & & & $\mathbf{U}$ & 5 & NS & $\mu g / L$ \\
\hline 1,1-Dichloroethane & GE & & & $\mathbf{U}$ & 1 & NS & $\mu \mathrm{g} / \mathrm{L}$ \\
\hline 1,1-Dichloroethylene & WA & & & $\mathbf{U}$ & 5 & 7 & $\mu \mathrm{g} / \mathrm{L}$ \\
\hline 1,1-Dichloroethylene & GE & & & $\mathbf{U}$ & 1 & 7 & $\mu \mathrm{g} / \mathrm{L}$ \\
\hline 1,2,3-Trichloropropane & WA & & & $\mathbf{U}$ & 10 & NS & $\mu \mathrm{g} / \mathrm{L}$ \\
\hline 1,2,3-Trichloropropane & GE & & & U & 1 & NS & $\mu \mathrm{g} / \mathrm{L}$ \\
\hline 1,2-Dibromo-3-chloropropane & WA & & & $\mathbf{U}$ & 20 & 0.2 & $\mu \mathrm{g} / \mathrm{L}$ \\
\hline 1,2-Dibromo-3-chloropropane & GE & & & $\mathrm{U}$ & 1 & 0.2 & $\mu \mathrm{g} / \mathrm{L}$ \\
\hline 1,2-Dibromoethane & WA & & & $\mathrm{U}$ & 20 & NS & $\mu g / L$ \\
\hline 1,2-Dibromoethane & GE & & & U & 20 & NS & $\mu \mathrm{g} / \mathrm{L}$ \\
\hline 1,2-Dichloroethane & WA & & & $\mathrm{U}$ & 5 & 5 & $\mu \mathrm{g} / \mathrm{L}$ \\
\hline 1,2-Dichloroethane & GE & & & $\mathbf{U}$ & 1 & 5 & $\mu \mathrm{g} / \mathrm{L}$ \\
\hline 1,2-Dichloroethylene (total) & WA & & & $\mathbf{U}$ & 5 & 100 & $\mu \mathrm{g} / \mathrm{L}$ \\
\hline 1,2-Dichloroethylene (total) & GE & & & $\mathbf{U}$ & 1 & 100 & $\mu \mathrm{g} / \mathrm{L}$ \\
\hline 1,2-Dichloropropane & WA & & & $\mathbf{U}$ & 5 & 5 & $\mu \mathrm{g} / \mathrm{L}$ \\
\hline 1,2-Dichloropropane & GE & & & $\mathbf{U}$ & 1 & 5 & $\mu \mathrm{g} / \mathrm{L}$ \\
\hline 2-Hexanone & WA & & & U & 10 & NS & $\mu \mathrm{g} / \mathrm{L}$ \\
\hline 2-Hexanone & GE & & & $\mathbf{U}$ & 1 & NS & $\mu \mathrm{g} / \mathrm{L}$ \\
\hline 4-Methyl-2-pentanone & WA & & & $\mathbf{U}$ & 10 & NS & $\mu g / L$ \\
\hline Acetone & WA & & & & 14.2 & NS & $\mu \mathrm{g} / \mathrm{L}$ \\
\hline Acetone & GE & & & $\mathbf{U}$ & 100 & NS & $\mu \mathrm{g} / \mathrm{L}$ \\
\hline Acetonitrile & WA & & & $\mathbf{U}$ & 20 & NS & $\mu g / L$ \\
\hline Acetonitrile & GE & & & $\mathbf{U}$ & 1 & NS & $\mu g / L$ \\
\hline Acidity & WA & & & & 28 & NS & $\mathrm{mg} / \mathrm{L}$ \\
\hline Acrolein & WA & & & $\mathbf{U}$ & 10 & NS & $\mu \mathrm{g} / \mathrm{L}$ \\
\hline Acrolein & GE & & & $\mathbf{U}$ & 20 & NS & $\mu \mathrm{g} / \mathrm{L}$ \\
\hline Acrylonitrile & WA & & & $\mathrm{U}$ & 10 & NS & $\mu \mathrm{g} / \mathrm{L}$ \\
\hline Acrylonitrile & $\mathrm{GE}$ & & & $\mathrm{U}$ & 20 & NS & $\mu \mathrm{g} / \mathrm{L}$ \\
\hline Actinium-228 & EP & & & U & 30 & NS & $\mathrm{pCi} / \mathrm{L}$ \\
\hline Alkalinity & WA & & & & 2 & NS & $\mathrm{mg} / \mathrm{L}$ \\
\hline Alkalinity & GE & & NA & & 2.88 & NS & $\mathrm{mg} / \mathrm{L}$ \\
\hline Alkalinity & $\mathrm{GE}$ & & & & 3.36 & NS & $\mathrm{mg} / \mathrm{L}$ \\
\hline Allyl chloride & WA & & & $\mathbf{U}$ & 100 & NS & $\mu \mathrm{g} / \mathrm{L}$ \\
\hline Allyl chloride & $\mathrm{GE}$ & & & $\mathrm{U}$ & 50 & NS & $\mu \mathrm{g} / \mathrm{L}$ \\
\hline Aluminum & WA & & & $\mathbf{U}$ & 200 & NS & $\mu \mathrm{g} / \mathrm{L}$ \\
\hline Aluminum & $\mathrm{GE}$ & & & & 123 & NS & $\mu \mathrm{g} / \mathrm{L}$ \\
\hline Antimony & WA & & & $\mathbf{U}$ & 60 & 6 & $\mu g / L$ \\
\hline Antimony & GE & & & $\mathbf{U}$ & 2 & 6 & $\mu \mathrm{g} / \mathrm{L}$ \\
\hline Antimony-125 & $\mathrm{EP}$ & & & $\mathbf{U}$ & 20 & 300 & $\mathrm{pCi} / \mathrm{L}$ \\
\hline Arsenic & WA & & & $\mathbf{U}$ & 10 & 50 & $\mu \mathrm{g} / \mathrm{L}$ \\
\hline Arsenic & GE & & & $\mathbf{U}$ & 2 & 50 & $\mu \mathrm{g} / \mathrm{L}$ \\
\hline
\end{tabular}


Sampling and Analysis of Water From Upper Three Runs And Its Wetlands Near Tank 16 and the Mixed Waste Management Facility

UTR 147 Laboratory Analyses (cont'd)

\begin{tabular}{|c|c|c|c|c|c|c|c|c|}
\hline Analyte & Lab & Rep & Avg & Qualifier & Result & Acc & Stand & Units \\
\hline Barium & WA & & & $\mathbf{U}$ & 200 & & 2000 & $\mu \mathrm{g} / \mathrm{L}$ \\
\hline Barium & $\mathrm{GE}$ & & & & 11.3 & & 2000 & $\mu \mathrm{g} / \mathrm{L}$ \\
\hline Benzene & WA & & & $\mathbf{U}$ & 5 & & 5 & $\mu g / L$ \\
\hline Benzene & GE & & & $\mathbf{U}$ & 1 & & 5 & $\mu \mathrm{g} / \mathrm{L}$ \\
\hline Beryllium & WA & & & $\mathbf{U}$ & 5 & & $4^{7}$ & $\mu \mathrm{g} / \mathrm{L}$ \\
\hline Beryllium & GE & & & $\mathbf{U}$ & 3 & $\cdot$ & 4 & $\mu g / L$ \\
\hline Bis(2-chloro-1-methylethyl)ether & GE & & & $\mathbf{U}$ & 10 & & NS & $\mu g / L$ \\
\hline Bromodichloromethane & WA & & & $\mathbf{U}$ & 5 & & NS & $\mu \mathrm{g} / \mathrm{L}$ \\
\hline Bromodichloromethane & GE & & & $\mathbf{U}$ & 1 & & NS & $\mu \mathrm{g} / \mathrm{L}$ \\
\hline Bromoform & WA & & & $\mathbf{U}$ & 5 & & NS & $\mu g / L$ \\
\hline Bromoform & GE & & & $\mathbf{U}$ & 1 & & NS & $\mu g / L$ \\
\hline Cadmium & WA & & & $\mathbf{U}$ & 5 & & 5 & $\mu g / L$ \\
\hline Cadmium & GE & & & $\mathbf{U}$ & 2 & & 5 & $\mu g / L$ \\
\hline Calcium & WA & & & $\mathbf{U}$ & 5000 & & NS & $\mu \mathrm{g} / \mathrm{L}$ \\
\hline Calcium & GE & & & $\mathbf{J}$ & 523 & & NS & $\mu \mathrm{g} / \mathrm{L}$ \\
\hline Carbon disulfide & WA & & & $\mathbf{U}$ & 5 & & NS & $\mu g / L$ \\
\hline Carbon disulfide & GE & & & $\mathbf{U}$ & 1 & & NS & $\mu \mathrm{g} / \mathrm{L}$ \\
\hline Carbon tetrachloride & WA & & & $\mathbf{U}$ & 5 & & 5 & $\mu g / L$ \\
\hline Carbon tetrachloride & GE & & & $\mathbf{U}$ & 1 & & 5 & $\mu g / L$ \\
\hline Cerium-144 & EP & & & $\mathbf{U}$ & 60 & & 261 & $\mathrm{pCi} / \mathrm{L}$ \\
\hline Cesium-134 & EP & & & $\mathbf{U}$ & 10 & & 81.3 & $\mathrm{pCi} / \mathrm{L}$ \\
\hline Cesium-137 & TM & & & $\mathbf{U}$ & 9.6 & & 200 & $\mathrm{pCi} / \mathrm{L}$ \\
\hline Cesium-137 & EP & & & $\mathbf{U}$ & 10 & & 200 & $\mathrm{pCi} / \mathrm{L}$ \\
\hline Chloride & WA & & & & 2.79 & & NS & $\mathrm{mg} / \mathrm{L}$ \\
\hline Chloride & GE & & & & 2.32 & & NS & $\mathrm{mg} / \mathrm{L}$ \\
\hline Chlorobenzene & WA & & & $\mathbf{U}$ & 5 & & 100 & $\mu \mathrm{g} / \mathrm{L}$ \\
\hline Chlorobenzene & GE & & & $\mathbf{U}$ & 1 & & 100 & $\mu g / L$ \\
\hline Chloroethane & WA & & & $\mathbf{U}$ & 10 & & NS & $\mu \mathrm{g} / \mathrm{L}$ \\
\hline Chloroethane & $\mathrm{GE}$ & & & $\mathbf{U}$ & 1 & & NS & $\mu g / L$ \\
\hline Chloroform & WA & & & $\mathbf{U}$ & 5 & & 100 & $\mu \mathrm{g} / \mathrm{L}$ \\
\hline Chloroform & GE & & & $\mathbf{U}$ & 1 & & 100 & $\mu g / L$ \\
\hline Chloroprene & WA & & & $\mathbf{U}$ & 20 & & NS & $\mu g / L$ \\
\hline Chloroprene & GE & & & $\mathbf{U}$ & 200 & & NS & $\mu g / L$ \\
\hline Chromium & WA & & & $\mathbf{U}$ & 10 & & 100 & $\mu \mathrm{g} / \mathrm{L}$ \\
\hline Chromium & GE & & & $\mathbf{U}$ & 4 & & 100 & $\mu g / L$ \\
\hline cis-1,3-Dichloropropene & WA & & & $\mathbf{U}$ & 5 & & NS & $\mu \mathrm{g} / \mathrm{L}$ \\
\hline cis-1,3-Dichloropropene & GE & & & $\mathbf{U}$ & 1 & & NS & $\mu \mathrm{g} / \mathrm{L}$ \\
\hline Cobalt & WA & & & $\mathbf{U}$ & 50 & & NS & $\mu g / L$ \\
\hline Cobalt & GE & & & $\mathbf{U}$ & 4 & & NS & $\mu \mathrm{g} / \mathrm{L}$ \\
\hline Cobalt-57 & EP & & & $\mathbf{U}$ & 10 & & 1000 & $\mathrm{pCi} / \mathrm{L}$ \\
\hline Cobalt-60 & EP & & & $\mathbf{U}$ & 10 & & 100 & $\mathrm{pCi} / \mathrm{L}$ \\
\hline Copper & WA & & & $\mathbf{U}$ & 25 & & 1000 & $\mu \mathrm{g} / \mathrm{L}$ \\
\hline Copper & $\mathrm{GE}$ & & & $\mathbf{U}$ & 4 & & 1000 & $\mu g / L$ \\
\hline Cyanide & WA & & & $\mathbf{U}$ & 10 & & 200 & $\mu \mathrm{g} / \mathrm{L}$ \\
\hline Cyanide & GE & & & $\mathbf{U}$ & 5 & & 200 & $\mu \mathrm{g} / \mathrm{L}$ \\
\hline Dibromochloromethane & WA & & & $\mathbf{U}$ & 5 & & NS & $\mu g / L$ \\
\hline Dibromochloromethane & GE & & & $\mathbf{U}$ & 1 & & NS & $\mu g / L$ \\
\hline Dichlorodifluoromethane & WA & & & $\mathbf{U}$ & 10 & & NS & $\mu \mathrm{g} / \mathrm{L}$ \\
\hline Dichlorodifluoromethane & GE & & & $\mathbf{U}$ & 1 & & NS & $\mu g / L$ \\
\hline Ethylbenzene & WA & & & $\mathbf{U}$ & 5 & & 700 & $\mu g / L$ \\
\hline Ethylbenzene & GE & & & $\mathbf{U}$ & 1 & & 700 & $\mu \mathrm{g} / \mathrm{L}$ \\
\hline Europium-152 & EP & & & $\mathbf{U}$ & 40 & & NS & $\mathrm{pCi} / \mathrm{L}$ \\
\hline Europium-154 & EP & & & $\mathbf{U}$ & 20 & & 200 & $\mathrm{pCi} / \mathrm{L}$ \\
\hline Europium-155 & EP & & & $\mathbf{U}$ & 30 & & 600 & $\mathrm{pCi} / \mathrm{L}$ \\
\hline Fluoride & WA & & & $\mathbf{U}$ & 0.1 & & 4 & $\mathrm{mg} / \mathrm{L}$ \\
\hline Fluoride & GE & & & $\mathbf{U}$ & 0.1 & & 4 & $\mathrm{mg} / \mathrm{L}$ \\
\hline
\end{tabular}


UTR147 Laboratory Analyses (cont'd)

\begin{tabular}{|c|c|c|c|c|c|c|c|c|c|}
\hline Analyte & Lab & Rep & Avg & Qualifier & & Result & Acc & Stand & Units \\
\hline Gross alpha & TM & & & & & 1.2 & 1.1 & 15 & $\overline{\mathrm{pCi} / \mathrm{L}}$ \\
\hline Gross alpha & $\mathrm{GE}$ & & & $\mathbf{U}$ & & 2 & & 15 & $\mathrm{pCi} / \mathrm{L}$ \\
\hline Iron & WA & & & & & 142 & & 300 & $\mu g / L$ \\
\hline Iron & $\mathrm{GE}$ & & & & & 95.1 & & 300 & $\mu g / L$ \\
\hline Isobutyl alcohol & WA & & & $\mathbf{U}$ & & 20 & & NS & $\mu \mathrm{g} / \mathrm{L}$ \\
\hline Isobutyl alcohol & $\mathrm{GE}$ & & & $\mathbf{U}$ & & 100 & • & NS & $\mu \mathrm{g} / \mathrm{L}$ \\
\hline Lead & WA & & & $\mathbf{U}$ & & 3 & & 50 & $\mu g / L$ \\
\hline Lead & GE & & & $\mathbf{U}$ & & 3 & & 50 & $\mu g / L$ \\
\hline Lead-212 & EP & & & $\mathbf{U}$ & & 15 & & NS & $\mathrm{pCi} / \mathrm{L}$ \\
\hline Magnesium & WA & & & $\mathbf{U}$ & & 5000 & & NS & $\mu g / L$ \\
\hline Magnesium & GE & & & & & 396 & & NS & $\mu g / L$ \\
\hline Manganese & WA & & & & & 26 & & 50 & $\mu g / L$ \\
\hline Manganese & GE & & & & & 27.7 & & 50 & $\mu g / L$ \\
\hline Manganese-54 & $\overline{\mathrm{EP}}$ & & & $\mathbf{U}$ & & 10 & & 300 & $\mathrm{pCi} / \mathrm{L}$ \\
\hline Mercury & WA & & & $\mathbf{U}$ & & 0.2 & & 2 & $\mu \mathrm{g} / \mathrm{L}$ \\
\hline Mercury & GE & & . & $\mathbf{U}$ & & 0.2 & & 2 & $\mu g / L$ \\
\hline Methacrylonitrile & WA & & & $\mathbf{U}$ & & 20 & & NS & $\mu g / L$ \\
\hline Methacrylonitrile & GE & & & $\mathrm{U}$ & & 50 & & NS & $\mu g / L$ \\
\hline Methyl bromide & WA & & & $\mathbf{U}$ & & 10 & & NS & $\mu g / L$ \\
\hline Methyl bromide & GE & & & $\mathbf{U}$ & & 1 & & NS & $\mu \mathrm{g} / \mathrm{L}$ \\
\hline Methyl chloride & WA & & & $\mathbf{U}$ & & 10 & & NS & $\mu g / L$ \\
\hline Methyl chloride & GE & & & $\mathbf{U}$ & & 1 & & NS & $\mu g / L$ \\
\hline Methyl ethyl ketone & WA & & & $\mathbf{U}$ & & 10 & & NS & $\mu g / L$ \\
\hline Methyl ethyl ketone & $\mathrm{GE}$ & & & U & & 1 & & NS & $\mu g / L$ \\
\hline Methyl iodide & WA & & & $\mathbf{U}$ & & 10 & & NS & $\mu g / L$ \\
\hline Methyl iodide & GE & & & $\mathbf{U}$ & & 15 & & NS & $\mu \mathrm{g} / \mathrm{L}$ \\
\hline Methyl isobutyl ketone & GE & & & $\mathbf{U}$ & & 1 & & NS & $\mu \mathrm{g} / \mathrm{L}$ \\
\hline Methylene bromide & WA & & & $\mathbf{U}$ & & 10 & & NS & $\mu g / L$ \\
\hline Methylene bromide & GE & & & $\mathbf{U}$ & & 1 & & NS & $\mu g / L$ \\
\hline Methylene chloride & WA & & & $\mathbf{U}$ & & 5 & & 5 & $\mu \mathrm{g} / \mathrm{L}$ \\
\hline Methylene chloride & GE & & & $\mathrm{U}$ & & 1 & & 5 & $\mu g / L$ \\
\hline Nickel & WA & & & $\mathbf{U}$ & & 40 & & 100 & $\mu \mathrm{g} / \mathrm{L}$ \\
\hline Nickel & $\mathrm{GE}$ & & & $\mathbf{U}$ & & 4 & & 100 & $\mu \mathrm{g} / \mathrm{L}$ \\
\hline Nitrate as nitrogen & WA & & & & & 0.037 & & 10 & $\mathrm{mg} / \mathrm{L}$ \\
\hline Nitrate as nitrogen & GE & & & & & 0.07 & & 10 & $\mathrm{mg} / \mathrm{L}$ \\
\hline Nonvolatile beta & $\mathrm{TM}$ & & & & & 1.3 & 1.6 & 50 & $\mathrm{pCi} / \mathrm{L}$ \\
\hline Nonvolatile beta & GE & & & $\mathbf{U}$ & & 2 & & 50 & $\mathrm{pCi} / \mathrm{L}$ \\
\hline $\mathrm{pH}$ & GE & & & $\mathbf{J}$ & $\mathbf{Q}$ & 5.27 & & NS & PH \\
\hline Potassium & WA & & & $\mathbf{U}$ & & 5000 & & NS & $\mu g / L$ \\
\hline Potassium & GE & & & $\mathbf{U}$ & & 500 & & NS & $\mu \mathrm{g} / \mathrm{L}$ \\
\hline Potassium-40 & $\mathbf{E P}$ & & & $\mathbf{U}$ & & 110 & & 300 & $\mathrm{pCi} / \mathrm{L}$ \\
\hline Promethium-144 & $\mathbf{E P}$ & & & $\mathbf{U}$ & & 10 & & NS & $\mathrm{pCi} / \mathrm{L}$ \\
\hline Promethium-146 & EP & & & $\mathbf{U}$ & & 10 & & NS & $\mathrm{pCi} /$ \\
\hline Propionitrile & WA & & & $\mathrm{U}$ & & 50 & & NS & $\mu \mathrm{g} / \mathrm{L}$ \\
\hline Propionitrile & $\mathbf{G E}$ & & & $\mathbf{U}$ & & 200 & & NS & $\mu \mathrm{g} / \mathrm{L}$ \\
\hline Ruthenium-106 & EP & & & $\mathbf{U}$ & & 90 & & 30 & $\mathrm{pCi} / \mathrm{L}$ \\
\hline Selenium & WA & & & $\mathbf{U}$ & & 5 & & 50 & $\mu \mathrm{g} / \mathrm{L}$ \\
\hline Selenium & GE & & & UJ & $\mathbf{L}$ & 2 & & 50 & $\mu g / L$ \\
\hline Silver & WA & & & $\mathbf{U}$ & & 10 & & 50 & $\mu \mathrm{g}$ \\
\hline Silver & GE & & & $\mathbf{U}$ & & 2 & & 50 & $\mu g$ \\
\hline Sodium & WA & & & $\mathbf{U}$ & & 5000 & & NS & $\mu \mathrm{g}$ \\
\hline Sodium & GE & & & & & 2240 & & NS & $\mu \mathrm{g}$ \\
\hline Sodium-22 & $\mathbf{E P}$ & & & $\mathbf{U}$ & & 10 & & 466 & $\mathrm{pCi} /$ \\
\hline Styrene & WA & & & $\mathbf{U}$ & & 5 & & 100 & $\mu \mathrm{g} /$ \\
\hline Styrene & GE & & & $\mathbf{U}$ & & 1 & & 100 & $\mu \mathrm{g}$ \\
\hline
\end{tabular}


UTR147 Laboratory Analyses (cont'd)

\begin{tabular}{|c|c|c|c|c|c|c|c|c|}
\hline Analyte & Lab & Rep & Avg & Qualifier & Result & Acc & Stand & Units \\
\hline Sulfate & WA & & & $\mathbf{U}$ & 2.5 & & 400 & $\overline{\mathrm{mg} / \mathrm{L}}$ \\
\hline Sulfate & GE & & & & 2.42 & & 400 & $\mathrm{mg} / \mathrm{L}$ \\
\hline Sulfide & WA & & & $\mathbf{U}$ & 0.1 & & NS & $\mathrm{mg} / \mathrm{L}$ \\
\hline Sulfide & GE & & $\mathbf{N A}$ & $\mathbf{U}$ & 1 & & NS & $\mathrm{mg} / \mathrm{L}$ \\
\hline Sulfide & GE & & & $\mathbf{U}$ & 1 & & NS & $\mathrm{mg} / \mathrm{L}$ \\
\hline Tetrachloroethylene & WA & & & $\mathbf{U}$ & 5 & . & 5 & $\mu \mathrm{g} / \mathrm{L}$ \\
\hline Tetrachloroethylene & GE & & & $\mathbf{U}$ & 1 & & 5 & $\mu g / L$ \\
\hline Thallium & WA & & & $\mathbf{U}$ & 10 & & 2 & $\mu \mathrm{g} / \mathrm{L}$ \\
\hline Thallium & GE & & & $\mathbf{U}$ & 2 & & 2 & $\mu g / L$ \\
\hline Thorium-234 & EP & & & $\mathbf{U}$ & 350 & & 401 & $\mathrm{pCi} / \mathrm{L}$ \\
\hline Toluene & WA & & & $\mathbf{U}$ & 5 & & 1000 & $\mu g / L$ \\
\hline Toluene & GE & & & $\mathbf{U}$ & 1 & & 1000 & $\mu \mathrm{g} / \mathrm{L}$ \\
\hline Total hardness & WA & & & $\mathbf{U}$ & 0.1 & & NS & $\mathrm{mg} / \mathrm{L}$ \\
\hline Total hardness & GE & & & & 4.5 & & NS & $\mathrm{mg} / \mathrm{L}$ \\
\hline Total organic carbon & WA & & & & 2.92 & & NS & $\mathrm{mg} / \mathrm{L}$ \\
\hline Total organic carbon & GE & & & & 2.75 & & NS & $\mathrm{mg} / \mathrm{L}$ \\
\hline Total suspended solids & WA & & & $\mathbf{U}$ & 5 & & NS & $\mathrm{mg} / \mathrm{L}$ \\
\hline Total suspended solids & GE & & NA & $\mathbf{U}$ & 1 & & NS & $\mathrm{mg} / \mathrm{L}$ \\
\hline Total suspended solids & GE & & & & 1 & & NS & $\mathrm{mg} / \mathrm{L}$ \\
\hline trans-1,3-Dichloropropene & WA & & & $\mathbf{U}$ & 5 & & NS & $\mu g / L$ \\
\hline trans-1,3-Dichloropropene & GE & & & U & 1 & & NS & $\mu g / L$ \\
\hline trans-1,4-Dichloro-2-butene & WA & & & $\mathbf{U}$ & 100 & & NS & $\mu \mathrm{g} / \mathrm{L}$ \\
\hline trans-1,4-Dichloro-2-butene & GE & & & $\mathbf{U}$ & 30 & & NS & $\mu g / L$ \\
\hline Trichloroethylene & WA & & & $\mathbf{U}$ & 5 & & 5 & $\mu \mathrm{g} / \mathrm{L}$ \\
\hline Trichloroethylene & GE & & & $\mathrm{U}$ & 1 & & 5 & $\mu \mathrm{g} / \mathrm{L}$ \\
\hline Trichlorofluoromethane & WA & & & $\mathbf{U}$ & 5 & & NS & $\mu \mathrm{g} / \mathrm{L}$ \\
\hline Trichlorofluoromethane & GE & & & & 1.6 & & NS & $\mu \mathrm{g} / \mathrm{L}$ \\
\hline Tritium & TM & & & & 5 & 2.3 & 20 & $\mathrm{pCi} / \mathrm{ml}$ \\
\hline Tritium & GE & & & & 6.63 & & 20 & $\mathrm{pCi} / \mathrm{ml}$ \\
\hline Turbidity & WA & & $\mathbf{A}$ & & 0.48 & & NS & NTU \\
\hline Turbidity & GE & & A & & 0.44 & & NS & NTU \\
\hline Vanadium & WA & & & $\mathbf{U}$ & 50 & & NS & $\mu \mathrm{g} / \mathrm{L}$ \\
\hline Vanadium & GE & & & $\mathbf{U}$ & 8 & & NS & $\mu g / L$ \\
\hline Vinyl acetate & WA & & & $\mathbf{U}$ & 10 & & NS & $\mu \mathrm{g} / \mathrm{L}$ \\
\hline Vinyl acetate & GE & & & $\mathbf{U}$ & 1 & & NS & $\mu \mathrm{g} / \mathrm{L}$ \\
\hline Vinyl chloride & WA & & & $\mathbf{U}$ & 10 & & 2 & $\mu \mathrm{g} / \mathrm{L}$ \\
\hline Vinyl chloride & GE & & & $\mathbf{U}$ & 1 & & 2 & $\mu \mathrm{g} /$ \\
\hline Xylenes (total) & WA & & & $\mathbf{U}$ & 5 & & 10000 & $\mu \mathrm{g} / \mathrm{L}$ \\
\hline Xylenes (total) & $\mathrm{GE}$ & & & $\mathbf{U}$ & 2 & & 10000 & $\mu \mathrm{g} / \mathrm{L}$ \\
\hline Yttrium-88 & EP & & & $\mathbf{U}$ & 60 & & NS & $\mathrm{pCi} / \mathrm{L}$ \\
\hline Zinc & WA & & & & 20.3 & & 5000 & $\mu g / L$ \\
\hline Zinc & $\mathrm{GE}$ & & & & 9.41 & & 5000 & $\mu \mathrm{g} / \mathrm{L}$ \\
\hline Zinc-65 & EP & & & $\mathbf{U}$ & 20 & & 300 & $\mathrm{pCi} / \mathrm{L}$ \\
\hline
\end{tabular}




\section{UTR155}

Field Measurements:

Sample Date: 4/22/93

pH: 5.69

Specific Conductance: $45 \mu \mathrm{S} / \mathrm{cm}$

Red/Oxid Potential: $147 \mathrm{mv}$

Dissolved Oxygen: $1.2 \mathrm{mg} / \mathrm{L}$

\begin{tabular}{|c|c|c|c|c|c|c|c|}
\hline Analyte & Lab & Rep & Qualifier & Result & Acc & Stand & Units \\
\hline 1,1,1,2-Tetrachloroethane & WA & & $\mathbf{U}$ & 10 & & NS & $\mu \mathrm{g} / \mathrm{L}$ \\
\hline 1,1,1-Trichloroethane & WA & & $\mathbf{U}$ & 5 & & 200 & $\mu g / L$ \\
\hline 1,1,2,2-Tetrachloroethane & WA & & $\mathbf{U}$ & 5 & & NS & $\mu \mathrm{g} / \mathrm{L}$ \\
\hline 1,1,2-Trichloroethane & WA & & $\mathbf{U}$ & 5 & & 5 & $\mu \mathrm{g} / \mathrm{L}$ \\
\hline 1,1-Dichloroethane & WA & & $\mathbf{U}$ & 5 & & NS & $\mu g / L$ \\
\hline 1,1-Dichloroethylene & WA & & U & 5 & & 7 & $\mu g / L$ \\
\hline 1,2,3-Trichloropropane & WA & & $\mathbf{U}$ & 10 & & NS & $\mu g / L$ \\
\hline 1,2-Dibromo-3-chloropropane & WA & & $\mathrm{U}$ & 20 & & 0.2 & $\mu g / L$ \\
\hline 1,2-Dibromoethane & WA & & $\mathbf{U}$ & 20 & & NS & $\mu g / L$ \\
\hline 1,2-Dichloroethane & WA & & $\mathbf{U}$ & 5 & & 5 & $\mu g / L$ \\
\hline 1,2-Dichloroethylene (total) & WA & & U & 5 & & 100 & $\mu \mathrm{g} / \mathrm{L}$ \\
\hline 1,2-Dichloropropane & WA & & U & 5 & & 5 & $\mu g / L$ \\
\hline 2-Hexanone & WA & & $\mathbf{U}$ & 10 & & NS & $\mu g / L$ \\
\hline 4-Methyl-2-pentanone & WA & & U & 10 & & NS & $\mu g / L$ \\
\hline Acetone & WA & & U & 10 & & NS & $\mu g / L$ \\
\hline Acetonitrile & WA & & $\mathrm{U}$ & 20 & & NS & $\mu g / L$ \\
\hline Acidity & WA & & & 17 & & NS & $\mathrm{mg} / \mathrm{L}$ \\
\hline Acrolein & WA & & U & 10 & & NS & $\mu g / L$ \\
\hline Acrylonitrile & WA & & U & 10 & & NS & $\mu \mathrm{g} / \mathrm{L}$ \\
\hline Alkalinity & WA & & $\mathrm{U}$ & 0.5 & & NS & $\mathrm{mg} / \mathrm{L}$ \\
\hline Allyl chloride & WA & & $\mathrm{U}$ & 100 & & NS & $\mu \mathrm{g} / \mathrm{L}$ \\
\hline Aluminum & WA & & & 808 & & NS & $\mu g / L$ \\
\hline Antimony & WA & & $\mathrm{U}$ & 60 & & 6 & $\mu g / L$ \\
\hline Arsenic & WA & & $\mathbf{U}$ & 10 & & 50 & $\mu g / L$ \\
\hline Barium & WA & & $\mathrm{U}$ & 200 & & 2000 & $\mu g / L$ \\
\hline Benzene & WA & & $\mathrm{U}$ & 5 & & 5 & $\mu g / L$ \\
\hline Beryllium & WA & & $\mathrm{U}$ & 5 & & 4 & $\mu \mathrm{g} / \mathrm{L}$ \\
\hline Bromodichloromethane & WA & & $\mathrm{U}$ & 5 & & NS & $\mu g / L$ \\
\hline Bromoform & WA & & $\mathrm{U}$ & 5 & & NS & $\mu g / L$ \\
\hline Cadmium & WA & & U & 5 & & 5 & $\mu g / L$ \\
\hline Calcium & WA & & $\mathrm{U}$ & 5000 & & NS & $\mu g / L$ \\
\hline Carbon disulfide & WA & & $\mathrm{U}$ & 5 & & NS & $\mu g / L$ \\
\hline Carbon tetrachloride & WA & & U & 5 & & 5 & $\mu \mathrm{g} / \mathrm{L}$ \\
\hline Cesium-137 & $\mathrm{TM}$ & & & 3.7 & 7.6 & 200 & $\mathrm{pCi} / \mathrm{L}$ \\
\hline Chloride & WA & & & 3.03 & & NS & $\mathrm{mg} / \mathrm{L}$ \\
\hline Chlorobenzene & WA & & $\mathrm{U}$ & 5 & & 100 & $\mu \mathrm{g} / \mathrm{L}$ \\
\hline Chloroethane & WA & & $\mathrm{U}$ & 10 & & NS & $\mu g / L$ \\
\hline Chloroform & WA & & $\mathrm{U}$ & 5 & & 100 & $\mu g / L$ \\
\hline Chloroprene & WA & & $\mathbf{U}$ & 20 & & NS & $\mu \mathrm{g} / \mathrm{L}$ \\
\hline Chromium & WA & & $\mathrm{U}$ & 10 & & 100 & $\mu g / L$ \\
\hline cis-1,3-Dichloropropene & WA & & U & 5 & & NS & $\mu \mathrm{g} / \mathrm{L}$ \\
\hline Cobalt & WA & & U & 50 & & NS & $\mu \mathrm{g} / \mathrm{L}$ \\
\hline Copper & WA & & U & 25 & & 1000 & $\mu g / L$ \\
\hline Cyanide & WA & & $\mathrm{U}$ & 10 & & 200 & $\mu g / L$ \\
\hline Dibromochloromethane & WA & & $U$ & 5 & & NS & $\mu \mathrm{g} / \mathrm{L}$ \\
\hline Dichlorodifluoromethane & WA & & U & 10 & & NS & $\mu g / L$ \\
\hline Ethylbenzene & WA & & U & 5 & & 700 & $\mu \mathrm{g} / \mathrm{L}$ \\
\hline Fluoride & WA & & $\mathrm{U}$ & 0.1 & & 4 & $\mathrm{mg} / \mathrm{L}$ \\
\hline
\end{tabular}


UTR155 Laboratory Analyses (cont'd)

\begin{tabular}{|c|c|c|c|c|c|c|c|c|}
\hline Analyte & Lab & Rep. & Avg & Qualifier & Result & Acc & Stand & Units \\
\hline Gross alpha & TM & & & & 1.8 & 1.3 & 15 & $\overline{\mathrm{pCi} / \mathrm{L}}$ \\
\hline Iron & WA & & & & 415 & & 300 & $\mu g / L$ \\
\hline Isobutyl alcohol & WA & & & $\mathbf{U}$ & 20 & & NS & $\mu \mathrm{g} / \mathrm{L}$ \\
\hline Lead & WA & & & $\mathbf{U}$ & 3 & & 50 & $\mu g / L$ \\
\hline Magnesium & WA & & & $\mathbf{U}$ & 5000 & & NS & $\mu g / L$ \\
\hline Manganese & WA & & & & 133 & - & 50 & $\mu \mathrm{g} / \mathrm{L}$ \\
\hline Mercury & WA & & & $\mathbf{U}$ & 0.2 & & 2 & $\mu \mathrm{g} / \mathrm{L}$ \\
\hline Methacrylonitrile & WA & & & $\mathbf{U}$ & 20 & & NS & $\mu \mathrm{g} / \mathrm{L}$ \\
\hline Methyl bromide & WA & & & $\mathbf{U}$ & 10 & & NS & $\mu \mathrm{g} / \mathrm{L}$ \\
\hline Methyl chloride & WA & & & $\mathbf{U}$ & 10 & & NS & $\mu g / L$ \\
\hline Methyl ethyl ketone & WA & & & $\mathbf{U}$ & 10 & & NS & $\mu g / L$ \\
\hline Methyl iodide & WA & & & $\mathbf{U}$ & 10 & & NS & $\mu g / L$ \\
\hline Methylene bromide & WA & & & $\mathbf{U}$ & 10 & & NS & $\mu g / L$ \\
\hline Methylene chloride & WA & & & $\mathbf{U}$ & 5 & & 5 & $\mu g / L$ \\
\hline Nickel & WA & & . & $\mathbf{U}$ & 40 & & 100 & $\mu g / L$ \\
\hline Nitrate as nitrogen & WA & & & & 1.3 & & 10 & $\mathrm{mg} / \mathrm{L}$ \\
\hline Nonvolatile beta & TM & & & & 5.6 & 2 & 50 & $\mathrm{pCi} / \mathrm{L}$ \\
\hline Potassium & WA & & & $\mathbf{U}$ & 5000 & & NS & $\mu g / L$ \\
\hline Propionitrile & WA & & & $\mathbf{U}$ & 50 & & NS & $\mu g / L$ \\
\hline Selenium & WA & & & $\mathbf{U}$ & 5 & & 50 & $\mu g / L$ \\
\hline Silver & WA & & & $\mathbf{U}$ & 10 & & 50 & $\mu g / L$ \\
\hline Sodium & WA & & & $\mathbf{U}$ & 5000 & & NS & $\mu g / L$ \\
\hline Styrene & WA & & & $\mathbf{U}$ & 5 & & 100 & $\mu g / L$ \\
\hline Sulfate & WA & & & $\mathbf{U}$ & 0.25 & & 400 & $\mathrm{mg} / \mathrm{L}$ \\
\hline Sulfide & WA & & & $\mathbf{U}$ & 0.1 & & NS & $\mathrm{mg} / \mathrm{L}$ \\
\hline Tetrachloroethylene & WA & & & $\mathbf{U}$ & 5 & & 5 & $\mu g / L$ \\
\hline Thallium & WA & & & $\mathbf{U}$ & 10 & & 2 & $\mu g / L$ \\
\hline Toluene & WA & & & $\mathbf{U}$ & 5 & & 1000 & $\mu g / L$ \\
\hline Total hardness & WA & · & $\mathbf{A}$ & & 10 & & NS & $\mathrm{mg} / \mathrm{L}$ \\
\hline Total organic carbon & WA & & & & 24.1 & 8 & NS & $\mathrm{mg} / \mathrm{L}$ \\
\hline Total suspended solids & WA & & $\mathbf{A}$ & & 19.5 & ' & NS & $\mathrm{mg} / \mathrm{L}$ \\
\hline trans-1,3-Dichloropropene & WA & & & $\mathbf{U}$ & 5 & & NS & $\mu \mathrm{g} / \mathrm{L}$ \\
\hline trans-1,4-Dichloro-2-butene & WA & & & $\mathbf{U}$ & 100 & & NS & $\mu \mathrm{g} / \mathrm{L}$ \\
\hline Trichloroethylene & WA & & & & 5.09 & & 5 & $\mu \mathrm{g} / \mathrm{L}$ \\
\hline Trichlorofluoromethane & WA & & & $\mathbf{U}$ & 5 & & NS & $\mu \mathrm{g} / \mathrm{L}$ \\
\hline Tritium & $\mathrm{TM}$ & & & & 30.6 & 14.1 & 20 & $\mathrm{pCi} / \mathrm{ml}$ \\
\hline Turbidity & WA & & $\mathbf{A}$ & & 12.3 & & NS & NTU \\
\hline Vanadium & WA & & & $\mathbf{U}$ & 50 & & NS & $\mu \mathrm{g} / \mathrm{L}$ \\
\hline Vinyl acetate & WA & & & $\mathbf{U}$ & 10 & & NS & $\mu \mathrm{g} / \mathrm{L}$ \\
\hline Vinyl chloride & WA & & & $\mathbf{U}$ & 10 & & 2 & $\mu g / L$ \\
\hline Xylenes (total) & WA & & & $\mathbf{U}$ & 5 & & 10000 & $\mu g / L$ \\
\hline Zinc & WA & & & $\mathbf{U}$ & 20 & & 5000 & $\mu \mathrm{g} /$ \\
\hline
\end{tabular}




\section{UQA006}

(Equipment Blank - Filter)

Field Measurements:

Sample Date: 4/13/93

\begin{tabular}{|c|c|c|c|c|c|c|c|c|}
\hline Analyte & Lab & Rep & Avg & Qualifier & Result & Acc & Stand & Units \\
\hline 1,1,1,2-Tetrachloroethane & WA & & & $\mathbf{U}$ & 10 & . & NS & $\mu g / L$ \\
\hline 1,1,1,2-Tetrachloroethane & WA & & NA & $\mathbf{U}$ & 10 & & NS & $\mu g / L$ \\
\hline $1,1,1,2$-Tetrachloroethane & WA & & NA & $\mathbf{U}$ & 10 & & NS & $\mu g / L$ \\
\hline 1,1,1-Trichloroethane & WA & & & $\mathbf{U}$ & 5 & & 200 & $\mu g / L$ \\
\hline 1,1,1-Trichloroethane & WA & & NA & $\mathbf{U}$ & 5 & & 200 & $\mu g / L$ \\
\hline 1,1,1-Trichloroethane & WA & & NA & $\mathbf{U}$ & 5 & & 200 & $\mu g / L$ \\
\hline 1,1,2,2-Tetrachloroethane & WA & & & $\mathbf{U}$ & 5 & & NS & $\mu \mathrm{g} / \mathrm{L}$ \\
\hline 1,1,2,2-Tetrachloroethane & WA & & NA & $\mathbf{U}$ & 5 & & NS & $\mu g / L$ \\
\hline 1,1,2,2-Tetrachloroethane & WA & & NA & $\mathbf{U}$ & 5 & & NS & $\mu \mathrm{g} / \mathrm{L}$ \\
\hline 1,1,2-Trichloroethane & WA & & & $\mathbf{U}$ & 5 & & 5 & $\mu g / L$ \\
\hline 1,1,2-Trichloroethane & WA & & NA & $\mathbf{U}$ & 5 & & 5 & $\mu \mathrm{g} / \mathrm{L}$ \\
\hline 1,1,2-Trichloroethane & WA & & NA & $\mathbf{U}$ & 5 & & 5 & $\mu g / L$ \\
\hline 1,1-Dichloroethane & WA & & & $\mathbf{U}$ & 5 & & NS & $\mu g / L$ \\
\hline 1,1-Dichloroethane & WA & & NA & $\mathbf{U}$ & 5 & & NS & $\mu g / L$ \\
\hline 1,1-Dichloroethane & WA & & NA & $\mathbf{U}$ & 5 & & NS & $\mu g / L$ \\
\hline 1,1-Dichloroethylene & WA & & & $\mathbf{U}$ & 5 & & 7 & $\mu \mathrm{g} / \mathrm{L}$ \\
\hline 1,2,3-Trichloropropane & WA & & & $\mathbf{U}$ & 10 & & NS & $\mu g / L$ \\
\hline 1,2,3-Trichloropropane & WA & & NA & $\mathbf{U}$ & 10 & & NS & $\mu g / L$ \\
\hline 1,2,3-Trichloropropane & WA & & NA & $\mathbf{U}$ & 10 & & NS & $\mu g / L$ \\
\hline 1,2-Dibromo-3-chloropropane & WA & & & $\mathbf{U}$ & 20 & & 0.2 & $\mu g / L$ \\
\hline 1,2-Dibromo-3-chloropropane & WA & & NA & $\mathbf{U}$ & 20 & & 0.2 & $\mu g / L$ \\
\hline 1,2-Dibromo-3-chloropropane & WA & & NA & $\mathbf{U}$ & 20 & & 0.2 & $\mu g / L$ \\
\hline 1,2-Dibromoethane & WA & & & $\mathbf{U}$ & 20 & & NS & $\mu \mathrm{g} / \mathrm{L}$ \\
\hline 1,2-Dibromoethane & WA & & NA & $\mathbf{U}$ & 20 & & NS & $\mu g / L$ \\
\hline 1,2-Dibromoethane & WA & & NA & $\mathbf{U}$ & 20 & & NS & $\mu g / L$ \\
\hline 1,2-Dichloroethane & WA & & & $\mathbf{U}$ & 5 & & 5 & $\mu g / L$ \\
\hline 1,2-Dichloroethane & WA & & NA & $\mathbf{U}$ & 5 & & 5 & $\mu \mathrm{g} / \mathrm{L}$ \\
\hline 1,2-Dichloroethane & WA & & NA & $\mathbf{U}$ & 5 & & 5 & $\mu \mathrm{g} / \mathrm{L}$ \\
\hline 1,2-Dichloroethylene (total) & WA & & & $\mathbf{U}$ & 5 & & 100 & $\mu g / L$ \\
\hline 1,2-Dichloroethylene (total) & WA & & NA & $\mathbf{U}$ & 5 & & 100 & $\mu g / L$ \\
\hline 1,2-Dichloroethylene (total) & WA & & NA & $\mathbf{U}$ & 5 & & 100 & $\mu \mathrm{g} / \mathrm{L}$ \\
\hline 1,2-Dichloropropane & WA & & & $\mathbf{U}$ & 5 & & 5 & $\mu g / L$ \\
\hline 1,2-Dichloropropane & WA & & NA & $\mathbf{U}$ & 5 & & 5 & $\mu \mathrm{g} / \mathrm{L}$ \\
\hline 1,2-Dichloropropane & WA & & NA & $\mathbf{U}$ & 5 & & 5 & $\mu \mathrm{g} / \mathrm{L}$ \\
\hline 2-Hexanone & WA & & & $\mathbf{U}$ & 10 & & NS & $\mu g / L$ \\
\hline 2-Hexanone & WA & & NA & $\mathbf{U}$ & 10 & & NS & $\mu g / L$ \\
\hline 2-Hexanone & WA & & NA & $\mathbf{U}$ & 10 & & NS & $\mu g / L$ \\
\hline 4-Methyl-2-pentanone & WA & & & $\mathbf{U}$ & 10 & & NS & $\mu \mathrm{g} / \mathrm{L}$ \\
\hline 4-Methyl-2-pentanone & WA & & NA & $\mathbf{U}$ & 10 & & NS & $\mu \mathrm{g} / \mathrm{L}$ \\
\hline 4-Methyl-2-pentanone & WA & & NA & $\mathbf{U}$ & 10 & & NS & $\mu g / L$ \\
\hline Acetone & WA & & & & 10.7 & & NS & $\mu g / L$ \\
\hline Acetone & WA & & NA & $\mathbf{U}$ & 10 & & NS & $\mu g / L$ \\
\hline Acetone & WA & & NA & $\mathbf{J}$ & 7.12 & & NS & $\mu \mathrm{g} / \mathrm{L}$ \\
\hline Acetonitrile & WA & & & $\mathbf{U}$ & 20 & & NS & $\mu \mathrm{g} / \mathrm{L}$ \\
\hline Acetonitrile & WA & & $\mathbf{N A}$ & $\mathbf{U}$ & 20 & & NS & $\mu g / L$ \\
\hline Acetonitrile & WA & & NA & $\mathbf{U}$ & 20 & & NS & $\mu \mathrm{g} / \mathrm{L}$ \\
\hline Acidity & WA & & & & 3 & & NS & $\mathrm{mg} / \mathrm{L}$ \\
\hline
\end{tabular}


UQA006 Laboratory Analyses (cont'd)

\begin{tabular}{|c|c|c|c|c|c|c|c|c|}
\hline Analyte & Lab & Rep & Avg & Qualifier & Result & Acc & Stand & Units \\
\hline Acrolein & WA & & & U & 10 & & NS & $\mu g / L$ \\
\hline Acrolein & WA & & NA & $\mathbf{U}$ & 10 & & NS & $\mu g / L$ \\
\hline Acrolein & WA & & NA & $\mathbf{U}$ & 10 & & NS & $\mu \mathrm{g} / \mathrm{L}$ \\
\hline Acrylonitrile & WA & & & $\mathbf{U}$ & 10 & & NS & $\mu g / L$ \\
\hline Acrylonitrile & WA & & NA & $\mathbf{U}$ & 10 & & NS & $\mu g / L$ \\
\hline Acrylonitrile & WA & & NA & $\mathbf{U}$ & 10 & - & NS & $\mu \mathrm{g} / \mathrm{L}$ \\
\hline Alkalinity & WA & & & $\mathbf{U}$ & 0.5 & & NS & $\mathrm{mg} / \mathrm{L}$ \\
\hline Allyl chloride & WA & & & $\mathbf{U}$ & 100 & & NS & $\mu g / L$ \\
\hline Allyl chloride & WA & & NA & $\mathbf{U}$ & 100 & & NS & $\mu g / L$ \\
\hline Allyl chloride & WA & & NA & $\mathbf{U}$ & 100 & & NS & $\mu g / L$ \\
\hline Aluminum & WA & & & $\mathbf{U}$ & 200 & & NS & $\mu g / L$ \\
\hline Antimony & WA & & & $\mathbf{U}$ & 60 & & 6 & $\mu g / L$ \\
\hline Arsenic & WA & & & $\mathbf{U}$ & 10 & & 50 & $\mu g / L$ \\
\hline Barium & WA & & & $\mathbf{U}$ & 200 & & 2000 & $\mu g / L$ \\
\hline Benzene & WA & & . & $\mathbf{U}$ & 5 & & 5 & $\mu g / L$ \\
\hline Beryllium & WA & & & $\mathbf{U}$ & 5 & & 4 & $\mu g / L$ \\
\hline Bromodichloromethane & WA & & & $\mathbf{U}$ & 5 & & NS & $\mu g / L$ \\
\hline Bromodichloromethane & WA & & NA & $\mathbf{U}$ & 5 & & NS & $\mu g / L$ \\
\hline Bromodichloromethane & WA & & NA & $\mathbf{U}$ & 5 & & NS & $\mu g / L$ \\
\hline Bromoform & WA & & & $\mathbf{U}$ & 5 & & NS & $\mu g / L$ \\
\hline Bromoform & WA & & NA & $\mathbf{U}$ & 5 & & NS & $\mu g / L$ \\
\hline Bromoform & WA & & NA & $\mathbf{U}$ & 5 & & NS & $\mu g / L$ \\
\hline Cadmium & WA & & & $\mathbf{U}$ & 5 & & 5 & $\because g / L$ \\
\hline Calcium & WA & & & $\mathbf{U}$ & 5000 & & NS & $i . \mathrm{g} / \mathrm{L}$ \\
\hline Carbon disulfide & WA & & & $\mathbf{U}$ & 5 & & NS & $\mu g / L$ \\
\hline Carbon disulfide & WA & . & NA & $\mathbf{U}$ & 5 & & NS & $\mu g / L$ \\
\hline Carbon disulfide & WA & & NA & $\mathbf{U}$ & 5 & & NS & $\mu g / L$ \\
\hline Carbon tetrachloride & WA & & & $\mathbf{U}$ & 5 & & 5 & $\mu \mathrm{g} / \mathrm{L}$ \\
\hline Carbon tetrachloride & WA & & NA & $\mathbf{U}$ & 5 & & 5 & $\mu g / L$ \\
\hline Carbon tetrachloride & WA & & NA & $\mathbf{U}$ & 5 & & 5 & $\mu g / L$ \\
\hline Cesium-137 & TM & & & $\mathbf{U}$ & 10 & & 200 & $\mathrm{pCi} / \mathrm{L}$ \\
\hline Chloride & WA & & & & 0.652 & & NS & $\mathrm{mg} / \mathrm{L}$ \\
\hline Chlorobenzene & WA & & & $\mathbf{U}$ & 5 & & 100 & $\mu \mathrm{g} / \mathrm{L}$ \\
\hline Chloroethane & WA & & & $\mathrm{U}$ & 10 & & NS & $\mu g / L$ \\
\hline Chloroethane & WA & & NA & $\mathrm{U}$ & 10 & & NS & $\mu \mathrm{g} / \mathrm{L}$ \\
\hline Chloroethane & WA & & NA & $\mathbf{U}$ & 10 & & NS & $\mu g / L$ \\
\hline Chloroform & WA & & & $\mathrm{U}$ & 5 & & 100 & $\mu g / L$ \\
\hline Chloroform & WA & & NA & $\mathrm{U}$ & 5 & & 100 & $\mu g / L$ \\
\hline Chloroform & WA & & NA & $\mathrm{U}$ & 5 & & 100 & $\mu g / L$ \\
\hline Chloroprene & WA & & & $\mathrm{U}$ & 20 & & NS & $\mu g / L$ \\
\hline Chloroprene & WA & & NA & $\mathbf{U}$ & 20 & & NS & $\mu g / L$ \\
\hline Chloroprene & WA & & NA & $\mathbf{U}$ & 20 & & NS & $\mu g / L$ \\
\hline Chromium . & WA & & & $\mathrm{U}$ & 10 & & 100 & $\mu g / L$ \\
\hline cis-1,3-Dichloropropene & WA & & & U & 5 & & NS & $\mu g / L$ \\
\hline cis-1,3-Dichloropropene & WA & & NA & $\mathrm{U}$ & 5 & & NS & $\mu g / L$ \\
\hline cis-1,3-Dichloropropene & WA & & NA & U & 5 & & NS & $\mu g / L$ \\
\hline Cobalt & WA & & & $U$ & 50 & & NS & $\mu \mathrm{g} / \mathrm{L}$ \\
\hline Copper & WA & & & $\mathrm{U}$ & 25 & & 1000 & $\mu g / L$ \\
\hline Cyanide & WA & & & $\mathrm{U}$ & 10 & & 200 & $\mu g / L$ \\
\hline Dibromochloromethane & WA & & & $\mathbf{U}$ & 5 & & NS & $\mu \mathrm{g} / \mathrm{L}$ \\
\hline Dibromochloromethane & WA & & NA & $\mathbf{U}$ & 5 & & NS & $\mu g / L$ \\
\hline Dibromochloromethane & WA & & NA & $\mathbf{U}$ & 5 & & NS & $\mu \mathrm{g} / \mathrm{L}$ \\
\hline Dichlorodifluoromethane & WA & & & $\mathbf{U}$ & 10 & & NS & $\mu \mathrm{g} / \mathrm{L}$ \\
\hline Dichlorodifluoromethane & WA & & NA & $\mathbf{U}$ & 10 & & NS & $\mu g / L$ \\
\hline Dichlorodifluoromethane & WA & & NA & $\mathbf{U}$ & 10 & & NS & $\mu g / L$ \\
\hline
\end{tabular}


UQA006 Laboratory Analyses (cont'd)

\begin{tabular}{|c|c|c|c|c|c|c|c|}
\hline Analyte & Lab & Rep & Qu vifier & Result & Acc & Stand & Units \\
\hline Ethylbenzene & WA & & $\mathbf{U}$ & 5 & & 700 & $\mu g / L$ \\
\hline Ethylbenzene & WA & NA & $\mathbf{U}$ & 5 & & 700 & $\mu g / L$ \\
\hline Ethylbenzene & WA & NA & $\mathbf{U}$ & 5 & & 700 & $\mu g / L$ \\
\hline Fluoride & WA & & $\mathbf{U}$ & 0.1 & & 4 & $\mathrm{mg} / \mathrm{L}$ \\
\hline Gross alpha & TM & & $\mathbf{U}$ & 0.4 & & 15 & $\mathrm{pCi} / \mathrm{L}$ \\
\hline Iron & WA & & $\mathbf{U}$ & 100 & • & 300 & $\mu g / L$ \\
\hline Isobutyl alcohol & WA & & U & 20 & & NS & $\mu g / L$ \\
\hline Isobutyl alcohol & WA & NA & U & 20 & & NS & $\mu g / L$ \\
\hline Isobutyl alcohol & WA & NA & $\mathbf{U}$ & 20 & & NS & $\mu g / L$ \\
\hline Lead & WA & & $\mathbf{U}$ & 3 & & 50 & $\mu \mathrm{g} / \mathrm{L}$ \\
\hline Magnesium & WA & & $\mathbf{U}$ & 5000 & & NS & $\mu g / L$ \\
\hline Manganese & WA & & $\mathbf{U}$ & 15 & & 50 & $\mu g / L$ \\
\hline Mercury & WA & & $\mathbf{U}$ & 0.2 & & 2 & $\mu g / L$ \\
\hline Methacrylonitrile & WA & & $\mathbf{U}$ & 20 & & NS & $\mu g / L$ \\
\hline Methacrylonitrile & WA & NA & $\mathbf{U}$ & 20 & & NS & $\mu g / L$ \\
\hline Methacrylonitrile & WA & NA & U & 20 & & NS & $\mu g / L$ \\
\hline Methyl bromide & WA & & U & 10 & & NS & $\mu g / L$ \\
\hline Methyl bromide & WA & NA & U & 10 & & NS & $\mu g / L$ \\
\hline Methyl bromide & WA & NA & $\mathbf{U}$ & 10 & & NS & $\mu g / L$ \\
\hline Methyl chloride & WA & & $\mathbf{U}$ & 10 & & NS & $\mu g / L$ \\
\hline Methyl chloride & WA & NA & $\mathbf{U}$ & 10 & & NS & $\mu \mathrm{g} / \mathrm{L}$ \\
\hline Methyl chloride & WA & NA & $\mathbf{U}$ & 10 & & NS & $\mu g / L$ \\
\hline Methyl ethyl ketone & WA & & $\mathbf{J}$ & 6.91 & & NS & $\mu g / L$ \\
\hline Methyl ethyl ketone & WA & NA & $\mathbf{J}$ & 5.73 & & NS & $\mu g / L$ \\
\hline Methyl ethyl ketone & WA & NA & $\mathbf{J}$ & 8.44 & & NS & $\mu g / L$ \\
\hline Methyl iodide & WA & & $\mathrm{U}$ & 10 & & NS & $\mu g / L$ \\
\hline Methyl iodide & WA & NA & $\mathbf{U}$ & 10 & & NS & $\mu g / L$ \\
\hline Methyl iodide & WA & NA & U & 10 & & NS & $\mu g / L$ \\
\hline Methylene bromide & WA & & U & 10 & & NS & $\mu \mathrm{g} / \mathrm{L}$ \\
\hline Methylene bromide & WA & NA & $\mathbf{U}$ & 10 & & NS & $\mu g / L$ \\
\hline Methylene bromide & WA & NA & $\mathbf{U}$ & 10 & & NS & $\mu g / L$ \\
\hline Methylene chloride & WA & & U & 5 & & 5 & $\mu g / L$ \\
\hline Methylene chloride & WA & NA & $\mathrm{U}$ & 5 & & 5 & $\mu g / L$ \\
\hline Methylene chloride & WA & NA & $\mathbf{U}$ & 5 & & 5 & $\mu g / L$ \\
\hline Nickel & WA & & $\mathbf{U}$ & 40 & & 100 & $\mu g / L$ \\
\hline Nitrate as nitrogen & WA & & U & 0.1 & & 10 & $\mathrm{mg} / \mathrm{L}$ \\
\hline Nonvolatile beta & TM & & $\mathrm{U}$ & 0.7 & & 50 & $\mathrm{pCi} / L$ \\
\hline Potassium & WA & & U & 5000 & & NS & $\mu \mathrm{g} / \mathrm{L}$ \\
\hline Propionitrile & WA & & $\mathbf{U}$ & 50 & & NS & $\mu g / L$ \\
\hline Propionitrile & WA & NA & $\mathrm{U}$ & 50 & & NS & $\mu \mathrm{g} / \mathrm{L}$ \\
\hline Propionitrile & WA & NA & $\mathrm{U}$ & 50 & & NS & $\mu g / L$ \\
\hline Selenium & $\mathrm{W} / \mathrm{s}$ & & $\mathbf{U}$ & 5 & & 50 & $\mu g / L$ \\
\hline Silver & WA & & U & 10 & & 50 & $\mu g / 2$ \\
\hline Sodium & WA & & $\mathrm{U}$ & 5000 & & NS & $\mu \mathrm{g} / \mathrm{L}$ \\
\hline Styrene & WA & & U & 5 & & 100 & $\mu \mathrm{g} / \mathrm{L}$ \\
\hline Styrene & WA & NA & U & 5 & & 100 & $\mu \mathrm{g} / \mathrm{L}$ \\
\hline Styrene & WA & NA & $\mathrm{U}$ & 5 & & 100 & $\mu g / L$ \\
\hline Sulfate & WA & & $\mathbf{U}$ & 2.5 & & 400 & $\mathrm{mg} / \mathrm{L}$ \\
\hline Sulfide & WA & & $\mathbf{U}$ & 0.1 & & NS & $\mathrm{mg} / \mathrm{L}$ \\
\hline Tetrachloroethylene & WA & & $\mathrm{U}$ & 5 & & 5 & $\mu g / L$ \\
\hline Tetrachloroethylene & WA & NA & $\mathbf{U}$ & 5 & & 5 & $\mu \mathrm{g} / \mathrm{L}$ \\
\hline Tetrachloroethylene & WA & NA & $\mathbf{U}$ & 5 & & 5 & $\mu g / L$ \\
\hline Thallium & WA & & U & 10 & & 2 & $\mu \mathrm{g} / \mathrm{L}$ \\
\hline Toluene & WA & & $\mathbf{U}$ & 5 & & 1000 & $\mu \mathrm{g} / \mathrm{L}$ \\
\hline
\end{tabular}


UQA006 Laboratory Analyses (cont'd)

\begin{tabular}{|c|c|c|c|c|c|c|c|c|}
\hline Analyte & Lab & Rep & Avg & Qualifier & Result & Acc & Stand & Units \\
\hline Total hardness & WA & & & $\mathrm{U}$ & 2 & & NS & $m g / L$ \\
\hline Total hardness & WA & & NA & $\mathbf{U}$ & 2 & & NS & $\mathbf{m g} / \mathbf{L}$ \\
\hline Total organic carbon & WA & & & $\mathbf{U}$ & 0.5 & & NS & $\mathrm{mg} / \mathrm{L}$ \\
\hline Total suspended solids & WA & & & $\mathbf{U}$ & 5 & & NS & $\mathrm{mg} / \mathrm{L}$ \\
\hline trans-1,3-Dichloropropene & WA & & & $\mathbf{U}$ & 5 & & NS & $\mu g / L$ \\
\hline trans-1,3-Dichloropropene & WA & & NA & $\mathbf{U}$ & 5 & . & NS & $\mu \mathrm{g} / \mathrm{L}$ \\
\hline trans-1,3-Dichloropropene & WA & & NA & $\mathbf{U}$ & 5 & & NS & $\mu \mathrm{g} / \mathrm{L}$ \\
\hline trans-1,4-Dichlora-2-butene & WA & & & $\mathbf{U}$ & 100 & & NS & $\mu g / L$ \\
\hline trans-1,4-Dichloro-2-butene & WA & & NA & $U$ & 100 & & NS & $\mu g / L$ \\
\hline trans-1,4-Dichloro-2-butene & WA & & NA & $\mathbf{U}$ & 100 & & NS & $\mu g / L$ \\
\hline Trichloroethylene & WA & & & $\mathbf{U}$ & 5 & & 5 & $\mu g / L$ \\
\hline Trichlorofluoromethane & WA & & & $\mathbf{U}$ & 5 & & NS & $\mu g / L$ \\
\hline Trichlorofluoromethane & WA & & NA & $\mathbf{U}$ & 5 & & NS & $\mu g / L$ \\
\hline Trichlorofluoromethane & WA & & NA & $\mathbf{U}$ & 5 & & NS & $\mu g / L$ \\
\hline Tritium & $\mathbf{T M}$ & & & $\mathbf{U}$ & 0.5 & & 20 & $\mathrm{pCi} / \mathrm{ml}$ \\
\hline Turbidity & WA & & & $\mathbf{U}$ & 0.2 & & NS & NTU \\
\hline Vanadium & WA & & & $\mathbf{U}$ & 50 & & NS & $\mu g / L$ \\
\hline Vinyl acetate & WA & & & $\mathbf{U}$ & 10 & & NS & $\mu \mathrm{g} / \mathrm{L}$ \\
\hline Vinyl acetate & WA & & NA & $\mathbf{U}$ & 10 & & NS & $\mu g / L$ \\
\hline Vinyl acetate & WA & & NA & $\mathbf{U}$ & 10 & & NS & $\mu g / L$ \\
\hline Vinyl chloride & WA & & & $\mathbf{U}$ & 10 & & 2 & $\mu g / L$ \\
\hline Vinyl chloride & WA & & NA & $\mathbf{U}$ & 10 & & 2 & $\mu g / L$ \\
\hline Vinyl chloride & WA & & NA & $\mathbf{U}$ & 10 & & 2 & $\mu g / L$ \\
\hline Xylenes (total) & WA & & & $\mathbf{U}$ & 5 & & 10000 & $\mu \mathrm{g} / \mathrm{L}$ \\
\hline Xylenes (total) & WA & & $\mathbf{N A}$ & $\mathbf{U}$ & 5 & & 10000 & $\mu g / L$ \\
\hline Xylenes (total) & WA & & NA & $\mathbf{U}$ & 5 & & 10000 & $\mu \mathrm{g} / \mathrm{L}$ \\
\hline Zinc & WA & & & $\mathbf{U}$ & 20 & & 5000 & $\mu g / L$ \\
\hline
\end{tabular}




\section{UQA007}

(Equipment Blank - Shovel)

Field Measurements:

Sample Date: 4/19/93

\begin{tabular}{|c|c|c|c|c|c|c|c|c|}
\hline Analyte & Lab & Rep & Avg & Qualifier & Result & Acc & Stand & Units \\
\hline 1,1,1,2-Tetrachloroethane & WA & & & $\mathbf{U}$ & 10 & • & NS & $\mu g / L$ \\
\hline 1,1,1-Trichloroethane & WA & & & $\mathbf{U}$ & 5 & & 200 & $\mu g / L$ \\
\hline 1,1,2,2-Tetrachloroethane & WA & & & $\mathbf{U}$ & 5 & & NS & $\mu g / L$ \\
\hline 1,1,2-Trichloroethane & WA & & & $\mathbf{U}$ & 5 & & 5 & $\mu g / L$ \\
\hline 1,1-Dichloroethane & WA & & & $\mathbf{U}$ & 5 & & NS & $\mu g / L$ \\
\hline 1,1-Dichloroethylene & WA & & & $\mathbf{U}$ & 5 & & 7 & $\mu g / L$ \\
\hline 1,2,3-Trichloropropane & WA & & & $\mathbf{U}$ & 10 & & NS & $\mu g / L$ \\
\hline 1,2-Dibromo-3-chloropropane & WA & & & $\mathbf{U}$ & 20 & & 0.2 & $\mu g / L$ \\
\hline 1,2-Dibromoethane & WA & & & $\mathbf{U}$ & 20 & & NS & $\mu g / L$ \\
\hline 1,2-Dichloroethane & WA & & & $\mathbf{U}$ & 5 & & 5 & $\mu g / L$ \\
\hline 1,2-Dichloroethylene (total) & WA & & . & $\mathbf{U}$ & 5 & & 100 & $\mu g / L$ \\
\hline 1,2-Dichloropropane & WA & & & $\mathbf{U}$ & 5 & & 5 & $\mu g / L$ \\
\hline 2-Hexanone & WA & & & $\mathbf{U}$ & 10 & & NS & $\mu g / L$ \\
\hline 4-Methyl-2-pentanone & WA & & & $\mathbf{U}$ & 10 & & NS & $\mu g / L$ \\
\hline Acetone & WA & & & & 12.6 & & NS & $\mu g / L$ \\
\hline Acetonitrile & WA & & & $\mathbf{U}$ & 20 & & NS & $\mu g / L$ \\
\hline Acidity & WA & & & & 1.5 & & NS & $\mathrm{mg} / \mathrm{L}$ \\
\hline Acrolein & WA & & & $\mathbf{U}$ & 10 & & NS & $\mu g / L$ \\
\hline Acrylonitrile & WA & & & $\mathbf{U}$ & 10 & & NS & $\mu g / L$ \\
\hline Alkalinity & WA & & & & 0.5 & & NS & $\mathrm{mg} / \mathrm{L}$ \\
\hline Allyl chloride & WA & & & $\mathbf{U}$ & 100 & & NS & $\mu \mathrm{g} / \mathrm{L}$ \\
\hline Aluminum & WA & & & $\mathbf{U}$ & 200 & & NS & $\mu \mathrm{g} / \mathrm{L}$ \\
\hline Antimony & WA & & & $\mathbf{U}$ & 60 & & 6 & $\mu \mathrm{g} / \mathrm{L}$ \\
\hline Arsenic & WA & & & $\mathbf{U}$ & 10 & & 50 & $\mu g / L$ \\
\hline Barium & WA & & & $\mathbf{U}$ & 200 & & 2000 & $\mu \mathrm{g} / \mathrm{L}$ \\
\hline Benzene & WA & & & $\mathbf{U}$ & 5 & & 5 & $\mu \mathrm{g} / \mathrm{L}$ \\
\hline Beryllium & WA & & & $\mathbf{U}$ & 5 & & 4 & $\mu \mathrm{g} / \mathrm{L}$ \\
\hline Bromodichloromethane & WA & & & $\mathbf{U}$ & 5 & & NS & $\mu \mathrm{g} / \mathrm{L}$ \\
\hline Bromoform & WA & & & $\mathbf{U}$ & 5 & & NS & $\mu g / L$ \\
\hline Cadmium & WA & & & $\mathbf{U}$ & 5 & & 5 & $\mu g / L$ \\
\hline Calcium & WA & & & $\mathbf{U}$ & 5000 & & NS & $\mu g / L$ \\
\hline Carbon disulfide & WA & & & $\mathbf{U}$ & 5 & & NS & $\mu g / L$ \\
\hline Carbon tetrachloride & WA & & & $\mathbf{U}$ & 5 & & 5 & $\mu \mathrm{g} / \mathrm{L}$ \\
\hline Cesium-137 & TM & & & $\mathbf{U}$ & 5.8 & & 200 & $\mathrm{pCi} / \mathrm{L}$ \\
\hline Chloride & WA & & & & 0.293 & & NS & $\mathrm{mg} / \mathrm{L}$ \\
\hline Chlorobenzene & WA & & & $\mathbf{U}$ & 5 & & 100 & $\mu g / L$ \\
\hline Chloroethane & WA & & & $\mathbf{U}$ & 10 & & NS & $\mu \mathrm{g} / \mathrm{L}$ \\
\hline Chloroform & WA & & & $\mathbf{U}$ & 5 & & 100 & $\mu \mathrm{g} / \mathrm{L}$ \\
\hline Chloroprene - & WA & & & $\mathbf{U}$ & 20 & & NS & $\mu \mathrm{g} / \mathrm{L}$ \\
\hline Chromium & WA & & & $\mathrm{U}$ & 10 & & 100 & $\mu \mathrm{g} / \mathrm{L}$ \\
\hline cis-1,3-Dichloropropene & WA & & & $\mathbf{U}$ & 5 & & NS & $\mu \mathrm{g} / \mathrm{L}$ \\
\hline Cobalt & WA & & & $\mathbf{U}$ & 50 & & NS & $\mu g / L$ \\
\hline Cobalt-60 & TM & & & & 4.2 & 1.4 & 100 & $\mathrm{pCi} / \mathrm{L}$ \\
\hline Copper & WA & & & U & 25 & & 1000 & $\mu g / L$ \\
\hline Cyanide & WA & & & $U$ & 10 & & 200 & $\mu \mathrm{g} / \mathrm{L}$ \\
\hline Dibromochloromethane & WA & & & $\mathbf{U}$ & 5 & & NS & $\mu \mathrm{g} / \mathrm{L}$ \\
\hline Dichlorodifluoromethane & WA & & & $\mathbf{U}$ & 10 & & NS & $\mu g / L$ \\
\hline Ethylbenzene & WA & & & $\mathbf{U}$ & 5 & & 700 & $\mu \mathrm{g} / \mathrm{L}$ \\
\hline Fluoride & WA & & & $\mathbf{U}$ & 0.1 & & 4 & $\mathrm{mg} / \mathrm{L}$ \\
\hline
\end{tabular}


UQA007 Laboratory Analyses (cont'd)

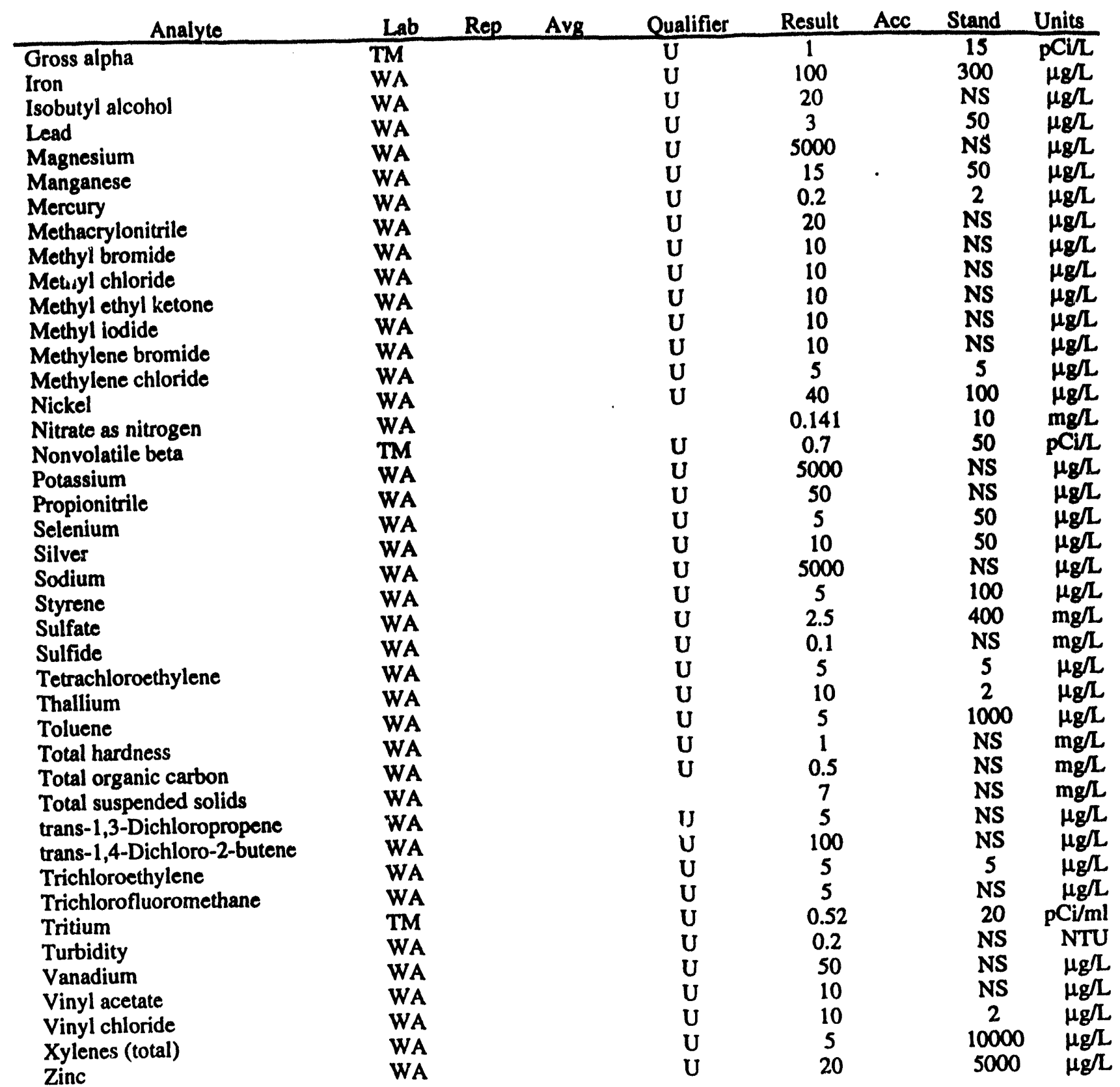




\section{UQA008}

(Equipment Blank - Shuvel)

Field Measurements:

Sample Date: 4/19/93

\begin{tabular}{|c|c|c|c|c|c|c|c|c|}
\hline Analyte & Lab & Rep & Avg & Qualifier & Result & Acc & Stand & Units \\
\hline 1,1,1,2-Tetrachloroethane & $\overline{\text { WA }}$ & & & $\overline{\mathrm{U}}$ & 10 & & NS & $\mu \mathrm{g} / \mathrm{L}$ \\
\hline 1,1,1,2-Tetrachloroethane & WA & & NA & U & 10 & & NS & $\mu g / L$ \\
\hline $1,1,1,2$-Tetrachloroethane & WA & & NA & $\mathbf{U}$ & 10 & & NS & $\mu g / L$ \\
\hline 1,1,1-Trichloroethane & WA & & & $\mathbf{U}$ & 5 & & 200 & $\mu \mathrm{g} / \mathrm{L}$ \\
\hline 1,1,1-Trichloroethane & WA & & NA & $\mathbf{U}$ & 5 & & 200 & $\mu g / L$ \\
\hline $1,1,1$-Trichloroethane & WA & & NA & U & 5 & & 200 & $\mu g / L$ \\
\hline $1,1,2,2$-Tetrachloroethane & WA & & & U & 5 & & NS & $\mu g / \Omega$ \\
\hline 1,1,2,2-Tetrachloroethane & WA & & NA & $\mathbf{U}$ & 5 & & NS & $\mu g / L$ \\
\hline $1,1,2,2$-Tetrachloroethane & WA & & NA & U & 5 & & NS & $\mu \mathrm{g} / \mathrm{L}$ \\
\hline 1,1,2-Trichloroethane & WA & & & $\mathbf{U}$ & 5 & & 5 & $\mu g / L$ \\
\hline 1,1,2-Trichloroethane & WA & & NA & $\mathbf{U}$ & 5 & & 5 & $\mu g / L$ \\
\hline 1,1,2-Trichloroethane & WA & & NA & $\mathbf{U}$ & 5 & & 5 & $\mu g / L$ \\
\hline 1,1-Dichloroethane & WA & & & $\mathrm{U}$ & 5 & & NS & $\mu g / L$ \\
\hline 1,1-Dichloroethane & WA & & NA & $\mathbf{U}$ & 5 & & NS & $\mu g / L$ \\
\hline 1,1-Dichloroethane & WA & & NA & $\mathbf{U}$ & 5 & & NS & $\mu g / L$ \\
\hline 1,1-Dichloroethylene & WA & & & $\mathbf{U}$ & 5 & & 7 & $\mu \mathrm{g} / \mathrm{L}$ \\
\hline 1,2,3-Trichloropropane & WA & & & $\mathbf{U}$ & 10 & & NS & $\mu g /$ \\
\hline 1,2,3-Trichloropropane & WA & & NA & $\mathrm{U}$ & 10 & & NS & $\mu g / L$ \\
\hline 1,2,3-Trichloropropane & WA & & NA & $\mathrm{U}$ & 10 & & NS & $\mu g / L$ \\
\hline 1,2-Dibromo-3-chloropropane & WA & & & U & 20 & & 0.2 & $\mu g / L$ \\
\hline 1,2-Dibromo-3-chloropropane & WA & & NA & $\mathbf{U}$ & 20 & & 0.2 & $\mu \mathrm{g} / \mathrm{L}$ \\
\hline 1,2-Dibromo-3-chloropropane & WA & & NA & $\mathbf{U}$ & 20 & & 0.2 & $\mu g / L$ \\
\hline 1,2-Dibromoethane & WA & & & U & 20 & & NS & $\mu \mathrm{g} / \mathrm{L}$ \\
\hline 1,2-Dibromoethane & WA & & NA & $\mathbf{U}$ & 20 & & NS & $\mu g / L$ \\
\hline 1,2-Dibromoethane & WA & & NA & $\mathbf{U}$ & 20 & & NS & $\mu g / L$ \\
\hline 1,2-Dichloroethane & WA & & & U & 5 & & 5 & $\mu \mathrm{g} / \mathrm{L}$ \\
\hline 1,2-Dichloroethane & WA & & NA & $\mathbf{U}$ & 5 & & 5 & $\mu g / L$ \\
\hline 1,2-Dichloroethane & WA & & NA & $\mathbf{U}$ & 5 & & 5 & $\mu g / L$ \\
\hline 1,2-Dichloroethylene (total) & WA & & & $\mathbf{U}$ & 5 & & 100 & $\mu \mathrm{g} / \mathrm{L}$ \\
\hline 1,2-Dichloroethylene (total) & WA & & NA & $\mathbf{U}$ & 5 & & 100 & $\mu g / L$ \\
\hline 1,2-Dichloroethylene (total) & WA & & NA & U & 5 & & 100 & $\mu g / L$ \\
\hline 1,2-Dichloropropane & WA & & & $\mathbf{U}$ & 5 & & 5 & $\mu g / L$ \\
\hline 1,2-Dichloropropaite & WA & & NA & $\mathbf{U}$ & 5 & & 5 & $\mu g / L$ \\
\hline 1,2-Dichloropropane & WA & & NA & U & 5 & & 5 & $\mu \mathrm{g} / \mathrm{L}$ \\
\hline 2-Hexanone & WA & & & $\mathrm{U}$ & 10 & & NS & $\mu \mathrm{g} / \mathrm{L}$ \\
\hline 2-Hexanone & WA & & NA & $\mathrm{U}$ & 10 & & NS & $\mu g / L$ \\
\hline 2-Hexanone & WA & & NA & $\mathbf{U}$ & 10 & & NS & $\mu \mathrm{g} /$ \\
\hline 4-Methyl-2-pentanone & WA & & & U & 10 & & NS & $\mu \mathrm{g}$ \\
\hline 4-Methyl-2-pentanone & WA & & NA & $\mathbf{U}$ & 10 & & NS & $\mu g / L$ \\
\hline 4-Methyl-2-pentanone & WA & & NA & $\mathrm{U}$ & 10 & & NS & \\
\hline Acetone & WA & & & $\mathrm{U}$ & 10 & & NS & $\mu \mathrm{g} / \mathrm{L}$ \\
\hline Acetone & WA & & NA & J & 9.75 & & NS & $\mu g$ \\
\hline Acetone & WA & & NA & J & 4.36 & & NS & $\mu \mathrm{g}$ \\
\hline Acetonitrile & WA & & & U & 20 & & NS & $\mu \mathrm{g} / \mathrm{L}$ \\
\hline Acetonitrile & WA & & NA & U & 20 & & NS & \\
\hline Acetonitrile & WA & & NA & U & 20 & & NS & $\mu g / L$ \\
\hline Acidity & WA & & & & 1.5 & & NS & \\
\hline
\end{tabular}



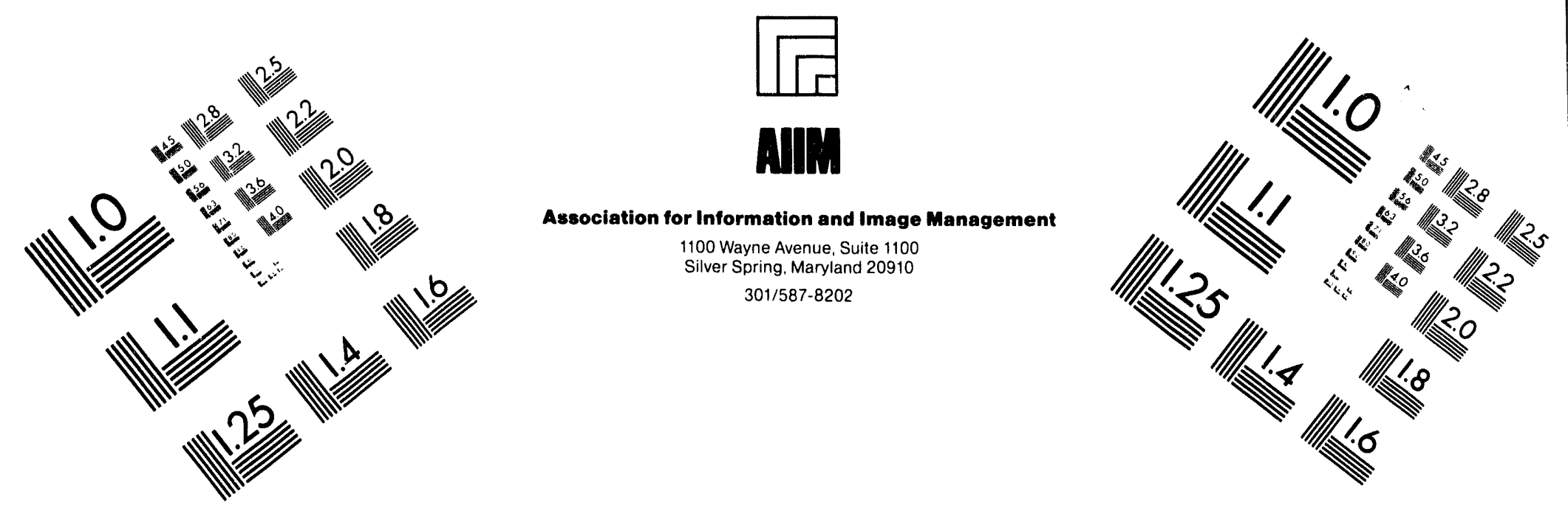

\section{Centimeter}

$\begin{array}{llllllllllllllll}1 & 2 & 3 & 4 & 5 & 6 & 7 & 8 & 9 & 10 & 11 & 12 & 13 & 14 & 15 & \mathrm{~mm}\end{array}$

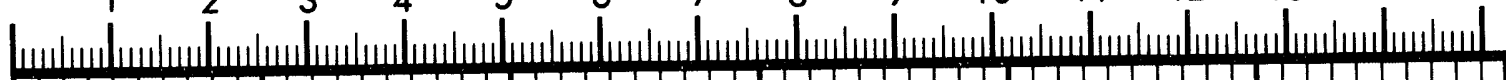

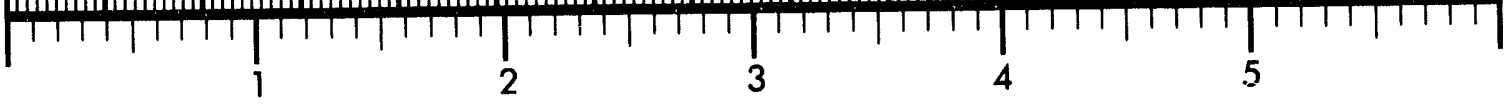
Inches
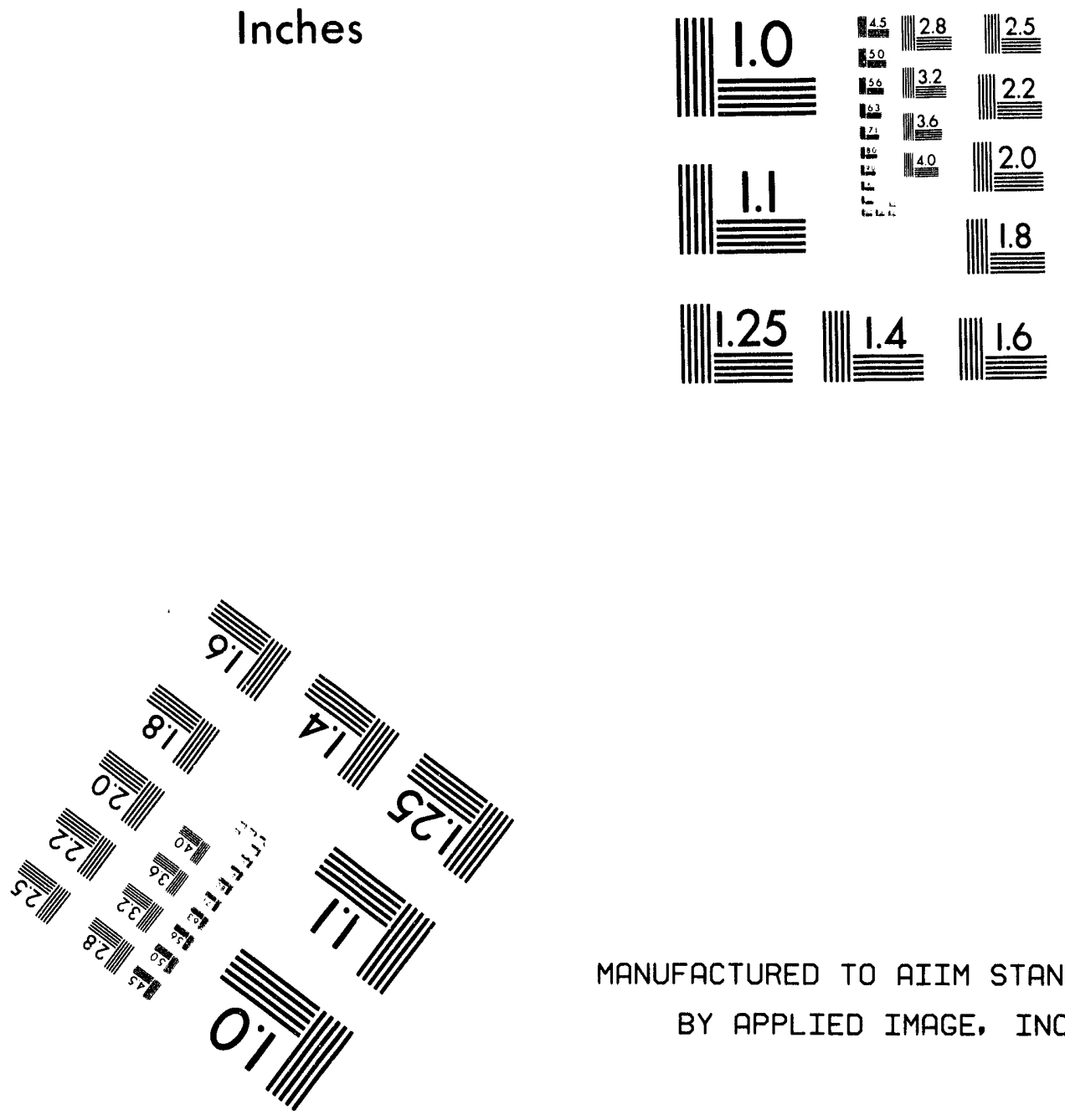

MANUFACTURED TO AIIM STANDARDS BY APPLIED IMAGE. INC.

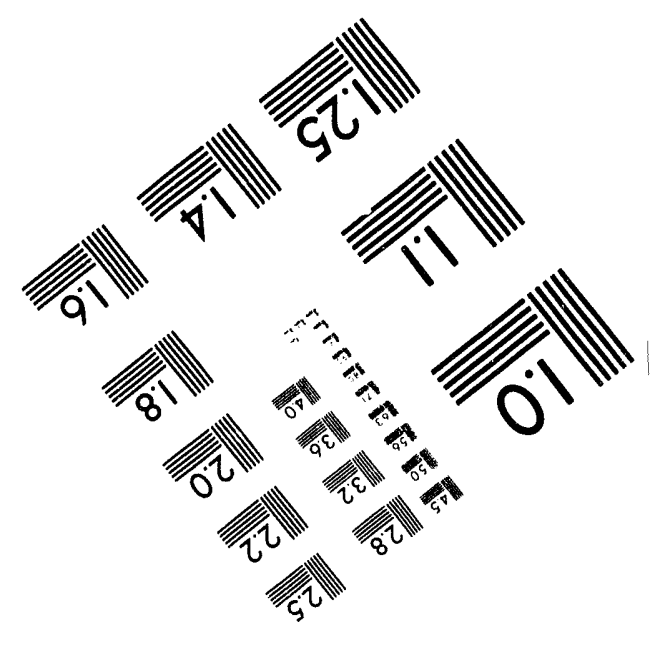



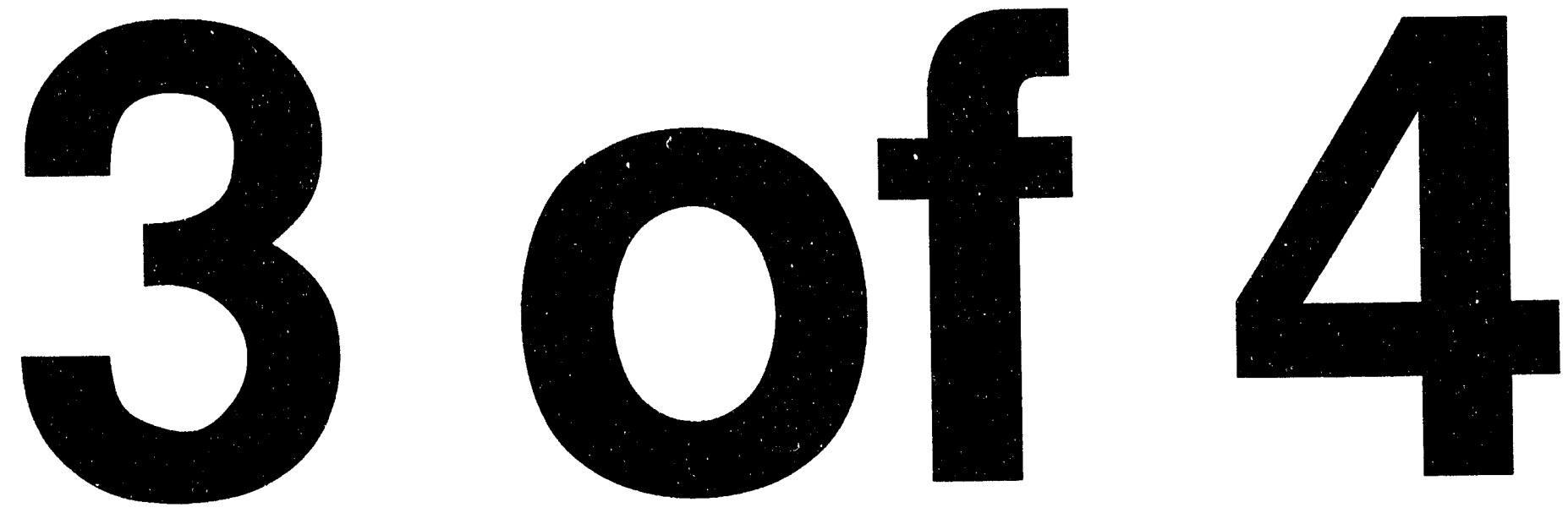
UQA008 Laboratory Analyses (cont'd)

\begin{tabular}{|c|c|c|c|c|c|c|c|c|}
\hline Analyte & Lab & Rep & Avg & Qualifier & Result & Acc & Stand & Units \\
\hline Acrolein & WA & & & $\mathbf{U}$ & 10 & & NS & $\mu g / L$ \\
\hline Acrolein & WA & & NA & $\mathbf{U}$ & 10 & & NS & $\mu g / L$ \\
\hline Acrolein & WA & & NA & $\mathbf{U}$ & 10 & & NS & $\mu \mathrm{g} / \mathrm{L}$ \\
\hline Acrylonitrile & WA & & & $\mathbf{U}$ & 10 & & NS & $\mu \mathrm{g} / \mathrm{L}$ \\
\hline Acrylonitrile & WA & & NA & U & 10 & & NS & $\mu \mathrm{g} / \mathrm{L}$ \\
\hline Acrylonitrile & WA & & NA & U & 10 & & NS & $\mu \mathrm{g} / \mathrm{L}$ \\
\hline Alkalinity & WA & & & & 0.5 & & NS & $\mathrm{mg} / \mathrm{L}$ \\
\hline Allyl chloride & WA & & & $\mathbf{U}$ & 100 & & NS & $\mu \mathrm{g} / \mathrm{L}$ \\
\hline Allyl chloride & WA & & NA & U & 100 & & NS & $\mu g / L$ \\
\hline Allyl chloride & WA & & NA & $\mathbf{U}$ & 100 & & NS & $\mu g / L$ \\
\hline Aluminum & WA & & & $\mathbf{U}$ & 200 & & NS & $\mu g / L$ \\
\hline Antimony & WA & & & $\mathbf{U}$ & 60 & & 6 & $\mu \mathrm{g} / \mathrm{L}$ \\
\hline Arsenic & WA & & & $\mathbf{U}$ & 10 & & 50 & $\mu \mathrm{g} / \mathrm{L}$ \\
\hline Barium & WA & & & $\mathbf{U}$ & 200 & & 2000 & $\mu \mathrm{g} / \mathrm{L}$ \\
\hline Benzene & WA & & & $U$ & 5 & & 5 & $\mu g / L$ \\
\hline Beryllium & WA & & & U & 5 & & 4 & $\mu g / L$ \\
\hline Bromodichloromethane & WA & & & $\mathbf{U}$ & 5 & & NS & $\mu g / L$ \\
\hline Bromodichloromethane & WA & & NA & $\mathbf{U}$ & 5 & & NS & $\mu \mathrm{g} / \mathrm{L}$ \\
\hline Bromodichloromethane & WA & & NA & $\mathbf{U}$ & 5 & & NS & $\mu \mathrm{g} / \mathrm{L}$ \\
\hline Bromoform & WA & & & $\mathbf{U}$ & 5 & & NS & $\mu g / L$ \\
\hline Bromoform & WA & & NA & $\mathbf{U}$ & 5 & & NS & $\mu g / L$ \\
\hline Bromoform & WA & & NA & U & 5 & & NS & $\mu g / L$ \\
\hline Cadmium & WA & & & $\mathbf{U}$ & 5 & & 5 & $\mu g / L$ \\
\hline Calcium & WA & & & $\mathbf{U}$ & 5000 & & NS & $\mu g / L$ \\
\hline Carbon disulfide & WA & & & $\mathrm{U}$ & 5 & & NS & $\mu g / L$ \\
\hline Carbon disulfide & WA & & NA & $\mathbf{U}$ & 5 & & NS & $\mu g / L$ \\
\hline Carbon disulfide & WA & & NA & $\mathbf{U}$ & 5 & & NS & $\mu g / L$ \\
\hline Carbon tetrachloride & WA & & & $\mathbf{U}$ & 5 & & 5 & $\mu g / L$ \\
\hline Carbon tetrachloride & WA & & NA & U & 5 & & 5 & $\mu g / L$ \\
\hline Carbon tetrachloride & WA & & NA & $\mathbf{U}$ & 5 & & 5 & $\mu g / \Lambda$ \\
\hline Cesium-137 & TM & & & $\mathbf{U}$ & 10 & & 200 & $\mathrm{pCi} / \mathrm{L}$ \\
\hline Chloride & WA & & & & 0.274 & & NS & $\mathrm{mg} / \mathrm{L}$ \\
\hline Chlorobenzene & WA & & & $\mathbf{U}$ & 5 & & 100 & $\mu g / \mathrm{L}$ \\
\hline Chloroethane & WA & & & $\mathbf{U}$ & 10 & & NS & $\mu g / L$ \\
\hline Chloroethane & WA & & NA & $\mathbf{U}$ & 10 & & NS & $\mu g / L$ \\
\hline Chloroethane & WA & & NA & U & 10 & & NS & $\mu g / L$ \\
\hline Chloroform & WA & & & $\mathbf{U}$ & 5 & & 100 & $\mu \mathrm{g} / \mathrm{L}$ \\
\hline Chloroform & WA & & NA & $\mathbf{U}$ & 5 & & 100 & $\mu g / L$ \\
\hline Chloroform & WA & & NA & $\mathbf{U}$ & 5 & & 100 & $\mu g / L$ \\
\hline Chloroprene & WA & & & $\mathrm{U}$ & 20 & & NS & $\mu \mathrm{g} / \mathrm{L}$ \\
\hline Chloroprene & WA & & NA & $\mathbf{U}$ & 20 & & NS & $\mu \mathrm{g} / \mathrm{L}$ \\
\hline Chloroprene & WA & & NA & $U$ & 20 & & NS & $\mu \mathrm{g} / \mathrm{L}$ \\
\hline Chromium & WA & & & $\mathrm{U}$ & 10 & & 100 & $\mu g / L$ \\
\hline cis-1,3-Dichloropropene & WA & & & $\mathrm{U}$ & 5 & & NS & $\mu \mathrm{g} / \mathrm{L}$ \\
\hline cis-1,3-Dichloropropene & WA & & NA & $\mathrm{U}$ & 5 & & NS & $\mu g / L$ \\
\hline cis-1,3-Dichloropropene & WA & & NA & $\mathbf{U}$ & 5 & & NS & $\mu \mathrm{g} / \mathrm{L}$ \\
\hline Cobalt & WA & & & $\mathbf{U}$ & 50 & & NS & $\mu \mathrm{g} / \mathrm{L}$ \\
\hline Copper & WA & & & U & 25 & & 1000 & $\mu \mathrm{g} / \mathrm{L}$ \\
\hline Cyanide & WA & & & U & 10 & & 200 & $\mu \mathrm{g} / \mathrm{L}$ \\
\hline Dibromochloromethane & WA & & & $\mathrm{U}$ & 5 & & NS & $\mu \mathrm{g} / \mathrm{L}$ \\
\hline Dibromochloromethane & WA & & NA & $\mathbf{U}$ & 5 & & NS & $\mu \mathrm{g} / \mathrm{L}$ \\
\hline Dibromochloromethane & WA & & NA & $\mathbf{U}$ & 5 & & NS & $\mu \mathrm{g} / \mathrm{L}$ \\
\hline Dichlorodifluoromethane & WA & & & $\mathbf{U}$ & 10 & & NS & $\mu g / L$ \\
\hline Dichlorodifluoromethane & WA & & NA & $\mathbf{U}$ & 10 & & NS & $\mu g / L$ \\
\hline Dichlorodifluoromethane & WA & & NA & $\mathbf{U}$ & 10 & & NS & \\
\hline
\end{tabular}


UQA008 Laboratory Analyses (cont'd)

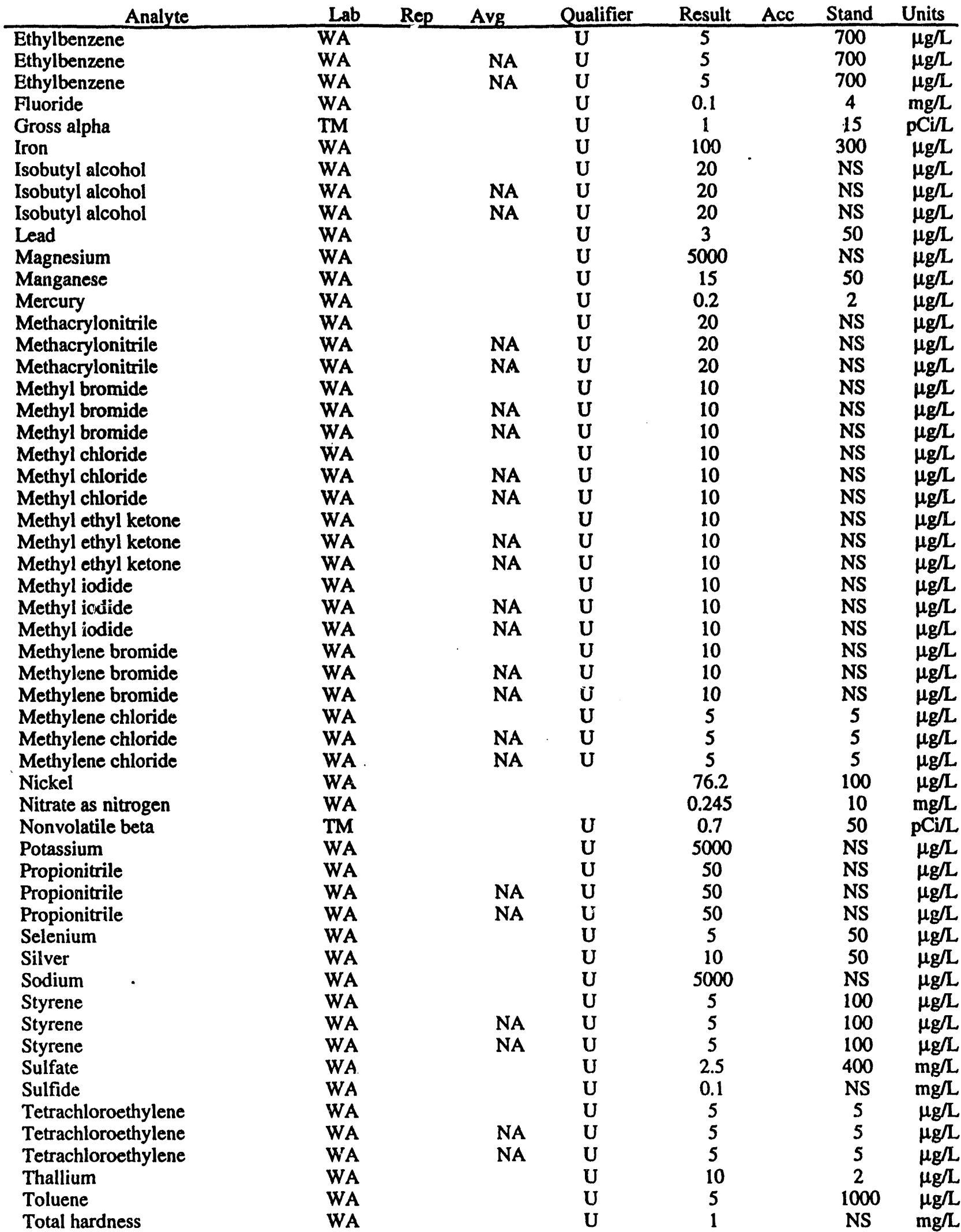


UQA008 Laboratory Analyses (cont'd)

\begin{tabular}{|c|c|c|c|c|c|c|c|c|}
\hline Analyte & Lab & Rep & Avg & Qualifier & Result & Acc & Stand & Units \\
\hline Total organic carbon & $\overline{\text { WA }}$ & & & $\mathbf{U}$ & 0.5 & & NS & $\overline{\mathrm{mg} / \mathrm{L}}$ \\
\hline Total suspended solids & WA & & & & 7 & & NS & $\mathrm{mg} / \mathrm{L}$ \\
\hline trans-1,3-Dichloropropene & WA & & & $\mathbf{U}$ & 5 & & NS & $\mu \mathrm{g} / \mathrm{L}$ \\
\hline trans-1,3-Dichloropropene & WA & & NA & $\mathbf{U}$ & 5 & & NS & $\mu g / L$ \\
\hline trans-1,3-Dichloropropene & WA & & NA & $\mathbf{U}$ & 5 & & NS & $\mu g / L$ \\
\hline trans-1,4-Dichloro-2-butene & WA & & & $\mathbf{U}$ & 100 & - & NS & $\mu g / L$ \\
\hline trans-1,4-Dichloro-2-butene & WA & & NA & $\mathbf{U}$ & 100 & & NS & $\mu \mathrm{g} / \mathrm{L}$ \\
\hline trans-1,4-Dichloro-2-butene & WA & & NA & $\mathbf{U}$ & 100 & & NS & $\mu g / L$ \\
\hline Trichloroethylene & WA & & & $\mathbf{U}$ & 5 & & 5 & $\mu g / L$ \\
\hline Trichlorofluoromethane & WA & & & $\mathbf{U}$ & 5 & & NS & $\mu \mathrm{g} / \mathrm{L}$ \\
\hline Trichlorofluoromethane & WA & & $\mathbf{N A}$ & $\mathbf{U}$ & 5 & & NS & $\mu g / L$ \\
\hline Trichlorofluoromethane & WA & & $\mathbf{N A}$ & $\mathbf{U}$ & 5 & & NS & $\mu g / L$ \\
\hline Tritium & TM & & & $\mathbf{U}$ & 0.51 & & 20 & $\mathrm{pCi} / \mathrm{ml}$ \\
\hline Turbidity & WA & & & $\mathbf{U}$ & 0.2 & & NS & NTU \\
\hline Vanadium & WA & & & $\mathbf{U}$ & 50 & & NS & $\mu g / L$ \\
\hline Vinyl acetate & WA & & & $\mathbf{U}$ & 10 & & NS & $\mu \mathrm{g} / \mathrm{L}$ \\
\hline Vinyl acetate & WA & & NA & $\mathbf{U}$ & 10 & & NS & $\mu g / L$ \\
\hline Vinyl acetate & WA & & NA & $\mathbf{U}$ & 10 & & NS & $\mu g / L$ \\
\hline Vinyl chloride & WA & & & $\mathbf{U}$ & 10 & & 2 & $\mu \mathrm{g} / \mathrm{L}$ \\
\hline Vinyl chloride & WA & & NA & $\mathbf{U}$ & 10 & & 2 & $\mu \mathrm{g} / \mathrm{L}$ \\
\hline Vinyl chloride & WA & & NA & $\mathbf{U}$ & 10 & & 2 & $\mu g / L$ \\
\hline Xylenes (total) & WA & & & $\mathbf{U}$ & 5 & & 10000 & $\mu g / L$ \\
\hline Xylenes (total) & WA & & NA & $\mathbf{U}$ & 5 & & 10000 & $\mu g / L$ \\
\hline Xylenes (total) & WA & & NA & $\mathbf{U}$ & 5 & & 10000 & $\mu g / L$ \\
\hline Zinc & WA & & & $\mathbf{U}$ & 20 & & 5000 & $\mu g / L$ \\
\hline
\end{tabular}


UQA009

(Equipment Blank - Shovel)

Field Measurements:

Sample Date: 4/21/93

\begin{tabular}{|c|c|c|c|c|c|c|c|}
\hline Analyte & Lab & Rep & Qualifier & Result & Acc & Stand & Units \\
\hline 1,1,1,2-Tetrachloroethane & WA & & $\mathbf{U}$ & 10 & & NS & $\mu g / L$ \\
\hline 1,1,1-Trichloroethane & WA & & $\mathbf{U}$ & 5 & & 200 & $\mu \mathrm{g} / \mathrm{L}$ \\
\hline 1,1,2,2-Tetrachloroethane & WA & & $\mathbf{U}$ & 5 & & NS & $\mu g / L$ \\
\hline 1,1,2-Trichloroethane & WA & & $\mathbf{U}$ & 5 & & 5 & $\mu \mathrm{g} / \mathrm{L}$ \\
\hline 1,1-Dichloroethane & WA & & $\mathbf{U}$ & 5 & & NS & $\mu g / L$ \\
\hline 1,1-Dichloroethylene & WA & & $\mathbf{U}$ & 5 & & 7 & $\mu g / L$ \\
\hline 1,2,3-Trichloropropane & WA & & U & 10 & & NS & $\mu g / L$ \\
\hline 1,2-Dibromo-3-chloropropane & WA & & $\mathbf{U}$ & 20 & & 0.2 & $\mu \mathrm{g} / \mathrm{L}$ \\
\hline 1,2-Dibromoethane & WA & & $\mathbf{U}$ & 20 & & NS & $\mu g / L$ \\
\hline 1,2-Dichloroethane & WA & & $\mathbf{U}$ & 5 & & 5 & $\mu g / L$ \\
\hline 1,2-Dichloroethylene (total) & WA & & $\mathbf{U}$ & 5 & & 100 & $\mu g / L$ \\
\hline 1,2-Dichloropropane & WA & & $\mathbf{U}$ & 5 & & 5 & $\mu g / L$ \\
\hline 2-Hexanone & WA & & $\mathbf{U}$ & 10 & & NS & $\mu g / L$ \\
\hline 4-Methyl-2-pentanone & WA & & $\mathbf{U}$ & 10 & & NS & $\mu g / L$ \\
\hline Acetone & WA & & $\mathbf{U}$ & 10 & & NS & "g/L \\
\hline Acetonitrile & WA & & $\mathbf{U}$ & 20 & & NS & $\mu \mathrm{g} / \mathrm{L}$ \\
\hline Acidity & WA & & & 2 & & NS & $\mathrm{mg} / \mathrm{L}$ \\
\hline Acrolein & WA & & $\mathbf{U}$ & 10 & & NS & $\mu \mathrm{g} / \mathrm{L}$ \\
\hline Acrylonitrile & WA & & U & 10 & & NS & $\mu \mathrm{g} / \mathrm{L}$ \\
\hline Alkalinity & WA & & & 1 & & NS & $\mathrm{mg} / \mathrm{L}$ \\
\hline Allyl chloride & WA & & $\mathbf{U}$ & 100 & & NS & $\mu \mathrm{g} / \mathrm{L}$ \\
\hline Aluminum & WA & & $\mathbf{U}$ & 200 & & NS & $\mu g / L$ \\
\hline Antimony & WA & & $\mathbf{U}$ & 60 & & 6 & $\mu g / L$ \\
\hline Arsenic & WA & & $\mathbf{U}$ & 10 & & 50 & $\mu \mathrm{g} / \mathrm{L}$ \\
\hline Barium & WA & & $\mathbf{U}$ & 200 & & 2000 & $\mu \mathrm{g} / \mathrm{L}$ \\
\hline Benzene & WA & & $\mathbf{U}$ & 5 & & 5 & $\mu g / L$ \\
\hline Beryllium & WA & & $\mathbf{U}$ & 5 & & 4 & $\mu \mathrm{g} / \mathrm{L}$ \\
\hline Bromodichloromethane & WA & & U & 5 & & NS & $\mu \mathrm{g} / \mathrm{L}$ \\
\hline Bromoform & WA & & $\mathbf{U}$ & 5 & & NS & $\mu g / L$ \\
\hline Cadmium & WA & & $\mathbf{U}$ & 5 & & 5 & $\mu \mathrm{g} / \mathrm{L}$ \\
\hline Calcium & WA & & $\mathbf{U}$ & 5000 & & NS & $\mu \mathrm{g} / \mathrm{L}$ \\
\hline Carbon disulfide & WA & & $\mathbf{U}$ & 5 & & NS & $\mu \mathrm{g} / \mathrm{L}$ \\
\hline Carbon tetrachloride & WA & & $\mathbf{U}$ & 5 & & 5 & $\mu \mathrm{g} / \mathrm{L}$ \\
\hline Cesium-137 & TM & & & 3.2 & 5.4 & 200 & $\mathrm{pCi} / \mathrm{L}$ \\
\hline Chloride & WA & & & 0.392 & & NS & $\mathrm{mg} / \mathrm{L}$ \\
\hline Chlorobenzene & WA & & U & 5 & & 100 & $\mu \mathrm{g} / \mathrm{L}$ \\
\hline Chloroethane & WA & & U & 10 & & NS & $\mu \mathrm{g} / \mathrm{L}$ \\
\hline Chloroform & WA & & $\mathbf{U}$ & 5 & & 100 & $\mu \mathrm{g} / \mathrm{L}$ \\
\hline Chloroprene & WA & & $\mathrm{U}$ & 20 & & NS & $\mu \mathrm{g} / \mathrm{L}$ \\
\hline Chromium & WA & & $\mathbf{U}$ & 10 & & 100 & $\mu \mathrm{g} / \mathrm{L}$ \\
\hline cis-1,3-Dichloropropene & WA & & $\mathbf{U}$ & 5 & & NS & $\mu g / L$ \\
\hline Cobalt & WA & & $\mathbf{U}$ & 50 & & NS & $\mu g / L$ \\
\hline Copper & WA & & $\mathbf{U}$ & 25 & & 1000 & $\mu g / L$ \\
\hline Cyanide & WA & & U & 10 & & 200 & $\mu g / L$ \\
\hline Dibromochloromethane & WA & & $\mathrm{U}$ & 5 & & NS & $\mu \mathrm{g} / \mathrm{L}$ \\
\hline Dichlorodifluoromethane & WA & & $\mathbf{U}$ & 10 & & NS & $\mu \mathrm{g} / \mathrm{L}$ \\
\hline Ethylbenzene & WA & & U & 5 & & 700 & $\mu \mathrm{g} / \mathrm{L}$ \\
\hline Fluoride & WA & & $\mathbf{U}$ & 0.1 & & 4 & $\mathrm{mg} / \mathrm{L}$ \\
\hline Gross alpha & TM & & & 34 & 4.1 & 15 & $\mathrm{pCi} / \mathrm{L}$ \\
\hline
\end{tabular}


UQA009 Laboratory Analyses (cont'd)

\begin{tabular}{|c|c|c|c|c|c|c|c|c|}
\hline Analyte & Lab & Rep & Avg & Qualifier & Result & Acc & Stand & Units \\
\hline Iron & WA & & & $\mathbf{U}$ & 100 & & 300 & $\mu \mathrm{g} / \mathrm{L}$ \\
\hline Isobutyl alcohol & WA & & & $\mathbf{U}$ & 20 & & NS & $\mu \mathrm{g} / \mathrm{L}$. \\
\hline Lead & WA & & & & 3 & & 50 & $\mu g / L$ \\
\hline Magnesium & WA & & & $\mathbf{U}$ & 5000 & & NS & $\mu \mathrm{g} / \mathrm{L}$ \\
\hline Manganese & WA & & & $\mathbf{U}$ & 15 & & 50 & $\mu \mathrm{g} / \mathrm{L}$ \\
\hline Mercury & WA & & & $\mathbf{U}$ & 0.2 & . & 2 & $\mu g / L$ \\
\hline Methacrylonitrile & WA & & & $\mathbf{U}$ & 20 & & NS & $\mu g / L$ \\
\hline Methyl bromide & WA & & & $\mathbf{U}$ & 10 & & NS & $\mu \mathrm{g} / \mathrm{L}$ \\
\hline Methyl chloride & WA & & & $\mathbf{U}$ & 10 & & NS & $\mu g / L$ \\
\hline Methyl ethyl ketone & WA & & & $\mathbf{U}$ & 10 & & NS & $\mu \mathrm{g} / \mathrm{L}$ \\
\hline Methyl iodide & WA & & & $\mathbf{U}$ & 10 & & NS & $\mu \mathrm{g} / \mathrm{L}$ \\
\hline Methylene bromide & WA & & & $\mathbf{U}$ & 10 & & NS & $\mu g / L$ \\
\hline Methylene chloride & WA & & & $\mathbf{U}$ & 5 & & 5 & $\mu g / L$ \\
\hline Nickel & WA & & & $\mathbf{U}$ & 40 & & 100 & $\mu g / L$ \\
\hline Nitrate as nitrogen & WA & & & & 0.025 & & 10 & $\mathrm{mg} / \mathrm{L}$ \\
\hline Nonvolatile beta & TM & & & & 841 & 17.2 & 50 & $\mathrm{pCi} / \mathrm{L}$ \\
\hline Potassium & WA & & & $\mathbf{U}$ & 5000 & & NS & $\mu g / L$ \\
\hline Propionitrile & WA & & & $\mathbf{U}$ & 50 & & NS & $\mu g / L$ \\
\hline Selenium & WA & & & $\mathbf{U}$ & 5 & & 50 & $\mu g / L$ \\
\hline Silver & WA & & & $\mathbf{U}$ & 10 & & 50 & $\mu g /$. \\
\hline Sodium & WA & & & U & 5000 & & NS & $\mu \mathrm{g} / \mathrm{L}$ \\
\hline Styrene & WA & & & $\mathbf{U}$ & 5 & & 100 & $\mu \mathrm{g} / \mathrm{L}$ \\
\hline Sulfate & WA & & & $\mathbf{U}$ & 2.5 & & 400 & $\mathrm{mg} / \mathrm{L}$ \\
\hline Sulfide & WA & & & $\mathbf{U}$ & 0.1 & & NS & $\mathrm{mg} / \mathrm{L}$ \\
\hline Tetrachloroethylene & WA & & & $\mathbf{U}$ & 5 & & 5 & $\mu g / L$ \\
\hline Thallium & WA & & & $\mathbf{U}$ & 10 & & 2 & $\mu g / L$ \\
\hline Toluene & WA & & & $\mathbf{U}$ & 5 & & 1000 & $\mu g / L$ \\
\hline Total hardness & WA & & & U & 0.1 & & NS & $\mathrm{mg} / \mathrm{L}$ \\
\hline Total organic carbon & WA & & & & 0.67 & & NS & $\mathrm{mg} / \mathrm{L}$ \\
\hline Total suspended solids & WA & & & $\mathbf{U}$ & 5 & & NS & $\mathrm{mg} / \mathrm{h}$ \\
\hline trans-1,3-Dichloropropene & WA & & & $\mathbf{U}$ & 5 & & NS & $\mu g / L$ \\
\hline trans-1,4-Dichloro-2-butene & WA & & & $\mathbf{U}$ & 100 & & NS & $\mu g / 2$ \\
\hline Trichloroethylene & WA & & & $\mathrm{U}$ & 5 & & 5 & $\mu g / \mathrm{L}$ \\
\hline Trichlorofluoromethane & WA & & & $\mathbf{U}$ & 5 & & NS & $\mu \mathrm{g} / \mathrm{L}$ \\
\hline Tritium & $\mathrm{TM}$ & & & $\mathbf{U}$ & 0.48 & & 20 & $\mathrm{pCi} / \mathrm{ml}$ \\
\hline Turbidity & WA & & & $\mathbf{U}$ & 0.2 & & NS & NTU \\
\hline Vanadium & WA & & & $\mathbf{U}$ & 50 & & NS & $\mu \mathrm{g} / \mathrm{L}$ \\
\hline Vinyl acetate & WA & & & $\mathbf{U}$ & 10 & & NS & $\mu g / \mathrm{L}$ \\
\hline Vinyl chloride & WA & & & $\mathbf{U}$ & 10 & & 2 & $\mu \mathrm{g} / \mathrm{L}$ \\
\hline Xylenes (total) & WA & & & $\mathbf{U}$ & 5 & & 10000 & $\mu g / L$ \\
\hline Zinc & WA & & & $\mathbf{U}$ & 20 & & 5000 & $\mu g / L$ \\
\hline
\end{tabular}


UQA010

(Equipment Blank - Shovel)

Field Measurements:

Sample Date: 4/21/93

\begin{tabular}{|c|c|c|c|c|c|c|c|}
\hline Analyte & Lab & $\operatorname{Rep}$ & Qualifier & Result & Acc & Stand & Units \\
\hline 1,1,1,2-Tetrachloroethane & WA & & $\mathbf{U}$ & 10 & & NS & $\mu \mathrm{g} / \mathrm{L}$ \\
\hline 1,1,1-Trichloroethane & WA & & $\mathbf{U}$ & 5 & & 200 & $\mu g / \Omega$ \\
\hline 1,1,2,2-Tetrachloroethane & WA & & $\mathbf{U}$ & 5 & & NS & $\mu \mathrm{g} / \mathrm{L}$ \\
\hline 1,1,2-Trichloroethane & WA & & $\mathbf{U}$ & 5 & & 5 & $\mu g / L$ \\
\hline 1,1-Dichloroethane & WA & & U & 5 & & NS & $\mu g / L$ \\
\hline 1,1-Dichloroethylene & WA & & U & 5 & & 7 & $\mu g / L$ \\
\hline 1,2,3-Trichloropropane & WA & & $\mathbf{U}$ & 10 & & NS & $\mu \mathrm{g} / \mathrm{L}$ \\
\hline 1,2-Dibromo-3-chloropropane & WA & & $\mathbf{U}$ & 20 & & 0.2 & $\mu \mathrm{g} / \mathrm{L}$ \\
\hline 1,2-Dibromoethane & WA & & $\mathbf{U}$ & 20 & & NS & $\mu \mathrm{g} / \mathrm{L}$ \\
\hline 1,2-Dichloroethane & WA & & U & 5 & & 5 & $\mu \mathrm{g} / \mathrm{L}$ \\
\hline 1,2-Dichloroethylene (total) & WA & & $\mathbf{U}$ & 5 & & 100 & $\mu \mathrm{g} / \mathrm{L}$ \\
\hline 1,2-Dichloropropane & WA & & $\mathbf{U}$ & 5 & & 5 & $\mu g / L$ \\
\hline 2-Hexanone & WA & & $\mathbf{U}$ & 10 & & NS & $\mu \mathrm{g} / \mathrm{L}$ \\
\hline 4-Methyl-2-pentanone & WA & & $\mathbf{U}$ & 10 & & NS & $\mu \mathrm{g} / \mathrm{L}$. \\
\hline Acetone & WA & & & 12 & & NS & $\mu g / L$ \\
\hline Acetonitrile & WA & & $\mathbf{U}$ & 20 & & NS & $\mu g / L$ \\
\hline Acidity & WA & & & 2.5 & & NS & $\mathrm{mg} / \mathrm{L}$ \\
\hline Acrolein & WA & & $\mathbf{U}$ & 10 & & NS & $\mu g / L$ \\
\hline Acrylonitrile & WA & & $\mathbf{U}$ & 10 & & NS & $\mu \mathrm{g} / \mathrm{L}$ \\
\hline Alkalinity & WA & & $\mathbf{U}$ & 0.5 & & NS & $\mathrm{mg} / \mathrm{L}$ \\
\hline Allyl chloride & WA & & $\mathbf{U}$ & 100 & & NS & $\mu g / L$ \\
\hline Aluminum & WA & & $\mathbf{U}$ & 200 & & NS & $\mu g / L$ \\
\hline Antimony & WA & & U & 60 & & 6 & $\mu \mathrm{g} / \mathrm{L}$ \\
\hline Arsenic & WA & & $\mathbf{U}$ & 10 & & 50 & $\mu \mathrm{g} / \mathrm{L}$ \\
\hline Barium & WA & & $\mathbf{U}$ & 200 & & 2000 & $\mu g / L$ \\
\hline Benzene & WA & & $\mathbf{U}$ & 5 & & 5 & $\mu g / L$ \\
\hline Beryllium & WA & & $\mathbf{U}$ & 5 & & 4 & $\mu g / L$ \\
\hline Bromodichloromethane & WA & & U & 5 & & NS & $\mu \mathrm{g} / \mathrm{L}$ \\
\hline Bromoform & WA & & $\mathbf{U}$ & 5 & & NS & $\mu \mathrm{g} / \mathrm{L}$ \\
\hline Cadmium & WA & & & 5.9 & & 5 & $\mu g / L$ \\
\hline Calcium & WA & & $\mathbf{U}$ & 5000 & & NS & $\mu \mathrm{g} / \mathrm{L}$ \\
\hline Carbon disulfide & WA & & $\mathbf{U}$ & 5 & & NS & $\mu g / L$ \\
\hline Carbon tetrachloride & WA & & $\mathbf{U}$ & 5 & & 5 & $\mu g / L$ \\
\hline Cesium-137 & TM & & $\mathbf{U}$ & 9.5 & & 200 & $\mathrm{pCi} / \mathrm{L}$ \\
\hline Chloride & WA & & & 0.326 & & NS & $\mathrm{mg} / \mathrm{L}$ \\
\hline Chlorobenzene & WA & & $\mathbf{U}$ & 5 & & 100 & $\mu g / L$ \\
\hline Chloroethane & WA & & $\mathbf{U}$ & 10 & & NS & $\mu g / L$ \\
\hline Chloroform & WA & & U & 5 & & 100 & $\mu \mathrm{g} / \mathrm{L}$ \\
\hline Chloroprene - & WA & & $\mathbf{U}$ & 20 & & NS & $\mu g / L$ \\
\hline Chromium & WA & & $\mathbf{U}$ & 10 & & 100 & $\mu g / L$ \\
\hline cis-1,3-Dichloropropene & WA & & $\mathbf{U}$ & 5 & & NS & $\mu g / L$ \\
\hline Cobalt & WA & & U & 50 & & NS & $\mu \mathrm{g} / \mathrm{L}$ \\
\hline Copper & WA & & $\mathbf{U}$ & 25 & & 1000 & $\mu g / L$ \\
\hline Cyanide & WA & & $\mathbf{U}$ & 10 & & 200 & $\mu g / L$ \\
\hline Dibromochloromethane & WA & & U & 5 & & NS & $\mu g / L$ \\
\hline Dichlorodifluoromethane & WA & & U & 10 & & NS & $\mu g / L$ \\
\hline Ethylbenzene & WA & & $\mathbf{U}$ & 5 & & 700 & $\mu g / L$ \\
\hline Fluoride & WA & & $\mathbf{U}$ & 0.1 & & 4 & $\mathrm{mg} / \mathrm{L}$ \\
\hline Gross alpha & TM & & $\mathbf{U}$ & 0.4 & & 15 & $\mathrm{pCi} / \mathrm{L}$ \\
\hline
\end{tabular}


UQA010 Laboratory Analyses (cont'd)

\begin{tabular}{|c|c|c|c|c|c|c|c|c|}
\hline Analyte & $\mathrm{Lab}$ & Rep & Avg & Qualifier & Result & Acc & Stand & Units \\
\hline Iron & WA & & & $\bar{U}$ & 100 & & 300 & $\mu g / L$ \\
\hline Isobutyl alcohol & WA & & & U & 20 & & NS & $\mu \mathrm{g} / \mathrm{L}$ \\
\hline Lead & WA & & & $\mathrm{U}$ & 3 & & 50 & $\mu \mathrm{g} / \mathrm{L}$ \\
\hline Magnesium & WA & & & $\mathbf{U}$ & 5000 & & NS & $\mu \mathrm{g} / \mathrm{L}$ \\
\hline Manganese & WA & & & $\mathbf{U}$ & 15 & & 50 & $\mu \mathrm{g} / \mathrm{L}$ \\
\hline Mercury & WA & & & $\mathbf{U}$ & 0.2 & . & 2 & $\mu \mathrm{g} / \mathrm{L}$ \\
\hline Methacrylonitrile & WA & & & $\mathbf{U}$ & 20 & & NS & $\mu \mathrm{g} / \mathrm{L}$ \\
\hline Methyl bromide & WA & & & $\mathbf{U}$ & 10 & & NS & $\mu g / L$ \\
\hline Methyl chloride & WA & & & $\mathbf{U}$ & 10 & & NS & $\mu \mathrm{g} / \mathrm{L}$ \\
\hline Methyl ethyl ketone & WA & & & $\mathbf{U}$ & 10 & & NS & $\mu \mathrm{g} / \mathrm{L}$ \\
\hline Methyl iodide & WA & & & $\mathbf{U}$ & 10 & & NS & $\mu \mathrm{g} / \mathrm{L}$ \\
\hline Methylene bromide & WA & & & $\mathbf{U}$ & 10 & & NS & $\mu g / 2$ \\
\hline Methylene chloride & WA & & & $\mathbf{U}$ & 5 & & 5 & $\mu g / \mathrm{L}$ \\
\hline Nickel & WA & & & $\mathbf{U}$ & 40 & & 100 & $\mu g / L$ \\
\hline Nitrate as nitrogen & WA & & & & 0.063 & & 10 & $\mathrm{mg} / \mathrm{L}$ \\
\hline Nonvolatile beta & TM & & & & 4.6 & 1.9 & 50 & $\mathrm{pCi} / \mathrm{L}$ \\
\hline Potassium & WA & & & $\mathbf{U}$ & 5000 & & NS & $\mu g / L$ \\
\hline Propionitrile & WA & & & U & 50 & & NS & $\mu g / L$ \\
\hline Selenium & WA & & & $\mathbf{U}$ & 5 & & 50 & $\mu \mathrm{g} / \mathrm{L}$ \\
\hline Silver & WA & & & $\mathbf{U}$ & 10 & & 50 & $\mu \mathrm{g} / \mathrm{L}$ \\
\hline Sodium & WA & & & U & 5000 & & NS & $\mu g / \mathrm{L}$ \\
\hline Styrene & WA & & & $\mathbf{U}$ & 5 & & 100 & $\mu g / \mathrm{L}$ \\
\hline Sulfate & WA & & & $\mathbf{U}$ & 2.5 & & 400 & $\mathrm{mg} / \mathrm{L}$ \\
\hline Sulfide & WA & & & $\mathrm{U}$ & 0.1 & & NS & $\mathrm{mg} / \mathrm{L}$ \\
\hline Sulfide & WA & & NA & $\mathrm{U}$ & 0.1 & & NS & $\mathrm{mg} / \mathrm{L}$ \\
\hline Tetrachloroethylene & WA & & & $\mathbf{U}$ & 5 & & 5 & $\mu g / L$ \\
\hline Thallium & WA & & & $\mathrm{U}$ & 10 & & 2 & $\mu g / L$ \\
\hline Toluene & WA & & & U & 5 & & 1000 & $\mu g / L$ \\
\hline Total hardness & WA & & & $\mathrm{U}$ & 0.1 & & NS & $\mathrm{mg} / \mathrm{L}$ \\
\hline Total organic carbon & WA & & & $\mathbf{U}$ & 0.5 & & NS & $\mathrm{mg} / \mathrm{L}$ \\
\hline Total suspended solids & WA & & & U & 5 & & NS & $\mathrm{mg} / \mathrm{L}$ \\
\hline trans-1,3-Dichloropropene & WA & & & U & 5 & & NS & $\mu g / \mathrm{L}$ \\
\hline trans-1,4-Dichloro-2-butene & WA & & & U & 100 & & NS & $\mu \mathrm{g} / \mathrm{L}$ \\
\hline Trichloroethylene & WA & & & $\mathbf{U}$ & 5 & & 5 & $\mu g / L$ \\
\hline Trichlorofluoromethane & WA & & & $\mathbf{U}$ & 5 & & NS & $\mu g / \mathrm{L}$ \\
\hline Tritium & TM & & & U & 0.48 & & 20 & $\mathrm{pCi} / \mathrm{ml}$ \\
\hline Turbidity & WA & & & $\mathbf{U}$ & 0.2 & & NS & NTU \\
\hline Vanadium & WA & & & $\mathbf{U}$ & 50 & & NS & $\mu \mathrm{g} / \mathrm{L}$ \\
\hline Vinyl acetate & WA & & & $\mathbf{U}$ & 10 & & NS & $\mu g / \mathrm{L}$ \\
\hline Vinyl chloride & WA & & & $\mathbf{U}$ & 10 & & 2 & $\mu g / \mathrm{L}$ \\
\hline Xylenes (total) & WA & & & $\mathbf{U}$ & 5 & & 10000 & $\mu \mathrm{g} / \mathrm{L}$ \\
\hline Zinc & WA & & & & 36.6 & & 5000 & $\mu \mathrm{g} / \mathrm{L}$ \\
\hline
\end{tabular}




\section{UQA011}

(Trip Blank)

Field Measurements:

Sample Date: 4/13/93

\begin{tabular}{|c|c|c|c|c|c|c|c|c|}
\hline Analyte & Lab & Rep & Avg & Qualifier & Result & Acc & Stand & Units \\
\hline 1,1,1,2-Tetrachloroethane & WA & & & $\bar{U}$ & 10 & & NS & $\mu g / L$ \\
\hline 1,1,1-Trichloroethane & WA & & & $\mathbf{U}$ & 5 & $\cdot$ & 200 & $\mu \mathrm{g} / \mathrm{L}$ \\
\hline 1,1,2,2-Tetrachloroethane & WA & & & $\mathbf{U}$ & 5 & & NS & $\mu g / L$ \\
\hline 1,1,2-Trichloroethane & WA & & & $\mathbf{U}$ & 5 & & 5 & $\mu g / L$ \\
\hline 1,1-Dichloroethane & WA & & & $\mathbf{U}$ & 5 & & NS & $\mu g / L$ \\
\hline 1,1-Dichloroethylene & WA & & & $\mathbf{U}$ & 5 & & 7 & $\mu g / L$ \\
\hline 1,2,3-Trichloropropane & WA & & & $\mathbf{U}$ & 10 & & NS & $\mu g / L$ \\
\hline 1,2-Dibromo-3-chloropropane & WA & & & $\mathbf{U}$ & 20 & & 0.2 & $\mu g / L$ \\
\hline 1,2-Dibromoethane & WA & & & $\mathbf{U}$ & 20 & & NS & $\mu g / L$ \\
\hline 1,2-Dichloroethane & WA & & & $\mathbf{U}$ & 5 & & 5 & $\mu g / L$ \\
\hline 1,2-Dichloroethylene (total) & WA & & & $\mathbf{U}$ & 5 & & 100 & $\mu g / L$ \\
\hline 1,2-Dichloropropane & WA & & & $\mathbf{U}$ & 5 & & 5 & $\mu g / L$ \\
\hline 2-Hexanone & WA & & & $\mathbf{U}$ & 10 & & NS & $\mu g / L$ \\
\hline 4-Methyl-2-pentanone & WA & & & $\mathbf{U}$ & 10 & & NS & $\mu g / L$ \\
\hline Acetone & WA & & & & 10.6 & & NS & $\mu g / L$ \\
\hline Acetonitrile & WA & & & $\mathbf{U}$ & 20 & & NS & $\mu g / L$ \\
\hline Acidity & WA & & & & 2 & & NS & $\mathrm{mg} / \mathrm{L}$ \\
\hline Acrolein & WA & & & $\mathbf{U}$ & 10 & & NS & $\mu g / L$ \\
\hline Acrylonitrile & WA & & & $\mathbf{U}$ & 10 & & NS & $\mu \mathrm{g} / \mathrm{L}$ \\
\hline Alkalinity & WA & & & $\mathbf{U}$ & 0.5 & & NS & $\mathrm{mg} / \mathrm{L}$ \\
\hline Allyl chloride & WA & & & $\mathbf{U}$ & 100 & & NS & $\mu g / L$ \\
\hline Aluminum & WA & & & $\mathrm{U}$ & 200 & & NS & $\mu g / L$ \\
\hline Antimony & WA & & & $\mathbf{U}$ & 60 & & 6 & $\mu g / L$ \\
\hline Arsenic & WA & & & $\mathbf{U}$ & 10 & & 50 & $\mu g / L$ \\
\hline Barium & WA & & & $\mathbf{U}$ & 200 & & 2000 & $\mu g / L$ \\
\hline Benzene & WA & & & $\mathbf{U}$ & 5 & & 5 & $\mu g / L$ \\
\hline Beryllium & WA & & & $\mathbf{U}$ & 5 & & 4 & $\mu g / L$ \\
\hline Bromodichloromethane & WA & & & $\mathbf{U}$ & 5 & & NS & $\mu g / L$ \\
\hline Bromoform & WA & & & $\mathbf{U}$ & 5 & & NS & $\mu g / L$ \\
\hline Cadmium & WA & & & $\mathbf{U}$ & 5 & & 5 & $\mu \mathrm{g} / \mathrm{L}$ \\
\hline Calcium & WA & & & $\mathbf{U}$ & 5000 & & NS & $\mu g / L$ \\
\hline Carbon disulfide & WA & & & $\mathbf{U}$ & 5 & & NS & $\mu g / L$ \\
\hline Carbon tetrachloride & WA & & & $\mathbf{U}$ & 5 & & 5 & $\mu \mathrm{g} / \mathrm{L}$ \\
\hline Cesium-137 & $\mathbf{T M}$ & & & $\mathbf{U}$ & 9.7 & & 200 & $\mathrm{pCi} / \mathrm{L}$ \\
\hline Chloride & WA & & & $\mathbf{U}$ & 0.25 & & NS & $\mathrm{mg} / \mathrm{L}$ \\
\hline Chlorobenzene & WA & & & $\mathbf{U}$ & 5 & & 100 & $\mu g / L$ \\
\hline Chloroethane & WA & & & $\mathbf{U}$ & 10 & & NS & $\mu g / L$ \\
\hline Chloroform & WA & & & $\mathbf{U}$ & 5 & & 100 & $\mu g / L$ \\
\hline Chloroprene . & WA & & & $\mathbf{U}$ & 20 & & NS & $\mu \mathrm{g} / \mathrm{L}$ \\
\hline Chromium & WA & & & $\mathbf{U}$ & 10 & & 100 & $\mu \mathrm{g} / \mathrm{L}$ \\
\hline cis-1,3-Dichloropropene & WA & & & $\mathbf{U}$ & 5 & & NS & $\mu g / L$ \\
\hline Cobalt & WA & & & $\mathbf{U}$ & 50 & & NS & $\mu \mathrm{g} / \mathrm{L}$ \\
\hline Copper & WA & & & $\mathbf{U}$ & 25 & & 1000 & $\mu \mathrm{g} / \mathrm{L}$ \\
\hline Cyanide & WA & & & $\mathbf{U}$ & 10 & & 200 & $\mu \mathrm{g} / \mathrm{L}$ \\
\hline Dibromochloromethane & WA & & & $\mathbf{U}$ & 5 & & NS & $\mu g / L$ \\
\hline Dichlorodifluoromethane & WA & & & $\mathbf{U}$ & 10 & & NS & $\mu g / L$ \\
\hline Ethylbenzene & WA & & & $\mathbf{U}$ & 5 & & 700 & $\mu \mathrm{g} / \mathrm{L}$ \\
\hline
\end{tabular}


UQA011 Laboratory Analyses (cont'd)

\begin{tabular}{|c|c|c|c|c|c|c|c|c|}
\hline Analyte & Lab & Rep & Avg & Qualifier & Result & Acc & Stand & Units \\
\hline Fluoride & WA & & & $\mathrm{U}$ & 0.1 & & 4 & $\overline{\mathrm{mg} / \mathrm{L}}$ \\
\hline Fluoride & WA & & $\mathbf{N A}$ & $\mathbf{U}$ & 0.1 & & 4 & $\mathrm{mg} / \mathrm{L}$ \\
\hline Gross alpha & TM & & & $\mathbf{U}$ & 0.6 & & 15 & $\mathrm{pCi} / \mathrm{L}$ \\
\hline Iron & WA & & & $\mathbf{U}$ & 100 & & 300 & $\mu g / L$ \\
\hline Isobutyl alcohol & WA & & & $\mathbf{U}$ & 20 & & NS & $\mu \mathrm{g} / \mathrm{L}$ \\
\hline Lead & WA & & & $\mathbf{U}$ & 3 & . & 50 & $\mu \mathrm{g} / \mathrm{L}$ \\
\hline Magnesium & WA & & & $\mathbf{U}$ & 5000 & & NS & $\mu \mathrm{g} / \mathrm{L}$ \\
\hline Manganese & WA & & & $\mathbf{U}$ & 15 & & 50 & $\mu g / L$ \\
\hline Mercury & WA & & & & 0.242 & & 2 & $\mu \mathrm{g} / \mathrm{L}$ \\
\hline Methacrylonitrile & WA & & & $\mathbf{U}$ & 20 & & NS & $\mu g / L$ \\
\hline Methyl bromide & WA & & & $\mathbf{U}$ & 10 & & NS & $\mu g / L$ \\
\hline Methyl chloride & WA & & & $\mathbf{U}$ & 10 & & NS & $\mu g / L$ \\
\hline Methyl ethyl ketone & WA & & & $\mathbf{U}$ & 10 & & NS & $\mu g / L$ \\
\hline Methyl iodide & WA & & & $\mathbf{U}$ & 10 & & NS & $\mu g / L$ \\
\hline Methylene bromide & WA & & & $\mathbf{U}$ & 10 & & NS & $\mu \mathrm{g} / \mathrm{L}$ \\
\hline Methylene chloride & WA & & & $\mathbf{U}$ & 5 & & 5 & $\mu g / L$ \\
\hline Nickel & WA & & & $\mathbf{U}$ & 40 & & 100 & $\mu g / L$ \\
\hline Nitrate as nitrogen & WA & & & $\mathbf{U}$ & 0.02 & & 10 & $\mathrm{mg} / \mathrm{L}$ \\
\hline Nitrate as nitrogen & WA & & $\mathbf{N A}$ & $\mathbf{U}$ & 0.02 & & 10 & $\mathrm{mg} / \mathrm{L}$ \\
\hline Nonvolatile beta & TM & & & $\mathbf{U}$ & 0.8 & & 50 & $\mathrm{pCi} / \mathrm{L}$ \\
\hline Potassium & WA & & & $\mathbf{U}$ & 5000 & & NS & $\mu g / L$ \\
\hline Propionitrile & WA & & & $\mathbf{U}$ & 50 & & NS & $\mu g / L$ \\
\hline Selenium & WA & & & $\mathbf{U}$ & 5 & & 50 & $\mu g / L$ \\
\hline Si.ver & WA & & & $\mathbf{U}$ & 10 & & 50 & $\mu g / L$ \\
\hline Sodium & WA & & & $\mathbf{U}$ & 5000 & & NS & $\mu g / L$ \\
\hline Styrene & WA & & & $\mathbf{U}$ & 5 & & 100 & $\mu g / L$ \\
\hline Sulfate & WA & & & $\mathbf{U}$ & 2.5 & & 400 & $\mathrm{mg} / \mathrm{L}$ \\
\hline Sulfide & WA & & & $\mathbf{U}$ & 0.1 & & NS & $\mathrm{mg} / \mathrm{L}$ \\
\hline Tetrachloroethylene & WA & & & $\mathbf{U}$ & 5 & & 5 & $\mu \mathrm{g} / \mathrm{L}$ \\
\hline Thallium & WA & & & $\mathbf{U}$ & 10 & & 2 & $\mu g / L$ \\
\hline Toluene & WA & & & $\mathbf{U}$ & 5 & & 1000 & $\mu g / L$ \\
\hline Total hardness & WA & & & $\mathbf{U}$ & 0.1 & & NS & $\mathrm{mg} / \mathrm{L}$ \\
\hline Total organic carbon & WA & & & $\mathbf{U}$ & 0.5 & & NS & $\mathbf{m g} / \mathbf{L}$ \\
\hline Total suspended solids & WA & & & & 8 & & NS & $\mathrm{mg} / \mathrm{L}$ \\
\hline trans-1,3-Dichloropropene & WA & & & $\mathbf{U}$ & 5 & & NS & $\mu g / L$ \\
\hline trans-1,4-Dichloro-2-butene & WA & & & $\mathbf{U}$ & 100 & & NS & $\mu g / L$ \\
\hline Trichloroethylene & WA & & & $\mathbf{U}$ & 5 & & 5 & $\mu g / L$ \\
\hline Trichlorofluoromethane & WA & & & $\mathbf{U}$ & 5 & & NS & $\mu g / L$ \\
\hline Tritium & TM & & & $\mathbf{U}$ & 0.46 & & 20 & $\mathrm{pCi} / \mathrm{ml}$ \\
\hline Turbidity & WA & & & $\mathbf{U}$ & 0.2 & & NS & NTU \\
\hline Vanadium & WA & & & $\mathbf{U}$ & 50 & & NS & $\mu g / L$ \\
\hline Vinyl acetate & WA & & & $\mathbf{U}$ & 10 & & NS & $\mu g / L$ \\
\hline Vinyl chloride. & WA & & & $\mathrm{U}$ & 10 & & 2 & $\mu g / L$ \\
\hline Xylenes (total) & WA & & & $\mathbf{U}$ & 5 & & 10000 & $\mu g / L$ \\
\hline Zinc & WA & & & & 40.7 & & 5000 & $\mu g / L$ \\
\hline
\end{tabular}




\section{Volatile Trip Blank}

Field Measurements:

Sample Date: 4/13/93

\begin{tabular}{|c|c|c|c|c|c|c|c|}
\hline Analyte & Lab & Avg & Qualifier & Result & Acc & Stand & Units \\
\hline 1,1,1,2-Tetrachloroethane & WA & & $\mathbf{U}$ & 10 & & NS & $\mu g / L$ \\
\hline 1,1,1-Trichloroethane & WA & & $\mathbf{U}$ & 5 & • & 200 & $\mu g / L$ \\
\hline 1,1,2,2-Tetrachloroethane & WA & & $\mathbf{U}$ & 5 & & NS & $\mu g / L$ \\
\hline 1,1,2-Trichloroethane & WA & & $\mathbf{U}$ & 5 & & 5 & $\mu g / L$ \\
\hline 1,1-Dichloroethane & WA & & $\mathbf{U}$ & 5 & & NS & $\mu g / L$ \\
\hline 1,1-Dichloroethylene & WA & & $\mathbf{U}$ & 5 & & 7 & $\mu g / L$ \\
\hline 1,2,3-Trichloropropane & WA & & $\mathbf{U}$ & 10 & & NS & $\mu g / L$ \\
\hline 1,2-Dibromo-3-chloropropane & WA & & $\mathbf{U}$ & 20 & & 0.2 & $\mu g / L$ \\
\hline 1,2-Dibromoethane & WA & & U & 20 & & NS & $\mu g / L$ \\
\hline 1,2-Dichloroethane & WA & & $\mathbf{U}$ & 5 & & 5 & $\mu g / L$ \\
\hline 1,2-Dichloropropane & WA & & $\mathbf{U}$ & 5 & & 5 & $\mu g / L$ \\
\hline 2-Hexanone & WA & & $\mathbf{U}$ & 10 & & NS & $\mu g / L$ \\
\hline 4-Methyl-2-pentanone & WA & & $\mathbf{U}$ & 10 & & NS & $\mu g / L$ \\
\hline Acetone & WA & & & 19 & & NS & $\mu g / L$ \\
\hline Acetonitrile & WA & & U & 20 & & NS & $\mu g / L$ \\
\hline Acrolein & WA & & $\mathbf{U}$ & 10 & & NS & $\mu g / L$ \\
\hline Acrylonitrile & WA & & $\mathbf{U}$ & 10 & & NS & $\mu g / L$ \\
\hline Allyl chloride & WA & & $\mathbf{U}$ & 100 & & NS & $\mu g / L$ \\
\hline Benzene & WA & & $\mathbf{U}$ & 5 & & 5 & $\mu g / L$ \\
\hline Bromodichloromethane & WA & & $\mathbf{U}$ & 5 & & NS & $\mu g / L$ \\
\hline Bromoform & WA & & $\mathbf{U}$ & 5 & & NS & $\mu g / L$ \\
\hline Carbon disulfide & WA & & $\mathbf{U}$ & 5 & & NS & $\mu g / L$ \\
\hline Carbon tetrachloride & WA & & $\mathbf{U}$ & 5 & & 5 & $\mu g / L$ \\
\hline Chlorobenzene & WA & & $\mathbf{U}$ & 5 & & 100 & $\mu g / L$ \\
\hline Chloroethane & WA & & $\mathbf{U}$ & 10 & & NS & $\mu g / L$ \\
\hline Chloroform & WA & & $\mathbf{U}$ & 5 & & 100 & $\mu g / L$ \\
\hline Chloroprene & WA & & $\mathbf{U}$ & 20 & & NS & $\mu g / L$ \\
\hline cis-1,3-Dichloropropene & WA & & $\mathbf{U}$ & 5 & & NS & $\mu g / L$ \\
\hline Dibromochloromethane & WA & & $\mathbf{U}$ & 5 & & NS & $\mu g / L$ \\
\hline Dichlorodifluoromethane & WA & & $\mathbf{U}$ & 10 & & NS & $\mu g / L$ \\
\hline Ethylbenzene & WA & & $\mathbf{U}$ & 5 & & 700 & $\mu g / L$ \\
\hline Isobutyl alcohol & WA & & $\mathbf{U}$ & 20 & & NS & $\mu g / L$ \\
\hline Methacrylonitrile & WA & & $\mathbf{U}$ & 20 & & NS & $\mu g / L$ \\
\hline Methyl bromide & WA & & $\mathbf{U}$ & 10 & & NS & $\mu g / L$ \\
\hline Methyl chloride & WA & & $\mathbf{U}$ & 10 & & NS & $\mu g / L$ \\
\hline Methyl ethyl ketone & WA & & $\mathbf{U}$ & 10 & & NS & $\mu g / L$ \\
\hline Methyl iodide & WA & & $\mathbf{U}$ & 10 & & NS & $\mu g / L$ \\
\hline Methylene bromide & WA & & $\mathbf{U}$ & 10 & & NS & $\mu g / L$ \\
\hline Methylene chloride & WA & & $\mathbf{J}$ & 3.92 & & 5 & $\mu g / L$ \\
\hline Propionitrile - & WA & & $\mathbf{U}$ & 50 & & NS & $\mu g / L$ \\
\hline Styrene & WA & & $\mathbf{U}$ & 5 & & 100 & $\mu \mathrm{g} / \mathrm{L}$ \\
\hline Tetrachloroethylene & WA & & $\mathbf{U}$ & 5 & & 5 & $\mu \mathrm{g} / \mathrm{L}$ \\
\hline Toluene & WA & & $\mathbf{U}$ & 5 & & 1000 & $\mu \mathrm{g} / \mathrm{L}$ \\
\hline trans-1,2-Dichloroethylene (total) & WA & & $\mathbf{U}$ & 5 & & 100 & $\mu \mathrm{g} / \mathrm{L}$ \\
\hline trans-1,3-Dichloropropene & WA & & $\mathbf{U}$ & 5 & & NS & $\mu g / L$ \\
\hline trans-1,4-Dichloro-2-butene & WA & & $\mathbf{U}$ & 100 & & NS & $\mu \mathrm{g} / \mathrm{L}$ \\
\hline Trichloroethylene & WA & & $\mathbf{U}$ & 5 & & 5 & $\mu g / L$ \\
\hline Trichlorofluoromethane & WA & & $\mathbf{U}$ & 5 & & NS & $\mu \mathrm{g} / \mathrm{L}$ \\
\hline Vinyl acetate & WA & & $\mathbf{U}$ & 10 & & NS & $\mu g / L$ \\
\hline Vinyl chloride & WA & & $\mathbf{U}$ & 10 & & NS & $\mu g / L$ \\
\hline Xylenes (total) & WA & & $\mathbf{U}$ & 5 & & 10000 & $\mu g / L$ \\
\hline
\end{tabular}




\section{Volatile Trip Blank}

Field Measurements:

Sample Date: $4 / 15 / 93$

\begin{tabular}{|c|c|c|c|c|c|c|c|c|}
\hline Analyte & Lab & Rep & Avg & Qualifier & Result & Acc & Stand & Units \\
\hline 1,1,1,2-Tetrachloroethane & WA & & & $\bar{U}$ & 10 & & NS & $\mu_{g} \sqrt{L}$ \\
\hline 1,1,1-Trichloroethane & WA & & & $\mathbf{U}$ & 5 & - & 200 & $\mu \mathrm{g} / \mathrm{L}$ \\
\hline $1,1,2,2$-Tetrachloroethane & WA & & & $\mathbf{U}$ & 5 & & NS & $\mu \mathrm{g} / \mathrm{L}$ \\
\hline 1,1,2-Trichloroethane & WA & & & $\mathbf{U}$ & 5 & & 5 & $\mu g / L$ \\
\hline 1,1-Dichloroethane & WA & & & $\mathbf{U}$ & 5 & & NS & $\mu g / L$ \\
\hline 1,1-Dichloroethylene & WA & & & $\mathbf{U}$ & 5 & & 7 & $\mu g / L$ \\
\hline 1,2,3-Trichloropropane & WA & & & $\mathbf{U}$ & 10 & & NS & $\mu g / L$ \\
\hline 1,2-Dibromo-3-chloropropane & WA & & & $\mathbf{U}$ & 20 & & 0.2 & $\mu g / L$ \\
\hline 1,2-Dibromoethane & WA & & & $\mathbf{U}$ & 20 & & NS & $\mu \mathrm{g} / \mathrm{L}$ \\
\hline 1,2-Dichloroethane & WA & & & $\mathbf{U}$ & 5 & & 5 & $\mu \mathrm{g} / \mathrm{L}$ \\
\hline 1,2-Dichloropropane & WA & & & $\mathbf{U}$ & 5 & & 5 & $\mu g / L$ \\
\hline 2-Hexanone & WA & & & U & 10 & & NS & $\mu g / L$ \\
\hline 4-Methyl-2-pentanone & WA & & & $\mathbf{U}$ & 10 & & NS & $\mu g / L$ \\
\hline Acetone & W.A & & & & 10.9 & & NS & $\mu g / L$ \\
\hline Acetonitrile & WA & & & $\mathbf{U}$ & 20 & & NS & $\mu g / L$ \\
\hline Acrolein & WA & & & $\mathbf{U}$ & 10 & & NS & $\mu g / L$ \\
\hline Acrylonitrile & WA & & & $\mathbf{U}$ & 10 & & NS & $\mu g / L$ \\
\hline Allyl chloride & WA & & & $\mathbf{U}$ & 100 & & NS & $\mu \mathrm{g} / \mathrm{L}$ \\
\hline Benzene & WA & & & U & 5 & & 5 & $\mu g / L$ \\
\hline Bromodichloromethane & WA & & & $\mathbf{U}$ & 5 & & NS & $\mu g / L$ \\
\hline Bromoform & WA & & & $\mathbf{U}$ & 5 & & NS & $\mu \mathrm{g} / \mathrm{L}$ \\
\hline Carbon disulfide & WA & & & $\mathbf{U}$ & 5 & & NS & $\mu g / L$ \\
\hline Carbon tetrachloride & WA & & & $\mathbf{U}$ & 5 & & 5 & $\mu \mathrm{g} / \mathrm{L}$ \\
\hline Chlorobenzene & WA & & & $\mathbf{U}$ & 5 & & 100 & $\mu g / L$ \\
\hline Chloroethane & WA & & & $\mathbf{U}$ & 10 & & NS & $\mu g / L$ \\
\hline Chloroform & WA & & & $\mathbf{U}$ & 5 & & 100 & $\mu g / L$ \\
\hline Chloroprene & WA & & & $\mathbf{U}$ & 20 & & NS & $\mu g / L$ \\
\hline cis-1,3-Dichloropropene & WA & & & $\mathbf{U}$ & 5 & & NS & $\mu g / L$ \\
\hline Dibromochloromethane & WA & & & $\mathbf{U}$ & 5 & & NS & $\mu g / L$ \\
\hline Dichlorodifluoromethane & WA & & & $\mathbf{U}$ & 10 & & NS & $\mu \mathrm{g} / \mathrm{L}$ \\
\hline Ethylbenzene & WA & & & $\mathbf{U}$ & 5 & & 700 & $\mu \mathrm{g} / \mathrm{L}$ \\
\hline Isobutyl alcohol & WA & & & $\mathrm{U}$ & 20 & & NS & $\mu g / L$ \\
\hline Methacrylonitrile & WA & & & U & 20 & & NS & $\mu \mathrm{g} / \mathrm{L}$ \\
\hline Methyl bromide & WA & & & $\mathbf{U}$ & 10 & & NS & $\mu \mathrm{g} / \mathrm{L}$ \\
\hline Methyl chloride & WA & & & $\mathbf{U}$ & 10 & & NS & $\mu g / L$ \\
\hline Methyl ethyl ketone & WA & & & $\mathrm{U}$ & 10 & & NS & $\mu \mathrm{g} / \mathrm{L}$ \\
\hline Methyl iodide & WA & & & $\mathbf{U}$ & 10 & & NS & $\mu g / L$ \\
\hline Methylene bromide & WA & & & $\mathbf{U}$ & 10 & & NS & $\mu \mathrm{g} / \mathrm{L}$ \\
\hline Methylene chloride & WA & & & $\mathbf{J}$ & 4.23 & & 5 & $\mu g / L$ \\
\hline Propionitrile & WA & & & U & 50 & & NS & $\mu g / L$ \\
\hline Styrene & WA & & & $\mathbf{U}$ & 5 & & 100 & $\mu \mathrm{g} / \mathrm{L}$ \\
\hline Tetrachloroethylene & WA & & & $\mathrm{U}$ & 5 & & 5 & $\mu \mathrm{g} / \mathrm{L}$ \\
\hline Toluene & WA & & & $\mathbf{U}$ & 5 & & 1000 & $\mu \mathrm{g} / \mathrm{L}$ \\
\hline trans-1,2-Dichloroethylene (total) & WA & & & $\mathbf{U}$ & 5 & & 100 & $\mu g / L$ \\
\hline trans-1,3-Dichloropropene & WA & & & $\mathbf{U}$ & 5 & & NS & $\mu g / L$ \\
\hline trans-1,4-Dichloro-2-butene & WA & & & $\mathbf{U}$ & 100 & & NS & $\mu \mathrm{g} / \mathrm{L}$ \\
\hline Trichloroethylene & WA & & & $\mathbf{U}$ & 5 & & 5 & $\mu \mathrm{g} / \mathrm{L}$ \\
\hline Trichlorofluoromethane & WA & & & $\mathbf{U}$ & 5 & & NS & $\mu g / L$ \\
\hline Vinyl acetate & WA & & & $\mathbf{U}$ & 10 & & NS & $\mu \mathrm{g} / \mathrm{L}$ \\
\hline Vinyl chloride & WA & & & $\mathbf{U}$ & 10 & & NS & $\mu \mathrm{g} / \mathrm{L}$ \\
\hline Xylenes (total) & WA & & & $\mathbf{U}$ & 5 & & 10000 & $\mu g / L$ \\
\hline
\end{tabular}


Volatile Trip Blank

Field Measurements:

Sample Date: 4/21/93

\begin{tabular}{|c|c|c|c|c|c|c|c|c|}
\hline Analyte & Lab & Rep & Avg & Qualifier & Result & Acc & Stand & Units \\
\hline 1,1,1,2-Tetrachloroethane & WA & & & $\mathbf{U}$ & 10 & & NS & $\mu g / L$ \\
\hline 1,1,1-Trichloroethane & WA & & & $\mathbf{U}$ & 5 & & 200 & $\mu g / L$ \\
\hline 1,1,2,2-Tetrachloroethane & WA & & & $\mathbf{U}$ & 5 & & NS & $\mu \mathrm{g} / \mathrm{L}$ \\
\hline 1,1,2-Trichloroethane & WA & & & $\mathbf{U}$ & 5 & & 5 & $\mu \mathrm{g} / \mathrm{L}$ \\
\hline 1,1-Dichloroethane & WA & & & $\mathbf{U}$ & 5 & & NS & $\mu g / L$ \\
\hline 1,1-Dichloroethylene & WA & & & $\mathbf{U}$ & 5 & & 7 & $\mu \mathrm{g} / \mathrm{L}$ \\
\hline 1,2,3-Trichloropropane & WA. & & & $\mathbf{U}$ & 10 & & NS & $\mu \mathrm{g} / \mathrm{L}$ \\
\hline 1,2-Dibromo-3-chloropropane & WA & & & $\mathbf{U}$ & 20 & & 0.2 & $\mu g / L$ \\
\hline 1,2-Dibromoethane & WA & & & $\mathbf{U}$ & 20 & & NS & $\mu \mathrm{g} / \mathrm{L}$ \\
\hline 1,2-Dichloroethane & WA & & & $\mathbf{U}$ & 5 & & 5 & $\mu \mathrm{g} / \mathrm{L}$ \\
\hline 1,2-Dichloropropane & WA & & & $\mathbf{U}$ & 5 & & 5 & $\mu \mathrm{g} / \mathrm{L}$ \\
\hline 2-Hexanone & WA & & . & $\mathbf{U}$ & 10 & & NS & $\mu g / L$ \\
\hline 4-Methyl-2-pentanone & WA & & & $\mathbf{U}$ & 10 & & NS & $\mu g / L$ \\
\hline Acetone & WA & & & $\mathbf{U}$ & 10 & & NS & $\mu g / L$ \\
\hline Acetonitrile & WA & & & $\mathbf{U}$ & 20 & & NS & $\mu \xi / L$ \\
\hline Acrolein & WA & & & $\mathbf{U}$ & 10 & & NS & $\mu g T$ \\
\hline Acrylonitrile & WA & & & $\mathbf{U}$ & 10 & & NS & $\mu g D$ \\
\hline Allyl chloride & WA & & & $\mathbf{U}$ & 100 & & NS & $\mu g / L$ \\
\hline Benzene & WA & & & $\mathbf{U}$ & 5 & & 5 & $\mu g / L$ \\
\hline Bromodichloromethane & WA & & & $\mathbf{U}$ & 5 & & NS & $\mu \mathrm{g} / \mathrm{L}$ \\
\hline Bromoform & WA & & & $\mathbf{U}$ & 5 & & NS & $\mu \mathrm{g} / \mathrm{L}$ \\
\hline Carbon disulfide & WA & & & $\mathbf{U}$ & 5 & & NS & $\mu g / L$ \\
\hline Carbon tetrachloride & WA & & & $\mathbf{U}$ & 5 & & 5 & $\mu g / L$ \\
\hline Chlorobenzene & WA & & & $\mathbf{U}$ & 5 & & 100 & $\mu g / L$ \\
\hline Chloroethane & WA & & & $\mathbf{U}$ & 10 & & NS & $\mu \mathrm{g} / \mathrm{L}$ \\
\hline Chloroform & WA & & & $\mathbf{U}$ & 5 & & 100 & $\mu \mathrm{g} / \mathrm{L}$ \\
\hline Chloroprene & WA & & & $\mathbf{U}$ & 20 & & NS & $\mu g / L$ \\
\hline cis-1,3-Dichloropropene & WA & & & $\mathbf{U}$ & 5 & & NS & $\mu g / L$ \\
\hline Dibromochloromethane & WA & & & $\mathbf{U}$ & 5 & & NS & $\mu g / L$ \\
\hline Dichlorodifluoromethane & WA & & & $\mathbf{U}$ & 10 & & NS & $\mu g / L$ \\
\hline Ethylbenzene & WA & & & $\mathbf{U}$ & 5 & & 700 & $\mu g / L$ \\
\hline Isobutyl alcohol & WA & & & $\mathbf{U}$ & 20 & & NS & $\mu g / L$ \\
\hline Methacrylonitrile & WA & & & $\mathbf{U}$ & 20 & & NS & $\mu g / L$ \\
\hline Methyl bromide & WA & & & $\mathbf{U}$ & 10 & & NS & $\mu g / L$ \\
\hline Methyl chloride & WA & & & $\mathbf{U}$ & 10 & & NS & $\mu \mathrm{g} / \mathrm{L}$ \\
\hline Methyl ethyl ketone & WA & & & $\mathbf{U}$ & 10 & & NS & $\mu \mathrm{g} / \mathrm{L}$ \\
\hline Methyl iodide & WA & & & $\mathbf{U}$ & 10 & & NS & $\mu \mathrm{g} / \mathrm{L}$ \\
\hline Methylene bromide & WA & & & $\mathbf{U}$ & 10 & & NS & $\mu g / L$ \\
\hline Methylene chloride & WA & & & $\mathbf{U}$ & 5 & & 5 & $\mu g / L$ \\
\hline Propionitrile - & WA & & & $\mathbf{U}$ & 50 & & NS & $\mu g / L$ \\
\hline Styrene & WA & & & $\mathbf{U}$ & 5 & & 100 & $\mu g / L$ \\
\hline Tetrachloroethylene & W/A & & & $\mathbf{U}$ & 5 & & 5 & $\mu \mathrm{g} / \mathrm{L}$ \\
\hline Toluene & WA & & & J & 2.08 & & 1000 & $\mu g / L$ \\
\hline trans-1,2-Dichloroethylene (total) & WA & & & $\mathbf{U}$ & 5 & & 100 & $\mu g / L$ \\
\hline trans-1,3-Dichloropropene & WA & & & $\mathbf{U}$ & 5 & & NS & $\mu g / L$ \\
\hline trans-1,4-Dichloro-2-butene & WA & & & $\mathbf{U}$ & 100 & & NS & $\mu g / L$ \\
\hline Trichloroethylene & WA & & & $\mathbf{U}$ & 5 & & 5 & $\mu g / L$ \\
\hline Trichlorofluoromethane & WA & & & $\mathbf{U}$ & 5 & & NS & $\mu \mathrm{g} / \mathrm{L}$ \\
\hline Vinyl acetate & WA & & & $\mathbf{U}$ & 10 & & NS & $\mu g / L$ \\
\hline Vinyl chloride & WA & & & $\mathbf{U}$ & 10 & & NS & $\mu g / L$ \\
\hline Xylenes (total) & WA & & & $\mathbf{U}$ & 5 & & 1000 & $\mu g / L$ \\
\hline
\end{tabular}




\section{Volatile Trip Blank}

Field Measurements:

Sample Date: 4/22/93

\begin{tabular}{|c|c|c|c|c|c|c|c|c|}
\hline Analyte & Lab & Rep & Avg & Qualifier & Result & Acc & Stand & Units \\
\hline 1,1,1,2-Tetrachloroethane & $\overline{\text { WA }}$ & & & $\bar{U}$ & 10 & & NS & $\mu g / L$ \\
\hline $1,1,1$-Trichloroethane & WA & & & $\mathbf{U}$ & 5 & · & 200 & $\mu \mathrm{g} / \mathrm{L}$ \\
\hline 1,1,2,2-Tetrachloroethane & WA & & & $\mathbf{U}$ & 5 & & NS & $\mu \mathrm{g} / \mathrm{L}$ \\
\hline 1,1,2-Trichloroethane & WA & & & $\mathbf{U}$ & 5 & & 5 & $\mu g / L$ \\
\hline 1,1-Dichloroethane & WA & & & $\mathbf{U}$ & 5 & & NS & $\mu \mathrm{g} / \mathrm{L}$ \\
\hline 1,1-Dichloroethylene & WA & & & $\mathbf{U}$ & 5 & & 7 & $\mu g / L$ \\
\hline 1,2,3-Trichloropropane & WA & & & $\mathbf{U}$ & 10 & & NS & $\mu g / L$ \\
\hline 1,2-Dibromo-3-chloropropane & WA & & & $\mathbf{U}$ & 20 & & 0.2 & $\mu g / L$ \\
\hline 1,2-Dibromoethane & WA & & & $\mathbf{U}$ & 20 & & NS & $\mu \mathrm{g} / \mathrm{L}$ \\
\hline 1,2-Dichloroethane & WA & & & $\mathbf{U}$ & 5 & & 5 & $\mu g / L$ \\
\hline 1,2-Dichloropropane & WA & & . & $\mathbf{U}$ & 5 & & 5 & $\mu g / L$ \\
\hline 2-Hexanone & WA & & & $\mathbf{U}$ & 10 & & NS & $\mu g / L$ \\
\hline 4-Methyl-2-pentanone & WA & & & $\mathbf{U}$ & 10 & & NS & $\mu g / L$ \\
\hline Acetone & WA & & & & 9.94 & & NS & $\mu g / L$ \\
\hline Acetonitrile & WA & & & $\mathbf{U}$ & 20 & & NS & $\mu g / L$ \\
\hline Acrolein & WA & & & $\mathbf{U}$ & 10 & & NS & $\mu g / L$ \\
\hline Acrylonitrile & WA & & & $\mathbf{U}$ & 10 & & NS & $\mu g / L$ \\
\hline Allyl chloride & WA & & & $\mathbf{U}$ & 100 & & NS & $\mu \mathrm{g} / \mathrm{L}$ \\
\hline Benzene & WA & & & $\mathbf{U}$ & 5 & & 5 & $\mu \mathrm{g} / \mathrm{L}$ \\
\hline Bromodichloromethane & WA & & & $\mathbf{U}$ & 5 & & NS & $\mu g / L$ \\
\hline Bromoform & WA & & & $\mathbf{U}$ & 5 & & NS & $\mu g / L$ \\
\hline Carbon disulfide & WA & & & $\mathbf{U}$ & 5 & & NS & $\mu g / L$ \\
\hline Carbon tetrachloride & WA & & & $\mathbf{U}$ & 5 & & 5 & $\mu g / L$ \\
\hline Chlorobenzene & WA & & & $\mathbf{U}$ & 5 & & 100 & $\mu g / L$ \\
\hline Chloroethane & WA & & & $\mathbf{U}$ & 10 & & NS & $\mu g / L$ \\
\hline Chloroform & WA & & & $\mathbf{U}$ & 5 & & 100 & $\mu g / L$ \\
\hline Chloroprene & WA & & & $\mathbf{U}$ & 20 & & NS & $\mu g / L$ \\
\hline cis-1,3-Dichloropropene & WA & & & $\mathbf{U}$ & 5 & & NS & $\mu g / L$ \\
\hline Dibromochloromethane & WA & & & $\mathbf{U}$ & 5 & & NS & $\mu g / L$ \\
\hline Dichlorodifluoromethane & WA & & & $\mathbf{U}$ & 10 & & NS & $\mu g / L$ \\
\hline Ethylbenzene & WA & & & $\mathbf{U}$ & 5 & & 700 & $\mu \mathrm{g} / \mathrm{L}$ \\
\hline Isobutyl alcohol & WA & & & $\mathbf{U}$ & 20 & & NS & $\mu g / L$ \\
\hline Methacrylonitrile & WA & & & $\mathbf{U}$ & 20 & & NS & $\mu \mathrm{g} / \mathrm{L}$ \\
\hline Methyl bromide & WA & & & $\mathbf{U}$ & 10 & & NS & $\mu g / L$ \\
\hline Methyl chloride & WA & & & $\mathbf{U}$ & 10 & & NS & $\mu g / L$ \\
\hline Methyl ethyl ketone & WA & & & $\mathbf{U}$ & 10 & & NS & $\mu g / L$ \\
\hline Methyl iodide & WA & & & $\mathbf{U}$ & 10 & & NS & $\mu \mathrm{g} / \mathrm{L}$ \\
\hline Methylene bromide & WA & & & $\mathbf{U}$ & 10 & & NS & $\mu g / L$ \\
\hline Methylene chloride & WA & & & $\mathbf{U}$ & 5 & & 5 & $\mu g / L$ \\
\hline Propionitrile & WA & & & $\mathbf{U}$ & 50 & & NS & $\mu g / L$ \\
\hline Styrene & WA & & & $\mathbf{U}$ & 5 & & 100 & $\mu \mathrm{g} / \mathrm{L}$ \\
\hline Tetrachloroethylene & WA & & & $\mathbf{U}$ & 5 & & 5 & $\mu \mathrm{g} / \mathrm{L}$ \\
\hline Toluene & WA & & & $\mathbf{U}$ & 5 & & 1000 & $\mu g / L$ \\
\hline trans-1,2-Dichloroethylene (total) & WA & & & $\mathbf{U}$ & 5 & & 100 & $\mu \mathrm{g} / \mathrm{L}$ \\
\hline trans-1,3-Dichloropropene & WA & & & $\mathbf{U}$ & 5 & & NS & $\mu \mathrm{g} / \mathrm{L}$ \\
\hline trans-1,4-Dichloro-2-butene & WA & & & $\mathbf{U}$ & 100 & & NS & $\mu \mathrm{g} / \mathrm{L}$ \\
\hline Trichloroethylene & WA & & & $\mathbf{U}$ & 5 & & 5 & $\mu \mathrm{g} / \mathrm{L}$ \\
\hline Trichlorofluoromethane & WA & & & $\mathbf{U}$ & 5 & & NS & $\mu g / L$ \\
\hline Vinyl acetate & WA & & & $\mathbf{U}$ & 10 & & NS & $\mu g / L$ \\
\hline Vinyl chloride & WA & & & $\mathbf{U}$ & 10 & & NS & $\mu g / L$ \\
\hline Xylenes (totai) & WA & & & $\mathbf{U}$ & 5 & & 10000 & $\mu \mathrm{g} / \mathrm{L}$ \\
\hline
\end{tabular}


Volatile Trip Blank

Field Measurements:

Sample Date: 4/22/93

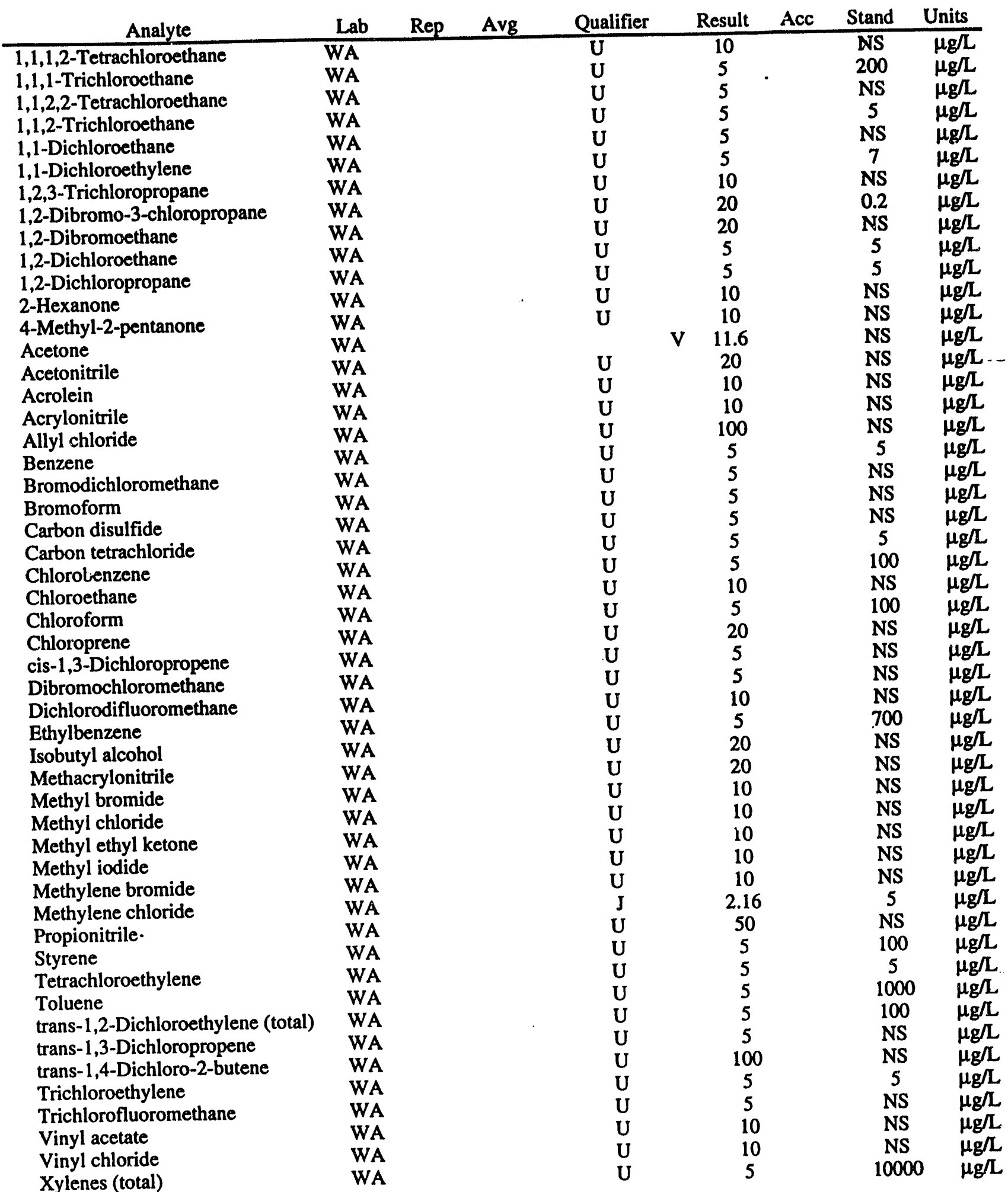


Volatile Trip Blank

Field Measurements:

Sample Date: 4/26/93

\begin{tabular}{|c|c|c|c|c|c|c|c|c|}
\hline Analyte & Lab & Rep & Avg & Qualifier & Result & Acc & Stand & Units \\
\hline 1,1,1,2-Tetrachloroethane & WA & & & $\mathbf{U}$ & 10 & & NS & $\mu \mathrm{g} / \mathrm{L}$ \\
\hline 1,1,1-Trichloroethane & WA & & & $\mathbf{U}$ & 5 & & 200 & $\mu g / L$ \\
\hline 1,1,2,2-Tetrachloroethane & WA & & & $\mathbf{U}$ & 5 & & NS & $\mu g / L$ \\
\hline 1,1,2-Trichloroethane & WA & & & $\mathbf{U}$ & 5 & & 5 & $\mu g / L$ \\
\hline 1,1-Dichloroethane & WA & & & $\mathbf{U}$ & 5 & & NS & $\mu g / L$ \\
\hline 1,1-Dichloroethylene & WA & & & $\mathbf{U}$ & 5 & & 7 & $\mu g / L$ \\
\hline 1,2,3-Trichloropropane & WA & & & $\mathbf{U}$ & 10 & & NS & $\mu g / L$ \\
\hline 1,2-Dibromo-3-chloropropane & WA & & & $\mathbf{U}$ & 20 & & 0.2 & $\mu g / L$ \\
\hline 1,2-Dibromoethane & WA & & & $\mathbf{U}$ & 20 & & NS & $\mu g / L$ \\
\hline 1,2-Dichloroethane & WA & & & $\mathbf{U}$ & 5 & & 5 & $\mu g / L$ \\
\hline 1,2-Dichloropropane & WA & & & $\mathbf{U}$ & 5 & & 5 & $\mu g / L$ \\
\hline 2-Hexanone & WA & & & $\mathbf{U}$ & 10 & & NS & $\mu g / L$ \\
\hline 4-Methyl-2-pentanone & WA & & & $\mathbf{U}$ & 10 & & NS & $\mu g / L$ \\
\hline Acetone & WA & & & $\mathbf{U}$ & 10 & & NS & $\mu g / L$ \\
\hline Acetonitrile & WA & & & $\mathbf{U}$ & 20 & & NS & $\mu g / L$ \\
\hline Acrolein & WA & & & $\mathbf{U}$ & 10 & & NS & $\mu g / L$ \\
\hline Acrylonitrile & WA & & & $\mathbf{U}$ & 10 & & NS & $\mu g / L$ \\
\hline Allyl chloride & WA & & & $\mathbf{U}$ & 100 & & NS & $\mu g / L$ \\
\hline Benzene & WA & & & $\mathbf{U}$ & 5 & & 5 & $\mu g / L$ \\
\hline Bromodichloromethane & WA & & & $\mathbf{U}$ & 5 & & NS & $\mu \mathrm{g} / \mathrm{L}$ \\
\hline Bromoform & WA & & & $\mathbf{U}$ & 5 & & NS & $\mu \mathrm{g} / \mathrm{L}$ \\
\hline Carbon disulfide & WA & & & $\mathbf{U}$ & 5 & & NS & $\mu g / L$ \\
\hline Carbon tetrachloride & WA & & & $\mathbf{U}$ & 5 & & 5 & $\mu g / L$ \\
\hline Chlorobenzene & WA & & & $\mathbf{U}$ & 5 & & 100 & $\mu g / L$ \\
\hline Chloroethane & WA & & & $\mathbf{U}$ & 10 & & NS & $\mu \mathrm{g} / \mathrm{L}$ \\
\hline Chloroform & WA & & & $\mathbf{U}$ & 5 & & 100 & $\mu g / L$ \\
\hline Chloroprene & WA & & & $\mathbf{U}$ & 20 & & NS & $\mu g / L$ \\
\hline cis-1,3-Dichloropropene & WA & & & $\mathbf{U}$ & 5 & & NS & $\mu \mathrm{g} / \mathrm{L}$ \\
\hline Dibromochloromethane & WA & & & $\mathbf{U}$ & 5 & & NS & $\mu g / L$ \\
\hline Dichlorodifluoromethane & WA & & & $\mathbf{U}$ & 10 & & NS & $\mu \mathrm{g} / \mathrm{L}$ \\
\hline Ethylbenzene & WA & & & $\mathbf{U}$ & 5 & & 700 & $\mu \mathrm{g} / \mathrm{L}$ \\
\hline Isobutyl alcohol & WA & & & $\mathbf{U}$ & 20 & & NS & $\mu \mathrm{g} / \mathrm{L}$ \\
\hline Methacrylonitrile & WA & & & $\mathbf{U}$ & 20 & & NS & $\mu g / L$ \\
\hline Methyl bromide & WA & & & $\mathbf{U}$ & 10 & & NS & $\mu g / L$ \\
\hline Methyl chloride & WA & & & $\mathbf{U}$ & 10 & & NS & $\mu g / L$ \\
\hline Methyl ethyl ketone & WA & & & $U$ & 10 & & NS & $\mu \mathrm{g} / \mathrm{L}$ \\
\hline Methyl iodide & WA & & & $\mathrm{U}$ & 10 & & NS & $\mu g / L$ \\
\hline Methylene bromide & WA & & & $\mathrm{U}$ & 10 & & NS & $\mu \mathrm{g} / \mathrm{L}$ \\
\hline Methylene chloride & WA & & & $\mathbf{U}$ & 5 & & 5 & $\mu \mathrm{g} / \mathrm{L}$ \\
\hline Propionitrile & WA & & & $\mathbf{U}$ & 50 & & NS & $\mu g / L$ \\
\hline Styrene & WA & & & $\mathbf{U}$ & 5 & & 100 & $\mu \mathrm{g} / \mathrm{L}$ \\
\hline Tetrachloroethylene & WA & & & $\mathrm{U}$ & 5 & & 5 & $\mu \mathrm{g} / \mathrm{L}$. \\
\hline Toluene & WA & & & J & 2.37 & & 1000 & $\mu \mathrm{g} / \mathrm{L}$ \\
\hline trans-1,2-Dichloroethylene (total) & WA & & & $\mathbf{U}$ & 5 & & 100 & $\mu g / L$ \\
\hline trans-1,3-Dichloropropene & WA & & & $\mathbf{U}$ & 5 & & NS & $\mu g / L$ \\
\hline trans-1,4-Dichloro-2-butene & WA & & & $\mathbf{U}$ & 100 & & NS & $\mu \mathrm{g} / \mathrm{L}$ \\
\hline Trichloroethylene & WA & & & U & 5 & & 5 & $\mu g / L$ \\
\hline Trichlorofluoromethane & WA & & & $\mathbf{U}$ & 5 & & NS & $\mu g / L$ \\
\hline Vinyl acetate & WA & & & $\mathbf{U}$ & 10 & & NS & $\mu g / L$ \\
\hline Vinyl chloride & WA & & & $\mathbf{U}$ & 10 & & NS & $\mu g / L$ \\
\hline Xylenes (total) & WA & & & $\mathbf{U}$ & 5 & & 10000 & $\mu g / L$ \\
\hline
\end{tabular}




\section{Volatile Trip Blank}

Field Measurements:

Sample Date: 4/26/93

\begin{tabular}{|c|c|c|c|c|c|c|c|c|}
\hline Analyte & Lab & $\operatorname{Rep}$ & Avg & Qualifier & Result & Acc & Stand & Units \\
\hline 1,1,1,2-Tetrachloroethane & WA & & & $\bar{U}$ & 10 & & NS & $\mu g / L$ \\
\hline 1,1,1-Trichloroethane & WA & & & $\mathbf{U}$ & 5 & - & 200 & $\mu g / L$ \\
\hline 1,1,2,2-Tetrachloroethane & WA & & & $\mathbf{U}$ & 5 & & NS & $\mu g / L$ \\
\hline 1,1,2-Trichloroethane & WA & & & $\mathbf{U}$ & 5 & & 5 & $\mu g / L$ \\
\hline 1,1-Dichloroethane & WA & & & $\mathbf{U}$ & 5 & & NS & $\mu g / L$ \\
\hline 1,1-Dichloroethylene & WA & & & $\mathbf{U}$ & 5 & & 7 & $\mu g / L$ \\
\hline 1,2,3-Trichloropropane & WA & & & $\mathbf{U}$ & 10 & & NS & $\mu g / L$ \\
\hline 1,2-Dibromo-3-chloropropane & WA & & & $\mathbf{U}$ & 20 & & 0.2 & $\mu g / L$ \\
\hline 1,2-Dibromoethane & WA & & & $\mathbf{U}$ & 20 & & NS & $\mu g / L$ \\
\hline 1,2-Dichloroethane & WA & & & $\mathbf{U}$ & 5 & & 5 & $\mu g / L$ \\
\hline 1,2-Dichloropropane & WA & & & $\mathbf{U}$ & 5 & & 5 & $\mu g / L$ \\
\hline 2-Hexanone & WA & & $v^{\circ}$ & $\mathbf{U}$ & 10 & & NS & $\mu g / L$ \\
\hline 4-Methyl-2-pentanone & WA & & & $\mathbf{U}$ & 10 & & NS & $\mu g / L$ \\
\hline Acetone & WA & & & & 15.1 & & NS & $\mu \mathrm{g} / \mathrm{L}$ \\
\hline Acetonitrile & WA & & & $\mathbf{U}$ & 20 & & NS & $\mu g / L$ \\
\hline Acrolein & WA & & & $\mathbf{U}$ & 10 & & NS & $\mu g / L$ \\
\hline Acrylonitrile & WA & & & $\mathbf{U}$ & 10 & & NS & $\mu g / L$ \\
\hline Allyl chloride & WA & & & $\mathbf{U}$ & 100 & & NS & $\mu g / L$ \\
\hline Benzene & WA & & & $\mathbf{U}$ & 5 & & 5 & $\mu g / L$ \\
\hline Bromodichloromethane & WA & & & $\mathbf{U}$ & 5 & & NS & $\mu g / L$ \\
\hline Bromoform & WA & & & $\mathbf{U}$ & 5 & & NS & $\mu \mathrm{g} / \mathrm{L}$ \\
\hline Carbon disulfide & WA & & & $\mathbf{U}$ & 5 & & NS & $\mu g / L$ \\
\hline Carbon tetrachloride & WA & & & $\mathbf{U}$ & 5 & & 5 & $\mu g / L$ \\
\hline Chlorobenzene & WA & & & $\mathbf{U}$ & 5 & & 100 & $\mu g / L$ \\
\hline Chloroethane & WA & & & $\mathbf{U}$ & 10 & & NS & $\mu g / L$ \\
\hline Chloroform & WA & & & $\mathbf{U}$ & 5 & & 100 & $\mu \mathrm{g} / \mathrm{L}$ \\
\hline Chloroprene & WA & & & $\mathbf{U}$ & 20 & & NS & $\mu g / L$ \\
\hline cis-1,3-Dichloropropene & WA & & & $\mathbf{U}$ & 5 & & NS & $\mu g / L$ \\
\hline Dibromochloromethane & WA & & & $\mathbf{U}$ & 5 & & NS & $\mu g / L$ \\
\hline Dichlorodifluoromethane & WA & & & $\mathbf{U}$ & 10 & & NS & $\mu g / L$ \\
\hline Ethylbenzene & WA & & & $\mathbf{U}$ & 5 & & 700 & $\mu g / L$ \\
\hline Isobutyl alcohol & WA & & & $\mathbf{U}$ & 20 & & NS & $\mu g / L$ \\
\hline Methacrylonitrile & WA & & & $\mathbf{U}$ & 20 & & NS & $\mu g / L$ \\
\hline Methyl bromide & WA & & & $\mathbf{U}$ & 10 & & NS & $\mu g / L$ \\
\hline Methyl chloride & WA & & & $\mathbf{U}$ & 10 & & NS & $\mu g / L$ \\
\hline Methyl ethyl ketone & WA & & & $\mathbf{U}$ & 10 & & NS & $\mu g / L$ \\
\hline Methyl iodide & WA & & & $\mathbf{U}$ & 10 & & NS & $\mu g / L$ \\
\hline Methylene bromide & WA & & & $\mathbf{U}$ & 10 & & NS & $\mu g / L$ \\
\hline Methylene chloride & WA & & & $\mathrm{J}$ & 2.1 & & 5 & $\mu g / L$ \\
\hline Propionitrile . & WA & & & $\mathbf{U}$ & 50 & & NS & $\mu g / L$ \\
\hline Styrene & WA & & & $\mathbf{U}$ & 5 & & 100 & $\mu g / L$ \\
\hline Tetrachloroethylene & WA & & & $\mathbf{U}$ & 5 & & 5 & $\mu g / L$ \\
\hline Toluene & WA & & & $\mathbf{J}$ & 3.25 & & 1000 & $\mu \mathrm{g} / \mathrm{L}$ \\
\hline trans-1,2-Dichloroethylene (total) & WA & & & $\mathbf{U}$ & 5 & & 100 & $\mu g / L$ \\
\hline trans-1,3-Dichloropropene & WA & & & $\mathbf{U}$ & 5 & & NS & $\mu g / L$ \\
\hline trans-1,4-Dichloro-2-butene & WA & & & $\mathbf{U}$ & 100 & & NS & $\mu \mathrm{g} / \mathrm{L}$ \\
\hline Trichloroethylene & WA & & & $\mathbf{U}$ & 5 & & 5 & $\mu g / L$ \\
\hline Trichlorofluoromethane & WA & & & $\mathbf{U}$ & 5 & & NS & $\mu g / L$ \\
\hline Vinyl acetate & WA & & & $\mathbf{U}$ & 10 & & NS & $\mu g / L$ \\
\hline Vinyl chloride & WA & & & $\mathbf{U}$ & 10 & & NS & $\mu \mathrm{g} / \mathrm{L}$ \\
\hline Xylenes (total) & WA & & & $\mathbf{U}$ & 5 & & 10000 & $\mu g / L$ \\
\hline
\end{tabular}


Volatile Trip Blank

Field Measurements:

Sample Date: 4/26/93

\begin{tabular}{|c|c|c|c|c|c|c|c|c|}
\hline Analyte & Lab & Rep & Avg & Qualifier & Result & Acc & Stand & Units \\
\hline 1,1,1,2-Tetrachloroethane & WA & & & $\mathbf{U}$ & 10 & & NS & $\overline{\mu g / L}$ \\
\hline 1,1,1-Trichloroethane & WA & & & $\mathbf{U}$ & 5 & . & 200 & $\mu \mathrm{g} / \mathrm{L}$ \\
\hline 1,1,2,2-Tetrachloroethane & WA & & & $\mathbf{U}$ & 5 & & NS & $\mu \mathrm{g} / \mathrm{L}$ \\
\hline 1,1,2-Trichloroethane & WA & & & $\mathbf{U}$ & 5 & & 5 & $\mu g / L$ \\
\hline 1,1-Dichloroethane & WA & & & $\mathbf{U}$ & 5 & & NS & $\mu g / L$ \\
\hline 1,1-Dichloroethylene & WA & & & $\mathbf{U}$ & 5 & & 7 & $\mu g / L$ \\
\hline 1,2,3-Trichloropropane & WA & & & $\mathbf{U}$ & 10 & & NS & $\mu \mathrm{g} / \mathrm{L}$ \\
\hline 1,2-Dibromo-3-chloropropane & WA & & & $\mathbf{U}$ & 20 & & 0.2 & $\mu g / L$ \\
\hline 1,2-Dibromoethane & WA & & & $\mathbf{U}$ & 20 & & NS & $\mu \mathrm{g} / \mathrm{L}$ \\
\hline 1,2-Dichloroethane & WA & & & $\mathbf{U}$ & 5 & & 5 & $\mu g / L$ \\
\hline 1,2-Dichloropropane & WA & & & $\mathbf{U}$ & 5 & & 5 & $\mu g / L$ \\
\hline 2-Hexanone & WA & & & $\mathbf{U}$ & 10 & & NS & $\mu g / L$ \\
\hline 4-Methyl-2-pentanone & WA & & & $\mathbf{U}$ & 10 & & NS & $\mu g / L$ \\
\hline Acetone & WA & & & $\mathbf{J}$ & 8.08 & & NS & $\mu \mathrm{g} / \mathrm{L}$ \\
\hline Acetonitrile & WA & & & $\mathbf{U}$ & 20 & & NS & $\mu g / L$ \\
\hline Acrolein & WA & & & $\mathbf{U}$ & 10 & & NS & $\mu g / L$ \\
\hline Acrylonitrile & WA & & & $\mathbf{U}$ & 10 & & NS & $\mu g / L$ \\
\hline Allyl chloride & WA & & & $\mathbf{U}$ & 100 & & NS & $\mu g / L$ \\
\hline Benzene & WA & & & $\mathbf{U}$ & 5 & & 5 & $\mu g / L$ \\
\hline Bromodichloromethane & WA & & & $\mathbf{U}$ & 5 & & NS & $\mu g / L$ \\
\hline Bromoform & WA & & & $\mathbf{U}$ & 5 & & NS & $\mu g / L$ \\
\hline Carbon disulfide & WA & & & $\mathbf{U}$ & 5 & & NS & $\mu g / L$ \\
\hline Carbon tetrachloride & WA & & & $\mathbf{U}$ & 5 & & 5 & $\mu g / L$ \\
\hline Chlorobenzene & WA & & & $\mathbf{U}$ & 5 & & 100 & $\mu g / L$ \\
\hline Chloroethane & WA & & & $\mathbf{U}$ & 10 & & NS & $\mu g / L$ \\
\hline Chloroform & WA & & & $\mathbf{U}$ & 5 & & 100 & $\mu g / L$ \\
\hline Chloroprene & WA & & & $\mathbf{U}$ & 20 & & NS & $\mu \mathrm{g} / \mathrm{L}$ \\
\hline cis-1,3-Dichloropropene & WA & & & $\mathbf{U}$ & 5 & & NS & $\mu \mathrm{g} / \mathrm{L}$ \\
\hline Dibromochloromethane & WA & & & $\mathbf{U}$ & 5 & & NS & $\mu g / L$ \\
\hline Dichlorodifluoromethane & WA & & & $\mathbf{U}$ & 10 & & NS & $\mu g / L$ \\
\hline Ethylbenzene & WA & & & $\mathbf{U}$ & 5 & & 700 & $\mu g / L$ \\
\hline Isobutyl alcohol & WA & & & $\mathbf{U}$ & 20 & & NS & $\mu g / L$ \\
\hline Methacrylonitrile & WA & & & $\mathbf{U}$ & 20 & & NS & $\mu g / L$ \\
\hline Methyl bromide & WA & & & $\mathbf{U}$ & 10 & & NS & $\mu \mathrm{g} / \mathrm{L}$ \\
\hline Methyl chloride & WA & & & $\mathbf{U}$ & 10 & & NS & $\mu g / L$ \\
\hline Methyl ethyl ketone & WA & & & $\mathbf{U}$ & 10 & & NS & $\mu g / L$ \\
\hline Methyl iodide & WA & & & $\mathbf{U}$ & 10 & & NS & $\mu g / L$ \\
\hline Methylene brumide & WA & & & $\mathbf{U}$ & 10 & & NS & $\mu g / L$ \\
\hline Methylene chloride & WA & & & $\mathbf{J}$ & 1.79 & & 5 & $\mu g / L$ \\
\hline Propionitrile & WA & & & $\mathrm{U}$ & 50 & & NS & $\mu \mathrm{g} / \mathrm{L}$ \\
\hline Styrene & WA & & & $\mathbf{U}$ & 5 & & 100 & $\mu g / L$ \\
\hline Tetrachloroethylene & WA & & & $\mathbf{U}$ & 5 & & 5 & $\mu \mathrm{g} / \mathrm{L}$ \\
\hline Toluene & WA & & & $\mathbf{J}$ & 2.34 & & 1000 & $\mu g / L$ \\
\hline trans-1,2-Dichloroethylene (total) & WA & & & $\mathbf{U}$ & 5 & & 100 & $\mu g / L$ \\
\hline trans-1,3-Dichloropropene & WA & & & $\mathbf{U}$ & 5 & & NS & $\mu g / L$ \\
\hline trans-1,4-Dichloro-2-butene & WA & & & $\mathbf{U}$ & 100 & & NS & $\mu \mathrm{g} / \mathrm{L}$ \\
\hline Trichloroethylene & WA & & & $\mathbf{U}$ & 5 & & 5 & $\mu g / L$ \\
\hline Trichlorofluoromethane & WA & & & $\mathbf{U}$ & 5 & & NS & $\mu g / L$ \\
\hline Vinyl acetate & WA & & & $\mathbf{U}$ & 10 & & NS & $\mu g / L$ \\
\hline Vinyl chloride & WA & & & $\mathbf{U}$ & 10 & & NS & $\mu g / L$ \\
\hline Xylenes (total) & WA & & & $\mathbf{U}$ & 5 & & 10000 & $\mu g / L$ \\
\hline
\end{tabular}




\section{Volatile Trip Blank}

Field Measurements:

Sample Date: 4/28/93

\begin{tabular}{|c|c|c|c|c|c|c|c|c|}
\hline Analyte & Lab & Rep & Avg & Qualifier & Result & Acc & Stand & Units \\
\hline 1,1,1,2-Tetrachloroethane & WA & & & $\mathbf{U}$ & 10 & & $\mathbf{N S}$ & $\mu g / L$ \\
\hline 1,1,1-Trichloroethane & WA & & & $\mathbf{U}$ & 5 & & 200 & $\mu g / L$ \\
\hline 1,1,2,2-Tetrachloroethane & WA & & & $\mathbf{U}$ & 5 & $\cdot$ & NS & $\mu \mathrm{g} / \mathrm{L}$ \\
\hline 1,1,2-Trichloroethane & WA & & & $\mathbf{U}$ & 5 & & 5 & $\mu g / L$ \\
\hline 1,1-Dichloroethane & WA & & & $\mathbf{U}$ & 5 & & NS & $\mu g / L$ \\
\hline 1,1-Dichloroethylene & WA & & & $\mathbf{U}$ & 5 & & 7 & $\mu g / L$ \\
\hline 1,2,3-Trichloropropane & WA & & & $\mathbf{U}$ & 10 & & NS & $\mu \mathrm{g} / \mathrm{L}$ \\
\hline 1,2-Dibromo-3-chloropropane & WA & & & $\mathbf{U}$ & 20 & & 0.2 & $\mu g / L$ \\
\hline 1,2-Dibromoethane & WA & & & $\mathbf{U}$ & 20 & & NS & $\mu g / L$ \\
\hline 1,2-Dichloroethane & WA & & & $\mathbf{U}$ & 5 & & 5 & $\mu g / L$ \\
\hline 1,2-Dichloropropane & WA & & & $\mathbf{U}$ & 5 & & 5 & $\mu g / L$ \\
\hline 2-Hexanone & WA & & & $\mathbf{U}$ & 10 & & NS & $\mu g / L$ \\
\hline 4-Methyl-2-pentanone & WA & & & $\mathbf{U}$ & 10 & & NS & $\mu g / L$ \\
\hline Acetone & WA & & & & 19.2 & & NS & $\mu g / L$ \\
\hline Acetonitrile & WA & & & $\mathbf{U}$ & 20 & & NS & $\mu \mathrm{g} / \mathrm{L}$ \\
\hline Acrolein & WA & & & $\mathbf{U}$ & 10 & & NS & $\mu g / L$ \\
\hline Acrylonitrile & WA & & & $\mathbf{U}$ & 10 & & NS & $\mu g / L$ \\
\hline Allyl chloride & WA & & & $\mathbf{U}$ & 100 & & NS & $\mu g / L$ \\
\hline Benzene & WA & & & $\mathbf{U}$ & 5 & & 5 & $\mu \mathrm{g} / \mathrm{L}$ \\
\hline Bromodichloromethane & WA & & & $\mathbf{U}$ & 5 & & NS & $\mu g / L$ \\
\hline Bromoform & WA & & & $\mathbf{U}$ & 5 & & NS & $\mu g / L$ \\
\hline Carbon disulfide & WA & & & $\mathbf{U}$ & 5 & & NS & $\mu g / L$ \\
\hline Carbon tetrachloride & WA & & & $\mathbf{U}$ & 5 & & 5 & $\mu g / L$ \\
\hline Chlorobenzene & WA & & & $\mathbf{U}$ & 5 & & 100 & $\mu \mathrm{g} / \mathrm{L}$ \\
\hline Chloroethane & WA & & & $\mathbf{U}$ & 10 & & NS & $\mu \mathrm{g} / \mathrm{L}$ \\
\hline Chloroform & WA & & & $\mathbf{U}$ & 5 & & 100 & $\mu g / L$ \\
\hline Chloroprene & WA & & & $\mathbf{U}$ & 20 & & NS & $\mu g / L$ \\
\hline cis-1,3-Dichloropropene & WA & & & $\mathbf{U}$ & 5 & & NS & $\mu \mathrm{g} / \mathrm{L}$ \\
\hline Dibromochloromethane & WA & & & $\mathbf{U}$ & 5 & & NS & $\mu \mathrm{g} / \mathrm{L}$ \\
\hline Dichlorodifluoromethane & WA & & & $\mathbf{U}$ & 10 & & NS & $\mu g / L$ \\
\hline Ethylbenzene & WA & & & $\mathbf{U}$ & 5 & & 700 & $\mu \mathrm{g} / \mathrm{L}$ \\
\hline Isobutyl alcohol & WA & & & $\mathbf{U}$ & 20 & & NS & $\mu g / L$ \\
\hline Methacrylonitrile & WA & & & $\mathbf{U}$ & 20 & & NS & $\mu \mathrm{g} / \mathrm{L}$ \\
\hline Methyl broinide & WA & & & $\mathbf{U}$ & 10 & & NS & $\mu g / L$ \\
\hline Methyl chloride & WA & & & $\mathbf{U}$ & 10 & & NS & $\mu \mathrm{g} / \mathrm{L}$ \\
\hline Methyl ethyl ketone & WA & & & $\mathbf{U}$ & 10 & & NS & $\mu g / L$ \\
\hline Methyl iodide & WA & & & $\mathbf{U}$ & 10 & & NS & $\mu \mathrm{g} / \mathrm{L}$ \\
\hline Methylene bromide & WA & & & $\mathbf{U}$ & 10 & & NS & $\mu g / L$ \\
\hline Methylene chloride & WA & & & $\mathbf{J}$ & 1.72 & & 5 & $\mu \mathrm{g} / \mathrm{L}$ \\
\hline Propionitrile . & WA & & & $\mathbf{U}$ & 50 & & NS & $\mu \mathrm{g} / \mathrm{L}$ \\
\hline Styrene & WA & & & $\mathbf{U}$ & 5 & & 100 & $\mu \mathrm{g} / \mathrm{L}$ \\
\hline Tetrachloroethylene & WA & & & $\mathbf{U}$ & 5 & & 5 & $\mu \mathrm{g} / \mathrm{L}$ \\
\hline Toluene & WA & & & J & 1.49 & & 1000 & $\mu \mathrm{g} / \mathrm{L}$ \\
\hline trans-1,2-Dichloroethylene (total) & WA & & & $\mathbf{U}$ & 5 & & 100 & $\mu \mathrm{g} / \mathrm{L}$ \\
\hline trans-1,3-Dichloropropene & WA & & & $\mathbf{U}$ & 5 & & NS & $\mu \mathrm{g} / \mathrm{L}$ \\
\hline trans-1,4-Dichloro-2-butene & WA & & & $\mathbf{U}$ & 100 & & NS & $\mu \mathrm{g} / \mathrm{L}$ \\
\hline Trichloroethylene & WA & & & $\mathbf{U}$ & 5 & & 5 & $\mu \mathrm{g} / \mathrm{L}$ \\
\hline Trichlorofluoromethane & WA & & & $\mathbf{U}$ & 5 & & NS & $\mu \mathrm{g} / \mathrm{L}$ \\
\hline Vinyl acetate & WA & & & $\mathbf{U}$ & 10 & & NS & $\mu g / L$ \\
\hline Vinyl chloride & WA & & & $\mathbf{U}$ & 10 & & NS & $\mu g / L$ \\
\hline Xylenes (total) & WA & & & $\mathbf{U}$ & 5 & & 10000 & $\mu \mathrm{g} / \mathrm{L}$ \\
\hline
\end{tabular}




\section{Appendix B}

\section{Analytical Results}

Fall Sampling Event 



\section{APPENDIX B}

\section{Analytical Results - Fall Sampling Event}

Analytical results from the fall sampling event of the UTR wetlands sampling project are presented in this section. The analytical data for each location (wetland and stream) are presented in tabular format with the background samples presented first followed by the stream samples, wetland samples, and QAVC samples. Locations ending with the suffix "D" designate drainage, or stream sampling locations. These drainage, or stream, samples were collected from perennial tributaries to UTR rather than the main channel of UTR. All other location IDs, excluding QA samples, designate wetland locations (i.e. UTR or BGS). Location identification numbers beginning with the prefix "BG" designate background locations.

For each sampling location, the sampling date, field measurements including pH, conductivity, etc., and all laboratory analyses are presented (excluding lab blanks and standards). Included in the laburatory analyses tables for each location and analyte is the lab that performed the analyses, any result or analytical qualifiers, the analytical results, the accuracy of the result (for radiological analyses only), any standard (i.e. PDWS, MCL, SDWS), and the units of measurement.

The following is a list of abbreviations used in the tables:

$\begin{array}{ll}\text { Lab } & \begin{array}{l}\text { Laboratory performing analysis } \\ \text { Modifier }\end{array} \\ \begin{array}{l}\text { Result or analysis qualifier } \\ \text { Stand }\end{array} & \begin{array}{l}\text { Standard } \\ \text { Rep }\end{array} \\ \text { Rvglication sample (denoted by "R") } \\ \text { Average code }\end{array}$

Result and Analysis Qualifiers

\begin{tabular}{|l|l|}
\hline Result Qualifiers \\
\hline CODE & DESCRIPTION \\
\hline (BLANK) & Data not remarked. Number should be interpreted exactly as reported. \\
\hline $\mathrm{J}$ & The analytical result is an estimated quantity. \\
\hline $\mathrm{R}$ & $\begin{array}{l}\text { Rejected because of performance requirements in the sample or associated quality contro } \\
\text { analyses were not met. The analyte may or may not be present. }\end{array}$ \\
\hline $\mathrm{U}$ & Material analyzed for but not detected. Value reported is the sample quantification limit. \\
\hline
\end{tabular}

\begin{tabular}{|l|l|}
\hline \multicolumn{2}{|l|}{ Analysis Qualifiers } \\
\hline CODE & DESCRIPTION \\
\hline $\mathrm{Q}$ & $\begin{array}{l}\text { Sample held beyond normal holding time. If holding time is exceeded by less than } 30 \text { das } \\
\text { used with "J". If holding time is exceeded by more than } 30 \text { days, used with "R". }\end{array}$ \\
\hline $\mathrm{L}$ & Analytical factor causing bias. The associated result may under estimate the true value. \\
\hline $\mathrm{H}$ & Analytical factor causing bias. The associated result may over estimate the true value. \\
\hline $\mathrm{P}$ & Analytical factor causing bias. The associated result may be of high variability.. \\
\hline $\mathrm{V}$ & Indicates the analyte was detected in both the sample and associated method blank. \\
\hline
\end{tabular}




\section{BGD001}

Field Measurements:

Stream Discharge: 0.015 cfs

Sample Date: 9/1/93

pH: 5.26

Spevific Conductance: $31 \mu \mathrm{S} / \mathrm{cm}$

Red/Oxid Potential: $161 \mathrm{mv}$

Dissolved Oxygen: $3.91 \mathrm{mg} / \mathrm{L}$

\begin{tabular}{|c|c|c|c|c|c|c|c|c|}
\hline Analyte & Lab & Rep & Avg & Modifier & Result & Acc & Stand & Units \\
\hline 1,1,1,2-Tetrachloroethane & WA & & & $\mathbf{U}$ & 10 & & NS & $\mu g / L$ \\
\hline 1,1,1-Trichloroethane & WA & & & $\mathbf{U}$ & 5 & & 200 & $\mu g / L$ \\
\hline $1,1,2,2$-Tetrachloroethane & WA & & & $\mathbf{U}$ & 5 & & NS & $\mu g / L$ \\
\hline 1,1,2-Trichloroethane & WA & & & $\mathbf{U}$ & 5 & & 5 & $\mu g / L$ \\
\hline 1,1-Dichloroethane & WA & & & $\mathbf{U}$ & 5 & & NS & $\mu g / L$ \\
\hline 1,1-Dichloroethylene & WA & & & $\mathbf{U}$ & 5 & & 7 & $\mu g / L$ \\
\hline 1,2,3-Trichloropropane & WA & & & $\mathbf{U}$ & 10 & & NS & $\mu g / L$ \\
\hline 1,2-Dibromo-3-chloropropane & WA & & & $\mathbf{U}$ & 20 & & 0.2 & $\mu g / L$ \\
\hline 1,2-Dibromoethane & WA & & & $\mathbf{U}$ & 20 & & NS & $\mu g / L$ \\
\hline 1,2-Dichloroethane & WA & & & $\mathbf{U}$ & 5 & & 5 & $\mu g / L$ \\
\hline 1,2-Dichloroethylene (total) & WA & & & $\mathbf{U}$ & 5 & & 7 & $\mu g / L$ \\
\hline 1,2-Dichloropropane & WA & & & $\mathbf{U}$ & 5 & & 5 & $\mu g / L$ \\
\hline 2-Hexanone & WA & & & $\mathbf{U}$ & 10 & & NS & $\mu g / L$ \\
\hline 4-Methyl-2-pentanone & WA & & & $\mathbf{U}$ & 10 & & NS & $\mu g / L$ \\
\hline Acetone & WA & & & & 14.8 & & NS & $\mu g / L$ \\
\hline Acetonitrile & WA & & & $\mathbf{U}$ & 20 & & NS & $\mu g / L$ \\
\hline Acidity & WA & & $\mathbf{A}$ & & 27 & & NS & $\mathrm{mg} / \mathrm{L}$ \\
\hline Acrolein & WA & & & $\mathbf{U}$ & 10 & & NS & $\mu g / L$ \\
\hline Acrylonitrile & WA & & & $\mathbf{U}$ & 10 & & NS & $\mu g / L$ \\
\hline Alkalinity & WA & & $\mathbf{A}$ & & 1.25 & & NS & $\mathrm{mg} / \mathrm{L}$ \\
\hline Allyl chloride & WA & & & $\mathbf{U}$ & 100 & & NS & $\mu g / L$ \\
\hline Aluminum & WA & & A & & 474.5 & & NS & $\mu \mathrm{g} / \mathrm{L}$ \\
\hline Antimony & WA & & & $\mathbf{U}$ & 60 & & 6 & $\mu g / L$ \\
\hline Antimony & WA & & NA & $\mathbf{U}$ & 60 & & 6 & $\mu g / L$ \\
\hline Arsenic & WA & & & $\mathbf{U}$ & 10 & & 50 & $\mu \mathrm{g} / \mathrm{L}$ \\
\hline Arsenic & WA & & NA & $\mathbf{U}$ & 10 & & 50 & $\mu g / L$ \\
\hline Barium & WA & & & $\mathbf{U}$ & 200 & & 2000 & $\mu g / L$ \\
\hline Barium & WA & & $\mathbf{N A}$ & $\mathbf{U}$ & 200 & & 2000 & $\mu g / L$ \\
\hline Benzene & WA & & & $\mathbf{U}$ & 5 & & 5 & $\mu g / L$ \\
\hline Beryllium & WA & & & $\mathbf{U}$ & 5 & & 4 & $\mu g / L$ \\
\hline Beryllium & WA & & NA & $\mathbf{U}$ & 5 & & 4 & $\mu \mathrm{g} / \mathrm{L}$ \\
\hline Bromodichloromethane & WA & & & $\mathbf{U}$ & 5 & & NS & $\mu \mathrm{g} / \mathrm{L}$ \\
\hline Bromoform & WA & & & $\mathbf{U}$ & 5 & & NS & $\mu \mathrm{g} / \mathrm{L}$ \\
\hline Cadmium & WA & & & $\mathbf{U}$ & 5 & & 5 & $\mu g / L$ \\
\hline Cadmium & WA & & NA & $\mathbf{U}$ & 5 & & 5 & $\mu g / L$ \\
\hline Calcium & WA & & & $\mathbf{U}$ & 5000 & & NS & $\mu g / L$ \\
\hline Calcium & WA & & $\mathbf{N A}$ & $\mathbf{U}$ & 5000 & & NS & $\mu g / L$ \\
\hline Carbon disulfide & WA & & & $\mathbf{U}$ & 5 & & NS & $\mu g / L$ \\
\hline Carbon tetrachloride & WA & & & $\mathbf{U}$ & 5 & & 5 & $\mu g / L$ \\
\hline Cesium -137 & TM & & NA & $\mathbf{U}$ & 5.9 & & 200 & $\mathrm{pCi} / \mathrm{L}$ \\
\hline Cesium-137 & $\mathbf{T M}$ & & & $\mathbf{U}$ & 5.8 & & 200 & $\mathrm{pCi} / \mathrm{L}$ \\
\hline Chloride & WA & & & & 1.47 & & NS & $\mathrm{mg} / \mathrm{L}$ \\
\hline Chlorobenzene & WA & & & $\mathbf{U}$ & 5 & & 100 & $\mu g / L$ \\
\hline Chloroethane & WA & & & $\mathbf{U}$ & 10 & & NS & $\mu g / L$ \\
\hline Chloroform & WA & & & $\mathbf{U}$ & 5 & & 100 & $\mu g / L$ \\
\hline Chloroprene & WA & & & $\mathbf{U}$ & 20 & & NS & $\mu g / L$ \\
\hline
\end{tabular}


BGD001 Laboratory Analyses (cont'd)

\begin{tabular}{|c|c|c|c|c|c|c|c|c|c|}
\hline Analyte & Lab & Rep & Avg & Modifier & & Result & Acc & Stand & Units \\
\hline Chromium & WA & & & $U$ & & 10 & & 100 & $\overline{\mu g / L}$ \\
\hline Chromium & WA & & NA & $\mathbf{U}$ & & 10 & & 100 & $\mu g / L$ \\
\hline cis-1,3-Dichloropropene & WA & & & $\mathbf{U}$ & & 5 & & NS & $\mu g / L$ \\
\hline Cobalt & WA & & & $\mathbf{U}$ & & 50 & & NS & $\mu g / L$ \\
\hline Cobalt & WA & & NA & $\mathbf{U}$ & & 50 & & NS & $\mu g / L$ \\
\hline Copper & WA & & & $\mathbf{U}$ & & 25 & & 1000 & $\mu g / L$ \\
\hline Copper & WA & & NA & $\mathbf{U}$ & & 25 & & 1000 & $\mu \mathrm{g} / \mathrm{L}$ \\
\hline Dibromochloromethane & WA & & & $\mathbf{U}$ & & 5 & & NS & $\mu g / L$ \\
\hline Dichlorodifluoromethane & WA & & & U & & 10 & & NS & $\mu g / L$ \\
\hline Ethylbenzene & WA & & & $\mathbf{U}$ & & 5 & & 700 & $\mu \mathrm{g} / \mathrm{L}$ \\
\hline Fluoride & WA & & & $\mathbf{U}$ & & 0.1 & & 4 & $\mathrm{mg} / \mathrm{L}$ \\
\hline Gross alpha & TM & & & & & 1.4 & 1.1 & 15 & $\mathrm{pCi} / \mathrm{L}$ \\
\hline Gross alpha & TM & & A & & & 1.6 & 1.15 & 15 & $\mathrm{pCi} / \mathrm{L}$ \\
\hline Iron & WA & & A & & & 1745 & & 300 & $\mu g / L$ \\
\hline Isobutyl alcohol & WA & & & $\mathbf{U}$ & & 20 & & NS & $\mu \mathrm{g} / \mathrm{L}$ \\
\hline Lead & WA & & & $\mathbf{U}$ & & 3 & & 50 & $\mu \mathrm{g} / \mathrm{L}$ \\
\hline Lead & WA & & NA & & & 3.6 & & 50 & $\mu g / L$ \\
\hline Magnesium & WA & & & $\mathbf{U}$ & & 5000 & & NS & $\mu g / L$ \\
\hline Magnesium & WA & & NA & $\mathbf{U}$ & & 5000 & & NS & $\mu \mathrm{g} / \mathrm{L}$ \\
\hline Manganese & WA & & A & & & 90.6 & & 50 & $\mu g / L$ \\
\hline Mercury & WA & & & $\mathbf{U}$ & & 0.2 & & 2 & $\mu \mathrm{g} / \mathrm{L}$ \\
\hline Mercury & WA & & NA & $\mathbf{U}$ & & 0.2 & & 2 & $\mu g / L$ \\
\hline Methacrylonitrile & WA & & & $\mathbf{U}$ & & 20 & & NS & $\mu g / L$ \\
\hline Methyl bromide & WA & & & U & & 10 & & NS & $\mu g / L$ \\
\hline Methyl chloride & WA & & & U & & 10 & & NS & $\mu \mathrm{g} / \mathrm{L}$ \\
\hline Methyl ethyl ketone & WA & & & $\mathbf{U}$ & & 10 & & NS & $\mu g / L$ \\
\hline Methyl iodide & WA & & & $\mathbf{U}$ & & 10 & & NS & $\mu g / L$ \\
\hline Methylene bromide & WA & & & $\mathbf{U}$ & & 10 & & NS & $\mu \mathrm{g} / \mathrm{L}$ \\
\hline Methylene chloride & WA & & & $\mathbf{J}$ & V & 3.71 & & 5 & $\mu \mathrm{g} / \mathrm{L}$ \\
\hline Nickel & WA & & & $\mathbf{U}$ & & 40 & & 100 & $\mu g / L$ \\
\hline Nickel & WA & & NA & $\mathbf{U}$ & & 40 & & 100 & $\mu g / L$ \\
\hline Nitrate as nitrogen & WA & & & $\mathbf{J}$ & $\mathbf{Q}$ & 0.047 & & 10 & $\mathrm{mg} / \mathrm{L}$ \\
\hline Nonvolatile beta & TM & & $\mathbf{A}$ & & & 1.45 & 2.45 & 50 & $\mathrm{pCi} / \mathrm{L}$ \\
\hline Potassium & WA & & & $\mathbf{U}$ & & 5000 & & NS & $\mu \mathrm{g} / \mathrm{L}$ \\
\hline Potassium & WA & & NA & $\mathbf{U}$ & & 5000 & & NS & $\mu g / L$ \\
\hline Propionitrile & WA & & & $\mathbf{U}$ & & 50 & & NS & $\mu g / L$ \\
\hline Selenium & WA & & & $\mathbf{U}$ & & 5 & & 50 & $\mu g / L$ \\
\hline Selenium & WA & & NA & $\mathbf{U}$ & & 5 & & 50 & $\mu \mathrm{g} / \mathrm{L}$ \\
\hline Silver & WA & & & $\mathbf{U}$ & & 10 & & 50 & $\mu \mathrm{g} / \mathrm{L}$ \\
\hline Silver & WA & & NA & $\mathbf{U}$ & & 10 & & 50 & $\mu g / L$ \\
\hline Sodium & WA & & & $\mathrm{U}$ & & 5000 & & NS & $\mu g / L$ \\
\hline Sodium & WA & & NA & $\mathbf{U}$ & & 5000 & & NS & $\mu g / L$ \\
\hline Styrene & WA & & & $\mathbf{U}$ & & 5 & & 100 & $\mu \mathrm{g} / \mathrm{L}$ \\
\hline Sulfate & WA & & & U & & 2.5 & & 400 & $\mathrm{mg} / \mathrm{L}$ \\
\hline Sulfide & WA & & & $\mathbf{U}$ & & 0.1 & & NS & $\mathrm{mg} / \mathrm{L}$ \\
\hline Tetrachloroethylene & WA & & & $\mathbf{U}$ & & 5 & & 5 & $\mu g / L$ \\
\hline Thallium & WA & & & U & & 10 & & 2 & $\mu \mathrm{g} / \mathrm{L}$ \\
\hline Thallium & WA & & NA & $\mathbf{U}$ & & 10 & & 2 & $\mu \mathrm{g} / \mathrm{L}$ \\
\hline Toluene & WA & & & $\mathbf{U}$ & & 5 & & 1000 & $\mu \mathrm{g} / \mathrm{L}$ \\
\hline Total hardness & WA & & & & & 2.9 & & NS & $\mathrm{mg} / \mathrm{L}$ \\
\hline Total organic carbon & WA & & & & & 2.55 & & NS & $\mathrm{mg} / \mathrm{L}$ \\
\hline Total suspended solids & WA & & & & & 20 & & NS & $\mathrm{mg} / \mathrm{L}$ \\
\hline trans-1,3-Dichloropropene & WA & & & $\mathbf{U}$ & & 5 & & NS & $\mu g / L$ \\
\hline trans-1,4-Dichloro-2-butene & WA & & & U & & 100 & & NS & $\mu \mathrm{g} / \mathrm{L}$ \\
\hline Trichloroethylene & WA & & & $\mathbf{U}$ & & 5 & & 5 & $\mu g / L$ \\
\hline
\end{tabular}


Sampling And Analysis of Water From Upper Three Runs And lis Wetlands Near Tank 16 and the Mixed Waste Management Facility

BGD001 Laboratory Analyses (cont'd)

\begin{tabular}{|c|c|c|c|c|c|c|c|c|c|}
\hline Analyte & Lab & Rep & Avg & Modifier & & Result & Acc & Stand & Units \\
\hline Trichlorofluoromethane & WA & & & $\mathrm{U}$ & & 5 & & NS & $\mu g /$ \\
\hline Tritium & TM & & A & & & 1.79 & 0.36 & 20 & $\mathrm{pCi} / \mathrm{ml}$ \\
\hline Turbidity & WA & & & $\mathbf{J}$ & $\mathbf{Q}$ & 22.4 & & NS & NTU \\
\hline Vanadium & WA & & & $\mathrm{U}$ & & 50 & & NS & $\mu \mathrm{g} / \mathrm{L}$ \\
\hline Vanadium & WA & & NA & $\mathbf{U}$ & & 50 & & NS" & $\mu \mathrm{g} / \mathrm{L}$ \\
\hline Vinyl acetate & WA & & & $\mathbf{U}$ & & 10 & . & NS & $\mu g /$ \\
\hline Vinyl chloride & WA & & & $\mathbf{U}$ & & 10 & & 2 & $\mu \mathrm{g} \Omega$ \\
\hline Xylenes (total) & WA & & & $\mathbf{U}$ & & 5 & & 10000 & $\mu g / L$ \\
\hline Zinc & WA & & & & & 37.6 & & 5000 & $\mu \mathrm{g} / \mathrm{L}$ \\
\hline Zinc & WA & & NA & * & & 21.4 & & 5000 & $\mu g \Omega$ \\
\hline
\end{tabular}




\section{BGD002}

Field Measurements:

Stream Discharge: 0.0082 cfs

Sample Date: 9/1/93

pH: 5.41

Specific Conductance: $79 \mu \mathrm{S} / \mathrm{cm}$

Red/Oxid Potential: $197 \mathrm{mv}$

Dissolved Oxygen: $3.7 \mathrm{mg} / \mathrm{L}$

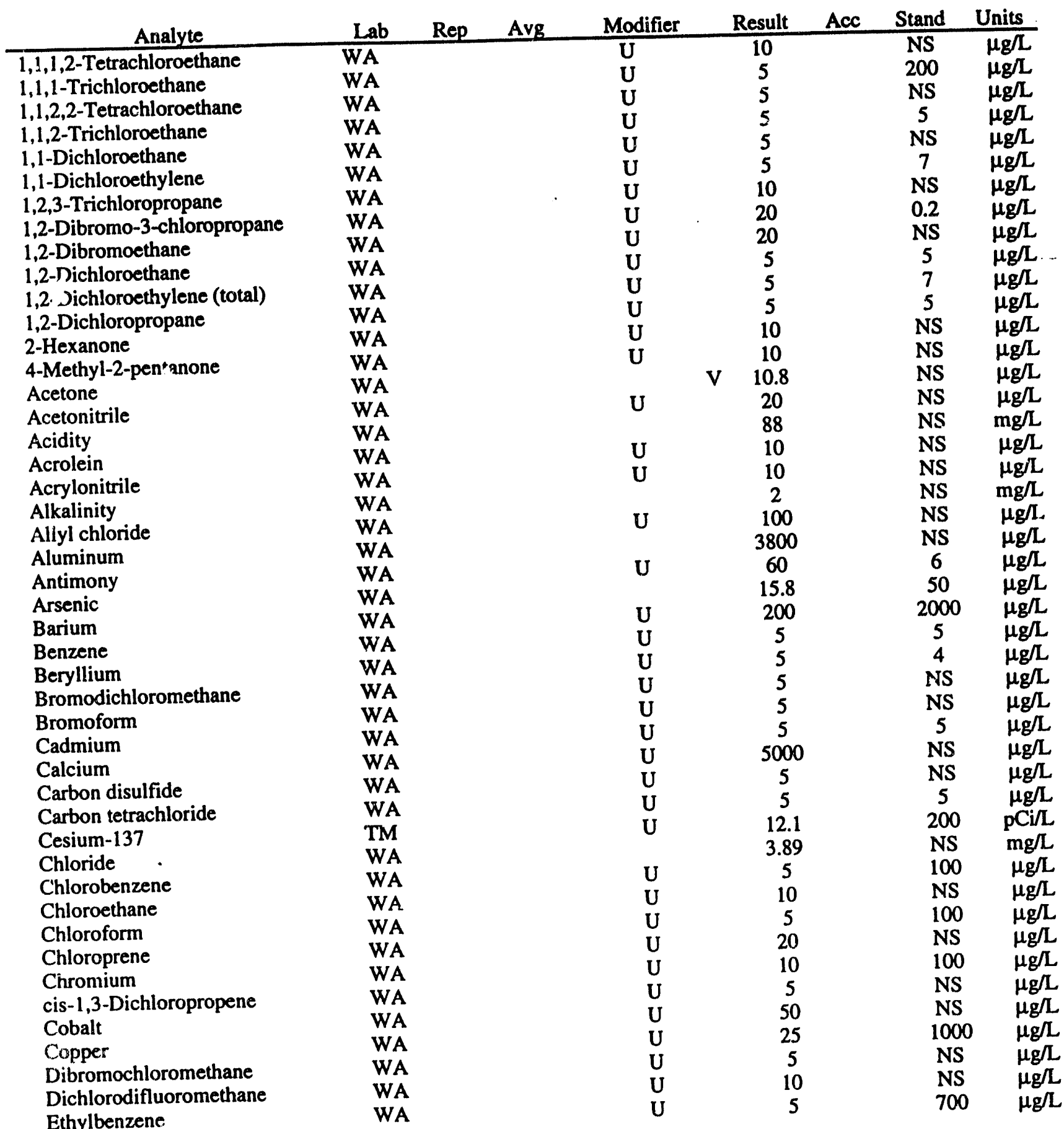


Sampling And Analysis of Water From Upper Three Runs And Its Wetlands Near Tank 16 and the Mixed Waste Management Facility

BGD002 Laboratory Analyses (cont'd)

\begin{tabular}{|c|c|c|c|c|c|c|c|c|c|}
\hline Analyte & Lab & Rep & Avg & Modifier & & Result & Acc & Stand & Units \\
\hline Fluoride & WA & & & $\bar{U}$ & & 0.1 & & 4 & $\mathrm{mg} / \mathrm{L}$ \\
\hline Fluoride & WA & & NA & U & & 0.1 & & 4 & $\mathrm{mg} / \mathrm{L}$ \\
\hline Gross alpha & TM & & & & & 0.8 & 1.4 & 15 & $\mathrm{p} \overline{\mathrm{Ci}} / \mathrm{L}$ \\
\hline Iron & WA & & & & & 146000 & & 300 & $\mu g / L$ \\
\hline Isobutyl alcohol & WA & & & $\mathbf{U}$ & & 20 & & NS & $\mu \mathrm{g} / \mathrm{L}$ \\
\hline Lead & WA & & & & & 4.2 & & 50 & $\mu \mathrm{g} / \mathrm{L}$ \\
\hline Magnesium & WA & & & $U$ & & 5000 & & NS & $\mu \mathrm{g} / \mathrm{L}$ \\
\hline Manganese & WA & & & & & 278 & & 50 & $\mu g / L$ \\
\hline Mercury & WA & & & $\mathbf{U}$ & & 0.2 & & 2 & $\mu \mathrm{g} / \mathrm{L}$ \\
\hline Methacrylonitrile & WA & & & $\mathrm{U}$ & & 20 & & NS & $\mu g / L$ \\
\hline Methyl bromide & WA & & & $\mathbf{U}$ & & 10 & & NS & $\mu g / L$ \\
\hline Methyl chloride & WA & & & $\mathbf{U}$ & & 10 & & NS & $\mu \mathrm{g} / \mathrm{L}$ \\
\hline Methyl ethyl ketone & WA & & & $\mathbf{U}$ & & 10 & & NS & $\mu g / L$ \\
\hline Methyl iodide & WA & & & $\mathbf{U}$ & & 10 & & NS & $\mu \mathrm{g} / \mathrm{L}$ \\
\hline Methylene bromide & WA & & & U & & 10 & & NS & $\mu \mathrm{g} / \mathrm{L}$ \\
\hline Methylene chloride & WA & & & $\mathbf{J}$ & V & 3.88 & & 5 & $\mu \mathrm{g} / \mathrm{L}$ \\
\hline Nickel & WA & & & $\mathbf{U}$ & & 40 & & 100 & $\mu g / L$ \\
\hline Nitrate as nitrogen & WA & & & $\mathbf{J}$ & $\mathbf{Q}$ & 0.034 & & 10 & $\mathrm{mg} / \mathrm{L}$ \\
\hline Nonvolatile beta & TM & & & & & 2.3 & 3.3 & 50 & $\mathrm{pCi} / \mathrm{L}$ \\
\hline Potassium & WA & & & $\mathbf{U}$ & & 5000 & & NS & $\mu g / L$ \\
\hline Propionitrile & WA & & & $\mathbf{U}$ & & 50 & & NS & $\mu g / L$ \\
\hline Selenium & WA & & & U & & 5 & & 50 & $\mu g / L$ \\
\hline Silver & WA & & & $\mathrm{U}$ & & 10 & & 50 & $\mu g / L$ \\
\hline Sodium & WA & & & $\mathrm{U}$ & & 5000 & & NS & $\mu g / L$ \\
\hline Styrene & WA & & & U & & 5 & & 100 & $\mu \mathrm{g} / \mathrm{L}$ \\
\hline Sulfate & WA & & & U & & 2.5 & & 400 & $\mathrm{mg} / \mathrm{L}$ \\
\hline Sulfide & WA & & & $\mathrm{U}$ & & 0.1 & & NS & $\mathrm{mg} / \mathrm{L}$ \\
\hline Tetrachloroethylene & WA & & & $U$ & & 5 & & 5 & $\mu \mathrm{g} / \mathrm{L}$ \\
\hline Thallium & WA & & & U & & 10 & & 2 & $\mu g / L$ \\
\hline Toluene & WA & & & U & & 5 & & 1000 & $\mu g / L$ \\
\hline Total hardness & WA & & & & & 3.9 & & NS & $\mathrm{mg} / \mathrm{L}$ \\
\hline Total organic carbon & WA & & & & & 6.11 & & NS & $\mathrm{mg} / \mathrm{L}$ \\
\hline Total suspended solids & WA & & & & & 143 & & NS & $\mathrm{mg} / \mathrm{L}$ \\
\hline trans-1,3-Dichloropropene & WA & & & U & & 5 & & NS & $\mu \mathrm{g} / \mathrm{L}$ \\
\hline trans-1,4-Dichloro-2-butene & WA & & & $\mathbf{U}$ & & 100 & & NS & $\mu \mathrm{g} / \mathrm{L}$ \\
\hline Trichloroethylene & WA & & & U & & 5 & & 5 & $\mu \mathrm{g} / \mathrm{L}$ \\
\hline Trichlorofluoromethane & WA & & & U & & 5 & & NS & $\mu \mathrm{g} / \mathrm{L}$ \\
\hline Tritium & TM & & & & & 2.16 & 0.39 & 20 & $\mathrm{pCi} / \mathrm{ml}$ \\
\hline Turbidity & WA & & & $\mathbf{J}$ & $\mathbf{Q}$ & 16.1 & & NS & NTU \\
\hline Vanadium & WA & & & $\mathbf{U}$ & & 50 & & NS & $\mu \mathrm{g} / \mathrm{L}$ \\
\hline Vinyl acetate & WA & & & $\mathrm{U}$ & & 10 & & NS & $\mu \mathrm{g} / \mathrm{L}$ \\
\hline Vinyl chloride & WA & & & $\mathbf{U}$ & & 10 & & 2 & $\mu g / L$ \\
\hline Xylenes (total) & WA & & & $\mathbf{U}$ & & 5 & & 10000 & $\mu g / L$ \\
\hline Zinc & WA & & & $\mathbf{U}$ & & 20 & & 5000 & $\mu g / L$ \\
\hline
\end{tabular}




\section{BGS002}

Field Measurements:

Sample Date: 9/1/93

pH: 5.48

Specific Conductance: $281 \mu \mathrm{S} / \mathrm{cm}$

Red/Oxid Potential: $281 \mathrm{mv}$

Dissolved Oxygen: $1 \mathrm{mg} / \mathrm{L}$

\begin{tabular}{|c|c|c|c|c|c|c|c|c|}
\hline Analyte & Lab & Rep & Avg & Modifier & Result & Acc & Stand & Units \\
\hline 1,1,1,2-Tetrachloroethane & WA & & & $\mathbf{U}$ & 10 & & NS & $\mu g / L$ \\
\hline 1,1,1-Trichloroethane & WA & & & $\mathbf{U}$ & 5 & & 200 & $\mu \mathrm{g} / \mathrm{L}$ \\
\hline 1,1,2,2-Tetrachloroethane & WA & & & $\mathbf{U}$ & 5 & & NS & $\mu g / L$ \\
\hline 1,1,2-Trichloroethane & WA & & & $\mathbf{U}$ & 5 & & 5 & $\mu \mathrm{g} / \mathrm{L}$ \\
\hline 1,1-Dichloroethane & WA & & & $\mathbf{U}$ & 5 & & NS & $\mu \mathrm{g} / \mathrm{L}$ \\
\hline 1,1-Dichloroethylene & WA & & & $\mathbf{U}$ & 5 & & 7 & $\mu g / L$ \\
\hline 1,2,3-Trichloropropane & WA & & & $\mathbf{U}$ & 10 & & NS & $\mu g / L$ \\
\hline 1,2-Dibromo-3-chloropropane & WA & & . & $\mathbf{U}$ & 20 & & 0.2 & $\mu g / L$ \\
\hline 1,2-Dibromoethane & WA & & & $\mathbf{U}$ & 20 & & NS & $\mu \mathrm{g} / \mathrm{L}$ \\
\hline 1,2-Dichloroethane & WA & & & $\mathbf{U}$ & 5 & & 5 & $\mu \mathrm{g} / \mathrm{L}$ \\
\hline 1,2-Dichloroethylene (total) & WA & & & $\mathbf{U}$ & 5 & & 7 & $\mu \mathrm{g} / \mathrm{L}$ \\
\hline 1,2-Dichloropropane & WA & & & $\mathbf{U}$ & 5 & & 5 & $\mu g / L$ \\
\hline 2-Hexanone & WA & & & $\mathbf{U}$ & 10 & & NS & $\mu \mathrm{g} / \mathrm{L}$ \\
\hline 4-Methyl-2-pentanone & WA & & & $\mathbf{U}$ & 10 & & NS & $\mu \mathrm{g} / \mathrm{L}$ \\
\hline Acetone & WA & & & & 12.4 & & NS & $\mu \mathrm{g} / \mathrm{L}$ \\
\hline Acetonitrile & WA & & & $\mathbf{U}$ & 20 & & NS & $\mu g / L$ \\
\hline Acidity & WA & & & & 84 & & NS & $\mathrm{mg} / \mathrm{L}$ \\
\hline Acrolein & WA & & & $\mathbf{U}$ & 10 & & NS & $\mu g / L$ \\
\hline Acrylonitrile & WA & & & $\mathbf{U}$ & 10 & & NS & $\mu \mathrm{g} / \mathrm{L}$ \\
\hline Alkalinity & WA & & & $\mathbf{U}$ & 0.5 & & NS & $\mathrm{mg} / \mathrm{L}$ \\
\hline Allyl chloride & WA & & & $\mathbf{U}$ & 100 & & NS & $\mu g / L$ \\
\hline Aluminum & WA & & & & 6060 & & NS & $\mu \mathrm{g} / \mathrm{L}$ \\
\hline Antimony & WA & & & $\mathbf{U}$ & 60 & & 6 & $\mu \mathrm{g} / \mathrm{L}$ \\
\hline Arsenic & WA & & & $\mathbf{U}$ & 10 & & 50 & $\mu g / L$ \\
\hline Barium & WA & & & $\mathbf{U}$ & 200 & & 2000 & $\mu \mathrm{g} / \mathrm{L}$ \\
\hline Benzene & WA & & & $\mathbf{U}$ & 5 & & 5 & $\mu \mathrm{g} / \mathrm{L}$ \\
\hline Beryllium & WA & & & $\mathbf{U}$ & 5 & & 4 & $\mu g / L$ \\
\hline Bromodichloromethane & WA & & & $\mathbf{U}$ & 5 & & NS & $\mu g / L$ \\
\hline Bromoform & WA & & & $\mathbf{U}$ & 5 & & NS & $\mu \mathrm{g} / \mathrm{L}$ \\
\hline Cadmium & WA & & & $\mathbf{U}$ & 5 & & 5 & $\mu g / L$ \\
\hline Calcium & WA & & & $\mathbf{U}$ & 5000 & & NS & $\mu g / L$ \\
\hline Carbon disulfide & WA & & & $\mathbf{U}$ & 5 & & NS & $\mu \mathrm{g} / \mathrm{L}$ \\
\hline Carbon tetrachloride & WA & & & $\mathbf{U}$ & 5 & & 5 & $\mu \mathrm{g} / \mathrm{L}$ \\
\hline Cesium-137 & TM & & & $\mathbf{U}$ & 9.2 & & 200 & $\mathrm{pCi} / \mathrm{L}$ \\
\hline Chloride & WA & & & & 7.43 & & NS & $\mathrm{mg} / \mathrm{L}$ \\
\hline Chlorobenzene & WA & & & $\mathbf{U}$ & 5 & & 100 & $\mu \mathrm{g} / \mathrm{L}$ \\
\hline Chloroethane & WA & & & $\mathbf{U}$ & 10 & & NS & $\mu \mathrm{g} / \mathrm{L}$ \\
\hline Chloroform & WA & & & $\mathrm{U}$ & 5 & & 100 & $\mu g / L$ \\
\hline Chloroprene & WA & & & $\mathbf{U}$ & 20 & & NS & $\mu g / \Lambda$ \\
\hline Chromium & WA & & & $\mathbf{U}$ & 10 & & 100 & $\mu g / L$ \\
\hline cis-1,3-Dichloropropene & WA & & & $\mathbf{U}$ & 5 & & NS & $\mu \mathrm{g} / \mathrm{L}$ \\
\hline Cobalt & WA & & & $\mathbf{U}$ & 50 & & NS & $\mu \mathrm{g} / \mathrm{L}$ \\
\hline Copper & WA & & & $\mathbf{U}$ & 25 & & 1000 & $\mu g / L$ \\
\hline Dibromochloromethane & WA & & & $\mathbf{U}$ & 5 & & NS & $\mu g / L$ \\
\hline Dichlorodifluoromethane & WA & & & $\mathbf{U}$ & 10 & & NS & $\mu \mathrm{g} / \mathrm{L}$ \\
\hline Ethylbenzene & WA & & & $U$ & 5 & & 700 & $\mu g / L$ \\
\hline Fluoride & WA & & & $\mathbf{U}$ & 0.1 & & 4 & $\mathrm{mg} /$ \\
\hline
\end{tabular}


BGS002 Laboratory Analyses (cont'd)

\begin{tabular}{|c|c|c|c|c|c|c|c|c|c|}
\hline Analyte & Lab & Rep & Avg & Modifier & & Result & Acc & Stand & Units \\
\hline Gross alpha & $\mathbf{T M}$ & & & & & 8.2 & 2.3 & 15 & $\mathrm{pCi} / \mathrm{L}$ \\
\hline Iron & WA & & & & & 7690 & & 300 & $\mu g / L$ \\
\hline Isobutyl alcohol & WA & & & $\mathbf{U}$ & & 20 & & NS & $\mu g / L$ \\
\hline Lead & WA & & & & & 4.6 & & 50 & $\mu g / L$ \\
\hline Magnesium & WA & & & $\mathbf{U}$ & & 5000 & & NS & $\mu g / L$ \\
\hline Manganese & WA & & & & & 45.3 & - & 50 & $\mu g / L$ \\
\hline Mercury & WA & & & $\mathbf{U}$ & & 0.2 & & 2 & $\mu g / L$ \\
\hline Methacrylonitrile & WA & & & $\mathbf{U}$ & & 20 & & NS & $\mu g / L$ \\
\hline Methyl bromide & WA & & & $\mathbf{U}$ & & 10 & & NS & $\mu g / L$ \\
\hline Methyl chloride & WA & & & $\mathbf{U}$ & & 10 & & NS & $\mu g / L$ \\
\hline Methyl ethyl ketone & WA & & & $\mathbf{U}$ & & 10 & & NS & $\mu g / L$ \\
\hline Methyl iodide & WA & & & $\mathbf{U}$ & & 10 & & NS & $\mu g / L$ \\
\hline Methylene bromide & WA & & & $\mathbf{U}$ & & 10 & & NS & $\mu g / L$ \\
\hline Methylene chloride & WA & & & $\mathbf{J}$ & $\mathbf{V}$ & 3.76 & & 5 & $\mu g / L$ \\
\hline Nickel & WA & & & $\mathbf{U}$ & & 40 & & 100 & $\mu g / L$ \\
\hline Nitrate as nitrogen & WA & & & JU & $\mathbf{Q}$ & 0.02 & & 10 & $\mathrm{mg} / \mathrm{L}$ \\
\hline Nonvolatile beta & $\mathbf{T M}$ & & & & & 8.9 & 3.5 & 50 & $\mathrm{pCi} / \mathrm{L}$ \\
\hline Potassium & WA & & & $\mathbf{U}$ & & 5000 & & NS & $\mu g / L$ \\
\hline Propionitrile & WA & & & $\mathbf{U}$ & & 50 & & NS & $\mu g / L$ \\
\hline Selenium & WA & & & $\mathbf{U}$ & & 5 & & 50 & $\mu g / L$ \\
\hline Silver & WA & & & $\mathbf{U}$ & & 10 & & 50 & $\mu g / L$ \\
\hline Sodium & WA & & & $\mathbf{U}$ & & 5000 & & NS & $\mu g / L$ \\
\hline Styrene & WA & & & $\mathbf{U}$ & & 5 & & 100 & $\mu g / L$ \\
\hline Sulfate & WA & & & & & 3.1 & & 400 & $\mathrm{mg} / \mathrm{L}$ \\
\hline Sulfide & WA & & & $\mathbf{U}$ & & 0.1 & & NS & $\mathrm{mg} / \mathrm{L}$ \\
\hline Tetrachloroethylene & WA & & & $\mathbf{U}$ & & 5 & & 5 & $\mu g / L$ \\
\hline Thallium & WA & & & $\mathbf{U}$ & & 10 & & 2 & $\mu g / L$ \\
\hline Toluene & WA & & & $\mathbf{U}$ & & 5 & & 1000 & $\mu g / L$ \\
\hline Total hardness & WA & & & & & 15.5 & & NS & $\mathrm{mg} / \mathrm{L}$ \\
\hline Total organic carbon & WA & & & & & 14.2 & & NS & $\mathrm{mg} / \mathrm{L}$ \\
\hline Total suspended solids & WA & & & & & 206 & & NS & $\mathbf{m g} / \mathbf{L}$ \\
\hline trans-1,3-Dichloropropene & WA & & & $\mathbf{U}$ & & 5 & & NS & $\mu g / L$ \\
\hline trans-1,4-Dichloro-2-butene & WA & & & $\mathbf{U}$ & & 100 & & NS & $\mu g / L$ \\
\hline Trichloroethylene & WA & & & $\mathbf{U}$ & & 5 & & 5 & $\mu g / L$ \\
\hline Trichlorofluoromethane & WA & & & $\mathbf{U}$ & & 5 & & NS & $\mu g / L$ \\
\hline Tritium & $\mathbf{T M}$ & & & & & 1.38 & 0.34 & 20 & $\mathrm{pCi} / \mathrm{ml}$ \\
\hline Turbidity & WA & & & J & $\mathbf{Q}$ & 126 & & NS & NTU \\
\hline Vanadium & WA & & & $\mathbf{U}$ & & 50 & & NS & $\mu g /$ \\
\hline Vinyl acetate & WA & & & $\mathbf{U}$ & & 10 & & NS & $\mu \mathrm{g} /$ \\
\hline Vinyl chloride & WA & & & $\mathbf{U}$ & & 10 & & 2 & $\mu \mathrm{g} /$ \\
\hline Xylenes (total) & WA & & & $\mathbf{U}$ & & 5 & & 10000 & $\mu \mathrm{g} /$ \\
\hline Zinc & WA & & & & & 84.4 & & 5000 & $\mu \mathrm{g} /$ \\
\hline
\end{tabular}




\section{BGS003}

Field Measurements:

Sample Date: 9/1/93

pH: 7.53

Specific Conductance: $51 \mu \mathrm{S} / \mathrm{cm}$

Red/Oxid Potential: $170 \mathrm{mv}$

Dissolved Oxygen: $2.1 \mathrm{mg} / \mathrm{L}$

\begin{tabular}{|c|c|c|c|c|c|c|c|}
\hline Analyte & Lab & Avg & Modifier & Result & Acc & Stand & Units \\
\hline 1,1,1,2-Tetrachloroethane & WA & & $\mathbf{U}$ & 10 & & NS & $\mu g / L$ \\
\hline 1,1,1-Trichloroethane & WA & & $\mathbf{U}$ & 5 & & 200 & $\mu g / L$ \\
\hline $1,1,2,2-$ Tetrachloroethane & WA & & $\mathbf{U}$ & 5 & & NS & $\mu g / L$ \\
\hline 1,1,2-Trichloroethane & WA & & $\mathbf{U}$ & 5 & & 5 & $\mu \mathrm{g} / \mathrm{L}$ \\
\hline 1,1-Dichloroethane & WA & & $\mathbf{U}$ & 5 & & NS & $\mu \mathrm{g} / \mathrm{L}$ \\
\hline 1,1-Dichloroethylene & WA & & $\mathbf{U}$ & 5 & & 7 & $\mu \mathrm{g} / \mathrm{L}$ \\
\hline 1,2,3-Trichloropropane & WA & & $\mathbf{U}$ & 10 & & NS & $\mu \mathrm{g} / \mathrm{L}$ \\
\hline 1,2-Dibromo-3-chloropropane & WA & . & $\mathbf{U}$ & 20 & & 0.2 & $\mu g / L$ \\
\hline 1,2-Dibromoethane & WA & & $\mathbf{U}$ & 20 & & NS & $\mu \mathrm{g} / \mathrm{L}$ \\
\hline 1,2-Dichloroethane & WA & & $\mathbf{U}$ & 5 & & 5 & $\mu g / L$ \\
\hline 1,2-Dichloroethylene (total) & WA & & $\mathbf{U}$ & 5 & & 7 & $\mu g / L$. \\
\hline 1,2-Dichloropropane & WA & & $\mathbf{U}$ & 5 & & 5 & $\mu \mathrm{g} / \mathrm{L}$ \\
\hline 2-Hexanone & WA & & $\mathbf{U}$ & 10 & & NS & $\mu g / L$ \\
\hline 4-Methyl-2-pentanone & WA & & $\mathbf{U}$ & 10 & & NS & $\mu g / L$ \\
\hline Acetone & WA & & $\mathbf{J}$ & 7.24 & & NS & $\begin{array}{l}\mu g / L \\
\mu g / L\end{array}$ \\
\hline Acetonitrile & WA & & $\dot{U}$ & 20 & & NS & $\begin{array}{l}\mu \mathrm{g} / \mathrm{L} \\
\mu \mathrm{g} / \mathrm{L}\end{array}$ \\
\hline Acidity & WA & & & 68 & & NS & $\mathrm{mg} / \mathrm{L}$ \\
\hline Acrolein & WA & & $\mathbf{U}$ & 10 & & NS & $\mu g / L$ \\
\hline Acrylonitrile & WA & & $\mathbf{U}$ & 10 & & NS & $\begin{array}{l}\mu g / L \\
\mu g / L\end{array}$ \\
\hline Allyl chloride & WA & & $\mathbf{U}$ & 100 & & NS & $\mu g / L$ \\
\hline Aluminum & WA & & & 234 & & NS & $\begin{array}{l}\mu g / L \\
\mu g / L\end{array}$ \\
\hline Antimony & WA & & $\mathbf{U}$ & 60 & & 6 & $\begin{array}{l}\mu \mathrm{g} / \mathrm{L} \\
\mu \mathrm{g} / \mathrm{L}\end{array}$ \\
\hline Arsenic & WA & & $\mathbf{U}$ & 10 & & 50 & $\begin{array}{l}\mu g / L \\
\mu g / L\end{array}$ \\
\hline Barium & WA & & $\mathbf{U}$ & 200 & & 2000 & $\begin{array}{l}\mu g / L \\
\mu g / L\end{array}$ \\
\hline Benzene & WA & & $\mathbf{U}$ & 5 & & 5 & $\mu g / L$ \\
\hline Beryllium & WA & & $\mathbf{U}$ & 5 & & 4 & $\mu g / L$ \\
\hline Bromodichloromethane & WA & & $\mathbf{U}$ & 5 & & NS & $\mu g / L$ \\
\hline Bromoform & WA & & $\mathbf{U}$ & 5 & & NS & $\mu g / L$ \\
\hline Cadmium & WA & & $\mathrm{U}$ & 5 & & 5 & $\mu g / L$ \\
\hline Calcium & WA & & $\mathbf{U}$ & 5000 & & NS & $\mu g / L$ \\
\hline Carbon disulfide & WA & & $\mathbf{U}$ & 5 & & NS & $\mu g / L$ \\
\hline Carbon tetrachloride & WA & & $\mathbf{U}$ & 5 & & 5 & $\mu g / L$ \\
\hline Cesium-137 & $\mathbf{T M}$ & & $\mathbf{U}$ & 9.7 & & 200 & $\mathrm{pCi} / \mathrm{L}$ \\
\hline Chloride & WA & & & 8.05 & & NS & $\mathrm{mg} / \mathrm{L}$ \\
\hline Chlorobenzene & WA & & $\mathbf{U}$ & 5 & & 100 & $\mu \mathrm{g} / \mathrm{L}$ \\
\hline Chloroethane. & WA & & $\mathrm{U}$ & 10 & & NS & $\mu \mathrm{g} / \mathrm{L}$ \\
\hline Chloroform & WA & & $\mathbf{U}$ & 5 & & 100 & $\mu \mathrm{g} / \mathrm{L}$ \\
\hline Chloroprene & WA & & $\mathrm{U}$ & 20 & & NS & $\mu \mathrm{g} / \mathrm{L}$ \\
\hline Chromium & WA & & $\mathbf{U}$ & 10 & & 100 & $\mu \mathrm{g} / \mathrm{L}$ \\
\hline cis-1,3-Dichloropropene & WA & & $\mathrm{U}$ & 5 & & NS & $\mu \mathrm{g} / \mathrm{L}$ \\
\hline Cobalt & WA & & $\mathbf{U}$ & 50 & & NS & $\mu \mathrm{g} / \mathrm{L}$ \\
\hline Copper & WA & & $\mathbf{U}$ & 25 & & 1000 & $\mu \mathrm{g} / \mathrm{L}$ \\
\hline Dibromochloromethane & WA & & $\mathbf{U}$ & 5 & & NS & $\mu g / L$ \\
\hline Dichlorodifluoromethane & WA & & U & 10 & & NS & $\mu g / L$ \\
\hline Ethylbenzene & WA & & $\mathbf{U}$ & 5 & & 700 & $\mu g / L$ \\
\hline Fluoride & WA & & $\mathbf{U}$ & 0.1 & & 4 & $\mathrm{mg} / \mathrm{L}$ \\
\hline Gross alpha & TM & & & 0.8 & 1 & 15 & $\mathrm{pCi} / \mathrm{L}$ \\
\hline
\end{tabular}


BGS003 Laboratory Analyses (cont'd)

\begin{tabular}{|c|c|c|c|c|c|c|c|c|c|}
\hline Analyte & Lab & Rep & Avg & Modifier & & Result & Acc & Stand & Units \\
\hline Iron & WA & & & & & 580 & & 300 & $\mu g / L$ \\
\hline Isobutyl alcohol & WA & & & $\mathbf{U}$ & & 20 & & NS & $\mu g / L$ \\
\hline Lead & WA & & & $\mathbf{U}$ & & 3 & & 50 & $\mu g / L$ \\
\hline Magnesium & WA & & & $\mathbf{U}$ & & 5000 & & NS & $\mu g / L$ \\
\hline Manganese & WA & & & & & 96.1 & & 50 & $\mu g / L$ \\
\hline Mercury & WA & & & $\mathbf{U}$ & & 0.2 & . & 2 & $\mu g / L$ \\
\hline Methacrylonitrile & WA & & & $\mathbf{U}$ & & 20 & & NS & $\mu g / L$ \\
\hline Methyl bromide & WA & & & $\mathbf{U}$ & & 10 & & NS & $\mu g / L$ \\
\hline Methyl chloride & WA & & & $\mathbf{U}$ & & 10 & & NS & $\mu g / L$ \\
\hline Methyl ethyl ketone & WA & & & $\mathbf{U}$ & & 10 & & NS & $\mu g / L$ \\
\hline Methyl iodide & WA & & & $\mathbf{U}$ & & 10 & & NS & $\mu g / L$ \\
\hline Methylene bromide & WA & & & $\mathbf{U}$ & & 10 & & NS & $\mu g / L$ \\
\hline Methylene chloride & WA & & & $\mathbf{J}$ & $\mathbf{V}$ & 3.53 & & 5 & $\mu g / L$ \\
\hline Nickel & WA & & & $\mathbf{U}$ & & 40 & & 100 & $\mu g / L$ \\
\hline Nitrate as nitrogen & WA & & & $\mathbf{J}$ & Q & 0.149 & & 10 & $\mathrm{mg} / \mathrm{L}$ \\
\hline Nonvolatile beta & TM & & & $\mathbf{U}$ & & 0.6 & & 50 & $\mathrm{pCi} / \mathrm{L}$ \\
\hline Potassium & WA & & & $\mathbf{U}$ & & 5000 & & NS & $\mu g / L$ \\
\hline Propionitrile & WA & & & $\mathbf{U}$ & & 50 & & NS & $\mu g / L$ \\
\hline Selenium & WA & & & $\mathbf{U}$ & & 5 & & 50 & $\mu g / L$ \\
\hline Silver & WA & & & $\mathbf{U}$ & & 10 & & 50 & $\mu g / L$ \\
\hline Sodium & WA & & & $\mathbf{U}$ & & 5000 & & NS & $\mu g / L$ \\
\hline Styrene & WA & & & $\mathbf{U}$ & & 5 & & 100 & $\mu g / L$ \\
\hline Sulfate & WA & & & $\mathbf{U}$ & & 2.5 & & 400 & $\mathrm{mg} / \mathrm{L}$ \\
\hline Sulfide & WA & & & $\mathbf{U}$ & & 0.1 & & NS & $\mathrm{mg} / \mathrm{L}$ \\
\hline Tetrachloroethylene & WA & & & $\mathbf{U}$ & & 5 & & 5 & $\mu g / L$ \\
\hline Thallium & WA & & & $\mathbf{U}$ & & 10 & & 2 & $\mu g / L$ \\
\hline Toluene & WA & & & $\mathbf{U}$ & & 5 & & 1000 & $\mu g / L$ \\
\hline Total hardness & WA & & & & & 13.6 & & NS & mg/L \\
\hline Total organic carbon & WA & & & & & 5.45 & & NS & mg/L \\
\hline Total suspended solids & WA & & & & & 10 & & NS & $\mathrm{mg} / \mathrm{L}$ \\
\hline trans-1,3-Dichloropropene & WA & & & $\mathbf{U}$ & & 5 & & NS & $\mu g / L$ \\
\hline trans-1,4-Dichloro-2-butene & WA & & & $\mathbf{U}$ & & 100 & & NS & $\mu g / L$ \\
\hline Trichloroethylene & WA & & & $\mathbf{U}$ & & 5 & & 5 & $\mu g / L$ \\
\hline Trichlorofluoromethane & WA & & & $\mathbf{U}$ & & 5 & & NS & $\mu g / L$ \\
\hline Tritium & TM & & & & & 1.79 & 0.36 & 20 & $\mathrm{pCi} / \mathrm{ml}$ \\
\hline Turbidity & WA & & & $\mathbf{J}$ & $\mathbf{Q}$ & 7.05 & & NS & NTU \\
\hline Vanadium & WA & & & $\mathbf{U}$ & & 50 & & NS & $\mu g / L$ \\
\hline Vinyl acetate & WA & & & $\mathbf{U}$ & & 10 & & NS & $\mu g / L$ \\
\hline Vinyl chloride & WA & & & $\mathbf{U}$ & & 10 & & 2 & $\mu g / L$ \\
\hline Xylenes (total) & WA & & & $\mathbf{U}$ & & 5 & & 10000 & $\mu g / L$ \\
\hline Zinc & WA & & & & & 22.2 & & 5000 & $\mu g / L$ \\
\hline
\end{tabular}




\section{UTD001}

Field Measurements:

Stream Discharge: $0.24 \mathrm{cfs}$

Sample Date: 9/1/93

pH: 5.68

Specific Conductance: $42 \mu \mathrm{S} / \mathrm{cm}$

Red/Oxid Potential: $210 \mathrm{mv}$

Dissolved Oxygen: $7.85 \mathrm{mg} / \mathrm{L}$

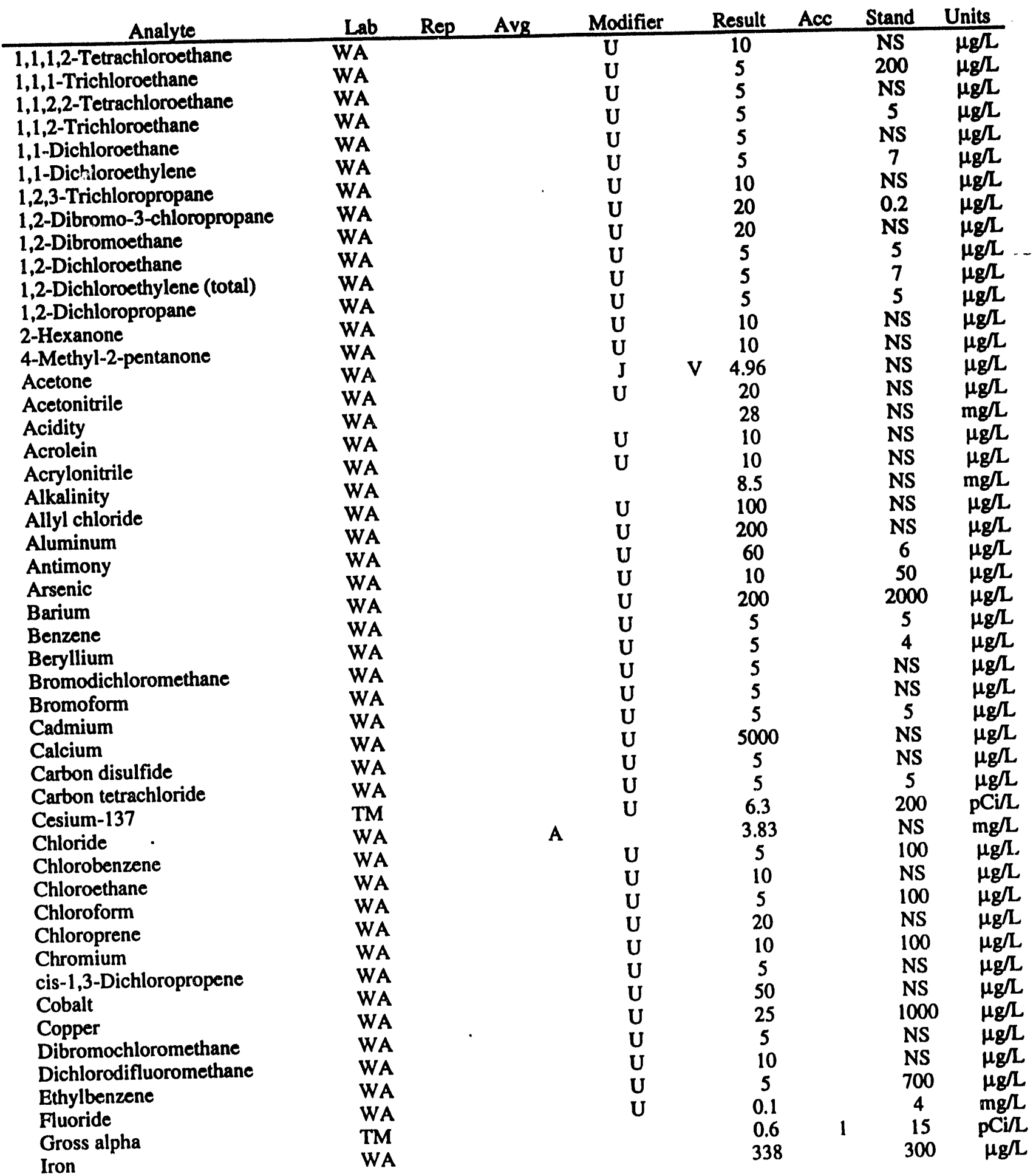


UTD001 Laboratory Analyses (cont'd)

\begin{tabular}{|c|c|c|c|c|c|c|c|c|c|}
\hline Analyte & Lab & Rep & Avg & Modifier & & Result & Acc & Stand & Units \\
\hline Isobutyl alcohol & WA & & & $\mathbf{U}$ & & 20 & & NS & $\mu g / L$ \\
\hline Lead & WA & & & $\mathbf{U}$ & & 3 & & 50 & $\mu g / L$ \\
\hline Magnesium & WA & & & $\mathbf{U}$ & & 5000 & & NS & $\mu g / L$ \\
\hline Manganese & WA & & & $\mathbf{U}$ & & 15 & & 50 & $\mu g / L$ \\
\hline Mercury & WA & & & $\mathbf{U}$ & & 0.2 & & $2^{7}$ & $\mu g / L$ \\
\hline Methacrylonitrile & WA & & & $\mathbf{U}$ & & 20 & . & NS & $\mu g / L$ \\
\hline Methyl bromide & WA & & & $\mathbf{U}$ & & 10 & & NS & $\mu g / L$ \\
\hline Methyl chloride & WA & & & $\mathbf{U}$ & & 10 & & NS & $\mu g / L$ \\
\hline Methyl ethyl ketone & WA & & & $\mathbf{U}$ & & 10 & & NS & $\mu g / L$ \\
\hline Methyl iodide & WA & & & $\mathbf{U}$ & & 10 & & NS & $\mu g / L$ \\
\hline Methylene bromide & WA & & & $\mathbf{U}$ & & 10 & & NS & $\mu g / L$ \\
\hline Methylene chloride & WA & & & $\mathbf{J}$ & V & 3.7 & & 5 & $\mu g / L$ \\
\hline Nickel & WA & & & $\mathbf{U}$ & & 40 & & 100 & $\mu g / L$ \\
\hline Nitrate as nitrogen & WA & & & $\mathbf{J}$ & $\mathbf{Q}$ & 0.132 & & 10 & $\mathrm{mg} / \mathrm{L}$ \\
\hline Nonvolatile beta & $\mathbf{T M}$ & & & $\mathbf{U}$ & & 0.6 & & 50 & $\mathrm{pCi} / \mathrm{L}$ \\
\hline Potassium & WA & & & $\mathbf{U}$ & & 5000 & & NS & $\mu g / L$ \\
\hline Propionitrile & WA & & & $\mathbf{U}$ & & 50 & & NS & $\mu g / L$ \\
\hline Selenium & WA & & & $\mathbf{U}$ & & 5 & & 50 & $\mu g / L$ \\
\hline Silver & WA & & & $\mathbf{U}$ & & 10 & & 50 & $\mu g / L$ \\
\hline Sodium & WA & & & & & 7280 & & NS & $\mu g /$ \\
\hline Styrene & WA & & & $\mathbf{U}$ & & 5 & & 100 & $\mu g / L$ \\
\hline Sulfate & WA & & & & & 4.96 & & 400 & mg/L \\
\hline Sulfide & WA & & & $\mathbf{U}$ & & 0.1 & & NS & $\mathbf{m g} / \mathrm{L}$ \\
\hline Tetrachloroethylene & WA & & & $\mathbf{U}$ & & 5 & & 5 & $\mu g / L$ \\
\hline Thallium & WA & & & $\mathbf{U}$ & & 10 & & 2 & $\mu g / L$ \\
\hline Toluene & WA & & & $\mathbf{U}$ & & 5 & & 1000 & $\mu g / L$ \\
\hline Total hardness & WA & & & $\mathbf{U}$ & & 1 & & NS & $\mathrm{mg} / \mathrm{L}$ \\
\hline Total organic carbon & WA & & & & & 1.43 & & NS & $\mathrm{mg} / \mathrm{L}$ \\
\hline Total suspended solids & WA & & & & & 12 & & NS & $\mathrm{mg} / \mathrm{L}$ \\
\hline trans-1,3-Dichloropropene & WA & & & $\mathbf{U}$ & & 5 & & NS & $\mu g / L$ \\
\hline trans-1,4-Dichloro-2-butene & WA & & & $\mathbf{U}$ & & 100 & & NS & $\mu g /$ \\
\hline Trichloroethylene & WA & & & $\mathbf{U}$ & & 5 & & 5 & $\mu g / L$ \\
\hline Trichlorofluoromethane & WA & & & $\mathbf{U}$ & & 5 & & NS & $\mu g / L$ \\
\hline Tritium & TM & & & & & 7.03 & 0.62 & 20 & $\mathrm{pCi} / \mathrm{ml}$ \\
\hline Turbidity & WA & & & $\mathbf{J}$ & $\mathbf{Q}$ & 3.34 & & NS & NTU \\
\hline Vanadium & WA & & & $\mathbf{U}$ & & 50 & & NS & $\mu g / L$ \\
\hline Vinyl acetate & WA & & & $\mathbf{U}$ & & 10 & & NS & $\mu g / L$ \\
\hline Vinyl chloride & WA & & & $\mathbf{U}$ & & 10 & & 2 & $\mu g / L$ \\
\hline Xylenes (total) & WA & & . & $\mathbf{U}$ & & 5 & & 10000 & $\mu g / L$ \\
\hline Zinc & WA & & & $\mathbf{U}$ & & 20 & & 5000 & $\mu g / L$ \\
\hline
\end{tabular}




\section{UTDO02}

Field Measurements:

Stream Discharge: 0.15 cfs

Sample Date: 9/1/93

pH: 5.76

Specific Conductance: $55 \mu \mathrm{S} / \mathrm{cm}$

Red/Oxid Potential: $121 \mathrm{mv}$

Dissolved Oxygen: $7.81 \mathrm{mg} / \mathrm{L}$

\begin{tabular}{|c|c|c|c|c|c|c|c|c|}
\hline Analyte & Lab & Rep & Avg & Modifier & Result & Acc & Stand & Units \\
\hline 1,1,1,2-Tetrachloroethane & WA & & & $\mathbf{U}$ & 10 & & NS & $\mu g / L$ \\
\hline 1,1,1-Trichloroethane & WA & & & $\mathbf{U}$ & 5 & & 200 & $\mu g / L$ \\
\hline $1,1,2,2-$ Tetrachloroethane & WA & & & $\mathbf{U}$ & 5 & & NS & $\mu g / L$ \\
\hline 1,1,2-Trichloroethane & WA & & & $\mathbf{U}$ & 5 & & 5 & $\mu g / L$ \\
\hline 1,1-Dichloroethane & WA & & & $\mathbf{U}$ & 5 & & NS & $\mu g / L$ \\
\hline 1,1-Dichloroethylene & WA & & & $\mathbf{U}$ & 5 & & 7 & $\mu g / L$ \\
\hline 1,2,3-Trichloropropane & WA & & . & $\mathbf{U}$ & 10 & & NS & $\mu g / L$ \\
\hline 1,2-Dibromo-3-chloropropane & WA & & & $\mathbf{U}$ & 20 & & 0.2 & $\mu g / L$ \\
\hline 1,2-Dibromoethane & WA & & & $\mathbf{U}$ & 20 & & NS & $\mu g / L$ \\
\hline 1,2-Dichloroethane & WA & & & $\begin{array}{l}\mathbf{U} \\
\mathbf{U}\end{array}$ & $\begin{array}{l}5 \\
5\end{array}$ & & 5 & $\mu g / L$ \\
\hline $\begin{array}{l}\text { 1,2-Dichloroethylene (total) } \\
\text { 1,2-Dichloropropane }\end{array}$ & WA & & & $\underset{\mathbf{U}}{\mathbf{U}}$ & $\begin{array}{l}5 \\
5\end{array}$ & & 7 & $\mu g / L$ \\
\hline $\begin{array}{l}\text { 1,2-Dichloropropane } \\
\text { 2-Hexanone }\end{array}$ & WA & & & $\begin{array}{l}\mathbf{U} \\
\mathbf{U}\end{array}$ & $\begin{array}{l}5 \\
10\end{array}$ & & 5 & $\mu g / L$ \\
\hline $\begin{array}{l}\text { 2-Hexanone } \\
\text { 4-Methyl-2-pentanone }\end{array}$ & WA & & & $\underset{\mathbf{U}}{\mathbf{U}}$ & $\begin{array}{l}10 \\
10\end{array}$ & & NS & $\mu g / L$ \\
\hline $\begin{array}{l}\text { 4-Methyl-2-pentanone } \\
\text { Acetone }\end{array}$ & $\begin{array}{l}\text { WA } \\
\text { WA }\end{array}$ & & & $\mathbf{U}$ & $\begin{array}{c}10 \\
7.46\end{array}$ & & NS & $\mu g / L$ \\
\hline $\begin{array}{l}\text { Acetone } \\
\text { Acetonitrile }\end{array}$ & $\begin{array}{l}\text { WA } \\
\text { WA }\end{array}$ & & & $\underset{\mathbf{U}}{\mathbf{J}}$ & $\begin{array}{c}7.46 \\
20\end{array}$ & & NS & $\mu g / L$ \\
\hline $\begin{array}{l}\text { Acetonitrile } \\
\text { Acidity }\end{array}$ & $\begin{array}{l}\text { WA } \\
\text { WA }\end{array}$ & & $\mathbf{A}$ & $\mathbf{U}$ & $\begin{array}{l}20 \\
94\end{array}$ & & NS & $\mu \mathrm{g} / \mathrm{L}$ \\
\hline $\begin{array}{l}\text { Acidity } \\
\text { Acrolein }\end{array}$ & $\begin{array}{l}\text { WA } \\
\text { WA }\end{array}$ & & & & $\begin{array}{l}94 \\
10\end{array}$ & & NS & $\mathrm{mg} / \mathrm{L}$ \\
\hline $\begin{array}{l}\text { Acrolein } \\
\text { Acrylonitrile }\end{array}$ & $\begin{array}{l}\text { WA } \\
\text { WA }\end{array}$ & & & $\begin{array}{l}\mathbf{U} \\
\mathbf{U}\end{array}$ & $\begin{array}{l}10 \\
10\end{array}$ & & NS & $\mu g / L$ \\
\hline $\begin{array}{l}\text { Acrylonitrile } \\
\text { Alkalinity }\end{array}$ & $\begin{array}{l}\text { WA } \\
\text { WA }\end{array}$ & & & $\mathbf{U}$ & $\begin{array}{c}10 \\
7\end{array}$ & & NS & $\mu g / L$ \\
\hline $\begin{array}{l}\text { Alkalinity } \\
\text { Allyl chloride }\end{array}$ & $\begin{array}{l}\text { WA } \\
\text { WA }\end{array}$ & & & & $\stackrel{7}{100}$ & & NS & $\mathrm{mg} / \mathrm{L}$ \\
\hline $\begin{array}{l}\text { Allyl chloride } \\
\text { Aluminum }\end{array}$ & $\begin{array}{l}\text { WA } \\
\text { WA }\end{array}$ & & & $\mathbf{U}$ & 100 & & NS & $\mu g / L$ \\
\hline $\begin{array}{l}\text { Aluminum } \\
\text { Antimony }\end{array}$ & $\begin{array}{l}\text { WA } \\
\text { WA }\end{array}$ & & & $\mathbf{U}$ & 200 & & NS & $\mu g /$ \\
\hline $\begin{array}{l}\text { Antimony } \\
\text { Arsenic }\end{array}$ & $\begin{array}{l}\text { WA } \\
\text { WA }\end{array}$ & & & $\mathbf{U}$ & 60 & & 6 & $\mu g / L$ \\
\hline $\begin{array}{l}\text { Arsenic } \\
\text { Barium }\end{array}$ & $\begin{array}{l}\text { WA } \\
\text { WA }\end{array}$ & & & $\mathbf{U}$ & 10 & & 50 & $\mu \varepsilon / L$ \\
\hline $\begin{array}{l}\text { Barium } \\
\text { Benzene }\end{array}$ & WA & & & $\mathbf{U}$ & 200 & & 2000 & $\mu g / L$ \\
\hline $\begin{array}{l}\text { Benzene } \\
\text { Bervllium }\end{array}$ & WA & & & $\mathbf{U}$ & 5 & & 5 & $\mu g / L$ \\
\hline $\begin{array}{l}\text { Beryllium } \\
\text { Bromodichloromethane }\end{array}$ & WA & & & $\mathbf{U}$ & 5 & & 4 & $\mu g / L$ \\
\hline $\begin{array}{l}\text { Bromodichloromethane } \\
\text { Bromoform }\end{array}$ & WA & & & $\mathbf{U}$ & 5 & & NS & $\mu g / L$ \\
\hline Bromoform & WA & & & $\mathbf{U}$ & 5 & & NS & $\mu g / L$ \\
\hline Cadmium & WA & & & $\mathbf{U}$ & 5 & & 5 & $\mu g / L$ \\
\hline Calcium & WA & & & $\mathbf{U}$ & 5000 & & NS & $\mu g / L$ \\
\hline Carbon disulfide & WA & & & $\mathbf{U}$ & 5 & & NS & $\mu g / L$ \\
\hline Carbon tetrachloride & WA & & & $\mathbf{U}$ & 5 & & 5 & $\mu \mathrm{g} / \mathrm{L}$ \\
\hline Cesium-137 & TM & & & $\mathbf{U}$ & 9.2 & & 200 & $\mathrm{pCi} / \mathrm{L}$ \\
\hline Chloride & WA & & & & 1.5 & & NS & $\mathrm{mg} / \mathrm{L}$ \\
\hline Chlorobenzene & WA & & & $\mathbf{U}$ & 5 & & 100 & $\mu g / L$ \\
\hline Chloroethane & WA & & & $\mathbf{U}$ & 10 & & NS & $\mu g / L$ \\
\hline Chloroform & WA & & & $\mathbf{U}$ & 5 & & 100 & $\mu \mathrm{g} / \mathrm{L}$ \\
\hline Chloroprene & WA & & & $\mathbf{U}$ & 20 & & NS & $\mu g / L$ \\
\hline Chromium & WA & & & $\mathbf{U}$ & 10 & & 100 & $\mu g / L$ \\
\hline cis-1,3-Dichloropropene & WA & & & $\mathbf{U}$ & 5 & & NS & $\mu g / L$ \\
\hline Cobalt & WA & & & $\mathbf{U}$ & 50 & & NS & $\begin{array}{l}\mu g / L \\
\mu g / L\end{array}$ \\
\hline Copper & WA & & & $\mathbf{U}$ & 25 & & 1000 & $\mu g / L$ \\
\hline Dibromochloromethane & WA & & & $\mathbf{U}$ & 5 & & NS & $\mu g / L$ \\
\hline Dichlorodifluoromethane & WA & & & $\mathrm{U}$ & 10 & & NS & $\mu g / L$ \\
\hline Ethylben & WA & & & $\mathbf{U}$ & 5 & & 700 & $\mu g / L$ \\
\hline
\end{tabular}


UTD002 Laboratory Analyses (cont'd)

\begin{tabular}{|c|c|c|c|c|c|c|c|c|c|}
\hline Analyte & Lab & Rep & Avg & Modifier & & Result & Acc & Stand & Units \\
\hline Fluoride & WA & & & $\overline{\mathbf{U}}$ & & 0.1 & & 4 & $\mathrm{mg} / \mathrm{L}$ \\
\hline Gross alpha & TM & & & & & 0.7 & 1 & 15 & $\mathrm{pCi} / \mathrm{L}$ \\
\hline Iron & WA & & & & & 849 & & 300 & $\mu \mathrm{g} / \mathrm{L}$ \\
\hline Isobutyl alcohol & WA & & & $\mathbf{U}$ & & 20 & & NS & $\mu g /$ \\
\hline Lead & WA & & & $\mathbf{U}$ & & 3 & & 50 & $\mu g / L$ \\
\hline Magnesium & WA & & & $\mathbf{U}$ & & 5000 & & NS & $\mu g /$ \\
\hline Manganese & WA & & & & & 22.3 & & 50 & $\mu g / L$ \\
\hline Mercury & WA & & & $\mathbf{U}$ & & 0.2 & & 2 & $\mu g /$ \\
\hline Methacrylonitrile & WA & & & $\mathbf{U}$ & & 20 & & NS & $\mu g / L$ \\
\hline Methyl bromide & WA & & & $\mathbf{U}$ & & 10 & & NS & $\mu g / L$ \\
\hline Methyl chloride & WA & & & $\mathbf{U}$ & & 10 & & NS & $\mu g / L$ \\
\hline Methyl ethyl ketone & WA & & & $\mathbf{U}$ & & 10 & & NS & $\mu g / L$ \\
\hline Methyl iodide & WA & & & $\mathbf{U}$ & & 10 & & NS & $\mu g / \alpha$ \\
\hline Methylene bromide & WA & & & $\mathbf{U}$ & & 10 & & NS & $\mu g \Omega$ \\
\hline Methylene chloride & WA & & & $\mathbf{J}$ & v & 3.5 & & 5 & $\mu g / L$ \\
\hline Nickel & WA & & & $\mathbf{U}$ & & 40 & & 100 & $\mu g / L$ \\
\hline Nitrate as nitrogen & WA & & & J & $\mathbf{Q}$ & 0.088 & & 10 & $\mathrm{mg} / \mathrm{h}$ \\
\hline Nonvolatile beta & TM & & & $\mathbf{U}$ & & 0.6 & & 50 & pCin \\
\hline Potassium & WA & & & $\mathbf{U}$ & & 5000 & & NS & $\mu g / L$ \\
\hline Propionitrile & WA & & & $\mathbf{U}$ & & 50 & & NS & $\mu g / 2$ \\
\hline Selenium & WA & & & $\mathbf{U}$ & & 5 & & 50 & $\mu g / L$ \\
\hline Silver & WA & & & $\mathbf{U}$ & & 10 & & 50 & $\mu g / L$ \\
\hline Sodium & WA & & & $\mathbf{U}$ & & 5000 & & NS & $\mu g / L$ \\
\hline Styrene & WA & & & $\mathbf{U}$ & & 5 & & 100 & $\mu \mathrm{g} / \mathrm{L}$ \\
\hline Sulfate & WA & & & $\mathbf{U}$ & & 2.5 & & 400 & $\mathrm{mg} / \mathrm{h}$ \\
\hline Sulfate & WA & & NA & $\mathbf{U}$ & & 2.5 & & 400 & $\mathrm{mg} / \mathrm{L}$ \\
\hline Sulfide & WA & & & $\mathbf{U}$ & & 0.1 & & NS & $\mathrm{mg} / \mathrm{L}$ \\
\hline Tetrachloroethylene & WA & & & $\mathbf{U}$ & & 5 & & 5 & $\mu \mathrm{g} / \mathrm{L}$ \\
\hline Thallium & WA & & & $\mathbf{U}$ & & 10 & & 2 & $\mu g / L$ \\
\hline Toluene & WA & & & $\mathbf{U}$ & & 5 & & 1000 & $\mu g / L$ \\
\hline Total hardness & WA & & $\mathbf{A}$ & & & 5.3 & & NS & $\mathrm{mg} / \mathrm{L}$ \\
\hline Total organic carbon & WA & & & & & 2.17 & & NS & $\mathrm{mg} / \mathrm{L}$ \\
\hline Total suspended solids & WA & & & & & 23 & & NS & $\mathrm{mg} / \mathrm{L}$ \\
\hline trans-1,3-Dichloropropene & WA & & & $\mathbf{U}$ & & 5 & & NS & $\mu g / L$ \\
\hline trans-1,4-Dichloro-2-butene & WA & & & $\mathrm{U}$ & & 100 & & NS & $\mu g / \Omega$ \\
\hline Trichloroethylene & WA & & & $\mathbf{U}$ & & 5 & & 5 & $\mu g / L$ \\
\hline Trichlorofluoromethane & WA & & & $\mathbf{U}$ & & 5 & & NS & $\mu g / L$ \\
\hline Tritium & $\mathbf{T M}$ & & & & & 55.2 & 1.65 & 20 & $\mathrm{pCi} / \mathrm{ml}$ \\
\hline Turbidity & WA & & & J & $\mathbf{Q}$ & 1.77 & & NS & NTU \\
\hline Vanadium & WA & & & $\mathbf{U}$ & & 50 & & NS & $\mu g / L$ \\
\hline Vinyl acetate & WA & & & $\mathbf{U}$ & & 10 & & NS & $\mu g / L$ \\
\hline Vinyl chloride & WA & & & $\mathbf{U}$ & & 10 & & 2 & $\mu g / L$ \\
\hline Xylenes (total). & WA & & & $\mathbf{U}$ & & 5 & & 10000 & $\mu g / L$ \\
\hline Zinc & WA & & & & & 38.4 & & 5000 & $\mu g / L$ \\
\hline
\end{tabular}




\section{UTD003}

Field Measurements:

Stream Discharge: $0.03 \mathrm{cfs}$

Sample Date: 9/1/93

pH: 6.11

Specific Conductance: $38 \mu \mathrm{S} / \mathrm{cm}$

Red/Oxid Potential: $199 \mathrm{mv}$

Dissolved Oxygen: $6.56 \mathrm{mg} / \mathrm{L}$

Analyte

1,1,1,2-Tetrachloroethane

1,1,1,2-Tetrachloroethane

1,1,1-Trichloroethane

1,1,1-Trichloroethane

1,1,2,2-Tetrachloroethane

1,1,2,2-Tetrachloroethane

1,1,2-Trichloroethane

1,1,2-Trichloroethane

1,1-Dichloroethane

1,1-Dichloroethane

1,1-Dichloroethylene

1,1-Dichloroethylene

1,2,3-Trichloropropane

1,2,3-Trichloropropane

1,2-Dibromo-3-chloropropane

1,2-Dibromo-3-chloropropane

1,2-Dibromoethane

1,2-Dibromoethane

1,2-Dichloroethane

1,2-Dichloroethane

1,2-Dichloroethylene (total)

1,2-Dichloropropane

1,2-Dichloropropane

2-Hexanone

2-Hexanone

4-Methyl-2-pentanone

Acetone

Acetone

Acetonitrile

Acetonitrile (Methyl cyanide)

Acidity

Acrolein

Acrolein

Acrylonitrile

Acrylonitrile .

Alkalinity

Alkalinity

Allyl chloride

Allyl chloride

Aluminum

Aluminum

Aluminum

Antimony

Antimony

Antimony

Lab Rep Avg

GE

WA

GE

WA

GE

WA

GE

WA

GE

WA

GE

WA

GE

WA

GE

WA

GE

WA

GE

WA

WA

GE

WA

GE

WA

WA

GE

WA

GE

WA

WA

GE

WA

GE

WA

GE

WA

GE

WA

GE

GE

WA

GE

GE

Uesult Acc Stand Units

$\begin{array}{llll}U & 10 & \text { NS } & \mu \& / L\end{array}$

$\begin{array}{lll}U & 1 & \text { NS } \mu g / L\end{array}$

$\begin{array}{llll}U & 5 & 200 & \mu g / L\end{array}$

U $\quad 1 \quad 200 \quad \mu g / L$

$\begin{array}{lll}U & 5 & N S \mu / L\end{array}$

$\begin{array}{lll}U & 1 & \text { NS } \mu g / L\end{array}$

$\begin{array}{llll}U & 5 & 5 & \mu g / L\end{array}$

$\begin{array}{llll}U & 1 & 5 & \mu g / L\end{array}$

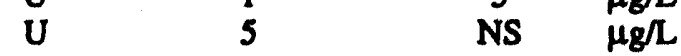

U 1 NS $\mu g / L$

$\begin{array}{llll}U & 5 & 7 & \mu g / L\end{array}$

$\begin{array}{llll}\mathrm{U} & 1 & 7 & \mu g / L\end{array}$

$\begin{array}{lccc}U & 10 & N S & \mu g / L \\ U & 1 & N S & \mu g / L\end{array}$

$\begin{array}{llll}U & 20 & 0.2 & \mu g / L\end{array}$

$\begin{array}{llll}U & 1 & 0.2 & \mu g / L\end{array}$

U 20 NS $\mu g / L$

$\begin{array}{llll}U & 20 & & \mu g / L\end{array}$

$\begin{array}{llll}U & 5 & 5 & \mu g / L\end{array}$

$\begin{array}{llll}U & 1 & 5 & \mu g / L\end{array}$

$\begin{array}{llll}U & 5 & 7 & \mu g / L\end{array}$

$\begin{array}{llll}U & 5 & 5 & \mu g / L\end{array}$

$\begin{array}{llll}U & 1 & 5 & \mu g / L\end{array}$

U 10 NS $\mu g / L$

UJ $V \quad 1 \quad$ NS $\mu g / L$

$U \quad 10 \quad N S \quad \mu g / L$

$\begin{array}{llll}V & 27.8 & N S \quad \mu g / L\end{array}$

$U \quad 100 \quad$ NS $\mu g / L$

$\begin{array}{lll}U & 20 & \text { NS } \mu g / L\end{array}$

$\begin{array}{llll}\mathrm{U} & 1 & \mathrm{NS} & \mu \mathrm{g} / \mathrm{L}\end{array}$

102 NS $\mathrm{mg} / \mathrm{L}$

$\begin{array}{ccc}102 & \mathrm{NS} & \mathrm{mg} / \mathrm{L} \\ 10 & \mathrm{NS} & \mu \mathrm{g} / \mathrm{L}\end{array}$

20 NS $\mu \mathrm{g} / \mathrm{L}$

10 NS $\mu g / L$

20 NS $\mu \mathrm{g} / \mathrm{L}$

7 NS $\mathrm{mg} / \mathrm{L}$

10 NS $\mathrm{mg} / \mathrm{L}$

$\begin{array}{ccc}10 & \mathrm{NS} & \mathrm{mg} / \mathrm{L} \\ 100 & \mathrm{NS} & \mu \mathrm{g} / \mathrm{L}\end{array}$

50 NS $\mu g / L$

200 NS $\mu g / L$

$\begin{array}{llll}\mathrm{H} & 62.1 & \text { NS } & \mu \mathrm{g} / \mathrm{L} \\ \mathrm{H} & 63.2 & \mathrm{NS} & \mu \mathrm{g} / \mathrm{L}\end{array}$

$60 \quad 6 \quad \mu g / L$

L $22 \quad 6 \quad \mu g / L$

$\begin{array}{llllll}\text { NA UJ } & L & 2 & 6 & \mu g / L\end{array}$


UTD003 Laboratory Analyses (cont'd)

\begin{tabular}{|c|c|c|c|c|c|c|c|c|}
\hline Analyte & Lab & Rep & Avg & Modifier & Result & Acc & Stand & Units \\
\hline Arsenic & WA & & & $\mathbf{U}$ & 10 & & 50 & $\mu g / L$ \\
\hline Arsenic & GE & & & $\mathbf{U}$ & 2 & & 50 & $\mu g / \Omega$ \\
\hline Arsenic & GE & & NA & $\mathbf{U}$ & 2 & & 50 & $\mu g / L$ \\
\hline Barium & WA & & & $\mathbf{U}$ & 200 & & 2000 & $\mu g / \Omega$ \\
\hline Barium & GE & & A & & 18.1 & & 2000 & $\mu g / h$ \\
\hline Benzene & WA & & & $\mathbf{U}$ & 5 & - & 5 & $\mu g / 2$ \\
\hline Benzene & $\mathrm{GE}$ & & & $\mathbf{U}$ & 1 & & 5 & $\mu g / L$ \\
\hline Beryllium & WA & & & U & 5 & & 4 & $\mu g / L$ \\
\hline Beryllium & GE & & & $\mathbf{U}$ & 3 & & 4 & $\mu g /$ \\
\hline Beryllium & GE & & NA & $\mathbf{U}$ & 3 & & 4 & $\mu g / L$ \\
\hline Bis(2-chloro-1-methylethyl)ether & $G E$ & & & $\mathbf{U}$ & 10 & & NS & $\mu g / \Lambda$ \\
\hline Bromodichloromethane & WA & & & $\mathbf{U}$ & 5 & & NS & $\mu g / \Lambda$ \\
\hline Bromodichloromethane & GE & & & $\mathbf{U}$ & 1 & & NS & $\mu g /$ \\
\hline Bromoform & WA & & & $\mathbf{U}$ & 5 & & NS & $\mu \mathrm{g} / \mathrm{L}$ \\
\hline Bromoform & GE & & & $\mathbf{U}$ & 1 & & NS & $\mu g / L$ \\
\hline Cadmium & WA & & & $\mathbf{U}$ & 5 & & 5 & $\mu g / \Omega$ \\
\hline Cadmium & GE & & & $\mathbf{U}$ & 2 & & 5 & $\mu g / \Omega$ \\
\hline Cadmium & GE & & NA & $\mathbf{U}$ & 2 & & 5 & $\mu g /$ \\
\hline Calcium & WA & & & $\mathbf{U}$ & 5000 & & NS & $\mu g /$ \\
\hline Calcium & GE & & A & & 3440 & & NS & $\mu g / h$ \\
\hline Carbon disulfide & WA & & & $\mathbf{U}$ & 5 & & NS & $\mu g / L$ \\
\hline Carbon disulfide & GE & & & $\mathbf{U}$ & 1 & & NS & $\mu g \Omega$ \\
\hline Carbon tetrachloride & WA & & & $\mathbf{U}$ & 5 & & 5 & $\mu \mathrm{g} / \mathrm{L}$ \\
\hline Carbon tetrachloride & GE & & & $\mathbf{U}$ & 1 & & 5 & $\mu g / L$ \\
\hline Cesium-137 & TM & & & U & 9.9 & & 200 & $\mathrm{pCi} / \mathrm{L}$ \\
\hline Chloride & WA & & & & 1.48 & & NS & $\mathrm{mg} / \mathrm{L}$ \\
\hline Chloride & GE & & & & 2.15 & & NS & $\mathrm{mg} / \mathrm{L}$ \\
\hline Chlorobenzene & WA & & & $\mathbf{U}$ & 5 & & 100 & $\mu g / L$ \\
\hline Chlorobenzene & GE & & & $\mathbf{U}$ & 1 & & 100 & $\mu g / L$ \\
\hline Chloroethane & WA & & & $\mathbf{U}$ & 10 & & NS & $\mu g / L$ \\
\hline Chloroethane & GE & & & $\mathbf{U}$ & 1 & & NS & $\mu g / L$ \\
\hline Chloroform & WA & & & $\mathbf{U}$ & 5 & & 100 & $\mu g / L$ \\
\hline Chloroform & GE & & & $\mathbf{U}$ & 1 & & 100 & $\mu g / L$ \\
\hline Chloroprene & WA & & & $\mathbf{U}$ & 20 & & NS & $\mu g / L$ \\
\hline Chloroprene & GE & & & $\mathbf{U}$ & 200 & & NS & $\mu g / L$ \\
\hline Chromium & WA & & & U & 10 & & 100 & $\mu \mathrm{g} / \mathrm{L}$ \\
\hline Chromium & GE & & & $\mathbf{U}$ & 4 & & 100 & $\mu g / L$ \\
\hline Chromium & GE & & NA & $\mathbf{U}$ & 4 & & 100 & $\mu \mathrm{g} / \mathrm{L}$ \\
\hline cis-1,3-Dichloropropene & WA & & & $\mathbf{U}$ & 5 & & NS & $\mu g / L$ \\
\hline cis-1,3-Dichloropropene & GE & & & $\mathbf{U}$ & 1 & & NS & $\mu g / L$ \\
\hline Cobalt & WA & & & U & 50 & & NS & $\mu g / L$ \\
\hline Cobalt & GE & & & $\mathbf{U}$ & 4 & & NS & $\mu_{g / L}$ \\
\hline Cobalt & $\mathrm{GE}$ & & NA & U & 4 & & NS & $\mu g / L$ \\
\hline Copper & WA & & & $\mathrm{U}$ & 25 & & 1000 & $\mu g / L$ \\
\hline Copper & GE & & & $\mathbf{U}$ & 4 & & 1000 & $\mu_{g} / L$ \\
\hline Copper & GE & & NA & $\mathbf{U}$ & 4 & & 1000 & $\mu g / L$ \\
\hline Cyanide & GE & & & UJ & $\mathbf{Q}$ & & 200 & $\mu g / L$ \\
\hline Dibromochloromethane & WA & & & U & 5 & & NS & $\mu g / L$ \\
\hline Dibromochloromethane & GE & & & $\mathbf{U}$ & 1 & & NS & $\mu g / L$ \\
\hline Dichlorodifluoromethane & WA & & & $\mathbf{U}$ & 10 & & NS & $\mu g / L$ \\
\hline Dichlorodifluoromethane & GE & & & $\mathbf{U}$ & 1 & & NS & $\mu g / L$ \\
\hline Ethylbenzene & WA & & & $\mathbf{U}$ & 5 & & 700 & L. \\
\hline Ethylbenzene & GE & & & $\mathbf{U}$ & 1 & & 700 & \\
\hline Fluoride & WA & & & $\mathbf{U}$ & 0.1 & & 4 & $g / 2$ \\
\hline Fluoride & $\mathbf{G E}$ & & & $\mathbf{U}$ & 0.1 & & 4 & \\
\hline
\end{tabular}


UTD003 Laboratory Analyses (cont'd)

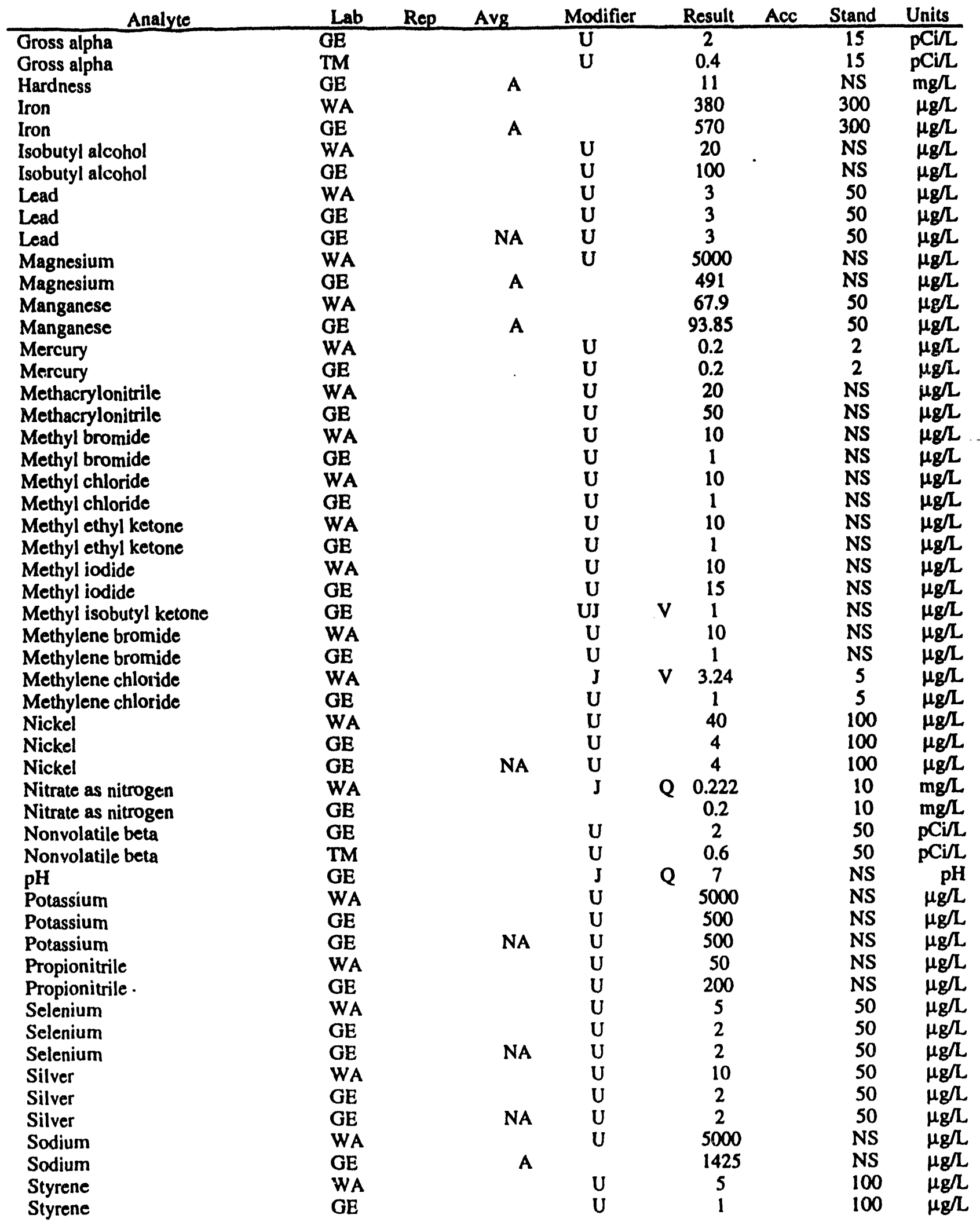


UTD003 Laboratory Analyses (cont'd)

\begin{tabular}{|c|c|c|c|c|c|c|c|c|c|}
\hline Analyte & Lab & Rep & Avg & Modifier & & Result & Acc & Stand & Units \\
\hline Sulfate & WA & & & $\overline{\mathbf{U}}$ & & 2.5 & & 400 & $\overline{m g / L}$ \\
\hline Sulfate & $\mathrm{GE}$ & & & $\mathbf{U}$ & & 1 & & 400 & $\mathrm{mg} / \mathrm{L}$ \\
\hline Sulfide & WA & & & $\mathbf{U}$ & & 0.1 & & NS & $\mathrm{mg} / \mathrm{L}$ \\
\hline Sulfide & GE & & & $\mathbf{U}$ & & 1 & & NS & $\mathrm{mg} / \mathrm{L}$ \\
\hline Sulfide & $\mathrm{GE}$ & & NA & $\mathbf{U}$ & & 1 & & NS & $\mathrm{mg} / \mathrm{L}$ \\
\hline Tetrachloroethylene & WA & & & $\mathbf{U}$ & & 5 & . & 5 & $\mu g / L$ \\
\hline Tetrachloroethylene & $\mathbf{G E}$ & & & $\mathbf{U}$ & & 1 & & 5 & $\mu g / L$ \\
\hline Thallium & WA & & & $\mathbf{U}$ & & 10 & & 2 & $\mu g / L$ \\
\hline Thallium & $\mathbf{G E}$ & & & $\mathbf{U}$ & & 2 & & 2 & $\mu g / L$ \\
\hline Thallium & $\overline{G E}$ & & NA & $\mathbf{U}$ & & 2 & & 2 & $\mu g / L$ \\
\hline Toluene & WA & & & $\mathbf{U}$ & & 5 & & 1000 & $\mu g / L$ \\
\hline Toluene & GE & & & $\mathbf{U}$ & & 1 & & 1000 & $\mu g / L$ \\
\hline Total hardness & WA & & & & & 6.7 & & NS & $\mathrm{mg} / \mathrm{L}$ \\
\hline Total organic carbon & WA & & & & & 1.81 & & NS & $\mathrm{mg} / \mathrm{L}$ \\
\hline Total organic carbon & GE & & & $\mathbf{U}$ & & 1 & & NS & $\mathrm{mg} / \mathrm{L}$ \\
\hline Total suspended solids & WA & & & & & 20 & & NS & $\mathrm{mg} / \mathrm{L}$ \\
\hline Total suspended solids & $\mathbf{G E}$ & & & $\mathbf{U}$ & & 1 & & NS & $\mathrm{mg} / \mathrm{L}$ \\
\hline trans-1,2-Dichloroethylene (total) & $\mathbf{G E}$ & & & $\mathbf{U}$ & & 1 & & 100 & $\mu g / L$ \\
\hline trans-1,3-Dichloropropene & WA & & & $\mathbf{U}$ & & 5 & & NS & $\mu \mathrm{g} / \mathrm{L}$ \\
\hline trans-1,3-Dichloropropene & $\mathbf{G E}$ & & & $\mathbf{U}$ & & 1 & & NS & $\mu g / L$ \\
\hline trans-1,4-Dichloro-2-butene & WA & & & $\mathbf{U}$ & & 100 & & NS & $\mu g / L$ \\
\hline trans-1,4-Dichloro-2-butene & GE & & & $\mathbf{U}$ & & 30 & & NS & $\mu g / L$ \\
\hline Trichloroethylene & WA & & & $\mathbf{U}$ & & 5 & & 5 & $\mu g / L$ \\
\hline Trichloroethylene & GE & & & $\mathbf{U}$ & & 1 & & 5 & $\mu g / L$ \\
\hline Trichlorofluoromethane & WA & & & $\mathbf{U}$ & & 5 & & NS & $\mu g / L$ \\
\hline Trichlorofluoromethane & GE & & & $\mathbf{U}$ & & 1 & & NS & $\mu g / L$ \\
\hline Tritium & GE & & & & & 93.1 & & 20 & $\mathrm{pCi} / \mathrm{ml}$ \\
\hline Tritium & $\overrightarrow{T M}$ & & & & & 103.5 & 2.23 & 20 & $\mathrm{pCi} / \mathrm{ml}$ \\
\hline Turbidity & WA & & & $\mathbf{J}$ & $\mathbf{Q}$ & 3.14 & & NS & NTU \\
\hline Turbidity & GE & & $\mathbf{A}$ & & & 4.64 & & NS & NTU \\
\hline Vanadium & WA & & & $\mathbf{U}$ & & 50 & & NS & $\mu g / L$ \\
\hline Vanadium & GE & & & $\mathbf{U}$ & & 8 & & NS & $\mu \mathrm{g} / \mathrm{L}$ \\
\hline Vanadium & GE & & NA & $\mathbf{U}$ & & 8 & & NS & $\mu g / L$ \\
\hline Vinyl acetate & WA & & & $\mathbf{U}$ & & 10 & & NS & $\mu \mathrm{g} / \mathrm{L}$ \\
\hline Vinyl acetate & GE & & & $\mathbf{U}$ & & 1 & & NS & $\mu \mathrm{g} / \mathrm{L}$ \\
\hline Vinyl chloride & WA & & & $\mathbf{U}$ & & 10 & & 2 & $\mu g / L$ \\
\hline Vinyl chloride & GE & & & $\mathbf{U}$ & & 1 & & 2 & $\mu \mathrm{g} / \mathrm{L}$ \\
\hline Xylenes (total) & WA & & & $\mathbf{U}$ & & 5 & & 10000 & $\mu g / L$ \\
\hline Xylenes (total) & GE & & & $\mathbf{U}$ & & 2 & & 10000 & $\mu g / L$ \\
\hline Zinc & WA & & & U & & 20 & & 5000 & $\mu g / L$ \\
\hline Zinc & GE & & & $\mathbf{U}$ & & 2 & & 5000 & $\mu g / L$ \\
\hline Zinc & GE & & NA & $\mathbf{U}$ & & 2 & & 5000 & $\mu g / L$ \\
\hline
\end{tabular}




\section{UTDO04}

Field Measurements:

Stream Discharge: 0.24 cfs

Sample Date: 9/1/93

pH: 6.01

Specific Conductance: $40 \mu \mathrm{S} / \mathrm{cm}$

Red/Oxid Potential: $359 \mathrm{mv}$

Dissolved Oxygen: $8.0 \mathrm{mg} / \mathrm{L}$

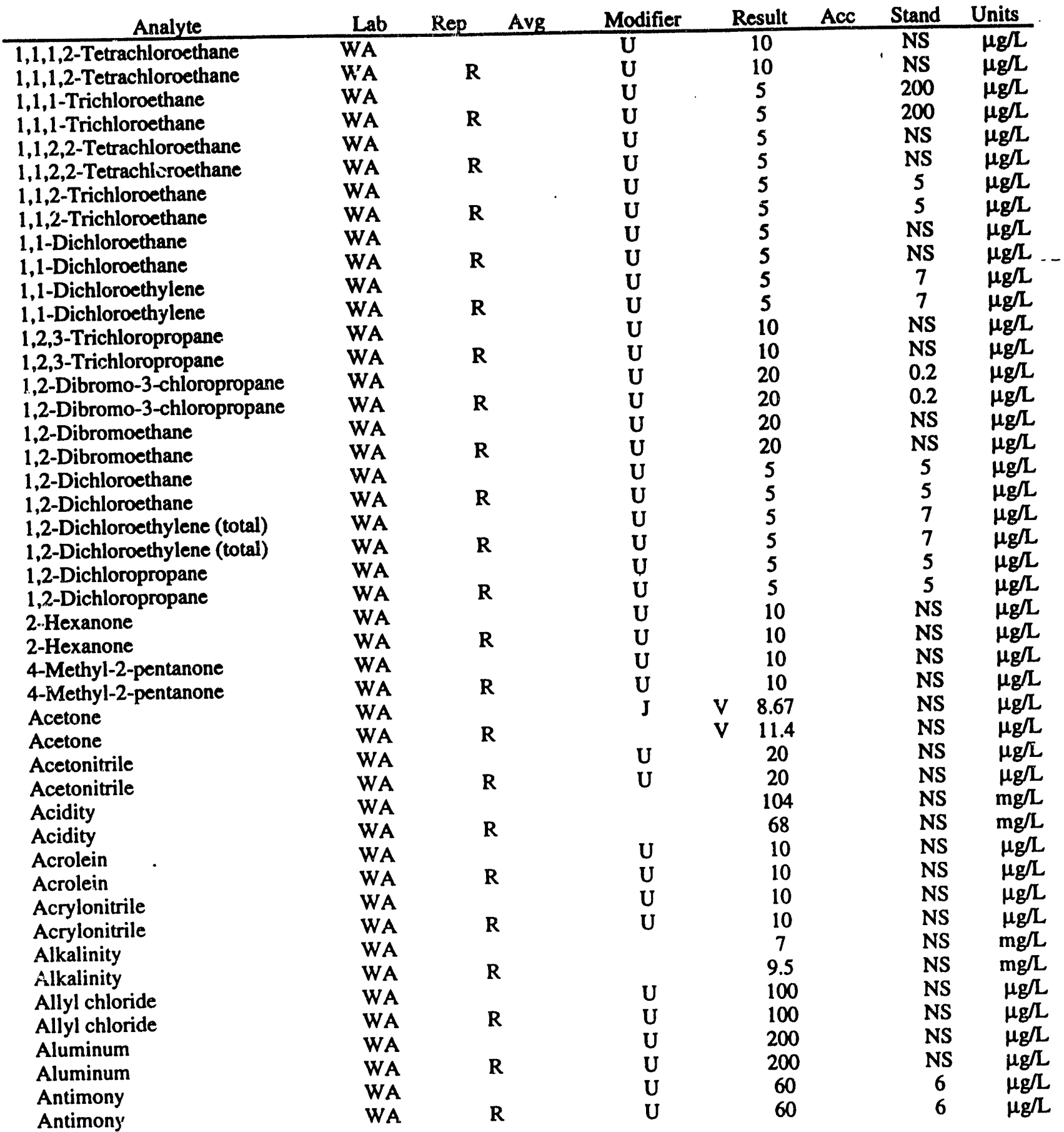


Sampling And Analysis of Water From Upper Three Runs And Its Wetlands Near Tank 16 and the Mixed Waste Management Facility

UTD004 Laboratory Analyses (cont'd)

\begin{tabular}{|c|c|c|c|c|c|c|c|c|}
\hline Analyte & Lab & Rep & Avg & Modifier & Result & Acc & Stand & Units \\
\hline Arsenic & WA & & & $\bar{U}$ & 10 & & 50 & $\mu g / L$ \\
\hline Arsenic & WA & $\mathbf{R}$ & & $\mathbf{U}$ & 10 & & 50 & $\mu \mathrm{g} / \mathrm{L}$ \\
\hline Barium & WA & & & $\mathbf{U}$ & 200 & & 2000 & $\mu g / L$ \\
\hline Barium & WA & $\mathbf{R}$ & & $\mathbf{U}$ & 200 & & 2000 & $\mu g / L$ \\
\hline Benzene & WA & & & $\mathbf{U}$ & 5 & & $5^{7}$ & $\mu \mathrm{g} / \mathrm{L}$ \\
\hline Benzene & WA & $\mathbf{R}$ & & $\mathbf{U}$ & 5 & . & 5 & $\mu \mathrm{g} / \mathrm{L}$ \\
\hline Beryllium & WA & & & U & 5 & & 4 & $\mu \mathrm{g} / \mathrm{L}$ \\
\hline Beryllium & WA & $\mathbf{R}$ & & $\mathbf{U}$ & 5 & & 4 & $\mu \mathrm{g} / \mathrm{L}$ \\
\hline Bromodichloromethane & WA & & & $\mathbf{U}$ & 5 & & NS & $\mu \mathrm{g} / \mathrm{L}$ \\
\hline Bromodichloromethane & WA & $\mathbf{R}$ & & $\mathbf{U}$ & 5 & & NS & $\mu g / L$ \\
\hline Bromoform & WA & & & $\mathbf{U}$ & 5 & & NS & $\mu g / \mathrm{L}$ \\
\hline Bromoform & WA & $\mathbf{R}$ & & $\mathbf{U}$ & 5 & & NS & $\mu \mathrm{g} / \mathrm{L}$ \\
\hline Cadmium & WA & & & $\mathrm{U}$ & 5 & & 5 & $\mu \mathrm{g} / \mathrm{L}$ \\
\hline Cadmium & WA & $\mathbf{R}$ & & $\mathbf{U}$ & 5 & & 5 & $\mu \mathrm{g} / \mathrm{L}$ \\
\hline Calcium & WA & & & $\mathbf{U}$ & 5000 & & NS & $\mu g / L$ \\
\hline Calcium & WA & $\mathbf{R}$ & & $\mathbf{U}$ & 5000 & & NS & $\mu g / L$ \\
\hline Carbon disulfide & WA & & & $\mathbf{U}$ & 5 & & NS & $\mu \mathrm{g} / \mathrm{L}$ \\
\hline Carbon disulfide & WA & $\mathbf{R}$ & & $\mathbf{U}$ & 5 & & NS & $\mu g / \mathrm{L}$ \\
\hline Carbon tetrachloride & WA & & & $\mathbf{U}$ & 5 & & 5 & $\mu \mathrm{g} / \mathrm{L}$ \\
\hline Carbon tetrachloride & WA & $\mathbf{R}$ & & $\mathbf{U}$ & 5 & & 5 & $\mu g / L$ \\
\hline Cesium-137 & TM & & & $\mathbf{U}$ & 5.8 & & 200 & $\mathrm{pCi} / \mathrm{L}$ \\
\hline Cesium-137 & TM & $\mathbf{R}$ & & $\mathbf{U}$ & 6.2 & & 200 & $\mathrm{pCi} / \mathrm{L}$ \\
\hline Chloride & WA & & & & 4.32 & & NS & $\mathrm{mg} / \mathrm{L}$ \\
\hline Chloride & WA & $\mathbf{R}$ & A & & 4.305 & & NS & $\mathrm{mg} / \mathrm{L}$ \\
\hline Chlorobenzene & WA & & & $\mathbf{U}$ & 5 & & 100 & $\mu \mathrm{g} / \mathrm{L}$ \\
\hline Chlorobenzene & WA & $\mathbf{R}$ & & $\mathrm{U}$ & 5 & & 100 & $\mu g / L$ \\
\hline Chloroethane & WA & & & $\mathbf{U}$ & 10 & & NS & $\mu g / L$ \\
\hline Chloroethane & WA & $\mathbf{R}$ & & $\mathbf{U}$ & 10 & & NS & $\mu \mathrm{g} / \mathrm{L}$ \\
\hline Chloroform & WA & & & $\mathbf{U}$ & 5 & & 100 & $\mu \mathrm{g} / \mathrm{L}$ \\
\hline Chloroform & WA & $\mathbf{R}$ & & $\mathbf{U}$ & 5 & & 100 & $\mu g / L$ \\
\hline Chloroprene & WA & & & $\mathbf{U}$ & 20 & & NS & $\mu \mathrm{g} / \mathrm{L}$ \\
\hline Chloroprene & WA & $\mathbf{R}$ & & $\mathbf{U}$ & 20 & & NS & $\mu \mathrm{g} / \mathrm{L}$ \\
\hline Chromium & WA & & & $\mathbf{U}$ & 10 & & 100 & $\mu \mathrm{g} / \mathrm{L}$ \\
\hline Chromium & WA & $\mathbf{R}$ & & $\mathbf{U}$ & 10 & & 100 & $\mu g / L$ \\
\hline cis-1,3-Dichloropropene & WA & & & $\mathbf{U}$ & 5 & & NS & $\mu g / L$ \\
\hline cis-1,3-Dichloropropene & WA & $\mathbf{R}$ & & $\mathbf{U}$ & 5 & & NS & $\mu g / L$ \\
\hline Cobalt & WA & & & $\mathbf{U}$ & 50 & & NS & $\mu \mathrm{g} / \mathrm{L}$ \\
\hline Cobalt & WA & $\mathbf{R}$ & & U & 50 & & NS & $\mu g / L$ \\
\hline Copper & WA & & & $\mathbf{U}$ & 25 & & 1000 & $\mu \mathrm{g} / \mathrm{L}$ \\
\hline Copper & WA & $\mathbf{R}$ & & $\mathbf{U}$ & 25 & & 1000 & $\mu g / L$ \\
\hline Dibromochloromethane & WA & & & $\mathrm{U}$ & 5 & & NS & $\mu g / L$ \\
\hline Dibromochloromethane & WA & $\mathbf{R}$ & & $\mathbf{U}$ & 5 & & NS & $\mu g / L$ \\
\hline Dichlorodifluoromethane & WA & & & $\mathrm{U}$ & 10 & & NS & $\mu \mathrm{g} / \mathrm{L}$ \\
\hline Dichlorodifluoromethane & WA & $\mathbf{R}$ & & $\mathrm{U}$ & 10 & & NS & $\mu g / L$ \\
\hline Ethylbenzene & WA & & & 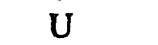 & 5 & & 700 & $\mu \mathrm{g} / \mathrm{L}$ \\
\hline Ethylbenzene & WA & $\mathbf{R}$ & & $\mathrm{U}$ & 5 & & 700 & $\mu \mathrm{g}$ \\
\hline Fluoride & WA & & & $\mathrm{U}$ & 0.1 & & 4 & $\mathrm{mg} / \mathrm{L}$ \\
\hline Fluoride & WA & $\mathbf{R}$ & & $\mathbf{U}$ & 0.1 & & 4 & $\mathrm{mg} / \mathrm{L}$ \\
\hline Gross alpha & TM & & & & 1.4 & 1.2 & 15 & $\mathrm{pCi} / \mathrm{L}$ \\
\hline Gross alpha & TM & $\mathbf{k}$ & & & 0.6 & 1 & 15 & $\mathrm{pCi} / \mathrm{L}$ \\
\hline Iron & WA & & & & 372 & & 300 & $\mu g / \mathrm{L}$ \\
\hline Iron & WA & $\mathbf{R}$ & & & 369 & & 300 & $\mu \mathrm{g}$ \\
\hline Isobutyl alcohol & WA & & & $\mathbf{U}$ & 20 & & NS & $\mu \mathrm{g} / \mathrm{L}$ \\
\hline Isobutyl alcohol & WA & $\mathbf{R}$ & & $\mathbf{U}$ & 20 & & NS & $h$ \\
\hline
\end{tabular}


UTD004 Laboratory Analyses (cont'd)

\begin{tabular}{|c|c|c|c|c|c|c|c|c|c|}
\hline Analyte & Lab & Rep & Avg & Modifier & & Result & Acc & Stand & Units \\
\hline Lead & WA & & & & & 7.8 & & 50 & $\mu g / L$ \\
\hline Lead & WA & $\mathbf{R}$ & & & & 7.4 & & 50 & $\mu g / L$ \\
\hline Magnesium & WA & & & $\mathbf{U}$ & & 5000 & & NS & $\mu \mathrm{g} / \mathrm{L}$ \\
\hline Magnesium & WA & $\mathbf{R}$ & & $\mathbf{U}$ & & 5000 & & NS & $\mu \mathrm{g} / \mathrm{L}$ \\
\hline Manganese & WA & & & $\mathbf{U}$ & & 15 & & 50 & $\mu \mathrm{g} / \mathrm{L}$ \\
\hline Manganese & WA & $\mathbf{R}$ & & $\mathbf{U}$ & & 15 & & 50 & $\mu \mathrm{g} / \mathrm{L}$ \\
\hline Mercury & WA & & & $\mathbf{U}$ & & 0.2 & & 2 & $\mu \mathrm{g} / \mathrm{L}$ \\
\hline Mercury & WA & $\mathbf{R}$ & & $\mathbf{U}$ & & 0.2 & & 2 & $\mu g / L$ \\
\hline Methacrylonitrile & WA & & & $\mathbf{U}$ & & 20 & & NS & $\mu g / L$ \\
\hline Methacrylonitrile & WA & $\mathbf{R}$ & & $\mathbf{U}$ & & 20 & & NS & $\mu g / L$ \\
\hline Methyl bromide & WA & & & $\mathbf{U}$ & & 10 & & NS & $\mu g / L$ \\
\hline Methyl bromide & WA & $\mathbf{R}$ & & $\mathbf{U}$ & & 10 & & NS & $\mu g / L$ \\
\hline Methyl chloride & WA & & & $\mathbf{U}$ & & 10 & & NS & $\mu g / L$ \\
\hline Methyl chloride & WA & $\mathbf{R}$ & & $\mathbf{U}$ & & 10 & & NS & $\mu \mathrm{g} / \mathrm{L}$ \\
\hline Methyl ethyl ketone & WA & & & $\mathbf{U}$ & & 10 & & NS & $\mu g / L$ \\
\hline Methyl ethyl ketone & WA & $\mathbf{R}$ & & $\mathbf{U}$ & & 10 & & NS & $\mu g / L$ \\
\hline Methyl iodide & WA & & & $\mathbf{U}$ & & 10 & & NS & $\mu \mathrm{g} / \mathrm{L}$ \\
\hline Methyl iodide & WA & $\mathbf{R}$ & & U & & 10 & & NS & $\mu g / L$ \\
\hline Methylene bromide & WA & & & $\mathbf{U}$ & & 10 & & NS & $\mu \mathrm{g} / \mathrm{L}$. \\
\hline Methylene bromide & WA & $\mathbf{R}$ & & $\mathbf{U}$ & & 10 & & NS & $\mu g / L$ \\
\hline Methylene chloride & WA & & & $\mathbf{J}$ & V & 3.78 & & 5 & $\mu g / L$ \\
\hline Methylene chloride & WA & $\mathbf{R}$ & & $\mathbf{J}$ & V & 3.61 & & 5 & $\mu g / L$ \\
\hline Nickel & WA & & & $\mathbf{U}$ & & 40 & & 100 & $\mu g / L$ \\
\hline Nickel & WA & $\mathbf{R}$ & & $\mathbf{U}$ & & 40 & & 100 & $\mu g / L$ \\
\hline Nitrate as nitrogen & WA & & & J & $\mathbf{Q}$ & 0.249 & & 10 & $\mathrm{mg} / \mathrm{L}$ \\
\hline Nitrate as nitrogen & WA & $\mathbf{R}$ & & $\mathbf{J}$ & $\mathbf{Q}$ & 0.285 & & 10 & $\mathrm{mg} / \mathrm{L}$ \\
\hline Nonvolatile beta & TM & & & & & 1 & 2.5 & 50 & pCi/L \\
\hline Nonvolatile beta & TM & $\mathbf{R}$ & & $\mathbf{U}$ & & 0.6 & & 50 & $\mathrm{pCi} / \mathrm{L}$ \\
\hline Potassium & WA & & & U & & 5000 & & NS & $\mu g / L$ \\
\hline Potassium & WA & $\mathbf{R}$ & & $\mathbf{U}$ & & 5000 & & NS & $\mu g / L$ \\
\hline Propionitrile & WA & & & $\mathbf{U}$ & & 50 & & NS & $\mu g / L$ \\
\hline Propionitrile & WA & $\mathbf{R}$ & & U & & 50 & & NS & $\mu g / L$ \\
\hline Selenium & WA & & & $U$ & & 5 & & 50 & $\mu \mathrm{g} / \mathrm{L}$ \\
\hline Selenium & WA & $\mathbf{R}$ & & U & & 5 & & 50 & $\mu g / L$ \\
\hline Silver & WA & & & $\mathbf{U}$ & & 10 & & 50 & $\mu g / L$ \\
\hline Silver & WA & $\mathbf{R}$ & & $\mathbf{U}$ & & 10 & & 50 & $\mu g / L$ \\
\hline Sodium & WA & & & & & 6640 & & NS & $\mu \mathrm{g} / \mathrm{L}$ \\
\hline Sodium & WA & $\mathbf{R}$ & & & & 6720 & & NS & $\mu g / L$ \\
\hline Styrene & WA & & & $\mathbf{U}$ & & 5 & & 100 & $\mu g / L$ \\
\hline Styrene & WA & $\mathbf{R}$ & & $\mathbf{U}$ & & 5 & & 100 & $\mu \mathrm{g} / \mathrm{L}$ \\
\hline Sulfate & WA & & & & & 4.27 & & 400 & $\mathrm{mg} / \mathrm{L}$ \\
\hline Sulfate & WA & $\mathbf{R}$ & & & & 3.66 & & 400 & $\mathrm{mg} / \mathrm{L}$ \\
\hline Sulfide & WA & & & $\mathbf{U}$ & & 0.1 & & NS & $\mathrm{mg} / \mathrm{L}$ \\
\hline Sulfide & WA & $\mathbf{R}$ & & $\mathbf{U}$ & & 0.1 & & NS & $\mathrm{mg} / \mathrm{L}$ \\
\hline Tetrachloroethylene & WA & & & $\mathbf{U}$ & & 5 & & 5 & $\mu \mathrm{g} / \mathrm{L}$ \\
\hline Tetrachloroethylene & WA & $\mathbf{R}$ & & $\mathbf{U}$ & & 5 & & 5 & $\mu g / L$ \\
\hline Thallium & WA & & & $\mathbf{U}$ & & 10 & & 2 & $\mu g / L$ \\
\hline Thallium & WA & $\mathbf{R}$ & & U & & 10 & & 2 & $\mu \mathrm{g} / \mathrm{L}$ \\
\hline Toluene & WA & & & $\mathbf{U}$ & & 5 & & 1000 & $\mu \mathrm{g} / \mathrm{L}$ \\
\hline Toluene & WA & $\mathbf{R}$ & & $\mathrm{U}$ & & 5 & & 1000 & $\mu g / L$ \\
\hline Total hardness & WA & & & & & 4.8 & & NS & $\mathrm{mg} / \mathrm{L}$ \\
\hline Total hardness & WA & $\mathbf{R}$ & & & & 1.9 & & NS & $\mathrm{mg} / \mathrm{L}$ \\
\hline Total organic carbon & WA & & & & & 1.81 & & NS & $\mathrm{mg} / \mathrm{L}$ \\
\hline Total organic carbon & WA & $\mathbf{R}$ & $\mathbf{A}$ & & & 1.68 & & NS & $\mathrm{mg} / \mathrm{L}$ \\
\hline
\end{tabular}


UTD004 Laboratory Analyses (cont'd)

\begin{tabular}{|c|c|c|c|c|c|c|c|c|c|}
\hline Analyte & Lab & Rep & Avg & Modifier & & Result & Acc & Stand & Units \\
\hline Total suspended solids & WA & & & & & 23 & & NS & $\mathrm{mg} / \mathrm{L}$ \\
\hline Total suspended solids & WA & $\mathbf{R}$ & & & & 20 & & NS & $\mathrm{mg} / \mathrm{L}$ \\
\hline trans-1,3-Dichloropropene & WA & & & $\mathbf{U}$ & & 5 & & NS & $\mu \mathrm{g} / \mathrm{L}$ \\
\hline trans-1,3-Dichloropropene & WA & $\mathbf{R}$ & & $\mathbf{U}$ & & 5 & & NS & $\mu g / L$ \\
\hline trans-1,4-Dichloro-2-butene & WA & & & $\mathbf{U}$ & & 100 & & NS & $\mu g / L$ \\
\hline trans-1,4-Dichloro-2-butene & WA & $\mathbf{R}$ & & $\mathbf{U}$ & & 100 & . & NS & $\mu g / L$ \\
\hline Trichloroethylene & WA & & & $\mathbf{U}$ & & 5 & & 5 & $\mu \mathrm{g} / \mathrm{L}$ \\
\hline Trichloroethylene & WA & $\mathbf{R}$ & & $\mathbf{U}$ & & 5 & & 5 & $\mu g / L$ \\
\hline Trichlorofluoromethane & WA & & & $\mathbf{U}$ & & 5 & & NS & $\mu g / L$ \\
\hline Trichlorofluoromethane & WA & $\mathbf{R}$ & & $\mathbf{U}$ & & 5 & & NS & $\mu g / L$ \\
\hline Tritium & TM & & & & & 53.45 & 1.65 & 20 & $\mathrm{pCi} / \mathrm{ml}$ \\
\hline Tritium & TM & $\mathbf{R}$ & & & & 51.29 & 1.59 & 20 & $\mathrm{pCi} / \mathrm{ml}$ \\
\hline Turbidity & WA & & & $\mathbf{J}$ & $\mathbf{Q}$ & 6.6 & & NS & NTU \\
\hline Turbidity & WA & $\mathbf{R}$ & & $\mathbf{J}$ & $\mathbf{Q}$ & 2.95 & & NS & NTU \\
\hline Vanadium & WA & & & $\mathbf{U}$ & & 50 & & NS & $\mu g / L$ \\
\hline Vanadium & WA & $\mathbf{R}$ & & $\mathbf{U}$ & & 50 & & NS & $\mu g / L$ \\
\hline Vinyl acetate & WA & & & $\mathbf{U}$ & & 10 & & NS & $\mu g / L$ \\
\hline Vinyl acetate & WA & $\mathbf{R}$ & & U & & 10 & & NS & $\mu g / L$ \\
\hline Vinyl chloride & WA & & & $\mathbf{U}$ & & 10 & & 2 & $\mu g / L$ \\
\hline Vinyl chloride & WA & $\mathbf{R}$ & & $\mathbf{U}$ & & 10 & & 2 & $\mu \mathrm{g} / \mathrm{L}$ \\
\hline Xylenes (total) & WA & & & $\mathbf{U}$ & & 5 & & 10000 & $\mu g / L$ \\
\hline Xylenes (total) & WA & $\mathbf{R}$ & & $\mathbf{U}$ & & 5 & & 10000 & \\
\hline Zinc & WA & & & $\mathrm{U}$ & & 20 & & 5000 & L \\
\hline Zinc & WA & $\mathbf{R}$ & & $\mathbf{U}$ & & 20 & & 5000 & \\
\hline
\end{tabular}




\section{UTD005}

Field Measurements:

Stream Discharge: 0.13 cfs

Sample Date: 9/1/93

pH: 7.82

Specific Conductance: $74 \mu \mathrm{S} / \mathrm{cm}$

Red/Oxid Potential: $50 \mathrm{mv}$

Dissolved Oxygen: $6.2 \mathrm{mg} / \mathrm{L}$

\begin{tabular}{|c|c|c|c|c|c|c|c|}
\hline Analyte & $\mathrm{Lab}$ & Avg & Modifier & Result & Acc & Stand & Units \\
\hline 1,1,1,2-Tetrachloroethane & WA & & $\mathbf{U}$ & 10 & & NS & $\mu g / L$ \\
\hline 1,1,1-Trichloroethane & WA & & $\mathbf{U}$ & 5 & & 200 & $\mu g / L$ \\
\hline $1,1,2,2-$ Tetrachloroethane & WA & & $\mathbf{U}$ & 5 & & NS & $\mu g / L$ \\
\hline 1,1,2-Trichloroethane & WA & & $\mathbf{U}$ & 5 & & 5 & $\mu g / L$ \\
\hline 1,1-Dichloroethane & WA & & $\mathbf{U}$ & 5 & & NS & $\mu \mathrm{g} / \mathrm{L}$ \\
\hline 1,1-Dichloroethylene & WA & & $\mathbf{U}$ & 5 & & 7 & $\mu g / L$ \\
\hline 1,2,3-Trichloropropane & WA & & $\mathbf{U}$ & 10 & & NS & $\mu g / L$ \\
\hline 1,2-Dibromo-3-chloropropane & WA & & $\mathbf{U}$ & 20 & & 0.2 & $\mu \mathrm{g} / \mathrm{L}$ \\
\hline 1,2-Dibromoethane & WA & & $\mathbf{U}$ & 20 & & NS & $\mu g / L$ \\
\hline 1,2-Dichloroethane & WA & & $\mathrm{U}$ & 5 & & 5 & $\mu g / L$ \\
\hline 1,2-Dichloroethylene (total) & WA & & $\mathbf{U}$ & 5 & & 7 & $\mu g / L$ \\
\hline 1,2-Dichloropropane & WA & & U & 5 & & 5 & $\mu g / L$ \\
\hline 2-Hexanone & WA & & $\mathrm{U}$ & 10 & & NS & $\mu g / L$ \\
\hline 4-Methyl-2-pentanone & WA & & $\mathbf{U}$ & 10 & & NS & $\mu g / L$ \\
\hline Acetone & WA & & & 19.8 & & NS & $\mu \mathrm{g} / \mathrm{L}$ \\
\hline Acetonitrile & WA & & $\mathbf{U}$ & 20 & & NS & $\mu g / L$ \\
\hline Acidity & WA & & & 74 & & NS & $\mathrm{mg} / \mathrm{L}$ \\
\hline Acrolein & WA & & $\mathrm{U}$ & 10 & & NS & $\mu \mathrm{g} / \mathrm{L}$ \\
\hline Acrylonitrile & WA & & $\mathrm{U}$ & 10 & & NS & $\mu \mathrm{g} / \mathrm{L}$ \\
\hline Alkalinity & WA & & & 26 & & NS & $\mathrm{mg} / \mathrm{L}$ \\
\hline Allyl chloride & WA & & $\mathbf{U}$ & 100 & & NS & $\mu g / L$ \\
\hline Aluminum & WA & & $\mathbf{U}$ & 200 & & NS & $\begin{array}{l}\mu g L \\
\mu g / L\end{array}$ \\
\hline Americium-241 & TM & & & 17.8 & 4 & 6.34 & $\mathrm{pCi} / \mathrm{L}$ \\
\hline Antimony & WA & & $\mathbf{U}$ & 60 & & 6 & $\mu g / L$ \\
\hline $\begin{array}{l}\text { Anumony } \\
\text { Arsenic }\end{array}$ & WA & & $\mathbf{U}$ & 10 & & 50 & $\mu g / L$ \\
\hline Barium & WA & & $\mathbf{U}$ & 200 & & 2000 & $\mu g / L$ \\
\hline $\begin{array}{l}\text { Banum } \\
\text { Benzene }\end{array}$ & WA & & U & 5 & & 5 & $\begin{array}{l}\mu g / L \\
\mu g / L\end{array}$ \\
\hline $\begin{array}{l}\text { Benzene } \\
\text { Beryllium }\end{array}$ & WA & & $\mathbf{U}$ & 5 & & 4 & $\begin{array}{l}\mu \mathrm{g} / \mathrm{L} \\
\mu \mathrm{g} / \mathrm{L}\end{array}$ \\
\hline Bromodichloromethane & WA & & $\mathbf{U}$ & 5 & & NS & $\begin{array}{l}\mu g / L \\
\mu g / L\end{array}$ \\
\hline Bromoform & WA & & $\mathbf{U}$ & 5 & & NS & $\mu g / L$ \\
\hline Cadmium & WA & & $\mathbf{U}$ & 5 & & 5 & $\mu g / L$ \\
\hline Calcium & WA & & $\mathbf{U}$ & 5000 & & NS & $\mu g / L$ \\
\hline Carbon disulfide & WA & & $\mathbf{U}$ & 5 & & NS & $\mu g / L$ \\
\hline Carbon tetrachloride & WA & & $\mathbf{U}$ & 5 & & 5 & $\mu \mathrm{g} / \mathrm{L}$ \\
\hline Cesium-137 . & TM & & $\mathbf{U}$ & 10.1 & & 200 & $\mathrm{pCi} / \mathrm{L}$ \\
\hline Chloride & WA & & & 10.3 & & NS & $\mathrm{mg} / \mathrm{L}$ \\
\hline Chlorobenzene & WA & & $\mathbf{U}$ & 5 & & 100 & $\mu g / L$ \\
\hline Chloroethane & WA & & $\mathbf{U}$ & 10 & & NS & $\mu \mathrm{g} / \mathrm{L}$ \\
\hline Chloroform & WA & & $\mathbf{U}$ & 5 & & 100 & $\mu g / L$ \\
\hline Chloroprene & WA & & $\mathrm{U}$ & 20 & & NS & $\mu \mathrm{g} / \mathrm{L}$ \\
\hline Chromium & WA & & $\mathbf{U}$ & 10 & & 100 & $\mu \mathrm{g} / \mathrm{L}$ \\
\hline cis-1,3-Dichloropropene & WA & & $\mathbf{U}$ & 5 & & NS & $\mu \mathrm{g} / \mathrm{L}$ \\
\hline 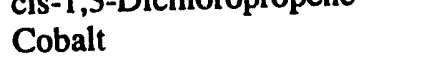 & WA & & $\mathbf{U}$ & 50 & & NS & $\mu \mathrm{g} / \mathrm{L}$ \\
\hline Copper & WA & & $\mathbf{U}$ & 25 & & 1000 & $\mu \mathrm{g} / \mathrm{L}$ \\
\hline Dibromochloromethane & WA & & $\mathbf{U}$ & 5 & & NS & $\mu g / L$ \\
\hline Dichlorodifluoromethane & WA & & $\mathbf{U}$ & 10 & & NS & $\mu g / L$ \\
\hline
\end{tabular}


UTD005 Laboratory Analyses (cont'd)

\begin{tabular}{|c|c|c|c|c|c|c|c|c|c|}
\hline Analyte & Lab & Rep & Avg & Modifier & & Result & Acc & Stand & Units \\
\hline Ethylbenzene & $\overline{\text { WA }}$ & & & $\mathbf{U}$ & & 5 & & 700 & $\overline{\mu g / L}$ \\
\hline Fluoride & WA & & & $\mathbf{U}$ & & 0.1 & & 4 & $\mathrm{mg} / \mathrm{L}$ \\
\hline Gross alpha & TM & & & & & 1.4 & 1.3 & 15 & $\mathrm{pCi} / \mathrm{L}$ \\
\hline Iron & WA & & & & & 1420 & & 300 & $\mu \mathrm{g} / \mathrm{L}$ \\
\hline Isobutyl alcohol & WA & & & $\mathbf{U}$ & & 20 & & NS & $\mu \mathrm{g} / \mathrm{L}$ \\
\hline Lead & WA & & & U & & 3 & . & 50 & $\mu g / \mathrm{L}$ \\
\hline Magnesium & WA & & & $\mathbf{U}$ & & 5000 & & NS & $\mu g / L$ \\
\hline Manganese & WA & & & & & 79.1 & & 50 & $\mu g / \mathrm{L}$ \\
\hline Mercury & WA & & & $\mathbf{U}$ & & 0.2 & & 2 & $\mu \mathrm{g} / \mathrm{L}$ \\
\hline Methacrylonitrile & WA & & & $\mathbf{U}$ & & 20 & & NS & $\mu \mathrm{g} / \mathrm{L}$ \\
\hline Methyl bromide & WA & & & $\mathbf{U}$ & & 10 & & NS & $\mu \mathrm{g} / \mathrm{L}$ \\
\hline Methyl chloride & WA & & & $\mathbf{U}$ & & 10 & & NS & $\mu g / L$ \\
\hline Methyl ethyl ketone & WA & & & $\mathbf{U}$ & & 10 & & NS & $\mu g / L$ \\
\hline Methyl iodide & WA & & & $\mathbf{U}$ & & 10 & & NS & $\mu \mathrm{g} / \mathrm{L}$ \\
\hline Methylene bromide & WA & & & $\mathbf{U}$ & & 10 & & NS & $\mu g / L$ \\
\hline Methylene chloride & WA & & & $\mathbf{J}$ & v & 3.73 & & 5 & $\mu g / L$ \\
\hline Nickel & WA & & & U & & 40 & & 100 & $\mu g / L$ \\
\hline Nitrate as nitrogen & WA & & & $\mathbf{J}$ & $\mathbf{Q}$ & 0.225 & & 10 & $\mathrm{mg} / \mathrm{L}$ \\
\hline Nonvolatile beta & TM & & & & & 4.6 & 2.8 & 50 & $\mathrm{pCi} / \mathrm{L}$ \\
\hline Potassium & WA & & & $\mathbf{U}$ & & 5000 & & NS & $\mu \mathrm{g} / \mathrm{L}$ \\
\hline Propionitrile & WA & & & $\mathbf{U}$ & & 50 & & NS & $\mu \mathrm{g} / \mathrm{L}$ \\
\hline Selenium & WA & & & $\mathbf{U}$ & & 5 & & 50 & $\mu \mathrm{g} / \mathrm{L}$ \\
\hline Silver & WA & & & $\mathbf{U}$ & & 10 & & 50 & $\mu g / L$ \\
\hline Sodium & WA & & & & & 18300 & & NS & $\mu g / L$ \\
\hline Styrene & WA & & & $\mathbf{U}$ & & 5 & & 100 & $\mu g / L$ \\
\hline Sulfate & WA & & & $\mathbf{U}$ & & 2.5 & & 400 & $\mathrm{mg} / \mathrm{L}$ \\
\hline Sulfide & WA & & & $\mathbf{U}$ & & 0.1 & & NS & $\mathrm{mg} / \mathrm{h}$ \\
\hline Tetrachloroethylene & WA & & & U & & 5 & & 5 & $\mu \mathrm{g} / \mathrm{L}$ \\
\hline Thallium & WA & & & $\mathbf{U}$ & & 10 & & 2 & $\mu \mathrm{g} /$ \\
\hline Toluene & WA & & & $\mathbf{U}$ & & 5 & & 1000 & $\mu g / L$ \\
\hline Total hardness & WA & & & & & 6.7 & & NS & $\mathrm{mg} / \mathrm{L}$ \\
\hline Total organic carbon & WA & & & & & 3.41 & & NS & $\mathrm{mg} / \mathrm{h}$ \\
\hline Total suspended solids & WA & & & & & 33 & & NS & $\mathrm{mg} / \mathrm{L}$ \\
\hline trans-1,3-Dichloropropene & WA & & & $\mathbf{U}$ & & 5 & & NS & $\mu g / L$ \\
\hline trans-1,4-Dichloro-2-butene & WA & & & $\mathbf{U}$ & & 100 & & NS & $\mu \mathrm{g} / \mathrm{L}$ \\
\hline Trichloroethylene & WA & & & $\mathbf{U}$ & & 5 & & 5 & $\mu g / L$ \\
\hline Trichlorofluoromethane & WA & & & $\mathbf{U}$ & & 5 & & NS & $\mu g$ \\
\hline Tritium & TM & & & & & 28.2 & 1.2 & 20 & $\mathrm{pCi} / \mathrm{ml}$ \\
\hline Turbidity & WA & & & $\mathbf{J}$ & $\mathbf{Q}$ & 11.9 & & NS & NTU \\
\hline Turbidity & WA & & NA & $\mathbf{J}$ & Q & 11.8 & & NS & NTU \\
\hline Vanadium & WA & & & $\mathrm{U}$ & & 50 & & NS & $\mu \mathrm{g} / \mathrm{L}$ \\
\hline Vinyl acetate & WA & & & $\mathrm{U}$ & & 10 & & NS & $\mu \mathrm{g}$ \\
\hline Vinyl chloride & WA & & & U & & 10 & & 2 & $\mu g / L$ \\
\hline Xylenes (total). & WA & & & U & & 5 & & 10000 & $\mu g /$ \\
\hline Zinc & WA & & & & & 24.6 & & 5000 & 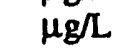 \\
\hline
\end{tabular}




\section{UTD006}

Field Measurements:

Stream Discharge: $0.05 \mathrm{cfs}$

Sample Date: 9/1/93

pH: 7.04

Specific Conductance: $79 \mu \mathrm{S} / \mathrm{cm}$

Red/Oxid Potential: $25 \mathrm{mv}$

Dissolved Oxygen: $3.2 \mathrm{mg} / \mathrm{L}$

\begin{tabular}{|c|c|c|c|c|c|c|c|}
\hline Analyte & Lab & Rep & Modifier & Result & Acc & Stand & Units \\
\hline 1,1,1,2-Tetrachloroethane & WA & & $\mathbf{U}$ & 10 & & NS & $\mu g / L$ \\
\hline 1,1,1-Trichloroethane & WA & & $\mathbf{U}$ & 5 & & 200 & $\mu g / L$ \\
\hline 1,1,2,2-Tetrachloroethane & WA & & $\mathbf{U}$ & 5 & & NS & $\mu g / L$ \\
\hline 1,1,2-Trichloroethane & WA & & $\mathbf{U}$ & 5 & & 5 & $\mu g / L$ \\
\hline 1,1-Dichloroethane & WA & & $\mathbf{U}$ & 5 & & NS & $\mu g / L$ \\
\hline 1,1-Dichloroethylene & WA & & U & 5 & & 7 & $\mu g / L$ \\
\hline 1,2,3-Trichloropropane & WA & & $\mathbf{U}$ & 10 & & NS & $\mu g / L$ \\
\hline 1,2-Dibromo-3-chloropropane & WA & & $\mathbf{U}$ & 20 & & 0.2 & $\mu g / L$ \\
\hline 1,2-Dibromoethane & WA & & $\mathbf{U}$ & 20 & & NS & $\mu g / L$ \\
\hline 1,2-Dichloroethane & WA & & $\mathbf{U}$ & 5 & & 5 & \\
\hline 1,2-Dichloroethylene (total) & WA & & $\mathrm{U}$ & 5 & & 7 & $\mu g / L$ \\
\hline 1,2-Dichloropropane & WA & & U & 5 & & 5 & $\mu g / L$ \\
\hline 2-Hexanone & WA & & $\mathbf{U}$ & 10 & & NS & $\mu g / L$ \\
\hline 4-Methyl-2-pentanone & WA & & $\mathbf{U}$ & 10 & & NS & $\mu g / L$ \\
\hline Acetone & WA & & & 18.2 & & NS & $\mu g / L$ \\
\hline Acetonitrile & WA & & U & 20 & & NS & $\mu g / L$ \\
\hline Acidity & WA & & & 84 & & NS & $\mathrm{mg} / \mathrm{L}$ \\
\hline Acrolein & WA & & $\mathbf{U}$ & 10 & & NS & $\mu g / L$ \\
\hline Acrylonitrile & WA & & U & 10 & & NS & $\mu g / L$ \\
\hline Alkalinity & WA & & & 24 & & NS & $\mathrm{mg} / \mathrm{L}$ \\
\hline Allyl chloride & WA & & $\mathbf{U}$ & 100 & & NS & $\mu g / L$ \\
\hline Aluminum & WA & & & 214 & & NS & $\mu g / L$ \\
\hline Antimony & WA & & $\mathbf{U}$ & 60 & & 6 & $\mu g / L$ \\
\hline Arsenic & WA & & $\mathbf{U}$ & 10 & & 50 & $\mu g / L$ \\
\hline Barium & WA & & U & 200 & & 2000 & $\mu g / L$ \\
\hline Benzene & WA & & $\mathbf{U}$ & 5 & & 5 & $\mu \mathrm{g} / \mathrm{L}$ \\
\hline Beryllium & WA & & $\mathrm{U}$ & 5 & & 4 & $\mu g / L$ \\
\hline Bromodichloromethane & WA & & U & 5 & & NS & $\mu g / L$ \\
\hline Bromoform & WA & & $\mathbf{U}$ & 5 & & NS & $\mu g / L$ \\
\hline Cadmium & WA & & $\mathbf{U}$ & 5 & & 5 & $\mu g / L$ \\
\hline Calcium & WA & & $\mathbf{U}$ & 5000 & & NS & $\mu \mathrm{g} / \mathrm{L}$ \\
\hline Carbon disulfide & WA & & $\mathbf{U}$ & 5 & & NS & $\mu g / L$ \\
\hline Carbon tetrachloride & WA & & $\mathbf{U}$ & 5 & & 5 & $\mu g / L$ \\
\hline Cesium-137 & TM & & $\mathbf{U}$ & 9.7 & & 200 & $\mathrm{pCi} / \mathrm{L}$ \\
\hline Chloride & WA & & & 9.28 & & NS & $\mathrm{mg} / \mathrm{L}$ \\
\hline Chlorobenzene & WA & & $\mathbf{U}$ & 5 & & 100 & $\mu g / L$ \\
\hline Chloroethane & WA & & $\mathbf{U}$ & 10 & & NS & $\mu g / L$ \\
\hline Chloroform & WA & & $\mathbf{U}$ & 5 & & 100 & $\mu g / L$ \\
\hline Chloroprene & WA & & $\mathbf{U}$ & 20 & & NS & $\mu \mathrm{g} / \mathrm{L}$ \\
\hline Chromium & WA & & $\mathbf{U}$ & 10 & & 100 & $\mu g / L$ \\
\hline cis-1,3-Dichloropropene & WA & & $\mathbf{U}$ & 5 & & NS & $\mu \mathrm{g} / \mathrm{L}$ \\
\hline Cobalt & WA & & $\mathbf{U}$ & 50 & & NS & $\mu g / L$ \\
\hline Copper & WA & & $\mathbf{U}$ & 25 & & 1000 & $\mu \mathrm{g} / \mathrm{L}$ \\
\hline Dibromochloromethane & WA & & $\mathbf{U}$ & 5 & & NS & $\mu g / L$ \\
\hline Dichlorodifluoromethane & WA & & $\mathbf{U}$ & 10 & & NS & $\mu g / L$ \\
\hline Ethylbenzene & WA & & $\mathbf{U}$ & 5 & & 700 & $\mu_{\varepsilon}$ \\
\hline
\end{tabular}


UTD006 Laboratory Analyses (cont'd)

\begin{tabular}{|c|c|c|c|c|c|c|c|c|c|}
\hline Analyte & Lab & Rep & Avg & Modifier & & Result & Acc & Stand & Units \\
\hline Fluoride & $\overline{\text { WA }}$ & & & $\bar{U}$ & & 0.1 & & 4 & $\mathrm{mg} / \mathrm{L}$ \\
\hline Fluoride & WA & & NA & $\mathbf{U}$ & & 0.1 & & 4 & $\mathrm{mg} / \mathrm{L}$ \\
\hline Gross alpha & TM & & & & & 0.8 & 1.2 & 15 & $\mathrm{pCi} / \mathrm{L}$ \\
\hline & WA & & & & & 4770 & & 300 & $\mu g / L$ \\
\hline Isobutyl alcohol & WA & & & $\mathbf{U}$ & & 20 & & NS & $\mu_{g} / \mathrm{L}$ \\
\hline Lead & WA & & & $\mathbf{U}$ & & 3 & & 50 & $\mu \mathrm{g} / \mathrm{L}$ \\
\hline Magnesium & WA & & & $\mathbf{U}$ & & 5000 & & NS & $\mu g / L$ \\
\hline Manganese & WA & & & & & 103 & & 50 & $\mu g / L$ \\
\hline Mercury & WA & & & $\mathbf{U}$ & & 0.2 & & 2 & $\mu_{g} / \mathrm{L}$ \\
\hline Methacrylonitrile & WA & & & $\mathbf{U}$ & & 20 & & NS & $\mu g / L$ \\
\hline Methyl bromide & WA & & & $\mathbf{U}$ & & 10 & & NS & $\mu g / L$ \\
\hline Methyl chloride & WA & & & $\mathbf{U}$ & & 10 & & NS & $\mu g / L$ \\
\hline Methyl ethyl ketone & WA & & & $\mathbf{U}$ & & 10 & & NS & $\mu g / L$ \\
\hline Methyl iodide & WA & & & $\mathbf{U}$ & & 10 & & NS & $\mu g / L$ \\
\hline Methylene bromide & WA & & & $\mathbf{U}$ & & 10 & & NS & $\mu g / L$ \\
\hline Methylene chloride & WA & & & J & V & 3.53 & & 5 & $\mu g / \Omega$ \\
\hline Nickel & WA & & & $\mathbf{U}$ & & 40 & & 100 & $\mu g / L$ \\
\hline Nitrate as nitrogen & WA & & & $\mathbf{J}$ & $\mathbf{Q}$ & 0.103 & & 10 & $\mathrm{mg} / \mathrm{L}$ \\
\hline Nonvolatile beta & TM & & & & & 3 & 2.6 & 50 & pCi/L \\
\hline Potassium & WA & & & $\mathbf{U}$ & & 5000 & & NS & $\mu g / L$ \\
\hline Propionitrile & WA & & & $\mathbf{U}$ & & 50 & & NS & $\mu \mathrm{g} / \mathrm{L}$ \\
\hline Selenium & WA & & & $\mathbf{U}$ & & 5 & & 50 & $\mu g / L$ \\
\hline Silver & WA & & & $\mathbf{U}$ & & 10 & & 50 & $\mu g / L$ \\
\hline Sodium & WA & & & & & 14300 & & NS & $\mu g / \Lambda$ \\
\hline Styrene & WA & & & $\mathbf{U}$ & & 5 & & 100 & $\mu g / L$ \\
\hline Sulfate & WA & & & & & 4.18 & & 400 & $\mathrm{mg} / \mathrm{L}$ \\
\hline Sulfide & WA & & & $\mathbf{U}$ & & 0.1 & & NS & $\mathrm{mg} / \mathrm{L}$ \\
\hline Tetrachloroethylene & WA & & & $\mathbf{U}$ & & 5 & & 5 & $\mu g / L$ \\
\hline Thallium & WA & & & $\mathbf{U}$ & & 10 & & 2 & $\mu \mathrm{g} / \mathrm{L}$ \\
\hline Toluene & WA & & & $\mathbf{U}$ & & 5 & & 1000 & $\mu g / L$ \\
\hline Total hardness & WA & & & & & 13.4 & & NS & $\mathrm{mg} / \mathrm{L}$ \\
\hline Total organic carbon & WA & & $\mathbf{A}$ & & & 3.32 & & NS & $\mathrm{mg} / \mathrm{L}$ \\
\hline Total suspended solids & WA & & & & & 25 & & NS & $\mathrm{mg} / \mathrm{L}$ \\
\hline trans-1,3-Dichloropropene & WA & & & $\mathbf{U}$ & & 5 & & NS & $\mu \mathrm{g}$ \\
\hline trans-1,4-Dichloro-2-butene & WA & & & $\mathbf{U}$ & & 100 & & NS & $\mu g$ \\
\hline Trichloroethylene & WA & & & $\mathbf{U}$ & & 5 & & 5 & $\mu \mathrm{g}$ \\
\hline Trichlorofluoromethane & WA & & & $\mathrm{U}$ & & 5 & & NS & $\mu_{g}$ \\
\hline Tritium & TM & & & & & 26.76 & 1.16 & 20 & $\mathrm{pCi} / \mathrm{ml}$ \\
\hline Turbidity & WA & & & $\mathbf{J}$ & $\mathbf{Q}$ & 10.2 & & NS & NT \\
\hline Vanadium & WA & & & $\mathbf{U}$ & & 50 & & NS & $\mu$ \\
\hline Vinyl acetate & WA & & & $\mathbf{U}$ & & 10 & & NS & $\mu \mathrm{g}$ \\
\hline Vinyl chloride & WA & & & U & & 10 & & 2 & $\mu_{\mathrm{g}}$ \\
\hline Xylenes (total) & WA & & & U & & 5 & & 10000 & \\
\hline Zinc & WA & & & U & & 20 & & 5000 & $\mu g$ \\
\hline
\end{tabular}




\section{UTR011}

Field Measurements:

Sample Date: 8/24/93

pH: 5.03

Specific Conductance: $16 \mu \mathrm{S} / \mathrm{cm}$

Red/Oxid Potential: $110 \mathrm{mv}$

Dissolved Oxygen: $1.9 \mathrm{mg} / \mathrm{L}$

\begin{tabular}{|c|c|c|c|c|c|c|c|c|}
\hline Analyte & Lab & Rep & Avg & Modifier & Result & Acc & Stand & Units \\
\hline 1,1,1,2-Tetrachloroethane & WA & & & $\mathbf{U}$ & 10 & & NS & $\mu g / L$ \\
\hline 1,1,1,2-Tetrachloroethane & WA & $\mathbf{R}$ & & $\mathbf{U}$ & 10 & & NS & $\mu g / L$ \\
\hline 1,1,1-Trichloroethane & WA & & & $U$ & 5 & & 200 & $\mu g / L$ \\
\hline 1,1,1-Trichloroethane & WA & $\mathbf{R}$ & & $\mathbf{U}$ & 5 & & 200 & $\boldsymbol{\mu} \boldsymbol{g} \mathbf{L}$ \\
\hline 1,1,2,2-Tetrachloroethane & WA & & & U & 5 & & NS & $\mu g / L$ \\
\hline 1,1,2,2-Tetrachloroethane & WA & $\mathbf{R}$ & & $\mathbf{U}$ & 5 & & NS & $\mu g / L$ \\
\hline 1,1,2-Trichloroethane & WA & & & $\mathbf{U}$ & 5 & & 5 & $\mu g / L$ \\
\hline 1,1,2-Trichloroethane & WA & $\mathbf{R}$ & & $\mathbf{U}$ & 5 & & 5 & $\mu g / L$ \\
\hline 1,1-Dichloroethane & WA & & & $\mathbf{U}$ & 5 & & NS & $\mu g / L$ \\
\hline 1,1-Dichloroethane & WA & $\mathbf{R}$ & & $\mathbf{U}$ & 5 & & NS & $\mu \boldsymbol{g} / \mathbf{L}$ \\
\hline 1,1-Dichloroethylene & WA & & & $\mathbf{U}$ & 5 & & 7 & $\mu g / L$ \\
\hline 1,1-Dichloroethylene & WA & $\mathbf{R}$ & & $\mathbf{U}$ & 5 & & 7 & $\mu g / L$ \\
\hline 1,2,3-Trichloropropane & WA & & & $\mathbf{U}$ & 10 & & NS & $\mu g / L$ \\
\hline 1,2,3-Trichloropropane & WA & $\mathbf{R}$ & & $\mathbf{U}$ & 10 & & NS & $\mu g / L$ \\
\hline 1,2-Dibromo-3-chloropropane & WA & & & $\mathbf{U}$ & 20 & & 0.2 & $\mu g / L$ \\
\hline 1,2-Dibromo-3-chloropropane & WA & $\mathbf{R}$ & & $\mathbf{U}$ & 20 & & 0.2 & $\mu g / L$ \\
\hline 1,2-Dibromoethane & WA & & & $\mathbf{U}$ & 20 & & NS & $\mu g / L$ \\
\hline 1,2-Dibromoethane & WA & $\mathbf{R}$ & & $\mathbf{U}$ & 20 & & NS & $\mu g / L$ \\
\hline 1,2-Dichloroethane & WA & & & $\mathbf{U}$ & 5 & & 5 & $\mu g / L$ \\
\hline 1,2-Dichloroethane & WA & $\mathbf{R}$ & & $\mathbf{U}$ & 5 & & 5 & $\mu g / L$ \\
\hline 1,2-Dichloroethylene (total) & WA & & & $\mathbf{U}$ & 5 & & 7 & $\mu g / L$ \\
\hline 1,2-Dichloroethylene (total) & WA & $\mathbf{R}$ & & U & 5 & & 7 & $\mu g / L$ \\
\hline 1,2-Dichloropropane & WA & & & $\mathbf{U}$ & 5 & & 5 & $\mu \mathrm{g} / \mathrm{L}$ \\
\hline 1,2-Dichloropropane & WA & $\mathbf{R}$ & & $\mathbf{U}$ & 5 & & 5 & $\mu g / L$ \\
\hline 2-Hexanone & WA & & & $\mathbf{U}$ & 10 & & NS & $\mu g / L$ \\
\hline 2-Hexanone & WA & $\mathbf{R}$ & & $\mathbf{U}$ & 10 & & NS & $\mu g / L$ \\
\hline 4-Methyl-2-pentanone & WA & & & $\mathbf{U}$ & 10 & & NS & $\mu \mathrm{g} / \mathrm{L}$ \\
\hline 4-Methyl-2-pentanone & WA & $\mathbf{R}$ & & $\mathbf{U}$ & 10 & & NS & $\mu g / L$ \\
\hline Acetone & WA & & & $\mathbf{J}$ & 6.3 & & NS & $\mu g / L$ \\
\hline Acetone & WA & $\mathbf{R}$ & & J & 9.11 & & NS & $\mu g / L$ \\
\hline Acetonitrile & WA & & & $\mathbf{U}$ & 20 & & NS & $\mu g / L$ \\
\hline Acetonitrile & WA & $\mathbf{R}$ & & $\mathbf{U}$ & 20 & & NS & $\mu \boldsymbol{g} / \mathbf{L}$ \\
\hline Acidity & WA & & $\mathbf{A}$ & & 109 & & NS & $\mathrm{mg} / \mathrm{L}$ \\
\hline Acidity & WA & $\mathbf{R}$ & & & 40 & & NS & $\mathbf{m g} / \mathbf{L}$ \\
\hline Acrolein & WA & & & $\mathbf{U}$ & 10 & & NS & $\mu g / L$ \\
\hline Acrolein & WA & $\mathbf{R}$ & & $\mathbf{U}$ & 10 & & NS & $\mu g / L$ \\
\hline Acrylonitrile & WA & & & $\mathbf{U}$ & 10 & & NS & $\mu g / L$ \\
\hline Acrylonitrile & WA & $\mathbf{R}$ & & $\mathrm{U}$ & 10 & & NS & $\mu g / L$ \\
\hline Allyl chloride & WA & & & $\mathbf{U}$ & 100 & & NS & $\mu g / L$ \\
\hline Allyl chloride & WA & $\mathbf{R}$ & & $\mathbf{U}$ & 100 & & NS & $\mu g / L$ \\
\hline Aluminum & WA & & & $\mathbf{U}$ & 200 & & NS & $\mu \mathrm{g} / \mathrm{L}$ \\
\hline Aluminum & WA & $\mathbf{R}$ & & U & 200 & & NS & $\mu g / L$ \\
\hline Antimony & WA & & & $\mathbf{U}$ & 60 & & 6 & $\mu \mathrm{g} / \mathrm{L}$ \\
\hline Antimony & WA & $\mathbf{R}$ & & $\mathbf{U}$ & 60 & & 6 & $\mu g / L$ \\
\hline Arsenic & WA & & & $\mathbf{U}$ & 10 & & 50 & $\mu g / L$ \\
\hline Arsenic & WA & $\mathbf{R}$ & & $\mathbf{U}$ & 10 & & 50 & $\mu g / L$ \\
\hline
\end{tabular}


UTR011 Laboratory Analyses (cont'd)

\begin{tabular}{|c|c|c|c|c|c|c|c|c|}
\hline Analyte & $\underset{\text { WA }}{\text { Lab }}$ & Rep & Avg & $\underset{\mathbf{U}}{\text { Modifier }}$ & $\begin{array}{c}\text { Result } \\
200\end{array}$ & Acc & $\begin{array}{l}\text { Stand } \\
2000\end{array}$ & $\begin{array}{l}\text { Units } \\
\mu g / L\end{array}$ \\
\hline Barium & WA & $\mathbf{R}$ & & $\mathbf{U}$ & 200 & & 2000 & $\mu g / L$ \\
\hline Benzene & WA & & & $\mathbf{U}$ & 5 & & 5 & $\mu g / L$ \\
\hline Benzene & WA & $\mathbf{R}$ & & $\mathbf{U}$ & 5 & & 5 & $\mu g / L$ \\
\hline Beryllium & WA & & & $\mathbf{U}$ & 5 & & 4 & $\mu \mathrm{g} / \mathrm{L}$ \\
\hline Beryllium & WA & $\mathbf{R}$ & & $\mathbf{U}$ & 5 & . & 4 & $\mu_{g} / L$ \\
\hline Bromodichloromethane & WA & & & $\mathbf{U}$ & 5 & & NS & $\mu g / L$ \\
\hline Bromodichloromethane & WA & $\mathbf{R}$ & & $\mathbf{U}$ & 5 & & NS & $\mu_{g} / L$ \\
\hline Bromoform & WA & & & $\mathbf{U}$ & 5 & & NS & $\mu_{g} / L$ \\
\hline Bromoform & WA & $\mathbf{R}$ & & $\mathbf{U}$ & 5 & & NS & $\mu g / L$ \\
\hline Cadmium & WA & & & U & 5 & & 5 & $\mu g / L$ \\
\hline Cadmium & WA & $\mathbf{R}$ & & $\mathbf{U}$ & 5 & & 5 & $\mu g / L$ \\
\hline Calcium & WA & & & $\mathbf{U}$ & 5000 & & NS & $\mu g / L$ \\
\hline Calcium & WA & $\mathbf{R}$ & & $\mathbf{U}$ & 5000 & & NS & $\mu g / L$ \\
\hline Carbon disulfide & WA & & & $\mathbf{U}$ & 5 & & NS & $\mu g / L$ \\
\hline Carbon disulfide & WA & $\mathbf{R}$ & & $\mathbf{U}$ & 5 & & NS & $\mu g / L$ \\
\hline Carbon tetrachloride & WA & & & $\mathbf{U}$ & 5 & & 5 & $\mu g / L$ \\
\hline Carbon tetrachloride & WA & $\mathbf{R}$ & & $\mathbf{U}$ & 5 & & 5 & $\mu g / L$ \\
\hline Chloride & WA & & & & 4.46 & & NS & $\mathrm{mg} / \mathrm{L}$ \\
\hline Chloride & WA & $\mathbf{R}$ & & & 4.63 & & NS & $\mathrm{mg} / \mathrm{L}$ \\
\hline Chlorobenzene & WA & & & $\mathbf{U}$ & 5 & & 100 & $\mu g / L$ \\
\hline Chlorobenzene & WA & $\mathbf{R}$ & & $\mathbf{U}$ & 5 & & 100 & $\mu g / L$ \\
\hline Chloroethane & WA & & & $\mathbf{U}$ & 10 & & NS & $\mu g / L$ \\
\hline Chloroethane & WA & $\mathbf{R}$ & & $\mathbf{U}$ & 10 & & NS & $\mu g / L$ \\
\hline Chloroform & WA & & & $\mathbf{U}$ & 5 & & 100 & $\mu g / L$ \\
\hline Chloroform & WA & $\mathbf{R}$ & & $\mathbf{U}$ & 5 & & 100 & $\mu \mathrm{g} / \mathrm{L}$ \\
\hline Chloroprene & WA & & & $\mathbf{U}$ & 20 & & NS & $\mu g / L$ \\
\hline Chloroprene & WA & $\mathbf{R}$ & & $\mathbf{U}$ & 20 & & NS & $\mu \mathrm{g} / \mathrm{L}$ \\
\hline Chromium & WA & & & $\mathbf{U}$ & 10 & & 100 & $\mu g / L$ \\
\hline Chromium & WA & $\mathbf{R}$ & & $\mathbf{U}$ & 10 & & 100 & $\mu g / L$ \\
\hline cis-1,3-Dichloropropene & WA & & & $\mathbf{U}$ & 5 & & NS & $\mu g / L$ \\
\hline cis-1,3-Dichloropropene & WA & $\mathbf{R}$ & & $\mathbf{U}$ & 5 & & NS & $\mu \mathrm{g} / \mathrm{L}$ \\
\hline Cobalt & WA & & & $\mathbf{U}$ & 50 & & NS & $\mu g / L$ \\
\hline Cobalt & WA & $\mathbf{R}$ & & $\mathbf{U}$ & 50 & & NS & $\mu g / L$ \\
\hline Copper & WA & & & $\mathbf{U}$ & 25 & & 1000 & $\mu g / L$ \\
\hline Copper & WA & $\mathbf{R}$ & & $\mathbf{U}$ & 25 & & 1000 & $\mu g / L$ \\
\hline Dibromochloromethane & WA & & & $\mathbf{U}$ & 5 & & NS & $\mu g / L$ \\
\hline Dibromochloromethane & WA & $\mathbf{R}$ & & $\mathbf{U}$ & 5 & & NS & $\mu g / L$ \\
\hline Dichlorodifluoromethane & WA & & & U & 10 & & NS & $\mu g / L$ \\
\hline Dichlorodifluoromethane & WA & $\mathbf{R}$ & & $\mathbf{U}$ & 10 & & NS & $\mu g / L$ \\
\hline Ethylbenzene & WA & & & $\mathbf{U}$ & 5 & & 700 & $\mu g / L$ \\
\hline Ethylbenzene & WA & $\mathbf{R}$ & & $\mathbf{U}$ & 5 & & 700 & $\mu \mathrm{g} / \mathrm{L}$ \\
\hline Fluoride & WA & & & $\mathbf{U}$ & 0.1 & & 4 & $\mathrm{mg} / \mathrm{L}$ \\
\hline Fluoride & WA & $\mathbf{R}$ & & $\mathbf{U}$ & 0.1 & & 4 & $\mathrm{mg} / \mathrm{L}$ \\
\hline Gross alpha & TM & & $\mathbf{A}$ & & 1.8 & 1.1 & 15 & $\mathrm{pCi} / \mathrm{L}$ \\
\hline Gross alpha & TM & $\mathbf{R}$ & & & 2.3 & 1.5 & 15 & $\mathrm{pCi} / 2$ \\
\hline Iron & WA & & & & 722 & & 300 & $\mu g / L$ \\
\hline Iron & WA & $\mathbf{R}$ & & & 915 & & 300 & $\mu g / L$ \\
\hline Isobutyl alcohol & WA & & & $\mathbf{U}$ & 20 & & NS & $\mu g / L$ \\
\hline Isobutyl alcohol & WA & $\mathbf{R}$ & & $\mathbf{U}$ & 20 & & NS & $\mu g / L$ \\
\hline Lead & WA & & & $\mathbf{U}$ & 3 & & 50 & $\mu g / L$ \\
\hline Lead & WA & $\mathbf{R}$ & & $\mathbf{U}$ & 3 & & 50 & $\mu g / L$ \\
\hline Magnesium & WA & & & $\mathbf{U}$ & 5000 & & NS & $\mu g / L$ \\
\hline Magnesium & WA & $\mathbf{R}$ & & $\mathbf{U}$ & 5000 & & NS & $\mu g / L$ \\
\hline
\end{tabular}


UTR011 Laboratory Analyses (cont'd)

\begin{tabular}{|c|c|c|c|c|c|c|c|c|c|}
\hline Analyte & Lab & Rep & Avg & Modifier & & Result & Acc & Stand & Units \\
\hline Manganese & $\overline{\text { WA }}$ & & & $\bar{U}$ & & 15 & & 50 & $\mu \mathrm{g} / \mathrm{L}$ \\
\hline Manganese & WA & $\mathbf{R}$ & & & & 15.4 & & 50 & $\mu g / L$ \\
\hline Mercury & WA & & & $\mathbf{U}$ & & 0.2 & & 2 & $\mu \mathrm{g} / \mathrm{L}$ \\
\hline Mercury & WA & & NA & $\mathbf{U}$ & & 0.2 & & 2 & $\mu g / L$ \\
\hline Mercury & WA & $\mathbf{R}$ & & $\mathbf{U}$ & & 0.2 & & 2 & $\mu g / L$ \\
\hline Methacrylonitrile & WA & & & U & & 20 & & NS & $\mu g / L$ \\
\hline Methacrylonitrile & WA & $\mathbf{R}$ & & U & & 20 & • & NS & $\mu g / L$ \\
\hline Methyl bromide & WA & & & U & & 10 & & NS & $\mu g / \Omega$ \\
\hline Methyl bromide & WA & $\mathbf{R}$ & & $\mathbf{U}$ & & 10 & & NS & $\mu g / L$ \\
\hline Methyl chloride & WA & & & $\mathbf{U}$ & & 10 & & NS & $\mu g / \Omega$ \\
\hline Methyl chloride & WA & $\mathbf{R}$ & & $\mathbf{U}$ & & 10 & & NS & $\mu g / L$ \\
\hline Methyl ethyl ketone & WA & & & U & & 10 & & NS & $\mu g \Omega$ \\
\hline Methyl ethyl ketone & WA & $\mathbf{R}$ & & U & & 10 & & NS & $\mu g / \Omega$ \\
\hline Methyl iodide & WA & & & $\mathbf{U}$ & & 10 & & NS & $\mu g / L$ \\
\hline Methyl iodide & WA & $\mathbf{R}$ & & $\mathbf{U}$ & & 10 & & NS & $\mu g / L$ \\
\hline Methylene bromide & WA & & . & $\mathbf{U}$ & & 10 & & NS & $\mu g / L$ \\
\hline Methylene bromide & WA & $\mathbf{R}$ & & $\mathbf{U}$ & & 10 & & NS & $\mu g / L$ \\
\hline Methylene chloride & WA & & & $\mathbf{J}$ & V & 4.11 & & 5 & $\mu g / 2$ \\
\hline Methylene chloride & WA & $\mathbf{R}$ & & J & V & 3.73 & & 5 & $\mu g / L$ \\
\hline Nickel & WA & & & $\mathbf{U}$ & & 40 & & 100 & $\mu g / L$ \\
\hline Nickel & WA & $\mathbf{R}$ & & U & & 40 & & 100 & $\mu g /$ \\
\hline Nitrate as nitrogen & WA & & & $\mathbf{J}$ & $\mathbf{Q}$ & 0.186 & & 10 & $\mathrm{mg} / \mathrm{L}$ \\
\hline Nitrate as nitrogen & WA & $\mathbf{R}$ & & J & $\mathbf{Q}$ & 0.196 & & 10 & $\mathrm{mg} / \mathrm{L}$ \\
\hline Nonvolatile beta & $\mathrm{TM}$ & & & & & 2.6 & 1.7 & 50 & $\mathrm{pCi} / \mathrm{L}$ \\
\hline Nonvolatile beta & TM & $\mathbf{R}$ & & & & 2.3 & 1.7 & 50 & $\mathrm{pCi} / \mathrm{L}$ \\
\hline Potassium & WA & & & $\mathbf{U}$ & & 5000 & & NS & $\mu g / L$ \\
\hline Potassium & WA & $\mathbf{R}$ & & $\mathrm{U}$ & & 5000 & & NS & $\mu g /$ \\
\hline Propionitrile & WA & & & $\mathrm{U}$ & & 50 & & NS & $\mu \mathrm{g} / \mathrm{L}$ \\
\hline Propionitrile & WA & $\mathbf{R}$ & & $\mathbf{U}$ & & 50 & & NS & $\mu g /$ \\
\hline Selenium & WA & & & $\mathbf{U}$ & & 5 & & 50 & $\mu g / L$ \\
\hline Selenium & WA & $\mathbf{R}$ & & $\mathbf{U}$ & & 5 & & 50 & $\mu g / L$ \\
\hline Silver & WA & & & $\mathbf{U}$ & & 10 & & 50 & $\mu \mathrm{g} / \mathrm{L}$ \\
\hline Silver & WA & $\mathbf{R}$ & & $\mathbf{U}$ & & 10 & & 50 & $\mu g /$ \\
\hline Sodium & WA & & & U & & 5000 & & NS & $\mu g / L$ \\
\hline Sodium & WA & $\mathbf{R}$ & & $\mathbf{U}$ & & 5000 & & NS & $\mu g / L$ \\
\hline Styrene & WA & & & $\mathbf{U}$ & & 5 & & 100 & $\mu g / L$ \\
\hline Styrene & WA & $\mathbf{R}$ & & $\mathbf{U}$ & & 5 & & 100 & $\mu g / L$ \\
\hline Sulfate & WA & & & & & 2.66 & & 400 & $\mathrm{mg} / \mathrm{L}$ \\
\hline Sulfate & WA & $\mathbf{R}$ & & $\mathbf{U}$ & & 2.5 & & 400 & $\mathrm{mg} / \mathrm{L}$ \\
\hline Sulfide & WA & & & $\mathbf{U}$ & & 0.1 & & NS & $\mathrm{mg} \Omega$ \\
\hline Sulfide & WA & & NA & $\mathbf{U}$ & & 0.1 & & NS & $\mathrm{mg} / \mathrm{L}$ \\
\hline Sulfide & WA & $\mathbf{R}$ & & $\mathbf{U}$ & & 0.1 & & NS & $\mathrm{mg} / \mathrm{L}$ \\
\hline Tetrachloroethylene & WA & & & $\mathbf{U}$ & & 5 & & 5 & $\mu g / L$ \\
\hline Tetrachloroethylene & WA & $\mathbf{R}$ & & $\mathbf{U}$ & & 5 & & 5 & $\mu g / L$ \\
\hline Thallium & WA & & & $\mathbf{U}$ & & 10 & & 2 & $\mu g / L$ \\
\hline Thallium & WA & $\mathbf{R}$ & & 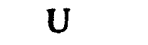 & & 10 & & 2 & $\mu g / L$ \\
\hline Toluene & WA & & & $\mathbf{J}$ & & 1.88 & & 1000 & $\mu g / L$ \\
\hline Toluene & WA & $\mathbf{R}$ & & & & 5.08 & & 1000 & \\
\hline Total hardness & WA & & & U & & 1 & & NS & $\mathrm{mg} / \mathrm{L}$ \\
\hline Total hardness & WA & & NA & $\mathbf{U}$ & & 1 & & NS & $\mathrm{mg} / \mathrm{L}$ \\
\hline Total hardness & WA & $\mathbf{R}$ & & & & 4.9 & & NS & mg \\
\hline Total organic carbon & WA & & & & & 1.72 & & NS & $\mathrm{mg} / \mathrm{L}$ \\
\hline Total organic carbon & WA & $\mathbf{R}$ & & & & 3.13 & & NS & \\
\hline Total suspended solids & WA & & & & & 14 & & NS & $\mathbf{L}$ \\
\hline Total suspended solids & WA & $\mathbf{R}$ & & & & 8 & & NS & \\
\hline
\end{tabular}


UTR011 Laboratory Analyses (cont'd)

\begin{tabular}{|c|c|c|c|c|c|c|c|c|}
\hline Analyte & Lab & $\operatorname{Rep}$ & Avg & Modifier & Result & Acc & Stand & Units \\
\hline trans-1,3-Dichloropropene & WA & & & $\mathbf{U}$ & 5 & & NS & $\mu g / L$ \\
\hline trans-1,3-Dichloropropene & WA & $\mathbf{R}$ & & $\mathbf{U}$ & 5 & & NS & $\mu g / L$ \\
\hline trans-1,4-Dichloro-2-butene & WA & & & $\mathbf{U}$ & 100 & & NS & $\mu g / L$ \\
\hline trans-1,4-Dichloro-2-butene & WA & $\mathbf{R}$ & & $\mathbf{U}$ & 100 & & NS & $\mu g / L$ \\
\hline Trichloroethylene & WA & & & $\mathbf{U}$ & 5 & & 5 & $\mu g / L$ \\
\hline Trichloroethylene & WA & $\mathbf{R}$ & & $\mathbf{U}$ & 5 & . & 5 & $\mu g / L$ \\
\hline Trichlorofluoromethane & WA & & & $\mathbf{U}$ & 5 & & NS & $\mu g / L$ \\
\hline Trichlorofluoromethane & WA & $\mathbf{R}$ & & $\mathbf{U}$ & 5 & & NS & $\mu g / L$ \\
\hline Tritium & $\mathbf{T M}$ & $\mathbf{R}$ & & & 17.6 & 0.95 & 20 & $\mathrm{pCi} / \mathrm{ml}$ \\
\hline Tritium & $\mathrm{TM}$ & & $\mathbf{A}$ & & 18.7 & 1.0 & 20 & $\mathrm{pCi} / \mathrm{ml}$ \\
\hline Turbidity & WA & & & & 0.86 & & NS & NIU \\
\hline Turbidity & WA & $\mathbf{R}$ & & & 0.41 & & NS & NIU \\
\hline Vanadium & WA & & & $\mathbf{U}$ & 50 & & NS & $\mu_{g} / L$ \\
\hline Vanadium & WA & $\mathbf{R}$ & & $\mathbf{U}$ & 50 & & NS & $\mu_{g} / L$ \\
\hline Vinyl acetate & WA & & & $\mathbf{U}$ & 10 & & NS & $\mu g / L$ \\
\hline Vinyl acetate & WA & $\mathbf{R}$ & & $\mathbf{U}$ & 10 & & NS & $\mu g / L$ \\
\hline Vinyl chloride & WA & & & $\mathbf{U}$ & 10 & & 2 & $\mu g / L$ \\
\hline Vinyl chloride & WA & $\mathbf{R}$ & & $\mathbf{U}$ & 10 & & 2 & $\mu g / L$ \\
\hline Xylenes (total) & WA & & & $\mathbf{U}$ & 5 & & 10000 & $\mu g / L$ \\
\hline Xylenes (total) & WA & $\mathbf{R}$ & & $\mathbf{U}$ & 5 & & 10000 & $\mu g / L$ \\
\hline Zinc & WA & & & $\mathbf{U}$ & 20 & & 5000 & $\mu g /$ \\
\hline Zinc & WA & $\mathbf{R}$ & & $\mathbf{U}$ & 20 & & 5000 & $\mu g / L$ \\
\hline
\end{tabular}




\section{UTR020}

Field Measurements:

Sample Date: 8/31/93

pH: 6.78

Specific Conductance: $62 \mu \mathrm{S} / \mathrm{cm}$

Red/Oxid Potential: $176 \mathrm{mv}$

Dissolved Oxygen: $1.9 \mathrm{mg} / \mathrm{L}$

\begin{tabular}{|c|c|c|c|c|c|c|c|c|}
\hline Analyte & Lab & Rep & Avg & Modifier & Result & Acc & Stand & Units \\
\hline 1,1,1,2-Tetrachloroethane & WA & & & $\mathbf{U}$ & 10 & & NS & $\mu g / L$ \\
\hline 1,1,1-Trichloroethane & WA & & & $\mathbf{U}$ & 5 & & 200 & $\mu g / L$ \\
\hline $1,1,2,2-$ Tetrachloroethane & WA & & & $\mathbf{U}$ & 5 & & NS & $\mu g / L$ \\
\hline 1,1,2-Trichloroethane & WA & & & $\mathbf{U}$ & 5 & & 5 & $\mu g / L$ \\
\hline 1,1-Dichloroethane & WA & & & $\mathbf{U}$ & 5 & & NS & $\mu g / L$ \\
\hline 1,1-Dichloroethylene & WA & & & $\mathbf{U}$ & 5 & & 7 & $\mu g / L$ \\
\hline 1,2,3-Trichloropropane & WA & & & $\mathbf{U}$ & 10 & & NS & $\mu g / L$ \\
\hline 1,2-Dibromo-3-chloropropane & WA & & & $\mathbf{U}$ & 20 & & 0.2 & $\mu_{g} / L$ \\
\hline 1,2-Dibromoethane & WA & & & $\mathbf{U}$ & 20 & & NS & $\mu g / L$ \\
\hline 1,2-Dichloroethane & WA & & & $\mathbf{U}$ & 5 & & 5 & $\mu g / L$ \\
\hline 1,2-Dichloroethylene (total) & WA & & & $\mathbf{U}$ & 5 & & 7 & $\mu g / L$ \\
\hline 1,2-Dichloropropane & WA & & & $\mathbf{U}$ & 5 & & 5 & $\mu g / L$ \\
\hline 2-Hexanone & WA & & & $\mathbf{U}$ & 10 & & NS & $\mu g / L$ \\
\hline 4-Methyl-2-pentanone & WA & & & $\mathbf{U}$ & 10 & & NS & $\mu g / L$ \\
\hline Acetone & WA & & & & 18.4 & & NS & $\mu g / L$ \\
\hline Acetonitrile & WA & & & $\mathbf{U}$ & 20 & & NS & $\mu g / L$ \\
\hline Acidity & WA & & $\mathbf{A}$ & & 158 & & NS & $\mathrm{mg} / \mathrm{L}$ \\
\hline Acrolein & WA & & & $\mathbf{U}$ & 10 & & NS & $\mu g / L$ \\
\hline Acrylonitrile & WA & & & $\mathbf{U}$ & 10 & & NS & $\mu g / L$ \\
\hline Alkalinity & WA & & & & 6 & & NS & $\mathrm{mg} / \mathrm{L}$ \\
\hline Allyl chloride & WA & & & $\mathbf{U}$ & 100 & & NS & $\mu g / L$ \\
\hline Aluminum & WA & & $\mathbf{A}$ & & 5040 & & NS & $\mu g / L$ \\
\hline Antimony & WA & & & $\mathbf{U}$ & 60 & & 6 & $\mu g / L$ \\
\hline Antimony & WA & & NA & $\mathbf{U}$ & 60 & & 6 & $\mu g / L$ \\
\hline Arsenic & WA & & & $\mathbf{U}$ & 10 & & 50 & $\mu g / L$ \\
\hline Arsenic & WA & & NA & $\mathbf{U}$ & 10 & & 50 & $\mu g / L$ \\
\hline Barium & WA & & & $\mathbf{U}$ & 200 & & 2000 & $\mu g / L$ \\
\hline Barium & WA & & NA & $\mathbf{U}$ & 200 & & 2000 & $\mu g / L$ \\
\hline Benzene & WA & & & $\mathbf{U}$ & 5 & & 5 & $\mu g / L$ \\
\hline Beryllium & WA & & & $\mathbf{U}$ & 5 & & 4 & $\mu g / L$ \\
\hline Beryllium & WA & & NA & $\mathbf{U}$ & 5 & & 4 & $\mu g / L$ \\
\hline Bromodichloromethane & WA & & & $\mathbf{U}$ & 5 & & NS & $\mu g / L$ \\
\hline Bromoform & WA & & & $\mathbf{U}$ & 5 & & NS & $\mu g / L$ \\
\hline Cadmium & WA & & & $\mathbf{U}$ & 5 & & 5 & $\mu g / L$ \\
\hline Cadmium & WA & & NA & $\mathbf{U}$ & 5 & & 5 & $\mu \mathrm{g} / \mathrm{L}$ \\
\hline Calcium & WA & & & $\mathbf{U}$ & 5000 & & NS & $\mu \mathrm{g} / \mathrm{L}$ \\
\hline Calcium & WA & & NA & $\mathbf{U}$ & 5000 & & NS & $\mu \mathrm{g} / \mathrm{L}$ \\
\hline Carbon disulfide & WA & & & $\mathbf{U}$ & 5 & & NS & $\mu \mathrm{g} / \mathrm{L}$ \\
\hline Carbon tetrachloride & WA & & & $\mathbf{U}$ & 5 & & 5 & $\mu g / L$ \\
\hline Cesium-137 & TM & & NA & $\mathbf{U}$ & 6.1 & & 200 & $\mathrm{pCi} / \mathrm{L}$ \\
\hline Cesium-137 & TM & & & $\mathbf{U}$ & 6 & & 200 & $\mathrm{pCi} / \mathrm{L}$ \\
\hline Chloride & WA & & & & 4.29 & & NS & $\mathrm{mg} / \mathrm{L}$ \\
\hline Chlorobenzene & WA & & & $\mathbf{U}$ & 5 & & 100 & $\mu \mathrm{g} / \mathrm{L}$ \\
\hline Chloroethane & WA & & & U & 10 & & NS & $\mu \mathrm{g} / \mathrm{L}$ \\
\hline Chloroform & WA & & & $\mathbf{U}$ & 5 & & 100 & $\mu \mathrm{g} / \mathrm{L}$ \\
\hline Chloroprene & WA & & & $\mathbf{U}$ & 20 & & NS & $\mu \mathrm{g} / \mathrm{L}$ \\
\hline
\end{tabular}


UTR020 Laboratory Analyses (cont'd)

\begin{tabular}{|c|c|c|c|c|c|c|c|c|c|}
\hline Analyte & Lab & Rep & Avg & Modifier & & Result & Acc & Stand & Units \\
\hline Chromium & WA & & & $\bar{U}$ & & 10 & & 100 & $\mu g / L$ \\
\hline Chromium & WA & & NA & $\mathbf{U}$ & & 10 & & 100 & $\mu g / L$ \\
\hline cis-1,3-Dichloropropene & WA & & & U & & 5 & & NS & $\mu \mathrm{g} / \mathrm{L}$ \\
\hline Cobalt & WA & & & $\mathrm{U}$ & & 50 & & NS & $\mu \mathrm{g} / \mathrm{L}$ \\
\hline Cobalt & WA & & NA & $\mathbf{U}$ & & 50 & & NS & $\mu g / L$ \\
\hline Copper & WA & & & $\mathbf{U}$ & & 25 & & 1000 & $\mu \mathrm{g} /$ \\
\hline Copper & WA & & NA & $\mathbf{U}$ & & 25 & & 1000 & $\mu g / L$ \\
\hline Dibromochloromethane & WA & & & $\mathbf{U}$ & & 5 & & NS & $\mu g / L$ \\
\hline Dichlorodifluoromethane & WA & & & $\mathbf{U}$ & & 10 & & NS & $\mu g \Lambda$ \\
\hline Ethylbenzene & WA & & & $\mathbf{U}$ & & 5 & & 700 & $\mu \mathrm{g} / \mathrm{L}$ \\
\hline Fluoride & WA & & & $\mathbf{U}$ & & 0.1 & & 4 & $\mathrm{mg} / \mathrm{h}$ \\
\hline Gross alpha & $\mathbf{T M}$ & & NA & $\mathbf{U}$ & & 0.6 & & 15 & $\mathrm{pCi} / \mathrm{L}$ \\
\hline Gross alpha & TM & & & & & 0.7 & 1.1 & 15 & $\mathrm{pCi} / 2$ \\
\hline Iron & WA & & $\mathbf{A}$ & & & 2720 & & 300 & $\mu \mathrm{g} / \mathrm{L}$ \\
\hline Isobutyl alcohol & WA & & & $\mathbf{U}$ & & 20 & & NS & $\mu g / L$ \\
\hline Lead & WA & & & $\mathbf{U}$ & & 3 & & 50 & $\mu g /$ \\
\hline Lead & WA & & NA & & & 3.6 & & 50 & $\mu g / L$ \\
\hline Magnesium & WA & & & $\mathbf{U}$ & & 5000 & & NS & $\mu \mathrm{g} / \mathrm{L}$ \\
\hline Magnesium & WA & & NA & $\mathbf{U}$ & & 5000 & & NS & $\mu \mathrm{g} / \mathrm{h}$ \\
\hline Manganese & WA & & $\mathbf{A}$ & & & 17.9 & & 50 & $\mu g \Omega$ \\
\hline Mercury & WA & & & U & & 0.2 & & 2 & $\mu g / \mathrm{L}$ \\
\hline Mercury & WA & & NA & $\mathbf{U}$ & & 0.2 & & 2 & $\mu g / L$ \\
\hline Methacrylonitrile & WA & & & $\mathbf{U}$ & & 20 & & NS & $\mu g \Lambda$ \\
\hline Methyl bromide & WA & & & $\mathbf{U}$ & & 10 & & NS & $\mu \mathrm{g} \Omega$ \\
\hline Methyl chloride & WA & & & $\mathbf{U}$ & & 10 & & NS & $\mu \mathrm{g} / \mathrm{L}$ \\
\hline Methyl cthyl ketone & WA & & & $\mathbf{U}$ & & 10 & & NS & $\mu \mathrm{g} / \mathrm{L}$ \\
\hline Methyl iodide & WA & & & $\mathbf{U}$ & & 10 & & NS & $\mu g / L$ \\
\hline Methylene bromide & WA & & & U & & 10 & & NS & $\mu \mathrm{g} / \mathrm{L}$ \\
\hline Methylene chloride & WA & & & $\mathbf{J}$ & V & 3.68 & & 5 & $\mu g / L$ \\
\hline Nickel & WA & & & $\mathrm{U}$ & & 40 & & 100 & $\mu g / \mathrm{L}$ \\
\hline Nickel & WA & & NA & $\mathbf{U}$ & & 40 & & 100 & $\mu \mathrm{g} / \mathrm{h}$ \\
\hline Nitrate as nitrogen & WA & & & JU & $\mathbf{Q}$ & 0.02 & & 10 & $\mathrm{mg} / \mathrm{L}$ \\
\hline Nonvolatile beta & TM & & $\mathbf{A}$ & & & 2.75 & 2.75 & 50 & $\mathrm{pCi} / \mathrm{L}$ \\
\hline Potassium & WA & & & U & & 5000 & & NS & $\mu g / L$ \\
\hline Potassium & WA & & NA & $\mathbf{U}$ & & 5000 & & NS & $\mu g / \mathrm{L}$ \\
\hline Propionitrile & WA & & & $\mathbf{U}$ & & 50 & & NS & $\mu g / \mathrm{L}$ \\
\hline Selenium & WA & & & U & & 5 & & 50 & $\mu \mathrm{g} / \mathrm{L}$ \\
\hline Selenium & WA & & NA & U & & 5 & & 50 & $\mu g / \mathrm{L}$ \\
\hline Silver & WA & & & $\mathrm{U}$ & & 10 & & 50 & $\mu \mathrm{g} / \mathrm{L}$ \\
\hline Silver & WA & & NA & $\mathbf{U}$ & & 10 & & 50 & $\mu \mathrm{g} / \mathrm{L}$ \\
\hline Sodium & WA & & $\mathbf{A}$ & & & 5945 & & NS & $\mu g / L$ \\
\hline Styrene & WA & & & $\mathrm{U}$ & & 5 & & 100 & $\mu g /$ \\
\hline Sulfate & WA & & & $\mathbf{U}$ & & 2.5 & & 400 & $\mathrm{mg} / \mathrm{L}$ \\
\hline Sulfide & WA & & & $\mathrm{U}$ & & 0.1 & & NS & $\mathrm{mg} / \mathrm{L}$ \\
\hline Tetrachloroethylene & WA & & & $\mathrm{U}$ & & 5 & & 5 & $\mu \mathrm{g} / \mathrm{L}$ \\
\hline Thallium & WA & & & $\mathrm{U}$ & & 10 & & 2 & $\mu g / L$ \\
\hline Thallium & WA & & NA & $\mathrm{U}$ & & 10 & & 2 & $\mu g / \mathrm{L}$ \\
\hline Toluene & WA & & & $\mathrm{U}$ & & 5 & & 1000 & $\mu g / \mathrm{L}$ \\
\hline Total hardness & WA & & & & & 1.9 & & NS & $\mathrm{mg} / \mathrm{L}$ \\
\hline Total organic carbon & WA & & & & & 5.8 & & NS & $\mathrm{mg} / \mathrm{L}$ \\
\hline Total suspended solids & WA & & & & & 19 & & NS & $\mathrm{mg} / \mathrm{L}$ \\
\hline trans-1,3-Dichloropropene & WA & & & $\mathrm{U}$ & & 5 & & NS & $\mu g / L$ \\
\hline trans-1,4-Dichloro-2-butene & WA & & & U & & 100 & & NS & $\mu \mathrm{g}$ \\
\hline Trichloroethylene & WA & & & U & & 5 & & 5 & $r$ \\
\hline
\end{tabular}


UTR020 Laboratory Analyses (cont'd)

\begin{tabular}{|c|c|c|c|c|c|c|c|c|}
\hline Analyte & Lab & $\operatorname{Rep}$ & Avg & Modifier & Result & Acc & Stand & Units \\
\hline Trichlorofluoromethane & WA & & & $\bar{U}$ & 5 & & NS & $\mu g / L$ \\
\hline Tritium & TM & & A & & 32.24 & 1.28 & 20 & $\mathrm{pCi} / \mathrm{ml}$ \\
\hline Turbidity & WA & & & & 170 & & NS & NTU \\
\hline Vanadium & WA & & & $\mathbf{U}$ & 50 & & NS & $\mu g / L$ \\
\hline Vanadium & WA & & NA & $\mathbf{U}$ & 50 & & NS & $\mu \mathrm{g} / \mathrm{L}$ \\
\hline Vinyl acetate & WA & & & $\mathbf{U}$ & 10 & & NS & $\mu g / L$ \\
\hline Vinyl chloride & WA & & & $\mathbf{U}$ & 10 & - & 2 & $\mu \mathrm{g} / \mathrm{L}$ \\
\hline Xylenes (total) & WA & & & $\mathbf{U}$ & 5 & & 10000 & $\mu g / L$ \\
\hline Zinc & WA & & $\mathbf{A}$ & & 96.5 & & 5000 & 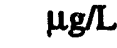 \\
\hline
\end{tabular}




\section{UTR022}

Field Measurements:

Sample Date: 8/25/93

pH: $\mathbf{5 . 8 0}$

Specific Conductance: $42 \mu \mathrm{S} / \mathrm{cm}$

Red/Oxid Potential: $85 \mathrm{mv}$

Dissolved Oxygen: $2.2 \mathrm{mg} / \mathrm{L}$

\begin{tabular}{|c|c|c|c|c|c|c|c|c|}
\hline Analyte & Lab & Rep & Avg & Modifier & Result & Acc & Stand & Units \\
\hline 1,1,1,2-Tetrachloroethane & WA & & & $\mathrm{U}$ & 10 & & NS & $\mu g / L$ \\
\hline 1,1,1-Trichloroethane & WA & & & $\mathbf{U}$ & 5 & & 200 & $\mu g / L$ \\
\hline 1,1,2,2-Tetrachloroethane & WA & & & $\mathbf{U}$ & 5 & & NS & $\mu g / L$ \\
\hline 1,1,2-Trichloroethane & WA & & & $\mathbf{U}$ & 5 & & 5 & $\mu g / L$ \\
\hline 1,1-Dichloroethane & WA & & & $\mathbf{U}$ & 5 & & NS & $\mu g / L$ \\
\hline 1,1-Dichloroethylene & WA & & & $\mathbf{U}$ & 5 & & 7 & $\mu g / L$ \\
\hline 1,2,3-Trichloropropane & WA & & & $\mathbf{U}$ & 10 & & NS & $\mu g / L$ \\
\hline 1,2-Dibromo-3-chloropropane & WA & & . & $\mathbf{U}$ & 20 & & 0.2 & $\mu g / L$ \\
\hline 1,2-Dibromoethane & WA & & & $\mathbf{U}$ & 20 & & NS & $\mu g / L$ \\
\hline 1,2-Dichloroethane & WA & & & $\mathbf{U}$ & 5 & & 5 & $\mu g / L$ \\
\hline 1,2-Dichloroethylene (total) & WA & & & & 9.67 & & 7 & $\mu g / L$ \\
\hline 1,2-Dichloropropane & WA & & & $\mathbf{U}$ & 5 & & 5 & $\mu g / L$ \\
\hline 2-Hexanone & WA & & & $\mathbf{U}$ & 10 & & NS & $\mu g / L$ \\
\hline 4-Methyl-2-pentanone & WA & & & $\mathbf{U}$ & 10 & & NS & $\mu g / L$ \\
\hline Acetone & WA & & & $\mathbf{U}$ & 9.9 & & NS & $\mu g / L$ \\
\hline Acetonitrile & WA & & & $\mathbf{U}$ & 20 & & NS & $\mu g / L$ \\
\hline Acidity & WA & & & & 120 & & NS & $\mathrm{mg} / \mathrm{L}$ \\
\hline Acrolein & WA & & & $\mathbf{U}$ & 10 & & NS & $\mu g / L$ \\
\hline Acrylonitrile & WA & & & $\mathbf{U}$ & 10 & & NS & $\mu g / L$ \\
\hline Alkalinity & WA & & & & 7 & & NS & $\mathrm{mg} / \mathrm{L}$ \\
\hline Allyl chloride & WA & & & $\mathbf{U}$ & 100 & & NS & $\mu g / L$ \\
\hline Aluminum & WA & & & & 580 & & NS & $\mu \mathrm{g} / \mathrm{L}$ \\
\hline Antimony & WA & & & $\mathbf{U}$ & 60 & & 6 & $\mu \mathrm{g} / \mathrm{L}$ \\
\hline Arsenic & WA & & & $\mathbf{U}$ & 10 & & 50 & $\mu g / L$ \\
\hline Barium & WA & & & $\mathbf{U}$ & 200 & & 2000 & $\mu g / L$ \\
\hline Benzene & WA & & & $\mathbf{U}$ & 5 & & 5 & $\mu g / L$ \\
\hline Beryllium & WA & & & $\mathbf{U}$ & 5 & & 4 & $\mu g / L$ \\
\hline Bromodichloromethane & WA & & & $\mathbf{U}$ & 5 & & NS & $\mu g / L$ \\
\hline Bromoform & WA & & & $\mathbf{U}$ & 5 & & NS & $\mu g / L$ \\
\hline Cadmium & WA & & & $\mathbf{U}$ & 5 & & 5 & $\mu \mathrm{g} / \mathrm{L}$ \\
\hline Calcium & WA & & & $\mathbf{U}$ & 5000 & & NS & $\mu \mathrm{g} / \mathrm{L}$ \\
\hline Carbon disulfide & WA & & & $\mathbf{U}$ & 5 & & NS & $\mu g / L$ \\
\hline Carbon tetrachloride & WA & & & $\mathbf{U}$ & 5 & & 5 & $\mu \mathrm{g} / \mathrm{L}$ \\
\hline Cesium-137 & TM & & NA & $\mathbf{U}$ & 6 & & 200 & $\mathrm{pCi} / \mathrm{L}$ \\
\hline Cesium-137 & TM & & & $\mathbf{U}$ & 9.6 & & 200 & $\mathrm{pCi} / \mathrm{L}$ \\
\hline Chloride & WA & & & & 8.43 & & NS & $\mathrm{mg} / \mathrm{L}$ \\
\hline Chlorobenzene & WA & & & $\mathbf{U}$ & 5 & & 100 & $\mu g / L$ \\
\hline Chloroethane & WA & & & $\mathbf{U}$ & 10 & & NS & $\mu g / L$ \\
\hline Chloroform & WA & & & $\mathbf{U}$ & 5 & & 100 & $\mu g / L$ \\
\hline Chloroprene & WA & & & $\mathbf{U}$ & 20 & & NS & $\mu \mathrm{g} / \mathrm{L}$ \\
\hline Chromium & WA & & & $\mathbf{U}$ & 10 & & 100 & $\mu \mathrm{g} / \mathrm{L}$ \\
\hline cis-1,3-Dichloropropene & WA & & & $\mathbf{U}$ & 5 & & NS & $\mu \mathrm{g} / \mathrm{L}$ \\
\hline Cobalt & WA & & & $\mathbf{U}$ & 50 & & NS & $\mu g / L$ \\
\hline Copper & WA & & & $\mathbf{U}$ & 25 & & 1000 & $\mu g / L$ \\
\hline Dibromochloromethane & WA & & & $\mathbf{U}$ & 5 & & NS & $\mu \mathrm{g} / \mathrm{L}$ \\
\hline Dichlorodifluoromethane & WA & & & $\mathbf{U}$ & 10 & & NS & $\mu g / L$ \\
\hline Ethylbenzene & WA & & & $\mathbf{U}$ & 5 & & 700 & $\mu g / L$ \\
\hline
\end{tabular}


UTR022 Laboratory Analyses (cont'd)

\begin{tabular}{|c|c|c|c|c|c|c|c|c|}
\hline Analyte & Lab & Rep & Avg & Modifier & Result & Acc & Stand & Units \\
\hline $\begin{array}{l}\text { Fluoride } \\
\text { Gross alpha }\end{array}$ & WA & & & $\mathbf{U}$ & 0.1 & & 4 & $\mathrm{mg} / \mathrm{L}$ \\
\hline $\begin{array}{l}\text { Gross alpha } \\
\text { Iron }\end{array}$ & $\begin{array}{l}\text { TM } \\
\text { WA }\end{array}$ & & $\mathbf{A}$ & & $\begin{array}{c}1.8 \\
3390\end{array}$ & 1.45 & $\begin{array}{c}15 \\
300\end{array}$ & $\begin{array}{l}\mathrm{PCH/L} \\
\mu \mathrm{g} / \mathrm{L}\end{array}$ \\
\hline $\begin{array}{l}\text { Iron } \\
\text { Isobutyl alcohol }\end{array}$ & WA & & & $\mathbf{U}$ & 20 & & NS & $\mu \mathrm{g} / \mathrm{L}$ \\
\hline Lead & WA & & & $\mathbf{U}$ & 3 & & 50 & $\mu g / L$ \\
\hline Magnesium & WA & & & $\mathbf{U}$ & 5000 & & NS & $\mu g / L$ \\
\hline Manganese & WA & & & & 308 & & 50 & $\mu g / L$ \\
\hline Mercury & WA & & & U & 0.2 & & 2 & $\mu g / L$ \\
\hline Methacrylonitrile & WA & & & $\mathbf{U}$ & 20 & & NS & $\mu g / L$ \\
\hline Methyl bromide & WA & & & $\mathbf{U}$ & 10 & & NS & $\mu g / L$ \\
\hline Methyl chloride & WA & & & U & 10 & & NS & $\mu g / L$ \\
\hline Methyl ethyl ketone & WA & & & U & 10 & & NS & $\mu g / L$ \\
\hline Methyl iodide & WA & & & $\mathbf{U}$ & 10 & & NS & $\mu \mathrm{g} / \mathrm{L}$ \\
\hline Methylene bromide & WA & & & $\mathbf{U}$ & 10 & & NS & $\mu g / L$ \\
\hline Methylene chloride & WA & & & $\mathbf{J}$ & V 3.77 & & 5 & $\mu g / L$ \\
\hline Nickel & WA & & . & $\mathbf{U}$ & 40 & & 100 & $\mu g / L$ \\
\hline Nitrate as nitrogen & WA & & 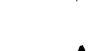 & J & Q 0.161 & & 10 & $\mathrm{mg} / \mathrm{L}$ \\
\hline Nonvolatile beta & TM & & A & & 6.1 & 2.9 & 50 & $\mathrm{pCi} / \mathrm{L}$ \\
\hline Potassium & WA & & & $\mathbf{U}$ & 5000 & & NS & $\mu g / L$ \\
\hline Propionitrile & WA & & & $\mathbf{U}$ & 50 & & NS & $\mu g / L$ \\
\hline Selenium & WA & & & $\mathbf{U}$ & 5 & & 50 & $\mu g / L$ \\
\hline Silver & WA & & & U & 10 & & 50 & $\mu g / L$ \\
\hline Sodium & WA & & & U & 5000 & & NS & $\mu g / L$ \\
\hline Styrene & WA & & & $\mathbf{U}$ & 5 & & 100 & $\mu g / L$ \\
\hline Sulfate & WA & & & & 5.25 & & 400 & $\mathrm{mg} / \mathrm{L}$ \\
\hline Sulfide & WA & & & $\mathbf{U}$ & 0.1 & & NS & $\mathrm{mg} / \mathrm{L}$ \\
\hline Tetrachloroethylene & WA & & & $\mathbf{U}$ & 5 & & 5 & $\mu g / L$ \\
\hline Thallium & WA & & & $\mathbf{U}$ & 10 & & 2 & $\mu g / L$ \\
\hline Toluene & WA & & & $\mathrm{U}$ & 5 & & 1000 & $\mu g / L$ \\
\hline Total hardness & WA & & & & 9.8 & & NS & $\mathrm{mg} / \mathrm{L}$ \\
\hline Total organic carbon & WA & & & & 4.12 & & NS & $\mathrm{mg} / \mathrm{L}$ \\
\hline Total suspended solids & WA & & & & 38 & & NS & $\mathrm{mg} / \mathrm{L}$ \\
\hline trans-1,3-Dichloropropene & WA & & & $\mathbf{U}$ & 5 & & NS & $\mu g / L$ \\
\hline trans-1,4-Dichloro-2-butene & WA & & & $\mathbf{U}$ & 100 & & NS & $\mu g / L$ \\
\hline Trichloroethylene & WA & & & $\mathbf{U}$ & 5 & & 5 & $\mu \mathrm{g} / \mathrm{L}$ \\
\hline Trichlorofluoromethane & WA & & & U & 5 & & NS & $\mu g / L$ \\
\hline Tritium & TM & & $\mathbf{A}$ & & 132.05 & 2.54 & 20 & $\mathrm{pCi} / \mathrm{ml}$ \\
\hline Turbidity & WA & & & & 18.2 & & NS & NTU \\
\hline Vanadium & WA & & & $U$ & 50 & & NS & $; g / L$ \\
\hline Vinyl acetate & WA & & & U & 10 & & NS & $\mu g / L$ \\
\hline Vinyl chloride & WA & & & $\mathbf{U}$ & 10 & & 2 & $\mu \mathrm{g} / \mathrm{L}$ \\
\hline Xylenes (total) & WA & & & $\mathbf{U}$ & 5 & & 10000 & $\mu g / h$ \\
\hline & WA & & & & 206 & & 5000 & $\mu g / L$ \\
\hline
\end{tabular}




\section{UTR024}

Field Measuremen :

Sample Date: 8/24/93

pH: 5.79

Specific Conductance: $17 \mu \mathrm{S} / \mathrm{cm}$

Red/Oxid Potential: $131 \mathrm{mv}$

Dissolved Oxygen: $2.9 \mathrm{mg} / \mathrm{L}$

\begin{tabular}{|c|c|c|c|c|c|c|c|c|c|}
\hline Analyte & Lab & Rep & Avg & Modifier & & Result & Acc & Stand & Units \\
\hline 1,1,1,2-Tetrachloroethane & WA & & & $\mathbf{U}$ & & 10 & & NS & $\mu g / L$ \\
\hline 1,1,1-Trichloroethane & WA & & & $\mathbf{U}$ & & 5 & & 200 & $\mu g / L$ \\
\hline 1,1,2,2-Tetrachloroethane & WA & & & $\mathbf{U}$ & & 5 & & NS & $\mu \mathrm{g} / \mathrm{L}$ \\
\hline 1,1,2-Trichloroethane & WA & & & $\mathbf{U}$ & & 5 & & 5 & $\mu g / L$ \\
\hline 1,1-Dichloroethane & WA & & & $\mathbf{U}$ & & 5 & & NS & $\mu g / L$ \\
\hline 1,1-Dichloroethylene & WA & & & $\mathbf{U}$ & & 5 & & 7 & $\mu \mathrm{g} / \mathrm{L}$ \\
\hline 1,2,3-Trichloropropane & WA & & & $\mathbf{U}$ & & 10 & & NS & $\mu g / L$ \\
\hline 1,2-Dibromo-3-chloropropane & WA & & & $\mathbf{U}$ & & 20 & & 0.2 & $\mu g / L$ \\
\hline 1,2-Dibromoethane & WA & & & $\mathbf{U}$ & & 20 & & NS & $\mu g / L$ \\
\hline 1,2-Dichloroethane & WA & & & $\mathbf{U}$ & & 5 & & 5 & $\mu g / L$ \\
\hline 1,2-Dichloroethylene (total) & WA & & & $\mathbf{U}$ & & 5 & & 7 & $\mu g / L$ \\
\hline 1,2-Dichloropropane & WA & & & $\mathbf{U}$ & & 5 & & 5 & $\mu g / L$ \\
\hline 2-Hexanone & WA & & & $\mathbf{U}$ & & 10 & & NS & $\mu \mathrm{g} / \mathrm{L}$ \\
\hline 4-Methyl-2-pentanone & WA & & & $\mathbf{U}$ & & 10 & & NS & $\mu g / L$ \\
\hline Acetone & WA & & & $\mathbf{J}$ & V & 7.98 & & NS & $\mu g / L$ \\
\hline Acetonitrile & WA & & & $\mathbf{U}$ & & 20 & & NS & $\mu \mathrm{g} / \mathrm{L}$ \\
\hline Acidity & WA & & & & & 106 & & NS & $\mathrm{mg} / \mathrm{L}$ \\
\hline Acrolein & WA & & & $\mathbf{U}$ & & 10 & & NS & $\mu \mathrm{g} / \mathrm{L}$ \\
\hline Acrylonitrile & WA & & & $\mathbf{U}$ & & 10 & & NS & $\mu \mathrm{g} / \mathrm{L}$ \\
\hline Allyl chloride & WA & & & $\mathbf{U}$ & & 100 & & NS & $\mu \mathrm{g} / \mathrm{L}$ \\
\hline Aluminum & WA & & & $\mathbf{U}$ & & 200 & & NS & $\mu g / L$ \\
\hline Antimony & WA & & & $\mathbf{U}$ & & 60 & & 6 & $\mu \mathrm{g} / \mathrm{L}$ \\
\hline Arsenic & WA & & & $\mathbf{U}$ & & 10 & & 50 & $\mu \mathrm{g} / \mathrm{L}$ \\
\hline Barium & WA & & & $\mathbf{U}$ & & 200 & & 2000 & $\mu g / L$ \\
\hline Benzene & WA & & & $\mathbf{U}$ & & 5 & & 5 & $\mu g / L$ \\
\hline Beryllium & WA & & & $\mathbf{U}$ & & 5 & & 4 & $\mu g / L$ \\
\hline Bromodichloromethane & WA & & & $\mathbf{U}$ & & 5 & & NS & $\mu \mathrm{g} / \mathrm{L}$ \\
\hline Bromoform & WA & & & $\mathbf{U}$ & & 5 & & NS & $\mu g / L$ \\
\hline Cadmium & WA & & & $\mathbf{U}$ & & 5 & & 5 & $\mu g / L$ \\
\hline Calcium & WA & & & $\mathbf{U}$ & & 5000 & & NS & $\mu g / L$ \\
\hline Carbon disulfide & WA & & & $\mathbf{U}$ & & 5 & & NS & $\mu g / L$ \\
\hline Carbon tetrachloride & WA & & & $\mathbf{U}$ & & 5 & & 5 & $\mu g / L$ \\
\hline Chloride & WA & & & & & 3.66 & & NS & $\mathrm{mg} / \mathrm{L}$ \\
\hline Chlorobenzene & WA & & & $U$ & & 5 & & 100 & $\mu g / L$ \\
\hline Chloroethane & WA & & & $\mathbf{U}$ & & 10 & & NS & $\mu \mathrm{g} / \mathrm{L}$ \\
\hline Chloroform & WA & & & $\mathbf{U}$ & & 5 & & 100 & $\mu \mathrm{g} / \mathrm{L}$ \\
\hline Chloroprene & WA & & & $\mathbf{U}$ & & 20 & & NS & $\mu \mathrm{g} / \mathrm{L}$ \\
\hline Chromium & WA & & & $\mathbf{U}$ & & 10 & & 100 & $\mu \mathrm{g} / \mathrm{L}$ \\
\hline cis-1,3-Dichloropropene & WA & & & $\mathbf{U}$ & & 5 & & NS & $\mu g / L$ \\
\hline Cobalt & WA & & & $\mathbf{U}$ & & 50 & & NS & $\mu g / L$ \\
\hline Copper & WA & & & $\mathbf{U}$ & & 25 & & 1000 & $\mu g / L$ \\
\hline Dibromochloromethane & WA & & & $\mathbf{U}$ & & 5 & & NS & $\mu g / L$ \\
\hline Dichlorodifluoromethane & WA & & & $\mathbf{U}$ & & 10 & & NS & $\mu g / L$ \\
\hline Ethylbenzene & WA & & & $\mathbf{U}$ & & 5 & & 700 & $\mu g / L$ \\
\hline Fluoride & WA & & & $\mathbf{U}$ & & 0.1 & & 4 & $\mathrm{mg} / \mathrm{L}$ \\
\hline Gross alpha & TM & & & & & 0.9 & 0.9 & 15 & $\mathrm{pCi} / \mathrm{L}$ \\
\hline
\end{tabular}


UTR024 Laboratory Analyses (cont'd)

\begin{tabular}{|c|c|c|c|c|c|c|c|c|c|}
\hline Analyte & Lab & Rep & Avg & Modifier & & Result & Acc & Stand & Units \\
\hline Iron & WA & & & & & 145 & & 300 & $\overline{\mu g / L}$ \\
\hline Isobutyl alcohol & WA & & & $\mathbf{U}$ & & 20 & & NS & $\mu g / L$ \\
\hline Lead & WA & & & $\mathbf{U}$ & & 3 & & 50 & $\mu g / L$ \\
\hline Magnesium & WA & & & U & & 5000 & & NS & $\mu g / L$ \\
\hline Manganese & WA & & & $\mathbf{U}$ & & 15 & & 50 & $\mu g / L$ \\
\hline Mercury & WA & & & $\mathbf{U}$ & & 0.2 & & 2 & $\mu g / L$ \\
\hline Methacrylonitrile & WA & & & $\mathbf{U}$ & & 20 & $\cdot$ & NS & $\mu g / L$ \\
\hline Methyl bromide & WA & & & $\mathbf{U}$ & & 10 & & NS & $\mu g / L$ \\
\hline Methyl chloride & WA & & & $\mathbf{U}$ & & 10 & & NS & $\mu g / L$ \\
\hline Methyl ethyl ketone & WA & & & $\mathbf{U}$ & & 10 & & NS & $\mu g / L$ \\
\hline Methyl iodide & $\mathbf{W A}$ & & & $\mathbf{U}$ & & 10 & & NS & $\mu g / L$ \\
\hline Methylene bromide & WA & & & $\mathbf{U}$ & & 10 & & NS & $\mu g / L$ \\
\hline Methylene chloride & WA & & & $\mathbf{J}$ & V & 3.87 & & 5 & $\mu g / L$ \\
\hline Nickel & WA & & & $\mathbf{U}$ & & 40 & & 100 & $\mu g / L$ \\
\hline Nitrate as nitrogen & WA & & & $\mathbf{J}$ & $\mathbf{Q}$ & 0.158 & & 10 & $\mathrm{mg} / \mathrm{L}$ \\
\hline Nonvolatile beta & TM & & & & & 3.4 & 1.8 & 50 & $\mathrm{pCi} / \mathrm{L}$ \\
\hline Potassium & WA & & & $U$ & & 5000 & & NS & $\mu g / L$ \\
\hline Propionitrile & WA & & & $\mathbf{U}$ & & 50 & & NS & $\mu g / L$ \\
\hline Selenium & WA & & & $\mathbf{U}$ & & 5 & & so & $\mu g / L$ \\
\hline Silver & WA & & & $\mathbf{U}$ & & 10 & & 50 & $\mu g / L$ \\
\hline Sodium & WA & & & $\mathbf{U}$ & & 5000 & & NS & $\mu g / L$ \\
\hline Styrene & WA & & & $\mathbf{U}$ & & 5 & & 100 & $\mu g / L$ \\
\hline Sulfate & WA & & & $\mathbf{U}$ & & 2.5 & & 400 & $\mathrm{mg} / \mathrm{L}$ \\
\hline Sulfide & WA & & & $\mathbf{U}$ & & 0.1 & & NS & $\mathrm{mg} / \mathrm{L}$ \\
\hline Tetrachloroethylene & WA & & & $\mathbf{U}$ & & 5 & & 5 & $\mu g / L$ \\
\hline Thallium & WA & & & $\mathbf{U}$ & & 10 & & 2 & $\mu g / L$ \\
\hline Toluene & WA & & & $\mathbf{U}$ & & 5 & & 1000 & $\mu g / L$ \\
\hline Total hardness & WA & & & $\mathbf{U}$ & & 1 & & NS & $\mathrm{mg} / \mathrm{L}$ \\
\hline Total organic carbon & WA & & & & & 1.28 & & NS & $\mathrm{mg} / \mathrm{L}$ \\
\hline Total suspended solids & WA & & & & & 14 & & NS & $\mathrm{mg} / \mathrm{L}$ \\
\hline trans-1,3-Dichloropropene & WA & & & $\mathbf{U}$ & & 5 & & NS & $\mu g / L$ \\
\hline trans-1,4-Dichloro-2-butene & WA & & & $\mathbf{U}$ & & 100 & & NS & $\mu g / L$ \\
\hline Trichloroethylene & WA & & & $\mathbf{U}$ & & 5 & & 5 & $\mu g / L$ \\
\hline Trichlorofluoromethane & WA & & & $\mathbf{U}$ & & 5 & & NS & $\mu g / L$ \\
\hline Tritium & TM & & & & & 28.4 & 1.2 & 20 & $\mathrm{pCi} / \mathrm{ml}$ \\
\hline Turbidity & WA & & & & & 0.28 & & NS & NTU \\
\hline Vanadium & WA & & & $\mathbf{U}$ & & 50 & & NS & $\mu g / L$ \\
\hline Vinyl acetate & WA & & & $\mathbf{U}$ & & 10 & & NS & $\mu g / L$ \\
\hline Vinyl chloride & WA & & & $\overline{\mathbf{U}}$ & & 10 & & 2 & $\mu \mathrm{g} / \mathrm{L}$ \\
\hline Xylenes (total) & WA & & & $\mathbf{U}$ & & 5 & & 10000 & $\mu g / L$ \\
\hline Zinc & WA & & & $\mathbf{U}$ & & 20 & & 5000 & $\mu g / L$ \\
\hline
\end{tabular}




\section{UTR029}

Field Measurements:

Sample Date: 8/24/93

pH: 5.60

Specific Conductance: $82 \mu \mathrm{S} / \mathrm{cm}$

Red/Oxid Potential: $179 \mathrm{mv}$

Dissolved Oxygen: $0.08 \mathrm{mg} / \mathrm{L}$

\begin{tabular}{|c|c|c|c|c|c|c|c|c|c|}
\hline Analyte & Lab & Rep & Avg & Modifier & & Result & Acc & Stand & Units \\
\hline 1,1,1,2-Tetrachloroethane & WA & & & $\mathbf{U}$ & & 10 & & NS & $\mu g / L$ \\
\hline 1,1,1-Trichloroethane & WA & & & $\mathbf{U}$ & & 5 & & 200 & $\mu g / L$ \\
\hline 1,1,2,2-Tetrachloroethane & WA & & & $\mathbf{U}$ & & 5 & & NS & $\mu g /$ \\
\hline 1,1,2-Trichloroethane & WA & & & $\mathbf{U}$ & & 5 & & 5 & $\mu g / L$ \\
\hline 1,1-Dichloroethane & WA & & & $\mathbf{U}$ & & 5 & & NS & $\mu \mathrm{g} / \mathrm{L}$ \\
\hline 1,1-Dichloroethylene & WA & & & $\mathbf{U}$ & & 5 & & 7 & $\mu \mathrm{g} / \mathrm{L}$ \\
\hline 1,2,3-Trichloropropane & WA & & & $\mathbf{U}$ & & 10 & & NS & $\mu \mathrm{g} / \mathrm{L}$ \\
\hline 1,2-Dibromo-3-chloropropane & WA & & & $\mathbf{U}$ & & 20 & & 0.2 & $\mu \mathrm{g} / \mathrm{L}$ \\
\hline 1,2-Dibromoethane & WA & & & $\mathbf{U}$ & & 20 & & NS & $\mu g / L$ \\
\hline 1,2-Dichloroethane & WA & & & $\mathbf{U}$ & & 5 & & 5 & $\mu \mathrm{g} / \mathrm{L}$ \\
\hline 1,2-Dichloroethylene (total) & WA & & & $\mathbf{U}$ & & 5 & & 7 & $\mu g / L$ \\
\hline 1,2-Dichloropropane & WA & & & $\mathbf{U}$ & & 5 & & 5 & $\mu g / L$ \\
\hline 2-Hexanone & WA & & & $\mathbf{U}$ & & 10 & & NS & $\mu g / L$ \\
\hline 4-Methyl-2-pentanone & WA & & & $\mathbf{U}$ & & 10 & & NS & $\mu g / L$ \\
\hline Acetone & WA & & & & V & 10.2 & & NS & $\mu \mathrm{g} / \mathrm{L}$ \\
\hline Acetonitrile & WA & & & U & & 20 & & NS & $\mu \mathrm{g} / \mathrm{L}$ \\
\hline Acidity & WA & & & & & 134 & & NS & $\mathrm{mg} / \mathrm{L}$ \\
\hline Acrolein & WA & & & $\mathbf{U}$ & & 10 & & NS & $\mu g / L$ \\
\hline Acrylonitrile & WA & & & $\mathbf{U}$ & & 10 & & NS & $\mu g / L$ \\
\hline Alkalinity & WA & & A & & & 20 & & NS & $\mathrm{mg} / \mathrm{L}$ \\
\hline Allyl chloride & WA & & & $\mathbf{U}$ & & 100 & & NS & $\mu g / L$ \\
\hline Aluminum & WA & & & & & 14800 & & NS & $\mu g / L$ \\
\hline Antimony & WA & & & $\mathbf{U}$ & & 60 & & 6 & $\mu g / L$ \\
\hline Arsenic & WA & & & $\mathbf{U}$ & & 10 & & 50 & $\mu g \Omega$ \\
\hline Barium & WA & & & $\mathbf{U}$ & & 200 & & 2000 & $\mu \mathrm{g} / \mathrm{L}$ \\
\hline Benzene & WA & & & $\mathrm{U}$ & & 5 & & 5 & $\mu \mathrm{g} / \mathrm{L}$ \\
\hline Beryllium & WA & & & $\mathbf{U}$ & & 5 & & 4 & $\mu \mathrm{g} / \mathrm{L}$ \\
\hline Bromodichloromethane & WA & & & $\mathbf{U}$ & & 5 & & NS & $\mu g / L$ \\
\hline Bromoform & WA & & & $\mathrm{U}$ & & 5 & & NS & $\mu g / L$ \\
\hline Cadmium & WA & & & $\mathrm{U}$ & & 5 & & 5 & $\mu g / L$ \\
\hline Calcium & WA & & & $\mathrm{U}$ & & 5000 & & NS & $\mu g / L$ \\
\hline Carbon disulfide & WA & & & $U$ & & 5 & & NS & $\mu g / L$ \\
\hline Carbon tetrachloride & WA & & & $\mathrm{U}$ & & 5 & & 5 & $\mu \mathrm{g} / \mathrm{L}$ \\
\hline Chloride & WA & & & & & 6.27 & & NS & $\mathrm{mg} / \mathrm{L}$ \\
\hline Chlorobenzene & WA & & & U & & 5 & & 100 & $\mu g / L$ \\
\hline Chloroethane & WA & & & U & & 10 & & NS & $\mu \mathrm{g} /$ \\
\hline Chloroform & WA & & & U & & 5 & & 100 & $\mu \mathrm{g} / \mathrm{L}$ \\
\hline Chloroprene & WA & & & U & & 20 & & NS & $\mu \mathrm{g} / \mathrm{L}$ \\
\hline Chromium & WA & & & & & 12.6 & & 100 & $\mu \mathrm{g} /$ \\
\hline cis-1,3-Dichloropropene & WA & & & $\mathbf{U}$ & & 5 & & NS & $\mu \mathrm{g} /$ \\
\hline Cobalt & WA & & & $\mathbf{U}$ & & 50 & & NS & $\mu \mathrm{g}$ \\
\hline Copper & WA & & & $\mathbf{U}$ & & 25 & & 1000 & $\mu g / L$ \\
\hline Dibromochloromethane & WA & & & $\mathbf{U}$ & & 5 & & NS & $\mu \mathrm{g} /$ \\
\hline Dichlorodifluoromethane & WA & & & $\mathbf{U}$ & & 10 & & NS & in \\
\hline Ethylbenzene & WA & & & $\mathbf{U}$ & & 5 & & 700 & $\lg / \mathrm{L}$ \\
\hline Fluoride & WA & & & $\mathbf{U}$ & & 0.1 & & 4 & $\mathrm{mg}$ \\
\hline Gross alpha & TM & & & & & 3.6 & 1.8 & 15 & $\mathrm{pCi} / \mathrm{L}$ \\
\hline
\end{tabular}


UTR029 Laboratory Analyses (cont'd)

\begin{tabular}{|c|c|c|c|c|c|c|c|c|c|}
\hline Analyte & Lab & Rep & Avg & Modifier & & Result & Acc & Stand & Units \\
\hline Iron & WA & & & & & 11300 & & 300 & $\mu g / L$ \\
\hline Isobutyl alcohol & WA & & & $\mathbf{U}$ & & 20 & & NS & $\mu g / L$ \\
\hline Lead & WA & & & & & 6.1 & & 50 & $\mu \mathrm{g} / \mathrm{L}$ \\
\hline Magnesium & WA & & & $\mathbf{U}$ & & 5000 & & NS & $\mu g / L$ \\
\hline Manganese & WA & & & & & 795 & & 50 & $\mu \mathrm{g} / \mathrm{L}$ \\
\hline Mercury & WA & & & $\mathbf{U}$ & & 0.2 & & 2 & $\mu g / L$ \\
\hline Methacrylonitrile & WA & & & $\mathbf{U}$ & & 20 & $\cdot$ & NS & $\mu g / L$ \\
\hline Methyl bromide & WA & & & $\mathbf{U}$ & & 10 & & NS & $\mu g / L$ \\
\hline Methyl chloride & WA & & & $\mathbf{U}$ & & 10 & & NS & $\mu g / L$ \\
\hline Methyl ethyl ketone & WA & & & $\mathbf{U}$ & & 10 & & NS & $\mu g / L$ \\
\hline Methyl iodide & WA & & & $\mathbf{U}$ & & 10 & & NS & $\mu g / L$ \\
\hline Methylene bromide & WA & & & $\mathbf{U}$ & & 10 & & NS & $\mu g / L$ \\
\hline Methylene chloride & WA & & & $\mathbf{J}$ & $\mathbf{V}$ & 4.1 & & 5 & $\mu g / L$ \\
\hline Nickel & WA & & & $\mathbf{U}$ & & 40 & & 100 & $\mu g / L$ \\
\hline Nitrate as nitrogen & WA & & & $\mathbf{J}$ & $\mathbf{Q}$ & 0.734 & & 10 & $\mathrm{mg} / \mathrm{L}$ \\
\hline Nonvolatile beta & TM & & & & & 5.90 & 2.90 & 50 & $\mathrm{pCi} / \mathrm{L}$ \\
\hline Potassium & WA & & & $\mathbf{U}$ & & 5000 & & NS & $\mu g / L$ \\
\hline Propionitrile & WA & & & $\mathbf{U}$ & & 50 & & NS & $\mu g / L$ \\
\hline Selenium & WA & & & $\mathbf{U}$ & & 5 & & 50 & $\mu g / L$ \\
\hline Silver & WA & & & $\mathbf{U}$ & & 10 & & 50 & $\mu \mathrm{g} / \mathrm{L}$ \\
\hline Sodium & WA & & & & & 8300 & & NS & $\mu g / L$ \\
\hline Styrene & WA & & & $\mathbf{U}$ & & 5 & & 100 & $\mu \mathrm{g} / \mathrm{L}$ \\
\hline Sulfate & WA & & & & & 12.6 & & 400 & $\mathrm{mg} / \mathrm{L}$ \\
\hline Sulfide & WA & & & $\mathbf{U}$ & & 0.1 & & NS & $\mathrm{mg} / \mathrm{L}$ \\
\hline Tetrachloroethylene & WA & & & $\mathbf{U}$ & & 5 & & 5 & $\mu g / L$ \\
\hline Thallium & WA & & & $\mathbf{U}$ & & 10 & & 2 & $\mu g / L$ \\
\hline Toluene & WA & & & $\mathbf{J}$ & & 1.71 & & 1000 & $\mu g / L$ \\
\hline Total hardness & WA & & & & & 29.4 & & NS & $\mathrm{mg} / \mathrm{L}$ \\
\hline Total organic carbon & WA & & & & & 6.42 & & NS & $\mathrm{mg} / \mathrm{L}$ \\
\hline Total suspended solids & WA & & $\mathbf{A}$ & & & 191.5 & & NS & $\mathrm{mg} / \mathrm{L}$ \\
\hline trans-1,3-Dichloropropene & WA & & & $\mathbf{U}$ & & 5 & & NS & $\mu \mathrm{g} / \mathrm{L}$ \\
\hline trans-1,4-Dichloro-2-butene & WA & & & $\mathbf{U}$ & & 100 & & NS & $\mu g / L$ \\
\hline Trichloroethylene & WA & & & $\mathbf{U}$ & & 5 & & 5 & $\mu \mathrm{g} / \mathrm{L}$ \\
\hline Trichlorofluoromethane & WA & & & $\mathbf{U}$ & & 5 & & NS & $\mu \mathrm{g} / \mathrm{L}$ \\
\hline Tritium & TM & & & & & 23.1 & 1.1 & 20 & $\mathrm{pCi} / \mathrm{ml}$ \\
\hline Turbidity & WA & & & & & 119 & & NS & NTU \\
\hline Vanadium & WA & & & $\mathbf{U}$ & & 50 & & NS & $\mu \mathrm{g} / \mathrm{L}$ \\
\hline Vinyl acetate & WA & & & $\mathbf{U}$ & & 10 & & NS & $\mu \mathrm{g} / \mathrm{L}$ \\
\hline Vinyl chloride & WA & & & $\mathbf{U}$ & & 10 & & 2 & $\mu \mathrm{g} / \mathrm{L}$ \\
\hline Xylenes (total) & WA & & & $\mathbf{U}$ & & 5 & & 10000 & $\mu \mathrm{g} / \mathrm{L}$ \\
\hline Zinc & WA & & & & & 78 & & 5000 & $\mu \mathrm{g} / \mathrm{L}$ \\
\hline
\end{tabular}




\section{UTR033}

Field Measurements:

Sample Date: 8/24/93

pH: 5.58

Specific Conductance: $19 \mu \mathrm{S} / \mathrm{cm}$

Red/Oxid Potential: $165 \mathrm{mv}$

Dissolved Oxygen: $1.1 \mathrm{mg} / \mathrm{L}$

\begin{tabular}{|c|c|c|c|c|c|c|c|c|c|}
\hline Analyte & Lab & Rep & Avg & Modifier & & Result & Acc & Stand & Units \\
\hline 1,1,1,2-Tetrachloroethane & WA & & & $\mathrm{U}$ & & 10 & & NS & $\mu g /$ \\
\hline $1,1,1,2$-Tetrachloroethane & WA & $\mathbf{R}$ & & $\mathbf{U}$ & & 10 & & NS & $\mu g / L$ \\
\hline 1,1,1-Trichloroethane & WA & & & $\mathbf{U}$ & & 5 & & 200 & $\mu g / L$ \\
\hline 1,1,1-Trichloroethane & WA & $\mathbf{R}$ & & $\mathbf{U}$ & & 5 & & 200 & $\mu g / L$ \\
\hline 1,1,2,2-Tetrachloroethane & WA & & & $\mathbf{U}$ & & 5 & & NS & $\mu g / L$ \\
\hline 1,1,2,2-Tetrachloroethane & WA & $\mathbf{R}$ & & $\mathbf{U}$ & & 5 & & NS & $\mu g / L$ \\
\hline 1,1,2-Trichloroethane & WA & & & $\mathbf{U}$ & & 5 & & 5 & $\mu g / L$ \\
\hline 1,1,2-Trichloroethane & WA & $\mathbf{R}$ & & $\mathbf{U}$ & & 5 & & 5 & $\mu g / L$ \\
\hline 1,1-Dichloroethane & WA & & & $\mathbf{U}$ & & 5 & & NS & $\mu g / L$ \\
\hline 1,1-Dichloroethane & WA & $\mathbf{R}$ & & $\mathbf{U}$ & & 5 & & NS & $\mu g / L$ \\
\hline 1,1-Dichloroethylene & WA & & & $\mathbf{U}$ & & 5 & & 7 & $\mu g / L$ \\
\hline 1,1-Dichloroethylene & WA & $\mathbf{R}$ & & $\mathbf{U}$ & & 5 & & 7 & $\mu \mathrm{g} / \mathrm{L}$ \\
\hline 1,2,3-Trichloropropane & WA & & & $\mathbf{U}$ & & 10 & & NS & $\mu g / L$ \\
\hline 1,2,3-Trichloropropane & WA & $\mathbf{R}$ & & $\mathbf{U}$ & & 10 & & NS & $\mu g / L$ \\
\hline 1,2-Dibromo-3-chloropropane & WA & & & $\mathbf{U}$ & & 20 & & 0.2 & $\mu \mathrm{g} / \mathrm{L}$ \\
\hline 1,2-Dibromo-3-chloropropane & WA & $\mathbf{R}$ & & $\mathbf{U}$ & & 20 & & 0.2 & $\mu \mathrm{g} / \mathrm{L}$ \\
\hline 1,2-Dibromoethane & WA & & & $\mathbf{U}$ & & 20 & & NS & $\mu g / L$ \\
\hline 1,2-Dibromoethane & WA & $\mathbf{R}$ & & $\mathbf{U}$ & & 20 & & NS & $\mu \mathrm{g} / \mathrm{L}$ \\
\hline 1,2-Dichloroethane & WA & & & $\mathbf{U}$ & & 5 & & 5 & $\mu \mathrm{g} / \mathrm{L}$ \\
\hline 1,2-Dichloroethane & WA & $\mathbf{R}$ & & $\mathbf{U}$ & & 5 & & 5 & $\mu \mathrm{g} / \mathrm{L}$ \\
\hline 1,2-Dichloroethylene (total) & WA & & & $\mathbf{U}$ & & 5 & & 7 & $\mu \mathrm{g} / \mathrm{L}$ \\
\hline 1,2-Dichloroethylene (total) & WA & $\mathbf{R}$ & & $\mathbf{U}$ & & 5 & & 7 & $\mu g / L$ \\
\hline 1,2-Dichloropropane & WA & & & $\mathbf{U}$ & & 5 & & 5 & $\mu g / L$ \\
\hline 1,2-Dichloropropane & WA & $\mathbf{R}$ & & $\mathbf{U}$ & & 5 & & 5 & $\mu \mathrm{g} / \mathrm{L}$ \\
\hline 2-Hexanone & WA & & & $\mathbf{U}$ & & 10 & & NS & $\mu g / L$ \\
\hline 2-Hexanone & WA & $\mathbf{R}$ & & $\mathbf{U}$ & & 10 & & NS & $\mu \mathrm{g} / \mathrm{L}$ \\
\hline 4-Methyl-2-pentanone & WA & & & $\mathbf{U}$ & & 10 & & NS & $\mu g / L$ \\
\hline 4-Methyl-2-pentanone & WA & $\mathbf{R}$ & & $\mathbf{U}$ & & 10 & & NS & $\mu g / L$ \\
\hline Acetone & WA & & & $\mathbf{J}$ & V & 1.37 & & NS & $\mu g / L$ \\
\hline Acetone & WA & $\mathbf{R}$ & & J & V & 7.15 & & NS & $\mu g / L$ \\
\hline Acetonitrile & WA & & & $\mathbf{U}$ & & 20 & & NS & $\mu \mathrm{g} / \mathrm{L}$ \\
\hline Acetonitrile & WA & $\mathbf{R}$ & & $\mathbf{U}$ & & 20 & & NS & $\mu \mathrm{g} / \mathrm{L}$ \\
\hline Acidity & WA & & A & & & 74 & & NS & $\mathrm{mg} / \mathrm{L}$ \\
\hline Acidity & WA & $\mathbf{R}$ & & & & 60 & & NS & $\mathrm{mg} / \mathrm{L}$ \\
\hline Acrolein & WA & & & $\mathbf{U}$ & & 10 & & NS & $\mu g / L$ \\
\hline Acrolein & WA & $\mathbf{R}$ & & $\mathbf{U}$ & & 10 & & NS & $\mu g / L$ \\
\hline Acrylonitrile & WA & & & $\mathbf{U}$ & & 10 & & NS & $\mu \mathrm{g} / \mathrm{L}$ \\
\hline Acrylonitrile & WA & $\mathbf{R}$ & & $\mathbf{U}$ & & 10 & & NS & $\mu g / L$ \\
\hline Alkalinity & WA & & & & & 2 & & NS & $\mathrm{mg} / \mathrm{L}$ \\
\hline Allyl chloride & WA & & & $\mathbf{U}$ & & 100 & & NS & $\mu g / L$ \\
\hline Allyl chloride & WA & $\mathbf{R}$ & & $\mathbf{U}$ & & 100 & & NS & $\mu g / L$ \\
\hline Aluminum & WA & & & $\mathbf{U}$ & & 200 & & NS & $\mu \mathrm{g} / \mathrm{L}$ \\
\hline Aluminum & WA & $\mathbf{R}$ & & $\mathbf{U}$ & & 200 & & NS & $\mu \mathrm{g} / \mathrm{L}$ \\
\hline Antimony & WA & & & $\mathbf{U}$ & & 60 & & 6 & $\mu \mathrm{g} / \mathrm{L}$ \\
\hline Antimony & WA & $\mathbf{R}$ & & $\mathbf{U}$ & & 60 & & 6 & $\mu \mathrm{g} / \mathrm{L}$ \\
\hline Arsenic & WA & & & $\mathbf{U}$ & & 10 & & 50 & $\mu g / L$ \\
\hline Arsenic & WA & $\mathbf{R}$ & & $\mathbf{U}$ & & 10 & & 50 & $\mu \mathrm{g}$ \\
\hline
\end{tabular}


UTR033 Laboratory Analyses (cont'd)

\begin{tabular}{|c|c|c|c|c|c|c|c|c|}
\hline Analyte & Lab & Rep & Avg & Modifier & Result & Acc & Stand & Units \\
\hline Barium & WA & & & $\bar{U}$ & 200 & & 2000 & $\overline{\mu g / L}$ \\
\hline Barium & WA & $\mathbf{R}$ & & $\mathrm{U}$ & 200 & & 2000 & $\mu \mathrm{g} / \mathrm{L}$ \\
\hline Benzene & WA & & & $\mathbf{U}$ & 5 & & 5 & $\mu g / L$ \\
\hline Benzene & WA & $\mathbf{R}$ & & $\mathbf{U}$ & 5 & & 5 & $\mu g / L$ \\
\hline Beryllium & WA & & & $\mathbf{U}$ & 5 & & 4 & $\mu g / L$ \\
\hline Beryllium & WA & $\mathbf{R}$ & & $\mathbf{U}$ & 5 & & 4 & $\mu g / L$ \\
\hline Bromodichloromethane & WA & & & $\mathbf{U}$ & 5 & - & NS & $\mu g / L$ \\
\hline Bromodichloromethane & WA & $\mathbf{R}$ & & $\mathbf{U}$ & 5 & & NS & $\mu g / L$ \\
\hline Bromoform & WA & & & $\mathbf{U}$ & 5 & & NS & $\mu g / L$ \\
\hline Bromoform & WA & $\mathbf{R}$ & & $\mathbf{U}$ & 5 & & NS & $\mu g / L$ \\
\hline Cadmium & WA & & & $\mathbf{U}$ & 5 & & 5 & $\mu g / L$ \\
\hline Cadmium & WA & $\mathbf{R}$ & & $\mathbf{U}$ & 5 & & 5 & $\mu g / L$ \\
\hline Calcium & WA & & & $\mathbf{U}$ & 5000 & & NS & $\mu g / L$ \\
\hline Calcium & WA & $\mathbf{R}$ & & $\mathbf{U}$ & 5000 & & NS & $\mu g / L$ \\
\hline Carbon disulfide & WA & & & $\mathbf{U}$ & 5 & & NS & $\mu g / L$ \\
\hline Carbon disulfide & WA & $\mathbf{R}$ & & $\mathbf{U}$ & 5 & & NS & $\mu g / L$ \\
\hline Carbon tetrachloride & WA & & & $\mathbf{U}$ & 5 & & 5 & $\mu g / L$ \\
\hline Carbon tetrachloride & WA & $\mathbf{R}$ & & $\mathbf{U}$ & 5 & & 5 & $\mu g / L$ \\
\hline Chloride & WA & & & & 3.11 & & NS & $\mathrm{mg} / \mathrm{L}$ \\
\hline Chloride & WA & $\mathbf{R}$ & & & 4.36 & & NS & $\mathrm{mg} / \mathrm{L}$ \\
\hline Chlorobenzene & WA & & & $\mathbf{U}$ & 5 & & 100 & $\mu g / L$ \\
\hline Chlorobenzene & WA & $\mathbf{R}$ & & $\mathbf{U}$ & 5 & & 100 & $\mu g / L$ \\
\hline Chloroethane & WA & & & $\mathbf{U}$ & 10 & & NS & $\mu g / L$ \\
\hline Chloroethane & WA & $\mathbf{R}$ & & $\mathbf{U}$ & 10 & & NS & $\mu g / L$ \\
\hline Chloroform & WA & & & $\mathbf{U}$ & 5 & & 100 & $\mu g / L$ \\
\hline Chloroform & WA & $\mathbf{R}$ & & $\mathbf{U}$ & 5 & & 100 & $\mu g / L$ \\
\hline Chloroprene & WA & & & $\mathbf{U}$ & 20 & & NS & $\mu g / L$ \\
\hline Chloroprene & WA & $\mathbf{R}$ & & $\mathbf{U}$ & 20 & & NS & $\mu \mathrm{g} / \mathrm{L}$ \\
\hline Chromium & WA & & & U & 10 & & 100 & $\mu g / L$ \\
\hline Chromium & WA & $\mathbf{R}$ & & $\mathbf{U}$ & 10 & & 100 & $\mu g / L$ \\
\hline cis-1,3-Dichloropropene & WA & & & $\mathbf{U}$ & 5 & & NS & $\mu \mathrm{g} / \mathrm{L}$ \\
\hline cis-1,3-Dichloropropene & WA & $\mathbf{R}$ & & $\mathbf{U}$ & 5 & & NS & $\mu g / L$ \\
\hline Cobalt & WA & & & $\mathrm{U}$ & 50 & & NS & $\mu g / L$ \\
\hline Cobalt & WA & $\mathbf{R}$ & & $\mathbf{U}$ & 50 & & NS & $\mu g / L$ \\
\hline Copper & WA & & & U & 25 & & 1000 & $\mu g / L$ \\
\hline Copper & WA & $\mathbf{R}$ & & $\mathbf{U}$ & 25 & & 1000 & $\mu g / L$ \\
\hline Dibromochloromethane & WA & & & $\mathbf{U}$ & 5 & & NS & $\mu \mathrm{g} / \mathrm{L}$ \\
\hline Dibromochloromethane & WA & $\mathbf{R}$ & & $\mathbf{U}$ & 5 & & NS & $\mu g / L$ \\
\hline Dichlorodifluoromethane & WA & & & $\mathbf{U}$ & 10 & & NS & $\mu g / L$ \\
\hline Dichlorodifluoromethane & WA & $\mathbf{R}$ & & $\mathbf{U}$ & 10 & & NS & $\mu g / L$ \\
\hline Ethylbenzene & WA & & & $\mathbf{U}$ & 5 & & 700 & $\mu g / L$ \\
\hline Ethylbenzene & WA & $\mathbf{R}$ & & $\mathbf{U}$ & 5 & & 700 & $\mu g / L$ \\
\hline Fluoride & WA & & & $\mathbf{U}$ & 0.1 & & 4 & $\mathrm{mg} / \mathrm{L}$ \\
\hline Fluoride & WA & $\mathbf{R}$ & & $\mathbf{U}$ & 0.1 & & 4 & $\mathrm{mg} / \mathrm{L}$ \\
\hline Gross alpha & TM & & & $\mathbf{U}$ & 0.6 & & 15 & $\mathrm{pCi} / \mathrm{L}$ \\
\hline Gross alpha & TM & $\mathbf{R}$ & & & 1.3 & 1.3 & 15 & $\mathrm{pCi} / \mathrm{L}$ \\
\hline Iron & WA & & & $\mathbf{U}$ & 100 & & 300 & $\mu g / L$ \\
\hline Iron & WA & $\mathbf{R}$ & & & 174 & & 300 & $\mu \mathrm{g} / \mathrm{L}$ \\
\hline Isobutyl alcohol & WA & & & $\mathbf{U}$ & 20 & & NS & $\mu g / L$ \\
\hline Isobutyl alcohol & WA & $\mathbf{R}$ & & $\mathbf{U}$ & 20 & & NS & $\mu g / L$ \\
\hline Lead & WA & & & $\mathbf{U}$ & 3 & & 50 & $\mu \mathrm{g} / \mathrm{L}$ \\
\hline Lead & WA & $\mathbf{R}$ & & $\mathbf{U}$ & 3 & & 50 & $\mu \mathrm{g} / \mathrm{L}$ \\
\hline Magnesium & WA & & & $\mathbf{U}$ & 5000 & & NS & $\mu g / L$ \\
\hline Magnesium & WA & $\mathbf{R}$ & & $\mathbf{U}$ & 5000 & & NS & $\mu g / L$ \\
\hline
\end{tabular}


UTR033 Laboratory Analyses (cont'd)

\begin{tabular}{|c|c|c|c|c|c|c|c|c|c|}
\hline Analyte & Lab & Rep & Avg & Modifier & & Result & Acc & Stand & Units \\
\hline Manganese & WA & & & $\bar{U}$ & & 15 & & 50 & $\mu \mathrm{g} / \mathrm{L}$ \\
\hline Manganese & WA & $\mathbf{R}$ & & $\mathbf{U}$ & & 15 & & 50 & $\mu g / L$ \\
\hline Mercury & WA & & & $\mathbf{U}$ & & 0.2 & & 2 & $\mu g / \Omega$ \\
\hline Mercury & WA & $\mathbf{R}$ & & $\mathbf{U}$ & & 0.2 & & 2 & $\mu \mathrm{g} / \mathrm{L}$ \\
\hline Methacrylonitrile & WA & & & $\mathbf{U}$ & & 20 & & NS & $\mu \mathrm{g} / \mathrm{L}$ \\
\hline Methacrylonitrile & WA & $\mathbf{R}$ & & U & & 20 & . & NS & $\mu g / L$ \\
\hline Methyl bromide & WA & & & $\mathbf{U}$ & & 10 & & NS & $\mu \mathrm{g} / \mathrm{L}$ \\
\hline Methyl bromide & WA & $\mathbf{R}$ & & $\mathbf{U}$ & & 10 & & NS & $\mu g /$ \\
\hline Methyl chloride & WA & & & $\mathbf{U}$ & & 10 & & NS & $\mu g / L$ \\
\hline Methyl chloride & WA & $\mathbf{R}$ & & $\mathbf{U}$ & & 10 & & NS & $\mu g /$ \\
\hline Methyl ethyl ketone & WA & & & $\mathbf{U}$ & & 10 & & NS & $\mu g / \mathrm{L}$ \\
\hline Methyl ethyl ketone & WA & $\mathbf{R}$ & & $\mathbf{U}$ & & 10 & & NS & $\mu g / L$ \\
\hline Methyl iodide & WA & & & $\mathbf{U}$ & & 10 & & NS & $\mu g / L$ \\
\hline Methyl iodide & WA & $\mathbf{R}$ & & $\mathbf{U}$ & & 10 & & NS & $\mu \mathrm{g} / \mathrm{L}$ \\
\hline Methylene bromide & WA & & & $\mathbf{U}$ & & 10 & & NS & $\mu \mathrm{g} / \mathrm{L}$ \\
\hline Methylene bromide & WA & $\mathbf{R}$ & & $\mathbf{U}$ & & 10 & & NS & $\mu g / L$ \\
\hline Methylene chloride & WA & & & $\mathbf{J}$ & V & 3.36 & & 5 & $\mu g / L$ \\
\hline Methylene chloride & WA & $\mathbf{R}$ & & $\mathbf{J}$ & V & 3.52 & & 5 & $\mu g / L$ \\
\hline Nickel & WA & & & $\mathbf{U}$ & & 40 & & 100 & $\mu g / L$ \\
\hline Nickel & WA & $\mathbf{R}$ & & $\mathbf{U}$ & & 40 & & 100 & $\mu g / L$ \\
\hline Nitrate as nitrogen & WA & & & $\mathbf{J}$ & $\mathbf{Q}$ & 0.518 & & 10 & $\mathrm{mg} / \mathrm{L}$ \\
\hline Nitrate as nitrogen & WA & $\mathbf{R}$ & & & & 0.463 & & 10 & $\mathrm{mg} / \mathrm{L}$ \\
\hline Nonvolatile beta & TM & & & & & 2.3 & 1.7 & 50 & pCi/L \\
\hline Nonvolatile beta & TM & $\mathbf{R}$ & & & & 3.6 & 2.7 & 50 & $\mathrm{pCi} / \mathrm{L}$ \\
\hline Potassium & WA & & & U & & 5000 & & NS & $\mu \mathrm{g} / \mathrm{L}$ \\
\hline Potassium & WA & $\mathbf{R}$ & & $\mathbf{U}$ & & 5000 & & NS & $\mu g / L$ \\
\hline Propionitrile & WA & & & $\mathbf{U}$ & & 50 & & NS & $\mu g / L$ \\
\hline Propionitrile & WA & $\mathbf{R}$ & & $\mathbf{U}$ & & 50 & & NS & $\mu g / L$ \\
\hline Selenium & WA & & & $\mathbf{U}$ & & 5 & & 50 & $\mu \mathrm{g} / \mathrm{L}$ \\
\hline Selenium & WA & $\mathbf{R}$ & & $\mathbf{U}$ & & 5 & & 50 & $\mu g / L$ \\
\hline Silver & WA & & & $\mathbf{U}$ & & 10 & & 50 & $\mu \mathrm{g} / \mathrm{L}$ \\
\hline Silver & WA & $\mathbf{R}$ & & $\mathbf{U}$ & & 10 & & 50 & $\mu g / L$ \\
\hline Sodium & WA & & & $\mathbf{U}$ & & 5000 & & NS & $\mu g / L$ \\
\hline Sodium & WA & $\mathbf{R}$ & & $\mathbf{U}$ & & 5000 & & NS & $\mu g / L$ \\
\hline Styrene & WA & & & $\mathbf{U}$ & & 5 & & 100 & $\mu g / L$ \\
\hline Styrene & WA & $\mathbf{R}$ & & $\mathbf{U}$ & & 5 & & 100 & $\mu \mathrm{g} / \mathrm{L}$ \\
\hline Sulfate & WA & & & $\mathbf{U}$ & & 2.5 & & 400 & $\mathrm{mg} / \mathrm{L}$ \\
\hline Sulfate & WA & & NA & $\mathbf{U}$ & & 2.5 & & 400 & $\mathrm{mg} / \mathrm{L}$ \\
\hline Sulfate & WA & $\mathbf{R}$ & & $\mathbf{U}$ & & 2.5 & & 400 & $\mathrm{mg} / \mathrm{L}$ \\
\hline Sulfide & WA & & & $\mathbf{U}$ & & 0.1 & & NS & $\mathrm{mg} / \mathrm{L}$ \\
\hline Sulfide & WA & $\mathbf{R}$ & & $\mathbf{U}$ & & 0.1 & & NS & $\mathrm{mg} / \mathrm{L}$ \\
\hline Tetrachloroethylene & WA & & & $\mathbf{U}$ & & 5 & & 5 & $\mu g / L$ \\
\hline Tetrachloroethylene & WA & $\mathbf{R}$ & & $\mathbf{U}$ & & 5 & & 5 & $\mu \mathrm{g} / \mathrm{L}$ \\
\hline Thallium & WA & & & $\mathbf{U}$ & & 10 & & 2 & $\mu g / L$ \\
\hline Thallium & WA & $\mathbf{R}$ & & $\mathbf{U}$ & & 10 & & 2 & $\mu \mathrm{g}$ \\
\hline Toluene & WA & & & $\mathbf{J}$ & & 1.31 & & 1000 & $\mu g / L$ \\
\hline Toluene & WA & $\mathbf{R}$ & & $\mathbf{U}$ & & 5 & & 1000 & $\mu g / L$ \\
\hline Total hardness & WA & & & $\mathbf{U}$ & & 1 & & NS & $\mathrm{mg} / \mathrm{L}$ \\
\hline Total hardness & WA & & NA & $\mathbf{U}$ & & 1 & & NS & $\mathrm{mg} / \mathrm{L}$ \\
\hline Total hardness & WA & $\mathbf{R}$ & & & & 1.96 & & NS & $\mathrm{mg} / \mathrm{L}$ \\
\hline Total organic carbon & WA & & & & & 0.809 & & NS & $\mathrm{mg} / \mathrm{L}$ \\
\hline Total organic carbon & WA & $\mathbf{R}$ & & $\mathbf{J}$ & $\mathbf{Q}$ & 0.519 & & NS & $m g / L$ \\
\hline Total suspended solids & WA & & & & & 11 & & NS & $\mathrm{mg} / \mathrm{L}$ \\
\hline Total suspended solids & WA & $\mathbf{R}$ & & & & 11 & & NS & mg \\
\hline
\end{tabular}


UTR033 Laboratory Analyses (cont'd)

\begin{tabular}{|c|c|c|c|c|c|c|c|c|}
\hline Analyte & $\mathrm{Lab}$ & $\operatorname{Rep}$ & Avg & Modifier & Result & Acc & Stand & Units \\
\hline trans-1,3-Dichloropropene & WA & & & $\mathbf{U}$ & 5 & & NS & $\mu g / L$ \\
\hline trans-1,3-Dichloropropene & WA & $\mathbf{R}$ & & $\mathbf{U}$ & 5 & & NS & $\mu g / L$ \\
\hline trans-1,4-Dichloro-2-butene & WA & & & $\mathbf{U}$ & 100 & & NS & $\mu g / L$ \\
\hline trans-1,4-Dichloro-2-butene & WA & $\mathbf{R}$ & & $\mathbf{U}$ & 100 & & NS & $\mu g / L$ \\
\hline Trichloroethylene & WA & & & $\mathbf{U}$ & 5 & & 5 & $\mu g / L$ \\
\hline Trichloroethylene & WA & $\mathbf{R}$ & & $\mathbf{U}$ & 5 & & 5 & $\mu g / L$ \\
\hline Trichlorofluoromethane & WA & & & $\mathbf{U}$ & 5 & & NS & $\mu \mathrm{g} / \mathrm{L}$ \\
\hline Trichlorofluoromethane & WA & $\mathbf{R}$ & & $\mathbf{U}$ & 5 & & NS & $\mu g / L$ \\
\hline Tritium & TM & & & & 20.2 & 1 & 20 & $\mathrm{pCi} / \mathrm{ml}$ \\
\hline Tritium & TM & $\mathbf{R}$ & & & 20.3 & 1 & 20 & $\mathrm{pCi} / \mathrm{ml}$ \\
\hline Turbidity & WA & & & $\mathbf{U}$ & 0.2 & & NS & NTU \\
\hline Turbidity & WA & $\mathbf{R}$ & $\mathbf{A}$ & & 0.46 & & NS & NTU \\
\hline Vanadium & WA & & & $\mathbf{U}$ & 50 & & NS & $\mu g / L$ \\
\hline Vanadium & WA & $\mathbf{R}$ & & $\mathbf{U}$ & 50 & & NS & $\mu g / L$ \\
\hline Vinyl acetate & WA & & & $\mathbf{U}$ & 10 & & NS & $\mu g / L$ \\
\hline Vinyl acetate & WA & $\mathbf{R}$ & . & $\mathbf{U}$ & 10 & & NS & $\mu \mathrm{g} / \mathrm{L}$ \\
\hline Vinyl chloride & WA & & & $\mathbf{U}$ & 10 & & 2 & $\mu \mathrm{g} / \mathrm{L}$ \\
\hline Vinyl chloride & WA & $\mathbf{R}$ & & $\mathbf{U}$ & 10 & & 2 & $\mu g / L$ \\
\hline Xylenes (total) & WA & & & $\mathbf{U}$ & 5 & & 10000 & $\mu g / L$ \\
\hline Xylenes (total) & WA & $\mathbf{R}$ & & $\mathbf{U}$ & 5 & & 10000 & $\mu \mathrm{g} / \mathrm{L}$ \\
\hline Zine & WA & & & $\mathbf{U}$ & 20 & & 5000 & $\mu g / L$ \\
\hline Zinc & WA & $\mathbf{R}$ & & $\mathbf{U}$ & 20 & & 5000 & $\mu g / L$ \\
\hline
\end{tabular}




\section{UTR036}

Field Measurements:

Sample Date: $8 / 24 / 93$

pH: 4.04

Specific Conductance: $24 \mu \mathrm{S} / \mathrm{cm}$

Red/Oxid Potential: $182 \mathrm{mv}$

Dissolved Oxygen: $1.2 \mathrm{mg} / \mathrm{L}$

\begin{tabular}{|c|c|c|c|c|c|c|c|c|c|}
\hline Analyte & Lab & Rep & Avg & Modifier & & Result & Acc & Stand & Units \\
\hline 1,1,1,2-Tetrachloroethane & WA & & & $\mathrm{U}$ & & 10 & & NS & $\mu g / L$ \\
\hline 1,1,1-Trichloroethane & WA & & & $\mathbf{U}$ & & 5 & & 200 & $\mu \mathrm{g} / \mathrm{L}$ \\
\hline $1,1,2,2$-Tetrachloroethane & WA & & & $\mathbf{U}$ & & 5 & & NS & $\mu \mathrm{g} / \mathrm{L}$ \\
\hline 1,1,2-Trichloroethane & WA & & & $\mathbf{U}$ & & 5 & & 5 & $\mu \mathrm{g} / \mathrm{L}$ \\
\hline 1,1-Dichloroethane & WA & & & $\mathbf{U}$ & & 5 & & NS & $\mu \mathrm{g} / \mathrm{L}$ \\
\hline 1,1-Dichloroethylene & WA & & & $\mathbf{U}$ & & 5 & & 7 & $\mu \mathrm{g} / \mathrm{L}$ \\
\hline 1,2,3-Trichloropropane & WA & & & $\mathbf{U}$ & & 10 & & NS & $\mu g / L$ \\
\hline 1,2-Dibromo-3-chloropropane & WA & & & $\mathbf{U}$ & & 20 & & 0.2 & $\mu g / L$ \\
\hline 1,2-Dibromoethane & WA & & & $\mathbf{U}$ & & 20 & & NS & $\mu g / L$ \\
\hline 1,2-Dichloroethane & WA & & & $U$ & & 5 & & 5 & $\mu g / L$ \\
\hline 1,2-Dichloroethylene (total) & WA & & & $\mathbf{U}$ & & 5 & & 7 & $\mu \mathrm{g} / \mathrm{L}$ \\
\hline 1,2-Dichloropropane & WA & & & $\mathbf{U}$ & & 5 & & 5 & $\mu g / L$ \\
\hline 2-Hexanone & WA & & & $\mathrm{U}$ & & 10 & & NS & $\mu g / L$ \\
\hline 4-Methyl-2-pentanone & WA & & & $U$ & & 10 & & NS & $\mu g / L$ \\
\hline Acetone & WA & & & J & $\mathbf{V}$ & 6.84 & & NS & $\mu g / L$ \\
\hline Acetonitrile & WA & & & $\mathbf{U}$ & & 20 & & NS & $\mu \mathrm{g} / \mathrm{L}$ \\
\hline Acidity & WA & & & & & 90 & & NS & $\mathrm{mg} / \mathrm{L}$ \\
\hline Acrolein & WA & & & $\mathbf{U}$ & & 10 & & NS & $\mu g / L$ \\
\hline Acrylonitrile & WA & & & $\mathbf{U}$ & & 10 & & NS & $\mu g / L$ \\
\hline Alkalinity & WA & & & & & 2 & & NS & $\mathrm{mg} / \mathrm{h}$ \\
\hline Allyl chloride & WA & & & $\mathbf{U}$ & & 100 & & NS & $\mu g / L$ \\
\hline Aluminum & WA & & & $\mathrm{U}$ & & 200 & & NS & $\mu \mathrm{g} / \mathrm{L}$ \\
\hline Antimony & WA & & & $\mathbf{U}$ & & 60 & & 6 & $\mu g / L$ \\
\hline Arsenic & WA & & & $\mathrm{U}$ & & 10 & & 50 & $\mu g / L$ \\
\hline Barium & WA & & & $\mathbf{U}$ & & 200 & & 2000 & $\mu g / L$ \\
\hline Benzene & WA & & & $\mathbf{U}$ & & 5 & & 5 & $\mu g / L$ \\
\hline Beryllium & WA & & & $\mathbf{U}$ & & 5 & & 4 & $\mu g / L$ \\
\hline Bromodichloromethane & WA & & & $\mathrm{U}$ & & 5 & & NS & $\mu \mathrm{g} / \mathrm{L}$ \\
\hline Bromoform & WA & & & $\mathrm{U}$ & & 5 & & NS & $\mu g / L$ \\
\hline Cadmium & WA & & & $\mathbf{U}$ & & 5 & & 5 & $\mu g / L$ \\
\hline Calcium & WA & & & $\mathrm{U}$ & & 5000 & & NS & $\mu g / L$ \\
\hline Carbon disulfide & WA & & & $\mathbf{U}$ & & 5 & & NS & $\mu \mathrm{g} / \mathrm{L}$ \\
\hline Carbon tetrachloride & WA & & & $\mathbf{U}$ & & 5 & & 5 & $\mu g / L$ \\
\hline Chloride & WA & & & $\mathbf{U}$ & & 0.25 & & NS & $\mathrm{mg} / \mathrm{L}$ \\
\hline Chlorobenzene & WA & & & $\mathbf{U}$ & & 5 & & 100 & $\mu \mathrm{g} / \mathrm{L}$ \\
\hline Chloroethane & WA & & & $\mathbf{U}$ & & 10 & & NS & $\mu \mathrm{g} / \mathrm{L}$ \\
\hline Chiloroform & WA & & & $\mathbf{U}$ & & 5 & & 100 & $\mu g / L$ \\
\hline Chloroprene & WA & & & $\mathbf{U}$ & & 20 & & NS & $\mu g / L$ \\
\hline Chromium & WA & & & $\mathbf{U}$ & & 10 & & 100 & $\mu g / L$ \\
\hline cis-1,3-Dichloropropene & WA & & & $\mathbf{U}$ & & 5 & & NS & $\mu g / L$ \\
\hline Cobalt & WA & & & $\mathbf{U}$ & & 50 & & NS & $\mu g / L$ \\
\hline Copper & WA & & & $\mathbf{U}$ & & 25 & & 1000 & $\mu g / L$ \\
\hline Dibromochloromethane & WA & & & $\mathbf{U}$ & & 5 & & NS & $\mu g / L$ \\
\hline Dichlorodifluoromethane & WA & & & $\mathbf{U}$ & & 10 & & NS & $\mu g / L$ \\
\hline Ethylbenzene & WA & & & $\mathbf{U}$ & & 5 & & 700 & $\mu g / L$ \\
\hline Fluoride & WA & & & $\mathbf{U}$ & & 0.1 & & 4 & $\mathrm{mg} / \mathrm{L}$ \\
\hline Gross alpha & $\mathrm{TM}$ & & & & & 0.8 & 0.9 & 15 & $\mathrm{pCi} / \mathrm{L}$ \\
\hline
\end{tabular}


UTR036 Laboratory Analyses (cont'd)

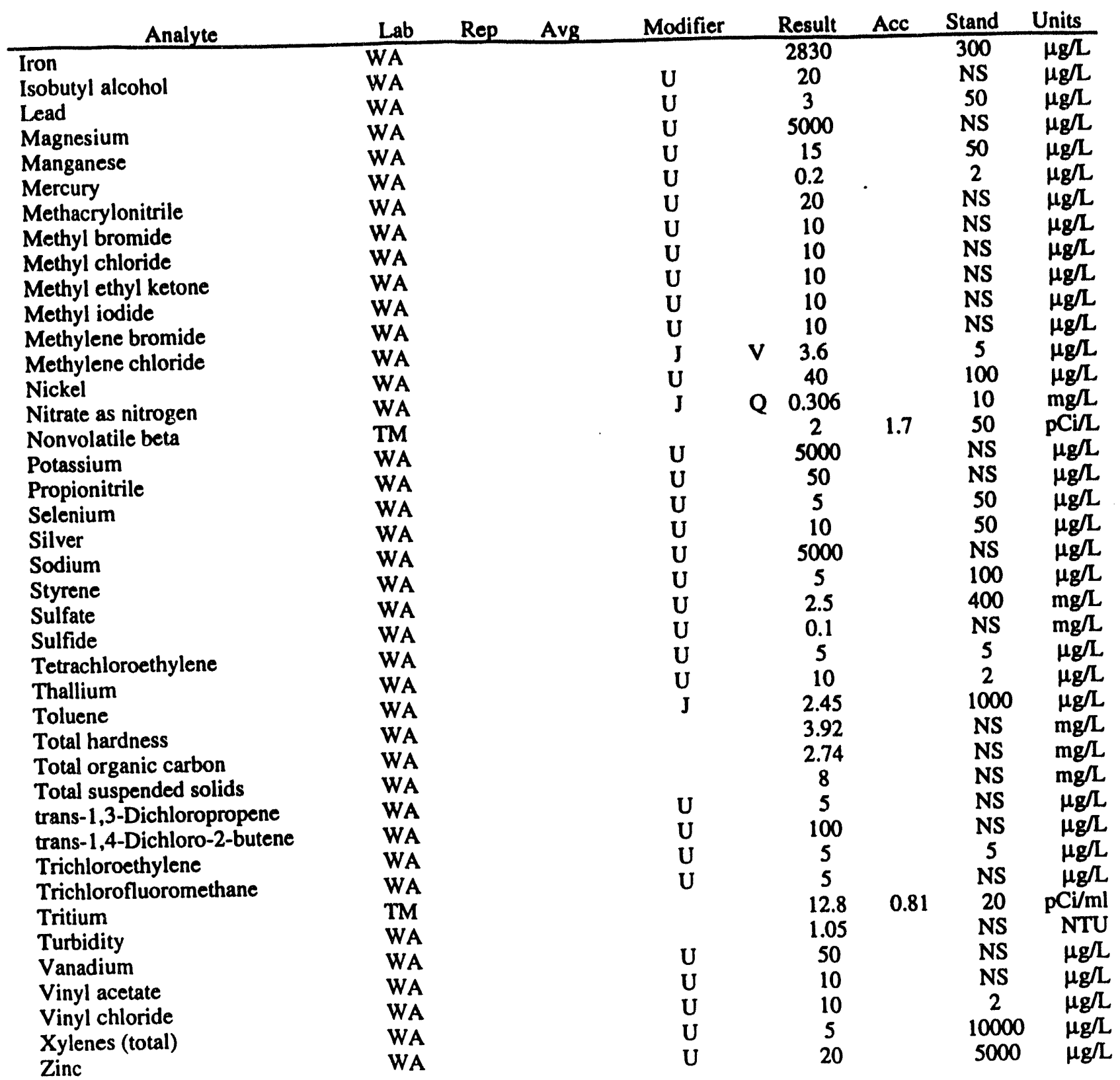


UTR039

Field Measurements:

Sample Date: 8/25/93

pH: 6.46

Specific Conductance: $24 \mu \mathrm{S} / \mathrm{cm}$

Red/Oxid Potential: $203 \mathrm{mv}$

Dissolved Oxygen: $2.1 \mathrm{mg} / \mathrm{L}$

\begin{tabular}{|c|c|c|c|c|c|c|c|c|}
\hline Analyte & Lab & Rep & Avg & Modifier & Result & Acc & Stand & Units \\
\hline 1,1,1,2-Tetrachloroethane & WA & & & $\bar{U}$ & 10 & & NS & $\mu g / L$ \\
\hline 1,1,1-Trichloroethane & WA & & & $\mathbf{U}$ & 5 & & 200 & $\mu g / L$ \\
\hline 1,1,2,2-Tetrachloroethane & WA & & & $\mathbf{U}$ & 5 & & NS & $\mu g / L$ \\
\hline 1,1,2-Trichloroethane & WA & & & $\mathbf{U}$ & 5 & & 5 & $\mu g / L$ \\
\hline 1,1-Dichloroethane & WA & & & $\mathbf{U}$ & 5 & & NS & $\mu g / L$ \\
\hline 1,1-Dichloroethylene & WA & & & $\mathbf{U}$ & 5 & & 7 & $\mu \mathrm{g} / \mathrm{L}$ \\
\hline 1,2,3-Trichloropropane & WA & & . & $\mathbf{U}$ & 10 & & NS & $\mu \mathrm{g} / \mathrm{L}$ \\
\hline 1,2-Dibromo-3-chloropropane & WA & & & $\mathbf{U}$ & 20 & & 0.2 & $\mu g / L$ \\
\hline 1,2-Dibromoethane & WA & & & $\mathbf{U}$ & 20 & & NS & $\mu g / L$ \\
\hline 1,2-Dichloroethane & WA & & & $\mathbf{U}$ & 5 & & 5 & $\mu \mathrm{g} / \mathrm{L}$ \\
\hline 1,2-Dichloroethylene (total) & WA & & & $\mathbf{U}$ & 5 & & 7 & $\mu g / L$ \\
\hline 1,2-Dichloropropane & WA & & & $\mathbf{U}$ & 5 & & 5 & $\mu g / L$ \\
\hline 2-Hexanone & WA & & & $\mathbf{U}$ & 10 & & NS & $\mu g / L$ \\
\hline 4-Methyl-2-pentanone & WA & & & $\mathbf{U}$ & 10 & & NS & $\mu g / L$ \\
\hline Acetone & WA & & & $\mathbf{U}$ & 10 & & NS & $\mu g / L$ \\
\hline Acetonitrile & WA & & & $\mathbf{U}$ & 20 & & NS & $\mu g / L$ \\
\hline Acidity & WA & & & & 64 & & NS & $\mathrm{mg} / \mathrm{L}$ \\
\hline Acrolein & WA & & & $\mathbf{U}$ & 10 & & NS & $\mu g / L$ \\
\hline Acrylonitrile & WA & & & $\mathbf{U}$ & 10 & & NS & $\mu g / L$ \\
\hline Alkalinity & WA & & & $\mathrm{U}$ & 0.5 & & NS & $\mathrm{mg} / \mathrm{L}$ \\
\hline Alkalinity & WA & & NA & $U$ & 0.5 & & NS & $\mathrm{mg} / \mathrm{L}$ \\
\hline Allyl chloride & WA & & & $\overline{\mathbf{U}}$ & 100 & & NS & $\mu g / L$ \\
\hline Aluminum & WA & & & $\mathbf{U}$ & 200 & & NS & $\mu g / L$ \\
\hline Antimony & WA & & & $\mathbf{U}$ & 60 & & 6 & $\mu g / L$ \\
\hline Arsenic & WA & & & $\mathbf{U}$ & 10 & & 50 & $\mu g / L$ \\
\hline Barium & WA & & & U & 200 & & 2000 & $\mu \mathrm{g} / \mathrm{L}$ \\
\hline Benzene & WA & & & $\mathbf{U}$ & 5 & & 5 & $\mu g / L$ \\
\hline Beryllium & WA & & & $\mathbf{U}$ & 5 & & 4 & $\mu g / L$ \\
\hline Bromodichloromethane & WA & & & $\mathrm{U}$ & 5 & & NS & $\mu g / L$ \\
\hline Bromoform & WA & & & $\mathbf{U}$ & 5 & & NS & $\mu g / L$ \\
\hline Cadmium & WA & & & $\mathrm{U}$ & 5 & & 5 & $\mu \mathrm{g} / \mathrm{L}$ \\
\hline Calcium & WA & & & $\mathrm{U}$ & 5000 & & NS & $\mu g / L$ \\
\hline Carbon disulfide & WA & & & $\mathbf{U}$ & 5 & & NS & $\mu \mathrm{g} / \mathrm{L}$ \\
\hline Carbon tetrachloride & WA & & & $\mathbf{U}$ & 5 & & 5 & $\mu g / L$ \\
\hline Cesium-137 & TM & & & $\mathrm{U}$ & 9.8 & & 200 & $\mathrm{pCi} / \mathrm{L}$ \\
\hline Chloride & WA & & & & 2.96 & & NS & $\mathrm{mg} / \mathrm{L}$ \\
\hline Chlorobenzene & WA & & & $\mathbf{U}$ & 5 & & 100 & $\mu \mathrm{g} / \mathrm{L}$ \\
\hline Chloroethane & WA & & & $\mathrm{U}$ & 10 & & NS & $\mu \mathrm{g} / \mathrm{L}$ \\
\hline Chloroform & WA & & & $\mathbf{U}$ & 5 & & 100 & $\mu \mathrm{g} / \mathrm{L}$ \\
\hline Chloroprene & WA & & & $\mathrm{U}$ & 20 & & NS & $\mu \mathrm{g} / \mathrm{L}$ \\
\hline Chromium & WA & & & $\mathbf{U}$ & 10 & & 100 & $\mu \mathrm{g} / \mathrm{L}$ \\
\hline cis-1,3-Dichloropropene & WA & & & $\mathbf{U}$ & 5 & & NS & $\mu \mathrm{g} / \mathrm{L}$ \\
\hline Cobalt & WA & & & $\mathbf{U}$ & 50 & & NS & $\mu \mathrm{g} / \mathrm{L}$ \\
\hline Copper & WA & & & $\mathbf{U}$ & 25 & & 1000 & $\mu \mathrm{g} / \mathrm{L}$ \\
\hline Dibromochloromethane & WA & & & U & 5 & & NS & $\mu g / L$ \\
\hline Dichlorodifluoromethane & WA & & & $\mathrm{U}$ & 10 & & NS & $\mu \mathrm{g} / \mathrm{L}$ \\
\hline Ethylbenzene & WA & & & $\mathbf{U}$ & 5 & & 700 & $\mu \mathrm{g} / \mathrm{L}$ \\
\hline
\end{tabular}


UTR039 Laboratory Analyses (cont'd)

\begin{tabular}{|c|c|c|c|c|c|c|c|c|c|}
\hline Analyte & Lab & Rep & Avg & Modifier & & Result & Acc & Stand & Units \\
\hline Fluoride & WA & & & $\bar{U}$ & & 0.1 & & 4 & $\mathrm{mg} / \mathrm{L}$ \\
\hline Fluoride & WA & & NA & $\mathbf{U}$ & & 0.1 & & 4 & $\mathrm{mg} / \mathrm{L}$ \\
\hline Gross alpha & TM & & & & & 1.6 & 1.4 & 15 & $\mathrm{pCi} / \mathrm{L}$ \\
\hline Iron & WA & & & $\mathbf{U}$ & & 100 & & 300 & $\mu \mathrm{g} / \mathrm{L}$ \\
\hline Isobutyl alcohol & WA & & & $\mathbf{U}$ & & 20 & & NS & $\mu \mathrm{g} / \mathrm{L}$ \\
\hline Lead & WA & & & $\mathbf{U}$ & & 3 & & 50 & $\mu \mathrm{g} / \mathrm{L}$ \\
\hline Magnesium & WA & & & $\mathbf{U}$ & & 5000 & & NS & $\mu \mathrm{g} / \mathrm{L}$ \\
\hline Manganese & WA & & & U & & 15 & & 50 & $\begin{array}{l}\mu \mathrm{g} / \mathrm{L} \\
\mu \mathrm{g} / \mathrm{L}\end{array}$ \\
\hline Mercury & WA & & & U & & 0.2 & & 2 & $\begin{array}{l}\mu g / L \\
\mu g / L\end{array}$ \\
\hline $\begin{array}{l}\text { Mercury } \\
\text { Methacrylonitrile }\end{array}$ & WA & & & U & & 20 & & NS & $\begin{array}{l}\mu g / L \\
\mu g / L\end{array}$ \\
\hline Methyl bromide & WA & & & $\mathbf{U}$ & & $\begin{array}{l}20 \\
10\end{array}$ & & NS & $\mu g / L$ \\
\hline Methyl chloride & WA & & & $\mathbf{U}$ & & $\begin{array}{l}10 \\
10\end{array}$ & & NS & $\mu \mathrm{g} / \mathrm{L}$ \\
\hline Methyl ethyl ketone & WA & & & U & & $\begin{array}{l}10 \\
10\end{array}$ & & NS & $\mu g / L$ \\
\hline Methyl iodide & WA & & & $\mathbf{U}$ & & $\begin{array}{l}10 \\
10\end{array}$ & & NS & $\mu g / L$ \\
\hline Methylene bromide & WA & & & $\mathbf{U}$ & & $\begin{array}{l}10 \\
10\end{array}$ & & NS & $\mu g / L$ \\
\hline Methylene chloride & WA & & & J & & $\begin{array}{c}10 \\
3.92\end{array}$ & & NS & $\mu g / L$ \\
\hline Nickel & WA & & $\cdot$ & U & V & $\begin{array}{c}3.92 \\
40\end{array}$ & & 5 & $\mu g / L$ \\
\hline Nitrate as nitrogen & WA & & & $\mathbf{J}$ & & $\begin{array}{c}40 \\
089 ?\end{array}$ & & 100 & $\mu g / L$ \\
\hline Nonvolatile beta & TM & & & & $\mathbf{Q}$ & 0.892 & & 10 & $\mathrm{mg} / \mathrm{L}$ \\
\hline Potassium & WA & & & $\mathbf{U}$ & & 1.8 & 2.6 & 50 & $\mathrm{pCi} / \mathrm{L}$ \\
\hline Propionitrile & WA & & & $\mathbf{U}$ & & 5000 & & NS & $\mu g / L$ \\
\hline Selenium & WA & & & $\mathbf{U}$ & & 50 & & NS & $\mu \mathrm{g} / \mathrm{L}$ \\
\hline $\begin{array}{l}\text { Selenumin } \\
\text { Silver }\end{array}$ & WA & & & U & & 5 & & 50 & $\mu g / L$ \\
\hline $\begin{array}{l}\text { Silver } \\
\text { Sodium }\end{array}$ & WA & & & $\mathrm{U}$ & & 10 & & 50 & $\mu \mathrm{g} / \mathrm{L}$ \\
\hline $\begin{array}{l}\text { Sodium } \\
\text { Styrene }\end{array}$ & WA & & & U & & 5000 & & NS & $\mu \mathrm{g} / \mathrm{L}$ \\
\hline $\begin{array}{l}\text { Styrene } \\
\text { Sulfate }\end{array}$ & WA & & & $\mathrm{U}$ & & 5 & & 100 & $\mu g / L$ \\
\hline $\begin{array}{l}\text { Sulfate } \\
\text { Sulfide }\end{array}$ & WA & & & $\begin{array}{l}\mathbf{U} \\
\mathbf{U}\end{array}$ & & 2.5 & & 400 & $\mathrm{mg} / \mathrm{L}$ \\
\hline $\begin{array}{l}\text { Sulfide } \\
\text { Tetrachloroethylene }\end{array}$ & WA & & & $\begin{array}{l}\mathbf{U} \\
\mathbf{U}\end{array}$ & & 0.1 & & NS & $\mathrm{mg} / \mathrm{L}$ \\
\hline $\begin{array}{l}\text { Tetrachloroethylene } \\
\text { Thallium }\end{array}$ & WA & & & $\begin{array}{l}U \\
U\end{array}$ & & 5 & & 5 & $\mu g / L$ \\
\hline $\begin{array}{l}\text { Thallium } \\
\text { Toluene }\end{array}$ & WA & & & U & & 10 & & 2 & $\mu \mathrm{g} / \mathrm{L}$ \\
\hline $\begin{array}{l}\text { Toluene } \\
\text { Total hardness }\end{array}$ & WA & & & $\mathbf{J}$ & & 1.91 & & 1000 & $\mu \mathrm{g} / \mathrm{L}$ \\
\hline $\begin{array}{l}\text { Total hardness } \\
\text { Total organic carbon }\end{array}$ & WA & & & & & 1.96 & & NS & $\mathrm{mg} / \mathrm{L}$ \\
\hline $\begin{array}{l}\text { Total organic carbon } \\
\text { Total suspended solids }\end{array}$ & WA & & A & & & 0.906 & & NS & $\mathrm{mg} / \mathrm{L}$ \\
\hline & WA & & & U & & 5 & & NS & $\mathrm{mg} / \mathrm{L}$ \\
\hline $\begin{array}{l}\text { trans-1,3-Dichloropropene } \\
\text { trans-1,4-Dichloro-2-butene }\end{array}$ & WA & & & $\mathbf{U}$ & & 5 & & NS & $\mu g / L$ \\
\hline $\begin{array}{l}\text { trans-1,4-Dichloro-2-butene } \\
\text { Trichloroethylene }\end{array}$ & WA & & & $\mathbf{U}$ & & 100 & & NS & $\mu g / L$ \\
\hline & WA & & & $\mathbf{U}$ & & 5 & & 5 & $\mu \mathrm{g} / \mathrm{L}$ \\
\hline $\begin{array}{l}\text { Trichiorofluoromethane } \\
\text { Tritium }\end{array}$ & $\mathrm{TM}$ & & & $\mathrm{U}$ & & 5 & & NS & $\mu g / L$ \\
\hline $\begin{array}{l}\text { Tritium } \\
\text { Turbidity }\end{array}$ & WA & & & & & 14.49 & 0.84 & 20 & $\mathrm{pCi} / \mathrm{ml}$ \\
\hline $\begin{array}{l}\text { Turbidity } \\
\text { Vanadium }\end{array}$ & WA & & & & & 0.37 & & NS & NTU \\
\hline $\begin{array}{l}\text { Vanadium } \\
\text { Vinyl acetate }\end{array}$ & WA & & & U & & 50 & & NS & $\mu \mathrm{g} / \mathrm{L}$ \\
\hline $\begin{array}{l}\text { Vinyl acetate } \\
\text { Vinyl chloride }\end{array}$ & WA & & & $\mathbf{U}$ & & 10 & & NS & $\mu \mathrm{g} / \mathrm{L}$ \\
\hline & WA & & & $\mathrm{U}$ & & 10 & & 2 & $\mu \mathrm{g} / \mathrm{L}$ \\
\hline $\begin{array}{l}\text { Xylenes (total) } \\
\text { Zinc }\end{array}$ & WA & & & $\mathrm{U}$ & & 5 & & 10000 & $\mu \mathrm{g} / \mathrm{L}$ \\
\hline$=$ & & & & & & 26 & & 5000 & $\mu \mathrm{g} / \mathrm{L}$ \\
\hline
\end{tabular}




\section{UTR042}

Field Measurements:

Sample Date: $8 / 24 / 93$

pH: 5.46

Specific Conductance: $20 \mu \mathrm{S} / \mathrm{cm}$

Red/Oxid Potential: $87 \mathrm{mv}$

Dissolved Oxygen: $1.4 \mathrm{mg} / \mathrm{L}$

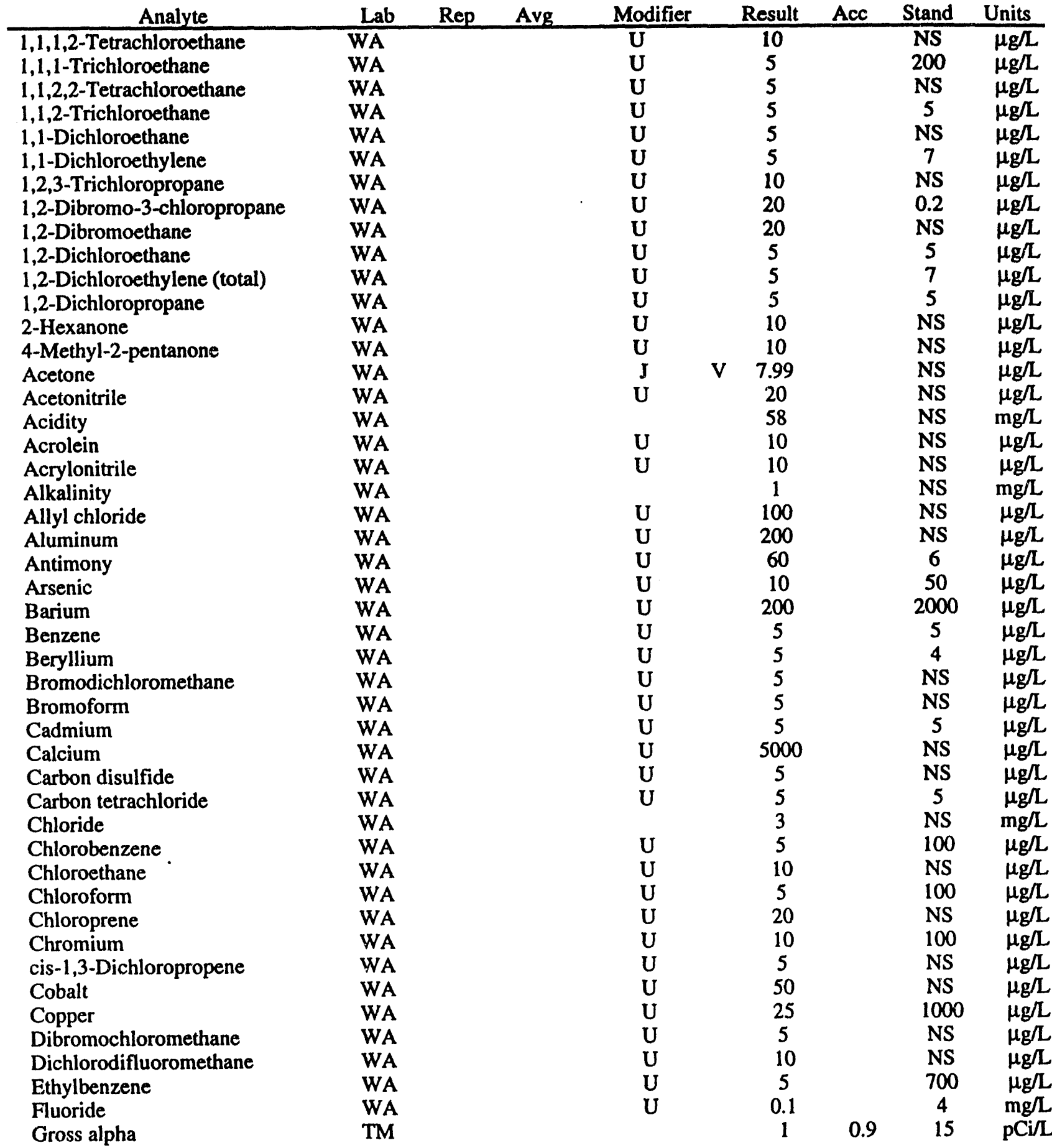


UTR042 Laboratory Analyses (cont'd)

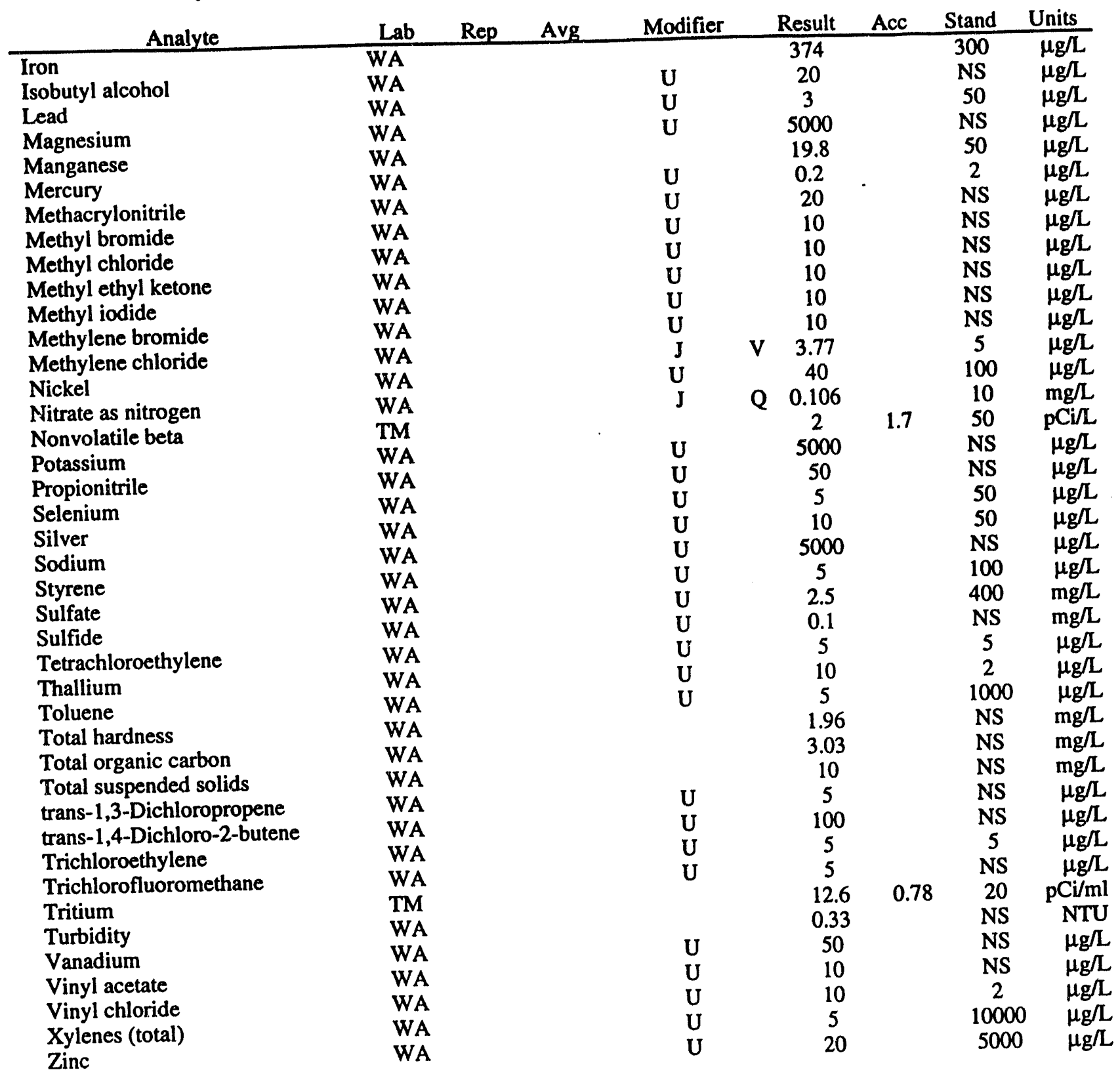




\section{UTR043}

Field Measurements:

Sample Date: $8 / 31 / 93$

pH: 5.67

Specific Conductance: $21 \mu \mathrm{S} / \mathrm{cm}$

Red/Oxid Potential: $201 \mathrm{mv}$

Dissolved Oxygen: $1.9 \mathrm{mg} / \mathrm{L}$

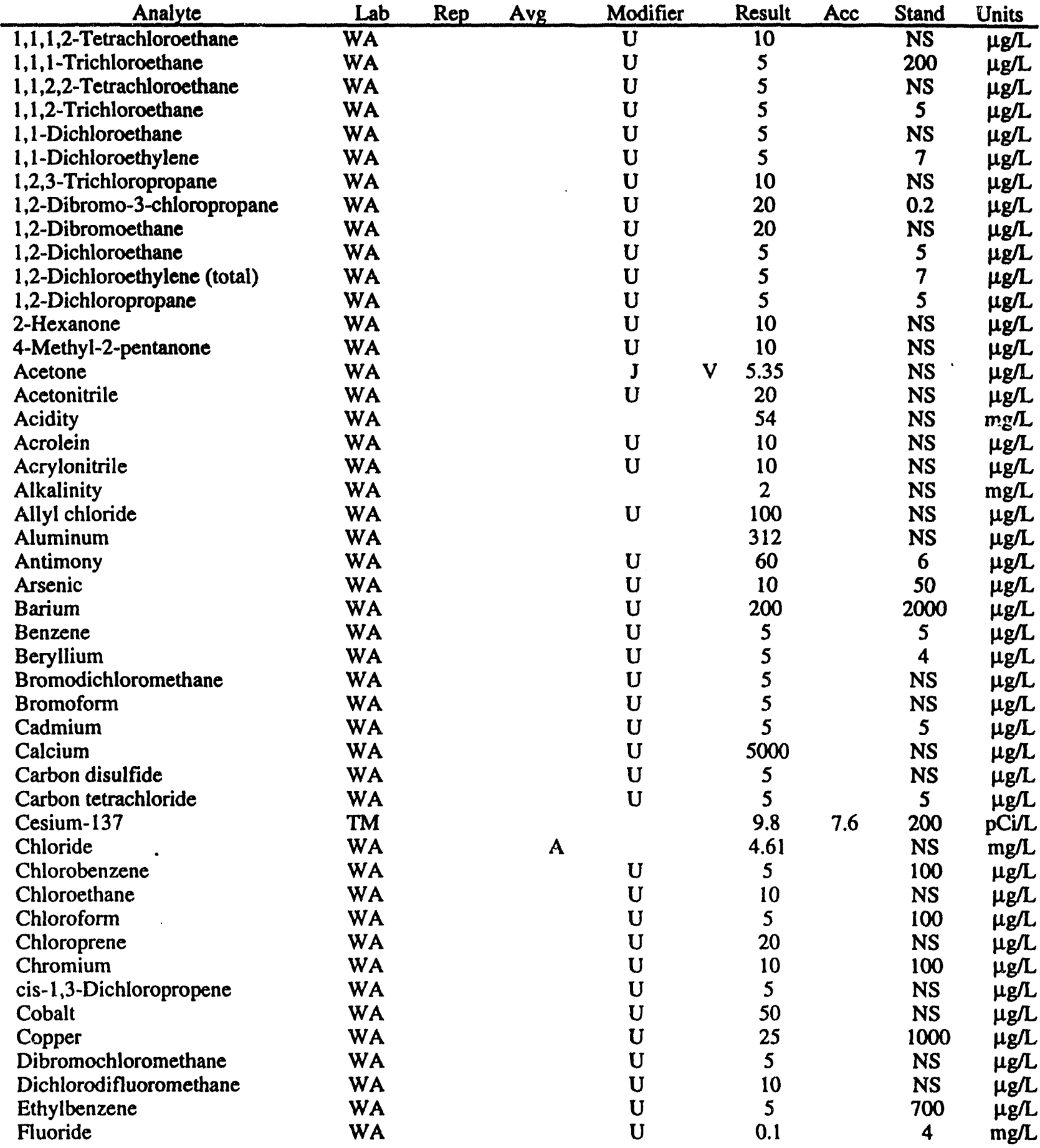


UTR043 Laboratory Analyses (cont'd)

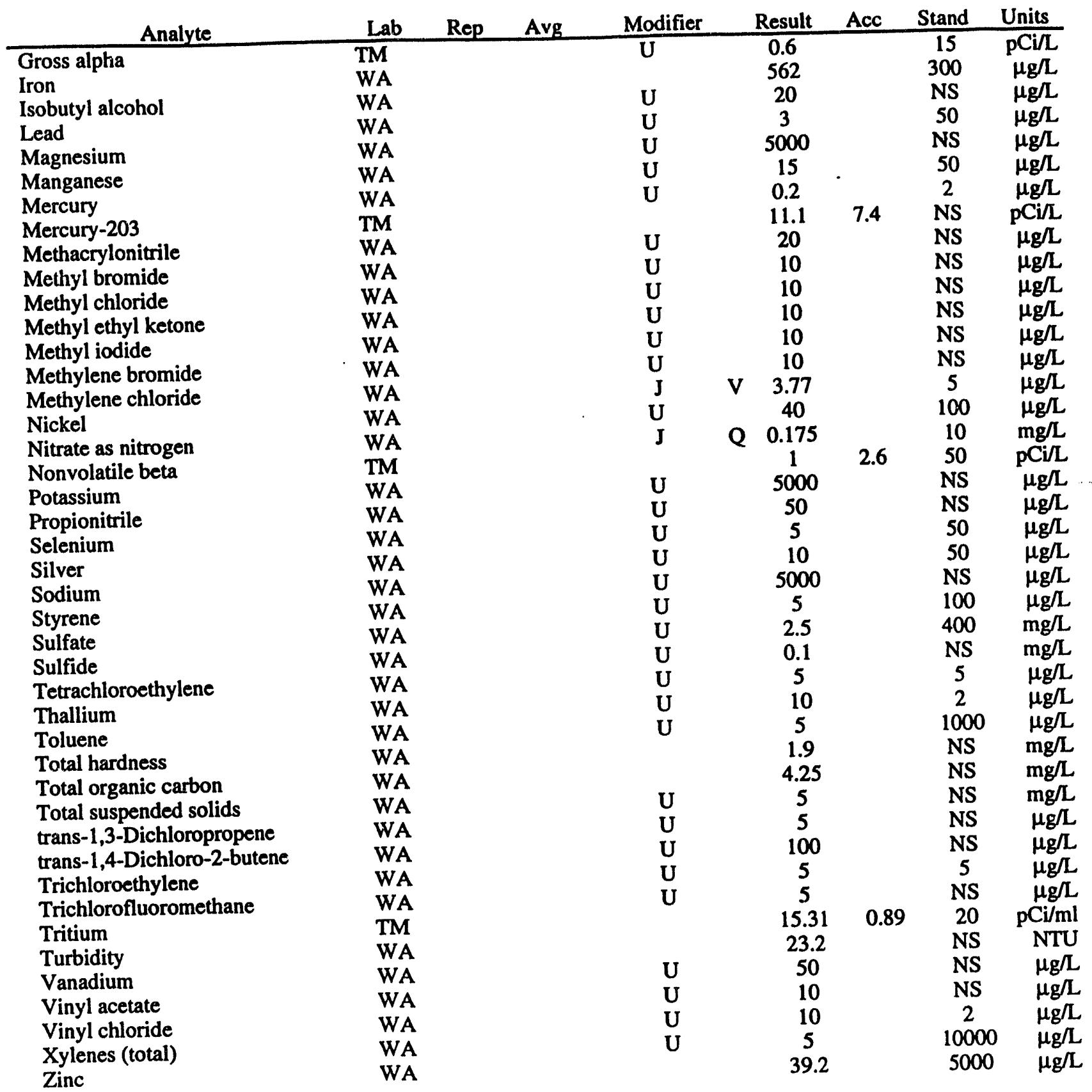




\section{UTR045}

Field Measurements:

Sample Date: $8 / 24 / 93$

pH: 5.28

Specific Conductance: $15 \mu \mathrm{S} / \mathrm{cm}$

Red/Oxid Potential: $165 \mathrm{mv}$

Dissolved Oxygen: $1.3 \mathrm{mg} / \mathrm{L}$

\begin{tabular}{|c|c|c|c|c|c|c|}
\hline Analyte & Lab & Avg & Modifier & Result & Stand & Units \\
\hline 1,1,1,2-Tetrachloroethane & WA & & $\mathbf{U}$ & 10 & NS & $\mu g / L$ \\
\hline $1,1,1,2$-Tetrachloroethane & GE & & $\mathbf{U}$ & 1 & NS & $\mu g / L$ \\
\hline 1,1,1-Trichloroethane & WA & & $\mathbf{U}$ & 5 & 200 & $\mu g / L$ \\
\hline 1,1,1-Trichloroethane & GE & & $\mathbf{U}$ & 1 & 200 & $\mu g / L$ \\
\hline $1,1,2,2$-Tetrachloroethane & WA & & $\mathbf{U}$ & 5 & NS & $\mu g / L$ \\
\hline $1,1,2,2$-Tetrachloroethane & GE & & $\mathbf{U}$ & 1 & NS & $\mu \mathrm{g} / \mathrm{L}$ \\
\hline 1,1,2-Trichloroethane & WA & & $\mathbf{U}$ & 5 & 5 & $\mu g / L$ \\
\hline 1,1,2-Trichloroethane & $G E$ & & $\mathbf{U}$ & 1 & 5 & $\mu g / L$ \\
\hline 1,1-Dichloroethane & WA & & $\mathbf{U}$ & 5 & NS & $\mu g / L$ \\
\hline 1,1-Dichloroethane & GE & & $\mathbf{U}$ & i & NS & $\mu g / L$ \\
\hline 1,1-Dichloroethylene & WA & & $\mathbf{U}$ & 5 & 7 & $\mu g / \Lambda$ \\
\hline 1,1-Dichloroethylene & GE & & $\mathbf{U}$ & i & 7 & $\mu g / L$ \\
\hline 1,2,3-Trichloropropane & WA & & $\mathbf{U}$ & 10 & NS & $\mu \mathrm{g} / \mathrm{L}$ \\
\hline 1,2,3-Trichloropropane & GE & & $\mathbf{U}$ & 1 & NS & $\mu g / L$ \\
\hline 1,2-Dibromo-3-chloropropane & WA & & $\mathbf{U}$ & 20 & 0.2 & $\mu g / L$ \\
\hline 1,2-Dibromo-3-chloropropane & GE & & $\mathbf{U}$ & 1 & 0.2 & $\mu \mathrm{g} / \mathrm{L}$ \\
\hline 1,2-Dibromoethane & WA & & $\mathbf{U}$ & 20 & NS & $\mu g / L$ \\
\hline 1,2-Dibromoethane & GE & & $\mathbf{U}$ & 20 & 7 & $\mu \mathrm{g} / \mathrm{L}$ \\
\hline 1,2-Dichloroethane & WA & & $\mathbf{U}$ & 5 & 5 & $\mu g / L$ \\
\hline 1,2-Dichloroethane & GE & & $\mathbf{U}$ & 1 & 5 & $\mu \mathrm{g} / \mathrm{L}$ \\
\hline 1,2-Dichloroethylene (total) & WA & & $\mathbf{U}$ & 5 & 7 & $\mu \mathrm{g} / \mathrm{L}$ \\
\hline 1,2-Dichloropropane & WA & & $\mathbf{U}$ & 5 & 5 & $\mu \mathrm{g} / \mathrm{L}$ \\
\hline 1,2-Dichloropropane & GE & & $\mathbf{U}$ & 1 & 5 & $\mu g / L$ \\
\hline 2-Hexanone & WA & & $\mathbf{U}$ & 10 & NS & $\mu \mathrm{g} / \mathrm{L}$ \\
\hline 2-Hexanone & GE & & $\mathbf{U}$ & 1 & NS & $\mu \mathrm{g} / \mathrm{L}$ \\
\hline 4-Methyl-2-pentanone & WA & & $\mathbf{U}$ & 10 & NS & $\mu \mathrm{g} / \mathrm{L}$ \\
\hline Acetone & WA & & $\mathbf{J}$ & 7.3 & NS & $\mu \mathrm{g} / \mathrm{L}$ \\
\hline Acetone & GE & & $\mathbf{U}$ & 100 & NS & $\mu \mathrm{g} / \mathrm{L}$ \\
\hline Acetonitrile & WA & & $\mathbf{U}$ & 20 & NS & $\mu \mathrm{g} / \mathrm{L}$ \\
\hline Acetonitrile (Methyl cyanide) & GE & & $\mathbf{U}$ & 1 & NS & $\mu g / L$ \\
\hline Acidity & WA & & & 44 & NS & $\mathrm{mg} / \mathrm{L}$ \\
\hline Acrolein & WA & & $\mathbf{U}$ & 10 & NS & $\mu g / L$ \\
\hline Acrolein & GE & & $\mathbf{U}$ & 20 & NS & $\mu \mathrm{g} / \mathrm{L}$ \\
\hline Acrylonitrile & WA & & $\mathbf{U}$ & 10 & NS & $\mu \mathrm{g} / \mathrm{L}$ \\
\hline Acrylonitrile & GE & & $\mathbf{U}$ & 20 & NS & $\mu \mathrm{g} / \mathrm{L}$ \\
\hline Alkalinity & WA & & & 1 & NS & $\mathrm{mg} / \mathrm{L}$ \\
\hline Alkalinity & GE & & & V & NS & $\mathrm{mg} / \mathrm{L}$ \\
\hline Allyl chloride & WA & & $\mathbf{U}$ & 100 & NS & $\mu \mathrm{g} / \mathrm{L}$ \\
\hline Allyl chloride & GE & & $\mathbf{U}$ & 50 & NS & $\mu \mathrm{g} / \mathrm{L}$ \\
\hline Aluminum & WA & & $\mathbf{U}$ & 200 & NS & $\mu \mathrm{g} / \mathrm{L}$ \\
\hline Aluminum & GE & & & 51.1 & NS & $\mu g / L$ \\
\hline Antimony & WA & & $\mathbf{U}$ & 60 & 6 & $\mu g / L$ \\
\hline Antimony & $\mathrm{GE}$ & & UJ & $\mathbf{L}$ & 6 & $\mu \mathrm{g} / \mathrm{L}$ \\
\hline Arsenic & WA & & $\mathbf{U}$ & 10 & 50 & $\mu \mathrm{g} / \mathrm{L}$ \\
\hline Arsenic & GE & & $\mathbf{U}$ & 2 & 50 & $\mu \mathrm{g} / \mathrm{L}$ \\
\hline Barium & WA & & $\mathbf{U}$ & 200 & 2000 & $\mu g / L$ \\
\hline Barium & GE & & & 19.8 & 2000 & $\mu \mathrm{g} / \mathrm{L}$ \\
\hline
\end{tabular}


UTR045 Laboratory Analyses (cont'd)

\begin{tabular}{|c|c|c|c|c|c|c|c|c|}
\hline Analyte & Lab & Rep & Avg & Modifier & Result & Acc & Stand & Units \\
\hline Benzene & WA & & & $\overline{\mathbf{U}}$ & 5 & & 5 & $\overline{\mu g / L}$ \\
\hline Benzene & GE & & & $\mathbf{U}$ & 1 & & 5 & $\mu g / L$ \\
\hline Beryllium & WA & & & $\mathbf{U}$ & 5 & & 4 & $\mu g / L$ \\
\hline Beryllium & GE & & & $\mathbf{U}$ & 3 & & 4 & $\mu g / L$ \\
\hline Bis(2-chloro-1-methylethyl)ether & GE & & & $\mathbf{U}$ & 10 & & NS & $\mu g / L$ \\
\hline Bromodichloromethane & WA & & & $\mathbf{U}$ & 5 & & NS & $\mu \mathrm{g} / \mathrm{L}$ \\
\hline Bromodichloromethane & GE & & & $\mathbf{U}$ & 1 & & NS & $\mu g / L$ \\
\hline Bromoform & WA & & & $\mathbf{U}$ & 5 & & NS & $\mu g / L$ \\
\hline Bromoform & GE & & & $\mathbf{U}$ & 1 & & NS & $\mu g / L$ \\
\hline Cadmium & WA & & & $\mathbf{U}$ & 5 & & 5 & $\mu g \Omega$ \\
\hline Cadmium & GE & & & $\mathbf{U}$ & 2 & & 5 & $\mu g /$ \\
\hline Calcium & WA & & & $\mathbf{U}$ & 5000 & & NS & $\mu g / L$ \\
\hline Calcium & GE & & & & 1620 & & NS & $\mu \mathrm{g} / \mathrm{L}$ \\
\hline Carbon disulfide & WA & & & $\mathbf{U}$ & 5 & & NS & $\mu g / L$ \\
\hline Carbon disulfide & GE & & & $\mathbf{U}$ & 1 & & NS & $\mu \mathrm{g} / \mathrm{L}$ \\
\hline Carbon tetrachloride & WA & & & $\mathbf{U}$ & 5 & & 5 & $\mu g / L$ \\
\hline Carbon tetrachloride & GE & & & $\mathbf{U}$ & 1 & & 5 & $\mu g / h$ \\
\hline Chloride & WA & & & & 4.44 & & NS & $\mathrm{mg} / \mathrm{L}$ \\
\hline Chloride & GE & & & & 5.74 & & NS & $\mathrm{mg} / \mathrm{L}$ \\
\hline Chlorobenzene & WA & & & $\mathbf{U}$ & 5 & & 100 & $\mu \mathrm{g} / \mathrm{L}$ \\
\hline Chlorobenzene & GE & & & $\mathbf{U}$ & 1 & & 100 & $\mu g / L$ \\
\hline Chloroethane & WA & & & $\mathbf{U}$ & 10 & & NS & $\mu \mathrm{g} / \mathrm{L}$ \\
\hline Chloroethane & $\mathrm{GE}$ & & & U & 1 & & NS & $\mu g / L$ \\
\hline Chloroform & WA & & & $\mathbf{U}$ & 5 & & 100 & $\mu g / L$ \\
\hline Chloroform & $\mathrm{GE}$ & & & $\mathbf{U}$ & 1 & & 100 & $\mu g / L$ \\
\hline Chlorop:ene & WA & & & $\mathbf{U}$ & 20 & & NS & $\mu \mathrm{g} / \mathrm{L}$ \\
\hline Chloroprene & $\mathrm{GE}$ & & & $\mathbf{U}$ & 200 & & NS & $\mu g / L$ \\
\hline Chromium & WA & & & $\mathbf{U}$ & 10 & & 100 & $\mu g / L$ \\
\hline Chromium & GE & & & $\mathbf{U}$ & 4 & & 100 & $\mu g / L$ \\
\hline cis-1,3-Dichloropropene & WA & & & $\mathbf{U}$ & 5 & & NS & $\mu g / L$ \\
\hline cis-1,3-Dichloropropene & GE & & & $\mathbf{U}$ & 1 & & NS & $\mu g / \Omega$ \\
\hline Cobalt & WA & & & $\mathbf{U}$ & 50 & & NS & $\mu g / L$ \\
\hline Cobalt & GE & & & $\mathbf{U}$ & 4 & & NS & $\mu \mathrm{g} / \mathrm{L}$ \\
\hline Copper & WA & & & $\mathbf{U}$ & 25 & & 1000 & $\mu \mathrm{g} / \mathrm{L}$ \\
\hline Copper & GE & & & $\mathbf{U}$ & 4 & & 1000 & $\mu g / L$ \\
\hline Cyanide & GE & & & $\mathbf{U}$ & 5 & & 200 & $\mu g / L$ \\
\hline Dibromochloromethane & WA & & & U & 5 & & NS & $\mu g / L$ \\
\hline Dibromochloromethane & GE & & & $\mathbf{U}$ & 1 & & NS & $\mu g / L$ \\
\hline Dichlorodifluoromethane & WA & & & $\mathbf{U}$ & 10 & & NS & $\mu g / L$ \\
\hline Dichlorodifluoromethane & GE & & & $\mathbf{U}$ & 1 & & NS & $\mu \mathrm{g} / \mathrm{L}$ \\
\hline Ethylbenzene & WA & & & $\mathbf{U}$ & 5 & & 700 & $\mu \mathrm{g} / \mathrm{L}$ \\
\hline Ethylbenzene & GE & & & $\mathbf{U}$ & 1 & & 700 & $\mu \mathrm{g} / \mathrm{L}$ \\
\hline Fluoride & WA & & & $\mathbf{U}$ & 0.1 & & 4 & $\mathrm{mg} / \mathrm{L}$ \\
\hline Fluoride & $\mathrm{GE}$ & & & U & 0.1 & & 4 & $\mathrm{mg} / \mathrm{L}$ \\
\hline Gross alpha & $G E$ & & & $\mathrm{U}$ & 2 & & 15 & $\mathrm{pCi} / \mathrm{L}$ \\
\hline Gross alpha & TM & & & & 1.5 & 1.1 & 15 & $\mathrm{pCi} / \mathrm{L}$ \\
\hline Hardness & $\mathrm{GE}$ & & & & 12 & & NS & $\mathrm{mg} / \mathrm{L}$ \\
\hline Iron & WA & & & & 534 & & 300 & $\mu \mathrm{g} / \mathrm{L}$ \\
\hline Iron & GE & & & & 1300 & & 300 & $\mu \mathrm{g} / \mathrm{L}$ \\
\hline Isobutyl alcohol & WA & & & $U$ & 20 & & NS & $\mu g / L$ \\
\hline Isobutyl alcohol & $\mathrm{GE}$ & & & U & 100 & & NS & \\
\hline Lead & WA & & & $\mathbf{U}$ & 3 & & 50 & $\mu \mathrm{g} / \mathrm{L}$ \\
\hline Lead & GE & & & $\mathbf{U}$ & 3 & & 50 & $\mu g / L$ \\
\hline Magnesium & WA & & & $\mathbf{U}$ & 5000 & & NS & $\mu \mathrm{g} / \mathrm{L}$ \\
\hline Magnesium & $\mathrm{GE}$ & & & & 652 & & NS & $L$ \\
\hline
\end{tabular}


UTR045 Laboratory Analyses (cont'd)

\begin{tabular}{|c|c|c|c|c|c|c|c|}
\hline Analyte & Lab & Avg & Modifier & Result & Acc & Stand & Units \\
\hline Manganese & WA & & $\bar{U}$ & 15 & & 50 & $\overline{\mu g / L}$ \\
\hline Manganese & GE & & & 19 & & 50 & $\mu g / L$ \\
\hline Mercury & WA & & $\mathbf{U}$ & 0.2 & & 2 & $\mu g / L$ \\
\hline Mercury & GE & & $\mathbf{U}$ & 0.2 & & 2 & $\mu g / L$ \\
\hline Methacrylonitrile & WA & & $\mathbf{U}$ & 20 & & NS & $\mu g / L$ \\
\hline Methacrylonitrile & GE & & $\mathbf{U}$ & 50 & . & NS & $\mu g / L$ \\
\hline Methyl bromide & WA & & $\mathbf{U}$ & 10 & & NS & $\mu g / L$ \\
\hline Methyl bromide & GE & & $\mathbf{U}$ & 1 & & NS & $\mu \mathrm{g} / \mathrm{L}$ \\
\hline Methyl chloride & WA & & $\mathbf{U}$ & 10 & & NS & $\mu g / L$ \\
\hline Methyl chloride & $\mathrm{GE}$ & & U & 1 & & NS & $\mu \mathrm{g} / \mathrm{L}$ \\
\hline Methyl ethyl ketone & WA & & $\mathbf{U}$ & 10 & & NS & $\mu g / L$ \\
\hline Methyl ethyl ketone & GE & & $\mathbf{U}$ & 1 & & NS & $\mu g / L$ \\
\hline Methyl iodide & WA & & $\mathbf{U}$ & 10 & & NS & $\mu g / L$ \\
\hline Methyl iodide & GE & & $\mathrm{U}$ & 15 & & NS & $\mu \mathrm{g} / \mathrm{L}$ \\
\hline Methyl isobutyl ketone & GE & & $\mathbf{U}$ & 1 & & NS & $\mu g /$ \\
\hline Methylene bromide & WA & & $\mathbf{U}$ & 10 & & NS & $\mu \mathrm{g} / \mathrm{L}$ \\
\hline Methylene bromide & GE & & $\mathbf{U}$ & 1 & & NS & $\mu g / L$ \\
\hline Methylene chloride & WA & & $\mathbf{J}$ & 3.74 & & 5 & $\mu \mathrm{g} / \mathrm{L}$ \\
\hline Methylene chloride & GE & & $\mathbf{U}$ & 1 & & 5 & $\mu g / L$ \\
\hline Nickel & WA & & $\mathrm{U}$ & 40 & & 100 & $\mu \mathrm{g} / \mathrm{h}$ \\
\hline Nickel & GE & & $\mathbf{U}$ & 4 & & 100 & $\mu g / L$ \\
\hline Nitrate as nitrogen & WA & & $\mathrm{J}$ & Q $\quad 0.225$ & & 10 & $\mathrm{mg} / \mathrm{L}$ \\
\hline Nitrate as nitrogen & GE & & & 0.26 & & 10 & $\mathrm{mg} / \mathrm{L}$ \\
\hline Nonvolatile beta & TM & & & 1.8 & 1.6 & 50 & $\mathrm{pCi} / \mathrm{L}$ \\
\hline Nonvolatile beta & $\mathrm{GE}$ & & $\mathbf{U}$ & 2 & & 50 & $\mathrm{pCi} / \mathrm{L}$ \\
\hline $\mathrm{pH}$ & $\mathrm{GE}$ & & $\mathbf{J}$ & Q $\quad 5.33$ & & NS & pH \\
\hline Potassium & WA & & $\mathrm{U}$ & 5000 & & NS & $\mu g / L$ \\
\hline Potassium & GE & & U & 500 & & NS & $\mu \mathrm{g} / \mathrm{L}$ \\
\hline Propionitrile & WA & & $\mathrm{U}$ & 50 & & NS & $\mu g / L$ \\
\hline Propionitrile & GE & & $\mathbf{U}$ & 200 & & NS & $\mu g / L$ \\
\hline Selenium & WA & & $\mathbf{U}$ & 5 & & 50 & $\mu g / L$ \\
\hline Selenium & GE & & UJ & $\mathbf{L}$ & & 50 & $\mu g / L$ \\
\hline Silver & WA & & $\mathrm{U}$ & 10 & & 50 & $\mu \mathrm{g} / \mathrm{L}$ \\
\hline Silver & GE & & $\mathbf{U}$ & 2 & & 50 & $\mu \mathrm{g} / \mathrm{L}$ \\
\hline Sodium & WA & & $\mathbf{U}$ & 5000 & & NS & $\mu g / L$ \\
\hline Sodium & $\mathrm{GE}$ & & & 1220 & & NS & $\mu \mathrm{g} / \mathrm{L}$ \\
\hline Styrene & WA & & $\mathbf{U}$ & 5 & & 100 & $\mu \mathrm{g} / \mathrm{L}$ \\
\hline Styrene & GE & & $\mathbf{U}$ & 1 & & 100 & $\mu \mathrm{g} / \mathrm{L}$ \\
\hline Sulfate & WA & & $\mathbf{U}$ & 2.5 & & 400 & $\mathrm{mg} / \mathrm{L}$ \\
\hline Sulfate & GE & & & 1.02 & & 400 & $\mathrm{mg} / \mathrm{L}$ \\
\hline Sulfide & WA & & $\mathbf{U}$ & 0.1 & & NS & $\mathrm{mg} / \mathrm{L}$ \\
\hline Sulfide & GE & & $\mathrm{U}$ & 1 & & NS & $\mathrm{mg} / \mathrm{L}$ \\
\hline Sulfide & GE & NA & $\mathrm{U}$ & $i$ & & NS & $\mathrm{mg} / \mathrm{L}$ \\
\hline Tetrachloroethylene & WA & & $\mathrm{U}$ & 5 & & 5 & $\mu \mathrm{g} / \mathrm{L}$ \\
\hline Tetrachloroethylene & $\mathrm{GE}$ & & $\mathrm{U}$ & 1 & & 5 & $\mu \mathrm{g} / \mathrm{L}$ \\
\hline Thallium & WA & & $\mathrm{U}$ & 10 & & 2 & $\mu \mathrm{g} / \mathrm{L}$ \\
\hline Thallium & GE & & $\mathrm{U}$ & 2 & & 2 & $\mu \mathrm{g} / \mathrm{L}$ \\
\hline Toluene & WA & & $\mathrm{U}$ & 5 & & 1000 & $\mu \mathrm{g} / \mathrm{L}$ \\
\hline Toluene & GE & & $U$ & 1 & & 1000 & $\mu \mathrm{g} / \mathrm{L}$ \\
\hline Total hardness & WA & & & 2.94 & & NS & $\mathrm{mg} / \mathrm{L}$ \\
\hline Total organic carbon & WA & & & 1.49 & & NS & $\mathrm{mg} / \mathrm{L}$ \\
\hline Total organic carbon & $\mathrm{GE}$ & & & 2.33 & & NS & $\mathrm{mg} / \mathrm{L}$ \\
\hline Total suspended solids & WA & & & 10 & & NS & $\mathrm{mg} / \mathrm{L}$ \\
\hline Total suspended solids & GE & & & 13 & & NS & $\mathrm{mg} / \mathrm{L}$ \\
\hline trans-1,2-Dichloroethylene (total) & GE & & $\mathrm{U}$ & 1 & & 100 & $\mu \mathrm{g} / \mathrm{L}$ \\
\hline
\end{tabular}


UTR045 Laboratory Analyses (cont'd)

\begin{tabular}{|c|c|c|c|c|c|c|c|c|}
\hline Analyte & Lab & Rep & Avg & Modifier & Result & Acc & Stand & Units \\
\hline trans-1,3-Dichloropropene & WA & & & $\mathrm{U}$ & 5 & & NS & $\mu g / L$ \\
\hline trans-1,3-Dichloropropene & GE & & & U & 1 & & NS & $\mu g / L$ \\
\hline trans-1,4-Dichloro-2-butene & WA & & & $U$ & 100 & & NS & $\mu g / L$ \\
\hline trans-1,4-Dichloro-2-butene & GE & & & $\mathbf{U}$ & 30 & & NS & $\mu g / L$ \\
\hline Trichloroethylene & WA & & & $\mathbf{U}$ & 5 & & 5 & $\mu \mathrm{g} / \mathrm{L}$ \\
\hline Trichloroethylene & $\mathrm{GE}$ & & & $\mathbf{U}$ & 1 & & 5 & $\mu g / L$ \\
\hline Trichlorofluoromethane & WA & & & $\mathbf{U}$ & 5 & • & NS & $\mu g / L$ \\
\hline Trichlorofluoromethane & $\mathrm{GE}$ & & & $\mathbf{U}$ & 1 & & NS & $\mu g / L$ \\
\hline Tritium & TM & & & & 16.6 & 0.92 & 20 & $\mathrm{pCi} / \mathrm{ml}$ \\
\hline Tritium & GE & & & & 14.9 & & 20 & $\mathrm{pCl} / \mathrm{ml}$ \\
\hline Turbidity & WA & & & & 0.36 & & NS & NTU \\
\hline Turbidity & GE & & & & 1.36 & & NS & NTU \\
\hline Vanadium & WA & & & $\mathbf{U}$ & 50 & & NS & $\mu g / L$ \\
\hline Vanadium & GE & & & $\mathbf{U}$ & 8 & & NS & $\mu g / L$ \\
\hline Vinyl acetate & WA & & & $\mathbf{U}$ & 10 & & NS & $\mu g / L$ \\
\hline Vinyl acetate & $\mathrm{GE}$ & & & $\mathbf{U}$ & 1 & & NS & $\mu \mathrm{g} / \mathrm{L}$ \\
\hline Vinyl chloride & WA & & & $\mathbf{U}$ & 10 & & 2 & $\mu g / L$ \\
\hline Vinyl chloride & GE & & & $\mathbf{U}$ & 1 & & 2 & $\mu g / L$ \\
\hline Xylenes (total) & WA & & & $\mathrm{U}$ & 5 & & 10000 & $\mu \mathrm{g} / \mathrm{L}$ \\
\hline Xylenes (total) & GE & & & $\mathbf{U}$ & 2 & & 10000 & $\mu g / L$ \\
\hline Zinc & WA & & & & 33 & & 5000 & $\mu g / L$ \\
\hline Zinc & GE & & & & 10.1 & & 5000 & $\mu g / L$ \\
\hline
\end{tabular}




\section{UTR049}

Field Measurements:

Sample Date: $8 / 25 / 93$

pH: 5.78

Specific Conductance: $61 \mu \mathrm{S} / \mathrm{cm}$

Red/Oxid Potential: $152 \mathrm{mv}$

Dissolved Oxygen: $1.6 \mathrm{mg} / \mathrm{L}$

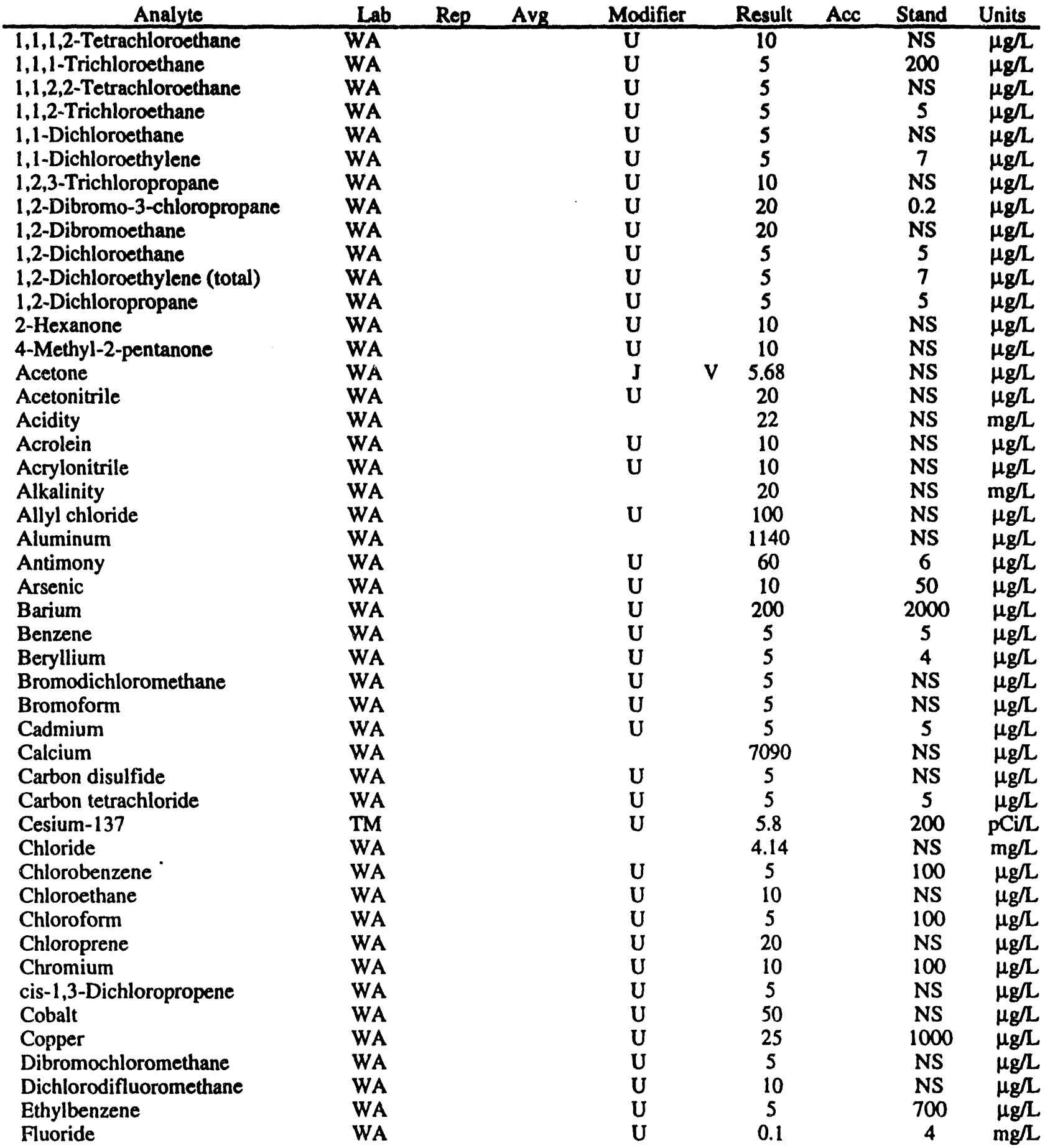


UTR049 Laboratory Analyses (cont'd)

\begin{tabular}{|c|c|c|c|c|c|c|c|c|c|}
\hline Analyte & Lab & Rep & Avg & Modifier & & Result & Acc & Stand & Units \\
\hline Gross alpha & TM & & & $\mathrm{U}$ & & 0.5 & & 15 & $\mathrm{pCi} / \mathrm{L}$ \\
\hline Iron & WA & & & & & 234 & & 300 & $\mu \mathrm{g} / \mathrm{L}$ \\
\hline Isobutyl alcohol & WA & & & $U$ & & 20 & & NS & $\mu g / L$ \\
\hline Lead & WA & & & $\mathrm{U}$ & & 3 & & 50 & $\mu \mathrm{g} / \mathrm{L}$ \\
\hline Magnesium & WA & & & $\mathrm{U}$ & & 5000 & & NS & $\mu g / L$ \\
\hline Manganese & WA & & & & & 43.8 & & 50 & $\mu \mathrm{g} / \mathrm{L}$ \\
\hline Mercury & WA & & & $U$ & & 0.2 & & 2 & $\mu \mathrm{g} / \mathrm{L}$ \\
\hline Methacrylonitrile & WA & & & $U$ & & 20 & & NS & $\mu g / L$ \\
\hline Methyl bromide & WA & & & $\mathbf{U}$ & & 10 & & NS & $\mu g / L$ \\
\hline Methyl chloride & WA & & & $\mathbf{U}$ & & 10 & & NS & $\mu g / L$ \\
\hline Methyl ethyl ketone & WA & & & U & & 10 & & NS & $\mu g / L$ \\
\hline Methyl iodide & WA & & & U & & 10 & & NS & $\mu g / L$ \\
\hline Methylene bromide & WA & & & $\mathbf{U}$ & & 10 & & NS & $\mu g / L$ \\
\hline Methylene chloride & WA & & & $\mathbf{J}$ & V & 4.25 & & 5 & $\mu g / L$ \\
\hline Nickel & WA & & & U & & 40 & & 100 & $\mu g / L$ \\
\hline Nitrate as nitrogen & WA & & & J & $\mathbf{Q}$ & 0.181 & & 10 & $\mathrm{mg} / \mathrm{L}$ \\
\hline Nitrate as nitrogen & WA & & NA & J & $\mathbf{Q}$ & 0.182 & & 10 & $\mathrm{mg} / \mathrm{L}$ \\
\hline Nonvolatile beta & TM & & & & & 3.9 & 2.7 & 50 & $\mathrm{pCi} / \mathrm{L}$ \\
\hline Potassium & WA & & & $\mathbf{U}$ & & 5000 & & NS & $\mu g / L$ \\
\hline Propionitrile & WA & & & $\mathbf{U}$ & & 50 & & NS & $\mu g / L$ \\
\hline Selenium & WA & & & $\mathbf{U}$ & & 5 & & 50 & $\mu g / L$ \\
\hline Silver & WA & & & $\mathbf{U}$ & & 10 & & 50 & $\mu \mathrm{g} / \mathrm{L}$ \\
\hline Sodium & WA & & & $\mathbf{U}$ & & 5000 & & NS & $\mu g / L$ \\
\hline Styrene & WA & & & $\mathbf{U}$ & & 5 & & 100 & $\mu \mathrm{g} / \mathrm{L}$ \\
\hline Sulfate & WA & & & & & 5.82 & & 400 & $\mathrm{mg} / \mathrm{L}$ \\
\hline Sulfide & WA & & & $\mathbf{U}$ & & 0.1 & & NS & $\mathrm{mg} / \mathrm{L}$ \\
\hline Sulfide & WA & & NA & U & & 0.1 & & NS & $\mathrm{mg} / \mathrm{L}$ \\
\hline Tetrachloroethylene & WA & & & U & & 5 & & 5 & $\mu g / L$ \\
\hline Thallium & WA & & & U & & 10 & & 2 & $\mu \mathrm{g} / \mathrm{L}$ \\
\hline Toluene & WA & & & $\mathrm{U}$ & & 5 & & 1000 & $\mu g / L$ \\
\hline Total hardness & WA & & & & & 8.82 & & NS & $\mathrm{mg} / \mathrm{L}$ \\
\hline Total organic carbon & WA & & & & & 2.94 & & NS & $\mathrm{mg} / \mathrm{L}$ \\
\hline Total suspended solids & WA & & & & & 13 & & NS & $\mathrm{mg} / \mathrm{L}$ \\
\hline trans-1,3-Dichloropropene & WA & & & $\mathbf{U}$ & & 5 & & NS & $\mu g / L$ \\
\hline trans-1,4-Dichloro-2-butene & WA & & & U & & 100 & & NS & $\mu g / L$ \\
\hline Trichloroethylene & WA & & & $\mathrm{U}$ & & 5 & & 5 & $\mu \mathrm{g} / \mathrm{L}$ \\
\hline Trichlorofluoromethane & WA & & & $\mathbf{U}$ & & 5 & & NS & $\mu g / L$ \\
\hline Tritium & TM & & & & & 9.96 & 0.72 & 20 & $\mathrm{pCi} / \mathrm{ml}$ \\
\hline Turbidity & WA & & & & & 28.3 & & NS & NTU \\
\hline Vanadium & WA & & & $U$ & & 50 & & NS & $\mu g / L$ \\
\hline Vinyl acetate & WA & & & $\mathrm{U}$ & & 10 & & NS & $\mu \mathrm{g} / \mathrm{L}$ \\
\hline Vinyl chloride & WA & & & U & & 10 & & 2 & $\mu g / L$ \\
\hline Xylenes (total) & WA & & & $\mathrm{U}$ & & 5 & & 10000 & $\mu g / L$ \\
\hline Zinc & WA & & & & & 77.1 & & 5000 & $\mu g / L$ \\
\hline
\end{tabular}




\section{UTROS1A}

Field Measurements:

Sample Date: $8 / 25 / 93$

pH: 7.41

Specific Conductance: $20 \mu \mathrm{S} / \mathrm{cm}$

Red/Oxid Potential: $162 \mathrm{mv}$

Dissolved Oxygen: $2.3 \mathrm{mg} / \mathrm{L}$

\begin{tabular}{|c|c|c|c|c|c|c|c|}
\hline Analyte & Lab & Avg & Modifier & Result & Acc & Stand & Units \\
\hline 1,1,1,2-Tetrachloroethane & WA & & $\mathbf{U}$ & 10 & & NS & $\mu g / L$ \\
\hline 1,1,1-Trichloroethane & WA & & $\mathbf{U}$ & 5 & & 200 & $\mu g / L$ \\
\hline 1,1,2,2-Tetrachloroethane & WA & & $\mathbf{U}$ & 5 & & NS & $\mu g / L$ \\
\hline 1,1,2-Trichloroethane & WA & & $\mathbf{U}$ & 5 & & 5 & $\mu g / L$ \\
\hline 1,1-Dichloroethane & WA & & $\mathbf{U}$ & 5 & & NS & $\mu \mathrm{g} / \mathrm{L}$ \\
\hline 1,1-Dichloroethylene & WA & & $\mathbf{U}$ & 5 & & 7 & $\mu g / L$ \\
\hline 1,2,3-Trichloropropane & WA & & $\mathbf{U}$ & 10 & & NS & $\mu g / L$ \\
\hline 1,2-Dibromo-3-chloropropane & WA & & $\mathbf{U}$ & 20 & & 0.2 & $\mu g / L$ \\
\hline 1,2-Dibromoethane & WA & & $\mathbf{U}$ & 20 & & NS & $\mu g / L$ \\
\hline 1,2-Dichloroethane & WA & & $\mathbf{U}$ & 5 & & 5 & $\mu g / L$ \\
\hline 1,2-Dichloroethylene (total) & WA & & $\mathbf{U}$ & 5 & & 7 & $\mu g / L$ \\
\hline 1,2-Dichloropropane & WA & & $\mathbf{U}$ & 5 & & 5 & $\mu g / L$ \\
\hline 2-Hexanone & WA & & $\mathbf{U}$ & 10 & & NS & $\mu g / L$ \\
\hline 4-Methyl-2-pentanone & WA & & $\mathbf{U}$ & 10 & & NS & $\mu g / L$ \\
\hline Acetone & WA & & V & 16 & & NS & $\mu \mathrm{g} / \mathrm{L}$ \\
\hline Acetonitrile & WA & & $\mathbf{U}$ & 20 & & NS & $\mu g / L$ \\
\hline Acidity & WA & $\mathbf{A}$ & & 41 & & NS & $\mathrm{mg} / \mathrm{L}$ \\
\hline Acrolein & WA & & $\mathbf{U}$ & 10 & & NS & $\mu g / L$ \\
\hline Acrylonitrile & WA & & $\mathbf{U}$ & 10 & & NS & $\mu g / L$ \\
\hline Alkalinity & WA & & & 5 & & NS & $\mathrm{mg} / \mathrm{L}$ \\
\hline Allyl chloride & WA & & $\mathbf{U}$ & 100 & & NS & $\mu g / L$ \\
\hline Aluminum & $\mathbf{W A}$ & & & 412 & & NS & $\mu g / L$ \\
\hline Antimony & WA & & $\mathbf{U}$ & 60 & & 6 & $\mu g / !$. \\
\hline Arsenic & WA & & $\mathbf{U}$ & 10 & & 50 & $\mu g / L$ \\
\hline Barium & WA & & $\mathbf{U}$ & 200 & & 2000 & $\mu g / L$ \\
\hline Benzene & WA & & $\mathbf{U}$ & 5 & & 5 & $\mu g / L$ \\
\hline Beryllium & WA & & $\mathbf{U}$ & 5 & & 4 & $\mu g / L$ \\
\hline Bromodichloromethane & WA & & $\mathbf{U}$ & 5 & & NS & $\mu g / L$ \\
\hline Bromoform & WA & & $\mathbf{U}$ & 5 & & NS & $\mu g / L$ \\
\hline Cadmium & WA & & $\mathbf{U}$ & 5 & & 5 & $\mu \mathrm{g} / \mathrm{L}$ \\
\hline Calcium & WA & & $\mathbf{U}$ & 5000 & & NS & $\mu g / L$ \\
\hline Carbon disulfide & WA & & $\mathbf{U}$ & 5 & & NS & $\mu g / L$ \\
\hline Carbon tetrachloride & WA & & $\mathbf{U}$ & 5 & & 5 & $\mu g / L$ \\
\hline Cesium-137 & TM & & & 0.39 & 7.8 & 200 & $\mathrm{pCi} / \mathbf{L}$ \\
\hline Chloride & WA & & & 2.96 & & NS & $\mathrm{mg} / \mathrm{L}$ \\
\hline Chlorobenzene & WA & & $\mathbf{U}$ & 5 & & 100 & $\mu g / L$ \\
\hline Chloroethane & WA & & $\mathbf{U}$ & 10 & & NS & $\mu \mathrm{g} / \mathrm{L}$ \\
\hline Chloroform & WA & & $\mathbf{U}$ & 5 & & 100 & $\mu g / L$ \\
\hline Chloroprene & WA & & $\mathrm{U}$ & 20 & & NS & $\mu g / L$ \\
\hline Chromium & WA & & $\mathbf{U}$ & 10 & & 100 & $\mu g / L$ \\
\hline cis-1,3-Dichloropropene & WA & & $\mathbf{U}$ & 5 & & NS & $\mu g / L$ \\
\hline Cobalt & WA & & $\mathbf{U}$ & 50 & & NS & $\mu \mathrm{g} / \mathrm{L}$ \\
\hline Copper & WA & & $\mathbf{U}$ & 25 & & 1000 & $\mu g / L$ \\
\hline Dibromochloromethane & WA & & U & 5 & & NS & $\mu \mathrm{g} / \mathrm{L}$ \\
\hline Dichlorodifluoromethane & WA & & U & 10 & & NS & $\mu g / L$ \\
\hline Ethylbenzene & WA & & $\mathbf{U}$ & 5 & & 700 & $\mu g / L$ \\
\hline Fluoride & WA & & $\mathbf{U}$ & 0.1 & & 4 & $\mathrm{mg} / \mathrm{L}$ \\
\hline
\end{tabular}


UTR051A Laboratory Analyses (cont'd)

\begin{tabular}{|c|c|c|c|c|c|c|c|c|c|}
\hline Analyte & Lab & Rep & Avg & Modifier & & Result & Acc & Stand & Units \\
\hline Gross alpha & TM & & & & & 0.7 & 1.2 & 15 & $\mathrm{pCi} / \mathrm{L}$ \\
\hline Iron & WA & & & & & 399 & & 300 & $\mu g / L$ \\
\hline Isobutyl alcohol & WA & & & $\mathbf{U}$ & & 20 & & NS & $\mu g / L$ \\
\hline Lead & WA & & & $\mathbf{U}$ & & 3 & & 50 & $\mu g / L$ \\
\hline Magnesium & WA & & & $\mathbf{U}$ & & 5000 & & NS & $\mu g / L$ \\
\hline Manganese & WA & & & & & 52.9 & & 50 & $\mu g / L$ \\
\hline Mercury & WA & & & $\mathbf{U}$ & & 0.2 & & 2 & $\mu g / L$ \\
\hline Methacrylonitrile & WA & & & $\mathbf{U}$ & & 20 & & NS & $\mu g / L$ \\
\hline Methyl bromide & WA & & & $\mathbf{U}$ & & 10 & & NS & $\mu g / L$ \\
\hline Methyl chloride & WA & & & $\mathbf{U}$ & & 10 & & NS & $\mu g /$ \\
\hline Methyl ethyl ketone & WA & & & $\mathbf{U}$ & & 10 & & NS & $\mu g / L$ \\
\hline Methyl iodide & WA & & & $\mathbf{U}$ & & 10 & & NS & $\mu g / L$ \\
\hline Methylene bromide & WA & & & $\mathbf{U}$ & & 10 & & NS & $\mu g / L$ \\
\hline Methylene chloride & WA & & & $\mathbf{J}$ & $\mathbf{V}$ & 4.18 & & 5 & $\mu g / L$ \\
\hline Nickel & WA & & & $\mathbf{U}$ & & 40 & & 100 & $\mu g / L$ \\
\hline Nitrate as nitrogen & WA & & & $\mathbf{J}$ & $\mathbf{Q}$ & 0.23 & & 10 & $\mathrm{mg} / \mathrm{L}$ \\
\hline Nonvolatile beta & TM & & & & & 2.8 & 2.7 & 50 & pCi/L \\
\hline Potassium & WA & & & $\mathbf{U}$ & & 5000 & & NS & $\mu g / \Omega$ \\
\hline Propionitrile & WA & & & U & & 50 & & NS & $\mu g / L$ \\
\hline Selenium & WA & & & $\mathbf{U}$ & & 5 & & 50 & $\mu g / L$ \\
\hline Silver & WA & & & $\mathbf{U}$ & & 10 & & 50 & $\mu \mathrm{g} / \mathrm{L}$ \\
\hline Sodium & WA & & & $\mathbf{U}$ & & 5000 & & NS & $\mu g / L$ \\
\hline Styrene & WA & & & $\mathbf{U}$ & & 5 & & 100 & $\mu g / L$ \\
\hline Sulfate & WA & & & & & 4.7 & & 400 & $\mathrm{mg} / \mathrm{L}$ \\
\hline Sulfide & WA & & & $\mathbf{U}$ & & 0.1 & & NS & $\mathrm{mg} / \mathrm{L}$ \\
\hline Tetrachloroethylene & WA & & & $\mathbf{U}$ & & 5 & & 5 & $\mu g / L$ \\
\hline Thallium & WA & & & $\mathbf{U}$ & & 10 & & 2 & $\mu g / L$ \\
\hline Toluene & WA & & & $\mathbf{U}$ & & 5 & & 1000 & $\mu g / L$ \\
\hline Total hardness & WA & & & & & 7.84 & & NS & $\mathrm{mg} / \mathrm{L}$ \\
\hline Total organic carbon & WA & & & & & 1.58 & & NS & $m g / L$ \\
\hline Total suspended solids & WA & & & & & 8 & & NS & $\mathrm{mg} / \mathrm{L}$ \\
\hline trans-1,3-Dichloropropene & WA & & & $\mathbf{U}$ & & 5 & & NS & $\mu g / L$ \\
\hline trans-1,4-Dichloro-2-butene & WA & & & $\mathbf{U}$ & & 100 & & NS & $\mu g / L$ \\
\hline Trichloroethylene & WA & & & $\mathbf{U}$ & & 5 & & 5 & $\mu \mathrm{g} / \mathrm{L}$ \\
\hline Trichlorofluoromethane & WA & & & $\mathbf{U}$ & & 5 & & NS & $\mu g / L$ \\
\hline Tritium & TM & & & & & 12.14 & 0.8 & 20 & $\mathrm{pCi} / \mathrm{ml}$ \\
\hline Turbidity & WA & & & & & 23.8 & & NS & NTU \\
\hline Vanadium & WA & & & $\mathrm{U}$ & & 50 & & NS & $\mu \mathrm{g} / \mathrm{L}$ \\
\hline Vinyl acetate & WA & & & $\mathbf{U}$ & & 10 & & NS & $\mu \mathrm{g} / \mathrm{L}$ \\
\hline Vinyl chloride & WA & & & $\mathbf{U}$ & & 10 & & 2 & $\mu \mathrm{g} / \mathrm{L}$ \\
\hline Xylenes (total) & WA & & & $\mathbf{U}$ & & 5 & & 10000 & $\mu g / L$ \\
\hline Zinc & WA & & & & & 24.8 & & 5000 & $\mu \mathrm{g} / \mathrm{L}$ \\
\hline
\end{tabular}




\section{UTROS3}

Field Measurements:

Sample Date: 8/25/93

pH: 5.27

Specific Conductance: $31 \mu \mathrm{S} / \mathrm{cm}$

Red/Oxid Potential: $155 \mathrm{mv}$

Dissolved Oxygen: $4.9 \mathrm{mg} / \mathrm{L}$

\begin{tabular}{|c|c|c|c|c|c|c|c|}
\hline Analyte & Lab & Avg & Modifier & Result & Acc & Stand & Units \\
\hline 1,1,1,2-Tetrachloroethane & WA & & $\mathbf{U}$ & 10 & & NS & $\mu g / L$ \\
\hline 1,1,1-Trichloroethane & WA & & $\mathbf{U}$ & 5 & & 200 & $\mu g / L$ \\
\hline 1,1,2,2-Tetrachloroethane & WA & & $\mathbf{U}$ & 5 & & NS & $\mu g / L$ \\
\hline 1,1,2-Trichloroethane & WA & & $\mathbf{U}$ & 5 & & 5 & $\mu g / L$ \\
\hline 1,1-Dichloroethane & WA & & $\mathbf{U}$ & 5 & & NS & $\mu g / L$ \\
\hline 1,1-Dichloroethylene & WA & & $\mathbf{U}$ & 5 & & 7 & $\mu \mathrm{g} / \mathrm{L}$ \\
\hline 1,2,3-Trichloropropane & WA & & $\mathbf{U}$ & 10 & & NS & $\mu g / L$ \\
\hline 1,2-Dibromo-3-chloropropane & WA & & $\mathbf{U}$ & 20 & & 0.2 & $\mu g /$ \\
\hline 1,2-Dibromoethane & WA & & $\mathbf{U}$ & 20 & & NS & $\mu g / L$ \\
\hline 1,2-Dichloroethane & WA & & $\mathbf{U}$ & 5 & & 5 & $\mu g / L$ \\
\hline 1,2-Dichloroethylene (total) & WA & & $\mathbf{U}$ & 5 & & 7 & $\mu g / L$ \\
\hline 1,2-Dichloropropane & WA & & $\mathbf{U}$ & 5 & & 5 & $\mu g / L$ \\
\hline 2-Hexanone & WA & & $\mathbf{U}$ & 10 & & NS & $\mu g / L$ \\
\hline 4-Methyl-2-pentanone & WA & & $\mathbf{U}$ & 10 & & NS & $\mu g / L$ \\
\hline Acetone & WA & & J & 5.68 & & NS & $\mu g / L$ \\
\hline Acetonitrile & WA & & $\mathbf{U}$ & 20 & & NS & $\mu \mathrm{g} / \mathrm{L}$ \\
\hline Acidity & WA & & & 34 & & NS & $\mathrm{mg} / \mathrm{L}$ \\
\hline Acrolein & WA & & $\mathbf{U}$ & 10 & & NS & $\mu \mathrm{g} / \mathrm{L}$ \\
\hline Acrylonitrile & WA & & $\mathbf{U}$ & 10 & & NS & $\mu \mathrm{g} / \mathrm{L}$ \\
\hline Alkalinity & WA & & & 5.5 & & NS & $\mathrm{mg} / \mathrm{L}$ \\
\hline Allyl chloride & WA & & $\mathbf{U}$ & 100 & & NS & $\mu g / L$ \\
\hline Aluminum & WA & & $\mathbf{U}$ & 200 & & NS & $\mu g / L$ \\
\hline Antimony & WA & & $\mathbf{U}$ & 60 & & 6 & $\mu \mathrm{g} / \mathrm{L}$ \\
\hline Arsenic & WA & & $\mathbf{U}$ & 10 & & 50 & $\mu g / L$ \\
\hline Barium & WA & & $\mathbf{U}$ & 200 & & 2000 & $\mu \mathrm{g} / \mathrm{L}$ \\
\hline Benzene & WA & & $\mathbf{U}$ & 5 & & 5 & $\mu \mathrm{g} / \mathrm{L}$ \\
\hline Beryllium & WA & & $\mathbf{U}$ & 5 & & 4 & $\mu g / L$ \\
\hline Bromodichloromethane & WA & & $\mathrm{U}$ & 5 & & NS & $\mu \mathrm{g} / \mathrm{L}$ \\
\hline Bromoform & WA & & $\mathbf{U}$ & 5 & & NS & $\mu \mathrm{g} / \mathrm{L}$ \\
\hline Cadmium & WA & & $\mathrm{U}$ & 5 & & 5 & $\mu \mathrm{g} / \mathrm{L}$ \\
\hline Calcium & WA & & & 6820 & & NS & $\mu \mathrm{g} / \mathrm{L}$ \\
\hline Carbon disulfide & WA & & $\mathbf{U}$ & 5 & & NS & $\mu g / L$ \\
\hline Carbon tetrachloride & WA & & $\mathbf{U}$ & 5 & & 5 & $\mu g / L$ \\
\hline Cesium-137 & TM & & $\mathbf{U}$ & 10 & & 200 & $\mathrm{pCi} / \mathrm{L}$ \\
\hline Chloride & WA & & & 2.73 & & NS & $\mathrm{mg} / \mathrm{L}$ \\
\hline Chlorobenzene & WA & & $\mathbf{U}$ & 5 & & 100 & $\mu g / L$ \\
\hline Chloroethane & WA & & $\mathrm{U}$ & 10 & & NS & $\mu g / L$ \\
\hline Chloroform & WA & & U & 5 & & 100 & $\mu \mathrm{g} / \mathrm{L}$ \\
\hline Chloroprene & WA & & $\mathbf{U}$ & 20 & & NS & $\mu \mathrm{g} / \mathrm{L}$ \\
\hline Chromium & WA & & $\mathbf{U}$ & 10 & & 100 & $\mu \mathrm{g} / \mathrm{L}$ \\
\hline cis-1,3-Dichloropropene & WA & & $\mathbf{U}$ & 5 & & NS & $\mu g / L$ \\
\hline Cobalt & WA & & $\mathbf{U}$ & 50 & & NS & $\mu \mathrm{g} / \mathrm{L}$ \\
\hline Copper & WA & & $\mathrm{U}$ & 25 & & 1000 & $\mu \mathrm{g} / \mathrm{L}$ \\
\hline Dibromochloromethane & WA & & $\mathbf{U}$ & 5 & & NS & $\mu g / L$ \\
\hline Dichlorodifluoromethane & WA & & U & 10 & & NS & $\mu g / L$ \\
\hline Ethylbenzene & WA & & $\mathbf{U}$ & 5 & & 700 & $\mu \mathrm{g} / \mathrm{L}$ \\
\hline Fluoride & WA & & $\mathbf{U}$ & 0.1 & & 4 & $\mathrm{mg} / \mathrm{L}$ \\
\hline
\end{tabular}


UTR053 Laboratory Analyses (cont'd)

\begin{tabular}{|c|c|c|c|c|c|c|c|c|c|}
\hline Analyte & Lab & Rep & Avg & Modifier & & Result & Acc & Stand & Units \\
\hline Gross alpha & TM & & & $\mathrm{U}$ & & 0.5 & & 15 & $\mathrm{pCi} / \mathrm{L}$ \\
\hline Iron & WA & & & $\mathbf{U}$ & & 100 & & 300 & $\mu g / L$ \\
\hline Isobutyl alcohol & WA & & & $\mathbf{U}$ & & 20 & & NS & $\mu \mathrm{g} / \mathrm{L}$ \\
\hline Lead & WA & & & $\mathbf{U}$ & & 3 & & 50 & $\mu \mathrm{g} / \mathrm{L}$ \\
\hline Magnesium & WA & & & $\mathbf{U}$ & & 5000 & & NS & $\mu g / L$ \\
\hline Manganese & WA & & & $\mathbf{U}$ & & 15 & & 50 & $\mu g / L$ \\
\hline Mercury & WA & & & $\mathbf{U}$ & & 0.2 & $\cdot$ & 2 & $\mu \mathrm{g} / \mathrm{L}$ \\
\hline Methacrylonitrile & WA & & & $\mathbf{U}$ & & 20 & & NS & $\mu \mathrm{g} / \mathrm{L}$ \\
\hline Methyl bromide & WA & & & U & & 10 & & NS & $\mu \mathrm{g} / \mathrm{L}$ \\
\hline Methyl chloride & WA & & & $\mathbf{U}$ & & 10 & & NS & $\mu g / L$ \\
\hline Methyl ethyl ketone & WA & & & $\mathbf{U}$ & & 10 & & NS & $\mu \mathrm{g} / \mathrm{L}$ \\
\hline Methyl iodide & WA & & & $\mathbf{U}$ & & 10 & & NS & $\mu g / L$ \\
\hline Methylene bromide & WA & & & $\mathbf{U}$ & & 10 & & NS & $\mu \mathrm{g} / \mathrm{L}$ \\
\hline Methylene chloride & WA & & & U & V & 4.52 & & 5 & $\mu g / L$ \\
\hline Nickel & WA & & & $\mathbf{U}$ & & 40 & & 100 & $\mu g / L$ \\
\hline Nitrate as nitrogen & WA & & & $\mathbf{J}$ & $\mathbf{Q}$ & 0.436 & & 10 & $\mathrm{mg} / \mathrm{L}$ \\
\hline Nonvolatile beta & TM & & & $\mathbf{U}$ & & 0.7 & & 50 & $\mathrm{pCi} / \mathrm{L}$ \\
\hline Potassium & WA & & & $\mathbf{U}$ & & 5000 & & NS & $\mu g / L$ \\
\hline Propionitrile & WA & & & $\mathbf{U}$ & & 50 & & NS & $\mu \mathrm{g} / \mathrm{L}$ \\
\hline Selenium & WA & & & $\mathbf{U}$ & & 5 & & 50 & $\mu g / L$ \\
\hline Silver & WA & & & $\mathbf{U}$ & & 10 & & 50 & $\mu g / L$ \\
\hline Sodium & WA & & & $\mathbf{U}$ & & 5000 & & NS & $\mu g / L$ \\
\hline Styrene & WA & & & $U$ & & 5 & & 100 & $\mu \mathrm{g} / \mathrm{L}$ \\
\hline Sulfate & WA & & & $\mathbf{U}$ & & 2.5 & & 400 & $\mathrm{mg} / \mathrm{L}$ \\
\hline Sulfide & WA & & & $\mathbf{U}$ & & 0.1 & & NS & $\mathrm{mg} / \mathrm{L}$ \\
\hline Tetrachloroethylene & WA & & & $\mathrm{U}$ & & 5 & & 5 & $\mu g / L$ \\
\hline Thallium & WA & & & $\mathbf{U}$ & & 10 & & 2 & $\mu \mathrm{g} / \mathrm{L}$ \\
\hline Toluene & WA & & & $\mathrm{U}$ & & 5 & & 1000 & $\mu g / L$ \\
\hline Total hardness & WA & & & & & 9.8 & & NS & $\mathrm{mg} / \mathrm{L}$ \\
\hline Total organic carbon & WA & & & & & 0.519 & & NS & $\mathrm{mg} / \mathrm{L}$ \\
\hline Total suspended solids & WA & & & & & 7 & & NS & $\mathrm{mg} / \mathrm{L}$ \\
\hline trans-1,3-Dichloropropene & WA & & & $U$ & & 5 & & NS & $\mu g / L$ \\
\hline trans-1,4-Dichloro-2-butene & WA & & & $\mathbf{U}$ & & 100 & & NS & $\mu g / L$ \\
\hline Trichloroethylene & WA & & & $\mathbf{U}$ & & 5 & & 5 & $\mu \mathrm{g} / \mathrm{L}$ \\
\hline Trichlorofluoromethane & WA & & & $\mathbf{U}$ & & 5 & & NS & $\mu g / L$ \\
\hline Tritium & TM & & & & & 10.75 & 0.74 & 20 & $\mathrm{pCi} / \mathrm{ml}$ \\
\hline Turbidity & WA & & & & & 0.45 & & NS & NTU \\
\hline Vanadium & WA & & & $\mathbf{U}$ & & 50 & & NS & $\mu \mathrm{g} / \mathrm{L}$ \\
\hline Vinyl acetate & WA & & & $\mathrm{U}$ & & 10 & & NS & $\mu \mathrm{g} / \mathrm{L}$ \\
\hline Vinyl chloride & WA & & & $\mathrm{U}$ & & 10 & & 2 & $\mu g / L$ \\
\hline Xylenes (total) & WA & & & $\mathbf{U}$ & & 5 & & 10000 & $\mu \mathrm{g} / \mathrm{L}$ \\
\hline Zinc & WA & & & $\mathbf{U}$ & & 20 & & 5000 & $\mu \mathrm{g} / \mathrm{L}$ \\
\hline
\end{tabular}




\section{UTR056}

Field Measurements:

Sample Date: 8/25/93

pH: 5.64

Specific Conductance: $33 \mu \mathrm{S} / \mathrm{cm}$

Red/Oxid Potential: $176 \mathrm{mv}$

Dissolved Oxygen: $1.1 \mathrm{mg} / \mathrm{L}$

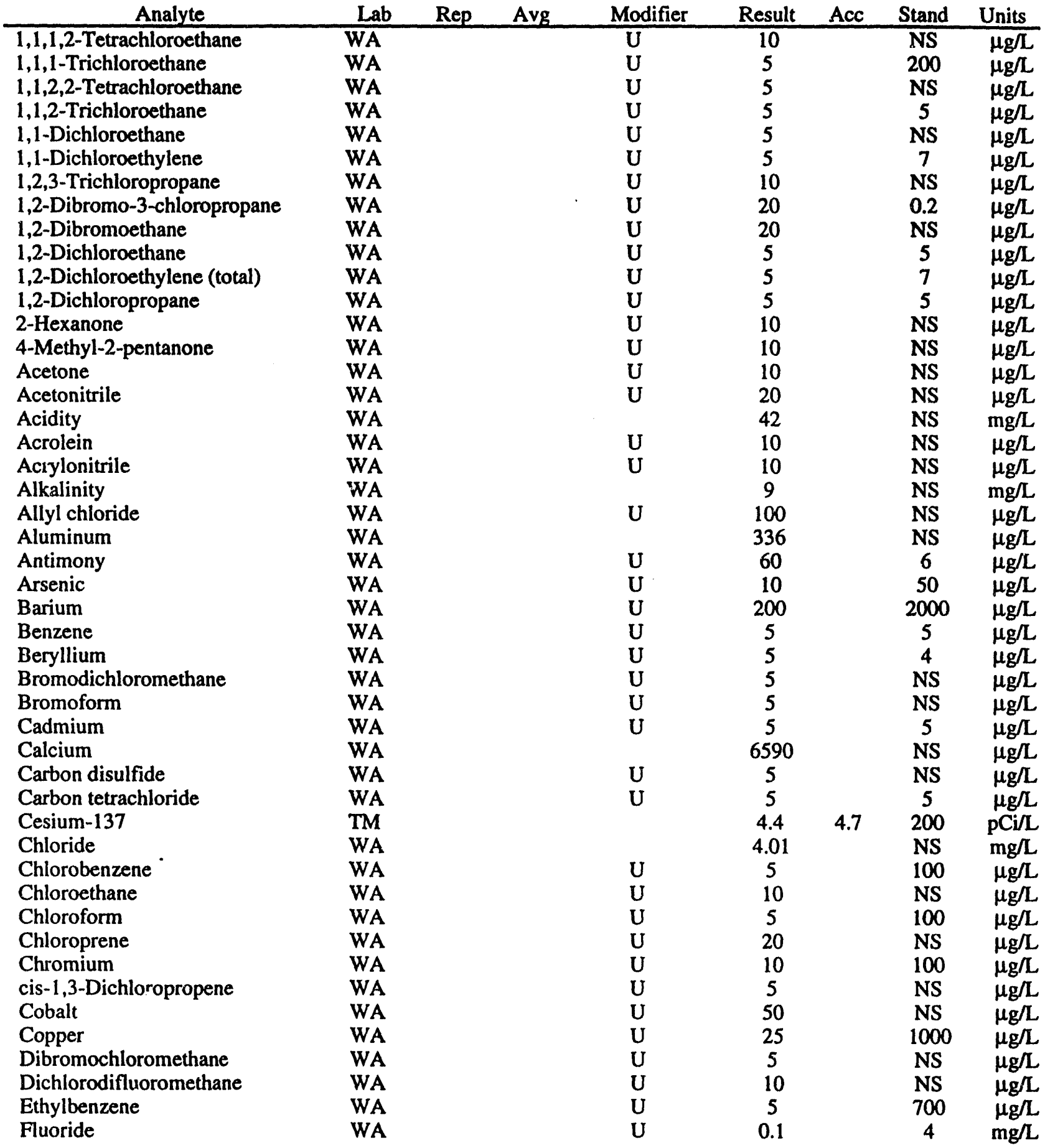


UTR056 Laboratory Analyses (cont'd)

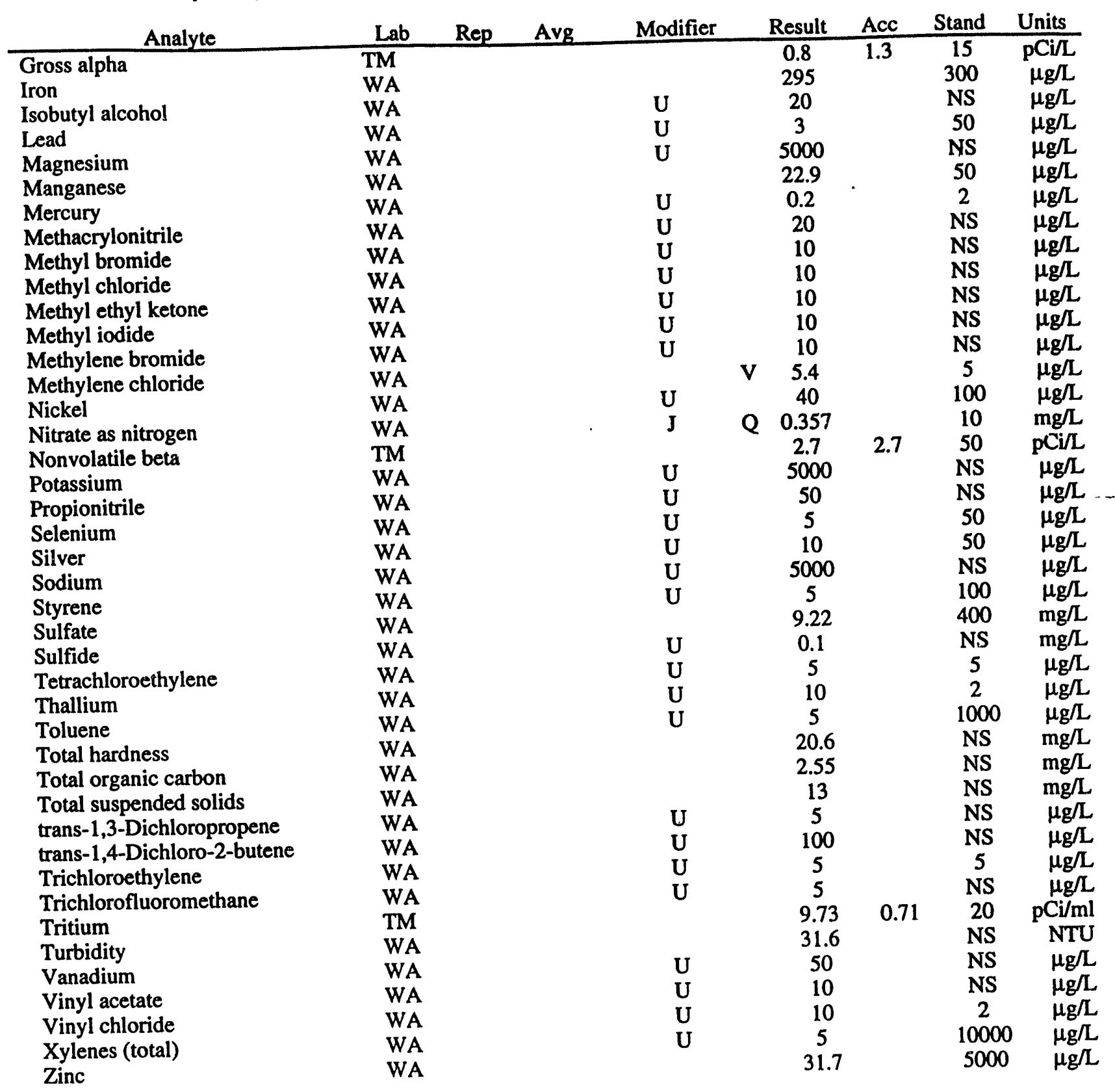




\section{UTR060}

Field Measurements:

Sample Date: $8 / 25 / 93$

$\mathrm{pH}: 5.86$

Specific Conductance: $31 \mu \mathrm{S} / \mathrm{cm}$

Red/Oxid Potential: $181 \mathrm{mv}$

Dissolved Oxygen: $1.4 \mathrm{mg} / \mathrm{L}$

\begin{tabular}{|c|c|c|c|c|c|c|c|c|}
\hline Analyte & Lab & Rep & Avg & Modifier & Result & Acc & Stand & Units \\
\hline 1,1,1,2-Tetrachloroethane & WA & & & $\mathbf{U}$ & 10 & & NS & $\mu g / L$ \\
\hline 1,1,1-Trichloroethane & WA & & & $\mathbf{U}$ & 5 & & 200 & $\mu g / L$ \\
\hline 1,1,2,2-Tetrachloroethane & WA & & & $\mathbf{U}$ & 5 & & NS & $\mu g / L$ \\
\hline 1,1,2-Trichloroethane & WA & & & $\mathbf{U}$ & 5 & & 5 & $\mu \mathrm{g} / \mathrm{L}$ \\
\hline 1,1-Dichloroethane & WA & & & $\mathbf{U}$ & 5 & & NS & $\mu g / L$ \\
\hline 1,1-Dichloroethylene & WA & & & $\mathrm{U}$ & 5 & & 7 & $\mu \mathrm{g} / \mathrm{L}$ \\
\hline 1,2,3-Trichloropropane & WA & & & $\mathbf{U}$ & 10 & & NS & $\mu g / L$ \\
\hline 1,2-Dibromo-3-chloropropane & WA & & & $\mathbf{U}$ & 20 & & 0.2 & $\mu g / L$ \\
\hline 1,2-Dibromoethane & WA & & & $\mathbf{U}$ & 20 & & NS & $\mu g / L$ \\
\hline 1,2-Dichloroethane & WA & & & $\mathbf{U}$ & 5 & & 5 & $\mu \mathrm{g} / \mathrm{L}$ \\
\hline 1,2-Dichloroethylene (total) & WA & & & $\mathbf{U}$ & 5 & & 7 & $\mu g / L$ \\
\hline 1,2-Dichloropropane & WA & & & $\mathbf{U}$ & 5 & & 5 & $\mu \mathrm{g} / \mathrm{L}$ \\
\hline 2-Hexanone & WA & & & $\mathbf{U}$ & 10 & & NS & $\mu \mathrm{g} / \mathrm{L}$ \\
\hline 4-Methyl-2-pentanone & WA & & & $\mathbf{U}$ & 10 & & NS & $\mu g / L$ \\
\hline Acetone & WA & & & $\mathbf{U}$ & 9.65 & & NS & $\mu \mathrm{g} / \mathrm{L}$ \\
\hline Acetonitrile & WA & & & $\mathrm{U}$ & 20 & & NS & $\mu \mathrm{g} / \mathrm{L}$ \\
\hline Acidity & WA & & & & 58 & & NS & $\mathrm{mg} / \mathrm{L}$ \\
\hline Acrolein & WA & & & $\mathbf{U}$ & 10 & & NS & $\mu g / L$ \\
\hline Acrylonitrile & WA & & & $\mathbf{U}$ & 10 & & NS & $\mu g / L$ \\
\hline Alkalinity & WA & & & & 4 & & NS & $\mathrm{mg} / \mathrm{L}$ \\
\hline Allyl chloride & WA & & & $U$ & 100 & & NS & $\mu \mathrm{g} / \mathrm{L}$ \\
\hline Aluminum & WA & & & U & 200 & & NS & $\mu \mathrm{g} / \mathrm{L}$ \\
\hline Antimony & WA & & & $\mathbf{U}$ & 60 & & 6 & $\mu \mathrm{g} / \mathrm{L}$ \\
\hline Arsenic & WA & & & $\mathbf{U}$ & 10 & & 50 & $\mu \mathrm{g} / \mathrm{L}$ \\
\hline Barium & WA & & & $\mathbf{U}$ & 200 & & 2000 & $\mu g / L$ \\
\hline Benzene & WA & & & $\mathbf{U}$ & 5 & & 5 & $\mu \mathrm{g} / \mathrm{L}$ \\
\hline Beryllium & WA & & & $\mathrm{U}$ & 5 & & 4 & $\mu \mathrm{g} / \mathrm{L}$ \\
\hline Bromodichloromethane & WA & & & $\mathbf{U}$ & 5 & & NS & $\mu \mathrm{g} / \mathrm{L}$ \\
\hline Bromoform & WA & & & $\mathrm{U}$ & 5 & & NS & $\mu \mathrm{g} / \mathrm{L}$ \\
\hline Cadmium & WA & & & $\mathbf{U}$ & 5 & & 5 & $\mu g / L$ \\
\hline Calcium & WA & & & $U$ & 5000 & & NS & $\mu \mathrm{g} / \mathrm{L}$ \\
\hline Carbon disulfide & WA & & & $\mathbf{U}$ & 5 & & NS & $\mu \mathrm{g} / \mathrm{L}$ \\
\hline Carbon tetrachloride & WA & & & U & 5 & & 5 & $\mu \mathrm{g} / \mathrm{L}$ \\
\hline Cesium-137 & TM & & & $\mathbf{U}$ & 9.2 & & 200 & $\mathrm{pCi} / \mathrm{L}$ \\
\hline Chloride & WA & & & & 4.09 & & NS & $\mathrm{mg} / \mathrm{L}$ \\
\hline Chlorobenzene & WA & & & $\mathrm{U}$ & 5 & & 100 & $\mu \mathrm{g} / \mathrm{L}$ \\
\hline Chloroethane & WA & & & $\mathrm{U}$ & 10 & & NS & $\mu \mathrm{g} / \mathrm{L}$ \\
\hline Chloroform & WA & & & $\mathbf{U}$ & 5 & & 100 & $\mu \mathrm{g} / \mathrm{L}$ \\
\hline Chloroprene & WA & & & $\mathbf{U}$ & 20 & & NS & $\mu \mathrm{g} / \mathrm{L}$ \\
\hline Chromium & WA & & & U & 10 & & 100 & $\mu \mathrm{g} / \mathrm{L}$ \\
\hline cis-1,3-Dichloropropene & WA & & & $\mathbf{U}$ & 5 & & NS & $\mu \mathrm{g} / \mathrm{L}$ \\
\hline Cobalt & WA & & & $\mathbf{U}$ & 50 & & NS & $\mu \mathrm{g} / \mathrm{L}$ \\
\hline Copper & WA & & & $\mathbf{U}$ & 25 & & 1000 & $\mu g / L$ \\
\hline Dibromochloromethane & WA & & & $\mathbf{U}$ & 5 & & NS & $\mu \mathrm{g} / \mathrm{L}$ \\
\hline Dichlorodifluoromethane & WA & & & $\mathbf{U}$ & 10 & & NS & $\mu \mathrm{g} / \mathrm{L}$ \\
\hline Ethylbenzene & WA & & & $\mathbf{U}$ & 5 & & 700 & $\mu \mathrm{g} / \mathrm{L}$ \\
\hline Fluoride & WA & & & $\mathbf{U}$ & 0.1 & & 4 & $\mathrm{mg} / \mathrm{L}$ \\
\hline
\end{tabular}


UTR060 Laboratory Analyses (cont'd)

\begin{tabular}{|c|c|c|c|c|c|c|c|c|c|}
\hline Analyte & Lab & Rep & Avg & Modifier & & Result & Acc & Stand & Units \\
\hline Gross alpha & TM & & & $\bar{U}$ & & 0.5 & & 15 & $\overline{\mathrm{pCi} / \mathrm{L}}$ \\
\hline Iron & WA & & & & & 252 & & 300 & $\mu g / L$ \\
\hline Isobutyl alcohol & WA & & & $\mathbf{U}$ & & 20 & & NS & $\mu g / L$ \\
\hline Lead & WA & & & $\mathbf{U}$ & & 3 & & 50 & $\mu g / L$ \\
\hline Magnesium & WA & & & $\mathbf{U}$ & & 5000 & & NS & $\mu g / L$ \\
\hline Manganese & WA & & & & & 19.8 & & 50 & $\mu \mathrm{g} / \mathrm{L}$ \\
\hline Mercury & WA & & & $\mathbf{U}$ & & 0.2 & $\cdot$ & 2 & $\mu g / L$ \\
\hline Methacrylonitrile & WA & & & $\mathbf{U}$ & & 20 & & NS & $\mu \mathrm{g} / \mathrm{L}$ \\
\hline Methyl bromide & WA & & & $\mathbf{U}$ & & 10 & & NS & $\mu \mathrm{g} / \mathrm{L}$ \\
\hline Methyl chloride & WA & & & $\mathbf{U}$ & & 10 & & NS & $\mu \mathrm{g} / \mathrm{L}$ \\
\hline Methyl ethyl ketone & WA & & & $\mathbf{U}$ & & 10 & & NS & $\mu g / L$ \\
\hline Methyl iodide & WA & & & $\mathbf{U}$ & & 10 & & NS & $\mu \mathrm{g} / \mathrm{L}$ \\
\hline Methylene bromide & WA & & & $\mathrm{U}$ & & 10 & & NS & $\mu g / L$ \\
\hline Methylene chloride & WA & & & & V & 5.05 & & 5 & $\mu g / L$ \\
\hline Nickel & WA & & & $\mathrm{J}$ & & 40 & & 100 & $\mu g / L$ \\
\hline Nitrate as nitrogen & WA & & . & $\mathbf{J}$ & Q & 0.094 & & 10 & $\mathrm{mg} / \mathrm{L}$ \\
\hline Nonvolatile beta & TM & & & $\mathbf{U}$ & & 0.8 & & 50 & $\mathrm{pCi} / \mathrm{L}$ \\
\hline Potassium & WA & & & $\mathbf{U}$ & & 5000 & & NS & $\mu \mathrm{g} / \mathrm{L}$ \\
\hline Propionitrile & WA & & & $\mathbf{U}$ & & 50 & & NS & $\mu g / L$ \\
\hline Selenium & WA & & & $\mathbf{U}$ & & 5 & & 50 & $\mu g / L$ \\
\hline Silver & WA & & & $\mathrm{U}$ & & 10 & & 50 & $\mu g / L$ \\
\hline Sodium & WA & & & $\mathbf{U}$ & & 5000 & & NS & $\mu g / L$ \\
\hline Styrene & WA & & & $\mathrm{U}$ & & 5 & & 100 & $\mu g / L$ \\
\hline Sulfate & WA & & & $\mathbf{U}$ & & 2.5 & & 400 & $\mathrm{mg} / \mathrm{L}$ \\
\hline Sulfide & WA & & & $\mathbf{U}$ & & 0.1 & & NS & $\mathrm{mg} / \mathrm{L}$ \\
\hline Tetrachloroethylene & WA & & & $\mathrm{U}$ & & 5 & & 5 & $\mu g / L$ \\
\hline Thallium & WA & & & $\mathbf{U}$ & & 10 & & 2 & $\mu g / L$ \\
\hline Toluene & WA & & & $\mathbf{U}$ & & 5 & & 1000 & $\mu \mathrm{g} / \mathrm{L}$ \\
\hline Total hardness & WA & & & $\mathbf{U}$ & & 1 & & NS & $\mathrm{mg} / \mathrm{L}$ \\
\hline Total organic carbon & WA & & & & & 1.87 & & NS & $\mathrm{mg} / \mathrm{L}$ \\
\hline Total suspended solids & WA & & & & & 13 & & NS & $\mathrm{mg} / \mathrm{L}$ \\
\hline trans-1,3-Dichloropropene & WA & & & $\mathrm{U}$ & & 5 & & NS & $\mu \mathrm{g} / \mathrm{L}$ \\
\hline trans-1,4-Dichloro-2-butene & WA & & & $\mathbf{U}$ & & 100 & & NS & $\mu g / L$ \\
\hline Trichloroethylene & WA & & & $\mathbf{U}$ & & 5 & & 5 & $\mu g / L$ \\
\hline Trichlorofluoromethane & WA & & & $\mathbf{U}$ & & 5 & & NS & $\mu \mathrm{g} / \mathrm{L}$ \\
\hline Tritium & TM & & & & & 15.2 & 0.88 & 20 & $\mathrm{pCi} / \mathrm{ml}$ \\
\hline Turbidity & WA & & & & & 0.23 & & NS & NTU \\
\hline Vanadium & WA & & & $\mathbf{U}$ & & 50 & & NS & $\mu g / L$ \\
\hline Vinyl acetate & WA & & & $\mathrm{U}$ & & 10 & & NS & $\mu g / L$ \\
\hline Vinyl chloride & WA & & & $\mathbf{U}$ & & 10 & & 2 & $\mu \mathrm{g} / \mathrm{L}$ \\
\hline Xylenes (total) & WA & & & $\mathbf{U}$ & & 5 & & 10000 & $\mu \mathrm{g} / \mathrm{L}$ \\
\hline Zinc & WA & & & $\mathrm{U}$ & & 20 & & 5000 & $\mu \mathrm{g} / \mathrm{L}$ \\
\hline
\end{tabular}




\section{UTR062}

Field Measurements:

Sample Date: $8 / 26 / 93$

$\mathrm{pH}: 5.86$

Specific Conductance: $45 \mu \mathrm{S} / \mathrm{cm}$

Red/Oxid Potential: $134 \mathrm{mv}$

Dissolved Oxygen: $1.0 \mathrm{mg} / \mathrm{L}$

\begin{tabular}{|c|c|c|c|c|c|c|c|c|}
\hline Analyte & Lab & Rep & Avg & Modifier & Result & Acc & Stand & Units \\
\hline 1,1,1,2-Tetrachloroethane & WA & & & $\overline{\mathbf{U}}$ & 10 & & NS & $\mu g / L$ \\
\hline 1,1,1,2-Tetrachloroethane & WA & & NA & $\mathbf{U}$ & 10 & & NS & $\mu g / L$ \\
\hline 1,1,1,2-Tetrachloroethane & WA & & NA & $\mathbf{U}$ & 10 & & NS & $\mu \mathrm{g} / \mathrm{L}$ \\
\hline 1,1,1-Trichloroethane & WA & & & $\mathbf{U}$ & 5 & & 200 & $\mu g / L$ \\
\hline 1,1,1-Trichloroethane & WA & & NA & $\mathbf{U}$ & 5 & & 200 & $\mu \mathrm{g} / \mathrm{L}$ \\
\hline 1,1,1-Trichloroethane & WA & & NA & $\mathbf{U}$ & 5 & & 200 & $\mu g / L$ \\
\hline 1,1,2,2-Tetrachloroethane & WA & & & $\mathbf{U}$ & 5 & & NS & $\mu g / L$ \\
\hline 1,1,2,2-Tetrachloroethane & WA & & $\mathbf{N A}$ & $\mathbf{U}$ & 5 & & NS & $\mu \mathrm{g} / \mathrm{L}$ \\
\hline 1,1,2,2-Tetrachloroethane & WA & & NA & $\mathbf{U}$ & 5 & & NS & $\mu g / L$ \\
\hline 1,1,2-Trichloroethane & WA & & & $\mathbf{U}$ & 5 & & 5 & $\mu g / L$ \\
\hline 1,1,2-Trichloroethane & WA & & NA & $\mathbf{U}$ & 5 & & 5 & $\mu g / L$ \\
\hline 1,1,2-Trichloroethane & WA & & NA & $\mathbf{U}$ & 5 & & 5 & $\mu \mathrm{g} / \mathrm{L}$ \\
\hline 1,1-Dichloroethane & WA & & & $\mathbf{U}$ & 5 & & NS & $\mu g / L$ \\
\hline 1,1-Dichloroethane & WA & & NA & $\mathbf{U}$ & 5 & & NS & $\mu g / L$ \\
\hline 1,1-Dichloroethane & WA & & $\mathbf{N A}$ & $\mathbf{U}$ & 5 & & NS & $\mu g / L$ \\
\hline 1,1-Dichloroethylene & WA & & & $\mathbf{U}$ & 5 & & 7 & $\mu g / L$ \\
\hline 1,2,3-Trichloropropane & WA & & & $\mathbf{U}$ & 10 & & NS & $\mu \mathrm{g} / \mathrm{L}$ \\
\hline 1,2,3-Trichloropropane & WA & & NA & U & 10 & & NS & $\mu g / L$ \\
\hline 1,2,3-Trichloropropane & WA & & NA & $\mathbf{U}$ & 10 & & NS & $\mu g / L$ \\
\hline 1,2-Dibromo-3-chloropropane & WA & & & $\mathbf{U}$ & 20 & & 0.2 & $\mu \mathrm{g} / \mathrm{L}$ \\
\hline 1,2-Dibromo-3-chloropropane & WA & & NA & $\mathbf{U}$ & 20 & & 0.2 & $\mu g / L$ \\
\hline 1,2-Dibromo-3-chloropropane & WA & & NA & $\mathbf{U}$ & 20 & & 0.2 & $\mu g / L$ \\
\hline 1,2-Dibromoethane & WA & & & $\mathbf{U}$ & 20 & & NS & $\mu \mathrm{g} / \mathrm{L}$ \\
\hline 1,2-Dibromoethane & WA & & NA & $\mathbf{U}$ & 20 & & NS & $\mu \mathrm{g} / \mathrm{L}$ \\
\hline 1,2-Dibromoethane & WA & & NA & $\mathbf{U}$ & 20 & & NS & $\mu \mathrm{g} / \mathrm{L}$ \\
\hline 1,2-Dichloroethane & WA & & & $\mathbf{U}$ & 5 & & 5 & $\mu \mathrm{g} / \mathrm{L}$ \\
\hline 1,2-Dichloroethane & WA & & NA & $\mathbf{U}$ & 5 & & 5 & $\mu \mathrm{g} / \mathrm{L}$ \\
\hline 1,2-Dichloroethane & WA & & NA & $\mathbf{U}$ & 5 & & 5 & $\mu \mathrm{g} / \mathrm{L}$ \\
\hline 1,2-Dichloroethylene (total) & WA & & & $\mathrm{U}$ & 5 & & 7 & $\mu g / L$ \\
\hline 1,2-Dichloroethylene (total) & WA & & NA & $\mathbf{U}$ & 5 & & 7 & $\mu \mathrm{g} / \mathrm{L}$ \\
\hline 1,2-Dichloroethylene (total) & WA & & NA & $\mathbf{U}$ & 5 & & 7 & $\mu g / L$ \\
\hline 1,2-Dichloropropane & WA & & & $\mathbf{U}$ & 5 & & 5 & $\mu g / L$ \\
\hline 1,2-Dichloropropane & WA & & NA & $\mathrm{U}$ & 5 & & 5 & $\mu \mathrm{g} / \mathrm{L}$ \\
\hline 1,2-Dichloropropane & WA & & NA & $\mathrm{U}$ & 5 & & 5 & $\mu \mathrm{g} / \mathrm{L}$ \\
\hline 2-Hexanone & WA & & & $\mathrm{U}$ & 10 & & NS & $\mu \mathrm{g} / \mathrm{L}$ \\
\hline 2-Hexanone & WA & & NA & $\mathrm{U}$ & 10 & & NS & $\mu \mathrm{g} / \mathrm{L}$ \\
\hline 2-Hexanone & WA & & NA & $\mathbf{U}$ & 10 & & NS & $\mu \mathrm{g} / \mathrm{L}$ \\
\hline 4-Methyl-2-pentanone & WA & & & $\mathrm{U}$ & 10 & & NS & $\mu \mathrm{g} / \mathrm{L}$ \\
\hline 4-Methyl-2-pentanone & WA & & NA & U & 10 & & NS & $\mu \mathrm{g} / \mathrm{L}$ \\
\hline 4-Methyl-2-pentanone & WA & & NA & $\mathbf{U}$ & 10 & & NS & $\mu g / L$ \\
\hline Acetone & WA & & & $\mathbf{J}$ & 4.67 & & NS & $\mu \mathrm{g} / \mathrm{L}$ \\
\hline Acetone & WA & & NA & $\mathbf{J}$ & 4.91 & & NS & $\mu \mathrm{g} / \mathrm{L}$ \\
\hline Acetone & WA & & NA & $\mathbf{J}$ & 3.46 & & NS & $\mu g / L$ \\
\hline Acetonitrile & WA & & & $\mathbf{U}$ & 20 & & NS & $\mu \mathrm{g} / \mathrm{L}$ \\
\hline Acetonitrile & WA & & NA & $\mathbf{U}$ & 20 & & NS & $\mu \mathrm{g} / \mathrm{L}$ \\
\hline Acetonitrile & WA & & NA & $\mathbf{U}$ & 20 & & NS & $\mu \mathrm{g} / \mathrm{L}$ \\
\hline Acidity & WA & & & & 36 & & NS & $\mathrm{mg} / \mathrm{L}$ \\
\hline
\end{tabular}


UTR062 Laboratory Analyses (cont'd)

\begin{tabular}{|c|c|c|c|c|c|c|c|c|}
\hline Analyte & Lab & Rep & Avg & Modifier & Result & Acc & Stand & Units \\
\hline Acrolein & WA & & & $\mathrm{U}$ & 10 & & NS & $\mu g / L$ \\
\hline Acrolein & WA & & NA & $\mathbf{U}$ & 10 & & NS & $\mu \mathrm{g} / \mathrm{L}$ \\
\hline Acrolein & WA & & NA & $\mathbf{U}$ & 10 & & NS & $\mu \mathrm{g} / \mathrm{L}$ \\
\hline Acrylonitrile & WA & & & $\mathbf{U}$ & 10 & & NS & $\mu \mathrm{g} / \mathrm{L}$ \\
\hline Acrylonitrile & WA & & NA & $\mathbf{U}$ & 10 & & NS & $\mu \mathrm{g} / \mathrm{L}$ \\
\hline Acrylonitrile & WA & & $\mathrm{NA}$ & $\mathrm{U}$ & 10 & & NS & $\mu \mathrm{g} / \mathrm{L}$ \\
\hline Alkalinity & WA & & $\mathbf{A}$ & & 10.75 & • & NS & $\mathrm{mg} / \mathrm{L}$ \\
\hline Allyl chloride & WA & & & $\mathbf{U}$ & 100 & & NS & $\mu \mathrm{g} / \mathrm{L}$ \\
\hline Allyl chloride & WA & & NA & $\mathbf{U}$ & 100 & & NS & $\mu g / L$ \\
\hline Allyl chloride & WA & & NA & $\mathrm{U}$ & 100 & & NS & $\mu g / L$ \\
\hline Aluminum & WA & & & $\mathbf{U}$ & 200 & & NS & $\mu \mathrm{g} / \mathrm{L}$ \\
\hline Aluminum & WA & & NA & $\mathbf{U}$ & 200 & & NS & $\mu g / L$ \\
\hline Antimony & WA & & & $\mathbf{U}$ & 60 & & 6 & $\mu g / L$ \\
\hline Antimony & WA & & NA & U & 60 & & 6 & $\mu g / L$ \\
\hline Arsenic & WA & & & $\mathbf{U}$ & 10 & & 50 & $\mu \mathrm{g} / \mathrm{L}$ \\
\hline Arsenic & WA & & $\mathbf{N A}$ & $\mathbf{U}$ & 10 & & 50 & $\mu g / L$ \\
\hline Barium & WA & & & $\mathrm{U}$ & 200 & & 2000 & $\mu g / L$ \\
\hline Barium & WA & & NA & $\mathbf{U}$ & 200 & & 2000 & $\mu g / L$ \\
\hline Benzene & WA & & & $\mathbf{U}$ & 5 & & 5 & $\mu \mathrm{g} / L$ \\
\hline Beryllium & WA & & & $\mathbf{U}$ & 5 & & 4 & $\mu \mathrm{g} / \mathrm{L}$ \\
\hline Beryllium & WA & & NA & $\mathrm{U}$ & 5 & & 4 & $\mu g / L$ \\
\hline Bromodichloromethane & WA & & & $\mathbf{U}$ & 5 & & NS & $\mu g / L$ \\
\hline Bromodichloromethane & WA & & NA & $\mathbf{U}$ & 5 & & NS & $\mu g / L$ \\
\hline Bromodichloromethane & WA & & NA & $\mathrm{U}$ & 5 & & NS & $\mu \mathrm{g} / \mathrm{L}$ \\
\hline Bromoform & WA & & & U & 5 & & NS & $\mu \mathrm{g} / \mathrm{L}$ \\
\hline Bromoform & WA & & NA & $U$ & 5 & & NS & $\mu \mathrm{g} / \mathrm{L}$ \\
\hline Bromoform & WA & & NA & $\mathrm{U}$ & 5 & & NS & $\mu \mathrm{g} / \mathrm{L}$ \\
\hline Cadmium & WA & & & $\mathbf{U}$ & 5 & & 5 & $\mu \mathrm{g} / \mathrm{L}$ \\
\hline Cadmium & WA & & NA & $\mathrm{U}$ & 5 & & 5 & $\mu \mathrm{g} / \mathrm{L}$ \\
\hline Calcium & WA & & & $\mathrm{U}$ & 5000 & & NS & $\mu \mathrm{g} / \mathrm{L}$ \\
\hline Calcium & WA & & NA & $\mathbf{U}$ & 5000 & & NS & $\mu \mathrm{g} / \mathrm{L}$ \\
\hline Carbon disulfide & WA & & & $\mathbf{U}$ & 5 & & NS & $\mu g / L$ \\
\hline Carbon disulfide & WA & & NA & $U$ & 5 & & NS & $\mu \mathrm{g} / \mathrm{L}$ \\
\hline Carbon disulfide & WA & & NA & $\mathrm{U}$ & 5 & & NS & $\mu g / L$ \\
\hline Carbon tetrachloride & WA & & & $\mathrm{U}$ & 5 & & 5 & $\mu \mathrm{g} / \mathrm{L}$ \\
\hline Carbon tetrachloride & WA & & NA & $\mathbf{U}$ & 5 & & 5 & $\mu g / L$ \\
\hline Carbon tetrachloride & WA & & NA & $\mathbf{U}$ & 5 & & 5 & $\mu g / L$ \\
\hline Cesium-137 & TM & & NA & U & 9.9 & & 200 & $\mathrm{pCi} / \mathrm{L}$ \\
\hline Cesium-137 & $\mathrm{TM}$ & & & $\mathrm{U}$ & 9.8 & & 200 & $\mathrm{pCi} / \mathrm{L}$ \\
\hline Chloride & WA & & A & & 4.25 & & NS & $\mathrm{mg} / \mathrm{L}$ \\
\hline Chlorobenzene & WA & & & $\mathbf{U}$ & 5 & & 100 & $\mu \mathrm{g} / \mathrm{L}$ \\
\hline Chloroethane & WA & & & $\mathrm{U}$ & 10 & & NS & $\mu \mathrm{g} / \mathrm{L}$ \\
\hline Chloroethane & WA & & NA & $\mathbf{U}$ & 10 & & NS & $\mu \mathrm{g} / \mathrm{L}$ \\
\hline Chloroethane. & WA & & NA & $\mathrm{U}$ & 10 & & NS & $\mu \mathrm{g} / \mathrm{L}$ \\
\hline Chloroform & WA & & & $\mathbf{U}$ & 5 & & 100 & $\mu \mathrm{g} / \mathrm{L}$ \\
\hline Chloroform & WA & & NA & $\mathrm{U}$ & 5 & & 100 & $\mu \mathrm{g} / \mathrm{L}$ \\
\hline Chloroform & WA & & NA & $\mathbf{U}$ & 5 & & 100 & $\mu \mathrm{g} / \mathrm{L}$ \\
\hline Chloroprene & WA & & & $\mathrm{U}$ & 20 & & NS & $\mu \mathrm{g} / \mathrm{L}$ \\
\hline Chloroprene & WA & & NA & $\mathbf{U}$ & 20 & & NS & $\mu \mathrm{g} / \mathrm{L}$ \\
\hline Chloroprene & WA & & NA & $\mathrm{U}$ & 20 & & NS & $\mu \mathrm{g} / \mathrm{L}$ \\
\hline Chromium & WA & & & $\mathbf{U}$ & 10 & & 100 & $\mu \mathrm{g} / \mathrm{L}$ \\
\hline Chromium & WA & & NA & $\mathbf{U}$ & 10 & & 100 & $\mu \mathrm{g} / \mathrm{L}$ \\
\hline cis-1,3-Dichloropropene & WA & & & $\mathbf{U}$ & 5 & & NS & $\mu \mathrm{g} / \mathrm{L}$ \\
\hline cis-1,3-Dichloropropene & WA & & NA & $\mathbf{U}$ & 5 & & NS & $\mu \mathrm{g} / \mathrm{L}$ \\
\hline cis-1,3-Dichloropropene & WA & & NA & $\mathbf{U}$ & 5 & & NS & $\mu \mathrm{g} / \mathrm{L}$ \\
\hline
\end{tabular}


UTR062 Laboratory Analyses (cont'd)

\begin{tabular}{|c|c|c|c|c|c|c|c|c|}
\hline Analyte & Lab & Rep $\mid$ Avg $\mid$ & Modifier & & Result I & Acc & Stand & Units \\
\hline Cobalt & WA & & $\mathbf{U}$ & & 50 & & NS & $\mu g / L$ \\
\hline Cobalt & WA & NA & $\mathbf{U}$ & & 50 & & NS & $\mu g / L$ \\
\hline Copper & WA & & $\mathbf{U}$ & & 25 & & 1000 & $\mu \mathrm{g} / \mathrm{L}$ \\
\hline Copper & WA & NA & U & & 25 & & 1000 & $\mu \mathrm{g} / \mathrm{L}$ \\
\hline Dibromochloromethane & WA & & $\mathbf{U}$ & & 5 & & NS & $\mu g / L$ \\
\hline Dibromochloromethane & WA & NA & $\mathbf{U}$ & & 5 & & NS & $\mu \mathrm{g} / \mathrm{L}$ \\
\hline Dibromochloromethane & WA & NA & $\mathbf{U}$ & & 5 & & NS & $\mu \mathrm{g} / \mathrm{L}$ \\
\hline Dichlorodifluoromethane & WA & & $\mathbf{U}$ & & 10 & & NS & $\mu \mathrm{g} / \mathrm{L}$ \\
\hline Dichlorodifluoromethane & WA & NA & $\mathbf{U}$ & & 10 & & NS & $\mu g / L$ \\
\hline Dichlorodifluoromethane & WA & NA & $\mathbf{U}$ & & 10 & & NS & $\mu g / L$ \\
\hline Ethylbenzene & WA & & $\mathbf{U}$ & & 5 & & 700 & $\mu g / L$ \\
\hline Ethylbenzene & WA & NA & $\mathbf{U}$ & & 5 & & 700 & $\mu g / L$ \\
\hline Ethylbenzene & WA & NA & $\mathbf{U}$ & & 5 & & 700 & $\mu \mathrm{g} / \mathrm{L}$ \\
\hline Fluoride & WA & & $\mathbf{U}$ & & 0.1 & & 4 & $\mathrm{mg} / \mathrm{L}$ \\
\hline Gross alpha & TM & NA & $\mathbf{U}$ & & 0.7 & & 15 & $\mathrm{pCi} / \mathrm{L}$ \\
\hline Gross alpha & TM & & $\mathbf{U}$ & & 0.7 & & 15 & $\mathrm{pCi} / \bar{L}$ \\
\hline Iron & WA & A & & & 220 & & 300 & $\mu g / L$ \\
\hline Isobutyl alcohol & WA & & $\mathbf{U}$ & & 20 & & NS & $\mu g / L$ \\
\hline Isobutyl alcohol & WA & NA & $\mathbf{U}$ & & 20 & & NS & $\mu g / L$ \\
\hline Isobutyl alcohol & WA & NA & $\mathbf{U}$ & & 20 & & NS & $\mu g / L$ \\
\hline Lead & WA & & $\mathbf{U}$ & & 3 & & 50 & $\mu g / L$ \\
\hline Lead & WA & NA & $\mathbf{U}$ & & 3 & & 50 & $\mu \mathrm{g} / \mathrm{L}$ \\
\hline Magnesium & WA & & U & & 5000 & & NS & $\mu \mathrm{g} / \mathrm{L}$ \\
\hline Magnesium & WA & NA & $\mathbf{U}$ & & 5000 & & NS & $\mu \mathrm{g} / \mathrm{L}$ \\
\hline Manganese & WA & & $\mathbf{U}$ & & 15 & & 50 & $\mu \mathrm{g} / \mathrm{L}$ \\
\hline Manganese & WA & NA & $U$ & & 15 & & 50 & $\mu \mathrm{g} / \mathrm{L}$ \\
\hline Mercury & WA & & $\mathbf{U}$ & & 0.2 & & 2 & $\mu \mathrm{g} / \mathrm{L}$ \\
\hline Mercury & WA & NA & $\mathbf{U}$ & & 0.2 & & 2 & $\mu g / L$ \\
\hline Methacrylonitrile & WA & & $\mathbf{U}$ & & 20 & & NS & $\mu \mathrm{g} / \mathrm{L}$ \\
\hline Methacrylonitrile & WA & NA & $\mathbf{U}$ & & 20 & & NS & $\mu g / L$ \\
\hline Methacrylonitrile & WA & NA & $\mathbf{U}$ & & 20 & & NS & $\mu g / L$ \\
\hline Methyl bromide & WA & & $\mathbf{U}$ & & 10 & & NS & $\mu \mathrm{g} / \mathrm{L}$ \\
\hline Methyl bromide & WA & NA & $\mathbf{U}$ & & 10 & & NS & $\mu \mathrm{g} / \mathrm{L}$ \\
\hline Methyl bromide & WA & NA & $\mathbf{U}$ & & 10 & & NS & $\mu \mathrm{g} / \mathrm{L}$ \\
\hline Methyl chloride & WA & & $\mathrm{U}$ & & 10 & & NS & $\mu g / L$ \\
\hline Methyl chloride & WA & NA & $\mathbf{U}$ & & 10 & & NS & $\mu g / L$ \\
\hline Methyl chloride & WA & NA & $\mathbf{U}$ & & 10 & & NS & $\mu g / L$ \\
\hline Methyl ethyl ketone & WA & & U & & 10 & & NS & $\mu \mathrm{g} / \mathrm{L}$ \\
\hline Methyl ethyl ketone & WA & NA & $\mathbf{U}$ & & 10 & & NS & $\mu \mathrm{g} / \mathrm{L}$ \\
\hline Methyl ethyl ketone & WA & NA & $\mathbf{U}$ & & 10 & & NS & $\mu g / L$ \\
\hline Methyl iodide & WA & & $\mathbf{U}$ & & 10 & & NS & $\mu \mathrm{g} / \mathrm{L}$ \\
\hline Methyl iodide & WA & NA & $\mathbf{U}$ & & 10 & & NS & $\mu \mathrm{g} / \mathrm{L}$ \\
\hline Methyl iodide & WA & NA & $\mathbf{U}$ & & 10 & & NS & $\mu g / L$ \\
\hline Methylene bromide & WA & & $\mathbf{U}$ & & 10 & & NS & $\mu g / L$ \\
\hline Methylene bromide & WA & NA & $\mathbf{U}$ & & 10 & & NS & $\mu \mathrm{g} / \mathrm{L}$ \\
\hline Methylene bromide & WA & NA & $\mathbf{U}$ & & 10 & & NS & $\mu g / L$ \\
\hline Methylene chloride & WA & $\mathbf{A}$ & & V & 10.58 & & 5 & $\mu g / L$ \\
\hline Nickel & WA & & U & & 40 & & 100 & $\mu \mathrm{g} / \mathrm{L}$ \\
\hline Nickel & WA & NA & U & & 40 & & 100 & $\mu \mathrm{g} / \mathrm{L}$ \\
\hline Nitrate as nitrogen & WA & & $\mathbf{J}$ & Q & 0.13 & & 10 & $\mathrm{mg} / \mathrm{L}$ \\
\hline Nonvolatile beta & TM & $\mathbf{A}$ & & & 1.55 & 1.75 & 50 & pCi/L \\
\hline Potassium & WA & & $\mathbf{U}$ & & 5000 & & NS & $\mu \mathrm{g} / \mathrm{L}$ \\
\hline Potassium & WA & NA & $\mathbf{U}$ & & 5000 & & NS & $\mu g / L$ \\
\hline
\end{tabular}


UTR062 Laboratory Analyses (cont'd)

\begin{tabular}{|c|c|c|c|c|c|c|c|}
\hline Analyte & Lab & Rep & Modifier & Result & Acc & Stand & Units \\
\hline Propionitrile & WA & & $\mathbf{U}$ & 50 & & NS & $\mu g / L$ \\
\hline Propionitrile & WA & NA & $\mathbf{U}$ & 50 & & NS & $\mu g / L$ \\
\hline Propionitrile & WA & NA & $\mathbf{U}$ & 50 & & NS & $\mu g / L$ \\
\hline Selenium & WA & & $\mathbf{U}$ & 5 & & 50 & $\mu \mathrm{g} / \mathrm{L}$ \\
\hline Selenium & WA & NA & $\mathbf{U}$ & 5 & & 50 & $\mu \mathrm{g} / \mathrm{L}$ \\
\hline Silver & WA & & $\mathbf{U}$ & 10 & & 50 & $\mu g / L$ \\
\hline Silver & WA & NA & $\mathbf{U}$ & 10 & & 50 & $\mu g / L$ \\
\hline Sodium & WA & & $\mathbf{U}$ & 5000 & & NS & $\mu g / L$ \\
\hline Sodium & WA & NA & $\mathbf{U}$ & 5000 & & NS & $\mu g / L$ \\
\hline Styrene & WA & & $\mathbf{U}$ & 5 & & 100 & $\mu g / L$ \\
\hline Styrene & WA & NA & $\mathbf{U}$ & 5 & & 100 & $\mu \mathrm{g} / \mathrm{L}$ \\
\hline Styrene & WA & NA & $\mathbf{U}$ & 5 & & 100 & $\mu g / L$ \\
\hline Sulfate & WA & & $\mathbf{U}$ & 2.5 & & 400 & $\mathrm{mg} / \mathrm{L}$ \\
\hline Sulfide & WA & & $\mathbf{U}$ & 0.1 & & NS & $\mathrm{mg} / \mathrm{L}$ \\
\hline Sulfide & WA & NA & $\mathbf{U}$ & 0.1 & & NS & $\mathrm{mg} / \mathrm{L}$ \\
\hline Tetrachloroethylenc & WA & . & $\mathbf{U}$ & 5 & & 5 & $\mu \mathrm{g} / \mathrm{L}$ \\
\hline Tetrachloroethylene & WA & NA & $\mathbf{U}$ & 5 & & 5 & $\mu \mathrm{g} / \mathrm{L}$ \\
\hline Tetrachloroethylene & WA & NA & $\mathbf{U}$ & 5 & & 5 & $\mu \mathrm{g} / \mathrm{L}$ \\
\hline Thallium & WA & & $\mathbf{U}$ & 10 & & 2 & $\mu \mathrm{g} / \mathrm{L}$ \\
\hline Thallium & WA & NA & $\mathbf{U}$ & 10 & & 2 & $\mu \mathrm{g} / \mathrm{L}$ \\
\hline Toluene & WA & & $\mathbf{U}$ & 5 & & 1000 & $\mu \mathrm{g} / \mathrm{L}$ \\
\hline Total hardness & WA & & & 10.8 & & NS & $\mathrm{mg} / \mathrm{L}$ \\
\hline Total organic carbon & WA & & & 2.45 & & NS & $\mathrm{mg} / \mathrm{L}$ \\
\hline Total suspended solids & WA & & $\mathbf{U}$ & 5 & & NS & $\mathrm{mg} / \mathrm{L}$ \\
\hline trans-1,3-Dichloropropene & WA & & $\mathrm{U}$ & 5 & & NS & $\mu \mathrm{g} / \mathrm{L}$ \\
\hline trans-1,3-Dichloropropene & WA & NA & $\mathbf{U}$ & 5 & & NS & $\mu \mathrm{g} / \mathrm{L}$ \\
\hline trans-1,3-Dichloropropene & WA & NA & $\mathbf{U}$ & 5 & & NS & $\mu \mathrm{g} / \mathrm{L}$ \\
\hline trans-1,4-Dichloro-2-butene & WA & & $\mathbf{U}$ & 100 & & NS & $\mu g / L$ \\
\hline trans-1,4-Dichloro-2-butene & WA & NA & $\mathbf{U}$ & 100 & & NS & $\mu \mathrm{g} / \mathrm{L}$ \\
\hline trans-1,4-Dichloro-2-butene & WA & NA & $\mathbf{U}$ & 100 & & NS & $\mu \mathrm{g} / \mathrm{L}$ \\
\hline Trichloroethylene & WA & & $\mathbf{U}$ & 5 & & 5 & $\mu g / L$ \\
\hline Trichlorofluoromethane & WA & & $\mathbf{U}$ & 5 & & NS & $\mu \mathrm{g} / \mathrm{L}$ \\
\hline Trichlorofluoromethane & WA & NA & $\mathrm{U}$ & 5 & & NS & $\mu \mathrm{g} / \mathrm{L}$ \\
\hline Trichlorofluoromethane & WA & NA & $\mathbf{U}$ & 5 & & NS & $\mu g / L$ \\
\hline Tritium & TM & A & & 16.38 & 0.89 & 20 & $\mathrm{pCi} / \mathrm{ml}$ \\
\hline Turbidity & WA & & $J$ & 0.57 & & NS & NTU \\
\hline Vanadium & WA & & $\mathrm{U}$ & 50 & & NS & $\mu g / L$ \\
\hline Vanadium & WA & NA & $\mathbf{U}$ & 50 & & NS & $\mu g / L$ \\
\hline Vinyl acetate & WA & & $\mathrm{U}$ & 10 & & NS & $\mu \mathrm{g} / \mathrm{L}$ \\
\hline Vinyl acetate & WA & NA & $\mathrm{U}$ & 10 & & NS & $\mu \mathrm{g} / \mathrm{L}$ \\
\hline Vinyl acetate & WA & NA & $\mathbf{U}$ & 10 & & NS & $\mu \mathrm{g} / \mathrm{L}$ \\
\hline Vinyl chloride & WA & & $\mathbf{U}$ & 10 & & 2 & $\mu \mathrm{g} / \mathrm{L}$ \\
\hline Vinyl chloride & WA & NA & $\mathbf{U}$ & 10 & & 2 & $\mu \mathrm{g} / \mathrm{L}$ \\
\hline Vinyl chloride & WA & NA & $\mathbf{U}$ & 10 & & 2 & $\mu \mathrm{g} / \mathrm{L}$ \\
\hline Xylenes (total) & WA & & $\mathbf{U}$ & 5 & & 10000 & $\mu \mathrm{g} / \mathrm{L}$ \\
\hline Xylenes (total) & WA & NA & $\mathbf{U}$ & 5 & & 10000 & $\mu g / L$ \\
\hline Xylenes (total) & WA & NA & $\mathrm{U}$ & 5 & & 10000 & $\mu \mathrm{g} / \mathrm{L}$ \\
\hline Zinc & WA & & & 24.2 & & 5000 & $\mu \mathrm{g} / \mathrm{L}$ \\
\hline Zinc & WA & NA & $\mathbf{U}$ & 20 & & 5000 & $\mu \mathrm{g} / \mathrm{L}$ \\
\hline
\end{tabular}




\section{UTR063}

Field Measurements:

Sample Date: 8/26/93

pH: 6.02

Specific Conductance: $25 \mu \mathrm{S} / \mathrm{cm}$

Red/Oxid Potential: $184 \mathrm{mv}$

Dissolved Oxygen: $1.7 \mathrm{mg} / \mathrm{L}$

\begin{tabular}{|c|c|c|c|c|c|c|c|c|}
\hline Analyte & Lab & Rep & Avg & Modifier & Result & Acc & Stand & Units \\
\hline 1,1,1,2-Tetrachloroethane & WA & & & $\mathrm{U}$ & 10 & & NS & $\mu g / L$ \\
\hline 1,1,1-Trichloroethane & WA & & & $\mathbf{U}$ & 5 & & 200 & $\mu g / L$ \\
\hline 1,1,2,2-Tetrachloroethane & WA & & & $\mathbf{U}$ & 5 & & NS & $\mu g / L$ \\
\hline 1,1,2-Trichloroethane & WA & & & $\mathbf{U}$ & 5 & & 5 & $\mu \mathrm{g} / \mathrm{L}$ \\
\hline 1,1-Dichloroethane & WA & & & $\mathbf{U}$ & 5 & & NS & $\mu g / L$ \\
\hline 1,1-Dichloroethylene & WA & & & $\mathbf{U}$ & 5 & & 7 & $\mu g / L$ \\
\hline 1,2,3-Trichloropropane & WA & & & $\mathbf{U}$ & 10 & & NS & $\mu g / L$ \\
\hline 1,2-Dibromo-3-chloropropane & WA & & & $\mathbf{U}$ & 20 & & 0.2 & $\mu g / L$ \\
\hline 1,2-Dibromoethane & WA & & & $\mathbf{U}$ & 20 & & NS & $\mu \mathrm{g} / \mathrm{L}$ \\
\hline 1,2-Dichloroethane & WA & & & $\mathbf{U}$ & 5 & & 5 & $\mu \mathrm{g} / \mathrm{L}$ \\
\hline 1,2-Dichloroethylene (total) & WA & & & $\mathbf{U}$ & 5 & & 7 & $\mu \mathrm{g} / \mathrm{L}$ \\
\hline 1,2-Dichloropropane & WA & & & $\mathbf{U}$ & 5 & & 5 & $\mu g / L$ \\
\hline 2-Hexanone & WA & & & $\mathbf{U}$ & 10 & & NS & $\mu g / L$ \\
\hline 4-Methyl-2-pentanone & WA & & & $\mathrm{U}$ & 10 & & NS & $\mu g / L$ \\
\hline Acetone & WA & & & & 14 & & NS & $\mu \mathrm{g} / \mathrm{L}$ \\
\hline Acetonitrile & WA & & & $\mathbf{U}$ & 20 & & NS & $\mu \mathrm{g} / \mathrm{L}$ \\
\hline Acidity & WA & & & & 124 & & NS & $\mathrm{mg} / \mathrm{L}$ \\
\hline Acrolein & WA & & & $\mathbf{U}$ & 10 & & NS & $\mu \mathrm{g} / \mathrm{L}$ \\
\hline Acrylonitrile & WA & & & $\mathbf{U}$ & 10 & & NS & $\mu \mathrm{g} / \mathrm{L}$ \\
\hline Alkalinity & WA & & & & 5.5 & & NS & $\mathrm{mg} / \mathrm{L}$ \\
\hline Allyl chloride & WA & & & $\mathbf{U}$ & 100 & & NS & $\mu \mathrm{g} / \mathrm{L}$ \\
\hline Aluminum & WA & & & $\mathbf{U}$ & 200 & & NS & $\mu g / L$ \\
\hline Antimony & WA & & & $\mathrm{U}$ & 60 & & 6 & $\mu g / L$ \\
\hline Arsenic & WA & & & $\mathbf{U}$ & 10 & & 50 & $\mu g / L$ \\
\hline Barium & WA & & & $\mathbf{U}$ & 200 & & 2000 & $\mu \mathrm{g} / \mathrm{L}$ \\
\hline Benzene & WA & & & $\mathrm{U}$ & 5 & & 5 & $\mu \mathrm{g} / \mathrm{L}$ \\
\hline Beryllium & WA & & & $\mathrm{U}$ & 5 & & 4 & $\mu \mathrm{g} / \mathrm{L}$ \\
\hline Bromodichloromethane & WA & & & $\mathbf{U}$ & 5 & & NS & $\mu \mathrm{g} / \mathrm{L}$ \\
\hline Bromoform & WA & & & U & 5 & & NS & $\mu \mathrm{g} / \mathrm{L}$ \\
\hline Cadmium & WA & & & $\mathbf{U}$ & 5 & & 5 & $\mu g / L$, \\
\hline Calcium & WA & & & $\mathbf{U}$ & 5000 & & NS & $\mu \mathrm{g} / \mathrm{L}$ \\
\hline Carbon disulfide & WA & & & $\mathbf{U}$ & 5 & & NS & $\mu \mathrm{g} / \mathrm{L}$ \\
\hline Carbon tetrachloride & WA & & & $U$ & 5 & & 5 & $\mu g / L$ \\
\hline Cesium-137 & TM & & & $\mathbf{U}$ & 5.9 & & 200 & $\mathrm{pCi} / \mathrm{L}$ \\
\hline Chloride & WA & & & & 3.36 & & NS & $\mathrm{mg} / \mathrm{L}$ \\
\hline Chlorobenzene & WA & & & $\mathbf{U}$ & 5 & & 100 & $\mu g / L$ \\
\hline Chloroethane & WA & & & $\mathbf{U}$ & 10 & & NS & $\mu \mathrm{g} / \mathrm{L}$ \\
\hline Chloroform & WA & & & $\mathbf{U}$ & 5 & & 100 & $\mu \mathrm{g} / \mathrm{L}$ \\
\hline Chloroprene & WA & & & $\mathbf{U}$ & 20 & & NS & $\mu \mathrm{g} / \mathrm{L}$ \\
\hline Chromium & WA & & & $\mathbf{U}$ & 10 & & 100 & $\mu \mathrm{g} / \mathrm{L}$ \\
\hline cis-1,3-Dichloropropene & WA & & & $\mathbf{U}$ & 5 & & NS & $\mu \mathrm{g} / \mathrm{L}$ \\
\hline Cobalt & WA & & & $\mathrm{U}$ & 50 & & NS & $\mu g / L$ \\
\hline Copper & WA & & & $\mathbf{U}$ & 25 & & 1000 & $\mu \mathrm{g} / \mathrm{L}$ \\
\hline Dibromochloromethane & WA & & & $\mathbf{U}$ & 5 & & NS & $\mu \mathrm{g} / \mathrm{L}$ \\
\hline Dichlorodifluoromethane & WA & & & $\mathbf{U}$ & 10 & & NS & $\mu g / L$ \\
\hline Ethylbenzene & WA & & & $\mathbf{U}$ & 5 & & 700 & $\mu \mathrm{g} / \mathrm{L}$ \\
\hline Fluoride & WA & & & $\mathbf{U}$ & 0.1 & & 4 & $\mathrm{mg} / \mathrm{L}$ \\
\hline
\end{tabular}


UTR063 Laboratory Analyses (cont'd)

\begin{tabular}{|c|c|c|c|c|c|c|c|c|c|}
\hline Analyte & Lab & Rep & Avg & Modifier & & Result & Acc & Stand & Units \\
\hline Gross alpha & TM & & & U & & 0.7 & & 15 & $\mathrm{pCi} / \mathrm{L}$ \\
\hline Iron & WA & & & & & 347 & & 300 & $\mu \mathrm{g} / \mathrm{L}$ \\
\hline Isobutyl alcohol & WA & & & U & & 20 & & NS & $\mu g / L$ \\
\hline Lead & WA & & & $\mathbf{U}$ & & 3 & & 50 & $\mu \mathrm{g} / \mathrm{L}$ \\
\hline Magnesium & WA & & & U & & 5000 & & NS & $\mu g / L$ \\
\hline Manganese & WA & & & U & & 15 & & 50 & $\mu g / L$ \\
\hline Mercury & WA & & & $\mathbf{U}$ & & 0.2 & & 2 & $\mu \mathrm{g} / \mathrm{L}$ \\
\hline Methacrylonitrile & WA & & & U & & 20 & & NS & $\mu g / L$ \\
\hline Methyl bromide & WA & & & U & & 10 & & NS & $\mu g / L$ \\
\hline Methyl chloride & WA & & & U & & 10 & & NS & $\mu \mathrm{g} / \mathrm{L}$ \\
\hline Methyl ethyl ketone & WA & & & U & & 10 & & NS & $\mu g / L$ \\
\hline Methyl iodide & WA & & & $\mathbf{U}$ & & 10 & & NS & $\mu g / L$ \\
\hline Methylene bromide & WA & & & $\mathbf{U}$ & & 10 & & NS & $\mu \mathrm{g} / \mathrm{L}$ \\
\hline Methylene chloride & WA & & & J & V & 3.9 & & 5 & $\mu g / L$ \\
\hline Nickel & WA & & & U & & 40 & & 100 & $\mu g / L$ \\
\hline Nitrate as nitrogen & WA & & & $\mathbf{J}$ & $\mathbf{Q}$ & 0.102 & & 10 & $\mathrm{mg} / \mathrm{L}$ \\
\hline Nonvolatile beta & TM & & & U & & 0.8 & & 50 & $\mathrm{pCi} / \mathrm{L}$ \\
\hline Potassium & WA & & & U & & 5000 & & NS & $\mu g / L$ \\
\hline Propionitrile & WA & & & $\mathbf{U}$ & & 50 & & NS & $\mu g / L$ \\
\hline Selenium & WA & & & $\mathbf{U}$ & & 5 & & 50 & $\mu \mathrm{g} / \mathrm{L}$ \\
\hline Silver & WA & & & U & & 10 & & 50 & $\mu g / L$ \\
\hline Sodium & WA & & & $\mathbf{U}$ & & 5000 & & NS & $\mu g / L$ \\
\hline Styrene & WA & & & $\mathbf{U}$ & & 5 & & 100 & $\mu g / L$ \\
\hline Sulfate & WA & & & $\mathbf{U}$ & & 2.5 & & 400 & $\mathrm{mg} / \mathrm{L}$ \\
\hline Sulfate & WA & & NA & U & & 2.5 & & 400 & $\mathrm{mg} / \mathrm{L}$ \\
\hline Sulfide & WA & & & U & & 0.1 & & NS & $\mathrm{mg} / \mathrm{L}$ \\
\hline Tetrachloroethylene & WA & & & $\mathbf{U}$ & & 5 & & 5 & $\mu g / L$ \\
\hline Thallium & WA & & & $\mathrm{U}$ & & 10 & & 2 & $\mu g / L$ \\
\hline Toluene & WA & & & $\mathbf{U}$ & & 5 & & 1000 & $\mu g / L$ \\
\hline Total hardness & WA & & & & & 7.8 & & NS & $\mathrm{mg} / \mathrm{L}$ \\
\hline Total organic carbon & WA & & & & & 1.9 & & NS & $\mathrm{mg} / \mathrm{L}$ \\
\hline Total suspended solids & WA & & & & & 12 & & NS & $\mathrm{mg} / \mathrm{L}$ \\
\hline trans-1,3-Dichloropropene & WA & & & $\mathbf{U}$ & & 5 & & NS & $\mu \mathrm{g} / \mathrm{L}$ \\
\hline trans-1,4-Dichloro-2-butene & WA & & & U & & 100 & & NS & $\mu g / L$ \\
\hline Trichloroethylene & WA & & & $\mathbf{U}$ & & 5 & & 5 & $\mu \mathrm{g} / \mathrm{L}$ \\
\hline Trichlorofluoromethane & WA & & & $\mathrm{U}$ & & 5 & & NS & $\mu \mathrm{g} / \mathrm{L}$ \\
\hline Tritium & TM & & & & & 19.41 & 0.96 & 20 & $\mathrm{pCi} / \mathrm{ml}$ \\
\hline Turbidity & WA & & & $\mathbf{J}$ & $\mathbf{Q}$ & 1.2 & & NS & NTU \\
\hline Vanadium & WA & & & $\mathbf{U}$ & & 50 & & NS & $\mu \mathrm{g} / \mathrm{L}$ \\
\hline Vinyl acetate & WA & & & $\mathbf{U}$ & & 10 & & NS & $\mu g / L$ \\
\hline Vinyl chloride & WA & & & U & & 10 & & 2 & $\mu g / L$ \\
\hline Xylenes (total) & WA & & & U & & 5 & & 10000 & $\mu \mathrm{g} / \mathrm{h}$ \\
\hline & WA & & & & & 27.5 & & 5000 & $\mu \mathrm{g} / \mathrm{L}$ \\
\hline
\end{tabular}




\section{UTR066}

Field Measurements:

Sample Date: 8/26/93

pH: 5.15

Specific Conductance: $23 \mu \mathrm{S} / \mathrm{cm}$

Red/Oxid Potential: $125 \mathrm{mv}$

Dissolved Oxygen: $0.02 \mathrm{mg} / \mathrm{L}$

\begin{tabular}{|c|c|c|c|c|c|c|c|c|}
\hline Analyte & Lab & Rep & Avg & Modifier & Result & Acc & Stand & Units \\
\hline 1,1,1,2-Tetrachloroethane & WA & & & $\mathbf{U}$ & 10 & & NS & $\mu g / L$ \\
\hline $1,1,1,2$-Tetrachloroethane & WA & $\mathbf{R}$ & & $\mathbf{U}$ & 10 & & NS & $\mu g / L$ \\
\hline 1,1,1-Trichloroethane & WA & & & $\mathbf{U}$ & 5 & & 200 & $\mu g / L$ \\
\hline 1,1,1-Trichloroethane & WA & $\mathbf{R}$ & & $\mathbf{U}$ & 5 & & 200 & $\mu g / L$ \\
\hline $1,1,2,2$-Tetrachloroethane & WA & & & $\mathbf{U}$ & 5 & & NS & $\mu g / L$ \\
\hline 1,1,2,2-Tetrachloroethane & WA & $\mathbf{R}$ & & $\mathbf{U}$ & 5 & & NS & $\mu g / L$ \\
\hline 1,1,2-Trichloroethane & WA & & & $\mathbf{U}$ & 5 & & 5 & $\mu g / L$ \\
\hline 1,1,2-Trichloroethane & WA & $\mathbf{R}$ & & $\mathbf{U}$ & 5 & & 5 & $\mu g / L$ \\
\hline 1,1-Dichloroethane & WA & & & $\mathbf{U}$ & 5 & & NS & $\mu g / L$ \\
\hline 1,1-Dichloroethane & WA & $\mathbf{R}$ & & $\mathbf{U}$ & 5 & & NS & $\mu g / L$ \\
\hline 1,1-Dichloroethylene & WA & & & $\mathbf{U}$ & 5 & & 7 & $\mu g / L$ \\
\hline 1,1-Dichloroethylene & WA & $\mathbf{R}$ & & $\mathbf{U}$ & 5 & & 7 & $\mu g / L$ \\
\hline 1,2,3-Trichloropropane & WA & & & $\mathbf{U}$ & 10 & & NS & $\mu g / L$ \\
\hline 1,2,3-Trichloropropane & WA & $\mathbf{R}$ & & $\mathbf{U}$ & 10 & & NS & $\mu g / L$ \\
\hline 1,2-Dibromo-3-chloropropane & WA & & & $\mathbf{U}$ & 20 & & 0.2 & $\mu \mathrm{g} / \mathrm{L}$ \\
\hline 1,2-Dibromo-3-chloropropane & WA & $\mathbf{R}$ & & U & 20 & & 0.2 & $\mu g / L$ \\
\hline 1,2-Dibromoethane & WA & & & $\mathbf{U}$ & 20 & & NS & $\mu g / L$ \\
\hline 1,2-Dibromoethane & WA & $\mathbf{R}$ & & $\mathbf{U}$ & 20 & & NS & $\mu g / L$ \\
\hline 1,2-Dichloroethane & WA & & & $\mathbf{U}$ & 5 & & 5 & $\mu \mathrm{g} / \mathrm{L}$ \\
\hline 1,2-Dichloroethane & WA & $\mathbf{R}$ & & $\mathbf{U}$ & 5 & & 5 & $\mu \mathrm{g} / \mathrm{L}$ \\
\hline 1,2-Dichloroethylene (total) & WA & & & $\mathbf{U}$ & 5 & & 7 & $\mu g / L$ \\
\hline 1,2-Dichloroethylene (total) & WA & $\mathbf{R}$ & & $\mathbf{U}$ & 5 & & 7 & $\mu \mathrm{g} / \mathrm{L}$ \\
\hline 1,2-Dichloropropane & WA & & & $\mathbf{U}$ & 5 & & 5 & $\mu g / L$ \\
\hline 1,2-Dichloropropane & WA & $\mathbf{R}$ & & $\mathbf{U}$ & 5 & & 5 & $\mu g / L$ \\
\hline 2-Hexanone & WA & & & $\mathbf{U}$ & 10 & & NS & $\mu g / L$ \\
\hline 2-Hexanone & WA & $\mathbf{R}$ & & $\mathbf{U}$ & 10 & & NS & $\mu g / L$ \\
\hline 4-Methyl-2-pentanone & WA & & & $\mathbf{U}$ & 10 & & NS & $\mu \mathrm{g} / \mathrm{L}$ \\
\hline 4-Methyl-2-pentanone & WA & $\mathbf{R}$ & & $\mathrm{U}$ & 10 & & NS & $\mu \mathrm{g} / \mathrm{L}$ \\
\hline Acetone & WA & & & J & 5.03 & & NS & $\mu \mathrm{g} / \mathrm{L}$ \\
\hline Acetone & WA & $\mathbf{R}$ & & $\mathbf{J}$ & 6.81 & & NS & $\mu g / L$ \\
\hline Acetonitrile & WA & & & $\mathbf{U}$ & 20 & & NS & $\mu g / L$ \\
\hline Acetonitrile & WA & $\mathbf{R}$ & & $\mathbf{U}$ & 20 & & NS & $\mu \mathrm{g} / \mathrm{L}$ \\
\hline Acidity & WA & & & & 112 & & NS & $\mathrm{mg} / \mathrm{L}$ \\
\hline Acidity & WA & $\mathbf{R}$ & & & 76 & & NS & $\mathrm{mg} / \mathrm{L}$ \\
\hline Acrolein & WA & & & U & 10 & & NS & $\mu g / L$ \\
\hline Acrolein & WA & $\mathbf{R}$ & & $\mathbf{U}$ & 10 & & NS & $\mu \mathrm{g} / \mathrm{L}$ \\
\hline Acrylonitrile & WA & & & $\mathrm{U}$ & 10 & & NS & $\mu g / L$ \\
\hline Acrylonitrile & WA & $\mathbf{R}$ & & $\mathbf{U}$ & 10 & & NS & $\mu \mathrm{g} / \mathrm{L}$ \\
\hline Alkalinity & WA & & & $\mathbf{U}$ & 0.5 & & NS & $\mathrm{mg} / \mathrm{L}$ \\
\hline Alkalinity & WA & $\mathbf{R}$ & & $\mathbf{U}$ & 0.5 & & NS & $\mathrm{mg} / \mathrm{L}$ \\
\hline Allyl chloride & WA & & & $\mathbf{U}$ & 100 & & NS & $\mu \mathrm{g} / \mathrm{L}$ \\
\hline Allyl chloride & WA & $\mathbf{R}$ & & $\mathbf{U}$ & 100 & & NS & $\mu \mathrm{g} / \mathrm{L}$ \\
\hline Aluminum & WA & & & $\mathbf{U}$ & 200 & & NS & $\mu \mathrm{g} / \mathrm{L}$ \\
\hline Aluminum & WA & $\mathbf{R}$ & & $\mathbf{U}$ & 200 & & NS & $\mu \mathrm{g} / \mathrm{L}$ \\
\hline Antimony & WA & & & $\mathbf{U}$ & 60 & & 6 & $\mu \mathrm{g} / \mathrm{L}$ \\
\hline Antimony & WA & $\mathbf{R}$ & & $\mathbf{U}$ & 60 & & 6 & $\mu \mathrm{g} / \mathrm{L}$ \\
\hline
\end{tabular}


UTR066 Laboratory Analyses (cont'd)

\begin{tabular}{|c|c|c|c|c|c|c|c|c|}
\hline Analyte & Lab & Rep & Avg & Modifier & Result & Acc & Stand & Units \\
\hline Arsenic & WA & & & $\bar{U}$ & 10 & & 50 & $\mu g / L$ \\
\hline Arsenic & WA & $\mathbf{R}$ & & U & 10 & & so & $\mu g / L$ \\
\hline Barium & WA & & & $\mathbf{U}$ & 200 & & 2000 & $\mu g / L$ \\
\hline Barium & WA & $\mathbf{R}$ & & 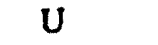 & 200 & & 2000 & $\mu g / L$ \\
\hline Benzene & WA & & & $U$ & 5 & & 5 & $\mu g / L$ \\
\hline Benzene & WA & $\mathbf{R}$ & & $\mathbf{U}$ & 5 & & 5 & $\mu \mathrm{g} / \mathrm{L}$ \\
\hline Beryllium & WA & & & $\mathbf{U}$ & 5 & & 4 & $\mu g / L$ \\
\hline Beryllium & WA & $\mathbf{R}$ & & $\mathbf{U}$ & 5 & & 4 & $\mu \mathrm{g} / \mathrm{L}$ \\
\hline Bromodichl romethane & WA & & & $\mathbf{U}$ & 5 & & NS & $\mu g / L$ \\
\hline Bromodichloromethane & WA & $\mathbf{R}$ & & $\mathbf{U}$ & 5 & & NS & $\mu g / \Omega$ \\
\hline Bromoform & WA & & & $\mathbf{U}$ & 5 & & NS & $\mu g / \mathrm{L}$ \\
\hline Bromoform & WA & $\mathbf{R}$ & & $U$ & 5 & & NS & $\mu g / L$ \\
\hline Cadmium & WA & & & U & 5 & & 5 & $\mu \mathrm{g} / \mathrm{L}$ \\
\hline Cadmium & WA & $\mathbf{R}$ & & $\mathbf{U}$ & 5 & & 5 & $\mu g /$ \\
\hline Calcium & WA & & & $\mathbf{U}$ & 5000 & & NS & $\mu \mathrm{g} / \mathrm{L}$ \\
\hline Calcium & WA & $\mathbf{R}$ & & $\mathbf{U}$ & 5000 & & NS & $\mu g / L$ \\
\hline Carbon disulfide & WA & & & $\mathbf{U}$ & 5 & & NS & $\mu g / L$ \\
\hline Carbon disulfide & WA & $\mathbf{R}$ & & U & 5 & & NS & $\mu g / L$ \\
\hline Carbon tetrachloride & WA & & & $\mathbf{U}$ & 5 & & 5 & $\mu g / L$ \\
\hline Carbon tetrachloride & WA & $\mathbf{R}$ & & $\mathbf{U}$ & 5 & & 5 & $\mu g / 2$ \\
\hline Cesium-137 & TM & & & $\mathbf{U}$ & 6.3 & & 200 & pCi/L \\
\hline Cesium-137 & TM & $\mathbf{R}$ & & $\mathbf{U}$ & 13 & & 200 & $\mathrm{pCi} / / \mathrm{L}$ \\
\hline Chloride & WA & & & & 3.17 & & NS & $\mathrm{mg} / \mathrm{L}$ \\
\hline Chloride & WA & $\mathbf{R}$ & & & 2.64 & & NS & $\mathrm{mg} / \mathrm{L}$ \\
\hline Chlorobenzene & WA & & & $\mathbf{U}$ & 5 & & 100 & $\mu g / L$ \\
\hline Chlorobenzene & WA & $\mathbf{R}$ & & $U$ & 5 & & 100 & $\mu g / L$ \\
\hline Chloroethane & WA & & & U & 10 & & NS & $\mu g / L$ \\
\hline Chloroethane & WA & $\mathbf{R}$ & & U & 10 & & NS & $\mu \mathrm{g} / \mathrm{L}$ \\
\hline Chloroform & WA & & & U & 5 & & 100 & $\mu g / L$ \\
\hline Chloroform & WA & $\mathbf{R}$ & & $\mathbf{U}$ & 5 & & 100 & $\mu \mathrm{g} / \mathrm{L}$ \\
\hline Chloroprene & WA & & & U & 20 & & NS & $\mu \mathrm{g} / \mathrm{L}$ \\
\hline Chloroprene & WA & $\mathbf{R}$ & & U & 20 & & NS & $\mu g / L$ \\
\hline Chromium & WA & & & $U$ & 10 & & 100 & $\mu \mathrm{g} / \mathrm{L}$ \\
\hline Chromium & WA & $\mathbf{R}$ & & $\mathrm{U}$ & 10 & & 100 & $\mu \mathrm{g} / \mathrm{L}$ \\
\hline cis-1,3-Dichloropropene & WA & & & $U$ & 5 & & NS & $\mu \mathrm{g} / \mathrm{L}$ \\
\hline cis-1,3-Dichloropropene & WA & $\mathbf{R}$ & & $\mathrm{U}$ & 5 & & NS & $\mu g / L$ \\
\hline Cobalt & WA & & & $\mathrm{U}$ & 50 & & NS & $\mu \mathrm{g} / \mathrm{L}$ \\
\hline Cobalt & WA & $\mathbf{R}$ & & U & 50 & & NS & $\mu \mathrm{g} / \mathrm{L}$ \\
\hline Copper & WA & & & $\mathbf{U}$ & 25 & & 1000 & $\mu \mathrm{g} / \mathrm{L}$ \\
\hline Copper & WA & $\mathbf{R}$ & & $U$ & 25 & & 1000 & $\mu \mathrm{g} / \mathrm{L}$ \\
\hline Dibromochloromethane & WA & & & U & 5 & & NS & $\mu g / L$ \\
\hline Dibromochloromethane & WA & $\mathbf{R}$ & & $\mathrm{U}$ & 5 & & NS & $\mu g / L$ \\
\hline Dichlorodifluorom ithane & WA & & & $\mathrm{U}$ & 10 & & NS & $\mu \mathrm{g} / \mathrm{L}$ \\
\hline Dichlorodifluoromethane & WA & $\mathbf{R}$ & & $\mathrm{U}$ & 10 & & NS & $\mu g / L$ \\
\hline Ethylbenzene & WA & & & $\mathrm{U}$ & 5 & & 700 & $\mu \mathrm{g} / \mathrm{L}$ \\
\hline Ethylbenzene & WA & $\mathbf{R}$ & & U & 5 & & 700 & $\mu \mathrm{g} / \mathrm{L}$ \\
\hline Fluoride & WA & & & U & 0.1 & & 4 & $\mathrm{mg} / \mathrm{L}$ \\
\hline Fluoride & WA & & NA & $\mathrm{U}$ & 0.1 & & 4 & $\mathrm{mg} / \mathrm{L}$ \\
\hline Fluoride & WA & $\mathbf{R}$ & & $\mathrm{U}$ & 0.1 & & 4 & $\mathrm{mg} / \mathrm{L}$ \\
\hline Gross alpha & $\mathbf{T M}$ & & & $\mathrm{U}$ & 0.7 & & 15 & $\mathrm{pCi} / \mathrm{L}$ \\
\hline Gross alpha & TM & $\mathbf{R}$ & & $\mathrm{U}$ & 0.7 & & 15 & $\mathrm{pCi} / \mathrm{L}$ \\
\hline Iron & WA & & & & 324 & & 300 & $\mu \mathrm{g} / \mathrm{L}$ \\
\hline Iron & WA & $\mathbf{R}$ & & & 404 & & 300 & $\mu \mathrm{g} / \mathrm{L}$ \\
\hline Isobutyl alcohol & WA & & & U & 20 & & NS & $\mu \mathrm{g} / \mathrm{L}$ \\
\hline Isobutyl alcohol & WA & $\mathbf{R}$ & & U & 20 & & NS & $\mu g / L$ \\
\hline
\end{tabular}


UTR066 Laboratory Analyses (cont'd)

\begin{tabular}{|c|c|c|c|c|c|c|c|c|}
\hline Analyte & Lab & Rep & Avg & Modifier & Result & Acc & Stand & Units \\
\hline Lead & $\overline{\text { WA }}$ & & & $\bar{U}$ & 3 & & 50 & $\mu \mathrm{g} / \mathrm{L}$ \\
\hline Lead & WA & $\mathbf{R}$ & & $\mathbf{U}$ & 3 & & 50 & $\mu g / L$ \\
\hline Magnesium & WA & & & $\mathbf{U}$ & 5000 & & NS & $\mu \mathrm{g} / \mathrm{L}$ \\
\hline Magnesium & WA & $\mathbf{R}$ & & $\mathbf{U}$ & 5000 & & NS & $\mu g / L$ \\
\hline Manganese & WA & & & $\mathbf{U}$ & 15 & & 50 & $\mu \mathrm{g} / \mathrm{L}$ \\
\hline Manganese & WA & $\mathbf{R}$ & & $\mathbf{U}$ & 15 & . & 50 & $\mu \mathrm{g} / \mathrm{L}$ \\
\hline Mercury & WA & & & $\mathbf{U}$ & 0.2 & & 2 & $\mu g / L$ \\
\hline Mercury & WA & $\mathbf{R}$ & & U & 0.2 & & 2 & $\mu \mathrm{g} / \mathrm{L}$ \\
\hline Methacrylonitrile & WA & & & $\mathbf{U}$ & 20 & & NS & $\mu g / L$ \\
\hline Methacrylonitrile & WA & $\mathbf{R}$ & & $\mathbf{U}$ & 20 & & NS & $\mu \mathrm{g} / \mathrm{L}$ \\
\hline Methyl bromide & WA & & & $\mathbf{U}$ & 10 & & NS & $\mu \mathrm{g} / \mathrm{L}$ \\
\hline Methyl bromide & WA & $\mathbf{R}$ & & $\mathbf{U}$ & 10 & & NS & $\mu \mathrm{g} / \mathrm{L}$ \\
\hline Methyl chloride & WA & & & $\mathbf{U}$ & 10 & & NS & $\mu g / L$ \\
\hline Methyl chloride & WA & $\mathbf{R}$ & & $\mathbf{U}$ & 10 & & NS & $\mu \mathrm{g} / \mathrm{L}$ \\
\hline Methyl ethyl ketone & WA & & & $\mathbf{U}$ & 10 & & NS & $\mu g / L$ \\
\hline Methyl ethyl ketone & WA & $\mathbf{R}$ & & $\mathbf{U}$ & 10 & & NS & $\mu \mathrm{g} / \mathrm{L}$ \\
\hline Methyl iodide & WA & & & $\mathbf{U}$ & 10 & & NS & $\mu g / L$ \\
\hline Methyl iodide & WA & $\mathbf{R}$ & & $\mathbf{U}$ & 10 & & NS & $\mu g / L$ \\
\hline Methylene bromide & WA & & & $\mathbf{U}$ & 10 & & NS & $\mu \mathrm{g} / \mathrm{L}$ \\
\hline Methylene bromide & WA & $\mathbf{R}$ & & $\mathbf{U}$ & 10 & & NS & $\mu g / L$ \\
\hline Methylene chloride & WA & & & J & 3.93 & & 5 & $\mu \mathrm{g} / \mathrm{L}$ \\
\hline Methylene chloride & WA & $\mathbf{R}$ & & J & 3.97 & & 5 & $\mu \mathrm{g} / \mathrm{L}$ \\
\hline Nickel & WA & & & $\mathrm{U}$ & 40 & & 100 & $\mu g / L$ \\
\hline Nickel & WA & $\mathbf{R}$ & & $\mathbf{U}$ & 40 & & 100 & $\mu \mathrm{g} / \mathrm{L}$ \\
\hline Nitrate as nitrogen & WA & & & J & Q 0.139 & & 10 & $\mathrm{mg} / \mathrm{L}$ \\
\hline Nitrate as nitrogen & WA & $\mathbf{R}$ & & $\mathbf{J}$ & Q $\quad 0.368$ & & 10 & $\mathrm{mg} / \mathrm{L}$ \\
\hline Nonvolatile beta & TM & & & & 1 & 1.7 & 50 & $\mathrm{pCi} / \mathrm{L}$ \\
\hline Nonvolatile beta & TM & $\mathbf{R}$ & & $\mathrm{U}$ & 0.8 & & 50 & $\mathrm{pCi} / \mathrm{L}$ \\
\hline Potassium & WA & & & $\mathbf{U}$ & 5000 & & NS & $\mu \mathrm{g} / \mathrm{L}$ \\
\hline Potassium & WA & $\mathbf{R}$ & & $\mathbf{U}$ & 5000 & & NS & $\mu g / L$ \\
\hline Propionitrile & WA & & & $\mathbf{U}$ & 50 & & NS & $\mu \mathrm{g} / \mathrm{L}$ \\
\hline Propionitrile & WA & $\mathbf{R}$ & & $\mathrm{U}$ & 50 & & NS & $\mu \mathrm{g} / \mathrm{L}$ \\
\hline Selenium & WA & & & $\mathrm{U}$ & 5 & & 50 & $\mu g / L$ \\
\hline Selenium & WA & $\mathbf{R}$ & & $\mathrm{U}$ & 5 & & 50 & $\mu \mathrm{g} / \mathrm{L}$ \\
\hline Silver & WA & & & $\mathrm{U}$ & 10 & & 50 & $\mu \mathrm{g} / \mathrm{L}$ \\
\hline Silver & WA & $\mathbf{R}$ & & $\mathbf{U}$ & 10 & & 50 & $\mu \mathrm{g} / \mathrm{L}$ \\
\hline Sodium & WA & & & $\mathrm{U}$ & 5000 & & NS & $\mu \mathrm{g} / \mathrm{L}$ \\
\hline Sodium & WA & $\mathbf{R}$ & & $\mathrm{U}$ & 5000 & & NS & $\mu \mathrm{g} / \mathrm{L}$ \\
\hline Styrene & WA & & & $\mathrm{U}$ & 5 & & 100 & $\mu \mathrm{g} / \mathrm{L}$ \\
\hline Styrene & WA & $\mathbf{R}$ & & $\mathrm{U}$ & 5 & & 100 & $\mu \mathrm{g} / \mathrm{L}$ \\
\hline Sulfate & WA & & & $\mathrm{U}$ & 2.5 & & 400 & $\mathrm{mg} / \mathrm{L}$ \\
\hline Sulfate & WA & $\mathbf{R}$ & & $\mathrm{U}$ & 2.5 & & 400 & $\mathrm{mg} / \mathrm{L}$ \\
\hline Sulfide & WA & & & U & 0.1 & & NS & $\mathrm{mg} / \mathrm{L}$ \\
\hline Sulfide & WA & $\mathbf{R}$ & & $\mathrm{U}$ & 0.1 & & NS & $\mathrm{mg} / \mathrm{L}$ \\
\hline Tetrachloroethylene & WA & & & $\mathrm{U}$ & 5 & & 5 & $\mu \mathrm{g} / \mathrm{L}$ \\
\hline Tetrachloroethylene & WA & $\mathbf{R}$ & & $\mathrm{U}$ & 5 & & 5 & $\mu \mathrm{g} / \mathrm{L}$ \\
\hline Thallium & WA & & & $\mathrm{U}$ & 10 & & 2 & $\mu \mathrm{g} / \mathrm{L}$ \\
\hline Thallium & WA & $\mathbf{R}$ & & $\mathrm{U}$ & 10 & & 2 & $\mu \mathrm{g} / \mathrm{L}$ \\
\hline Toluene & WA & & & $\mathbf{J}$ & 3.53 & & 1000 & $\mu g / L$ \\
\hline Toluene & WA & $\mathbf{R}$ & & J & 2.29 & & 1000 & $\mu g / L$ \\
\hline Total hardness & WA & & & $\mathrm{U}$ & 1 & & NS & $\mathrm{mg} / \mathrm{L}$ \\
\hline Total hardness & WA & $\mathbf{R}$ & & & 1.96 & & NS & $\mathrm{mg} / \mathrm{L}$ \\
\hline Total organic carbon & WA & & & & 1.9 & & NS & $\mathrm{mg} / \mathrm{L}$ \\
\hline Total organic carbon & WA & $\mathbf{R}$ & & & 1.9 & & NS & $\mathrm{mg} / \mathrm{L}$ \\
\hline
\end{tabular}



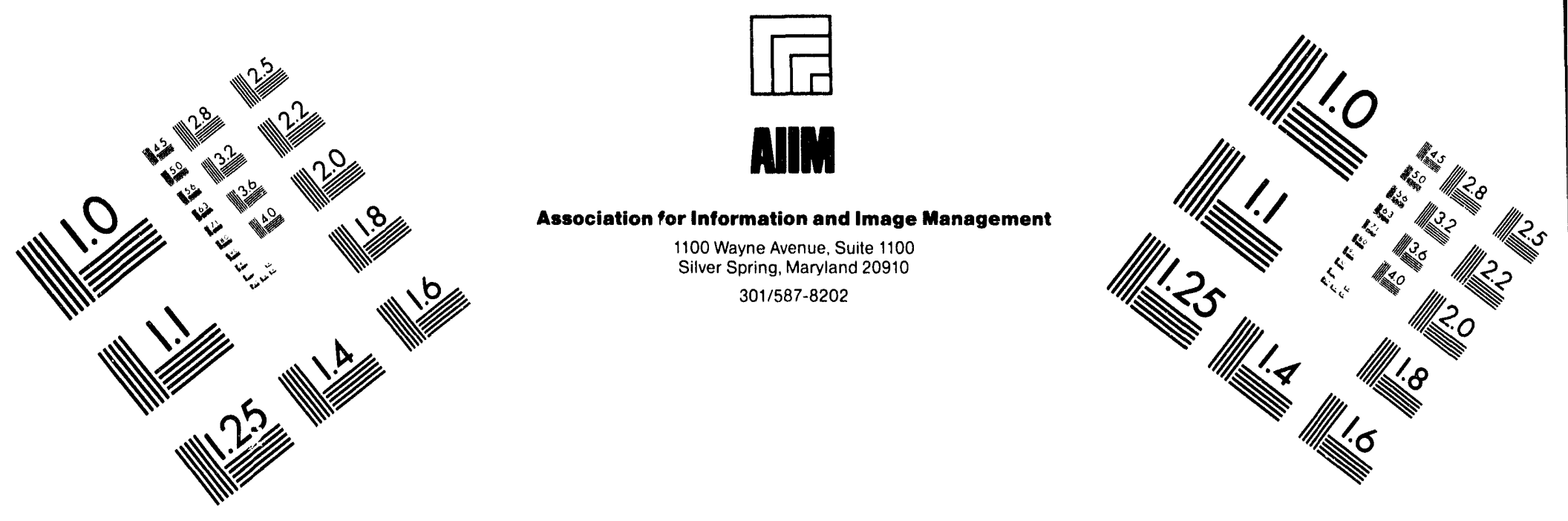

\section{Centimeter}

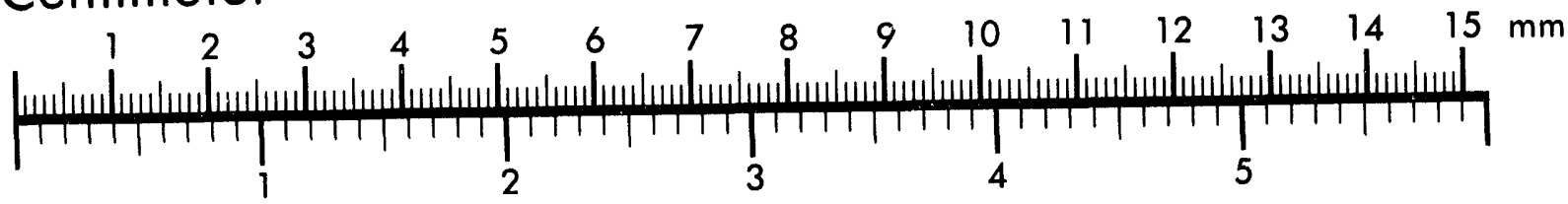

Inches
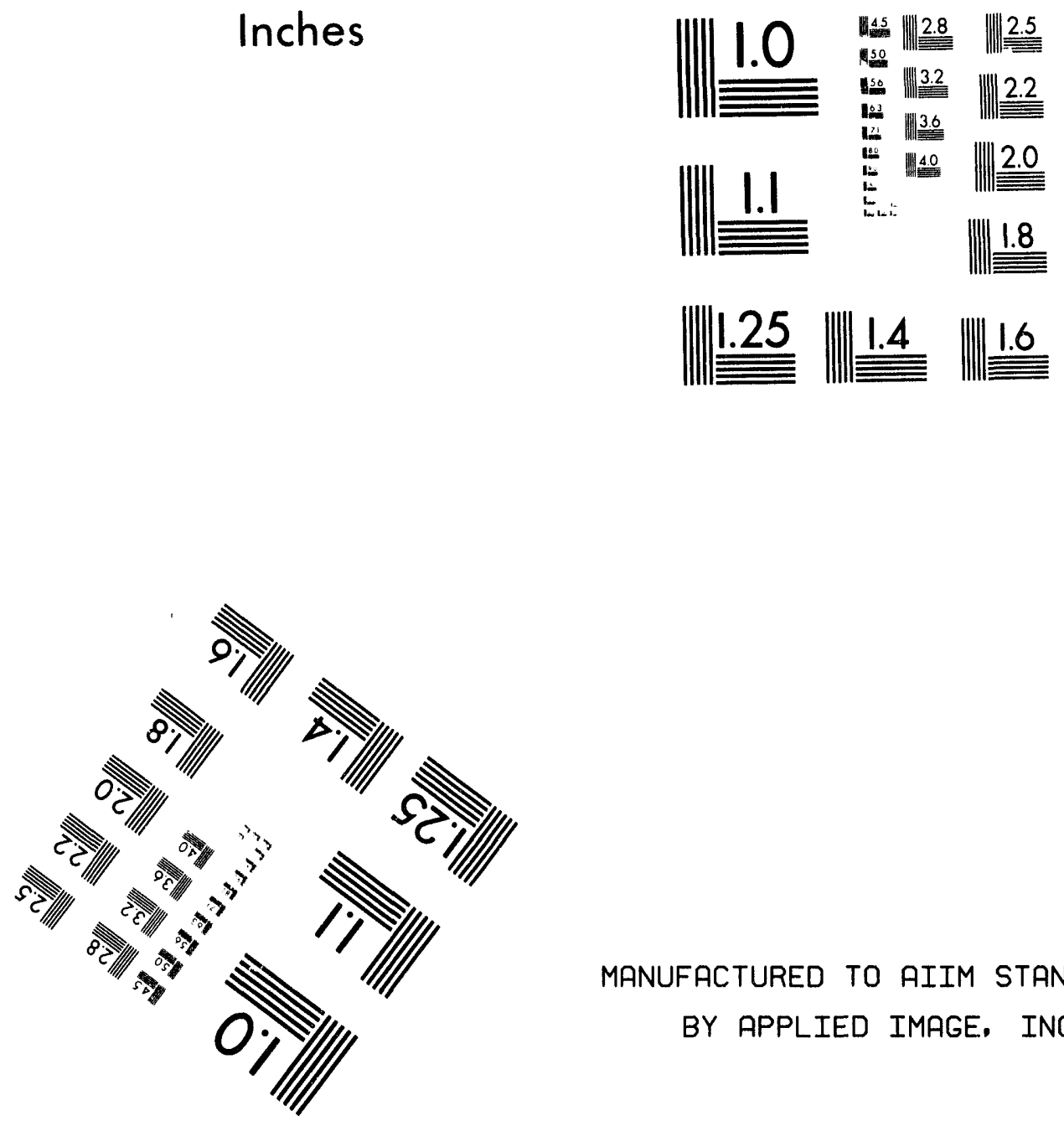

MANUFACTURED TO AIIM STANDFRDS BY APPLIED IMAGE, INC.

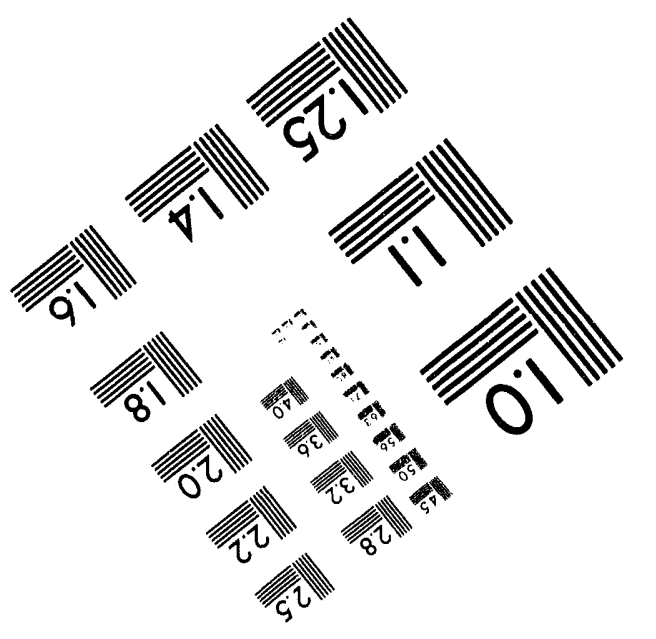



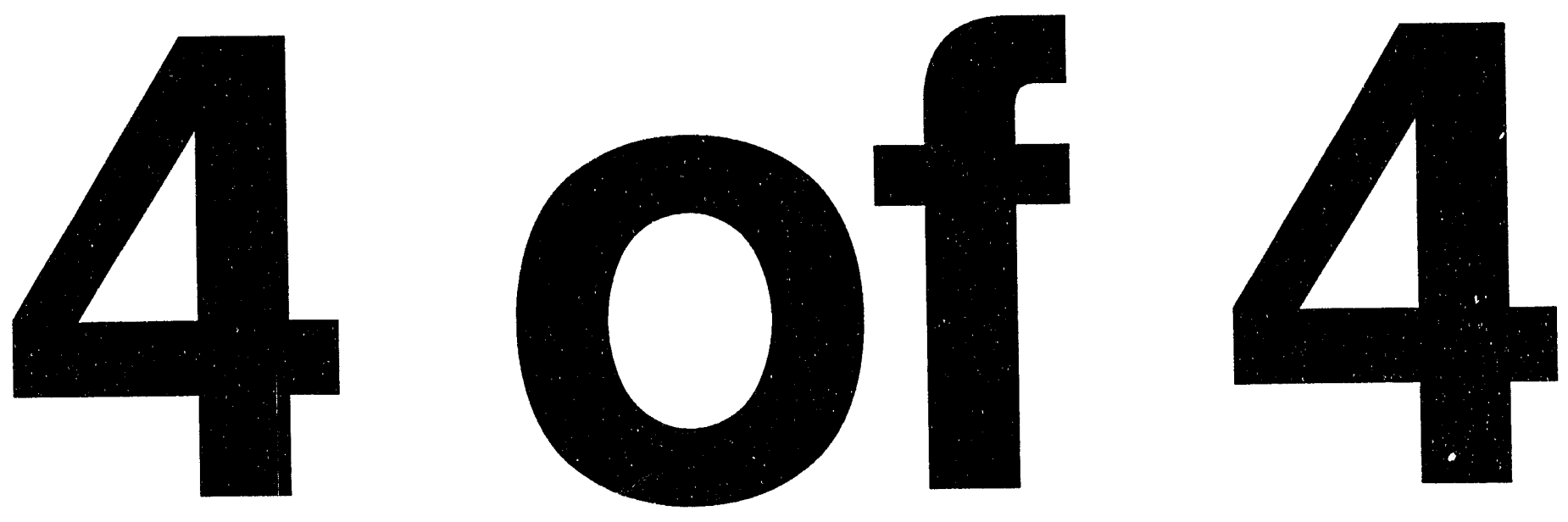
UTR066 Laboratory Analyses (cont'd)

\begin{tabular}{|c|c|c|c|c|c|c|c|c|}
\hline Analyte & Lab & Rep & Avg & Modifier & Result & Acc & Stand & Units \\
\hline Total suspended solids & WA & & & & 8 & & NS & $\mathrm{mg} / \mathrm{L}$ \\
\hline Total suspended solids & WA & $\mathbf{R}$ & & & 6 & & NS & $\mathrm{mg} / \mathrm{L}$ \\
\hline trans-1,3-Dichloropropene & WA & & & $\mathbf{U}$ & 5 & & NS & $\mu g / L$ \\
\hline trans-1,3-Dichloropropene & WA & $\mathbf{R}$ & & $\mathbf{U}$ & 5 & & NS & $\mu g / L$ \\
\hline trans-1,4-Dichloro-2-butene & WA & & & $\mathbf{U}$ & 100 & & NS & $\mu g / L$ \\
\hline trans-1,4-Dichloro-2-butene & WA & $\mathbf{R}$ & & U & 100 & & NS & $\mu g / L$ \\
\hline Trichloroethylene & WA & & & $\mathbf{U}$ & 5 & $\cdot$ & 5 & $\mu \mathrm{g} / \mathrm{L}$ \\
\hline Trichloroethylene & WA & $\mathbf{R}$ & & $\mathbf{U}$ & 5 & & 5 & $\mu g / L$ \\
\hline Trichlorofluoromethane & WA & & & $\mathbf{U}$ & 5 & & NS & $\mu g / L$ \\
\hline Trichlorofluoromethane & WA & $\mathbf{R}$ & & $\mathbf{U}$ & 5 & & NS & $\mu g / L$ \\
\hline Tritium & TM & & & & 27.3 & 1.14 & 20 & $\mathrm{pCi} / \mathrm{ml}$ \\
\hline Tritium & TM & $\mathbf{R}$ & & & 23.52 & 1.05 & 20 & $\mathrm{pCi} / \mathrm{ml}$ \\
\hline Turbidity & WA & & & J & 0.47 & & NS & NTU \\
\hline Turbidity & WA & $\mathbf{R}$ & & $\mathbf{J}$ & 1.1 & & NS & NTU \\
\hline Vanadium & WA & & & $\mathbf{U}$ & 50 & & NS & $\mu g / L$ \\
\hline Vanadium & WA & $\mathbf{R}$ & & $\mathbf{U}$ & 50 & & NS & $\mu g / L$ \\
\hline Vinyl acetate & WA & & & $\mathbf{U}$ & 10 & & NS & $\mu g / L$ \\
\hline Vinyl acetate & WA & $\mathbf{R}$ & & $\mathbf{U}$ & 10 & & NS & $\mu g / L$ \\
\hline Vinyl chloride & WA & & & $\mathbf{U}$ & 10 & & 2 & $\mu g / L$ \\
\hline Vinyl chloride & WA & $\mathbf{R}$ & & $\mathbf{U}$ & 10 & & 2 & $\mu g / L$ \\
\hline Xylenes (total) & WA & & & $\mathbf{U}$ & 5 & & 10000 & $\mu g / L$ \\
\hline Xylenes (total) & WA & $\mathbf{R}$ & & $\mathbf{U}$ & 5 & & 10000 & $\mu g / L$ \\
\hline Zinc & WA & & & & 31.4 & & 5000 & $\mu \mathrm{g} / \mathrm{L}$ \\
\hline Zinc & WA & $\mathbf{R}$ & & & 76.1 & & 5000 & $\mu \mathrm{g} / \mathrm{L}$ \\
\hline
\end{tabular}




\section{UTR068}

Field Measurements:

Sample Date: $8 / 26 / 93$

pH: 4.99

Specific Conductance: $24 \mu \mathrm{S} / \mathrm{cm}$

Red/Oxid Potential: $107 \mathrm{mv}$

Dissolved Oxygen: $1.6 \mathrm{mg} / \mathrm{L}$

\begin{tabular}{|c|c|c|c|c|c|c|c|}
\hline Analyte & Lab & Rep & Modifier & Result & Acc & Stand & Units \\
\hline 1,1,1,2-Tetrachloroethane & WA & & $\overline{\mathrm{U}}$ & 10 & & NS & $\overline{\mu g / L}$ \\
\hline 1,1,1,2-Tetrachloroethane & GE & & $\mathbf{U}$ & 1 & & NS & $\mu \mathrm{g} / \mathrm{L}$ \\
\hline 1,1,1-Trichloroethane & WA & & $\mathbf{U}$ & 5 & & 200 & $\mu \mathrm{g} / \mathrm{L}$ \\
\hline 1,1,1-Trichloroethane & GE & & $\mathbf{U}$ & 1 & & 200 & $\mu \mathrm{g} / \mathrm{L}$ \\
\hline 1,1,2,2-Tetrachloroethane & WA & & $\mathbf{U}$ & 5 & & NS & $\mu \mathrm{g} / \mathrm{L}$ \\
\hline $1,1,2,2$-Tetrachloroethane & GE & & $\mathbf{U}$ & 1 & & NS & $\mu \mathrm{g} / \mathrm{L}$ \\
\hline 1,1,2-Trichloroethane & WA & & $\mathbf{U}$ & 5 & & 5 & $\mu \mathrm{g} / \mathrm{L}$ \\
\hline 1,1,2-Trichloroethane & GE &. & $\mathbf{U}$ & 1 & & 5 & $\mu g / L$ \\
\hline 1,1-Dichloroethane & WA & & $\mathbf{U}$ & 5 & & NS & $\mu g / L$ \\
\hline 1,1-Dichloroethane & $\mathrm{GE}$ & & $\mathbf{U}$ & 1 & & NS & $\mu g / L$ \\
\hline 1,1-Dichloroethylene & WA & & $\mathbf{U}$ & 5 & & 7 & $\mu g / L$ \\
\hline 1,1-Dichloroethylene & GE & & $\mathbf{U}$ & 1 & & 7 & $\mu \mathrm{g} / \mathrm{L}$ \\
\hline 1,2,3-Trichloropropane & WA & & $\mathbf{U}$ & 10 & & NS & $\mu g / L$ \\
\hline 1,2,3-Trichloropropane & GE & & $\mathbf{U}$ & 1 & & NS & $\mu g / L$ \\
\hline 1,2-Dibromo-3-chloropropane & WA & & $\mathbf{U}$ & 20 & & 0.2 & $\mu \mathrm{g} / \mathrm{L}$ \\
\hline 1,2-Dibromo-3-chloropropane & GE & & $\mathbf{U}$ & 1 & & 0.2 & $\mu g / L$ \\
\hline 1,2-Dibromoethane & WA & & $\mathbf{U}$ & 20 & & NS & $\mu \mathrm{g} / \mathrm{L}$ \\
\hline 1,2-Dibromoethane & GE & & $\mathbf{U}$ & 20 & & 7 & $\mu \mathrm{g} / \mathrm{L}$ \\
\hline 1,2-Dichloroethane & WA & & $\mathrm{U}$ & 5 & & 5 & $\mu g / L$ \\
\hline 1,2-Dichloroethane & GE & & $\mathbf{U}$ & 1 & & 5 & $\mu \mathrm{g} / \mathrm{L}$ \\
\hline 1,2-Dichloroethylene (total) & WA & & $\mathbf{U}$ & 5 & & 7 & $\mu g / L$ \\
\hline 1,2-Dichloropropane & WA & & $\mathbf{U}$ & 5 & & 5 & $\mu \mathrm{g} / \mathrm{L}$ \\
\hline 1,2-Dichloropropane & GE & & $\mathrm{U}$ & 1 & & 5 & $\mu \mathrm{g} / \mathrm{L}$ \\
\hline 2-Hexanone & WA & & $\mathbf{U}$ & 10 & & NS & $\mu \mathrm{g} / \mathrm{L}$ \\
\hline 2-Hexanone & GE & & $\mathbf{U}$ & 1 & & NS & $\mu \mathrm{g} / \mathrm{L}$ \\
\hline 4-Methyl-2-pentanone & WA & & $\mathbf{U}$ & 10 & & NS & $\mu \mathrm{g} / \mathrm{L}$ \\
\hline Acetone & WA & & $\mathbf{J}$ & 6.25 & & NS & $\mu \mathrm{g} / \mathrm{L}$ \\
\hline Acetone & GE & & $\mathbf{U}$ & 100 & & NS & $\mu \mathrm{g} / \mathrm{L}$ \\
\hline Acetonitrile & WA & & $\mathbf{U}$ & 20 & & NS & $\mu \mathrm{g} / \mathrm{L}$ \\
\hline Acetonitrile (Methyl cyanide) & GE & & $\mathrm{U}$ & 1 & & NS & $\mu \mathrm{g} / \mathrm{L}$ \\
\hline Acidity & WA & & & 74 & & NS & $\mathrm{mg} / \mathrm{L}$ \\
\hline Acrolein & WA & & $\mathbf{U}$ & 10 & & NS & $\mu g / L$ \\
\hline Acrolein & GE & & $\mathbf{U}$ & 20 & & NS & $\mu \mathrm{g} / \mathrm{L}$ \\
\hline Acrylonitrile & WA & & $\mathbf{U}$ & 10 & & NS & $\mu \mathrm{g} / \mathrm{L}$ \\
\hline Acrylonitrile & GE & & $\mathbf{U}$ & 20 & & NS & $\mu \mathrm{g} / \mathrm{L}$ \\
\hline Alkalinity & GE & & $\mathrm{U}$ & $\mathrm{V}$ & & NS & $\mathrm{mg} / \mathrm{L}$ \\
\hline Allyl chloride & WA & & U & 100 & & NS & $\mu \mathrm{g} / \mathrm{L}$ \\
\hline Allyl chloride & GE & & $\mathbf{U}$ & 50 & & NS & $\mu \mathrm{g} / \mathrm{L}$ \\
\hline Aluminum & WA & & $\mathbf{U}$ & 200 & & NS & $\mu g / L$ \\
\hline Aluminum & $\mathrm{GE}$ & & & 64.3 & & NS & $\mu g / L$ \\
\hline Antimony & WA & & $\mathbf{U}$ & 60 & & 6 & $\mu g / L$ \\
\hline Antimony & $\mathrm{GE}$ & & UJ & L & & 6 & $\mu \mathrm{g} / \mathrm{L}$ \\
\hline Antimony & $\overline{G E}$ & NA & UJ & $\bar{L}$ & & 6 & $\mu \mathrm{g} / \mathrm{L}$ \\
\hline Arsenic & WA & & $\mathbf{U}$ & 10 & & 50 & $\mu g / L$ \\
\hline Arsenic & GE & & $\mathbf{U}$ & 2 & & 50 & $\mu \mathrm{g} / \mathrm{L}$ \\
\hline Arsenic & GE & NA & $\mathbf{U}$ & 2 & & 50 & $\mu g / L$ \\
\hline
\end{tabular}


UTR068 Laboratory Analyses (cont'd)

\begin{tabular}{|c|c|c|c|c|c|c|c|c|}
\hline Analyte & Lab & $\operatorname{Rep}$ & Avg & Modifier & Result & Acc & Stand & Units \\
\hline Barium & WA & & & $\mathrm{U}$ & 200 & & 2000 & $\mu g / L$ \\
\hline Barium & $\mathrm{GE}$ & & & & 13.4 & & 2000 & $\mu \mathrm{g} / \mathrm{L}$ \\
\hline Benzene & WA & & & $\mathrm{U}$ & 5 & & 5 & $\mu g / L$ \\
\hline Benzene & GE & & & $\mathrm{U}$ & 1 & & 5 & $\mu g / L$ \\
\hline Beryllium & WA & & & $\mathrm{U}$ & 5 & & 4 & $\mu \mathrm{g} / \mathrm{L}$ \\
\hline Beryllium & GE & & & $\mathrm{U}$ & 3 & & 4 & $\mu \mathrm{g} / \mathrm{L}$ \\
\hline Bis(2-chloro-1-methylethyl)ether & GE & & & $\mathrm{U}$ & 10 & - & NS & $\mu \mathrm{g} / \mathrm{L}$ \\
\hline Bromedichloromethane & WA & & & U & 5 & & NS & $\mu \mathrm{g} / \mathrm{L}$ \\
\hline Bromodichloromethane & GE & & & $\mathbf{U}$ & 1 & & NS & $\mu \mathrm{g} / \mathrm{L}$ \\
\hline Bromoform & WA & & & $\mathbf{U}$ & 5 & & NS & $\mu \mathrm{g} / \mathrm{L}$ \\
\hline Bromoform & GE & & & U & 1 & & NS & $\mu \mathrm{g} / \mathrm{L}$ \\
\hline Cadmium & WA & & & $\mathrm{U}$ & 5 & & 5 & $\mu \mathrm{g} / \mathrm{L}$ \\
\hline Cadmium & GE & & & $\mathrm{U}$ & 2 & & 5 & $\mu \mathrm{g} / \mathrm{L}$ \\
\hline Calcium & WA & & & $\mathrm{U}$ & 5000 & & NS & $\mu \mathrm{g} / \mathrm{L}$ \\
\hline Calcium & GE & & & & 203 & & NS & $\mu \mathrm{g} / \mathrm{L}$ \\
\hline Carbon disulfide & WA & & e & $\mathbf{U}$ & 5 & & NS & $\mu g / L$ \\
\hline Carbon disulfide & GE & & e & U & 1 & & NS & $\mu \mathrm{g} / \mathrm{L}$ \\
\hline Carbon tetrachloride & WA & & & U & 5 & & 5 & $\mu \mathrm{g} / \mathrm{L}$ \\
\hline Carbon tetrachloride & GE & & & U & 1 & & 5 & $\mu \mathrm{g} / \mathrm{L}$ \\
\hline Cesium-137 & TM & & & U & 6.2 & & 200 & $\mathrm{pCi} / \mathrm{L}$ \\
\hline Chloride & WA & & & & 2.92 & & NS & $\mathrm{mg} / \mathrm{L}$ \\
\hline Chloride & GE & & A & & 2.315 & & NS & $\mathrm{mg} / \mathrm{L}$ \\
\hline Chlorobenzene & WA & & & $\mathrm{U}$ & 5 & & 100 & $\mu g / L$ \\
\hline Chlorobenzene & GE & & & $\mathrm{U}$ & 1 & & 100 & $\mu \mathrm{g} / \mathrm{L}$ \\
\hline Chloroethane & WA & & & $\mathrm{U}$ & 10 & & NS & $\mu \mathrm{g} / \mathrm{L}$ \\
\hline Chloroethane & GE & & & U & 1 & & NS & $\mu \mathrm{g} / \mathrm{L}$ \\
\hline Chloroform & WA & & & U & 5 & & 100 & $\mu g / L$ \\
\hline Chloroform & GE & & & $\mathbf{U}$ & 1 & & 100 & $\mu g / L$ \\
\hline Chloroprene & WA & & & U & 20 & & NS & $\mu g / L$ \\
\hline Chloroprene & GE & & & $\mathrm{U}$ & 200 & & NS & $\mu \mathrm{g} / \mathrm{L}$ \\
\hline Chromium & WA & & & $\mathrm{U}$ & 10 & & 100 & $\mu \mathrm{g} / \mathrm{L}$ \\
\hline Chromium & GE & & & $\mathrm{U}$ & 4 & & 100 & $\mu g / L$ \\
\hline cis-1,3-Dichloropropene & WA & & & $\mathrm{U}$ & 5 & & NS & $\mu \mathrm{g} / \mathrm{L}$ \\
\hline cis-1,3-Dichloropropene & GE & & & U & 1 & & NS & $\mu \mathrm{g} / \mathrm{L}$ \\
\hline Cobalt & WA & & & $\mathrm{U}$ & 50 & & NS & $\mu \mathrm{g} / \mathrm{L}$ \\
\hline Cobalt & GE & & & $\mathrm{U}$ & 4 & & NS & $\mu \mathrm{g} / \mathrm{L}$ \\
\hline Copper & WA & & & $\mathrm{U}$ & 25 & & 1000 & $\mu \mathrm{g} / \mathrm{L}$ \\
\hline Copper & GE & & & $\mathrm{U}$ & 4 & & 1000 & $\mu \mathrm{g} / \mathrm{L}$ \\
\hline Cyanide & GE & & & U & 5 & & 200 & $\mu \mathrm{g} / \mathrm{L}$ \\
\hline Dibromochloromethane & WA & & & $\mathbf{U}$ & 5 & & NS & $\mu \mathrm{g} / \mathrm{L}$ \\
\hline Dibromochloromethane & GE & & & $\mathrm{U}$ & 1 & & NS & $\mu \mathrm{g} / \mathrm{L}$ \\
\hline Dichlorodifluoromethane & WA & & & $\mathrm{U}$ & 10 & & NS & $\mu \mathrm{g} / \mathrm{L}$ \\
\hline Dichlorodifluoromethane & GE & & & $\mathrm{U}$ & 1 & & NS & $\mu g / L$ \\
\hline Ethylbenzene. & WA & & & $\mathrm{U}$ & 5 & & 700 & $\mu \mathrm{g} / \mathrm{L}$ \\
\hline Ethylbenzene & GE & & & $\mathrm{U}$ & 1 & & 700 & $\mu g / L$ \\
\hline Fluoride & WA & & & $\mathrm{U}$ & 0.1 & & 4 & $\mathrm{mg} / \mathrm{L}$ \\
\hline Fluoride & GE & & & $\mathrm{U}$ & 0.1 & & 4 & $\mathrm{mg} / \mathrm{L}$ \\
\hline Gross alpha & GE & & & $\mathrm{U}$ & 2 & & 15 & $\mathrm{pCi} / \mathrm{L}$ \\
\hline Gross alpha & TM & & & $\mathrm{U}$ & 0.7 & & 15 & $\mathrm{pCi} / \mathrm{L}$ \\
\hline Hardness & GE & & & & 4 & & NS & $\mathrm{mg} / \mathrm{L}$ \\
\hline Iron & WA & & & & 682 & & 300 & $\mu \mathrm{g} / \mathrm{L}$ \\
\hline Iron & GE & & & & 501 & & 300 & $\mu g / L$ \\
\hline Isobutyl alcohol & WA & & & $\mathrm{U}$ & 20 & & NS & $\mu \mathrm{g} / \mathrm{L}$ \\
\hline Isobutyl alcohol & GE & & & U & 100 & & NS & $\mu g / L$ \\
\hline
\end{tabular}


Sampling And Analysis of Water From Upper Three Runs And Its Wetlands Near Tank 16 and the Mixed Waste Management Facility

UTR068 Laboratory Analyses (cont'd)

\begin{tabular}{|c|c|c|c|c|c|c|c|c|c|}
\hline Analyte & Lab & Rep & Avg & Modifier & & Result & Acc & Stand & Units \\
\hline Lead & WA & & & $\mathbf{U}$ & & 3 & & 50 & $\overline{\mu g / L}$ \\
\hline Lead & $\mathrm{GE}$ & & & $\mathbf{U}$ & & 3 & & 50 & $\mu \mathrm{g} / \mathrm{L}$ \\
\hline Lead & GE & & NA & $\mathbf{U}$ & & 3 & & 50 & $\mu g / L$ \\
\hline Magnesium & WA & & & $\mathbf{U}$ & & 5000 & & NS & $\mu \mathrm{g} / \mathrm{L}$ \\
\hline Magnesium & $\mathrm{GE}$ & & & & & 167 & & NS & $\mu \mathrm{g} / \mathrm{L}$ \\
\hline Manganese & WA & & & & & 17.2 & . & 50 & $\mu g / L$ \\
\hline Manganese & GE & & & & & 16.8 & & 50 & $\mu g / L$ \\
\hline Mercury & WA & & & $\mathbf{U}$ & & 0.2 & & 2 & $\mu \mathrm{g} / \mathrm{L}$ \\
\hline Mercury & GE & & & $\mathbf{U}$ & & 0.2 & & 2 & $\mu \mathrm{g} / \mathrm{L}$ \\
\hline Methacrylonitrile & WA & & & $\mathbf{U}$ & & 20 & & NS & $\mu \mathrm{g} / \mathrm{L}$ \\
\hline Methacrylonitrile & GE & & & $\mathbf{U}$ & & 50 & & NS & $\mu g / L$ \\
\hline Methyl bromide & WA & & & $\mathbf{U}$ & & 10 & & NS & $\mu \mathrm{g} / \mathrm{L}$ \\
\hline Methyl bromide & $\mathrm{GE}$ & & & $\mathbf{U}$ & & 1 & & NS & $\mu \mathrm{g} / \mathrm{L}$ \\
\hline Methyl chloride & WA & & & $\mathbf{U}$ & & 10 & & NS & $\mu \mathrm{g} / \mathrm{L}$ \\
\hline Methyl chloride & $\mathrm{GE}$ & & & $\mathbf{U}$ & & 1 & & NS & $\mu g / L$ \\
\hline Methyl ethyl ketone & WA & & . & $\mathbf{U}$ & & 10 & & NS & $\mu g / L$ \\
\hline Methyl ethyl ketone & GE & & & $\mathbf{U}$ & & 1 & & NS & $\mu g / L$ \\
\hline Methyl iodide & WA & & & $\mathbf{U}$ & & 10 & & NS & $\mu \mathrm{g} / \mathrm{L}$ \\
\hline Methyl iodide & $\mathrm{GE}$ & & & $\mathbf{U}$ & & 15 & & NS & $\mu g / L$ \\
\hline Methyl isobutyl ketone & GE & & & $\mathbf{U}$ & & 1 & & NS & $\mu g / L$ \\
\hline Methylene bromide & WA & & & $\mathbf{U}$ & & 10 & & NS & $\mu \mathrm{g} / \mathrm{L}$ \\
\hline Methylene bromide & GE & & & $\mathbf{U}$ & & 1 & & NS & $\mu g / L$ \\
\hline Methylene chloride & WA & & & $\mathbf{J}$ & V & 3.98 & & 5 & $\mu g / L$ \\
\hline Methylene chloride & $\mathrm{GE}$ & & & $\mathbf{U}$ & & 1 & & 5 & $\mu \mathrm{g} / \mathrm{L}$ \\
\hline Nickel & WA & & & $\mathbf{U}$ & & 40 & & 100 & $\mu g / L$ \\
\hline Nickel & GE & & & $\mathbf{U}$ & & 4 & & 100 & $\mu g / L$ \\
\hline Nitrate as nitrogen & WA & & & J & $\mathbf{Q}$ & 0.211 & & 10 & $\mathrm{mg} / \mathrm{L}$ \\
\hline Nitrate as nitrogen & GE & & & $\mathrm{U}$ & & 0.05 & & 10 & $\mathrm{mg} / \mathrm{L}$ \\
\hline Nonvolatile beta & GE & & & $\mathbf{U}$ & & 2 & & 50 & $\mathrm{pCi} / \mathrm{L}$ \\
\hline Nonvolatile beta & TM & & & $\mathbf{U}$ & & 0.8 & & 50 & $\mathrm{pCi} / \mathrm{L}$ \\
\hline pH & GE & & & $\mathbf{J}$ & Q & 4.84 & & NS & $\mathrm{pH}$ \\
\hline Potassium & WA & & & $\mathbf{U}$ & & 5000 & & NS & $\mu \mathrm{g} / \mathrm{L}$ \\
\hline Potassium & $\mathrm{GE}$ & & & $\mathbf{U}$ & & 500 & & NS & $\mu \mathrm{g} / \mathrm{L}$ \\
\hline Propionitrile & WA & & & $\mathbf{U}$ & & 50 & & NS & $\mu \mathrm{g} / \mathrm{L}$ \\
\hline Propionitrile & GE & & & $\mathbf{U}$ & & 200 & & NS & $\mu g / L$ \\
\hline Selenium & WA & & & $\mathbf{U}$ & & 5 & & 50 & $\mu \mathrm{g} / \mathrm{L}$ \\
\hline Selenium & GE & & & UJ & $\mathbf{L}$ & 2 & & 50 & $\mu g / L$ \\
\hline Selenium & $\overline{G E}$ & & NA & UJ & $\bar{L}$ & 2 & & 50 & $\mu \mathrm{g} / \mathrm{L}$ \\
\hline Silver & WA & & & $\mathrm{U}$ & & 10 & & 50 & $\mu \mathrm{g} / \mathrm{L}$ \\
\hline Silver & GE & & & $\mathrm{U}$ & & 2 & & 50 & $\mu g / L$ \\
\hline Sodium & WA & & & $\mathrm{U}$ & & 5000 & & NS & $\mu g / L$ \\
\hline Sodium & GE & & & & & 1230 & & NS & $\mu \mathrm{g} / \mathrm{L}$ \\
\hline Styrene & WA & & & $\mathrm{U}$ & & 5 & & 100 & $\mu g / L$ \\
\hline Styrene & GE & & & $\mathrm{U}$ & & 1 & & 100 & $\mu g / L$ \\
\hline Sulfate & WA & & & $\mathbf{U}$ & $Q$ & 2.5 & & 400 & $\mathrm{mg} / \mathrm{L}$ \\
\hline Sulfate & $\mathrm{GE}$ & & & $\mathrm{U}$ & & 1 & & 400 & $\mathrm{mg} / \mathrm{L}$ \\
\hline Sulfate & $\mathrm{GE}$ & & NA & $\mathbf{U}$ & & 1 & & 400 & $\mathrm{mg} / \mathrm{L}$ \\
\hline Sulfide & WA & & & $\mathbf{U}$ & & 0.1 & & NS & $\mathrm{mg} / \mathrm{L}$ \\
\hline Sulfide & $\mathrm{GE}$ & & & $\mathrm{U}$ & & 1 & & NS & $\mathrm{mg} / \mathrm{L}$ \\
\hline Sulfide & $\overline{G E}$ & & NA & $U$ & & 1 & & NS & $\mathrm{mg} / \mathrm{L}$ \\
\hline Tetrachloroethylene & WA & & & $\mathbf{U}$ & & 5 & & 5 & $\mu g / L$ \\
\hline Tetrachloroethylene & $\mathrm{GE}$ & & & $\mathbf{U}$ & & 1 & & 5 & $\mu g / L$ \\
\hline Thallium & WA & & & $\mathbf{U}$ & & 10 & & 2 & $\mu g / L$ \\
\hline Thallium & GE & & & $\mathbf{U}$ & & 2 & & 2 & $\mu g / L$ \\
\hline Thallium & GE & & NA & $\mathbf{U}$ & & 2 & & 2 & $\mu \mathrm{g} / \mathrm{L}$ \\
\hline
\end{tabular}


UTR068 Laboratory Analyses (cont'd)

\begin{tabular}{|c|c|c|c|c|c|c|c|c|}
\hline Analyte & Lab & Rep & Avg & Modifier & Result & Acc & Stand & Units \\
\hline Toluene & WA & & & $\mathrm{U}$ & 5 & & 1000 & $\mu g / L$ \\
\hline Toluene & GE & & & $\mathbf{U}$ & 1 & & 1000 & $\mu \mathrm{g} / \mathrm{L}$ \\
\hline Total hardness & WA & & & $\mathbf{U}$ & 1 & & NS & $\mathrm{mg} / \mathrm{L}$ \\
\hline Total organic carbon & WA & & & & 2.26 & & NS & $\mathrm{mg} / \mathrm{L}$ \\
\hline Total organic carbon & GE & & A & & 2.16 & & NS & $\mathrm{mg} / \mathrm{L}$ \\
\hline Total suspended solids & WA & & $\mathbf{A}$ & & 10 & & NS & $\mathrm{mg} / \mathrm{L}$ \\
\hline Total suspended solids & $\mathrm{GE}$ & & & & 1 & & NS & mg/L. \\
\hline trans-1,2-Dichloroethylene (total) & GE & & & $\mathbf{U}$ & 1 & & 100 & $\mu g / L$ \\
\hline trans-1,3-Dichloropropene & WA & & & $\mathbf{U}$ & 5 & & NS & $\mu g / L$ \\
\hline trans-1,3-Dichloropropene & GE & & & $\mathrm{U}$ & 1 & & NS & $\begin{array}{l}\mu \mathrm{g} / \mathrm{L} \\
\mu \mathrm{g} / \mathrm{L}\end{array}$ \\
\hline trans-1,4-Dichloro-2-butene & WA & & & $\mathbf{U}$ & 100 & & NS & $\begin{array}{l}\mu g / L \\
\mu g / L\end{array}$ \\
\hline trans-1,4-Dichloro-2-butene & GE & & & $\mathbf{U}$ & 30 & & NS & $\mu g / L$ \\
\hline Trichloroethylene & WA & & & $\mathbf{U}$ & 5 & & 5 & $\mu \mathrm{g} / \mathrm{L}$ \\
\hline Trichloroethylene & GE & & & $\mathbf{U}$ & 1 & & 5 & $\mu g / L$ \\
\hline Trichlorofluoromethane & WA & & & $\mathbf{U}$ & 5 & & NS & $\mu \mathrm{g} / \mathrm{L}$ \\
\hline Trichlorofluoromethane & GE & & & $\mathrm{U}$ & 1 & & NS & $\mu g / L$ \\
\hline Tritium & GE & & & & 65.5 & & 20 & $\mathrm{pCi} / \mathrm{ml}$ \\
\hline Tritium & TM & & & & 73.84 & 1.83 & 20 & $\mathrm{pCi} / \mathrm{ml}$ \\
\hline Turbidity & WA & & & $\mathbf{J}$ & 0.47 & & NS & NTU \\
\hline Turbidity & GE & & & $\mathbf{U}$ & 0.1 & & NS & NTU \\
\hline Vanadium & WA & & & $\mathbf{U}$ & 50 & & NS & $\mu g / L$ \\
\hline Vanadium & GE & & & $\mathbf{U}$ & 8 & & NS & $\mu \mathrm{g} / \mathrm{L}$ \\
\hline Vinyl acetate & WA & & & $\mathbf{U}$ & 10 & & NS & $\mu \mathrm{g} / \mathrm{L}$ \\
\hline Vinyl acetate & GE & & & $\mathbf{U}$ & 1 & & NS & $\mu \mathrm{g} / \mathrm{L}$ \\
\hline Vinyl chloride & WA & & & U & 10 & & 2 & $\mu g / L$ \\
\hline Vinyl chloride & GE & & & $\mathbf{U}$ & 1 & & 2 & $\mu g / L$ \\
\hline Xylenes (total) & WA & & & $\mathrm{U}$ & 5 & & 10000 & $\mu \mathrm{g} / \mathrm{L}$ \\
\hline Xylenes (total) & GE & & & $\mathbf{U}$ & 2 & & 10000 & $\mu \mathrm{g} / \mathrm{L}$ \\
\hline Zinc & WA & & & & 29.1 & & 5000 & $\mu g / L$ \\
\hline Zinc & GE & & & & 3.62 & & 5000 & $\mu \mathrm{g} / \mathrm{L}$ \\
\hline
\end{tabular}




\section{UTR071}

Field Measurements:

Sample Date: $8 / 31 / 93$

pH: 6.78

Specific Conductance: $22 \mu \mathrm{S} / \mathrm{cm}$

Red/Oxid Potential: $196 \mathrm{mv}$

Dissolved Oxygen: $1.1 \mathrm{mg} / \mathrm{L}$

\begin{tabular}{|c|c|c|c|c|c|c|c|c|}
\hline Analyte & Lab & Rep & Avg & Modifier & Result & Acc & Stand & Units \\
\hline 1,1,1,2-Tetrachloroethane & WA & & & $\mathbf{U}$ & 10 & & NS & $\mu g / L$ \\
\hline 1,1,1-Trichloroethane & WA & & & $\mathbf{U}$ & 5 & & 200 & $\mu g / L$ \\
\hline 1,1,2,2-Tetrachloroethane & WA & & & $\mathbf{U}$ & 5 & & NS & $\mu \mathrm{g} / \mathrm{L}$ \\
\hline 1,1,2-Trichloroethane & WA & & & $\mathbf{U}$ & 5 & & 5 & $\mu g / L$ \\
\hline 1,1-Dichloroethane & WA & & & $\mathbf{U}$ & 5 & & NS & $\mu g / L$ \\
\hline 1,1-Dichloroethylene & WA & & & $\mathbf{U}$ & 5 & & 7 & $\mu g / L$ \\
\hline 1,2,3-Trichloropropane & WA & & & $\mathbf{U}$ & 10 & & NS & $\mu g / L$ \\
\hline 1,2-Dibromo-3-chloropropane & WA & & & $\mathbf{U}$ & 20 & & 0.2 & $\mu g / L$ \\
\hline 1,2-Dibromoethane & WA & & & $\mathbf{U}$ & 20 & & NS & $\mu g / L$ \\
\hline 1,2-Dichloroethane & WA & & & $\mathbf{U}$ & 5 & & 5 & $\mu \mathrm{g} / \mathrm{L}$ \\
\hline 1,2-Dichloroethylene (total) & WA & & & $\mathbf{U}$ & 5 & & 7 & $\mu g / L$ \\
\hline 1,2-Dichloropropane & WA & & & $\mathbf{U}$ & 5 & & 5 & $\mu g / L$ \\
\hline 2-Hexanone & WA & & & $\mathbf{U}$ & 10 & & NS & $\mu \mathrm{g} / \mathrm{L}$ \\
\hline 4-Methyl-2-pentanone & WA & & & $\mathbf{U}$ & 10 & & NS & $\mu g / L$ \\
\hline Acetone & WA & & & & 16.5 & & NS & $\mu g / L$ \\
\hline Acetonitrile & WA & & & $\mathrm{U}$ & 20 & & NS & $\mu g / L$ \\
\hline Acidity & WA & & & & 92 & & NS & $\mathrm{mg} / \mathrm{L}$ \\
\hline Acrolein & WA & & & $\mathrm{U}$ & 10 & & NS & $\mu \mathrm{g} / \mathrm{L}$ \\
\hline Acrylonitrile & WA & & & $\mathbf{U}$ & 10 & & NS & $\mu g / L$ \\
\hline Alkalinity & WA & & & U & 0.5 & & NS & $\mathrm{mg} / \mathrm{L}$ \\
\hline Allyl chloride & WA & & & $\mathbf{U}$ & 100 & & NS & $\mu g / L$ \\
\hline Aluminum & WA & & & & 2540 & & NS & $\mu \mathrm{g} / \mathrm{L}$ \\
\hline Antimony & WA & & & $U$ & 60 & & 6 & $\mu g / L$ \\
\hline Arsenic & WA & & & $\mathbf{U}$ & 10 & & 50 & $\mu g / L$ \\
\hline Barium & WA & & & $\mathbf{U}$ & 200 & & 2000 & $\mu g / L$ \\
\hline Benzene & WA & & & $\mathbf{U}$ & 5 & & 5 & $\mu \mathrm{g} / \mathrm{L}$ \\
\hline Beryllium & WA & & & $\mathbf{U}$ & 5 & & 4 & $\mu g / L$ \\
\hline Bromodichloromethane & WA & & & $\mathbf{U}$ & 5 & & NS & $\mu \mathrm{g} / \mathrm{L}$ \\
\hline Bromoform & WA & & & $\mathbf{U}$ & 5 & & NS & $\mu \mathrm{g} / \mathrm{L}$ \\
\hline Cadmium & WA & & & U & 5 & & 5 & $\mu \mathrm{g} / \mathrm{L}$ \\
\hline Calcium & WA & & & $\mathbf{U}$ & 5000 & & NS & $\mu g / L$ \\
\hline Carbon disulfide & WA & & & $\mathrm{U}$ & 5 & & NS & $\mu \mathrm{g} / \mathrm{L}$ \\
\hline Carbon tetrachloride & WA & & & $\mathrm{U}$ & 5 & & 5 & $\mu \mathrm{g} / \mathrm{L}$ \\
\hline Cesium-137 & $\mathrm{TM}$ & & & $\mathrm{U}$ & 9.5 & & 200 & $\mathrm{pCi} / \mathrm{L}$ \\
\hline Chloride & WA & & & & 3.86 & & NS & $\mathrm{mg} / \mathrm{L}$ \\
\hline Chlorobenzene & WA & & & $\mathbf{U}$ & 5 & & 100 & $\mu \mathrm{g} / \mathrm{L}$ \\
\hline Chloroethane & WA & & & $\mathbf{U}$ & 10 & & NS & $\mu \mathrm{g} / \mathrm{L}$ \\
\hline Chloroform & WA & & & $\mathrm{U}$ & 5 & & 100 & $\mu \mathrm{g} / \mathrm{L}$ \\
\hline Chloroprene & WA & & & $\mathbf{U}$ & 20 & & NS & $\mu \mathrm{g} / \mathrm{L}$ \\
\hline Chromium & WA & & & $\mathbf{U}$ & 10 & & 100 & $\mu \mathrm{g} / \mathrm{L}$ \\
\hline cis-1,3-Dichloropropene & WA & & & $\mathbf{U}$ & 5 & & NS & $\mu \mathrm{g} / \mathrm{L}$ \\
\hline Cobalt & WA & & & $\mathbf{U}$ & 50 & & NS & $\mu \mathrm{g} / \mathrm{L}$ \\
\hline Copper & WA & & & $\mathbf{U}$ & 25 & & 1000 & $\mu g / L$ \\
\hline Dibromochloromethane & WA & & & $\mathbf{U}$ & 5 & & NS & $\mu g / L$ \\
\hline Dichlorodifluoromethane & WA & & & $\mathbf{U}$ & 10 & & NS & $\mu \mathrm{g} / \mathrm{L}$ \\
\hline Ethylbenzene & WA & & & $\mathbf{U}$ & 5 & & 700 & $\mu \mathrm{g} / \mathrm{L}$ \\
\hline Fluoride & WA & & & $U$ & 0.1 & & 4 & $\mathrm{mg} / \mathrm{L}$ \\
\hline
\end{tabular}


UTR071 Laboratory Analyses (cont'd)

\begin{tabular}{|c|c|c|c|c|c|c|c|c|c|}
\hline Analyte & Lab & Rep & Avg & Modifier & & Result & Acc & Stand & Units \\
\hline Gross alpha & TM & & & $\mathbf{U}$ & & 0.6 & & 15 & $\mathrm{pCi} / \mathrm{L}$ \\
\hline Iron & WA & & & & & 655 & & 300 & $\mu g / L$ \\
\hline Isobutyl alcohol & WA & & & $\mathbf{U}$ & & 20 & & NS & $\mu \mathrm{g} / \mathrm{L}$ \\
\hline Lead & WA & & & $\mathbf{U}$ & & 3 & & 50 & $\mu \mathrm{g} / \mathrm{L}$ \\
\hline Magnesium & WA & & & $\mathbf{U}$ & & 5000 & & NS & $\mu g / L$ \\
\hline Manganese & WA & & & & & 53.1 & & 50 & $\mu \mathrm{g} / \mathrm{L}$ \\
\hline Mercury & WA & & & U & & 0.2 & . & 2 & $\mu \mathrm{g} / \mathrm{L}$ \\
\hline Methacrylonitrile & WA & & & $\mathbf{U}$ & & 20 & & NS & $\mu \mathrm{g} / \mathrm{L}$ \\
\hline Methyl bromide & WA & & & $\mathbf{U}$ & & 10 & & NS & $\mu \mathrm{g} / \mathrm{L}$ \\
\hline Methyl chloride & WA & & & $\mathbf{U}$ & & 10 & & NS & $\mu \mathrm{g} / \mathrm{L}$ \\
\hline Methyl ethyl ketone & WA & & & $\mathbf{U}$ & & 10 & & NS & $\mu \mathrm{g} / \mathrm{L}$ \\
\hline Methyl iodide & WA & & & $\mathbf{U}$ & & 10 & & NS & $\mu \mathrm{g} / \mathrm{L}$ \\
\hline Methylene brcmide & WA & & & $\mathbf{U}$ & & 10 & & NS & $\mu \mathrm{g} / \mathrm{L}$ \\
\hline Methylene chloride & WA & & & $\mathbf{J}$ & V & 3.4 & & 5 & $\mu \mathrm{g} / \mathrm{L}$ \\
\hline Nickel & WA & & & U & & 40 & & 100 & $\mu g / L$ \\
\hline Nitrate as nitrogen & WA & & & J & $\mathbf{Q}$ & 0.054 & & 10 & $\mathrm{mg} / \mathrm{L}$ \\
\hline Nonvolatile beta & TM & & & & & 2.4 & 2.7 & 50 & $\mathrm{pCi} / \mathrm{L}$ \\
\hline Potassium & WA & & & $\mathbf{U}$ & & 5000 & & NS & $\mu g / L$ \\
\hline Propionitrile & WA & & & $\mathbf{U}$ & & 50 & & NS & $\mu \mathrm{g} / \mathrm{L}$ \\
\hline Selenium & WA & & & $\mathbf{U}$ & & 5 & & 50 & $\mu \mathrm{g} / \mathrm{L}$ \\
\hline Silver & WA & & & $\mathbf{U}$ & & 10 & & 50 & $\mu \mathrm{g} / \mathrm{L}$ \\
\hline Sodium & WA & & & $\mathbf{U}$ & & 5000 & & NS & $\mu g / L$ \\
\hline Styrene & WA & & & $\mathbf{U}$ & & 5 & & 100 & $\mu g / L$ \\
\hline Sulfate & WA & & & $\mathbf{U}$ & & 2.5 & & 400 & $\mathrm{mg} / \mathrm{L}$ \\
\hline Sulfide & WA & & & $\mathbf{U}$ & & 0.1 & & NS & $\mathrm{mg} / \mathrm{L}$ \\
\hline Tetrachloroethylene & WA & & & $\mathrm{U}$ & & 5 & & 5 & $\mu \mathrm{g} / \mathrm{L}$ \\
\hline Thallium & WA & & & $\mathrm{U}$ & & 10 & & 2 & $\mu \mathrm{g} / \mathrm{L}$ \\
\hline Toluene & WA & & & $\mathrm{U}$ & & 5 & & 1000 & $\mu \mathrm{g} / \mathrm{L}$ \\
\hline Total hardness & WA & & & & & 7.7 & & NS & $\mathrm{mg} / \mathrm{L}$ \\
\hline Total organic carbon & WA & & & & & 4.03 & & NS & $\mathrm{mg} / \mathrm{L}$ \\
\hline Total suspended solids & WA & & & & & 27 & & NS & $\mathrm{mg} / \mathrm{L}$ \\
\hline trans-1,3-Dichloropropene & WA & & & $\mathbf{U}$ & & 5 & & NS & $\mu \mathrm{g} / \mathrm{L}$ \\
\hline trans-1,4-Dichloro-2-butene & WA & & & $\mathbf{U}$ & & 100 & & NS & $\mu \mathrm{g} / \mathrm{L}$ \\
\hline Trichloroethylene & WA & & & U & & 5 & & 5 & $\mu \mathrm{g} / \mathrm{L}$ \\
\hline Trichlorofluoromethane & WA & & & $\mathbf{U}$ & & 5 & & NS & $\mu \mathrm{g} / \mathrm{L}$ \\
\hline Tritium & TM & & & & & 44.4 & 1.5 & 20 & $\mathrm{pCi} / \mathrm{ml}$ \\
\hline Turbidity & WA & & & & & 137 & & NS & NTU \\
\hline Vanadium & WA & & & $\mathbf{U}$ & & 50 & & NS & $\mu \mathrm{g} / \mathrm{L}$ \\
\hline Vinyl acetate & WA & & & $\mathrm{U}$ & & 10 & & NS & $\mu \mathrm{g} / \mathrm{L}$ \\
\hline Vinyl chloride & WA & & & $\mathbf{U}$ & & 10 & & 2 & $\mu g / L$ \\
\hline Xylenes (total) & WA & & & $\mathbf{U}$ & & 5 & & 10000 & $\mu g / L$ \\
\hline Zinc & WA & & & $\mathrm{U}$ & & 20 & & 5000 & $\mu \mathrm{g} / \mathrm{L}$ \\
\hline
\end{tabular}




\section{UTR072}

Field Measurements:

Sample Date: 8/31/93

pH: 6.24

Specific Conductance: $21 \mu \mathrm{S} / \mathrm{cm}$

Red/Oxid Potential: $222 \mathrm{mv}$

Dissolved Oxygen: $2.5 \mathrm{mg} / \mathrm{L}$

\begin{tabular}{|c|c|c|c|c|c|c|c|c|}
\hline Analyte & Lab & Rep & Avg & Modifier & Result & Acc & Stand & Units \\
\hline 1,1,1,2-Tetrachloroethane & WA & & & $\mathrm{U}$ & 10 & & NS & $\mu g / L$ \\
\hline 1,1,1-Trichloroethane & WA & & & $\mathbf{U}$ & 5 & & 200 & $\mu \mathrm{g} / \mathrm{L}$ \\
\hline $1,1,2,2-$ Tetrachloroethane & WA & & & $\mathbf{U}$ & 5 & & NS & $\mu g / L$ \\
\hline 1,1,2-Trichloroethane & WA & & & $\mathbf{U}$ & 5 & & 5 & $\mu g / L$ \\
\hline 1,1-Dichloroethane & WA & & & $\mathbf{U}$ & 5 & & NS & $\mu \mathrm{g} / \mathrm{L}$ \\
\hline 1,1-Dichloroethylene & WA & & & $\mathbf{U}$ & 5 & & 7 & $\mu g / L$ \\
\hline 1,2,3-Trichloropropane & W'A & & & $\mathbf{U}$ & 10 & & NS & $\mu g / L$ \\
\hline 1,2-Dibromo-3-chloropropane & WA & & & $\mathbf{U}$ & 20 & & 0.2 & $\mu \mathrm{g} / \mathrm{L}$ \\
\hline 1,2-Dibromoethane & WA & & & $\mathbf{U}$ & 20 & & NS & $\mu \mathrm{g} / \mathrm{L}$ \\
\hline 1,2-Dichloroethane & WA & & & $\mathbf{U}$ & 5 & & 5 & $\mu \mathrm{g} / \mathrm{L}$ \\
\hline 1,2-Dichloroethylene (total) & WA & & & $\mathbf{U}$ & 5 & & 7 & $\mu g / L$ \\
\hline 1,2-Dichloropropane & WA & & & $\mathbf{U}$ & 5 & & 5 & $\mu \mathrm{g} / \mathrm{L}$ \\
\hline 2-Hexanone & WA & & & $\mathbf{U}$ & 10 & & NS & $\mu \mathrm{g} / \mathrm{L}$ \\
\hline 4-Methyl-2-pentanone & WA & & & $\mathbf{U}$ & 10 & & NS & $\mu g / L$ \\
\hline Acetone & WA & & & $\mathbf{J}$ & 6.29 & & NS & $\mu g / L$ \\
\hline Acetonitrile & WA & & & $\mathbf{U}$ & 20 & & NS & $\mu g / L$ \\
\hline Acidity & WA & & & & 142 & & NS & $\mathrm{mg} / \mathrm{L}$ \\
\hline Acrolein & WA & & & $\mathbf{U}$ & 10 & & NS & $\mu \mathrm{g} / \mathrm{L}$ \\
\hline Acrylonitrile & WA & & & $\mathbf{U}$ & 10 & & NS & $\mu g / L$ \\
\hline Allyl chloride & WA & & & $\mathbf{U}$ & 100 & & NS & $\mu \mathrm{g} / \mathrm{L}$ \\
\hline Aluminum & WA & & & $\mathbf{U}$ & 200 & & NS & $\mu \mathrm{g} / \mathrm{L}$ \\
\hline Antimony & WA & & & $\mathbf{U}$ & 60 & & 6 & $\mu g / L$ \\
\hline Arsenic & WA & & & $\mathbf{U}$ & 10 & & 50 & $\mu g / L$ \\
\hline Barium & WA & & & $\mathbf{U}$ & 200 & & 2000 & $\mu g / L$ \\
\hline Benzene & WA & & & $\mathbf{U}$ & 5 & & 5 & $\mu \mathrm{g} / \mathrm{L}$ \\
\hline Beryllium & WA & & & $\mathbf{U}$ & 5 & & 4 & $\mu \mathrm{g} / \mathrm{L}$ \\
\hline Bromodichloromethane & WA & & & $\mathbf{U}$ & 5 & & NS & $\mu \mathrm{g} / \mathrm{L}$ \\
\hline Bromoform & WA & & & $\mathrm{U}$ & 5 & & NS & $\mu \mathrm{g} / \mathrm{L}$ \\
\hline Cadmium & WA & & & $\mathrm{U}$ & 5 & & 5 & $\mu \mathrm{g} / \mathrm{L}$ \\
\hline Calcium & WA & & & U & 5000 & & NS & $\mu \mathrm{g} / \mathrm{L}$ \\
\hline Carbon disulfide & WA & & & $\mathbf{U}$ & 5 & & NS & $\mu g / L$ \\
\hline Carbon tetrachloride & WA & & & $\mathbf{U}$ & 5 & & 5 & $\mu \mathrm{g} / \mathrm{L}$ \\
\hline Cesium-137 & TM & & & $\mathbf{U}$ & 9.4 & & 200 & $\mathrm{pCi} / \mathrm{L}$ \\
\hline Chloride & WA & & & & 5.93 & & NS & $\mathrm{mg} / \mathrm{L}$ \\
\hline Chlorobenzene & WA & & & $\mathrm{U}$ & 5 & & 100 & $\mu g / L$ \\
\hline Chloroethane & WA & & & $\mathrm{U}$ & 10 & & NS & $\mu \mathrm{g} / \mathrm{L}$ \\
\hline Chloroform & WA & & & $\mathrm{U}$ & 5 & & 100 & $\mu \mathrm{g} / \mathrm{L}$ \\
\hline Chloroprene & WA & & & $U$ & 20 & & NS & $\mu \mathrm{g} / \mathrm{L}$ \\
\hline Chromium & WA & & & $U$ & 10 & & 100 & $\mu \mathrm{g} / \mathrm{L}$ \\
\hline cis-1,3-Dichloropropene & WA & & & $\mathrm{U}$ & 5 & & NS & $\mu \mathrm{g} / \mathrm{L}$ \\
\hline Cobalt & WA & & & $\mathrm{U}$ & 50 & & NS & $\mu \mathrm{g} / \mathrm{L}$ \\
\hline Copper & WA & & & $\mathrm{U}$ & 25 & & 1000 & $\mu \mathrm{g} / \mathrm{L}$ \\
\hline Dibromochloromethane & WA & & & $\mathrm{U}$ & 5 & & NS & $\mu \mathrm{g} / \mathrm{L}$ \\
\hline Dichlorodifluoromethane & WA & & & U & 10 & & NS & $\mu \mathrm{g} / \mathrm{L}$ \\
\hline Ethylbenzene & WA & & & $\mathrm{U}$ & 5 & & 700 & $\mu \mathrm{g} / \mathrm{L}$ \\
\hline Fluoride & WA & & & $\mathrm{U}$ & 0.1 & & 4 & $\mathrm{mg} / \mathrm{L}$ \\
\hline Gross alpha & $\mathrm{TM}$ & & & $\mathbf{U}$ & 0.6 & & 15 & $\mathrm{pCi} / /$. \\
\hline
\end{tabular}


UTR072 Laboratory Analyses (cont'd)

\begin{tabular}{|c|c|c|c|c|c|c|c|c|c|}
\hline Analyte & $\mathrm{Lab}$ & Rep & Avg & Modifier & & Result & Acc & Stand & Units \\
\hline Iron & WA & & & & & 1560 & & 300 & $\mu g / L$ \\
\hline Isobutyl alcohol & WA & & & $\mathbf{U}$ & & 20 & & NS & $\mu \mathrm{g} / \mathrm{L}$ \\
\hline Lead & WA & & & $\mathbf{U}$ & & 3 & & 50 & $\mu \mathrm{g} / \mathrm{L}$ \\
\hline Magnesium & WA & & & $\mathbf{U}$ & & 5000 & & NS & $\mu \mathrm{g} / \mathrm{L}$ \\
\hline Manganese & WA & & & & & 215 & & 50 & $\mu \mathrm{g} / \mathrm{L}$ \\
\hline Mercury & WA & & & $\mathbf{U}$ & & 0.2 & & 2 & $\mu g / L$ \\
\hline Methacrylonitrile & WA & & & $\mathbf{U}$ & & 20 & & NS & $\mu g / L$ \\
\hline Methyl bromide & WA & & & $\mathbf{U}$ & & 10 & & NS & $\mu g / L$ \\
\hline Methyl chloride & WA & & & $\mathbf{U}$ & & 10 & & NS & $\mu g / L$ \\
\hline Methyl ethyl ketone & WA & & & $\mathbf{U}$ & & 10 & & NS & $\mu g / L$ \\
\hline Methyl iodide & WA & & & $\mathbf{U}$ & & 10 & & NS & $\mu g / L$ \\
\hline Methylene bromide & WA & & & $\mathbf{U}$ & & 10 & & NS & $\mu \mathrm{g} / \mathrm{L}$ \\
\hline Methylene chloride & WA & & & $\mathbf{J}$ & V & 3.63 & & 5 & $\mu g / L$ \\
\hline Nickel & WA & & & $\mathbf{U}$ & & 40 & & 100 & $\mu g / L$ \\
\hline Nitrate as nitrogen & WA & & & J & $\mathbf{Q}$ & 0.142 & & 10 & $\mathrm{mg} / \mathrm{L}$ \\
\hline Nonvolatile beta & TM & & & $\mathrm{U}$ & & 0.8 & & 50 & $\mathrm{pCi} / \mathrm{L}$ \\
\hline Potassium & WA & & & $\mathbf{U}$ & & 5000 & & NS & $\mu g / L$ \\
\hline Propionitrile & WA & & & $\mathbf{U}$ & & 50 & & NS & $\mu \mathrm{g} / \mathrm{L}$ \\
\hline Selenium & WA & & & $\mathbf{U}$ & & 5 & & 50 & $\mu g / L$ \\
\hline Silver & WA & & & $U$ & & 10 & & 50 & $\mu g / L$ \\
\hline Sodium & WA & & & $\mathbf{U}$ & & 5000 & & NS & $\mu g / L$ \\
\hline Styrene & WA & & & $\mathbf{U}$ & & 5 & & 100 & $\mu g / L$ \\
\hline Sulfate & WA & & & $\mathbf{U}$ & & 2.5 & & 400 & $\mathrm{mg} / \mathrm{L}$ \\
\hline Sulfide & WA & & & $\mathbf{U}$ & & 0.1 & & NS & $\mathrm{mg} / \mathrm{L}$ \\
\hline Tetrachloroethylene & WA & & & $\mathrm{U}$ & & 5 & & 5 & $\mu g / L$ \\
\hline Thallium & WA & & & $\mathbf{U}$ & & 10 & & 2 & $\mu g / L$ \\
\hline Toluene & WA & & & $U$ & & 5 & & 1000 & $\mu g / L$ \\
\hline Total hardness & WA & & & & & 2.9 & & NS & $\mathrm{mg} / \mathrm{L}$ \\
\hline Total organic carbon & WA & & & & & 2.79 & & NS & $\mathrm{mg} / \mathrm{L}$ \\
\hline Total suspended solids & WA & & A & & & 9.5 & & NS & $\mathrm{mg} / \mathrm{L}$ \\
\hline trans-1,3-Dichloropropene & WA & & & $\mathbf{U}$ & & 5 & & NS & $\mu g / L$ \\
\hline trans-1,4-Dichloro-2-butene & WA & & & $\mathbf{U}$ & & 100 & & NS & $\mu g / L$ \\
\hline Trichloroethylene & WA & & & $\mathbf{U}$ & & 5 & & 5 & $\mu g / L$ \\
\hline Trichlorofluoromethane & WA & & & $\mathbf{U}$ & & 5 & & NS & $\mu \mathrm{g} / \mathrm{L}$ \\
\hline Tritium & TM & & & & & 34.79 & 1.31 & 20 & $\mathrm{pCi} / \mathrm{ml}$ \\
\hline Turbidity & WA & & & & & 1.3 & & NS & NTU \\
\hline Vanadium & WA & & & $\mathrm{U}$ & & 50 & & NS & $\mu g / L$ \\
\hline Vinyl acetate & WA & & & $\mathrm{U}$ & & 10 & & NS & $\mu \mathrm{g} / \mathrm{L}$ \\
\hline Vinyl chloride & WA & & & $\mathbf{U}$ & & 10 & & 2 & $\mu \mathrm{g} / \mathrm{L}$ \\
\hline Xylenes (total) & WA & & & $\mathbf{U}$ & & 5 & & 10000 & $\mu \mathrm{g} / \mathrm{L}$ \\
\hline Zinc & WA & & & $\mathrm{U}$ & & 20 & & 5000 & $\mu g / L$ \\
\hline
\end{tabular}




\section{UTR099}

Field Measurements:

Sample Date: 8/31/93

pH: 7.20

Specific Conductance: $76 \mu \mathrm{S} / \mathrm{cm}$

Red/Oxid Potential: $62 \mathrm{mv}$

Dissolved Oxygen: $1.1 \mathrm{mg} / \mathrm{L}$

\begin{tabular}{|c|c|c|c|c|c|c|c|c|}
\hline Analyte & Lab & Rep & Avg & Monifier & Result & Acc & Stand & Units \\
\hline 1,1,1,2-Tetrachloroethane & WA & & & $\bar{U}$ & 10 & & NS & $\mu g / L$ \\
\hline 1,1,1-Trichloroethane & WA & & & $\mathbf{U}$ & 5 & & 200 & $\mu \mathrm{g} / \mathrm{L}$ \\
\hline $1,1,2,2-$ Tetrachloroethane & WA & & & $\mathbf{U}$ & 5 & & NS & $\mu g / L$ \\
\hline 1,1,2-Trichloroethane & WA & & & $\mathbf{U}$ & 5 & & 5 & $\mu \mathrm{g} / \mathrm{L}$ \\
\hline 1,1-Dichloroethane & WA & & & $\mathbf{U}$ & 5 & & NS & $\mu g / L$ \\
\hline 1,1-Dichloroethylene & WA & & & $\mathbf{U}$ & 5 & & 7 & $\mu g / L$ \\
\hline 1,2,3-Trichloropropane & WA & & & $\mathbf{U}$ & 10 & & NS & $\mu g / L$ \\
\hline 1,2-Dibromo-3-chloropropane & WA & & & $\mathbf{U}$ & 20 & & 0.2 & $\mu g / L$ \\
\hline 1,2-Dibromoethane & WA & & & $\mathbf{U}$ & 20 & & NS & $\mu \mathrm{g} / \mathrm{L}$ \\
\hline 1,2-Dichloroethane & WA & & & $\mathbf{U}$ & 5 & & 5 & $\mu g / L$ \\
\hline 1,2-Dichloroethylene (total) & WA & & & $\mathbf{U}$ & 5 & & 7 & $\mu g / L$ \\
\hline 1,2-Dichloropropane & WA & & & $\mathbf{U}$ & 5 & & 5 & $\mu g / L$ \\
\hline 2-Hexanone & WA & & & $\mathbf{U}$ & 10 & & NS & $\mu \mathrm{g} / \mathrm{L}$ \\
\hline 4-Methyl-2-pentanone & WA & & & $\mathbf{U}$ & 10 & & NS & $\mu \mathrm{g} / \mathrm{L}$ \\
\hline Acetone & WA & & & $\mathrm{J}$ & 6.3 & & NS & $\mu \mathrm{g} / \mathrm{L}$ \\
\hline Acetonitrile & WA & & & $\mathrm{U}$ & 20 & & NS & $\mu \mathrm{g} / \mathrm{L}$ \\
\hline Acidity & WA & & & & 50 & & NS & $\mathrm{mg} / \mathrm{L}$ \\
\hline Acrolein & WA & & & $\mathbf{U}$ & 10 & & NS & $\mu g / L$ \\
\hline Acrylonitrile & WA & & & $\mathrm{U}$ & 10 & & NS & $\mu \mathrm{g} / \mathrm{L}$ \\
\hline Alkalinity & WA & & & & 6 & & NS & $\mathrm{mg} / \mathrm{L}$ \\
\hline Allyl chloride & WA & & & $\mathrm{U}$ & 100 & & NS & $\mu \mathrm{g} / \mathrm{L}$ \\
\hline Aluminum & WA & & & $\mathbf{U}$ & 200 & & NS & $\mu g / L$ \\
\hline Antimony & WA & & & $\mathbf{U}$ & 60 & & 6 & $\mu g / L$ \\
\hline Arsenic & WA & & & $\mathrm{U}$ & 10 & & 50 & $\mu \mathrm{g} / \mathrm{L}$ \\
\hline Barium & WA & & & $\mathbf{U}$ & 200 & & 2000 & $\mu g / L$ \\
\hline Benzene & WA & & & $\mathbf{U}$ & 5 & & 5 & $\mu g / L$ \\
\hline Beryllium & WA & & & $\mathbf{U}$ & 5 & & 4 & $\mu g / L$ \\
\hline Bromodichloromethane & WA & & & $\mathbf{U}$ & 5 & & NS & $\mu g / L$ \\
\hline Bromoform & WA & & & $\mathrm{U}$ & 5 & & NS & $\mu g / L$ \\
\hline Cadmium & WA & & & $\mathrm{U}$ & 5 & & 5 & $\mu g / L$ \\
\hline Calcium & WA & & & $\mathbf{U}$ & 5000 & & NS & $\mu g / L$ \\
\hline Carbon disulfide & WA & & & U & 5 & & NS & $\mu g / L$ \\
\hline Carbon tetrachloride & WA & & & $\mathbf{U}$ & 5 & & 5 & $\mu g / L$ \\
\hline Cesium-137 & TM & & & $\mathrm{U}$ & 6.6 & & 200 & $\mathrm{pCi} / \mathrm{L}$ \\
\hline Chloride & WA & & & & 4.44 & & NS & $\mathrm{mg} / \mathrm{L}$ \\
\hline Chlorobenzene & WA & & & $\mathbf{U}$ & 5 & & 100 & $\mu g / L$ \\
\hline Chloroethane & WA & & & $\mathbf{U}$ & 10 & & NS & $\mu g / L$ \\
\hline Chloroform & WA & & & $\mathbf{U}$ & 5 & & 100 & $\mu \mathrm{g} / \mathrm{L}$ \\
\hline Chloroprene & WA & & & $\mathbf{U}$ & 20 & & NS & $\mu \mathrm{g} / \mathrm{L}$ \\
\hline Chromium & WA & & & U & 10 & & 100 & $\mu \mathrm{g} / \mathrm{L}$ \\
\hline cis-1,3-Dichloropropene & WA & & & $\mathbf{U}$ & 5 & & NS & $\mu \mathrm{g} / \mathrm{L}$ \\
\hline Cobalt & WA & & & $\mathrm{U}$ & 50 & & NS & $\mu \mathrm{g} / \mathrm{L}$ \\
\hline Copper & WA & & & $\mathbf{U}$ & 25 & & 1000 & $\mu g / L$ \\
\hline Dibromochloromethane & WA & & & $\mathbf{U}$ & 5 & & NS & $\mu \mathrm{g} / \mathrm{L}$ \\
\hline Dichlorodifluoromethane & WA & & & $\mathrm{U}$ & 10 & & NS & $\mu \mathrm{g} / \mathrm{L}$ \\
\hline Ethylbenzene & WA & & & $\mathbf{U}$ & 5 & & 700 & $\mu g / L$ \\
\hline Fluoride & WA & & & $\mathbf{U}$ & 0.1 & & 4 & $\mathrm{mg} / \mathrm{L}$ \\
\hline
\end{tabular}


UTR099 Laboratory Analyses (cont'd)

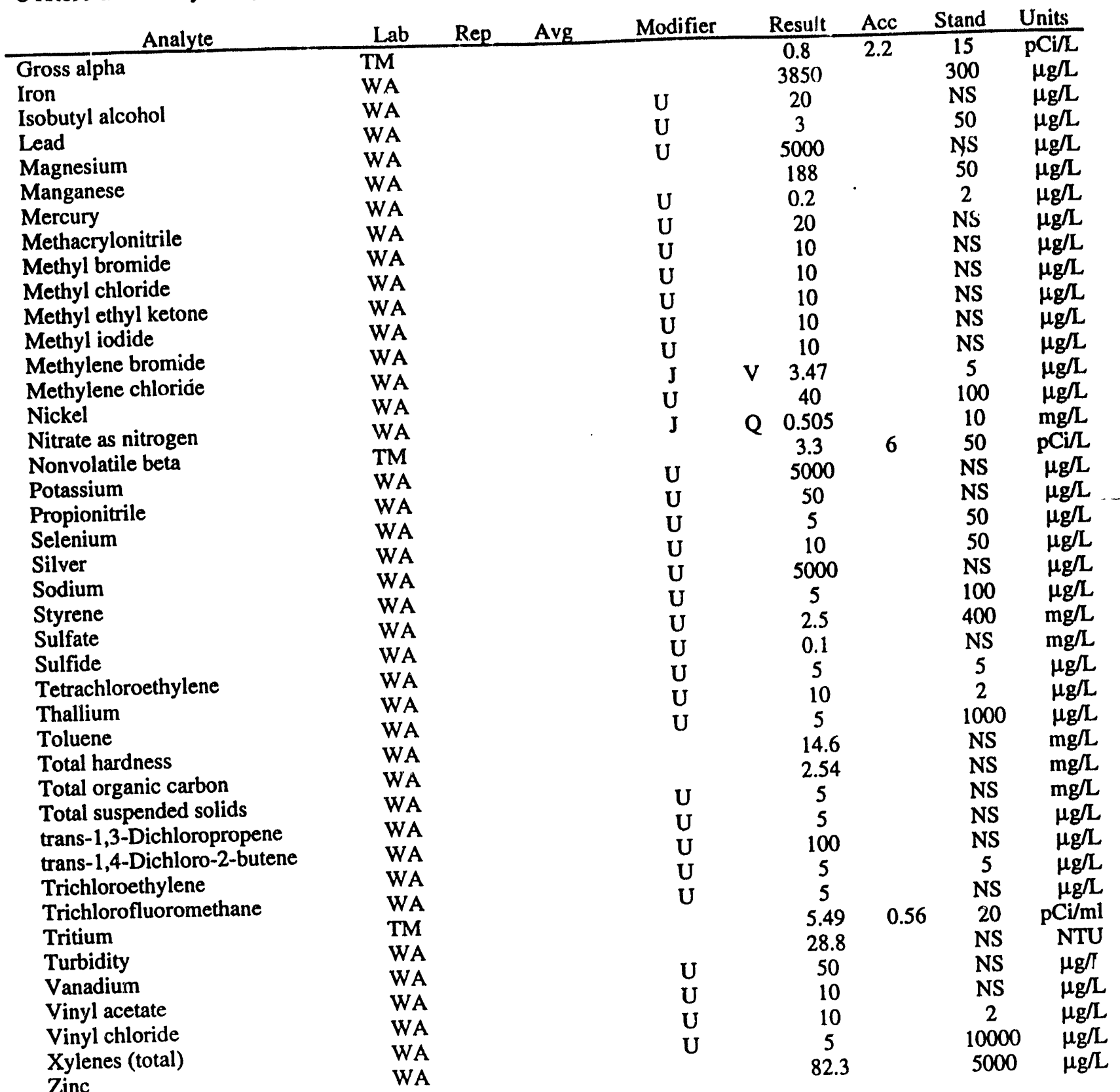




\section{UTR102}

Field Measurements:

Sample Date: 8/31/93

pH: 7.14

Specific Conductance: $16 \mu \mathrm{S} / \mathrm{cm}$

Red/Oxid Potential: $-44 \mathrm{mv}$

Dissolved Oxygen: $1.4 \mathrm{mg} / \mathrm{L}$

\begin{tabular}{|c|c|c|c|c|c|c|c|}
\hline Analyte & Lab & Avg & Modifier & Result & Acc & Stand & Units \\
\hline 1,1,1,2-Tetrachloroethane & $\overline{\text { WA }}$ & & $\mathbf{U}$ & 10 & & NS & $\mu g / L$ \\
\hline 1,1,1-Trichloroethane & WA & & $\mathbf{U}$ & 5 & & 200 & $\mu \mathrm{g} / \mathrm{L}$ \\
\hline 1,1,2,2-Tetrachloroethane & WA & & $\mathbf{U}$ & 5 & & NS & $\mu g / L$ \\
\hline 1,1,2-Trichloroethane & WA & & U & 5 & & 5 & $\mu g / L$ \\
\hline 1,1-Dichloroethane & WA & & $\mathbf{U}$ & 5 & & NS & $\mu \mathrm{g} / \mathrm{L}$ \\
\hline 1,1-Dichloroethylene & WA & & $\mathbf{U}$ & 5 & & 7 & $\mu \mathrm{g} / \mathrm{L}$ \\
\hline 1,2,3-Trichloropropane & WA & & $\mathbf{U}$ & 10 & & NS & $\mu \mathrm{g} / \mathrm{L}$ \\
\hline 1,2-Dibromo-3-chloropropane & WA & & $\mathbf{U}$ & 20 & & 0.2 & $\mu g / L$ \\
\hline 1,2-Dibromoethane & WA & & $\mathbf{U}$ & 20 & & NS & $\mu g / L$ \\
\hline 1,2-Dichloroethane & WA & & $\mathbf{U}$ & 5 & & 5 & $\mu \mathrm{g} / \mathrm{L}$ \\
\hline 1,2-Dichloroethylene (total) & W.A & & $\mathbf{U}$ & 5 & & 7 & $\mu \mathrm{g} / \mathrm{L}$ \\
\hline 1,2-Dichloropropane & WA & & $\mathbf{U}$ & 5 & & 5 & $\mu \mathrm{g} / \mathrm{L}$ \\
\hline 2-Hexanone & WA & & $\mathbf{U}$ & 10 & & $1 \mathrm{~S}$ & $\mu \mathrm{g} / \mathrm{L}$ \\
\hline 4-Methyl-2-pentanone & WA & & $\mathbf{U}$ & 10 & & $\mathrm{NS}$ & $\mu g / \mathrm{L}$ \\
\hline Acetone & WA & & $\mathbf{J}$ & 5.99 & & NS & $\mu g / L$ \\
\hline Acetonitrile & WA & & $\mathbf{U}$ & 20 & & NS & $\mu \mathrm{g} / \mathrm{L}$ \\
\hline Acidity & WA & & & 44 & & NS & $\mathrm{mg} / \mathrm{L}$ \\
\hline Acrolein & WA & & $\mathbf{U}$ & 10 & & NS & $\mu \mathrm{g} / \mathrm{L}$ \\
\hline Acrylonitrile & WA & & $\mathbf{U}$ & 10 & & NS & $\mu \mathrm{g} / \mathrm{L}$ \\
\hline Alkalinity & WA & & $\mathrm{U}$ & 0.5 & & NS & $\mathrm{mg} / \mathrm{L}$ \\
\hline Allyl chloride & WA & & $\mathrm{U}$ & 100 & & NS & $\mu \mathrm{g} / \mathrm{L}$ \\
\hline Aluminum & WA & & $\mathbf{U}$ & 200 & & NS & $\mu \mathrm{g} / \mathrm{L}$ \\
\hline Antimony & WA & & $\mathrm{U}$ & 60 & & 6 & $\mu \mathrm{g} / \mathrm{L}$ \\
\hline Arsenic & WA & & $\mathbf{U}$ & 10 & & 50 & $\mu \mathrm{g} / \mathrm{L}$ \\
\hline Barium & WA & & $\mathbf{U}$ & 200 & & 2000 & $\mu \mathrm{g} / \mathrm{L}$ \\
\hline Benzene & WA & & $\mathbf{U}$ & 5 & & 5 & $\mu \mathrm{g} / \mathrm{L}$ \\
\hline Beryllium & WA & & $\mathbf{U}$ & 5 & & 4 & $\mu \mathrm{g} / \mathrm{L}$ \\
\hline Bromodichloromethane & WA & & $\mathbf{U}$ & 5 & & NS & $\mu \mathrm{g} / \mathrm{L}$ \\
\hline Bromoform & WA & & $\mathbf{U}$ & 5 & & NS & $\mu \mathrm{g} / \mathrm{L}$ \\
\hline Cadmium & WA & & U & 5 & & 5 & $\mu \mathrm{g} / \mathrm{L}$ \\
\hline Calcium & WA & & $\mathbf{U}$ & 5000 & & NS & $\mu \mathrm{g} / \mathrm{L}$ \\
\hline Carbon disulfide & WA & & $\mathrm{U}$ & 5 & & NS & $\mu \mathrm{g} / \mathrm{L}$ \\
\hline Carbon tetrachloride & WA & & $\mathrm{U}$ & 5 & & 5 & $\mu g / L$ \\
\hline Cesium-137 & TM & & $\mathrm{U}$ & 9.4 & & 200 & $\mathrm{pCi} / \mathrm{L}$ \\
\hline Chloride & WA & & & 4.2 & & NS & $\mathrm{mg} / \mathrm{L}$ \\
\hline Chlorobenzenè & WA & & $\mathrm{U}$ & 5 & & 100 & $\mu g / L$ \\
\hline Chloroethane & WA & & $\mathrm{U}$ & 10 & & NS & $\mu \mathrm{g} / \mathrm{L}$ \\
\hline Chloroform & WA & & $\mathrm{U}$ & 5 & & 100 & $\mu \mathrm{g} / \mathrm{L}$ \\
\hline Chloroprene & WA & & $\mathrm{U}$ & 20 & & NS & $\mu \mathrm{g} / \mathrm{L}$ \\
\hline Chromium & WA & & $\mathbf{U}$ & 10 & & 100 & $\mu \mathrm{g} / \mathrm{L}$ \\
\hline cis-1,3-Dichloropropene & WA & & $\mathbf{U}$ & 5 & & NS & $\mu \mathrm{g} / \mathrm{L}$ \\
\hline Cobalt & WA & & $\mathbf{U}$ & 50 & & NS & $\mu \mathrm{g} / \mathrm{L}$ \\
\hline Copper & WA & & $\mathbf{U}$ & 25 & & 1000 & $\mu \mathrm{g} / \mathrm{L}$ \\
\hline Dibromochloromethane & WA & & $\mathbf{U}$ & 5 & & NS & $\mu \mathrm{g} / \mathrm{L}$ \\
\hline Dichlorodifluoromethane & WA & & $\mathrm{U}$ & 10 & & NS & $\mu g / L$ \\
\hline Ethylbenzene & WA & & $\mathbf{U}$ & 5 & & 700 & $\mu \mathrm{g} / \mathrm{L}$. \\
\hline Fluoride & WA & & $\mathbf{U}$ & 0.1 & & 4 & $\mathrm{mg} / \mathrm{L}$ \\
\hline
\end{tabular}


UTR102 Laboratory Analyses (cont'd)

\begin{tabular}{|c|c|c|c|c|c|c|c|c|c|}
\hline Analyte & Lab & Rep & Avg & Modifier & & Result & Acc & Stand & Units \\
\hline Gross alpha & TM & & & & & 1.1 & 1.2 & 15 & $\overline{\mathrm{pCi} / \mathrm{L}}$ \\
\hline Iron & WA & & & & & 844 & & 300 & $\mu g / L$ \\
\hline Isobutyl alcohol & WA & & & $\mathrm{U}$ & & 20 & & NS & $\mu \mathrm{g} / \mathrm{L}$ \\
\hline Lead & WA & & & $\mathrm{U}$ & & 3 & & 50 & $\mu \mathrm{g} / \mathrm{L}$ \\
\hline Magnesium & WA & & & $\mathrm{U}$ & & 5000 & & NS & $\mu \mathrm{g} / \mathrm{L}$ \\
\hline Manganese & WA & & & & & 17.3 & & 50 & $\mu \mathrm{g} / \mathrm{L}$ \\
\hline Mercury & WA & & & $\mathrm{U}$ & & 0.2 & - & 2 & $\mu g / L$ \\
\hline Methacrylonitrile & WA & & & $\mathbf{U}$ & & 20 & & NS & $\mu g / L$ \\
\hline Methyl bromide & WA & & & $\mathbf{U}$ & & 10 & & NS & $\mu g / L$ \\
\hline Methyl chloride & WA & & & $\mathrm{U}$ & & 10 & & NS & $\mu g / L$ \\
\hline Methyl ethyl ketone & WA & & & $\mathbf{U}$ & & 10 & & NN & $\mu g / L$ \\
\hline Methyl iodide & WA & & & $\mathbf{U}$ & & 10 & & NS & $\mu \mathrm{g} / \mathrm{L}$ \\
\hline Methylene bromide & WA & & & $\mathbf{U}$ & & 10 & & NS & $\mu g / L$ \\
\hline Methylene chloride & WA & & & J & $\mathbf{V}$ & 3.87 & & 5 & $\mu \mathrm{g} / \mathrm{L}$ \\
\hline Nickel & WA & & & $\mathbf{U}$ & & 40 & & 100 & $\mu \mathrm{g} / \mathrm{L}$ \\
\hline Nitrate as nitrogen & WA & & . & J & $\mathbf{Q}$ & 0.139 & & 10 & $\mathrm{mg} / \mathrm{L}$ \\
\hline Nonvolatile beta & $\mathrm{TM}$ & & & & & 0.9 & 2.6 & 50 & $\mathrm{pCi} / \mathrm{L}$ \\
\hline Potassium & WA & & & $\mathbf{U}$ & & 5000 & & NS & $\mu g / L$ \\
\hline Propionitrile & WA & & & $\mathrm{U}$ & & 50 & & NS & $\mu g / L$ \\
\hline Selenium & WA & & & $\mathrm{U}$ & & 5 & & 50 & $\mu \mathrm{g} / \mathrm{L}$ \\
\hline Silver & WA & & & $\mathbf{U}$ & & 10 & & 50 & $\mu g / L$ \\
\hline Sodium & WA & & & $\mathrm{U}$ & & 5000 & & NS & $\mu g / L$ \\
\hline Styrene & WA & & & $\mathrm{U}$ & & 5 & & 100 & $\mu g / L$ \\
\hline Sulfate & WA & & & $\mathrm{U}$ & & 2.5 & & 400 & $\mathrm{mg} / \mathrm{L}$ \\
\hline Sulfide & WA & & & U & & 0.1 & & NS & $\mathrm{mg} / \mathrm{L}$ \\
\hline Tetrachloroethylene & WA & & & $\mathrm{U}$ & & 5 & & 5 & $\mu \mathrm{g} / \mathrm{L}$ \\
\hline Thallium & WA & & & $\mathbf{U}$ & & 10 & & 2 & $\mu g / L$ \\
\hline Toluene & WA & & & $U$ & & 5 & & 1000 & $\mu \mathrm{g} / \mathrm{L}$ \\
\hline Total hardness & WA & & & $\mathbf{U}$ & & 1 & & NS & $\mathrm{mg} / \mathrm{L}$ \\
\hline Total organic carbon & WA & & & & & 2.18 & & NS & $\mathrm{mg} / \mathrm{L}$ \\
\hline Total suspended solids & WA & & & $\mathrm{U}$ & & 5 & & NS & $\mathrm{mg} / \mathrm{L}$ \\
\hline trans-1,3-Dichloropropene & WA & & & $\mathrm{U}$ & & 5 & & NS & $\mu \mathrm{g} / \mathrm{L}$ \\
\hline trans-1,4-Dichloro-2-butene & WA & & & $\mathrm{U}$ & & 100 & & NS & $\mu g / L$ \\
\hline Trichloroethylene & WA & & & $\mathrm{U}$ & & 5 & & 5 & $\mu \mathrm{g} / \mathrm{L}$ \\
\hline Trichlorofluoromethane & WA & & & $\mathrm{U}$ & & 5 & & NS & $\mu g / L$ \\
\hline Tritium & $\mathrm{TM}$ & & & & & 4.7 & 0.53 & 20 & $\mathrm{pCi} / \mathrm{ml}$ \\
\hline Turbidity & WA & & & & & 0.75 & & NS & NTU \\
\hline Vanadium & WA & & & $\mathrm{U}$ & & 50 & & NS & $\mu \mathrm{g} / \mathrm{L}$ \\
\hline Vinyl acetate & WA & & & $\mathrm{U}$ & & 10 & & NS & $\mu \mathrm{g} / \mathrm{L}$ \\
\hline Vinyl chloride & WA & & & $\mathrm{U}$ & & 10 & & 2 & $\mu \mathrm{g} / \mathrm{L}$ \\
\hline Xylenes (total) & WA & & & $\mathrm{U}$ & & 5 & & 10000 & $\mu \mathrm{g} / \mathrm{L}$ \\
\hline Zinc & WA & & & $\mathrm{U}$ & & 20 & & 5000 & $\mu \mathrm{g} / \mathrm{L}$ \\
\hline
\end{tabular}




\section{UTR108}

Field Measurements:

Sample Date: 8/26/93

pH: 6.06

Specific Conductance: $20 \mu \mathrm{S} / \mathrm{cm}$

Red/Oxid Potential: $74 \mathrm{mv}$

Dissolved Oxygen: $1.7 \mathrm{mg} / \mathrm{L}$

\begin{tabular}{|c|c|c|c|c|c|c|c|c|}
\hline Analyte & Lab & Rep & Avg & Modifier & Result & Acc & Stand & Units \\
\hline 1,1,1,2-Tetrachloroethane & WA & & & $\mathbf{U}$ & 10 & & NS & $\mu g / L$ \\
\hline $1,1,1$-Trichloroethane & WA & & & $\mathbf{U}$ & 5 & & 200 & $\mu \mathrm{g} / \mathrm{L}$ \\
\hline $1,1,2, ?-$ Tetrachloroethane & WA & & & $\mathbf{U}$ & 5 & & NS & $\mu g / L$ \\
\hline 1,1,2-Trichloroethane & WA & & & $\mathbf{U}$ & 5 & & 5 & $\mu g / L$ \\
\hline 1,1-Dichloroethane & WA & & & $\mathbf{U}$ & 5 & & NS & $\mu g / L$ \\
\hline 1,1-Dichloroethylene & WA & & & $\mathbf{U}$ & 5 & & 7 & $\mu g / L$ \\
\hline 1,2,3-Trichloropropane & WA & & & $\mathbf{U}$ & 10 & & NS & $\mu \mathrm{g} / \mathrm{L}$ \\
\hline 1,2-Dibromo-3-chloropropane & WA & & & $\mathbf{U}$ & 20 & & 0.2 & $\mu \mathrm{g} / \mathrm{L}$ \\
\hline 1,2-Dibromoethane & WA & & & $\mathbf{U}$ & 20 & & NS & $\mu \mathrm{g} / \mathrm{L}$ \\
\hline 1,2-Dichloroethane & WA & & & U & 5 & & 5 & $\mu g / L$ \\
\hline 1,2-Dichloroethylene (total) & WA & & & $\mathbf{U}$ & 5 & & 7 & $\mu \mathrm{g} / \mathrm{L}$ \\
\hline 1,2-Dichloropropane & WA & & & $\mathbf{U}$ & 5 & & 5 & $\mu g / L$ \\
\hline 2-Hexanone & WA & & & $\mathbf{U}$ & 10 & & NS & $\mu g / L$ \\
\hline 4-Methyl-2-pentanone & WA & & & $\mathbf{U}$ & 10 & & NS & $\mu g / L$ \\
\hline Acetone & WA & & & $\mathbf{J}$ & 4.86 & & NS & $\mu g / L$ \\
\hline Acetonitrile & WA & & & $\mathbf{U}$ & 20 & & NS & $\mu g / L$ \\
\hline Acidity & WA & & & & 64 & & NS & $\mathrm{mg} / \mathrm{L}$ \\
\hline Acrolein & WA & & & $\mathbf{U}$ & 10 & & NS & $\mu g / L$ \\
\hline Acrylonitrile & WA & & & $\mathbf{U}$ & 10 & & NS & $\mu g / L$ \\
\hline Alkalinity & WA & & & & 2 & & NS & $\mathrm{mg} / \mathrm{L}$ \\
\hline Allyl chloride & WA & & & $\mathbf{U}$ & 100 & & NS & $\mu g / L$ \\
\hline Aluminum & WA & & & & 871 & & NS & $\mu g / L$ \\
\hline Antimony & WA & & & $\mathbf{U}$ & 60 & & 6 & $\mu g / L$ \\
\hline Arsenic & WA & & & $\mathbf{U}$ & 10 & & 50 & $\mu g / L$ \\
\hline Barium & WA & & & $\mathbf{U}$ & 200 & & 2000 & $\mu g / L$ \\
\hline Benzene & WA & & & $\mathbf{U}$ & 5 & & 5 & $\mu g / L$ \\
\hline Beryllium & WA & & & $\mathbf{U}$ & 5 & & 4 & $\mu g / L$ \\
\hline Bromodichloromethane & WA & & & $\mathbf{U}$ & 5 & & NS & $\mu \mathrm{g} / \mathrm{L}$ \\
\hline Bromoform & WA & & & $\mathbf{U}$ & 5 & & NS & $\mu \mathrm{g} / \mathrm{L}$ \\
\hline Cadmium & WA & & & $\mathbf{U}$ & 5 & & 5 & $\mu \mathrm{g} / \mathrm{L}$ \\
\hline Calcium & WA & & & $\mathbf{U}$ & 5000 & & NS & $\mu g / L$ \\
\hline Carbon disulfide & WA & & & $\mathbf{U}$ & 5 & & NS & $\mu g / L$ \\
\hline Carbon tetrachloride & WA & & & $\mathbf{U}$ & 5 & & 5 & $\mu \mathrm{g} / \mathrm{L}$ \\
\hline Cesium-137 & TM & & & $\mathbf{U}$ & 10.2 & & 200 & $\mathrm{pCi} / \mathrm{L}$ \\
\hline Chloride & WA & & & & 5.42 & & NS & $\mathrm{mg} / \mathrm{L}$ \\
\hline Chlorobenzene & WA & & & $\mathbf{U}$ & 5 & & 100 & $\mu g / L$ \\
\hline Chloroethane & WA & & & $\mathbf{U}$ & 10 & & NS & $\mu g / L$ \\
\hline Chloroform & WA & & & $\mathbf{U}$ & 5 & & 100 & $\mu g / L$ \\
\hline Chloroprene & WA & & & $\mathbf{U}$ & 20 & & NS & $\mu \mathrm{g} / \mathrm{L}$ \\
\hline Chromium & WA & & & $\mathbf{U}$ & 10 & & 100 & $\mu g / L$ \\
\hline cis-1,3-Dichloropropene & WA & & & $\mathbf{U}$ & 5 & & NS & $\mu \mathrm{g} / \mathrm{L}$ \\
\hline Cobalt & WA & & & $\mathrm{U}$ & 50 & & NS & $\mu \mathrm{g} / \mathrm{L}$ \\
\hline Copper & WA & & & $\mathbf{U}$ & 25 & & 1000 & $\mu \mathrm{g} / \mathrm{L}$ \\
\hline Dibromochloromethane & WA & & & $\mathbf{U}$ & 5 & & NS & $\mu g / L$ \\
\hline Dichlorodifluoromethane & WA & & & $\mathbf{U}$ & 10 & & NS & $\mu \mathrm{g} / \mathrm{L}$ \\
\hline Ethylbenzene & WA & & & $\mathbf{U}$ & 5 & & 700 & $\mu \mathrm{g} / \mathrm{L}$ \\
\hline
\end{tabular}


UTR 108 Laboratory Analyses (cont'd)

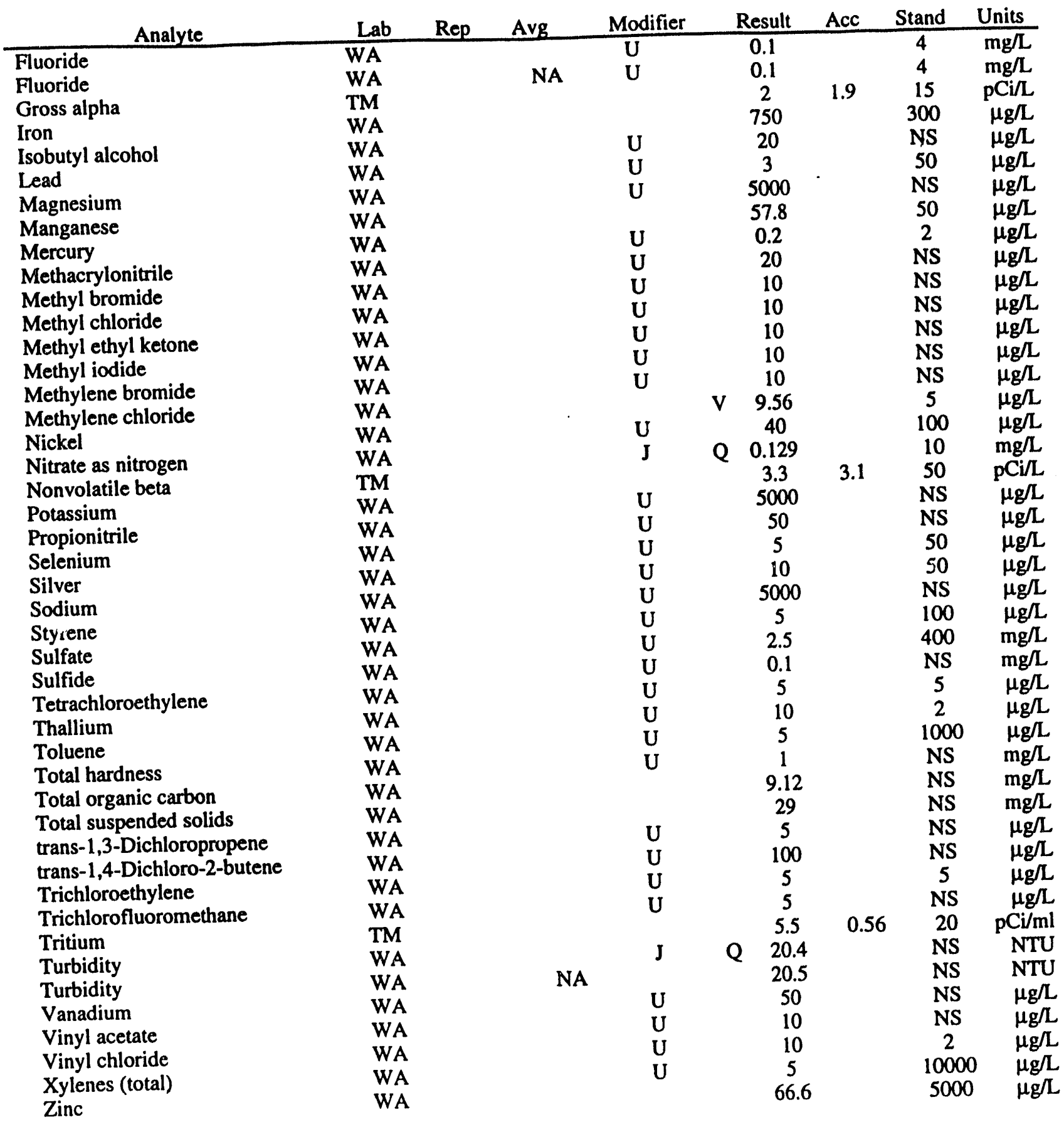




\section{UTR109}

Field Measurements:

Sample Date: $8 / 26 / 93$

pH: 6.57

Specific Conductance: $36 \mu \mathrm{S} / \mathrm{cm}$

Red/Oxid Potential: $170 \mathrm{mv}$

Dissolved Oxygen: $1.5 \mathrm{mg} / \mathrm{L}$

\begin{tabular}{|c|c|c|c|c|c|c|c|c|}
\hline Analyte & Lab & Rep & Avg & Modifier & Result & Acc & Stand & Units \\
\hline 1,1,1,2-Tetrachloroethane & WA & & & $\mathbf{U}$ & 10 & & NS & $\mu g / L$ \\
\hline 1,1,1-Trichloroethane & WA & & & $\mathbf{U}$ & 5 & & 200 & $\mu \mathrm{g} / \mathrm{L}$ \\
\hline $1,1,2,2$-Tetrachloroethane & WA & & & $\mathbf{U}$ & 5 & & NS & $\mu g / L$ \\
\hline 1,1,2-Trichloroethane & WA & & & $\mathbf{U}$ & 5 & & 5 & $\mu g / L$ \\
\hline 1,1-Dichloroethane & WA & & & $\mathbf{U}$ & 5 & & NS & $\mu g / L$ \\
\hline 1,1-Dichloroethylene & WA & & & $\mathbf{U}$ & 5 & & 7 & $\mu g / L$ \\
\hline 1,2,3-Trichloropropane & WA & & & $\mathbf{U}$ & 10 & & NS & $\mu g / L$ \\
\hline 1,2-Dibromo-3-chloropropane & WA & & & $\mathbf{U}$ & 20 & & 0.2 & $\mu g / L$ \\
\hline 1,2-Dibromoethane & WA & & & $\mathbf{U}$ & 20 & & NS & $\mu \mathrm{g} / \mathrm{L}$ \\
\hline 1,2-Dichloroethane & WA & & & $\mathbf{U}$ & 5 & & 5 & $\mu g / L$ \\
\hline 1,2-Dichloroethylene (total) & WA & & & $\mathbf{U}$ & 5 & & 7 & $\mu g / L$ \\
\hline 1,2-Dichloropropane & WA & & & $\mathbf{U}$ & 5 & & 5 & $\mu g / L$ \\
\hline 2-Hexanone & WA & & & $\mathbf{U}$ & 10 & & NS & $\mu g / L$ \\
\hline 4-Methyl-2-pentanone & WA & & & $\mathbf{U}$ & 10 & & NS & $\mu g / L$ \\
\hline Acetone & WA & & & $\mathbf{J}$ & 9.32 & & NS & $\mu \mathrm{g} / \mathrm{L}$ \\
\hline Acetonitrile & WA & & & $\mathbf{U}$ & 20 & & NS & $\mu \mathrm{g} / \mathrm{L}$ \\
\hline Acidity & WA & & & & 36 & & NS & $\mathrm{mg} / \mathrm{L}$ \\
\hline Acrolein & WA & & & $\mathbf{U}$ & 10 & & NS & $\mu g / L$ \\
\hline Acrylonitrile & WA & & & $\mathbf{U}$ & 10 & & NS & $\mu g / L$ \\
\hline Allyl chloride & WA & & & $\mathbf{U}$ & 100 & & NS & $\mu \mathrm{g} / \mathrm{L}$ \\
\hline Aluminum & WA & & & $\mathbf{U}$ & 200 & & NS & $\mu g / L$ \\
\hline Antimony & WA & & & $\mathrm{U}$ & 60 & & 6 & $\mu \mathrm{g} / \mathrm{L}$ \\
\hline Arsenic & WA & & & $\mathbf{U}$ & 10 & & 50 & $\mu g / L$ \\
\hline Barium & WA & & & $\mathbf{U}$ & 200 & & 2000 & $\mu g / L$ \\
\hline Benzene & WA & & & U & 5 & & 5 & $\mu g / L$ \\
\hline Beryllium & WA & & & $\mathbf{U}$ & 5 & & 4 & $\mu g / L$ \\
\hline Bromodichloromethane & WA & & & $\mathrm{U}$ & 5 & & NS & $\mu \mathrm{g} / \mathrm{L}$ \\
\hline Bromoform & WA & & & $\mathbf{U}$ & 5 & & NS & $\mu g / L$ \\
\hline Cadmium & WA & & & U & 5 & & 5 & $\mu g / L$ \\
\hline Calcium & WA & & & $\mathrm{U}$ & 5000 & & NS & $\mu \mathrm{g} / \mathrm{L}$ \\
\hline Carbon disulfide & WA & & & $\mathbf{U}$ & 5 & & NS & $\mu \mathrm{g} / \mathrm{L}$ \\
\hline Carbon tetrachloride & WA & & & $\mathrm{U}$ & 5 & & 5 & $\mu g / L$ \\
\hline Cesium-137 & TM & & & $\mathrm{U}$ & 6.3 & & 200 & $\mathrm{pCi} / \mathrm{L}$ \\
\hline Chloride & WA & & & & 4.35 & & NS & $\mathrm{mg} / \mathrm{L}$ \\
\hline Chlorobenzene. & WA & & & $\mathbf{U}$ & 5 & & 100 & $\mu \mathrm{g} / \mathrm{L}$ \\
\hline Chloroethane & WA & & & $U$ & 10 & & NS & $\mu \mathrm{g} / \mathrm{L}$ \\
\hline Chloroform & WA & & & $U$ & 5 & & 100 & $\mu \mathrm{g} / \mathrm{L}$ \\
\hline Chloroprene & WA & & & U & 20 & & NS & $\mu \mathrm{g} / \mathrm{L}$ \\
\hline Chromium & WA & & & $\mathbf{U}$ & 10 & & 100 & $\mu \mathrm{g} / \mathrm{L}$ \\
\hline cis-1,3-Dichloropropene & WA & & & $\mathbf{U}$ & 5 & & NS & $\mu \mathrm{g} / \mathrm{L}$ \\
\hline Cobalt & WA & & & $\mathbf{U}$ & 50 & & NS & $\mu \mathrm{g} / \mathrm{L}$ \\
\hline Copper & WA & & & $\mathbf{U}$ & 25 & & 1000 & $\mu \mathrm{g} / \mathrm{L}$ \\
\hline Dibromochloromethane & WA & & & $\mathbf{U}$ & 5 & & NS & $\mu g / L$ \\
\hline Dichlorodifluoromethane & WA & & & $\mathrm{U}$ & 10 & & NS & $\mu \mathrm{g} / \mathrm{L}$ \\
\hline Ethylbenzene & WA & & & $\mathrm{U}$ & 5 & & 700 & $\mu g / L$ \\
\hline Fluoride & WA & & & $\mathbf{U}$ & 0.1 & & 4 & $\mathrm{mg} / \mathrm{L}$ \\
\hline Gross alpha & TM & & & U & 0.7 & & 15 & $\mathrm{pCi} / \mathrm{L}$ \\
\hline
\end{tabular}


UTR109 Laboratory Analyses (cont'd)

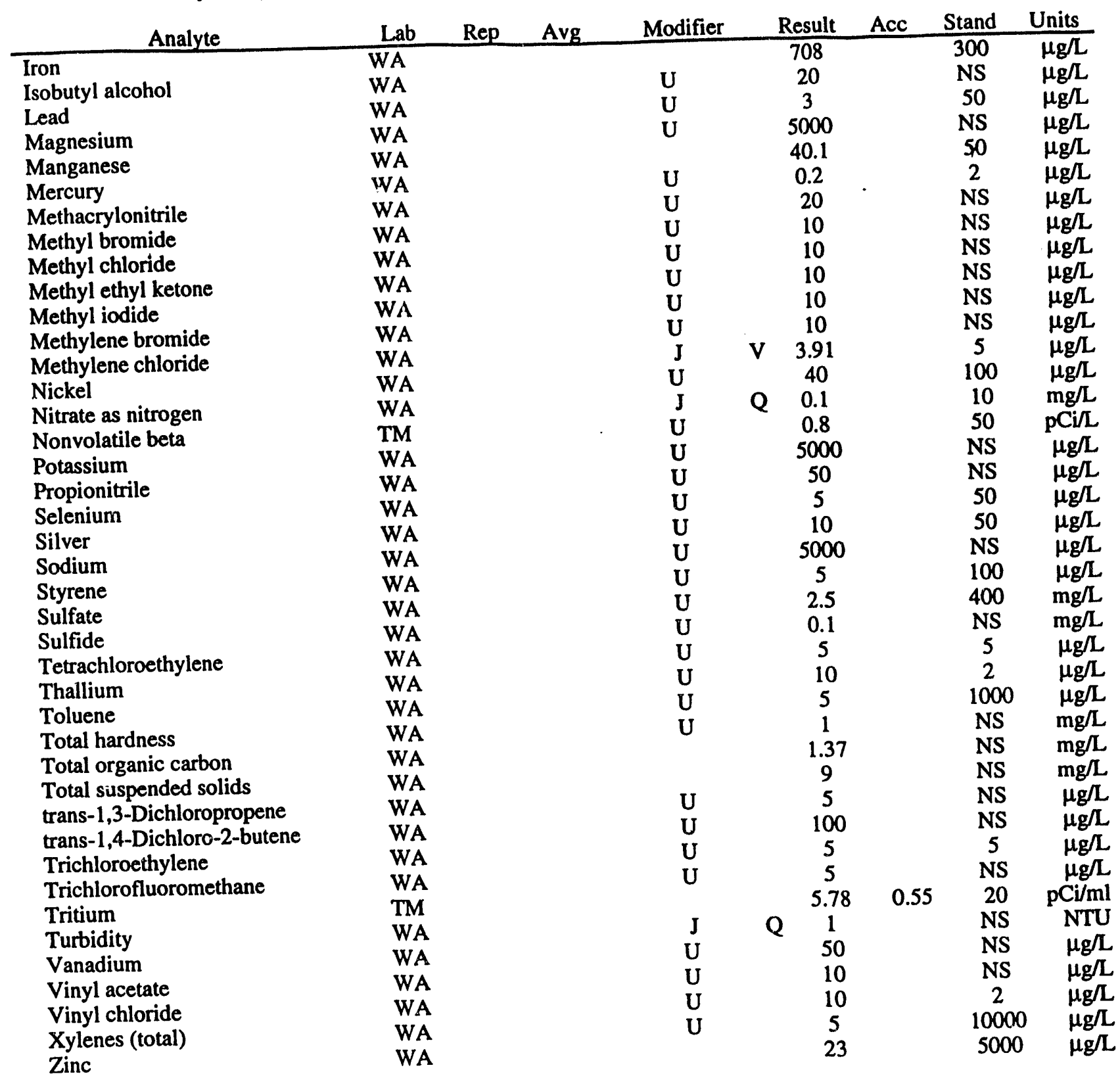




\section{UTR112}

Field Measurements:

Sample Date: 8/26/93

pH: 6.22

Specific Conductance: $49 \mu \mathrm{S} / \mathrm{cm}$

Red/Oxid Potential: -143 mv

Dissolved Oxygen: $3.5 \mathrm{mg} / \mathrm{L}$

\begin{tabular}{|c|c|c|c|c|c|c|c|c|}
\hline Analyte & Lab & Rep & Avg & Modifier & Result & Acc & Stand & Units \\
\hline 1,1,1,2-Tetrachloroethane & WA & & & $\mathbf{U}$ & 10 & & NS & $\overline{\mu g / L}$ \\
\hline 1,1,1-Trichloroethane & WA & & & $\mathbf{U}$ & 5 & & 200 & $\mu \mathrm{g} / \mathrm{L}$ \\
\hline 1,1,2,2-Tetrachloroethane & WA & & & $\mathbf{U}$ & 5 & & NS & $\mu \mathrm{g} / \mathrm{L}$ \\
\hline 1,1,2-Trichloroethane & WA & & & $\mathbf{U}$ & 5 & & 5 & $\mu \mathrm{g} / \mathrm{L}$ \\
\hline 1,1-Dichloroethane & WA & & & $\mathbf{U}$ & 5 & & NS & $\mu \mathrm{g} / \mathrm{L}$ \\
\hline 1,1-Dichloroethylene & WA & & & $\mathbf{U}$ & 5 & & 7 & $\mu \mathrm{g} / \mathrm{L}$ \\
\hline 1,2,3-Trichloropropane & WA & & & $\mathbf{U}$ & 10 & & NS & $\mu \mathrm{g} / \mathrm{L}$ \\
\hline 1,2-Dibromo-3-chloropropane & WA & & & $\mathbf{U}$ & 20 & & 0.2 & $\mu \mathrm{g} / \mathrm{L}$ \\
\hline 1,2-Dibromoethane & WA & & & $\mathbf{U}$ & 20 & & NS & $\mu g / L$ \\
\hline 1,2-Dichloroethane & WA & & & $\mathbf{U}$ & 5 & & 5 & $\mu \mathrm{g} / \mathrm{L}$ \\
\hline 1,2-Dichloroethylene (total) & WA & & & $\mathbf{U}$ & 5 & & 7 & $\mu \mathrm{g} / \mathrm{L}$ \\
\hline 1,2-Dichloropropane & WA & & & $\mathbf{U}$ & 5 & & 5 & $\mu g / L$ \\
\hline 2-Hexanone & WA & & & $\mathbf{U}$ & 10 & & NS & $\mu \mathrm{g} / \mathrm{L}$ \\
\hline 4-Methyl-2-pentanone & WA & & & $\mathbf{U}$ & 10 & & NS & $\mu \mathrm{g} / \mathrm{L}$ \\
\hline Acetone & WA & & & J & 6.83 & & NS & $\mu g / L$ \\
\hline Acetonitrile & WA & & & $\mathbf{U}$ & 20 & & NS & $\mu \mathrm{g} / \mathrm{L}$ \\
\hline Acidity & WA & & & & 70 & & NS & $\mathrm{mg} / \mathrm{L}$ \\
\hline Acrolein & WA & & & $\mathrm{U}$ & 10 & & NS & $\mu g / L$ \\
\hline Acrylonitrile & WA & & & $\mathbf{U}$ & 10 & & NS & $\mu \mathrm{g} / \mathrm{L}$ \\
\hline Alkalinity & WA & & & & 8 & & NS & $\mathrm{mg} / \mathrm{L}$ \\
\hline Allyl chloride & WA & & & $\mathbf{U}$ & 100 & & NS & $\mu \mathrm{g} / \mathrm{L}$ \\
\hline Aluminum & WA & & & $\mathbf{U}$ & 200 & & NS & $\mu g / L$ \\
\hline Antimony & WA & & & $\mathbf{U}$ & 60 & & 6 & $\mu g / L$ \\
\hline Arsenic & WA & & & $\mathbf{U}$ & 10 & & 50 & $\mu g / L$ \\
\hline Barium & WA & & & $\mathbf{U}$ & 200 & & 2000 & $\mu g / L$ \\
\hline Benzene & WA & & & $\mathrm{J}$ & 5 & & 5 & $\mu \mathrm{g} / \mathrm{L}$ \\
\hline Beryllium & WA & & & $\mathbf{U}$ & 5 & & 4 & $\mu g / L$ \\
\hline Bromodichloromethane & WA & & & $\mathbf{U}$ & 5 & & NS & $\mathrm{llg} / \mathrm{L}$ \\
\hline Bromoform & WA & & & $\mathbf{U}$ & 5 & & NS & $\mu \mathrm{g} / \mathrm{L}$ \\
\hline Cadmium & WA & & & $\mathbf{U}$ & 5 & & 5 & $\mu \mathrm{g} / \mathrm{L}$ \\
\hline Calcium & WA & & & $\mathbf{U}$ & 5000 & & NS & $\mu \mathrm{g} / \mathrm{L}$ \\
\hline Carbon disulfide & WA & & & $\mathbf{U}$ & 5 & & NS & $\mu g / L$ \\
\hline Carbon tetrachloride & WA & & & $\mathrm{U}$ & 5 & & 5 & $\mu g / L$ \\
\hline Cesium-137 & TM & & & $\mathbf{U}$ & 12.7 & & 200 & $\mathrm{pCi} / \mathrm{L}$ \\
\hline Chloride & WA & & & & 4.65 & & NS & $\mathrm{mg} / \mathrm{L}$ \\
\hline Chlorobenzenè & WA & & & $\mathbf{U}$ & 5 & & 100 & $\mu g / L$ \\
\hline Chloroethane & WA & & & $\mathbf{U}$ & 10 & & NS & $\mu \mathrm{g} / \mathrm{L}$ \\
\hline Chloroform & WA & & & $\mathbf{U}$ & 5 & & 100 & $\mu \mathrm{g} / \mathrm{L}$ \\
\hline Chloroprene & WA & & & U & 20 & & NS & $\mu \mathrm{g} / \mathrm{L}$ \\
\hline Chromium & WA & & & $\mathrm{U}$ & 10 & & 100 & $\mu g / L$ \\
\hline cis-1,3-Dichloropropene & WA & & & $\mathbf{U}$ & 5 & & NS & $\mu g / L$ \\
\hline Cobalt & WA & & & $\mathbf{U}$ & 50 & & NS & $\mu \mathrm{g} / \mathrm{L}$ \\
\hline Copper & WA & & & $\mathbf{U}$ & 25 & & 1000 & $\mu g / L$ \\
\hline Dibromochloromethane & WA & & & $\mathbf{U}$ & 5 & & NS & $\mu g / L$ \\
\hline Dichlorodifluoromethane & WA & & & $\mathbf{U}$ & 10 & & NS & $\mu \mathrm{g} / \mathrm{L}$ \\
\hline Ethylbenzene & WA & & & $\mathbf{U}$ & 5 & & 700 & $\mu g / L$ \\
\hline Fluoride & WA & & & $\mathbf{U}$ & 0.1 & & 4 & $\mathrm{mg} / \mathrm{L}$ \\
\hline
\end{tabular}


UTR 112 Laboratory Analyses (cont'd)

\begin{tabular}{|c|c|c|c|c|c|c|c|c|c|}
\hline Analyte & Lab & Rep & Avg & Modifier & & Result & Acc & Stand & Units \\
\hline Gross alpha & $\mathrm{TM}$ & & & & & 2.4 & 2 & 15 & $\overline{\mathrm{pCi} / \mathrm{L}}$ \\
\hline Iron & WA & & & & & 12700 & & 300 & $\mu g / L$ \\
\hline Isobutyl alcohol & WA & & & $\mathbf{U}$ & & 20 & & NS & $\mu g / L$ \\
\hline Lead & WA & & & $\mathbf{U}$ & & 3 & & 50 & $\mu \mathrm{g} / \mathrm{L}$ \\
\hline Magnesium & WA & & & $\mathrm{U}$ & & 5000 & & NS & $\mu g / L$ \\
\hline Manganese & WA & & & & & 73.9 & & 50 & $\mu g / L$ \\
\hline Mercury & WA & & & $\mathbf{U}$ & & 0.2 & & 2 & $\mu g / L$ \\
\hline Methacrylonitrile & WA & & & $\mathbf{U}$ & & 20 & & NS & $\mu g / L$ \\
\hline Methyl bromide & WA & & & $\mathbf{U}$ & & 10 & & NS & $\mu g / L$ \\
\hline Methyl chloride & WA & & & $\mathbf{U}$ & & 10 & & NS & $\mu g / L$ \\
\hline Methyl ethyl ketone & WA & & & $\mathrm{U}$ & & 10 & & NS & $\mu g / L$ \\
\hline Methyl iodide & WA & & & $\mathbf{U}$ & & 10 & & NS & $\mu \mathrm{g} / \mathrm{L}$ \\
\hline Methylene bromide & WA & & & $\mathbf{U}$ & & 10 & & NS & $\mu \mathrm{g} / \mathrm{L}$ \\
\hline Methylene chloride & WA & & & & $\mathbf{V}$ & 9.2 & & 5 & $\mu g / L$ \\
\hline Nickel & WA & & & $\mathbf{U}$ & & 40 & & 100 & $\mu \mathrm{g} / \mathrm{L}$ \\
\hline Nitrate as nitrogen & WA & & . & $\mathbf{J}$ & $\mathbf{Q}$ & 0.228 & & 10 & $\mathrm{mg} / \mathrm{L}$ \\
\hline Nonvolatile beta & TM & & & & & 2.5 & 3.1 & 50 & $\mathrm{pCi} / \mathrm{L}$ \\
\hline Potassium & WA & & & $\mathbf{U}$ & & 5000 & & NS & $\mu g / L$ \\
\hline Propionitrile & WA & & & $\mathbf{U}$ & & 50 & & NS & $\mu g / L$ \\
\hline Selenium & WA & & & $\mathbf{U}$ & & 5 & & 50 & $\mu g / L$ \\
\hline Silver & WA & & & $\mathbf{U}$ & & 10 & & 50 & $\mu \mathrm{g} / \mathrm{L}$ \\
\hline Sodium & WA & & & $\mathbf{U}$ & & 5000 & & NS & $\mu \mathrm{g} / \mathrm{L}$ \\
\hline Styrene & WA & & & $\mathbf{U}$ & & 5 & & 100 & $\mu \mathrm{g} / \mathrm{L}$ \\
\hline Sulfate & WA & & & & & 2.66 & & 400 & $\mathrm{mg} / \mathrm{L}$ \\
\hline Sulfide & WA & & & $\mathbf{U}$ & & 0.1 & & NS & $\mathrm{mg} / \mathrm{L}$ \\
\hline Tetrachloroethylene & WA & & & $\mathbf{U}$ & & 5 & & 5 & $\mu g / L$ \\
\hline Thallium & WA & & & $\mathbf{U}$ & & 10 & & 2 & $\mu \mathrm{g} / \mathrm{L}$ \\
\hline Toluene & WA & & & $\mathbf{U}$ & & 5 & & 1000 & $\mu \mathrm{g} / \mathrm{L}$ \\
\hline Total hardness & WA & & & & & 15.7 & & NS & $\mathrm{mg} / \mathrm{L}$ \\
\hline Total organic carbon & WA & & & & & 4.87 & & NS & $\mathrm{mg} / \mathrm{L}$ \\
\hline Total suspended solids & WA & & & & & 29 & & NS & $\mathrm{mg} / \mathrm{L}$ \\
\hline trans-1,3-Dichloropropene & WA & & & $\mathbf{U}$ & & 5 & & NS & $\mu g / L$ \\
\hline trans-1,4-Dichloro-2-butene & WA & & & $\mathbf{U}$ & & 100 & & NS & $\mu \mathrm{g} / \mathrm{L}$ \\
\hline Trichloroethylene & WA & & & $\mathbf{U}$ & & 5 & & 5 & $\mu \mathrm{g} / \mathrm{L}$ \\
\hline Trichlorofluoromethane & WA & & & $\mathbf{U}$ & & 5 & & NS & $\mu \mathrm{g} / \mathrm{L}$ \\
\hline Tritium & $\mathrm{TM}$ & & & & & 8.54 & 0.66 & 20 & $\mathrm{pCi} / \mathrm{ml}$ \\
\hline Turbidity & WA & & & $\mathbf{J}$ & $\mathbf{Q}$ & 7.66 & & NS & NTU \\
\hline Vanadium & WA & & & U & & 50 & & NS & $\mu \mathrm{g} / \mathrm{L}$ \\
\hline Vinyl acetate & WA & & & $\mathbf{U}$ & & 10 & & NS & $\mu g / L$ \\
\hline Vinyl chloride & WA & & & $\mathbf{U}$ & & 10 & & 2 & $\mu g / L$ \\
\hline Xylenes (total) & WA & & & $\mathbf{U}$ & & 5 & & 10000 & $\mu \mathrm{g} / \mathrm{L}$ \\
\hline Zinc & WA & & & $\mathbf{U}$ & & 20 & & 5000 & $\mu \mathrm{g} / \mathrm{L}$ \\
\hline
\end{tabular}




\section{UTR114}

Field Measurements:

Sample Date: $8 / 26 / 93$

pH: 5.78

Specific Conductance: $34 \mu \mathrm{S} / \mathrm{cm}$

Red/Oxid Potential: $130 \mathrm{mv}$

Dissolved Oxygen: $1.4 \mathrm{mg} / \mathrm{L}$

\begin{tabular}{|c|c|c|c|c|c|c|c|c|}
\hline Analyte & Lab & Rep & Avg & Modifier & Result & Acc & Stand & Units \\
\hline 1,1,1,2-Tetrachloroethane & WA & & & $\mathbf{U}$ & 10 & & NS & $\mu g / L$ \\
\hline 1,1,1,2-Tetrachloroethane & GE & & & $\mathbf{U}$ & 1 & & NS & $\mu g / L$ \\
\hline 1,1,1-Trichloroethane & WA & & & $\mathbf{U}$ & 5 & & 200 & $\mu \mathrm{g} / \mathrm{L}$ \\
\hline 1,1,1-Trichloroethane & GE & & & $\mathbf{U}$ & 1 & & 200 & $\mu g / L$ \\
\hline 1,1,2,2-Tetrachloroethane & WA & & & $\mathbf{U}$ & 5 & & NS & $\mu g / L$ \\
\hline 1,1,2,2-Tetrachloroethane & GE & & & $\mathbf{U}$ & 1 & & NS & $\mu g / L$ \\
\hline 1,1,2-Trichloroethane & WA & & & $\mathbf{U}$ & 5 & & 5 & $\mu g / L$ \\
\hline $1,1,2$-Trichloroethane & GE & & & $\mathbf{U}$ & 1 & & 5 & $\mu g / L$ \\
\hline 1,1-Dichloroethane & WA & & & $\mathbf{U}$ & 5 & & NS & $\mu g / L$ \\
\hline 1,1-Dichloroethane & GE & & & $\mathbf{U}$ & 1 & & NS & $\mu g / L$ \\
\hline 1,1-Dichloroethylene & WA & & & $\mathbf{U}$ & 5 & & 7 & $\mu g / L$ \\
\hline 1,1-Dichloroethylene & $\mathrm{GE}$ & & & $\mathbf{U}$ & 1 & & 7 & $\mu g / L$ \\
\hline 1,2,3-Trichloropropane & WA & & & $\mathbf{U}$ & 10 & & NS & $\mu g / L$ \\
\hline 1,2,3-Trichloropropane & $\mathrm{GE}$ & & & $\mathbf{U}$ & 1 & & NS & $\mu g / L$ \\
\hline 1,2-Dibromo-3-chloropropane & WA & & & $\mathbf{U}$ & 20 & & 0.2 & $\mu g / L$ \\
\hline 1,2-Dibromo-3-chloropropane & GE & & & $\mathbf{U}$ & 1 & & 0.2 & $\mu \mathrm{g} / \mathrm{L}$ \\
\hline 1,2-Dibromoethane & WA & & & $\mathbf{U}$ & 20 & & NS & $\mu g / L$ \\
\hline 1,2-Dibromoethane & GE & & & $\mathbf{U}$ & 20 & & 7 & $\mu \mathrm{g} / \mathrm{L}$ \\
\hline 1,2-Dichloroethane & WA & & & $\mathbf{U}$ & 5 & & 5 & $\mu \mathrm{g} / \mathrm{L}$ \\
\hline 1,2-Dichloroethane & GE & & & $\mathbf{U}$ & 1 & & 5 & $\mu g / L$ \\
\hline 1,2-Dichloroethylene (total) & WA & & & $\mathbf{U}$ & 5 & & 7 & $\mu \mathrm{g} / \mathrm{L}$ \\
\hline 1,2-Dichloropropane & WA & & & $\mathbf{U}$ & 5 & & 5 & $\mu g / L$ \\
\hline 1,2-Dichloropropane & GE & & & $\mathbf{U}$ & 1 & & 5 & $\mu g / L$ \\
\hline 2-Hexanone & WA & & & $\mathbf{U}$ & 10 & & NS & $\mu \mathrm{g} / \mathrm{L}$ \\
\hline 2-Hexanone & GE & & & $\mathbf{U}$ & 1 & & NS & $\mu g / L$ \\
\hline 4-Methyl-2-pentanone & WA & & & $\mathbf{U}$ & 10 & & NS & $\mu g / L$ \\
\hline Acetone & WA & & & $\mathbf{U}$ & 10 & & NS & $\mu g / L$ \\
\hline Acetone & GE & & & $\mathbf{U}$ & 100 & & NS & $\mu g / L$ \\
\hline Acetonitrile & WA & & & $\mathbf{U}$ & 20 & & NS & $\mu g / L$ \\
\hline Acetonitrile (Methyl cyanide) & GE & & & U & 1 & & NS & $\mu \mathrm{g} / \mathrm{L}$ \\
\hline Acidity & WA & & & & 112 & & NS & $\mathrm{mg} / \mathrm{L}$ \\
\hline Acrolein & WA & & & $\mathbf{U}$ & 10 & & NS & $\mu \mathrm{g} / \mathrm{L}$ \\
\hline Acrolein & $\mathrm{GE}$ & & & $\mathbf{U}$ & 20 & & NS & $\mu \mathrm{g} / \mathrm{L}$ \\
\hline Acrylonitrile & WA & & & $\mathbf{U}$ & 10 & & NS & $\mu g / L$ \\
\hline Acrylonitrile & GE & & & $\mathbf{U}$ & 20 & & NS & $\mu \mathrm{g} / \mathrm{L}$ \\
\hline Alkalinity & $\mathrm{GE}$ & & & & V & & NS & $\mathrm{mg} / \mathrm{L}$ \\
\hline Allyl chloride & WA & & & $\mathbf{U}$ & 100 & & NS & $\mu \mathrm{g} / \mathrm{L}$ \\
\hline Allyl chloride & GE & & & $\mathbf{U}$ & 50 & & NS & $\mu \mathrm{g} / \mathrm{L}$ \\
\hline Aluminum & WA & & & & 1130 & & NS & $\mu \mathrm{g} / \mathrm{L}$ \\
\hline Aluminum & $\mathrm{GE}$ & & & & 496 & & NS & $\mu \mathrm{g} / \mathrm{L}$ \\
\hline Antimony & WA & & & $\mathbf{U}$ & 60 & & 6 & $\mu \mathrm{g} / \mathrm{L}$ \\
\hline Antimony & GE & & & UJ & L & & 6 & $\mu \mathrm{g} / \mathrm{L}$ \\
\hline Arsenic & WA & & & $\mathbf{U}$ & 10 & & 50 & $\mu \mathrm{g} / \mathrm{L}$ \\
\hline Arsenic & GE & & & $\mathbf{U}$ & 2 & & 50 & $\mu \mathrm{g} / \mathrm{L}$ \\
\hline Barium & WA & & & U & 200 & & 2000 & $\mu \mathrm{g} / \mathrm{L}$ \\
\hline Barium & GE & & & & 15.5 & & 2000 & $\mu \mathrm{g} / \mathrm{L}$ \\
\hline
\end{tabular}


UTR114 Laboratory Analyses (cont'd)

\begin{tabular}{|c|c|c|c|c|c|c|c|}
\hline Analyte & $\mathrm{Lab}$ & Rep & Modifier & Result & Acc & Stand & Units \\
\hline Benzene & WA & & U & 5 & & 5 & $\mu \mathrm{g} / \mathrm{L}$ \\
\hline Benzene & GE & & U & 1 & & 5 & $\mu \mathrm{g} / \mathrm{L}$ \\
\hline Beryllium & WA & & $\mathbf{U}$ & 5 & & 4 & $\mu g / L$ \\
\hline Beryllium & GE & & $\mathbf{U}$ & 3 & & 4 & $\mu \mathrm{g} / \mathrm{L}$ \\
\hline Bis(2-chloro-1-methylethyl)ether & GE & & $\mathbf{U}$ & 10 & & NS & $\mu \mathrm{g} / \mathrm{L}$ \\
\hline Bromodichloromethane & WA & & $\mathbf{U}$ & 5 & & NS & $\mu g / L$ \\
\hline Bromodichloromethane & GE & & $\mathbf{U}$ & 1 & $\cdot$ & NS & $\mu \mathrm{g} / \mathrm{L}$ \\
\hline Bromoform & WA & & $\mathbf{U}$ & 5 & & NS & $\mu g / L$ \\
\hline Bromoform & GE & & $\mathbf{U}$ & 1 & & NS & $\mu g / L$ \\
\hline Cadmium & WA & & $\mathbf{U}$ & 5 & & 5 & $\mu g / L$ \\
\hline Cadmium & GE & & $\mathbf{U}$ & 2 & & 5 & $\mu g / L$ \\
\hline Calcium & WA & & $\mathbf{U}$ & 5000 & & NS & $\mu \mathrm{g} / \mathrm{L}$ \\
\hline Calcium & GE & & & 787 & & NS & $\mu \mathrm{g} / \mathrm{L}$ \\
\hline Carbon disulfide & WA & & $\mathbf{U}$ & 5 & & NS & $\mu \mathrm{g} / \mathrm{L}$ \\
\hline Carbon disulfide & GE & & $\mathbf{U}$ & 1 & & NS & $\mu \mathrm{g} / \mathrm{L}$ \\
\hline Carbon tetrachloride & WA & & $\mathbf{U}$ & 5 & & 5 & $\mu g / L$ \\
\hline Carbon tetrachloride & GE & & $\mathbf{U}$ & 1 & & 5 & $\mu g / L$ \\
\hline Cesium-137 & TM & & $\mathbf{U}$ & 9.6 & & 200 & $\mathrm{pCi} / \mathrm{L}$ \\
\hline Chloride & WA & & & 3.93 & & NS & $\mathrm{mg} / \mathrm{L}$ \\
\hline Chloride & GE & & & 3.98 & & NS & $\mathrm{mg} / \mathrm{L}$ \\
\hline Chlorobenzene & WA & & $\mathbf{U}$ & 5 & & 100 & $\mu g / L$ \\
\hline Chlorobenzene & GE & & $\mathbf{U}$ & 1 & & 100 & $\mu \mathrm{g} / \mathrm{L}$ \\
\hline Chloroethane & WA & & U & 10 & & NS & $\mu g / L$ \\
\hline Chloroethane & GE & & $\mathbf{U}$ & 1 & & NS & $\mu \mathrm{g} / \mathrm{L}$ \\
\hline Chloroform & WA & & $\mathbf{U}$ & 5 & & 100 & $\mu g / L$ \\
\hline Chloroform & GE & & $\mathrm{U}$ & 1 & & 100 & $\mu g / L$ \\
\hline Chloroprene & WA & & $\mathbf{U}$ & 20 & & NS & $\mu \mathrm{g} / \mathrm{L}$ \\
\hline Chloroprene & GE & & $\mathbf{U}$ & 200 & & NS & $\mu \mathrm{g} / \mathrm{L}$ \\
\hline Chromium & WA & & $\mathrm{U}$ & 10 & & 100 & $\mu \mathrm{g} / \mathrm{L}$ \\
\hline Chromium & $\mathrm{GE}$ & & $\mathbf{U}$ & 4 & & 100 & $\mu \mathrm{g} / \mathrm{L}$ \\
\hline cis-1,3-Dichloropropene & WA & & $\mathbf{U}$ & 5 & & NS & $\mu \mathrm{g} / \mathrm{L}$ \\
\hline cis-1,3-Dichloropropene & GE & & $\mathbf{U}$ & 1 & & NS & $\mu \mathrm{g} / \mathrm{L}$ \\
\hline Cobalt & WA & & $\mathbf{U}$ & 50 & & NS & $\mu g / L$ \\
\hline Cobalt & $\mathrm{GE}$ & & $\mathbf{U}$ & 4 & & NS & $\mu \mathrm{g} / \mathrm{L}$ \\
\hline Copper & WA & & $\mathbf{U}$ & 25 & & 1000 & $\mu \mathrm{g} / \mathrm{L}$ \\
\hline Copper & $\mathrm{GE}$ & & $\mathrm{U}$ & 4 & & 1000 & $\mu \mathrm{g} / \mathrm{L}$ \\
\hline Cyanide & $\mathrm{GE}$ & & $\mathbf{U}$ & 5 & & 200 & $\mu \mathrm{g} / \mathrm{L}$ \\
\hline Dibromochloromethane & WA & & $\mathbf{U}$ & 5 & & NS & $\mu \mathrm{g} / \mathrm{L}$ \\
\hline Dibromochloromethane & GE & & $\mathbf{U}$ & 1 & & NS & $\mu g / L$ \\
\hline Dichlorodifluoromethane & WA & & $\mathbf{U}$ & 10 & & NS & $\mu \mathrm{g} / \mathrm{L}$ \\
\hline Dichlorodifluoromethane & $\mathrm{GE}$ & & $\mathbf{U}$ & 1 & & NS & $\mu \mathrm{g} / \mathrm{L}$ \\
\hline Ethylbenzene & WA & & $\mathbf{U}$ & 5 & & 700 & $\mu \mathrm{g} / \mathrm{L}$ \\
\hline Ethylbenzene & $\mathrm{GE}$ & & $\mathrm{U}$ & 1 & & 700 & $\mu g / L$ \\
\hline Fluoride & WA & & $\mathbf{U}$ & 0.1 & & 4 & $\mathrm{mg} / \mathrm{L}$ \\
\hline Fluoride & GE & & $\mathbf{U}$ & 0.1 & & 4 & $\mathrm{mg} / \mathrm{L}$ \\
\hline Gross alpha & $\mathrm{GE}$ & & $\mathbf{U}$ & 2 & & 15 & $\mathrm{pCi} / \mathrm{L}$ \\
\hline Gross alpha & $\mathrm{TM}$ & & $\mathbf{U}$ & 0.9 & & 15 & $\mathrm{pCi} / \mathrm{L}$ \\
\hline Hardness & $\mathrm{GE}$ & & & 6 & & NS & $\mathrm{mg} / \mathrm{L}$ \\
\hline Iron & WA & & & 4340 & & 300 & $\mu \mathrm{g} / \mathrm{L}$ \\
\hline Iron & $\mathrm{GE}$ & & & 4520 & & 300 & $\mu \mathrm{g} / \mathrm{L}$ \\
\hline Isobutyl alcohol & WA & & $\mathbf{U}$ & 20 & & NS & $\mu g / L$ \\
\hline Isobutyl alcohol & GE & & $\mathbf{U}$ & 100 & & NS & $\mu \mathrm{g} / \mathrm{L}$ \\
\hline Lead & WA & & & 3.3 & & 50 & $\mu g / L$ \\
\hline Lead & GE & & $\mathbf{U}$ & 3 & & 50 & $\mu \mathrm{g} / \mathrm{L}$ \\
\hline
\end{tabular}


UTR114 Laboratory Analyses (cont'd)

\begin{tabular}{|c|c|c|c|c|c|c|c|c|c|}
\hline Analyte & Lab & Rep & Avg & Modifier & & Result & Acc & Stand & Units \\
\hline Magnesium & WA & & & $\bar{U}$ & & 5000 & & NS & $\mu \mathrm{g} / \mathrm{L}$ \\
\hline Magnesium & GE & & & & & 531 & & NS & $\mu \mathrm{g} / \mathrm{L}$ \\
\hline Manganese & WA & & & & & 29.1 & & 50 & $\mu \mathrm{g} / \mathrm{L}$ \\
\hline Manganese & GE & & & & & 33.5 & & 50 & $\mu g / L$ \\
\hline Mercury & WA & & & $\mathbf{U}$ & & 0.2 & & 2 & $\mu \mathrm{g} / \mathrm{L}$ \\
\hline Mercury & GE & & & $\mathrm{U}$ & & 0.2 & & 2 & $\mu g / L$ \\
\hline Mercury & GE & & NA & $\mathbf{U}$ & & 0.2 & & 2 & $\mu \mathrm{g} / \mathrm{L}$ \\
\hline Methacrylonitrile & WA & & & $\mathbf{U}$ & & 20 & & NS & $\mu \mathrm{g} / \mathrm{L}$ \\
\hline Methacrylonitrile & GE & & & $\mathbf{U}$ & & 50 & & NS & $\mu \mathrm{g} / \mathrm{L}$ \\
\hline Methyl bromide & WA & & & $\mathbf{U}$ & & 10 & & NS & $\mu \mathrm{g} / \mathrm{L}$ \\
\hline Methyl bromide & GE & & & $\mathbf{U}$ & & 1 & & NS & $\mu g / L$ \\
\hline Methyl chloride & WA & & & $\mathbf{U}$ & & 10 & & NS & $\mu \mathrm{g} / \mathrm{L}$ \\
\hline Methyl chloride & GE & & & $\mathbf{U}$ & & 1 & & NS & $\mu \mathrm{g} / \mathrm{L}$ \\
\hline Methyl ethyl ketone & WA & & & $\mathbf{U}$ & & 10 & & NS & $\mu g / \mathrm{L}$ \\
\hline Methyl ethyl ketone & GE & & & $\mathbf{U}$ & & 1 & & NS & $\mu \mathrm{g} / \mathrm{L}$ \\
\hline Methyl iodide & WA & & & $\mathbf{U}$ & & 10 & & NS & $\mu g / L$ \\
\hline Methyl iodide & GE & & & $\mathbf{U}$ & & 15 & & NS & $\mu \mathrm{g} / \mathrm{L}$ \\
\hline Methyl isobutyl ketone & GE & & & U & & 1 & & NS & $\mu \mathrm{g} / \mathrm{L}$ \\
\hline Methylene bromide & WA & & & $\mathbf{U}$ & & 10 & & NS & $\mu g / L$ \\
\hline Methylene bromide & GE & & & $\mathbf{U}$ & & 1 & & NS & $\mu g / L$ \\
\hline Methylene chloride & WA & & & $\mathrm{J}$ & $\mathrm{v}$ & 3.78 & & 5 & $\mu \mathrm{g} / \mathrm{L}$ \\
\hline Methylene chloride & GE & & & U & & 1 & & 5 & $\mu g / L$ \\
\hline Nickel & WA & & & U & & 40 & & 100 & $\mu g / L$ \\
\hline Nickel & GE & & & U & & 4 & & 100 & $\mu \mathrm{g} / \mathrm{L}$ \\
\hline Nitrate as nitrogen & WA & & & $\mathrm{J}$ & Q & 0.463 & & 10 & $\mathrm{mg} / \mathrm{L}$ \\
\hline Nitrate as nitrogen & GE & & & & & 0.41 & & 10 & $\mathrm{mg} / \mathrm{L}$ \\
\hline Nonvolatile beta & $\mathrm{GE}$ & & & $\mathbf{U}$ & & 2 & & 50 & $\mathrm{pCi} / \mathrm{L}$ \\
\hline Nonvolatile beta & TM & & & $\mathbf{U}$ & & 1 & & 50 & $\mathrm{pCi} / \mathrm{L}$ \\
\hline pH & GE & & & $\mathrm{J}$ & Q & 5.22 & & NS & $\mathrm{pH}$ \\
\hline Potassium & WA & & & U & & 5000 & & NS & $\mu \mathrm{g} / \mathrm{L}$ \\
\hline Potassium & GE & & & $\mathbf{U}$ & & 500 & & NS & $\mu g / L$ \\
\hline Propionitrile & WA & & & $\mathrm{U}$ & & 50 & & NS & $\mu \mathrm{g} / \mathrm{L}$ \\
\hline Propionitrile & $\mathbf{G E}$ & & & U & & 200 & & NS & $\mu g / L$ \\
\hline Selenium & WA & & & U & & 5 & & 50 & $\mu g / L$ \\
\hline Selenium & GE & & & UJ & L & 2 & & 50 & $\mu \mathrm{g} / \mathrm{L}$ \\
\hline Silver & WA & & & $\mathrm{U}$ & & 10 & & 50 & $\mu \mathrm{g} / \mathrm{L}$ \\
\hline Silver & $\mathrm{GE}$ & & & U & & 2 & & 50 & $\mu g / L$ \\
\hline Sodium & WA & & & U & & 5000 & & NS & $\mu \mathrm{g} / \mathrm{L}$ \\
\hline Sodium & $\mathrm{GE}$ & & & & & 1700 & & NS & $\mu \mathrm{g} / \mathrm{L}$ \\
\hline Styrene & WA & & & $\mathrm{U}$ & & 5 & & 100 & $\mu g / L$ \\
\hline Styrene & $G E$ & & & $\mathrm{U}$ & & 1 & & 100 & $\mu \mathrm{g} / \mathrm{L}$ \\
\hline Sulfate & WA & & & U & & 2.5 & & 400 & $\mathrm{mg} / \mathrm{L}$ \\
\hline Sulfate & $G E$ & & & & & 1.02 & & 400 & $\mathrm{mg} / \mathrm{L}$ \\
\hline Sulfide & WA & & & $\mathrm{U}$ & & 0.1 & & NS & $\mathrm{mg} / \mathrm{L}$ \\
\hline Sulfide & $\mathrm{GE}$ & & & $\mathrm{U}$ & & 1 & & NS & $\mathrm{mg} / \mathrm{L}$ \\
\hline Tetrachloroethylene & WA & & & $\mathrm{U}$ & & 5 & & 5 & $\mu \mathrm{g} / \mathrm{L}$ \\
\hline Tetrachloroethylene & $\mathrm{GE}$ & & & $\mathrm{U}$ & & 1 & & 5 & $\mu g / \mathrm{L}$ \\
\hline Thallium & WA & & & $\mathrm{U}$ & & 10 & & 2 & $\mu \mathrm{g} / \mathrm{h}$ \\
\hline Thallium & $\mathrm{GE}$ & & & $\mathrm{U}$ & & 2 & & 2 & $\mu \mathrm{g} / \mathrm{L}$ \\
\hline Toluene & WA & & & $\mathrm{U}$ & & 5 & & 1000 & $\mu \mathrm{g} / \mathrm{L}$ \\
\hline Toluene & GE & & & & & 1.3 & & 1000 & $\mu \mathrm{g} / \mathrm{L}$ \\
\hline Total hardness & WA & & & & & 8.82 & & NS & $\mathrm{mg} / \mathrm{L}$ \\
\hline Total organic carbon & WA & & & & & 3.14 & & NS & $\mathrm{mg} / \mathrm{L}$ \\
\hline Total organic carbon & $\mathrm{GE}$ & & & & & 3.84 & & NS & $\mathrm{mg} / \mathrm{L}$ \\
\hline
\end{tabular}


UTR1 14 Laboratory Analyses (cont'd)

\begin{tabular}{|c|c|c|c|c|c|c|c|c|c|}
\hline Analyte & Lab & Rep & Avg & Modifier & & Result & Acc & Stand & Units \\
\hline Total suspended solids & WA & & & & & 42 & & NS & $\mathrm{mg/L}$ \\
\hline Total suspended solids & $\mathrm{GE}$ & & $\mathbf{A}$ & & & 17 & & NS & $\mathrm{mg} / \mathrm{L}$ \\
\hline trans-1,2-Dichloroethylene (total) & GE & & & $\mathbf{U}$ & & 1 & & 100 & $\mu g / L$ \\
\hline trans-1,3-Dichloropropene & WA & & & $\mathbf{U}$ & & 5 & & NS & $\mu g / L$ \\
\hline trans-1,3-Dichloropropene & $\mathrm{GE}$ & & & $\mathbf{U}$ & & 1 & & NS & $\mu g / L$ \\
\hline trans-1,4-Dichloro-2-butene & WA & & & $\mathbf{U}$ & & 100 & & NS & $\mu g / L$ \\
\hline trans-1,4-Dichloro-2-butene & GE & & & $\mathbf{U}$ & & 30 & • & NS & $\mu g / L$ \\
\hline Trichloroethylene & WA & & & $\mathbf{U}$ & & 5 & & 5 & $\mu \mathrm{g} / \mathrm{L}$ \\
\hline Trichloroethylene & GE & & & $\mathbf{U}$ & & 1 & & 5 & $\mu g / L$ \\
\hline Trichlorofluoromethane & WA & & & $\mathbf{U}$ & & 5 & & NS & $\mu g / L$ \\
\hline Trichlorofluoromethane & GE & & & $\mathbf{U}$ & & 1 & & NS & $\mu g / L$ \\
\hline Tritium & GE & & & & & 243 & & 20 & $\mathrm{pCi} / \mathrm{ml}$ \\
\hline Tritium & TM & & & & & 272 & 3.48 & 20 & $\mathrm{pCi} / \mathrm{ml}$ \\
\hline Turbidity & WA & & & $\mathbf{J}$ & $Q$ & 88.3 & & NS & NTU \\
\hline Turbidity & GE & & & & & 95.5 & & NS & NTU \\
\hline Vanadium & WA & & & $\mathbf{U}$ & & 50 & & NS & $\mu g / L$ \\
\hline Vanadium & GE & & & $\mathbf{U}$ & & 8 & & NS & $\mu g / L$ \\
\hline Vinyl acetate & WA & & & $\mathbf{U}$ & & 10 & & NS & $\mu g / L$ \\
\hline Vinyl acetate & GE & & & $\mathbf{U}$ & & 1 & & NS & $\mu g / L$ \\
\hline Vinyl chloride & WA & & & $\mathbf{U}$ & & 10 & & 2 & $\mu g / L$ \\
\hline Vinyl chloride & GE & & & $\mathbf{U}$ & & 1 & & 2 & $\mu g / L$ \\
\hline Xylenes (total) & WA & & & $\mathbf{U}$ & & 5 & & 10000 & $\mu g / L$ \\
\hline Xylenes (total) & GE & & & $\mathbf{U}$ & & 2 & & 10000 & $\mu g / L$ \\
\hline Zinc & WA & & & & & 46.8 & & 5000 & $\mu g / L$ \\
\hline Zinc & GE & & & & & 14.3 & & 5000 & $\mu g / L$ \\
\hline
\end{tabular}




\section{UTR116}

Field Measurements:

Sample Date: $8 / 26 / 93$

pH: 6.98

Specific Conductance: $27 \mu \mathrm{S} / \mathrm{cm}$

Red/Oxid Potential: $55 \mathrm{mv}$

Dissolved Oxygen: $0.6 \mathrm{mg} / \mathrm{L}$

\begin{tabular}{|c|c|c|c|c|c|c|c|c|}
\hline Analyte & Lab & Rep & Avg & Modifier & Result & Acc & Stand & Units \\
\hline 1,1,1,2-Tetrachloroethane & $\overline{\text { WA }}$ & & & $\mathrm{U}$ & 10 & & NS & $\mu \mathrm{g} / \mathrm{L}$ \\
\hline 1,1,1,2-Tetrachloroethane & WA & $\mathbf{R}$ & & $\mathbf{U}$ & 10 & & NS & $\mu \mathrm{g} / \mathrm{L}$ \\
\hline 1,1,1-Trichloroethane & WA & & & $\mathbf{U}$ & 5 & & 200 & $\mu \mathrm{g} / \mathrm{L}$ \\
\hline 1,1,1-Trichloroethane & WA & $\mathbf{R}$ & & $\mathbf{U}$ & 5 & & 200 & $\mu g / L$ \\
\hline 1,1,2,2-Tetrachloroethane & WA & & & $\mathbf{U}$ & 5 & & NS & $\mu g / L$ \\
\hline $1,1,2,2$-Tetrachloroethane & WA & $\mathbf{R}$ & & $\mathbf{U}$ & 5 & & NS & $\mu g / L$ \\
\hline 1,1,2-Trichloroethane & WA & & & $\mathbf{U}$ & 5 & & 5 & $\mu g / L$ \\
\hline 1,1,2-Trichloroethane & WA & $\mathbf{R}$ & & $\mathbf{U}$ & 5 & & 5 & $\mu g / L$ \\
\hline 1,1-Dichloroethane & WA & & & $\mathbf{U}$ & 5 & & NS & $\mu g / L$ \\
\hline 1,1-Dichloroethane & WA & $\mathbf{R}$ & & $\mathbf{U}$ & 5 & & NS & $\mu \mathrm{g} / \mathrm{L}$. \\
\hline 1,1-Dichloroethylene & WA & & & $\mathbf{U}$ & 5 & & 7 & $\mu \mathrm{g} / \mathrm{L}$ \\
\hline 1,1-Dichloroethylene & WA & $\mathbf{R}$ & & $\mathbf{U}$ & 5 & & 7 & $\mu \mathrm{g} / \mathrm{L}$ \\
\hline 1,2,3-Trichloropropane & WA & & & $\mathbf{U}$ & 10 & & NS & $\mu g / L$ \\
\hline 1,2,3-Trichloropropane & WA & $\mathbf{R}$ & & $\mathbf{U}$ & 10 & & NS & $\mu \mathrm{g} / \mathrm{L}$ \\
\hline 1,2-Dibromo-3-chloropropane & WA & & & $\mathbf{U}$ & 20 & & 0.2 & $\mu g / L$ \\
\hline 1,2-Dibromo-3-chloropropane & WA & $\mathbf{R}$ & & $\mathbf{U}$ & 20 & & 0.2 & $\mu \mathrm{g} / \mathrm{L}$ \\
\hline 1,2-Dibromoethane & WA & & & $\mathrm{U}$ & 20 & & NS & $\mu g / L$ \\
\hline 1,2-Dibromoethane & WA & $\mathbf{R}$ & & $\mathrm{U}$ & 20 & & NS & $\mu \mathrm{g} / \mathrm{L}$ \\
\hline 1,2-Dichloroethane & WA & & & $\mathrm{U}$ & 5 & & 5 & $\mu \mathrm{g} / \mathrm{L}$ \\
\hline 1,2-Dichloroethane & WA & $\mathbf{R}$ & & $\mathbf{U}$ & 5 & & 5 & $\mu g / L$ \\
\hline 1,2-Dichloroethylene (total) & WA & & & $\mathbf{U}$ & 5 & & 7 & $\mu \mathrm{g} / \mathrm{L}$ \\
\hline 1,2-Dichloroethylene (total) & WA & $\mathbf{R}$ & & $\mathbf{U}$ & 5 & & 7 & $\mu g / L$ \\
\hline 1,2-Dichloropropane & WA & & & $\mathbf{U}$ & 5 & & 5 & $\mu \mathrm{g} / \mathrm{L}$ \\
\hline 1,2-Dichloropropane & WA & $\mathbf{R}$ & & U & 5 & & 5 & $\mu g / L$ \\
\hline 2-Hexanone & WA & & & $\mathbf{U}$ & 10 & & NS & $\mu \mathrm{g} / \mathrm{L}$ \\
\hline 2-Hexanone & WA & $\mathbf{R}$ & & $\mathbf{U}$ & 10 & & NS & $\mu \mathrm{g} / \mathrm{L}$ \\
\hline 4-Methyl-2-pentanone & WA & & & $\mathrm{U}$ & 10 & & NS & $\mu \mathrm{g} / \mathrm{L}$ \\
\hline 4-Methyl-2-pentanone & WA & $\mathbf{R}$ & & $\mathbf{U}$ & 10 & & NS & $\mu \mathrm{g} / \mathrm{L}$ \\
\hline Acetone & WA & & & $\mathbf{J}$ & 7.22 & & NS & $\mu \mathrm{g} / \mathrm{L}$ \\
\hline Acetone & WA & $\mathbf{R}$ & & $\mathbf{J}$ & 3.46 & & NS & $\mu \mathrm{g} / \mathrm{L}$ \\
\hline Acetonitrile & WA & & & $\mathbf{U}$ & 20 & & NS & $\mu g / L$ \\
\hline Acetonitrile & WA & $\mathbf{R}$ & & $\mathbf{U}$ & 20 & & NS & $\mu \mathrm{g} / \mathrm{L}$ \\
\hline Acidity & WA & & & & 86 & & NS & $\mathrm{mg} / \mathrm{L}$ \\
\hline Acidity & WA & $\mathbf{R}$ & & & 80 & & NS & $\mathrm{mg} / \mathrm{L}$ \\
\hline Acrolein & WA & & & $\mathbf{U}$ & 10 & & NS & $\mu g / L$ \\
\hline Acrolein & WA & $\mathbf{R}$ & & $\mathbf{U}$ & 10 & & NS & $\mu \mathrm{g} / \mathrm{L}$ \\
\hline Acrylonitrile & WA & & & $\mathbf{U}$ & 10 & & NS & $\mu \mathrm{g} / \mathrm{L}$ \\
\hline Acrylonitrile & WA & $\mathbf{R}$ & & U & 10 & & NS & $\mu \mathrm{g} / \mathrm{L}$ \\
\hline Alkalinity & WA & $\mathbf{R}$ & & & 2.5 & & NS & $\mathrm{mg} / \mathrm{L}$ \\
\hline Allyl chloride & WA & & & $\mathbf{U}$ & 100 & & NS & $\mu \mathrm{g} / \mathrm{L}$ \\
\hline Allyl chloride & WA & $\mathbf{R}$ & & $\mathbf{U}$ & 100 & & NS & $\mu \mathrm{g} / \mathrm{L}$ \\
\hline Aluminum & WA & & & $\mathbf{U}$ & 200 & & NS & $\mu \mathrm{g} / \mathrm{L}$ \\
\hline Aluminum & WA & $\mathbf{R}$ & & $\mathbf{U}$ & 200 & & NS & $\mu \mathrm{g} / \mathrm{L}$ \\
\hline Antimony & WA & & & $\mathbf{U}$ & 60 & & 6 & $\mu \mathrm{g} / \mathrm{L}$ \\
\hline Antimony & WA & $\mathbf{R}$ & & $\mathbf{U}$ & 60 & & 6 & $\mu \mathrm{g} / \mathrm{L}$ \\
\hline Arsenic & WA & & & $\mathbf{U}$ & 10 & & 50 & $\mu \mathrm{g} / \mathrm{L}$ \\
\hline Arsenic & WA & $\mathbf{R}$ & & $\mathrm{U}$ & 10 & & 50 & $\mu \mathrm{g} / \mathrm{L}$ \\
\hline
\end{tabular}


UTR 116 Laboratory Analyses (cont'd)

\begin{tabular}{|c|c|c|c|c|c|c|c|c|}
\hline Analyte & Lab & Rep & Avg & Modifier & Result & Acc & Stand & Units \\
\hline Barium & WA & & & U & 200 & & 2000 & $\mu \mathrm{g} / \mathrm{L}$ \\
\hline Barium & WA & $\mathbf{R}$ & & $U$ & 200 & & 2000 & $\mu \mathrm{g} / \mathrm{L}$ \\
\hline Benzene & WA & & & U & 5 & & 5 & $\mu \mathrm{g} / \mathrm{L}$ \\
\hline Benzene & WA & $\mathbf{R}$ & & U & 5 & & 5 & $\mu \mathrm{g} / \mathrm{L}$ \\
\hline Beryllium & WA & & & $\mathbf{U}$ & 5 & & 4 & $\mu g / L$ \\
\hline Beryllium & WA & $\mathbf{R}$ & & U & 5 & & 4 & $\mu \mathrm{g} / \mathrm{L}$ \\
\hline Bromodichloromethane & WA & & & $\mathbf{U}$ & 5 & & NS & $\mu g / L$ \\
\hline Bromodichloromethane & WA & $\mathbf{R}$ & & U & 5 & & NS & $\mu g / L$ \\
\hline Bromoform & WA & & & $\mathbf{U}$ & 5 & & NS & $\mu g / L$ \\
\hline Bromoform & WA & $\mathbf{R}$ & & U & 5 & & NS & $\mu g / L$ \\
\hline Cadmium & WA & & & $\mathbf{U}$ & 5 & & 5 & $\mu g / L$ \\
\hline Cadmium & WA & $\mathbf{R}$ & & $\mathbf{U}$ & 5 & & 5 & $\mu \mathrm{g} / \mathrm{L}$ \\
\hline Calcium & WA & & & $\mathbf{U}$ & 5000 & & NS & $\mu g / L$ \\
\hline Calcium & WA & $\mathbf{R}$ & & $\mathbf{U}$ & 5000 & & NS & $\mu g / L$ \\
\hline Carbon disulfide & WA & & & $\mathbf{U}$ & 5 & & NS & $\mu \mathrm{g} / \mathrm{L}$ \\
\hline Carbon disulfide & WA & $\mathbf{R}$ & & $\mathbf{U}$ & 5 & & NS & $\mu \mathrm{g} / \mathrm{L}$ \\
\hline Carbon tetrachloride & WA & & & $\mathbf{U}$ & 5 & & 5 & $\mu g / L$ \\
\hline Carbon tetrachloride & WA & $\mathbf{R}$ & & $\mathbf{U}$ & 5 & & 5 & $\mu \mathrm{g} / \mathrm{L}$ \\
\hline Cesium-137 & TM & & & $\mathrm{U}$ & 6.1 & & 200 & $\mathrm{pCi} / \mathrm{L}$ \\
\hline Cesium-137 & TM & $\mathbf{R}$ & & $U$ & 6.2 & & 200 & $\mathrm{pCi} / \mathrm{L}$ \\
\hline Chloride & WA & & & & 4.32 & & NS & $\mathrm{mg} / \mathrm{L}$ \\
\hline Chloride & WA & $\mathbf{R}$ & & & 1.77 & & NS & $\mathrm{mg} / \mathrm{L}$ \\
\hline Chlorobenzene & WA & & & $\mathbf{U}$ & 5 & & 100 & $\mu g / L$ \\
\hline Chlorobenzene & WA & $\mathbf{R}$ & & $\mathbf{U}$ & 5 & & 100 & $\mu \mathrm{g} / \mathrm{L}$ \\
\hline Chloroethane & WA & & & $\mathbf{U}$ & 10 & & NS & $\mu g / L$ \\
\hline Chloroethane & WA & $\mathbf{R}$ & & U & 10 & & NS & $\mu g / L$ \\
\hline Chloroform & WA & & & $\mathbf{U}$ & 5 & & 100 & $\mu g / L$ \\
\hline Chloroform & WA & $\mathbf{R}$ & & $\mathbf{U}$ & 5 & & 100 & $\mu g / L$ \\
\hline Chloroprene & WA & & & $\mathrm{U}$ & 20 & & NS & $\mu g / L$ \\
\hline Chloroprene & WA & $\mathbf{R}$ & & U & 20 & & NS & $\mu g / L$ \\
\hline Chromium & WA & & & $\mathrm{U}$ & 10 & & 100 & $\mu g / L$ \\
\hline Chromium & WA & $\mathbf{R}$ & & U & 10 & & 100 & $\mu g / L$ \\
\hline cis-1,3-Dichloropropene & WA & & & $\mathbf{U}$ & 5 & & NS & $\mu \mathrm{g} / \mathrm{L}$ \\
\hline cis-1,3-Dichloropropene & WA & $\mathbf{R}$ & & $\mathrm{U}$ & 5 & & NS & $\mu \mathrm{g} / \mathrm{L}$ \\
\hline Cobalt & WA & & & $U$ & 50 & & NS & $\mu \mathrm{g} / \mathrm{L}$ \\
\hline Cobalt & WA & $\mathbf{R}$ & & $\mathbf{U}$ & 50 & & NS & $\mu g / L$ \\
\hline Copper & WA & & & U & 25 & & 1000 & $\mu g / L$ \\
\hline Copper & WA & $\mathbf{R}$ & & $\mathbf{U}$ & 25 & & 1000 & $\mu g / L$ \\
\hline Dibromochloromethane & WA & & & $\mathbf{U}$ & 5 & & NS & $\mu \mathrm{g} / \mathrm{L}$ \\
\hline Dibromochloromethane & WA & $\mathbf{R}$ & & U & 5 & & NS & $\mu g / L$ \\
\hline Dichlorodifluoromethane & WA & & & $\mathbf{U}$ & 10 & & NS & $\mu g / L$ \\
\hline Dichlorodifluoromethane & WA & $\mathbf{R}$ & & $\mathrm{U}$ & 10 & & NS & $\mu \mathrm{g} / \mathrm{L}$ \\
\hline Ethylbenzene & WA & & & $\mathrm{U}$ & 5 & & 700 & $\mu g / L$ \\
\hline Ethylbenzene - & WA & $\mathbf{R}$ & & $\mathrm{U}$ & 5 & & 700 & $\mu g / L$ \\
\hline Fluoride & WA & & & U & 0.1 & & 4 & $\mathrm{mg} / \mathrm{L}$ \\
\hline Fluoride & WA & $\mathbf{R}$ & & $\mathrm{U}$ & 0.1 & & 4 & $\mathrm{mg} / \mathrm{L}$ \\
\hline Fluoride & WA & $\mathbf{R}$ & NA & U & 0.1 & & 4 & $\mathrm{mg} / \mathrm{L}$ \\
\hline Gross alpha & TM & & & & 1.7 & 1.9 & 15 & $\mathrm{pCi} / \mathrm{L}$ \\
\hline Gross alpha & TM & $\mathbf{R}$ & & U & 0.9 & & 15 & $\mathrm{pCi} / \mathrm{L}$ \\
\hline Iron & WA & & & & 566 & & 300 & $\mu \mathrm{g} / \mathrm{L}$ \\
\hline Iron & WA & $\mathbf{R}$ & & & 553 & & 300 & $\mu \mathrm{g} / \mathrm{L}$ \\
\hline Isobutyl alcohol & WA & & & $\mathrm{U}$ & 20 & & NS & $\mu g / L$ \\
\hline Isobutyl alcohol & WA & $\mathbf{R}$ & & U & 20 & & NS & $\mu \mathrm{g} / \mathrm{L}$ \\
\hline Lead & WA & & & U & 3 & & 50 & $\mu \mathrm{g} / \mathrm{L}$ \\
\hline Lead & WA & $\mathbf{R}$ & & $\mathbf{U}$ & 3 & & 50 & $\mu g / L$ \\
\hline
\end{tabular}


UTR116 Laboratory Analyses (cont'd)

\begin{tabular}{|c|c|c|c|c|c|c|c|c|c|}
\hline Analyte & Lab & Rep & Avg & Modifier & & Result & Acc & Stand & Units \\
\hline Magnesium & WA & & & $\mathrm{U}$ & & 5000 & & NS & $\mu \mathrm{g} / \mathrm{L}$ \\
\hline Magnesium & WA & $\mathbf{R}$ & & $\mathbf{U}$ & & 5000 & & NS & $\mu \mathrm{g} / \mathrm{L}$ \\
\hline Manganese & WA & & & & & 15.1 & & 50 & $\mu \mathrm{g} / \mathrm{L}$ \\
\hline Manganese & WA & $\mathbf{R}$ & & & & 18.7 & & 50 & $\mu \mathrm{g} / \mathrm{L}$ \\
\hline Mercury & WA & & & $\mathbf{U}$ & & 0.2 & & 2 & $\mu \mathrm{g} / \mathrm{L}$ \\
\hline Mercury & WA & $\mathbf{R}$ & & $\mathbf{U}$ & & 0.2 & & 2 & $\mu \mathrm{g} / \mathrm{L}$ \\
\hline Methacrylonitrile & WA & & & $\mathbf{U}$ & & 20 & & NS & $\mu \mathrm{g} / \mathrm{L}$ \\
\hline Methacrylonitrile & WA & $\mathbf{R}$ & & $\mathbf{U}$ & & 20 & & NS & $\mu \mathrm{g} / \mathrm{L}$ \\
\hline Methyl bromide & WA & & & $\mathbf{U}$ & & 10 & & NS & $\mu \mathrm{g} / \mathrm{L}$ \\
\hline Methyl bromide & WA & $\mathbf{R}$ & & U & & 10 & & NS & $\mu \mathrm{g} / \mathrm{L}$ \\
\hline Methyl chloride & WA & & & $\mathbf{U}$ & & 10 & & NS & $\mu g / L$ \\
\hline Methyl chloride & WA & $\mathbf{R}$ & & $\mathbf{U}$ & & 10 & & NS & $\mu \mathrm{g} / \mathrm{L}$ \\
\hline Methyl ethyl ketone & WA & & & $\mathbf{U}$ & & 10 & & NS & $\mu \mathrm{g} / \mathrm{L}$ \\
\hline Methyl ethyl ketone & WA & $\mathbf{R}$ & & $U$ & & 10 & & NS & $\mu g / L$ \\
\hline Methyl iodile & WA & & & $\mathbf{U}$ & & 10 & & NS & $\mu g / L$ \\
\hline Methyl iodids & WA & $\mathbf{R}$ & & $\mathbf{U}$ & & 10 & & NS & $\mu \mathrm{g} / \mathrm{L}$ \\
\hline Methylene bromide & WA & & & $\mathbf{U}$ & & 10 & & NS & $\mu \mathrm{g} / \mathrm{L}$ \\
\hline Methylene bromide & WA & $\mathbf{R}$ & & $\mathbf{U}$ & & 10 & & NS & $\mu g / L$ \\
\hline Methylene chloride & WA & & & J & V & 4.1 & & 5 & $\mu g / L$ \\
\hline Methylene chloride & WA & $\mathbf{R}$ & & $\mathbf{J}$ & V & 4.17 & & 5 & $\mu g / L$ \\
\hline Nickel & WA & & & $\mathbf{U}$ & & 40 & & 100 & $\mu g / L$ \\
\hline Nickel & WA & $\mathbf{R}$ & & $\mathbf{U}$ & & 40 & & 100 & $\mu g / L$ \\
\hline Nitrate as nitrogen & WA & & & $\mathbf{J}$ & $\mathbf{Q}$ & 0.103 & & 10 & $\mathrm{mg} / \mathrm{L}$ \\
\hline Nitrate as nitrogen & WA & $\mathbf{R}$ & & J & Q & 0.146 & & 10 & $\mathrm{mg} / \mathrm{L}$ \\
\hline Nonvolatile beta & TM & & & & & 2.5 & 3 & 50 & $\mathrm{pCi} / \mathrm{L}$ \\
\hline Nonvolatile beta & TM & $\mathbf{R}$ & & & & 2.2 & 3 & 50 & $\mathrm{pCi} / \mathrm{L}$ \\
\hline Potassium & WA & & & $\mathbf{U}$ & & 5000 & & NS & $\mu g / L$ \\
\hline Potassium & WA & $\mathbf{R}$ & & $\mathbf{U}$ & & 5000 & & NS & $\mu \mathrm{g} / \mathrm{L}$ \\
\hline Propionitrile & WA & & & U & & 50 & & NS & $\mu g / L$ \\
\hline Propionitrile & WA & $\mathbf{R}$ & & $\mathbf{U}$ & & 50 & & NS & $\mu g / L$ \\
\hline Selenium & WA & & & $\mathbf{U}$ & & 5 & & 50 & $\mu \mathrm{g} / \mathrm{L}$ \\
\hline Selenium & WA & $\mathbf{R}$ & & $\mathbf{U}$ & & 5 & & 50 & $\mu \mathrm{g} / \mathrm{L}$ \\
\hline Silver & WA & & & $\mathbf{U}$ & & 10 & & 50 & $\mu \mathrm{g} / \mathrm{L}$ \\
\hline Silver & WA & $\mathbf{R}$ & & $\mathbf{U}$ & & 10 & & 50 & $\mu g / L$ \\
\hline Sodium & WA & & & $\mathbf{U}$ & & 5000 & & NS & $\mu g / L$ \\
\hline Sodium & WA & $\mathbf{R}$ & & $\mathbf{U}$ & & 5000 & & NS & $\mu g / L$ \\
\hline Styrene & WA & & & $\mathbf{U}$ & & 5 & & 100 & $\mu \mathrm{g} / \mathrm{L}$ \\
\hline Styrene & WA & $\mathbf{R}$ & & $\mathbf{U}$ & & 5 & & 100 & $\mu \mathrm{g} / \mathrm{L}$ \\
\hline Sulfate & WA & & & $\mathbf{U}$ & & 2.5 & & 400 & $\mathrm{mg} / \mathrm{L}$ \\
\hline Sulfate & WA & $\mathbf{R}$ & & $\mathbf{U}$ & & 2.5 & & 400 & $\mathrm{mg} / \mathrm{L}$ \\
\hline Sulfide & WA & & & $\mathbf{U}$ & & 0.1 & & NS & $\mathrm{mg} / \mathrm{L}$ \\
\hline Sulfide & WA & $\mathbf{R}$ & & $\mathbf{U}$ & & 0.1 & & NS & $\mathrm{mg} / \mathrm{L}$ \\
\hline Tetrachloroethylene & WA & & & $\mathbf{U}$ & & 5 & & 5 & $\mu g / L$ \\
\hline Tetrachloroethylene & WA & $\mathbf{R}$ & & U & & 5 & & 5 & $\mu \mathrm{g} / \mathrm{L}$ \\
\hline Thallium & WA & & & $\mathbf{U}$ & & 10 & & 2 & $\mu \mathrm{g} / \mathrm{L}$ \\
\hline Thallium & WA & $\mathbf{R}$ & & $\mathrm{U}$ & & 10 & & 2 & $\mu \mathrm{g} / \mathrm{L}$ \\
\hline Toluene & WA & & & $\mathbf{U}$ & & 5 & & 1000 & $\mu g / L$ \\
\hline Toluene & $\because A$ & $\mathbf{R}$ & & $\mathbf{J}$ & & 1.02 & & 1000 & $\mu \mathrm{g} / \mathrm{L}$ \\
\hline Total hardness & WA & & & & & 1.96 & & NS & $\mathrm{mg} / \mathrm{L}$ \\
\hline Total hardness & WA & $\mathbf{R}$ & & $\mathbf{U}$ & & 1 & & NS & $\mathrm{mg} / \mathrm{L}$. \\
\hline Total organic carbon & WA & & & & & 1.9 & & NS & $\mathrm{mg} / \mathrm{L}$ \\
\hline Total organic carbon & WA & $\mathbf{R}$ & & & & 1.99 & & NS & $\mathrm{mg} / \mathrm{L}$ \\
\hline Total suspended solids & WA & & & & & 11 & & NS & $\mathrm{mg} / \mathrm{L}$ \\
\hline Total suspended solids & WA & $\mathbf{R}$ & & $\mathbf{U}$ & & 5 & & NS & $\mathrm{mg} / \mathrm{L}$ \\
\hline Total suspended solids & WA & $\mathbf{R}$ & NA & & & 5 & & NS & $\mathrm{mg}$ \\
\hline
\end{tabular}


UTR1 16 Laboratory Analyses (cont'd)

\begin{tabular}{|c|c|c|c|c|c|c|c|c|}
\hline Analyte & Lab & Rep & Avg & Modifier & Result & Acc & Stand & Units \\
\hline trans-1,3-Dichloropropene & WA & & & $\mathrm{U}$ & 5 & & NS & $\overline{\mu g / L}$ \\
\hline trans-1,3-Dichloropropene & WA & $\mathbf{R}$ & & $\mathbf{U}$ & 5 & & NS & $\mu g / L$ \\
\hline trans-1,4-Dichloro-2-butene & WA & & & $\mathbf{U}$ & 100 & & NS & $\mu \mathrm{g} / \mathrm{L}$ \\
\hline trans-1,4-Dichloro-2-butene & WA & $\mathbf{R}$ & & $\mathrm{U}$ & 100 & & NS & $\mu g / L$ \\
\hline Trichloroethylene & WA & & & $\mathbf{U}$ & 5 & & 5 & $\mu \mathrm{g} / \mathrm{L}$ \\
\hline Trichloroethylene & WA & $\mathbf{R}$ & & $\mathbf{U}$ & 5 & & 5 & $\mu g / L$ \\
\hline Trichlorofluoromethane & WA & & & $\mathbf{U}$ & 5 & & NS & $\mu \mathrm{g} / \mathrm{L}$ \\
\hline Trichlorofluoromethane & WA & $\mathbf{R}$ & & $\mathbf{U}$ & 5 & & NS & $\mu \mathrm{g} / \mathrm{L}$ \\
\hline Tritium & TM & & & & 111.9 & 2.25 & 20 & $\mathrm{pCi} / \mathrm{ml}$ \\
\hline Tritium & TM & $\mathbf{R}$ & & & 104.6 & 2.16 & 20 & $\mathrm{pCi} / \mathrm{ml}$ \\
\hline Turbidity & WA & & & $\mathbf{J}$ & 2.6 & & NS & NTU \\
\hline Turbidity & WA & $\mathbf{R}$ & & $\mathbf{J}$ & 1.92 & & NS & NTU \\
\hline Vanadium & WA & & & $\mathbf{U}$ & 50 & & NS & $\mu g / L$ \\
\hline Vanadium & WA & $\mathbf{R}$ & & $\mathrm{U}$ & 50 & & NS & $\mu g / L$ \\
\hline Vinyl acetate & WA & & & $\mathbf{U}$ & 10 & & NS & $\mu \mathrm{g} / \mathrm{L}$ \\
\hline Vinyl acetate & WA & $\mathbf{R}$ & & $\mathbf{U}$ & 10 & & NS & $\mu g / L$ \\
\hline Vinyl chloride & WA & & & $\mathbf{U}$ & 10 & & 2 & $\mu g / L$ \\
\hline Vinyl chloride & WA & $\mathbf{R}$ & & $\mathbf{U}$ & 10 & & 2 & $\mu g / L$ \\
\hline Xylenes (total) & WA & & & U & 5 & & 10000 & $\mu g / L$ \\
\hline Xylenes (total) & WA & $\mathbf{R}$ & & $\mathbf{U}$ & 5 & & 10000 & $\mu g / L$ \\
\hline Zinc & WA & & & & 67.8 & & 5000 & $\mu \mathrm{g} / \mathrm{L}$ \\
\hline Zinc & WA & $\mathbf{R}$ & & & 71.1 & & 5000 & $\mu g / L$ \\
\hline
\end{tabular}


UTR120

Field Measurements:

Sample Date: $9 / 2 / 93$

pH: 7.54

Specific Conductance: $47 \mu \mathrm{S} / \mathrm{cm}$

Red/Oxid Potential: $-10 \mathrm{mv}$

Dissolved Oxygen: $4.7 \mathrm{mg} / \mathrm{L}$

\begin{tabular}{|c|c|c|c|c|c|c|c|c|}
\hline Analyte & Lab & Rep & Avg & Modifier & Result & Acc & Stand & Units \\
\hline 1,1,1,2-Tetrachloroethane & $\overline{\text { WA }}$ & & & $\mathbf{U}$ & 10 & & NS & $\mu g / L$ \\
\hline 1,1,1-Trichloroethane & WA & & & $\mathbf{U}$ & 5 & & 200 & $\mu g / L$ \\
\hline 1,1,2,2-Tetrachloroethane & WA & & & $\mathbf{U}$ & 5 & & NS & $\mu g / L$ \\
\hline 1,1,2-Trichloroethane & WA & & & $\mathbf{U}$ & 5 & & 5 & $\mu g / L$ \\
\hline 1,1-Dichloroethane & WA & & & $\mathbf{U}$ & 5 & & NS & $\mu \mathrm{g} / \mathrm{L}$ \\
\hline 1,1-Dichloroethylene & WA & & & $\bar{U}$ & 5 & & 7 & $\mu g / L$ \\
\hline 1,2,3-Trichloropropane & WA & & & $\mathbf{U}$ & 10 & & NS & $\mu g / L$ \\
\hline 1,2-Dibromo-3-chloropropane & WA & & & $\mathbf{U}$ & 20 & & 0.2 & $\mu g / L$ \\
\hline 1,2-Dibromoethane & WA & & & $\mathbf{U}$ & 20 & & NS & $\mu \mathrm{g} / \mathrm{L}$ \\
\hline 1,2-Dichloroethane & WA & & & $\mathbf{U}$ & 5 & & 5 & $\mu \mathrm{g} / \mathrm{L}$ \\
\hline 1,2-Dichloroethylene (total) & WA & & & $\mathbf{U}$ & 5 & & 7 & $\mu \mathrm{g} / \mathrm{L}$ \\
\hline 1,2-Dichloropropane & WA & & & $\mathbf{U}$ & 5 & & 5 & $\mu g / L$ \\
\hline 2-Hexanone & WA & & & $\mathbf{U}$ & 10 & & NS & $\mu \mathrm{g} / \mathrm{L}$ \\
\hline 4-Methyl-2-pentanone & WA & & & $\mathbf{U}$ & 10 & & NS & $\mu \mathrm{g} / \mathrm{L}$ \\
\hline Acetone & WA & & & $\mathbf{J}$ & 2.28 & & NS & $\mu \mathrm{g} / \mathrm{L}$ \\
\hline Acetonitrile & WA & & & $\mathbf{U}$ & 20 & & NS & $\mu \mathrm{g} / \mathrm{L}$ \\
\hline Acidity & WA & & A & & 55 & & NS & $\mathrm{mg} / \mathrm{L}$ \\
\hline Acrolein & WA & & & $\mathbf{U}$ & 10 & & NS & $\mu \mathrm{g} / \mathrm{L}$ \\
\hline Acrylonitrile & WA & & & $\mathbf{U}$ & 10 & & NS & $\mu g / L$ \\
\hline Alkalinity & WA & & $\mathbf{A}$ & & 1 & & NS & $\mathrm{mg} / \mathrm{L}$ \\
\hline Allyl chloride & WA & & & $\mathbf{U}$ & 100 & & NS & $\mu \mathrm{g} / \mathrm{L}$ \\
\hline Aluminum & WA & & & $\mathbf{U}$ & 200 & & NS & $\mu \mathrm{g} / \mathrm{L}$ \\
\hline Aluminum & WA & & NA & $\mathbf{U}$ & 200 & & NS & $\mu g / L$ \\
\hline Antimony & WA & & & $\mathbf{U}$ & 60 & & 6 & $\mu g / L$ \\
\hline Antimony & WA & & NA & $\mathbf{U}$ & 60 & & 6 & $\mu g / L$ \\
\hline Arsenic & WA & & & $\mathbf{U}$ & 10 & & 50 & $\mu \mathrm{g} / \mathrm{L}$ \\
\hline Arsenic & WA & & NA & $\mathbf{U}$ & 10 & & 50 & $\mu g / L$ \\
\hline Barium & WA & & & $\mathbf{U}$ & 200 & & 2000 & $\mu g / L$ \\
\hline Barium & WA & & NA & $\mathbf{U}$ & 200 & & 2000 & $\mu g / L$ \\
\hline Benzene & WA & & & $\mathbf{U}$ & 5 & & 5 & $\mu \mathrm{g} / \mathrm{L}$ \\
\hline Beryllium & WA & & & $\mathbf{U}$ & 5 & & 4 & $\mu \mathrm{g} / \mathrm{L}$ \\
\hline Beryllium & WA & & NA & $\mathbf{U}$ & 5 & & 4 & $\mu g / L$ \\
\hline Bromodichloromethane & WA & & & $\mathbf{U}$ & 5 & & NS & $\mu \mathrm{g} / \mathrm{L}$ \\
\hline Bromoform & WA & & & U & 5 & & NS & $\mu \mathrm{g} / \mathrm{L}$ \\
\hline Cadmium & WA & & & $\mathrm{U}$ & 5 & & 5 & $\mu \mathrm{g} / \mathrm{L}$ \\
\hline Cadmium & WA & & NA & $\mathbf{U}$ & 5 & & 5 & $\mu \mathrm{g} / \mathrm{L}$ \\
\hline Calcium & WA & & & $\mathrm{U}$ & 5000 & & NS & $\mu \mathrm{g} / \mathrm{L}$ \\
\hline Calcium & WA & & NA & $\mathbf{U}$ & 5000 & & NS & $\mu \mathrm{g} / \mathrm{L}$ \\
\hline Carbon disulfide & WA & & & $\mathbf{U}$ & 5 & & NS & $\mu \mathrm{g} / \mathrm{L}$ \\
\hline Carbon tetrachloride & WA & & & $\mathbf{U}$ & 5 & & 5 & $\mu \mathrm{g} / \mathrm{L}$ \\
\hline Cesium-137 & TM & & NA & $\mathbf{U}$ & 9.87 & & 200 & $\mathrm{pCi} / \mathrm{L}$ \\
\hline Cesium-137 & TM & & & $\mathbf{U}$ & 9.68 & & 200 & $\mathrm{pCi} / \mathrm{L}$ \\
\hline Chloride & WA & & A & & 2.995 & & NS & $\mathrm{mg} / \mathrm{L}$ \\
\hline Chlorobenzene & WA & & & $\mathbf{U}$ & 5 & & 100 & $\mu \mathrm{g} / \mathrm{L}$ \\
\hline Chloroethane & WA & & & $\mathbf{U}$ & 10 & & NS & $\mu \mathrm{g} / \mathrm{L}$ \\
\hline Chloroform & WA & & & $\mathbf{U}$ & 5 & & 100 & $\mu g / L$ \\
\hline Chloroprene & WA & & & $\mathbf{U}$ & 20 & & NS & $\mu g / L$ \\
\hline
\end{tabular}


UTR 120 Laboratory Analyses (cont'd)

\begin{tabular}{|c|c|c|c|c|c|c|c|}
\hline Analyte & Lab & Rep & Modifier & Result & Acc & Stand & Units \\
\hline Chromium & WA & & $\mathbf{U}$ & 10 & & 100 & $\mu g / L$ \\
\hline Chromium & WA & NA & $\mathbf{U}$ & 10 & & 100 & $\mu \mathrm{g} / \mathrm{L}$ \\
\hline cis-1,3-Dichloropropene & WA & & $\mathbf{U}$ & 5 & & NS & $\mu g / L$ \\
\hline Cobalt & WA & & $\mathbf{U}$ & 50 & & NS & $\mu \mathrm{g} / \mathrm{L}$ \\
\hline Cobalt & WA & NA & $\mathbf{U}$ & 50 & & NS & $\mu \mathrm{g} / \mathrm{L}$ \\
\hline Copper & WA & & $\mathbf{U}$ & 25 & & 1000 & $\mu g / L$ \\
\hline Copper & WA & NA & $\mathbf{U}$ & 25 & $\cdot$ & 1000 & $\mu g / L$ \\
\hline Dibromochloromethane & WA & & $\mathbf{U}$ & 5 & & NS & $\mu g / L$ \\
\hline Dichlorodifluoromethane & WA & & $\mathbf{U}$ & 10 & & NS & $\mu g / L$ \\
\hline Ethylbenzene & WA & & $\mathbf{U}$ & 5 & & 700 & $\mu \mathrm{g} / \mathrm{L}$ \\
\hline Fluoride & WA & & $\mathbf{U}$ & 0.1 & & 4 & $\mathrm{mg} / \mathrm{L}$ \\
\hline Gross alpha & TM & $\mathbf{A}$ & & 4.35 & 1.75 & 15 & $\mathrm{pCi} / \mathrm{L}$ \\
\hline Iron & WA & A & & 267.5 & & 300 & $\mu g / L$ \\
\hline Isobutyl alcohol & WA & & $\mathbf{U}$ & 20 & & NS & $\mu g / L$ \\
\hline Lead & WA & & $\mathbf{U}$ & 3 & & 50 & $\mu g / L$ \\
\hline Lead & WA & NA & $\mathbf{U}$ & 3 & & 50 & $\mu g / L$ \\
\hline Magnesium & WA & & $\mathbf{U}$ & 5000 & & NS & $\mu \mathrm{g} / \mathrm{L}$ \\
\hline Magnesium & WA & NA & $\mathbf{U}$ & 5000 & & NS & $\mu g / L$ \\
\hline Manganese & WA & & $\mathbf{U}$ & 15 & & 50 & $\mu g / L$ \\
\hline Manganese & WA & NA & $\mathbf{U}$ & 15 & & 50 & $\mu g / L$ \\
\hline Mercury & WA & & $\mathbf{U}$ & 0.2 & & 2 & $\mu g / L$ \\
\hline Methacrylonitrile & WA & & $\mathbf{U}$ & 20 & & NS & $\mu \mathrm{g} / \mathrm{L}$ \\
\hline Methyl bromide & WA & & $\mathbf{U}$ & 10 & & NS & $\mu g / L$ \\
\hline Methyl chloride & WA & & $\mathbf{U}$ & 10 & & NS & $\mu \mathrm{g} / \mathrm{L}$ \\
\hline Methyl ethyl ketone & WA & & $\mathbf{U}$ & 10 & & NS & $\mu \mathrm{g} / \mathrm{L}$ \\
\hline Methyl iodide & WA & & $\mathrm{U}$ & 10 & & NS & $\mu g / L$ \\
\hline Methylene bromide & WA & & $\mathbf{U}$ & 10 & & NS & $\mu g / L$ \\
\hline Methylene chloride & WA & & $\mathbf{U}$ & 5 & & 5 & $\mu g / L$ \\
\hline Nickel & WA & & $\mathbf{U}$ & 40 & & 100 & $\mu g / L$ \\
\hline Nickel & WA & NA & $\mathbf{U}$ & 40 & & 100 & $\mu \mathrm{g} / \mathrm{L}$ \\
\hline Nitrate as nitrogen & WA & & $\mathbf{Q}$ & 0.866 & & 10 & $\mathrm{mg} / \mathrm{L}$ \\
\hline Nonvolatile beta & TM & $\mathbf{A}$ & & 11 & 2.9 & 50 & $\mathrm{pCi} / \mathrm{L}$ \\
\hline Potassium & WA & & $\mathbf{U}$ & 5000 & & NS & $\mu g / L$ \\
\hline Potassium & WA & NA & $\mathbf{U}$ & 5000 & & NS & $\mu \mathrm{g} / \mathrm{L}$ \\
\hline Propionitrile & WA & & $\mathbf{U}$ & 50 & & NS & $\mu \mathrm{g} / \mathrm{L}$ \\
\hline Selenium & WA & & $\mathbf{U}$ & 5 & & 50 & $\mu \mathrm{g} / \mathrm{L}$ \\
\hline Selenium & WA & NA & $\mathbf{U}$ & 5 & & 50 & $\mu \mathrm{g} / \mathrm{L}$ \\
\hline Silver & WA & & $\mathbf{U}$ & 10 & & 50 & $\mu \mathrm{g} / \mathrm{L}$ \\
\hline Silver & WA & NA & $\mathbf{U}$ & 10 & & 50 & $\mu \mathrm{g} / \mathrm{L}$ \\
\hline Sodium & WA & & $\mathbf{U}$ & 5000 & & NS & $\mu \mathrm{g} / \mathrm{L}$ \\
\hline Sodium & WA & NA & $\mathbf{U}$ & 5000 & & NS & $\mu \mathrm{g} / \mathrm{L}$ \\
\hline Styrene & WA & & $\mathrm{U}$ & 5 & & 100 & $\mu \mathrm{g} / \mathrm{L}$ \\
\hline Sulfate & WA & & U & 2.5 & & 400 & $\mathrm{mg} / \mathrm{L}$ \\
\hline Sulfide & WA & & U & 0.1 & & NS & $\mathrm{mg} / \mathrm{L}$ \\
\hline Sulfide & WA & NA & $\mathbf{U}$ & 0.1 & & NS & $\mathrm{mg} / \mathrm{L}$ \\
\hline Tetrachloroethylene & WA & & $\mathbf{U}$ & 5 & & 5 & $\mu \mathrm{g} / \mathrm{L}$ \\
\hline Thallium & WA & & U & 10 & & 2 & $\mu \mathrm{g} / \mathrm{L}$ \\
\hline Thallium & WA & NA & $\mathbf{U}$ & 10 & & 2 & $\mu g / L$ \\
\hline Toluene & WA & & $\mathbf{U}$ & 5 & & 1000 & $\mu \mathrm{g} / \mathrm{L}$ \\
\hline Total hardness & WA & & & 4.8 & & NS & $\mathrm{mg} / \mathrm{L}$ \\
\hline Total organic carbon & WA & & & 1.16 & & NS & $\mathrm{mg} / \mathrm{L}$ \\
\hline Total suspended solids & WA & & & 6 & & NS & $\mathrm{mg} / \mathrm{L}$ \\
\hline trans-1,3-Dichloropropene & WA & & $\mathbf{U}$ & 5 & & NS & $\mu \mathrm{g} / \mathrm{L}$ \\
\hline trans-1,4-Dichloro-2-butene & WA & & $\mathbf{U}$ & 100 & & NS & $\mu g / L$ \\
\hline
\end{tabular}


UTR120 Laboratory Analyses (cont'd)

Trichloroethylene

Trichlorofluoromethane

Tritium

Turbidity

Vanadium

Vanadium

Vinyl acetate

Vinyl chloride

Xylenes (total)

Zinc

Zinc
Lab

WA

WA

TM

WA

WA

WA

WA

WA

WA

WA

WA
Rep Avg Modifier

Resul

\begin{tabular}{lccr} 
ult & Acc & Stand & Units \\
\hline & & 5 & $\mu \mathrm{g} / \mathrm{L}$ \\
48 & 1.59 & $\mathrm{NS}$ & $\mu \mathrm{g} / \mathrm{L}$ \\
31 & & $\mathrm{NS}$ & $\mathrm{pCi} / \mathrm{ml}$ \\
0 & & $\mathrm{NS}$ & $\mu \mathrm{NT} / \mathrm{L}$ \\
0 & & $\mathrm{NS}$ & $\mu \mathrm{g} / \mathrm{L}$ \\
0 & & $\mathrm{NS}$ & $\mu \mathrm{g} / \mathrm{L}$ \\
0 & & 2 & $\mu \mathrm{g} / \mathrm{L}$ \\
5 & & 10000 & $\mu \mathrm{g} / \mathrm{L}$ \\
0 & & 5000 & $\mu \mathrm{g} / \mathrm{L}$ \\
20 & & 5000 & $\mu \mathrm{g} / \mathrm{L}$
\end{tabular}




\section{UTR122}

Field Measurements:

Sample Date: 9/2/93

pH: 6.50

Specific Conductance: $54 \mu \mathrm{S} / \mathrm{cm}$

Red/Oxid Potential: $131 \mathrm{mv}$

Dissolved Oxygen: $2.1 \mathrm{mg} / \mathrm{L}$

\begin{tabular}{|c|c|c|c|c|c|c|c|c|}
\hline Analyte & Lab & Rep & Avg & Modifier & Result & Acc & Stand & Units \\
\hline 1,1,1,2-Tetrachloroethane & $\overline{W A}$ & & & $\mathbf{U}$ & 10 & & NS & $\mu g / L$ \\
\hline 1,1,1-Trichloroethane & WA & & & $\mathbf{U}$ & 5 & & 200 & $\mu g / L$ \\
\hline 1,1,2,2-Tetrachloroethane & WA & & & $\mathbf{U}$ & 5 & & NS & $\mu g / L$ \\
\hline 1,1,2-Trichloroethane & WA & & & $\mathbf{U}$ & 5 & & 5 & $\mu \mathrm{g} / \mathrm{L}$ \\
\hline 1,1-Dichloroethane & WA & & & $U$ & 5 & & NS & $\mu \mathrm{g} / \mathrm{L}$ \\
\hline 1,1-Dichloroethylene & WA & & & $\mathbf{U}$ & 5 & & 7 & $\mu g / L$ \\
\hline 1,2,3-Trichloropropane & WA & & & $\mathbf{U}$ & 10 & & NS & $\mu g / L$ \\
\hline 1,2-Dibromo-3-chloropropane & WA & & & $\mathbf{U}$ & 20 & & 0.2 & $\mu g / L$ \\
\hline 1,2-Dibromoethane & WA & & & $\mathbf{U}$ & 20 & & NS & $\mu g / L$ \\
\hline 1,2-Dichloroethane & WA & & & $\mathbf{U}$ & 5 & & 5 & $\mu \mathrm{g} / \mathrm{L}$ \\
\hline 1,2-Dichloroethylene (total) & WA & & & $\mathbf{U}$ & 5 & & 7 & $\mu g / L$ \\
\hline 1,2-Dichloropropane & WA & & & $\mathbf{U}$ & 5 & & 5 & $\mu g / L$ \\
\hline 2-Hexanone & WA & & & $\mathbf{U}$ & 10 & & NS & $\mu \mathrm{g} / \mathrm{L}$ \\
\hline 4-Methyl-2-pentanone & WA & & & $\mathbf{U}$ & 10 & & NS & $\mu \mathrm{g} / \mathrm{L}$ \\
\hline Acetone & WA & & & & 15.8 & & NS & $\mu \mathrm{g} / \mathrm{L}$ \\
\hline Acetonitrile & WA & & & $\mathbf{U}$ & 20 & & NS & $\mu \mathrm{g} / \mathrm{L}$ \\
\hline Acidity & WA & & & & 96 & & NS & $\mathrm{mg} / \mathrm{L}$ \\
\hline Acrolein & WA & & & $\mathbf{U}$ & 10 & & NS & $\mu g / L$ \\
\hline Acrylonitrile & WA & & & $\mathbf{U}$ & 10 & & NS & $\mu \mathrm{g} / \mathrm{L}$ \\
\hline Alkalinity & WA & & & & 0.5 & & NS & $\mathrm{mg} / \mathrm{L}$ \\
\hline Allyl chloride & WA & & & $\mathbf{U}$ & 100 & & NS & $\mu g / L$ \\
\hline Aluminum & WA & & & & 471 & & NS & $\mu \mathrm{g} / \mathrm{L}$ \\
\hline Antimony & WA & & & $\mathbf{U}$ & 60 & & 6 & $\mu g / L$ \\
\hline Arsenic & WA & & & $\mathbf{U}$ & 10 & & 50 & $\mu g / L$ \\
\hline Barium & WA & & & $\mathbf{U}$ & 200 & & 2000 & $\mu g / L$ \\
\hline Benzene & WA & & & $\mathbf{U}$ & 5 & & 5 & $\mu g / L$ \\
\hline Beryllium & WA & & & $\mathbf{U}$ & 5 & & 4 & $\mu g / L$ \\
\hline Bromodichloromethane & WA & & & $\mathbf{U}$ & 5 & & NS & $\mu g / L$ \\
\hline Bromoform & WA & & & $\mathbf{U}$ & 5 & & NS & $\mu g / L$ \\
\hline Cadmium & WA & & & $\mathbf{U}$ & 5 & & 5 & $\mu g / L$ \\
\hline Calcium & WA & & & U & 5000 & & NS & $\mu g / L$ \\
\hline Carbon disulfide & WA & & & $\mathbf{U}$ & 5 & & NS & $\mu g / L$ \\
\hline Carbon tetrachloride & WA & & & $\mathbf{U}$ & 5 & & 5 & $\mu g / L$ \\
\hline Cesium-137 & TM & & & $\mathbf{U}$ & 6.3 & & 200 & $\mathrm{pCi} / \mathrm{L}$ \\
\hline Chloride & WA & & & & 5.65 & & NS & $\mathrm{mg} / \mathrm{L}$ \\
\hline Chlorobenzene & WA & & & $\mathbf{U}$ & 5 & & 100 & $\mu g / L$ \\
\hline Chloroethane & WA & & & $\mathbf{U}$ & 10 & & NS & $\mu \mathrm{g} / \mathrm{L}$ \\
\hline Chloroform & WA & & & $\mathbf{U}$ & 5 & & 100 & $\mu \mathrm{g} / \mathrm{L}$ \\
\hline Chloroprene & WA & & & $\mathbf{U}$ & 20 & & NS & $\mu \mathrm{g} / \mathrm{L}$ \\
\hline Chromium & WA & & & & 10.4 & & 100 & $\mu \mathrm{g} / \mathrm{L}$ \\
\hline cis-1,3-Dichloropropene & WA & & & $\mathbf{U}$ & 5 & & NS & $\mu g / L$ \\
\hline Cobalt & WA & & & U & 50 & & NS & $\mu g / L$ \\
\hline Copper & WA & & & U & 25 & & 1000 & $\mu \mathrm{g} / \mathrm{L}$ \\
\hline Dibromochloromethane & WA & & & $\mathbf{U}$ & 5 & & NS & $\mu g / L$ \\
\hline Dichlorodifluoromethane & WA & & & $\mathbf{U}$ & 10 & & NS & $\mu g / L$ \\
\hline Ethylbenzene & WA & & & $\mathbf{U}$ & 5 & & 700 & $\mu \mathrm{g} / \mathrm{L}$ \\
\hline Fluoride & WA & & & $\mathbf{U}$ & 0.1 & & 4 & $\mathrm{mg} / \mathrm{L}$ \\
\hline
\end{tabular}


UTR 122 Laboratory Analyses (cont'd)

\begin{tabular}{|c|c|c|c|c|c|c|c|c|c|}
\hline Analyte & Lab & Rep & Avg & Modifier & & Result & Acc & Stand & Units \\
\hline Gross alpha & TM & & & & & 1.4 & 1.2 & 15 & $\mathrm{pCi} / \mathrm{L}$ \\
\hline Iron & WA & & & & & 1780 & & 300 & $\mu g / L$ \\
\hline Isobutyl alcohol & WA & & & $\mathbf{U}$ & & 20 & & NS & $\mu g / L$ \\
\hline Lead & WA & & & $U$ & & 3 & & 50 & $\mu \mathrm{g} / \mathrm{L}$ \\
\hline Magnesium & WA & & & $\mathbf{U}$ & & 5000 & & NS̈ & $\mu \mathrm{g} / \mathrm{L}$ \\
\hline Manganese & WA & & & & & 143 & . & 50 & $\mu \mathrm{g} / \mathrm{L}$ \\
\hline Mercury & WA & & & $\mathbf{U}$ & & 0.2 & & 2 & $\mu \mathrm{g} / \mathrm{L}$ \\
\hline Methacrylonitrile & WA & & & $\mathbf{U}$ & & 20 & & NS & $\mu \mathrm{g} / \mathrm{L}$ \\
\hline Methyl bromide & WA & & & $\mathbf{U}$ & & 10 & & NS & $\mu \mathrm{g} / \mathrm{L}$ \\
\hline Methyl chloride & WA & & & $\mathbf{U}$ & & 10 & & NS & $\mu g / L$ \\
\hline Methyl ethyl ketone & WA & & & $\mathbf{U}$ & & 10 & & NS & $\mu \mathrm{g} / \mathrm{L}$ \\
\hline Methyl iodide & WA & & & $\mathbf{U}$ & & 10 & & NS & $\mu g / L$ \\
\hline Methylene bromide & WA & & & U & & 10 & & NS & $\mu g / L$ \\
\hline Methylene chloride & WA & & & $\mathbf{J}$ & V & 3.63 & & 5 & $\mu g / L$ \\
\hline Nickel & WA & & & $\mathbf{U}$ & & 40 & & 100 & $\mu g / L$ \\
\hline Nitrate as nitrogen & WA & & & J & $\mathbf{Q}$ & 0.024 & & 10 & $\mathrm{mg} / \mathrm{L}$ \\
\hline Nitrate as nitrogen & WA & & NA & J & $\mathrm{Q}$ & 0.032 & & 10 & $\mathrm{mg} / \mathrm{L}$ \\
\hline Nonvolatile beta & TM & & & $\mathbf{U}$ & & 0.8 & & 50 & $\mathrm{pCi} / \mathrm{L}$ \\
\hline Potassium & WA & & & $\mathbf{U}$ & & 5000 & & NS & $\mu g / L$ \\
\hline Propionitrile & WA & & & $\mathbf{U}$ & & 50 & & NS & $\mu g / L$ \\
\hline Selenium & WA & & & $\mathbf{U}$ & & 5 & & 50 & $\mu \mathrm{g} / \mathrm{L}$ \\
\hline Silver & WA & & & $\mathbf{U}$ & & 10 & & 50 & $\mu \mathrm{g} / \mathrm{L}$ \\
\hline Sodium & WA & & & $\mathbf{U}$ & & 5000 & & NS & $\mu \mathrm{g} / \mathrm{L}$ \\
\hline Styrene & WA & & & $U$ & & 5 & & 100 & $\mu g / L$ \\
\hline Suifate & WA & & & $\mathrm{U}$ & & 2.5 & & 400 & $\mathrm{mg} / \mathrm{L}$ \\
\hline Sulfide & WA & & & $\mathrm{U}$ & & 0.1 & & NS & $\mathrm{mg} / \mathrm{L}$ \\
\hline Tetrachloroethylene & WA & & & $\mathbf{U}$ & & 5 & & 5 & $\mu \mathrm{g} / \mathrm{L}$ \\
\hline Thallium & WA & & & $\mathrm{U}$ & & 10 & & 2 & $\mu \mathrm{g} / \mathrm{L}$ \\
\hline Toluene & WA & & & $\mathrm{U}$ & & 5 & & 1000 & $\mu \mathrm{g} / \mathrm{L}$ \\
\hline Total hardness & WA & & & & & 1.9 & & NS & $\mathrm{mg} / \mathrm{L}$ \\
\hline Total organic carbon & WA & & & & & 2.27 & & NS & $\mathrm{mg} / \mathrm{L}$ \\
\hline Total suspended solids & WA & & & & & 15 & & NS & $\mathrm{mg} / \mathrm{L}$ \\
\hline trans-1,3-Dichloropropene & WA & & & $\mathbf{U}$ & & 5 & & NS & $\mu \mathrm{g} / \mathrm{L}$ \\
\hline trans-1,4-Dichloro-2-butene & WA & & & $\mathbf{U}$ & & 100 & & NS & $\mu g / L$ \\
\hline Trichloroethylene & WA & & & $\mathrm{U}$ & & 5 & & 5 & $\mu \mathrm{g} / \mathrm{L}$ \\
\hline Trichlorofluoromethane & WA & & & $\mathbf{U}$ & & 5 & & NS & $\mu \mathrm{g} / \mathrm{L}$ \\
\hline Tritium & TM & & & & & 19.83 & 0.97 & 20 & $\mathrm{pCi} / \mathrm{ml}$ \\
\hline Turbidity & WA & & & $\mathrm{J}$ & $Q$ & 16.4 & & NS & NTU \\
\hline Vanadium & WA & & & $\mathrm{U}$ & & 50 & & NS & $\mu \mathrm{g} / \mathrm{L}$ \\
\hline Vinyl acetate & WA & & & $\mathrm{U}$ & & 10 & & NS & $\mu g / L$ \\
\hline Vinyl chloride & WA & & & $\mathrm{U}$ & & 10 & & 2 & $\mu \mathrm{g} / \mathrm{L}$ \\
\hline Xylenes (total) & WA & & 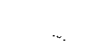 & $\mathrm{U}$ & & 5 & & 10000 & $\mu g / L$ \\
\hline Zinc & WA & & & & & 97.8 & & 5000 & $\mu g / L$ \\
\hline
\end{tabular}




\section{UTR127}

Field Measurements: Sample Date: 9/2/93 pH: 6.92

Specific Conductance: $59 \mu \mathrm{S} / \mathrm{cm}$

Red/Oxid Potential: - $180 \mathrm{mv}$

Dissolved Oxygen: $1.2 \mathrm{mg} / \mathrm{L}$

\begin{tabular}{|c|c|c|c|c|c|c|}
\hline Analyte & Lab & Rep & Modifier & Result & Stand & Units \\
\hline 1,1,1,2-Tetrachloroethane & $\overline{\mathbf{W A}}$ & & $\overline{\mathbf{U}}$ & 10 & NS & $\mu g / L$ \\
\hline $1,1,1,2$-Tetrachloroethane & WA & NA & $\mathbf{U}$ & 10 & NS & $\mu g / L$ \\
\hline $1,1,1,2$-Tetrachloroethane & WA & NA & $\mathbf{U}$ & 10 & NS & $\mu g / L$ \\
\hline 1,1,1-Trichloroethane & WA & & $\mathbf{U}$ & 5 & 200 & $\mu g / L$ \\
\hline 1,1,1-Trichloroethane & WA & NA & $\mathbf{U}$ & 5 & 200 & $\mu g / L$ \\
\hline 1,1,1-Trichloroethane & WA & NA & $\mathbf{U}$ & 5 & 200 & $\mu g / L$ \\
\hline $1,1,2,2$-Tetrachloroethane & WA & & $\mathbf{U}$ & 5 & NS & $\mu \mathrm{g} / \mathrm{L}$ \\
\hline 1,1,2,2-Tetrachloroethane & WA & NA & $\mathbf{U}$ & 5 & NS & $\mu g / L$ \\
\hline 1,1,2,2-Tetrachloroethane & WA & $\mathbf{N A}$ & $\mathbf{U}$ & 5 & NS & $\mu g / L$ \\
\hline 1,1,2-Trichloroethane & WA & & $\mathbf{U}$ & 5 & 5 & $\mu g / L$ \\
\hline 1,1,2-Trichloroethane & WA & NA & $\mathbf{U}$ & 5 & 5 & $\mu \mathrm{g} / \mathrm{L}$ \\
\hline 1,1,2-Trichloroethane & WA & NA & $\mathbf{U}$ & 5 & 5 & $\mu g / L$ \\
\hline 1,1-Dichloroethane & WA & & $\mathbf{U}$ & 5 & NS & $\mu g / L$ \\
\hline 1,1-Dichloroethane & WA & NA & $\mathbf{U}$ & 5 & NS & $\mu g / L$ \\
\hline 1,1-Dichloroethane & WA & NA & $\mathbf{U}$ & 5 & NS & $\mu \mathrm{g} / \mathrm{L}$ \\
\hline 1,1-Dichloroethylene & WA & & $\mathbf{U}$ & 5 & 7 & $\mu \mathrm{g} / \mathrm{L}$ \\
\hline 1,2,3-Trichloropropane & WA & & $\mathbf{U}$ & 10 & NS & $\mu g / L$ \\
\hline 1,2,3-Trichloropropane & WA & NA & $\mathbf{U}$ & 10 & NS & $\mu \mathrm{g} / \mathrm{L}$ \\
\hline 1,2,3-Trichloropropane & WA & NA & $\mathrm{U}$ & 10 & NS & $\mu \mathrm{g} / \mathrm{L}$ \\
\hline 1,2-Dibromo-3-chloropropane & WA & & $\mathbf{U}$ & 20 & 0.2 & $\mu g / L$ \\
\hline 1,2-Dibromo-3-chloropropane & WA & NA & $\mathbf{U}$ & 20 & 0.2 & $\mu \mathrm{g} / \mathrm{L}$ \\
\hline 1,2-Dibromo-3-chloropropane & WA & NA & $\mathbf{U}$ & 20 & 0.2 & $\mu g / L$ \\
\hline 1,2-Dibromoethane & WA & & $\mathbf{U}$ & 20 & NS & $\mu \mathrm{g} / \mathrm{L}$ \\
\hline 1,2-Dibromoethane & WA & NA & $\mathbf{U}$ & 20 & NS & $\mu g / L$ \\
\hline 1,2-Dibromoethane & WA & NA & $\mathbf{U}$ & 20 & NS & $\mu \mathrm{g} / \mathrm{L}$ \\
\hline 1,2-Dichloroethane & WA & & $\mathbf{U}$ & 5 & 5 & $\mu g / L$ \\
\hline 1,2-Dichloroethane & WA & NA & $\mathbf{U}$ & 5 & 5 & $\mu g / L$ \\
\hline 1,2-Dichloroethane & WA & NA & $\mathbf{U}$ & 5 & 5 & $\mu \mathrm{g} / \mathrm{L}$ \\
\hline 1,2-Dichloroethylene (total) & WA & & $\mathrm{U}$ & 5 & 7 & $\mu g / L$ \\
\hline 1,2-Dichloroethylene (total) & WA & NA & U & 5 & 7 & $\mu g / L$ \\
\hline 1,2-Dichloroethylene (total) & WA & NA & $\mathbf{U}$ & 5 & 7 & $\mu g / L$ \\
\hline 1,2-Dichloropropane & WA & & $\mathbf{U}$ & 5 & 5 & $\mu \mathrm{g} / \mathrm{L}$ \\
\hline 1,2-Dichloropropane & WA & NA & $\mathrm{U}$ & 5 & 5 & $\mu \mathrm{g} / \mathrm{L}$ \\
\hline 1,2-Dichloropropane & WA & NA & $\mathbf{U}$ & 5 & 5 & $\mu \mathrm{g} / \mathrm{L}$ \\
\hline 2-Hexanone & WA & & $\mathbf{U}$ & 10 & NS & $\mu g / L$ \\
\hline 2-Hexanone & WA & NA & $\mathbf{U}$ & 10 & NS & $\mu \mathrm{g} / \mathrm{L}$ \\
\hline 2-Hexanone & WA & NA & $\mathbf{U}$ & 10 & NS & $\mu \mathrm{g} / \mathrm{L}$ \\
\hline 4-Methyl-2-pentanone & WA & & $\mathbf{U}$ & 10 & NS & $\mu g / L$ \\
\hline 4-Methyl-2-pentanone & WA & NA & $\mathbf{U}$ & 10 & NS & $\mu \mathrm{g} / \mathrm{L}$ \\
\hline 4-Methyl-2-pentanone & WA & NA & $\mathbf{U}$ & 10 & NS & $\mu g / L$ \\
\hline Acetone & WA & & $\mathbf{J}$ & 5.76 & NS & $\mu \mathrm{g} / \mathrm{L}$ \\
\hline Acetone & WA & NA & $\mathbf{J}$ & 3.89 & NS & $\mu \mathrm{g} / \mathrm{L}$ \\
\hline Acetone & WA & NA & $\mathbf{J}$ & 4.2 & NS & $\mu \mathrm{g} / \mathrm{L}$ \\
\hline Acetonitrile & WA & & $\mathbf{U}$ & 20 & NS & $\mu \mathrm{g} / \mathrm{L}$ \\
\hline Acetonitrile & WA & NA & $\mathbf{U}$ & 20 & NS & $\mu g / L$ \\
\hline Acetonitrile & WA & NA & $\mathbf{U}$ & 20 & NS & $\mu g / L$ \\
\hline Acidity & WA & & & 58 & NS & $\mathrm{mg} / \mathrm{L}$ \\
\hline
\end{tabular}


UTR 127 Laboratory Analyses (cont'd)

\begin{tabular}{|c|c|c|c|c|c|c|c|c|}
\hline Analyte & Lab & Rep & Avg & Modifier & Result & Acc & Stand & Units \\
\hline Acrolein & $\overline{\text { WA }}$ & & & $\bar{U}$ & 10 & & NS & $\mu g / L$ \\
\hline Acrolein & WA & & NA & $\mathbf{U}$ & 10 & & NS & $\mu_{g} / \mathrm{L}$ \\
\hline Acrolein & WA & & NA & U & 10 & & NS & $\mu g / L$ \\
\hline Acrylonitrile & WA & & & $\mathbf{U}$ & 10 & & NS & $\mu g / L$ \\
\hline Acrylonitrile & WA & & NA & U & 10 & & NŚ & $\mu \mathrm{g} / \mathrm{L}$ \\
\hline Acrylonitrile & WA & & NA & U & 10 & . & NS & $\mu_{g} / L$ \\
\hline Alkalinity & WA & & & & 1 & & NS & $\mathrm{mg} / \mathrm{L}$ \\
\hline Allyl chloride & WA & & & $\mathbf{U}$ & 100 & & NS & $\mu \mathrm{g} / \mathrm{L}$ \\
\hline Allyl chloride & WA & & NA & $\mathbf{U}$ & 100 & & NS & $\mu g / \Omega$ \\
\hline Allyl chloride & WA & & NA & $\mathbf{U}$ & 100 & & NS & $\mu g / L$ \\
\hline Aluminum & WA & & & $\mathbf{U}$ & 200 & & NS & $\mu g / L$ \\
\hline Antimony & WA & & & $\mathbf{U}$ & 60 & & 6 & $\mu g / L$ \\
\hline Arsenic & WA & & & $\mathbf{U}$ & 10 & & 50 & $\mu g / L$ \\
\hline Barium & WA & & & $\mathbf{U}$ & 200 & & 2000 & $\mu \mathrm{g} / \mathrm{L}$ \\
\hline Benzene & WA & & & $\mathbf{U}$ & 5 & & 5 & $\mu g / L$ \\
\hline Beryllium & WA & & & $\mathbf{U}$ & 5 & & 4 & $\mu g / L$ \\
\hline Bromodichloromethane & WA & & & $\mathbf{U}$ & 5 & & NS & $\mu g / L$ \\
\hline Bromodichloromethane & WA & & NA & $\mathbf{U}$ & 5 & & NS & $\mu \mathrm{g} / \mathrm{L}$ \\
\hline Bromodichloromethane & WA & & NA & $\mathbf{U}$ & 5 & & NS & $\mu \mathrm{g} / \mathrm{L}$ \\
\hline Bromoform & WA & & & $\mathbf{U}$ & 5 & & NS & $\mu g / L$ \\
\hline Bromoform & WA & & NA & $\mathbf{U}$ & 5 & & NS & $\mu \mathrm{g} / \mathrm{L}$ \\
\hline Bromoform & WA & & NA & U & 5 & & NS & $\mu \mathrm{g} / \mathrm{L}$ \\
\hline Cadmium & WA & & & $\mathbf{U}$ & 5 & & 5 & $\mu g / L$ \\
\hline Calcium & WA & & & $\mathbf{U}$ & 5000 & & NS & $\mu \mathrm{g} / \mathrm{L}$ \\
\hline Carbon disulfide & WA & & & U & 5 & & NS & $\mu \mathrm{g} / \mathrm{L}$ \\
\hline Carbon disulfide & WA & & NA & $\mathbf{U}$ & 5 & & NS & $\mu \mathrm{g} / \mathrm{L}$ \\
\hline Carbon disulfide & WA & & NA & $\mathbf{U}$ & 5 & & NS & $\mu \mathrm{g} / \mathrm{L}$ \\
\hline Carbon tetrachloride & WA & & & $\mathbf{U}$ & 5 & & 5 & $\mu \mathrm{g} / \mathrm{L}$ \\
\hline Carbon tetrachloride & WA & & NA & $\mathbf{U}$ & 5 & & 5 & $\mu g / L$ \\
\hline Carbon tetrachloride & WA & & NA & U & 5 & & 5 & $\mu g / L$ \\
\hline Cesium-137 & TM & & & $\mathbf{U}$ & 6.25 & & 200 & $\mathrm{pCi} / \mathrm{L}$ \\
\hline Chloride & WA & & & & 5.63 & & NS & $\mathrm{mg} / \mathrm{L}$ \\
\hline Chlorobenzene & WA & & & $\mathbf{U}$ & 5 & & 100 & $\mu \mathrm{g} / \mathrm{L}$ \\
\hline Chloroethane & WA & & & $\mathbf{U}$ & 10 & & NS & $\mu \mathrm{g} / \mathrm{L}$ \\
\hline Chloroethane & WA & & NA & $\mathrm{U}$ & 10 & & NS & $\mu \mathrm{g} / \mathrm{L}$ \\
\hline Chloroethane & WA & & NA & $\mathbf{U}$ & 10 & & NS & $\mu g / L$ \\
\hline Chloroform & WA & & & $\mathbf{U}$ & 5 & & 100 & $\mu \mathrm{g} / \mathrm{L}$ \\
\hline Chloroform & WA & & NA & $\mathbf{U}$ & 5 & & 100 & $\mu \mathrm{g} / \mathrm{L}$ \\
\hline Chloroform & WA & & NA & U & 5 & & 100 & $\mu g / L$ \\
\hline Chloroprene & WA & & & $\mathbf{U}$ & 20 & & NS & $\mu g / L$ \\
\hline Chloroprene & WA & & NA & $\mathbf{U}$ & 20 & & NS & $\mu g / L$ \\
\hline Chloroprene & WA & & NA & $\mathrm{U}$ & 20 & & NS & $\mu \mathrm{g} / \mathrm{L}$ \\
\hline Chromium & WA & & & $\mathrm{U}$ & 10 & & 100 & $\mu \mathrm{g} / \mathrm{L}$ \\
\hline cis-1,3-Dichloropropene & WA & & & $\mathbf{U}$ & 5 & & NS & $\mu g / L$ \\
\hline cis-1,3-Dichloropropene & WA & & NA & $\mathrm{U}$ & 5 & & NS & $\mu g / L$ \\
\hline cis-1,3-Dichloropropene & WA & & NA & $\mathrm{U}$ & 5 & & NS & $\mu g / L$ \\
\hline Cobalt & WA & & & $\mathbf{U}$ & 50 & & NS & $\mu g / L$ \\
\hline Copper & WA & & & $\mathrm{U}$ & 25 & & 1000 & $\mu g / L$ \\
\hline Dibromochloromethane & WA & & & $\mathbf{U}$ & 5 & & NS & $\mu \mathrm{g} / \mathrm{L}$ \\
\hline Dibronıochloromethane & WA & & NA & $\mathbf{U}$ & 5 & & NS & $\mu g / L$ \\
\hline Dibromochloromethane & WA & & NA & $\mathbf{U}$ & 5 & & NS & $\mu g / L$ \\
\hline Dichlorodifluoromethane & WA & & & $\mathbf{U}$ & 10 & & NS & $\mu g / L$ \\
\hline Dichlorodifluoromethane & WA & & NA & $\mathbf{U}$ & 10 & & NS & $\mu g / L$ \\
\hline Dichlorodifluoromethane & WA & & NA & $\mathbf{U}$ & 10 & & NS & $\mu \mathrm{g} / \mathrm{L}$ \\
\hline
\end{tabular}


UTR 127 Laboratory Analyses (cont'd)

\begin{tabular}{|c|c|c|c|c|c|c|c|c|c|}
\hline Analyte & Lab & Rep & Avg & Modifier & & Result & Acc & Stand & Units \\
\hline Ethylbenzene & WA & & & $\mathbf{U}$ & & 5 & & 700 & $\overline{\mu g / L}$ \\
\hline Ethylbenzene & WA & & NA & $\mathbf{U}$ & & 5 & & 700 & $\mu_{\varepsilon} / L$ \\
\hline Ethylbenzene & WA & & NA & $\mathbf{U}$ & & 5 & & 700 & $\mu g / L$ \\
\hline Fluoride & WA & & & $\mathbf{U}$ & & 0.1 & & 4 & $\mathrm{mg} / \mathrm{L}$ \\
\hline Gross alpha & TM & & & & & 0.8 & 1 & 15 & $\mathrm{pCi} / \mathrm{L}$ \\
\hline Iron & WA & & & & & 1030 & & 300 & $\mu g / L$ \\
\hline Isobutyl alcohol & WA & & & $\mathbf{U}$ & & 20 & & NS & $\mu g / L$ \\
\hline Isobutyl alcohol & WA & & NA & $\mathbf{U}$ & & 20 & & NS & $\mu g / L$ \\
\hline Isobutyl alcohol & WA & & NA & $\mathbf{U}$ & & 20 & & NS & $\mu g / L$ \\
\hline Lead & WA & & & $\mathbf{U}$ & & 3 & & 50 & $\mu g / L$ \\
\hline Magnesium & WA & & & $\mathbf{U}$ & & 5000 & & NS & $\mu g / L$ \\
\hline Manganese & WA & & & & & 60.7 & & 50 & $\mu g / L$ \\
\hline Mercury & WA & & & $\mathbf{U}$ & & 0.2 & & 2 & $\mu g / L$ \\
\hline Methacrylonitrile & WA & & & $\mathbf{U}$ & & 20 & & NS & $\mu g / L$ \\
\hline Methacrylonitrile & WA & & NA & $\mathbf{U}$ & & 20 & & NS & $\mu g / L$ \\
\hline Methacrylonitrile & WA & & NA & $\mathbf{U}$ & & 20 & & NS & $\mu g / L$ \\
\hline Methyl bromide & WA & & & $\mathbf{U}$ & & 10 & & NS & $\mu g / L$ \\
\hline Methyl bromide & WA & & NA & $\mathbf{U}$ & & 10 & & NS & $\mu g / L$ \\
\hline Methyl bromide & WA & & NA & $\mathbf{U}$ & & 10 & & NS & $\mu g / L$ \\
\hline Methyl chloride & WA & & & $\mathbf{U}$ & & 10 & & NS & $\mu g / L$ \\
\hline Methyl chloride & WA & & NA & $\mathbf{U}$ & & 10 & & NS & $\mu g / L$ \\
\hline Methyl chloride & WA & & $\mathbf{N A}$ & $\mathbf{U}$ & & 10 & & NS & $\mu g / L$ \\
\hline Methyl ethyl ketone & WA & & & $\mathbf{U}$ & & 10 & & NS & $\mu g / L$ \\
\hline Methyl ethyl ketone & WA & & NA & $\mathbf{U}$ & & 10 & & NS & $\mu g / L$ \\
\hline Methyl ethyl ketone & WA & & NA & $\mathbf{U}$ & & 10 & & NS & $\mu g / L$ \\
\hline Methyl iodide & WA & & & $\mathbf{U}$ & & 10 & & NS & $\mu g / L$ \\
\hline Methyl iodide & WA & & NA & $\mathbf{U}$ & & 10 & & NS & $\mu \mathrm{g} / \mathrm{L}$ \\
\hline Methyl iodide & WA & & NA & $\mathbf{U}$ & & 10 & & NS & $\mu g / L$ \\
\hline Methylene bromide & WA & & & $\mathbf{U}$ & & 10 & & NS & $\mu g / L$ \\
\hline Methylene bromide & WA & & NA & $\mathbf{U}$ & & 10 & & NS & $\mu g / L$ \\
\hline Methylene bromide & WA & & NA & $\mathbf{U}$ & & 10 & & NS & $\mu g / L$ \\
\hline Methylene chloride & WA & & & $\mathbf{U}$ & & 5 & & 5 & $\mu \mathrm{g} / \mathrm{L}$ \\
\hline Methylene chloride & WA & & NA & $\mathbf{U}$ & & 5 & & 5 & $\mu g / L$ \\
\hline Methylene chloride & WA & & NA & $\mathbf{U}$ & & 5 & & 5 & $\mu g / L$ \\
\hline Nickel & WA & & & $\boldsymbol{U}$ & & 40 & & 100 & $\mu \mathrm{g} / \mathrm{L}$ \\
\hline Nitrate as nitrogen & WA & & & J & $\mathbf{Q}$ & 0.043 & & 10 & $\mathrm{mg} / \mathrm{L}$ \\
\hline Nonvolatile beta & TM & & & & & 1.9 & 2.2 & 50 & $\mathrm{pCi} / \mathrm{L}$ \\
\hline Potassium & WA & & & $\mathbf{U}$ & & 5000 & & NS & $\mu g / L$ \\
\hline Propionitrile & WA & & & $\mathbf{U}$ & & 50 & & NS & $\mu g / L$ \\
\hline Propionitrile & WA & & NA & $\mathbf{U}$ & & 50 & & NS & $\mu g / L$ \\
\hline Propionitrile & WA & & NA & $\mathbf{U}$ & & 50 & & NS & $\mu \mathrm{g} / \mathrm{L}$ \\
\hline Selenium & WA & & & $\mathbf{U}$ & & 5 & & 50 & $\mu g / L$ \\
\hline Silver & WA & & & $\mathbf{U}$ & & 10 & & 50 & $\mu \mathrm{g} / \mathrm{L}$ \\
\hline Sodium & WA & & & & & 5230 & & NS & $\mu \mathrm{g} / \mathrm{L}$ \\
\hline Styrene & WA & & & $\mathbf{U}$ & & 5 & & 100 & $\mu \mathrm{g} / \mathrm{L}$ \\
\hline Styrene & WA & & NA & $\mathbf{U}$ & & 5 & & 100 & $\mu \mathrm{g} / \mathrm{L}$ \\
\hline Styrene & WA & & NA & $\mathbf{U}$ & & 5 & & 100 & $\mu \mathrm{g} / \mathrm{L}$ \\
\hline Sulfate & WA & & & & & 3.28 & & 400 & $\mathrm{mg} / \mathrm{L}$ \\
\hline Sulfide & WA & & & $\mathbf{U}$ & & 0.1 & & NS & $\mathrm{mg} / \mathrm{L}$ \\
\hline Tetrachloroethylene & WA & & & $\mathbf{U}$ & & 5 & & 5 & $\mu g / L$ \\
\hline Tetrachloroethylene & WA & & NA & $\mathbf{U}$ & & 5 & & 5 & $\mu \mathrm{g} / \mathrm{L}$ \\
\hline Tetrachloroethylene & WA & & NA & $\mathbf{U}$ & & 5 & & 5 & $\mu \mathrm{g} / \mathrm{L}$ \\
\hline Thallium & WA & & & $\mathbf{U}$ & & 10 & & 2 & $\mu \mathrm{g} / \mathrm{L}$ \\
\hline Toluene & WA & & & $\mathbf{J}$ & & 1.31 & & 1000 & $\mu \mathrm{g} / \mathrm{L}$ \\
\hline Total hardness & WA & & & & & 9.7 & & NS & $\mathrm{mg} / \mathrm{L}$ \\
\hline
\end{tabular}


UTR127 Laboratory Analyses (cont'd)

\begin{tabular}{|c|c|c|c|c|c|c|c|c|c|}
\hline Analyte & Lab & Rep & Avg & Modifier & & Result & Acc & Stand & Units \\
\hline Total organic carbon & WA & & & & & 3.58 & & NS & $\mathrm{mg} / \mathrm{L}$ \\
\hline Total suspended solids & WA & & & & & 5 & & NS & $\mathrm{mg} / \mathrm{L}$ \\
\hline Total suspended solids & WA & & NA & $\mathbf{U}$ & & 5 & & NS & $\mathrm{mg} / \mathrm{L}$ \\
\hline trans-1,3-Dichloropropene & WA & & & $\mathbf{U}$ & & 5 & & NS & $\mu \mathrm{g} / \mathrm{L}$ \\
\hline trans-1,3-Dichloropropene & WA & & NA & $\mathbf{U}$ & & 5 & & NS & $\mu \mathrm{g} / \mathrm{L}$ \\
\hline trans-1,3-Dichloropropene & WA & & NA & $\mathbf{U}$ & & 5 & . & NS & $\mu g / L$ \\
\hline trans-1,4-Dichloro-2-butene & WA & & & $\mathbf{U}$ & & 100 & & NS & $\mu g / L$ \\
\hline trans-1,4-Dichloro-2-butene & WA & & NA & $\mathbf{U}$ & & 100 & & NS & $\mu g / L$ \\
\hline trans-1,4-Dichloro-2-butene & WA & & NA & $\mathbf{U}$ & & 100 & & NS & $\mu g / L$ \\
\hline Trichloroethylene & WA & & & $\mathbf{U}$ & & 5 & & 5 & $\mu \mathrm{g} / \mathrm{L}$ \\
\hline Trichlorofluoromethane & WA & & & $\mathbf{U}$ & & 5 & & NS & $\mu g / L$ \\
\hline Trichlorofluoromethane & WA & & NA & $\mathbf{U}$ & & 5 & & NS & $\mu g / L$ \\
\hline Trichlorofluoromethane & WA & & NA & $\mathbf{U}$ & & 5 & & NS & $\mu g / L$ \\
\hline Tritium & TM & & & & & 8.45 & 0.65 & 20 & $\mathrm{pCi} / \mathrm{ml}$ \\
\hline Turbidity & WA & & & J & $\mathbf{Q}$ & 1.06 & & NS & NTU \\
\hline Vanadium & WA & & & $\mathbf{U}$ & & 50 & & NS & $\mu g / L$ \\
\hline Vinyl acetate & WA & & & $\mathbf{U}$ & & 10 & & NS & $\mu g / L$ \\
\hline Vinyl acetate & WA & & $\mathbf{N A}$ & $\mathbf{U}$ & & 10 & & NS & $\mu g / L$ \\
\hline Vinyl acetate & WA & & NA & $\mathbf{U}$ & & 10 & & NS & $\mu g /$ \\
\hline Vinyl chloride & WA & & & $\mathbf{U}$ & & 10 & & 2 & $\mu g / L$ \\
\hline Vinyl chloride & WA & & $\mathbf{N A}$ & $\mathbf{U}$ & & 10 & & 2 & $\mu g / L$ \\
\hline Vinyl chloride & WA & & NA & $\mathbf{U}$ & & 10 & & 2 & $\mu \mathrm{g} / \mathrm{L}$ \\
\hline Xylenes (total) & WA & & & $\mathbf{U}$ & & 5 & & 10000 & $\mu g / L$ \\
\hline Xylenes (total) & WA & & NA & $\mathbf{U}$ & & 5 & & 10000 & $\mu \mathrm{g} / \mathrm{L}$ \\
\hline Xylenes (total) & WA & & NA & $\mathbf{U}$ & & 5 & & 10000 & $\mu \mathrm{g} / \mathrm{L}$ \\
\hline Zinc & WA & & & $\mathbf{U}$ & & 20 & & 5000 & $\mu g / L$ \\
\hline
\end{tabular}




\section{UTR133}

Field Measurements:

Sample Date: 9/2/93

$\mathrm{pH}: 6.72$

Specific Conductance: $115 \mu \mathrm{S} / \mathrm{cm}$

Red/Oxid Potential: $39 \mathrm{mv}$

Dissolved Oxygen: $1.1 \mathrm{mg} / \mathrm{L}$

\begin{tabular}{|c|c|c|c|c|c|c|c|}
\hline Analyte & Lab & Avg & Modifier & Result & Acc & Stand & Units \\
\hline 1,1,1,2-Tetrachloroethane & WA & & $\mathbf{U}$ & 10 & & NS & $\mu g / L$ \\
\hline 1,1,1-Trichloroethane & WA & & $\mathbf{U}$ & 5 & & 200 & $\mu g / L$ \\
\hline $1,1,2,2$-Tetrachloroethane & WA & & $\mathbf{U}$ & 5 & & NS & $\mu g / L$ \\
\hline 1,1,2-Trichloroethane & WA & & $\mathbf{U}$ & 5 & & 5 & $\mu g / L$ \\
\hline 1,1-Dichloroethane & WA & & $\mathbf{U}$ & 5 & & NS & $\mu g / L$ \\
\hline 1,1-Dichloroethylene & WA & & $\mathbf{U}$ & 5 & & 7 & $\mu g / L$ \\
\hline 1,2,3-Trichloropropane & WA & & $\mathbf{U}$ & 10 & & NS & $\mu g / L$ \\
\hline 1,2-Dibromo-3-chloropropane & WA & & $\mathbf{U}$ & 20 & & 0.2 & $\mu g / L$ \\
\hline 1,2-Dibromoethane & WA & & $\mathbf{U}$ & 20 & & NS & $\mu g / L$ \\
\hline 1,2-Dichloroethane & WA & & $\mathbf{U}$ & 5 & & 5 & $\mu g / L$ \\
\hline 1,2-Dichloroethylene (total) & WA & & $\mathbf{U}$ & 5 & & 7 & $\mu g / L$ \\
\hline 1,2-Dichloropropane & WA & & $\mathbf{U}$ & 5 & & 5 & $\mu g / L$ \\
\hline 2-Hexanone & WA & & $\mathbf{U}$ & 10 & & NS & $\mu g / L$ \\
\hline 4-Methyl-2-pentanone & WA & & $\mathbf{U}$ & 10 & & NS & $\mu g / L$ \\
\hline Acetone & WA & & & 19.6 & & NS & $\mu g / L$ \\
\hline Acetonitrile & WA & & $\mathbf{U}$ & 20 & & NS & $\mu g / L$ \\
\hline Acidity & WA & & & 102 & & NS & $\mathrm{mg} / \mathrm{L}$ \\
\hline Acrolein & WA & & $\mathbf{U}$ & 10 & & NS & $\mu \mathrm{g} / \mathrm{L}$ \\
\hline Acrylonitrile & WA & & $\mathbf{U}$ & 10 & & NS & $\mu g / L$ \\
\hline Alkalinity & WA & & & 7.5 & & NS & $\mathrm{mg} / \mathrm{L}$ \\
\hline Allyl chloride & WA & & $\mathbf{U}$ & 100 & & NS & $\mu \mathrm{g} / \mathrm{L}$ \\
\hline Aluminum & WA & & $\mathbf{U}$ & 200 & & NS & $\mu g / L$ \\
\hline Antimony & WA & & $\mathbf{U}$ & 60 & & 6 & $\mu g / L$ \\
\hline Arsenic & WA & & $\mathbf{U}$ & 10 & & 50 & $\mu g / L$ \\
\hline Barium & WA & & $\mathbf{U}$ & 200 & & 2000 & $\mu g / L$ \\
\hline Benzene & WA & & $\mathbf{U}$ & 5 & & 5 & $\mu g / L$ \\
\hline Beryllium & WA & & $\mathbf{U}$ & 5 & & 4 & $\mu g / L$ \\
\hline Bromodichloromethane & WA & & $\mathbf{U}$ & 5 & & NS & $\mu g / L$ \\
\hline Bromoform & WA & & $\mathbf{U}$ & 5 & & NS & $\mu g / L$ \\
\hline Cadmium & WA & & $\mathbf{U}$ & 5 & & 5 & $\mu \mathrm{g} / \mathrm{L}$ \\
\hline Calcium & WA & & $\mathbf{U}$ & 5000 & & NS & $\mu g / L$ \\
\hline Carbon disulfide & WA & & $\mathbf{U}$ & 5 & & NS & $\mu g / L$ \\
\hline Carbon tetrachloride & WA & & $\mathbf{U}$ & 5 & & 5 & $\mu \mathrm{g} / \mathrm{L}$ \\
\hline Cesium-137 & $T M$ & & $\mathbf{U}$ & 5.79 & & 200 & $\mathrm{pCi} / \mathrm{L}$ \\
\hline Chloride & WA & & & 8.44 & & NS & $\mathrm{mg} / \mathrm{L}$ \\
\hline Chlorobenzene & WA & & U & 5 & & 100 & $\mu \mathrm{g} / \mathrm{L}$ \\
\hline Chloroethane & WA & & $\mathbf{U}$ & 10 & & NS & $\mu \mathrm{g} / \mathrm{L}$ \\
\hline Chloroform & WA & & $\mathbf{U}$ & 5 & & 100 & $\mu \mathrm{g} / \mathrm{L}$ \\
\hline Chloroprene & WA & & $\mathbf{U}$ & 20 & & NS & $\mu \mathrm{g} / \mathrm{L}$ \\
\hline Chromium & WA & & & 51.4 & & 100 & $\mu \mathrm{g} / \mathrm{L}$ \\
\hline cis-1,3-Dichloropropene & WA & & $\mathrm{U}$ & 5 & & NS & $\mu \mathrm{g} / \mathrm{L}$ \\
\hline Cobalt & WA & & $\mathrm{U}$ & 50 & & NS & $\mu \mathrm{g} / \mathrm{L}$ \\
\hline Copper & WA & & $\mathbf{U}$ & 25 & & 1000 & $\mu g / L$ \\
\hline Dibromochloromethane & WA & & $\mathbf{U}$ & 5 & & NS & $\mu \mathrm{g} / \mathrm{L}$ \\
\hline Dichlorodifluoromethane & WA & & $\mathbf{U}$ & 10 & & NS & $\mu \mathrm{g} / \mathrm{L}$ \\
\hline Ethylbenzene & WA & & U & 5 & & 700 & $\mu g / L$ \\
\hline Fluoride & WA & & $\mathbf{U}$ & 0.1 & & 4 & $\mathrm{mg} / \mathrm{L}$ \\
\hline
\end{tabular}


UTR 133 Laboratory Analyses (cont'd)

\begin{tabular}{|c|c|c|c|c|c|c|c|c|}
\hline Analyte & Lab & Avg & Modifier & & Result & Acc & Stand & Units \\
\hline Gross alpha & TM & & & & 0.8 & 1 & 15 & $\mathrm{pCi} / \mathrm{L}$ \\
\hline Iron & WA & & & & 1360 & & 300 & $\mu \mathrm{g} / \mathrm{L}$ \\
\hline Isobutyl alcohol & WA & & $\mathbf{U}$ & & 20 & & NS & $\mu g / L$ \\
\hline Lead & WA & & $\mathbf{U}$ & & 3 & & 50 & $\mu g / L$ \\
\hline Magnesium & WA & & $\mathbf{U}$ & & 5000 & & NS & $\mu g / L$ \\
\hline Manganese & WA & & & & 24.9 & . & 50 & $\mu g / L$ \\
\hline Mercury & WA & & $\mathbf{U}$ & & 0.2 & & 2 & $\mu \mathrm{g} / \mathrm{L}$ \\
\hline Methacrylonitrile & WA & & $\mathbf{U}$ & & 20 & & NS & $\mu \mathrm{g} / \mathrm{L}$ \\
\hline Methyl bromide & WA & & $\mathbf{U}$ & & 10 & & NS & $\mu g / L$ \\
\hline Methyl chloride & WA & & $\mathbf{U}$ & & 10 & & NS & $\mu g / L$ \\
\hline Methyl ethyl ketone & WA & & $\mathbf{U}$ & & 10 & & NS & $\mu g / L$ \\
\hline Methyl iodide & WA & & $\mathbf{U}$ & & 10 & & NS & $\mu g / L$. \\
\hline Methylene bromide & WA & & $\mathbf{U}$ & & 10 & & NS & $\mu \mathrm{g} / \mathrm{L}$ \\
\hline Methylene chloride & WA & & J & $\mathbf{V}$ & 3.86 & & 5 & $\mu g / L$ \\
\hline Nickel & WA & & $\mathbf{U}$ & & 40 & & 100 & $\mu g / L$ \\
\hline Nitrate as nitrogen & WA & & $\mathbf{J}$ & $\mathbf{Q}$ & 0.052 & & 10 & $\mathrm{mg} / \mathrm{L}$ \\
\hline Nonvolatile beta & TM & & $\mathbf{U}$ & & 0.8 & & 50 & $\mathrm{pCi} / L$ \\
\hline Potassium & WA & & $\mathbf{U}$ & & 5000 & & NS & $\mu g / L$ \\
\hline Propionitrile & WA & & $\mathbf{U}$ & & 50 & & NS & $\mu g / L$ \\
\hline Selenium & WA & & $\mathbf{U}$ & & 5 & & 50 & $\mu g / L$ \\
\hline Silver & WA & & $\mathbf{U}$ & & 10 & & 50 & $\mu g / L$ \\
\hline Sodium & WA & & & & 13500 & & NS & $\mu \mathrm{g} / \mathrm{L}$ \\
\hline Styrene & WA & & $\mathbf{U}$ & & 5 & & 100 & $\mu g / L$ \\
\hline Sulfate & WA & & & & 8.45 & & 400 & $\mathrm{mg} / \mathrm{L}$ \\
\hline Sulfide & WA & & $\mathbf{U}$ & & 0.1 & & NS & $\mathrm{mg} / \mathrm{L}$ \\
\hline Tetrachloroethylene & WA & & $\mathbf{U}$ & & 5 & & 5 & $\mu g / L$ \\
\hline Thallium & WA & & $\mathbf{U}$ & & 10 & & 2 & $\mu g / L$ \\
\hline Toluene & WA & & J & & 3.79 & & 1000 & $\mu g / L$ \\
\hline Total hardness & WA & & & & 2.9 & & NS & $\mathrm{mg} / \mathrm{L}$ \\
\hline Total organic carbon & WA & & & & 3.23 & & NS & $\mathrm{mg} / \mathrm{L}$ \\
\hline Total suspended solids & WA & & & & 13 & & NS & $\mathrm{mg} / \mathrm{L}$ \\
\hline trans-1,3-Dichloropropene & WA & & $\mathbf{U}$ & & 5 & & NS & $\mu g / L$ \\
\hline trans-1,4-Dichloro-2-butene & WA & & $\mathbf{U}$ & & 100 & & NS & $\mu g / L$ \\
\hline Trichloroethylene & WA & & $\mathbf{U}$ & & 5 & & 5 & $\mu \mathrm{g} / \mathrm{L}$ \\
\hline Trichlorofluoromethane & WA & & $\mathbf{U}$ & & 5 & & NS & $\mu \mathrm{g} / \mathrm{L}$ \\
\hline Tritium & TM & & & & 4.59 & 0.52 & 20 & $\mathrm{pCi} / \mathrm{ml}$ \\
\hline Turbidity & WA & & J & $Q$ & 2.55 & & NS & NTU \\
\hline Vanadium & WA & & $\mathbf{U}$ & & 50 & & NS & $\mu \mathrm{g} / \mathrm{L}$ \\
\hline Vinyl acetate & WA & & $\mathbf{U}$ & & 10 & & NS & $\mu \mathrm{g} / \mathrm{L}$ \\
\hline Vinyl chloride & WA & & $\mathbf{U}$ & & 10 & & 2 & $\mu g / L$ \\
\hline Xylenes (total) & WA & & $\mathbf{U}$ & & 5 & & 10000 & $\mu g / L$ \\
\hline Zinc & WA & & $U$ & & 20 & & 5000 & $\mu \mathrm{g} / \mathrm{L}$ \\
\hline
\end{tabular}




\section{UTR145}

Field Measurements:

Sample Date: 8/31/93

pH: 7.72

Specific Conductance: $19 \mu \mathrm{S} / \mathrm{cm}$

Red/Oxid Potential: $183 \mathrm{mv}$

Dissolved Oxygen: $5.5 \mathrm{mg} / \mathrm{L}$

\begin{tabular}{|c|c|c|c|c|c|c|c|c|}
\hline Analyte & Lab & Rep & Avg & Modifier & Result & Acc & Stand & Units \\
\hline 1,1,1,2-Tetrachloroethane & WA & & & $\mathbf{U}$ & 10 & & NS & $\mu g / L$ \\
\hline 1,1,1-Trichloroethane & WA & & & $\mathbf{U}$ & 5 & & 200 & $\mu g / L$ \\
\hline 1,1,2,2-Tetrachloroethane & WA & & & $\mathbf{U}$ & 5 & & NS & $\mu g / L$ \\
\hline 1,1,2-Trichloroethane & WA & & & $\mathbf{U}$ & 5 & & 5 & $\mu \mathrm{g} / \mathrm{L}$ \\
\hline 1,1-Dichloroethane & WA & & & $\mathbf{U}$ & 5 & & NS & $\mu g / L$ \\
\hline 1,1-Dichloroethylene & WA & & & $\mathbf{U}$ & 5 & & 7 & $\mu g / L$ \\
\hline 1,2,3-Trichloropropane & WA & & & $\mathbf{U}$ & 10 & & NS & $\mu \mathrm{g} / \mathrm{L}$ \\
\hline 1,2-Dibromo-3-chloropropane & WA & & & $\mathbf{U}$ & 20 & & 0.2 & $\mu \mathrm{g} / \mathrm{L}$ \\
\hline 1,2-Dibromoethane & WA & & & $\mathbf{U}$ & 20 & & NS & $\mu g / L$ \\
\hline 1,2-Dichloroethane & WA & & & $\mathbf{U}$ & 5 & & 5 & $\mu \mathrm{g} / \mathrm{L}$ \\
\hline 1,2-Dichloroethylene (total) & WA & & & $\mathbf{U}$ & 5 & & 7 & $\mu g / L$ \\
\hline 1,2-Dichloropropane & WA & & & $\mathbf{U}$ & 5 & & 5 & $\mu g / L$ \\
\hline 2-Hexanone & WA & & & $\mathbf{U}$ & 10 & & NS & $\mu g / L$ \\
\hline 4-Methyl-2-pentanone & WA & & & $\mathrm{U}$ & 10 & & NS & $\mu g / L$ \\
\hline Acetone & WA & & & J & 6.58 & & NS & $\mu g / L$ \\
\hline Acetonitrile & WA & & & $\mathbf{U}$ & 20 & & NS & $\mu g / L$ \\
\hline Acidity & WA & & & & 40 & & NS & $\mathrm{mg} / \mathrm{L}$ \\
\hline Acrolein & WA & & & $\mathbf{U}$ & 10 & & NS & $\mu \mathrm{g} / \mathrm{L}$ \\
\hline Acrylonitrile & WA & & & $\mathbf{U}$ & 10 & & NS & $\mu g / L$ \\
\hline Alkalinity & WA & & & & 1.5 & & NS & $\mathrm{mg} / \mathrm{L}$ \\
\hline Allyl chloride & WA & & & $\mathbf{U}$ & 100 & & NS & $\mu g / L$ \\
\hline Aluminum & WA & & & & 289 & & NS & $\mu \mathrm{g} / \mathrm{L}$ \\
\hline Antimony & WA & & & $\mathbf{U}$ & 60 & & 6 & $\mu g / L$ \\
\hline Arsenic & WA & & & $\mathbf{U}$ & 10 & & 50 & $\mu \mathrm{g} / \mathrm{L}$ \\
\hline Barium & WA & & & $\mathbf{U}$ & 200 & & 2000 & $\mu \mathrm{g} / \mathrm{L}$ \\
\hline Benzene & WA & & & $\mathbf{U}$ & 5 & & 5 & $\mu \mathrm{g} / \mathrm{L}$ \\
\hline Beryllium & WA & & & $\mathbf{U}$ & 5 & & 4 & $\mu \mathrm{g} / \mathrm{L}$ \\
\hline Bromodichloromethane & WA & & & $\mathbf{U}$ & 5 & & NS & $\mu \mathrm{g} / \mathrm{L}$ \\
\hline Bromoform & WA & & & $\mathbf{U}$ & 5 & & NS & $\mu \mathrm{g} / \mathrm{L}$ \\
\hline Cadmium & WA & & & $\mathbf{U}$ & 5 & & 5 & $\mu \mathrm{g} / \mathrm{L}$ \\
\hline Calcium & WA & & & $\mathbf{U}$ & 5000 & & NS & $\mu g / L$ \\
\hline Carbon disulfide & WA & & & $\mathbf{U}$ & 5 & & NS & $\mu \mathrm{g} / \mathrm{L}$ \\
\hline Carbon tetrachloride & WA & & & $\mathbf{U}$ & 5 & & 5 & $\mu \mathrm{g} / \mathrm{L}$ \\
\hline Cesium-137 & TM & & & U & 5.8 & & 200 & $\mathrm{pCi} / \mathrm{L}$ \\
\hline Chloride & WA & & & & 3.52 & & NS & $\mathrm{mg} / \mathrm{L}$ \\
\hline Chlorobenzene & WA & & & $\mathbf{U}$ & 5 & & 100 & $\mu g / L$ \\
\hline Chloroethane & WA & & & $\mathbf{U}$ & 10 & & NS & $\mu g / L$ \\
\hline Chloroform & WA & & & $\mathbf{U}$ & 5 & & 100 & $\mu . g / L$ \\
\hline Chloroprene & WA & & & $\mathbf{U}$ & 20 & & NS & $\mu \mathrm{g} / \mathrm{L}$ \\
\hline Chromium & WA & & & $\mathbf{U}$ & 10 & & 100 & $\mu \mathrm{g} / \mathrm{L}$ \\
\hline cis-1,3-Dichloropropene & WA & & & $\mathbf{U}$ & 5 & & NS & $\mu \mathrm{g} / \mathrm{L}$ \\
\hline Cobalt & WA & & & $\mathbf{U}$ & 50 & & NS & $\mu \mathrm{g} / \mathrm{L}$ \\
\hline Copper & WA & & & $\mathbf{U}$ & 25 & & 1000 & $\mu \mathrm{g} / \mathrm{L}$ \\
\hline Dibromochloromethane & WA & & & $\mathbf{U}$ & 5 & & NS & $\mu \mathrm{g} / \mathrm{L}$ \\
\hline Dichlorodifluoromethane & WA & & & $\mathbf{U}$ & 10 & & NS & $\mu g / L$ \\
\hline Ethylbenzene & WA & & & $\mathbf{U}$ & 5 & & 700 & $\mu g / L$ \\
\hline Fluoride & WA & & & $\mathrm{U}$ & 0.1 & & 4 & $\mathrm{mg} / \mathrm{L}$ \\
\hline
\end{tabular}


Sampling And Analysis of Water From Upper Three Runs And Its Wetlands Near Tank 16 and the Mixed Waste Management Facility

UTR 145 Laboratory Analyses (cont'd)

\begin{tabular}{|c|c|c|c|c|c|c|c|c|c|}
\hline Analyte & Lab & Rep & Avg & Modifier & & Result & Acc & Stand & Units \\
\hline Gross alpha & TM & & & & & 0.9 & 1.2 & 15 & $\overline{\mathrm{pCi} / \mathrm{L}}$ \\
\hline Iron & WA & & & & & 319 & & 300 & $\mu \mathrm{g} / \mathrm{L}$ \\
\hline Isobutyl alcohol & WA & & & $\mathbf{U}$ & & 20 & & NS & $\mu \mathrm{g} / \mathrm{L}$ \\
\hline Lead & WA & & & $\mathbf{U}$ & & 3 & & 50 & $\mu \mathrm{g} / \mathrm{L}$ \\
\hline Magnesium & WA & & & $\mathbf{U}$ & & 5000 & & $\mathrm{NS}$ & $\mu g / L$ \\
\hline Manganese & WA & & & $\mathbf{U}$ & & 15 & . & 50 & $\mu \mathrm{g} / \mathrm{L}$ \\
\hline Mercury & WA & & & $\mathbf{U}$ & & 0.2 & & 2 & $\mu g / L$ \\
\hline Methacrylonitrile & WA & & & $\mathbf{U}$ & & 20 & & NS & $\mu g / L$ \\
\hline Methyl bromide & WA & & & $\mathbf{U}$ & & 10 & & NS & $\mu \mathrm{g} / \mathrm{L}$ \\
\hline Methyl chloride & WA & & & $\mathbf{U}$ & & 10 & & NS & $\mu g / L$ \\
\hline Methyi ethyl ketone & WA & & & $\mathbf{U}$ & & 10 & & INS & $\mu g / L$ \\
\hline Methyl iodide & WA & & & $\mathbf{U}$ & & 10 & & NS & $\mu \mathrm{g} / \mathrm{L}$ \\
\hline Methylene bromide & WA & & & U & & 10 & & NS & $\mu \mathrm{g} / \mathrm{L}$ \\
\hline Methylene chloride & WA & & & $\mathbf{J}$ & V & 3.63 & & 5 & $\mu \mathrm{g} / \mathrm{L}$ \\
\hline Nickel & WA & & . & $\mathbf{U}$ & & 40 & & 100 & $\mu \mathrm{g} / \mathrm{L}$ \\
\hline Nitrate as nitrogen & WA & & & $\mathbf{J}$ & $\mathbf{Q}$ & 0.331 & & 10 & $\mathrm{mg} / \mathrm{L}$ \\
\hline Nonvolatile beta & TM & & & $\mathbf{U}$ & & 0.8 & & 50 & $\mathrm{pCi} / \mathrm{L}$ \\
\hline Potassium & WA & & & $\mathbf{U}$ & & 5000 & & NS & $\mu \mathrm{g} / \mathrm{L}$ \\
\hline Propionitrile & WA & & & $\mathbf{U}$ & & 50 & & NS & $\mu g / L$ \\
\hline Selenium & WA & & & $\mathbf{U}$ & & 5 & & 50 & $\mu g / L$ \\
\hline Silver & WA & & & $\mathbf{U}$ & & 10 & & 50 & $\mu g / L$ \\
\hline Sodium & WA & & & $\mathbf{U}$ & & 5000 & & NS & $\mu g / L$ \\
\hline Styrene & WA & & & $\mathbf{U}$ & & 5 & & 100 & $\mu g / L$ \\
\hline Sulfate & WA & & & & & 3.28 & & 400 & $\mathrm{mg} / \mathrm{L}$ \\
\hline Sulfide & WA & & & $\mathbf{U}$ & & 0.1 & & NS & $\mathrm{mg} / \mathrm{L}$ \\
\hline Tetrachloroethylene & WA & & & U & & 5 & & 5 & $\mu \mathrm{g} / \mathrm{L}$ \\
\hline Thallium & WA & & & $\mathbf{U}$ & & 10 & & 2 & $\mu g / L$ \\
\hline Toluene & WA & & & $\mathbf{U}$ & & 5 & & 1000 & $\mu \mathrm{g} / \mathrm{L}$ \\
\hline Total hardness & WA & & A & & & 5.8 & & NS & $\mathrm{mg} / \mathrm{L}$ \\
\hline Total organic carbon & WA & & & & & 1.55 & & NS & $\mathrm{mg} / \mathrm{L}$ \\
\hline Total suspended solids & WA & & & $\mathbf{U}$ & & 5 & & NS & $\mathrm{mg} / \mathrm{L}$ \\
\hline trans-1,3-Dichloropropene & WA & & & U & & 5 & & NS & $\mu \mathrm{g} / \mathrm{L}$ \\
\hline trans-1,4-Dichloro-2-butene & WA & & & $\mathbf{U}$ & & 100 & & NS & $\mu \mathrm{g} / \mathrm{L}$ \\
\hline Trichloroethylene & WA & & & $\mathbf{U}$ & & 5 & & 5 & $\mu g / L$ \\
\hline Trichlorofluoromethane & WA & & & $\mathbf{U}$ & & 5 & & NS & $\mu g / L$ \\
\hline Tritium & TM & & & & & 4.77 & 0.53 & 20 & $\mathrm{pCi} / \mathrm{ml}$ \\
\hline Turbidity & WA & & A & & & 14.9 & & NS & NTU \\
\hline Vanadium & WA & & & $\mathrm{U}$ & & 50 & & NS & $\mu g / L$ \\
\hline Vinyl acetate & WA & & & 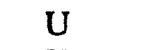 & & 10 & & NS & $\mu \mathrm{g} / \mathrm{L}$ \\
\hline Vinyl chloride & WA & & & $\mathrm{U}$ & & 10 & & 2 & $\mu \mathrm{g} / \mathrm{L}$ \\
\hline Xylenes (total) & WA & & & $\mathrm{U}$ & & 5 & & 10000 & $\mu g / L$ \\
\hline Zinc & WA & & & $\mathbf{U}$ & & 20 & & 5000 & $\mu g / \Lambda$ \\
\hline
\end{tabular}




\section{UTR147}

Field Measurements:

Sample Date: 9/2/93

pH: 7.18

Specific Conductance: $59 \mu \mathrm{S} / \mathrm{cm}$

Red/Oxid Potential: $224 \mathrm{mv}$

Dissolved Oxygen: $4.6 \mathrm{mg} / \mathrm{L}$

\begin{tabular}{|c|c|c|c|c|c|c|c|}
\hline Analyte & Lab & Rep & Modifier & Result & Acc & Stand & Units \\
\hline $1,1,1,2$-Tetrachloroethane & WA & & $\mathbf{U}$ & 10 & & NS & $\mu g / L$ \\
\hline $1,1,1,2-$ Tetrachloroethane & GE & & $\mathbf{U}$ & 1 & & NS & $\mu \mathrm{g} / \mathrm{L}$ \\
\hline 1,1,1-Trichloroethane & WA & & $\mathbf{U}$ & 5 & & 200 & $\mu \mathrm{g} / \mathrm{L}$ \\
\hline 1,1,1-Trichloroethane & GE & & $\mathbf{U}$ & 1 & & 200 & $\mu g / L$ \\
\hline $1,1,2,2-$ Tetrachloroethane & WA & & $\mathbf{U}$ & 5 & & NS & $\mu g / L$ \\
\hline $1,1,2,2$-Tetrachloroethane & GE & & $\mathbf{U}$ & 1 & & NS & $\mu g / L$ \\
\hline 1,1,2-Trichloroethane & WA & & $\mathbf{U}$ & 5 & & 5 & $\begin{array}{l}\mu g / L \\
\mu g / L\end{array}$ \\
\hline 1,1,2-Trichloroethane & $\mathrm{GE}$ & & $\mathbf{U}$ & 1 & & 5 & \\
\hline 1,1-Dichloroethane & WA & & $\mathbf{U}$ & $\begin{array}{l}1 \\
5\end{array}$ & & NS & $\mu g / L$ \\
\hline 1,1-Dichloroethane & $\mathrm{GE}$ & & $\mathbf{U}$ & 1 & & $\begin{array}{l}\text { NS } \\
\text { NS }\end{array}$ & $\mu g / L$ \\
\hline 1,1-Dichloroethylene & WA & & $\mathbf{U}$ & 5 & & NS & $\mu g / L$ \\
\hline 1,1-Dichloroethylene & $\mathrm{GE}$ & & $\mathbf{U}$ & 1 & & 7 & $\mu g / L$ \\
\hline 1,2,3-Trichloropropane & WA & & $\mathbf{U}$ & $\begin{array}{c}1 \\
10\end{array}$ & & 7 & $\mu \mathrm{g} / \mathrm{L}$ \\
\hline 1,2,3-Trichloropropane & GE & & $\mathbf{U}$ & 1 & & NS & $\mu . g / L$ \\
\hline 1,2-Dibromo-3-chloropropane & WA & & $\mathbf{U}$ & 20 & & NS & $\mu g / L$ \\
\hline 1,2-Dibromo-3-chloropropane & GE & & $\mathbf{U}$ & 1 & & 0.2 & $\mu g / L$ \\
\hline 1,2-Dibromoethane & WA & & $\mathbf{U}$ & 20 & & 0.2 & $\mu g / L$ \\
\hline 1,2-Dibromoethane & $\mathrm{GE}$ & & $\mathbf{U}$ & 20 & & NS & $\mu g / L$ \\
\hline 1,2-Dichloroethane & WA & & $\mathbf{U}$ & $\begin{array}{c}20 \\
5\end{array}$ & & 7 & $\mu g / L$ \\
\hline 1,2-Dichloroethane & $\mathrm{GE}$ & & $\mathbf{U}$ & & & 5 & $\mu \mathrm{g} / \mathrm{L}$ \\
\hline 1,2-Dichloroethylene (total) & WA & & $\mathbf{U}$ & $\begin{array}{l}1 \\
5\end{array}$ & & 5 & $\mu \mathrm{g} / \mathrm{L}$ \\
\hline 1,2-Dichloropropane & WA & & $\mathbf{U}$ & $\begin{array}{l}5 \\
5\end{array}$ & & 7 & $\mu g / L$ \\
\hline 1,2-Dichloropropane & GE & & $\mathbf{U}$ & $\begin{array}{l}5 \\
1\end{array}$ & & 5 & $\mu g / L$ \\
\hline 2-Hexanone & WA & & $\mathbf{U}$ & $\begin{array}{c}1 \\
10\end{array}$ & & 5 & $\mu g / L$ \\
\hline 2-Hexanone & GE & & UI & $\begin{array}{c}10 \\
1\end{array}$ & & NS & $\mu g / L$ \\
\hline 4-Methyl-2-pentanone & WA & & $\mathbf{U}$ & $\begin{array}{c}1 \\
10\end{array}$ & & NS & $\mu \mathrm{g} / \mathrm{L}$ \\
\hline $\begin{array}{l}\text { 4-Metnyl-2-pentanonc } \\
\text { Acetone }\end{array}$ & WA & & J & $\begin{array}{c}10 \\
3.95\end{array}$ & & NS & $\mu g / L$ \\
\hline $\begin{array}{l}\text { Acetone } \\
\text { Acetone }\end{array}$ & GE & & $\mathbf{U}$ & 3.95 & & NS & $\mu \mathrm{g} / \mathrm{L}$ \\
\hline Acetonitrile & WA & & $\mathbf{U}$ & 100 & & NS & $\mu g / L$ \\
\hline Acetonitrile (Methyl cyanide) & $\mathrm{GE}$ & & $\mathbf{U}$ & 20 & & NS & $\mu g / L$ \\
\hline Acidity & WA & & $\mathbf{U}$ & 1 & & NS & $\mu \mathrm{g} / \mathrm{L}$ \\
\hline Acrolein & WA & & $\mathbf{U}$ & 80 & & NS & $\mathrm{mg} / \mathrm{L}$ \\
\hline Acrolein & GE & & $\begin{array}{l}\mathbf{U} \\
\mathbf{U}\end{array}$ & 10 & & NS & $\mu \mathrm{g} / \mathrm{L}$ \\
\hline Acrylonitrile & WA & & $\mathbf{U}$ & 20 & & NS & $\mu \mathrm{g} / \mathrm{L}$ \\
\hline Acrylonitrile & $\mathrm{GE}$ & & U & 10 & & NS & $\mu \mathrm{g} / \mathrm{L}$ \\
\hline Alkalinity & WA & & $\mathbf{U}$ & 20 & & NS & $\mu g / L$ \\
\hline Alkalinity & $\begin{array}{l}\text { WA } \\
\text { GE }\end{array}$ & & & 2.5 & & NS & $\mathrm{mg} / \mathrm{L}$ \\
\hline Allyl chloride & $\begin{array}{l}\text { GE } \\
\text { WA }\end{array}$ & & & 13 & & NS & $\mathrm{mg} / \mathrm{L}$ \\
\hline Allyl chloride & WA & & U & 100 & & NS & $\mu g / L$ \\
\hline Aluminum & GE & & $\mathbf{U}$ & 50 & & NS & $\mu \mathrm{g} / \mathrm{L}$ \\
\hline $\begin{array}{l}\text { Aluminum } \\
\text { Aluminum }\end{array}$ & WA & & $\mathbf{U}$ & 200 & & NS & $\mu \mathrm{g} / \mathrm{L}$ \\
\hline $\begin{array}{l}\text { Aluminum } \\
\text { Antimony }\end{array}$ & GE & & & 97.4 & & NS & $\mu \mathrm{g} / \mathrm{L}$ \\
\hline $\begin{array}{l}\text { Antimony } \\
\text { Antimony }\end{array}$ & WA & & $\mathbf{U}$ & 60 & & 6 & $\mu \mathrm{g} / \mathrm{L}$ \\
\hline $\begin{array}{l}\text { Antimony } \\
\text { Arsenic }\end{array}$ & $\mathrm{GE}$ & & $\mathbf{U}$ & 2 & & 6 & $\mu \mathrm{g} / \mathrm{L}$ \\
\hline $\begin{array}{l}\text { Arsenic } \\
\text { Arsenic }\end{array}$ & WA & & $\mathbf{U}$ & 10 & & 50 & $\mu g / L$ \\
\hline $\begin{array}{l}\text { Arsenic } \\
\text { Barium }\end{array}$ & GE & & $\mathrm{U}$ & 2 & & 50 & $\mu g / L$ \\
\hline $\begin{array}{l}\text { Barium } \\
\text { Barium }\end{array}$ & WA & & U & 200 & & 2000 & $\mu g / L$ \\
\hline & GE & & & 12.4 & & 2000 & $\mu g$ \\
\hline
\end{tabular}


UTR 147 Laboratory Analyses (cont'd)

\begin{tabular}{|c|c|c|c|c|c|c|c|c|}
\hline Analyte & Lab & Rep & Avg & Modifier & Result & Acc & Stand & Units \\
\hline Benzene & WA & & & $\mathbf{U}$ & 5 & & 5 & $\mu \mathrm{g} / \mathrm{L}$ \\
\hline Benzene & GE & & & $\mathbf{U}$ & 1 & & 5 & $\mu \mathrm{g} / \mathrm{L}$ \\
\hline Beryllium & WA & & & $\mathbf{U}$ & 5 & & 4 & $\mu \mathrm{g} / \mathrm{L}$ \\
\hline Beryllium & GE & & & $\mathbf{U}$ & 3 & & 4 & $\mu g / L$ \\
\hline Bis(2-chloro-1-methylethyl)ether & GE & & & $\mathrm{U}$ & 10 & & NS̀ & $\mu g / L$ \\
\hline Bromodichloromethane & WA & & & $\mathbf{U}$ & 5 & . & NS & $\mu g / L$ \\
\hline Bromodichloromethane & GE & & & $\mathbf{U}$ & 1 & & NS & $\mu \mathrm{g} / \mathrm{L}$ \\
\hline Bromoform & WA & & & $\mathrm{U}$ & 5 & & NS & $\mu \mathrm{g} / \mathrm{L}$ \\
\hline Bromoform & GE & & & $\mathbf{U}$ & 1 & & NS & $\mu g / L$ \\
\hline Cadmium & WA & & & $\mathbf{U}$ & 5 & & 5 & $\mu g / L$ \\
\hline Cadmium & GE & & & $\mathbf{U}$ & 2 & & 5 & $\mu g / L$ \\
\hline Calcium & WA & & & $\mathbf{U}$ & 5000 & & NS & $\mu g / L$ \\
\hline Calcium & GE & & & & 481 & & NS & $\mu g / L$ \\
\hline Carbon disulfide & WA & & & $\mathbf{U}$ & 5 & & NS & $\mu g / L$ \\
\hline Carbon disulfide & GE & & & $\mathbf{U}$ & 1 & & NS & $\mu g / L$ \\
\hline Carbon tetrachloride & WA & & & $\mathbf{U}$ & 5 & & 5 & $\mu g / L$ \\
\hline Carbon tetrachloride & GE & & & $\mathbf{U}$ & 1 & & 5 & $\mu \mathrm{g} / \mathrm{L}$ \\
\hline Cesium-137 & TM & & & $\mathbf{U}$ & 9.81 & & 200 & $\mathrm{pCi} / \mathrm{L}$ \\
\hline Chloride & WA & & & & 2.74 & & NS & $\mathrm{mg} / \mathrm{L}$ \\
\hline Chloride & GE & & & & 2.75 & & NS & $\mathrm{mg} / \mathrm{L}$ \\
\hline Chlorobenzene & WA & & & $\mathbf{U}$ & 5 & & 100 & $\mu g / L$ \\
\hline Chlorobenzene & GE & & & $\mathbf{U}$ & 1 & & 100 & $\mu g / L$ \\
\hline Chloroethane & WA & & & $\mathbf{U}$ & 10 & & NS & $\mu \mathrm{g} / \mathrm{L}$ \\
\hline Chloroethane & GE & & & $\mathbf{U}$ & 1 & & NS & $\mu g / L$ \\
\hline Chloroform & WA & & & $\mathbf{U}$ & 5 & & 100 & $\mu \mathrm{g} / \mathrm{L}$ \\
\hline Chloroform & GE & & & $\mathbf{U}$ & 1 & & 100 & $\mu g / L$ \\
\hline Chloroprene & WA & & & $\mathbf{U}$ & 20 & & NS & $\mu \mathrm{g} / \mathrm{L}$ \\
\hline Chloroprene & GE & & & $\mathbf{U}$ & 200 & & NS & $\mu g / L$ \\
\hline Chromium & WA & & & $\mathbf{U}$ & 10 & & 100 & $\mu g / L$ \\
\hline Chromium & GE & & & $\mathbf{U}$ & 4 & & 100 & $\mu \mathrm{g} / \mathrm{L}$ \\
\hline cis-1,3-Dichloropropene & WA & & & $\mathbf{U}$ & 5 & & NS & $\mu g / L$ \\
\hline cis-1,3-Dichloropropene & GE & & & $U$ & 1 & & NS & $\mu g / L$ \\
\hline Cobalt & WA & & & $\mathbf{U}$ & 50 & & NS & $\mu \mathrm{g} / \mathrm{L}$ \\
\hline Cobalt & GE & & & $\mathbf{U}$ & 4 & & NS & $\mu \mathrm{g} / \mathrm{L}$ \\
\hline Copper & WA & & & $\mathbf{U}$ & 25 & & 1000 & $\mu \mathrm{g} / \mathrm{L}$ \\
\hline Copper & GE & & & $\mathbf{U}$ & 4 & & 1000 & $\mu \mathrm{g} / \mathrm{L}$ \\
\hline Cyanide & GE & & & UJ & $Q$ & & 200 & $\mu \mathrm{g} / \mathrm{L}$ \\
\hline Dibromochloromethane & WA & & & $\mathbf{U}$ & 5 & & NS & $\mu \mathrm{g} / \mathrm{L}$ \\
\hline Dibromochloromethane & GE & & & $\mathbf{U}$ & 1 & & NS & $\mu \mathrm{g} / \mathrm{L}$ \\
\hline Dichlorodifluoromethane & WA & & & $\mathbf{U}$ & 10 & & NS & $\mu g / L$ \\
\hline Dichlorodifluoromethane & GE & & & $\mathbf{U}$ & 1 & & NS & $\mu \mathrm{g} / \mathrm{L}$ \\
\hline Ethylbenzene & WA & & & $\mathbf{U}$ & 5 & & 700 & $\mu \mathrm{g} / \mathrm{L}$ \\
\hline Ethylbenzene & GE & & & U & 1 & & 700 & $\mu \mathrm{g} / \mathrm{L}$ \\
\hline Fluoride & WA & & & U & 0.1 & & 4 & $\mathrm{mg} / \mathrm{L}$ \\
\hline Fluoride & GE & & & $\mathbf{U}$ & 0.1 & & 4 & $\mathrm{mg} / \mathrm{L}$ \\
\hline Gross alpha & GE & & & U & 2 & & 15 & $\mathrm{pCi} / \mathrm{L}$ \\
\hline Gross alpha & TM & & & & 2 & 1.4 & 15 & $\mathrm{pCi} / \mathrm{L}$ \\
\hline Hardness & GE & & A & & 2.5 & & NS & $\mathrm{mg} / \mathrm{L}$ \\
\hline Iron & WA & & & & 293 & & 300 & $\mu \mathrm{g} / \mathrm{L}$ \\
\hline Iron & GE & & & & 474 & & 300 & $\mu \mathrm{g} / \mathrm{L}$ \\
\hline Isobutyl alcohol & WA & & & $\mathbf{U}$ & 20 & & NS & $\mu \mathrm{g} / \mathrm{L}$ \\
\hline Isobutyl alcohol & GE & & & $\mathbf{U}$ & 100 & & NS & $\mu \mathrm{g} / \mathrm{L}$ \\
\hline Lead & WA & & & $\mathbf{U}$ & 3 & & 50 & $\mu \mathrm{g} / \mathrm{L}$ \\
\hline Lead & GE & & & $\mathrm{U}$ & 3 & & 50 & $\mu \mathrm{g} /$ \\
\hline
\end{tabular}


UTR147 Laboratory Analyses (cont'd)

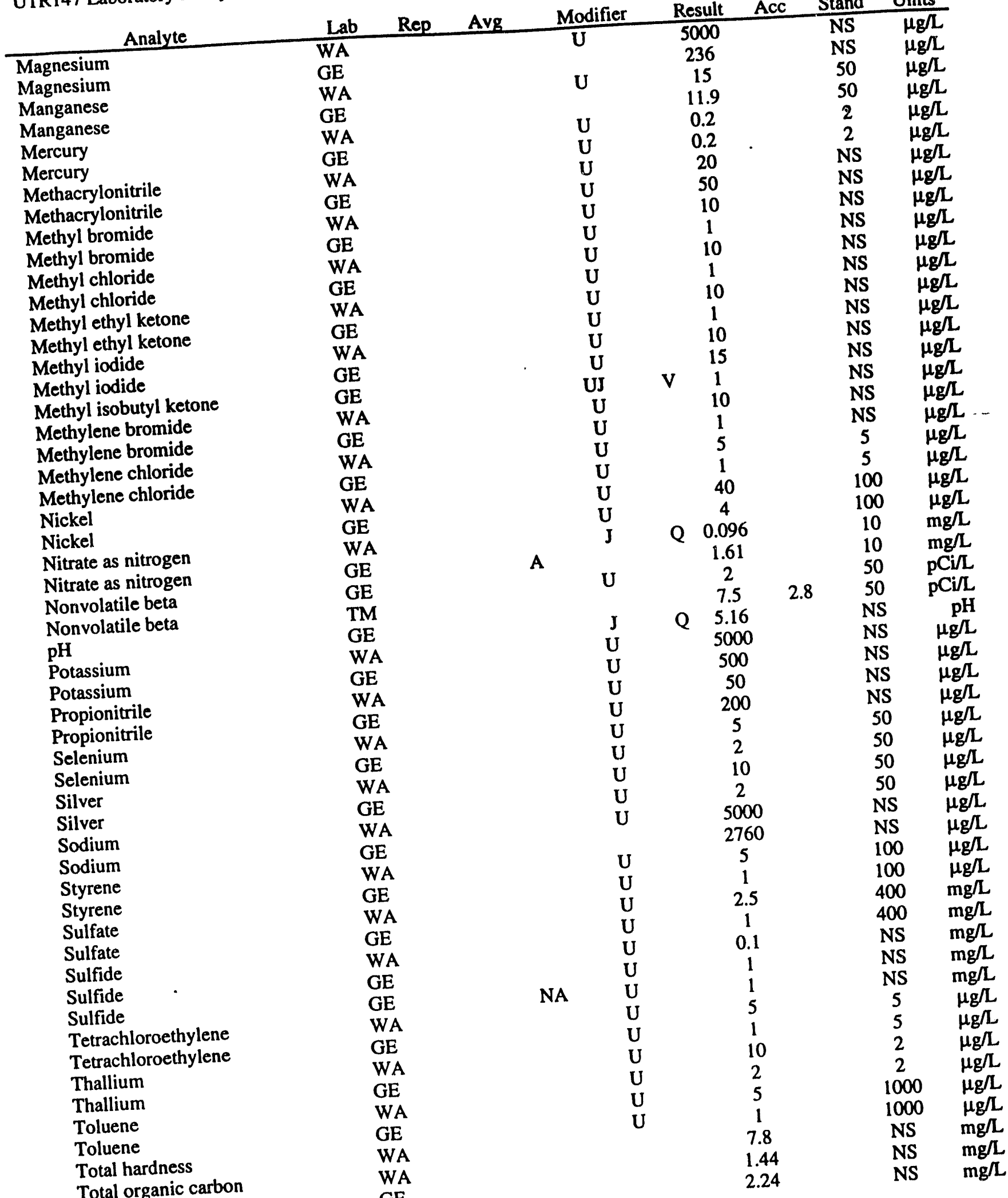


UTR147 Laboratory Analyses (cont'd)

\begin{tabular}{|c|c|c|c|c|c|c|c|c|c|}
\hline Analyte & $\mathrm{Lab}$ & Rep & Avg & Modifier & & Result & Acc & Stand & Units \\
\hline Total suspended solids & WA & & & & & 10 & & NS & $\mathrm{mg} / \mathrm{L}$ \\
\hline Total suspended solids & GE & & & & & 2 & & NS & $\mathrm{mg} / \mathrm{L}$ \\
\hline trans-1,2-Dichloroethylene (total) & GE & & & $\mathbf{U}$ & & $\overline{1}$ & & 100 & $\mu g / L$ \\
\hline trans-1,3-Dichloropropene & WA & & & $\mathbf{U}$ & & 5 & & NS & $\mu g / L$ \\
\hline trans-1,3-Dichloropropene & GE & & & $\mathbf{U}$ & & 1 & & NS & $\mu g / L$ \\
\hline trans-1,4-Dichloro-2-butene & WA & & & $\mathbf{U}$ & & 100 & . & NS & $\mu \mathrm{g} / \mathrm{L}$ \\
\hline trans-1,4-Dichloro-2-butene & GE & & & $\mathbf{U}$ & & 30 & & NS & $\mu g / L$ \\
\hline Trichloroethylene & WA & & & $\mathbf{U}$ & & 5 & & 5 & $\mu g / L$ \\
\hline Trichloroethylene & GE & & & $\mathbf{U}$ & & 1 & & 5 & $\mu g / L$ \\
\hline Trichlorofluoromethane & WA & & & $\mathbf{U}$ & & 5 & & NS & $\mu g / L$ \\
\hline Trichlorofluoromethane & GE & & & $\mathbf{U}$ & & 1 & & NS & $\mu g / L$ \\
\hline Tritium & GE & & & & & 5.23 & & 20 & $\mathrm{pCi} / \mathrm{mi}$ \\
\hline Tritium & TM & & & & & 21.18 & 0.99 & 20 & $\mathrm{pCi} / \mathrm{ml}$ \\
\hline Turbidity & WA & & & J & $\mathbf{Q}$ & 0.42 & & NS & NTU \\
\hline Turbidity & GE & & & & & 0.448 & & NS & NTU \\
\hline Vanadium & WA & & & $\mathbf{U}$ & & 50 & & NS & $\mu \mathrm{g} / \mathrm{L}$ \\
\hline Vanadium & GE & & & $\mathbf{U}$ & & 8 & & NS & $\mu g / L$ \\
\hline Vinyl acetate & WA & & & $\mathbf{U}$ & & 10 & & NS & $\mu g / L$ \\
\hline Vinyl acetate & GE & & & $\mathbf{U}$ & & 1 & & NS & $\mu \mathrm{g} / \mathrm{L}$ \\
\hline Vinyl chloride & WA & & & $\mathbf{U}$ & & 10 & & 2 & $\mu g / L$ \\
\hline Vinyl chloride & $\mathrm{GE}$ & & & $\mathbf{U}$ & & 1 & & 2 & $\mu g / L$ \\
\hline Xylenes (total) & WA & & & $\mathbf{U}$ & & 5 & & 10000 & $\mu g /$ \\
\hline Xylenes (total) & GE & & & $\mathbf{U}$ & & 2 & & 10000 & $\mu \mathrm{g} / \mathrm{L}$ \\
\hline Zinc & WA & & & & & 75.8 & & 5000 & $\mu g / L$ \\
\hline Zinc & $\mathrm{GE}$ & & & & & 12.1 & & 5000 & $\mu \mathrm{g} / \mathrm{L}$ \\
\hline
\end{tabular}




\section{UTR155}

Field Measurements: Sample Date: $9 / 2 / 93$

pH: 7.57

Specific Conductance: $50 \mu \mathrm{S} / \mathrm{cm}$

Red/Oxid Potential: $135 \mathrm{mv}$

Dissolved Oxygen: $4.0 \mathrm{mg} / \mathrm{L}$

\begin{tabular}{|c|c|c|c|c|c|c|c|c|c|}
\hline Analyte & Lab & Rep & Avg & Modifier & & Result & Acc & Stand & Units \\
\hline 1,1,1,2-Tetrachloroethane & WA & & & $\mathbf{U}$ & & 10 & & NS & $\mu g / L$ \\
\hline 1,1,1-Trichloroethane & WA & & & $\mathbf{U}$ & & 5 & & 200 & $\mu g / L$ \\
\hline 1,1,2,2-Tetrachloroethane & WA & & & $\mathbf{U}$ & & 5 & & NS & $\mu \mathrm{g} / \mathrm{L}$ \\
\hline 1,1,2-Trichloroethane & WA & & & $\mathbf{U}$ & & 5 & & 5 & $\mu g / L$ \\
\hline 1,1-Dichloroethane & WA & & & $\mathbf{U}$ & & 5 & & NS & $\mu \mathrm{g} / \mathrm{L}$ \\
\hline 1,1-Dichloroethylene & WA & & & $\mathbf{U}$ & & 5 & & 7 & $\mu \mathrm{g} / \mathrm{L}$ \\
\hline 1,2,3-Trichloropropane & WA & & & $\mathbf{U}$ & & 10 & & NS & $\mu \mathrm{g} / \mathrm{L}$ \\
\hline 1,2-Dibromo-3-chloropropane & WA & & 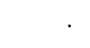 & $\mathbf{U}$ & & 20 & & 0.2 & $\mu g / L$ \\
\hline 1,2-Dibromoethane & WA & & & $\mathbf{U}$ & & 20 & & NS & $\mu g / L$ \\
\hline 1,2-Dichloroethane & WA & & & $\mathbf{U}$ & & 5 & & 5 & $\mu g / L$ \\
\hline 1,2-Dichloroethylene (total) & WA & & & $\mathbf{U}$ & & 5 & & 7 & $\mu g / L$ \\
\hline 1,2-Dichloropropane & WA & & & $\mathbf{U}$ & & 5 & & 5 & $\mu \mathrm{g} / \mathrm{L}$ \\
\hline 2-Hexanone & WA & & & $\mathbf{U}$ & & 10 & & NS & $\mu \mathrm{g} / \mathrm{L}$ \\
\hline 4-Methyl-2-pentanone & WA & & & $\mathbf{U}$ & & 10 & & NS & $\mu g / L$ \\
\hline Acetone & WA & & & $\mathbf{U}$ & $\mathbf{V}$ & 9.62 & & NS & $\mu g / L$ \\
\hline Acetonitrile & WA & & & $\mathbf{U}$ & & 20 & & NS & $\mu g / L$ \\
\hline Acidity & WA & & & & & 70 & & NS & $\mathrm{mg} / \mathrm{L}$ \\
\hline Acrolein & WA & & & $\mathbf{U}$ & & 10 & & NS & $\mu \mathrm{g} / \mathrm{L}$ \\
\hline Acrylonitrile & WA & & & $\mathbf{U}$ & & 10 & & NS & $\mu g / L$ \\
\hline Alkalinity & WA & & & & & 2 & & NS & $\mathrm{mg} / \mathrm{L}$ \\
\hline Allyl chloride & WA & & & $\mathbf{U}$ & & 100 & & NS & $\mu g / L$ \\
\hline Aluminum & WA & & & $\mathbf{U}$ & & 200 & & NS & $\mu g / L$ \\
\hline Antimony & WA & & & $\mathbf{U}$ & & 60 & & 6 & $\mu g / L$ \\
\hline Arsenic & WA & & & $\mathbf{U}$ & & 10 & & 50 & $\mu g / L$ \\
\hline Barium & WA & & & $\mathbf{U}$ & & 200 & & 2000 & $\mu \mathrm{g} / \mathrm{L}$ \\
\hline Benzene & WA & & & $\mathbf{U}$ & & 5 & & 5 & $\mu g / L$ \\
\hline Beryllium & WA & & & $\mathbf{U}$ & & 5 & & 4 & $\mu g / L$ \\
\hline Bromodichloromethane & WA & & & $\mathbf{U}$ & & 5 & & NS & $\mu g / L$ \\
\hline Bromoform & WA & & & $\mathbf{U}$ & & 5 & & NS & $\mu g / L$ \\
\hline Cadmium & WA & & & $\mathbf{U}$ & & 5 & & 5 & $\mu g / L$ \\
\hline Calcium & WA & & & $\mathbf{U}$ & & 5000 & & NS & $\mu \mathrm{g} / \mathrm{L}$ \\
\hline Carbon disulfide & WA & & & $\mathbf{U}$ & & 5 & & NS & $\mu g / L$ \\
\hline Carbon tetrachloride & WA & & & $\mathbf{U}$ & & 5 & & 5 & $\mu \mathrm{g} / \mathrm{L}$ \\
\hline Cesium-137 & TM & & & $\mathbf{U}$ & & 9.64 & & 200 & $\mathrm{pCi} / \mathrm{L}$ \\
\hline Chloride & WA & & & & & 3.19 & & NS & $\mathrm{mg} / \mathrm{L}$ \\
\hline Chlorobenzene & WA & & & $\mathbf{U}$ & & 5 & & 100 & $\mu \mathrm{g} / \mathrm{L}$ \\
\hline Chloroethane & WA & & & $\mathbf{U}$ & & 10 & & NS & $\mu g / L$ \\
\hline Chloroform & WA & & & $\mathbf{U}$ & & 5 & & 100 & $\mu \mathrm{g} / \mathrm{L}$ \\
\hline Chloroprene & WA & & & $\mathbf{U}$ & & 20 & & NS & $\mu \mathrm{g} / \mathrm{L}$ \\
\hline Chromium & WA & & & $\mathbf{U}$ & & 10 & & 100 & $\mu \mathrm{g} / \mathrm{L}$ \\
\hline cis-1,3-Dichloropropene & WA & & & $\mathbf{U}$ & & 5 & & NS & $\mu g / L$ \\
\hline Cobalt & WA & & & $\mathbf{U}$ & & 50 & & NS & $\mu \mathrm{g} / \mathrm{L}$ \\
\hline Copper & WA & & & $\mathbf{U}$ & & 25 & & 1000 & $\mu \mathrm{g} / \mathrm{L}$ \\
\hline Dibromochloromethane & WA & & & $\mathbf{U}$ & & 5 & & NS & $\mu \mathrm{g} / \mathrm{L}$ \\
\hline Dichlorodifluoromethane & WA & & & $\mathbf{U}$ & & 10 & & NS & $\mu \mathrm{g} / \mathrm{L}$ \\
\hline Ethylbenzene & WA & & & $\mathbf{U}$ & & 5 & & 700 & $\mu g / L$ \\
\hline Fluoride & WA & & & $\mathbf{U}$ & & 0.1 & & 4 & $\mathrm{mg} / \mathrm{L}$ \\
\hline
\end{tabular}


UTR.155 Laboratory Analyses (cont'd)

\begin{tabular}{|c|c|c|c|c|c|c|c|c|c|}
\hline Analyte & Lab & Rep & Avg & Modifier & & Result & Acc & Stand & Units \\
\hline Gross alpha & TM & & & & & 0.9 & 1.1 & 15 & $\mathrm{pCi} / \mathrm{L}$ \\
\hline Iron & WA & & & $\mathbf{U}$ & & 100 & & 300 & $\mu g / L$ \\
\hline Isobutyl alcohol & WA & & & $\mathbf{U}$ & & 20 & & NS & $\mu \mathrm{g} / \mathrm{L}$ \\
\hline Lead & WA & & & $\mathbf{U}$ & & 3 & & 50 & $\mu g / L$ \\
\hline Magnesium & WA & & & $\mathbf{U}$ & & 5000 & & NS & $\mu \mathrm{g} / \mathrm{L}$ \\
\hline Manganese & WA & & & & & 46.2 & . & 50 & $\mu g / L$ \\
\hline Mercury & WA & & & $\mathbf{U}$ & & 0.2 & & 2 & $\mu \mathrm{g} / \mathrm{L}$ \\
\hline Methacrylonitrile & WA & & & $\mathbf{U}$ & & 20 & & NS & $\mu \mathrm{g} / \mathrm{L}$ \\
\hline Methyl bromide & WA & & & $\mathbf{U}$ & & 10 & & NS & $\mu \mathrm{g} / \mathrm{L}$ \\
\hline Methyl chloride & WA & & & U & & 10 & & NS & $\mu g / L$ \\
\hline Methyl ethyl ketone & WA & & & $\mathbf{U}$ & & 10 & & NS & $\mu g / L$ \\
\hline Methyl iodide & WA & & & $\mathbf{U}$ & & 10 & & NS & $\mu \mathrm{g} / \mathrm{L}$ \\
\hline Methylene bromide & WA & & & $\mathbf{U}$ & & 10 & & NS & $\mu \mathrm{g} / \mathrm{L}$ \\
\hline Methylene chloride & WA & & & $\mathbf{J}$ & v & 3.89 & & 5 & $\mu g / L$ \\
\hline Nickel & WA & & & $\mathbf{u}$ & & 40 & & 100 & $\mu \mathrm{g} / \mathrm{L}$ \\
\hline Nitrate as nitrogen & WA & & & $\mathbf{J}$ & $\mathbf{Q}$ & 1.7 & & 10 & $\mathrm{mg} / \mathrm{L}$ \\
\hline Nonvolatile beta & TM & & & & & 1.8 & 2.2 & 50 & $\mathrm{pCi} / \mathrm{L}$ \\
\hline Potassium & WA & & & $\mathbf{U}$ & & 5000 & & NS & $\mu g / L$ \\
\hline Propionitrile & WA & & & $\mathbf{U}$ & & 50 & & NS & $\mu \mathrm{g} / \mathrm{L}$ \\
\hline Selenium & WA & & & $\mathbf{U}$ & & 5 & & 50 & $\mu \mathrm{g} / \mathrm{L}$ \\
\hline Silver & WA & & & $\mathbf{U}$ & & 10 & & 50 & $\mu \mathrm{g} / \mathrm{L}$ \\
\hline Sodium & WA & & & $\mathbf{U}$ & & 5000 & & NS & $\mu \mathrm{g} / \mathrm{L}$ \\
\hline Styrene & WA & & & $\mathbf{U}$ & & 5 & & 100 & $\mu g / L$ \\
\hline Sulfate & WA & & & $\mathbf{U}$ & & 2.5 & & 400 & $\mathrm{mg} / \mathrm{L}$ \\
\hline Sulfide & WA & & & $\mathbf{U}$ & & 0.1 & & NS & $\mathrm{mg} / \mathrm{h}$ \\
\hline Tetrachloroethylene & WA & & & $\mathbf{U}$ & & 5 & & 5 & $\mu g / L$ \\
\hline Thallium & WA & & & $U$ & & 10 & & 2 & $\mu \mathrm{g} / \mathrm{L}$ \\
\hline Toluene & WA & & & $\mathbf{J}$ & & 1.02 & & 1000 & $\mu \mathrm{g} / \mathrm{L}$ \\
\hline Total hardness & WA & & & & & 5.8 & & NS & $\mathrm{mg} / \mathrm{L}$ \\
\hline Total organic carbon & WA & & & & & 1.28 & & NS & $\mathrm{mg} / \mathrm{L}$ \\
\hline Total suspended solids & WA & & & & & 12 & & NS & $\mathrm{mg} / \mathrm{L}$ \\
\hline trans-1,3-Dichloropropene & WA & & & $\mathbf{U}$ & & 5 & & NS & $\mu g / L$ \\
\hline trans-1,4-Dichloro-2-butene & WA & & & $\mathbf{U}$ & & 100 & & NS & $\mu \mathrm{g} / \mathrm{L}$ \\
\hline Trichloroethylene & WA & & & $\mathrm{J}$ & & 4.66 & & 5 & $\mu \mathrm{g} / \mathrm{L}$ \\
\hline Trichlorofluoromethane & WA & & & $\mathbf{U}$ & & 5 & & NS & $\mu g / L$ \\
\hline Tritium & TM & & & & & 6.27 & 0.57 & 20 & $\mathrm{pCi} / \mathrm{ml}$ \\
\hline Turbidity & WA & & & $\mathbf{J}$ & $\mathbf{Q}$ & 0.45 & & NS & NTU \\
\hline Vanadium & WA & & & $\mathbf{U}$ & & 50 & & NS & $\mu \mathrm{g} / \mathrm{L}$ \\
\hline Vinyl acetate & WA & & & $\mathrm{U}$ & & 10 & & NS & $\mu \mathrm{g} / \mathrm{L}$ \\
\hline Vinyl chloride & WA & & & $\mathrm{U}$ & & 10 & & 2 & $\mu \mathrm{g} / \mathrm{L}$ \\
\hline Xylenes (total) & WA & & & U & & 5 & & 10000 & $\mu \mathrm{g} / \mathrm{L}$ \\
\hline Zinc & WA & & & $\mathbf{U}$ & & 20 & & 5000 & $\mu g / L$ \\
\hline
\end{tabular}




\section{UQA006}

(Equipment Blank - Shovel)

Field Measurements:

Sample Date: 8/24/93

\begin{tabular}{|c|c|c|c|c|c|c|c|c|c|}
\hline Analyte & Lab & Rep & Avg & Modifier & & Result & Acc & Stand & Units \\
\hline 1,1,1,2-Tetrachloroethane & WA & & & $\bar{U}$ & & 10 & & NS & $\mu g / L$ \\
\hline $1,1,1,2$-Tetrachloroethane & WA & & NA & $\mathbf{U}$ & & 10 & & NS & $\mu \mathrm{g} / \mathrm{L}$ \\
\hline 1,1,1,2-Tetrachloroethane & WA & & NA & $\mathbf{U}$ & & 10 & & NS & $\mu g / L$ \\
\hline 1,1,1-Trichloroethane & WA & & & $\mathbf{U}$ & & 5 & & 200 & $\mu g / L$ \\
\hline 1,1,1-Trichloroethane & WA & & NA & $\mathrm{U}$ & & 5 & & 200 & $\mu \mathrm{g} / \mathrm{L}$ \\
\hline 1,1,1-Trichloroethane & WA & & NA & $\mathbf{U}$ & & 5 & & 200 & $\mu g / L$ \\
\hline $1,1,2,2$-Tetrachloroethane & WA & & & $\mathbf{U}$ & & 5 & & NS & $\mu g / L$ \\
\hline 1,1,2,2-Tetrachloroethane & WA & & NA & U & & 5 & & NS & $\mu g / L$ \\
\hline 1,1,2,2-Tetrachloroethane & WA & & NA & $\mathbf{U}$ & & 5 & & NS & $\mu g / L$ \\
\hline 1,1,2-Trichloroethane & WA & & & $\mathbf{U}$ & & 5 & & 5 & $\mu g / L$ \\
\hline 1,1,2-Trichloroethane & WA & & NA & U & & 5 & & 5 & $\mu g / L$ \\
\hline 1,1,2-Trichloroethane & WA & & NA & $\mathbf{U}$ & & 5 & & 5 & $\mu g / L$ \\
\hline 1,1-Dichloroethane & WA & & & $\mathbf{U}$ & & 5 & & NS & $\mu g / L$ \\
\hline 1,1-Dichloroethane & WA & & NA & $\mathbf{U}$ & & 5 & & NS & $\mu g / L$ \\
\hline 1,1-Dichloroethane & WA & & NA & $\mathrm{U}$ & & 5 & & NS & $\mu g / L$ \\
\hline 1,1-Dichloroethylene & WA & & & $\mathbf{U}$ & & 5 & & 7 & $\mu \mathrm{g} / \mathrm{L}$ \\
\hline 1,2,3-Trichloropropane & WA & & & $\mathrm{U}$ & & 10 & & NS & $\mu g / L$ \\
\hline 1,2,3-Trichloropropane & WA & & NA & $\mathbf{U}$ & & 10 & & NS & $\mu \mathrm{g} / \mathrm{L}$ \\
\hline 1,2,3-Trichloropropane & WA & & NA & $\mathbf{U}$ & & 10 & & NS & $\mu \mathrm{g} / \mathrm{L}$ \\
\hline 1,2-Dibromo-3-chloropropane & WA & & & $\mathbf{U}$ & & 20 & & 0.2 & $\mu \mathrm{g} / \mathrm{L}$ \\
\hline 1,2-Dibromo-3-chloropropane & WA & & NA & $\mathrm{U}$ & & 20 & & 0.2 & $\mu g / L$ \\
\hline 1,2-Dibromo-3-chloropropane & WA & & NA & $\mathbf{U}$ & & 20 & & 0.2 & $\mu \mathrm{g} / \mathrm{L}$ \\
\hline 1,2-Dibromoethane & WA & & & $\mathbf{U}$ & & 20 & & NS & $\mu \mathrm{g} / \mathrm{L}$ \\
\hline 1,2-Dibromoethane & WA & & NA & $\mathbf{U}$ & & 20 & & NS & $\mu g / L$ \\
\hline 1,2-Dibromoethane & WA & & NA & $\mathbf{U}$ & & 20 & & NS & $\mu g / L$ \\
\hline 1,2-Dichloroethane & WA & & & $\mathbf{U}$ & & 5 & & 5 & $\mu g / L$ \\
\hline 1,2-Dichloroethane & WA & & NA & U & & 5 & & 5 & $\mu g / L$ \\
\hline 1,2-Dichloroethane & WA & & NA & $\mathbf{U}$ & & 5 & & 5 & $\mu g / L$ \\
\hline 1,2-Dichloroethylene (total) & WA & & & $\mathrm{U}$ & & 5 & & 7 & $\mu g / L$ \\
\hline 1,2-Dichloroethylene (total) & WA & & NA & $\mathrm{U}$ & & 5 & & 7 & $\mu g / L$ \\
\hline 1,2-Dichloroethylene (total) & WA & & NA & $\mathbf{U}$ & & 5 & & 7 & $\mu \mathrm{g} / \mathrm{L}$ \\
\hline 1,2-Dichloropropane & WA & & & $\mathbf{U}$ & & 5 & & 5 & $\mu g / L$ \\
\hline 1,2-Dichloropropane & WA & & NA & $\mathbf{U}$ & & 5 & & 5 & $\mu g / L$ \\
\hline 1,2-Dichloropropane & WA & & NA & U & & 5 & & 5 & $\mu g / L$ \\
\hline 2-Hexanone & WA & & & $\mathrm{U}$ & & 10 & & NS & $\mu \mathrm{g} / \mathrm{L}$ \\
\hline 2-Hexanone & WA & & NA & $\mathrm{U}$ & & 10 & & NS & $\mu g / L$ \\
\hline 2-Hexanone & WA & & NA & $\mathrm{U}$ & & 10 & & NS & $\mu g / L$ \\
\hline 4-Methyl-2-pentanone & WA & & & $\mathbf{U}$ & & 10 & & NS & $\mu g / L$ \\
\hline 4-Methyl-2-pentanone & WA & & NA & U & & 10 & & NS & $\mu \mathrm{g} / \mathrm{L}$ \\
\hline 4-Methyl-2-pentanone & WA & & NA & $\mathbf{U}$ & & 10 & & NS & $\mu \mathrm{g} / \mathrm{L}$ \\
\hline Acetone & WA & & & & V & 12.5 & & NS & $\mu \mathrm{g} / \mathrm{L}$ \\
\hline Acetone & WA & & NA & $\mathbf{J}$ & V & 7.32 & & NS & $\mu \mathrm{g} / \mathrm{L}$ \\
\hline Acetone & WA & & NA & & V & 10.5 & & NS & $\mu \mathrm{g} / \mathrm{L}$ \\
\hline Acetonitrile & WA & & & $\mathrm{U}$ & & 20 & & NS & $\mu \mathrm{g} / \mathrm{L}$ \\
\hline Acetonitrile & WA & & NA & $\mathbf{U}$ & & 20 & & NS & $\mu \mathrm{g} / \mathrm{L}$ \\
\hline Acetonitrile & WA & & NA & $\mathbf{U}$ & & 20 & & NS & $\mu \mathrm{g} / \mathrm{L}$ \\
\hline
\end{tabular}


UQA006 Laboratory Analyses (cont'd)

\begin{tabular}{|c|c|c|c|c|c|c|c|}
\hline Analyte & Lab & Avg & Modifier & Result & Acc & Stand & Units \\
\hline Acidity & WA & & & 30 & & NS & $\mathrm{mg} / \mathrm{L}$ \\
\hline Acrolein & WA & & $\mathbf{U}$ & 10 & & NS & $\mu g / L$ \\
\hline Acrolein & WA & NA & $\mathbf{U}$ & 10 & & NS & $\mu g / L$ \\
\hline Acrolein & WA & NA & $\mathbf{U}$ & 10 & & NS & $\mu g / L$ \\
\hline Acrylonitrile & WA & & $\mathbf{U}$ & 10 & & NS & $\mu g / L$ \\
\hline Acrylonitrile & WA & NA & $\mathbf{U}$ & 10 & & NS & $\mu \mathrm{g} / \mathrm{L}$ \\
\hline Acrylonitrile & WA & NA & $\mathbf{U}$ & 10 & & NS & $\mu \mathrm{g} / \mathrm{L}$ \\
\hline Alkalinity & WA & & $\mathbf{U}$ & 0.5 & & NS & $\mathrm{mg} / \mathrm{L}$ \\
\hline Allyl chloride & WA & & $\mathbf{U}$ & 100 & & NS & $\mu g / L$ \\
\hline Allyl chloride & WA & NA & $\mathbf{U}$ & 100 & & NS & $\mu g / L$ \\
\hline Allyl chloride & WA & NA & $\mathbf{U}$ & 100 & & NS & $\mu g / L$ \\
\hline Aluminum & WA & & $\mathbf{U}$ & 200 & & NS & $\mu g / L$ \\
\hline Aluminum & WA & NA & $\mathbf{U}$ & 200 & & NS & $\mu g / L$ \\
\hline Antimony & WA & & $\mathbf{U}$ & 60 & & 6 & $\mu g / L$ \\
\hline Antimony & WA & NA & $\mathbf{U}$ & 60 & & 6 & $\mu g / L$ \\
\hline Arsenic & WA & & $\mathbf{U}$ & 10 & & 50 & $\mu g / L$ \\
\hline Arsenic & WA & NA & $\mathbf{U}$ & 10 & & 50 & $\mu g / L$ \\
\hline Barium & WA & & $\mathbf{U}$ & 200 & & 2000 & $\mu g / L$ \\
\hline Barium & WA & NA & $\mathbf{U}$ & 200 & & 2000 & $\mu g / L$ \\
\hline Benzene & WA & & $\mathbf{U}$ & 5 & & 5 & $\mu g / L$ \\
\hline Beryllium & WA & & $\mathbf{U}$ & 5 & & 4 & $\mu g / L$ \\
\hline Beryllium & WA & NA & $\mathbf{U}$ & 5 & & 4 & $\mu g / L$ \\
\hline Bromodichloromethane & WA & & $\mathbf{U}$ & 5 & & NS & $\mu g / L$ \\
\hline Bromodichloromethane & WA & NA & $\mathbf{U}$ & 5 & & NS & $\mu g / L$ \\
\hline Bromodichloromethane & WA & NA & $\mathbf{U}$ & 5 & & NS & $\mu g / L$ \\
\hline Bromoform & WA & & $\mathbf{U}$ & 5 & & NS & $\mu g / L$ \\
\hline Bromoform & WA & NA & $\mathbf{U}$ & 5 & & NS & $\mu g / L$ \\
\hline Bromoform & WA & $\mathbf{N A}$ & $\mathbf{U}$ & 5 & & NS & $\mu g / L$ \\
\hline Cadmium & WA & & $\mathbf{U}$ & 5 & & 5 & $\mu g / L$ \\
\hline Cadmium & WA & NA & $\mathbf{U}$ & 5 & & 5 & $\mu g / L$ \\
\hline Calcium & WA & & $\mathbf{U}$ & 5000 & & NS & $\mu g / L$ \\
\hline Calcium & WA & NA & $\mathbf{U}$ & 5000 & & NS & $\mu g / L$ \\
\hline Carbon disulfide & WA & & $\mathbf{U}$ & 5 & & NS & $\mu g / L$ \\
\hline Carbon disulfide & WA & NA & $\mathbf{U}$ & 5 & & NS & $\mu \mathrm{g} / \mathrm{L}$ \\
\hline Carbon disulfide & WA & NA & $\mathbf{U}$ & 5 & & NS & $\mu g / L$ \\
\hline Carbon tetrachloride & WA & & $\mathbf{U}$ & 5 & & 5 & $\mu \mathrm{g} / \mathrm{L}$ \\
\hline Carbon tetrachloride & WA & NA & $\mathbf{U}$ & 5 & & 5 & $\mu \mathrm{g} / \mathrm{L}$ \\
\hline Carbon tetrachloride & WA & NA & $\mathbf{U}$ & 5 & & 5 & $\mu \mathrm{g} / \mathrm{L}$ \\
\hline Chloride & WA & & $\mathbf{U}$ & 0.25 & & NS & $\mathrm{mg} / \mathrm{L}$ \\
\hline Chloride & WA & NA & $\mathbf{U}$ & 0.25 & & NS & $\mathrm{mg} / \mathbf{L}$ \\
\hline Chlorobenzene & WA & & $\mathbf{U}$ & 5 & & 100 & $\mu g / L$ \\
\hline Chloroethane & WA & & $\mathbf{U}$ & 10 & & NS & $\mu g / L$ \\
\hline Chloroethane & WA & NA & $\mathrm{U}$ & 10 & & NS & $\mu g / L$ \\
\hline Chloroethane & WA & NA & $\mathbf{U}$ & 10 & & NS & $\mu g / L$ \\
\hline Chloroform & WA & & $\mathbf{U}$ & 5 & & 100 & $\mu \mathrm{g} / \mathrm{L}$ \\
\hline Chloroform & WA & NA & $\mathbf{U}$ & 5 & & 100 & $\mu \mathrm{g} / \mathrm{L}$ \\
\hline Chloroform & WA & NA & $\mathbf{U}$ & 5 & & 100 & $\mu g / L$ \\
\hline Chloroprene & WA & & $\mathbf{U}$ & 20 & & NS & $\mu g / L$ \\
\hline Chloroprene & WA & NA & $\bar{U}$ & 20 & & NS & $\mu \mathrm{g} / \mathrm{L}$ \\
\hline Chloroprene & WA & NA & $\mathbf{U}$ & 20 & & NS & $\mu g / L$ \\
\hline Chromium & WA & & $\mathbf{U}$ & 10 & & 100 & $\mu g / L$ \\
\hline Chromium & WA & NA & $\mathbf{U}$ & 10 & & 100 & $\mu \mathrm{g} / \mathrm{L}$ \\
\hline
\end{tabular}


UQA006 Laboratory Analyses (cont'd)

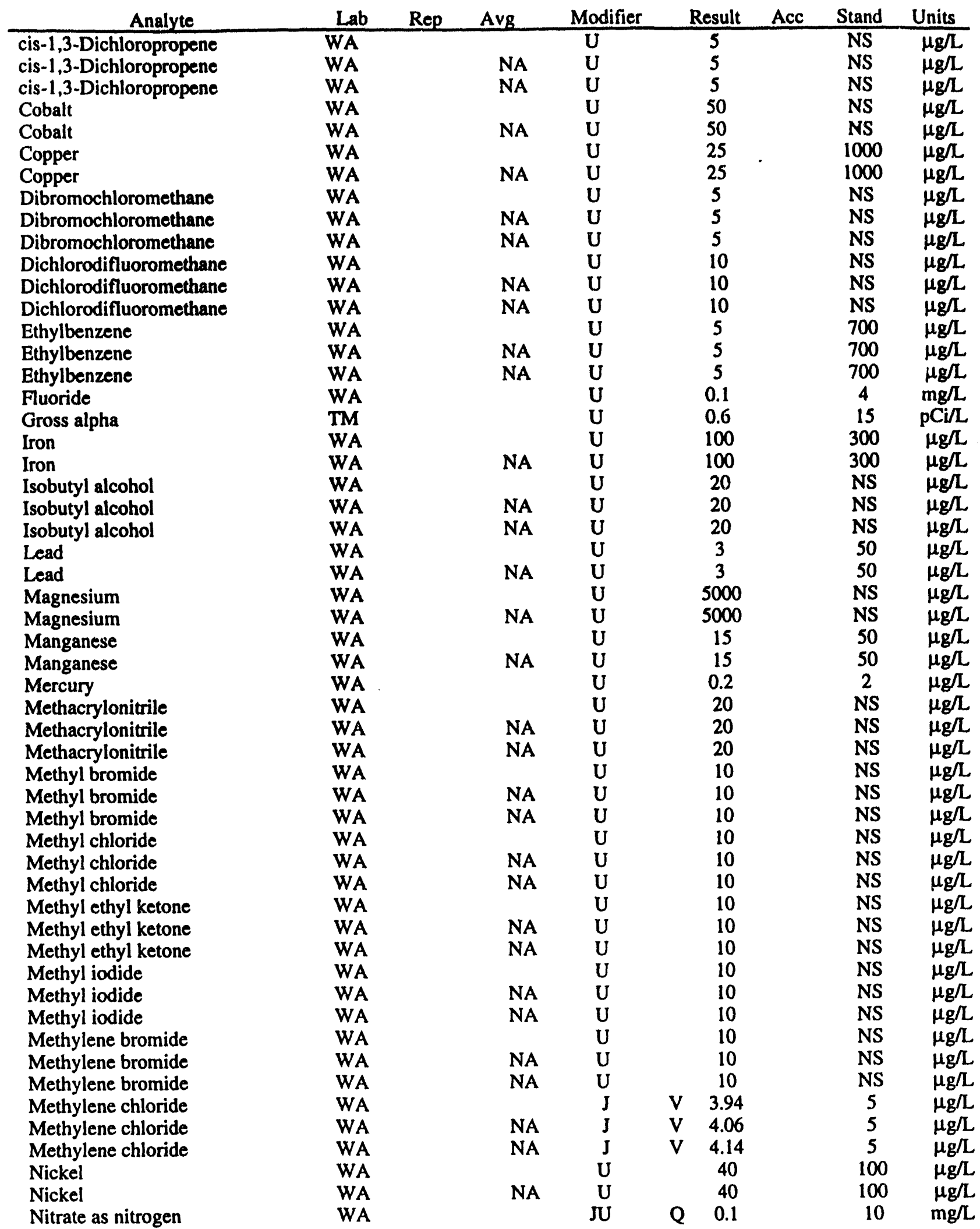


UQA006 Laboratory Analyses (cont'd)

\begin{tabular}{|c|c|c|c|c|c|c|c|}
\hline Analyte & Lab & Avg & Modifier & Result & Acc & Stand & Units \\
\hline Nonvolatile beta & TM & & & 0.8 & 1.5 & 50 & $\mathrm{pCi} / \mathrm{L}$ \\
\hline Potassium & WA & & $\mathbf{U}$ & 5000 & & NS & $\mu g / L$ \\
\hline Potassium & WA & NA & $\mathbf{U}$ & 5000 & & NS & $\mu g / L$ \\
\hline Propionitrile & WA & & $\mathbf{U}$ & 50 & & NS & $\mu g / L$ \\
\hline Propionitrile & WA & NA & $\mathbf{U}$ & 50 & & NS & $\mu g / L$ \\
\hline Propionitrile & WA & $\mathbf{N A}$ & $\mathbf{U}$ & 50 & . & NS & $\mu g / L$ \\
\hline Selenium & WA & & $\mathbf{U}$ & 5 & & 50 & $\mu \mathrm{g} / \mathrm{L}$ \\
\hline Selenium & WA & NA & $\mathbf{U}$ & 5 & & 50 & $\mu g / L$ \\
\hline Silver & WA & & $\mathbf{U}$ & 10 & & 50 & $\mu g / L$ \\
\hline Silver & WA & NA & $\mathbf{U}$ & 10 & & 50 & $\mu g / L$ \\
\hline Sodium & WA & & $\mathbf{U}$ & 5000 & & NS & $\mu g / L$ \\
\hline Sodium & WA & NA & $\mathbf{U}$ & 5000 & & NS & $\mu g / L$ \\
\hline Styrene & WA & & $\mathbf{U}$ & 5 & & 100 & $\mu g / L$ \\
\hline Styrene & WA & NA & $\mathbf{U}$ & 5 & & 100 & $\mu g / L$ \\
\hline Styrene & WA & NA & $\mathbf{U}$ & 5 & & 100 & $\mu g / L$ \\
\hline Sulfate & WA & & $\mathbf{U}$ & 2.5 & & 400 & $\mathrm{mg} / \mathrm{L}$ \\
\hline Sulfide & WA & & $\mathbf{U}$ & 0.1 & & NS & $\mathrm{mg} / \mathrm{L}$ \\
\hline Tetrachloroethylene & WA & & $\mathbf{U}$ & 5 & & 5 & $\mu g / L$ \\
\hline Tetrachloroethylene & WA & NA & $\mathbf{U}$ & 5 & & 5 & $\mu g / L$ \\
\hline Tetrachloroethylene & WA & NA & $\mathbf{U}$ & 5 & & 5 & $\mu \mathrm{g} / \mathrm{L}$ \\
\hline Thallium & WA & & $\mathbf{U}$ & 10 & & 2 & $\mu g / L$ \\
\hline Thallium & WA & NA & $\mathbf{U}$ & 10 & & 2 & $\mu g / L$ \\
\hline Toluene & WA & & $\mathbf{U}$ & 5 & & 1000 & $\mu \mathrm{g} / \mathrm{L}$ \\
\hline Total hardness & WA & & $\mathbf{U}$ & 1 & & NS & $\mathrm{mg} / \mathrm{L}$ \\
\hline Total organic carbon & WA & & & 0.616 & & NS & $\mathrm{mg} / \mathrm{L}$ \\
\hline Total suspended solids & WA & & $\mathbf{U}$ & 5 & & NS & $\mathrm{mg} / \mathrm{L}$ \\
\hline Total suspended solids & WA & NA & & 5 & & NS & $\mathrm{mg} / \mathrm{L}$ \\
\hline trans-1,3-Dichloropropene & WA & & $\mathrm{U}$ & 5 & & NS & $\mu \mathrm{g} / \mathrm{L}$ \\
\hline trans-1,3-Dichloropropene & WA & NA & $\mathbf{U}$ & 5 & & NS & $\mu \mathrm{g} / \mathrm{L}$ \\
\hline trans-1,3-Dichloropropene & WA & NA & $\mathbf{U}$ & 5 & & NS & $\mu \mathrm{g} / \mathrm{L}$ \\
\hline trans-1,4-Dichloro-2-butene & WA & & $\mathbf{U}$ & 100 & & NS & $\mu g / L$ \\
\hline trans-1,4-Dichloro-2-butene & WA & NA & $\mathbf{U}$ & 100 & & NS & $\mu g / L$ \\
\hline trans-1,4-Dichloro-2-butene & WA & NA & U & 100 & & NS & $\mu g / L$ \\
\hline Trichloroethylene & WA & & $\mathbf{U}$ & 5 & & 5 & $\mu \mathrm{g} / \mathrm{L}$ \\
\hline Trichlorofluoromethane & WA & & $\mathbf{U}$ & 5 & & NS & $\mu \mathrm{g} / \mathrm{L}$ \\
\hline Trichlorofluoromethane & WA & NA & $\mathbf{U}$ & 5 & & NS & $\mu g / L$ \\
\hline Trichlorofluoromethane & WA & NA & $\mathrm{U}$ & 5 & & NS & $\mu g / L$ \\
\hline Tritium & TM & & & 0.79 & 0.25 & 20 & $\mathrm{pCi} / \mathrm{ml}$ \\
\hline Turbidity & WA & & $\mathbf{U}$ & 0.2 & & NS & NTU \\
\hline Vanadium & WA & & $\mathbf{U}$ & 50 & & NS & $\mu g / L$ \\
\hline Vanadium & WA & NA & $\mathbf{U}$ & 50 & & NS & $\mu g / L$ \\
\hline Vinyl acetate & WA & & $\mathbf{U}$ & 10 & & NS & $\mu \mathrm{g} / \mathrm{L}$ \\
\hline Vinyl acetate & WA & $\mathbf{N A}$ & $\mathbf{U}$ & 10 & & NS & $\mu g / L$ \\
\hline Vinyl acetate & WA & NA & $\mathbf{U}$ & 10 & & NS & $\mu g / L$ \\
\hline Vinyl chloride & WA & & $\mathbf{U}$ & 10 & & 2 & $\mu g / L$ \\
\hline Vinyl chloride & WA & NA & $\mathbf{U}$ & 10 & & 2 & $\mu \mathrm{g} / \mathrm{L}$ \\
\hline Vinyl chloride & WA & NA & $\mathbf{U}$ & 10 & & 2 & $\mu g / L$ \\
\hline Xylenes (total) & WA & & $\mathbf{U}$ & 5 & & 10000 & $\mu g / L$ \\
\hline Xylenes (total) & WA & $\mathbf{N A}$ & $\mathbf{U}$ & 5 & & 10000 & $\mu \mathrm{g} / \mathrm{L}$ \\
\hline Xylenes (total) & WA & NA & $\mathbf{U}$ & 5 & & 10000 & $\mu g / L$ \\
\hline Zinc & WA & & $\mathbf{U}$ & 20 & & 5000 & $\mu g / L$ \\
\hline Zinc & WA & NA & $\mathbf{U}$ & 20 & & 5000 & $\mu g / L$ \\
\hline
\end{tabular}


UQA007

(Equipment Blank - Shovel)

Field Measurements:

Sample Date: 8/25/93

\begin{tabular}{|c|c|c|c|c|c|c|c|c|c|}
\hline Analyte & Lab & Rep & Avg & Modifier & & Result & Acc & Stand & Units \\
\hline 1,1,1,2-Tetrachloroethane & $\overline{\text { WA }}$ & & & $\bar{U}$ & & 10 & & NS & $\mu g / L$ \\
\hline 1,1,1,2-Tetrachloroethane & WA & & NA & $\mathbf{U}$ & & 10 & & NS & $\mu g / L$ \\
\hline 1,1,1,2-Tetrachloroethane & WA & & NA & $\mathbf{U}$ & & 10 & & NS & $\mu \mathrm{g} / \mathrm{L}$ \\
\hline 1,1,1-Trichloroethane & WA & & & $\mathbf{U}$ & & 5 & & 200 & $\mu g / L$ \\
\hline 1,1,1-Trichloroethane & WA & & NA & $\mathbf{U}$ & & 5 & & 200 & $\mu g / L$ \\
\hline 1,1,1-Trichloroethane & WA & & NA & $\mathbf{U}$ & & 5 & & 200 & $\mu g / L$ \\
\hline 1,1,2,2-Tetrachloroethane & WA & & & $\mathbf{U}$ & & 5 & & NS & $\mu g / L$ \\
\hline 1,1,2,2-Tetrachloroethane & WA & & NA & $\mathbf{U}$ & & 5 & & NS & $\mu g / L$ \\
\hline 1,1,2,2-Tetrachloroethane & WA & & NA & U & & 5 & & NS & $\mu g / L$ \\
\hline 1,1,2-Trichloroethane & WA & & & $U$ & & 5 & & 5 & $\mu g / L$ \\
\hline 1,1,2-Trichloroethane & WA & & NA & $\mathrm{U}$ & & 5 & & 5 & $\mu g / L$ \\
\hline 1,1,2-Trichloroethane & WA & & NA & U & & 5 & & 5 & $\mu g / L$ \\
\hline 1,1-Dichloroethane & WA & & & $\mathbf{U}$ & & 5 & & NS & $\mu g / L$ \\
\hline 1,1-Dichloroethane & WA & & NA & U & & 5 & & NS & $\mu g / L$ \\
\hline 1,1-Dichloroethane & WA & & NA & $\mathrm{U}$ & & 5 & & NS & $\mu_{g} / L$ \\
\hline 1,1-Dichloroethylene & WA & & & $\mathbf{U}$ & & 5 & & 7 & $\mu g / L$ \\
\hline 1,2,3-Trichloropropane & WA & & & $\mathrm{U}$ & & 10 & & NS & $\mu g / L$ \\
\hline 1,2,3-Trichloropropane & WA & & NA & U & & 10 & & NS & $\mu g / L$ \\
\hline 1,2,3-Trichloropropane & WA & & NA & $\mathbf{U}$ & & 10 & & NS & $\mu \mathrm{g} / \mathrm{L}$ \\
\hline 1,2-Dibromo-3-chloropropane & WA & & & $\mathbf{U}$ & & 20 & & 0.2 & $\mu g / L$ \\
\hline 1,2-Dibromo-3-chloropropane & WA & & NA & $\mathbf{U}$ & & 20 & & 0.2 & $\mu g / L$ \\
\hline 1,2-Dibromo-3-chloropropane & WA & & NA & $\mathrm{U}$ & & 20 & & 0.2 & $\mu \mathrm{g} / \mathrm{L}$ \\
\hline 1,2-Dibromoethane & WA & & & $\mathrm{U}$ & & 20 & & NS & $\mu g / L$ \\
\hline 1,2-Dibromoethane & WA & & NA & U & & 20 & & NS & $\mu \mathrm{g} / \mathrm{L}$ \\
\hline 1,2-Dibromoethane & WA & & NA & $\mathbf{U}$ & & 20 & & NS & $\mu g / L$ \\
\hline 1,2-Dichloroethane & WA & & & $\mathbf{U}$ & & 5 & & 5 & $\mu g / L$ \\
\hline 1,2-Dichloroethane & WA & & NA & $\mathbf{U}$ & & 5 & & 5 & $\mu g / L$ \\
\hline 1,2-Dichloroethane & WA & & NA & $\mathbf{U}$ & & 5 & & 5 & $\mu g / L$ \\
\hline 1,2-Dichloroethylene (total) & WA & & & $\mathrm{U}$ & & 5 & & 7 & $\mu g / L$ \\
\hline 1,2-Dichloroethylene (total) & WA & & NA & $\mathbf{U}$ & & 5 & & 7 & $\mu g / L$ \\
\hline 1,2-Dichloroethylene (total) & WA & & NA & U & & 5 & & 7 & $\mu g / L$ \\
\hline 1,2-Dichloropropane & WA & & & $\mathrm{U}$ & & 5 & & 5 & $\mu g \Omega$ \\
\hline 1,2-Dichloropropane & WA & & NA & $\mathrm{U}$ & & 5 & & 5 & $\mu g / L$ \\
\hline 1,2-Dichloropropane & WA & & NA & U & & 5 & & 5 & $\mu g / \alpha$ \\
\hline 2-Hexanone & WA & & & U & & 10 & & NS & $\mu g / L$ \\
\hline 2-Hexanone & WA & & NA & $\mathbf{U}$ & & 10 & & NS & $\mu g / L$ \\
\hline 2-Hexanone & WA & & NA & $\mathrm{U}$ & & 10 & & NS & $\mu g / L$ \\
\hline 4-Methyl-2-pentanone & WA & & & $\mathbf{U}$ & & 10 & & NS & $\mu \mathrm{g} / \mathrm{L}$ \\
\hline 4-Methyl-2-pentanone & WA & & NA & $\mathbf{U}$ & & 10 & & NS & $\mu \mathrm{g} / \mathrm{L}$ \\
\hline 4-Methyl-2-pentanone & WA & & NA & $\mathbf{U}$ & & 10 & & NS & $\mu \mathrm{g} / \mathrm{L}$ \\
\hline Acetone & WA & & & & $\mathrm{v}$ & 11.8 & & NS & $\mu \mathrm{g} / \mathrm{L}$ \\
\hline Acetone & WA & & NA & $\mathbf{J}$ & $\mathrm{V}$ & 3.45 & & NS & $\mu g /$. \\
\hline Acetone & WA & & NA & $\mathbf{J}$ & $\mathrm{v}$ & 8.08 & & NS & $\mu g / L$ \\
\hline Acetonitrile & WA & & & U & & 20 & & NS & $\mu \mathrm{g} / \mathrm{L}$ \\
\hline Acetonitrile & WA & & NA & $\mathbf{U}$ & & 20 & & NS & $\mu g / L$ \\
\hline Acetonitrile & WA & & NA & $U$ & & 20 & & NS & \\
\hline Acidity & WA & & & & & 22 & & NS & $\Lambda$ \\
\hline
\end{tabular}


UQA007 Laboratory Analyses (cont'd)

\begin{tabular}{|c|c|c|c|c|c|c|c|c|}
\hline Analyte & Lab & Rep & Avg & Modifier & Result & Acc & Stand & Units \\
\hline Acrolein & WA & & & $\mathbf{U}$ & 10 & & NS & $\overline{\mu g / L}$ \\
\hline Acrolein & WA & & NA & $\mathbf{U}$ & 10 & & NS & $\mu \mathrm{g} / \mathrm{L}$ \\
\hline Acrolein & WA & & NA & $\mathbf{U}$ & 10 & & NS & $\mu \mathrm{g} / \mathrm{L}$ \\
\hline Acrylonitrile & WA & & & $\mathbf{U}$ & 10 & & NS & $\mu g / L$ \\
\hline Acrylonitrile & WA & & NA & $\mathbf{U}$ & 10 & & NS & $\mu g / L$ \\
\hline Acrylonitrile & WA & & NA & U & 10 & . & NS & $\mu_{\mathrm{g}} / \mathrm{L}$ \\
\hline Alkalinity & WA & & & $\mathbf{U}$ & 0.5 & & NS & $\mathrm{mg} / \mathrm{L}$ \\
\hline Allyl chloride & WA & & & $\mathbf{U}$ & 100 & & NS & $\mu g / L$ \\
\hline Allyl chloride & WA & & NA & $\mathbf{U}$ & 100 & & NS & $\mu g / L$ \\
\hline Allyl chloride & WA & & NA & $\mathbf{U}$ & 100 & & NS & $\mu g / L$ \\
\hline Aluminum & WA & & & $\mathbf{U}$ & 200 & & NS & $\mu g / L$ \\
\hline Aluminum & WA & & NA & $\mathbf{U}$ & 200 & & NS & $\mu g / L$ \\
\hline Antimony & WA & & & $\mathbf{U}$ & 60 & & 6 & $\mu g / L$ \\
\hline Antimony & WA & & NA & $\mathbf{U}$ & 60 & & 6 & $\mu \mathrm{g} / \mathrm{L}$ \\
\hline Arsenic & WA & & & $\mathbf{U}$ & 10 & & 50 & $\mu g / L$ \\
\hline Arsenic & WA & & NA & $\mathbf{U}$ & 10 & & 50 & $\mu g / L$ \\
\hline Barium & WA & & & $\mathbf{U}$ & 200 & & 2000 & $\mu g / L$ \\
\hline Barium & WA & & NA & $\mathbf{U}$ & 200 & & 2000 & $\mu g / L$ \\
\hline Benzene & WA & & & $\mathbf{U}$ & 5 & & 5 & $\mu g / L$ \\
\hline Beryllium & WA & & & $\mathbf{U}$ & 5 & & 4 & $\mu g / L$ \\
\hline Beryllium & WA & & NA & $\mathbf{U}$ & 5 & & 4 & $\mu g / L$ \\
\hline Bromodichloromethane & WA & & & $\mathbf{U}$ & 5 & & NS & $\mu g / L$ \\
\hline Bromodichloromethane & WA & & NA & $\mathbf{U}$ & 5 & & NS & $\mu g / L$ \\
\hline Bromodichloromethane & WA & & NA & $\mathbf{U}$ & 5 & & NS & $\mu g / L$ \\
\hline Bromoform & WA & & & $\mathbf{U}$ & 5 & & NS & $\mu g / L$ \\
\hline Bromoform & WA & & NA & $\mathbf{U}$ & 5 & & NS & $\mu \mathrm{g} / \mathrm{L}$ \\
\hline Bromoform & WA & & NA & U & 5 & & NS & $\mu g / L$ \\
\hline Cadmium & WA & & & $\mathbf{U}$ & 5 & & 5 & $\mu g / L$ \\
\hline Cadmium & WA & & NA & $\mathbf{U}$ & 5 & & 5 & $\mu g / L$ \\
\hline Calcium & WA & & & $\mathbf{U}$ & 5000 & & NS & $\mu \mathrm{g} / \mathrm{L}$ \\
\hline Calcium & WA & & NA & $\mathbf{U}$ & 5000 & & NS & $\mu g / L$ \\
\hline Carbon disulfide & WA & & & U & 5 & & NS & $\mu_{g} / L$ \\
\hline Carbon disulfide & WA & & NA & $\mathrm{U}$ & 5 & & NS & $\mu g / L$ \\
\hline Carbon disulfide & WA & & $\mathbf{N A}$ & $\mathbf{U}$ & 5 & & NS & $\mu g / L$ \\
\hline Carbon tetrachloride & WA & & & $\mathbf{U}$ & 5 & & 5 & $\mu \mathrm{g} / \mathrm{L}$ \\
\hline Carbon tetrachloride & WA & & NA & $\mathrm{U}$ & 5 & & 5 & $\mu \mathrm{g} / \mathrm{L}$ \\
\hline Carbon tetrachloride & WA & & NA & U & 5 & & 5 & $\mu \mathrm{g} / \mathrm{L}$ \\
\hline Cesium-137 & TM & & & U & 6.2 & & 200 & $\mathrm{pCi} / \mathrm{L}$ \\
\hline Chloride & WA & & & $\mathrm{U}$ & 0.25 & & NS & $\mathrm{mg} / \mathrm{L}$ \\
\hline Chlorobenzene & WA & & & $\mathrm{U}$ & 5 & & 100 & $\mu \mathrm{g} / \mathrm{L}$ \\
\hline Chloroethane & WA & & & $\mathrm{U}$ & 10 & & NS & $\mu g / L$ \\
\hline Chloroethane & WA & & NA & U & 10 & & NS & $\mu \mathrm{g} / \mathrm{L}$ \\
\hline Chloroethane . & WA & & NA & $\mathbf{U}$ & 10 & & NS & $\mu \mathrm{g} / \mathrm{L}$ \\
\hline Chloroform & WA & & & U & 5 & & 100 & $\mu \mathrm{g} / \mathrm{L}$ \\
\hline Chloroform & WA & & NA & $\mathrm{U}$ & 5 & & 100 & $\mu \mathrm{g} / \mathrm{L}$ \\
\hline Chloroform & WA & & NA & $\mathbf{U}$ & 5 & & 100 & $\mu \mathrm{g} / \mathrm{L}$ \\
\hline Chloroprene & WA & & & $\mathrm{U}$ & 20 & & NS & $\mu g / L$ \\
\hline Chloroprene & WA & & NA & $\mathrm{U}$ & 20 & & NS & $\mu \mathrm{g} / \mathrm{L}$ \\
\hline Chloroprene & WA & & NA & $\mathbf{U}$ & 20 & & NS & $\mu \mathrm{g} / \mathrm{L}$ \\
\hline Chromium & WA & & & $\mathbf{U}$ & 10 & & 100 & $\mu \mathrm{g} / \mathrm{L}$ \\
\hline Chromium & WA & & NA & U & 10 & & 100 & $\mu \mathrm{g} / \mathrm{L}$ \\
\hline cis-1,3-Dichloropropene & WA & & & $\mathrm{U}$ & 5 & & NS & $\mu \mathrm{g} / \mathrm{L}$ \\
\hline cis-1,3-Dichloropropene & WA & & NA & $\mathbf{U}$ & 5 & & NS & $\mu \mathrm{g} / \mathrm{L}$ \\
\hline cis-1,3-Dichloropropene & WA & & NA & $\mathbf{U}$ & 5 & & NS & $\mu \mathrm{g} / \mathrm{L}$ \\
\hline
\end{tabular}


UQA007 Laboratory Analyses (cont'd)

\begin{tabular}{|c|c|c|c|c|c|c|c|}
\hline Analyte & Lab & Rep & Modifier & Result & Acc & Stand & Units \\
\hline Cobalt & WA & & $\bar{U}$ & 50 & & NS & $\mu g / L$ \\
\hline Cobalt & WA & NA & U & 50 & & NS & $\mu g / L$ \\
\hline Copper & WA & & $\mathbf{U}$ & 25 & & 1000 & $\mu g / L$ \\
\hline Copper & WA & NA & $\mathbf{U}$ & 25 & & 1000 & $\mu g / L$ \\
\hline Dibromochloromethane & WA & & $\mathbf{U}$ & 5 & & NS & $\mu g / \mathrm{L}$ \\
\hline Dibromochloromethane & WA & NA & $\mathbf{U}$ & 5 & & NS & $\mu g / \mathrm{L}$ \\
\hline Dibromochloromethane & WA & NA & $\mathbf{U}$ & 5 & & NS & $\mu g / L$ \\
\hline Dichlorodifluoromethane & WA & & $\mathbf{U}$ & 10 & & NS & $\mu g / L$ \\
\hline Dichlorodifluoromethane & WA & NA & $\mathbf{U}$ & 10 & & NS & $\mu g / L$ \\
\hline Dichlorodifluoromethane & WA & NA & $\mathbf{U}$ & 10 & & NS & $\mu g / L$ \\
\hline Ethylbenzene & WA & & $\mathbf{U}$ & 5 & & 700 & $\mu g / L$ \\
\hline Ethylbenzene & WA & NA & $\mathbf{U}$ & 5 & & 700 & $\mu g / L$ \\
\hline Ethylbenzene & WA & NA & $\mathbf{U}$ & 5 & & 700 & $\mu g / L$ \\
\hline Fluoride & WA & & $\mathbf{U}$ & 0.1 & & 4 & $m g / L$ \\
\hline Gross alpha & TM & & $\mathbf{U}$ & 0.5 & & 15 & $\mathrm{pCi} / \mathrm{L}$ \\
\hline Iron & WA & & U & 100 & & 300 & $\mu g / L$ \\
\hline Iron & WA & NA & $\mathbf{U}$ & 100 & & 300 & $\mu \mathrm{g} / \mathrm{L}$ \\
\hline Isobutyl alcohol & WA & & $\mathbf{U}$ & 20 & & NS & $\mu g / L$ \\
\hline Isobutyl alcohol & WA & NA & $\mathbf{U}$ & 20 & & NS & $\mu \mathrm{g} / \mathrm{L}$ \\
\hline Isobutyl alcohol & WA & NA & U & 20 & & NS & $\mu g / L$ \\
\hline Lead & WA & & $\mathbf{U}$ & 3 & & 50 & $\mu_{g} / L$ \\
\hline Lead & WA & NA & $\mathbf{U}$ & 3 & & 50 & $\mu g / L$ \\
\hline Magnesium & WA & & $\mathbf{U}$ & 5000 & & NS & $\mu g / L$ \\
\hline Magnesium & WA & NA & $\mathbf{U}$ & 5000 & & NS & $\mu g / L$ \\
\hline Manganese & WA & & $\mathbf{U}$ & 15 & & 50 & $\mu g / L$ \\
\hline Manganese & WA & NA & $\mathbf{U}$ & 15 & & 50 & $\mu g / L$ \\
\hline Mercury & WA & & $\mathrm{U}$ & 0.2 & & 2 & $\mu g / L$ \\
\hline Methacrylonitrile & WA & & U & 20 & & NS & $\mu g / L$ \\
\hline Methacrylonitrile & WA & NA & $\mathbf{U}$ & 20 & & NS & $\mu g / L$ \\
\hline Methacrylonitrile & WA & NA & $\mathbf{U}$ & 20 & & NS & $\mu g / L$ \\
\hline Methyl bromide & WA & & U & 10 & & NS & $\mu g / L$ \\
\hline Methyl bromide & WA & NA & $\mathbf{U}$ & 10 & & NS & $\mu g / L$ \\
\hline Methyl bromide & WA & NA & $\mathbf{U}$ & 10 & & NS & $\mu g / L$ \\
\hline Methyl chloride & WA & & $\mathbf{U}$ & 10 & & NS & $\mu g / L$ \\
\hline Methyl chloride & WA & NA & $\mathbf{U}$ & 10 & & NS & $\mu g / L$ \\
\hline Methyl chloride & WA & NA & $\mathbf{U}$ & 10 & & NS & $\mu \mathrm{g} / \mathrm{L}$ \\
\hline Methyl ethyl ketone & WA & & U & 10 & & NS & $\mu g / L$ \\
\hline Methyl ethyl ketone & WA & NA & $\mathrm{U}$ & 10 & & NS & $\mu \mathrm{g} / \mathrm{L}$ \\
\hline Methyl ethyl ketone & WA & NA & $\mathbf{U}$ & 10 & & NS & $\mu g / L$ \\
\hline Methyl iodide & WA & & $\mathbf{U}$ & 10 & & NS & $\mu \mathrm{g} / \mathrm{L}$ \\
\hline Methyl iodide & WA & NA & $\mathbf{U}$ & 10 & & NS & $\mu \mathrm{g} / \mathrm{L}$ \\
\hline Methyl iodide & WA & NA & U & 10 & & NS & $\mu \mathrm{g} / \mathrm{L}$ \\
\hline Methylene bromide & WA & & $\mathbf{U}$ & 10 & & NS & $\mu g / L$ \\
\hline Methylene bromide & WA & NA & $\mathbf{U}$ & 10 & & NS & $\mu \mathrm{g} / \mathrm{L}$ \\
\hline Methylene bromide & WA & NA & $\mathrm{U}$ & 10 & & NS & $\mu \mathrm{g} / \mathrm{L}$ \\
\hline Methylene chloride & WA & & & 5 & & 5 & $\mu \mathrm{g} / \mathrm{L}$ \\
\hline Methylene chloride & WA & NA & & V $\quad 5.17$ & & 5 & $\mu g / L$ \\
\hline Methylene chloride & WA & NA & $\mathrm{U}$ & 4.97 & & 5 & $\mu \mathrm{g} / \mathrm{L}$ \\
\hline Nickel & WA & & U & 40 & & 100 & \\
\hline Nickel & WA & NA & U & 40 & & 100 & $\mu_{8}$ \\
\hline Nitrate as nitrogen & WA & & J & Q $\quad 0.105$ & & 10 & $\mathrm{mg}$ \\
\hline Nonvolatile beta & TM & & & 1.7 & 2.6 & 50 & $\mathrm{pCi} / \mathrm{I}$ \\
\hline Potassium & WA & & $\mathbf{U}$ & 5000 & & NS & $\mu g / L$ \\
\hline Potassium & WA & NA & $U$ & 5000 & & NS & $\pi$ \\
\hline
\end{tabular}


UQA007 Laboratory Analyses (cont'd)

\begin{tabular}{|c|c|c|c|c|c|c|c|c|}
\hline Analyte & Lab & Rep & Avg & Modifier & Result & Acc & Stand & Units \\
\hline Propionitrile & WA & & & $\bar{U}$ & 50 & & NS & $\mu \mathrm{g} / \mathrm{L}$ \\
\hline Propionitrile & WA & & NA & $\mathrm{U}$ & 50 & & NS & $\mu g / L$ \\
\hline Propionitrile & WA & & NA & $U$ & 50 & & NS & $\mu \mathrm{g} / \mathrm{L}$ \\
\hline Selenium & WA & & & $U$ & 5 & & 50 & $\mu \mathrm{g} / \mathrm{L}$ \\
\hline Selenium & WA & & NA & U & 5 & & 50 & $\mu \mathrm{g} / \mathrm{L}$ \\
\hline Silver & WA & & & $\mathrm{U}$ & 10 & . & 50 & $\mu \mathrm{g} / \mathrm{L}$ \\
\hline Silver & WA & & NA & U & 10 & & 50 & $\mu g / L$ \\
\hline Sodium & WA & & & $\mathbf{U}$ & 5000 & & NS & $\mu \mathrm{g} / \mathrm{L}$ \\
\hline Sodium & WA & & NA & $\mathrm{U}$ & 5000 & & NS & $\mu \mathrm{g} / \mathrm{L}$ \\
\hline Styrene & WA & & & $\mathbf{U}$ & 5 & & 100 & $\mu g / L$ \\
\hline Styrene & WA & & NA & $\mathbf{U}$ & 5 & & 100 & $\mu \mathrm{g} / \mathrm{L}$ \\
\hline Styrene & WA & & NA & $\mathrm{U}$ & 5 & & 100 & $\mu g / L$ \\
\hline Sulfate & WA & & & $\mathrm{U}$ & 2.5 & & 400 & $\mathrm{mg} / \mathrm{L}$ \\
\hline Sulfide & WA & & & $\mathrm{U}$ & 0.1 & & NS & $\mathrm{mg} / \mathrm{L}$ \\
\hline Tetrachloroethylene & WA & & & U & 5 & & 5 & $\mu g / L$ \\
\hline Tetrachloroethylene & WA & & NA & U & 5 & & 5 & $\mu \mathrm{g} / \mathrm{L}$ \\
\hline Tetrachloroethylene & WA & & NA & $\mathbf{U}$ & 5 & & 5 & $\mu g / L$ \\
\hline Thallium & WA & & & $\mathbf{U}$ & 10 & & 2 & $\mu g / L$ \\
\hline Thallium & WA & & NA & $\mathbf{U}$ & 10 & & 2 & $\mu g / L$ \\
\hline Toluene & WA & & & $\mathbf{U}$ & 5 & & 1000 & $\mu g / L$ \\
\hline Total hardness & WA & & & U & 2 & & NS & $\mathrm{mg} / \mathrm{L}$ \\
\hline Total organic carton & WA & & & & 0.616 & & NS & $\mathrm{mg} / \mathrm{L}$ \\
\hline Total suspended solids & WA & & $\mathbf{A}$ & & 7 & & NS & $\mathrm{mg} / \mathrm{L}$ \\
\hline trans-1,3-Dichloropropene & WA & & & U & $z$ & & NS & $\mu \mathrm{g} / \mathrm{L}$ \\
\hline trans-1,3-Dichloropropene & WA & & NA & U & 5 & & NS & $\mu g / L$ \\
\hline trans-1,3-Dichloropropene & WA & & NA & U & 5 & & NS & $\mu \mathrm{g} / \mathrm{L}$ \\
\hline trans-1,4-Dichloro-2-butene & WA & & & $\mathbf{U}$ & 100 & & NS & $\mu \mathrm{g} / \mathrm{L}$ \\
\hline trans-1,4-Dichloro-2-butene & WA & & NA & $\mathrm{U}$ & 100 & & NS & $\mu g / L$ \\
\hline trans-1,4-Dichloro-2-butene & WA & & NA & $\mathbf{U}$ & 100 & & NS & $\mu \mathrm{g} / \mathrm{L}$ \\
\hline Trichloroethylene & WA & & & U & 5 & & 5 & $\mu \mathrm{g} / \mathrm{L}$ \\
\hline Trichlorofluoromethane & WA & & & $\mathbf{U}$ & 5 & & NS & $\mu \mathrm{g} / \mathrm{L}$ \\
\hline Trichlorofluoromethane & WA & & NA & $\mathbf{U}$ & 5 & & NS & $\mu g / L$ \\
\hline Trichlorofluoromethane & WA & & NA & $\mathbf{U}$ & 5 & & NS & $\mu g / L$ \\
\hline Tritium & TM & & & $\mathrm{U}$ & 0.15 & & 20 & $\mathrm{pCi} / \mathrm{ml}$ \\
\hline Turbidity & WA & & A & & 0.42 & & NS & NTU \\
\hline Vanadium & WA & & & U & 50 & & NS & $\mu g / L$ \\
\hline Vanadium & WA & & NA & $\mathrm{U}$ & 50 & & NS & $\mu g / L$ \\
\hline Vinyl acetate & WA & & & $\mathbf{U}$ & 10 & & NS & $\mu g / L$ \\
\hline Vinyl acetate & WA & & NA & $\mathrm{U}$ & 10 & & NS & $\mu g / \mathrm{L}$ \\
\hline Vinyl acetate & WA & & NA & $\mathrm{U}$ & 10 & & NS & $\mu g / L$ \\
\hline Vinyl chloride & WA & & & $\mathrm{U}$ & 10 & & 2 & $\mu g / L$ \\
\hline Vinyl chloride & WA & & NA & U & 10 & & 2 & $\mu g / L$ \\
\hline Vinyl chloride . & WA & & NA & $U$ & 10 & & 2 & $\mu g / L$ \\
\hline Xylenes (total) & WA & & & $U$ & 5 & & 10000 & $\mu \mathrm{g} / \mathrm{L}$ \\
\hline Xylenes (total) & WA & & NA & $\mathrm{U}$ & 5 & & 10000 & $\mu g / L$ \\
\hline Xylenes (total) & WA & & NA & $\mathrm{U}$ & 5 & & 10000 & $\mu \mathrm{g} / \mathrm{L}$ \\
\hline Zinc & WA & & & $\mathrm{U}$ & 20 & & 5000 & $\mu g / L$ \\
\hline Zinc & WA & & NA & $\mathrm{U}$ & 20 & & 5000 & $\mu g / L$ \\
\hline
\end{tabular}


UQA008

(Equipment Blank - Shovel)

Field Measurements:

Sample Date: 8/26/93

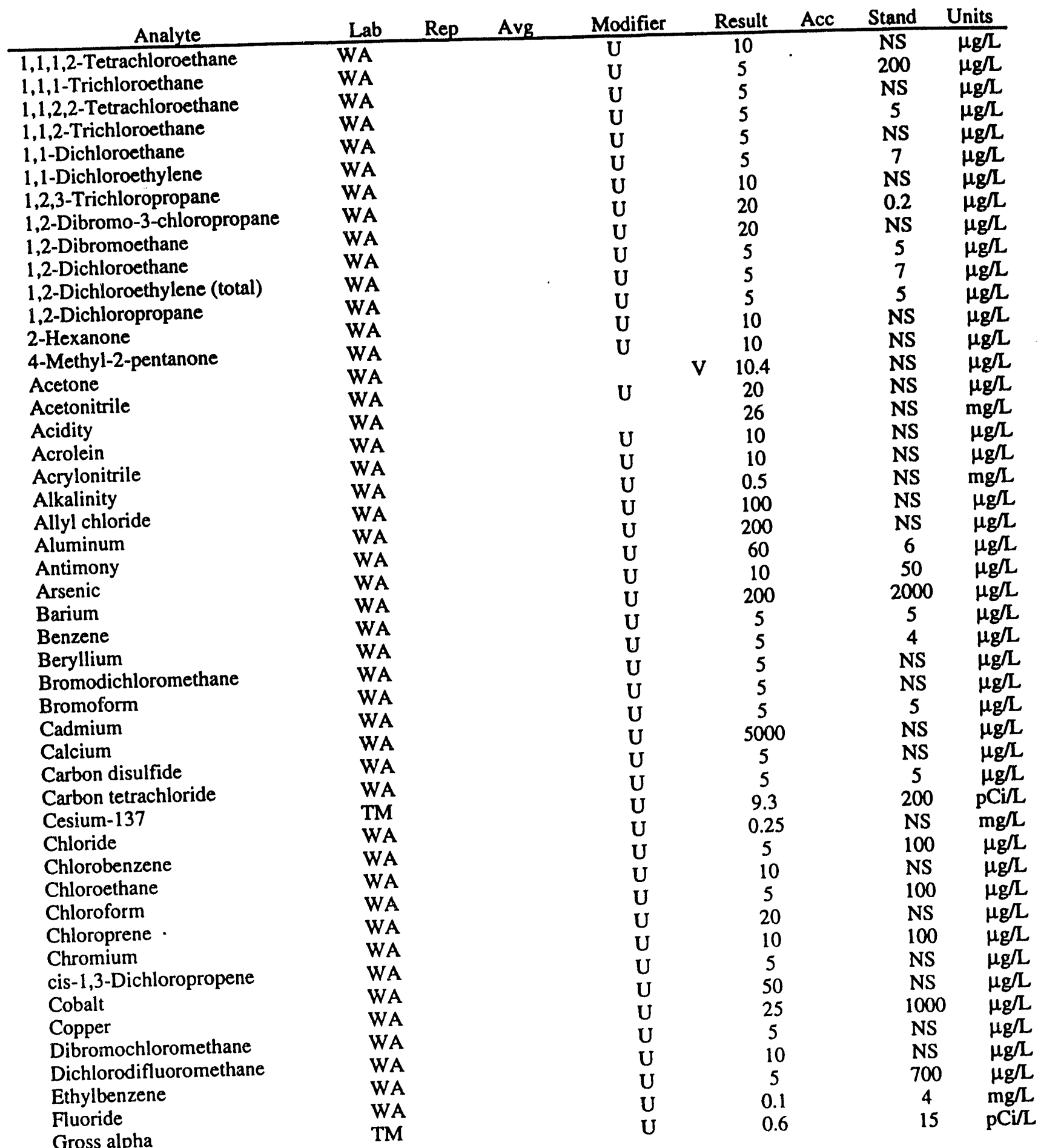


UQA008 Laboratory Analyses (cont'd)

\begin{tabular}{|c|c|c|c|c|c|c|c|c|c|}
\hline Analyte & Lab & Rep & Avg & Modifier & & Result & Acc & Stand & Units \\
\hline Iron & WA & & & $\bar{U}$ & & 100 & & 300 & $\mu g / \mathrm{L}$ \\
\hline Isobutyl alcohol & WA & & & $\mathbf{U}$ & & 20 & & NS & $\mu g / L$ \\
\hline Lead & WA & & & $\mathbf{U}$ & & 3 & & 50 & $\mu \mathrm{g} / \mathrm{L}$ \\
\hline Magnesium & WA & & & $\mathrm{U}$ & & 5000 & & NS & $\mu \mathrm{g} / \mathrm{L}$ \\
\hline Manganese & WA & & & $\mathbf{U}$ & & 15 & & 50 & $\mu g / L$ \\
\hline Mercury & WA & & & $\mathbf{U}$ & & 0.2 & . & 2 & $\mu \mathrm{g} / \mathrm{L}$ \\
\hline Methacrylonitrile & WA & & & $\mathbf{U}$ & & 20 & & NS & $\mu \mathrm{g} / \mathrm{L}$ \\
\hline Methyl bromide & WA & & & $\mathbf{U}$ & & 10 & & NS & $\mu g / L$ \\
\hline Methyl chloride & WA & & & U & & 10 & & NS & $\mu \mathrm{g} / \mathrm{L}$ \\
\hline Methyl ethyl ketone & WA & & & U & & 10 & & NS & $\mu g / L$ \\
\hline Methyl iodide & WA & & & $\mathbf{U}$ & & 10 & & NS & $\mu g / \mathrm{L}$ \\
\hline Methylene bromide & WA & & & $\mathbf{U}$ & & 10 & & NS & $\mu \mathrm{g} / \mathrm{L}$ \\
\hline Methylene chloride & WA & & & & $\mathbf{v}$ & 9.82 & & 5 & $\mu \mathrm{g} / \mathrm{L}$ \\
\hline Nickel & WA & & & $\mathbf{U}$ & & 40 & & 100 & $\mu g / L$ \\
\hline Nitrate as nitrogen & WA & & & J & $\mathbf{Q}$ & 0.133 & & 10 & $\mathrm{mg} / \mathrm{L}$ \\
\hline Nonvolatile beta & TM & & & $\mathbf{U}$ & & 0.8 & & 50 & $\mathrm{pCi} / \mathrm{L}$ \\
\hline Potassium & WA & & & U & & 5000 & & NS & $\mu g / L$ \\
\hline Propionitrile & WA & & & U & & 50 & & NS & $\mu g / L$ \\
\hline Selenium & WA & & & $\mathbf{U}$ & & 5 & & 50 & $\mu g / L$ \\
\hline Silver & WA & & & $\mathbf{U}$ & & 10 & & 50 & $\mu \mathrm{g} / \mathrm{L}$ \\
\hline Sodium & WA & & & $\mathbf{U}$ & & 5000 & & NS & $\mu g / L$ \\
\hline Styrene & WA & & & $\mathbf{U}$ & & 5 & & 100 & $\mu g / L$ \\
\hline Sulfate & WA & & & U & & 2.5 & & 400 & $\mathrm{mg} / \mathrm{L}$ \\
\hline Sulfide & WA & & & $\mathbf{U}$ & & 0.1 & & NS & $\mathrm{mg} / \mathrm{L}$ \\
\hline Tetrachloroethylene & WA & & & U & & 5 & & 5 & $\mu g / L$ \\
\hline Thallium & WA & & & $\mathbf{U}$ & & 10 & & 2 & $\mu \mathrm{g} / \mathrm{L}$ \\
\hline Toluene & WA & & & $\mathbf{U}$ & & 5 & & 1000 & $\mu \mathrm{g} / \mathrm{L}$ \\
\hline Total hardness & WA & & & $\mathbf{U}$ & & 1 & & NS & $\mathrm{mg} / \mathrm{L}$ \\
\hline Total organic carbon & WA & & & $\mathrm{U}$ & & 0.5 & & NS & $\mathrm{mg} / \mathrm{L}$ \\
\hline Total suspended solids & WA & & & & & 8 & & NS & $\mathrm{mg} / \mathrm{L}$ \\
\hline trans-1,3-Dichloropropene & WA & & & $\mathbf{U}$ & & 5 & & NS & $\mu \mathrm{g} / \mathrm{L}$ \\
\hline trans-1,4-Dichloro-2-butene & WA & & & $\mathrm{U}$ & & 100 & & NS & $\mu g / L$ \\
\hline Trichloroethylene & WA & & & $\mathrm{U}$ & & 5 & & 5 & $\mu g / h$ \\
\hline Trichlorofluoromethane & WA & & & $\mathbf{U}$ & & 5 & & NS & $\mu g / L$ \\
\hline Tritium & TM & & & $\mathrm{U}$ & & 0.17 & & 20 & $\mathrm{pCi} / \mathrm{ml}$ \\
\hline Turbidity & WA & & & $\mathbf{J}$ & $\mathbf{Q}$ & 0.79 & & NS & NTU \\
\hline Vanadium & WA & & & $\dot{U}$ & & 50 & & NS & \\
\hline Vinyl acetate & WA & & & $\mathrm{U}$ & & 10 & & NS & $\mu g / \mathrm{L}$ \\
\hline Vinyl chloride & WA & & & $\mathrm{U}$ & & 10 & & 2 & $\mu \mathrm{g} / \mathrm{L}$ \\
\hline Xylenes (total) & WA & & & $\mathrm{U}$ & & 5 & & 10000 & $\mu \mathrm{g} / \mathrm{L}$ \\
\hline Zinc & WA & & & U & & 20 & & 5000 & $\mu \mathrm{g} / \mathrm{L}$ \\
\hline
\end{tabular}




\section{UQA009}

(Equipment Blank - Tubing/Filter)

Field Measurements:

Sample Date: 9/2/93

\begin{tabular}{|c|c|c|c|c|c|c|c|}
\hline Analyte & Lab & Rep & Modifier & Result & Acc & Stand & Units \\
\hline 1,1,1,2-Tetrachloroethane & WA & & $\overline{\mathbf{U}}$ & 10 & & NS & $\overline{\mu g / L}$ \\
\hline 1,1,1-Trichloroethane & WA & & $\mathbf{U}$ & 5 & & 200 & $\mu \mathrm{g} / \mathrm{L}$ \\
\hline 1,1,2,2-Tetrachloroethane & WA & & $\mathbf{U}$ & 5 & & NS & $\mu g / L$ \\
\hline 1,1,2-Trichloroethane & WA & & $\mathbf{U}$ & 5 & & 5 & $\mu g / L$ \\
\hline 1,1-Dichloroethane & WA & & $\mathbf{U}$ & 5 & & NS & $\mu g / L$ \\
\hline 1,1-Dichloroethylene & WA & & $\mathbf{U}$ & 5 & & 7 & $\mu g / L$ \\
\hline 1,2,3-Trichloropropane & WA & & $\mathbf{U}$ & 10 & & NS & $\mu g / L$ \\
\hline 1,2-Dibromo-3-chloropropane & WA & & $\mathbf{U}$ & 20 & & 0.2 & $\mu g / L$ \\
\hline 1,2-Dibromoethane & WA & & $\mathbf{U}$ & 20 & & NS & $\mu g / L$ \\
\hline 1,2-Dichloroethane & WA & & $\mathbf{U}$ & 5 & & 5 & $\mu g / L$ \\
\hline 1,2-Dichloroethylene (total) & WA & & $\mathbf{U}$ & 5 & & 7 & $\mu g / L$ \\
\hline 1,2-Dichloropropane & WA & & $\mathbf{U}$ & 5 & & 5 & $\mu g / L$ \\
\hline 2-Hexanone & WA & & $\mathbf{U}$ & 10 & & NS & $\mu g / L$ \\
\hline 4-Methyl-2-pentanone & WA & & $\mathbf{U}$ & 10 & & NS & $\mu g / L$ \\
\hline Acetone & WA & & J & 6.46 & & NS & $\mu g / L$ \\
\hline Acetonitrile & WA & & $\mathbf{U}$ & 20 & & NS & $\mu g / L$ \\
\hline Acidity & WA & & & 30 & & NS & $\mathrm{mg} / \mathrm{L}$ \\
\hline Acrolein & WA & & $\mathbf{U}$ & 10 & & NS & $\mu g / L$ \\
\hline Acrylonitrile & WA & & $\mathbf{U}$ & 10 & & NS & $\mu g / L$ \\
\hline Alkalinity & WA & & $\mathbf{U}$ & 0.5 & & NS & $\mathrm{mg} / \mathrm{L}$ \\
\hline Allyl chloride & WA & & $\mathbf{U}$ & 100 & & NS & $\mu g / L$ \\
\hline Aluminum & WA & & $\mathbf{U}$ & 200 & & NS & $\mu g / L$ \\
\hline Antimony & WA & & $\mathbf{U}$ & 60 & & 6 & $\mu g / L$ \\
\hline Arsenic & WA & & $\mathbf{U}$ & 10 & & 50 & $\mu g / L$ \\
\hline Barium & WA & & $\mathbf{U}$ & 200 & & 2000 & $\mu g / L$ \\
\hline Benzene & WA & & $\mathbf{U}$ & 5 & & 5 & $\mu \mathrm{g} / \mathrm{L}$ \\
\hline Beryllium & WA & & $\mathbf{U}$ & 5 & & 4 & $\mu g / L$ \\
\hline Bromodichloromethane & WA & & $\mathbf{U}$ & 5 & & NS & $\mu g / L$ \\
\hline Bromoform & WA & & $\mathbf{U}$ & 5 & & NS & $\mu g / L$ \\
\hline Cadmium & WA & & $\mathbf{U}$ & 5 & & 5 & $\mu g / L$ \\
\hline Calcium & WA & & $\mathbf{U}$ & 5000 & & NS & $\mu g / L$ \\
\hline Carbon disulfide & WA & & $\mathbf{U}$ & 5 & & NS & $\mu g / L$ \\
\hline Carbon tetrachloride & WA & & $\mathbf{U}$ & 5 & & 5 & $\mu g / L$ \\
\hline Cesium-137 & TM & & $\mathbf{U}$ & 10 & & 200 & $\mathrm{pCi} / \mathrm{L}$ \\
\hline Chloride & WA & & $\mathbf{U}$ & 0.25 & & NS & $\mathrm{mg} / \mathrm{L}$ \\
\hline Chlorobenzene & WA & & $\mathbf{U}$ & 5 & & 100 & $\mu \mathrm{g} / \mathrm{L}$ \\
\hline Chloroethane & WA & & $\mathbf{U}$ & 10 & & NS & $\mu \mathrm{g} / \mathrm{L}$ \\
\hline Chloroform & WA & & $\mathbf{U}$ & 5 & & 100 & $\mu \mathrm{g} / \mathrm{L}$ \\
\hline Chloroprene . & WA & & $\mathbf{U}$ & 20 & & NS & $\mu \mathrm{g} / \mathrm{L}$ \\
\hline Chromium & WA & & $\mathbf{U}$ & 10 & & 100 & $\mu \mathrm{g} / \mathrm{L}$ \\
\hline cis-1,3-Dichloropropene & WA & & $\mathbf{U}$ & 5 & & NS & $\mu \mathrm{g} / \mathrm{L}$ \\
\hline Cobalt & WA & & $\mathbf{U}$ & 50 & & NS & $\mu g / L$ \\
\hline Copper & WA & & $\mathbf{U}$ & 25 & & 1000 & $\mu \mathrm{g} / \mathrm{L}$ \\
\hline Dibromochloromethane & WA & & $\mathbf{U}$ & 5 & & NS & $\mu \mathrm{g} / \mathrm{L}$ \\
\hline Dichlorodifluoromethane & WA & & $\mathbf{U}$ & 10 & & NS & $\mu \mathrm{g} / \mathrm{L}$ \\
\hline Ethylbenzene & WA & & $\mathbf{U}$ & 5 & & 700 & $\mu \mathrm{g} / \mathrm{L}$ \\
\hline Fluoride & WA & & $\mathbf{U}$ & 0.1 & & 4 & $\mathrm{mg} / \mathrm{L}$ \\
\hline Gross alpha & $\mathrm{TM}$ & & $\mathbf{U}$ & 0.6 & & 15 & $\mathrm{pCi} / \mathrm{L}$ \\
\hline
\end{tabular}


UQA009 Laboratory Analyses (cont'd)

\begin{tabular}{|c|c|c|c|c|c|c|c|c|c|}
\hline Analyte & Lab & Rep & Avg & Modifier & & Result & Acc & Stand & Units \\
\hline Iron & WA & & & $\mathrm{U}$ & & 100 & & 300 & $\mu g / L$ \\
\hline Isobutyl alcohol & WA & & & $\mathbf{U}$ & & 20 & & NS & $\mu g / L$ \\
\hline Lead & WA & & & $\mathbf{U}$ & & 3 & & 50 & $\mu g / L$ \\
\hline Magnesium & WA & & & $\mathbf{U}$ & & 5000 & & NS & $\mu g / L$ \\
\hline Manganese & WA & & & $\mathbf{U}$ & & 15 & & $50^{\circ}$ & $\mu \mathrm{g} / \mathrm{L}$ \\
\hline Mercury & WA & & & $\mathbf{U}$ & & 0.2 & . & 2 & $\mu \mathrm{g} / \mathrm{L}$ \\
\hline Methacrylonitrile & WA & & & $\mathbf{U}$ & & 20 & & NS & $\mu g / L$ \\
\hline Methyl bromide & WA & & & $\mathbf{U}$ & & 10 & & NS & $\mu g / L$ \\
\hline Methyl chloride & WA & & & $\mathbf{U}$ & & 10 & & NS & $\mu g / L$ \\
\hline Methyl ethyl ketone & WA & & & $\mathbf{U}$ & & 10 & & NS & $\mu g / L$ \\
\hline Methyl iodide & WA & & & $\mathbf{U}$ & & 10 & & NS & $\mu g / L$ \\
\hline Methylene bromide & WA & & & $\mathbf{U}$ & & 10 & & NS & $\mu g / L$ \\
\hline Methylene chloride & WA & & & $\mathbf{U}$ & & 5 & & 5 & $\mu \mathrm{g} / \mathrm{L}$ \\
\hline Nickel & .WA & & & $\mathbf{U}$ & & 40 & & 100 & $\mu g / L$ \\
\hline Nitrate as nitrogen & WA & & & J & $\mathbf{Q}$ & 0.037 & & 10 & $\mathrm{mg} / \mathrm{L}$ \\
\hline Nonvolatile beta & TM & & & & & 3.1 & 2.3 & 50 & $\mathrm{pCi} / \mathrm{L}$ \\
\hline Potassium & WA & & & $\mathbf{U}$ & & 5000 & & NS & $\mu g / L$ \\
\hline Propionitrile & WA & & & $\mathbf{U}$ & & 50 & & NS & $\mu g / L$ \\
\hline Selenium & WA & & & $\mathbf{U}$ & & 5 & & 50 & $\mu g / L$ \\
\hline Silver & WA & & & $\mathbf{U}$ & & 10 & & 50 & $\mu g / L$ \\
\hline Sodium & WA & & & $\mathbf{U}$ & & 5000 & & NS & $\mu g / L$ \\
\hline Styrene & WA & & & $\mathbf{U}$ & & 5 & & 100 & $\mu g / L$ \\
\hline Sulfate & WA & & & $\mathbf{U}$ & & 2.5 & & 400 & $\mathrm{mg} / \mathrm{L}$ \\
\hline Sulfide & WA & & & $\mathbf{U}$ & & 0.1 & & NS & $\mathrm{mg} / \mathrm{L}$ \\
\hline Tetrachloroethylene & WA & & & $\mathbf{U}$ & & 5 & & 5 & $\mu g / L$ \\
\hline Thallium & WA & & & $\mathbf{U}$ & & 10 & & 2 & $\mu g / L$ \\
\hline Toluene & WA & & & $\mathrm{U}$ & & 5 & & 1000 & $\mu g / L$ \\
\hline Total hardness & WA & & & U & & 1 & & NS & $\mathrm{mg} / \mathrm{L}$ \\
\hline Total organic carbon & WA & & & $\mathbf{U}$ & & 0.5 & & NS & $\mathrm{mg} / \mathrm{L}$ \\
\hline Total suspended solids & WA & & & & & 8 & & NS & $\mathrm{mg} / \mathrm{L}$ \\
\hline trans-1,3-Dichloropropene & WA & & & $\mathbf{U}$ & & 5 & & NS & $\mu g / L$ \\
\hline trans-1,4-Dichloro-2-butene & WA & & & $\mathrm{U}$ & & 100 & & NS & $\mu g / L$ \\
\hline Trichloroethylene & WA & & & $\mathbf{U}$ & & 5 & & 5 & $\mu g / L$ \\
\hline Trichlorofluoromethane & WA & & & $\mathbf{U}$ & & 5 & & NS & $\mu g / L$ \\
\hline Tritium & $\mathrm{TM}$ & & & & & 38.76 & 1.31 & 20 & $\mathrm{pCi} / \mathrm{ml}$ \\
\hline Turbidity & WA & & & UJ & $Q$ & 0.2 & & NS & NTU \\
\hline Vanadium & WA & & & $\mathbf{U}$ & & 50 & & NS & $\mu g / L$ \\
\hline Vinyl acetate & WA & & & $\mathrm{U}$ & & 10 & & NS & $\mu g / L$ \\
\hline Vinyl chloride & WA & & & $\mathbf{U}$ & & 10 & & 2 & $\mu g / L$ \\
\hline Xylenes (total) & WA & & & $\mathbf{U}$ & & 5 & & 10000 & $\mu g / L$ \\
\hline Zinc & WA & & & $\mathbf{U}$ & & 20 & & 5000 & $\mu g / L$ \\
\hline
\end{tabular}


UQA010

(Equipment Blank - Tubing/Filter)

Field Measurements:

Sample Date: 9/1/93

\begin{tabular}{|c|c|c|c|c|c|c|c|}
\hline Analyte & Lab & Avg & Modifier & Result & Acc & Stand & Units \\
\hline $1,1,1,2-$ Tetrachloroethane & WA & & $\mathbf{U}$ & 10 & & NS & $\mu g / L$ \\
\hline 1,1,1,2-Tetrachloroethane & WA & NA & $\mathbf{U}$ & 10 & ${ }^{\circ}$ & NS & $\mu g / L$ \\
\hline 1,1,1,2-Tetrachloroethane & WA & NA & $\mathbf{U}$ & 10 & & NS & $\mu g / L$ \\
\hline 1,1,1-Trichloroethane & WA & & $\mathbf{U}$ & 5 & & 200 & $\mu g / L$ \\
\hline 1,1,1-Trichloroethane & WA & NA & $\mathbf{U}$ & 5 & & 200 & $\mu g / L$ \\
\hline 1,1,1-Trichloroethane & WA & NA & $\mathbf{U}$ & 5 & & 200 & $\mu g / L$ \\
\hline $1,1,2,2-$ Tetrachloroethane & WA & & $\mathbf{U}$ & 5 & & NS & $\mu g / L$ \\
\hline $1,1,2,2$-Tetrachloroethane & WA & $\mathbf{N A}$ & $\mathbf{U}$ & 5 & & NS & $\mu g / L$ \\
\hline 1,1,2,2-Tetrachloroethane & WA & NA & $\mathbf{U}$ & 5 & & NS & $\mu g / L$ \\
\hline 1,1,2-Trichloroethane & WA & & $\mathbf{U}$ & 5 & & 5 & $\mu g / L$ \\
\hline 1,1,2-Trichloroethane & WA & NA & $\mathbf{U}$ & 5 & & 5 & $\mu g / L$ \\
\hline 1,1,2-Trichloroethane & WA & NA & $\mathbf{U}$ & 5 & & 5 & $\mu g / L$ \\
\hline 1,1-Dichloroethane & WA & & $\mathbf{U}$ & 5 & & NS & $\mu g / L$ \\
\hline 1,1-Dichloroethane & WA & NA & $\mathbf{U}$ & 5 & & NS & $\mu g / L$ \\
\hline 1,1-Dichloroethane & WA & NA & $\mathbf{U}$ & 5 & & NS & $\mu g / L$ \\
\hline 1,1-Dichloroethylene & WA & & $\mathbf{U}$ & 5 & & 7 & $\mu g / L$ \\
\hline 1,2,3-Trichloropropane & WA & & $\mathbf{U}$ & 10 & & NS & $\mu g / L$ \\
\hline 1,2,3-Trichloropropane & WA & NA & $\mathbf{U}$ & 10 & & NS & $\mu \mathrm{g} / \mathrm{L}$ \\
\hline 1,2,3-Trichloropropane & WA & NA & $\mathbf{U}$ & 10 & & NS & $\mu g / L$ \\
\hline 1,2-Dibromo-3-chloropropane & WA & & $\mathbf{U}$ & 20 & & 0.2 & $\mu g / L$ \\
\hline 1,2-Dibromo-3-chloropropane & WA & NA & $\mathbf{U}$ & 20 & & 0.2 & $\mu g / L$ \\
\hline 1,2-Dibromo-3-chloropropane & WA & NA & $\mathbf{U}$ & 20 & & 0.2 & $\mu \mathrm{g} / \mathrm{L}$ \\
\hline 1,2-Dibromoethane & WA & & $\mathbf{U}$ & 20 & & NS & $\mu g / L$ \\
\hline 1,2-Dibromoethane & WA & NA & $\mathbf{U}$ & 20 & & NS & $\mu g / L$ \\
\hline 1,2-Dibromoethane & WA & NA & $\mathbf{U}$ & 20 & & NS & $\mu g / L$ \\
\hline 1,2-Dichloroethane & WA & & $\mathbf{U}$ & 5 & & 5 & $\mu g / L$ \\
\hline 1,2-Dichloroethane & WA & NA & $\mathbf{U}$ & 5 & & 5 & $\mu g / L$ \\
\hline 1,2-Dichloroethane & WA & NA & $\mathbf{U}$ & 5 & & 5 & $\mu g / L$ \\
\hline 1,2-Dichloroethylene (total) & WA & & $\mathbf{U}$ & 5 & & 7 & $\mu g / L$ \\
\hline 1,2-Dichloroethylene (total) & WA & NA & $\mathbf{U}$ & 5 & & 7 & $\mu \mathrm{g} / \mathrm{L}$ \\
\hline 1,2-Dichloroethylene (total) & WA & NA & $\mathbf{U}$ & 5 & & 7 & $\mu \mathrm{g} / \mathrm{L}$ \\
\hline 1,2-Dichloropropane & WA & & $\mathbf{U}$ & 5 & & 5 & $\mu \mathrm{g} / \mathrm{L}$ \\
\hline 1,2-Dichloropropane & WA & NA & $\mathbf{U}$ & 5 & & 5 & $\mu \mathrm{g} / \mathrm{L}$ \\
\hline 1,2-Dichloropropane & WA & NA & $\mathbf{U}$ & 5 & & 5 & $\mu g / L$ \\
\hline 2-Hexanone & WA & & $\mathbf{U}$ & 10 & & NS & $\mu \mathrm{g} / \mathrm{L}$ \\
\hline 2-Hexanone & WA & NA & $\mathbf{U}$ & 10 & & NS & $\mu \mathrm{g} / \mathrm{L}$ \\
\hline 2-Hexanone & WA & NA & $\mathbf{U}$ & 10 & & NS & $\mu \mathrm{g} / \mathrm{L}$ \\
\hline 4-Methyl-2-pentanone & WA & & $\mathbf{U}$ & 10 & & NS & $\mu g / L$ \\
\hline 4-Methyl-2-pentanone & WA & NA & $\mathbf{U}$ & 10 & & NS & $\mu g / L$ \\
\hline 4-Methyl-2-pentanone & WA & NA & $\mathbf{U}$ & 10 & & NS & $\mu \mathrm{g} /$ \\
\hline Acetone & WA & & & 10.4 & & NS & $\mu \mathrm{g} / \mathrm{L}$ \\
\hline Acetone & WA & NA & J & 7.76 & & NS & $\mu \mathrm{g} / \mathrm{L}$ \\
\hline Acetone & WA & NA & & 11 & & NS & $\mu \mathrm{g} / \mathrm{L}$ \\
\hline Acetonitrile & WA & & $\mathbf{U}$ & 20 & & NS & $\mu g / L$ \\
\hline Acetonitrile & WA & NA & $\mathbf{U}$ & 20 & & NS & $\mu g / L$ \\
\hline Acetonitrile & WA & NA & $\mathbf{U}$ & 20 & & NS & $\mu \mathrm{g} / \mathrm{L}$ \\
\hline Acidity & WA & & & 26 & & NS & $\mathrm{mg} / \mathrm{L}$ \\
\hline
\end{tabular}


UQA010 Laboratory Analyses (cont'd)

\begin{tabular}{|c|c|c|c|c|c|c|c|c|}
\hline Analyte & Lab & Rep & Avg & Modifier & Result & Acc & Stand & Units \\
\hline Acrolein & WA & & & $\mathbf{U}$ & 10 & & NS & $\mu \mathrm{g} / \mathrm{L}$ \\
\hline Acrolein & WA & & NA & $\mathbf{U}$ & 10 & & NS & $\mu \mathrm{g} / \mathrm{L}$ \\
\hline Acrolein & WA & & NA & $\mathbf{U}$ & 10 & & NS & $\mu g / L$ \\
\hline Acrylonitrile & WA & & & $\mathbf{U}$ & 10 & & NS & $\mu \mathrm{g} / \mathrm{L}$ \\
\hline Acrylonitrile & WA & & NA & $\mathbf{U}$ & 10 & & NS & $\mu \mathrm{g} / \mathrm{L}$ \\
\hline Acrylonitrile & WA & & NA & $\mathrm{U}$ & 10 & . & NS & $\mu \mathrm{g} / \mathrm{L}$ \\
\hline Alkalinity & WA & & & $\mathbf{U}$ & 0.5 & & NS & $\mathrm{mg} / \mathrm{L}$ \\
\hline Allyl chloride & WA & & & $\mathbf{U}$ & 100 & & NS & $\mu g / L$ \\
\hline Allyl chloride & WA & & NA & $\mathbf{U}$ & 100 & & NS & $\mu g / L$ \\
\hline Allyl chloride & WA & & NA & $\mathbf{U}$ & 100 & & NS & $\mu \mathrm{g} / \mathrm{L}$ \\
\hline Aluminum & WA & & & $\mathbf{U}$ & 200 & & NS & $\mu \mathrm{g} / \mathrm{L}$ \\
\hline Americium-241 & TM & & & & 29.8 & 5.7 & 6.34 & $\mathrm{pCi} / \mathrm{L}$ \\
\hline Antimony & WA & & & $\mathbf{U}$ & 60 & & 6 & $\mu g / L$ \\
\hline Arsenic & WA & & & $\mathbf{U}$ & 10 & & 50 & $\mu g / L$ \\
\hline Barium & WA & & & $\mathbf{U}$ & 200 & & 2000 & $\mu g / L$ \\
\hline Benzene & WA & & & $\mathbf{U}$ & 5 & & 5 & $\mu g / L$ \\
\hline Beryllium & WA & & & $\mathbf{U}$ & 5 & & 4 & $\mu g / L$ \\
\hline Bromodichloromethane & WA & & & $\mathbf{U}$ & 5 & & NS & $\mu g / L$ \\
\hline Bromodichloromethane & WA & & NA & $\mathbf{U}$ & 5 & & NS & $\mu g /$ \\
\hline Bromodichloromethane & WA & & NA & $\mathbf{U}$ & 5 & & NS & $\mu g / L$ \\
\hline Bromoform & WA & & & $\mathbf{U}$ & 5 & & NS & $\mu \mathrm{g} / \mathrm{L}$ \\
\hline Bromoform & WA & & NA & $\mathbf{U}$ & 5 & & NS & $\mu \mathrm{g} / \mathrm{L}$ \\
\hline Bromoform & WA & & NA & $\mathbf{U}$ & 5 & & NS & $\mu \mathrm{g} / \mathrm{L}$ \\
\hline Cadmium & WA & & & $\mathrm{U}$ & 5 & & 5 & $\mu \mathrm{g} / \mathrm{L}$ \\
\hline Calcium & WA & & & $\mathbf{U}$ & 5000 & & NS & $\mu \mathrm{g} / \mathrm{L}$ \\
\hline Carbon disulfide & WA & & & $\mathbf{U}$ & 5 & & NS & $\mu g / L$ \\
\hline Carbon disulfide & WA & & NA & $\mathbf{U}$ & 5 & & NS & $\mu g / L$ \\
\hline Carbon disulfide & WA & & NA & $\mathbf{U}$ & 5 & & NS & $\mu \mathrm{g} / \mathrm{L}$ \\
\hline Carbon tetrachloride & WA & & & $\mathbf{U}$ & 5 & & 5 & $\mu g / L$ \\
\hline Carbon tetrachloride & WA & & NA & $\mathbf{U}$ & 5 & & 5 & $\mu g / L$ \\
\hline Carbon tetrachloride & WA & & NA & $\mathbf{U}$ & 5 & & 5 & $\mu g / L$ \\
\hline Cesium-137 & TM & & & $\mathrm{U}$ & 9.8 & & 200 & $\mathrm{pCi} / \mathrm{L}$ \\
\hline Chloride & WA & & & $\mathbf{U}$ & 0.25 & & NS & $\mathrm{mg} / \mathrm{L}$ \\
\hline Chlorobenzene & WA & & & $\mathbf{U}$ & 5 & & 100 & $\mu g / L$ \\
\hline Chloroethane & WA & & & $\mathbf{U}$ & 10 & & NS & $\mu g / L$ \\
\hline Chloroethane & WA & & NA & $\mathbf{U}$ & 10 & & NS & $\mu g / L$ \\
\hline Chloroethane & WA & & NA & U & 10 & & NS & $\mu \mathrm{g} / \mathrm{L}$ \\
\hline Chloroform & WA & & & $\mathbf{U}$ & 5 & & 100 & $\mu \mathrm{g} / \mathrm{L}$ \\
\hline Chloroform & WA & & NA & $\mathbf{U}$ & 5 & & 100 & $\mu g / L$ \\
\hline Chloroform & WA & & NA & $\mathbf{U}$ & 5 & & 100 & $\mu g / L$ \\
\hline Chloroprene & WA & & & $\mathbf{U}$ & 20 & & NS & $\mu g / L$ \\
\hline Chloroprene & WA & & NA & $\mathbf{U}$ & 20 & & NS & $\mu g / L$ \\
\hline Chloroprene & WA & & NA & $\mathbf{U}$ & 20 & & NS & $\mu \mathrm{g} / \mathrm{L}$ \\
\hline Chromium & WA & & & $\mathbf{U}$ & 10 & & 100 & $\mu \mathrm{g} / \mathrm{L}$ \\
\hline cis-1,3-Dichloropropene & WA & & & $\mathbf{U}$ & 5 & & NS & $\mu g / L$ \\
\hline cis-1,3-Dichloropropene & WA & & NA & $\mathbf{U}$ & 5 & & NS & $\mu \mathrm{g} / \mathrm{L}$ \\
\hline cis-1,3-Dichloropropene & WA & & NA & $\mathbf{U}$ & 5 & & NS & $\mu \mathrm{g} / \mathrm{L}$ \\
\hline Cobalt & WA & & & $\mathbf{U}$ & 50 & & NS & $\mu \mathrm{g} / \mathrm{L}$ \\
\hline Copper & WA & & & $\mathbf{U}$ & 25 & & 1000 & $\mu g / L$ \\
\hline Dibromochloromethane & WA & & & $\mathbf{U}$ & 5 & & NS & $\mu \mathrm{g} / \mathrm{L}$ \\
\hline Dibromochloromethane & WA & & NA & $\mathrm{U}$ & 5 & & NS & $\mu \mathrm{g} / \mathrm{L}$ \\
\hline Dibromochloromethane & WA & & NA & $\mathbf{U}$ & 5 & & NS & $\mu \mathrm{g} / \mathrm{L}$ \\
\hline Dichlorodifluoromethane & WA & & & $\mathbf{U}$ & 10 & & NS & $\mu g / L$ \\
\hline Dichlorodifluoromethane & WA & & NA & $\mathbf{U}$ & 10 & & NS & $\mu \mathrm{g} / \mathrm{L}$ \\
\hline Dichlorodifluoromethane & WA & & NA & $\mathbf{U}$ & 10 & & NS & $\mu \mathrm{g} / \mathrm{L}$ \\
\hline
\end{tabular}


UQA010 Laboratory Analyses (cont'd)

\begin{tabular}{|c|c|c|c|c|c|c|c|c|}
\hline Analyte & Lab & Rep & Avg & Modifier & Result & Acc & Stand & Units \\
\hline Ethylbenzene & WA & & & $\mathbf{U}$ & 5 & & 700 & $\mu g / L$ \\
\hline Ethylbenzene & WA & & NA & $\mathbf{U}$ & 5 & & 700 & $\mu \mathrm{g} / \mathrm{L}$ \\
\hline Ethylbenzene & WA & & NA & $\mathrm{U}$ & 5 & & 700 & $\mu \mathrm{g} / \mathrm{L}$ \\
\hline Fluoride & WA & & & $\mathbf{U}$ & 0.1 & & 4 & $\mathrm{mg} / \mathrm{L}$ \\
\hline Gross alpha & TM & & & & 0.7 & 1 & 15 & $\mathrm{pCi} / \mathrm{L}$ \\
\hline Iron & WA & & & $\mathbf{U}$ & 100 & 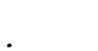 & 300 & $\mu g / L$ \\
\hline Isobutyl alcohol & WA & & & $\mathbf{U}$ & 20 & & NS & $\mu \mathrm{g} / \mathrm{L}$ \\
\hline Isobutyl alcohol & WA & & NA & $\mathbf{U}$ & 20 & & NS & $\mu g / L$ \\
\hline Isobutyl alcohol & WA & & NA & $\mathbf{U}$ & 20 & & NS & $\mu g / L$ \\
\hline Lead & WA & & & $\mathbf{U}$ & 3 & & 50 & $\mu g / L$ \\
\hline Magnesium & WA & & & $\mathbf{U}$ & 5000 & & NS & $\mu g / L$ \\
\hline Manganese & WA & & & $\mathbf{U}$ & 15 & & 50 & $\mu g / L$ \\
\hline Mercury & WA & & & $\mathbf{U}$ & 0.2 & & 2 & $\mu g / L$ \\
\hline Methacrylonitrile & WA & & & $\mathbf{U}$ & 20 & & NS & $\mu \mathrm{g} / \mathrm{L}$ \\
\hline Methacrylonitrile & WA & & NA & $\mathrm{U}$ & 20 & & NS & $\mu \mathrm{g} / \mathrm{L}$ \\
\hline Methacrylonitrile & WA & & NA & $\mathbf{U}$ & 20 & & NS & $\mu g / L$ \\
\hline Methyl bromide & WA & & & $\mathbf{U}$ & 10 & & NS & $\mu g / L$ \\
\hline Methyl bromide & WA & & NA & U & 10 & & NS & $\mu g / L$ \\
\hline Methyl bromide & WA & & NA & $\mathbf{U}$ & 10 & & NS & $\mu g / L$ \\
\hline Methyl chloride & WA & & & $\mathbf{U}$ & 10 & & NS & $\mu g / L$ \\
\hline Methyl chloride & WA & & NA & $\mathbf{U}$ & 10 & & NS & $\mu g / L$ \\
\hline Methyl chloride & WA & & NA & $\mathbf{U}$ & 10 & & NS & $\mu g / L$ \\
\hline Methyl ethyl ketone & WA & & & $\mathrm{U}$ & 10 & & NS & $\mu g / L$ \\
\hline Methyl ethyl ketone & WA & & NA & $\mathbf{U}$ & 10 & & NS & $\mu g / L$ \\
\hline Methyl ethyl ketone & WA & & NA & $\mathbf{U}$ & 10 & & NS & $\mu g / L$ \\
\hline Methyl iodide & WA & & & $\mathbf{U}$ & 10 & & NS & $\mu g / L$ \\
\hline Methyl iodide & WA & & NA & $\mathbf{U}$ & 10 & & NS & $\mu g / L$ \\
\hline Methyl iodide & WA & & NA & $\mathbf{U}$ & 10 & & NS & $\mu g / L$ \\
\hline Methylene bromide & WA & & & $\mathrm{U}$ & 10 & & NS & $\mu \mathrm{g} / \mathrm{L}$ \\
\hline Methylene bromide & WA & & NA & $\mathbf{U}$ & 10 & & NS & $\mu \mathrm{g} / \mathrm{L}$ \\
\hline Methylene bromide & WA & & NA & U & 10 & & NS & $\mu g / L$ \\
\hline Methylene chloride & WA & & & J & 3.6 & & 5 & $\mu g / L$ \\
\hline Methylene chloride & WA & & NA & J & 4.04 & & 5 & $\mu g / L$ \\
\hline Methylene chloride & WA & & NA & $\mathbf{J}$ & 3.51 & & 5 & $\mu g / L$ \\
\hline Nickel & WA & & & $\mathbf{U}$ & 40 & & 100 & $\mu g / L$ \\
\hline Nitrate as nitrogen & WA & & & J & Q $\quad 0.118$ & & 10 & $\mathrm{mg} / \mathrm{L}$ \\
\hline Nonvolatile beta & TM & & & $\mathbf{U}$ & 0.6 & & 50 & $\mathrm{pCi} / \mathrm{L}$ \\
\hline Potassium & WA & & & $\mathrm{U}$ & 5000 & & NS & $\mu g / L$ \\
\hline Propionitrile & WA & & & $\mathbf{U}$ & 50 & & NS & $\mu \mathrm{g} / \mathrm{L}$ \\
\hline Propionitrile & WA & & NA & $\mathbf{U}$ & 50 & & NS & $\mu g / L$ \\
\hline Propionitrile & WA & & NA & $\mathbf{U}$ & 50 & & NS & $\mu g / L$ \\
\hline Selenium & WA & & & $\mathrm{U}$ & 5 & & 50 & $\mu \mathrm{g} / \mathrm{L}$ \\
\hline Silver & WA & & & $\mathrm{U}$ & 10 & & 50 & $\mu \mathrm{g} / \mathrm{L}$ \\
\hline Sodium & WA & & & $\mathrm{U}$ & 5000 & & NS & $\mu \mathrm{g} / \mathrm{L}$ \\
\hline Styrene & WA & & & $\mathbf{U}$ & 5 & & 100 & $\mu \mathrm{g} / \mathrm{L}$ \\
\hline Styrene & WA & & NA & $\mathbf{U}$ & 5 & & 100 & $\mu \mathrm{g} / \mathrm{L}$ \\
\hline Styrene & WA & & NA & $\mathrm{U}$ & 5 & & 100 & $\mu g / L$ \\
\hline Sulfate & WA & & & $\mathbf{U}$ & 2.5 & & 400 & $\mathrm{mg} / \mathrm{L}$ \\
\hline Sulfide & WA & & & U & 0.1 & & NS & $\mathrm{mg} / \mathrm{L}$ \\
\hline Sulfide & WA & & NA & U & 0.1 & & NS & $\mathrm{mg} / \mathrm{L}$ \\
\hline Tetrachloroethylene & WA & & & U & 5 & & 5 & $\mu \mathrm{g} / \mathrm{L}$ \\
\hline Tetrachloroethylene & WA & & NA & $\mathrm{U}$ & 5 & & 5 & $\mu \mathrm{g} / \mathrm{L}$ \\
\hline Tetrachloroethylene & WA & & NA & $\mathrm{U}$ & 5 & & 5 & $\mu \mathrm{g} / \mathrm{L}$ \\
\hline Thallium & WA & & & $\mathbf{U}$ & 10 & & 2 & $\mu \mathrm{g} / \mathrm{L}$ \\
\hline Toluene & WA & & & $\mathbf{U}$ & 5 & & 1000 & $\mu \mathrm{g} / \mathrm{L}$ \\
\hline
\end{tabular}


Sampling And Analysis of Waser From Upper Three Runs And Its Wetlands Near Tank 16 and the Mixed Waste Management Facility

UQA010 Laboratory Analyses (cont'd)

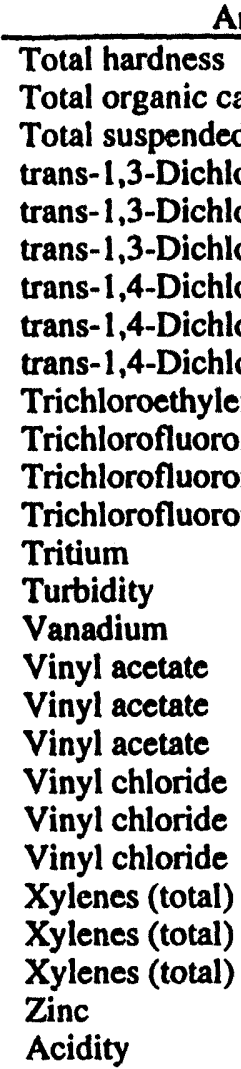

Analyte

carbon

ids

Total suspended solids

topropene

trans-1,4-Dichloro-2-butene

trans-1,4-Dichloro-2-butene

-1,4-Dichloro-2-butene

theromethane

The

Vanadium

Vinyl acetate

Vinyl acetate

Vinyl chloride

Vinyl chloride

Vinyl chloride

Xylenes (total)

Acidity

\begin{tabular}{|c|c|c|c|}
\hline \multirow{2}{*}{$\frac{\text { Lab }}{\text { WA }}$} & Rep & Avg & Modifi \\
\hline & & & $\mathbf{U}$ \\
\hline \multicolumn{4}{|l|}{ WA } \\
\hline \multicolumn{4}{|l|}{ WA } \\
\hline WA & & & $\mathbf{U}$ \\
\hline WA & & NA & $\mathbf{U}$ \\
\hline WA & & NA & $\mathbf{U}$ \\
\hline WA & & & $\mathbf{U}$ \\
\hline WA & & NA & $\mathbf{U}$ \\
\hline WA & & NA & $\mathbf{U}$ \\
\hline WA & & & $\mathbf{U}$ \\
\hline WA & & & $\mathbf{U}$ \\
\hline WA & & NA & $\mathbf{U}$ \\
\hline WA & & NA & $\mathbf{U}$ \\
\hline $\mathrm{TM}$ & & & $\mathbf{U}$ \\
\hline WA & & & $\mathbf{J}$ \\
\hline WA & & & $\mathbf{U}$ \\
\hline WA & & & $\mathbf{U}$ \\
\hline WA & & NA & $\mathbf{U}$ \\
\hline WA & & NA & $\mathbf{U}$ \\
\hline WA & & & $\mathbf{U}$ \\
\hline WA & & NA & $\mathbf{U}$ \\
\hline WA & & NA & $\mathbf{U}$ \\
\hline WA & & & $\mathbf{U}$ \\
\hline WA & & NA & $\mathbf{U}$ \\
\hline WA & & NA & $\mathbf{U}$ \\
\hline WA & & & $\mathbf{U}$ \\
\hline
\end{tabular}

$\begin{array}{llll}\text { WA QA11 } & 26 & \mathrm{mS} / \mathrm{L}\end{array}$

Result Acc Stand Units

NS $\mathrm{mg} / \mathrm{L}$

NS

9

$5 \quad$ NS $\mu g / L$

5 NS $\mu g / L$

5 . NS $\mu g / L$

$100 \quad N S \quad \mu g / L$

$100 \quad N S \quad \mu g / L$

$100 \quad$ NS $\mu g / L$

$5 \quad 5 \quad \mu g / L$

5 NS $\mu g / L$

5 NS $\mu g / L$

5 NS $\mu g / L$

$0.1420 \mathrm{pCi} / \mathrm{ml}$

$Q \quad 0.23 \quad$ NS NTU

$50 \quad N S \quad \mu g / L$

10 NS $\mu g / 2$

10 NS $\mu g / L$

10 NS $\mu g / L$

$102 \mathrm{Hg} / \mathrm{L}$

$10 \quad 2 \mu \mu g / L$

$10 \quad 2 \mu \mathrm{g} / \mathrm{L}$

$5 \quad 10000 \mu \mathrm{g} / \mathrm{L}$

$5 \quad 10000 \mu \mathrm{g} / \mathrm{L}$

$5 \quad 10000 \quad \mu g / L$

$20 \quad 5000 \quad \mu \mathrm{g} / \mathrm{L}$


UQA011

(Trip Blank)

Field Measurements:

Sample Date: 8/23/93

\begin{tabular}{|c|c|c|c|c|c|c|c|c|c|}
\hline Analyte & Lab & Rep & Avg & Modifier & & Result & Acc & Stand & Units \\
\hline Alkalinity & WA & & & & & 2 & & NS & $\mathrm{mg} / \mathrm{L}$ \\
\hline $\begin{array}{l}\text { Aluminum } \\
\text { Antimony }\end{array}$ & WA & & & U & & 200 & & NS & $\mu g / L$ \\
\hline $\begin{array}{l}\text { Antimony } \\
\text { Arsenic }\end{array}$ & WA & & & $\mathbf{U}$ & & 60 & & 6 & $\mu g / L$ \\
\hline $\begin{array}{l}\text { Arsenic } \\
\text { Barium }\end{array}$ & WA & & & $\mathbf{U}$ & & 10 & & 50 & $\mu g \Omega$ \\
\hline Barium & WA & & & $\mathbf{U}$ & & 200 & & 2000 & $\mu \mathrm{g} / \mathrm{L}$ \\
\hline Beryllium & WA & & & $\mathbf{U}$ & & 5 & & 4 & $\mu g / L$ \\
\hline $\begin{array}{l}\text { Cadmium } \\
\text { Calcium }\end{array}$ & WA & & & U & & 5 & & 5 & $\mu g / L$ \\
\hline $\begin{array}{l}\text { Calcium } \\
\text { Cesium-137 }\end{array}$ & WA & & & U & & 5000 & & NS & $\mu g / L$ \\
\hline Cesium-137 & TM & & & U & & 5.88 & & 200 & $\mathrm{pCi} / \mathrm{L}$ \\
\hline Chloride & WA & & & $\mathbf{U}$ & & 0.25 & & NS & $\mathrm{mg} / \mathrm{L}$ \\
\hline Chromium & WA & & & U & & 10 & & 100 & $\mu g / L$ \\
\hline Cobalt & WA & & & U & & 50 & & NS & $\mu g / L$ \\
\hline Copper & WA & & & U & & 25 & & 1000 & $\mu g / L$ \\
\hline Fluoride & WA & & & U & & 0.1 & & 4 & $\mathrm{mg} / \mathrm{L}$ \\
\hline Fluoride & WA & & NA & $U$ & & 0.1 & & 4 & $\mathrm{mgl}$ \\
\hline Gross alpha & TM & & & $\mathbf{U}$ & & 0.6 & & 15 & $\mathrm{pCi} / \mathrm{L}$ \\
\hline Iron & WA & & & U & & 100 & & 300 & $\mu g / L$ \\
\hline Lead & WA & & & U & & 3 & & 50 & $\mu g / L$ \\
\hline Magnesium & WA & & & U & & 5000 & & NS & $\mu g / L$ \\
\hline Manganese & WA & & & $\mathbf{U}$ & & 15 & & 50 & $\mu g / L$ \\
\hline Mercury & WA & & & $\mathrm{U}$ & & 0.2 & & 2 & $\mu g / L$ \\
\hline Nickel & WA & & & $\mathbf{U}$ & & 40 & & 100 & $\mu g / L$ \\
\hline Nitrate as nitrogen & WA & & & $\pi$ & Q & 0.02 & & 10 & $\mathrm{mg} / \mathrm{L}$ \\
\hline Nonvolatile beta & TM & & & & & 1.6 & 2.2 & 50 & $\mathrm{pCi} / \mathrm{L}$ \\
\hline Potassium & WA & & & U & & 5000 & & NS & $\mu \mathrm{g} / \mathrm{L}$ \\
\hline Selenium & WA & & & $\mathbf{U}$ & & 5 & & 50 & $\mu \mathrm{g} / \mathrm{L}$ \\
\hline Iver & WA & & & $\mathrm{U}$ & & 10 & & 50 & $\mu g / L$ \\
\hline Sodium & WA & & & $\mathbf{U}$ & & 5000 & & NS & $\mu g / L$ \\
\hline Sulfate & WA & & & U & $\mathbf{Q}$ & 2.5 & & 400 & $\mathrm{mg} / \mathrm{L}$ \\
\hline Sulfide & WA & & & $\mathbf{U}$ & $\mathbf{Q}$ & 0.1 & & NS & $\mathrm{mg} / \mathrm{L}$ \\
\hline Thallium & WA & & & $\mathbf{U}$ & & 10 & & 2 & $\mu g / L$ \\
\hline Total hardness & WA & & & U & & 1 & & NS & $\mathrm{mg} / \mathrm{L}$ \\
\hline Total organic carbon & WA & & & U & Q & 0.5 & & NS & $\mathrm{mg} / \mathrm{L}$ \\
\hline Total suspended solids & WA & & & JU & $\mathbf{Q}$ & 5 & & NS & $\mathrm{mg} / \mathrm{L}$ \\
\hline Total suspended solids & WA & & NA & JU & $Q$ & 5 & & NS & $\mathrm{mg} / \mathrm{L}$ \\
\hline Tritium & TM & & & U & & 0.17 & & 20 & $\mathrm{pCi} / \mathrm{ml}$ \\
\hline Turbidity & WA & & & JU & $\mathbf{Q}$ & 0.2 & & NS & NTU \\
\hline Vanadium & WA & & & $\mathrm{U}$ & & 50 & & NS & $\mu g / L$ \\
\hline Zinc & WA & & & $\mathbf{U}$ & & 20 & & 5000 & $\mu g$ \\
\hline
\end{tabular}


UVA001

(Volatile Trip Blank)

Sample Date: 8/25/93

Associated Station IDs: UTR01 1, UTR024, UTR029, UTR033

\begin{tabular}{|c|c|c|c|c|c|c|c|c|}
\hline Analyte & Lab & Avg & Modifier & & Result & Acc & Stand & Units \\
\hline 1,1,1,2-Tetrachloroethane & WA & & $\mathbf{U}$ & , & 10 & & NS & $\overline{\mu g / L}$ \\
\hline 1,1,1-Trichloroethane & WA & & $\mathbf{U}$ & & 5 & . & 200 & $\mu g / L$ \\
\hline 1,1,2,2-Tetrachloroethane & WA & & $\mathbf{U}$ & & 5 & & NS & $\mu g / L$ \\
\hline 1,1,2-Trichloroethane & WA & & $\mathbf{U}$ & & 5 & & 5 & $\mu g / L$ \\
\hline 1,1-Dichloroethane & WA & & $\mathbf{U}$ & & 5 & & NS & $\mu g / L$ \\
\hline 1,1-Dichloroethylene & WA & & $\mathbf{U}$ & & 5 & & 7 & $\mu g / L$ \\
\hline 1,2,3-Trichloropropane & WA & & $\mathbf{U}$ & & 10 & & NS & $\mu g / L$ \\
\hline 1,2-Dibromo-3-chloropropane & WA & & $\bar{U}$ & & 20 & & 0.2 & $\mu g / L$ \\
\hline 1,2-Dibromoethane & WA & & $\mathbf{U}$ & & 20 & & NS & $\mu g / L$ \\
\hline 1,2-Dichloroethane & WA & & $\mathbf{U}$ & & 5 & & 5 & $\mu g / L$ \\
\hline 1,2-Dichloroethylene (total) & WA & & $\mathbf{U}$ & & 5 & & 7 & $\mu g / L$ \\
\hline 1,2-Dichloropropane & WA & & $\mathbf{U}$ & & 5 & & 5 & $\mu g / L$ \\
\hline 2-Hexanone & WA & & $\mathbf{U}$ & & 10 & & NS & $\mu g / L$ \\
\hline 4-Methyl-2-pentanone & WA & & $\mathbf{U}$ & & 10 & & NS & $\mu \mathrm{g} / \mathrm{L}$ \\
\hline Acetone & WA & & & $\mathbf{V}$ & 15.8 & & NS & $\mu g / L$ \\
\hline Acetonitrile & WA & & $\mathbf{U}$ & & 20 & & NS & $\mu \mathrm{g} / \mathrm{L}$ \\
\hline Acrolein & WA & & $\mathbf{U}$ & & 10 & & NS & $\mu \mathrm{g} / \mathrm{L}$ \\
\hline Acrylonitrile & WA & & $\mathbf{U}$ & & 10 & & NS & $\mu g / L$ \\
\hline Allyl chloride & WA & & $\mathbf{U}$ & & 100 & & NS & $\mu \mathrm{g} / \mathrm{L}$ \\
\hline Benzene & WA & & $\mathbf{U}$ & & 5 & & 5 & $\mu \mathrm{g} / \mathrm{L}$ \\
\hline Bromodichloromethane & WA & & $\mathbf{U}$ & & 5 & & NS & $\mu g / L$ \\
\hline Bromoform & WA & & $\mathbf{U}$ & & 5 & & NS & $\mu \mathrm{g} / \mathrm{L}$ \\
\hline Carbon disulfide & WA & & $\mathbf{U}$ & & 5 & & NS & $\mu g / L$ \\
\hline Carbon tetrachloride & WA & & $\mathbf{U}$ & & 5 & & 5 & $\mu g / L$ \\
\hline Chlorobenzene & WA & & $\mathbf{U}$ & & 5 & & 100 & $\mu \mathrm{g} / \mathrm{L}$ \\
\hline Chloroethane & WA & & $\mathbf{U}$ & & 10 & & NS & $\mu g / L$ \\
\hline Chloroform & WA & & $\mathbf{U}$ & & 5 & & 100 & $\mu g / L$ \\
\hline Chloroprene & WA & & $\mathbf{U}$ & & 20 & & NS & $\mu g / L$ \\
\hline cis-1,3-Dichloropropene & WA & & $\mathbf{U}$ & & 5 & & NS & $\mu g / L$ \\
\hline Dibromochloromethane & WA & & $\mathbf{U}$ & & 5 & & NS & $\mu g / L$ \\
\hline Dichlorodifluoromethane & WA & & $\mathbf{U}$ & & 10 & & NS & $\mu \mathrm{g} / \mathrm{L}$ \\
\hline Ethylbenzene & WA & & $\mathbf{U}$ & & 5 & & 700 & $\mu \mathrm{g} / \mathrm{L}$ \\
\hline Isobutyl alcohol & WA & & $\mathbf{U}$ & & 20 & & NS & $\mu \mathrm{g} / \mathrm{L}$ \\
\hline Methacrylonitrile & WA & & $\mathbf{U}$ & & 20 & & NS & $\mu \mathrm{g} / \mathrm{L}$ \\
\hline Methyl bromide & WA & & $\mathbf{U}$ & & 10 & & NS & $\mu \mathrm{g} / \mathrm{L}$ \\
\hline Methyl chloride & WA & & $\mathbf{U}$ & & 10 & & NS & $\mu \mathrm{g} / \mathrm{L}$ \\
\hline Methyl ethyl ketone & WA & & $\mathbf{U}$ & & 10 & & NS & $\mu g / L$ \\
\hline Methyl iodide & WA & & $\mathbf{U}$ & & 10 & & NS & $\mu \mathrm{g} / \mathrm{L}$ \\
\hline Methylene bromide & WA & & $\mathbf{U}$ & & 10 & & NS & $\mu g / L$ \\
\hline Methylene chloride & WA & & & V & 8.82 & & 5 & $\mu g / L$ \\
\hline Propionitrile & WA & & $\mathbf{U}$ & & 50 & & NS & $\mu \mathrm{g} / \mathrm{L}$ \\
\hline Styrene & WA & & $\mathbf{U}$ & & 5 & & 100 & $\mu \mathrm{g} / \mathrm{L}$ \\
\hline Tetrachloroethylene & WA & & $\mathbf{U}$ & & 5 & & 5 & $\mu \mathrm{g} / \mathrm{L}$ \\
\hline Toluene & WA & & $\mathbf{U}$ & & 5 & & 1000 & $\mu \mathrm{g} / \mathrm{L}$ \\
\hline trans-1,3-Dichloropropene & WA & & $\mathbf{U}$ & & 5 & & NS & $\mu \mathrm{g} / \mathrm{L}$ \\
\hline trans-1,4-Dichloro-2-butene & WA & & $\mathbf{U}$ & & 100 & & NS & $\mu \mathrm{g} \Omega$ \\
\hline Trichloroethylene & WA & & $\mathbf{U}$ & & 5 & & 5 & $\mu \mathrm{g} / \mathrm{L}$ \\
\hline Trichlorofluoromethane & WA & & $\mathbf{U}$ & & 5 & & NS & $\mu g / L$ \\
\hline Vinyl acetate & WA & & $\mathbf{U}$ & & 10 & & NS & $\mu \mathrm{g} / \mathrm{L}$ \\
\hline Vinyl chloride & WA & & $\mathbf{U}$ & & 10 & & 2 & $\mu \mathrm{g} / \mathrm{L}$ \\
\hline Xylenes (total) & WA & & $\mathbf{U}$ & & 5 & & 10000 & $\mu \mathrm{g} / \mathrm{L}$ \\
\hline
\end{tabular}


UVA002

(Volatile Trip Blank)

Sample Date: $8 / 25 / 93$

Associated Station IDs: UTR036, UTR042, UTR045

\begin{tabular}{|c|c|c|c|c|c|c|c|c|}
\hline Analyte & Lab & Avg & Modifier & & Result & Acc & Stand & Units \\
\hline 1,1,1,2-Tetrachloroethane & WA & & $\bar{U}$ & & 10 & & NS & $\mu g / L$ \\
\hline 1,1,1-Trichloroethane & WA & & $\mathbf{U}$ & & 5 & & 200 & $\mu_{B} / L$ \\
\hline 1,1,2,2-Tetrachloroethane & WA & & $\mathbf{U}$ & & 5 & $\cdot$ & NS & $\mu g / L$ \\
\hline 1,1,2-Trichloroethane & WA & & $\mathbf{U}$ & & 5 & & 5 & $\mu g / L$ \\
\hline 1,1-Dichloroethane & WA & & $\mathbf{U}$ & & 5 & & NS & $\mu g / L$ \\
\hline 1,1-Dichloroethylene & WA & & $\mathbf{U}$ & & 5 & & 7 & $\mu g / L$ \\
\hline 1,2,3-Trichloropropane & WA & & $\mathbf{U}$ & & 10 & & NS & $\mu g / L$ \\
\hline 1,2-Dibromo-3-chloropropane & WA & & $\mathbf{U}$ & & 20 & & 0.2 & $\mu g / L$ \\
\hline 1,2-Dibromoethane & WA & & $\mathbf{U}$ & & 20 & & NS & $\mu g / L$ \\
\hline 1,2-Dichloroethane & WA & & $\mathbf{U}$ & & 5 & & 5 & $\mu g / L$ \\
\hline 1,2-Dichloroethylene (total) & WA & & U & & 5 & & 7 & $\mu g / L$ \\
\hline 1,2-Dichloropropane & WA & & $\mathbf{U}$ & & 5 & & 5 & $\mu g / L$ \\
\hline 2-Hexanone & WA & & $\mathbf{U}$ & & 10 & & NS & $\mu g / L$ \\
\hline 4-Methyl-2-pentanone & WA & & $\mathbf{U}$ & & 10 & & NS & $\mu g / L$ \\
\hline Acetone & WA & & & $\mathbf{V}$ & 12.5 & & NS & $\mu \& \Omega$ \\
\hline Acetonitrile & WA & & $\mathbf{U}$ & & 20 & & NS & $\mu \mathrm{g} / \mathrm{L}$ \\
\hline Acrolein & WA & & $\mathbf{U}$ & & 10 & & NS & $\mu g / L$ \\
\hline Acrylonitrile & WA & & $\mathbf{U}$ & & 10 & & NS & $\mu g / L$ \\
\hline Allyl chloride & WA & & $\mathbf{U}$ & & 100 & & NS & $\mu g / L$ \\
\hline Benzene & WA & & $\mathbf{U}$ & & 5 & & 5 & $\mu g / L$ \\
\hline Bromodichloromethane & WA & & $\mathbf{U}$ & & 5 & & NS & $\mu g / L$ \\
\hline Bromoform & WA & & $\mathbf{U}$ & & 5 & & NS & $\mu g / L$ \\
\hline Carbon disulfide & WA & & $\mathbf{U}$ & & 5 & & NS & $\mu \mathrm{g} / \mathrm{L}$ \\
\hline Carbon tetrachloride & WA & & $\mathbf{U}$ & & 5 & & 5 & $\mu g / L$ \\
\hline Chlorobenzene & WA & & $\mathbf{U}$ & & 5 & & 100 & $\mu g / L$ \\
\hline Chloroethane & WA & & $\mathbf{U}$ & & 10 & & NS & $\mu g / L$ \\
\hline Chloroform & WA & & $\mathbf{U}$ & & 5 & & 100 & $\mu \mathrm{g} / \mathrm{L}$ \\
\hline Chloroprene & WA & & U & & 20 & & NS & $\mu g / L$ \\
\hline cis-1,3-Dichloropropene & WA & & $\mathbf{U}$ & & 5 & & NS & $\mu \mathrm{g} / \mathrm{L}$ \\
\hline Dibromochloromethane & WA & & U & & 5 & & NS & $\mu g / L$ \\
\hline Dichlorodifluoromethane & WA & & $\mathbf{U}$ & & 10 & & NS & $\mu g / L$ \\
\hline Ethylbenzene & WA & & $\mathbf{U}$ & & 5 & & 700 & $\mu g / L$ \\
\hline Isobutyl alcohol & WA & & $\mathrm{U}$ & & 20 & & NS & $\mu g / L$ \\
\hline Methacrylonitrile & WA & & $\mathrm{U}$ & & 20 & & NS & $\mu g / L$ \\
\hline Methyl bromide & WA & & U & & 10 & & NS & $\mu g / L$ \\
\hline Methyl chloride & WA & & $\mathrm{U}$ & & 10 & & NS & $\mu g / L$ \\
\hline Methyl ethyl ketone & WA & & $\mathrm{U}$ & & 10 & & NS & $\mu \mathrm{g} / \mathrm{L}$ \\
\hline Methyl iodide & WA & & $\mathbf{U}$ & & 10 & & NS & $\mu \mathrm{g} / \mathrm{L}$ \\
\hline Methylene bromide & WA & & U & & 10 & & NS & $\mu \mathrm{g} / \mathrm{L}$ \\
\hline Methylene chloride & WA & & & $\mathbf{V}$ & 8.7 & & 5 & $\mu g / L$ \\
\hline Propionitrile & WA & & $\mathbf{U}$ & & 50 & & NS & $\mu g / L$ \\
\hline Styrene & WA & & $\mathbf{U}$ & & 5 & & 100 & $\mu g / L$ \\
\hline Tetrachloroethylene & WA & & $\mathbf{U}$ & & 5 & & 5 & $\mu \mathrm{g} / \mathrm{L}$ \\
\hline Toluene & WA & & U & & 5 & & 1000 & $\mu \mathrm{g} / \mathrm{L}$ \\
\hline trans-1,3-Dichloropropene & WA & & $\mathbf{U}$ & & 5 & & NS & $\mu \mathrm{g} / \mathrm{L}$ \\
\hline trans-1,4-Dichloro-2-butene & WA & & $\mathbf{U}$ & & 100 & & NS & $\mu g / L$ \\
\hline Trichloroethylene & WA & & $\mathbf{U}$ & & 5 & & 5 & $\mu g / L$ \\
\hline Trichlorofluoromethane & WA & & $\mathbf{U}$ & & 5 & & NS & $\mu \mathrm{g} / \mathrm{L}$ \\
\hline Vinyl acetate & WA & & $\mathbf{U}$ & & 10 & & NS & $\mu g / L$ \\
\hline Vinyl chloride & WA & & $U$ & & 10 & & 2 & $\mu g / L$ \\
\hline Xylenes (total) & WA & & $\mathbf{U}$ & & 5 & & 10000 & $\mu g /$ \\
\hline
\end{tabular}




\section{UVA003}

(Volatile Trip Blank)

Sample Date: 8/25/93

Associated Station IDs: UQA001, UQA002, UQA006

\begin{tabular}{|c|c|c|c|c|c|c|c|c|}
\hline Analyte & Lab & Avg & Modifier & & Result & Acc & Stand & Units \\
\hline 1,1,1,2-Tetrachloroethane & WA & & $\bar{U}$ & & 10 & & NS & $\mu g / L$ \\
\hline 1,1,1-Trichloroethane & WA & & $\mathbf{U}$ & & 5 & . & 200 & $\mu g / L$ \\
\hline 1,1,2,2-Tetrachloroethane & WA & & $\mathbf{U}$ & & 5 & & NS & $\mu g / L$ \\
\hline 1,1,2-Trichloroethane & WA & & U & & 5 & & 5 & $\mu g / L$ \\
\hline 1,1-Dichloroethane & WA & & $\mathbf{U}$ & & 5 & & NS & $\mu g / L$ \\
\hline 1,1-Dichloroethylene & WA & & $\mathbf{U}$ & & 5 & & 7 & $\mu g / L$ \\
\hline 1,2,3-Trichloropropane & WA & & $\mathbf{U}$ & & 10 & & NS & $\mu g / L$ \\
\hline 1,2-Dibromo-3-chloropropane & WA & & $\mathbf{U}$ & & 20 & & 0.2 & $\mu g /$ \\
\hline 1,2-Dibromoethane & WA & & $\mathbf{U}$ & & 20 & & NS & $\mu g / L$ \\
\hline 1,2-Dichloroethane & WA & & $\mathbf{U}$ & & 5 & & 5 & $\mu g / L$ \\
\hline 1,2-Dichloroethylene (total) & WA & & $\mathbf{U}$ & & 5 & & 7 & $\mu \mathrm{g} \Omega$ \\
\hline 1,2-Dichloropropane & WA & & $\mathbf{U}$ & & 5 & & 5 & $\mu g / L$ \\
\hline 2-Hexanone & WA & & $\mathbf{U}$ & & 10 & & NS & $\mu \mathrm{g} / \mathrm{L}$ \\
\hline 4-Methyl-2-pentanone & WA & & $\mathbf{U}$ & & 10 & & NS & $\mu g / L$ \\
\hline Acetone & WA & & & V & 10.7 & & NS & $\mu g / L$ \\
\hline Acetonitrile & WA & & $\mathbf{U}$ & & 20 & & NS & $\mu g / L$ \\
\hline Acrolein & WA & & $\mathbf{U}$ & & 10 & & NS & $\mu g / L$ \\
\hline Acrylonitrile & WA & & $\mathbf{U}$ & & 10 & & NS & $\mu g / L$ \\
\hline Allyl chloride & WA & & $\mathbf{U}$ & & 100 & & NS & $\mu g / L$ \\
\hline Benzene & WA & & $\mathbf{U}$ & & 5 & & 5 & $\mu g / L$ \\
\hline Bromodichloromethane & WA & & $\mathbf{U}$ & & 5 & & NS & $\mu g / 2$ \\
\hline Bromoform & WA & & $\mathbf{U}$ & & 5 & & NS & $\mu g / L$ \\
\hline Carbon disulfide & WA & & $\mathrm{U}$ & & 5 & & NS & $\mu \mathrm{g} / \mathrm{L}$ \\
\hline Carbon tetrachloride & WA & & $\mathbf{U}$ & & 5 & & 5 & $\mu \mathrm{g} / \mathrm{L}$ \\
\hline Chlorobenzene & WA & & $\mathbf{U}$ & & 5 & & 100 & $\mu g / L$ \\
\hline Chloroethane & WA & & $\mathbf{U}$ & & 10 & & NS & $\mu g / L$ \\
\hline Chloroform & WA & & $\mathbf{U}$ & & 5 & & 100 & $\mu g / L$ \\
\hline Chloroprene & WA & & U & & 20 & & NS & $\mu g / L$ \\
\hline cis-1,3-Dichloropropene & WA & & $\mathbf{U}$ & & 5 & & NS & $\mu \mathrm{g} / \mathrm{L}$ \\
\hline Dibromochloromethane & WA & & $\mathbf{U}$ & & 5 & & NS & $\mu g / L$ \\
\hline Dichlorodifluoromethane & WA & & $\mathbf{U}$ & & 10 & & NS & $\mu g / L$ \\
\hline Ethylbenzene & WA & & $\mathbf{U}$ & & 5 & & 700 & $\mu g / L$ \\
\hline Isobutyl alcohol & WA & & $\mathbf{U}$ & & 20 & & NS & $\mu g / L$ \\
\hline Methacrylonitrile & WA & & $\mathrm{U}$ & & 20 & & NS & $\mu \mathrm{g} / \mathrm{L}$ \\
\hline Methyl bromide & WA & & U & & 10 & & NS & $\mu \mathrm{g} / \mathrm{L}$ \\
\hline Methyl chloride & WA & & $\mathbf{U}$ & & 10 & & NS & $\mu \mathrm{g} / \mathrm{L}$ \\
\hline Methyl ethyl ketone & WA & & $\mathrm{U}$ & & 10 & & NS & $\mu g / L$ \\
\hline Methyl iodide & WA & & $\mathrm{U}$ & & 10 & & NS & $\mu \mathrm{g} / \mathrm{L}$ \\
\hline Methylene bromide & WA & & U & & 10 & & NS & $\mu \mathrm{g} / \mathrm{L}$ \\
\hline Methylene chloride & WA & & & $\mathrm{V}$ & 9.44 & & 5 & $\mu \mathrm{g} / \mathrm{L}$ \\
\hline Propionitrile & WA & & $\mathrm{U}$ & & 50 & & NS & $\mu g / L$ \\
\hline Styrene & WA & & $\mathrm{U}$ & & 5 & & 100 & $\mu \mathrm{g} \Omega$ \\
\hline Tetrachloroethylene & WA & & $\mathrm{U}$ & & 5 & & 5 & $\mu g / L$ \\
\hline Toluene & WA & & $\mathrm{U}$ & & 5 & & 1000 & $\mu \mathrm{g} / \mathrm{L}$ \\
\hline trans-1,3-Dichloropropene & WA & & U & & 5 & & NS & $\mu \mathrm{g} / \mathrm{L}$ \\
\hline trans-1,4-Dichloro-2-butene & WA & & $\mathrm{U}$ & & 100 & & NS & $\mu \mathrm{g} / \mathrm{L}$ \\
\hline Trichloroethylene & WA & & $\mathrm{U}$ & & 5 & & 5 & $\mu g / L$ \\
\hline Trichlorofluoromethane & WA & & U & & 5 & & NS & $\mu g / L$ \\
\hline Vinyl acetate & WA & & U & & 10 & & NS & $\mu \mathrm{g} / \mathrm{L}$ \\
\hline Vinyl chloride & WA & & $\mathrm{U}$ & & 10 & & 2 & $\mu g / L$ \\
\hline Xylenes (total) & WA & & U & & 5 & & 10000 & $\mu \mathrm{g} / \mathrm{L}$ \\
\hline
\end{tabular}


UVA004

(Volatile Trip Blank)

Sample Date: $8 / 26 / 93$

Associated Station IDs: UTR022, UTR039, UTR049, UTR051A

\begin{tabular}{|c|c|c|c|c|c|c|c|c|c|}
\hline Analyte & Lab & Rep & Avg & Modifier & & Result & Acc & Stand & Units \\
\hline 1,1,1,2-Tetrachloroethane & WA & & & $\mathbf{U}$ & & 10 & & NS & $\mu g / L$ \\
\hline 1,1,1-Trichloroethane & WA & & & $\mathbf{U}$ & & 5 & & 200 & $\mu g / L$ \\
\hline 1,1,2,2-Tetrachloroethane & WA & & & $\mathbf{U}$ & & 5 & & NS & $\mu g / L$ \\
\hline 1,1,2-Trichloroethane & WA & & & $\mathbf{U}$ & & 5 & & 5 & $\mu g / L$ \\
\hline 1,1-Dichloroethane & WA & & & U & & 5 & & NS & $\mu g / L$ \\
\hline 1,1-Dichloroethylene & WA & & & $\mathbf{U}$ & & 5 & & 7 & $\mu g / L$ \\
\hline 1,2,3-Trichloropropane & WA & & & $\mathbf{U}$ & & 10 & & NS & $\mu g / L$ \\
\hline 1,2-Dibromo-3-chloropropane & WA & & & $\mathbf{U}$ & & 20 & & 0.2 & $\mu g / L$ \\
\hline 1,2-Dibromoethane & WA & & & $\mathbf{U}$ & & 20 & & NS & $\mu g / L$ \\
\hline 1,2-Dichloroethane & WA & & & $\mathbf{U}$ & & 5 & & 5 & $\mu g / L$ \\
\hline 1,2-Dichloroethylene (total) & WA & & & $\mathbf{U}$ & & 5 & & 7 & $\mu g / L$ \\
\hline 1,2-Dichloropropane & WA & & & $\mathbf{U}$ & & 5 & & 5 & $\mu g / L$ \\
\hline 2-Hexanone & WA & & & $\mathbf{U}$ & & 10 & & NS & $\mu g / L$ \\
\hline 4-Methyl-2-pentanone & WA & & & $\mathbf{U}$ & & 10 & & NS & $\mu g / L$ \\
\hline Acetone & WA & & & J & $\mathbf{V}$ & 3.04 & & NS & $\mu g / L$ \\
\hline Acetonitrile & WA & & & $\mathbf{U}$ & & 20 & & NS & $\mu g / L$ \\
\hline Acrolein & WA & & & $\mathbf{U}$ & & 10 & & NS & $\mu g / L$ \\
\hline Acrylonitrile & WA & & & U & & 10 & & NS & $\mu g / L$ \\
\hline Allyl chloride & WA & & & $\mathbf{U}$ & & 100 & & NS & $\mu g / L$ \\
\hline Benzene & WA & & & $\mathrm{U}$ & & 5 & & 5 & $\mu g / L$ \\
\hline Bromodichloromethane & WA & & & $\mathbf{U}$ & & 5 & & NS & $\mu g / L$ \\
\hline Bromoform & WA & & & $\mathbf{U}$ & & 5 & & NS & $\mu g / L$ \\
\hline Carbon disulfide & WA & & & $\mathrm{U}$ & & 5 & & NS & $\mu g / L$ \\
\hline Carbon tetrachloride & WA & & & U & & 5 & & 5 & $\mu g / L$ \\
\hline Chlorobenzene & WA & & & $\mathbf{U}$ & & 5 & & 100 & $\mu g / L$ \\
\hline Chloroethane & WA & & & $\mathbf{U}$ & & 10 & & NS & $\mu g / L$ \\
\hline Chloroform & WA & & & $\mathrm{U}$ & & 5 & & 100 & $\mu g / L$ \\
\hline Chloroprene & WA & & & $\mathbf{U}$ & & 20 & & NS & $\mu g / L$ \\
\hline cis-1,3-Dichloropropene & WA & & & $\bar{U}$ & & 5 & & NS & $\mu g / L$ \\
\hline Dibromochloromethane & WA & & & $\mathbf{U}$ & & 5 & & NS & $\mu g / L$ \\
\hline Dichlorodifluoromethane & WA & & & $\mathbf{U}$ & & 10 & & NS & $\mu g / L$ \\
\hline Ethylbenzene & WA & & & $\mathbf{U}$ & & 5 & & 700 & $\mu g / L$ \\
\hline Isobutyl alcohol & WA & & & U & & 20 & & NS & $\mu \mathrm{g} / \mathrm{L}$ \\
\hline Methacrylonitrile & WA & & & U & & 20 & & NS & $\mu \mathrm{g} / \mathrm{L}$ \\
\hline Methyl bromide & WA & & & $\mathbf{U}$ & & 10 & & NS & $\mu g / L$ \\
\hline Methyl chloride & WA & & & $\mathbf{U}$ & & 10 & & NS & $\mu g / L$ \\
\hline Methyl ethyl ketone & WA & & & $\mathbf{U}$ & & 10 & & NS & $\mu g / L$ \\
\hline Methyl iodide & WA & & & $\mathrm{U}$ & & 10 & & NS & $\mu g / L$ \\
\hline Methylene bromide & WA & & & U & & 10 & & NS & $\mu g / L$ \\
\hline Methylene chloride & WA & & & & V & 9.32 & & 5 & $\mu \mathrm{g} / \mathrm{L}$ \\
\hline Propionitrile & WA & & & $\mathbf{U}$ & & 50 & & NS & $\mu \mathrm{g} / \mathrm{L}$ \\
\hline Styrene & WA & & & $\mathbf{U}$ & & 5 & & 100 & $\mu g / L$ \\
\hline Tetrachloroethylene & WA & & & U & & 5 & & 5 & $\mu \mathrm{g} / \mathrm{L}$ \\
\hline Toluene & WA & & & $\mathrm{U}$ & & 5 & & 1000 & $\mu g / L$ \\
\hline trans-1,3-Dichloropropene & WA & & & $\mathrm{U}$ & & 5 & & NS & $\mu \mathrm{g} / \mathrm{L}$ \\
\hline trans-1,4-Dichloro-2-butene & WA & & & U & & 100 & & NS & $\mu \mathrm{g} / \mathrm{L}$ \\
\hline Trichloroethylene & WA & & & $\mathbf{U}$ & & 5 & & 5 & $\mu g / L$ \\
\hline Trichlorofluoromethane & WA & & & $\mathbf{U}$ & & 5 & & NS & $\mu \mathrm{g} / \mathrm{L}$ \\
\hline Vinyl acetate & WA & & & $\mathrm{U}$ & & 10 & & NS & $\mu \mathrm{g}$ \\
\hline Vinyl chloride & WA & & & $\mathrm{U}$ & & 10 & & 2 & $\mu g /$ \\
\hline Xylenes (total) & WA & & & $\mathbf{U}$ & & 5 & & 10000 & $\mu g$ \\
\hline
\end{tabular}


UVA005

(Volatile Trip Blank)

Sample Date: $8 / 26 / 93$

Associated Station IDs: UTR053, UTR056, UTR060, UQA007

\begin{tabular}{|c|c|c|c|c|c|c|c|c|}
\hline Analyte & Lab & Rep & Avg & Modifier & Result & Acc & Stand & Units \\
\hline 1,1,1,2-Tetrachloroethane & WA & & & $\mathbf{U}$ & 10 & & NS & $\mu \mathrm{g} / \mathrm{L}$ \\
\hline 1,1,1-Trichloroethane & WA & & & $\mathbf{U}$ & 5 & & 200 & $\mu g / L$ \\
\hline 1,1,2,2-Tetrachloroethane & WA & & & $\mathbf{U}$ & 5 & & NS & $\mu \mathrm{g} / \mathrm{L}$ \\
\hline 1,1,2-Trichloroethane & WA & & & $\mathbf{U}$ & 5 & & 5 & $\mu g / L$ \\
\hline 1,1-Dichloroethane & WA & & & $\mathbf{U}$ & 5 & & NS & $\mu \mathrm{g} / \mathrm{L}$ \\
\hline 1,1-Dichloroethylene & WA & & & $\mathbf{U}$ & 5 & & 7 & $\mu g / L$ \\
\hline 1,2,3-Trichloropropane & WA & & & $\mathbf{U}$ & 10 & & NS & $\mu \mathrm{g} / \mathrm{L}$ \\
\hline 1,2-Dibromo-3-chloropropane & WA & & & $\mathbf{U}$ & 20 & & 0.2 & $\mu \mathrm{g} / \mathrm{L}$ \\
\hline 1,2-Dibromoethane & WA & & & $\mathbf{U}$ & 20 & & NS & $\mu \mathrm{g} / \mathrm{L}$ \\
\hline 1,2-Dichloroethane & WA & & & $\mathbf{U}$ & 5 & & 5 & $\mu \mathrm{g} / \mathrm{L}$ \\
\hline 1,2-Dichloroethylene (total) & WA & & . & $\mathbf{U}$ & 5 & & 7 & $\mu \mathrm{g} / \mathrm{L}$ \\
\hline 1,2-Dichloropropane & WA & & & $\mathbf{U}$ & 5 & & 5 & $\mu \mathrm{g} / \mathrm{L}$ \\
\hline 2-Hexanone & WA & & & $\mathbf{U}$ & 10 & & NS & $\mu g / L$ \\
\hline 4-Methyl-2-pentanone & WA & & & $\mathbf{U}$ & 10 & & NS & $\mu g / L$ \\
\hline Acetone & WA & & & $\mathbf{V}$ & 13.1 & & NS & $\mu g / L$ \\
\hline Acetonitrile & WA & & & $\mathbf{U}$ & 20 & & NS & $\mu g / L$ \\
\hline Acrolein & WA & & & $\mathbf{U}$ & 10 & & NS & $\mu \mathrm{g} / \mathrm{L}$ \\
\hline Acrylonitrile & WA & & & $\mathbf{U}$ & 10 & & NS & $\mu \mathrm{g} / \mathrm{L}$ \\
\hline Allyl chloride & WA & & & $\mathbf{U}$ & 100 & & NS & $\mu \mathrm{g} / \mathrm{L}$ \\
\hline Benzene & WA & & & $\mathbf{U}$ & 5 & & 5 & $\mu \mathrm{g} / \mathrm{L}$ \\
\hline Bromodichloromethane & WA & & & $\mathbf{U}$ & 5 & & NS & $\mu \mathrm{g} / \mathrm{L}$ \\
\hline Bromoform & WA & & & $\mathbf{U}$ & 5 & & NS & $\mu \mathrm{g} / \mathrm{L}$ \\
\hline Carbon disulfide & WA & & & $\mathbf{U}$ & 5 & & NS & $\mu \mathrm{g} / \mathrm{L}$ \\
\hline Carbon tetrachloride & WA & & & r & 5 & & 5 & $\mu \mathrm{g} / \mathrm{L}$ \\
\hline Chlorobenzene & WA & & & $\mathbf{U}$ & 5 & & 100 & $\mu g / L$ \\
\hline Chloroethane & WA & & & U] & 10 & & NS & $\mu g / L$ \\
\hline Chloroform & WA & & & $\mathbf{U}$ & 5 & & 100 & $\mu \mathrm{g} / \mathrm{L}$ \\
\hline Chloroprene & WA & & & $\mathbf{U}$ & 20 & & NS & $\mu \mathrm{g} / \mathrm{L}$ \\
\hline cis-1,3-Dichloropropene & WA & & & $\mathbf{U}$ & 5 & & NS & $\mu g / L$ \\
\hline Dibromochloromethane & WA & & & $\mathbf{U}$ & 5 & & NS & $\mu g / L$ \\
\hline Dichlorodifluoromethane & WA & & & $\mathbf{U}$ & 10 & & NS & $\mu \mathrm{g} / \mathrm{L}$ \\
\hline Ethylbenzene & WA & & & $\mathbf{U}$ & 5 & & 700 & $\mu \mathrm{g} / \mathrm{L}$ \\
\hline Isobutyl alcohol & WA & & & $\mathbf{U}$ & 20 & & NS & $\mu \mathrm{g} / \mathrm{L}$ \\
\hline Methacrylonitrile & WA & & & $\mathbf{U}$ & 20 & & NS & $\mu g / L$ \\
\hline Methyl bromide & WA & & & $\mathbf{U}$ & 10 & & NS & $\mu \mathrm{g} / \mathrm{L}$ \\
\hline Methyl chloride & WA & & & $\mathbf{U}$ & 10 & & NS & $\mu \mathrm{g} / \mathrm{L}$ \\
\hline Methyl ethyl ketone & WA & & & $\mathbf{U}$ & 10 & & NS & $\mu \mathrm{g} / \mathrm{L}$ \\
\hline Methyl iodide & WA & & & $\mathbf{U}$ & 10 & & NS & $\mu \mathrm{g} / \mathrm{L}$ \\
\hline Methylene bromide & WA & & & $\mathbf{U}$ & 10 & & NS & $\mu \mathrm{g} / \mathrm{L}$ \\
\hline Methylene chloride & WA & & & V & 9.38 & & 5 & $\mu \mathrm{g} / \mathrm{L}$ \\
\hline Propionitrile & WA & & & $\mathbf{U}$ & 50 & & NS & $\mu \mathrm{g} / \mathrm{L}$ \\
\hline Styrene & WA & & & $\mathbf{U}$ & 5 & & 100 & $\mu \mathrm{g} / \mathrm{L}$ \\
\hline Tetrachloroethylene & WA & & & $\mathbf{U}$ & 5 & & 5 & $\mu \mathrm{g} / \mathrm{L}$ \\
\hline Toluene & WA & & & $\mathbf{U}$ & 5 & & 1000 & $\mu \mathrm{g} / \mathrm{L}$ \\
\hline trans-1,3-Dichloropropene & WA & & & $\mathbf{U}$ & 5 & & NS & $\mu \mathrm{g} / \mathrm{L}$ \\
\hline trans-1,4-Dichloro-2-butene & WA & & & $\mathbf{U}$ & 100 & & NS & $\mu \mathrm{g} / \mathrm{L}$ \\
\hline Trichioroethylene & WA & & & $\mathbf{U}$ & 5 & & 5 & $\mu \mathrm{g} / \mathrm{L}$ \\
\hline Trichlorofluoromethane & WA & & & $\mathrm{U}$ & 5 & & NS & $\mu \mathrm{g} / \mathrm{L}$ \\
\hline Vinyl acetate & WA & & & $\mathbf{U}$ & 10 & & NS & $\mu \mathrm{g} / \mathrm{L}$ \\
\hline Vinyl chloride & WA & & & $\mathrm{U}$ & 10 & & 2 & $\mu \mathrm{g} / \mathrm{L}$ \\
\hline Xylenes (total) & WA & & & $\mathbf{U}$ & 5 & & 10000 & $\mu \mathrm{g} / \mathrm{L}$ \\
\hline
\end{tabular}


UVA006

(Volatile Trip Blank)

Sample Date: 8/27/93

Associated Station IDs: UTR062, UTR063, UTR066

\begin{tabular}{|c|c|c|c|c|c|c|c|}
\hline Analyte & Lab & Avg & Modifier & & Result & Stand & Units \\
\hline 1,1,1,2-Tetrachloroethane & WA & & $\overline{\mathrm{U}}$ & & 10 & NS & $\mu g / L$ \\
\hline 1,1,1-Trichloroethane & WA & & U & & 5 & 200 & $\mu g / L$ \\
\hline 1,1,2,2-Tetrachloroethane & WA & & U & & 5 & NS & $\mu \mathrm{g} / \mathrm{L}$ \\
\hline 1,1,2-Trichloroethane & WA & & $\mathrm{U}$ & & 5 & 5 & $\mu \mathrm{g} / \mathrm{L}$ \\
\hline 1,1-Dichloroethane & WA & & $\mathrm{U}$ & & 5 & NS & $\mu g / L$ \\
\hline 1,1-Dichloroethylene & WA & & $\mathrm{U}$ & & 5 & 7 & $i g / L$ \\
\hline 1,2,3-Trichloropropane & WA & & $\mathrm{U}$ & & 10 & NS & $\mu \mathrm{g} / \mathrm{L}$ \\
\hline 1,2-Dibromo-3-chloropropane & WA & & U & & 20 & 0.2 & $\mu \mathrm{g} / \mathrm{L}$ \\
\hline 1,2-Dibromoethane & WA & & $\mathrm{U}$ & & 20 & NS & $\mu \mathrm{E} / \mathrm{L}$ \\
\hline 1,2-Dichloroethane & WA & & $\mathrm{U}$ & & 5 & 5 & $\mu g / L$ \\
\hline 1,2-Dichloroethylene (total) & WA & & $\mathrm{U}$ & & 5 & 7 & $\mu \mathrm{g} / \mathrm{L}$ \\
\hline 1,2-Dichloropropane & WA & & U & & 5 & 5 & $\mu \mathrm{g} / \mathrm{L}$ \\
\hline 2-Hexanone & WA & & $\mathbf{U}$ & & 10 & NS & $\mu \mathrm{g} / \mathrm{L}$ \\
\hline 4-Methyl-2-pentanone & WA & & $\mathrm{U}$ & .. & 10 & NS & $\mu \mathrm{g} / \mathrm{L}$ \\
\hline Acetone & WA & & $\mathrm{J}$ & V & 7.16 & NS & $\mu \mathrm{g} / \mathrm{L}$ \\
\hline Acetonitrile & WA & & $\mathrm{U}$ & & 20 & NS & $\mu \mathrm{g} / \mathrm{L}$ \\
\hline Acrolein & WA & & $\mathrm{U}$ & & 10 & NS & $\mu \mathrm{g} / \mathrm{L}$ \\
\hline Acrylonitrile & WA & & $\mathrm{U}$ & & 10 & NS & $\mu g / L$ \\
\hline Allyl chloride & WA & & $\mathrm{U}$ & & 100 & NS & $\mu \mathrm{g} / \mathrm{L}$ \\
\hline Benzene & WA & & $\mathbf{U}$ & & 5 & 5 & $\mu \mathrm{g} / \mathrm{L}$ \\
\hline Bromodichloromethane & WA & & $\mathbf{U}$ & & 5 & NS & $\mu \mathrm{g} / \mathrm{L}$ \\
\hline Bromoform & WA & & $\mathrm{U}$ & & 5 & NS & $\mu \mathrm{g} / \mathrm{L}$ \\
\hline Carbon disulfide & WA & & $\mathrm{U}$ & & 5 & NS & $\mu \mathrm{g} / \mathrm{L}$ \\
\hline Carbon tetrachloride & WA & & $\mathrm{U}$ & & 5 & 5 & $\mu \mathrm{g} / \mathrm{L}$ \\
\hline Chlorobenzene & WA & & $\mathrm{U}$ & & 5 & 100 & $\mu g / L$ \\
\hline Chloroethane & WA & & $\mathrm{U}$ & & 10 & NS & $\mu g / L$ \\
\hline Chloroform & WA & & $\mathrm{U}$ & & 5 & 100 & $\mu \mathrm{g} / \mathrm{L}$ \\
\hline Chloroprene & WA & & $\mathrm{U}$ & & 20 & NS & $\mu \mathrm{g} / \mathrm{L}$ \\
\hline cis-1,3-Dichloropropene & WA & & $\mathrm{U}$ & & 5 & NS & $\mu g / L$ \\
\hline Dibromochloromethane & WA & & $\mathrm{U}$ & & 5 & NS & $\mu \mathrm{g} / \mathrm{L}$ \\
\hline Dichlorodifluoromethane & WA & & $\mathrm{U}$ & & 10 & NS & $\mu \mathrm{g} / \mathrm{L}$ \\
\hline Ethylbenzene & WA & & $\mathrm{U}$ & & 5 & 700 & $\mu \mathrm{g} / \mathrm{L}$ \\
\hline Isobutyl alcohol & WA & & $\mathrm{U}$ & & 20 & NS & $\mu \mathrm{g} / \mathrm{L}$ \\
\hline Methacrylonitrile & WA & & $\mathrm{U}$ & & 20 & NS & $\mu \mathrm{g} / \mathrm{L}$ \\
\hline Methyl bromide & WA & & $\mathrm{U}$ & & 10 & NS & $\mu \mathrm{g} / \mathrm{L}$ \\
\hline Methyl chloride & WA & & $\mathrm{U}$ & & 10 & NS & $\mu \mathrm{g} / \mathrm{L}$ \\
\hline Methyl ethyl ketone & WA & & $\mathrm{U}$ & & 10 & NS & $\mu \mathrm{g} / \mathrm{L}$ \\
\hline Methyl iodide & WA & & $\mathrm{U}$ & & 10 & NS & $\mu \mathrm{g} / \mathrm{L}$ \\
\hline Methylene bromide & WA & & $U$ & & 10 & NS & $\mu \mathrm{g} / \mathrm{L}$ \\
\hline Methylene chloride & WA & & & $\mathrm{V}$ & 14.3 & 5 & $\mu \mathrm{g} / \mathrm{L}$ \\
\hline Propionitrile & WA & & $\mathrm{U}$ & & 50 & NS & $\mu \mathrm{g} / \mathrm{L}$ \\
\hline Styrene & WA & & $\mathrm{U}$ & & 5 & 100 & $\mu \mathrm{g} / \mathrm{L}$ \\
\hline Tetrachloroethylene & WA & & $\mathrm{U}$ & & 5 & 5 & $\mu \mathrm{g} / \mathrm{L}$ \\
\hline Toluene & WA & & $\mathrm{U}$ & & 5 & 1000 & $\mu g / L$ \\
\hline trans-1,3-Dichloropropene & WA & & $\mathrm{U}$ & & 5 & NS & $\mu \mathrm{g} / \mathrm{L}$ \\
\hline trans-1,4-Dichloro-2-butene & WA & & $\mathrm{U}$ & & 100 & NS & $\mu \mathrm{g} / \mathrm{L}$ \\
\hline Trichloroethylene & WA & & $\mathrm{U}$ & & 5 & 5 & $\mu \mathrm{g} / \mathrm{L}$ \\
\hline Trichlorofluoromethane & WA & & U & & 5 & NS & $\mu \mathrm{g} / \mathrm{L}$ \\
\hline Vinyl acetate & WA & & $\mathbf{U}$ & & 10 & NS & $\mu g / L$ \\
\hline Vinyl chloride & WA & & $\mathrm{U}$ & & 10 & 2 & $\mu \mathrm{g} / \mathrm{L}$ \\
\hline Xylenes (total) & WA & & $\mathbf{U}$ & & 5 & 10000 & $\mu \mathrm{g} / \mathrm{L}$ \\
\hline
\end{tabular}


UVA007

(Volatile Trip Blank)

Sample Date:

Associated Station IDs:

\begin{tabular}{|c|c|c|c|c|c|c|c|c|c|}
\hline Analyte & Lab & Rep & Avg & Modifier & & Result & Acc & Stand & Units \\
\hline 1,1,1,2-Tetrachloroethane & WA & & & $\bar{U}$ & & 10 & & NS & $\overline{\mu g / L}$ \\
\hline 1,1,1-Trichloroethane & WA & & & $\mathbf{U}$ & & 5 & $\cdot$ & 200 & $\mu \mathrm{g} / \mathrm{L}$ \\
\hline 1,1,2,2-Tetrachloroethane & WA & & & $\mathbf{U}$ & & 5 & & NS & $\mu g / L$ \\
\hline 1,1,2-Trichloroethane & WA & & & $\mathbf{U}$ & & 5 & & 5 & $\mu g / L$ \\
\hline 1,1-Dichloroethane & WA & & & $\mathbf{U}$ & & 5 & & NS & $\mu g / L$ \\
\hline 1,1-Dichloroethylene & WA & & & $\mathbf{U}$ & & 5 & & 7 & $\mu g / L$ \\
\hline 1,2,3-Trichloropropane & WA & & & $\mathbf{U}$ & & 10 & & NS & $\mu \mathrm{g} / \mathrm{L}$ \\
\hline 1,2-Dibromo-3-chloropropane & WA & & & $\mathbf{U}$ & & 20 & & 0.2 & $\mu g / L$ \\
\hline 1,2-Dibromoethane & WA & & & $\mathbf{U}$ & & 20 & & NS & $\mu g / L$ \\
\hline 1,2-Dichloroethane & WA & & & $\mathbf{U}$ & & 5 & & 5 & $\mu g / L$ \\
\hline 1,2-Dichloroethylene (total) & WA & & & $\mathbf{U}$ & & 5 & & 7 & $\mu \mathrm{g} / \mathrm{L}$ \\
\hline 1,2-Dichloropropane & WA & & & $\mathbf{U}$ & & 5 & & 5 & $\mu \mathrm{g} / \mathrm{L}$ \\
\hline 2-Hexanone & WA & & & $\mathbf{U}$ & & 10 & & NS & $\mu g / L$ \\
\hline 4-Methyl-2-pentanone & WA & & & $\mathbf{U}$ & & 10 & & NS & $\mu g / L$ \\
\hline Acetone & WA & & & $\mathbf{J}$ & V & 6.89 & & NS & $\mu g / L$ \\
\hline Acetonitrile & WA & & & $\mathbf{U}$ & & 20 & & NS & $\mu \mathrm{g} / \mathrm{L}$ \\
\hline Acrolein & WA & & & $\mathbf{U}$ & & 10 & & NS & $\mu g / L$ \\
\hline Acrylonitrile & WA & & & $\mathbf{U}$ & & 10 & & NS & $\mu g / L$ \\
\hline Allyl chloride & WA & & & $\mathbf{U}$ & & 100 & & NS & $\mu g / L$ \\
\hline Benzene & WA & & & $\mathbf{U}$ & & 5 & & 5 & $\mu g / L$ \\
\hline Bromodichloromethane & WA & & & $\mathbf{U}$ & & 5 & & NS & $\mu g / L$ \\
\hline Bromoform & WA & & & $\mathbf{U}$ & & 5 & & NS & $\mu g / L$ \\
\hline Carbon disulfide & WA & & & $\mathbf{U}$ & & 5 & & NS & $\mu g / L$ \\
\hline Carbon tetrachloride & WA & & & $\mathbf{U}$ & & 5 & & 5 & $\mu \mathrm{g} / \mathrm{L}$ \\
\hline Chlorobenzene & WA & & & $\mathbf{U}$ & & 5 & & 100 & $\mu g / L$ \\
\hline Chloroethane & WA & & & $\mathbf{U}$ & & 10 & & NS & $\mu g / L$ \\
\hline Chloroform & WA & & - & $\mathbf{U}$ & & 5 & & 100 & $\mu g / L$ \\
\hline Chloroprene & WA & & & $\mathbf{U}$ & & 20 & & NS & $\mu g / L$ \\
\hline cis-1,3-Dichloropropene & WA & & & $\mathbf{U}$ & & 5 & & NS & $\mu g / L$ \\
\hline Dibromochloromethane & WA & & & $\mathbf{U}$ & & 5 & & NS & $\mu \mathrm{g} / \mathrm{L}$ \\
\hline Dichlorodifluoromethane & WA & & & $\mathbf{U}$ & & 10 & & NS & $\mu \mathrm{g} / \mathrm{L}$ \\
\hline Ethylbenzene & WA & & & $\mathbf{U}$ & & 5 & & 700 & $\mu \mathrm{g} / \mathrm{L}$ \\
\hline Isobutyl alcohol & WA & & & $\mathbf{U}$ & & 20 & & NS & $\mu \mathrm{g} / \mathrm{L}$ \\
\hline Methacrylonitrile & WA & & & $\mathbf{U}$ & & 20 & & NS & $\mu \mathrm{g} / \mathrm{L}$ \\
\hline Methyl bromide & WA & & & $\mathbf{U}$ & & 10 & & NS & $\mu \mathrm{g} / \mathrm{L}$ \\
\hline Methyl chloride & WA & & & $\mathbf{U}$ & & 10 & & NS & $\mu g / L$ \\
\hline Methyl ethyl ketone & WA & & & $\mathbf{U}$ & & 10 & & NS & $\mu g / L$ \\
\hline Methyl iodide & WA & & & $\mathbf{U}$ & & 10 & & NS & $\mu g / L$ \\
\hline Methylene bromide & WA & & & $\mathbf{U}$ & & 10 & & NS & $\mu \mathrm{g} / \mathrm{L}$ \\
\hline Methylene chloride & WA & & & & V & 14 & & 5 & $\mu \mathrm{g} / \mathrm{L}$ \\
\hline Propionitrile & WA & & & $\mathbf{U}$ & & 50 & & NS & $\mu \mathrm{g} / \mathrm{L}$ \\
\hline Styrene & WA & & & $\mathbf{U}$ & & 5 & & 100 & $\mu \mathrm{g} / \mathrm{L}$ \\
\hline Tetrachloroethylene & WA & & & $\mathbf{U}$ & & 5 & & 5 & $\mu \mathrm{g} / \mathrm{L}$ \\
\hline Toluene & WA & & & $\mathbf{U}$ & & 5 & & 1000 & $\mu g / L$ \\
\hline trans-1,3-Dichloropropene & WA & & & $\mathbf{U}$ & & 5 & & NS & $\mu \mathrm{g} / \mathrm{L}$ \\
\hline trans-1,4-Dichloro-2-butene & WA & & & $\mathbf{U}$ & & 100 & & NS & $\mu g / L$ \\
\hline Trichloroethylene & WA & & & $\mathbf{U}$ & & 5 & & 5 & $\mu \mathrm{g} / \mathrm{L}$ \\
\hline Trichlorofluoromethane & WA & & & $\mathbf{U}$ & & 5 & & NS & $\mu g / L$ \\
\hline Vinyl acetate & WA & & & $\mathbf{U}$ & & 10 & & NS & $\mu g / L$ \\
\hline Vinyl chloride & WA & & & $\mathbf{U}$ & & 10 & & 2 & $\mu \mathrm{g} / \mathrm{L}$ \\
\hline Xylenes (total) & WA & & & $\mathbf{U}$ & & 5 & & 10000 & $\mu \mathrm{g} / \mathrm{L}$ \\
\hline
\end{tabular}


UVA008

(Volatile Trip Blank)

Sample Date: $8 / 27 / 93$

Associated Station IDs: UQA003, UQA004, UQA008

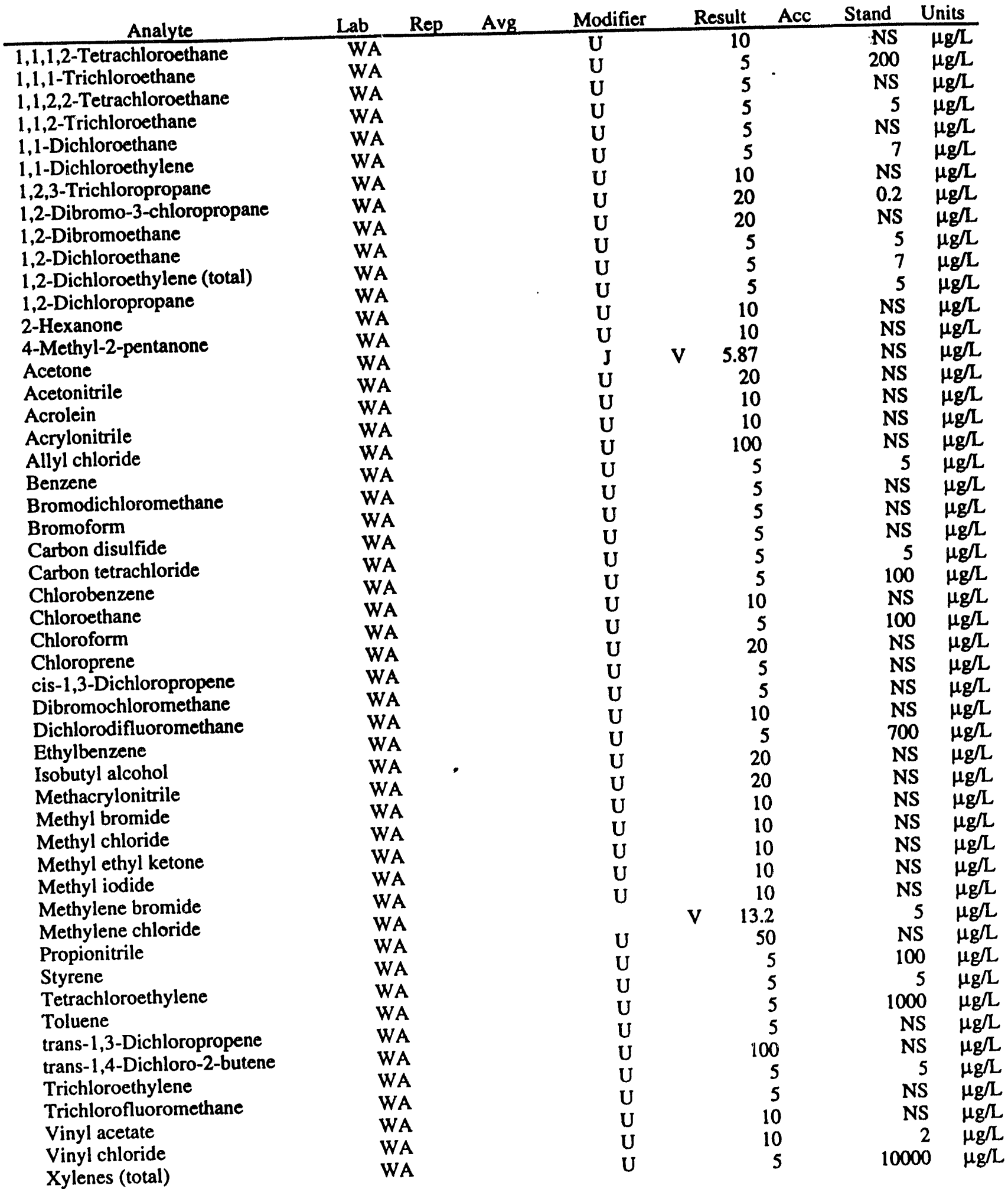


UVA009

(Volatile Trip Blank)

Sample Date: $8 / 27 / 93$

Associated Station IDs: UTR112, UTR114, UTR1 16

\begin{tabular}{|c|c|c|c|c|c|c|c|c|c|}
\hline Analyte & Lab & Rep & Avg & Modifier & & Result & Acc & Stand & Units \\
\hline 1,1,1,2-Tetrachloroethane & WA & & & $\mathbf{U}$ & & 10 & & NS & $\overline{\mu g / L}$ \\
\hline 1,1,1-Trichloroethane & WA & & & $\mathbf{U}$ & & 5 & . & 200 & $\mu \mathrm{g} / \mathrm{L}$ \\
\hline 1,1,2,2-Tetrachloroethane & WA & & & $\mathbf{U}$ & & 5 & & NS & $\mu g / L$ \\
\hline 1,1,2-Trichloroethane & WA & & & $\mathbf{U}$ & & 5 & & 5 & $\mu g / L$ \\
\hline 1,1-Dichloroethane & WA & & & $\mathbf{U}$ & & 5 & & NS & $\mu g / L$ \\
\hline 1,1-Dichloroethylene & WA & & & $\mathbf{U}$ & & 5 & & 7 & $\mu g / L$ \\
\hline 1,2,3-Trichloropropane & WA & & & $\mathbf{U}$ & & 10 & & NS & $\mu g / L$ \\
\hline 1,2-Dibromo-3-chloropropane & WA & & & $\mathbf{U}$ & & 20 & & 0.2 & $\mu g / L$ \\
\hline 1,2-Dibromoethane & WA & & & $\mathbf{U}$ & & 20 & & NS & $\mu \mathrm{g} / \mathrm{L}$ \\
\hline 1,2-Dichloroethane & WA & & & $\mathbf{U}$ & & 5 & & 5 & $\mu g / L$ \\
\hline 1,2-Dichloroethylene (total) & WA & & & $\mathbf{U}$ & & 5 & & 7 & $\mu g / L$ \\
\hline 1,2-Dichloropropane & WA & & & $\mathbf{U}$ & & 5 & & 5 & $\mu g / L$ \\
\hline 2-Hexanone & WA & & & $\mathbf{U}$ & & 10 & & NS & $\mu g / L$ \\
\hline 4-Methyl-2-pentanone & WA & & & $\mathbf{U}$ & & 10 & & NS & $\mu g / L$ \\
\hline Acetone & WA & & & $\mathbf{J}$ & V & 5.98 & & NS & $\mu g / L$ \\
\hline Acetonitrile & WA & & & $\mathbf{U}$ & & 20 & & NS & $\mu \mathrm{g} / \mathrm{L}$ \\
\hline Acrolein & WA & & & $\mathbf{U}$ & & 10 & & NS & $\mu \mathrm{g} / \mathrm{L}$ \\
\hline Acrylonitrile & WA & & & $\mathbf{U}$ & & 10 & & NS & $\mu g / L$ \\
\hline Allyl chloride & WA & & & $\mathbf{U}$ & & 100 & & NS & $\mu g / L$ \\
\hline Benzene & WA & & & $\mathrm{U}$ & & 5 & & 5 & $\mu \mathrm{g} / \mathrm{L}$ \\
\hline Bromodichloromethane & WA & & & $\mathbf{U}$ & & 5 & & NS & $\mu g / L$ \\
\hline Bromoform & WA & & & $\mathbf{U}$ & & 5 & & NS & $\mu g / L$ \\
\hline Carbon disulfide & WA & & & $\mathbf{U}$ & & 5 & & NS & $\mu \mathrm{g} / \mathrm{L}$ \\
\hline Carbon tetrachloride & WA & & & $\mathbf{U}$ & & 5 & & 5 & $\mu \mathrm{g} / \mathrm{L}$ \\
\hline Chlorobenzene & WA & & & $\mathbf{U}$ & & 5 & & 100 & $\mu \mathrm{g} / \mathrm{L}$ \\
\hline Chloroethane & WA & & & $\mathbf{U}$ & & 10 & & NS & $\mu \mathrm{g} / \mathrm{L}$ \\
\hline Chloroform & WA & & & $\mathbf{U}$ & & 5 & & 100 & $\mu \mathrm{g} / \mathrm{L}$ \\
\hline Chloroprene & WA & & & $\mathbf{U}$ & & 20 & & NS & $\mu \mathrm{g} / \mathrm{L}$ \\
\hline cis-1,3-Dichloropropene & WA & & & $\mathbf{U}$ & & 5 & & NS & $\mu \mathrm{g} / \mathrm{L}$ \\
\hline Dibromochloromethane & WA & & & $\mathbf{U}$ & & 5 & & NS & $\mu g / L$ \\
\hline Dichlorodifluoromethane & WA & & & $\mathbf{U}$ & & 10 & & NS & $\mu g / L$ \\
\hline Ethylbenzene & WA & & & $\mathrm{U}$ & & 5 & & 700 & $\mu g / L$ \\
\hline Isobutyl alcohol & WA & & & $\mathbf{U}$ & & 20 & & NS & $\mu \mathrm{g} / \mathrm{L}$ \\
\hline Methacrylonitrile & WA & & & $\mathbf{U}$ & & 20 & & NS & $\mu \mathrm{g} / \mathrm{L}$ \\
\hline Methyl bromide & WA & & & $\mathbf{U}$ & & 10 & & NS & $\mu \mathrm{g} / \mathrm{L}$ \\
\hline Methyl chloride & WA & & & $\mathbf{U}$ & & 10 & & NS & $\mu \mathrm{g} / \mathrm{L}$ \\
\hline Methyl ethyl ketone & WA & & & $\mathrm{U}$ & & 10 & & NS & $\mu \mathrm{g} / \mathrm{L}$ \\
\hline Methyl iodide & WA & & & $\mathbf{U}$ & & 10 & & NS & $\mu \mathrm{g} / \mathrm{L}$ \\
\hline Methylene bromide & WA & & & $\mathbf{U}$ & & 10 & & NS & $\mu g / L$ \\
\hline Methylene chloride & WA & & & & V & 12.3 & & 5 & $\mu g / L$ \\
\hline Propionitrile & WA & & & $\mathbf{U}$ & & 50 & & NS & $\mu g / L$ \\
\hline Styrene & WA & & & $\mathrm{U}$ & & 5 & & 100 & $\mu g / L$ \\
\hline Tetrachloroethylene & WA & & & $\mathrm{U}$ & & 5 & & 5 & $\mu \mathrm{g} / \mathrm{L}$ \\
\hline Toluene & WA & & & $\mathbf{U}$ & & 5 & & 1000 & $\mu \mathrm{g} / \mathrm{L}$ \\
\hline trans-1,3-Dichloropropene & WA & & & U & & 5 & & NS & $\mu g / L$ \\
\hline trans-1,4-Dichloro-2-butene & WA & & & $\mathrm{U}$ & & 100 & & NS & $\mu g / L$ \\
\hline Trichloroethylene & WA & & & $\mathrm{U}$ & & 5 & & 5 & $\mu \mathrm{g} / \mathrm{L}$ \\
\hline Trichlorofluoromethane & WA & & & $\mathbf{U}$ & & 5 & & NS & $\mu g / L$ \\
\hline Vinyl acetate & WA & & & $\mathbf{U}$ & & 10 & & NS & $\mu \mathrm{g} / \mathrm{L}$ \\
\hline Vinyl chloride & WA & & & $\mathbf{U}$ & & 10 & & 2 & $\mu \mathrm{g} / \mathrm{L}$ \\
\hline Xylenes (total) & WA & & & $\mathbf{U}$ & & 5 & & 10000 & $\mu \mathrm{g} / \mathrm{L}$ \\
\hline
\end{tabular}


UVA010

(Volatile Trip Blank)

Sample Date: 9/1/93

Associated Station IDs: UTR020, UTR043, UTR 145

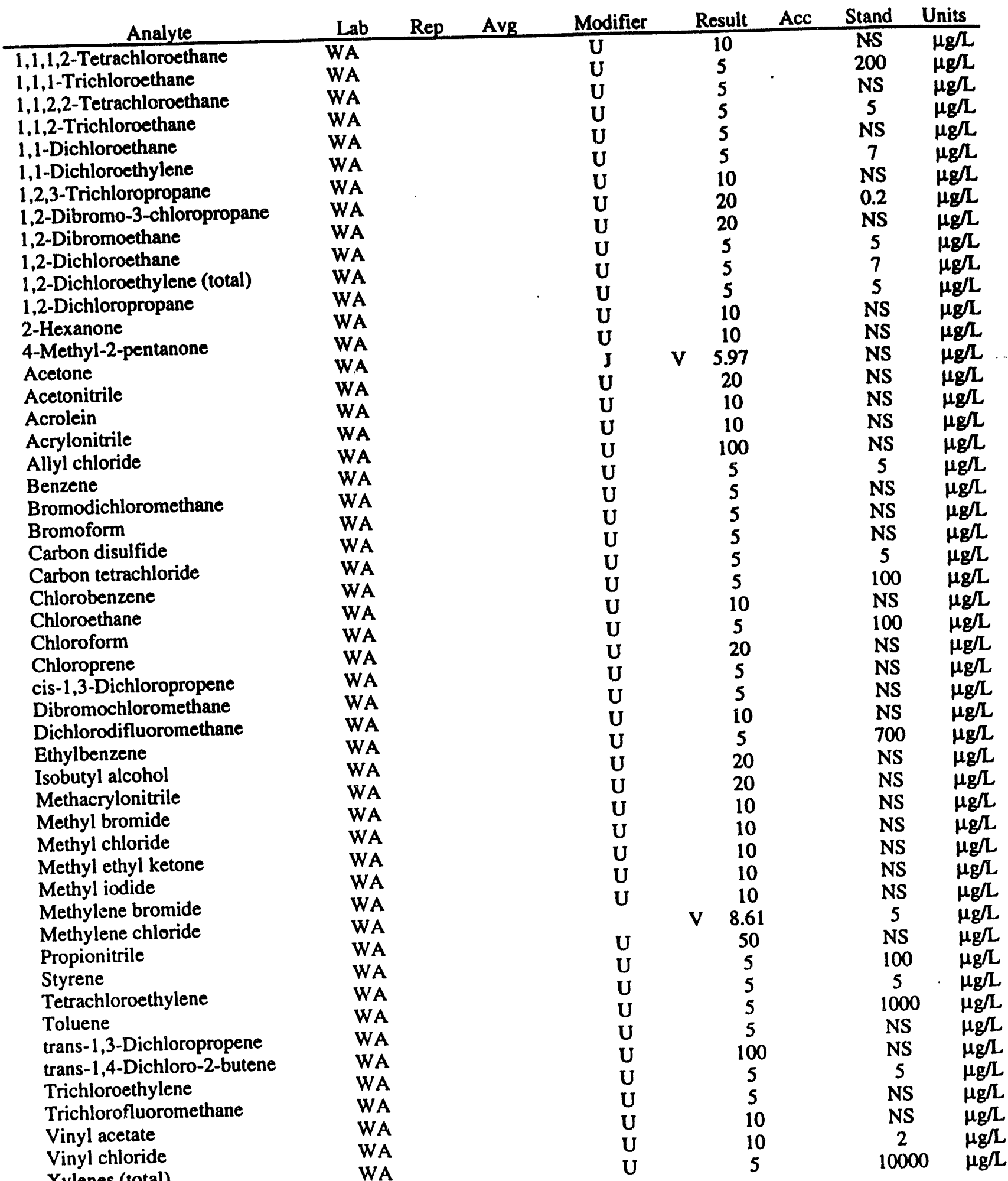


UVA011

(Volatile Trip Blank)

Sample Date: $9 / 1 / 93$

Associated Station IDs: UTR071, UTR072, UTR099, UTR102

\begin{tabular}{|c|c|c|c|c|c|c|c|c|c|}
\hline Analyte & Lab & Rep & Avg & Moditier & & Result & Acc & Stand & Units \\
\hline 1,1,1,2-Tetrachloroethane & WA & & & $\bar{U}$ & & 10 & & NS & $\mu g / L$ \\
\hline 1,1,1-Trichloroethane & WA & & & $\mathbf{U}$ & & 5 & . & 200 & $\mu \mathrm{g} / \mathrm{L}$ \\
\hline 1,1,2,2-Tetrachloroethane & WA & & & $\mathbf{U}$ & & 5 & & NS & $\mu g / L$ \\
\hline 1,1,2-Trichloroethane & WA & & & $\mathbf{U}$ & & 5 & & 5 & $\mu g / L$ \\
\hline 1,1-Dichloroethane & WA & & & $\mathbf{U}$ & & 5 & & NS & $\mu g / L$ \\
\hline 1,1-Dichloroethylene & WA & & & $\mathbf{U}$ & & 5 & & 7 & $\mu g / L$ \\
\hline 1,2,3-Trichloropropane & WA & & & $\mathbf{U}$ & & 10 & & NS & $\mu g / L$ \\
\hline 1,2-Dibromo-3-chloropropane & WA & & & $\mathbf{U}$ & & 20 & & 0.2 & $\mu g / L$ \\
\hline 1,2-Dibromoethane & WA & & & $\mathbf{U}$ & & 20 & & NS & $\mu g / L$ \\
\hline 1,2-Dichloroethane & WA & & & $\mathrm{U}$ & & 5 & & 5 & $\mu g / L$ \\
\hline 1,2-Dichloroethylene (total) & WA & & & $\mathbf{U}$ & & 5 & & 7 & $\mu g / L$ \\
\hline 1,2-Dichloropropane & WA & & & $\mathbf{U}$ & & 5 & & 5 & $\mu g / L$ \\
\hline 2-Hexanone & WA & & & $\mathbf{U}$ & & 10 & & NS & $\mu g / L$ \\
\hline 4-Methyl-2-pentanone & WA & & & $\mathbf{U}$ & & 10 & & NS & $\mu g / L$ \\
\hline Acetone & WA & & & & V & 16.3 & & NS & $\mu g / L$ \\
\hline Acetonitrile & WA & & & $\mathbf{U}$ & & 20 & & NS & $\mu g / L$ \\
\hline Acrolein & WA & & & $\mathrm{U}$ & & 10 & & NS & $\mu \mathrm{g} / \mathrm{L}$ \\
\hline Acrylonitrile & WA & & & $\mathbf{U}$ & & 10 & & NS & $\mu g / L$ \\
\hline Allyl chloride & WA & & & $\mathbf{U}$ & & 100 & & NS & $\mu \mathrm{g} / \mathrm{L}$ \\
\hline Benzene & WA & & & $\mathbf{U}$ & & 5 & & 5 & $\mu g / L$ \\
\hline Bromodichloromethane & WA & & & $\mathbf{U}$ & & 5 & & NS & $\mu \mathrm{g} / \mathrm{L}$ \\
\hline Bromoform & WA & & & U & & 5 & & NS & $\mu g / L$ \\
\hline Carbon disulfide & WA & & & $\mathrm{U}$ & & 5 & & NS & $\mu g / L$ \\
\hline Carbon tetrachloride & WA & & & $\mathbf{U}$ & & 5 & & 5 & $\mu \mathrm{g} / \mathrm{L}$ \\
\hline Chlorobenzene & WA & & & $\mathbf{U}$ & & 5 & & 100 & $\mu g / L$ \\
\hline Chloroethane & WA & & & $\mathbf{U}$ & & 10 & & NS & $\mu g / L$ \\
\hline Chloroform & WA & & & $\mathbf{U}$ & & 5 & & 100 & $\mu \mathrm{g} / \mathrm{L}$ \\
\hline Chloroprene & WA & & & $\mathrm{U}$ & & 20 & & NS & $\mu g / L$ \\
\hline cis-1,3-Dichloropropene & WA & & & $\mathbf{U}$ & & 5 & & NS & $\mu g / L$ \\
\hline Dibromochloromethane & WA & & & $\mathrm{U}$ & & 5 & & NS & $\mu g / L$ \\
\hline Dichlorodifluoromethane & WA & & & $U$ & & 10 & & NS & $\mu g / L$ \\
\hline Ethylbenzene & WA & & & U & & 5 & & 700 & $\mu g / L$ \\
\hline Isobutyl alcohol & WA & & & $\mathrm{U}$ & & 20 & & NS & $\mu g / L$ \\
\hline Methacrylonitrile & WA & & & $\mathbf{U}$ & & 20 & & NS & $\mu g / L$ \\
\hline Methyl bromide & WA & & & $U$ & & 10 & & NS & $\mu g / L$ \\
\hline Methyl chloride & WA & & & U & & 10 & & NS & $\mu \mathrm{g} / \mathrm{L}$ \\
\hline Methyl ethyl ketone & WA & & & $\mathbf{U}$ & & 10 & & NS & $\mu \mathrm{g} / \mathrm{L}$ \\
\hline Methyl iodide & WA & & & $\mathrm{U}$ & & 10 & & NS & $\mu g / L$ \\
\hline Methylene bromide & WA & & & $\mathbf{U}$ & & 10 & & NS & $\mu g / L$ \\
\hline Methylene chloride & WA & & & & V & 8.59 & & 5 & $\mu g / L$ \\
\hline Propionitrile & WA & & & $U$ & & 50 & & NS & $\mu \mathrm{g} / \mathrm{L}$ \\
\hline Styrene & WA & & & $\mathbf{U}$ & & 5 & & 100 & $\mu \mathrm{g} / \mathrm{L}$ \\
\hline Tetrachloroethylene & WA & & & $\mathrm{U}$ & & 5 & & 5 & $\mu g / L$ \\
\hline Toluene & WA & & & U & & 5 & & 1000 & $\mu g / L$ \\
\hline trans-1,3-Dichloropropene & WA & & & $\mathbf{U}$ & & 5 & & NS & $\mu g / L$ \\
\hline trans-1,4-Dichloro-2-butene & WA & & & $\mathbf{U}$ & & 100 & & NS & $\mu \mathrm{g} / \mathrm{L}$ \\
\hline Trichloroethylene & WA & & & $\mathbf{U}$ & & 5 & & 5 & $\mu \mathrm{g} / \mathrm{L}$ \\
\hline Trichlorofluoromethane & WA & & & $\mathbf{U}$ & & 5 & & NS & $\mu \mathrm{g} / \mathrm{L}$ \\
\hline Vinyl acetate & WA & & & $\mathbf{U}$ & & 10 & & NS & $\mu g / L$ \\
\hline Vinyl chloride & WA & & & $\mathbf{U}$ & & 10 & & 2 & $\mu \mathrm{g} / \mathrm{L}$ \\
\hline Xylenes (total) & WA & & & U & & 5 & & 10000 & $\mu \mathrm{g} / \mathrm{L}$ \\
\hline
\end{tabular}


UVA012

(Volatile Trip Blank)

Sample Date: 9/2/93

Associated Station IDs: BGD001, BGD002, BGSO02

\begin{tabular}{|c|c|c|c|c|c|c|c|c|c|}
\hline Analyte & Lab & Rep & Avg & Modifier & & Result & Acc & Stand & Units \\
\hline 1,1,1,2-Tetrachloroethane & WA & & & $\mathbf{U}$ & & 10 & & NS & $\mu g / L$ \\
\hline 1,1,1-Trichloroethane & WA & & & $\mathbf{U}$ & & 5 & & 200 & $\mu g / L$ \\
\hline 1,1,2,2-Tetrachloroethane & WA & & & $\mathbf{U}$ & & 5 & $\cdot$ & NS & $\mu g / L$ \\
\hline 1,1,2-Trichloroethane & WA & & & $\mathbf{U}$ & & 5 & & 5 & $\mu g / L$ \\
\hline 1,1-Dichloroethane & WA & & & $\mathbf{U}$ & & 5 & & NS & $\mu g / L$ \\
\hline 1,1-Dichloroethylene & WA & & & $\mathbf{U}$ & & 5 & & 7 & $\mu g / L$ \\
\hline 1,2,3-Trichloropropane & WA & & & $\mathbf{U}$ & & 10 & & NS & $\mu g / L$ \\
\hline 1,2-Dibromo-3-chloropropane & WA & & & $\mathbf{U}$ & & 20 & & 0.2 & $\mu g / L$ \\
\hline 1,2-Dibromoethane & WA & & & $\mathbf{U}$ & & 20 & & NS & $\mu g / L$ \\
\hline 1,2-Dichloroethane & WA & & & $\mathbf{U}$ & & 5 & & 5 & $\mu g / L$ \\
\hline 1,2-Dichloroethylene (total) & WA & & & $\mathbf{U}$ & & 5 & & 7 & $\mu g / L$ \\
\hline 1,2-Dichloropropane & WA & & & $\mathbf{U}$ & & 5 & & 5 & $\mu g / L$ \\
\hline 2-Hexanone & WA & & & $\mathbf{U}$ & & 10 & & NS & $\mu g / L$ \\
\hline 4-Methyl-2-pentanone & WA & & & $\mathbf{U}$ & & 10 & & NS & $\mu g / L$ \\
\hline Acetone & WA & & & $\mathbf{J}$ & V & 5.84 & & NS & $\mu g / L$ \\
\hline Acetonitrile & WA & & & $\mathbf{U}$ & & 20 & & NS & $\mu g / L$ \\
\hline Acrolein & WA & & & $\mathbf{U}$ & & 10 & & NS & $\mu g / L$ \\
\hline Acrylonitrile & WA & & & $\mathbf{U}$ & & 10 & & NS & $\mu g / L$ \\
\hline Allyl chloride & WA & & & $\mathbf{U}$ & & 100 & & NS & $\mu g / L$ \\
\hline Benzene & WA & & & $\mathbf{U}$ & & 5 & & 5 & $\mu \mathrm{g} / \mathrm{L}$ \\
\hline Bromodichloromethane & WA & & & $\mathbf{U}$ & & 5 & & NS & $\mu g / L$ \\
\hline Bromoform & WA & & & $\mathbf{U}$ & & 5 & & NS & $\mu g / L$ \\
\hline Carbon disulfide & WA & & & $\mathbf{U}$ & & 5 & & NS & $\mu g / L$ \\
\hline Carbon tetrachloride & WA & & & $\mathbf{U}$ & & 5 & & 5 & $\mu g / L$ \\
\hline Chlorobenzene & WA & & & $\mathbf{U}$ & & 5 & & 100 & $\mu g / L$ \\
\hline Chloroethane & WA & & & $\mathbf{U}$ & & 10 & & NS & $\mu g / L$ \\
\hline Chloroform & WA & & & $\mathbf{U}$ & & 5 & & 100 & $\mu g / L$ \\
\hline Chloroprene & WA & & & $\mathbf{U}$ & & 20 & & NS & $\mu g / L$ \\
\hline cis-1,3-Dichloropropene & WA & & & $\mathbf{U}$ & & 5 & & NS & $\mu g / L$ \\
\hline Dibromochloromethane & WA & & & $\mathbf{U}$ & & 5 & & NS & $\mu g / L$ \\
\hline Dichlorodifluoromethane & WA & & & $U$ & & 10 & & NS & $\mu \mathrm{g} / \mathrm{L}$ \\
\hline Ethylbenzene & WA & & & $U$ & & 5 & & 700 & $\mu g / L$ \\
\hline Isobutyl alcohol & WA & & & $\mathbf{U}$ & & 20 & & NS & $\mu g / L$ \\
\hline Methacrylonitrile & WA & & & $\mathbf{U}$ & & 20 & & NS & $\mu \mathrm{g} / \mathrm{L}$ \\
\hline Methyl bromide & WA & & & $\mathbf{U}$ & & 10 & & NS & $\mu \mathrm{g} / \mathrm{L}$ \\
\hline Methyl chloride & WA & & & $\mathbf{U}$ & & 10 & & NS & $\mu g / L$ \\
\hline Methyl ethyl ketone & WA & & & $\mathbf{U}$ & & 10 & & NS & $\mu \mathrm{g} / \mathrm{L}$ \\
\hline Methyl iodide & WA & & & U & & 10 & & NS & $\mu g / L$ \\
\hline Methylene bromide & WA & & & U & & 10 & & NS & $\mu \mathrm{g} / \mathrm{L}$ \\
\hline Methylene chloride & WA & & & & V & 8.47 & & 5 & $\mu \mathrm{g} / \mathrm{L}$ \\
\hline Propionitrile & WA & & & $\mathbf{U}$ & & 50 & & NS & $\mu \mathrm{g} / \mathrm{L}$ \\
\hline Styrene & WA & & & $\mathbf{U}$ & & 5 & & 100 & $\mu g / L$ \\
\hline Tetrachloroethylene & WA & & & U & & 5 & & 5 & $\mu \mathrm{g} / \mathrm{L}$ \\
\hline Toluene & WA & & & $\mathbf{U}$ & & 5 & & 1000 & $\mu g / L$ \\
\hline trans-1,3-Dichloropropene & WA & & & $\mathbf{U}$ & & 5 & & NS & $\mu \mathrm{g} / \mathrm{L}$ \\
\hline trans-1,4-Dichloro-2-butene & WA & & & $\mathbf{U}$ & & 100 & & NS & $\mu \mathrm{g} / \mathrm{L}$ \\
\hline Trichloroethylene & WA & & & $\mathbf{U}$ & & 5 & & 5 & $\mu \mathrm{g} / \mathrm{L}$ \\
\hline Trichlorofluoromethane & WA & & & $\mathbf{U}$ & & 5 & & NS & $\mu g / L$ \\
\hline Vinyl acetate & WA & & & $\mathbf{U}$ & & 10 & & NS & $\mu \mathrm{g} / \mathrm{L}$ \\
\hline Vinyl chloride & WA & & & $\mathbf{U}$ & & 10 & & 2 & $\mu \mathrm{g} / \mathrm{L}$ \\
\hline Xylenes (total) & WA & & & $\mathbf{U}$ & & 5 & & 10000 & $\mu \mathrm{g} / \mathrm{L}$ \\
\hline
\end{tabular}


UVA013

(Volatile Trip Blank)

Sample Date: 9/2/93

Associated Station IDs: BGS003, UQA005, UQA010

\begin{tabular}{|c|c|c|c|c|c|c|c|c|c|}
\hline Analyte & Lab & Rep & Avg & Modifier & & Result & Acc & Stand & Units \\
\hline 1,1,1,2-Tetrachloroethane & $\overline{\text { WA }}$ & & & $\mathrm{U}$ & & 10 & & NS & $\overline{\mu g / L}$ \\
\hline 1,1,1-Trichloroethane & WA & & & $\mathbf{U}$ & & 5 & . & 200 & $\mu g / L$ \\
\hline 1,1,2,2-Tetrachloroethane & WA & & & $\mathbf{U}$ & & 5 & & NS & $\mu g / L$ \\
\hline 1,1,2-Trichloroethane & WA & & & $\mathbf{U}$ & & 5 & & 5 & $\mu \mathrm{g} / \mathrm{L}$ \\
\hline 1,1-Dichloroethane & WA & & & $\mathbf{U}$ & & 5 & & NS & $\mu g / L$ \\
\hline 1,1-Dichloroethylene & WA & & & $\mathbf{U}$ & & 5 & & 7 & $\mu g / L$ \\
\hline 1,2,3-Trichloropropane & WA & & & $\mathbf{U}$ & & 10 & & NS & $\mu g / L$ \\
\hline 1,2-Dibromo-3-chloropropane & WA & & & $\mathbf{U}$ & & 20 & & 0.2 & $\mu g / L$ \\
\hline 1,2-Dibromoethane & WA & & & $\mathbf{U}$ & & 20 & & NS & $\mu g / L$ \\
\hline 1,2-Dichloroethane & WA & & & $\mathbf{U}$ & & 5 & & 5 & $\mu g / L$ \\
\hline 1,2-Dichloroethylene (total) & WA & & & $\mathbf{U}$ & & 5 & & 7 & $\mu g / \Lambda$ \\
\hline 1,2-Dichloropropane & WA & & & $\mathbf{U}$ & & 5 & & 5 & $\mu g / L$ \\
\hline 2-Hexanone & WA & & & $\mathbf{U}$ & & 10 & & NS & $\mu \mathrm{g} / \mathrm{L}$ \\
\hline 4-Methyl-2-pentanone & WA & & & $\mathbf{U}$ & & 10 & & NS & $\mu g / L$ \\
\hline Acetone & WA & & & & $\mathbf{V}$ & 16.5 & & NS & $\mu g / L$ \\
\hline Acetonitrile & WA & & & $\mathbf{U}$ & & 20 & & NS & $\mu g / L$ \\
\hline Acrolein & WA & & & $\mathbf{U}$ & & 10 & & NS & $\mu g / L$ \\
\hline Acrylonitrile & WA & & & $\mathbf{U}$ & & 10 & & NS & $\mu g / L$ \\
\hline Allyl chloride & WA & & & $\mathbf{U}$ & & 100 & & NS & $\mu g / L$ \\
\hline Benzene & WA & & & $\mathbf{U}$ & & 5 & & 5 & $\mu g / L$ \\
\hline Bromodichloromethane & WA & & & $\mathbf{U}$ & & 5 & & NS & $\mu g / L$ \\
\hline Bromoform & WA & & & $\mathbf{U}$ & & 5 & & NS & $\mu g / L$ \\
\hline Carbon disulfide & WA & & & $\mathbf{U}$ & & 5 & & NS & $\mu g / L$ \\
\hline Carbon tetrachloride & WA & & & $\mathbf{U}$ & & 5 & & 5 & $\mu \mathrm{g} / \mathrm{L}$ \\
\hline Chlorobenzene & WA & & & $\mathbf{U}$ & & 5 & & 100 & $\mu \mathrm{g} / \mathrm{L}$ \\
\hline Chloroethane & WA & & & $\mathbf{U}$ & & 10 & & NS & $\mu g / L$ \\
\hline Chloroform & WA & & & $\mathbf{U}$ & & 5 & & 100 & $\mu g / L$ \\
\hline Chloroprene & WA & & & $\mathbf{U}$ & & 20 & & NS & $\mu g / L$ \\
\hline cis-1,3-Dichloropropene & WA & & & $\mathbf{U}$ & & 5 & & NS & $\mu \mathrm{g} / \mathrm{L}$ \\
\hline Dibromochloromethane & WA & & & $\mathbf{U}$ & & 5 & & NS & $\mu g / L$ \\
\hline Dichlorodifluoromethane & WA & & & $\mathrm{U}$ & & 10 & & NS & $\mu g / L$ \\
\hline Ethylbenzene & WA & & & $\mathbf{U}$ & & 5 & & 700 & $\mu g / L$ \\
\hline Isobutyl alcohol & WA & & & $\mathbf{U}$ & & 20 & & NS & $\mu g / L$ \\
\hline Methacrylonitrile & WA & & & $\mathrm{U}$ & & 20 & & NS & $\mu g / L$ \\
\hline Methyl bromide & WA & & & $\mathrm{U}$ & & 10 & & NS & $\mu \mathrm{g} / \mathrm{L}$ \\
\hline Methyl chloride & WA & & & $\mathbf{U}$ & & 10 & & NS & $\mu g / L$ \\
\hline Methyl ethyl ketone & WA & & & $\mathbf{U}$ & & 10 & & NS & $\mu g / L$ \\
\hline Methyl iodide & WA & & & $\mathbf{U}$ & & 10 & & NS & $\mu g / L$ \\
\hline Methylene bromide & WA & & & $\mathrm{U}$ & & 10 & & NS & $\mu g / L$ \\
\hline Methylene chloride & WA & & & & V & 8.56 & & 5 & $\mu \mathrm{g} / \mathrm{L}$ \\
\hline Propionitrile & WA & & & $\mathbf{U}$ & & 50 & & NS & $\mu g / L$ \\
\hline Styrene & WA & & & $\mathbf{U}$ & & 5 & & 100 & $\mu g / L$ \\
\hline Tetrachloroethylene & WA & & & $\mathrm{U}$ & & 5 & & 5 & $\mu g / L$ \\
\hline Toluene & WA & & & $\mathrm{U}$ & & 5 & & 1000 & $\mu \mathrm{g} / \mathrm{L}$ \\
\hline trans-1,3-Dichloropropene & WA & & & $\mathrm{U}$ & & 5 & & NS & $\mu g / L$ \\
\hline trans-1,4-Dichloro-2-butene & WA & & & $\mathrm{U}$ & & 100 & & NS & $\mu g / L$ \\
\hline Trichloroethylene & WA & & & $\mathbf{U}$ & & 5 & & 5 & $\mu \mathrm{g} / \mathrm{L}$ \\
\hline Trichlorofluoromethane & WA & & & $U$ & & 5 & & NS & $\mu \mathrm{g} / \mathrm{L}$ \\
\hline Vinyl acetate & WA & & & $\mathrm{U}$ & & 10 & & NS & $\mu g / L$ \\
\hline Vinyl chloride & WA & & & $\mathrm{U}$ & & 10 & & 2 & $\mu \mathrm{g} / \mathrm{L}$ \\
\hline Xylenes (total) & WA & & & $\mathrm{U}$ & & 5 & & 10000 & $\mu \mathrm{g} / \mathrm{L}$ \\
\hline
\end{tabular}


UVA014

(Volatile Trip Blank)

Sample Date: 9/2/93

Associated Station IDs: UTD001, UTD002, UTD003

\begin{tabular}{|c|c|c|c|c|c|c|c|}
\hline Analyte & Lab & Avg & Modifier & Result & Acc & Stand & Units \\
\hline 1,1,1,2-Tetrachloroethane & WA & & $\mathbf{U}$ & 10 & & NS & $\mu g / L$ \\
\hline 1,1,1-Trichloroethane & WA & & $\mathbf{U}$ & 5 & & 200 & $\mu g / L$ \\
\hline 1,1,2,2-Tetrachloroethane & WA & & $\mathbf{U}$ & 5 & $\cdot$ & NS & $\mu g / L$ \\
\hline 1,1,2-Trichloroethane & WA & & $\mathbf{U}$ & 5 & & 5 & $\mu g / L$ \\
\hline 1,1-Dichloroethane & WA & & $\mathbf{U}$ & 5 & & NS & $\mu g / L$ \\
\hline 1,1-Dichloroethylene & WA & & $\mathbf{U}$ & 5 & & 7 & $\mu g / L$ \\
\hline 1,2,3-Trichloropropane & WA & & $\mathbf{U}$ & 10 & & NS & $\mu g / L$ \\
\hline 1,2-Dibromo-3-chloropropane & WA & & $\mathbf{U}$ & 20 & & 0.2 & $\mu g / L$ \\
\hline 1,2-Dibromoethane & WA & & $\mathbf{U}$ & 20 & & NS & $\mu g / L$ \\
\hline 1,2-Dichloroethane & WA & & $\mathbf{U}$ & 5 & & 5 & $\mu g / L$ \\
\hline 1,2-Dichloroethylene (total) & WA & & $\mathbf{U}$ & 5 & & 7 & $\mu g / L$ \\
\hline 1,2-Dichloropropane & WA & & $\mathbf{U}$ & 5 & & 5 & $\mu g / L$ \\
\hline 2-Hexanone & WA & & $\mathbf{U}$ & 10 & & NS & $\mu g / L$ \\
\hline 4-Methyl-2-pentanone & WA & & $\mathbf{U}$ & 10 & & NS & $\mu g / L$ \\
\hline Acetone & WA & & $\mathbf{V}$ & 12 & & NS & $\mu g / L$ \\
\hline Acetonitrile & WA & & $\mathbf{U}$ & 20 & & NS & $\mu g / L$ \\
\hline Acrolein & WA & & $\mathbf{U}$ & 10 & & NS & $\mu g / L$ \\
\hline Acrylonitrile & WA & & $\mathbf{U}$ & 10 & & NS & $\mu g / L$ \\
\hline Allyl chloride & WA & & $\mathbf{U}$ & 100 & & NS & $\mu g / L$ \\
\hline Benzene & WA & & U & 5 & & 5 & $\mu \mathrm{g} / \mathrm{L}$ \\
\hline Bromodichloromethane & WA & & $\mathbf{U}$ & 5 & & NS & $\mu g / L$ \\
\hline Bromoform & WA & & $\mathbf{U}$ & 5 & & NS & $\mu g / L$ \\
\hline Carbon disulfide & WA & & $\mathbf{U}$ & 5 & & NS & $\mu g / L$ \\
\hline Carbon tetrachloride & WA & & $\mathbf{U}$ & 5 & & 5 & $\mu g / L$ \\
\hline Chlorobenzene & WA & & $\mathbf{U}$ & 5 & & 100 & $\mu g / L$ \\
\hline Chloroethane & WA & & $\mathbf{U}$ & 10 & & NS & $\mu g / L$ \\
\hline Chloroform & WA & & $\mathbf{U}$ & 5 & & 100 & $\mu g / L$ \\
\hline Chloroprene & WA & & $\mathbf{U}$ & 20 & & NS & $\mu g / L$ \\
\hline cis-1,3-Dichloropropene & WA & & $\mathbf{U}$ & 5 & & NS & $\mu g / L$ \\
\hline Dibromochloromethane & WA & & $\mathbf{U}$ & 5 & & NS & $\mu g / L$ \\
\hline Dichlorodifluoromethane & WA & & $\mathbf{U}$ & 10 & & NS & $\mu g / L$ \\
\hline Ethylbenzene & WA & & U & 5 & & 700 & $\mu g / L$ \\
\hline Isobutyl alcohol & WA & & $\mathbf{U}$ & 20 & & NS & $\mu g / L$ \\
\hline Methacrylonitrile & WA & & $\mathbf{U}$ & 20 & & NS & $\mu \mathrm{g} / \mathrm{L}$ \\
\hline Methyl bromide & WA & & $\mathbf{U}$ & 10 & & NS & $\mu g / L$ \\
\hline Methyl chloride & WA & & U & 10 & & NS & $\mu g / L$ \\
\hline Methyl ethyl ketone & WA & & $\mathbf{U}$ & 10 & & NS & $\mu g / L$ \\
\hline Methyl iodide & WA & & $\mathbf{U}$ & 10 & & NS & $\mu g / L$ \\
\hline Methylene bromide & WA & & $\mathbf{U}$ & 10 & & NS & $\mu g / L$ \\
\hline Methylene chloride & WA & & V & 8.6 & & 5 & $\mu g / L$ \\
\hline Propionitrile & WA & & $\mathbf{U}$ & 50 & & NS & $\mu g / L$ \\
\hline Styrene & WA & & 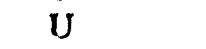 & 5 & & 100 & $\mu \mathrm{g} / \mathrm{L}$ \\
\hline Tetrachloroethylene & WA & & $\mathbf{U}$ & 5 & & 5 & $\mu g / L$ \\
\hline Toluene & WA & & $\mathbf{U}$ & 5 & & 1000 & $\mu \mathrm{g} / \mathrm{L}$ \\
\hline trans-1,3-Dichloropropene & WA & & $\mathbf{U}$ & 5 & & NS & $\mu \mathrm{g} / \mathrm{L}$ \\
\hline trans-1,4-Dichloro-2-butene & WA & & $\mathbf{U}$ & 100 & & NS & $\mu \mathrm{g} / \mathrm{L}$ \\
\hline Trichloroethylene & WA & & $\mathbf{U}$ & 5 & & 5 & $\mu \mathrm{g} / \mathrm{L}$ \\
\hline Trichlorofluoromethane & WA & & $\mathbf{U}$ & 5 & & NS & $\mu \mathrm{g} / \mathrm{L}$ \\
\hline Vinyl acetate & WA & & $\mathbf{U}$ & 10 & & NS & $\mu g / L$ \\
\hline Vinyl chloride & WA & & $\mathbf{U}$ & 10 & & 2 & $\mu g / L$ \\
\hline Xylenes (total) & WA & & $\mathbf{U}$ & 5 & & 10000 & $\mu g / L$ \\
\hline
\end{tabular}


UVA015

(Volatile Trip Blank)

Sample Date: 9/2/93

Associated Station IDs: UTD004, UTD005, UTD006

\begin{tabular}{|c|c|c|c|c|c|c|c|}
\hline Analyte & Lab & Avg & Modifier & Result & Acc & Stand & Units \\
\hline 1,1,1,2-Tetrachloroethane & WA & & $\bar{U}$ & 10 & & NS & $\mu g / L$ \\
\hline 1,1,1-Trichloroethane & WA & & $\mathbf{U}$ & 5 & . & 200 & $\mu \mathrm{g} / \mathrm{L}$ \\
\hline 1,1,2,2-Tetrachloroethane & WA & & $\mathbf{U}$ & 5 & & NS & $\mu g / L$ \\
\hline 1,1,2-Trichloroethane & WA & & $\mathbf{U}$ & 5 & & 5 & $\mu g / L$ \\
\hline 1,1-Dichloroethane & WA & & $\mathbf{U}$ & 5 & & NS & $\mu g / L$ \\
\hline 1,1-Dichloroethylene & WA & & $\mathbf{U}$ & 5 & & 7 & $\mu g / L$ \\
\hline 1,2,3-Trichloropropane & WA & & $\mathbf{U}$ & 10 & & NS & $\mu g / L$ \\
\hline 1,2-Dibromo-3-chloropropane & WA & & $\mathbf{U}$ & 20 & & 0.2 & $\mu g / L$ \\
\hline 1,2-Dibromoethane & WA & & $\mathbf{U}$ & 20 & & NS & $\mu g / L$ \\
\hline 1,2-Dichloroethane & WA & & $\mathbf{U}$ & 5 & & 5 & $\mu g / L$ \\
\hline 1,2-Dichloroethylene (total) & WA & & $\mathbf{U}$ & 5 & & 7 & $\mu g / L$ \\
\hline 1,2-Dichloropropane & WA & & $\mathbf{U}$ & 5 & & 5 & $\mu g / L$ \\
\hline 2-Hexanone & WA & & $\mathbf{U}$ & 10 & & NS & $\mu g / L$ \\
\hline 4-Methyl-2-pentanone & WA & & $\mathbf{U}$ & 10 & & NS & $\mu g / L$ \\
\hline Acetone & WA & & V & 15.5 & & NS & $\mu g / L$ \\
\hline Acetonitrile & WA & & $\mathbf{U}$ & 20 & & NS & $\mu g / L$ \\
\hline Acrolein & WA & & $\mathbf{U}$ & 10 & & NS & $\mu g / L$ \\
\hline Acrylonitrile & WA & & $\mathbf{U}$ & 10 & & NS & $\mu g / L$ \\
\hline Allyl chloride & WA & & $\mathbf{U}$ & 100 & & NS & $\mu \mathrm{g} / \mathrm{L}$ \\
\hline Benzene & WA & & $\mathbf{U}$ & 5 & & 5 & $\mu \mathrm{g} / \mathrm{L}$ \\
\hline Bromodichloromethane & WA & & $\mathbf{U}$ & 5 & & NS & $\mu g / L$ \\
\hline Bromoform & WA & & $\mathbf{U}$ & 5 & & NS & $\mu g / L$ \\
\hline Carbon disulfide & WA & & $\mathbf{U}$ & 5 & & NS & $\mu g / L$ \\
\hline Carbon tetrachloride & WA & & $\mathbf{U}$ & 5 & & 5 & $\mu g / L$ \\
\hline Chlorobenzene & WA & & $\mathbf{U}$ & 5 & & 100 & $\mu \mathrm{g} / \mathrm{L}$ \\
\hline Chloroethane & WA & & $\mathbf{U}$ & 10 & & NS & $\mu g / L$ \\
\hline Chloroform & WA & & $\mathbf{U}$ & 5 & & 100 & $\mu g / L$ \\
\hline Chloroprene & WA & & $\mathbf{U}$ & 20 & & NS & $\mu g / L$ \\
\hline cis-1,3-Dichloropropene & WA & & $\mathbf{U}$ & 5 & & NS & $\mu g / L$ \\
\hline Dibromochloromethane & WA & & $\mathbf{U}$ & 5 & & NS & $\mu g / L$ \\
\hline Dichlorodifluoromethane & WA & & $\mathbf{U}$ & 10 & & NS & $\mu \mathrm{g} / \mathrm{L}$ \\
\hline Ethylbenzene & WA & & $\mathbf{U}$ & 5 & & 700 & $\mu g / L$ \\
\hline Isobutyl alcohol & WA & & $\mathbf{U}$ & 20 & & NS & $\mu g / L$ \\
\hline Methacrylonitrile & WA & & $\mathbf{U}$ & 20 & & NS & $\mu g / L$ \\
\hline Methyl bromide & WA & & $\mathbf{U}$ & 10 & & NS & $\mu g / L$ \\
\hline Methyl chloride & WA & & $\mathbf{U}$ & 10 & & NS & $\mu \mathrm{g} / \mathrm{L}$ \\
\hline Methyl ethyl ketone & WA & & $\mathbf{U}$ & 10 & & NS & $\mu g / L$ \\
\hline Methyl iodide & WA & & $\mathrm{U}$ & 10 & & NS & $\mu g / L$ \\
\hline Methylene bromide & WA & & $\mathbf{U}$ & 10 & & NS & $\mu g / L$ \\
\hline Methylene chloride & WA & & V & 8.53 & & 5 & $\mu g / L$ \\
\hline Propionitrile & WA & & $\mathbf{U}$ & 50 & & NS & $\mu \mathrm{g} / \mathrm{L}$ \\
\hline Styrene & WA & & $\mathbf{U}$ & 5 & & 100 & $\mu g / L$ \\
\hline Tetrachloroethylene & WA & & $\mathbf{U}$ & 5 & & 5 & $\mu g / L$ \\
\hline Toluene & WA & & $\mathbf{U}$ & 5 & & 1000 & $\mu \mathrm{g} / \mathrm{L}$ \\
\hline trans-1,3-Dichloropropene & WA & & $\mathbf{U}$ & 5 & & NS & $\mu \mathrm{g} / \mathrm{L}$ \\
\hline trans-1,4-Dichloro-2-butene & WA & & $\mathbf{U}$ & 100 & & NS & $\mu \mathrm{g} / \mathrm{L}$ \\
\hline Trichloroethylene & WA & & $\mathbf{U}$ & 5 & & 5 & $\mu \mathrm{g} / \mathrm{L}$ \\
\hline Trichlorofluoromethane & WA & & $\mathbf{U}$ & 5 & & NS & $\mu g / L$ \\
\hline Vinyl acetate & WA & & $\mathbf{U}$ & 10 & & NS & $\mu \mathrm{g} / \mathrm{L}$ \\
\hline Vinyl chloride & WA & & $\mathbf{U}$ & 10 & & 2 & $\mu \mathrm{g} / \mathrm{L}$ \\
\hline Xylenes (total) & WA & & $\mathbf{U}$ & 5 & & 10000 & $\mu \mathrm{g} / \mathrm{L}$ \\
\hline
\end{tabular}




\section{UVA016}

(Volatile Trip Blank)

Sample Date: 9/2/93

Associated Station IDs: UTR120, UTR122, UTR127

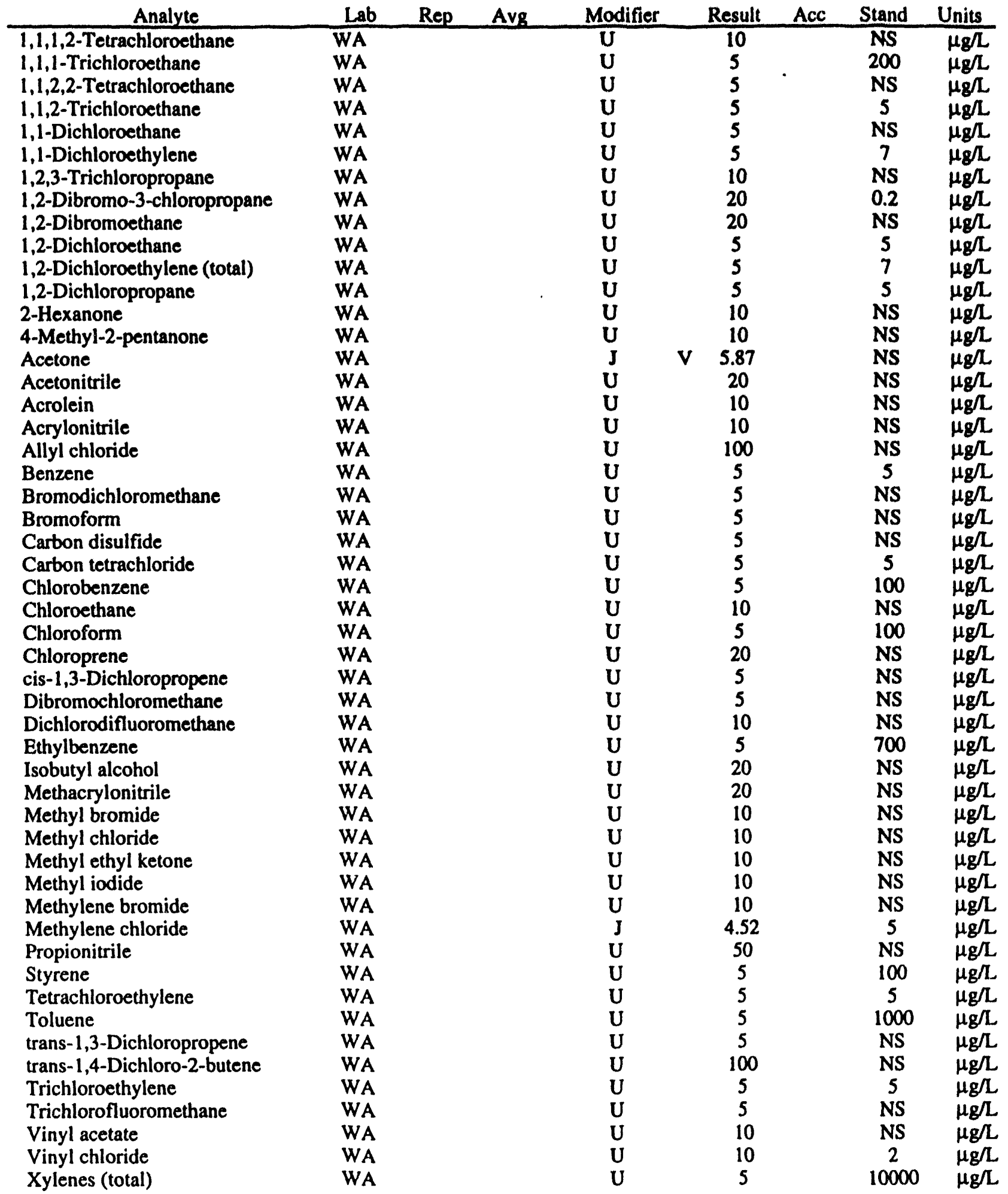


UVA017

(Volatile Trip Blank)

Sample Date: $9 / 3 / 93$

Associated Station IDs: UTR133, UTR 147, UTR155

\begin{tabular}{|c|c|c|c|c|c|c|c|c|}
\hline Analyte & Lab & Rep & Avg & Modifier & Result & Acc & Stand & Units \\
\hline 1,1,1,2-Tetrachloroethane & WA & & & $\mathrm{U}$ & 10 & & NS & $\mu g / L$ \\
\hline 1,1,1-Trichloroethane & WA & & & $\mathbf{U}$ & 5 & . & 200 & $\mu g / L$ \\
\hline 1,1,2,2-Tetrachloroethane & WA & & & $\mathbf{U}$ & 5 & & NS & $\mu \mathrm{g} / \mathrm{L}$ \\
\hline 1,1,2-Trichloroethane & WA & & & $\mathbf{U}$ & 5 & & 5 & $\mu g / L$ \\
\hline 1,1-Dichloroethane & WA & & & $\mathbf{U}$ & 5 & & NS & $\mu g / L$ \\
\hline 1,1-Dichloroethylene & WA & & & $\mathbf{U}$ & 5 & & 7 & $\mu \mathrm{g} / \mathrm{L}$ \\
\hline 1,2,3-Trichloropropane & WA & & & $\mathbf{U}$ & 10 & & NS & $\mu \mathrm{g} / \mathrm{L}$ \\
\hline 1,2-Dibromo-3-chloropropane & WA & & & $\mathbf{U}$ & 20 & & 0.2 & $\mu \mathrm{g} / \mathrm{L}$ \\
\hline 1,2-Dibromoethane & WA & & & $\mathbf{U}$ & 20 & & NS & $\mu g / L$ \\
\hline 1,2-Dichloroethane & WA & & & $\mathbf{U}$ & 5 & & 5 & $\mu g / L$ \\
\hline 1,2-Dichloroethylene (total) & WA & & & $\mathbf{U}$ & 5 & & 7 & $\mu g / L$ \\
\hline 1,2-Dichloropropane & WA & & & $\mathbf{U}$ & 5 & & 5 & $\mu g / L$ \\
\hline 2-Hexanone & WA & & & $\mathbf{U}$ & 10 & & NS & $\mu g / L$ \\
\hline 4-Methyl-2-pentanone & WA & & & $\mathbf{U}$ & 10 & & NS & $\mu g / L$ \\
\hline Acetone & WA & & & $\mathbf{J}$ & $\mathbf{V}$ & & NS & $\mu g / L$ \\
\hline Acetonitrile & WA & & & $\mathbf{U}$ & 20 & & NS & $\mu g / L$ \\
\hline Acrolein & WA & & & $\mathbf{U}$ & 10 & & NS & $\mu g / L$ \\
\hline Acrylonitrile & WA & & & $\mathbf{U}$ & 10 & & NS & $\mu g / L$ \\
\hline Allyl chloride & WA & & & $\mathbf{U}$ & 100 & & NS & $\mu g / L$ \\
\hline Benzene & WA & & & $\mathbf{U}$ & 5 & & 5 & $\mu g / L$ \\
\hline Bromodichloromethane & WA & & & $\mathbf{U}$ & 5 & & NS & $\mu g / L$ \\
\hline Bromoform & WA & & & $\mathbf{U}$ & 5 & & NS & $\mu g / L$ \\
\hline Carbon disulfide & WA & & & $\mathbf{U}$ & 5 & & NS & $\mu g / L$ \\
\hline Carbon tetrachloride & WA & & & $\mathbf{U}$ & 5 & & 5 & $\mu g / L$ \\
\hline Chlorobenzene & WA & & & $\mathbf{U}$ & 5 & & 100 & $\mu g / L$ \\
\hline Chloroethane & WA & & & $\mathbf{U}$ & 10 & & NS & $\mu \mathrm{g} / \mathrm{L}$ \\
\hline Chloroform & WA & & & $\mathbf{U}$ & 5 & & 100 & $\mu \mathrm{g} / \mathrm{L}$ \\
\hline Chloroprene & WA & & & $\mathbf{U}$ & 20 & & NS & $\mu g / L$ \\
\hline cis-1,3-Dichloropropene & WA & & & $\mathbf{U}$ & 5 & & NS & $\mu g / L$ \\
\hline Dibromochloromethane & WA & & & $\mathbf{U}$ & 5 & & NS & $\mu g / L$ \\
\hline Dichlorodifluoromethane & WA & & & $\mathbf{U}$ & 10 & & NS & $\mu g / L$ \\
\hline Ethylbenzene & WA & & & $\mathrm{U}$ & 5 & & 700 & $\mu \mathrm{g} / \mathrm{L}$ \\
\hline Isobutyl alcohol & WA & & & U & 20 & & NS & $\mu \mathrm{g} / \mathrm{L}$ \\
\hline Methacrylonitrile & WA & & & U & 20 & & NS & $\mu g / L$ \\
\hline Methyl bromide & WA & & & $\mathbf{U}$ & 10 & & NS & $\mu g / L$ \\
\hline Methyl chloride & WA & & & $\mathbf{U}$ & 10 & & NS & $\mu g / L$ \\
\hline Methyl ethyl ketone & WA & & & $\mathbf{U}$ & 10 & & NS & $\mu \mathrm{g} / \mathrm{L}$ \\
\hline Methyl iodide & WA & & & $\mathbf{U}$ & 10 & & NS & $\mu g / L$ \\
\hline Methylene bromide & WA & & & $\mathbf{U}$ & 10 & & NS & $\mu \mathrm{g} / \mathrm{L}$ \\
\hline Methylene chloride & WA & & & & 5.09 & & 5 & $\mu g / L$ \\
\hline Propionitrile & WA & & & $\mathbf{U}$ & 50 & & NS & $\mu \mathrm{g} / \mathrm{L}$ \\
\hline Styrene & WA & & & $\mathbf{U}$ & 5 & & 100 & $\mu g / L$ \\
\hline Tetrachloroethylene & WA & & & $\mathbf{U}$ & 5 & & 5 & $\mu \mathrm{g} / \mathrm{L}$ \\
\hline Toluene & WA & & & $\mathbf{U}$ & 5 & & 1000 & $\mu \mathrm{g} / \mathrm{L}$ \\
\hline trans-1,3-Dichloropropene & WA & & & $\mathbf{U}$ & 5 & & NS & $\mu \mathrm{g} / \mathrm{L}$ \\
\hline trans-1,4-Dichloro-2-butene & WA & & & $\mathbf{U}$ & 100 & & NS & $\mu \mathrm{g} / \mathrm{L}$ \\
\hline Trichloroethylene & WA & & & $\mathrm{U}$ & 5 & & 5 & $\mu g / L$ \\
\hline Trichlorofluoromethane & WA & & & $\mathbf{U}$ & 5 & & NS & $\mu \mathrm{g} / \mathrm{L}$ \\
\hline Vinyl acetate & WA & & & $\mathbf{U}$ & 10 & & NS & $\mu_{g} / L$ \\
\hline Vinyl chloride & WA & & & $\mathbf{U}$ & 10 & & 2 & $\mu \mathrm{g} / \mathrm{L}$ \\
\hline Xylenes (total) & WA & & & $\mathbf{U}$ & 5 & & 10000 & $\mu \mathrm{g} / \mathrm{L}$ \\
\hline
\end{tabular}


UVA918

(Vclatile Trip Blank)

Sample Date: 9/3/93

Associated Station IDs: UQA009, UQA011

\begin{tabular}{|c|c|c|c|c|c|c|c|c|c|}
\hline Analyte & Lab & Rep & Avg & Modifier & & Result & Acc & Stand & Units \\
\hline 1,1,1,2-Tetrachloroethane & WA & & & $\bar{U}$ & & 10 & & NS & $\mu g / L$ \\
\hline 1,1,1-Trichloroethane & WA & & & $\mathbf{U}$ & & 5 & & 200 & $\mu g / L$ \\
\hline $1,1,2,2$-Tetrachloroethane & WA & & & $\mathbf{U}$ & & 5 & & NS & $\mu g / L$ \\
\hline 1,1,2-Trichloroethane & WA & & & $\mathbf{U}$ & & 5 & & 5 & $\mu g / L$ \\
\hline 1,1-Dichloroethane & WA & & & $\mathbf{U}$ & & 5 & & NS & $\mu g / L$ \\
\hline 1,1-Dichloroethylene & WA & & & $\mathbf{U}$ & & 5 & & 7 & $\mu g / L$ \\
\hline 1,2,3-Trichloropropane & WA & & & $\mathbf{U}$ & & 10 & & NS & $\mu g / L$ \\
\hline 1,2-Dibromo-3-chloropropane & WA & & & $\mathbf{U}$ & & 20 & & 0.2 & $\mu \mathrm{g} / \mathrm{L}$ \\
\hline 1,2-Dibromoethane & WA & & & $\mathbf{U}$ & & 20 & & NS & $\mu g / L$ \\
\hline 1,2-Dichloroethane & WA & & & $\mathbf{U}$ & & 5 & & 5 & $\mu \mathrm{g} / \mathrm{L}$ \\
\hline 1,2-Dichloroethylene (total) & WA & & & $\mathbf{U}$ & & 5 & & 7 & $\mu g / L$ \\
\hline 1,2-Dichloropropane & WA & & & $\mathbf{U}$ & & 5 & & 5 & $\mu g / L$ \\
\hline 2-Hexanone & WA & & & $\mathbf{U}$ & & 10 & & NS & $\mu g / L$ \\
\hline 4-Methyl-2-pentanone & WA & & & $\mathbf{U}$ & & 10 & & NS & $\mu g / L$ \\
\hline Acetone & WA & & & $\mathbf{J}$ & V & 7.11 & & NS & $\mu g / L$ \\
\hline Acetonitrile & WA & & & $\mathbf{U}$ & & 20 & & NS & $\mu g / L$ \\
\hline Acrolein & WA & & & $\mathbf{U}$ & & 10 & & NS & $\mu \mathrm{g} / \mathrm{L}$ \\
\hline Acrylonitrile & WA & & & $\mathbf{U}$ & & 10 & & NS & $\mu \mathrm{g} / \mathrm{L}$ \\
\hline Allyl chloride & WA & & & $\mathbf{U}$ & & 100 & & NS & $\mu g / L$ \\
\hline Benzene & WA & & & $\mathbf{U}$ & & 5 & & 5 & $\mu \mathrm{g} / \mathrm{L}$ \\
\hline Bromodichloromethane & WA & & & $\mathbf{U}$ & & 5 & & NS & $\mu g / L$ \\
\hline Bromoform & WA & & & $\mathbf{U}$ & & 5 & & NS & $\mu g / L$ \\
\hline Carbon disulfide & WA & & & $\mathbf{U}$ & & 5 & & NS & $\mu g / L$ \\
\hline Carbon tetrachloride & WA & & & $\mathbf{U}$ & & 5 & & 5 & $\mu g / L$ \\
\hline Chlorobenzene & WA & & & $\mathbf{U}$ & & 5 & & 100 & $\mu g / L$ \\
\hline Chloroethane & WA & & & $\mathbf{U}$ & & 10 & & NS & $\mu g / L$ \\
\hline Chloroform & WA & & & $\mathbf{U}$ & & 5 & & 100 & $\mu g / L$ \\
\hline Chloroprene & WA & & & $\mathbf{U}$ & & 20 & & NS & $\mu g / L$ \\
\hline cis-1,3-Dichloropropene & WA & & & $\mathbf{U}$ & & 5 & & NS & $\mu g / L$ \\
\hline Dibromochloromethane & WA & & & $\mathbf{U}$ & & 5 & & NS & $\mu \mathrm{g} / \mathrm{L}$ \\
\hline Dichlorodifluoromethane & WA & & & $\mathbf{U}$ & & 10 & & NS & $\mu g / L$ \\
\hline Ethylbenzene & WA & & & $\mathrm{U}$ & & 5 & & 700 & $\mu g / L$ \\
\hline Isobutyl alcohol & WA & & & $\mathbf{U}$ & & 20 & & NS & $\mu \mathrm{g} / \mathrm{L}$ \\
\hline Methacrylonitrile & WA & & & $\mathbf{U}$ & & 20 & & NS & $\mu g / L$ \\
\hline Methyl bromide & WA & & & $\mathrm{U}$ & & 10 & & NS & $\mu g / L$ \\
\hline Methyl chloride & WA & & & $\mathbf{U}$ & & 10 & & NS & $\mu g / L$ \\
\hline Methyl ethyl ketone & WA & & & $\mathbf{U}$ & & 10 & & NS & $\mu \mathrm{g} / \mathrm{L}$ \\
\hline Methyl iodide & WA & & & $\mathbf{U}$ & & 10 & & NS & $\mu \mathrm{g} / \mathrm{L}$ \\
\hline Methylene bromide & WA & & & $\mathbf{U}$ & & 10 & & NS & $\mu g / L$ \\
\hline Methylene chlaride & WA & & & & & 5.24 & & 5 & $\mu g / L$ \\
\hline Propionitrile & WA & & & $\mathrm{U}$ & & 50 & & NS & $\mu g / L$ \\
\hline Styrene & WA & & & $\mathbf{U}$ & & 5 & & 100 & $\mu \mathrm{g} / \mathrm{L}$ \\
\hline Tetrachloroethylene & WA & & & U & & 5 & & 5 & $\mu \mathrm{g} / \mathrm{L}$ \\
\hline Toluene & WA & & & $\mathbf{U}$ & & 5 & & 1000 & $\mu \mathrm{g} / \mathrm{L}$ \\
\hline trans-1,3-Dichloropropene & WA & & & U & & 5 & & NS & $\mu \mathrm{g} / \mathrm{L}$ \\
\hline trans-1,4-Dichloro-2-butene & WA & & & $\mathbf{U}$ & & 100 & & NS & $\mu \mathrm{g} / \mathrm{L}$ \\
\hline Trichloroethylene & WA & & & $\mathbf{U}$ & & 5 & & 5 & $\mu \mathrm{g} / \mathrm{L}$ \\
\hline Trichlorofluoromethane & WA. & & & $\mathrm{U}$ & & 5 & & NS & $\mu \mathrm{g} / \mathrm{L}$ \\
\hline Vinyl acetate & WA & & & $\mathrm{U}$ & & 10 & & NS & $\mu \mathrm{g} / \mathrm{L}$ \\
\hline Vinyl chloride & WA & & & $\mathbf{U}$ & & 10 & & 2 & $\mu \mathrm{g} / \mathrm{L}$ \\
\hline Xylenes (total) & WA & & & $\mathbf{U}$ & & 5 & & 10000 & $\mu \mathrm{g} / \mathrm{L}$ \\
\hline
\end{tabular}


UVA019

(Volatile Trip Blank)

Sample Date: 9/3/93

Associated Station IDs: FHB014, FHB015, FHB020

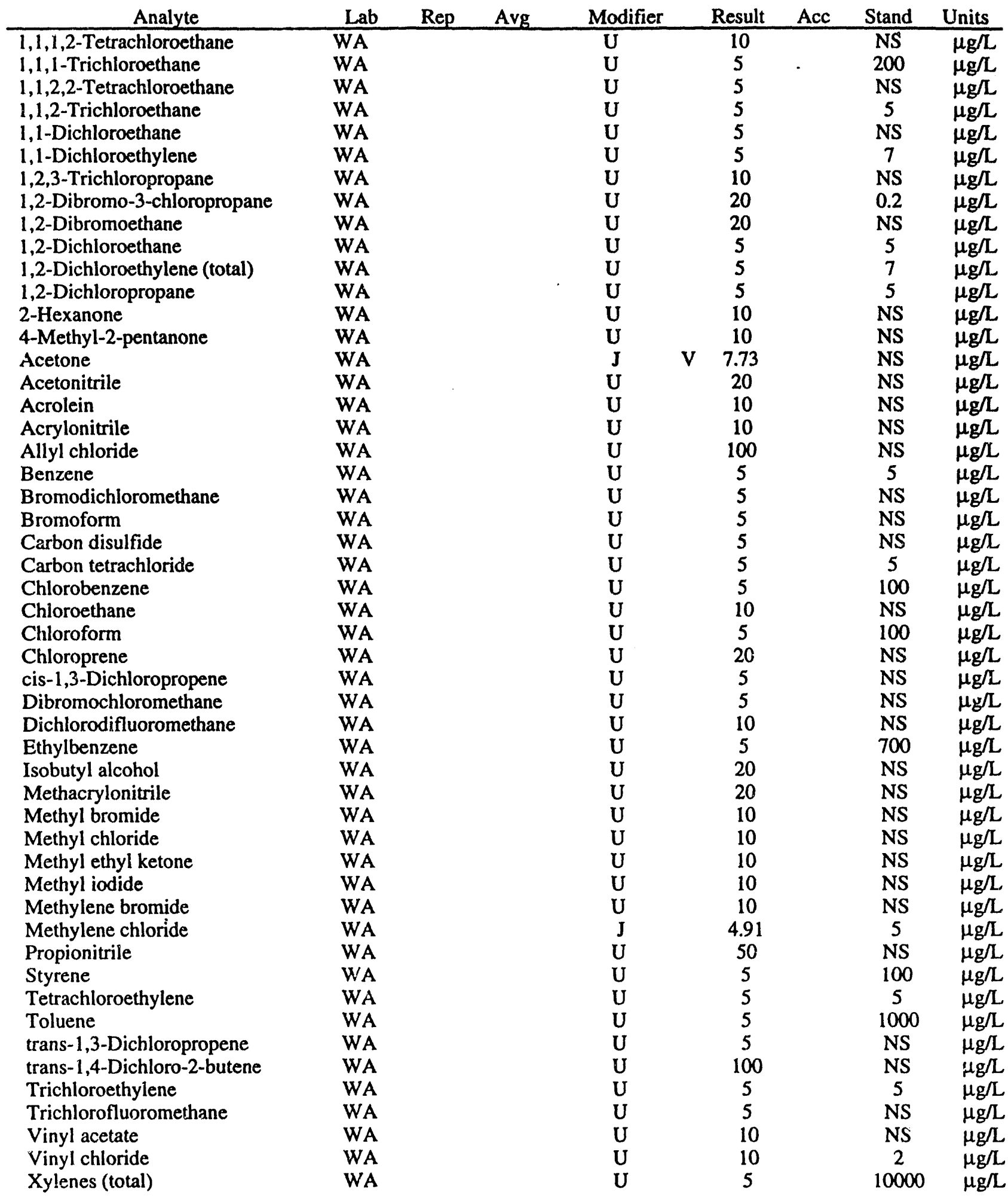


WSRC-TR-94-0277 


\section{Appendix $C$}

\section{Shipping Trip Blanks}


Shipping Trip Blanks And Their Associated Sampling Locations.

\begin{tabular}{|c|c|c|c|}
\hline $\begin{array}{l}\text { Shipping Trip } \\
\text { Blank ID }\end{array}$ & $\begin{array}{l}\text { Associated Sampling } \\
\text { Locations }\end{array}$ & $\begin{array}{l}\text { Shipping Trip } \\
\text { Blank ID }\end{array}$ & $\begin{array}{l}\text { Associated Sampling } \\
\text { Locations }\end{array}$ \\
\hline UVA001 & $\begin{array}{l}\text { UTR011 } \\
\text { UTR024 } \\
\text { UTR029 } \\
\text { UTR033 }\end{array}$ & UVA011 & $\begin{array}{l}\text { UTR071 } \\
\text { UTR072 } \\
\text { UTR099 } \\
\text { UTR102 }\end{array}$ \\
\hline UVA002 & $\begin{array}{l}\text { UTR036 } \\
\text { UTR042 } \\
\text { UTR045 }\end{array}$ & UVA012 & $\begin{array}{l}\text { BGD001 } \\
\text { BGD002 } \\
\text { BGS002 }\end{array}$ \\
\hline UVA003 & UQA006 & UVA013 & $\begin{array}{l}\text { BGS003 } \\
\text { UQA010 }\end{array}$ \\
\hline UVA004 & $\begin{array}{l}\text { UTR022 } \\
\text { UTR039 } \\
\text { UTR049 } \\
\text { UTR051A }\end{array}$ & UVA014 & $\begin{array}{l}\text { UTD001 } \\
\text { UTD002 } \\
\text { UTD003 }\end{array}$ \\
\hline UVA005 & $\begin{array}{l}\text { UTR053 } \\
\text { UTR056 } \\
\text { UTR060 } \\
\text { UQA007 }\end{array}$ & UVA015 & $\begin{array}{l}\text { UTD004 } \\
\text { UTD005 } \\
\text { UTD006 }\end{array}$ \\
\hline UVA006 & $\begin{array}{l}\text { UTR062 } \\
\text { UTR063 } \\
\text { UTR066 }\end{array}$ & UVA016 & $\begin{array}{l}\text { UTR120 } \\
\text { UTR122 } \\
\text { UTR127 }\end{array}$ \\
\hline UVA008 & UQA008 & UVA017 & $\begin{array}{l}\text { UTR133 } \\
\text { UTR147 } \\
\text { UTR155 }\end{array}$ \\
\hline UVA009 & $\begin{array}{l}\text { UTR } 112 \\
\text { UTR } 114 \\
\text { UTR116 }\end{array}$ & UVA018 & $\begin{array}{l}\text { UQA009 } \\
\text { UQA011 }\end{array}$ \\
\hline UVA010 & $\begin{array}{l}\text { UTR020 } \\
\text { UTR043 } \\
\text { UTR145 }\end{array}$ & & \\
\hline
\end{tabular}

${ }^{1}$ Shipping trip blanks were analyzed for volatile organics only. 
Appendix D

Information provided to ESS on Electroninc Data Diskettes 


\section{Information provided to ESS on Electroninc Data Diskettes}

\begin{tabular}{|lll|}
\hline Sample name & Laboratory sample number & Accuracy \\
Chain of custody number & Laboratory replicate & Number of dilutions \\
Sample date & Testname & Dilution factor \\
Received date & Extraction/digestion method & Instrument \\
Extraction date & Analysis method & Analyst \\
Extraction time & Detection limit & Nominal concentration \\
Analysis date & Result qualifier & \\
Analysis time & Analysis qualifier & \\
Analysis batch & Bias & \\
Laboratory code & Analytical result & \\
\hline
\end{tabular}



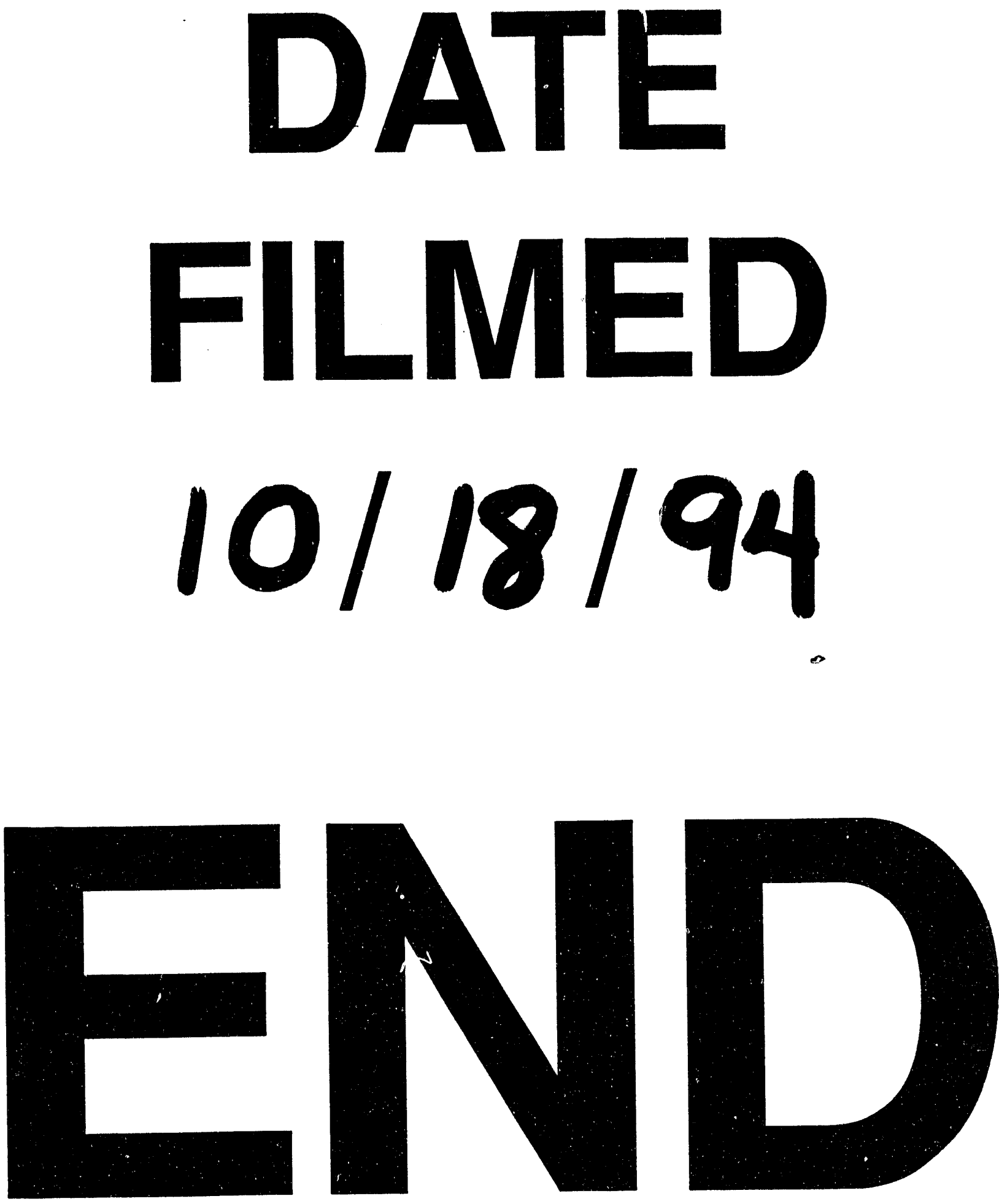
